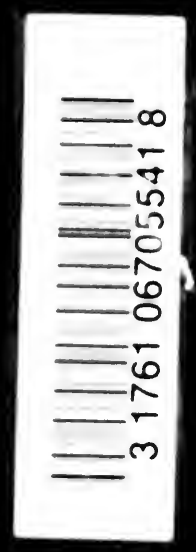




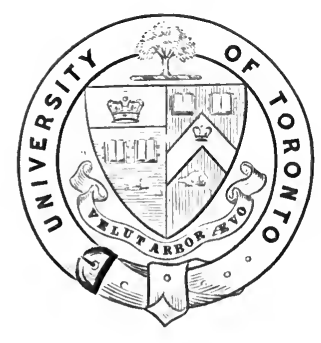

presented to

The $\mathfrak{I} i b r a r y$

of the

University of Toronto

by

nus. W.B. Handy 

Digitized by the Internet Archive in 2007 with funding from Microsoft Corporation 



\title{
TREATMENT
}

or

\section{INTERNAL DISEASES}

\section{FOR PHYSICIANS AND STUDENTS}

\author{
Br \\ DR. NORBERT ORTNER \\ OP THE UNIVERSTY OF VIExNA
}

IDITED or

NATHANIEL BOWDITCH POTTER, M.D.

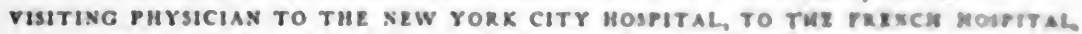
AND TO THE HOSPITAL FOK RUPTCKED AXD CRIPPLA I INITACTOE IN MEDICINE, COLUMBIA UXIVERSTY

TRANSLATED BY

FREDERIC H. BARTLETT, M.D.

FROM THE YOURTH GERMAV IOITION
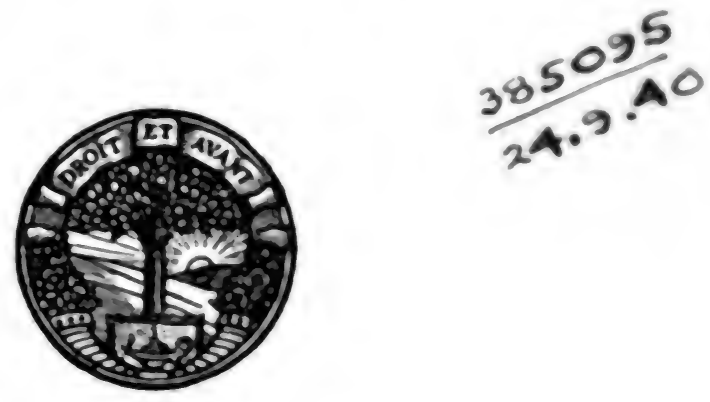

PHILADELPHIA \& LONDON

J. B. LIPPINCOT'T COMPANY 
Copyright, 1908

By J. B. Lippincott Company

ELECTROTYPED AND PRINTED BY J. B. LIPPINCOTT COMPANY, WASHINGTON SQUARE PRESS, PHILADELPHIA, U.S.A. 


\section{EDITOR'S PREFACE}

The popularity of the preceding three editions of Ortner's Lectures upon the Therapeuties of Internal Medicine is sufficient to justify the appearance of this English translation from the last, the fourth edition. The scope of the book is indicated in its title; prophylaxis is discussed in but few sections. The author initiates the reader into as much of the pathological physiology of the diseases discussed as bears upon their rational treatment. He very properly emphasizes throughout the volume the importance of the mechanical, dietetic, climatic, hydrotherapeutic, and other extra-medicinal methods, with judicious reasons for those selected, and follows these with a discussion of the applicability of certain drugs, their respective advantages, disadvantages, and limitations, adds useful prescriptions from his own experience and that of others, and leaves the reader better armed to meet etiological indications and the various contingencies which arise and require symptomatic treatment.

Dr. Bartlett has translated the German text into idiomatic English, and, without losing the spirit or the details of the original, he has materially condensed the work.

The prescriptions have been altered to conform to the American Pharmacopœia, and the equivalents in the English scale of measures have been added to the metric quantities. All temperatures have been transposed into the Fahrenheit scale. The references in climatology, hygiene, and dietetics have been adapted to the needs of the American student and practitioner. The medicinal waters recommended by the author have been tabulated, and to them have been added tables of the corresponding and most important American waters. Some of the lists of drugs have also been arranged in tables convenient for ready reference. Numerous American sanatoria for patients with tuberculosis have been mentioned. 
The sections upon membranous enteritis, diphtheria, and portions of a few others have been rewritten. Elsewhere I have allowed the original to stand unaltered but have added copious notes to suggest differences in opinion and to contribute further therapeutic resources from authoritative American or English clinicians. Such criticisms, suggestions, or additions are enclosed in brackets and signed thus: [.-Ed.]. I have also added a section upon the treatment of neurasthenia.

I have called the reader's attention several times to what I consider the only fault of the book; i.e., the profusion of prescriptions and the author's apparently perfect trust in so many drugs. In these days of therapeutic nihilism few practitioners need be cautioned not to depend upon drugs, and many will be glad to have at hand what seems an excess of measures aimed to relieve symptoms of disease.

My thanks are due and are very gratefully tendered to my assistant, Dr. Howard H. Mason, to St. C. R. Gay, formerly Pharmacist to the French Hospital, and to my secretary, Miss Revel Tobin, for their painstaking help in the preparation of this edition.

Nathl. Bowditch Potter. 


\section{AUTHOR'S PREFACE TO THE FOURTH EDITION}

In the fourth edition of these lectures I have noted the advances made in the therapy of internal diseases during the four years that have elapsed since the appearance of the third edition. Some new matter has been inserted, and some earlier suggestions, which have not stood the test of time and of my own experience, have been dropped.

I am indebted to Herr Victor Zinser for a painstaking revision of the table of contents.

VranNA, September, 1906.

ORTNER. 



\section{AUTHOR'S PREFACE 'TO THE FIRST EDITION}

After nine years' uninterrupted hospital service I venture to offer the results of my experience in these "Lectures on the Therapy of Internal Diseases." I am well aware that there exist much more extensive treatises and systems on the same subject, but I am inclined to believe that their very size and compass prevent them from being perfectly suited to the everyday needs of the average student and practitioner.

I have endeavored especially: (1) to explain briefly but clearly the rationale and the technic of every variety of therapeutic measure useful at the bedside; (2) to give explicit directions in regard to the choice of mineral waters and baths suited to each individual condition, with especial emphasis upon the method of carrying out such treatment in the patient's home; and (3) to furnish not merely lists of prescriptions but, whenever possible, a brief account of the action of each drug, with the hope of making medication less an affair of the memory and more one of the reason.

I have purposely omitted all discussion of the diseases of the nervous system, as their treatment is falling more and more into the hands of the specialist.

My friend and colleague, Herr Docent Dr. Frühwald, has kindly added the concluding chapters on the specific infectious diseases, in which his experience has been far more extensive than mine. I am also especially indebted to Herr Frank and Herr Dr. Foekl, who have aided me with the revision of the manuscript.

May the book prove of use to the student of medicine and the general practitioner.

Ortner.

Lisz, September, 1897. 


\section{CONTENTS}

\section{I.-THERAPY OF DISEASES OF THE CIRCULATORY ORGANS. PAGE}

Therapy of Valvular Diseases of the Heart.................. 1

Therapy of Compensated Valvular Lesions.................. 1

Therapy of Valvular Lesions in the Stage of Broken Compensation..... 11

Therapy of Hydrops and Venous Congestion in Broken Compensation... . 26

Therapy of Hydrops in Broken Compensation............... 27

Therapy of Congestion of the Viscera in Broken Compensation..... 47

Therapy of Compensated Symptomatic Heart Lesions.............. 68

Therapy of Diseases of the Blood Vessels.................. 72

Therapy of Chronic Arteritis.......................... 73

Therapy of Arteriosclerosis........................... 73

Therapy of Angina Pectoris.......................... 77

Therapy of Syphilitic Arteritis........................... 81

Therapy of Aortic Aneurism......................... 81

Therapy of Diseases of the Cardiac Muscle ................ 85

Therapy of Fatty Heart ............................ 85

Therapy of Idiopathic Enlarged Heart .................. 86

Therapy of Chronic Myocarditis....................... 87

Therapy of AcUte Endocarditis......................... 87

Therapy of Pericarditis................................ 90

Therapy of Adherent Pericardium ........................... 93

Therapy of Neuroses of the Heart........................ 93

Therapy of Palpitatio Cordis............................ 93

Therapy of Paroxysmal Tachycardia....................... 95

Therapy of Syncope................................. 96

\section{II.-THERAPY OF DISEASES OF THE GENITO-URINARY TRACT.}

Therapy of Diseases of the Kidneys........................ 98

Therapy of Acute Bright's Disease...................... 98

Therapy of Chronic Parenchymatous Bright's Disease............ 108

Therapy of Chronic Interstitial Nephritis..................... 118

Therapy of Uræmia .............................. 122

Therapy of Nephrolithiasts............................ 129

Therapy of Nephrolithiasis Urica....................... 130

Therapy of Nephrolithiasis Oxalurica..................... 137

Therapy of Lithiasis Phosphaturica...................... 138

Therapy of the Complications of Nephrolithiasis Urica, Nephrolithiasis

Oxalurica, and Lithiasis Phosphaturica .................. 140

Therapy of Pyelitis and Pyelonephritis..................... 142

Therapy of Movable Kidney ............................. 145

Therapy of Cystitis....................................... 147 
III.-THERAPY OF DISEASES OF THE BLOOD. PAGE

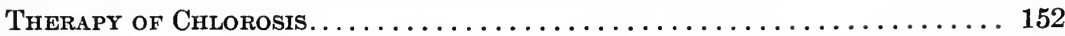

Therapy of Progressive Pernicious An femia.................. 168

Therapy of Leukemia and Pseudoleukamia................... 174

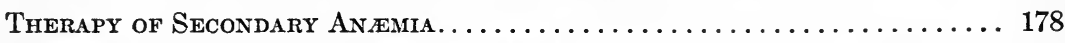

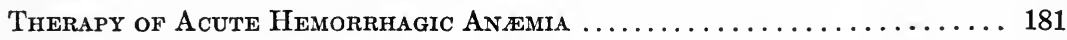

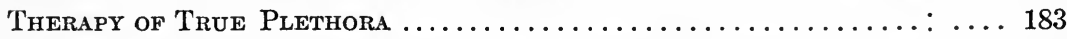

Therapy of Hamophilia and of Purpura Hemorrhagica . . . . . . . . . . . 184

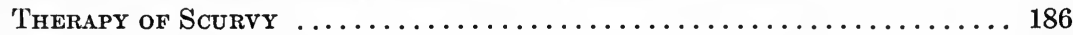

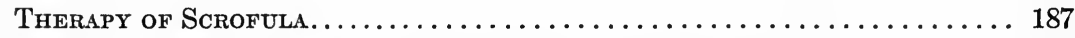

Therapy of Myxcedema, Cretinism, and Exophthalmic Goitre . . . . . . 197

Therapy of Myxœdema and Sporadic Cretinism.............. 197

Therapy of Exophthalmic Goitre . . . . . . . . . . . . . . . 199

\section{IV.-THERAPY OF DISEASES OF METABOLISM.}

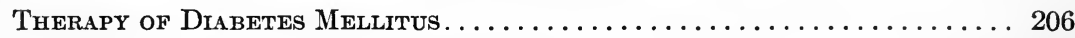

Principles Underlying the Science of Human Nutrition . . . . . . . . . 207

Dietetic Therapy of Diabetes Mellitus.................. 215

Dietetic Treatment of the Mild Form of Diabetes Mellitus ....... 217

Dietetic Treatment of Moderately Severe Form of Diabetes Mellitus 222

Dietetic Treatment of the Severe Form of Diabetes Mellitus . . . . . 224

Other General Principles of the Dietetic Therapy............. 224

Hygienic, Etiological and Medicinal Therapy............. 225

Therapy of Complications and Different Forms of Diabetes Mellitus 229

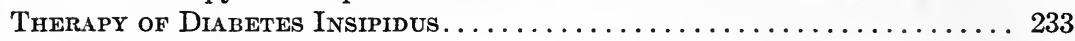

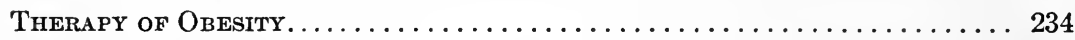

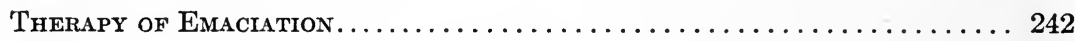

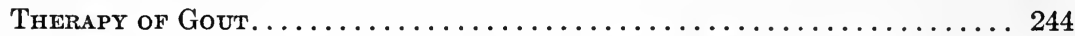

Therapy of Chronic Uric Acid Diathesis and its Manifestations ...... 244

Therapy of an Acute Attack of Gout .................... 251

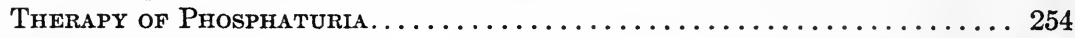

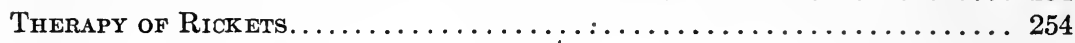

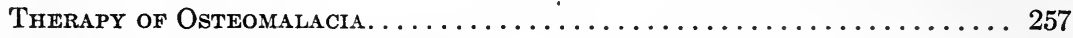

Therapy of Chronic Rheumatism........................ 259

\section{V.-THERAPY OF DISEASES OF THE MOUTH.}

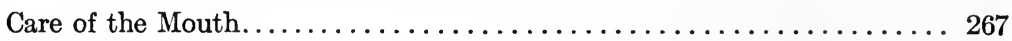

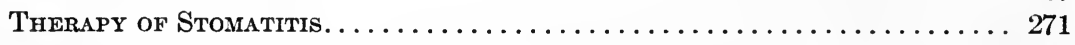

Therapy of Diseases of the Nose and Throat............... 273

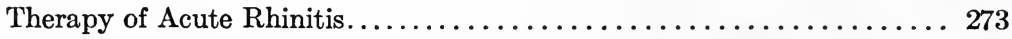

Therapy of Acute Catarrhal Pharyngitis and Tonsillitis.......... 276

\section{VI.-THERAPY OF DISEASES OF THE LARYNX.}

Therapy of Acute Laryngitis. . . . . . . . . . . . . . . . . 278

Therapy of Pseudocroup . . . . . . . . . . . . . . . . . . . 280

Therapy of Tuberculosis of the Larynx.................. 280

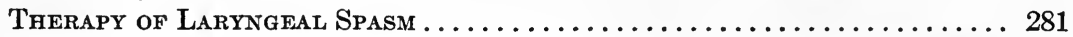


Therapy of Acute Bronchitis. . . . . . . . . . . . . . . . . 283

Therapy of Acute Tracheobronchitis..................... 283

Therapy of Acute Deep Bronchitis and Capillary Bronchitis ......... 291

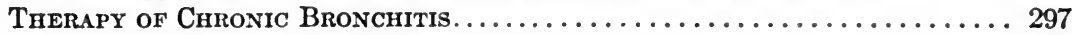

Therapy of Dry Catarrhal Bronchitis .................... 297

Therapy of Chronic Catarrhal Bronchitis with a Purulent Secretion . . . . 300

Therapy of Putrid Bronchitis and of Bronchiectasis............. 303

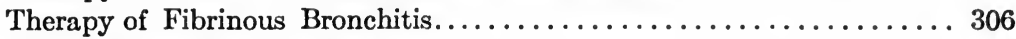

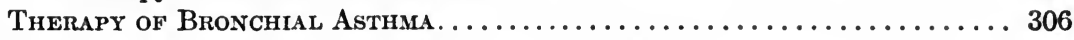

\section{VIII.-THERAPY OF DISEASES OF THE LUNGS.}

Therapy of Croupous Pneumonia....................... 312

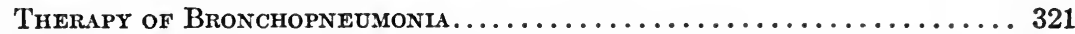

Therapy of Interstitial Pneumonia...................... 322

Therapy of Hemorrhagic Infarct, of Abscess, and of Gangrene in

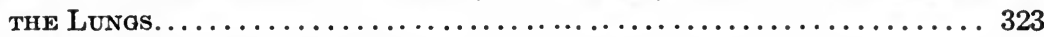

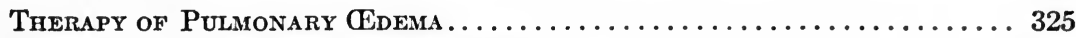

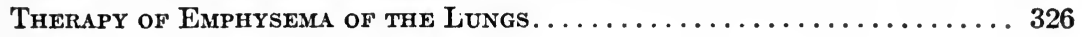

Therapy of Pulmonary Tuberculosis...................... 329

Medicinal Therapy of Pulmonary Tuberculosis................ 344

Symptomatic Therapy of Pulmonary Tuberculosis.............. 349

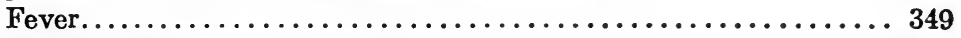

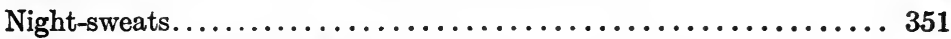

Coughing and Expectoration.......................... 353

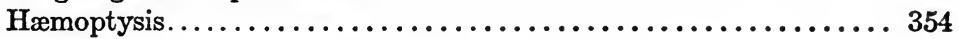

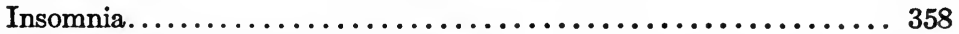

Disturbances of the Digestive Organs.................. 359

\section{IX.-THERAPY OF DISEASES OF THE PLEURA.}

Therapy of Pleurisy ................................... 363

Therapy of Acute Fibrinous Pleurisy...................... 363

Therapy of Pleurisy with Effusion..................... 364

Therapy of Fibrous Pleurisy......................... 368

Therapy of Suppurative and Purulent Pleurisy............... 369

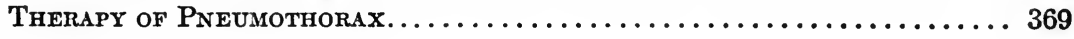

X.-GENERAL THERAPY OF DISEASES OF THE STOMACH AND INTESTINES.

General Principles Underlying the Therapy of the Diseases of the Stomach and Intestines......................... 370

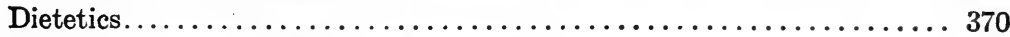

Nutrient Enemas and Cleansing Enemas.................. 394

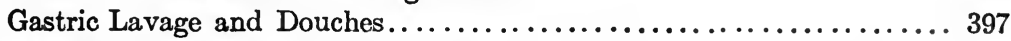

Massage of the Stomach and Abdomen.................. 400 
Diseases of the Stomach and Intestines.-Continued. Page

Electrotherapy................................. 403

Hydrotherapy................................. 404

Mineral Waters in Diseases of the Stomach and Intestines......... 406

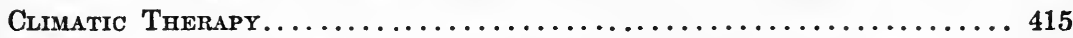

Medication in Diseases of the Stomach and Intestines.......... 415

\section{XI.-SPECIAL THERAPY OF DISEASES OF THE STOMACH AND} INTESTINES.

SPECIAL THERAPY OF DISEASES OF THE STOMACH .......... 432

Therapy of Acute Gastritis......................... 432

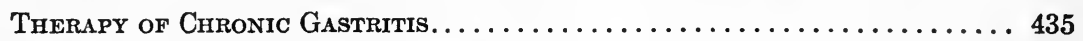

Therapy of Ulcer of the Stomach...................... 439

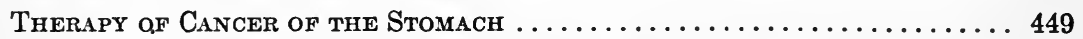

Therapy of Mechanical Insufficiency of the Stomach (Atony aNd

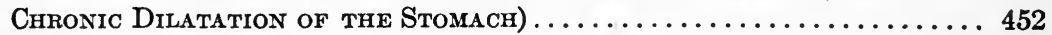

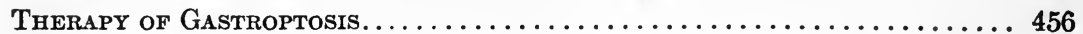

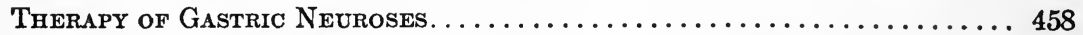

Therapy of Gastralgia........................... 459

Therapy of Nervous Eructation and Pneumatosis............. 461

Therapy of Nervous Vomiting. ..................... 462

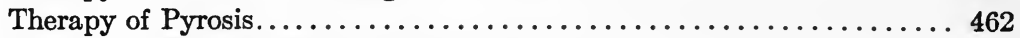

Therapy of Hypersecretion of Gastric Juice.................. 463

Therapy of Hyposecretion of Gastric Juice................. 465

Therapy of Nervous Dyspepsia...................... 466

SPECIAL THERAPY OF INTESTINAL DISEASES ............. 468

Therapy of Acute Intestinal Catarrh................... 468

Therapy of Chronic Intestinal Catarrh................. 470

Therapy of Membranous Enteritis.................... 476

Therapy of Intestinal Ulcer....................... 478

Therapy of Intestinal Stricture and Obstruction $\ldots \ldots \ldots \ldots \ldots \ldots \ldots .480$

Therapy of Chronic Constipation..................... 488

Therapy of Diarrhœa............................ 502

Therapy of Flatulency (Meteorismus) .................... 504

Therapy of Neoplasms of the Intestinal Wall, Enteroptosis, and Intestinal Colic................................... 505

Therapy of Intestinal Parasitic Diseases................. 506

\section{XII.-THERAPY OF DISEASES OF THE PERITONEUM.}

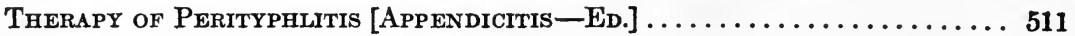

Therapy of Acute Diffuse Peritonitis................... 517

Therapy of Chronic Tuberculous Peritonitis................ 518

\section{XIII.-THERAPY OF DISEASES OF THE LIVER AND GALL PASSAGES.}

Therapy of Catarrhal Jaundice..................... 520

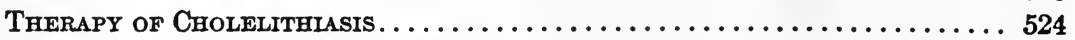

TherApy of CirRHosis of THE LIVER.................... 528 


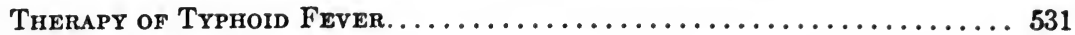

Therapy of Dysentery............................... 540

THERAPY OF INFLUENZA.............................. 543

Therapy of Acute Articular Rheumatism.................. 544

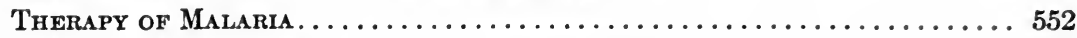

Therapy of Tetanus. . . . . . . . . . . . . . . . . 555

Therapy of the Most Common Acute Poisonings............. 557

XV.-APPENDIX.-THERAPY OF ACUTE INFECTIOUS DISEASES, BY PROF. FRÜHWALD.

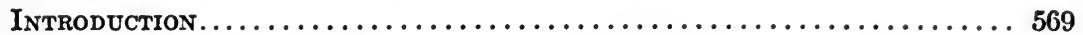

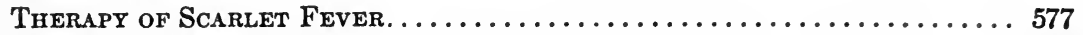

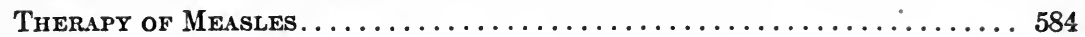

Therapy of German Measles......................... 587

Therapy of Chicken-Pox $\ldots \ldots \ldots \ldots \ldots \ldots \ldots \ldots \ldots \ldots \ldots \ldots \ldots . \ldots \ldots$

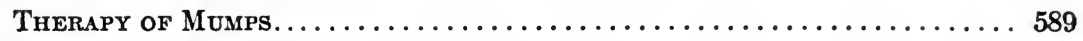

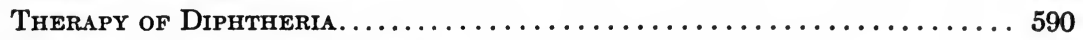

THERAPY of WHOOPING-COUGH........................... 601

XVI.-THERAPY OF NEURASTHENIA, BY DR. POTTER ..........615

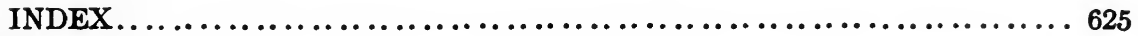




\section{TREATMENT}

OF

\section{INTERNAL DISEASES}

I.

\section{THERAPY OF DISEASES OF THE CIRCULATORY ORGANS}

DISEASES of the circulatory organs may be divided into those of the heart, acute and chronic, and those of the peripheral vessels. The former will be taken up first, beginning with the most common and important,- - the valvular diseases.

\section{THERAPY OF VALVULAR DISEASES OF THE HEART.}

From the therapeutic standpoint, valvular lesions which are fully compensated must be sharply distinguished from those in which the compensation is more or less imperfect. In the first case, the disease is latent, and presents no symptoms; in the second, the cardiac insufficiency becomes evident. This distinction applies to all valvular lesions, no matter which valve is affected. Between these two types a third may exist, a regurgitation at the aortic valve,--or much less frequently at the mitral valve,-in which there is neither absence of all symptoms, as in the first case, nor disturbance of compensation, as in the second case. It presents peculiar subjective symptoms as the result of changes in the peripheral circulation, especially in the arterial system. These peculiar conditions demand a special therapy.

\section{THERAPY OF COMPENSATED VALVULAR LESIONS.}

In a fully compensated lesion, which has been discovered by chance, it is wholly useless and often harmful to employ the ordinary heart stimulants, especially digitalis. In such a case the therapy should be wholly dietetic and mechanical, for as yet we cannot be sure that any drug, even potassium iodide or the new preparations, thiosinamin and fibrolysin, has a direct healing effect on the lesion due to the endocarditis. 
Dietetic Therapy: Object.-In every heart lesion, no matter how perfectly compensated, there are increased demands upon the cardiac muscle. The object of the dietetic therapy is, then, (1) to maintain and if possible to increase the strength of the heart muscle, and (2) to avoid anything which might increase the demand upon it. It is clear that the old method of keeping the patient on a low diet could do nothing but harm. On the contrary, the food should be as strengthening as possible. It is best given every 2 hours in relatively small amounts, in order to avoid the strain on the heart produced by too great distention of the stomach and intestines, which would crowd up the diaphragm and cause hyperæmia of the digestive organs, especially the liver, with consequent relatively increased flow of venous blood into the right auricle. [In compensated valvular lesions I have never found such frequent feedings necessary or advisable, and prefer to adhere to three regular meals with the third a light one. Throughout this book the reader will be struck with the author's generous dietary. Germans and Austrians eat more and oftener than we do. Without going to the opposite extreme, we cannot fail to be influenced by such striking experiments as those of Chittenden and others.-Ed.] Naturally all foods that irritate the heart must be avoided (condiments, vanilla, cinnamon, mace, coffee, tea, alcohol (see below), and too hot food). All foods that tend to cause flatulency must also be forbidden, as they dilate the stomach, and so mechanically and reflexly increase the heart's work, as shown by shortness of breath and palpitation. Examples of such foods are sauerkraut, cabbage, black bread, peas, beans, potatoes, and drinks charged with carbon dioxide. Carbon dioxide has the further disadvantage of increasing the peripheral blood pressure, and so adding to the work of the cardiac muscle. The patient should stay in bed until 8 or 9 A.M., having breakfast as early as his inclination directs.

For this first breakfast he may have a pint of milk with some bread, and perhaps butter. If he dislikes milk (sweet or sour), he may have added to it salt or a little brandy (a liqueur glass to a pint of milk), or a very small amount of weak tea or coffee,-better, malt or barley coffee. [If coffee is chosen as a flavoring there is no special disadvantage in permitting a very small amount-a demitasse-of freshly prepared black coffee. It certainly tastes better and is useful if constipation exists. Brandy in milk early in the morning would hardly prove advantageous. If such a patient awakes at 5 to $7 \mathrm{A.M}$., and is accustomed to remain awake, a cup of hot milk, with or without flavoring, hot gruel, or hot broth upon awakening, will generally counteract the fatigue of dressing. Old people without eardiac lesion find such a plan very helpful.-Ed.] Or he may begin with a smaller quantity of milk and gradually increase the amount. He may have two soft-boiled eggs or a piece of white meat. The latter, however, is better reserved for the second breakfast, two or three hours later. This may consist of a cup of bouillon, bread, and a small piece of fowl, chicken, pheasant, or partridge, 
roast veal or veal cutlet, ham, or beef fillet. The mid-day meal should come between 1 and 3 P.M., and should include soup, a little white meat, fish which is not too fat, with vegetables and a light pudding. The vegetables to be preferred are spinach, Brussels' sprouts, lettuce, (stewed) cauliflower, carrots, beets, peas in purée form, and potatoes made into soup, mashed, or riced. Apple-sauce and stewed figs or cherries are good, on account of their laxative effect. Uncooked fruit is less advisable. Supper should come not later than 8 o'clock and should consist of some white meat, bread, and butter.

[I consider this dietary too rich in meat proteid. While storing up energy for the heart muscle we must not forget that most eardiac cases depend upon their peripheral vessels and upon their kidneys for a long lease of life. The second breakfast could well be changed to a glass of milk, koumiss, matzoon, or buttermilk, and bread and butter. If fish is eaten at dinner, meat can well be omitted. As far as white meat is concerned, I believe that "white meat, less meat" expresses the true state of affairs. We are to-day, perhaps, cutting down meat too much, but this is, I believe, a safer fault than the reverse. Too much carbohydrate, however, produces fermentation, which is a serious drawback to cardiac cases.-Ed.]

Reduction of Fluids Ingested.-As for drinks, Oertel has shown that in cases of simple heart muscle disease, the patient does better with small quantities of liquid food. With insufficient heart activity, not more than 800-1000 c.c. (11/2-2 pt.) in 24 hours should be given, though at first it is very hard for the patient to be limited to so small a quantity. Oertel explains the consequent good results by the fact that a diminished fluid intake corresponds to a diminished quantity of blood and hence less work for the heart. Zuntz finds a possible explanation in the fact that the blood becomes more concentrated-richer in oxygen-and hence able to give the heart better nourishment. He draws an analogy between reducing the ingested fluid in heart disease, and reducing the fluid in the body by sweating, in training horses and athletes. The author considers, however, that with a fully compensated heart such an extreme reduction is not necessary. The patient may be allowed $1500-2000$ c.c. $\left(1 \frac{1}{2}-2\right.$ qt.) in 24 hours. [Variations between Oertel's lowest and the author's highest limit ( 800 and 2000 c.c.) must be decided in the individual case. The season, certainly, should be regarded. Even cardiac cases require more fluid during the hot months than in the winter.-Ed.]

Liquids Forbidden and Allowed.-Alcohol in general should be forbidden. If, however, the patient is strongly wedded to its daily use, a small quantity of wine (a wineglass daily of table wine diluted with water, or a liqueur glass of heavier wine, e.g., sherry, Madeira, port, or Hungarian wine) may be permitted. Beer must be avoided on account of its carbon dioxide, and also because it dilates the stomach, and has been shown by Bauer and Bollinger to have an especially injurious effect on the healthy heart muscle. Mineral waters containing carbon dioxide 
can be permitted only when absolutely necessary, (e.g., if no good water is obtainable), and then they should be uncorked several hours before they are drunk, to let the greater part of the gas escape, and stirred to get rid of the rest. Champagne, of course, is forbidden for the same reason. Tea and coffee have too stimulating an effect on the heart and should be used only in such individual cases as apparently are not affected by them, and then only in very diluted form.

Tobacco.-Tobacco is forbidden, as it is likely to cause increased heart activity, irregularity, and intermission, and sometimes attacks of angina pectoris. [Exceptions may be made in the case of old smokers in whom moderate smoking, upon observation, has been found to have no injurious effect.-Ed.] The patient should avoid even remaining in a room full of tobacco smoke, the effect of which is almost as bad as that of smoking itself.

Salt.-It has recently been strongly recommended, especially by French authors, that common salt be avoided in the diet of a person with a heart lesion, even in the stage of full compensation, or with involvement of the cardiac muscle; for the retention of chlorine, with its accompanying symptoms (dyspnœa, œdema, bronchitis, pulmonary œdema), is a forerunner of broken compensation. [The work of Widal and other French authors is certainly very important, but we should not forget, in our enthusiasm over a scientifically proved point of dietetics, that the palate must also be catered to. With so generous a meat diet much more salt is, of course, ingested with the cooked food. -Ed.]

Rest AFTER EATing-The patient should keep still for at least an hour after eating, in spite of the advice often given to the contrary. It is evidently bad for the heart if, at the height of digestion, almost twothirds of the blood in the gastro-intestinal vessels is suddenly drawn off to the peripheral muscles, thus causing increased arterial pressure and congestion in the veins and lungs. Two or three hours should elapse after eating before much exercise is taken. [In latent well-compensated valvular lesions we can safely allow more latitude than this, both in resting after meals and in exercising.-Ed.]

ExERCISE.-The exercise should be very carefully chosen so as not to cause any disturbance of the heart or lungs; e.g., dyspnœa. The patient may take walks over level ground or very easy grades, their duration to be determined by his own feelings, but never to exceed an hour or an hour and a half. With a fully compensated mitral insufficiency and an otherwise intact heart and vessels, he may ride a bicycle if he has learned to ride before his heart was at all affected, and if he experiences, with this exercise, no trace of dizziness, palpitation, or dyspnœa.

[The problem of out-of-door exercise can hardly be thus summarily dismissed. Many factors of importance in our decision will occur to all of us in establishing rules for such a patient's life. Age makes a decided difference. Each case must be balanced upon its own merits, 
but in a growing boy or young adult, even with an aortic leak (provided it is well compensated), I am convinced that more latitude should be allowed, especially some 16 or 20 months after all signs of acute endocarditis have disappeared, and no subsequent dilatation has supervened. A boy with such a condition at school is made so miserable deprived of all exercise, that I have found it wiser to allow such sports as golf, canoeing, rowing, bicycling, tennis, and sometimes even foot-ball, provided, of course, that he can be examined by a competent physician after such exercise and from time to time to see that no mischief is being accomplished. We must remember that at this age the patient will naturally exceed our permission, and so we must limit the exercise sharply. With reasonable care such a plan keeps a growing boy's nutrition and digestion in much better condition and often prevents his becoming introspective and later a timid, morbid invalid. The practical point is to avoid overfatigue, i.e., fatigue not readily overcome in a few minutes by rest in the horizontal position.

Patton does not prohibit bicycle riding; he gives the following instructions to cardiopaths who ride wheels: Do not ride over six miles an hour; confine riding to a boulevard or smooth gravel road, if possible; do not ride steep grades; do not ride against a strong wind; adjust the saddle well forward so that the weight of the body can be utilized in propelling the wheel; raise saddle so that the rider's leg is almost straight when pedal is low; use wide handle bars. He does not allow wheel riding in cases of mitral stenosis or marked aortic regurgitation.-Ed.]

Oertel's Terraincur.-Oertel has a special method of treatment by systematic exercise, called a " terraincur." It will be discussed more fully in the chapter on diseases of the heart muscle. Suffice it to say here that in general it is not suited to valvular lesions, even in the stage of complete compensation.

Zander's Gymnastic Method.-Proper gymnastics are most warmly to be recommended for many patients. Zander has devised a suitable method, consisting of active and passive movements and mechanical massage, all three carried out by means of special machines run by steam or a motor, and carefully supervised by the physician. The amount of muscular work demanded can be exactly measured.

Swedish Movements.-The " Swedish movements," introduced by Ling, also consist of active and passive movements and of a kind of massage, and aim at an exact measurement of the muscular work involved; but they differ from Zander's method in needing no special room or apparatus, the gymnasium director or physician taking the place of the machine in the passive and active movements.

The effect of both these methods can be explained only by the fact that by the movements of the muscles, especially the active movements, the blood current at the periphery through the veins towards the right heart is lightened, the peripheral blood pressure is reduced, the work of the heart lessened, and the heart therefore relieved. At the same time, 
thanks to the increased activity of the respiratory muscles, the blood stream from the right heart through the lungs to the left heart and the arteries is increased, the heart muscle receives a reflex impulse, and is stimulated to stronger contractions. The heart is thereby strengthened and, possibly, its muscular volume increased. It must be added that some patients are injured by gymnastics, so that their advisability should be decided in each special case by experiment.

Massage.-Massage works in the same way, especially massage of the abdomen and extremities. The blood stream at the periphery is accelerated because the veins of the extremities and of the venæ portæ discharge their blood into the right heart more promptly and more readily, and so further the capillary and arterial blood stream. The arterial pressure is lowered, thus sparing the heart and assisting the peripheral circulation. Precordial effleurage and gentle tapping seem to help in regulating, quieting, and strengthening the heart action. We, however, seldom advise massage in valvular lesions on account of the danger of tearing loose a thrombotic particle. Only when we are sure that this possibility is excluded by a perfectly healed valvular lesion, is massage indicated.

If gymnasties and massage are to have any good results, they must be continued every day for at least three months. The movements of walking, not forced but adjusted to the reserve strength of the heart, exert a similar though less marked effect, and have the great advantage of keeping the patient in the fresh air. He should be out of doors for as many hours in the day as practicable, unless it is extraordinarily bad weather.

Altitude.-In deciding the question of the most suitable altitude, we should remember (according to Hoffmann) that the higher the altitude, the less the barometric pressure, and the greater, therefore, the amount of water and gas given off from the skin and lungs. This increases the action of the respiratory muscles and hence demands a slight increase of metabolism, which, again, means an increased demand upon the heart and exercise of the heart muscle, if it is capable of it. Recent investigations have shown furthermore that the blood of animals and of men, even after a few days in a high altitude, shows an increase in the amount of hæmoglobin and in the number of red corpuscles. This favorable effect of a high altitude has been proved by Schaumann and Rosenqvist, in spite of the objections of Grawitz. It is best, therefore, for a patient with a fully compensated heart to choose a moderately high altitude (1500-2500 ft.). It is risky to go higher, for no one can foretell whether the heart can compensate enough to meet the increased demands. If it proves able to meet only a very moderate increase in work, a sheltered valley with an even temperature is better. [The question of the most suitable altitude in compensated heart disease is interesting but difficult. Many factors of importance enter into consideration; e.g., condition of right heart, of kidneys, of blood pressure, 
of patient's ability to exercise, etc. When asked to decide whether a patient with a compensated lesion should run the risk of travelling across the Rocky Mountains, I have usually acquiesced, at the same time cautioning the patient to remain recumbent in his berth while the train was at an altitude of $3000 \mathrm{ft}$; and if the lesion was serious or imperfectly compensated, I have advised selecting the route with the lowest elevation. When asked whether a change of residence, on account of business or other reasons, to an altitude of 3000-5000 ft. would be prudent or not, I have usually suggested a temporary change, eautioning the patient to remain very quiet for some weeks at first, until the heart, circulation, and lungs should become gradually accustomed to the new conditions. I have had no reason to regret this conservative advice and I know no definite rules to enable us to select one case, say a mitral insufficiency, as being perfectly capable of withstanding any altitude, and to condemn another, say a mitral stenosis, to remain at or near the sea-level. Babcock gives similar advice. He has discussed the question in an interesting fashion, and from his experiences with some 12 to 15 cases, is inclined to object to such a journey for cases of aortic stenosis and for compensated valvular lesions with pericardial or much pleural adhesions, or with much renal change. He expresses the wish for more available observations upon the blood pressure of such patients and of normal individuals at an altitude, because some cases which have experienced serious discomfort have been accompanied by a very low pressure. Gardiner and Hoagland, acting upon his suggestion, estimated the blood pressure of normal individuals at $8000 \mathrm{ft}$. altitude and found it lowered 8-10 mm.-Ed.]

Temperature.-In regard to temperature, a hot climate is without doubt depressing and relaxing to the entire system, and hence of course to the muscles and nerves of the circulatory system. A cold climate has the disadvantage of demanding increased heat from the body and therefore increased work on the part of the heart. As, however, the amount of heat radiated from the body ean be decreased by proper clothing and heating of houses, a cold climate is much better than a hot one. The ideal, however, is that which is moderate and equable without any extremes of heat and cold. If necessary the patient should go south for the winter; e.g., to the Riviera. [In the United States, to Southern California, Camden, Aiken, North Augusta, Summerville.-Ed.]

BATHs.-Until recently, it was believed that all baths were harmful for patients with heart disease, but experiences at certain baths, especially Nauheim (Hesse) and Cudowa (Silesia), have made it necessary to consider the question more carefully. The author here follows Hoffmann's observations.

Tepid Baths.-Tepid baths $\left(88^{\circ}-95^{\circ}\right)$ of $10-15$ minutes' duration may be used, except in case of certain contraindications to be presently mentioned. The patient should wear a cold compress on the head. in order to avoid congestion or overstimulation of the brain. The addition 
of $1-3 \mathrm{~kg}$. $\left(2 \frac{1}{2}-71 / 2 \mathrm{lb}\right.$.) of rock salt to a bath of about 75 gallons is of advantage, or a content of carbon dioxide, such as is found in the Sprudel baths at Nauheim $\left(82^{\circ}-95^{\circ}\right)$.

The effect of tepid baths is explained as follows: Any bath which causes an increase of metabolism demands work of the heart. Too hot baths do this by increasing the internal heat and preventing its radiation. Cold baths do it by causing an abnormal radiation of heat from the body to the cold water, and hence an abnormal production of heat and an increased metabolism. Baths at such a temperature that the body gives out to the water only as much heat as it generally does to the air, so that its temperature is not changed (the indifferent point), have no particular effect on the heart, although they may be slightly stimulating to the peripheral and central nervous systems. Experiments on animals carried out in Pflüger's laboratory have proved that at certain temperatures warm baths cause the animal to give out less earbon dioxide and take in less oxygen, so that its metabolism is decreased. Physicians find the same thing in their practice. Warm baths can be chosen of such a temperature that the loss of heat from the skin is diminished, and yet there is no heat retention in the body. This can happen only through decreased production of heat, which means less work for the heart. Hence it follows that patients with compensated heart lesions may use these baths without injury.

The bath-room should be of such a size as not to become filled with warm vapor, as this hinders the giving out of heat through the respiratory system. The patient should dress in an adjoining room.

Danger of Tepid Baths.-Although in compensated lesions tepid baths directly relieve the heart, there is one danger connected with them. After the bath the vessels of the skin are dilated and their tone is lowered, while at the same time the skin is wet and fitted to give off water vapor. If now, the patient goes immediately into cooler air, the vessels of the skin cannot contract quickly enough to prevent some abnormal loss of heat and of water. Even if they could, such a sudden contraction would change the blood pressure so quickly as to have a detrimental effect on the heart. As a matter of fact the vessels regain their tone only gradually. To protect the patient from catching cold he should be wrapped in warm coverings and rubbed down, as we do not dare to permit a cold sponge to restore the normal tone of the vessels, as in the case of a well man. Above all, he should wear woollen underclothing, which protects the skin from abnormal perspiration and by its rough surface keeps up a slight grade of hyperæmia with the accompanying good tone in the vessels. [During the past three or four years, a number of my patients have changed to linen-mesh underwear, and have convinced me that since this change they have kept in better health and suffered from fewer colds. I still believe that for cardiopaths or patients with tendencies toward lumbago, gout, or so-called chronic rheumatism, the best undergarment is an extremely thin woollen, inter- 
woven with enough silk or linen thread to prevent its shrinking from washing. Good ones are expensive. In overheated city houses, ordinary flannel or underclothing of any thick material keeps the body bathed in perspiration and predisposes towards colds, rheumatic pains, etc.-Ed.]

Explanation of the Effect of Nauheim.-The beneficial effect of the Nauheim Sprudel baths in fully compensated heart lesions is due to the fact that their carbon dioxide inhibits the relaxing effect of the warm bath. Moreover the carbon dioxide stimulates the peripheral nervous system; the stimulation proceeds to the centre, and thence to the cardiac nervous system, so that the heart is capable of increased work. The presence of rock salt in a bath has a similar effect. Gräupner explains the action of the Nauheim baths by saying that, since their temperature is below the indifferent point, they work reflexly through the skin on the vagus centre, stimulate this, and so increase the work of the heart. But the carbon dioxide tends to diminish the tension of the peripheral capillaries; therefore their good effect is due to (1) an increase of strength on the part of the heart muscle, and (2) a decrease of the peripheral resistance. Frankenhäuser has recently explained their good results by the marked contrast between the thermic effect of water and that of carbon dioxide. For water has a much higher indifferent point than carbon dioxide, so that, while the bath water causes a stimulation of cold, the bubbles of the carbon dioxide, being heated above their indifferent point, cause a stimulation of warmth.

Contraindications to Warm Baths.-The use of warm baths is contraindicated for patients with a valvular lesion on account of the accompanying heat retention. Even if there is no valvular lesion but rather a nervous irritability of the heart, warm baths are not advisable. In this condition they may lead to palpitation of the heart, for the warm bath makes unusual demands on the vasomotor centre, causing at first abnormal dilatation of the peripheral vessels, which must gradually contract again. A warm bath is, therefore, contraindicated in the case of an unusually irritable centre. For the baths suitable to this condition, see p. 94 (Cardiac Neuroses).

One practical point to be remembered is that there are frequently cases where an objective examination shows unmistakably complete compensation, but where there are present subjective symptoms of a neurasthenic nature (restlessness, palpitation of the heart, etc.). Here alcohol rubs, sponge baths, and somewhat cooler tub baths $\left(89.6^{\circ}-78.8^{\circ}\right)$ are of use. All except the last are of course suitable also to compensated lesions without subjective symptoms.

Medication.-As has been said, medication plays a very subordinate role. The only treatment that must be discussed in detail is that designed to tone up the whole system. In the first place, arsenic may be recommended in all cases when the compensated lesion is accompanied by anæmia. Arsenic is a valuable general tonic and a stomachic. Its good effect on the blood has been proved in many cases of chlorosis in 
which iron was of no avail, although we cannot demonstrate directly by our methods of investigation any change in the blood after its administration. We prescribe Fowler's solution, as follows :

B Liq. potassii arsenitis,

M. fiat mist.

Tinct. valerianæ................... āā $10.00 \ldots \ldots z$ iiss

Sig. -4 drops in water 2-3 times a day after meals. Increase 1 drop per day until 8 drops are taken.

Or we may give Fowler's solution in the form of compressed tablets, each of which contains from 1 to 3 drops; or we may prescribe arsenious acid, best in the form of tablets, $3-6$ a day, each containing $0.002 \mathrm{G}$. $(1 / 30$ gr.) of the acid. Or we may give Asiatic pills, as follows:

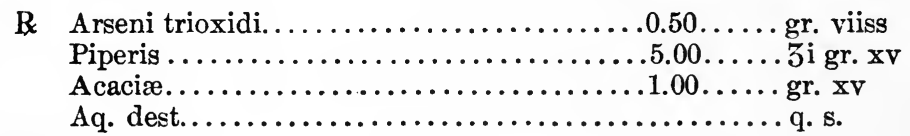

M. fiat pil. no. c.

Sig. - 1 pill 3 times a day. (Arseni trioxidum $0.005\left(\frac{1}{12} \mathrm{gr}\right.$.) pro dosi, 0.015 ( $\frac{1}{4}$ gr. $)$ pro die.)

Finally, arsenic may be given in the mineral waters containing it; e.g., the Guberquelle, and the Roncegno and Levico waters. The dose should be a small teaspoonful in the middle of the day and at night, then a tablespoonful, gradually increasing to 3 tablespoonfuls, always mixed with twice its quantity of water. The Mitterbader Quelle and the Quelle La Bourboule can also be used. (See Therapy of Progressive Pernicious Anæmia, p. 170.) All these waters and all preparations of arsenic should be taken during or after eating, in order to avoid the irritating effect of the arsenic on the mucous membrane of the stomach, as demonstrated especially by Nothnagel and Rossbach.

A similar but not nearly so effective a tonic in the condition under consideration is quinine, which may be prescribed as the compound tincture of cinchona, or else we may combine tincture of gentian compound with it, according to the following prescriptions:

R Tinct. cinchonæ comp..............120.00.... 3 iv

Or :

Sig. $-\frac{1}{2}-1$ teaspoonful in a little water 3 times a day before meals.

B Tinct. cinchonæ comp,

Tinct. gentianæ comp...............āā $60.00 \ldots \ldots$ z ii

M. fiat. sol.

Sig. $-1-1$ teaspoonful in a little water 3 times a day before meals.

We can also use a wine of iron and quinine (Serravallo), a tablespoonful or a liqueur glassful. 
The author does not recommend iron alone in cases of compensated lesions. If it has a good effect, it is rather on the anæmic condition of the heart than on the valvular lesion.

The bowels should move regularly, if necessary by the help of an enema or mild laxative; e.g.:

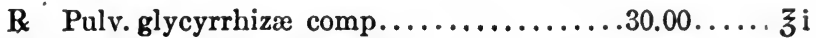

Sig. -1-2 teaspoonfuls at night.

\section{THERAPY OF VALVULAR LESIONS IN THE STAGE OF BROKEN COMPENSATION.}

Every valvular lesion demands increased work on the part of the heart. The right ventricle can endure this strain only imperfectly and for a short time, while the left ventricle can carry on the increased work much longer and more satisfactorily. Hence lesions of the mitral valve, or of any of the valves of the right heart, are much more often found in the stage of broken compensation than are those of the aortic valve. The following therapy, therefore, is particularly suited to mitral lesions and to those of the right heart, and applies to aortic valvular lesions only when the left ventricle is incapable of doing its work and there is congestion of the right heart. In that case it would be advisable even if the pulse were tolerably strong, and the heart action regular, only slightly hastened, or possibly even slowed.

REST.-With broken compensation, even moderate exercise must be given up; the patient must have absolute rest, thus reducing, as Zuntz maintains, the work of the heart almost a third. Except under special conditions, - cerebral anæmia, for example, in which the patient naturally lies down,--he should sit rather than lie in bed, even if there is slight dyspnœa, as sitting or standing diminishes the blood pressure. As this enforced rest is very likely to cause constipation, special care must be taken to see that the bowels move regularly.

DIET.-As long as there is no derangement of digestion or hydrops, the same diet may be followed as in fully compensated lesions, but as soon as these symptoms appear, the diet must consist in great part or, tentatively for a time, wholly of milk Aside from its diuretic effect (see p. 33) milk seems to be extraordinarily easy to digest, and, thanks to its composition of casein, milk sugar, and cream (proteid, carbohydrate, fat), it fulfills better than any other food the requirements of (1) slight demands upon the organs of digestion, and (2) a satisfactory chemical combination. [During broken compensation a purely milk diet gives too much fluid and overtasks the heart. I limit fluids to 1000 or 1500 c.c. in 24 hours and supply most of this quantity with milk, and then increase the nutritive value by adding very well cooked cereals, dextrinized starchy food, such as shredded-wheat biscuits, crisped bread, toasted crackers, or zwieback, and unsalted butter. Later, soft-boiled or poached eggs, broiled honeycomb tripe, lamb and veal chops, chicken, quail, 
partridge, broiled scrod and halibut, and raw oysters may be given with benefit.-Ed.] It should, however, be added that, according to the most recent investigations, the diet when œdema is present should be so chosen as to contain a relatively very small amount of common salt. In many cases, though not in all, such a diet, as the author has proved, causes an almost complete recession of the œdema. Suitable articles of food are: meat, roasted or boiled, potatoes, unsalted butter, green vegetables, and bread made without salt. Cream, milk, eggs, and rice the author, contrary to the opinions of many authors, does not consider as belonging to such a dietary. A milk diet would not, therefore, be indicated in the presence of œdema. With these dietary precautions and rest, the heart often returns to the stage of compensation. If not, therapy directed at strengthening the heart must be begun.

LOCAL CoLD.- The first means to be mentioned is local cold. An icebag, Leiter's coil, or cold compress may be laid over the precordia. The first two may be kept on for days at a time, being removed every third hour. The cold compresses, however, must be renewed the moment they begin to get the least warm.

Heart Stimulants.-A variety of drugs may also be employed, the object of them all being to strengthen the heart and make it fit to undertake its extra amount of work.

Digitalis.-The first of these heart stimulants is digitalis, the physiological action of which is as follows: The normal heart does not use all its reserve force. This is not because it is incompletely emptied, but because the diastolic filling of the chamber is not a maximum one, since the pressure in the auricle is not great enough to cause the largest possible diastolic dilatation of the chamber. The ventricle during normal diastole is expanded only partially, and therefore does not do its full quota of work. In the stage of broken compensation, the systolic emptying is not complete and the heart is permanently stretched, while the greater and lesser circulation show signs of evident congestion. A drug to regulate the disturbed function must increase the heart's power to contract during systole and at the same time must, in general, accomplish a better filling of the peripheral arteries and an increase of the blood pressure. This is just what digitalis does in small doses. In large doses it does just the opposite, reducing the strength of the heart muscle, even paralyzing it, and bringing it to a diastolic standstill. In addition digitalis contracts the splanchnic vessels, causes a partly reflex, partly active expansion of the peripheral vessels, ${ }^{1}$ and with it undoubtedly better circulatory conditions, and slows the heart action, in conse-

1 [Cushny says that digitalin, strophanthin, and convallamarin, as contrasted with digitoxin, have a dilating effect upon the peripheral vessels, partly owing to the increased pressure in the interior overcoming the contraction of the walls, but mainly to be ascribed to a reflex stimulation of the vasodilator centre induced by the contraction of the abdominal vessels. I do not think we are justified in assuming that digitalis, by which word the author presumably includes any of its preparations, produces this carefully selective action.-Ed] 
quence of its effect on the vagus centre and on the inhibitory apparatus in the cardiac muscle itself. The combination of these different effects makes digitalis by far the best of all our heart stimulants.

Cumulative and Toxic Effects of Digitalis.-The cumulative effect of digitalis should be kept in mind. Even 9 days after its use, we can detect its effect in a slowing of the pulse. This may be explained by the fact that, used for some time, it contracts the vessels of the kidneys by raising the blood pressure, and so hinders its excretion (Brunton). We should also remember that it has toxic properties. Nausea, vomiting, diarrhœa, pain in the heart, giddiness, slowing or quickening of the pulse, early allorrhythmia (especially bigemina), arrhythmia, sometimes weakness of the pulse, diminution of the amount of urine, sleeplessness, and nightmare are generally the first symptoms of digitalis poisoning. In severer form it leads to fatigue of the heart muscle, a true "delirium cordis," and a "veritable therapeutic asystole" (Dujardin). All these bad effects will disappear very soon after the drug is stopped. Therefore in prescribing it we should give only relatively small (not too small) doses, should use it only for a limited time, should sometimes decrease the dose, and should immediately stop giving it as soon as symptoms of intoxication appear, especially slowing of the pulse below 60, extra systoles, or gastric symptoms.

The best form in which to administer digitalis is that by maceration:

$\mathrm{B}$ Inf. digitalis................... $320.00 \ldots \ldots$ iv

Sig.-Tablespoonful every 2 hours.

The tincture may be employed, but it is not so effective or so sure as the preparation mentioned. We order 5-15 drops 3 times a day, or prescribe as follows:

R Tinct. digitalis, Tinct. scillæ.................. àa $7.50 \ldots \ldots z$ ii

M. fiat sol.

Sig. $-20-30$ drops 3 times a day.

Finally we may give digitalis as powder or in the form of pills. Neither is as good as the other forms of digitalis, as both are liable to irritate the mucous membrane of the stomach and cause inflammation. The usual dose is $0.20 \mathrm{G}$. ( $3 \mathrm{gr}$.) of the powder up to $0.60 \mathrm{G}$. (9 gr.) per diem. For example:

R Pulv. digitalis.......................... iss

Sacchar. lactis........................... $\nabla$

M. frat pulv. Dent. tal. dos. no. x.

Sig. -1 powder every 3 hours.

Such doses should not usually be continued for more than 4 or 5 days. If the treatment is to be kept up longer, the medication should 
be reduced to two-thirds or one-half the original quantity after the first two days. The author takes exception to the large doses often given.

Relative Intolerance for Digitalis.-If the patient cannot bear the infusion of digitalis, especially if it brings on stomach trouble, he may be given the tincture, or powder, or digitalis itself in pills. There are some people, however, who cannot take digitalis by the mouth in any form whatever, without suffering from loss of appetite, nausea, and vomiting. Sometimes they are helped by giving cognac, aromatic drinks, or acetic ether directly after the digitalis. The author also approves of Fräntzel's method of giving the infusion in such cases in very small doses, ice-cold, with pieces of ice, a procedure which can be tried where congestive catarrh of the stomach would contraindicate digitalis. If all these methods fail, digitalis may be given in an enema as follows:

R Inf. digitalis.................... $120.00 \ldots \ldots$ iv

Sig. -1 tablespoonful in 4 oz. of water, by rectum, repeated as directed.

One or two enemas, heated to the body temperature, may be given daily, the rectum being cleansed before each administration.

Or the digitalis leaves may be given as a suppository:

R Pulv. digitalis.................. $2.00-3.00 \ldots \ldots$ gr. $x x x-g r . x l v$ Ol. theobromatis...................... 8 .

Div. in suppos. no. $x$.

Sig.-Insert 2-4 suppositories daily.

The infusion of digitalis may also be injected subcutaneously, 0.30 1 G. (5-15 gr.) 2 or 3 times a day. The author has given up this method of administration, finding that it causes relatively severe pain and is less efficacious than the ordinary method. [In urgent cases I often inject 10-20 $\mathrm{min}$. of the tincture or 1-2 $\mathrm{min}$. of the fluidextract deep into the muscles.-Ed.]

It should be remembered that, no matter in what form digitalis is given, its activity depends upon the age of the leaves or the time of year at which they were gathered. The new leaves of the second halfyear are as much as four times as strong as the old leaves of the first half-year. The fresh leaves should therefore be gathered, immediately dried, and kept in air-tight receptacles. Or they may be immediately worked up into a drug of constant strength. Several good preparations are made in this way, among which the author has found the "digitalis dialysatum (Golaz)" particularly useful. Its uniformly good effect places it next to the maceration and the powder. The dose is 20 drops 3 or 4 times a day ( 60 drops $=1 \mathrm{G}$. pulv. digitalis). [Among the most constant preparations I have found Squibb's Acetic Acid Tincture and a fat-free tincture put up by Kelly and Durkee (Boston). Parke, Davis \& Company's tincture and other fat-free tinctures are well spoken of. The object of employing a fat-free tincture is to prevent the unpleasant effect upon the stomach.-Ed.] 
Digitoxin.-Theoretically the glucosides of digitalis are also distinguished by the constancy of their effect, though the author does not consider them equal to the digitalis leaves. Two of them he has used with satisfactory results. First, the digitoxin crystals (Merck), a reliable substitute for the mother-plant. One or 2 tablets, $0.0005 \mathrm{G}$. $\left(1 /{ }_{120} \mathrm{gr}\right.$.) each, may be given daily, or the drug may be administered by rectum as follows :

B Digitoxin. cryst. (Merck) .................. $\frac{1}{6}$

Alcohol ..................................

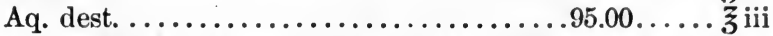

M. et sig. - Wash out rectum first. Take $\frac{1}{10}$ of above $B$ and mix with $60.00 \mathrm{G}$. ( $3 \mathrm{ii}$ ) of water for rectal injection. 1-2 enemas daily.

Digitalin.-The second glucoside is digitalin (Böhringer), which the author has repeatedly used with good effect.

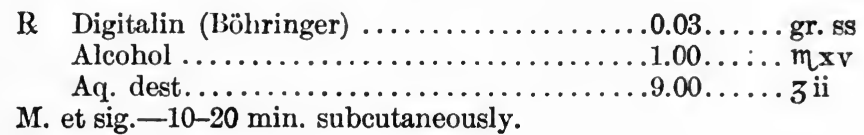

If, directly after the injection, acetic acid clay is applied to the spot affected, very little irritation will be caused.

Digalen.-The subcutaneous injection of the new soluble digitoxin (Cloetta), or " digalen," is more painful. This is an aqueous solution with 25 per cent. glycerin; $0.50-1.5$ c.c. is the usual dose ( 1 c.c. $=0.003$ G. $(1 / 20$ gr.) of digitoxin). The same quantity may be used for intravenous injection in case of acute cardiac insufficiency. It may also be given by the rectum or internally (0.50-1 c.c. 3 times a day). The author has not had a very extensive experience in its use, but considers it worthy of further trial. [I have used digalen for the past 18 months upon selected cases. It is worth a trial if the ordinary preparations do not seem to produce the desired effect or if a more rapid result is imperative. I should caution against too large, too frequent, or too continued dosage. I generally use 0.50 c.c. hypodermically and await its effect for 12 hours before administering another dose.-Ed.]

Digalen and digitalin are both particularly suited to those cases in which digitalis cannot be given internally (i.e., in consequence of vomiting). In such an event a subcutaneous or, if a very quick effect is needed, an intravenous injection is preferable to the administration by the rectum. [Digitalone, where the subcutaneous administration of digitalis has been indicated, has been used with great success at the New York Hospital. It may be given in $15 \mathrm{~min}$. doses every 4 hours.-Ed.]

Greater Effect of Digitalis in Mitral as Compared with Aortic Lesions. - The valvular lesions in which, in practice. we use digitalis or its glucosides are those of broken compensation. It must be borne in mind that it is much less constant in its effect in aortic valvular lesions, par- 
ticularly aortic insufficiency, than in mitral valvular lesions. The reason for this appears to be that an aortic insufficiency reaches the stage of broken compensation only when the heart muscle has suffered by fatty degeneration, fibrous myocarditis, or arteriosclerosis of the coronary arteries. [The use of digitalis in aortic insufficiency has been attended with anxiety upon the part of the physician since Corrigan discountenanced its employment in this lesion. Fothergill and Morrison, among others, agreed with him. Gibson considers that digitalis or one of its congeners is imperatively demanded when cardiac failure makes its appearance. Burr and Balfour before him urged its employment. The former suggested that the objections to its use depend largely upon a priori teaching in pharmacology. The latter always employs digitalis for broken compensation, no matter what the lesion, and thinks that for aortic insufficiency the dose should be 3 times as large as that required for mitral lesions. He does not believe that sudden death is ever due to the digitalis where it is being employed in the former lesion. $\mathrm{He}$ combines it with some vasodilator. Allbutt gives digitalis in aortic regurgitation where the muscle is sound. He administers one dose of the tincture (10 min.) every second day and watches the effect upon the flow of urine and the rate and rhythm of the pulse. In a later stage, when the right side of the heart is involved, he believes the drug is rarely harmful. Babcock agrees with Broadbent and gives digitalis freely in this lesion when dropsy is present, but with great caution when the loss of compensation is shown by left ventricular feebleness rather than by œdema. I have found digitalis of much less value in the broken compensation of aortic insufficiency than in that of mitral lesions, perhaps partly because I have generally been afraid to employ the drug in the doses that Balfour recommends, usually because so many of my cases (New York City Hospital) have been at the end of their tether, with very little healthy muscular fibre. Broken compensation to me, however, indicates first, absolute rest; second, drug treatment; third, diet; and often at this stage I frankly acknowledge that I am not able accurately to localize the lesion or lesions. Unless there is some contraindication, I usually begin my drug treatment with 5-15 gr. of calomel, followed by I/2-1 oz. of Epsom salts or some other saline; then administer 10-15 min. of the tincture or $2-4 \mathrm{oz}$. of the infusion of digitalis every 3 to 6 hours until decided diuresis is established or the pulse rate (at the apex) is less than 65.-Ed.]

Contraindications to Digitalis.-A diseased heart muscle, especially of the left ventricle, is a classical contraindication to digitalis. If the greater part of the muscular fibres have become degenerated, digitalis ean do no good and not infrequently does harm.

In all cases, then, of uncompensated lesions, particularly of the aortic valve, digitalis should be given with great caution if there is any degeneration of the heart muscle. It should be abandoned entirely if we are sure of fatty degeneration or chronic fibrous myocarditis. In all other 
cases, when we are not sure of the condition of the heart muscle, it may be given as a diagnostic experiment. If in 4 or 5 days it produces no effect (increased strength of pulse, diminution of peripheral arterial tension and of the height of the heart wave, fewer symptoms of congestion, increased diuresis), the heart muscle may be considered to be degenerated. All further administration of digitalis is then not only useless but directly dangerous. [In many cases of quite advanced fibrous and fatty myocarditis, I have obtained at least a temporary benefit from carefully administered doses of digitalis. Where such cases can be carefully watched, the drug, in my opinion, is often worth trying.-Ed.] In addition to fatty degeneration and chronic myocarditis, which are often accompanied by an abnormally weak pulse not to be explained by the lesion itself, especially aortic insufficiency, a further contraindication to digitalis therapy is an abnormal slowness of the cardiac contractions. The heart itself need not be slow, for in uncompensated lesions extra contractions which do not reach the periphery often occur and simulate true bradycardia, thus sometimes keeping us from the use of digitalis just where it would be most useful. If there is a true slowing of the pulse, atropine should be added to the digitalis so as to obtain all the effects of digitalis except the slowing of the pulse. [Cushny apparently does not believe in this combination. On account of its very slight inhibitive action, he tried helleborein in a few cases, but with no success, because its local irritant action produced nausea and diarrhœa.-Ed.] We may prescribe it as follows:

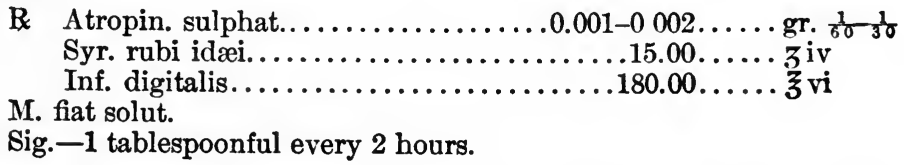

If a heart lesion, generally an aortic insufficiency, has developed by the extension of an atheromatous process to the aortic valves, ergotin (as Rosenbach has recommended), may be added to the digitalis, especially if the heart action is not conspicuously quickened (see p. 61). The author considers digitalis alone dangerous in the case of very rigid vessels, although uncomplicated arteriosclerosis is not a contraindication. Marked arteriosclerosis, when repeated rupture of the vessels has occurred, is.

One practical point, commented on especially by Eichhorst, is that very much run down individuals are often not affected by digitalis unless it has been preceded by large doses of alcohol.

If digitalis, administered for a stated number of days, or until the first appearance of symptoms of intoxication, has a plainly good effect upon the symptoms of broken compensation without wholly removing them, its use should be suspended for from 10 to 15 days, and then tried a second and a third time. Meanwhile some of its familiar substitutes may be employed, although they are not so efficacious in their tonic effect upon the heart and peripheral vessels. 
Strophanthus.-The first of these is strophanthus, either in the form of the seed, or in that of its essential principle, strophanthin. It strengthens the heart action, slows the pulse, and, although to a much less extent than digitalis, increases the arterial tension. Most authorities credit it with a diuretic action, but in the author's opinion this is not at all marked. [Osler recommends strophanthus as a diuretic in acute Bright's disease, but states that it is not to be compared to digitalis when dropsy is present in heart disease.-Ed.] Unlike digitalis, it produces no cumulative effect, so that it can be given for any length of time, unless stomach symptoms (nausea and vomiting) appear. It produces a noticeable effect on the heart muscle much more quickly than digitalis,-in 24 hours instead of $36-48$ or even more. Occasionally it has a deleterious effect on the kidneys. Dujardin-Beaumetz records a case where it caused hæmaturia, and Lemoine has observed that it increases albuminuria in Bright's disease. Bright's disease is therefore a contraindication for its use, as is also, according to G. Sée, true angina pectoris. The author's experience does not confirm the latter statement, [nor does the editor's experience confirm either statement-Ed.]

It is usually given as a tincture ( $1: 10$ alcohol $), 6-10$ drops 3 times a day alone, or 20-30 drops a day in a mixture.

R Tinct. strophanthi.............gtt. xv-gtt. $x x \ldots \ldots$ gtt. $x v-g t t . x x$

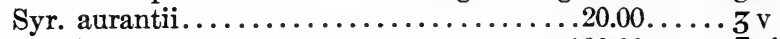

Aq. dest........................ $180.00 \ldots$ vi

M. fiat mist.

Or :

Sig.-1 tablespoonful every 2 hours.

B Tinct. strophanthi,

Tinct. valerianæ.............. āa $10.00 \ldots \ldots z$ iiss

M. et sig. $-10-20$ drops 3 times a day.

The "extractum strophanthi", (Catillon) (0.001 G. ( $1 /{ }_{64}$ gr.) granules up to 3 a day) is an exceedingly good preparation, perhaps even more reliable than the tincture.

Strophanthin is also given, in doses of $0.001-0.002$ G. $\left(1 /{ }_{64}^{-1} /{ }^{32}\right.$ gr.) a day.

R Strophanthini (Wurtz) $\ldots \ldots \ldots \ldots \ldots \ldots \ldots .0 .02 \ldots \ldots$ gr. $\frac{1}{3}$

Glycyrrhizæ............................. q.

M. fiat pil. no. $\mathrm{xx}$.

Sig.-Take 1 pill at noon and at night.

Or, according to Zangger-Eichhorst:

B Strophanthini (Niehaus)..............gtt. vi......gtt. vi

Syr. aurantii........................... $45.00 \ldots \ldots$ ziss

M. et sig. -1 teaspoonful in water 3 times a day.

The author agrees with Ziemssen in recommending strophanthin as a reliable glucoside. [Gibson considers strophanthus of very real value 
when a rapid effect is required, and in dilated senile hearts. Yeo prefers strophanthus in cases of cardiac feebleness with gouty tendencies-with corpulency, flatulence, and dyspepsia-or following acute illness. He also substitutes it for digitalis to avoid the latter's cumulative effect. He has also found it to suceed in producing diuresis after digitalis has failed. (Perhaps a delayed action of digitalis.-Ed.) Patton recommends strophanthus, 7-12 drops, in the minor degrees of ataxia of the heart exhibited by nervous excitable persons, and in controlling the dyspnœa and palpitation of mitral stenosis. Babcock uses strophanthus to supplement the action of digitalis and frequently as a substitute for the latter drug when he wishes to avoid arteriole contraction or digitalis poisoning. He uses it more freely than do most authorities. Cushny considers that the details of the digitalis congeners are still to be worked out.-Ed.]

Sparteine.-Sparteine is another heart tonic, uncertain in its effects. It is obtained from Cystisus scoparius, and given only in the form of sparteine sulphate. It is recommended by G. Sée as strengthening and regulating the heart and, like atropine, quickening the pulse. This is confirmed by Voigt, (Nothnagel's clinic) but disputed by Pawinsky. The dose is $0.08-0.10$ G. (1 $1 / 4-1 \frac{1}{2}$ gr.) in solution, powders, or pills; for example:

B Sparteinæ sulphatis......................... vi

Syr. aurantii........................ $300.00 \ldots$

M. et sig. -1 tablespoonful in water $3-4$ times a day. (Each table-

Or : spoonful contains $0.02 \mathrm{G}$. ( $\frac{1}{3} \mathrm{gr}$.) spartein. sulph.)

R Sparteinæ sulphatis................... 0.20....gr. iii

Sacchar. lactis.........................00....gr. xlv

M. fiat cachets no. $x$.

Sig. -1 cachet $3-4$ times daily.

Or :

B Sparteinæ sulphatis...................... vi

Glycyrrhizæ............................

M. fiat pil. no. $\mathbf{x x}$.

Sig. $-2-4$ pills daily. (Each pill contains 0.02 (gr. $\frac{1}{3}$ ) spartein. sulph.)

The subcutaneous injection is painful and seems to the author unadvisable. It is prescribed as follows:

B Sparteinæ sulphatis................... 0.20....gr. iii

Aq. dest.......................... 3 iiss

Sig.-For subcutaneous injection, $15 \mathrm{~min}$. once or twice daily.

Convallaria Majalis.-Another unreliable substitute for digitalis is convallaria majalis. The leaves and flowers (containing the essential principle, convallamarin) are used. It slows the heart action, increases the arterial pressure, and apparently has a diuretic effect. It is well 
borne by the stomach, has no cumulative effect, and is a mild laxative and stomachic. It may be questioned whether Sée is right in attributing to it a peculiar efficacy in all cases of heart irregularity of nervous origin. [With us it may be given as an extract or as a tincture.-Ed.]

B Tinct. convallariæ...

Sig. -20 drops 3 times a day.

Or 1.5-2 G. (20-30 min.) of the extract a day :

B Ext. convallariæ fl.................6.00... 3 iss Syr. aurantii,

Syr. scillæ ...................āā $75.00 \ldots \ldots$ ろ iiss

M. et sig. - 1 tablespoonful in water 3 times a day. (Squills used on account of its diuretic action.)

Adonis Vernalis.-With convallaria majalis may be grouped adonis vernalis, a drug which especially increases the arterial pressure, and strengthens and often slows the heart action. On account of its effect on the arterial pressure, it is contraindicated whenever the pressure is already high (interstitial nephritis, arteritis, and arteriosclerosis with secondary heart symptoms).

It is given either as an infusion:

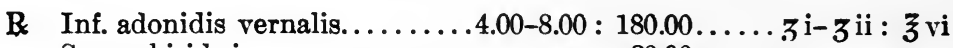

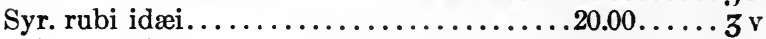

M. et sig. -1 tablespoonful every 2 hours.

Or as a tincture, $2-5$ G. (30-75 min.) a day:

R Tinct. adonidis vernalis............... $10.00 \ldots \ldots$ iiss

Syr. aurantii.................... $140.00 \ldots \ldots$ z $\mathrm{iv} \mathbf{3} \mathbf{v}$

M. et sig. -1 tablespoonful 3 times a day in water.

Or its essential principle, adonidin, may be prescribed as follows :

R Adonidini............................ $0.10 \ldots .$. gr. iss

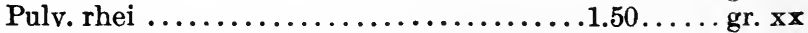

Ext. gentianæ .............................. s.

M. et fiat pil. no. $\mathbf{x x}$.

Sig. -1 pill $3-4$ times a day. (Each pill contains $0.005-\frac{1}{12}$ gr.)

The author, however, has seen no good results from 2 or even $3 \mathrm{mg}$. of adonidin a day. On the other hand, he was astonished at the good results in his hospital practice from dialyzed adonidin (Golaz) in cases of slight disturbances of compensation (almost always in mitral lesions). In a few days, the patients being kept in bed, the disturbances vanished.

R Adonidini dialysat. (Golaz) .......... 10.00.... z iiss

Sig. -5-8 drops an hour, or 20 drops 3 or 4 times (up to 100-150 drops) daily.

[I have not tried any of the last three drugs for some years, because I usually succeed with digitalis, strophanthus, or caffeine. I wish to call 
again the reader's attention to the editor's Preface. Professor Ortner is an enthusiastic therapeutist. He has tried many drugs of which I am entirely ignorant. Some of these he extols warmly with, in my judgment, insufficient reason. It would change the character of the book too materially if I criticized each drug or prescription which Professor Ortner selects; but I caution the reader again that the ready command of a long list of drugs and prescriptions does not make the best and certainly not the safest physician. I heartily commend Professor Ortner's aim to furnish the student and practitioner with a better armament for relieving symptoms. Fewer drugs, however, studied more carefully, and used more cautiously according to the individual peculiarities of the patient or condition, yield at my hands better results.-Ed.]

Caffeine.-A much better substitute for digitalis is caffeine (see p. 34). In physiological doses it strengthens the heart muscle, expands the coronary vessels, ${ }^{2}$ increases the peripheral arterial pressure, quickens the pulse at first and then slows it, stimulates the central functions, and above all increases diuresis by direct stimulation of the renal epithelium. [Cushny states that it is still disputed how caffeine causes diuresis, whether it acts directly upon the renal cells (Schroeder) or produces local changes in the circulation. Its action is, however, entirely dissimilar to that of digitalis. Its reputation in cardiac disease is mainly due to the removal of dropsy through its diuretic action. Combined with digitalis it works marvellously, though the digitalis relieves the cardiac dilatation, the caffeine merely aiding in the removal of the fluid which is obstructing the circulation by its pressure. Loewi has recently furnished experimental evidence to show that caffeine diuresis occurs only as a result of an increased blood supply to the kidneys.-Ed.]

Toxic Symptoms.-Relatively small doses of caffeine may produce toxic symptoms, particularly insomnia in many individuals, and even marked delirium and hallucination in convalescents from severe illnesses, in alcoholics, neurasthenics, and aged persons. It is the best substitute for digitalis if the blood pressure is low-never if it is high-and is particularly advisable if marked alterations in the heart muscle contraindicate digitalis. It has an effect in the last stage of cardiac insufficiency, the stage of asystole, when all other heart stimulants are useless.

Administration.-It is almost always given in one of its loose double compounds,-caffeine-sodium benzoate, caffeine-sodium cinnamylate, caffeine-sodium salicylate. If it is used as a heart stimulant, the dose of

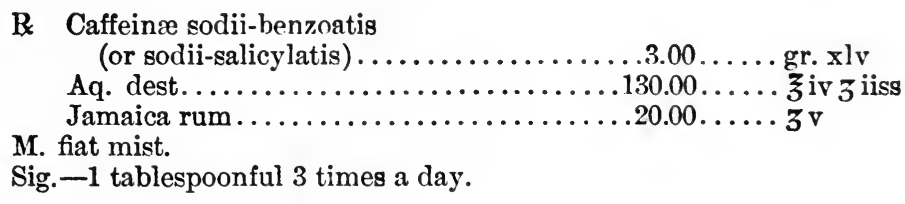

${ }^{2}$ ["Theobromine causes some dilatation of the coronary arteries when it is perfused through the heart, while caffeine is devoid of this action."-Cushry.] 
any one of these is $0.60-1$ G. (10-15 gr.) daily. If, however, a diuretic effect is desired, the dose must be increased to $1.5-2$ G. $(22-30 \mathrm{gr}$.). In the former case we may use the prescription on page 21.

A solution is preferable to a powder, as caffeine in the solid form causes in many individuals discomfort or pain in the stomach, nausea, and vomiting. If, however, the powder is selected, it may be given as follows :

R Caffeinæ sodii-benzoatis (or sodii-salicylatis) ............ $0.20-0.30 \ldots \ldots$ gr. iii-gr. $v$

Sacchar. lactis..................... $v$

M. et fiat pulv. Dent. tal. dos. no. $\mathbf{x}$.

Sig. - 1 powder in water 3 times a day.

Caffeine has a stronger effect when injected subcutaneously, and is naturally given in this way, or per rectum, when it causes gastric symptoms if given by the mouth. We prescribe as follows :

R Caffeinæ sodii-benzoatis..................... 3 ss Aq. dest.,

Glycerini ................... āā $5.00 \ldots \ldots 3$ im xv

M. et sig. $-15-20 \mathrm{~min}$. subcutaneously.

Caffeine and digitalis are often combined either as a mixture or a powder :

R Inf. digitalis...................... $180.00 \ldots$ ri

Caffeinæ sodii-benzoatis ................ $1.00 \ldots \ldots$ gr. $\mathbf{x v}$

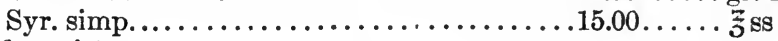

M. fiat mist.

Sig. -1 tablespoonful every 2 hours.

This combination of digitalis and caffeine appears exceedingly rational, as Braun has shown from experiments on animals that caffeine fully counterbalances the tendency of digitalis to contract the coronary vessels of the heart. [I frequently use digitalis and caffeine at the same time, but usually prescribe two separate solutions so that I may alter the dose of one or the other drug at will.-Ed.]

Semen Kolæ.-Kola is similar in its effect to caffeine, its action being due in fact principally to its content of the latter. It has, besides, a markedly stimulating effect on the nervous system, and is for this reason used by the Soudanese as we use coffee and tea. Its peculiar therapeutic field is neurasthenia, but it may be used in cardiac insufficiency instead of caffeine, although personally the author has not been successful with it. It is prescribed as follows:

R Tinct. kolæ......................

Or:

Sig. -2 teaspoonfuls twice a day. (20\% tincture.)

R Vin. kolæ....................... $120.00 \ldots \ldots$ iv

Sig.-A wineglassful twice a day. (10\% wine.) 
Or

B Sem. kolæ pulv............................ 3 i-gr. $\mathbf{x v}$

Ext. glycyrrhizæ ....................... 8.

M. fiat pil. no. 1 .

Or :

Sig. $-9-10$ pills a day.

B Sem. kolæ pulv.......................... gr. $x$

Massæ cacao.............................. 8.

M. et fiat trochisi no. $\mathbf{x x x}$.

Sig.-1-10 pastilles a day.

Finally, we may use Stoll's Elixir (2-3 liqueur glasses a day) or the French preparation, Astier's Granulated Kola (2 teaspoonfuls a day.) [I rarely use kola. Continental physicians prescribe it frequently for neurasthenics. I do not believe that it is wise to stimulate so constantly a weak nervous system, and I object quite as decidedly to the similar use of strychnine, especially in large doses. It requires a long time for an overstimulated neurasthenic to recover from its effects and the withdrawal of its stimulation.-Ed.]

Quinine.-Quinine may be used in cases of very slight insufficiency or sometimes in combination with other heart stimulants; e.g., caffeine.

Nerium Oleander.-The author cannot recommend the newest heart stimulant, nerium oleander, any more than he can kola (inf. nerii oleandris, 1 teaspoonful every 2 hours).

Apocynum.-The fluidextract of apocynum cannabinum (Canadian hemp), on the contrary, has often proved of good service as a heart stimulant, particularly as a diuretic. The dose is 10-30 drops generally 3 times a day.

Strychnine.-Strychnine is used successfully by some physicians as a heart stimulant. Its action is confined to raising the peripheral arterial pressure by its influence on the vasomotor centre of the medulla. The author uses it (or the tincture of nux vomica), either in addition to the stimulants already mentioned or after digitalis, to keep the peripheral blood pressure as high as possible. (See p.250.) [I do not doubt that many of us use strychnine rather loosely, either alone or in combination with digitalis or other heart stimulants. Much of its good effect in these conditions is perhaps due to its general action upon the nervous system, and upon the gastro-intestinal tract. Whether it increases the blood pressure or not is still in doubt. Cabot failed to find any increase after its use in a number of conditions. Babcock considers that it stimulates the heart through its action upon the cardiac motor ganglia. $\mathrm{He}$ has employed $1 / 30$ gr. hypodermically every $2-3$ hours until $7-8$ doses have been administered in the day and continues this dosage for days. $\mathrm{He}$ acknowledges patients' dependence upon the drug and the acquirement of a definite habit. Curtin, agreeing with Sansom and Lauder Brunton, objects to so large a dose. They employ $1 /{ }_{60}-1 /{ }^{\circ 0}$ gr. 3 or 4 times a day. Balfour, on the other hand, believes in pushing the drug. $\mathrm{He}$ employs 5 min. liq. strychninæ hydrochloridi $(=1 / 26$ gr. strychnine) twice a day for any length of time until the patient is toxic. Although he considers 
the drug cumulative to some extent, he finds that most individuals can take this dose for months or years. He cautions against large doses in anæmic patients, who, he finds, are generally intolerant.-Ed.]

It is often a good plan to use quinine and strychnine as an addition to digitalis, when coexisting symptoms of gastric congestion (e.g., nausea, vomiting, etc.) make one fear an intolerance for digitalis. For example, we may prescribe:

R Pulv. digitalis,

Quininæ hydrochloratis..............āā $2.00 \ldots \ldots 3$ ss

Ext. nucis vomicæ................... $0.40 \ldots \ldots$ gr. vi

M. et fiat mass. Div. in pil. no. xl. Consperg.

Sig. $-1-3$ pills 3 times a day.

This concludes the list of heart stimulants, strictly considered. As has already been said (p. 16), the best of them, digitalis, is sometimes contraindicated, and caffeine is often substituted in cases of extreme cardiac insufficiency with extensive fatty degeneration or myocarditis.

But where the symptoms of cardiac weakness are extraordinarily severe, the peripheral pulse hardly palpable, the heart tones weak, and the extremities cold and livid-in short, where the signs of asystole are present, we will do well to employ an intravenous injection of 1-1.5 c.c. of digalen, or an analeptic to avert the imminent danger of collapse and death. We may use strong wine (especially champagne), cognac, ${ }^{3}$ hot tea or coffee, wine or vinegar enemas, or, perhaps best of all, camphor.

Camphor.-With an exquisite effect on the nervous system, camphor combines in medicinal doses a prompt quickening and strengthening of the heart action. It is best injected subcutaneously:

B Camphoræ..........................2.00.... 3 ss

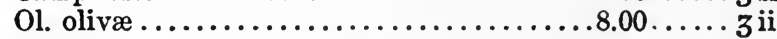

M. fiat solut.

Or:

Sig.-Subcutan. injection, 5 min. as necessary.

B Camphoræ...................... $0.03-0.20 \ldots \ldots$ gr. ss-gr. iii

Sacchar. lactis..................... $0.40 \ldots \ldots$ gr. vi

M. fiat pulv. no. i. Dent. tal. dos. no. x (chartulæ ceratæ).

Or:

Sig. - 1 powder every hour or two.

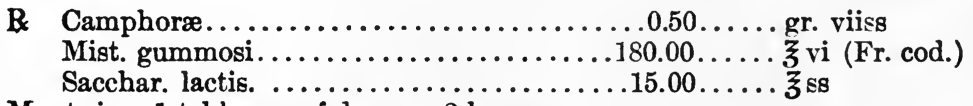

M. et sig. -1 tablespoonful every 2 hours.

Edlefsen particulariy recommends the use of camphor with digitalis for the treatment of heart lesions without advanced insufficiency. Eichhorst-Zangger have recently also recommended this combination for its

${ }^{3}$ [A very old natural whiskey, or, as recommended by Wm. Murray, a small riqueur glass of brandy made from the champagne grape in the Charente district has a much more striking effect than ordinary cognac or strong wine.-ED.] 
marked diuretic effect. (see p. 34). The author has used with great success $0.10 \mathrm{G}$. ( $1 \frac{1}{2} \mathrm{gr}$.) of powdered digitalis combined with $0.05-0.10$ G. $\left(3 / 4-1 \frac{1}{2}\right.$ gr. $)$ of camphor, 3 or 4 times a day.

Ether.-Another analeptic is ether, which, given in small doses, has a stimulating effect on the nervous system; 5-10 drops of sulphuric ether or acetic ether (the latter with a less unpleasant odor) may be given on sugar, in sweetened water, in syrups, or in gelatine capsules. Or sulphuric ether may be injected subcutaneously (15-30 min.). It is apt, however, to be followed by paralysis of the nerves and gangrene at the punctured spot, to say nothing of being painful. Ether can also be given in an enema, 4 G. ( $1 \mathrm{dr}$.) of sulphuric ether in $100 \mathrm{G}$. (3 oz.) of water. The spirits of ether are oftener used than pure ether ( 1 part ether to 3 parts spirits, the so-called Hoffmann's drops). The dose is 2-12 e.c. $(1 / 2-3 \mathrm{dr}$. $)$ with sugar or in tea.

Although ether is in general inferior to camphor, it acts more quickly. In a case of urgent necessity, the author uses ether followed by an injection of camphor in oil, or else an injection of ether and camphor in oil, together, in equal parts or in the ratio of 1:2 (1-3 Pravaz's syringes).

Moschus.-Mosehus is now rarely used. It is inferior to camphor, is much more expensive, and to many individuals is very unpleasant on account of its odor. It is given sometimes as a powder, sometimes as a tincture.

R Moschi..................... 0.05-0.30....gr. $\frac{3}{4}-$ gr. v

Sacchar. lactis.................... vi

M. fiat cachet no. i. Dent. tal. dos. no. $\mathbf{x}$.

Or:

Sig. -1 cachet every 2 hours.

B. Camphoræ,

Moschi ......................āa 0.03-0.10...... gr. ss-gr. ii

Sacchar. lactis.............................. gr. vi

M. fiat pulv. Dent. tal. dos. no. x. Dispens. in cachets.

Or :

Sig. -1 cachet every 2 hours.

R Tinct. moschi ...................... $15.00 \ldots \ldots$

Sig. $-10-30$ drops on sugar or in mixture every $2-3$ hours.

Ammonia as Liquor Ammonii Anisatus.-The last stimulant to be noted is ammonia, usually used in Germany as the liquor ammonii anisatus ( $5 \mathrm{G}$. $(1 \mathrm{1} / 4 \mathrm{dr}$.) of ethereal oil of anise, $25 \mathrm{G} .(6 \mathrm{r} / 4 \mathrm{dr}$.) of ammonia water, and $100 \mathrm{G}$. ( $3 \mathrm{oz}$.) of 90 per cent. alcohol).

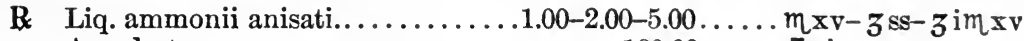

Aq. dest........................... $180.00 \ldots \ldots$ vi

Syr. aurantii .......................

Or :

M. et sig. -1 tablespoonful every 2 hours.

B Liq. ammonii anisati.................5.00.... 3 im $\times$

Aq. amygdal. amaræ......................... $35.00 \ldots$

Syr. acaciæ $\ldots \ldots \ldots \ldots \ldots \ldots \ldots \ldots \ldots \ldots, \ldots \ldots \ldots, \ldots \ldots$ iiss

M. fiat mist.

Sig. -2 teaspoonfuls $2-4$ times a day. 
Or: $\quad$ R Liq. ammonii anisati,

R. Liq. ammonii anisati,
Spir. ætheris.....................āa $10.00 \ldots \ldots z$ iiss
M. et sig.--8-10 drops in oatmeal gruel every hour.

[I usually employ aromatic spirits of ammonia in doses of 1 or 2 teaspoonfuls in 1 or 2 tablespoonfuls of water.-Ed.]

Valerian.-There remains one very mild stimulant, valerian, which the author agrees with Fräntzel in recommending in very mild cases of broken compensation, where digitalis is not called for:

Or:

R Inf. valerianæ............... $10.00: 180.00 \ldots \ldots z$ iiss : $\xi$ vi

(Spir. ætheris nitrosi...............2.00.... $3 \mathrm{ss}$ )

Syr. rubi idæi....................... $20.00 \ldots \mathbf{~ v ~}$

M. et sig. -1 tablespoonful every 2 hours.

B Tinct. valerianæ....................30.00.... $\xi_{\mathrm{i}}$

Or:

Sig. $-20-30$ drops 3-4 times a day.

$\mathrm{R}$ Tinct. valerianæ ammoniatæ ............ $30.00 \ldots \ldots \tilde{z} \mathrm{i}$

Sig. $-10-15$ drops 3-4 times a day.

A much stronger effect is obtained by using the valerianic acid menthol, validol, in the form of validolum camphoratum, 5-10 drops several times a day.

It only remains to be emphasized that a patient with broken compensation must remain quiet in bed. In mild cases, rest alone will often bring back full compensation, and, if stimulants are used, they must be helped by complete rest, as every movement involves work on the part of the heart muscle. [I think the value of rest should be emphasized even more forcibly than the author states; physical and mental rest should be absolute.-Ed.]

\section{THERAPY OF HYDROPS AND VENOUS CONGESTION IN BROKEN COMPENSATION.}

Origin and Significance of Symptoms of Hydrops.-By treating broken compensation with one of the heart stimulants just discussed, we may restore the power of the heart muscle without removing pathological processes in other organs, which have been set up by the cardiac insufficiency. So long as these processes continue, they may destroy the effect of the heart stimulants in spite of the rehabilitation of the cardiac muscle. Among such processes accompanying broken compensation is the symptom of congestion developed in the lesser circulation and in the veins of the greater. In consequence of the damming up of blood in the vena cava inferior, especially in the case of a mitral lesion, there may develop passive hyperæmia of the liver and kidneys. From the hepatic and portal veins results congestion of the spleen, stomach, and intestines. Swelling of the lower extremities, the scrotum, the skin of the penis 
and abdomen, and collections of fluid in the peritoneal, pleural, and pericardial cavities, symptoms of venous hyperæmia of the brain and its coverings, are all signs of congestion of the blood in the trunk and branches of both venæ cavæ and of the venæ magnæ cordis.

If such symptoms are present, they must be combated at the same time as or even before heart stimulants are used. We, therefore, next discuss the therapy of hydrops and of congestion of the various internal organs in broken compensation; and begin with the therapy of hydrops, which will evidently help or at least not hinder the relief of the congestion, since hydrops is only an advanced stage of local venous hyperæmia and stasis.

\section{Therapy of Hydrops in Broken Compensation.}

If the œdema is very slight, appearing only at the end of the day around the ankles and disappearing during the night, rest in bed for several days will be enough to reduce it, especially if the patient be limited to a milk diet.

If, however, 'transudates have collected in the subcutaneous tissues and serous cavities, we possess three methods of expelling these fluids from the body: (1) through the kidney, (2) through the intestines, (3) through the skin. In other words, we may have recourse in hydrops to diuretics, to purgatives, and to diaphoretics.

Diuretics: Renal, Circulatory.-Diuretics may be classified as follows :

1. Direct, renal diuretics:

a. Those stimulating the renal epithelium without irritating and injuring it.

$b$. Those stimulating the renal epithelium and injuring it.

2. Indirect, circulatory diuretics:

a. Cardiovascular diuretics (increasing diuresis, either by a direct effect upon the heart or by increase of the peripheral arterial pressure and consequent increase of the rapidity of the blood current in the renal arteries).

b. Plasmogenic diuretics (increasing diuresis by increasing the fluid portion of the blood).

The Heart Stimulants as Diuretics.-It is evident that the cardiovascular diuretics are the most important, for they strike directly at the root of the trouble. Digitalis is, therefore, the best diuretic as well as the best heart stimulant and all that has been previously said (see pp. 12-17) about its administration, indications, and contraindications applies here also. The author, as has already been mentioned, cannot agree with many authors, Dujardin-Beaumetz for example, in assigning a peculiarly marked diuretic power to strophanthus. Other diuretics already mentioned are the tincture and extract of convallaria, sparteine, adonis vernalis, and caffeine, the last being by far the best, and sometimes even proving effective where digitalis itself has failed, although in general 
inferior to it. We give sodium-salicylate or sodium-benzoate of caffeine in large doses (1.5-2 G.-20-30 gr. daily) in solution rather than in powder form or subcutaneously.

Diuretin.-Theobromine, closely related to caffeine, may be ranked with it and digitalis as a diuretic. It is often given as the sodiumsalicylate of theobromine, or the so-called diuretin. This drug belongs to the first subdivision of the renal diuretics, exciting diuresis without injuring the renal epithelium. Diuretin, except in very large doses, has no toxic properties or any effect on the nervous system or-at least according to many authorities - on the circulatory system. Patients often complain of its unpleasant taste and in some instances even of nausea and vomiting after taking it. The digestive disturbances may be best avoided by not exceeding $0.50 \mathrm{G}$. (71/2 gr.) in a single dose. As diuretin is not soluble in cold water, it is generally given in powder form, 4-6 G. (1-1 $1 / 2$ dr.) per diem.

$\mathrm{R}$
Dent. tal. dos. no. $\mathrm{xx}(\mathrm{chartulæ}$ ceratæ).

Sig. -1 powder every 2 hours up to 6 powders.

It seems better to the author, however, to follow Gram and Koritschoner (Schrötter's elinic) and to dissolve the diuretin in hot water, a solution that remains clear even after cooling.

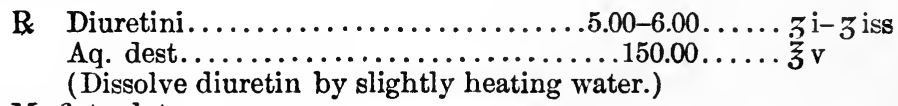

M. fiat solut.

Sig. -1 tablespoonful every 2 hours.

As the hydrochloric acid of the stomach makes it harder for the drug to be absorbed, Koritschoner recommends not giving it till 3 hours after a meal, or prescribing it with a dose of bicarbonate of soda in milk. Some patients show increased diuresis after the first day of the treatment. In case no effect is obtained after 4 or 5 days, a further trial is useless. [Patton states that less than $90 \mathrm{gr}$. of diuretin in 24 hours is not effective and that small and continuous dosage is useless. He prescribes $20 \mathrm{gr}$. every 4 hours until 6 doses are taken (120 gr. in all), and if this quantity does not cause diuresis he stops. Babcock recommends for cardiac dropsy $1 / 2$ oz. of infusion of digitalis (English leaves freshly prepared) every 4 hours up to 8 ounces. If the heart's contractions reach 60 or less, if nausea supervenes, or if the amount of urine falls off after it has first been increased, he stops the drug. Only when this remedy fails to remove dropsy does he employ diuretin in the same dose as Patton. He suggests counteracting the unpleasant taste and effects of the diuretin by adding to each dose 1-2 dr. of essence of pepsin.-Ed.]

Theobromine.-The pure theobromine is given in powder form and in somewhat smaller doses-generally $2-3$ G. (30-45 gr.), seldom 6 G. (1 I $/ 2$ 
dr.) daily. The author has found this successful in a large number of cases. It sometimes has a secondary effect on the stomach and on the nervous system (one of the author's patients complained of a constant desire to sleep, which disappeared when the drug was discontinued). Huchard advises the addition of $0.25 \mathrm{G}$. ( $33 / 4 \mathrm{gr}$.) of sodium phosphate to each $0.50 \mathrm{G}$. $(7 \mathrm{r} / 2 \mathrm{gr}$.) of powdered theobromine to counteract any unpleasant effect upon the stomach.

Uropherin.-There is also a third drug of similar derivation, the socalled uropherin (theobromine-lithium salicylate), a white crystalline powder with a slightly salty taste. It is given in $0.50-1 \mathrm{G}$. $(7 \mathrm{1} / 2-15 \mathrm{gr}$.) doses up to 3-6 G. (45-90 gr.) a day. Whether given alone or with digitalis, it is not conspicuously better than diuretin, but it undoubtedly increases diuresis, even in the minimum daily dose. Very exceptionally it too brings on an unpleasant feeling of pressure in the stomach and slight nausea.

Agurin.-Uropherin and pure theobromine have, however, been recently replaced to a great extent by a double salt of theobromine, similar to diuretin, theobromine-sodium acetate, or agurin. The author has often tried it in doses of 1-3 G. (15-45 gr.) and more a day in powder form, sometimes with a less, sometimes with an equal, and sometimes with a more marked effect than that of diuretin, which was tried first in each case.

Theophylline or Theocine.-Generally, if not always, both diuretin and agurin seem surpassed in effect by the synthetically prepared theophylline or theocine. It is given in doses of $0.30-0.40 \mathrm{G}$. (4-6 gr.) 3 times a day in powder form, or in the form of its easily soluble compounds, theocine-sodium and theocine-sodium acetate, the dose of the former being $0.20-0.40$ G. (3-6 gr.) 3 times a day, and that of the latter, $0.30-0.50$ G. (4-7 $1 / 2 \mathrm{gr}$.) of the powder, or $0.90-1 \mathrm{G}$. (13-15 gr.) of the solution, 3-4 times a day. From an extensive experience, the author considers theocine-sodium acetate the best of the diuretics in most cases. None of these preparations should be given on an empty stomach. If theocine is not well borne, it should be given oftener, in smaller doses $(0.15$ G. (21/4 gr.) 6 times a day). Some authorities, especially H. Schlesinger, attribute to theocine the frequent occurrence of convulsions epileptic in character. The author has seen one such case in a complicated lesion, but in general is inclined to attribute the convulsions to the valvular disease rather than to the theocine, the latter serving only as the immediate factor in starting them. 'To prevent the occurrence of such cerebral symptoms, adonis vernalis may be added to theocine. It must be acknowledged that the effect of theocine wears off almost uniformly in 3-4 days, so that it must be replaced by some other diuretic for a time. Agurin also loses its effect after a time, obliging us to increase the dose gradually.

Calomel.-In certain cases calomel produces remarkably satisfactory results. This, too, is a renal diuretic and does not injure the epithelium. The 24-hour amount of urine is often increased to 4 or 5 or even $10 \mathrm{qt}$. 
by the use of calomel, sometimes even in cases when digitalis is no longer effectual. Usually the effect of calomel is shown only three or four days after the beginning of the treatment, and generally lasts several days after the treatment has been stopped. In many cases, however, calomel produces no diuresis, often causing painful or severe symptoms of mercurial poisoning (salivation, extensive stomatitis, even scurvy, or severe diarrhœa, sometimes with an admixture of blood from intestinal ulcers). This occurs when the renal epithelium is no longer capable of responding to the stimulation.

Contraindication.-Hence a clear contraindication for the use of calomel is a coexistent nephritis. [Cushny attributes more value to calomel in cardiac, than in hepatic or renal dropsy, but does not consider that it is contraindicated in chronic nephritis. I employ calomel constantly in ehronic nephritis with dropsy and rarely meet with enough salivation to cause me to regret its selection. I have noticed salivation after its use more frequently in chronic nephritis without dropsy. Whenever the kidneys are diseased, I prescribe an active saline after 48 hours' administration of calomel.-Ed.] It should also be avoided when the heart muscle has been weak for so long a time that the kidneys have suffered extensive and serious injury from protracted lack of nourishment.

Administration.-We employ Jendrassik's prescription, to whom belongs the credit of bringing calomel into use again as a diuretic:

R Hydrarg. chloridi mitis................ $0.20 \ldots \ldots$ gr. iii

Sacchar. lactis.................... $0.40 \ldots \ldots$ gr. vi

M. fiat pulv. no. i. Dent. tal. dos. no. ix-xii.

Sig. -3 powders daily.

To obviate the danger of salivation the following should be used at least every hour:

$\mathrm{B}$ Potassii chloratis....................8.00.... 3 ii

Aq. dest........................ Oi

M. fiat solut.

Sig.-Mouth-wash.

Hydrogen peroxide is as good or better. As we need a 2 per cent. solution and it comes in a 10 per cent. solution, we prescribe as follows :

B Aq. hydrogenii dioxidi..............50.00... 3 iss

Sig.-2 teaspoonfuls to a half-glass of water. Use as a mouth-wash.

If the gums are slightly spongy or bleed, it is best to paint them with some astringent. This may be done in any case as a precaution.

R Tinct. krameriæ,

Tinct. gallæ.................... āa $15.00 \ldots \ldots$ zss

M. et sig. - Rub on the gums, and use $1 \mathrm{dr}$. to half a wineglass of water as a mouth-wash. 
Or: B Tinc. myrrhæ,

Tinct. gambiris comp.............ä $10.00 \ldots \ldots z$ iiss

Or :

M. et sig.-As above.

B Tinct. krameriæ,

Tinct. myrrhæ...

M. et sig.-As above. .$a \bar{a} 20.00 \ldots \cdots 3 \mathrm{v}$

Or :
B Tinct. myrrhæ,
Tinct. cinchonæ comp...............äa $2.00 \ldots \ldots 3$ ss
Ol. menth. piper.....................gtt. vi .....gtt. vi
Alcohol dil........................100.00.....

M. et sig.-As above.

Calomel should be given only 3 or 4 days, the mouth-wash being used meanwhile, and then should be discontinued for at least as long a time. If necessary, it may be given again and even a third time.

Diarrhœa.-If it produces diuresis but brings on diarrhœa at the same time, 0.05 G. ( $3 / 4$ gr.) of pulvis opii may be added to each dose.

Constipation.-If, on the other hand, constipation is present in spite of the calomel, other cathartics must be given, to avert the danger of mercurial poisoning. Cachetic individuals should not be treated with calomel. [Patton administers digitalis for 2 or 3 days if the pulse is weak and intermittent, before giving calomel in $3 \mathrm{gr}$. doses 3 times a day for 2 days. To prevent the bowels from moving during the exhibition of calomel, he preseribes 5 drops of deodorized tincture of opium $1 / 2$ hour after each dose of calomel. The diuresis may be delayed for 12 hours after the last dose of calomel; it usually begins, however, in the second day of its administration and lasts some time.-Ed.]

There are a number of other drugs which may be useful, although they are not such effective diuretics as those already mentioned.

Squills. - The first is squills. Like digitalis it brings on diuresis by strengthening and slowing the heart contractions and at the same time raising the peripheral blood pressure. It is, therefore, contraindicated when the blood pressure is already high.

Administration.-We give the dried scales in powder or pills, $0.10-0.30$ G. (1 $1 / 2-41 / 2$ gr. $)$, several times a day.

B Scillæ...............................00.... $z^{\mathbf{i}}$

Pulv. glycyrrhizæ $\ldots \ldots \ldots \ldots \ldots \ldots \ldots \ldots . .200 \ldots \ldots z_{\text {ss }}$

Ext. gentianæ $\ldots \ldots \ldots \ldots \ldots \ldots \ldots \ldots \ldots \ldots \ldots \ldots$ q. 8.

M. fiat mass. Div. in pil. no. $\mathbf{x x}$.

Sig. $-3-4$ pills daily. $\quad(1$ pill $=$ about 3 gr. $)$

Or the infusion (less good) :

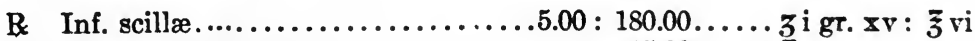

Syr. simplicis ........................

M. et sig. -1 tablespoonful every 2 hours. 
Or we may use preparations of these scales, acetum scillæ or oxymel scillæ (15-30 G. (4-71/2 dr.) a day in mixtures or saturations) ; tinctura scillæ (10-20 drops 3-4 times a day); extractum scillæ, 1 G. (15 gr.) or less a day, in doses of $0.20 \mathrm{G}$. ( $3 \mathrm{gr}$.) and under, preferably in pills :

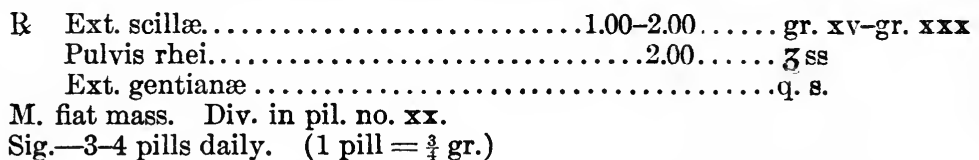

[The well-known Addison's or Niemeyer's pill is a happy combination of digitalis, calomel, and squills (āà gr. i). We employ 3 or 4 pills a day for 4 or 5 days at a time. A word of warning in its selection may be timely,-I have observed more salivation from its use than from the use of calomel alone; probably because it is given too long at a time.-Ed.]

Nitrates of Potassium and Sodium.-Next come the nitrates of potassium and sodium. Potassium nitrate is much oftener used than sodium, although neither is valued as highly as formerly. We do not know exactly how its diuretic effect is produced, but it probably stimulates the renal epithelium directly. Its action is temporary and generally slight, without bad effect, unless the mistake is made of giving it on an empty stomach, in too large doses, or for too long a time. The amount should not exceed $10 \mathrm{G}$. $(2 \mathrm{r} / 2 \mathrm{dr}$.) a day, given in the solution or the diuretic tea prescribed on p. 106.

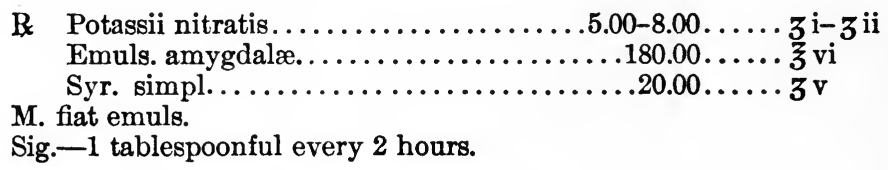

Potassium Acetate.-Potassium acetate is often used as a diuretic. On account of its attraction for water and consequent liquefaction when exposed to the air, it is used in a 40 per cent. solution as liquor potass. acetatis or potass. acetatis solut. Oxidized in the organism wholly or in part into a carbonate, it increases the alkaline reaction of the urine and therefore appears contraindicated in alkaline urine or in phosphaturia. Not more than 30 G. $(1 \mathrm{oz}$.$) a day should be given, in mixture or added$ to a diuretic tea. For example:

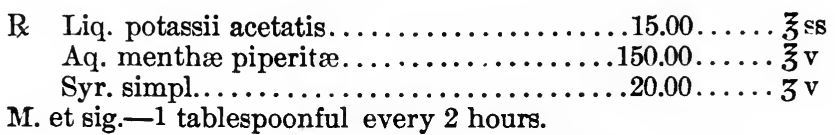

Plasmogenic Diuretics-Diuretic Tea.-We shall conclude the list of diuretics with mentioning the diuretic tea. It has but little or no effect in cardiac dropsy and therefore will be discussed at greater length elsewhere (see p. 106). Like mineral water its diuretic effect is due to 
its increasing the amount of fluid in the blood and therefore the amount of water excreted. The drugs themselves, of which there are a great number to choose from, have very little influence. The following are suitable formulas:

B Bacc. juniperi,

Rad. asparagi (both are to be omitted if the kidneys have acute inflammation),

Rad. ononidis spinosæ,

Rad. levistici,

Rad. petroselini.................. āa $15.00 \ldots \ldots \bar{z} \mathrm{ss}$

Or :

M. et sig. -2 teaspoonfuls to make tea. 1 cupful 2-3 times a day.

B Rad. apii graveolensis,

Tritici,

Rad. glycyrrhizæ,

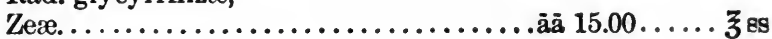

M. et sig.-As above.

Milk as a Diuretic.-W'There remain the dietetic diuretics, milk and milk sugar. Milk has an undoubted diuretic action, due not merely to the quantity of fluid ingested, as it far surpasses that of an equal quantity of water. It is better not to give milk exclusively for very long, but to give also a mixed diet, or at least eggs and all forms of food made with milk, or (see p. 113) a diet poor in common salt. A vegetable diet is better than an animal diet, because it contains more water. The daily quantity of milk should be 2 qt., to which may be added a large glass of some alkaline mineral water, e.g., Radein, Giesshübl, or Seltzer, the carbon dioxide being allowed to escape. This amount of milk cannot of course be given exclusively for any great length of time (5-7 days). After this we may give in addition foods made with milk and eggs, and very digestible farinaceous foods, such as zwieback, erackers, etc. A milk diet is naturally contraindicated when a large quantity of fluid is dangerous on account of a much weakened heart muscle or asystole of the heart. Hence it is important to know to what especial constituent of milk its diuretic action is due.

Lactose.-This has been proved (especially by Sée's investigations) to be its milk sugar. But Dujardin-Beaumetz has shown that all the other sugars, as saccharose and dextrose, have a diuretic action like that of lactose. This seems reasonable, as all the sugars are converted into grape sugar in the liver.

Limitations of Effect.-The effect of all of them, as Sée has shown in the case of lactose, does not persist when inflammation of the kidney exists, or when the albumin content is above 0.08 per cent.

Dujardin recommends using dextrose in a syrup as follows:

B Dextros. purificat.................750.00..... lb. iss

Aq. dest............... s. ut fiat syrupi $1000.00 \ldots$... Oii

Adde: Tinct. aurant. q. s. to flavor.

M. et sig. -1 tablespoonful 5 times daily. 
If this has produced no effect in 8 or 9 days, it should be permanently discontinued; if it has increased the urine, it should be stopped for a few days and then tried again.

Digitalis, Diuretin, Scilla (Solution).-In practice we more often use a combination of diuretics than any one, such a combination being more effective. For example:

B Inf. digitalis......................180.00.... $\mathrm{z}$ vi

Diuretini.....................1.00. $5.00 \ldots \ldots$ gr. $\mathbf{x v - 3 i ~ g r . ~} \mathbf{x v}$

Oxymel scillæ or succi juniperi..........15.00.... $\xi_{\text {ss }}$

M. et sig. -1 tablespoonful every 2 hours.

\section{Diuretin, Digitalis (Powder).-Or:}

B Diuretini..................................

Pulv. digitalis............................ iss

M. fiat cachet no. i. Dent. tal. cachets no. $x$.

Sig. -1 cachet 3 times a day.

Digitalis, Camphor.-The following combination (Zangger) seems especially suited to cases where there is a severe lesion of the cardiac muscle :

B Pulv. digitalis ........................ $0.10 \ldots \ldots$ gr. iss

Camphoræ ....................0.05-0.10.....gr. ${ }^{3}$-iss

M. fiat pulv. no. i. Dent. tal. dos. no. x (chartulæ ceratæ).

Sig. $-3-4$ powders daily.

\section{Digitalis, Calomel.-Or:}

R Pulv. digitalis.......................... $0.10 \ldots \ldots$ gr. iss Hydrargyri chloridi mitis.............. $0.20 \ldots \ldots$ gr. ii

M. fiat pulv. no. i. Dent. tal. dos. no. ix.

Sig. -3 powders daily.

Digitalis, Strophanthus, Caffeine, Liq. Potass. Acet.-Fürbringer and Kohlschütter recommend:

B Inf. digitalis....................... $150.00 \ldots \ldots \mathrm{z}$

Tinct. strophanthi...................... $3.00 \ldots \ldots l \nabla$

Caffeinæ citratæ.............................

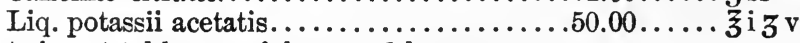

M. et sig. -1 tablespoonful every 2 hours.

The author combines the infusion of digitalis with a double salt of caffeine, and recently has often used theocine or its preparations with digitalis. About $4 \mathrm{G}$. $(1 \mathrm{dr}$.) of potassium nitrate is also often added to the daily quantity of diuretic tea to increase its effect.

Urea.-Recently. the author has tried successfully the simultaneous administration of diuretin (agurin) with another diuretic, urea, which so 
far has not been mentioned because in cardiac hydrops the author never uses it alone. The prescription is as follows:

B Diuretini,

Ureæ ............................āā 0.50.....gr. viiss

M. fiat pulv. no. i. Dent. tal. dos. no. xl (chartulæ ceratæ).

Sig. $-4-8$ powders daily.

Vinum Diureticum.-Trousseau recommends the so-called "vinum diureticum de l' Hôtel-Dieu":

B Digitalis ...........................10.00.... 3 iis

Scillæ................................5.00..... $\mathrm{z} \mathrm{igr.} \times \mathrm{x}$

Juniperi .............................5

Vin. albi.............................. $550.00 \ldots \ldots$ Oiss

Macerate for 4 days, then add :

Potassii acetatis .....................

Sig. $-1-4$ tablespoonfuls $3-4$ times daily.

Sirop des Cinque Racines.-The French employ a peculiar "sirop des cinque racines," similar to the following:

B Rad. apii graveolensis,

Rad. asparagi,

Rad. fœniculi,

Rad. petroselini,

Rad. rusci aculeati.................åa $10.00 \ldots \ldots z$ iiss

Make infusion, strain, add :

Aq. dest.......................... $8.150 .00 \ldots \ldots \tilde{z} \mathrm{v}$

Adde : Sacchar. lactis......300.00 ( $\bar{\xi} \times)$ ut fiat syrup.

Sig. -2 tablespoonfuls a day.

Hydragogin.-The new "hydragogin" is a combination of heart stimulant and diuretic, composed of tinct. digitalis, tinct. strophanthi, scillipicrin, scillitoxin, and oxysaponin. Ten drops in Seltzer water are given hourly for 2 days. The author has repeatedly tried it, without any remarkable success. It is contraindicated in nephritis.

Cathartics and Purges.-All these diuretics are of use only when the kidneys are intact and the heart muscle.only moderately affected. If this is not the case, we may have recourse to cathartics and purges. The cathartics (vegetable and saline) may also be used in milder cases of heart lesions, and must always be given if constipation is present. But in severe hydrops, drastic purges are necessary, since the object is not only to increase intestinal peristalsis, but also to increase the secretion of the intestinal juices to such a degree that the fluid collected in the connective tissues shall be drawn out. Peristalsis aids this by securing a quick passage of the intestinal contents (bile and pancreatic and intestinal secretions) through the colon, and so preventing the normal reabsorption of the intestinal juices. The blood is then left so poor in water and salt that it must draw water from the cedematous tissues. Both the intestinal 
juices and the blood, then, when drastic purges are used, take water from the organs affected (Nothnagel). Of these hydragogues, the chief are jalap, scammony, podophyllin, gamboge, and colocynth. Calomel might be included, though in hydrops resulting from heart lesions it is of much more use as a diuretic than as a purge.

Jalap.-Jalap is the purge most commonly used. Its action requires contact with an alkaline medium, and it has therefore no effect until it has entered the intestine. Even in small doses jalap produces several fluid stools within an average of $2-3$ hours. In moderate doses it may also cause nausea, vomiting, and colicky pains. To avoid this, it is well to add the extract of belladonna or of hyoscyamus $(0.01-0.03$ G. $-1 / 6-1 / 2$ gr.). It is of course contraindicated when the intestine is already irritated or inflamed. It is used in the powder, resin, tincture, or soap ; e.g.:

B Pulv. jalapæ $\ldots \ldots \ldots \ldots \ldots \ldots \ldots \ldots \ldots \ldots, 0.30 \ldots \ldots$. gr. $v$

Extr. hyoscyami....................... $0.03 \ldots \ldots$ gr. ss

Sacchar. lactis..................... $0.20 \ldots \ldots$ gr. ii

M. fiat pulv. no. i. Dent. tal. dos. no. $x$.

Sig. $-1-3$ powders daily.

Jalap is one of the principal constituents of our laxative pills (aloes, jalap, sapo, and anise), 2-10 pills (0.20 G.-3 gr. each) daily.

Resina jalapa is prescribed as follows $(0.10-0.50$ G. $-11 / 2-71 / 2$ gr. a day) :

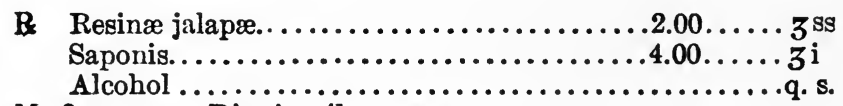

M. fiat mass. Div. in pil. no. $\mathbf{x x}$.

Sig. $-3-4$ pills daily.

The author recommends the tinctura jalapæ composita of the German pharmacopœia (pulv. jalapæ, 8.00 ; rad. turpethi, 1.00 ; scammoniæ halepens, 2.00; alcohol dil., 96.00); daily dose, 5-10 G. (1-2 teaspoonfuls). We may also use the composite tincture of jalap as follows:

B Tinct. jalapæ (Br. Ph.),

Syr. sennæ,

Syr. rhamni purshianæ............āā $30.00 \ldots \ldots$ żi

M. et sig. $-1-2$ tablespoonfuls daily.

[Babcock orders a tablespoonful of a saturated solution of sulphate of magnesia hourly until it begins to exert its effect and allows the patient to follow each dose with only a swallow of water, just enough to remove the bitter taste. The addition of 5-10 drops of Jamaica ginger to each dose will prevent the bad feeling in the stomach. Smaller doses should be persisted in day after day until the dropsy is removed. Hay recommends $2 \mathrm{oz}$. Epsom salts dissolved in $2 \mathrm{oz}$. water, to be given when the alimentary 
canal is empty after fasting and total abstinence from fluids for 12-18 hours. I usually employ Epsom salts in 1 dose of an ounce with as little water as possible. If that does not produce several watery evacuations, I use repeated doses as recommended by Babcock. Where satisfactory results do not follow or where the patient complains of the severity of the treatment, I usually try the compound jalap powder reinforced with cream of tartar as above. The important point to be urged is that we should persevere with our attempts until results have been assured, i.e., numerous copious watery discharges and reduction of the œdema.-Ed.]

Colocynth and gamboge the author seldom uses. They both, in somewhat large doses, irritate the mucous membrane and induce symptoms of gastro-enteritis. Colocynth may be prescribed as follows:
R Ext. colocynthidis,
Ext. rhei........................āā $1.00 \ldots \ldots$ gr. xv
Ext. gentianæ.............................. q.
M. fiat mass. Div. in pil. no. $\mathbf{x x}$.
Sig. $-3-5$ pills daily.

They are usually, however, combined; for instance, in Trousseau's pills:

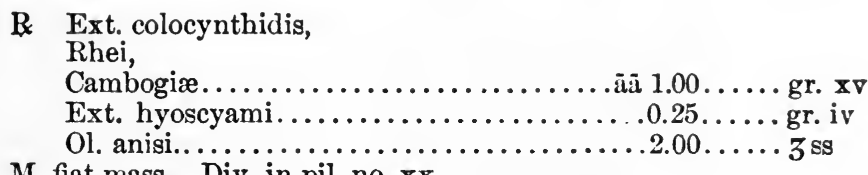

M. fiat mass. Div. in pil. no. $x x$.

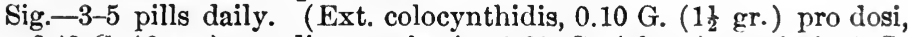

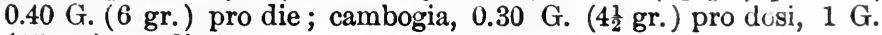
(15 gr.) pro die.)

The tincture of colocynth may be given in doses of 5-10 drops $(0.30 \mathrm{G}$. $\left(4 \frac{1}{2}\right.$ gr.) a day), either in some mucilaginous vehicle or combined with some diuretic.

Neither of these purges should be given if symptoms of intestinal inflammation are present. They must be either stopped entirely or much reduced in quantity if such symptoms appear.

Podophyllin.-Podophyllin the author uses oftener, especially for somewhat prolonged treatment:
R Podophyllini.........................1.00....gr. xv
Ext. belladonnæ....................... $0.40 \ldots \ldots$ gr. vi
Pulv. frangulæ $\ldots \ldots \ldots \ldots \ldots \ldots \ldots \ldots \ldots \ldots \ldots \ldots$. $.00 \ldots \ldots$ gr. $\mathbf{x x x}$
M. fiat mass. Div. in pil. no. $x x$.
Sig. -1-2 (or more) pills every evening.

Diaphoretics.-Finally, there is a third way to get rid of the accumulated fluids in hydrops-by diaphoresis. But pilocarpine, practically the only drug used as a diaphoretic, is contraindicated in heart lesions on account of its depressing influence on the arterial pressure and heart 
action (shown first by Kahler and Soyka). Hence in hydrops resulting from heart lesions, we are practically confined to diuretics and cathartics.

Surgical Therapy of Hydrops.-In many cases the accumulation of fluid in the serous cavities has reached such a point that surgical interference must be called in. This is partly operative, partly mechanical. The operative therapy is a double one, directed towards (1) the reduction of the cedema of the lower extremities, and (2) the removal of the fluid from the peritoneal, pleural, and pericardial cavities.

Incision.-There are three methods of relieving the œdema of the lower extremities: by simple incision, by aspiration, and by drainage. There is no consensus of opinion as to the best method. The author himself would choose the first two, giving the preference to aspiration rather than to incision, provided proper asepsis can be carried out. Simple incision is performed as follows: The patient should sit up for several hours before the incision is made, and afterwards until the fluid has ceased to flow from the wound. The effect of gravity is thus obtained. Both feet and legs should be scrubbed with soap and water, and the neighborhood of the place to be incised disinfected carefully with bichloride, alcohol, and ether. The spot chosen is generally below the external malleolus on the outer edge or the dorsum of the foot. An incision is made an inch or more long, through all the layers of the skin. The bleeding is stopped, and the foot is bound up in sterile or corrosive gauze and bandaged. The feet are then placed in a foot-tub containing some antiseptic solution or upon a rubber sheet arranged to collect and drain the escaping fluid. The bandage should be changed every day. If for other reasons the patient must stay in bed, the feet are placed lower than the rest of the body; otherwise the procedure remains the same. The wound generally heals without the necessity of a stitch. Ziemssen recommends a much longer and deeper incision, 4-6 inches long and an inch deep, penetrating at least into the subcutaneous tissues, and at the back or sides of both legs. Such an energetic procedure naturally gets rid of the fluid very quickly.

Puncture.-The second method, introduced by Southey, is that of trochar puncture. The author prefers it to the others because it is the most comfortable one for the patient. The spots selected are usually on the back or outer sides of both legs and are prepared as above. Several carefully sterilized trochars-generally four-are then thrust an inch or more into the subcutaneous tissue, up to the silver or gold enlargement on the cannula. The trochar is then drawn out and the free end of the cannula connected with a rubber tube previously filled with an antiseptic fluid. The other end of the tube hangs in a receiving vessel placed on the floor or on a stool if the patient is sitting up, or on the floor or between his legs if he is lying down. The skin around the puncture is painted with iodoform collodion or packed in salicylate gauze. This procedure will generally insure the closure of the wound after the removal of the cannula. [Sterilized dressings are sufficient, less irritating, and more 
agreeable.-Ed.] Each cannula is fixed by means of silk thread and surgeon's plaster. Southey's cannula has a circular lumen of about 1 $\mathrm{mm}$. in diameter; Curschmann's is elliptical with a two-edged lancetshaped stilette. He uses two of them instead of four.

Funnel Drainage.- The third method is that of funnel drainage, introduced by Straub, and modified by Curschmann and Buchwald. 'The wound is made in the thigh or the side of the leg. After scarification, a funnel of about $8-10 \mathrm{~cm}$. (3-4 in.) is applied. Its semicircularly bent waste-pipe is connected with a rubber tube a yard long, which may be closed by means of a stop-cock. The tube and the funnel are both filled with an antiseptic fluid, a paper being kept over the opening of the funnel until the apparatus is in place. Buchwald uses an empty glass funnel, which is filled when in place through an opening in the side. The force of suction, the strength of which depends on the level of the water in the tube, holds the funnel fast to the skin and sucks the skin into it, as in the process of cupping. The procedure is better in theory than in practice, for the funnel often falls off when the body is moved, and cannot be applied at all to certain parts of the body (hands, arms, feet).

Dangers and Advantages of Incision and Puncture.-There is danger of infection in both incision and puncture. This seems to the author to be less likely to arise in incision than in puncture, as in the former procedure there is no foreign body in the wound and the fluid flows out more rapidly and abundantly. He has, however, never seen a case of infection from puncture, although he has made very frequent use of the procedure. The second process is neater and less disagreeable for the patient than to have his extremities continually wet, but this advantage is much more than counterbalanced by the greater rapidity with which the œdematous fluid is removed by a large incision (often of the very greatest importance), and the relative slightness of the pain, especially if we follow Schurz's method. He considers puncture and drainage contraindicated in the case of scleroderma resulting from long. standing œdema, which presents, however, no obstacle to incision. $\mathrm{He}$ maintains that from three incisions 4452 c.c of fluid escape in 24 hours; from three cannulæ, only 3351 c.c. (according to Kopp, 2000 c.c.). This comparison, however, is of no great importance, as the number of incisions and of cannulæ can be increased at will. It remains to be said that the loss of so much fluid by any one of the methods and the accompanying loss of proteid often bring on symptoms of cerebral anæmia (giddiness, unconsciousness, nausea), conditions which demand immediate stoppage of drainage, and the administration of stimulants, the patient being laid flat in bed.

The operative therapy of hydrops in the serous cavities includes the puncture of the abdominal, the pleural, and, very rarely, the pericardial cavities.

Abdominal Puncture.-An abdominal puncture is indicated in all cases where, in addition to the development of large quantities of fluid in 
the subcutaneous tissues, the fluid has collected in the abdominal cavity in such quantities that the work of the weakened cardiac muscle is greatly increased, the dyspnœa is extremely marked, and the pressure on the stomach and intestines makes it impossible for the patient to receive proper nourishment.

There is a second class of cases in which we proceed to removal of the excessive ascites. We often find cardiac cases where the broken compensation has not proceeded very far, where there are only traces of œdema of the lower extremities, and where no fluid is present in the pleural and pericardial cavities, and yet where the ascites is very considerable. This is explained by the peculiarity of a so-called atrophic nutmeg liver produced by the intrahepatic compression of the portal branches, which compression is exerted by the connective tissue, newly formed as a result of stasis in the territory of the inferior vena cava and secondarily of the hepatic veins. A similar obstruction to the intrahepatic portal circulation often occurs when there is no new connective tissue around the hepatic veins, but only a diffuse distention of the branches of the hepatic veins in consequence of stasis. Such marked ascites may be accounted for in one of these ways. (The so-called "sugar liver" (Zuckergussleber) in synechia pericardii is not included in this discussion.) These cases of cardiac cirrhosis or atrophic nutmeg liver often require repeated punctures.

Technic of Abdominal Puncture.-We usually use a trochar fitted with a stop-cock and with a rubber tube. The point generally chosen lies in the perpendicular to the line between the umbilicus and symphysis pubis at its middle point, and as far to one side (usually the left) as possible. In perintoneal exudates the puncture is made in the middle of the collection of the fluid. The spot selected should be free from visible bloodvessels, especially veins. [I nearly always select a point in the median line just above the pubis,--first being sure that the bladder is empty. This necessitates a sitting posture, which is the one commonly assumed by the patient by choice on account of the pressure of the fluid Such a selection does away with the annoyance of bleeding.-Ed.] It is first cleaned with soap and brush, corrosive sublimate, ether, and alcohol. If œdema of the abdominal wall is present, it is reduced over the spot by massage. The trochar, held firmly between the palm of the hand and the thumb, is thrust in until suddenly no resistance is felt. Or else an incision $2 \mathrm{~mm}$. long is first made through the skin with a very sharp scalpel. This has the advantage of demanding much less strength in the thrust, since the skin offers the greater part of the resistance; it is, therefore, a less painful procedure. The needle is now withdrawn, the stopcock opened, and the fluid allowed to escape as long as it will spontaneously. Generally a sudden backward movement of the trochar shows when the fluid has stopped. The cannula is then quickly withdrawn, and the wound closed with iodoform gauze and iodoform collodion, and secured by several cross strips of iodoform gauze, fastened to the skin 
with collodion, the patient lying on the side opposite the puncture. This simple bandage prevents all oozing from the wound-a process very annoying to the patient-and is therefore strongly recommended by the author. [A simpler and perfectly satisfactory dressing is a piece of adhesive plaster previously soaked in bichloride solution or passed through the flame. I have never scen any bad results from its use. If the wound leaks, a more complicated dressing rarely prevents the oozing, which in any event is hardly more than a slight and transitory discomfort to the patient and trouble to the attendant.-Ed.]

Dangers of Puncture.-In any abdominal puncture, stimulants (wine, cognac, camphor) should be at hand, as the sudden expansion of the abdominal organs may cause slight cerebral anæmia, especially if the fluid escapes quickly. Otherwise the process is not at all dangerous. If proper asepsis is preserved, peritonitis is practically excluded. Injury to the intestine, especially in ascites, is almost impossible. There is somewhat more danger of it in a circumscribed peritonitis, but even here it may be avoided with certainty provided the abdomen has been carefully percussed and palpated beforehand to determine whether any fold of the intestine is adjacent to the abdominal wall at the spot chosen. If desired an exploratory puncture can be made first. Very rarely a large bloodvessel may be injured. Friedrich has reported a case of hæmatoma of the peritoneum.

In general the relief afforded by tapping is only temporary, but there are cases in which the ascites, caused by compression of the intrahepatic branches of the portal veins by markedly distended hepatic veins, does not recur for a long time. This is because the cardiac stimulants used after a puncture regulate the circulation in the region of the inferior vena cava and the portal veins, and so remove the basis for any fresh development of ascites. [Free catharsis exhibited promptly after aspiration and vigorous digitalis medication will often obviate or delay for some time the necessity of subsequent paracentesis.-Ed.]

Thoracentesis-Paracentesis of the Pericardium.-Puncture of the thorax is much more rarely undertaken than that of the abdomen, while the pericardium is almost never punctured. According to most authors, thoracentesis is resorted to in hydrothorax only when the pressure in the pleural cavity causes such extreme dyspnœa and increased work for the heart that life itself is directly threatened,-a condition of affairs that seldom arises. The author will therefore defer the discussion of this procedure until the therapy of pleural exudates is taken up (see p. 366). It has been recently suggested that a puncture should be made even in moderate cases of hydrothorax or a pleuritic fluid in broken compensation, it being claimed that the symptoms of cardiac insufficiency are thereby quickly relieved. The author is not ready to express any decided opinion on this point. [We remove fluid from the pleural cavity in cases of broken compensation earlier than Continental clinicians. I have never regretted early thoracentesis, but often have believed that I delayed too 
long. Forchheimer has recently urged very prompt removal in pleuritic effusions. There seems to me even less ground for delay where the mechanical effect is still more important, as in broken compensation with general anasarca, dyspnœa, and cyanosis. If one reasons that this accumulation is an effort of the organism to compensate for the weakness of the heart by temporarily withdrawing a certain amount of fluid from the blood mass in circulation, early thoracentesis seems imperative.-Ed.]

Mechanical Therapy and Hydrotherapy: Massage.-The mechanical therapy of hydrops is limited to massage, which has an excellent effect in moderate œdema of the lower extremities and also in many cases of ascites, provided they are taken at the very start. [The diagnosis of small amounts of fluid in the abdomen is so difficult and uncertain, that I do not believe that massage of the abdomen is of any particular service.-Ed.]

Hot Baths.-In relieving hydrops which arises from conditions other than cardiac insufficiency, hot baths are exceedingly efficacious. Recently many authors, particularly Kohlschütter, have contended that they may also be used to great advantage in the form of hydrops under consideration. They employ a tub bath at a uniform temperature of at least $97^{\circ}$, lasting on an average half an hour. [We should discourage the frequent use of baths hotter than $97^{\circ}$ by patients even with well compensated heart lesions.-Ed.] They admit that with certain patients symptoms of heart irritation (quick, irregular pulse) and of increased blood pressure in the brain (excitement, headache, giddiness, trembling of the eyelids, noises in the ears, throbbing of the carotid) are likely to occur, but they maintain that such symptoms also arise when the hydrops has a different origin, and that they generally may be overcome by the use of stimulants and the application of cold to the head during and after the bath, or may be avoided entirely by lowering the temperature and decreasing the duration of the bath. Only when these measures fail, would they consider it necessary to give up hot baths in treating hydrops arising from cardiac insufficiency. The author can only say that he almost never has had the courage to put such a patient into a hot bath, and therefore can give no information on the subject. [Beyond the general tonic effect of such a bath $I$ do not understand its action in reducing dropsy. Neutral baths $\left(94^{\circ}\right)$ prolonged for $3 / 4-11 / 2$ hours have been recently extolled by Strasser and Blumenkranz in nephritis. I have thought that they did good service in the few cases I have tried, and I should much prefer to use this temperature in eardiac cases with failing compensation.-Ed.] The only kind of heat that he can recommend is steam or hot air, which is often very well borne. The patient can be given a steam bath sitting in a cabinet, the head alone protruding, an ice-bag or cold compress being bound about it. Or the patient may lie in bed, and a tent be constructed over him by throwing a blanket over a wire frame, the head again protruding and being kept cool by ice or cold compresses. The steam is introduced through a pipe from a steaming 
kettle. The duration of the bath should be regulated by the condition of the patient, but may be an hour or more. Afterwards he should be wrapped in a blanket for an hour or two to sweat.

Hot-air Baths.-The best device in the author's opinion is the socalled phénix à l'air chaud. Here a basketwork frame is put over the patient, reaching from the feet to the breast or only to the abdomen, and hot air introduced from a heated kettle outside.

Such baths have no bad effect on the patient and (according to Dehio) often cause the disappearance of the cedema and consequently of the transudation into the serous cavities. The author considers them the only safe and rational way of producing diaphoresis in hydrops from cardiac insufficiency.

Medicated Baths.-The next therapeutic measure to be considered is medicated baths either alone or in connection with gymnastics. They may be used either in cases of slight lack of compensation or in those accompanied by symptoms of stasis. It may also be mentioned in anticipation that they are no less useful in diseases of the cardiac muscle. The baths are copied from those at Nauheim, Franzensbad, Marienbad, Cudowa, Kissingen, etc.

Salt Baths.-We begin with 1 per cent. common salt and 0.1-0.2 per cent. chloride of lime, later increasing to 2 or 3 per cent. common salt and 0.25-0.3 per cent. chloride of lime. Instead of the latter, mother-liquor containing calcium chloride may be used.

Carbon-dioxide Baths.-For a weak bath we use $1 / 2 \mathrm{lb}$. bicarbonate of soda and $3 / 4 \mathrm{lb}$. hydrochloric acid, increasing these quantities proportionately for a stronger bath. The large amount of bicarbonate of soda is intended to provide against any excess of acid, which may injure the skin of the patient. If a slow development of carbon dioxide is wanted, an open bottle filled with the acid is laid in the bottom of the tub and the acid allowed to escape gradually; if a quick development is wanted, the bottle is moved quickly to and fro just beneath the surface of the water, in which case the bath will be ready in a few minutes. The temperature varies between $92^{\circ}$ and $80^{\circ}$; generally we begin with $89^{\circ}-90^{\circ}$ and reduce it to $82^{\circ}-84^{\circ}$. The duration is 5 minutes, gradually increased to 20 minutes. At first the bath should be omitted every other day, then only every third day, till finally it may be given every day if the patient bears it well, as shown by an absence of dyspnœa during the process. After the bath the patient must be rubbed down with hot cloths, and care taken to keep up a good circulation in the extremities. The treatment may be kept up for $4-6$ weeks (25-30 baths) or even for 10 weeks and longer with longer pauses between the baths.

[Nauheim is too distant for many of our patients, so that a few details in regard to the preparation and employment of the baths at a patient's house or at some institution will be useful. When possible the latter should be selected, because the baths require a certain kind of tub,-slate, porcelain, enamel, or wood; because the patients need careful and skilled 
supervision at first, not only during but for some time after the bath; and because the psychical influence is still stronger away from home. At Watkins Glen, New York, the treatment is well given. A convenient method of preparing the baths at a patient's home is recommended by Satterthwaite. He employs American sea salt or Genesee dairy salt, and an acid sodium sulphate in place of the usual hydrochloric acid. The Cassebeer Pharmacal Co., 108 Fulton St., New York City, supply the carbonating ingredients in a wooden box, 8 by 6 by 4 inches, containing 8 discs of the acid sodium sulphate wrapped in heavy lead foil and 4 packages of sodium bicarbonate. The dises are broken into pieces with a hammer and put upon a plate, the soda is powdered over them, and the plate put into the tub. I prefer putting them upon three plates, at the middle and either end of the tub. He plans an ordinary course to last 6 weeks.

First Week.-Plain warm salt bath $\mathrm{I} / 2$ per cent. ( $2 \mathrm{lb}$. of bath salt to a 50 gal. bath). Temp., $98^{\circ}$. Duration, 4 minutes. Third and 6 th day of week bath omitted.

Second Week.-Warm salt bath $3 / 4$ per cent. ( $3 \mathrm{lb}$. to 50 gal.) plus $1 / 4$ per cent. carbonic acid gas ( 2 dises of acid sodium sulphate to 1 package of bicarbonate of soda). Temp., $97^{\circ}$. Duration, 6 minutes. Fourth day of week bath omitted.

Third Week.-Warm salt bath 1 per cent. ( $4 \mathrm{lb}$. to 50 gal.) plus $1 / 2$ per cent. $\mathrm{CO}_{2}$ ( 4 dises to 2 packages). Temp., $96^{\circ}$. Duration, 8 minutes. Omit bath on 4th day.

Fourth Week.-Warm salt bath 1/4/4 per cent. (5 lb. to 50 gal.) plus $3 / 4$ per cent. $\mathrm{CO}_{2}$ ( 6 dises to 3 packages) plus $6 \mathrm{oz}$. calcium chloride or $8 \mathrm{oz}$. Nauheim "Mutterlauge." Temp., 95. Duration, 10 minutes. Omit bath on 4th day.

Fifth Week.-Warm salt bath $11 / 2$ per cent. (6 lb. to 50 gal.) plus $3 / 4$ per cent. $\mathrm{CO}_{2}$ (6 dises to 3 packages), plus $12 \mathrm{oz}$. CaCl or $16 \mathrm{oz}$. "Mutterlauge." Temp., $94^{\circ}$. Duration, 12 minutes. Omit bath on 5th day.

Sixth Week.-Warm salt bath 2 per cent. ( $8 \mathrm{lb}$. to 50 gal.) plus 1 per cent. $\mathrm{CO}_{2}$ ( 8 discs to 4 packages) plus $12 \mathrm{oz}$. CaCl or $16 \mathrm{oz}$. "Mutterlauge." Temp., $93^{\circ}$. Duration, 14 minutes. Omit bath on 5th day.

In this method, the least troublesome I know, the expense of each bath is less than at Nauheim; but a cheaper method is one in which the bicarbonate of soda is dissolved in the warm water at the foot of the bath tub and allowed to diffuse gently through the bath. The salt and the calcium chloride are often omitted without apparently detracting much if any from the utility of the bath.-Ed.]

Both kinds of baths produce similar effects: stimulation of the cutaneous nerves, transmission of the stimulation to the vasomotor centre, stimulation of the heart action, exercise of the heart muscle, and slowing of the heart action. Whether the blood pressure is raised, unaffected, or lowered, depends upon the concentration and temperature of the bath; the stronger the concentration and the lower the temperature, the higher the blood pressure. 
When the compensation is far from intact, baths, gymnastics, and massage should not be used alone, but should be accompanied by heart tonies.

Gymnastics.-Schott lays down the following principles for his system of gymnastics, consisting of slow movements of the trunk and extremities against a slight resistance offered by the physician or some other person: (1) The movements should be slow, steady, and without any strain; (2) The same movements are never to be immediately repeated; (3) Each fresh exercise must bring into play new groups of muscles; (4) There must be a pause after each exercise; (5) The rate of respiration and the pulse must be controlled; [(6) The onset of palpitation, rapid respiration or dyspnœa, change in pulse tension, or nervousness, suggests that the movements should be stopped until such signs disappear; (7) The operator's resistance should be very slight at first and gradually increased with each subsequent treatment; (8) After the treatment the patient should rest; (9) The treatment should be given neither immediately before nor for some time after meals; (10) It is often wise to begin the treatment with the patient in a recumbent posture.-Ed.]

[The following list of Schott movements has been copied from Forchheimer :

I. The arms are extended in front of the body on a level with the shoulders, the palms of the hands meeting in front of the chest. They are now moved outward until in a line with the chest, the operator resisting by placing his hands back of the wrist of the patient. The arms are then moved back to their original position. This movement is resisted by placing the operator's hands on the palmar surface of the wrist.

II. Right arm hanging down, palm forward, is flexed until fingers touch shoulder, and is returned to original position. The upward movement is resisted by operator's hand on palmar surface of wrist, the downward movement by the operator's hand on the dorsal surface of the wrist. Repeat with left arm.

III. Both arms hanging down, palms forward, are raised outward without bending the elbow, until the thumbs meet above the head, and returned to original position. The upward movement is resisted by operator's hand on the radial side of the wrist, the downward movement by the operator's hand on the ulnar side of the wrist.

IV. Both arms hanging down, palms against thighs, are raised forward, without bending the elbows, until they are vertically extended, and returned to original position. The operator must study this movement with care in order to offer continuous resistance. In the beginning the fork of his thumb and forefinger should be applied to the radial side of the wrist; when the arm is at an angle of $45^{\circ}$ to the body the fingers must slide around the wrist until they are folded about the radial surface. Before the reverse movement begins he receives the ulnar aspect 
of the wrist in the fork of his hand. As the arm descends to $45^{\circ}$, the thumbs move outward and the fingers slide around until, when on a level with the shoulders, the ulnar surfaces of the patient's wrists rest upon and are resisted by the operator's hands.

$\mathrm{V}$. The trunk is flexed forward without bending the knees and brought back again to an erect position. The forward movement is resisted by one hand of the operator over the upper third of the sternum and the other supporting the middle of the lumbar region. The reverse movement is resisted by one hand of the operator over the junction of the cervical and dorsal portions of the spine.

VI. With both feet firmly planted on the floor the patient rotates his body, first to one side and then to the other, and back again. To resist this movement the operator places one hand in front of the advancing shoulder and one over the other shoulder.

VII. The trunk is bent as far as possible first to one side and then to the other, and is finally brought back to an upright position. To resist this movement the operator places one hand on the hip away from which the patient is bending, and with the other presses against the other side in the axilla.

VIII. This movement is like No. II except that the palms are turned outward and the fists are clinched.

IX. Taken in turn, each arm is extended downward, palm against thigh, and then makes a complete revolution from the shoulder-forward, upward, backward, downward. Before descending backward the palm must be turned outward. The operator folds the fingers of one hand around the radial side of patient's wrist. His other hand receives the wrist when it reaches the vertical position, and maintains the resistance until the arm has reached the original position.

$\mathrm{X}$. Both arms are extended downward, palms toward thighs, and are moved backward and forward as far as possible without bending the body. The upward movement is resisted by the fork of the hand on the ulnar surface of the wrist, and the downward movement by sliding the fingers around the radial surface.

XI. The patient holds on to a chair with one hand and flexes the other thigh as far as possible, then returns the foot to the ground. The leg should hang down from the knee-joint. The upward movement is resisted by one hand above the knee and the downward movement by one hand under the sole of the foot or below the lower part of the thigh.

XII. Supporting himself with one hand, the patient lifts one stiffy extended leg as far as he can to the front; then as far as he can to the back, and finally places the one foot beside the other. The forward movements are resisted in front of the ankle and above it, and the backward movements behind the ankle. Repeat with the other leg.

XIII. Resting one hand on a chair, the patient lifts the other extended leg as high as he can laterally. Resistance above the ankle. Repeat with the other leg.-Ed.] 
If the patient is taking baths at the same time, the exercises should be gone through with for a half hour once a day; if not, they may last for half an hour in the morning and 20 minutes late in the afternoon. If the patient shows symptoms of relapse (stasis, albuminuria, angina pectoris) the baths and gymnastics must be given up, medication being continued until he has recovered the lost ground.

\section{Therapy of Congestion of the Viscera in Broken Compensation.}

Congestion of the Viscera is much more Common in Mitral than in Aortic Lesions.-Congestion of the inner organs of the body (lungs, brain, abdominal viscera) occurs much more frequently in mitral than in aortic lesions, since in the former case the extra work is thrown directly upon the weak musculature of the right ventricle. But symptoms of stasis appear also in aortic insufficiency, showing themselves in the lesser circulation and in that of the venæ cavæ. They belong principally, but by no means exclusively, to the stage of broken compensation. The cause is purely mechanical : the dilatation of the left ventricle forces the septum between the chambers into the cavity of the right ventricle, so diminishing the volume of the latter. On account of the narrowing of the space in the right ventricle, disturbances of circulation in the region of both venæ cavæ result, as well as analogous symptoms of stasis.

Passive Cerebral Hyper memia.-These symptoms of stasis will be taken up according to their localization in the different organs, beginning with the brain (passive hyperæmia of the cerebrum and of the spinal cord).

Symptoms.-Clinically the symptoms of cerebral stasis may be divided into two directly opposite groups. Some patients are in a slight stupor, a half slumber, or are wholly apathetic, although their consciousness is otherwise intact. This condition is very apt to precede death. Other patients exhibit symptoms of sleeplessness, confusion, true psychic excitement, with perhaps irregular respiration (especially CheyneStokes breathing) or even mania. These symptoms do not justify an absolutely bad prognosis, as heart stimulants and analeptics may diminish or stop all of them (see p. 12).

One of the most torturing symptoms is sleeplessness. If not relieved, it will still further reduce the strength of the patient, who needs all the sleep he can get to fight the disease. It must, therefore, be treated symptomatically, and narcotics added to the heart stimulants.

Therapy of Sleeplessness.-One warning may be given to begin with: in sleeplessness resulting from hyperæmia of the brain, either in mitral insufficiency or, much more rarely, in aortic insufficiency with broken compensation of the left heart, opium or morphine should if possible be avoided or used only under special conditions to be mentioned later. For, even if Preisendörfer's investigations have shown that morphine in medicinal doses $(0.01-0.03$ G. ( $1 / 6-1 / 2$ gr.) subcutaneously) 
has no particular effect on the vessels, it is well known that opium and morphine reduce the tone of the vasomotor centre in the medulla, therefore decrease the pressure in the peripheral vessels and dilate them, and thereby cause congestion in the brain and cord, just the condition that underlies the sleeplessness. [Compare pp. 68 and 69 for the author's further views upon the employment of morphine in heart disease. Balfour recommends it highly as a hynotic in some cases; so does Patton, who is apt to combine $1 / 4$ gr. with 15 gr. of a bromide salt. Babcock also regards its use hypodermically in $1 / 10^{-1 / 8} \mathrm{gr}$. doses as imperative in many instances when other hypnotics fail to give the heart and the body the needed rest. He mentions in this connection its action as a cardiac tonic.-Ed.] The same is true of chloral. It has always been held that chloral reduces peripheral blood pressure through its effect partly on the vasomotor centre and partly on the heart ganglia. It has been considered to be a direct heart poison and therefore especially contraindicated in heart disease. More recently Geisböck's (in Müller's clinic) careful investigation of blood pressure has refuted this idea. $\mathrm{He}$ has shown that after chloral the decrease in blood pressure in heart disease is hardly appreciable. However, if the blood pressure is already very low, it is best not to prescribe chloral. [Balfour recommends large doses of chloral for sleeplessness dependent upon a senile heart; he employs up to $40 \mathrm{gr}$. an hour for 2 or 3 doses.-Ed.]

Among the suitable hypnotics we may recommend the bromides, sulphonal, trional, and veronal.

Bromides.-The bromides (sodium, potassium, and ammonium) are, to be sure, strictly speaking not hypnotics, since they do not directly induce sleep, but rather depress the reflex irritability of the nervous system and particularly of the brain. In this way many outer impressions, which otherwise would excite the brain, have no effect, and the brain is reduced to a condition of quietude which fosters sleep. [Insomnia in heart disease, as well as in most other conditions, will nearly always yield to the proper administration of the bromides. A single large dose at bedtime is, however, useless. I prescribe 5-15 gr. of strontium or sodium bromide 3 times daily, well diluted with water or milk (a tumblerful); and I usually order a hypnotic, such as veronal or amylene hydrate, for two or three nights until the patient has come partially under the influence of the bromide salt. If pain is at the bottom of the insomnia I add a small dose of codeine and rarely need to employ opium.-Ed.]

The effect of the bromides on the heart and circulation depends upon which salt we select. Sodium bromide has no detrimental effect on the heart. Potassium bromide, on the contrary, according to most authors (Dujardin-Beaumetz excepted) acts as a depressant. Taken either in single rather large doses (10 G.- $-2 \frac{1}{2} \mathrm{dr}$.) or in small doses for a considerable length of time, it not only slows the cardiac contraction (Kross), but also weakens the heart, and lowers the blood pressure. 
Nothnagel and Rossbach report that they were several times obliged with epilepties (and even with other patients with apparently sound hearts) to give up the use of potassium bromide after some months on account of its effect on the heart. In view of such facts potassium bromide should not be given (or at most for only a very short time) to patients with heart lesions, but should be replaced by sodium bromide, for apparently it is not the bromide but the potassium that is the cardiac depressant. Furthermore, the sodium is less liable to upset the digestion and can therefore be given in larger doses. It may be prescribed as follows :

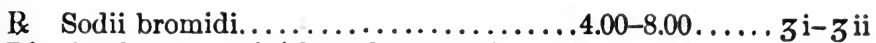

Div. in chart. no. vi (chartulæ ceratæ).

Sig. -1-2 powders in the evening.

This powder should be dissolved either in half a glass or a glass of milk or in an equal quantity of some sparkling mineral water (Erlenmeyer, carbonic, Apollinaris). If it is given in this way even for a considerable length of time, no symptoms of chronic bromism will result.

A second method of giving the bromides, especially sodium bromide, is in solution:

R Sodii bromidi $\ldots \ldots \ldots \ldots \ldots \ldots \ldots \ldots . . . . \ldots 0 \ldots \ldots$ ii

Syr. rubi idæi...................... $45.00 \ldots \ldots$ iss

Aq. dest....................... ad $120.00 \ldots \ldots$.

M. et sig. -1-2 tablespoonfuls in the evening. (1 tablespoonful contains $1.00 \mathrm{G}$. (15 gr.) sodium bromide.)

It should be borne in mind that sodium bromide induces sleep only after repeated doses, so that the patient should be warned in advance not to expect its full effect until after it has been taken, on an average, for four or five days. One to $2 \mathrm{G}$. (15-30 gr.) should be given about a quarter of an hour before the evening meal and the same quantity just before going to bed. [Bromipin in doses of $2-4 \mathrm{dr}$. in the afternoon and evening has proved of value in some of my cases where the bromide salts did not seem to satisfy the needs. It is well to remember that if salt is withheld from the food during the exhibition of the bromides, their effect is greater and smaller doses are required, while an excess of salt aids elimination. The latter fact is useful if symptoms of bromism are induced and we wish to secure rapid elimination.-Ed.]

Sulphonal.-Sulphonal induces a sleep almost exactly like natural sleep, lasting from four hours to the whole night. It undoubtedly acts directly on the brain cells, either in the cerebral cortex (Kast) or also in the cerebellum (Mairet). In rare cases the patient suffers afterward from weariness, slight nausea, giddiness, rumbling, etc. Sulphonal unfortunately is not so certain in its effect in heart lesions, being especially apt to fail in mitral insufficiency (See and Huchard). There have been rare cases in which it even had a bad after-effect on the heart. [Cushny 
says that sulphonal seems to have some deleterious action on the heart when used for long periods and is a much less certain hypnotic in cases of cardiac disease than in other conditions. I have seen two cases of sulphonal habit.-Ed.] If used at all, it must be given about three hours before it is expected to take effect, as the time required for its action varies from one to four hours. Sulphonal is excreted very slowly, so that a dose given 24 hours after a previous one has an increased effect, on account of the sulphonal still left in the body. It is also absorbed very slowly from the digestive organs (Kast), and should therefore be accompanied by a considerable quantity of a hot fluid. It may either be given in or be followed by hot tea, soup, or milk. The usual dose is $2 \mathrm{G}$. ( $30 \mathrm{gr}$.) for men and $1.5 \mathrm{G}$. ( $22 \mathrm{gr}$.) for women, but the author would never advise beginning with more than $1 \mathrm{G}$. (15 gr.) in any case. He prescribes it as follows:

B Sulphonal................ 1.00-1.50-2.00.....gr. xv-gr.xxii-gr. $x x x$

Dent. tal. dos. no. $x$. Dispens. in cachets.

Sig.-Take 1 cachet 3 hours before retiring, with a large cup of hot tea, milk, or soup.

Tetronal and Trional.-Two other narcotics are superior to sulphonal, the rarely used tetronal, and trional, the latter being everywhere preferred to tetronal on account of its greater solubility and its milder taste. Both are quicker in their action than sulphonal (trional takes effect in an hour or sometimes even in a quarter of an hour). Both are free from the after-effects of sleepiness and fatigue. The dose of trional is the same as that of sulphonal:

R Trional....................... 1.00-2.00....gr. xv-gr. $\mathbf{x x x}$

Dent. tal. pulv. no. $\mathbf{x}$.

Sig. -1 powder 1 hour before retiring in a half cup of hot tea, milk, or soup.

Veronal.-Veronal is at least equal to trional, in most cases even more effective. It is a diethylmalonyl urea. Its slightly bitter crystals easily form soluble alkaline salts and therefore it is easily absorbed in the intestine. The dose is $0.50-1 \mathrm{G}$. (7-15 gr.) taken in hot tea or milk; it induces in $1 / 2$ hour to 1 hour a sleep which generally lasts for several hours, almost always with no bad after-effects (headache, weariness, sleepiness).

Neuronal.-Neuronal (the diethylacetamid of bromine) appears to be as good as trional but inferior to veronal. It is a white crystalline powder with a bitter taste resembling that of menthol. The dose is 1-2 G. (15-30 gr.). Its good effect is due to its high content of bromine (40 per cent.).

Paraldehyde.-One of the best narcotics in broken compensation is paraldehyde, a polymer of acetaldehyde, first manufactured by Schmiedeberg, in the form of a colorless liquid. It has no bad effect on the heart 
and vessels even if they are seriously diseased, and lowers the blood pressure by only an insignificant amount. It has two minor disadvantages: it has an unpleasant taste, something like that of chloroform, which, however, may be disguised by the manner of prescription; and it is excreted mainly through the lungs, making the breath, often for 24 hours, smell like that of an intoxicated person. The dose is $2-4 \mathrm{G}$. $(1 / 2-1 \mathrm{dr}$.). The sleep comes quickly, often after ten minutes, and lasts from two to ten hours. [I have seen an alcoholic who substituted this drug and eventually acquired a paraldehyde habit.-Ed.]

B Paraldehydi.................. $10.00-20.00 \ldots \ldots z$ iiss $-3 \mathrm{~V}$ Aq. dest.........................

M. et sig. - 2-3 tablespoonfuls daily in a glass of sweetened water, mixed with rum or brandy.

Or:

B Paraldehydi..........................2.00... 3 हs

Tinct. vanillæ....................gtt.xx.....gtt. $\times x$

Aq. menthæ piperitæ................ $70.00 \ldots \ldots, 3$ ii 3 iiss

Syr. aurantii ............................ $30.00 \ldots \ldots 3^{3}$

M. et sig.-To be taken in the evening.

Or:

B Paraldehydi.......................2.00.... $\mathbf{z}_{\text {ss }}$

Aq. flor. aurantii,

Spir. vin. Gallici,

Syr. aurantii..................āa $20.00 \ldots \ldots$ 3

M. et sig.-To be taken in the evening.

Or it may be given in an enema:

R Paraldehydi.........................2.00.... $\mathbf{z}_{\text {ss }}^{\text {s }}$

Vitelli ovi..........................no. i..... no. i

Mucil. acaciæ.................... $120.00 \ldots \ldots$ iv

M. et sig.-To be used as an enema.

Urethane.-Another modern narcotic is urethane (crystals, very easily soluble in water). It was introduced as a narcotic by Schmiedeberg, tested practically and recommended by von Jaksch in Nothnagel's clinic. This drug also has no bad effects on the circulatory system; Lewin alone claims that it increases arterial tension and slows the pulse. If there are any unpleasant after-effects, they are merely slight disturbances of digestion. Only rather large doses bring on actual romiting. The ordinary dose is $3-4$ G. (45-60 gr.) ; its action, like that of paraldehyde, is rapid.

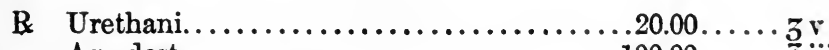

Aq. dest................................. $100.00 \ldots \ldots$ zii 3 iiss

M. et sig. $-\frac{1}{2}-1$ tablespoonful at night in a cup of orange tea.

Hedonal.-Hedonal (methylpropylearbinolurethane) is another narcotic that has come recently into use. It is nearly related to urethane and is a colorless powder, with a taste something like that of peppermint. 
The author has repeatedly used it, without noting any effects on the circulatory apparatus in those cases of broken compensation in which he has tried it. It takes effect in about half an hour.

R Hedonali.....................1.00-1.50.....gr. xv-gr. xxii

Dent. tal. dos. no. v. Dispens. in cachets.

Sig.-A cachet to be taken in the evening.

The author considers it particularly suitable for prolonged use in cases of persistent sleeplessness in heart disease, either alone or alternated with trional.

Amylene Hydroxide.-Amylene hydroxide has similar properties. Within 15 minutes (v.Mehring and Buschans) it brings on a deep sleep, lasting 6-10 hours. It only very rarely has unpleasant after-effects. In the author's extensive use of it, he has only once had the patient complain of its causing headache (in a case of aortic insufficiency with atheroma and myelodegeneration of the cord). It is a colorless liquid with an unpleasant taste, something like that of camphor and paraldehyde. It acts upon the cerebral cortex alone, having no effect upon the medulla or cord. But it affects both the cardiac ganglia and the peripheral vessels, so that it cannot be considered, as it was at first, to have no effect on the circulatory system. The dose is $2-3.5$ G. (30-50 min.) taken before going to bed.

R Amylenis hydroxidi.................6.00... 3 iss

Aq. dest.,

Syr. aurantii.................

M. et sig.- One-half to be taken in the evening.

Or:

B Amylenis hydroxidi........................ $m \times x v$

Dent. tal. dos. no. $x$. Dispens. in caps. molles.

Sig. $-2-3$ capsules to be taken in the evening.

Or as an enema:

B. Amylenis hydroxidi...............3.00-5.00.....m $\mathrm{mlv}-3 \mathrm{i} m \times \mathrm{xv}$

Mucil. acacie,

Aq. dest..................... a a $25.00 \ldots \ldots$ vi

M. et sig..-To be used :s an enema.

Dormiol.-A new compound of amylene hydrate with chloral hydrate, dormiol, has recently been put on the market. The author has often used it in heart disease with good effect and no bad consequences, except in one complicated lesion, where it caused a collapse. Apparently it should therefore be used with caution in heart lesions.

Chloralose.-Chloralose, a compound of chloral and glucose (anhydroglucochloral) has so far not come into wide use, in spite of its analgesic power and its lack of any effect on the circulatory system. The author recommends it, in powder form, beginning with small doses, increased if necessary. Its action is apt to be slow but it usually brings on a 5-6 
hours' sleep. [Balfour recommends it highly, as does Babcock; but the latter noticed that some of his patients (females) were apt to be rather nervous the next morning.-Ed.] It is prescribed as follows:

R Chloralosis................................ iss

(Not chloral hydrate!)

Sacchar. lactis.......................40.... vr. vi

M. fiat pulvis. Dent. tal. dos. no. $x$. Fiat cachets.

Sig. -1 powder the first day, on the following days 2 a day and never more than 3 a day. ( 3 powders $=0.30$ G. -5 gr.) in wafers.

Pellotine.-Another narcotic is pellotine hydrochlorate, a white, odorless, very bitter powder soluble in water. The author has found its effect prompt in the few cases in which he has been able to test it; but Langstein reported that in one case a small dose caused collapse. It may be given subcutaneously or by the mouth; dose, $0.04 \mathrm{G}$. $(3 / 4 \mathrm{gr}$.). It is prescribed as follows:

R Pellotinæ hydrochloridi...............0.04....gr. $\frac{3}{4}$

Sacchar. lactis.............................. gr. vi

M. fiat pulv. Dent. tal. dos. no. v.

Sig. -1 powder at night.

Salolum Tribromatum.-The author has also tried salolum tribromatum (cordol), with no bad effects and often good results, even when pain was present as well as sleeplessness. The dose is $0.50-2$ G. (730 gr.).

Isopral.-Isopral is a new preparation (trichlorisopropylalcohol). It is given in tablets, in doses of $0.50-0.75$ G. (7-12 gr.) and generally brings on a 7 hours' sleep in 15-30 minutes. It is contraindicated if the sleeplessness is due to circulatory disturbances, as it undoubtedly has an injurious effect on the heart. [I have tried isopral in a number of conditions with some success; but in one cardiorenal case, it apparently produced dyspnœa and cyanosis after three consecutive nights, and in a chronic myocardial case, symptoms of cardiac weakness.-Ed.]

The combination of two narcotics will sometimes be successful when either one alone has no effect. The author, for instance, often gives:

R Trional......................... $1.00-1.50 \ldots \ldots$ gr. $x$-grii

(or Veronal.......................... viii)

Codeinæ........................... $\frac{1}{3}$

M. fiat pulv. Dent. tal. dos. no. x.

Sig. -1 powder 3 hours before going to bed, followed by a cup of some hot drink.

[Even better effects may be expected from the following:

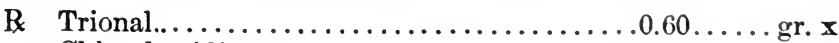

Chloralamidi.........................

Codeinæ sulphatis....................... ss

M. fiat pulv. Dent. tal. dos. no. $x$.

Sig. -1 powder $2-6$ hours before bedtime, to be followed immediately by a cup of some hot liquid.-Ed.] 
Undesirable Narcotics.-Of all the narcotics mentioned, the author practically always first tries veronal, trional, or hedonal, often in combination with codeine. He believes that the foregoing list contains all that are available in sleeplessness from passive cerebral hyperæmia. The use of all others is irrational, sometimes dangerous, and fortunately not necessary. He has given up hypnone, hypnal, methylal, and chloralamide in the treatment of sleeplessness from any cause.

The otherwise excellent narcotic, bromidia, a mixture of various sedatives and narcotics, among them chloral, must be used in heart lesions only with extreme caution, if at all. [I have a strong prejudice against any such remedy containing so many ingredients. Although it may prove a happy combination in many cases, one is apt to forget some of the constituents and perhaps to prescribe it when one or two of them are contraindicated. A little thought, attention, and trouble will produce as good and perhaps a more applicable composite prescription for each case.-Ed.]

Methylal (a colorless powder, soluble in water and alcohol, with an odor like that of chloroform) should not be used to induce sleep in cerebral hyperæmia, as it quickens the heart, and is decidedly injurious (Krafft-Ebing), its proper use being rather in cerebral anæmia.

As a narcotic, cannabis indica in extract, tincture, or alkaloidal form is directly contraindicated, as it increases the cardiac insufficiency (Lewin).

The following table recapitulates the foregoing discussion of narcotics in sleeplessness resulting from venous hyperæmia in heart disease:

\begin{tabular}{l|l|l}
\hline \multicolumn{1}{c|}{ Available. } & Available with great caution only, & \multicolumn{1}{c}{ Non-available. } \\
\cline { 3 - 3 } Sodium bromide & Potassium bromide & Opium \\
Paraldehyde & Sulphonal & Morphine \\
Trional & Bromidia & Hypnone \\
Tetronal & Chloral & Hypnal \\
Urethane & Dormiol & Methylal \\
Veronal & & Cannabis indica \\
Amylene hydroxide & & Isopral \\
Chloralose & & Chloralamide \\
Salolum tribromatum & & \\
Hedonal & & \\
Neuronal & & \\
\hline
\end{tabular}

[Babcock recommends highly the following:

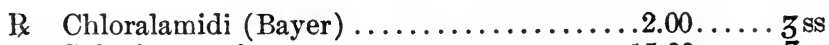

Spir. frumenti..........................

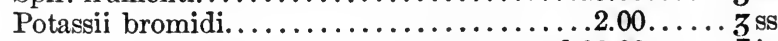

Syr. glycyrrhizæ.................. ad $30.00 \ldots \ldots$. $3 \mathrm{i}$

M. et sig.-This dose at bedtime.-Ed.] 
Of course in all cases, especially where we employ these drugs, the bowels should be cleaned out, even if necessary by drastic purges. [The author's complete discussion of the drugs available to relieve insomnia in heart disease leaves little to be desired. A few words might be added, however, to suggest various simple devices, mechanical, hydrotherapeutic, dietetic, electrical, psychical, etc., which should be attempted and persevered in before the physician turns to drug treatment. A warm drink or a eup of hot gruel just before bedtime, the change of the hearty meal from night to the middle of the day or vice versa, a neutral bath before retiring, a wet pack, and mild massage are examples in point.Ed.]

Bleeding.-Another therapeutic measure to be tried is bleeding either local or by venesection. The latter method will be discussed later (see p. 61).

Local bleeding often affords very noticeable relief. The technic of the process will be described here, although its use is by no means confined to passive cerebral hyperæmia in heart lesion, but is equally advantageous in venous hyperæmia of the brain in lung disease and in active cerebral hyperæmia resulting from plethora vera or plethora ad vasa (too much blood relative to the size of the arteries) in acute inflam. matory diseases.

The author has been strongly convinced by his own experience that local and, in a still greater degree, general bloodletting, have a place in modern therapy and are often of advantage when medication fails. $\mathrm{He}$ realizes that in most quarters bleeding is either ignored or denounced as antiquated or directly injurious, but. he nevertheless believes that the number of authorities who agree with him is constantly increasing.

The methods of local bleeding are as follows:

1. By leeches.

2. By cupping.

$a$. Dry cupping.

$b$. Wet cupping.

Dry cupping is not strictly a method of bloodletting, but for the sake of completeness it will be discussed here.

Dry Cupping.-The spot to be cupped is freed from hair and washed with warm water. The cup, a small glass bell, with a rather small opening, is first heated in hot water and dried. It is then held for a few seconds over the flame of an alcohol lamp placed near the body of the patient. The air in the cup having been thus rarefied, it is placed as quickly as possible over the place to be cupped, the edge being pressed closely to the skin. The skin is forced by the atmospheric pressure into the cup, and as a result of the dilatation of the vessels, becomes markedly hyperæmic. After a few minutes, the cup is removed by forcing a finger under it at one side and so letting in the outside air.

Dry cupping is indicated whenever a blister could be used,--therefore in various kinds of inflammatory and painful local affections. [A simple 
method, particularly where, as is often the case, several dry cups are required and very promptly, is to select small cups or tumblers (egg cups and small, thick whisky tumblers are good), smear the bottom and sides quickly with a swab wet with alcohol, wipe the edges dry, ignite, allow the alcohol adherent to burn for a few moments, and then clap one quickly to the spot desired.-Ed.]

Wet Cupping.- In wet cupping the spot is thoroughly cleaned and disinfected. The cup is then applied as before. But when the skin is thoroughly congested, the cup is removed, and an instrument used for the purpose placed on the spot. This consists of a metal case containing a number (usually 10) of small lancet blades, which spring out on the pressing of a spring and then immediately spring in again. The spring is let go, and at once a corresponding number of small wounds is made in the skin. If desired the instrument may then be turned and the same number of cuts be made crossing the first set at right angles. The cup is then warmed and replaced, the air having been rarefied. When the blood, sucked out by the atmospheric pressure, has filled two-thirds of the interior, the cup is removed as in dry cupping. The wounds are then cleaned and a dry antiseptic bandage is applied. The number of cups varies with the age and constitution of the patient and the part of the body to which they are applied. In general 8-12 are employed in dry cupping and 2-6 in wet cupping.

Leeches.-If leeches are used they must be perfectly fresh; that is, they must exhibit lively movements in water and draw themselves up when touched with the hand. The leech is placed in a small wineglass, cupping glass, or reagent glass and so put upon a selected spot, which has been previously shaved and carefully disinfected, and, to make the leech take better hold, smeared with sweetened water, milk, or blood. Generally the leech is left on till it falls off, but, in case only a small quantity of blood is desired, some salt is sprinkled on the other end of the leech, which will make it quickly let go. If the leech falls off spontaneously before enough blood has been withdrawn, the wound may be sprinkled with a warm antiseptic fluid, for instance salicylate solution, which will prolong the bleeding. If a leech should get into the stomach, rectum, or vagina, it should be killed by a salt solution. To prevent this accident, a thread may be tied to the leech beforehand and fastened to the skin outside. The number of leeches used at one time varies from one to ten; on an average a leech weighing $2-3 \mathrm{G}$. (30-45 gr.) will suck 5-6 G. (75-90 gr.) of blood.

Leeches have the advantage over cupping of being applicable to places like the neck, the arms, the abdomen, and behind the ear. On the other hand, it is sometimes hard to stop the bleeding from leeches. It may be necessary to scarify the wound, or use compression or styptic powders (alum) or liquids (liquor ferri sesquichloridi). Cupping has the further advantages of making it possible to measure exactly the blood drawn, and of being a cheaper procedure. 
Indications.-The indications for local bloodletting are: (1) Acute inflammatory diseases of the inner organs, as pneumonia, pleuritis, exudative pericarditis and fresh endocarditis, acute peritonitis (particularly if circumscribed, as appendicitis), acute nephritis, neuritis, and less often myelitis, meningitis, and acute otitis; (2) Congestion in the head occurring in plethoric individuals in venous hyperæmia, especially in heart lesions ; (3) Overloading of the right heart,- - that is, congestion of the blood in that chamber.

Site.-In cerebral diseases and congestion, the leeches are placed on the mastoid process; in perityphlitis, on the hip; in pericarditis, on the precordial region; and to relieve the right heart, on the neck at the level of the thyroid veins. In acute diseases of the lungs or the pleura, leeches or cups are placed on the sides or back of the thorax, between the vertebral column and the scapular line; in acute nephritis, on the lumbar region; and in the other above-mentioned diseases, on the place affected.

Bloodletting at a Distance.-Some authors (particularly Sacharjin) apply the leeches at a distance from the spot affected, basing their reasons for such a procedure on the observation that not infrequently marked and long continued symptoms of cerebral hyperæmia are relieved by hemorrhoidal bleeding, while much more copious bleeding from the nose has no such effect. They place four or five leeches at the anus or rather over the coccyx. The indications for this procedure are, according to Sacharjin: (1) Hyperæmia of the brain, with or without a coincident affection of the heart and vessels; (2) Hyperæmia of the cord and its coats; (3) Coughing up of blood without organic cause, in the case of sound heart and lungs, or in tuberculosis or heart disease, particularly affections of the mitral valve; (4) Stasis of the portal veins from any cause, particularly from congestion of the liver; (5) Inflammation of large hemorrhoids. Under (1) Sacharjin particularly mentions cases where the symptoms of cerebral hyperæmia (restless sleep, confused and excitable state of mind, heaviness in the head, pain in the neck and sometimes in the top of the head, weakness in walking, giddiness in bending over) are sometimes complicated by sudden congestions in the head (the face becoming quickly very red), which are like hemorrhoidal straining and threaten an apoplectic stroke. Before the leeches are applied the intestine must be emptied either by an enema or by a cathartic given several hours before. Subsequent hemorrhage may be prevented by washing the wound with warm salicylate solution until the blood, which is generally dark at first, comes out bright red.

Pulmonary Stasis.-This finishes the discussion of cerebral disturbances from congestion in heart lesions; we pass now to symptoms of congestion of the lungs.

Symptoms. - It has long been recognized that symptoms of pulmonary congestion are among the most frequent and the earliest signs of broken compensation, particularly in mitral lesions. A tendency 
toward periodically recurring bronchitis is an especially important symptom of an otherwise latent mitral insufficiency. We know further that these symptoms of pulmonary congestion consist of dyspnœa, and especially of bronchitis at the posterior base of the lungs with sometimes dry, sometimes moist râles, often accompanied by an exceedingly obstinate cough and by a varying amount of expectoration. We know, finally, that another symptom is hæmoptysis, varying in intensity and in duration, sometimes lasting for weeks.

General Therapy.-It should be repeated that heart stimulants, digitalis especially, should of course be given here. Revulsives are also indicated,--dry cupping, blisters, hot applications to the chest, and mustard plasters (see p. 65).

Special Therapy: Bronchitis.-Beside this more general therapy, we frequently have to employ a special therapy, directed against one or more of the above-mentioned symptoms. For instance, if dry bronchitis is present, we must increase the secretion of mucus and make it more fluid in its consistency. For this purpose, the author recommends the preparations of ammonia (liq. amm. anisat, 0.50-1 G. (7x/2-15 min.) a day, ammonium carbonate up to $2 \mathrm{G}$. (30 gr.) a day or ammonium chloride up to 5 G. ( 75 gr.) a day, in mixtures). The balsams, Peruvian balsam or (with a better taste) balsamum tolutanum, increase the expectoration and decrease the secretion. At present they are not often given internally. Still their syrups may be used, a teaspoonful to a tablespoonful several times a day or added to mixtures, 15-20 G. (4-5 dr.) a day. When there is an abundant secretion but little expectoration, expectorants must be used (tartar emetic, ipecac, senega, and apomorphine or euporphine).

Tartar emetic and sulphurated antimony (kermes mineral) should not, however, be used in bronchitis from congestion, even if it is the first and only symptom of cardiac insufficiency, for they both decrease the contractile power of the heart muscle. Apomorphine also appears to be rather contraindicated than indicated in this form of bronchitis, for its expectorant effects are accompanied by vomiting, which in heart lesions is dangerous, not so much on account of the passive cerebral anæmia so caused as on account of the possibility of cerebral hemorrhage. These dangers exist, only in a very much less degree, in the use of ipecac, which otherwise is most successful in its results. Given in conservative doses, it is certainly no more dangerous than senega, which latter is not so good an expectorant. For a more detailed discussion of these expectorants, see the Therapy of Bronchitis, p. 289.

Cough.-A most distressing symptom of pulmonary congestion is often a racking cough, which may be so bad that we are forced to use opium or morphine, in spite of the fact that they are contraindicated on general principles in broken compensation. To lessen the danger, a heart stimulant (digitalis) should be given at the same time. Bouchardat 
recommends the following mixture, in which atropine is used to counteract the opium, especially in its effect upon the brain:

I Morphinæ sulphatis...................... iii

Tinct. eucalypti........................... ii

Atropinæ sulphatis....................

Pulv. glycyrrhiza......................... $3 \mathrm{ss}$

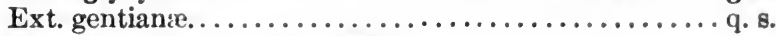

M. fiat mass. Div. in pil. no. $x x$.

Sig. $-1-2$ pills a day.

Codeine.-Before proceeding, however, to the use of either opium or morphine, codeine should be tried. We may employ the phosphate of codeine in mixture and added to a heart stimulant, as it is easily soluble in water. The dose is $0.015-0.03 \mathrm{G}$. $(1 / 4-1 / 2 \mathrm{gr}$.) up to $0.10-0.15 \mathrm{G}$. $\left(1 \frac{1}{2}-2 \frac{1}{4}\right.$ gr. $)$ daily. Or we may use tablets, pills, powder, or subcutaneous injection. For a more detailed discussion, see p. 285.

Heroine-Dionine.-Heroine and dionine, newer morphine derivatives, should be used cautiously on account of their effect on the heart muscle. They are as good as codeine but not quite so effective as morphine. Dionine and heroine hydrochlorate are quickly soluble in water, but heroine itself needs the addition of some acid. [Heroine in my hands has not proved as efficient as codeine; but has often produced a troublesome headache.-Ed.] The dose of heroine is like that of morphine (0.005-0.007 G. ( $1 / 12^{-1 / 10}$ gr.) a dose, 0.01-0.02 G. ( $1 / 6-1 / 3$ gr.) a day); that of dionine, like that of codeine $(0.01 \mathrm{G} .(\mathrm{I} / 6 \mathrm{gr}$.) a dose, 0.03 G. (1/2 gr.) a day).

B Heroini.............................. iiss

Pulv. glycyrrhizæ.....................

Ext. gentianæ...........................

M. fiat mass. Div. in. pil. no, xxx.

Sig. $-3-6$ pills a day.

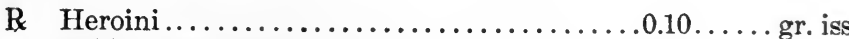

Acid. hydrochlorici diluti....................... ii

Aq. amygdal. amar......................

M. et sig. -10-15 drops 3-4 times a day.

B Dionini...................... $0.02-0.03 \ldots \ldots$ gr. $\frac{1}{3}-$ gr. $\frac{1}{2}$

Aq. dest...........................

Syr. simpl........................

M. et sig. -1 tablespoonful every 2 hours.

B Dionini........................... $\mathrm{v}$

Pulv. glycyrrhizæ......................

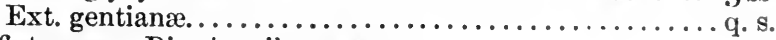

M. fiat mass. Div. in pil. no. xxx.

Sig. $-3-4$ pills a day.

Extracts of Belladonna and Hyoscyamus.-The extract of belladonna or of hyocyamus may also be given to quiet the cough. The maximum dose of the first is $0.03-0.10 \mathrm{G}$. ( $1 / 2-1 \mathrm{I} / 2 \mathrm{gr}$.) a day; and of the second, 
0.20-1 G. (3-15 gr.) a day. They are given in mixture, to which is best added the soothing syrup of althæa. For example, in bronchitis from mitral stenosis :

B Inf. digitalis.................. e $0.6-180.00 \ldots \ldots$.gr. $x-\xi$ vi

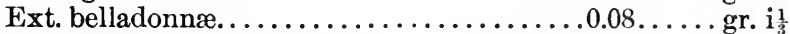

Codeinæ phosphatis.....................0......gr. ss

(or Morphinæ hydrochloratis.............0.01......gr. $\frac{1}{6}$ )

Syr. althææ,

Syr. senegæ.................. āi $10.00 \ldots \ldots z$ iiss

M. et sig. -1 tablespoonful every 2 hours.

Aqua Laurocerasi or Aq. Amygdalce Amara.-Dujardin-Beaumetz advises 1 teaspoonful of aqua laurocerasi in a cup of hot milk 3 times a day. The author also has found this an effective remedy in various cases of persistent cough from different causes.

Strümpell recommends chloralamide for the same purpose.

Dyspnœa.-Dyspnœa, the second symptom of pulmonary stasis in broken compensation, demands the same causal therapy as the cough (digitalis). Symptomatically we must often have recourse to narcotics and may use morphine or opium if we accompany them with a counteracting heart tonic.

In milder cases of cardiac asthma, not only from an uncompensated valvular lesion but from any muscular insufficiency of the heart, the theobromine preparations are often useful. They are combined in "dispnon," composed as follows:

B Diuretini........................... $0.25 \ldots \ldots$ gr. iiss

Agurini............................. $0.10 \ldots$ gr. iss

Dent. tal. dos. (caps. gelat.) no. 1.

Sig. -2 capsules $3-4$ times a day, after drinking hot milk or tea.

Hæmoptysis.-Another symptom of pulmonary stasis is hæmoptysis, manifested either by a slight tinge of blood to the mucus in the sputum or by an abundant expectoration of blood for several weeks. It should be said that, in mitral lesions especially, hæmoptysis may result from a hemorrhagic pulmonary infarct caused by embolism of the pulmonary veins or arteries, as well as from venous hyperæmia. In such a case the therapy is causal (digitalis, etc.). We may, however, with advantage use a styptic in addition. Only one will be mentioned here,-ergot, usually given as the fluidextract (extr. socal. cornut., Bombelon or Yvon or Denzel) subcutaneously. For example, we inject a Pravaz syringeful of a mixture of equal parts of ergotine (Bombelon) and distilled water. A syringeful of ergotine (Yvon) contains 1 G. (15 gr.) ergot, the maximum dose, which may, however, be repeated several times a day. Ergotine (Denzel) is prescribed as follows:

B Ergotini (Denzel)

(or Ergot. dialysat.)................2.00-3.00.....gr. xxx-gr. xlv

Aq. dest.......................... $7.00-8.00 \ldots \ldots .31^{3}-3$ ii

Acid. carbol....................... $0.10 \ldots \ldots$ gtt. ii

M. et sig. -1-2 syringes injected several times a day. 
Or: B Ext. ergotæ (Denzel) $\ldots \ldots \ldots \ldots \ldots \ldots \ldots \ldots \ldots \ldots \ldots \ldots$

Aq. menthæ piperitæ............... $180.00 \ldots \ldots$ z vi

Syr. aurantii...................... $20.00 \ldots$

M. et sig. -1 tablespoonful every 2 hours.

Rosenbach recommends in another connection the following combination :

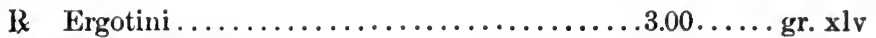

Pulv. digitalis............................ 3 ss

Pulv. glycyrrhizæe et ext. gentianæ................ q. s.

M. fiat mass. Div. in pil. no. $x / v$

Sig. $-2-3$ pills every 2 hours. ( 1 pill contains 1 gr. ergotine, $\frac{2}{3}$ gr. pulv. digitalis.)

Ergotinine.--Eulenburg recommends ergotinine subcutaneously in place of ergotine, as being less irritating and painful locally. It has the same general physiological effects as ergotine (slight slowing of pulse, decrease of tension, and regulation of the cardiac activity). The dose is 0.0007-0.001 G. $\left(1 /{ }_{100}-^{1} /{ }_{64}\right.$ gr. $)$, subcutaneously. [There is on the market a reliable and convenient prescription, "ergot-aseptic," each tube containing the average dose for hypodermic medication. Another good preparation is "ergotine" (Bonjean). Cushny questions whether ergot merits its reputation in these and other cases of local bleeding. Personally, I have never been able to obtain encouraging results from its use.-Ed.]

Ergotine and Ether.-Ether and ergotine should never be used together, as their physiological effects directly counteract each other, ether being a good antidote for acute ergotine poisoning.

Venesection.-One more therapeutic agent is very useful in pulmonary stasis from heart lesions,-venesection. The author will take this opportunity of discussing this procedure in general, thus bringing into one chapter the discussions of local and of general bleeding.

Indications.-The indications for venesection are as follows:

I. Broken Compensation.-The first general indication is broken compensation, particularly threatened pulmonary œedema, venous cerebral hyperæmia, and threatened heart paralysis. Pulmonary œdema in broken compensation has long been regarded as an indication for venesection. If local bleeding is helpful, venesection is effective in a much greater degree. The reduction of the blood mass and the stimulation of the circulation often make the greatly dilated heart once more perform its function. The same is true in cerebral hyperæmia. As for threatened heart paralysis venesection may be effective when stimulants and analeptics prove powerless and death is imminent, the patient being decidedly cyanotic, with blue and cold extremities and a thread-like pulse. If the symptoms of pulmonary and abdominal stasis develop so strongly or quickly (acute asystole) that there is no time to wait for the action of heart stimulants, venesection may give such temporary relief that digitalis or caffeine may then be often successfully used. 
II. Threatened Cerebral Hemorrhage, and Insolation.-The second principal indication for venesection is threatened cerebral hemorrhage, or insolation-to leave for the time being the subject of heart lesions. In the first case interstitial nephritis, especially with increased tension of the peripheral vessels and accentuated aortic tone, is a condition in which venesection is a good prophylactic measure. Also when cerebral hemorrhage is actually present (as a result of arteriosclerosis or nephritis), venesection often deserves being tried, for the sake of the instant relief it affords the vessel walls.

III. True Plethora.-The third chief indication for venesection is true plethora. Local bleeding or Sacharjin's bleeding at a distance (see p. 57) is often advantageous, but from the author's own experience venesection is fully as effective, especially when symptoms of active hyperæmia and congestion are conspicuous (face, cheeks, and ears intensely red, almost purple, calibre of peripheral vessels large, turgor of the skin, especially of the face, intense). It is particularly suitable if the history shows that the patients (generally men of 40-50 years, in easy circumstances and self-restrained sexually) have experienced relief from previous bleeding hemorrhoids. The newly recognized condition of polycythæmia with increased blood pressure, also receives at least temporary relief from repeated venesection.

IV. Croupous Pneumonia.-The fourth indication is croupous pneumonia. The author has often tried it when the patient was strong, with a full, almost bounding pulse, deep red or slightly cyanotic face, severe dyspnœa and pain, and abundant bloody sputum, from an acute pneumonia rapidly spreading all over one lung while the other showed diffuse catarrh, and when besides there was a marked accentuation of the second pulmonary tone, and often a relatively acute dilatation of the heart. Then venesection was regularly effective, even decreasing the subjective distress.

$V$. Pulmonary $G$ dema.-Another indication for venesection is pulmonary œdema (acute inflammatory œdema in croupous pneumonia of the opposite lung either at the start or at any time in a case of short duration, or œdema in nephritis or in heart lesions). If venesection does not succeed in the last instance, on account of the weakness of the heart, a subcutaneous injection of $0.20 \mathrm{G}$. (3 gr.) of the sodio-benzoate of caffeine should be tried.

$V I$. Kidney Diseases. - The sixth indication is found in certain forms of kidney disease: acute uræmia in chronic Bright's disease with good heart action, pulmonary œdema in Bright's disease, cerebral hemorrhage either threatened or actually present, and severe renal symptoms in all kinds of severe infections (typhoid, pneumonia, etc.). Sacaze warmly advocated phlebotomy in acute nephritis with acute uræmic symptoms, particularly convulsions. It is, however, also to be advocated in relatively slight uræmia (Dieulafoy), because it lessens the chances of the acute nephritis's passing over into the chronic form. The author (fol- 
lowing Baccelli's advice) draws blood from the vena pediæa, in order quickly to reduce the blood content of the urine in acute nephritis or an acute exacerbation of chronic nephritis.

VII. Diseases of the Blood: Chlorosis.-A seventh indication for venesection is found in diseases of the blood, particularly chlorosis (Dyes, Wilhelmi, Schubert). Vierordt discovered that slight losses of blood increase the number of red corpuscles; Lesser, that they increase the hæmoglobin content. We must assume (Neusser, 1891; Schiperowitsch, 1892) that the loss of blood stimulates the formation of new blood.

VIII. Transfusion of Blood and Poisoning.-Finally, venesection must be used in the rare procedure of the transfusion of blood, and in certain intoxications, such as illuminating gas and carbon monoxide poisoning. [In poisoning by illuminating gas the removal of 10-20 oz. of blood and immediate infusion of salt solution, 15-20 oz., followed by stimulating enemata and hypodermic stimulation, have proved of unquestionable value.-Ed.]

Method of Venesection.-The technic of venesection is as follows: The veins of the elbow (vena cephalica on the radial side, vena basilica on the ulnar, or the vena mediana) are carefully inspected to determine which is the most swollen and to be sure that it is not so close to the artery as to make an injury of the latter possible. The place is then earefully disinfected and the arm so bound above the elbow by a tourniquet that the subcutaneous veins of the hand and forearm stand out plainly and yet the radial pulse has not disappeared. The veins may be made to stand out more strongly by the patient's clenching the fist. The operator now holds the arm with his left hand and rests his right on it, the patient being in the decubitus posture and having his arm supported underneath. The operator holds the lancet with the thumb and index finger of the right hand and stabs the vein obliquely in order that the longitudinal and ring muscle fibres of the vessel may be cut instantaneously and a gaping wound be obtained. The blood is made to flow out quickly by the patient's clenching his hand and is caught in a measuring glass. When the required amount has been withdrawn, the bandage is removed, and the wound compressed with the finger and bound up in an antiseptic dressing drawn rather tight. The elbow is kept bent.

Amount of Blood Withdrawn.-The amount of blood taken is usually 2-4 G. for every $\mathrm{kg}$. of the patient's body weight (1-2 c.c. for every pound.) In chlorosis, when the process should be repeated every four. or in very weak individuals every eight weeks, the quantity is one-half as much, $1-2$ G. per $\mathrm{kg}$. ( $0.5-1$ c.c. per pound). In other cases the process may be repeated after 8-10 days. The quantity of blood drawn should never exceed 400 G. (12 oz.). [In uræmia and in enormous cardiac dilatation I have seen striking results from the abstraction of 20 oz.-Ed.] 
Accidents.-The following accidents may occur: (1) In an individual with much subcutaneous fat or anasarca, we may miss the vein. In that case we should either make another stab, or, opening up the cut, incise deeper into the vein. (2) The flow of blood may cease, and the patient become unconscious. The operation must then be immediately stopped, the wound bound up, and the head lowered. (3) If the spot is not correctly chosen, the brachial artery may be injured, which may lead to an arteriovenous aneurism. The author saw this accident occur in the case of an old woman, who eventually died from sepsis after an operation for the removal of the aneurism. (4) Oftener the median cutaneous nerve is injured, eausing numbness and pain, which, however, disappear in a few days.

The vein may be punctured instead of cut, a cannula somewhat larger than that of a Pravaz syringe being generally used.

Local or General Bloodletting.-The final question is whether to employ local bleeding or venesection if the indications permit of choice. The author is inclined to prefer venesection, other things being equal. Its advantages are the rapid effect, the direct opening up of the venous system, and the exact measuring of the blood taken.

CONGestion OF THE Liver.-Leaving the subject of venesection and returning to that of congestion in heart lesions, we pass from the subject of congestion of the lungs to that of congestion of the liver. This organ is very frequently congested in broken compensation, particularly if the lesion is situated in the mitral orifice or mitral valve, and often leads to most distressing symptoms. 'The reason for the quick appearance of congestion in the liver is the proximity of the hepatic veins to the heart, so that, since the pressure in them is always low, any backing up of the blood in the heart makes itself felt immediately. Congestion of the liver occurs not exclusively in heart lesions but also in all other diseases (diseases of the lungs or pleura, of the mediastinum, and of the thoracic portion of the vertebral column) in which the blood from the inferior vena cava enters the right auricle under difficulties, because the strength of the right ventricle has not been able to keep pace with the abnormally high pressure in the pulmonary arteries. Also in diseased conditions of the inferior vena cava (compression by mediastinal tumors), or of the hepatic veins themselves (periphlebitis of the hepatic veins with compression, or compression by an echinococcus cyst in the liver), passive congestion of the liver must naturally follow. The symptomatic therapy is the same, no matter what the etiology. From the therapeutic standpoint, the cases should be divided into those in which the liver is the only organ showing any sign of congestion, and the much larger group of those in which congestion appears in other organs also.

Causal Therapy.-Every hyperæmia of the liver must receive primarily a causal therapy; e.g., the stimulation of the heart by digitalis with or without calomel, the treatment of the primary pulmonary emphysema, or the administration of antiluetic specifies. 
Symptomatic Therapy.-But a causal therapy is often either impossible or so unsatisfactory that we are confined to treating the symptoms.

The first of these is pain in the epigastrium with a feeling of heaviness and pressure. This may be relieved by (1) revulsives; (2) purges; (3) blisters; (4) local eupping or leeches; (5) a mustard plaster; (6) an icebag (an ice-bag should never be filled more than half full and the air should be pressed out as much as possible before it is closed; it should never be placed directly on the skin without a compress between); (7) cupping.

Many patients get no relief from an ice-bag but are greatly helped by hot applications,-hot cloths, poultices, or the thermophor (see p. 72). Poultices may be made from flaxseed meal or mustard or bran mixed with water or scalded milk and spread $1 / 2-1$ inch thick on a linen eloth. The edges of the cloth are then folded completely over the mustard (or a bag may be made beforehand), and the poultice, its heat being first tested, is applied to the painful portion of the body, which should first be well greased. The effect of the poultice is the same as that of any form of dry heat, its special advantage being that it holds the heat for a long time.

If it is desired, a narcotic may be combined with an ordinary poultice. The narcotic, if in fluid form (tincture of opium or belladonna) or in the form of a salve, may be spread on a separate piece of cloth and placed between the poultice and the skin; or if the fluid narcotic is used, the under-side of the poultice may be wet with it.

The poultice should be covered with several layers of cloth or oiled silk, and left on for an hour or two. When it is changed the cold poultice is scraped off the cloth and added to the rest of the prepared material, which has been heated. When they are well mixed a new poultice is spread. New material should be prepared every 24 hours.

The application of leeches $(4-5)$ is better than cupping, and they are preferably placed over the coccyx, rather than over the spot affected.

The daily use of gentle, tepid showers over the hepatic region, followed by alcohol rubs, often relieves the patient of the distress caused by liver congestion.

Mineral Water Cures.-In cases of the first group, i.e., where the liver is the only organ to show symptoms of congestion, a careful course of mineral waters is often to be recommended (Mühlbrunn at Carlsbad, Kreuz or Ferdinandsbrunnen at Marienbad, Rakoczyquelle at Kissingen, Elisabethquelle, Kaiserquelle, and Ludwigsbrunnen at Homburg, and the Luziusquelle and Emeritaquelle at Tarasp). In this way the distress may often be fully relieved even if the cause is not at all affected. Frerichs reports a case of a patient with mitral stenosis, who got along for four winters quite comfortably by the use of the cooler Carlsbad springs; the author has repeatedly seen similar results from the use of Marienbad and Kissingen waters. [French Lick Springs._Ed.] 
Use of Mineral Waters at Home.-If the heart is not strong enough for the patient to think of taking the journey to one of these resorts and yet is not weakened enough to give rise to any other symptoms of stasis than congestion of the liver, any of these waters may be taken at home. To this list may be added the bitter waters (Ofnerbitter waters, Iunyadi János, Rakoczy, Franz Josef, and Ofner Koenigsbitterwasser, Sedlitz, Saidschitz, Püllna, and Friedrichshall bitter waters).

Vegetable Laxatives.-In the second group, i.e., when the liver hyperæmia is accompanied with other symptoms, even if it be only slight hydrops or kidney affection, the saline cathartics may also be used as well as the mild vegetable laxatives. (For a full discussion see p. 494 et seq.).

One or two prescriptions may be given here:

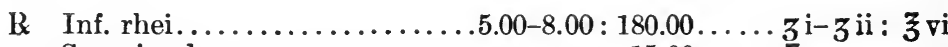

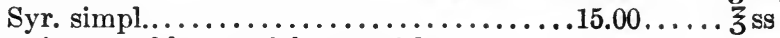

M. et sig. -1 tablespoonful every 2 hours.

B Ext. rhei pulv.,

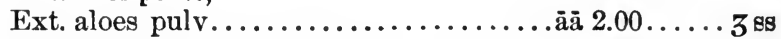

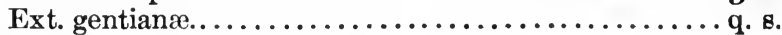

M. fiat mass. Div. in pil. no. xl.

Sig. -1-2 pills in the erening.

R Podophyllini.................... $0.40-1.00 \ldots .$. gr. vi-gr. $x v$

Ext. belladonnæ................ $0.10-0.15 \ldots \ldots$ gr. iss-iiss

Pulv. glycyrrhizæ.....................

M. fiat mass. Div. in pil. no. xl.

Sig. - 1-2 pills in the evening.

Cascara sagrada is one of the best laxatives for this purpose. It may be prescribed as tablets, $0.06-0.3 \mathrm{G}$. ( $1-5 \mathrm{gr}$.), as pastilles ( 1 pastille= $0.50-1 \mathrm{G}$. ( $7 \mathrm{I} / 2-15 \mathrm{gr}$.) of the extract), as the wine of cascara sagrada (1 to several liqueur glassfuls), or as the fluidextract (20-30 drops once or twice a day). Or we may write the prescription as follows:

B Ext. fluid. cascaræ sagradæ,

Syr. aurantii,

Aq. dest.................. a $10.00 \ldots \ldots g$ iss

M. et sig.-A teaspoonful 3 times a day.

If the liver is noticeably swollen and firm to palpation, but yet little or no ascites is present, we often employ advantageously two other drugs,-calomel in small doses $\left(0.03-0.04\right.$ G. $\left(1 / 2^{-3} / 5\right.$ gr. $), 3$ powders daily) continued for a long time, or potassium iodide, also in small doses (up to $0.50 \mathrm{G}$. ( $7 \mathrm{r} / 2 \mathrm{gr}$.) a day). One of these sometimes works when all the other methods of relief have failed.

Diet.-In both groups of liver hyperæmia, meat must be wholly given up or limited to as small an amount as possible, for meat taken into the 
stomach and intestines sets up an active hyperæmia in the liver. A milk diet is therefore the best for such patients, as far as it can be made to agree with them.

Congestion of the Kidney.-Another organ which shows signs of congestion in heart lesions almost as frequently as the liver, is the kidney. Here, although subjective symptoms hardly oblige us to proceed directly against pain, a milk diet plays an important part. We give 200-250 G. (a cupful) every hour for 12-15 hours in the day (about $3 \mathrm{qt}$.). If the kidneys are only very slightly affected, we may replace milk by lactose ( $100 \mathrm{G}$. : $2000 \mathrm{G}$. of water $-3 \mathrm{oz}: 2 \mathrm{qt}$.) in similar quantities as before. Nothing else can be done but to stimulate the heart and use diureties as far as we ean without irritating the kidneys. We can, for example, use the following prescription, in which the potassium iodide is used to dilate the vessels, and ergotin to contract the smooth muscle fibres of the vessel walls (Robin):

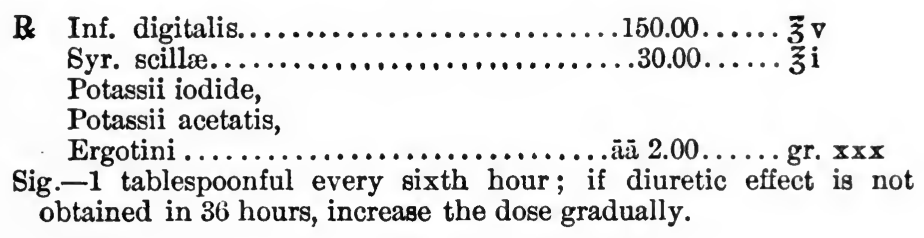

We may also use local bleeding, mild laxatives, cold colon irrigations, and hot foot-baths.

Congestion of the Stomach and Intestine.-Two more organs, the stomach and the intestine, are often congested. In consequence of the stasis in the hepatic veins, the circulation in the territory of the trunk and branches of the portal veins also suffers, only too often causing congestion in the mucous membrane of the stomach and intestine. This is the reason for the frequent catarrh of these organs in heart disease. The catarrh, of course, must be treated symptomatically (see p. 470). But this therapy must be accompanied by heart stimulants in order if possible to remove the cause. One fact may be noted, which has already been mentioned (see p. 14). Even where symptoms of congestion of the stomach are present (belching, nausea, vomiting), we are not obliged to give up digitalis but may prescribe it (Fräentzel) in very small amounts (1-1.5 G. (15-22 gr.) to $60 \mathrm{G}$. (2 oz.) of water), a small teaspoonful every 2 or 3 hours, ice cold and accompanied by lumps of ice or an ice cold liquid. Fräentzel and the author have both had good results from this. Another method is to give intravenous or subcutaneous injections of digalen or digitalin, or to use digalen, digitalis, or digitoxin in enemata or suppositories. The administration by the rectum, however, is of course the least effective of these methods.

In conclusion, attention may be called to the fact that some patients with heart lesions (or atheroma) know nothing of their heart trouble but 
complain only of indigestion (loss of appetite, slow digestion, fullness, pressure, burning, tightness after eating, relieved by loosing the waist band, and sometimes shortness of breath and palpitation). These symptoms depend upon slight circulatory disturbances in the region of the portal vein. Their therapy is not cardiac-digitalis often does more harm than good-but rather dietetic. The patient should be first put upon a milk diet, which is afterward changed gradually to a very light mixed diet. A half hour before eating he should take a glass of an alkaline or alkaline-sulphate water (Styriaquelle, (Rohitsch), Ferdinandsbrunnen (Marienbad), Vichy), and after eating, an antifermentative (menthol, naphthol, charcoal, see p. 424), followed 2-3 hours later by an irrigation of camomile or peppermint tea. The evening meal must be taken earlier and be smaller in quantity than usual.

Morphine in Heart Disease.-In conclusion, the use of morphine or opium in uncompensated valvular lesions or in pure muscular insufficiency must be briefly discussed. Although in general morphine is not to be used, yet under certain peculiar conditions it is an exceedingly im. portant agent at our command, to be employed not only as a sedative but as a direct help in relieving the condition. In such cases the widely prevalent prejudice against its use is most unfortunate. The author employs it internally, by rectum, or subcutaneously, where the patient is prevented from resting by severe dyspnœa, palpitation, cough, pain, mental excitement, or torturing symptoms of congestion. In such cases the loss of rest, the rapid falling off in the state of nutrition, and the general pain and torment, do much more harm than that attributed by many physicians to the use of morphine. Indeed the temporary rest and sleep afforded by morphine give the heart and nervous system a chance for recuperation, so that its use may be said to be directly curative. Morphine may be a cerebral and cardiac tonic. Practically, the author first tries an hypnotic; e.g., veronal with codeine. If this fails, he gives morphine, every evening if necessary. In one case of extreme insufficiency, hydrops, and torturing dyspnœa, when every other treatment did no good, the use of morphine, as advised by the author, brought about such an improvement that the patient was able to go back to his office work again. He died suddenly a year and a half later. It is to be hoped that the prevalent fear of morphine in heart disease will yield to its intelligent use in these cases, where it is so strongly indicated.

\section{THERAPY OF COMPENSATED SYMPTOMATIC HEART LESIONS.}

In dividing heart lesions into those with full compensation without symptoms and those with broken compensation (see p. 1) the author mentioned the existence of a third class,- - those with full compensation but with pathological symptoms caused by abnormal conditions of the circulation, particularly in the peripheral vessels. For the sake of brevity, he calls these "compensated symptomatic heart lesions." They 
are almost exclusively lesions of the aortic valve, generally insufficiency. These symptoms are (1) cerebral or (2) respiratory.

I. Cerebral Symptoms.-The cerebral symptoms are due to ischæmia of the brain, and to the pulsus celer of the cerebral arteries, caused by the quick and forcible streaming back of the blood from the arteries to the heart in disatole. They may occur in fully compensated aortic insufficiency.

In many respects, these symptoms are like those of neurasthenia (headache, sense of confusion, giddiness, often the typical fear of falling from a high place, excitability, brain fag). The patient's family and friends have often noticed for a long time his psychical sensitiveness, quick temper, and irritability. These are sometimes accompanied by obscurations of consciousness, and occasionally by insanity, oftener maniacal than of the melancholy type.

II. Respiratory Symptoms.-The principal respiratory symptom is nervous dyspnœa. It is caused either (1) by the transference to the pulmonary plexus of the stimulation-direct (periaortitis) or indirectof the cardiac plexus, or (2) by the direct irritation of the vagus from compression by the abnormally large heart.

Opium-Morphine.-The cerebral and the respiratory symptoms are both to be met by the same treatment,- the administration of opium or morphine, which are as preëminently the sole remedies in these cases as is digitalis in broken compensation. In suitable doses, opium, as we have said before, increases the supply of blood to the brain, but it also is a cerebral stimulant and a great aid in nervous dyspnœa. As Huchard expresses it, it makes one breathe, an effect due to its sedative influence upon the nervous system. There is one important contraindication to its use, however,- the presence of any kind of renal disease causing decreased functional activity, which would involve the danger of a serious intoxication from medicinal doses. In aortic insufficiency, especially, it is essential to make sure of the condition of the kidneys, since, in the author's experience, about one-fourth of the cases are associated with atypical parenchymatous nephritis. [Small doses 0.7 G. (10 gr.) of the bromide of sodium 3 times daily instead of opium or morphine will often allay these symptoms without causing the disagreeable effects of the morphine. The bromide is especially indicated also when, on account of renal insufficiency, opium is contraindicated.-Ed.]

If the kidneys are found to be intact, morphine is given, preferably subcutaneously, as in that case it is absorbed very quickly into the system, taking effect in 10 minutes, instead of in $1 / 4-1$ hour, the time required if taken by the mouth.

The maximum dose of morphinæ hydrochloridum, or of the much less useful morphinæ sulphas, is $0.03-0.12 \mathrm{G}$. ( $1 / 2-2 \mathrm{gr}$.).

Intolerance for Morphine in Certain Individuals.-Many individuals have an idiosyncrasy for morphine which cannot be foreseen; in others it is sufficiently typical to be anticipated (in nervous women, girls at the 
age of puberty, women at the climacteric, aged persons, and those inclined to cerebral hemorrhage). In such cases we must observe great caution, beginning with very small doses and slowly increasing them. Sometimes, when even the smallest doses of morphine produce symptoms of intoxication, especially vomiting, we can employ a mixture of morphine and atropine, in a hypodermic tablet containing $0.01 \mathrm{G}$. ( $1 / 6 \mathrm{gr}$.) morphine and $0.0005 \mathrm{G}$. ( $1 / 120 \mathrm{gr}$.) atropine.

If this will not work, or if, for any other reason (coincident intestinal symptoms), we prefer opium, we give it in the powder, extract, or tincture. For a fuller discussion see p. 284 (pulmonary diseases) and p. 479 (intestinal diseases). A few prescriptions are given here:

B Pulv. opii $\ldots \ldots \ldots \ldots \ldots \ldots \ldots \ldots \ldots, 0.01-0.03 \ldots \ldots$ gr. $\frac{1}{6}$-gr. $\frac{1}{2}$

Sacchar. lactis..................... $0.40 \ldots \ldots$ gr. vi

M. Dent. tal. pulv. no. $x$.

Or :

Sig. -1 powder every $2-3$ hours.

R Ext. opii................................... vi

Pulv. glycyrrhizæ...................2.00... 3 ss

Ext. gentianæ...........................

M. fiat mass. Div. in pil. no. $x \times$.

Or :

Sig. -1 pill every 2 hours.

B Tinct. opii,

Tinct. valerianæ,

Tinct. aromaticæ................. āa $10.00 \ldots \ldots z$ iiss

M. fiat mist.

Sig. $-15-20$ drops 2-3 times a day.

Maximum doses : Pulv. opii......G. $0.15-0.50 \ldots \ldots$ gr. $21-7 \frac{1}{2}$

Ext. opii ......... $0.10-0.40 \ldots$... gr. $1 \frac{1}{2}-6$

(Vogel recommends only half of this.)

Tinct. opii......G. 1.00-5.00..... m 15-75

[All these doses are much larger than I employ.-Ed.]

Bromides-Quinine.-Instead of opium, we may use bromide in cases of slight dyspnœa [see note above], or quinine in cases of cerebral disturbances.

Dyspnœa in Mitral Lesions.-Occasionally dyspnœa occurs also in fully compensated mitral lesions, in consequence of the increased amount of blood in the lungs, which manifests itself as pulmonary distention and rigidity (Basch). Here, of course, our aim must be to strengthen the heart muscle. (See p. 7 for the use of warm baths and p. 26 for the administration of valerian and its preparations.) The author has frequently found the long continued use of smaller doses of digitalis or strophanthus of great value in these cases, as well as in those mitral lesions, in which the slightest movement brings on subjective symptoms. The author has often given for this purpose $0.05-0.15$ G. $\left(3 / 4-2 \frac{1}{4}\right.$ gr.) of digitalis in any form preferred, or $0.20-0.50 \mathrm{G}$. $(3-7 \mathrm{I} / 2 \mathrm{gr}$.) of strophanthus a day, for several weeks. The so-called pneumatic treatment also seems worth trying in this group of mitral lesions, as well as 
in those just beginning to show signs of broken compensation. The author has no personal experience with the method, but it is recommended by such authorities as Rosenstein and Hoffmann. Rosenstein ranks it along with the use of digitalis, especially in lesions of the mitral orifice, although its effect is not so lasting. The patient is made to breathe compressed air by means of Waldenburg's apparatus or Geigel and Mayer's new "Schoepfradventilator" (better, according to Rieder). The effect in fully compensated mitral lesions which are still accompanicd by dyspnœa, or in those in the first stage of broken compensation manifesting itself merely by catarrh from the congestion of the respiratory tract, is thus explained by Hoffmann: The inhalation of compressed air, as experiments on animals show, undoubtedly causes pulmonary anæmia. This brings about a decreased stream of blood to the left heart, and a more difficult passage of blood from the right heart, thus lightening the work of the left heart. Since an oversupply of blood in the pulmonary circulation in compensated or almost compensated mitral lesions arises from a mitral insufficiency (even if it is very slight) in combination with a functionally perfect right heart, the good effect of compressed air in such cases is evident. [My colleague, Dr. Quimby, is a warm advocate of the cabinet treatment in heart disease. Since he introduced its use at the City Hospital, several cases, especially of aortic insufficiency, have shown remarkable improvement. For an interesting paper and discussion, see the Boston Medical and Surgical Journal, vol. cliv, no. 20, p. 549.-Ed.]

III. Painful Dyspepsia-Palpation.-There remain two more symptoms occurring with fully compensated lesions,- the "painful dyspepsia" often found in aortic insufficiency, and the "palpitation of the heart" accompanying either aortic or mitral lesions, but occurring even more frequently after broken compensation has begun.

There are not a few cases of aortic disease with perfect compensation, in which the patient complains of cramps in the epigastrium or occasionally of throbbing pain. These are better if the patient keeps still, worse if he makes any marked exertion or eats more than usual. The digestion is poor and the patient suffers from belching and pyrosis. These subjective symptoms are often accompanied by the objective symptom of a strong pulsation of the abdominal artery in the epigastric region. They are probably due to a neurosis of the cœliac ganglion or of the abdominal sympathetic caused by the permanent shaking of these nerve elements by the abdominal aorta.

The therapy of this cardialgesic form of aortic insufficiency is analogous to that of intense throbbing of the heart in full compensation. Ice-bags or Leiter's coils may be laid on the epigastrium or precordial region. Leiter's apparatus consists of a series of thin tubes made of rubber, aluminum, or very flexible lead covered with tin and bound together with bands. Ice-cold water is introduced into them by a rubber tube and escapes through another tube at the end of the coil. To start 
the flow of the water, the air is sucked out from the end of the escape tube with the mouth or, better, a syringe. For certain parts of the body (breast, head, abdomen), coils are made of a suitable shape.

Many patients are not helped, however, as much by cold as by hot applications (wet or dry) upon the epigastric region. In such cases we may use the thermophor, a rubber bag filled with sodium acetate. This is heated in boiling water for $6-10$ minutes, the salt being thus dissolved, and is then wrapped in hot wet eloths and laid on the patient. The crystallizing of the salt will keep the temperature at at least $113^{\circ}-122^{\circ}$ for four or five hours.

The medicinal therapy seeks to diminish the irritability of the heart (erethismus cordis). The bromides, with morphine or opium, are the most useful. The use of sodium bromide has already been discussed (see p. 48). Potassium bromide is not contraindicated in these cases, and is given in similar doses. Valerian may also be tried. The author has also often found quinine, in the form of the valerianate or hydrobromate, of use, especially if cerebral symptoms are present (see p. 70). [Cushny does not consider that the valerianate of quinine has any pharmacological action beyond that of the other salts of the acetic acid series.-Ed.]

B Quininæ valerianatis (or hydrobromidi) ...0.20-0.30 ....gr. iii-gr. v Dent. tal. dos. no. xv. Dispens. in cachets.

Sig. $-3-5$ cachets a day.

Mild hydropathic measures, such as those described in the therapy of compensated lesions (p. 7), are also beneficial.

If everything else fails, we may have recourse to chloroform water or cocaine (see p. 430), especially in severe cardialgia.

Aortic insufficiency, whether compensated or not, is often accompanied by many symptoms caused by the pathological condition of the blood vessels which lies at the root of the heart lesion. The therapy of these diseases of the vessels will next be discussed, particularly of such as give rise to aortic insufficiency through the extension of the pathological process from the walls of the aorta to the aortic valves.

\section{THERAPY OF DISEASES OF THE BLOOD VESSELS.}

The following diseases are excluded from this discussion: (1) All diseases of the veins, as they must be treated almost entirely by surgical interference; (2) acute inflammation of the peripheral arteries, for the same reason; (3) acute inflammation of the aorta, as it has been included under endocarditis (see p. 87) ; (4) very rare affections, such as congenital narrowing or dilation of the arteries or acquired narrowing of some one artery. There remain to be treated (1) chronic arteritis in its different forms, and (2) aneurism of the aorta. 


\section{THERAPY OF CHRONIC ARTERITIS.}

Arteriosclerosis.- The principal form of chronic arteritis is arteriosclerosis, which frequently, to be sure, begins with an acute process, but which later runs a latent course, so that when it makes itself manifest it is a chronic process of long standing. On account of its histological and anatomical peculiarities, as well as its special therapy, syphilitic endarteritis will be treated in a separate section. Periarteritis nodosa will be entirely omitted, as it causes no symptoms and is an exceedingly rare affection.

\section{Therapy of ARteriosclerosis.}

Causal Therapy.-Certain cases of arteriosclerosis demand, beside the general therapy of sclerosis as such, a special causal therapy. They are the cases of arteriosclerosis due to $(a)$ gout, $(b)$ diabetes mellitus, (c) obesity, $(d)$ alcoholism, and $(e)$ nicotinism.

(a) In Uric Acid Diathesis.-The causal therapy of the first is of course that of uric acid diathesis in general.

(b) In Diabetes Mellitus.-From the second group we must except those cases in which the diabetes is itself a result of a preëxisting arteriosclerosis arising in another way, namely, from sclerosis of the pancreatic artery. The author considers it probable that the rich proteid diet in diabetes, together with toxic factors and others not clearly understood, is accountable for the origin of the arterial atheroma. He therefore advises that in diabetes with prominent arteriosclerosis and heart symptoms (diabetic coma may be frequently explained by paralysis of the heart, according to Frerichs), the meat diet should be reduced as much as possible and more fat should be tentatively given.

(c) In Obesity.-The causal therapy of arteriosclerosis in obesity is of course that of the obesity itself.

(d) Alcohol.-As alcohol is an undoubted cause of arteriosclerosis, it should not be permitted to those suffering from this disease.

(c) Nicotine.-The same is true of smoking (Huchard), which in the author's opinion is not infrequently a cause of the disease.

In some other cases we may also eliminate the cause; e.g., working in lead, or too much bodily strain in hard work.

Traumatic, Toxic, and Post-Infectious Forms.-All forms of arteriosclerosis, those already named and all others (e.g., traumatic, toxic, and post-infectious), may be divided for therapeutic purposes into (1) those in which the heart muscle by hypertrophy is still able to com. pensate for the lessened elasticity of the arteries and their narrowing, and (2) those in which its strength is already partly paralyzed. In the latter case-cardiac insufficiency-we must, of course, use heart stimulants (digitalis and ergotine together, p. 61). It must be emphasized that arteriosclerosis, even with increased blood pressure, is no contraindication to the use of digtalis. If insufficiency of the heart muscle is 
present, we must use digitalis, and as a result we often see improvement of the heart's action and reduction of the blood pressure, a proof that this had been abnormally increased by the circulatory disturbances and the carbon dioxide intoxication connected with them. The therapy must furthermore aim at diuresis, as in hydrops in heart lesions. In the first group of cases, those with full compensation, our effort must be to reduce the excess of tension in the peripheral arteries and the increased blood pressure.

Diet.-The fact already mentioned, that too abundant a diet may cause arteriosclerosis, should be kept in mind. Moreover, the food should contain as small an amount of toxic substances as possible, as these, introduced into the body, cause a narrowing of the vessels (vasoconstriction) and therefore an increased blood pressure. The diet should consist of large quantities of milk in all forms, easily digestible vegetables (see p. 2), and the amount of meat necessary to keep up nitrogen equilibrium in the body. Game, sausages, all richly spiced food, strong broths, caviar, brains, sweetbreads, strong cheese, tea, coffee, chocolate, liqueurs, and all strongly alcoholic drinks should be absolutely forbidden. If practicable, smoking should also be prohibited, as nicotine is one of the strong vasoconstrictor toxins. As in compensated heart lesions (see p. 3), the amount of liquor allowed in 24 hours must be cut down. In the lighter cases only, very small quantities of alcohol (light Moselle wine mixed with water, or possibly a very little good beer) may be allowed.

Baths, Etc.-For the proper climate, the use of baths, etc., see the Therapy of Compensated Heart Lesions, p. 7. There are, however, some patients with arteriosclerosis who do well at $3000 \mathrm{ft}$. and over. [Very few, I believe.-Ed.] One important fact should be particularly emphasized,- - the danger in arteriosclerosis of any sudden alteration of the blood pressure, particularly of any sudden increase: Hot or very cold baths are interdicted therefore; the first because they may lead, aside from cerebral consequences, to sudden ischæmia and therefore paralysis of the heart, the second because they may cause only too easily rupture of the deep arteries, particularly those of the brain. On the contrary, the use of thermal baths in moderate cases of arteriosclerosis with compensated heart is often beneficial. Suitable resorts are Johannisbad (Bohemia), Schlangenbad (Hesse-Nassau), Teplitz (Bohemia), and Ragatz (Canton St. Gall, Switzerland). Gastein (Salzburg) is too high to be recommended (over $2000 \mathrm{ft}$.). The temperature of the baths is sometimes too high, and must be lowered to $75^{\circ}-80^{\circ}$ at first. [In America, no particularly well adapted resorts are available. General principles, however, lead us to advise the Hot Springs of Virginia during the spring and fall, the Berkshire Hills, the Adirondacks, the Maine coast, or the southern New England coast during the summer, Southern California, Georgia, or the Carolinas during the winter.-Ed.] The hot carbon dioxide baths at Nauheim also appear to be of use, although not so conspicuously so as in valvular lesions. 
Charged waters can be taken only if the carbon dioxide be first allowed to escape, and cold waters must be avoided, as either may lead to a sudden increase of pressure in the peripheral arteries and even to sudden death. If hot waters are taken, the temperature must be first reduced.

Gentle Hydriatic Procedures.-Gentle hydriatic measures are beneficial on account of their stimulating effect on metabolism, and their tonic influence on the nervous system (sponge baths or rubbing with alcohol or water at the temperature of the room). The author also places great reliance on regular and long continued massage of the abdomen and extremities (lowering of the blood pressure), and gymnastics.

Medication.-The bowels must be kept open by a mild vegetable or saline cathartic, if necessary.

An abundant diuresis must also be secured, since in arteriosclerosis the renal secretion suffers in consequence of the condition of the renal vessels, and toxins may be retained in the organism. Huchard advises theobromine, the diuretic effect of which (see p. 28) is due to a direct action on the renal epithelium:

$\mathrm{R}$ Theobromini $\ldots \ldots \ldots \ldots \ldots \ldots \ldots \ldots \ldots \ldots . \ldots \ldots \ldots \ldots \ldots$ gr. viii
Dent. tal. dos. no. xxx. Dispens. in gelatin caps.
Big. $-3-4-6$ caps. daily.

The author often uses diuretin, agurin, or theocin.

Another class of drugs usually given is the alkalies of iodine. Their effect is usually attributed (Huchard) to a dilatation of the peripheral vessels and a decrease of the blood pressure. Geigel lays great stress on a coincident expansion of the coronary arteries, while other authors attribute their favorable effect to a reduction of the viscosity of the blood and the consequent improvement of the circulation in the peripheral vessels, especially the capillaries. Although some authors deny any good effect from the alkalies of iodine, unless the arteriosclerosis is of luetic origin, the author differs from them, having had quite the opposite experience in his own practice, provided the iodine is continued long enough. Potassium or sodium iodide is given in 0.5-1.5 G. (7-22 gr.) daily doses, the amount being increased from week to week:

B Potassii (sodii) iodidi..........5.00-10.00-15.00......gr.lxxv- $\mathbf{3}$ iiss $-\overline{\mathbf{z}} \mathrm{ss}$ Sodii bicarbonatis...................5.00....gr. l l $x \times v$

Aq. menthæ piperitæ, Aq. dest......................... āā $75.00 \ldots \ldots$ z̃ iiss

M. fiat sol.

Sig. $-1-1 \frac{1}{2}$ tablespoonful daily after meals.

This treatment must be kept up for a long time, several years or so, being best intermitted every fourth week. The author usually adds about $5 \mathrm{G}$. (75 gr.) of sodium bicarbonate (see foregoing prescription), believing that iodine intoxication is thus better avoided. Or he gives 
0.25 G. (4 gr.) of potassium iodide in a little milk morning and night, during or just after meals. Instead of potassium or sodium iodide, we may use 6 per cent. iodine vasogen, 10 drops 3 times a day, increasing by 5 drops every other day, until we get it up to 30 drops 3 times a day, then decreasing the dose again. When internal administration is not possible, we may give iodipin subcutaneously or intramuscularly (1-5-10 c.c. ( $15 \mathrm{~min} .-1 \frac{1}{4} \mathrm{dr} .-2 \frac{1}{2} \mathrm{dr}$.) of the 25 per cent. preparation-a sesame oil of iodine-as an injection every 2-3 days). Since the iodine is absorbed from iodipin very slowly and gradually, there is almost no danger of iodine intoxication. In spite of this great advantage, iodipin cannot be recommended for internal use with most patients, on account of its almost unbearable taste. It must be borne in mind that some individuals cannot bear iodine in any form or get any advantage from it. With them it brings on disturbances of appetite and digestion, irritation, or loss of body weight, any one of which symptoms should be sufficient cause for the immediate cessation of the treatment. [Vierordt prescribes 2-3 gr. of iodide of sodium 2 or 3 times a day at first, then gradually increases the dose up to $15 \mathrm{gr}$. 3 times a day. He continues this medication for from 1 to 3 years, and claims to have obtained striking results. $\mathrm{He}$ omits the remedy one week in five and one month in five. Balfour advises small doses, 2 or 3 gr. every 12 hours over a long period. Babcock states that nothing is of greater service in cases of diffuse arteriosclerosis with secondary venous engorgement, than a periodic purge by means of calomel. The catharsis should be brisk.-Ed.]

A long continued use of arsenic is also often in place (see p. 10). [Balfour extols its employment.-Ed.]

Rumpf has recently recommended the treatment of arteriosclerosis by giving food with as little calcium as possible, and administering lactic acid; but the author, with other authorities, has not found the suggestion of any value.

Trunecek's Serum.-Recently there has been much discussion regarding the use of the serum anorganicum Trunecek in subcutaneous injections up to 10 c.c. daily. It is composed of a number of physiological blood salts (sodium chloride, sodium sulphate, sodium carbonate, calcium phosphate, magnesium phosphate). Its originator recommends its use only in the so-called arteriosclerotic meiopragia (dyspragia); other authorities confine its use to arterial hypertension in consequence of arteriosclerosis. The author has tried it in all possible varieties of the disease, without being .able to maintain that it has any reliable good effect, though it does no harm.

Nitrites.-There is another group of drugs, not so often used as the iodides, but having an effect upon the vessels similar to that which is supposed to be exerted by the iodides,-amyl nitrite, nitroglycerin, 'and sodium nitrite. The reason for their infrequent use is that their effect, though quick, disappears as quickly (in about 10 minutes). Hence, they are particularly suited to atheromatous processes involving the cardiac 
arteries and producing symptoms of angina pectoris. Before we pass to a discussion of this condition, another single symptom of atheroma, which is of the greatest diagnostic importance, will be taken up. It occurs particularly in chronic aortitis of the ascending aorta, perhaps in consequence of an acute or subacute exacerbation of this condition. We refer to retrosternal pain.

Retrosternal Pain.-This comes almost without exception when the patient is active,-going upstairs, for instance,-disappearing again when he is quiet. The first therapeutic measure is, naturally, to put the patient to bed. Secondly, we may use an ice-bag or, in some cases, preferably hot applications upon the seat of the pain, over the sternum. The good effect of this will be evident if one remembers that the pains due to an arteritis or periarteritis of the aorta are the result of irritation of the aortic plexus, caused by inflammation of the aortic wall. We may also use revulsives, such as blisters or the cautery over the origin of the aorta. For cauterization, the skin is lightly touched with a red hot cautery, making up to 60 or more tiny blisters.

Cerebral Symptoms in Atheroma.-Another special group of symptoms may be mentioned,- the cerebral symptoms in atheroma of the cerebral vessels (headache, giddiness, loss of consciousness, transient disturbances of speech, etc.). The author uses in such cases a combination of digitalis or sparteine with potassium iodide, or of an alkali of iodine with an alkali of bromine.

\section{Therapy of Angina Pectoris.}

The symptom complex of angina pectoris is made up of pain behind the sternum and precordial pains radiating into the left arm, in different directions through the abdomen, and to the teeth of the left lower jaw, accompanied by the severest anguish and even the sensation of impending death.

Causal and Prophylactic Therapy.-The attack itself is treated in the same manner no matter what its etiology, but the preventive therapy of course depends upon whether the angina pectoris is a symptom of a uric acid diathesis, of diabetes mellitus, of a syphilitic arteritis of the coronary arteries, or of arteriosclerosis of the coronary arteries. In the last case, the only one which interests us here, the preventive therapy consists of the iodide treatment (see p. 75) and the proper dietetic precautions (see p. 74). Many authorities (F. Frank and Potain) insist on an avoidance of all bodily strain and particularly of all quick and strong movements of the left arm. Smoking or being in an atmosphere of tobacco smoke is strictly forbidden, for nicotine has been definitely proved to contract the arteries (Claude Bernard, Basch, Oser; see also p. 4).

Therapy of THE AtTACK.-Passing to the treatment of the attack itself, we find a number of drugs at our command. The first of these is 
morphine. This takes effect best and quickest when given subcutaneously; like all the forms of opium, it does not work wholly symptomatically as an analgesic, but, by its power to dilate the capillary vessels, has also an effect on the cause of true angina pectoris, the ischæmia of the heart muscle. An injection of $0.01-0.03 \mathrm{G}$. ( $1 / 6-1 / 2 \mathrm{gr}$.) of morphine in many cases will cut the attack short; in severe attacks the injection may have to be repeated. If the pain begins, as it sometimes does, in the arm, an immediate injection may stop its spreading to the heart.

Chloral-Chloroform.-Chloral and the inhalation of chloroform are often recommended, but the author does not believe that either of them is free from danger if the angina pectoris is caused by an atheroma of the coronary arteries.

Nitrites: Amyl Nitrite.-Another group of drugs, frequently and successfully used, consists of amyl nitrite, nitroglycerin, and sodium nitrite.

Amyl nitrite, a yellowish-green, oily, exceedingly volatile and inflammable liquid, has an intense central and direct effect on the blood vessels, dilating them most strongly in the region of the skull and face, much less markedly in the extremities. It not only insures a better flow of blood by its vasodilatory properties, but also lowers the blood pressure by weakening the cardiac activity. Small doses are regularly followed by a quickening of the heart beat. The fact that these results follow in a few seconds after amyl nitrite is inhaled make it particularly suited to attacks of angina pectoris. To be sure, in the author's opinion, it is contraindicated in marked atheroma of the cerebral arteries, as in that ease the hyperæmia of the inner lining of the skull induced by amyl nitrite may easily lead to hemorrhage.

Five or 6 drops of amyl nitrite are poured upon a handkerchief or a small sponge covered with a handkerchief, and then inhaled through the mouth and nose. It is essential that the drug be fresh. A more convenient method, however, is to enclose the desired amount in a glass pearl, which can be easily broken; for instance:

R Amylitis nitritis................ $0.20-0.30 \ldots \ldots$ gtt. iii-gtt. $\nabla$

Dent. tal. dos. no. $x$. Dispens. in glass caps.

Sig. -1 pearl to be crushed in a handkerchief and the contents strongly inhaled.

As the system quickly adjusts itself to amyl nitrite, the amount to be inhaled will soon have to be increased. It should, however, never exceed 10 drops. It may be said in passing that amyl nitrite should always be kept in the dark, as exposure to the light brings about the development of hydrocyanic acid.

Nitroglycerin: Effect.-Nitroglyeerin has the same action as amyl nitrite (dilatation of the vessels, lowering of blood pressure, and quickening of the heart, but without weakening it), and its effect is slower and more lasting. Yet its action is only of moderate duration, as a 
recent work places it at only 10 minutes, while the longest time assigned by any authority (Manquat) is 2-3 hours. Nitroglycerin is given to mitigate or stop an attack of angina pectoris, and in the hope of deferring or preventing a recurrence. It may be prescribed as follows:

B Spir. glycerylis nitratis ..............5.00....ml $1 \times x \mathrm{~V}$

Sig. $-1-4$ drops several times a day on sugar.

Or :

B Spir. glycerylis nitratis................2.00.....gtt. $x \times x$

Aq. dest......................... $300.00 \ldots$

M. fiat sol.

Sig. -3 tablespoonfuls daily. ( 1 tablespoonful $=1 \frac{1}{2}$ drops of the solution.)

Or :

R Spir. glycerylis nitratis................0.03....gr. s8

Pulv. glycyrrhizæ.....................4.00..... $3^{\mathrm{i}}$

Ext. gentianæ.............................. s.

M. fiat mass. Div. in pil. no. $1 x$.

Sig. $-1-4$ pills daily.

The author has given up the use of prepared tablets, since Binz has shown that they often have wholly lost their content of nitroglycerin. [I agree heartily with the author. I have frequently tested the tablets at the New York City Hospital and others sold at excellent pharmacies, and found them almost if not quite inert. This method of administration is, however, so convenient in just the class of cases for whom we prescribe nitroglycerin that I continue to order them, but in very small quantities, freshly prepared each time. Forchheimer recommends the following: "One drop of a 1 per cent. alcoholic solution of nitroglycerin is dropped upon a plate; the experiment is then made to see how many simple sugar pills will absorb this drop; then a number of drops of nitroglycerin are put into a small vial, to which are added the requisite number of sugar pills." -Ed.] They may be preseribed in a manner analogous to the pills just mentioned. Or nitroglycerin may be given subcutaneously (Dujardin-Beaumetz).

Sodium Nitrite.-Sodium nitrite is less used than either of the others. It is given in doses of $0.03-0.05 \mathrm{G}$. $(1 / 2-3 / 4 \mathrm{gr}$.) 3 or 4 times a day.

B Sodii nitritis......................... $0.50 \ldots \ldots$ gr. viii

Aq. dest............................ $150.00 \ldots \mathrm{z}$

M. et sig. -1 tablespoonful $3-4$ times a day. $\quad(1$ tablespoonful $=$ 0.05 G. $-\frac{3}{4}$ gr.)

Erythrol Tetranitrate.-Turney, in Flintshire (England), has recently recommended another preparation, erythrol tetranitrate. The author has tried it in a number of cases, and finds that its effect is apparently more permanent than that of amyl nitrite or nitroglycerin. Sometimes the dose recommended by Turney ( 0.07 G. -1 gr.) brought on severe headache, with throbbing in the head, giddiness, and general dis- 
comfort. The author, therefore, generally reduced the dose to 0.010.02 G. ( $1 / 6-1 / 3 \mathrm{gr}$.), seldom exceeding $0.04 \mathrm{G}$. $(3 / 5 \mathrm{gr}$.). The dose was repeated if necessary an hour afterward, but the maximum daily amount was kept down to $0.05 \mathrm{G}$. ( $3 / 4 \mathrm{gr}$.). In the case of one patient, whose attacks came on in the arm, beginning with only a slightly painful paræsthesia, a pill at the beginning would sometimes avert the attack, but at others would have no results. Erythrol tetranitrate, then, aside from its more lasting effect, has apparently no advantage over amyl nitrite or nitroglycerin, so far as the effect upon a single attack is concerned. But it is very well suited for use after an attack (0.01-0.02 G. $(1 / 6-1 / 3$ gr. $)$ a day) to avert or at least to delay a repetition. [I have found $0.016 \mathrm{G}$. ( $1 / 4 \mathrm{gr}$.) too strong a dose for many people.-Ed.] The author's prescription is as follows:

B Erythrol tetranitratis.............. $0.10-0.20 \ldots \ldots$ gr. iss-gr, iii

Pulv. glycyrrhizæ.........................

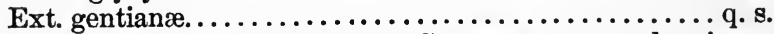

M. fiat mass. Div. in pil. no. xx. Consperg. cum pulv. cinnamomi.

Sig. -3 of the weaker pills daily between attacks; 2 of the stronger during an attack.

Theobromine.-To prevent as far as possible the return of an attack, the author has recently used-with Breuer-the theobromine preparations, with good success. They are also often useful in preventing the retrosternal pain in arteriosclerosis of the ascending aorta. We give 1.5-3.0 G. (20-45 gr.) of diuretin or agurin, and correspondingly smaller doses of theocin and its compounds, or the dispnon tablets. The effect of these preparations is probably due to their dilatation of the coronary arteries of the heart. [Forchheimer has had success with diuretin in many cases.-Ed.]

Antineuralgics.-Another group of drugs, the antineuralgics, have only a slight effect. Among them are antipyrine, antinervine (Radlauer), phenacetin, exalgin, maretin, and pyramidon. The dose of the first two is $0.5-1 \mathrm{G}$. (7-15 gr.) :

R Antipyrini (or antinervini) $\ldots \ldots \ldots \ldots \ldots . .50 \ldots \ldots$ gr. viii

Dent. tal. dos. no. $x$. Dispens. in gelatin caps.

Sig. $-1-2$ caps. at a time, to be repeated in 2 hours if necessary.

The dose of the others is one-half as much. Exalgin may also be given in an aqueous solution:

B Exalgini...........................2.50.....

Spir. menthæ piperitæ..................5.00.... m $\mathrm{lxxv}$

Aq. dest......................... 120.00..... క iv

Syr. aurantii flor........................30.00..... $\xi^{i}$

M. fiat mist.

Sig. -1 tablespoonful at morning and at night. ( 1 tablespoonful $=0.25$

G. (4 gr.) exalgin.)

[I should advise against any of these drugs.-Ed.] 
Revulsives.-Sometimes mustard plasters or hot applications over the cardiac region help to relieve the pain.

Diet.-The diet should be similar to that in arteriosclerosis (see p. 74), non-irritating, and one giving the heart as little work as possible. But the author does not believe in a too restricted diet (Entziehungskur), especially Tufnell's cure (see p. 82). He has seen two cases rapidly fall off under this treatment, while the number and intensity of the attacks was inereased, an effect probably due to the lowered blood pressure and depressed cardiac activity.

\section{THERAPY OF SYPHILITIC ARTERITIS.}

Iodides-Mercury.-All that need be said on this subject is that we must institute a thorough antiluetic treatment, using both iodides and mereury. The latter may be administered by inunction or be injected subcutaneously or into the veins. [I do not think that this important subject should be so briefly dismissed. In young or middle-aged adults an antisyphilitic cure is usually indicated; early in the disease mercury will prove serviceable, later the small repeated doses of the iodides. I question, however, the advisability of administering even the iodides in the arteriosclerosis of very late syphilis. We must recognize, I feel, that there comes a time in these cases when the process has advanced too far to be relieved medicinally._Ed.]

\section{THERAPY OF AORTIC ANEURISM.}

Aim of the Therapy.--In treating an aortic aneurism, our aim should be to coagulate the blood in the aneurism and to bring about the organization of the coagulated blood. Nature employs this method, as many cases of spontaneous healing show. This occurs usually, but not exclusively, where the aneurism has only a narrow opening into the aorta (sacculated aneurism). Naturally, the coagulation of the blood is furthered by a retarded blood current in the sac and a permanently lowered blood pressure.

Rest.-A rational therapeutic measure will therefore be long continued mental and physical rest. The patient should lie on a water bed for $2-21 / 2$ months without sitting up, receiving the best of care, with an ice-bag or cooling apparatus over the precordia to quiet the heart. The influence rest has upon the heart is shown by 'Tufnell's observations; he found that 40 minutes' rest reduced the pulse of a patient from 96 to 66 a minute (Bäumler), a reduction of 43,000 contractions in 24 hours.

Nourishment.-The reduction of the blood pressure is also brought about by reducing the amount of food, although to-day we do not subject the patient to the starvation diet which, with frequent bloodletting and rest in bed, was the classical treatment of aneurism, as proposed by Valsava and developed by Albertini. The English especially allow only a few kinds of food. 
Tufnell's Diet Scheme.-Tufnell recommends the following diet (total amount for 24 hours consists of only $300 \mathrm{G}$. (10 oz.) of solid and $240 \mathrm{G}$. $(8 \mathrm{oz}$.$) of liquid food) : Morning, 60 \mathrm{G}$. $(2 \mathrm{oz}$.) of milk or cocoa, equal amount of bread and butter; mid-day, $90 \mathrm{G}$. $(3 \mathrm{oz}$.$) of meat, same$ quantity of bread or potatoes, $120 \mathrm{G}$. ( $4 \mathrm{oz}$.) of water or of light Bordeaux wine; evening, 60 G. ( 2 oz.) tea and same quantity of bread and butter. If the patient gets restless and upset under the regime, this allowance should be slightly increased. Thirst may be quenched by pieces of ice. Pain and sleeplessness may be treated symptomatically. The author considers this diet too strict for the average patient with aneurism. He believes that in individual eases we may learn, by investigating the metabolism, how far the amount of food may be reduced without injury. If the patient is much run down, he must be built up by suitable and abundant food. Since the increase of the blood pressure must be carefully avoided, the quality and sometimes the quantity of the food should be the same as in arteriosclerosis (see p. 74). Too much meat, substances that might cause flatulency, and much liquid at meals are to be avoided for the same reason as in heart lesions.

The blood pressure in the aneurism is also lowered by drawing the blood away from it to other parts of the body; e.g., by keeping the legs warm, by sponge baths followed by rubbing, and by massage of the legs and abdomen.

Medication: Iodides.-Medication does not accomplish much toward curing aortic aneurism. The iodides, however, especially potassium iodide, are of great use in aneurism caused by syphilitic aortitis, although the reason for their effect is not clearly understood. The dose is 1-1.5 G. (15-20 gr.) a day, gradually increased to 5-6 G. (75-90 gr.). The treatment may be kept up for a long time. All other drugs,-for example, ergotine (recommended by Langenbeck), lead acetate, alum, digitalis, and opium,-may be successfully employed in treating certain symptoms of aneurism, but are of no use in combating the pathological process as such.

Lecithin.-Lecithin has been recommended, especially by English authorities, as having a strong coagulating effect on the blood. The author experimented with it in one case of aneurism, but without success.

Injection of Gelatin.-The French have recently claimed the same effect-increased coagulability of the blood-for an injection of gelatin into the gluteal region. They use a sterile $10 \mathrm{per}$ cent. gelatin solution (with the addition of common salt) and inject 40 c.c. (11/4 oz.) subcutaneously at the body temperature.

The method seems to have met with varying success in the hands of the French. The author has tried it in 7 cases of sacculated aneurism, giving 15-20 injections (1-2 a week) under the skin of the abdomen. In two cases the Roentgen rays showed that the aneurism had decidedly diminished; in one the compression neuralgia in the right arm and shoulder wholly vanished; while in the rest there was no marked effect 
of any kind. For the first $24-48$ hours, the injections cause considerable pain, and sometimes a rise of temperature, even to $103.4^{\circ}$. The method is certainly worth trying in sacculated, but is apparently useless in fusiform aneurisms.

Surgery.-The third method of treating an aortic aneurism is by surgery. The operations are something like those upon aneurisms of the peripheral arteries.

1. Compression.-Compression comes first. Naturally, this can be employed only when it is the abdominal aorta that is involved, but in such cases it has been successful. Proximal compression (between the sac and the heart) is to be preferred to distal. But the operation is by no means free from danger; cases have been known where it resulted in fatal peritonitis, or lesions of the organ compressed, such as the duodenum, pancreas, and cœliac plexus. The compression should be carried only to the point (usually under anæsthesia) where the pulsation in the aneurism disappears, and should be continued for not more than 5 hours. We may also use mild compression, if an aneurism of the ascending aorta pushes the chest wall outward, by employing adhesive plaster or, best, a collodion band or a convex pelotte over the pulsating tumor.

2. Introduction of Foreign Bodies.-All the other surgical methods aim at producing a coagulation of blood in the sac. The first is the introduction of foreign bodies into the sac of the aneurism. With or without the help of a trochar, a foreign body is introduced into the aneurism for the blood to coagulate about. This foreign body may be an iron or silver wire, a watch spring, horsehair, silk thread, or catgut. About 2-3 M. are generally introduced into the aneurism, although the originator of this method (Moore) employed even $23 \mathrm{M}$. of thin iron wire. The value of this method is disputed, but, at any rate, it is more successful where the aneurism is sacculated with only a small opening into the aorta. The author once saw Prof. Narath introduce over $1 \mathrm{M}$. of a thin watch spring into an aneurism as big as a small child's head, which was threatening to break through to the outside. The patient died 4 days later from heart failure, and the autopsy showed very little coagulation around the watch spring. To be sure, the aneurism had a wide opening into the aorta.

3. Acupuncture.-The third method is acupuncture. A fine needle is thrust into the aneurism and moved backward and forward, the scratch thus made serving as a starting point for coagulation. Or the needle may be left in for 48 hours, and the blood stream will move it sufficiently to cause the desired lesion. From the comparatively few records there seems to be some reason for trying this method.

4. Galvanopuncture.-The fourth method is galvanopuncture. A needle is introduced and connected with the anode of a battery, while the cathode, in the form of a large plate electrode, rests upon the patient's thorax. A current of 10-20 milliampères is employed for 10-40 minutes. Occasionally 2-6 needles are used, connected by pairs with the anode and 
the eathode, a procedure not unaccompanied by danger. The wound is closed with adhesive plaster or a collodion band, or an ice-bag is laid on it. A. cure is only very seldom effected, but an improvement is evident in a large number of cases, manifesting itself in the shrinking of the tumor, or the amelioration or disappearance of the subjective symptoms (pain, etc.).

5. Ligation of the Carotid and Subclavian.-In aneurisms of the ascending aorta, a fifth procedure may be tried, the tying of the common carotid and subclavian arteries. It is a last resort when a wide communication between the lumen of the aorta and the sac of the aneurism is suspected.

Ice-bag.-An ice-bag kept on the tumor for days and weeks may be tried as a means of inducing coagulation in the sac of the aneurism.

Of the surgical procedures, the most useful is the acupuncture, which the author believes should in practice always be tried when the proper diet, rest, and treatment with iodides or gelatin have failed.

Symptomatic Therapy.-As all these methods so often fail, it follows that we must often be reduced to a merely symptomatic therapy. The first symptom that we have to contend with is the frequent pain arising from the pressure of the aneurism on the neighboring nerve trunks. We can employ for this purpose any of the antineuralgics: antipyrine $(0.50$ G. $-7 \mathrm{r} / 2$ gr.), phenacetin ( 0.25 G. -4 gr.), antifebrin ( 0.25 G. -4 gr.), analgen ( 0.50 G. $-7 \frac{1}{2}$ gr.), malakin ( 0.50 G. $-71 / 2$ gr.), lactophenin ( 0.50 G. $-71 / 2$ gr.), pyramidon $(0.50 \mathrm{G} .-7 \mathrm{I} / 2 \mathrm{gr}$.), antinervine ( $0.50 \mathrm{G}$.$7 \mathrm{I} / 2 \mathrm{gr}$.), each to be given several times a day. The author prefers antipyrine, phenacetin, and particularly pyramidon. [I have found a smaller dose of pyramidon (1-4 gr.) quite efficient in controlling pain.Ed.] If the pain is situated directly in the aneurism, an ice-bag or Leiter's cooling apparatus is successful in many cases, or, if not, narcotics or analgesics may be used externally. For example, the author often uses the following:

B Mentholi......................... 2.00....gr. $\mathbf{x x x}$

Cocainæ hydrochloridi $\ldots \ldots \ldots \ldots \ldots \ldots \ldots .20 \ldots \ldots$ gr. iii

Morph. hydrochloridi ........................ gr. vi

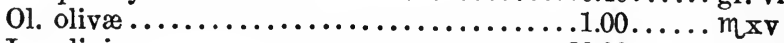

Lanolini............................

M. fiat ungt.

Sig.-Salve.

Or :

B Emplast. belladonnæ.................50.00.... $\boldsymbol{\zeta}_{\mathbf{i}} \mathbf{\mathrm { v }}$

(Ceræ flavæ.........................00..... $3^{\mathrm{i}}$

Terebinthinæ,

Ol. olivæ........................ āa $1.00 \ldots \ldots$ m $\mathrm{mv}$

While liquid adde

$3 \mathrm{ss})$

Sig.-Use as directed.

In both cases, however, we must often have recourse to narcotics. 
Dyspnœa.-Dyspnœa is another symptom which may accompany an aortic aneurism. It may arise from compression of the trachea or bronchus or from paralysis of the vocal cords, in which case it may be relieved by rest and narcoties. It may be of nervous origin, due to the pressure of the aneurism on the vagus or phrenic nerve. If it is due to the pressure on the vagus, opiates or bromides should be used to reduce the nervous irritability; if to pressure on the phrenic nerve, the latter should be galvanized at the neck. If the dyspnoea is of cardiac origin, heart stimulants should be employed. Again, it may be due to a progressive bronchitis arising from the local hyperæmia of the tracheal and bronchial membrane. In this case the therapy is that of bronchitis in general.

Hemorrhages, internal or external, are handled by the usual methods (see p. 60).

This finishes the discussion of the therapy of aortic aneurism, and with it, that of diseases of the blood vessels in general. A few other diseases of the circulatory system remain to be taken up.

\section{THERAPY OF DISEASES OF THE CARDIAC MUSCLE.}

Under this head will be discussed: (1) Fatty heart; (2) Idiopathic enlargement of the heart; (3) Myocarditis.

\section{THERAPY OF FATTY HEART.}

From a clinical and from a therapeutic point of view, we must distinguish between (1) fatty overgrowth, and (2) fatty infiltration and degeneration of the cardiac muscle. In the first class of cases, an abnormal amount of fat has collected in the epicardiac connective tissue, and often in the spaces between the muscle fibres. In many instances, this pathological condition is accompanied by atrophy of the muscle fibres of the heart, resulting in insufficiency.

FatTy Overgrow'rh: Diet.-Such a fatty heart is always accompanied by general obesity, of which it is merely one manifestation, the obesity being partly inherited, partly caused by too much food with too little activity. The therapy is, then, concerned principally with the diet. (See p. 234 for a full discussion.) It should be emphasized that the meals should be nearer together and less abundant than normal, and that little or no liquid should be taken with them or directly afterwards. These precautions reduce the amount of solids and liquids ingested.

Mineral Waters.-Laxative mineral waters are often very useful; for example, Marienbad (Kreuzbrunnen and Ferdinandsbrunnen). Carlsbad, Kissingen, and Tarasp. The author prefers the Karolinen- and Ambrosius-brunnen at Marienbad, on account of their iron, for in many cases iron is beneficial (e.g., Blaud's pills, see p. 159). The inhalation of oxygen (25-35 gal. a day) may also be helpful.

Oertel's Cure: Its Principle.-Oertel's cure is often indicated, although the author cannot recommend it in other heart diseases. Its 
essentials are: (1) The patient, besides having the especial diet suited to the reduction of fat, is, as a rule, limited to a quart or less of liquid, even when there is no hydrops; for the less the amount of blood circulating in the vessels, the less the cardiac exertion necessary to propel it, especially in the veins. If symptoms of congestion are present, reduction of the liquid given and increased excretion of water favor the process of oxidation in the body and the combustion of the fat, apart from relieving the venous system and the pulmonary circulation. The excretion of water is furthered by systematic exercise of the breathing muscles or by sweating. (2) The patient regularly goes through the exercise of hill climbing in a specially prepared locality, where there are all degrees of ascents from $0^{\circ}$ to $20^{\circ}$, the grade being suited to the condition of the heart. These exercises assist the flow of blood from the periphery to the centre, and give the heart sufficient exercise without straining it, besides quickening the breathing and so exercising the respiratory muscles and facilitating the flow of blood in the pulmonary circulation. Of course, if the heart action becomes very weak and the symptoms of insufficiency are threatening, this treatment must be abandoned and the patient be put to bed and given heart stimulants. (See Therapy of Valvular Diseases, p. 12.) Digitalis should not be used, however, as its action is too strong. Oertel's "terraincur" is provided for at Abbazia, Meran, Mais, BozenGries, Reichenhall, Ischl, Baden-Baden, Brenner-Gossensass, and Semmering. [Such a cure can be given by any thoughtful physician in the patient's own city or town, provided that it is reasonably hilly. At Virginia Hot Springs and Watkins Glen the method is in use.-Ed.]

Fatty Degeneration.- The second class of cases, those of fatty infiltration and degeneration of the heart muscle, must be handled very differently. In these the heart condition is generally secondary, occurring often in intoxications, anæmias, inanition, and infectious diseases. Here the therapy must be primarily directed at the weakness of the heart. We therefore give heart stimulants-alcohol in the form of brandy or heavy wine, and particularly camphor. Digitalis should be used only with the greatest caution. If in 2-3 days it does not produce a noticeable effect,particularly diuresis,-it should be given up, for fear the increased blood pressure which it causes may be too much for the weak heart and may bring on sudden paralysis of the cardiac muscle.

\section{THERAPY OF IDIOPATHIC ENLARGED HEART.}

The same treatment is indicated in the so-called idiopathic enlarged heart. The symptoms of cardiac insufficiency are almost always present, so that the treatment is the same as that of broken compensation. If excessive beer drinking is the cause of the condition (the so-called "Munich heart"), sudden heart failure is so imminent that the most energetic measures are demanded; for instance, subcutaneous injections of camphor, often repeated several times a day (10 or more syringes of camphor in oil). 
It should be noticed in passing that in the treatment of the first class of fatty hearts and also of idiopathic enlargement, gymnasties and massage are very successful. The massage should consist of trembling movements in the precordial region, of stroking along the vertebral column, and of tapping between the shoulders. It should not be kept up longer than 10 minutes at a time, and should be supplemented by breathing exercises (similar to artificial respiration).

\section{THERAPY OF CHRONIC MYOCARDITIS.}

So long as the heart has reserve force, the therapy of chronic myocarditis is the same as that of compensated valvular lesions and arteriosclerosis,-a proper diet, massage, gymnastics, iodides internally or externally, and small doses of digitalis or strophanthus or a preparation of theobromine, when there is very slight cardiac weakness. But if there are symptoms of pronounced insufficiency, the therapy is that of valvular lesions in the stage of broken compensation. It should be remarked that in the course of chronic myocarditis sudden attacks of heart failure may occur, in which case digitalis acts too slowly, and either caffeine, black coffee, or strong wine must be tried. If one has good reason to suspeet that the myocarditis is of syphilitic origin, an antiluetic treatment should naturally be adopted.

[Since Corvisart's time clinicians have repeatedly been surprised at the mistakes in the diagnosis of myocarditis which the post-mortem examination has revealed.

The author's subdivisions of myocardial disease, although theoretically and pathologically sound, cannot be differentiated clinically. Hence, therapeutic rules to fit each one of the types are useless and misleading. Forchheimer classifies the indications for the treatment much more practically under two heads, acute and chronic myocardial insufficiency, and adds a few special indications for treating the heart in arteriosclerosis and the fatty heart of obesity. We hardly distinguish the treatment of valvular lesions from that of myocardial disease, because in both instances our therapy is directed toward the same object, i.e., recovery from myocardial insufficiency.-Ed.]

\section{THERAPY OF ACUTE ENDOCARDITIS.}

The therapy of malignant ulcerative endocarditis can accomplish but very little. So far no effective causal treatment has been discovered. ${ }^{*}$ In a severe case, the author recently tried subcutaneous injections of orthocresol, which he had some years previously found successful in three cases of very severe puerperal pyæmia, caused by staphylococei and complicated by peritonitis and apparently by endocarditis. In the case at

4 [Douglas describes a case of streptococcus endocarditis with inrolvement of both sides of the heart, cured by inoculations of streptococci isolated from the patient's blood and killed by heat cultures.-Ed.] 
hand, however, this treatment proved useless, as it did also in several more recent cases of puerperal pyæmia, in which he tried it. In its place he now regularly tries collargol (argent. colloidale Credé), which appears to be the best remedy in all cases of sepsis, pyæmia, and bacteriæmia, although it is successful in only a fraction of the cases. He, unfortunately, has not found that in ulcerative endocarditis its effects are at all to be relied on. Eichhorst reports a case cured by a combined use of quinine and bichloride of mercury, as follows:

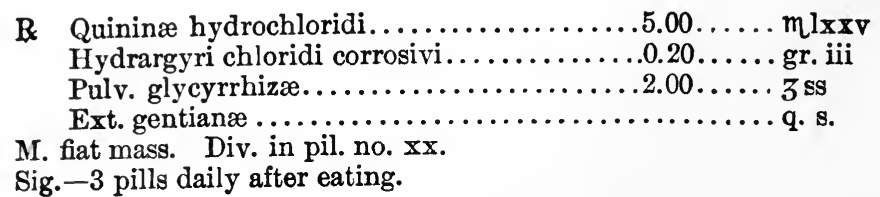

Hydrochloride of quinine alone is also of use $(0.50$ G. $-71 / 2$ gr. several times a day), to quiet an irritated and quickened heart action. In general, however, this is better accomplished by an ice-bag over the heart or by the administration of digitalis. If heart failure is threatened, we have recourse to camphor and strong alcohol.

In the second kind of acute endocarditis (verrucose), that of bacterial origin, more can be accomplished by proper therapy. To be sure, in rheumatic endocarditis the specific treatment with salicylates, which is of such great benefit in other forms of rheumatic inflammation of the serous membranes (synovia, pleura, peritoneum), is not so effective as we should expect it to be, although we should always give it a trial. Also, the theory that the growth upon the valves would be absorbed by the inhalation of $1 / 5$ per cent. solution of sodium carbonate or by ammonium chloride taken internally, has been proved erroneous. On the other hand, collargol appears in many cases to have a favorable effect. It is given by the rectum, by intravenous injection, by being rubbed into the skin (as in the treatment with mercurial ointment), and-rarely-internally. It apparently is most effective when injected subcutaneously. The author uses $10-20$ c.c. $(1 / 3-2 / 3$ oz.) of a $1 / 2$ per cent. sterile aqueous solution, or 2 c.c. $(1 / 2$ dr.) of a 5 per cent. solution. The latter is injected with a Pravaz syringe. The technic is very simple: After a thorough disinfecting of the spot chosen (on the arm), with soap, bichloride, alcohol, and ether, an Esmarch bandage is applied tightly enough to make the radial pulse just palpable. The skin on each side of the vein is then drawn tight and the cannula is inserted in the direction of the blood stream. When the blood dropping from the cannula indicates that the vein has been entered, the syringe-not entirely full-is inserted, the piston is pulled out to its fullest extent in order to bring up any air bubbles, and the contents are slowly injected, the arm being held horizontal. The bandage is then removed and the wound is closed with iodoform collodion. 
In the treatment with ointment, the part to be rubbed (upper and lower extremities, abdomen, back) is first washed with alcohol, then dried, and 3 G. (45 gr.) of 15 per cent. unguent. collargoli rubbed in carefully, so that the skin just shows a grayish-brown tinge.

For rectal use, we prescribe:

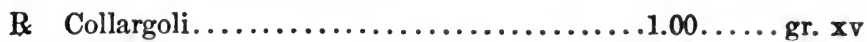

Aq. dest.......................200.00.... 3 vi $3 \mathrm{v}$

Sig.-Irrigate the rectum in the morning with a cleansing enema, then inject half of prescription, and the other half at the end of the day.

Very seldom, and only in cases where a local action on the digestive tract is desired (e.g., intestinal intoxication), collargol may be given internally, in the form of pills of $0.05 \mathrm{G}$. ( $3 / 4 \mathrm{gr}$.), 10 or more pills a day, or in corresponding doses of a mixture $(0.50: 100$ aq. ment.). This treatment is almost exclusively employed to supplement the rectal or cutaneous application.

Collargol is of undoubted use in mild cases of septicæmia, in which often 4-6 hours after the injection, the temperature begins to fall, a light sweating appears, and the subjective symptoms improve. Sometimes the chills disappear after the first injection, sometimes only after repeated injections. In pyæmia with primary abscess, a favorable effect is naturally to be expected only when the pus has been removed by surgical or natural means. In pyæmia with multiple metastatic abscesses, it is naturally often of no use, although the author has seen favorable results in some cases. It is evident that collargol may very often be employed to advantage in ulcerous endocarditis and verrucosa bacteritica. The author has even several times tried to avert the development of an endocarditis in acute joint rheumatism by the intravenous use of collargol, unfortunately with negative results.

Any further treatment of endocarditis must be merely symptomatic.

Local Therapy.-Externally, we employ an ice-bag or cooling apparatus, or, though not so effectively, a cold compress. It should be kept on continuously or for every other hour. Dry cold applications quiet the heart and may avert a threatened rupture of the valve, or arterial embolism. Many authorities also recommend (1) wet cupping, (2) cauterization (60-80 blisters), repeated after a week's intermission, and (3) local sinapism.

Medicinal Therapy.-As for medication, strong heart stimulants are in place when the heart is very weak, and digitalis where its action is rapid, weak, and irregular, or when symptoms of stasis have appeared. When the heart is evidently much irritated (tachycardia, pain in the precordial region), it is well to combine digitalis with aconite or bromine; e.g.:

$\mathrm{R}$ Tinct. digitalis......................6.00... 3 iss

Tinct. aconiti.............................. $3^{i}$

M. et sig. -10 drops 3-4 times a day. 
When the vegetative endocarditis is healed, in that it is confined to a circumseribed, fibrous, retracting process, potassium iodide is recommended by many authors, especially by the French. They maintain that it prevents the transition of the inflammatory deposit into firm connective tissue. It must be used cautiously, however, and alternated with digitalis.

The anæmia so often met with in convalescence from endocarditis may be combated by preparations of iron, which, however, do not prove very effective in this case; or perhaps better by preparations of quinine or the wine of iron and quinine, by arsenic, or by preparations of iron and manganese (the author considers the manganese superfluous) ; e.g.:

B Liq. ferri peptomanganati (Gude) $\ldots \ldots \ldots 360.00 \ldots \ldots$ zxii

Sig. -1 tablespoonful twice a day after eating.

The following is an example of a good combination of iron, arsenic, quinine, and wine:

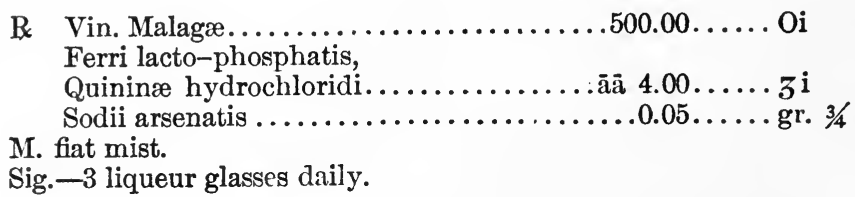

Of course, during the acute stage, the patient must be confined to bed and be limited to a liquid diet (milk, a little soup, and lemonade and other acid drinks). The bowels must be kept open.

All that has been said in regard to the therapy of endocarditis applies without change to the therapy of acute aortitis.

\section{THERAPY OF PERICARDITIS.}

When the pericarditis is of a fibrinous or serofibrinous type, the therapy agrees with that of simple endocarditis.

Rest.-The most important measure is absolute rest in bed, which markedly decreases the actual heart rate and therefore the inflammation of the pericardium, as the latter is kept up by the contractions of the heart. Locally, we may apply ice-bags or an ice coil, as in endocarditis (see p. 89). This treatment may be kept up for weeks, as very few patients object to it. In general, it causes the disappearance of the frequent pains, the turbulent heart action, and the sensation of pressure. At the first application of the ice-bag, a patient now and then shows traces of a chill, which soon gives way to the above-mentioned pleasant effects. A few patients, however, do prove intolerant to local applications of cold. With them we may try omitting the treatment for a while, or may let the water run through the coil at a somewhat higher temperature. If this does not work, we may use hot wet applications 
(Priessnitz's application) or a poultice, which, by causing a local paralysis of the vessels of the skin and then a local over-supply of blood, may have a slight derivative effect. For the same reason, we may-in the case of otherwise strong individuals-at the onset of a pericardial exudation, apply 6-8 leeches over the apex, or to the precordia, in the parasternal region of the fourth to the sixth intercostal spaces.

The diet is of course a liquid one, consisting essentially of milk and soup. [If the case yields within 10 or 20 days, this diet may be sufficient, but beyond a week or two it is well to add more nourishment for the sake of the heart muscle.-Ed.]

Medicinal Therapy.-Causal.-The medicinal therapy can be causal only in the case of a rheumatic pericarditis, where the salicylates must be given. This treatment, however, is far less successful than in joint rheumatism, perhaps because the pericardium is continually kept irritated by the contractions of the heart. The treatment with collargol is clearly often indicated.

Symptomatic.-The symptomatic therapy tries to quiet and strengthen the excessively rapid and perhaps also weak heart action. Digitalis is again our chief aid. (See p. 13 for the methods of giving digitalis, and for substitutes for it.) English authorities recommend tincture of aconite to reduce the abnormally rapid heart action; the author himself uses 5-8 drops 3 times a day or 1 drop every hour. [Of the new tincture (U. S. P.) 3 or 4 drops per hour.-Ed.]

Quinine is also recommended, especially by Schroetter, as being a good general tonic and, at the same time, especially valuable in correcting irregular heart action. A representative prescription is :

R Quininæ hydrochloridi................ $0.50 \ldots \ldots$ gr. viii

Dent. tal. cachets no. $x$.

Sig. -1-2 cachets daily.

Quinine may also be given with black coffee in pericarditis with symptoms of heart weakness; $0.50-1 \mathrm{G}$. (7-15 gr.) of quinine are mixed with $15 \mathrm{G}$. ( $1 / 2 \mathrm{oz}$.) of sugar and dissolved in coffee made by taking $10 \mathrm{G}$. $(\mathrm{I} / 3 \mathrm{oz}$.) of coffee beans to $100 \mathrm{G}$. (3 oz.) of water. It should be given hot.

Heart Stimulants and Narcotics.-Naturally, in many acute cases we must have recourse to heart stimulants, and (e.g., in severe dyspnœa) to narcotics, particularly morphine. But if the pericardial exudate has passed the acute stage, or if the case is one of chronic pericarditis, we must first of all give diuretics (usually in combination with digitalis). We may use diuretin, theocin, potassium acetate, calomel, diuretic tea, or suitable mineral waters (Wildungen or Bilin).

Surgery.-If, in spite of these measures, the exudate does not disappear, but gives rise to serere subjective and objective symptoms (cyanosis, venous swelling in the neck, orthopnœa), or if an acute pericarditis produces an exudate with such rapidity and in such an amount 
that life is threatened, or if the exudate is purulent, we must resort to surgery. In the case of a purulent exudate, we proceed at once to pericardiotomy, which, being a purely surgical procedure, need not be discussed here. In case of a dangerous serous or bloody exudate, however, the operation of puncture of the pericardial sac is indicated, and so belongs properly to the physician. The technic following Curschmann is as follows: The place chosen is generally the fifth or even the sixth intercostal space, according to the position of the diaphragm, usually in the left mammary line. [Curschmann's selection is probably the favorite with most clinicians and is generally safer. Romberg, F. C. Shattuck, and Forchheimer, among others, recommend it. The needle is inserted midway between the apex beat and outer line of absolute dulness. It is often impossible to determine the apex beat, even with the patient sitting well forward. If there is very much fluid, this determination is not necessary, as a point in the fifth or sixth space, a half inch to the right of the outer border of absolute dulness, will almost invariably prove satisfactory. If the amount of fluid is small, or if the fluid is encapsulated, it is well to have the patient lying down. Following F. C. Shattuck, I have usually selected this method. I have once wounded the left ventricle and once tapped a pleuritic effusion by mistake, although there was also fluid in the pericardial sac.

I have never used McDonnell's, Robert's, or Paton's favorite points, in the fifth interspace from 1 to $2 \mathrm{I} / 2$ inches to the left of the sternum. They avoid wounding the internal mammary artery but are liable to puncture the heart over the thin right auricle or ventricle.

Bristow's choice is my second, immediately to the left of the sternum in the fourth or fifth space. This avoids the artery, the pleura, and sometimes troublesome adhesions.

Rotah's choice, fifth or sixth space to right of sternum with the needle directed upward and inward, is more apt to succeed with children than with adults, except perhaps with very large effusions. The danger of wounding the thin right heart holds good.-Ed.] An exploratory puncture should always be made. [This is sometimes sufficient to establish absorption of the fluid. If the symptoms are not urgent, it is well to perform the second operation on the next day.-Ed.] The skin is first anæsthetized and a small cut (sometimes) made, into which the instrument is introduced and thrust cautiously forward until the resistance offered by the pericardium suddenly ceases. A trochar with a needle which can be withdrawn may be used, or better a flat two-edged instrument with a stop-cock. After the puncture has been made and the needle withdrawn, a tube filled with a sterile physiological salt solution is attached to the outlet of the trochar, the other end of the tube being placed in a receiving vessel also filled with the salt solution. The stopcock is then opened. The exudate should be allowed to flow only very gradually, and must be checked or stopped occasionally by turning back the stop-cock. We stop when no more fluid fiows out. From the nature 
of the disease, the operation must be performed with the upper part of the body raised. The danger lies in injury to the heart, sometimes causing the sudden death of the patient. The only way in which to avert it is to push the trochar in very slowly. [I have always used Potain's aspirator, selecting a smaller trochar than for tapping pleural effusions. -Ed.]

One more practical point may be mentioned: There is danger that a fibrinous or fibrino-exudative pericarditis may leave behind it an adherent pericardium, a serious and often fatal condition. Although we cannot be uniformly sure of success, we still may do much to avert this danger by giving digitalis from time to time during the whole stage of absorption. The resulting vigorous contractions of the heart minimize the chances of an adherent pericardium.

\section{THERAPY OF ADHERENT PERICARDIUM.}

If the heart totally adheres to the thoracic wall with a true systolic retraction and a diastolic thrust, so that the diagnosis of an adherent pericardium ean be made (a rare oceurrence), resection of the ribs and eardiolysis may be performed. Many good results have been reported concerning its effect on the circulatory disturbances. If the operation cannot be performed, the therapy must naturally be the same as in insufficiency of the cardiac muscle. [I have had no experience with such an operation and should hesitate to advise it.-Ed.]

\section{THERAPY OF NEUROSES OF THE HEART.}

\section{THERAPY OF PALPITATION OF THE HEART (PALPITATIO CORDIS).}

Palpitation is a symptom of several different conditions: (1) of an abnormal demand upon the normal heart, (2) of the beginning of broken compensation in an unsound heart, and (3) of a purely nervous affection.

In the first ease it usually disappears after the patient has kept quiet for a little while. If the strain was of long duration and more pronounced, so that the palpitation is the sign of an acute cardiac weakness, the treatment is the same as that of the latter condition: The patient is kept on his back with his head raised, the clothing is loosened, a mustard plaster is applied to the chest, the legs are rubbed, hot water bottles are put to the feet, and concentrated alcohol is given (brandy, rum, heavy wine, hot tea with brandy, black coffee with or without rum), or, if necessary, subcutaneous injections of ether or camphor are employed.

In many cases, where heart weakness is present as a congenital condition (paralytic thorax, phthisical diathesis) or in consequence of abuse of the heart, or where there are congenitally contracted vessels, palpitation is brought on by an amount of exereise that would have no effect on a normal person. In such cases of relative weakness of an otherwise sound heart, the best treatment for palpitation is earefully chosen and systematic exercise of the heart muscle (gymnastics of every kind, rowing, riding, 
swimming, careful climbing of gradual slopes), and hydriatic procedures (carbonated mineral water baths [Nauheim or Franzensbad-Ed.], and rubbing down the body with a cloth wrung out in water at the temperature of the room). Everything that may excite the heart must be avoided (coffee, tea, chocolate, strong alcohol, tobacco, digitalis, strophanthus, camphor, ether, etc.).

Nervous Palpitation.-In the third class of cases, those of nervous origin, palpitation may accompany heart lesions. The nervous origin is evident from the fact that the subjective symptoms are just the opposite from what the objective findings would lead us to suppose; for instance, palpitation may occur not only with the slightest movement but during absolute rest, and yet the examination may prove that there is perfect compensation. In such cases heart stimulants should be avoided, though the tincture of valerian (up to 3-5 teaspoonfuls) or the bromides may be used to advantage. We may also employ carbonated hot mineral water baths, tepid tub baths, and such mild hydriatic measures as those mentioned on p. 9. In many of these cases, however, the natural carbonated baths do not have a favorable effect; on the contrary, they are even irritating. Before sending a patient with a purely nervous palpitation of the heart to Nauheim or Franzensbad, it is therefore best to try the effect of weak carbonated baths at home.

In purely nervous cases we must discover the cause and try to remove it. Palpitation may be caused (1) by excessive smoking, in which case we must forbid smoking altogether or prohibit cigarettes and allow only 2 or 3 light cigars a day, which must never be smoked before mid-day or on an empty stomach, a cigar holder being always used and the smoke never inhaled; (2) by excessive use of tea or coffee; (3) by chlorosis or anæmia; (4) by intestinal parasites; (5) by gastro-intestinal disturbances, in which case a light diet, frequent meals, thorough mastication, and regular movements of the bowels are helpful, while all foods irritating to the heart should be avoided (tea, coffee, alcohol, tobacco, condiments) ; 6 ) by plethora abdominalis, where the palpitation may be relieved by drawing the blood away to the intestines (Marienbad waters, laxatives), or to the lower extremities (hot mustard foot-baths), or by employing leeches or venesection; (7) by disturbances in various other regions-e.g., those of the genito-urinary tract-where the local affection must receive proper treatment.

Finally, palpitation may be caused by a general neurotic condition (neurasthenia or hysteria), in which case it will generally disappear with the subsidence of the other symptoms of the neurosis. But it may be helped particularly by local cold (see p. 71), or, if the heart action is abnormally weak, by hot applications (see p. 72). The following drugs have proven of use: (1) Tincture of aconite (3-5 drops every hour); (2) Tincture of valerian (10-15 drops three times a day); (3) Camphora monobromata (0.10-0.50 G. (1 $1 / 2-7 \mathrm{~T} / 2$ gr.) daily) ; (4) Potassium bromide or a mixture of the bromides (see p. 48). With many patients 
antipyrine, phenacetin, etc., as well as the internal use of cocaine (0.01$0.02 \mathrm{G}$. (1/6-1/3 gr.) a day) or of morphine, have a good effect, although it must be remembered that these drugs used too often or in too great a quantity may themselves bring on toxic palpitation; other patients are helped by 10-20 drops of aqua laurocerasi during an attack. [I should use a larger dose, $1 / 2-1$ teaspoonful.-Ed.] To avert recurrence in patients susceptible to attacks, we may use tincture of valerian, or bromide; or, especially, hydrotherapy, particularly carbonated baths at Nauheim (with the above-mentioned precautions), or those artificially prepared at home (tepid); indifferent thermal springs; tepid baths $\left(90^{\circ}-\right.$ $95^{\circ}$ ) prolonged for a half hour or more followed by rest in bed, with an ice-bag to the precordium; and, with many patients, cold sitz baths, sponge baths, etc. Galvanic baths and four-cell baths are also of value. All heart irritants are of course forbidden; e.g., coffee, tea, chocolate, alcohol, tobacco, mental and physical strain, and excitement. For this reason the author advises against digitalis and, in general, strophanthus, although he admits having seen several patients with nervous palpitation helped by the latter. He has recently employed here, as well as in all cases of neurasthenia with heart distress, the syrupus kolæ compositus Hell, 3 teaspoonfuls daily after meals. It is made up as follows:

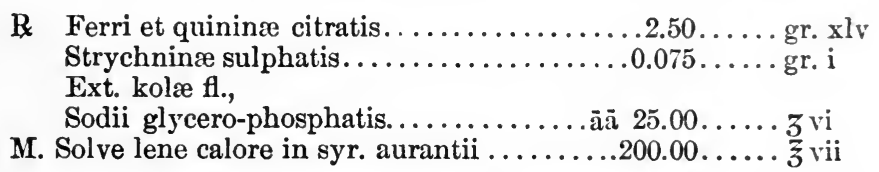

As for climatology, these patients do best in mild mountain climates. Compare Therapy of Exophthalmic Goitre, for a definite list of places (p. 199).

Finally, the recently invented "heart supporters" or "heart compressors " may be of use in cases of nervous palpitation, as well as in valvular lesions and in the local subjective disturbances in consequence of some cardiac affection (pressure or painful sensations). The author has seen marked suceess follow their use in several cases of palpitation; e.g., one in broken compensation in a mitral-aortic lesion, and one in a neurasthenic. Possibly this apparatus has a good effect on the abnormal mobility of the heart so frequently found; certainly it makes the rhythm of the heart-beat more regular (Gräupner). [I think the author emphasizes the treatment of this purely nervous palpitation more than is wise. Psychical treatment, as for neurasthenia, and an utter disregard of cardiac treatment, with proper diet, hygiene, and occupation, succeed better in my experience.-Ed.]

\section{THERAPY OF PAROXYSMAL TACHYCARDIA.}

So far as this is a neurosis, not accompanied by any affection of the heart (dilatation), the therapy is identical with that of nervous palpitation : avoidance of food in such quantity or of such quality or temperature 
as to irritate the heart; moderate exercise in the open air; hydropathic treatment, particularly charged mineral-water baths; residence in the mountains between attacks; and the administration of sedatives (tincture of valerian, bromine salts, zinc cyanide, or zinc valerianate). [Ovarian extract ( $5 \mathrm{gr}$. tablets) may be tried for women at the climacteric.-Ed.]

R Zinci valerianatis.................0.03-0.05.....gr. $\frac{1}{2}-$ gr.

Sacchar. lactis.....................0.40....gr. vi

M. fiat pulv. Dent. tal. cachets no. xv.

Or:

Sig. -3 cachets daily.

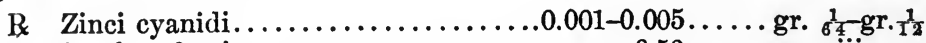

Sacchar. lactis..................... $0.50 \ldots \ldots$ gr. viii

M. fiat pulv. Dent. tal. caps. no. xv.

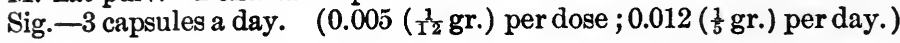

During the attack itself the patient should be laid on his back, the clothing loosened, pieces of ice put in the mouth, an ice-bag laid over the heart or on the neck, the neck sponged with ether or ethyl chloride (the medulla is often the starting point of paroxysmal tachycardia), and some narcotic given (bromides, opium, morphine, the latter by the mouth or subcutaneously). Galvanization of the vagus in the neck is sometimes successful.

If, however, the paroxysmal tachycardia goes along with severe dilatation of the heart, and attacks are repeated, champagne or some other form of strong alcohol should be used in addition to the remedies already mentioned. Digitalis or other heart stimulants are also indicated. Between attacks the therapy is the same as that of dilatation of the heart unaccompanied by tachycardial attacks.

In conclusion, there may be appended to this treatment of the two principal neuroses of the heart, a few words concerning the therapy of a disease which, in a certain sense, may be called a functional disturbance of the cardiac activity, due to cerebral anæmia; i.e., syncope.

\section{THERAPY OF SYNCOPE.}

Whether syncope is the result of anæmia, of some disease of the heart (pericarditis, fatty heart, myocarditis, valvular lesion), or of some severe nervous shock (severe stone colic, contusion of the testicles, great fright), the first thing to do is to lay the patient flat on his back or even to raise his feet somewhat higher than his head. The clothing should be loosened, and plenty of fresh air be given access to the patient. Next, the skin should be stimulated by rubbing, washing the face and epigastrium with vinegar or some liquid containing alcohol (e.g., cologne), sprinkling the face, neck, chest, and epigastrium with water, slapping the palms and soles with wet towels, and tickling the nose with a feather. Hot applications over the precordium should be used to stimulate the heart. If these measures succeed, the patient should not be allowed to stand or move too suddenly. The same precautions should be used as in allowing a patient 
to get up after a severe illness. He must not be allowed to stand until he has been gradually accustomed to sitting upright, and when he takes his first step, if the slightest sign of faintness occurs, he should be laid horizontal again, and wine, brandy, etc., be given to avert the threatened cerebral anæmia.

[In the therapy of all heart affections, too much attention cannot be given to the part obesity plays in the etiology. Therefore, it is safe to assume that the reduction of weight, life in the open air favorable to oxidation, and rest will accomplish much more than will drug treatment.-Ed.] 


\section{THERAPY OF DISEASES OF THE GENITO- URINARY TRACT}

\section{THERAPY OF DISEASES OF THE KIDNEYS.}

THE most common renal disease, and the one whose therapy belongs especially to the field of internal medicine, is inflammation of the kidneys. This affection can be divided diagnostically into an acute and a chronic parenchymatous nephritis, and a chronic interstitial nephritis. Therapeutically we distinguish, then, between acute Bright's disease, chronic parenchymatous Bright's disease, and granular atrophy of the kidneys.

\section{THERAPY OF ACUTE BRIGHT'S DISEASE.}

Causal Therapy.-In two varieties only do we treat acute nephritis by treating the disease to which it is secondary: (1) in the acute nephritis of malaria, and (2) in the acute or subacute nephritis of the secondary stage of syphilis. In the former, quinine has a direct effect upon the kidneys. The method of administration will be given in detail under the therapy of acute infectious diseases (see p. 552). In the latter, mercury has been shown to cause a complete and permanent subsidence of the inflammation, although in general mercury irritates the kidneys and causes a mercurial albuminuria. Iodides are less useful; they belong rather to the tertiary stage of syphilis.

In nephritis accompanying other diseases, particularly intoxications or acute infections, such as typhoid, pneumonia, scarlet fever, and diphtheria, we frequently employ the therapy appropriate to such diseases. Certain drugs, however, should be avoided if nephritis be present, particularly all kidney irritants, such as tar and styrax, naphthalin, carbolic acid, oil of turpentine, chlorate of potassium, and cantharides. Salicylic acid and its preparations also cause renal irritation (cylindruria and albuminuria); but they are not contraindicated under certain conditions (e.g., in acute articular rheumatism), as the symptoms of irritation recede in spite of a continued but moderate use of the salicylates. Alcohol, unless vitally indicated, must be given up, because it is apt to irritate the kidneys and may cause albuminuria or cylindruria (see p. 123). In typhoid with nephritis or marked renal hyperæmia cold baths should not be given, on account of the sudden cooling of the skin and consequent congestion of the kidneys; prolonged tepid baths should be substituted. It goes without saying that all food which may irritate the kidneys should be excluded from the diet. In all these cases, however, the nephritis itself 
calls for a separate therapy, as does also that form of nephritis the etiology of which is not clear, but which we attribute to catching cold. This therapy is purely symptomatic.

Symptomatic Therapy.-The severe pain can be relieved by the use of revulsives applied to the lumbar region. As has been said, the cantharides blister must be avoided, as it tends to cause active hyperæmia of the kidneys and even acute nephritis. The same thing is true, in a lesser degree, of mustard plasters. It is better to apply dry or, preferably, wet cups, leeches, or the actual cautery to the triangle of Petit, as the network of veins there accessible communicates directly with the perirenal veins. In severe acute congestion, Lemoine recommends the use of leeches every other day, and on the alternate days dry cupping morning and night, until the symptoms (marked diminution of urine, many red and white blood corpuscles in the urinary sediment, severe pain in the lumbar region, headache, and vomiting) have subsided. In less severe cases 1 or 2 dry euppings each day will suffice. The application of a biscuitshaped ice-bag to both kidneys for $2-3$ hours, with an intermission of 1 hour, also appears useful in relieving congestion.

With Baccelli, the author has repeatedly seen excellent results from venesection performed on the lower extremity (e.g., vena pediæa, $300 \mathrm{G}$. (10 oz.) of blood), often obtaining in this way the complete disappearance of blood from the urine in 24 hours. He strongly advises the procedure, especially where the blood content of the urine is high. [Herrick recommends 5-10 gr. of calcium chloride 3 times a day for persistent hæmaturia. I prefer the lactate of calcium, $10 \mathrm{gr}$. 4 times a day. It is worth trying.-Ed.]

The patient should remain in bed. Senator recommends that he lie on his stomach, in order to avoid a possible passive hyperæmia of the kidneys. [There is generally no objection to the use of a commode beside the bed-Ed.] The temperature of the room must be kept at about $62^{\circ}$, and every precaution must be taken against catching cold. The latter must be just as carefully avoided after the subsidence of the acute condition. The convalescent should always wear for a considerable time a woollen undergarment, and perhaps, in addition, a flannel band around the loins, and should keep his feet warm and dry. [A word about rest in bed. We have no very good index to tell us when a patient should be allowed to get up after an acute nephritis. My general rule is to keep him in bed as long as there is an appreciable amount of albumin, casts, or blood in the urine. If after from 5 to 7 weeks his general condition and these urinary findings remain stationary, I allow him to sit up in bed 2 or 3 days; then, if no contraindication develops, to sit up in an arm chair for from half an hour to an hour, and so on. Patients often improve much more rapidly and eat, sleep, and feel better after being allowed to sit up. Woollen night clothes are advisable, but not too many covers.-Ed.] 
Diet.-The diet is of supreme importance. In acute hemorrhagic nephritis all meat must be forbidden. Broths also must be given up, as they make great demands upon the kidneys. In acute nephritis the diet should consist largely of milk. An exclusively milk diet is not, however, generally advisable, even though there are many patients who can easily take $3-4$ qt. or more a day. Where the acute nephritis has given rise to symptoms of hydrops or of complications in the circulatory system, the $3 \mathrm{r} / 2-4 \mathrm{qt}$. of milk necessary to keep up a proper state of nutrition is directly harmful, as such an abnormally large amount of liquid only increases or even causes hydrops, lays an extra burden on the circulatory system, and furnishes the kidneys with too much urea, [as well as with too much phosphoric acid-Ed.] On an average the author gives only $1-1 \mathrm{~T} / 2$ qt. of pure milk, distributed over the day. [Von Noorden enriches 1500 c.c. of ordinary milk with 375 c.c. of a sweet cream (generally sterilized and up to 30 per cent. fat), and adds a small quantity of calcium carbonate, to unite with the phosphoric acid of the milk and be excreted by the intestines and so save the kidneys. This mixture contains about as many calories as are contained in $3 \mathrm{r} / 4 \mathrm{qt}$. of good milk, while the proteid percentage is about one-half that of the latter. Forchheimer prefers starch gruels from farina, arrow-root, rice, or fine flour, strained oatmeal jelly, crackers, rusks, and sugar to increase the caloric value of 3 pints of milk. I have been in the habit of combining the two methods, increasing the fat or diminishing the starch according to the individual and his symptoms,-corpulency, flatulency, constipation, tongue, appetite, etc. Pasteurizing or sterilizing the cream at home has usually sufficed with my patients, although in hot weather I have often wished for a good sterilized cream of standard strength. I even use sweet (unsalted) butter, very freshly prepared, upon rusks or toasted crackers, where I find that the patient's distaste for food needs correction. According to Strubell, we should be cautious with the administration of fats and select rather the carbohydrates; for he found that nephrectomized dogs fed upon carbohydrates showed milder symptoms of uræmia than did dogs in a state of starvation or those given albumin or fat.-Ed.] In case of an antipathy to milk, it is often better taken if given ice cold or with something added to it,-not brandy, of course, but some alkaline mineral water, lime water, cocoa, a small quantity of some aromatic substance, or a very small amount of coffee or tea. In such cases it is better to use fresh rather than boiled milk. Sometimes skimmed milk is better borne. The amounts should be very small, 1 or 2 tablespoonfuls every hour to begin with. Buttermilk or Gaertner's " fatmilk" may be added to ordinary milk as an experiment. [After considerable experience with Gaertner's " fat-milk" on the Continent, I have largely discarded its use. I do not know whether it can be obtained here readily. It is to me very distasteful, and many patients for whom it has been prescribed have complained to me. Fresh buttermilk is, on the contrary, generally well borne, and is sold everywhere. It is also laxa- 
tive and diuretic. At the bars of some of our large hotels in New York it can be obtained very fresh and palatable. Many of the dairies are careless about their product.-Ed.] Finally, koumiss or kefir may be tried, as they are taken by many patients with much less discomfort than is ordinary milk. Koumiss is mare's milk subjected to an alcoholic fermentation. It is much less used on the Continent than is kefir. The latter is cow's milk fermented by the addition of the kefir ferment, and may be easily prepared at home by adding kefir pastilles to ordinary milk ( 1 to a quart). Kefir 1 day old is laxative, 2 days old is indifferent, and 3 days old is constipating. About a quart should be taken each day, thoroughly shaken before drinking. On account of their content of alcohol, however, koumiss and kefir should not be used in nephritis, especially in its acute form, except in cases of absolute necessity. [I do not believe that the small amount of alcohol contained in koumiss or matzoon is enough to contraindicate their use in acute nephritis. I have often found them serviceable, especially as a means of alternating milk foods and so avoiding the distaste patients often aequire to all forms of milk. I agree with von Noorden that we should be more stringent in condemning alcohol for patients with a contracted kidney than for those suffering from an acute nephritis. Brush's or Daddirian's koumiss is made from cow's milk, and contains a large amount of carbonic acid gas. Matzoon or zoolak is thicker and not charged. Even in New York it is not always possible to obtain these preparations fresh, so that a recipe for their home manufacture is appended (see p. 441).-Ed.] Condensed milk may be used, either undiluted or diluted with water, a few tablespoonfuls a day. [Condensed milk is apt to cause too much fermentation.-Ed.]

To the 1-1/2 qt. of milk may be added eucasin (casein-ammonium), sanatogen, or nutrose (casein-sodium), 2-3 tablespoonfuls daily. Gruels and thick soups from prepared leguminous or starehy meals, starches, and fats in the form of cream or butter, may be allowed in order to keep up the strength without irritating the kidneys. To these gruels, as to milk, may be added eucasin or nutrose, from 2-3 teaspoonfuls to 2-3 tablespoonfuls a day. Both these preparations (loose compounds of undigested proteid) are very rich in albumin (85-90 per cent.) and free from nucleins and extractives (which irritate the kidneys), and are, therefore, suited to all varieties of Bright's disease. The same is true of sanatogen (casein, 95 per cent., sodium glycerophosphate, 5 per cent.). All these preparations should first be mixed with cold water and then stirred into the hot soup; otherwise they will lump. In other diseases they may be added in a similar way to broths, cocoa, and ehocolate. [In acute nephritis I rarely use either these preparations or similar American products, as plasmon, because I believe we obtain sufficient proteid from the milk mixture mentioned above and because their addition detracts from the palatability.-Ed.]

The best cereals are oatmeal, barley, and rice, preferably oatmeal, as it has not the slightly constipating effect of the other two. Two or 3 
tablespoonfuls of oatmeal should be mixed with a little cold water. The mixture should be boiled gently for half an hour with constant stirring. Rice and barley should be soaked 12 hours, and then boiled 2 hours, and strained. [I find gruels made from farina, hominy, and cornmeal very satisfactory to most patients. I do not agree with the author's directions about the preparation of the gruels and cereals (see p. 441 for definite directions). I believe many people will stand a more generous allowance of starchy foods, if they are very thoroughly cooked.-Ed.]

To prepare gruels from leguminous meals, we make use of some one of the numerous preparations on the market (Knorr, Liebe, Liebig, Hartenstein, Timpe), adding 1 or 2 tablespoonfuls to a cup of salted water, and heating, constantly stirring.

During convalescence, the change to a full diet must be made very slowly. We give junket, custards, mashed potatoes, apples, pears, and berries, cooked or raw, (fruit with stones, and cranberries, must be avoided on account of their hippuric acid,) bread and rolls, fatty cheese, butter, farinaceous foods, green vegetables, and eggs. Meat should not be given until the albuminuria has wholly disappeared, and then only in the middle of the day. [I believe this too sweeping a rule. Some patients continue to excrete a trace of albumin in the urine, and yet do well or better upon a moderate amount of well-chosen meat at the mid-day meal.-Ed.] All substances that irritate the kidneys (condiments, and especially alcohol) must be wholly forbidden for a long time.

When hydrops is prominent, the amount of liquid should be reduced to $1 \mathrm{qt}$. a day, and a diet poor in common salt chosen. (See p. 113.)

In the case of individuals who cannot take milk, on account of an antipathy for it, or more often on account of the digestive disturbances which it causes, there is nothing to be done but to give from the beginning other milky foods (if possible), gruels, zwieback or crackers, cream ( 1 glass a day), and the above-mentioned preparations.

Medicinal Therapy.-Passing to medication, we must confess that we possess no specific drug for Bright's disease. [Von Noorden urges against the employment of diuretin, caffeine, digitalis, and juniper in the early stages of nephritis, i.e., during the period of oliguria, in order to stimulate diuresis. These stimulants are like whip-lashes. (See p. 98 for list of drugs harmful to nephritics.) -Ed.]

Sodium Tannate.-Tannin has long been a favorite to reduce the albuminuria. It is strongly recommended by Pribam, of Prague, in the following prescription :

$\mathrm{R}$ Sodii tannatis................... $0.10-0.20 \ldots \ldots$ gr. iss-gr. iii

Dent. tal. cachets no. xv.

Sig. $-3-5$ cachets daily. (Not more than 1.00 (15 gr.) a day should be given.)

The author has repeatedly tried this preparation, but without marked success. 
Quinine Tannate.-In his experience with subacute nephritis after scarlet fever, in the children's hospital, he has often used quinine tannate as follows:

B Quininæ tannatis.................0.10-0.20....gr. iss-gr. iii

Dent. tal. cachets no. $\mathbf{x}$.

Sig. $-2,3$, or 4 cachets a day.

Tannalbin.-He has more recently used tannalbin, 2-3 G. (30-45 gr.) a day, but without affecting the progress of the disease as such.

B Tannalbini.................................. $\mathbf{x v}$

Dent. tal. cachets no. xv.

Sig. $-2-3$ cachets a day.

Alkalies.-But he now prefers the alkalies to tannin, particularly bicarbonate of soda, $6-8 \mathrm{G}$. (1 $1 / 2-2 \mathrm{dr}$.) a day, or lithium benzoate or carbonate, 1-2 G. (15-30 gr.). Their administration has repeatedly been followed by a rapid increase in the amount of urine and decrease in its blood content. The alkalies also reduce the congestion. Almost all other drugs recommended have even less in their favor than tannin and tannalbin. [I have never seen any good results from any of these drugs.-Ed.]

Methyl Violet-Sodium Benzoate.-Provisionally, methyl violet or methylene blue, $0.10 \mathrm{G}$. ( $1 \mathrm{r} / 2 \mathrm{gr}$.) in wafers, or sodium benzoate, may be employed for their bactericidal effect. Methyl violet possibly also reduces the albuminuria. Sodium benzoate may be prescribed as follows:

B Sodii benzoatis...................3.00-4.00.....gr. 45- $3^{i}$

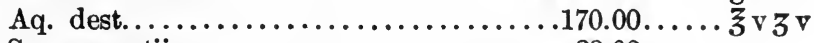

M. fiat sol.

Syr. aurantii......................20.00... $3 \mathrm{v}$

Sig. -1 tablespoonful every 2 hours.

Salts of Strontium.-The salts of strontium have been strongly recommended by French authors (Sée and Dujardin-Beaumetz) and have been tried in Vienna on Drasche's recommendation. Strontium bromide, strontium nitrate, and strontium lactate are used, the latter most frequently. It is prescribed as follows:

R Strontii lactatis .................6.00-8.00..... 3 iss -3 ii

Aq. dest.......................... $170.00 \ldots \ldots, \tilde{z} \mathrm{v} 3 \mathrm{v}$

Syr. aurantii $\ldots \ldots \ldots \ldots \ldots \ldots \ldots \ldots, 20.00 \ldots \ldots, 3 \mathrm{v}$

M. fiat sol.

Sig. -1 tablespoonful every 2 hours.

The author has never obtained any marked result from its use. Ergot is also used, especially in cases where severe hæmaturia is present. Leube advises relatively large amounts, prescribed as follows:

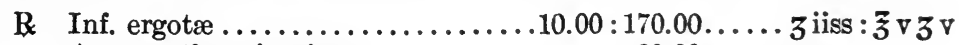

Aq. menthæ piperitæ...............20.00...3 $\mathbf{v}$

M. fiat mist.

Sig. -1 tablespoonful every 2 hours.

[I do not use any of these.-Ed.] 
The author considers that a venesection, taking 300 c.c. $(10 \mathrm{oz}$.$) of$ blood from the vena pediæa according to Baccelli's procedure, is more effective than is the use of any styptic. In a short time, sometimes in 24 hours, it will reduce a blood content of long standing to a mere microscopic trace, or even cause it to disappear entirely.

TheraPy of Oliguria with Hydrops.-Although we have no specific for acute nephritis, we are able to treat its most important symptom, oliguria. This need not, however, be attacked directly, unless it is accompanied by pronounced hydrops. Oliguria alone needs only rest, a suitable diet, and a reduction of the congestion. [Unquestionably it is frequently a conservative symptom, and is better left alone.-Ed.]

In most cases milk in large quantities is an excellent diuretic. [Large quantities of milk or water frequently increase rather than diminish the dropsy (see below). In acute nephritis we must first satisfy ourselves as to the cause of the oliguria and dropsy. If associated with a dilated heart, they are cardiac and should be treated promptly with digitalis or its congeners. If due to the marked inflammation of the glomeruli, the kidney needs rest at first, and rest from water as well as food, 2 or 3 or even 4 days with not more than 500 c.c. of fluid, sucking pieces of ice to slake the thirst, and daily hot-air baths. If the patient's œdema increases (as shown by careful weighing), or if his oliguria persists or becomes more pronounced when he is limited to 1000-1500 c.c. of fluid in 24 hours, this method, which von Noorden has urged so vigorously, is to me the only logical one; i.e., rest, with not more than 500 c.c. of water or milk and without food. Then when diuresis begins, and this will occur in nearly every case within 2 days, we can slowly increase the amount of milk and water.-Ed.]

Mineral Waters.-Alkaline and earthy mineral waters may be used alone or with milk to increase the amount of urine. Aufrecht recommends the Wildungen water especially, but we may choose from the Seltzer, Ems, Preblau, Bilin, Radein, and similar waters. Their use is in flushing out the kidney. [Poland and Waukesha are excellent pure waters with us. Nephritic patients do well at both resorts during the hot months. Strict instructions are necessary, however, in order to avoid the over-drinking so commonly observed at such springs.-Ed.] If they do not accomplish this, the diuresis not increasing correspondingly and an increase in body weight showing us that the fluid is being retained in the organism, then we must immediately stop their use, cut down the amount of liquid, and give a dietary poor in common salt, for hydrops is due not only to retention of fluid but also to the accumulation of salt in the organism. The author has several times seen striking success follow this precaution. [Every case of acute nephritis or of chronic uncompensated nephritis should be regularly and frequently weighedevery day or two. Since I began this as a routine measure 3 years ago at the City Hospital, I have been surprised how early we detect a slight accumulation of fluid, either in the subcutaneous tissues or in one of the 
serous sacs. I also urge upon my house staff the regular measurement of limbs and abdomen.-Ed.]

Diuretics: Caffeine Etc.-If mineral waters and a milk diet do not increase the amount of urine, recourse must be had to such diuretics as do not irritate the kidneys. The best of these are caffeine, theobromine, diuretin, and uropherin, agurin, and theocin. As the first, without injuring the kidneys, strengthens and stimulates the heart, it is a remarkably good diuretic for the purpose, as Leube has pointed out. Leube does not give more than $0.60 \mathrm{G}$. (9 gr.) daily, as $1 \mathrm{G}$. (15 gr.) or over may cause slight albuminuria and cylindruria in even healthy kidneys. It is best prescribed in the form of a double salt, generally caffeine-sodium salicylate. The same drug may be given in suppositories, prescribed as follows:

R Caffeinæ sodii-salicylatis...................... ii

Ol. theobromatis........................ 3 ss

M. fiat suppos. Dent. tal. suppos. no. xv.

Sig. - 3 suppositories daily.

For the administration of theobromine, diuretin, uropherin, agurin, and theocin, see p. 80.

Potassium Acetate.-Another diuretic is potassium acetate, a vegetable alkali, which in the organism is transformed into potassium carbonate. The author agrees with Immermann and Leube in prescribing large doses :

R Potassii acetatis...........................

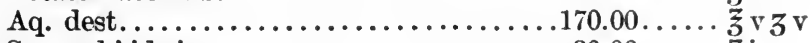

M. fiat sol.

Syr. rubi idæi.....................

Sig. -1 tablespoonful every 2 hours.

Tartar: Cream of Tartar.-Tartar is another good diuretic, as it is also laxative. In acute Bright's disease it is very important to keep the bowels open ; first, because toxins are thus passed off by way of the intestine instead of being absorbed into the blood and excreted through the kidneys, and secondly, because the excretion of urea and of toxins from the blood through the mucous membrane of the intestine is helped, thus delaying and diminishing the danger of uræmia. The following prescriptions may be used:

R Potassii bitartratis.................... 3 vi gr. $x v$

Elæosacchar. menthæ piperitæ.........5.00.... 3 i gr. $x y$

M. fiat pulv. Or :

Sig. -1 small teaspoonful (about 2.50 G. -40 gr.) 3 times a day.

B Potassii bitartratis......................... 3 iiss

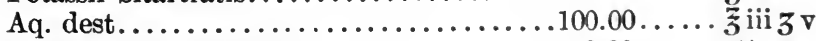

Spir. menthæ piperitæ.....................

Syr. rubi idæi..................... $20.00 \ldots \mathrm{v}$

M. fiat mist.

Sig. - To be taken in 3 doses during the day. 
Finally, there is the preparation that Bright himself preferred. It may be prescribed as follows, in quantities up to $10 \mathrm{G}$. $(2 \mathrm{I} / 2 \mathrm{dr}$.) a day:

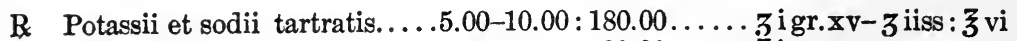
Syr. acid. citrici .................. $30.00 \ldots$

M. et sig. -1 tablespoonful every 2 hours.

Diuretic Teas.-A diuretic tea may be made from several components, but asparagus root, radix petroselina, radix apii graveolentis, radix ononis. spinosæ., and even juniper berries are not advisable, on account of their irritative qualities. The author recommends the following:

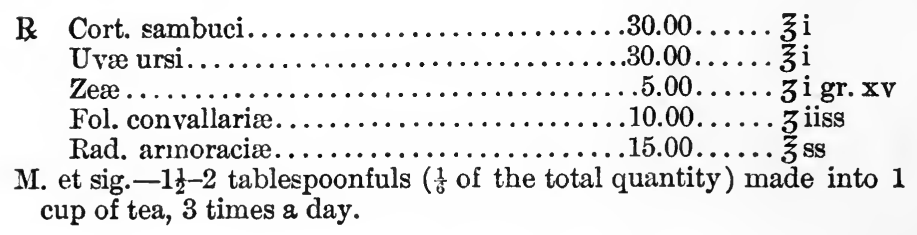

The author does not feel sure of the certain diuretic effect of birch leaves. Tea can be made from them directly, or $1 / 2$ small teaspoonful of folia dialysat. betulæ, Golaz, may be added to $1 / 2$ cup of warm water, 2-3 times a day.

[Von Noorden and Forchheimer do not employ diuretics under these conditions. Senator advises the so-called refrigerant diuretics, especially the carbonates and vegetable acid salts, which are converted into carbonates in the body, and claims that experience is altogether in favor of their efficiency. Since I have followed von Noorden's plan of starvation (food and liquid) I have rarely found it necessary to employ any of these diuretics.-Ed.]

Hydrotherapy.-Oliguria may also be treated by hydrotherapy, [and generally much more safely and efficiently.-Ed.]

Tepid Baths.-Hoffmann recommends tepid baths for almost every case of kidney disease, and says that none of the patients so treated became uræmic. The author advises in every case a tepid bath $\left(90^{\circ}-95^{\circ}\right)$ lasting 15-20 minutes each day. Lehmann's experience in particular has proved this to be the best form of hydrotherapy for increasing renal excretion. [Strasser and Blumenkranz have recently pointed to the favorable influence prolonged neutral baths have upon diuresis and the elimination of nitrogen and sodium chloride in nephritis. Temperature of $94^{\circ}$. Duration, 1-1 $1 / 2$ hours.-Ed.]

Hot Baths.-If increased excretion from the skin is desired, to get rid of the toxins circulating in the blood, the bath should be given hotter $\left(95^{\circ}-104^{\circ}\right)$ and should last from $1 / 2$ to 1 hour. Two or 3 a week may be ordered, or even 1 a day. The bath should be $95^{\circ}$ at the beginning and gradually raised to $104^{\circ}$ and kept there. 
Sweating-After the bath, the patient must be rubbed vigorously in order to contract the relaxed vessels of the skin. He should then lie in bed under sufficient covering. The old procedure of " sweating," according to which the patient, still wet, was wrapped tightly in blankets, has been almost abandoned, for the abundant perspiration involves the danger of concentration of the blood and resulting uræmia.

Wet Packs.-A wet pack is also useful. The patient is wrapped in a wrung-out blanket and then in a rubber blanket. During this procedure, as during the warm baths, an ice-bag should be placed on the head.

Cabinet Steam Baths.-Steam baths may also be utilized. The patient sits in a box with an opening for the head. The box is filled with steam by means of a metal tube communicating with a vessel of boiling water. The patient should remain in the steam for 15-20 minutes, then be vigorously dried, and go to bed.

Hot-Air Baths.-The pleasantest method of giving a sweat bath is by means of hot air, as in the so-called phénix à l'air chaud (see p. 43). The patient remains in this, with the air at $104^{\circ}-122^{\circ}$, for $10-15 \mathrm{~min}$ utes, or even an hour. The advantage of this method lies in the fact that patients with weak backs can stand it, as the position is horizontal, and only a part of the body need be exposed to the heat. A disadvantage is that many patients after repeated applications complain of painful burns on the lower extremities.

When there are symptoms of severe heart weakness, dyspnœa, marked œdema, or fever, the methods just described are generally hazardous, on account of the imperfect cardiac power and the atheroma of the vessels. The partial hot-air baths may be employed and also the hot pack, as recommended by Ziemssen.

Priessnitz Hot Application.-The patient is wrapped in a Priessnitz compress, the cloths being wrung out in very hot instead of cold water, and then in hot blankets. More blankets are laid over him and he remains quiet for 2-3 hours. If he is very short of breath, his head may be raised. [In institutions, and even in private houses, electric light baths may be conveniently substituted for all these measures. Von Noorden uses portable tunnels which can be applied to any bed; to their inner surface 6 to 10 incandescent bulbs are attached and the apparatus is connected with the electric fixture in the bedroom. This requires about half the time, avoids moving and so tiring the patient, and can be applied and attended to by one nurse.-Ed.]

During all these sweating processes an ice-bag should be kept on the head. If the patient is annoyed by thirst, he may drink cold water at will. Afterwards, it may be well to give a glass of wine.

Medicinal Diaphoretic Therapy; Pilocarpine.-The medical diaphoretic treatment consists of the subcutaneous injection of pilocarpine hydrochlorate, $0.01-0.02 \mathrm{G}$. ( $1 / 6-1 / 3 \mathrm{gr}$.). Its administration often produces results where hydrotherapy has failed. Often the œdema rapidly vanishes, a satisfactory diuresis returns, and threatening uræmic symp- 
toms are averted. Still, the author believes that its use should be confined to cases of severe hydrops, as it has unpleasant effects on the stomach and is decidedly dangerous to the heart. If the heart is not functionating properly, pilocarpine is directly contraindicated.

The author considers it a good plan, especially if there is any suspicion of weakness of the heart muscle, to accompany the injection with one of ether or with a dose of brandy.

Purging.-Another method of combating the hydrops in acute Bright's disease is by purgation. This does not seem advisable, except in cases of uræmia, as severe purging tends to irritate the intestine, and all irritation must be avoided in Bright's disease, which in itself may cause severe and even diphtheritic inflammation of the intestine. The author recommends, beside the preparations of tartar already mentioned (see p. 105), the cautious use of the saline cathartics, since the vegetable cathartics induce more or less hyperæmia of the abdominal organs and therefore renal inflammation. [I have come to depend more and more upon the effect of moderate or free catharsis in all forms of nephritis, including the acute. I have never produced a diphtheritic or severe inflammation of the intestines by my therapeutic efforts, but on the contrary I believe that I have relieved an inflamed intestine by the free use of cathartics. It appeals to my constant effort in therapy to follow nature's methods when possible. One of F. C. Shattuck's favorite combinations, which I have used more than any other, is the following:

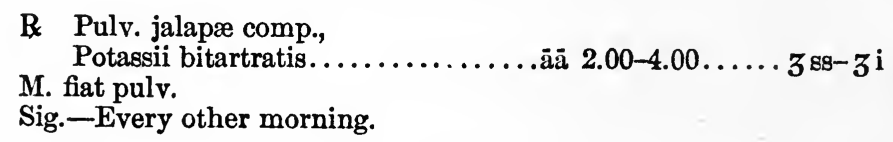

Purge one day, sweat the next.-Ed.]

Puncture.-When there is severe hydrops of the serous cavities, there is frequently only one thing to do, to remove the fluid by puncture. This is often followed by a marked improvement, or the disease remains at a stand-still.

Digitalis.-Whenever cedema is present, even in acute Bright's disease, it is necessary to make sure whether it is caused directly by the kidneys themselves or indirectly by their effect upon the heart. In uræmia the latter is often responsible, and in such cases digitalis may be given.

An acute exacerbation of chronic Bright's disease must receive the same treatment as the acute form.

\section{THERAPY OF CHRONIC PARENCHYMATOUS BRIGHT'S DISEASE.}

The therapy of chronic parenchymatous Bright's disease may be divided into dietetic, hygienic, and medicinal therapy. The former will be taken up in careful detail, as by a suitable diet much can be avoided and much ameliorated, while by an unsuitable diet much harm may be 
done. It may be added that the directions for the diet in chronic parenchymatous Bright's disease without marked œdema apply also, in almost all points, to chronic interstitial nephritis.

Dieteric Therapy.-The diet must be so chosen as to keep up the body weight and sometimes even to increase it; and, at the same time, it must make as slight a demand as possible on the renal activity and must be free from any article of food which might irritate the kidneys.

Insufficiency of an Exclusive Milk Diet.-Although some authorities still contend for an exclusive milk diet, it does not satisfy the conditions, for two reasons: (1) although it is the easiest of all foods for the kidneys (see p. 100), it is not fitted to keep the body nourished when used for a long time (for months or even several years), as it is too poor in carbohydrates; and (2) many patients cannot take 3-3r/2 qt. of milk a day for months. It frequently becomes very distasteful to the patient or upsets his digestion. The patient should, however, take 1-11/2 qt. of milk a day. If even this is unpleasant, we may modify the milk in various ways (see p. 100). One method may be mentioned here.

Before the fat and lactose of the milk can be absorbed in the intestine, they must be first acted on by the pancreatic juice. Such action occurs only in an alkaline medium. Normally, this condition is brought about by the sodium salts of the bile acid. These substances, however, are often lacking in Bright's disease, where the production of bile, with the liver functions in general, is abnormal. It becomes necessary to replace the missing alkali. This may be done by adding to the milk bicarbonate of soda, lime water, or an alkaline mineral water. Potain recommends giving a glass of milk every other hour (sometimes even during the night), and following it with $0.01-0.02 \mathrm{G}$. ( $1 / 6-\mathrm{T} / 3 \mathrm{gr}$.) of pancreatin in gelatin (or, better, glutoid) capsules (No. II, Sahli). [If useful, panereatin should be prescribed in much larger doses, 2-6 gr.-Ed.] One to $1 \mathrm{t} / 2$ qt. of cream of tartar whey may be used daily instead of the milk, if we wish to encourage diuresis; but we must remember that it has not a tenth as much proteid and fat as milk.

It must be admitted that certain individuals exhibit an absolute intolerance for milk, even if modified and given with the greatest caution. In such cases it is foolish to insist on its being taken, either in Bright's disease or in any other condition in which it ordinarily seems indicated.

Necessary Amount of Nourishment Besides Milk.-If the patient take only $1 \mathrm{r} / 2$ qt. of milk ( 875 calories) a day, he must get in addition about $30 \mathrm{G}$. ( $1 \mathrm{oz}$.$) of proteid (129 calories), and at least 350 \mathrm{G} .(10 \mathrm{oz}$.) of carbohydrates (1505 calories). A part of the latter may be replaced by fat (the caloric value of fat stands to that of proteid and carbohydrates in the ratio of $23: 10$ ).

Choice of Foods. - In just what form these necessary food elements shall be given depends partly on the physician, partly on the patient. The following discussion will try to show how the need may be met, and what particular foods should be forbidden. 
Meat.-Game must be entirely interdicted, as the amount of ptomaines and of extractives in the muscles of animals is increased by the process of hunting. Sausages, particularly if highly spiced, are on the forbidden list, as are all strong condiments, such as pepper, paprika, and mustard. The use of smoked meats should be strictly limited. "White meat" is allowed; the patient should get daily at least $150 \mathrm{G}$. ( $5 \mathrm{oz}$.) weighed raw, containing $30 \mathrm{G}$. $(1 \mathrm{oz}$.$) of proteid. Poultry, veal,$ and pork are permitted; liver, sweetbreads, brains, and kidneys forbidden. The author allows a free use of veal, although aware that some authorities object to it as being particularly rich in toxins, and, therefore, frequently giving rise to indigestion. He admits that this is true for many individuals. With some patients the use of veal regularly brings on diarrhœa. Beef, though forbidden by many authorities as " red meat," the author has found by repeated trials to be as permissible as white meat. If it is boiled, it is much less rich in extractives than is roasted white meat, and therefore at least no more irritating to the kidneys. [The question of veal in America is quite different from that on the Continent. With us it is killed younger, hung less time, and is less thoroughly cooked. Keeping these differences in mind, I have frequently ordered veal for my patients with nephritis and other diseases and have never found occasion to regret it. In recent years much of the prejudice against the employment of red meat has been transferred from doctors to laymen. If limited to white meat, we eat less meat; hence much of the value of this restriction. Forchheimer allows only white meat. Von Noorden, on the contrary, allows both red and white meats in chronic interstitial nephritis, basing this permission upon years of clinical experience and upon the researches of Kaufmann and Mohr. I have been accustomed to allow a similar latitude in choice, although I rarely permit as large amounts as von Noorden advises.-Ed.] Mutton, perhaps better boiled, may be given occasionally as a change, but lamb may be employed more freely. Calves' foot and calves' head jelly are permissible and useful, as is the occasional use of raw meat,- -scraped beef, for example. The danger of tapeworms should always be remembered.

Fish.-Sea-fish must not be eaten unless they can be obtained fresh, before various toxins, such as neuridine, guanine, and hydrocollidine, have had time to develop. Some fresh-water fish, such as salmon and sturgeon, are better avoided, their roe especially, as it is very rich in nitrogen. Eels are too fatty and difficult to digest. Other kinds of ordinary saltand fresh-water fish may be taken, provided they are perfectly fresh. Good sardines in oil may be used occasionally. Fresh oysters and frogs' legs are allowable, but crabs and lobsters are best entirely avoided, on account of their frequent content of toxins. [Widal allows fresh-water but not salt-water fish. I prefer to diminish the amount of $\mathrm{NaCl}$ in other ways and to allow fish, if very recently killed, from either fresh or salt water.-Ed.] Boiling is best, then steaming, roasting, broiling, and baking (see p. 371). No strong condiments (pepper, paprika, cinnamon, 
onion, mustard) should be used in cooking. [Neither fish nor meat is usually boiled long enough. Beef or mutton should be boiled 4-6 hours.-Ed.]

Eggs.- For many years it was a subject of great contention whether eggs were or were not advisable for a person with chronic Bright's disease. To-day it seems proved that raw eggs in any great quantity may cause albuminuria, but that 2 or 3 cooked eggs a day (soft-boiled, scrambled, in omelet, added to vegetables, etc.) are not only permissible but beneficial.

Vegetables.-Vegetables must be carefully selected. Onions, garlic, radishes, celery, and asparagus must be forbidden on account of the sharp oils that they contain; mushrooms, on account of their nitrogen; and tomatoes, on account of their oxalic acid. Other vegetables are useful; e.g., green and dried peas and beans, potatoes (especially in purée), cauliflower, turnips, carrots, and lettuce. If salad dressing is used, it should be made with a good deal of oil, and lemon juice rather than much vinegar. [Vinegar does not irritate the kidneys and I know of no reason for its prohibition in nephritis. Von Noorden allows it.-Ed.]

Carbohydrates.-Carbohydrates are not only permissible but directly indicated. Peas, beans, lentils, potatoes, cereals (particularly rice, barley, and farina), noodles, ${ }^{2}$ macaroni, and all light farinaceous foods are suitable. Bread can be used, perhaps best graham bread, though white and brown are both allowable.

Fat.-Fat, as has been said before, is highly necessary, but should be carefully selected, so as to be as digestible as possible. First on the list comes cream, especially as a substitute for milk, with butter, fatty cheese, and olive oil; next, lard and bacon, whose fat is more difficult to digest, because it is enclosed in animal cells. Cheeses which do not contain fat, particularly if they are fermenting, like Rochefort, Parmesan, Camembert, etc., are to be strictly interdicted, particularly on account of their alkaloids as well as their nitrogen. Aside from their nutritive value, the easily digestible fats have, when taken with other foods, the additional advantage of quickly causing a feeling of satiety and so suppressing the sensation of hunger. [Fat patients as a rule should be fed fats and very much restricted in carbohydrates. Lean patients should have both starch and fat in moderate quantities. We should remember that the constant presence of albumin and casts in a chronic nephritis does not contraindicate a diet in which each element appears in moderation. The only point to be kept constantly in mind is the individual toleration. A study should be made of the patient's toleration of the different kinds of fat (cream, butter, beef, mutton, bacon, olive oil). In the same manner starches should be tested, with proper regard to the patient's relish of the same.-Ed.]

2 [I rarely see noodles eaten in America. When well cooked-first boiled thoroughly, and then well baked with the addition of fresh butter-they are very nutritious and appetizing. Polenta, boiled, baked, and grilled, and Indian meal mush or hominy are useful additions to the nephritic's dietary.-Ed.] 
Fruit.-As it contains very little nitrogen, fruit, raw or cooked, may be given in spite of its salts of potassium and calcium. A choice should be exercised, however, similar to that in acute Bright's disease. [Theoretically, apples, pears, raspberries, and grapes are the most desirable fruits, because they contain so little benzoic acid. I permit moderate amounts of all fresh fruits except cranberries. In many well nourished patients a moderate "grape-cure" is indicated.-Ed.] Ices and icecream are not injurious, provided they are not too highly flavored.

Drinks.- However disastrous to the diseased kidneys a lack of liquid may be, too much is also disadvantageous, since it demands increased renal activity. The daily amount of liquid for a person with Bright's disease (good heart action being presupposed) should be 2 qt.

Alcohol.-Every strongly alcoholic drink is strictly forbidden, except as a heart stimulant if signs of weakness appear as a consequence of the renal disease, and even then it is better to employ some other stimulant, as camphor or digitalis.

Wines.-All wines are forbidden, except very small quantities of the very lightest white wines, diluted with water or mineral water. Their tartaric acid salts may have a slight diuretic action.

[In regard to the use of alcohol, I agree with von Noorden and prohibit it in chronic nephritis. His reasoning seems to me logical. Most patients with as chronic a disease as interstitial nephritis will not follow any physician's advice indefinitely. If in the ordinary course of the disease disagreeable or dangerous symptoms arise, they are apt to attribute these to his lack of care. If we allow them a glass of wine at dinner, they often take two or three and later on at luncheon as well. I believe in cutting off all liquor; then if the patient's digestion or appetite fail, in allowing a glass of Rhine or Moselle wine or a small drink of whiskey with the hearty meal of the day. When I have made this concession I have noticed that patients invariably take more than I allow them.-Ed.]

Unfermented Wines.-Chandeau is the most suitable. Unfermented grape juice may be substituted for wine.

Beer.-A small amount of beer with a low content of alcohol may be allowed. The English brown beer or the German malt beers are the best, as the long drying of the barley at a high temperature during the process of their manufacture makes them less irritating to the kidneys. Their nutritive value is also relatively high, on account of their content of so-called extractives (salts and dextrin). Their diastatic ferments help to digest the carbohydrates as well as sugar. The malt beer contains less alcohol (2-3 per cent.) than the English brown beer (5-6 per cent.). Both should be thinned with water or mineral water. Even these small quantities of alcohol should not be allowed, however, unless the patient finds it exceedingly hard to forego them.

Cider.-In spite of its diuretic action, cider is too irritating to the kidneys to be allowed. 
Tea, Coffee, etc.-Small amounts of tea and coffee taken with a good deal of milk or cream are allowable, especially for breakfast. The caffeine and theine may even be advantageous as diureties and cardiac stimulants. But the author especially recommends cocoa, or chocolate not flavored with vanilla. Mehring's "Kraftschokolade" (unflavored) is especially suitable, as its nutritive value is increased by the fact that the cocoa fat has been made emulsifiable, and therefore more easy of digestion, by the addition of a certain amount of free fatty acid. About $30 \mathrm{G}$. (1 oz.) of any kind of chocolate should be added to a cup of milk. Eucasin cocoa may also be used. [Plasmon cocoa and cacao l'avosie are other useful preparations.-Ed.]

Meat Soups.-All broths and all extracts of meat are to be rigorously excluded from the diet, on account of their extractives. Soups made from cereals must take their place. [The value of soup to promote the appetite must not be neglected, and patients should be furnished with numerous substitutes for broth and meat soups. The various purées,yellow and green pea and bean, rice, potato, barley, sago, corn, lettuce, squash, cauliflower,-clam broth topped with cream, and oyster broth are examples.-Ed.]

Lemonade.-Lemonade and fruit juices in general are to be permitted. The author does not, as do some authorities, find any valid objection to vinegar (see p. 111).

Salt.-As in compensated heart lesions, so in latent renal disease, a diet poor in common salt is warmly urged by many authors, especially when the relatively small amount of chlorine in the urine proves that it is being retained in the organism. [This has seemed to me a fitting place to emphasize the importance of the reduction of $\mathrm{NaCl}$ to nephriticsone of the most rational contributions to the treatment of Bright's disease. $\mathrm{NaCl}$ controls osmosis in the economy. When retained in excess in the blood, the latter becomes hypertonic, and less water is excreted by the skin, lungs, and kidneys. Dropsy then begins or increases; so does albuminuria. Most nephritics eliminate the $\mathrm{NaCl}$ ingested rather imperfectly, perhaps one-half the usual amount. Castaigne, in his recent monograph upon kidney diseases (1906), divides the diet for nephritics into (1) milk diet; (2) dechloridation diet. The discussion of the latter follows :

Raw meat contains $1 \mathrm{G}$. $\mathrm{NaCl}$ to the kilo. When boiled it loses the $\mathrm{NaCl}$ entirely. An egg contains $0.20-0.25 \mathrm{G}$. NaCl. The dry leguminous vegetables, potatoes, and grains contain less than $1 \mathrm{G}$. $\mathrm{NaCl}$ per kilo. Rice and hominy (la farine de froment) contain less than $0.10 \mathrm{G} . \mathrm{NaCl}$ per kilo. Among the green vegetables, spinach and caulifiower are rather rich in salt, while fresh green peas, boiled carrots, string beans, leeks, and lettuce contain very little and are permissible.

Ordinary bread always contains a good deal of salt, unless specially prepared. Potatoes, of course, do not. Fruits, sweets, desserts like puddings and creams, and cheese can be allowed freely. 
When most striking, peripheral œdema disappears, and with it the œdema (or its symptoms) of the viscera, lungs, brain, etc. This improvement coincides with an increased elimination of water and salt by the urine, and a diminution of weight $(1 / 2-11 / 2 \mathrm{lb}$. a day). Later on, the patient may return to a normal ingestion of salt without retention.

When favorable, the symptoms of retention disappear perhaps more slowly, but as soon as salt is ingested, even though less than the normal average, symptoms of retention reappear. This class comprises many uræmic types in which there is no dropsy (dry retention).

When unfavorable, dechloridation is not sufficient to reduce the œdema or to prevent the onset of uræmic symptoms.

'There are, of course, many cases of nephritis in which it is not necessary or even wise to decrease or omit $\mathrm{NaCl}$ from the diet, and many in which the deprivation should not be too prolonged. In some cases, after prolonged abstinence, an appearance or an increase of albuminuria has sometimes been observed, less resistance to infections has been noted, and anorexia or marked distaste for food has been seen. With these disadvantages in mind, the indications for dechloridation are as follows:

1. Acute and chronic nephritis with dropsy.

2. After several days of a milk diet (3 litres daily) $10 \mathrm{G}$. $(2 \mathrm{r} / 2 \mathrm{dr}$.) of $\mathrm{NaCl}$ are added. If now the patient's weight inereases, there must be retention, and so this imperfect regulation should be assisted by dechloridation.

Since Achard and Beaujard described cases of so-called "dry retention," who require dechloridation to prevent the onset of uræmic symptoms, it becomes necessary to obtain another criterion, because such cases exhibit no œdema and show no change in weight after submitting to such a test as described in (2) above. The patient, at absolute rest, is given for several days a diet poor in common salt (one of those cited above). In one case, the patient, although ingesting but 2-3 G. of salt, may excrete for the first few days a dozen, then reach a level and eliminate exactly the amount ingested. Such a patient does not require the dechloridation diet. In a second case, the patient, while on the low salt diet, continues to excrete considerable quantities of salt. The diet should therefore be continued, although under careful observation. In a third case, the patient upon small amounts of salt eliminates but little, yet after a number of days no salt equilibrium results. He continues to excrete more than he ingests, but with inexplicable diurnal variations. This condition is characteristic of dry retention, and the above diet will probably have to be continued for a long time. The latter are uræmic types.

After applying these principles to a patient, we begin to add a small amount of salt to his diet a few days after the œdema has disappeared, the weight has become fixed, and the balance between salt ingestion and excretion has been established. If such an addition does not produce any retention, we gradually increase the salt, until the diet has become 
normal again. Cases vary decidedly as to their retention. Some persist with retention for a few days only, others for weeks at a time, and still others, notably the dry type, continually.

These valuable studies explain what has always seemed to me difficult to understand; viz., the strikingly good results which follow in some cases of nephritis with œdema from decidedly diminishing the amount of milk in their diet and supplementing with solids, including meat. They also demonstrate that part, at least, of the value of a pure milk diet for nephritics depends upon its low salt content. One litre contains from 1.3-1.8 G., so that a patient upon 3 litres ingests but 4-5 G. of salt. Even so small an amount is more than some cases with œdema should ingest, since the œdema disappears more promptly and they improve more noticeably when allowed but $2-3$ G. in 24 hours. Despite these results, which I among many others have repeatedly confirmed during the past 3 years, milk is still and will continue to be the diet of selection for most uræmic types, because it is nourishing, it contains a large amount of fluid, it makes small demands upon the digestive functions, its nuclein is a paranuclein, it has a low salt content, and it contains only a small amount of toxins; but in the presence of marked œdema or with dry retention, better results will follow the selection of one of the above diets.-Ed.]

Where the nitrogen content of urine and feces proves the retention of urea, milk, carbohydrates, and fat should form the bulk of the diet, only 100-150 G. (3-5 oz.) of meat being given.

Meals Near Together.-In concluding the discussion of this important branch of the therapy of chronic Bright's disease, we may say that the meals should be relatively small in quantity and frequent in number. [Many such patients do better upon 3 or 4 moderate meals a day (see Compensated Heart Lesions, p. 2). I believe that the individual gastric motility is the decisive factor.-Ed.]

Hygiene.-To pass on to other hygienic measures. The patient mùst be guarded in every possible way from taking cold, and so possibly bringing on an acute exacerbation of the chronic inflammation.

Climate-Clothing.-He should keep his feet well protected, and wear woollen underwear, and sometimes in addition a flannel band over the loins, and should live in an even, warm climate, the South in winter, if possible. [For further details upon climate, see p. 120.-Ed.]

Dry Rubs.-To harden the skin against undue sensitiveness to unavoidable changes in temperature, it is well, as Semola suggests, to give the body a dry rub down every day. Cold, wet rubs the author agrees with Leube in considering contraindicated, as they may cause an acute congestion of the kidneys.

Baths.-Lukewarm baths $\left(90^{\circ}\right)$ or hotter baths $\left(95^{\circ}-100^{\circ}\right), 3$ or 4 times a week, followed by a light sweat, are advisable in order to keep up the skin secretion (see p. 106). Care should be taken, if atheroma exists. 
Exercise.-Moderate exercise in the open air is to be recommended, but should stop before the point of fatigue or the appearance of albuminuria. Mental strain and overwork also have a bad influence on the chronic condition. Massage is often useful.

Bowels.-It goes without saying that the bowels must be kept regularly open. If constipation is present, we should use saline cathartics (sodium sulphate, magnesium sulphate), or (though not advantageously) a combination of a vegetable and a saline purge. Leube, for example, prescribes:

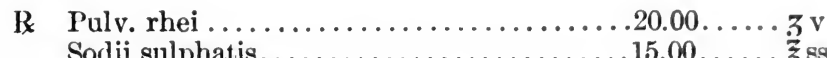

Sodii sulphatis...................... $15.00 \ldots \ldots$

M. fiat pulv.

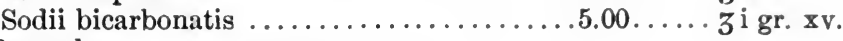

Sig. -1 teaspoonful in wafers at night.

Or:
B Pulv, rhei........................
Potassii bitartratis.................. $20.00 \ldots \mathrm{v}$
M. fiat pulv.
Sig. -1 teaspoonful in wafers at night.

[For these patients I employ compound jalap powder or Rochelle or Epsom salts more than other drugs.-Ed.]

Medication.-Any general medication fails entirely, so far as removing the cause or preventing death is concerned. All the drugs which have been recommended for chronic Bright's disease-lead acetate, saltpetre, preparations of arsenic, chloride of iron, and the strontium salts are powerless, or, at the very best, not to be depended on to prevent the fatal course.

The latter drugs, the salts of strontium and the iron and arsenic preparations, may be used in those cases in which the disease is latent,in the stage of compensation. Here a slight disturbance of the general health, some pallor, symptoms of arterial tension and hypertrophy of the left ventricle, but above all albuminuria and cylindruria, sufficiently indicate the presence of chronic Bright's disease, without such severe consequences of renal insufficiency as dropsy and uræmic intoxication. It seems to the author better, however, to have the patient inhale oxygen in such cases. This increases the appetite, diminishes the anæmia, increases the bodily strength, and prolongs the latent stage of the disease. At least as much as $71 / 2$ gallons (12-13 gallons on the average) should be inhaled, half at morning, half at night. [I have had no experience with this treatment. It is expensive.-Ed.]

Kola.-The preparations of the kola nut may be used as a tonic. The author's prescription is as follows :

R Ext. kolæ fluidi,

Ext. cocæ fluidi,

Ext. cinchonæ fluidi .............āa $20.00 \ldots \ldots 3^{v}$

M. et sig. -1 teaspoonful after eating, in a small quantity of table wine, or, preferably, a glass of an alkaline mineral water. 
Stoll's Elixir Kolæ, taken in exactly the same way, or Kola Astier, is good. (See p. 23.)

Iodine.-Bartel recommends the iodides, but the author has never had any success from their use, especially in the form of nephritis under consideration. Potassium iodide may be used in any of its forms, or the following prescription may be employed:

B Sodii phosphatis............................. $\mathbf{x x x}$

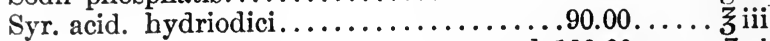

Aq. dest..................... s. ad $180.00 \ldots \ldots$.

M. fiat solut.

Sig. -1 teaspoonful 2-3 times a day.

Or we may give the easily digestible and rather palatable syrup of the iodide of iron, up to 3 tablespoonfuls daily, $1 / 2$ hour after meals. [This and the tincture of the chloride I have used with gratifying results. -Ed.]

Symptomatic Therapy.-The symptomatic therapy of chronic parenchymatous nephritis is directed oftenest against oliguria and the consequent hydrops. The therapy in this case is partly dietetic, partly medicinal (diuretic, diaphoretic, purgative), and partly hydropathic. In extreme cases surgery must be resorted to. The reduction of the amount of liquid ingested, including milk, and the exclusion of common salt, have caused in several of the author's obstinate cases an almost entire disappearance of hydrops, external and internal, in a few days. The whole subject of the therapy of hydrops has been treated in detail under acute Bright's disease. (See p. 104.)

Again, the therapy must often combat uræmia. This subject will be discussed fully in a section by itself. (See p. 122.)

We must occasionally combat directly an acute gastritis, diarrhœa arising from colitis, pulmonary cdema, inflammation of the serous membranes or of the lungs, or, finally, resulting brain complications. In all of these cases, the therapy does not differ from the usual treatment of the condition in question.

\section{THERAPY OF CHRONIC INTERSTITIAL NEPHRITIS.}

Iodides.-When interstitial nephritis is secondary to some other affection, as gout, chronic lead poisoning, diabetes mellitus, or chronic alcoholism, the therapy is directed first against the primary disease. Symptomatically, the treatment with iodides is of good service in such cases, particularly if there is a syphilitic endarteritis or a granular kidney, secondary to arteriosclerosis. The mercury treatment here seems directly contraindicated. The author has seen a case of granular kidney arising from syphilitic endarteritis, which grew much worse under mercury and improved only when put upon iodides. On the other hand, where the cause of the atrophied kidney is not clear, the author has seen the iodide treatment (1.5 G. ( $22 \mathrm{gr}$.) and more of potassium iodide a day) 
produce no benefit whatever. [In my experience, a nephritis with casts and a preponderating percentage of albumen, which complicates the secondary or late secondary stage of syphilis, requires and reacts well to mercury. In other forms some iodine salt is indicated. Drug treatment in any form of nephritis requires great discrimination. In chronic interstitial nephritis, a disease of very long duration, under ordinary circumstances the physician should prescribe as few drugs as possible and these for as short a time as possible. The following list may help in the discrimination. We should avoid:

(1) Drugs which act directly upon the kidney tissue (see list under Acute Nephritis, p. 98).

(2) The prolonged employment of diuretics, especially the purely renal diuretics. As soon as diuresis is established, it is well to omit the drug. If such drugs as digitalis, caffeine, diuretin, camphor, squills, etc., improve the cardiac power and so favor diuresis, they do no harm, certainly if not persisted in too long; but if their employment does not produce the expected improvement we should desist; otherwise aggravated symptoms will generally result, probably because of their difficult elimination and retention.

(3) Drugs which are slowly excreted by diseased kidneys. We know some, although not all of them. The most important are the iodine salts, salicylic acid, antipyrine, methylene blue, and boric acid. A cumulative drug like digitalis must also be watched carefully. Von Noorden found quinine qualitatively demonstrable in the urine, despite free diuresis, 8 days after the administration of $1 \mathrm{G}$. (15 gr.) of the hydrochlorate.

(4) Atropine is probably retained; it supposedly diminishes the urinary excretion, and because of its action upon the heart and vessels it should be very guardedly employed.

(5) Opium and morphine have been preached against in nephritis, although they are so commonly selected for uræmic convulsions. I employ them in moderate doses once or at most twice, and have never seen any serious results, but almost always a cessation of the convulsions. We have it on Cushny's authority that only with very large doses is any excreted by the kidneys, and that their use does not diminish the amount of urine. Senator, Herrick, Forchheimer, and others recommend it.

(6) Lead, copper, silver, mercury, and their salts should be used for a brief period only.

(7) Iodoform, resorcin, and hydroquinone are advised against by von Noorden.

(8) Ether and chloroform anæsthesia are both dangerous to patients with chronic nephritis. The choice between them is a point of very nice judgment. The old rule, ether should be avoided in Bright's and chloroform chosen, will not always apply. The condition of the heart and vessels, and probably the liver, is quite as important.-Ed.]

Diet.-In many points the therapy of chronic interstitial nephritis corresponds to that of chronic parenchymatous Bright's disease, from 
which secondary atrophied kidney, a form of interstitial nephritis, develops when the disease is of long standing. The diet is the same, except that in interstitial nephritis (polyuria) a very abundant or exclusive milk diet is in place only when there are signs of uræmia. On the contrary, the author agrees with von Noorden in attributing good results to the reduction of liquid to $1 \mathrm{qt}$. a day in cases where the atrophied kidney has led to beginning hydrops and cardiac insufficiency.

Hygiene of Latent Period.-The hygienic prophylaxis is also identical. One question, however, frequently arises, depending on the fact that chronic interstitial nephritis is often latent for years: How can we keep the disease stationary as long as possible in a patient in the latent stage?

Protection from Cold.-Our first care must be to keep the patient from catching cold. He must wear flannel or woollen underwear and furs in winter (see p. 8).

Climate.-When the disease is only moderately advanced he should be sent away for the winter to some warm climate which (1) exhibits no great changes of temperature during the 24 hours, and (2) has a relatively low humidity (the less the humidity, the more water excreted by the skin). Suitable climatic resorts are Egypt, especially Cairo, or better, Heluan, which is about 4 hours from Cairo and much better sheltered, [and a more typical desert climate. I am told that a competent German physician has a very good sanatorium at Heluan, situated just above Cairo in Egypt. There is a very good hotel at the French military post, Biskra, the first oasis in approaching the great desert from the town of Algiers. The steppes of southern Russia are credited with a very low relative moisture. Excellent dry climates in the United States are found in Texas, Arizona, and the New Mexico table-lands; but the comforts of living and the food are not well suited to feeble invalids. In southern California, among the foot-hills of Pasadena and Redlands, comfortable accommodations, pleasant society, and good medical attendance are available. These places are sufficiently distant from the sea, (Pasadena, altitude $1000 \mathrm{ft}$., 20 miles from the coast, 7 miles northeast of Los Angeles, light dry soil, good water supply, free from fog,) to offer a relatively dry climate. Although Santa Barbara is but a mile from the sea and is not so dry, there are many advantages in the way of comforts, hotels, cottages, good physicians, etc. P. K. Brown, of San Francisco, has the direction of a very well organized sanatorium just outside of Santa Barbara. Among the suitable fall and spring climates in the United States might be mentioned Aiken, Camden, Summerville, Thomasville, northern Florida, and Georgia. The soil is too full of clay and there are too many showers to recommend the Berkshires for such patients, although late in the spring and early in the fall it seems to suit many cases. The accommodations, amusements, society, and excellent medical attendance available, perhaps make up for the other drawbacks-Ed.]. On the Riviera are Cannes, Monte 
Carlo, San Remo, Mentone, and Hyères. Nice is not so favorable in either humidity or temperature changes. Corsica is good. [So is Nassau. Bermuda is windier and more changeable. The selection of the most suitable resort for a patient going to the Riviera is frequently best left to some competent Continental physician, sometimes to the patient's own choice. A few suggestions may not be amiss. Mentone, especially the East Bay, is the most sheltered and probably the warmest of the list. The soil is sandy. The hotels are comfortable, the living reasonable, villas for rent numerous, and life quiet, but there are many invalids, especially tuberculous patients. San Remo is similar, except that the soil is clay and there is more east wind. It is as quiet, and living is a little cheaper. Monte Carlo is one of the most beautiful and sheltered spots on the Riviera, but most people and patients I have known there spend too many hours of both day and night at the gaming tables. Living is excellent but expensive. Beaulieu is beautifully situated and sheltered from all points except the sea. It is half-way between Nice and Monte Carlo and is very quiet. Nice is a city, windy and dusty, hot and cold. Good theatres, excellent music, and the cosmopolitan society make it a very diverting place of residence for patients whose mental resources are limited and who require amusement and distraction. One can live there at any price. Cannes is very windy and dusty. Society is centred there, and there is much going on. Most invalids overdo and remain too much indoors. Living is more expensive. Hyères is the oldest resort. It has had its day. It is quiet and lies 3 miles inland; but it is windy and rather dusty. Living is more reasonable. Grasse, the seat of a large perfume industry, is still further inland, rather primitive, very quiet and dull; living is cheap and not so good. The surroundings are beautiful. There is less dust and wind. In south-western France there are a number of delightful resorts, such as Arcachon, Biarritz, St. Jean de Luz, etc. On the southern coast of England we might mention the Isle of Wight, Ventnor, Bournemouth, and Torquay.-Ed.] Tyrol is also beneficial, even if its humidity is somewhat higher ( 70 per cent.); it is suitable for winter and particularly for the spring and fall. The following places may be especially mentioned: Riva on Lake Garda, or, better protected, Salo or Gardone Riviera on the same lake, Arco (well sheltered), Gries, Meran, and especially Lugano. The patient should pass the summer in some place well sheltered from winds and protected by trees, so that the changes of temperature shall be moderate. [I have already mentioned Poland Springs and Waukesha as comfortable summer resorts, with pure, excellent spring water.-Ed.]

Balneotherapy.-Many patients with chronic interstitial and also with chronic parenchymatous nephritis show marked weakness and pallor. Such patients are benefited by the use of iron, dry rubbing, and mild laxative waters (Elster, Franzensbad, Cudowa, Koenigswart, and the Ambrosius and particularly the Carolin springs at Marienbad). For a chronic parenchymatous Bright's disease with oliguria, an earthy or 
alkaline water may be advised (Vichy, Carlsbad, Neuenahr). The hot springs of Gastein or Schlangenbad may be recommended for many patients with chronic renal inflammation, if one wishes to increase the activity of the skin by hot baths. Still, every chronic kidney inflammation,- that is, most cases of chronic interstitial nephritis and many of chronic parenchymatous nephritis,-is an absolute contraindication for the use of hot baths, which may bring on hypertrophy of the heart or arteriosclerosis, and in either case an inclination towards cerebral hemorrhage. Hot springs containing earbon dioxide (Nauheim, Franzensbad) may be used for the same purpose of increasing the activity of the skin. If a uric acid diathesis is at the bottom of the disease, our alkaline-saline waters (Carlsbad) are to be given. (See Therapy of Gout, p. 248.) [Perhaps the most desirable place with us is Hot Springs, Virginia. The arrangements, accommodations, and medical attendance there compare very favorably with the more popular European spas.-Ed.]

Care of the Heart.-A point in which the therapy of chronic interstitial nephritis is distinguished from that of chronic parenchymatous nephritis relates to the treatment of cardiac complications. In the former disease a compensatory hypertrophy takes place and the peripheral blood pressure is permanently raised. Whether from this cause or not, the peripheral vessel walls often become diseased, so that the patient is in permanent danger of cerebral hemorrhage. This danger should be combated by the physician in every way. Alcohol [excess of-Ed.], hot food, strong coffee or tea, too hot room temperature, over-exertion, horizontal postures or those with the head bent over [after eating -Ed.], violent coughing or vomiting, constipation, and hot baths, must all be strictly avoided. Any sign of heart weakness must be closely watched for. If such symptoms appear, uræmia is almost always imminent. It can often be averted by strengthening the heart muscle again.

\section{THERAPY OF URAMIA.}

Nature's Methods.-There are three ways in which the human organism tries to get rid of the toxins circulating in the blood as a result of renal insufficiency: through the skin, through the stomach and intestines, and through increased excretion from the kidneys. This increased renal excretion is brought about in chronic Bright's disease, and particularly in interstitial nephritis, by the hypertrophy of the heart, which increases the supply of blood to the kidneys. If uræmia, acute or chronic, develops, our therapy should try to aid nature in one of these three ways.

Diaphoresis.-In the first place, then, our therapy may be diaphoretic. It is clear that only a vigorous thorough sweating is of value. But this process (see p. 107) is not without danger. By it, more water, proportionately, is excreted than toxins, which are left in a more concentrated form in the more concentrated blood. This concentration of 
toxins after sweating is particularly to be feared in the œdema and hydrops so often accompanying Bright's disease, as the œdematous fluid, rich in toxins, may be absorbed by the blood to take the place of the lost water. The resulting excessively toxic blood may set up a severe acute uræmia. A vigorous diaphoresis, then, no matter how efficacious it may often be, must always be used with extreme caution, until it has been proved safe in the individual case. [Though very timely, Leube's caution in this respect has, I believe, been too carefully regarded. I employ diaphoresis in all such cases and in those without œdema, perhaps with a little more timidity than before I read Leube's paper, and rarely regret it. I know no rules for selection of appropriate cases.-Ed.]

Purgatives.-In the second place, our therapy may be directed towards the excretion of the toxins from the blood through the mucous membrane of the intestines by the use of purgatives. For this purpose we may employ the purges mentioned in the discussion of the treatment of hydrops in heart lesions; i.e., jalap, podophyllin, colocynth, and gamboge. But this therapy is often prevented by the presence of (1) uræmic vomiting, or (2) severe inflammation of the intestine. [Elaterin hypodermically, $1 /{ }_{60}-^{-1} /{ }_{10} \mathrm{gr}$. in acute cases, is often very useful.-Ed.]

Heart Tonics.- In the third place, our therapy may seek to increase the blood pressure in the kidney by stimulating the heart. This is suited to those cases of uremia with decreased blood pressure and a small or even irregular pulse. Digitalis is the usual stimulant. But, as its action is not felt for hours or perhaps for days, it is necessary in dangerous cases to employ with it our strongest heart stimulant, camphor. Large doses are necessary, $10 \mathrm{G}$. $(2 \mathrm{r} / 2 \mathrm{dr}$.) and more of a 10 per cent. solution of camphor in olive oil injected subcutaneously within 24 hours. In place of digitalis, especially in light cases, caffeine may be given by the mouth, or, perhaps, better, certainly when uræmic vomiting is present, by the rectum. To reinforce either digitalis or caffeine, strong alcohol (brandy or rum) may be given in spite of the renal inflammation. [I am apt to precede digitalis by caffeine or to combine the two.-Ed.]

Ingestion of Water.- In all cases of chronic parenchymatous Bright's disease, abundant water should be taken by the mouth or, if that is not possible, by the rectum. This is especially necessary if diaphoretic measures are being used. The danger of the latter therapy can be much diminished if a quart of water is ingested 2 or 3 times a day to replace the water lost by perspiration.

The author's practice is just the opposite in cases of granular kidney where uræmic symptoms appear, not from the kidney disease as such, but as a result of the lessened cardiac activity, with diminution of the amount of urine, which up to that time has been above normal. Here he agrees with von Noorden in prescribing rest and heart stimulants, and furthermore in calculating carefully the difference between the amount of liquid taken in and that excreted, and, if the latter is found 
to be less than the former, in reducing the quantity of liquid (even milk) to about a quart a day. The author has frequently seen this therapy result, in a few days, in the recovery of the heart and the disappearance of the uræmia.

Venesection with Infusion.-If these methods do not succeed, we may resort to venesection to get rid of the poisons in the blood. This procedure is indicated immediately in cases of uræmic convulsions, in which $1 / 2$ pt. of blood is taken; it is well to inject 400 c.c. of normal salt solution, in order to raise the reduced peripheral blood pressure. The author has repeatedly seen this procedure followed by complete cessation of uræmic convulsions, return of consciousness, and disappearance of the entire symptom complex for even 6 months, in spite of persistence of the renal disease. [ $I$ believe we delay venesection too long. It is wise not to wait for well-marked convulsions; I have never seen bleeding do any harm. If blood pressure is high or normal, I agree with Castaigne in withdrawing 400-500 c.c. of blood and purging and sweating; if the blood pressure is low and the heart is dilated, in employing digitalis.-Ed.]

Symptomatic Therapy.-To these therapeutic measures, directed against the disease as such, must be added the treatment of special symptoms.

Uræmic Vomiting: Cerium Oxalate--For constant and exhausting uræmic vomiting the author prescribes cerium oxalate (besides morphine, cocaine, and eucaine) :
B Cerii oxalatis........................ $1.00 \ldots \ldots$ gr. $\mathbf{x v}$
Sacchar. lactis......................... $\mathbf{3}^{\mathrm{ss}}$
M. Div. in pulv. no. vi.
Sig. -3 powders a day in wafers.

\section{Creosote.-}
B Creosoti............................. $0.60 \ldots \ldots$ gtt. $\mathbf{x}$
Emuls. amygdalæ..................50.00.... $3 \mathrm{z}_{3 \mathrm{z}}$
M. fiat emuls.

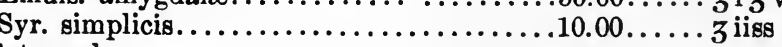
Sig. - A teaspoonful to a tablespoonful 3 times a day.

Tincture of Iodine-Chloroform-Calomel.-Or we give 2 drops of tincture of iodine or 5-10 drops of chloroform in a tablespoonful of water twice a day. This should be followed by milk. Similarly, we may prescribe:

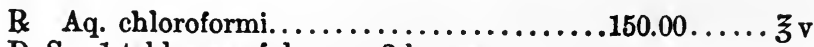

D. S. -1 tablespoonful every 2 hours.

[Small repeated doses of calomel are often very efficacious; $1 / 10 \mathrm{gr}$. every $1 / 2-1$ hour.-Ed.] 
Lactic Acid.-Lecorché and Talamon recommend lactic acid:

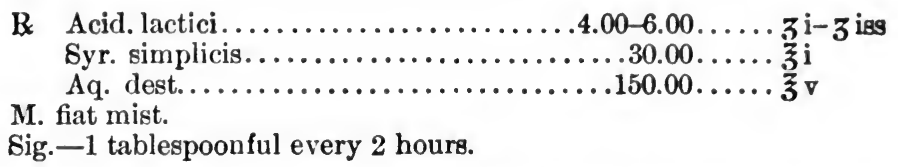

The patient should follow each dose of any of these drugs by swallowing bits of ice, or very small quantities of iced Seltzer water or champagne. Good results may often be obtained from washing out the stomach.

A small amount of vomiting is in itself therapeutic and should not be suppressed. On the contrary, we should assist it by a liberal use of warm water, as this will free the lining of the stomach from any adherent masses of mucus. [Unless contraindicated, a thorough gastric lavage with water at $105^{\circ}$ will often relieve the vomiting, and I usually prefer to try this method first and to employ drugs only when the vomiting is not relieved by the lavage.-Ed.]

Diarrhœa.-For uræmic diarrhœa we give either opium alone internally or a combination of opium and lead acetate:

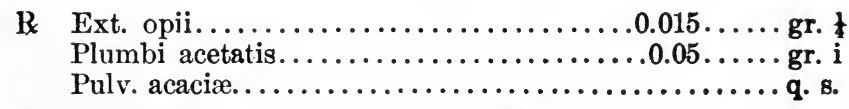

M. fiat pil. no. i. Dent. tal. pil. no. xii.

Sig. -1 pill 3 times a day.

[Unless the diarrhoea is very persistent and uncontrollable by irrigation, I think opium should not be emploved. Diarrhcea is one of nature's methods of relief which we do well in copying, not in hindering.-Ed.]

Tannalbin and tannigen may be tried, the latter as follows:

Re Tannigeni $.0 .50-1.00 \ldots . .$. gr. viii-gr. $x \nabla$

Dent. tal. cachets no. $x$.

Sig. -3 cachets daily.

Uræmic Asthma.-Uræmia may cause symptoms in the respiratory tract, as well as the digestive; i.e., uræmic asthma and uræmic pulmonary ndema. For the asthma we may employ antispasmodics, especially ether (10-30 drops of spirits of ether several times a day on sugar or in tea or sweetened water), or extract of valerian, as follows:

\footnotetext{
B Ext. valerianatis................ $0.30-0.50 \ldots \ldots$ gr. $\mathbf{r - g r}$. viii

Dent. tal. dos. (in caps. molles) no. xxx.

Sig. -1 capsule $3-5$ times a day, with water.
}

Sodium bromide, chloroform, morphine, or repeated inhalations of oxygen may also be used. 
The author has recently had good success in uræmic dyspncea from large doses of ether (following Lemoine and Gallois), either a deep hypodermic injection of 2 c.c. ( $1 / 2 \mathrm{dr}$.) every hour and a teaspoonful of ether in sweetened water given internally, or else two teaspoonfuls of ether internally every half hour, day and night. Relief generally comes in a few hours and the amount of urine increases, but the treatment must be kept up for several days, the doses being gradually decreased. The action of ether is two-fold, neurotonic and diuretic. [Castaigne recommends this method combined with morphine.-Ed.]

Pulmonary Edema.-The pulmonary œdema calls for a large mustard plaster on the chest and the internal use of lead acetate in large doses:

B Plumbi acetatis....................... i

Sacchar. lactis .............................. viii

M. fiat pulv. Dent. tal. dos. no. xv (chartæ ceratæ).

Sig. -1 powder every hour or two in a wafer.

The possibility of lead poisoning must be kept in mind. The author has seen 2 cases which followed only a relatively short use of the drug (colic, stomatitis, lead line).

Edema of the Glottis.-Edema may attack the glottis and call for ice-packs, ice internally, scarification, and, as a last resort, tracheotomy.

Cerebral Manifestations of Uræmia.-A final uræmic symptom group is connected with cerebral disturbances, often showing itself only in the form of very severe headaches. For these, we may use cerium oxalate, often with good results in the author's practice, or the common antineuralgics, migrainine, antipyrine, phenacetin, caffeine, antinervine, hemicranin, etc. In the most severe type, local or general bloodletting and local cold are effective. [Where the headache is accompanied by a very high blood pressure the following combination has often proved of service to me:

B Tinct. aconiti.............................. $m_{v}$

Chloralis hydratis...................... $0.30 \ldots .$. gr. $\mathrm{v}$

Aq. dest..................... s. ad $8.00 \ldots \ldots 3$ ii

M. fiat solut.

Sig.-Every hour until blood pressure falls.-Ed.]

The cerebral symptoms may also take the form of a high grade of restlessness, of psychic disturbances, of great excitement, and particularly of uræmic convulsions.

Narcotics.-In all manifestations, sedatives or narcotics may be cautiously used; but in case of eclampsia they are given not so much for the sake of the unconscious patient as for that of her friends.

Chloral Hydrate or Chloroform.-Chloral hydrate by rectum, or inhalations of chloroform, are contraindicated on account of their effect on the heart. 
Morphine and Camphor.-Morphine (0.01-0.02 G.-1/6-1/3 gr.) is better, accompanied or followed immediately by camphor or ether, all three to be given subcutaneously. [I have never seen chloroform carefully administered do any harm. It often stops a convulsion before the morphine can take effect.-Ed.]

Lumbar Puncture.-Recently lumbar puncture, repeated if necessary, has been used to relieve cerebral uræmia. In several cases the author has found it of decided, though only temporary, benefit. It relieves the uræmic coma, attacks of cramp, amaurosis, and torturing headache. It should be performed with the patient sitting or strongly bent forward. If this is impracticable, he should lie on his side, bringing the head and knees as near each other as possible in order to increase the spaces between the spinous processes of the lumbar vertebræ. We now connect the highest points of the iliac crests by a horizontal, and in the corresponding space between the spinous processes insert a special trochar and cannula, a little to one side of the middle line and pointed somewhat toward the head. When no further resistance is felt, the trochar is removed and the eerebrospinal fluid is withdrawn (generally 20-30 c.c. $\left(5\right.$ dr. -1 oz.), never more than 40 c.c. $\left(1 \frac{1}{4}\right.$ oz.) at once). [I have tried this procedure several times, occasionally with a very brief benefit only.-Ed.]

DiET.-The dict depends upon whether the uræmia is acute, subacute, or chronic. In acute uræmia, an exclusively milk diet is indicated. [Starvation is still better, or Renon's water diet, 1000-1500 c.c. daily with or without lactose for 2 or 3 days.-Ed.] In the subacute form, a diet similar to that in beginning Bright's disease; and in the chronic, a diet varied according to the special clinical manifestations of the renal disease. In the last case, we must be guided especially by the results of the quantitative analysis of the urine for chlorine and urea, and of the feces for nitrogen. If there is retention of chlorine, we give food poor in common salt; if retention of urea, a small amount of proteid (carbohydrates, fat, and only small amounts of milk). [Dilute hydrochloric acid, in doses of 1-3 teaspoonfuls in the 24 hours, has proved to be very useful to many of my patients with nephritis, particularly of the chronic interstitial or diffuse varieties, with constipation and digestive complaints. Von Noorden cites a case in which he administered 100 drops of dilute $\mathrm{HCl}$ (G. P.) daily, with the idea of increasing the nitrogen elimination in the form of ammonia. He found that the ammonia was increased from an average of 0.78 to $1.97 \mathrm{G}$., but the excretion of urea did not decrease correspondingly. Axisa (from Neusser's clinic) has recently furnished evidence of its applicability. He found hypo-acidity in 22 of 25 cases of nephritis.-Ed.]

Other Methods of Treatment: Colon Irrigation.-If only very slight uræmic symptoms are present (slight headache, restlessness, sleeplessness, cramps in the legs), hot colon irrigations are useful (a quart [or several quarts-Ed.] of water at about $102^{\circ}, 3$ times a day). These may 
also be tried in severe cases as an auxiliary measure, provided the heart action is good. Their effect is due to the washing out of the kidneys and the mild diaphoresis which results.

Stimulation of Saliva Secretion.-Finally, it may be mentioned that nature has a fourth way of aiding the elimination of the toxins,--through the salivary glands. Following Leube, the author has tried the stimulation of these glands by persistent use of ehewing-gum, but has met with no success, even in very mild cases of uræmia,-such, for instance, as would yield readily to hot irrigations. [I have tried this method many times, and though I have obtained as much as 800 c.c. of saliva in 24 hours, I have never seen any change in the symptoms, beyond increased thirst.-Ed.]

[Before leaving the treatment of kidney diseases, mention should be made of surgical treatment, a method tried more in America than elsewhere, of organotherapy, and of psychotherapy.

Surgical Treatment: Decapsulation.-My experience has been limited to 4 cases of far advanced chronic, diffuse, or parenchymatous nephritis. The results were not favorable. The operation does not appeal to my reason. I am inclined to class it with Talma's operation for cirrhosis of the liver, to which it has been compared by one of its warm advocates. All the records of experimental investigations which I have seen, point to its uselessness, and none show any proof or evidence of its claimed curative action. Modern researches show more and more conclusively that we are dealing with a general disease, not a local disease of the kidneys. Under appropriate medical treatment (including psychical), many cases of chronic nephritis exhibit extraordinary periods of improvement or even so-called cure; others, of chronic parenchymatous nephritis, as they improve are transformed into the interstitial variety and lose many of the evidences of renal mischief (albumin and casts). Despite these objections, some cases have improved after the operation and others have been reported cured,--whether correctly interpreted as to cause and effect, I am not certain. Many of the cases reported were not observed carefully or long enough. Castaigne recommends the operation (1) in acute nephritis with many leucocytes in the urinary sediment, which are daily increasing in quantity and when at the same time the patient's uræmic or infectious symptoms are not improving; (2) in acute nephritis with anuria, which resists all medical measures and which is beginning to be complicated by uræmic symptoms; (3) in chronic nephritis with a hæmaturia which resists all medical efforts and which endangers the patient's life or strength (in such a condition, if one kidney is at fault, a unilateral nephrotomy should be attempted); (4) in obstinate nephralgia, especially when associated with a movable kidney; (5) in chronic nephritis with uræmia, which has improved under medical treatment, relapsed, and finally failed to react. In some of these cases renal decapsulation may prolong life. 
Organotherapy.-I have had no personal experience in this line of treatment. Nephrine, a glycerin extract, has not proved of much service. Blood serum, drawn from the renal vein of a goat, proved of decided benefit in three cases reported by Tessier. Even if further observations confirm its value, it will be an expensive and difficult remedy to procure. The fresh pulp of pigs' kidneys, macerated and then acted upon by artificial gastric juice in order to free the extract from toxic substances, has been reported as useful in combating some of the serious symptoms of chronic nephritis. It is too soon to decide upon the value of any of these methods.

Psychotherapy.--In chronic Bright's disease, and especially in the interstitial variety, so much of success of whatever hygienic, climatic, dietetic, and medicinal therapy is instituted, depends upon the establishment and maintenance of a normal psychic and nervous condition with a cheerful, hopeful frame of mind, that we as physicians must not fail to do our part in this most important branch. The constant repetition of urinary examinations, of slight value in many cases, often depresses the patient. When it is evident that the patient's condition rises and falls upon the record of albumin and casts, such examinations are better omitted, and the physician should judge his patient by his symptoms and complaints. I make it a practice to tell my patients about the nature of their disease, about its curability, about patients with a similar or worse type who are living active, healthy lives. I encourage them to take up their profession or business if they have dropped it, or to continue in it, only warning them to live more rationally than has been their habit, with care in regard to diet, exercise, exposure, etc. I endeavor to find out some other interest or hobby, especially one taking them out of doors, and to persuade them to devote more time to its cultivation. In the matter of diet, I try to arrange that in such detail, so generously, with so many alternatives, that my diet sheets can be given to the cook or the housekeeper, so that the patient, if a man, soon forgets many of the restrictions. One principle especially governs my dietetic rules: I never tell a patient what he can't eat until I have told him so much that he can that I am satisfied his nutrition will not suffer. When a patient with chronic nephritis has his life carefully and systematically regulated, with excesses, indiscretions, fatigue, exposure, friction, and worry prevented; when he lives, in other words, a quiet, contented, happy life, he may live for many years and suffer less than many of his healthier friends.-Ed.]

\section{THERAPY OF NEPHROLITHIASIS.}

With the exception of the rare cystin and xanthin calculi, kidney stones may be divided into uric acid, oxalic acid, and phosphoric acid stones. The first two are formed in an acid medium; the third, in an alkaline one. The presence of phosphoric acid stones presupposes, then, a preëxistent affection of the ureter. Forerunners of the real stones, espe- 
cially the oxalate stones, are kidney sand, a fine powder precipitate, and kidney gravel, small concrements chemically like the true stones, easily passing through the ureter.

The therapy depends upon the chemical constitution of the stone.

\section{THERAPY OF NEPHROLITHIASIS URICA.}

Though the etiology of nephrolithiasis urica is not wholly clear, one point is certain, that it is closely connected with a uric acid diathesis and is frequently its earliest symptom. The presence of such a diathesis and an hereditary predilection to it, are the most common causes of uric acid stones.

It is clear, then, that their causal therapy must be identical with that of a uric acid diathesis (see p. 224). Only some special points will be added here. The objects must be (1) to check the precipitation of uric acid from the urine in the kidneys, or at least, (2) where kidney sand or gravel is present, to prevent its forming a stone, or finally, (3) if a stone has already been formed, to prevent its becoming larger, and either to dissolve it or to facilitate its excretion in small pieces. Two means are available, dietetic and medicinal.

Dietetic Therapy.-The aim of the dietetic therapy must be to deny all foods which cause an increased secretion of alloxuric bodies, particularly uric acid. The chief of such foods, as experiment and experience both prove, is meat. Taken freely, it leads to an increased excretion of alloxuric bodies. This is the reason for the long established practice of reducing the amount of meat in urolithiasis.

Vegetarianism.-A mixed diet, with a preponderance of vegetables, fats, and carbohydrates, occasionally with absolutely no meat, is indicated. Whether all meat should be prohibited, and, if so, for how long, depends upon (1) whether the body weight and strength of the patient keep up, and (2) whether an exclusively vegetarian diet brings on indigestion. It should be kept in mind that such a diet, long continued, may favor the formation of oxalic acid stones, or more often, of calcium phosphate stones, the latter because a vegetable diet leads to an alkaline reaction of the urine. A combined urate and oxalate stone or a uric acid stone surrounded with calcium phosphate is much worse to deal with than is a simple uric acid stone.

Generally, provided the patient bears it well, the author greatly reduces the amount of meat for about half a year, and then, if the patient begins to crave it again, tries a gradual increase, still keeping the other food elements predominant.

Choice of Animal Food.-The kind of animal food is very important. We must avoid all kinds rich in cell nuclei, as they furnish nuclein, the basic substance of all alloxuric bodies. Liver, brain, sweetbreads, kidneys, and fish roe are therefore forbidden; muscle, as Kossel has shown, is poor in nuclein and therefore allowable. 
Preparation.-As for the mode of preparation, boiling is best, especially if the meat be put into cold water first. In this way the extractives, injurious both in uric acid diathesis and kidney disease, are drawn out irto the water. Gout is much more common among people who eat raw or roasted meat than among those who prepare their meat by boiling. This process produces the least excretion of alloxuric bodies.

Forbidden Foods.-Theoretically every kind of muscle may be permitted, but practically we allow both dark and light meat, and avoid game, pickled fish, and shell-fish, as well as piquant sauces, too highly flavored foods, and mushrooms. (See Therapy of Bright's Disease, p. 110.) In particular, all broths, beef tea, and meat extracts must be wholly forbidden, as they are, so to speak, the concentrated form of extractives. Much salt and all salt fish and salt meat are best left alone, because the uric acid is precipitated more easily from urine containing an abundance of common salt. Even vegetables must be somewhat restricted, as some of them, asparagus for instance, increase the alloxuric bodies. The green vegetables, lettuce and other salads particularly, seem to be best.

Carbohydrates.-Carbohydrates are allowed, but the quantity must be moderate on account of the lactic acid fermentation that they cause and the consequent frequent indigestion.

Fat, milk, milk gruels, and eggs are exceedingly suitable forms of food. Although they contain nuclein, it does not, as Kossel has shown, give rise to alloxuric bodies, apparently because it is not derived from nuclei. The patient gets the needed amount of proteid from the foods already mentioned, with butter, cream cheese, gelatinous foods, peas and beans, and substitutes for meat which contain neither nuclein nor extractives, such as nutrose, sanatogen, eucasin, aleuronat [and roborat-Ed.].

Fruit.-Fruit is exceedingly good for patients with a uric acid diathesis, and therefore for those with urolithiasis. The vegetable acid alkalies are oxidized in the body, forming carbonates, and these, combining with uric acid, form urates, which are much more soluble in the urine than is uric acid.

Liquids.- The patient must take an abundant supply of liquid in order to keep the urine so dilute that precipitation will not easily occur. A good deal of milk and mineral water should be given. A glass of hot water in the morning on an empty stomach is advisable to increase diuresis. [An effort should be made to provide fluid enough to cause about 2000 c.c. of urine per day.-Ed.] All kinds of strongly alcoholic drinks must be forbidden, and even the lighter kinds should be limited. A glass of wine a day, or one-half to a pint of beer may often be allowed. The light Bohemian (Pilsener) beers are best, and the old Bordeaux and Moselle wines, the former being preferable as the less acid. The wine must always be well diluted. Cider need not be wholly interdicted if the kidneys are intact. Moderate amounts of tea, coffee, chocolate, or 
cocoa do no harm. Large quantities of coffee seem to increase the excretion of the alloxuric bodies.

Exercise--After eating, the patient may either rest or take gentle exercise, as seems to suit him best. As for exercise in general, patients with kidney stones [and colic-Ed.] will naturally stay in bed, but for those with sand or gravel, moderate exercise, such as walking, especially in somewhat hilly country, gymnastics, billiards, and massage are beneficial. Sports (riding, bicycling, boating, fencing, hunting) are too severe and markedly increase the alloxuric bodies.

RUBS AND BATHS.-Procedures which stimulate the functions of the skin are of great use, particularly in kidney gravel. The French use alcohol rubs and tepid douches. Cold baths are, of course, contraindicated. Tepid baths two or three times a week are very beneficial, as it can be proved (Klemperer) that they reduce the amount of alloxuric bodies. Hot baths, on the contrary, are injurious. Dry rubs are often advised.

MEDICATION : Alkalies.-Alkalies have long been employed and their usefulness fully proved, but the rationale of their administration is not very clear. To-day it scems probable that their good effects are due to their diminishing the acidity of the urine and so making it a better solvent for uric acid. The less the acidity of the urine, the greater its content of alkaline phosphates, and, therefore, the greater the quantity of uric acid combined with alkalies and so easily soluble.

Bicarbonate of Soda-Allaline Mineral Waters.--Various forms of alkali are recommended, particularly bicarbonate of soda. This should be given in such doses that the morning urine will be only weakly acid and its sediment on centrifugizing will be free from any uric acid crystals or amorphous urates. The amount generally needed is $2-4 \mathrm{G}$. $(\mathrm{I} / 2-1 \mathrm{dr}$.) in 24 hours, either in powder or dissolved in a pint of water. In its place alkaline mineral waters may be employed, such as Vichy, Fachingen, Bilin, Neuenahr. The author prefers the Célestines Vichy water in nephrolithiasis, the Quelle L'Hôpital in diseases of the alimentary canal, and La Grande Grille in diseases of the liver.

[The table on page 133 gives the constituents of the more important alkaline waters of Europe and the United States.-Ed.]

Alkaline Salt Waters.-Alkaline salt (muriatic) waters are also sometimes employed, although their chief use is in disease of the respiratory tract. The author agrees with Kobert in not recommending them, on account of their great amount of common salt; he believes that the more of this substance the urine contains, the less solvent it is for uric acid.

Hot Saline Springs: Carlsbad.-Of the alkaline-saline (sodium sulphate) waters only one is very commonly used in uric nephrolithiasis; i.e., Carlsbad. One or 2 glasses of the Mühlbrunnen are taken at about $122^{\circ}$, somewhat below the natural temperature. Instead of the water itself, we may employ a teaspoonful of the artificial or natural Carlsbad salts in a glass of warm water daily, generally on an empty stomach. 


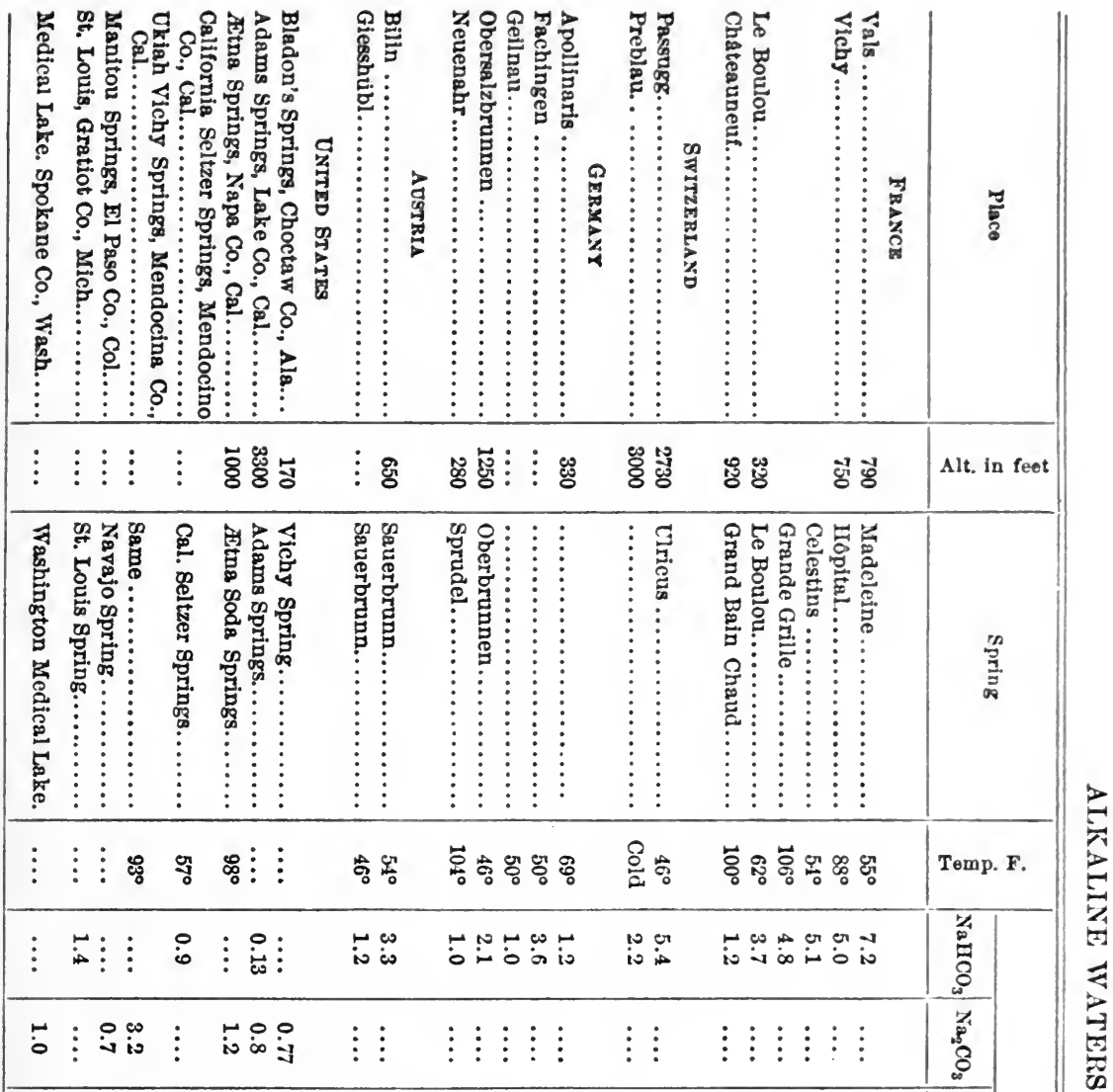

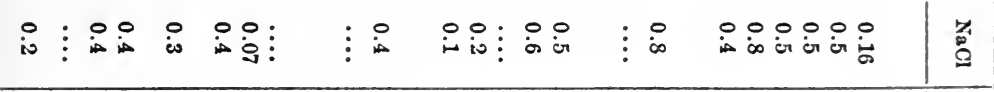

\begin{tabular}{|c|c|c|c|c|c|c|c|}
\hline$\vdots \stackrel{\circ}{\dot{\infty}} \vdots$ & $\vdots$ & 只 $\vdots \vdots$ & is: & $\vdots \stackrel{0}{\vdots} \vdots \vdots$ i & $\vdots \stackrel{\circ}{\dot{\infty}}$ & 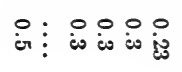 & 总 \\
\hline 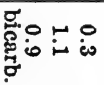 & is & i $\dot{i}$ & $\dot{0}$ & 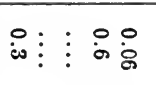 & $\vdots:$ & 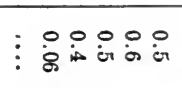 & ळ్ \\
\hline 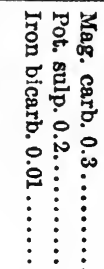 & $\begin{array}{c}\vdots \\
\vdots \\
\vdots \\
\vdots \\
\vdots \\
\vdots\end{array}$ & 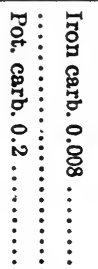 & 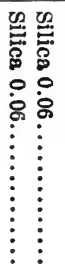 & 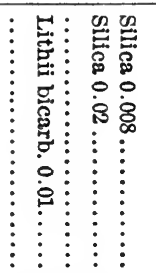 & $\begin{array}{c}\vdots \\
\vdots \\
\vdots \\
\vdots \\
\vdots \\
\vdots \\
\vdots \\
\vdots \\
\vdots \\
\vdots \\
\vdots \\
\vdots\end{array}$ & 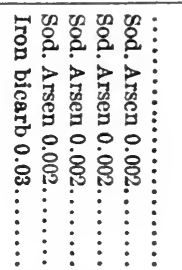 & 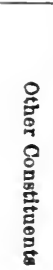 \\
\hline 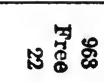 & $\checkmark$ & 농 葛芯 & 莕茄 & 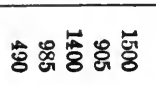 & $\%$ & 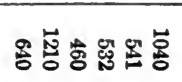 & ถి. \\
\hline
\end{tabular}


Artificial Mineral Waters.-It may be said here in passing that the artificial salts, while not so good as the natural ones, are very useful, especially in practice among the poorer classes. The following are the most important: Bilin, Ems, Fachingen, Friedrichshall, Kissing, Rakoczy, Marienbad, Hunyadi János, Salvator, Tarasp, Vichy, and Wildungen. About a teaspoonful to a tablespoonful of these is dissolved in the desired amount of water (a wineglass to a quart), or in a small quantity of soda water, which is then diluted to the required amount. Sandow's Salts may also be used as a substitute for mineral waters.

Uricedin.-Here may be also mentioned uricedin, a compound of sodium citrate, chloride, and sulphate, and citrate of lithium. One-half to 1 small teaspoonful dissolved in a glass of warm water is given in the morning on an empty stomach, and sometimes once or twice more during the day.

Preparations of Lithium.-In speaking of the use of mineral waters, their content of lithium salts was mentioned, because these salts have a long-standing and deserved reputation as a remedy in nephrolithiasis. Judging from experiment they owe their effect to their solvent action on uric acid, but this action appears problematic during life [probably to their diuretic action (Mendelsohn) - Ed.]. Which salt is given depends on the physician.

Lithium Carbonate.-One of the favorites is lithium carbonate. It is best given as a powder dissolved in a glass of carbonated water (being soluble only with difficulty in ordinary water) 1 to 75 . For example:

R Lithii carbonatis................ $0.10-0.20 \ldots \ldots$ gr. ii-gr. iv

M. fiat chart. Dent. tal. dos. no. $\mathbf{x}$.

Sig. -1 powder, in a wineglass of Seltzer water, 2 or 3 times a day.

Lithium Benzoate, Citrate, and Iodide--Other lithium salts, particularly lithium benzoate and lithium citrate, easily soluble in water, and (rarely) lithium iodide, may be given in this way. The daily dose should not exceed 1 G. (15 gr.), as larger amounts are apt to cause disturbances of digestion. The author personally prefers the citrate. [I usually employ either the benzoate or the effervescing citrate, of which latter there are several good preparations in the American market.-Ed.]

Lithium Carbonate Water.-If we wish to distribute the administration over the whole day, we may use lithium carbonate water, or, better, effervescent lithium carbonate, as follows:

B Lithii carbonatis..................... $0.20 \ldots \ldots$ gr. iv

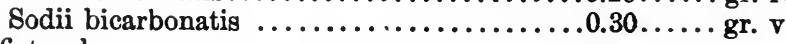

M. fiat pulv.

Sig.-No. 1.

$\mathrm{R} \quad$ Acid. citrici........................ $0.50 \ldots \ldots$ gr. viii

Sig. -No. 2.

Dent. tal. dos. Nos. 1 and 2 no. $\mathbf{x}$.

Sig. -1 of each powder in $\frac{1}{2}$ tumbler of water, 3 times a day, and drink while effervescing. 
Or: B Lithii carbonatis.................... iv

Sodii bicarbonatis.................... $\mathrm{g}$

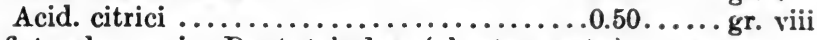

M. fiat pulv. no. i. Dent. tal. dos. (chartæ ceratæ) no. x.

Sig.-One 3 times a day in a tumbler of water, to be drunk while effervescing.-Ed.]

Lithium Waters: Radein, Salvator, Elster.-Lithium is also often given in the form of those natural mineral waters that contain it in considerable quantities. The following are the best: Radein, Salvator, Bonifacius, the Elster Koenigsquelle, and the Nathaliequelle in Franzensbad, [Geneva Lithia, Farmville Lithia, Buffalo Lithia-Ed.]. None of them, to be sure, contains over 0.02 per cent. of lithium. Hence the ordinary quantity of mineral water preseribed (about a quart daily) is hardly enough for its lithium to have any effect. [Theoretically calcium carbonate influences the relations between the monosodium and disodium phosphate in such a way as to enable the urine to retain in solution a large quantity of uric acid without losing its acid reaction. Influenced by J. Strauss and Kionka, Senator recommends a knife point of the salt in Seltzer water several times a day. Kohler recommends magnesix borocitricæ 50 G. $\left(1 \frac{1}{2}\right.$ oz. $)$, sacchar. lactis $10 \mathrm{G}$. $\left(2 \frac{1}{2}\right.$ dr. $)$, ol. limonis 1 G. (15 min.) ; M.S. -1 powder t. i. d. in sweetened water. Senator commends this and also lime water, one-half a teacupful with the same quantity of milk 3 or 4 times a day, especially when there is some catarrh of the passages.-Ed.]

Lysidine.-Next to alkalies and lithium, in the therapy of uric acid diathesis, may be mentioned two relatively new antiuratic drugs, lysidine and piperazine. The first the author cannot recommend. It not only has never done any good in his experience, but, once, after a few days' use, it caused an almost general acute, painful, and partly bullous eczema.

Piperazine.-On the contrary, piperazine (colorless crystals easily soluble in water) seems in some cases to be effective. Its action can be explained only by its power of dissolving uric acid. The author never prescribes it alone, but as follows:

B Piperazini ..................... $1.00-2.00 \ldots \ldots$ gr. $x v-g r . ~ x x x$

Dent. tal. dos. no. $x \nabla$ (dispens. in $\frac{1}{2} 3$ homeo. vials).

Sig.-Dissolve 1 powder in a bottle of Seltzer, Preblau, or soda water, and drink in small quantities during the day.

[Senator recommends 15-20 gr. of urotropine a day, to increase the dissolving power of the urine for uric acid, and " urosin," a combination of quinic acid and lithium citrate, in tablets, 6-10 a day.-Ed.]

Glycerin.-When the concrements have reached the size of real stones, the author finds the use of pure glycerin most beneficial. He has tried it in about 15 cases, in all with remarkable success. He agrees with the originator of this treatment, Herrmann, in attributing this result to 
the fact that the glycerin lubricates the ureters, so that the stones reach the bladder more easily. In one case, about a month after the administration of glycerin, a small uric acid stone about the size of a lentil was passed; in another case, about 20 small stones. It may be prescribed as follows:

B Glycerini,

Succi limonis....................āã $30.00 \ldots \ldots z^{i}$

M. S. -4 tablespoonfuls a day.

Or we may give it in lemonade, $30 \mathrm{G}$. (1 oz.) of glycerin being used instead of sugar to sweeten one quart. The daily dose of glycerin may be as high as $60 \mathrm{G}$. ( $2 \mathrm{oz}$.).

Urea.-Klemperer has recently argued in favor of the use of urea, as the only drug which really dissolves uric acid and has in addition a diuretic effect. He prescribes as follows:

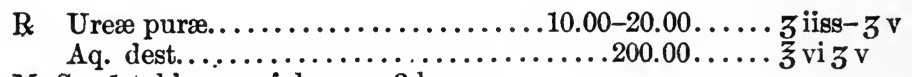

M. S. -1 tablespoonful every 2 hours.

Urea is particularly suited to those cases in which colic or hæmaturia occurs or stones are passed.

Benzoic Acid.-Benzoic acid is often credited with usefulness in uric acid nephrolithiasis. Its action is ascribed by one authority to the fact that it is changed in the kidneys into hippuric acid, and a large amount of hippuric is supposed to mean a small amount of uric acid, this supposition being based on the observation that the urine of herbivorous animals is very rich in hippuric acid and very poor in uric acid. Benzoic acid is also good as an antiseptic where a catarrhal affection of the ureter exists in consequence of the nephrolithiasis. It is prescribed either as sodium benzoate ( $4 \mathrm{G}$. $(1 \mathrm{dr}$.) a day), or as lithium benzoate (see p. 134).

Citric Acid.-Citric acid increases the alkalinity of the blood and hence of the urine. It is best given not as pure citric acid but as lemon juice. The juice from 2 or 3 lemons is taken at intervals during the day. Hot water or hot milk should be taken immediately afterwards. The treatment should last for weeks and months, the number of lemons being gradually raised to 10 or even 20 a day. The author does not approve of this "lemon cure," however, having repeatedly seen it bring on digestive disturbances.

INCREASED INGESTION OF LIQUID.-A final way to prevent the precipitation of uric acid, particularly the fresh precipitation after stones have once been formed, is to increase diuresis by the use of large quantities of milk and water. One or 2 glasses of water, cold, warm, or hot, should be taken upon rising and just before going to bed. Hot water is particularly indicated when there are other signs of a uric acid diathesis, 
such as constipation and hemorrhoids. Alkaline mineral water may be used instead of ordinary drinking water, as may also the various diuretic teas, already mentioned (see p. 32). Dujardin-Beaumetz prescribed the following tea made from herba arenariæ rubræ:

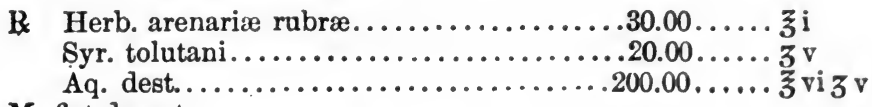

M. fiat decoct.

Sig. -1 tablespoonful every 2 hours.

This prescription is useful because of the benzoic acid contained in the ingredients. [The following prescription is better, as its content of benzoic acid is definite:

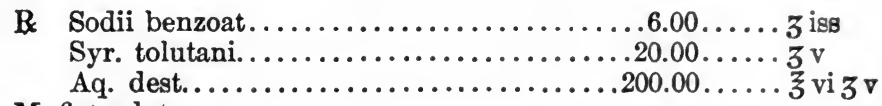

M. fiat solut.

Syr. tolutani.....................20.00.... $\mathrm{v}$

Sig. -1 tablespoonful every 2 hours.-Ed.]

To sum up the treatment of uric acid nephrolithiasis: we prescribe the appropriate diet and alkalies, and, if a stone is surely in the pelvis of the kidney, administer glycerin. The rest of the therapy is secondary.

\section{THERAPY OF NEPHROLITHIASIS OXALURICA.}

Etiology.-The etiology of this form of nephrolithiasis is not very clear, but, so far as we know, it arises from: (1) a general uric acid diathesis; (2) neurasthenia, especially nervous dyspepsia. The last is surely a cause of oxaluria, though the author does not consider it conclusively proved to be the cause of an oxalate stone. On the ground of recent chemical investigations, an alimentary oxaluria is denied. Practically, however, it seems best in both forms to limit the amount of food containing oxalic acid.

If the nephrolithiasis oxalurica springs from a general uric acid diathesis, the therapy is like that of uric acid nephrolithiasis, except that a strongly vegetable diet cannot be given.

When oxaluria, and perhaps the formation of oxalate stones, arise from nervous dyspepsia, our therapy must, of course, be directed against the latter. Hyperacidity of the gastric juice furthers the appearance of oxalates in the urine. For the method of combating hyperacidity, see p. 463.

Dietetic Therapy: Foods Forbidden.-The most prominent point in the dietetic therapy is total abstinence from sugar and starches: sugar itself, all kinds of sweets, fruits, cereals, potatoes, dried peas and beans. The injurious effect of the carbohydrates may be due to the increased acid fermentation they set up in the intestine. 
Foods that must be taken with caution are:

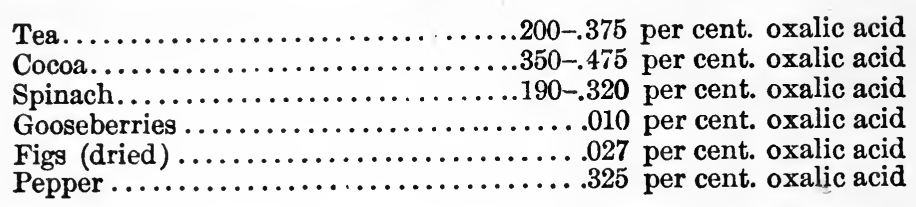

Coffee, chocolate, chicory, peas, beans, beets, [artichokes, rhubarb-Ed.,] and tomatoes should also be taken in small quantities. Beer should generally be forbidden.

The diet should consist in great part of animal food (meat, fish, eggs, fat) and, in addition, the green vegetables not mentioned above. Milk is allowed only in small quantities, but large amounts of water should be taken, in order to flush out the kidneys. [Senator permits apples and allows more carbohydrates; he objects to milk, eggs, and vegetables of the carbohydrate family because of their large percentage of calcium.-Ed.]

Medication.-The medicinal therapy is like that of uric acid nephrolithiasis, alkaline and diuretic. The patient should take at least $2 \frac{1}{2} \mathrm{qt}$. of liquid daily. According to Klemperer, $0.5 \mathrm{G}$. (7 gr.) of magnesium sulphate should be given 4 times a day, to keep the calcium oxalate, as far as possible, in solution. It should be remembered that rhubarb increases the excretion of calcium oxalate in the urine and should, therefore, not be prescribed. [In either of the above conditions, with calculi causing persistent pain, surgery can be resorted to if medicinal and dietetic measures prove unavailing (see below).-Ed.]

\section{THERAPY OF LITHIASIS PHOSPHATURICA.}

Etiology.-The author purposely avoids the term nephrolithiasis phosphaturica, because the phosphate stones (consisting of calcium phosphate and calcium carbonate, or, much oftener, of ammonio-magnesium phosphate) are much more frequently found in the bladder than in the kidneys. They almost always arise from an inflammatory condition of the urinary tract, which may be due either to the presence of calculi of one of the above types or to stagnation and bacterial alkaline decomposition of the urine. Sometimes they are due to an excessive alkalinity of the blood, which may be brought on, for example, by a prolonged administration of alkalies; sometimes to prolonged hyperacidity of the gastric juice. If other calculi-uric acid stones, for example-are the cause, the phosphate is usually in the form of an outer crust around the original stones. In such cases the therapy must be directed against the cause of the latter. Alkalies, however, must not be administered.

Medication: Mineral Acids.-If a primary lithiasis phosphaturica is present, showing itself not by the passing of actual stones but in an abundant excretion of crystals of ammonio-magnesium phosphate and 
calcium phosphate, it indicates urinary stagnation and decomposition and a resulting catarrhal condition of the ureter and bladder. In such cases, beside treating the existing inflammation and stagnation, we give mineral acids to combat the alkaline fermentation. They also appear to be indicated in the cases arising from excessive alkalinity of the blood. To be sure, we must not expect too great results from their use. They are preseribed as follows:

B Acid. phosphor. or hydrochlor. dil....4.00-5.00.... $z^{\mathrm{i}-} \mathrm{z}^{\mathrm{i}} \mathrm{im} \times \mathrm{v}$

Aq. dest........................... 180.00.... Syr. rubi idæi......................... $3^{\text {iv }}$

M. S.-Sufficient quantity for 1 day. A teaspoonful in a glass of water until the whole is taken.

Or the acids may be made into lemonade, insuring the consumption of a considerable quantity of liquid.

B Acid. phosphor. dil...................2.00.... $z$ ss

Aq. communis...................... $900.00 \ldots \ldots$ Oi $\zeta$ xiii

Syr. simplicis...................... $100.00 \ldots \ldots$ iii

M. S.-To be drunk during 1 day.

If hyperacidity of the gastric juice is the cause, hydrochloric acid must naturally be abandoned. Phosphoric acid is hardly advisable. We should rather treat the hyperacidity by atropine or silver nitrate. and also give nervina (see p. 465).

Diuretics.-Diuresis should be furthered by the use of large quantities of milk, water, or simple mineral waters.

Antifermentatives.-If a phosphate stone is due to bacterial decomposition, we must prescribe antifermentatives,-benzoic acid, sodium benzoate, resorcin, sodium borate, and salol (less suitable on account of its inflammatory effect on the urinary passages) :

R Sodii boratis...................1.00-2.00.....gr. $\mathbf{x \nabla - g r . ~} \mathbf{x x x}$

Aq. dest. ........................180.00....

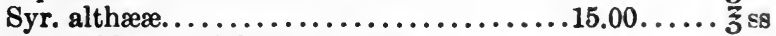

M. 8. -1 tablespoonful every 2 hours.

Or:

B Saloli............................... 0.50.....gr. viiss

Ol. menth. pip............................. q. s. to flavor

M. Dent. tal. cachets no. xv.

Or :

Sig. -1 cachet every 2 hours.

B Resorcinolis......................... $0.50 \ldots \ldots$ gr. viiss

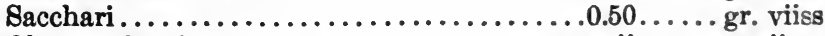

Ol. menth. pip......................gtt. ii.....gtt. ii

M. Dent. tal. cachets no. xx.

Sig. -3 cachets daily.

The author prefers to any of these, urotropine (formin, hexamethylenetetramine) and the nearly related helmitol. According to his experience, they exert the strongest effect in preventing the development of 
bacteria in the urine, owing to the large amount of formaldehyde which they give off. The former is prescribed in $0.5 \mathrm{G}$. (7 gr.) powders $(0.5-1.5$ G. (7-22 gr.) a day) or as follows:

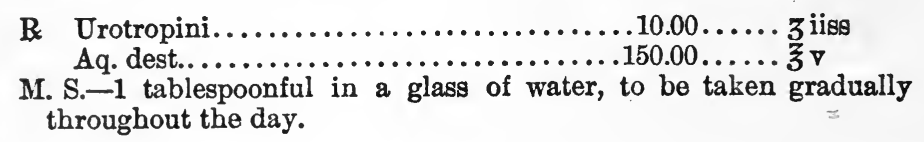

[I usually prescribe $5 \mathrm{gr}$. tablets, 1 to be dissolved in two-thirds of a tumbler of water and drunk slowly immediately after meals.-Ed.]

DIET.-The following foods are permitted: large amounts of meats, of broths, and milk in all forms, sugar, sweets, puddings and cereals, and water; the following are forbidden: fruit, acids [organic-Ed.], beer, wine, and liquors. Green vegetables must be used only sparingly. [The most suitable diet and medicinal therapy to prevent calculus formation, especially of this variety, has not been very accurately determined. Many authorities still prescribe alkaline waters, and sometimes evidently with rcason. At all events, such waters dilute the urine, stimulate diuresis, and flush out the urinary tract. Until we have more definite knowledge, it seems to me wise to treat the faulty condition almost always back of the calculus formation,-i.e., dyspepsia, hyperacidity, and neurasthenia,-and not to outline too strict dietary regulations. I believe that I have so succeeded better.-Ed.]

THERAPY OF THE COMPLICATIONS OF NEPHROLITHIASIS URICA, NEPHROLITHIASIS OXALURICA, AND LITHIASIS PHOSPHATURICA.

Only those complications of nephrolithiasis which can be treated by internal medication will be mentioned here. These include renal pain and colic, renal hemorrhage caused by stone, and inflammation of the urinary tract, particularly of the mucous membrane of the pelvis of the kidney.

Renal Pain.-To begin with the attacks of renal pain. They may assume the form either of (1) relatively slight pain in the region of the diseased kidney, or of (2) pure renal colic. Their therapy must be directed toward (1) the suppression of the pain, and (2) the removal of the cause, that is, the expedition of the passage of the stone.

When there is only a dull pain, we use hot wet applications, warm baths, oil of turpentine, or, especially, preparations of belladonna applied externally, and sodium salicylate given internally, which, by its action on the smooth muscle-fibres, will help to drive out the stone.

$\mathrm{R} \quad$ Linimenti terebinthinæ acetici.........60.00.... 3 ii

Sig. -To be used externally several times daily

$\mathrm{R} \quad$ Ungt. belladonnæ..................30.00.... $z^{\mathrm{i}}$

Sig.-To be applied externally once a day. 
Extract of belladonna may also be given internally, preferably in suppositories.

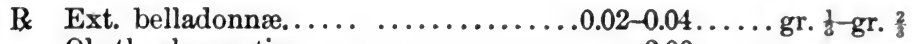

Ol. theobromatis ........................ 3 ss

M. fiat. suppos. Dent. tal. dos. no. v.

Sig. -1 or 2 suppositories a day.

The exit of the stone may also be hastened by increasing diuresis and by the use of glycerin (see p. 137, and particularly p. 135).

Renal Colic.-If the pain increases more or less suddenly to an excruciating intensity, with severe vomiting and symptoms similar to those of intestinal obstruction, or with a desire to pass urine and feces, the above mentioned measure will generally be ineffective. [Hot baths are sometimes very serviceable.-Ed.]

Morphine-Chloroform.-The colic pain is best controlled by subcutaneous injections of morphine, $0.01-0.02 \mathrm{G}$. ( $1 / 6-1 / 3 \mathrm{gr}$.), or, if there is no vomiting, by opium (internally or in suppositories), chloroform (internally), or chloral hydrate (internally or by rectum). The following prescriptions may be employed:

B Opii pulv. .......................... $0.05 \ldots \ldots$ gr. $\frac{3}{4}$

Ol. theobromatis...................... $1.00 \ldots \ldots$ gr. $x v$

M. fiat. suppos. no. i. Dent. tal. dos. no. v.

Sig. -1 suppository every 2 hours if necessary.

Or :

R Ext. opii...................... $0.05-0.10 \ldots \ldots$, gr. $\frac{3}{4}$-gr. $1 \frac{1}{2}$

Ext. belladonnæ................. $0.02-0.04 \ldots \ldots$ gr. $\frac{1}{3}-$ gr. $\frac{2}{3}$

Ol. theobromatis ............................ xv

II. fiat. suppos. no. i. Dent. tal. dos. no. x.

Sig. -1 suppository every 2 hours if necessary.

Or :

Ix Chloralis hydratis....................4.00.... $z^{\text {i }}$

Mucil. acaciæ ................... $100.00 \ldots \ldots$ iii 3 iiss

M. fiat. mist.

Sig.-Sufficient for 2 enemas.

[If an attack of renal colic is not relieved by a prompt immersion in a hot bath, $105^{\circ}-115^{\circ}$, for a half hour, I always employ a hypodermic of morphine, gr. $1 / 4-1 / 2$, and atropine, gr. $1 / 150^{-1 / 60}$. If this does not succeed, I repeat the morphine within an hour. Failing to relieve the pain, I attempt light chloroform anæsthesia. I rarely use suppositories or other medication. I consider it essential to watch the urine very carefully, so that sand, gravel, or stone may be collected and then examined. Our future treatment will depend upon the result of this examination, especially of the nucleus of a stone.-Ed.]

The colic is often accompanied by a severe reflex paralytic meteorism. Such cases are often relieved by an irrigation (e.g., chamomile tea, sometimes with the addition of $50-100 \mathrm{G}$. (11/2-3 oz.) of infusum sennæ compositum).

Renal Hemorrhage.-Bleeding, like the severe pain, is often caused by the rough surface of oxalate stones. It is of two grades: (1) a simple 
admixture of blood in the urine, (2) abundant renal hemorrhage. In the first case, the patient must be kept quiet and put on a milk diet with acid drinks. In the second, he must be kept absolutely still in bed, put on a milk diet, and his bowels be made to move freely. To stop the hemorrhage, we may employ fluidextract of ergot or ergotine subcutaneously.

One complication of the nephrolithiasis remains, pyelitis and pyolonephritis calculosa. But as the treatment of these conditions is, in general, the same, no matter what their etiology, they will be treated together in the next section.

\section{THERAPY OF PYELITIS AND PYELONEPHRITIS.}

Etiology.-Pyelitis is either a mucopurulent, a purulent, or, sometimes, a hemorrhagic inflammation. It is sometimes chronic, sometimes acute. Rarely it is a primary affection, like an acute infection, or a consequence of trauma. Generally it is secondary, due to an ascending inflammatory or suppurative process arising from the urethra, bladder, or, through these, from the female genitalia. It also accompanies other primary affections of the pelvis of the kidney, such as stone, and more rarely, new growths, and tuberculous and syphilitic disease of the kidney. The suppurative process may spread from the pelvis of the kidney to the parenchyma, just as inflammation of the kidney may spread to the pelvis. The pyelitis and pyelonephritis accompanying severe infectious diseases like smallpox are of only incidental interest.

Symptomatic Therapy.-Naturally, our therapy must as far as possible be causal, and therefore not properly to be discussed in this section.

Revulsives, etc.-The symptomatic therapy relieves the inflammation by local revulsives, preferably by leeches placed upon Petit's triangle, that is, over the middle of the iliac erest between the external oblique and the latissimus dorsi muscles, for the veins of this region communicate with those of the renal capsule. Excretion through the intestines should be secured to the fullest extent, in order to relieve the congested kidney, and purgatives should be employed to free the intestine of toxins and so avoid their excretion through the kidney, with its consequent irritation. The author advises vegetable cathartics or a combination of vegetable and mineral rather than the latter alone.

Of course, an exclusively milk diet is prescribed. The pain is to be combated, as in renal colic, by belladonna or opium (see p. 141).

Medication: Antiseptics.-The medicinal treatment for both acute and chronic forms is directed toward (1) reducing the inflammation, and (2) diluting the urine. The local inflammation is treated directly by antiseptics. Urotropine (see p. 149) and helmitol occupy the first place, benzoic acid and sodium benzoate the second. The first two should, however, be confined to cases where the reaction of the urine is alkaline. [Since the introduction of urotropine I have rarely used other remedies, and have succeeded with it even when the urine was acid. I have never met with unpleasant effects. Dr. Lewis Stimson told me of two cases in 
which 30 gr. a day caused marked hæmaturia. One of my patients has taken $15 \mathrm{gr}$. a day for over two years nearly continuously. She has a chronic pyelocystitis (colon infection). When she discontinues the drug the urine becomes slightly turbid and burns during micturition. When urotropine fails, Forchheimer recommends a capsule containing 11/2-3 gr. of methylene blue with enough grated nutmeg to prevent strangury, 3 times a day.-Ed.] Benzoic acid may reach a daily dose of 1-2 G. (15-30 gr.).

B Acid. benzoici $.0 .10-0.30 \ldots . .$. gr. ii-gr. $\nabla$

Fiat tabulla. Dent. tal. dos. no. xv.

Sig. -1 tablet every 2 hours.

Or 1-1.5 G. (15-20 gr.) of benzoic acid may be dissolved in 950 c.c. (a scant quart) of warm water, and $50 \mathrm{G}$. (11/2 oz.) of aqua cinnamomi added after cooling. This acid drink should be taken little by little throughout the day.

Sodium benzoate is not so apt to produce a slight renal irritation as is benzoic acid. Four G. ( $1 \mathrm{dr}$.) should be the maximum daily dose, as large amounts are apt to cause disturbances of digestion. To obviate this, it is best given in pills:

R Sodii benzoatis....................... $4.00 \ldots \ldots 3^{\text {i }}$

Ext. gentianæ ............................

M. fiat pil. no. xxx.

Or:

Sig. $-3-4$ pills every 2 hours (i.e., from 8 A. M. to 8 P. M.).

B Sodii benzoatis....................... $4.00 \ldots \ldots 3^{\mathrm{i}}$

Syr. rubi idæi,

Aq. menth. pip................. āā $25.00 \ldots \ldots z$ vi

M. S. -1 tablespoonful an hour after breakfast, 2 hours after the midday meal, and 2 hours after the evening meal (i.e., at the height of digestion) in a small cup of tea made from buchu leaves or rose leaves.

Sodium salicylate, salol, copaiba balsam, and eucalyptus oil are not advisable for prolonged use ; first, because they too often bring on disturbances of digestion (loss of appetite, burning, nausea, vomiting), and second, because taken in large quantities for a considerable length of time, they irritate the kidneys. Some empyreumatic oils are better, as oil of cade, juniper oil, and oil of birch. Oleum Haarlemense, made from equal parts of oil of cade and oil of juniper berries, is the author's favorite:

B Ol. Haarlemensis............................

Dent. tal. dos. no. xx. ad caps. molles.

Sig. $-3-4$ capsules daily.

Sodium borate is often used as an antifermentative.

Astringents.-A second group of drugs used to reduce the inflammation is the astringents (sodium tannate, tannigen, tannalbin, preparations of krameria, catechu, alum, iodotannic syrup). They should, however, 
be used interchangeably with the antiseptics or balsams, and laxatives. [Senator recommends as an astringent, lime water in 2-3 oz. doses 3-4 times a day, diluted with an equal quantity of milk. He also uses the following pill:

B Plumbi acetatis, Opii pulv........................ āā $2.00 \ldots \ldots$ gr. xxx

Ext. glycyrrhizæ...........................

M. fiat pil. no. $\mathrm{xxx}$.

Sig. $-1-2$ pills $3-4$ times a day. $-E d$.

Krameria may be given as a decoction:

B Krameriæ....................15.00-30.00..... $\xi_{\mathrm{ss}-\xi_{\mathrm{i}} \mathrm{i}}$

Syr. krameriæ......................30.00.... $\mathrm{z} i$

Aq. cinnamomi...................180.00.... 3 vi

M. fiat decoc.

Or :

Sig. -1 tablespoonful every 2 hours.

B Ext.krameriæ....................1.00-2.00.....gr. xv-gr. xxx

Sacchar. lactis.......................0.50.....gr. viii

M. fiat caps. Div. in dos. æq. no. $\mathbf{x}$.

Sig. $-3-5$ capsules daily.

Or as pills :

R Ext. krameriæ $\ldots \ldots \ldots \ldots \ldots \ldots \ldots \ldots \ldots, \ldots, \ldots \ldots \ldots 3^{\mathrm{i}}$

Ext. gentianæ .................................

M. fiat pil. no. $\mathbf{x x}$.

Sig. $-5-7$ pills daily.

Or :

B Tinct. krameriæ,

Tinct. cinnamomi................āa $10.00 \ldots \ldots z$ iiss

M. S. - 30 drops in oatmeal gruel every 2 hours.

Catechu is given as such or in the tincture, in which case it is prescribed exactly as is the tincture of krameria, 20-30 drops a day.

R Gambiris......................0.50-1.00.....gr. viii-gr. $\mathbf{x \nabla}$

Sacchar. lactis..................... $0.40 \ldots \ldots$ gr. vi

M. fiat caps. no. i. Dent. tal. dos. no. xv.

Sig. -1 capsule every 2 hours.

Alum is prescribed in the form of powders up to 3-5 G. (45-75 gr.) a day.

B Alumenis,

Acaciæ.........................āā 0.20......gr. iii

M. fiat caps. no. i. Dent. tal. dos. no. $x \mathbf{~}$

Big. -1 capsule every 2 hours.

Diuretics.-The second indication, the dilution of the urine, is brought about by the ingestion of large quantities of fluid (see p. 104).

If nephrolithiasis is the cause of the pyelitis, we employ, of course, appropriate mineral waters (already mentioned), provided the reaction of the urine does not contraindicate them. But if the pyelitis arises 
from other causes, it is best not to make use of waters (Carlsbad or Vichy) containing large quantities of minerals. Springs poor in alkalies (bicarbonate of soda, etc.) are better (Seltzer, Giesshübl, Krondorf, Neuenahr, or Rohitsch). The earthy waters seem especially useful, as Driburg (Hersterquelle), Lippspringe (Arminiusquelle) in Westphalia, Leuk (Canton Valais), Contrexéville (Dept. of the Vosges), Evian (south shore of the Lake of Geneva), and Wildungen. All these are cold springs, with the exception of the hot springs at Leuk $\left(122^{\circ}\right)$. Of the Wildungen waters, the Helenenquelle, which is very rich in sodium bicarbonate, is preferable where the urine has a strongly acid reaction; the Georg-Victorquelle, when the urine is neutral or alkaline and ferments rapidly. If these earthy waters are drunk at home, a glassful sliould be taken in the middle of the morning, another in the middle of the afternoon, and two glasses should be sipped for a half hour just before going to bed. If the patient is much run down, showing emaciation and pallor, chalybeate waters are indicated (as in chronic interstitial nephritis). A combined cure is often very successful, the patient taking the waters (11/2-2 pt. a day) first at one Spa and then at another, at Carlsbad for instance, and then at Franzensbad or Schwalbach. ${ }^{3}$

Diet.-The diet in general is the same as in Bright's disease. In acute pyelitis an exclusively milk diet should be tried.

Baths.-The patient should be carefully guarded from catching cold and should take baths at about $95^{\circ}$ two or three times a week.

Indications for Surgical Interference.-If the therapy fails and the inflammation spreads into the region around the kidney, or if pyelonephrosis occurs, recourse must be had to operation, nephrotomy or nephrectomy. Before the latter is attempted, however, it should be proved conclusively that the other kidney is functionating properly.

Casper has recently advocated washing out the pelvis of the kidney in female cases with antiseptic fluids by passing a catheter into the ureter. The author has had no personal experience with the method. [Ayer has reported success with this method. It does not appeal to me.-Ed.]

Of the other renal diseases, some (renal hæmophilia, chyluria, renal neuralgia) are too rare to require discussion, and others (neoplasms, echinococeus, tuberculosis) belong to the domain of surgery beyond the point at which their therapy ceases to be purely symptomatic; up to this point it would agree with that just given for pyelitis. The only one left to be treated here is movable kidney.

\section{THERAPY OF MOVABLE KIDNEY.}

Rest and Support.-If the patient's severe discomfort and pain have not made nephrorrhaphy seem nécessary, the therapy consists first of

' [All the tables of waters in this book were compiled largely from "Haggard's Handbook of Climatic Treatment" and Crook's "Mineral Waters of the United States."-Ed.] 


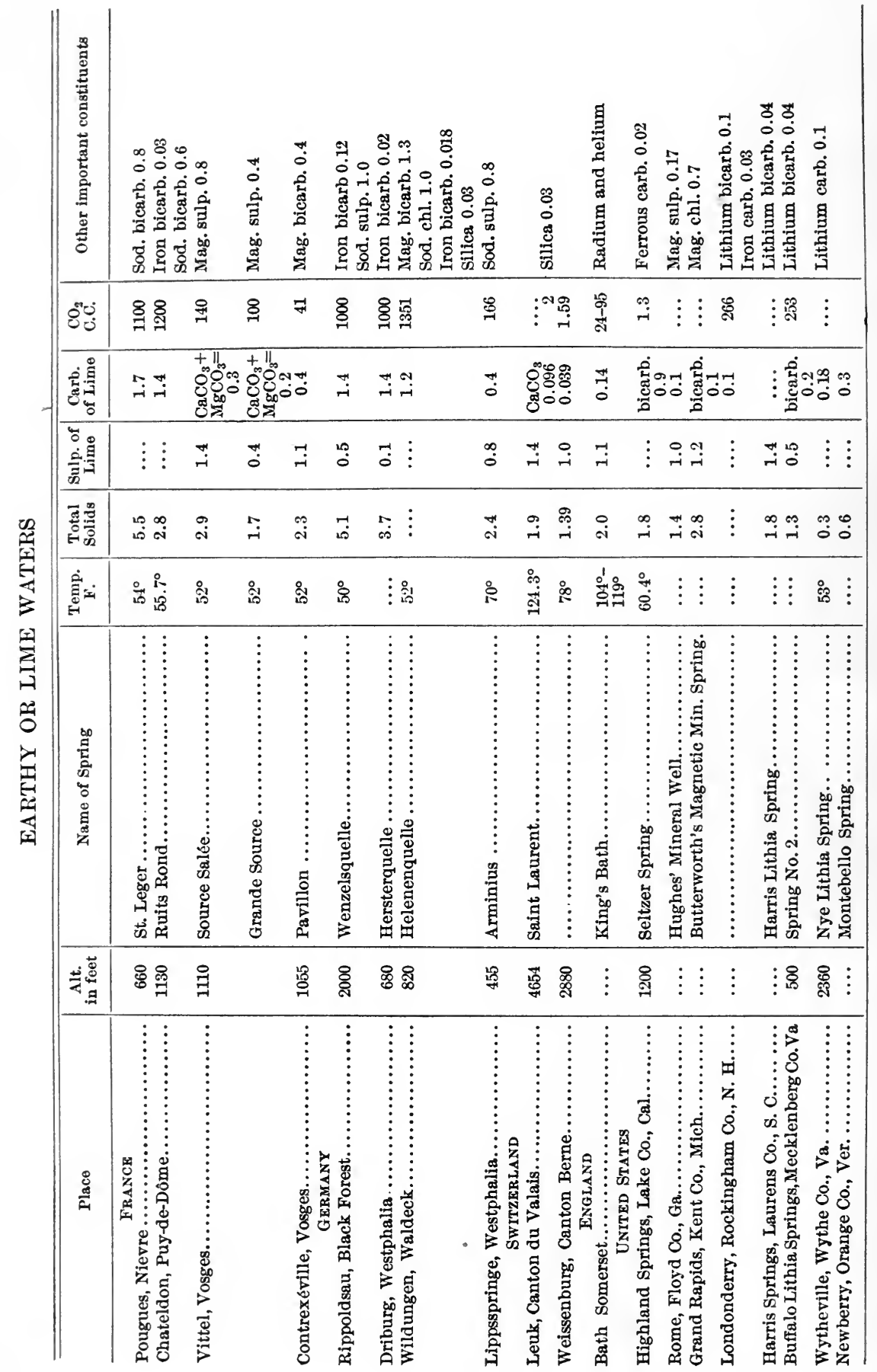


all in keeping the patient flat on his back for a long time, and secondly in proper bandaging. The bandage should press the contents of the abdomen upward evenly from the brim of the pelvis and hold the kidney in place. When the kidney is plainly palpable and can be put back into its natural position, it can sometimes be kept there by means of a specially adapted pad. [A properly fitting bandage is not easy to secure, especially for very thin patients. I rarely use a pad, but attempt to secure the retention of the kidney by a well fitting, long, low corset, or, in more difficult cases, by an elastic bandage encircling and sustaining the lower two-thirds of the abdomen. The physician must carefully examine such contrivances after being applied and worn by the patient. Of course, the success depends upon the amount of associated splanchnoptosis and the dyspepsia, neurasthenia, etc. No case should be operated upon, in my judgment, until every possible medical measure has been thoroughly tried.--Ed.]

Massage-Rest Cure.-Massage is often recommended, but, in the author's opinion, does far less by toning up the relaxed suspensory apparatus and making the fatty capsule snugger, than by relieving the frequent secondary nervous symptoms. A rest cure (see p. 387) is indicated in every case, as it relieves and often removes the distress. Gymnastics are also useful. [Unless the patient is aware of the movability the physician should not disclose the fact. Many a person is made hypochondriacal by such knowledge.-Ed.]

\section{THERAPY OF CYSTITIS.}

Acute Cystitis.-The therapy of acute eystitis is for the most part surgical. If we are obliged to treat cystitis medically, we look first for its cause. It is often secondary to diseases of the urethra, the prostate, or the bladder itself. Sometimes, as in acute gonorrhœal cystitis. loeal therapy is contraindicated; sometimes, as in stricture of the urethra, it is directly indicated. For the eystitis itself, whether it is idiopathic or secondary, we prescribe absolute rest in bed and an even temperature.

Diet.-The diet should consist almost exclusively of milk or its substitutes. Mineral waters poor in alkalies (e.g., Giesshübl, Seltzer) may be added, and sometimes nutrose or sanatogen; ordinary water or Russian tea may also be permitted. When the acute symptoms have subsided, solid food, without condiments, may be cautiously allowed. Alcohol must be absolutely prohibited, and also all drugs which irritate the bladder (cantharides, copaiba balsam).

Catharsis-Stupes.-The bowels should be kept open, if necessary by the help of oil or warm water enemas. Warm applications are made over the symphysis. The cloths may be wrung out in hot water and covered with rubber cloth and a broad flannel band; or we may use warm flaxseed poultices, compresses wrung out in hot chamomile tea, or hot flannel bandages. A few patients prefer ice-bags. 
Narcotics.-If these do not relieve the pain, we must have recourse to narcotics. We may inject into the skin of the abdomen $0.01 \mathrm{G}$. ( $1 / 6 \mathrm{gr}$.) of morphine hydrochlorate or we may use morphine and cocaine suppositories; for example:

B Morphinæ hydrochloratis............0.05-0.10.....gr. $\frac{3}{4}-$ gr. $1 \frac{1}{2}$

Cocainæ hydrochloratis............. $0.10-0.30 \ldots \ldots$ gr. $1 \frac{1}{2}$-gr. $4 \frac{1}{2}$

Ol. theobromatis............................ q.

M. fiat suppos. no. $\mathrm{x}$.

Sig. $-1-2$ suppositories a day.

Opium or morphine may be given internally, somewhat as follows:

B Morphinæ hydrochloratis................0.02....gr. $\frac{1}{3}$.

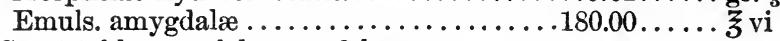

M. S. -1 tablespoonful every 2 hours.

Leeches.-If the pain and inflammation are both exceedingly intense, we may have to apply leeches, 8-10 on the perineum.

Baths.-If there is a strong desire to urinate, sitz baths or tub baths at $90^{\circ}-95^{\circ}$ are advisable. They should last at first only half an hour, but the time may be prolonged to an hour or even 2 hours. Many authorities object to baths on account of the necessary movement and stimulation, and prefer to use a soft eatheter not oftener than twice a day.

Mineral Waters.-As in pyelitis, the proper mineral waters are only the weakly alkaline or chalky waters (Wildungen, Contrexéville, Giesshübl, Seltzer, [Poland, Bethesda, Hygeia-Ed.] ). A pint and a half to a quart may be taken daily, always in small amounts and preferably warmed. If they increase diuresis too much and so irritate the bladder, they must be instantly stopped.

Chronic Cystitis: Diet.-The chronic condition, like the acute, demands an absolutely non-irritant diet. If the urine is not alkaline, alkaline or muriatic alkaline waters may be used (Preblau, Ems, Bilin, Giesshuibl, Seltzer, Fachingen). If the reaction is alkaline, especially if there is abundant secretion of mucus, the earthy mineral waters are best. They may owe their action to the formation of an easily soluble (and hence easily excreted) compound from the combination of their calcium with the mucus (Kisch). A liberal use of raw or cooked fruit does many patients good. In this condition a grape-cure may be recommended.

Baths.-The patient may take a course of treatment at the baths already mentioned, or at Vichy or Carlsbad.

Climate.-The patient should guard against taking cold. A warm, even temperature is best (Cairo, Pisa, Catania, Riviera di Ponente, [Southern California, Florida-Ed.]). Riding and long drives or railroad journeys are to be avoided.

Antiseptics.-Certain drugs may be given internally, to be excreted through the kidney and so to disinfect the bladder. Potassium chlorate 
is one of them, although the author does not like to use it on account of its destructive effect on the blood corpuscles. [Nor do I ever recommend it.-Ed.]

B. Potassii chloratis .................3.00-4.00.....gr. xlv-gr. lx

Syr. rubi idæei....................... $15.00 \ldots \ldots \xi_{3}$

Aq. dest. ....................... $180.00 \ldots \ldots$ z vi

M. S. -1 tablespoonful every 2 hours. (Never on an empty stomach.)

Or, if there is marked ammoniacal fermentation:

B Sodii salicylatis ...................... $0.60 \ldots \ldots$ gr. $x$

M. fiat cachet no. i. Dent. tal. dos. no. xx.

Sig.-Up to 10 cachets a day may be taken.

Salol is frequently used.

The author has had marked success with urotropine and helmitol (0.5-1.5 G. (7-22 gr.) a day), and prefers them to all other antiseptics (see p. 142).

Astringents.-Astringents are not of so much value, but folia uvæ ursi may be mentioned. It is best given as a tea, $10-15 \mathrm{G} \cdot(2 \mathrm{r} / 2-4 \mathrm{dr}$.) in about a quart of water, to be taken at different times during the day. But it may also be given as a decoction:

B Fol. uvæ ursi.................10.00-15.00.... $z$ iiss -3 iv

Aq. dest. ........................... $180.00 \ldots \ldots$, vi

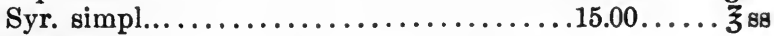

M. fiat decoc.

Sig. -1 tablespoonful every 2 hours.

If there is a decided purulent secretion, resins may be given; e.g., oil of turpentine, pix liquida, or copaiba balsam:

B Ol. terebinthinæ rectificat.............. $0.30 \ldots \ldots, m_{\mathrm{v}}$

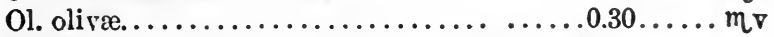

M. fiat caps. (molles) no. i. Dent. tal. caps. no. xv.

Sig.-Up to 5 capsules a day may be taken.

Or :

B Picis liquidæ..................0.05-0.10.... mi-mii

Dent. tal. caps. (molles) no. xv.

Sig. -1 or 2 capsules 3 times a day.

Or :

B Picis liquidæ............................. $\eta_{x \geq x}$

Ceræ albæ $\ldots \ldots \ldots \ldots \ldots \ldots \ldots \ldots \ldots \ldots, 1.00 \ldots \ldots$ gr. xv

Magnesii carbonatis........................ q. 8.

M. fiat pil. no. $\mathbf{x x x}$.

Sig. -2 pills 3 times a day.

Or :

B Copaibæ.......................... $0.50 \ldots \ldots$ m viii

Dent. tal. caps. (molles) no. xxx.

Sig. -2 capsules 3 times a day. 


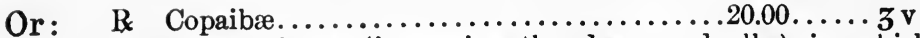

Sig. $-20-40$ drops (increasing the dose gradually) in whiskey or Madeira.

Catheterization-Irrigation.-A very important practical point is the method of emptying the bladder in case the patient cannot do so himself, or is only partially able to do so. A soft Nélaton eatheter, No. 10-12 F., should be disinfected with a 5 per cent. iodoform oil solution. [I usually boil the catheter to insure asepsis, and lubricate the same with one of the more modern sterile preparations, such as lubrichondrin.Ed.] After it has been passed and the bladder emptied, we inject by means of a syringe, irrigator, or funnel a disinfecting solution at $100^{\circ}$. The author recornmends for this purpose a physiological (0.6 per cent.) solution of common salt and boric acid if the secretion is mucous, and a 4 per cent. solution of boric acid if it is purulent. Not more than $150 \mathrm{G}$. $(4 \mathrm{~T} / 2 \mathrm{oz}$.) should be used, for fear of rupture of the bladder. It should be injected until a resisting pressure or pain is felt, and then be allowed to escape through the catheter. This process is repeated till the returning fluid is clear; some of it is then left in the bladder. The patient should be half raised, with his hips supported on a cushion.

Silver Nitrate.-Silver nitrate is peculiarly valuable, as it has an antiseptic and at the same time a directly healing effect on the diseased mucous membrane. Kaufmann gives the following directions for its use: The bladder should be washed out with warm boric acid solution and sompletely emptied. The catheter is then partly withdrawn, so that the injection may reach the pars prostatica. A solution of 1:2000-1:500 of silver nitrate (always beginning with a weak solution) is then injected and allowed to flow out spontaneously after two or three minutes. With sensitive patients this is followed by a physiological salt solution. In 2 or 3 days the operation is repeated with a $1: 1000$ solution. Morphine subcutaneously or in suppositories is used to allay any symptoms of inflammation. The time between the applications is later increased. The duration of the treatment is decided by the grade of inflammation, the effect on the frequency of micturition, the pain, and the condition of the urine. [The albuminous silver compounds, argyrol and protargol, have largely supplanted the nitrate. They are less liable to cause an unpleasant reaction.-Ed.]

Therapy of Paralysis of the Bladder.-If paralysis of the bladder is present, whether complicated by cystitis or not, it is most important to make sure that the bladder is emptied every 4-6 hours. Electricity may also be tried. If cystitis is not present, simple manual-expression of the urine should be attempted before proceeding to catheterization. This may frequently be done if the paralysis is of central origin (sphincter paresis). The physician sits by the bedside, facing the patient, with the hands laid over the symphysis, the thumbs directly over it and the fingers grasping the bladder from above. He then presses downward with the fingers so that their tips approach the positions of the thumbs. 
If the bladder is partly emptied, he must take a fresh grip, pressing the fingers downward and inward. The process may be carried out by the patient himself, and is quite safe. It is therefore to be preferred to catheterization, unless the paralysis is complicated by cystitis.

The genito-urinary diseases not already discussed belong to the domain of the surgeon or the specialist rather than to that of the general practitioner of internal medicine. 


\section{III.}

\section{THERAPY OF DISEASES OF THE BLOOD}

Clinically, diseases of the blood may be divided into two groups: (1) those in which there are qualitative changes in the composition of the different constituents of the blood, and (2) those in which it is merely or principally the quantity of blood that is abnormal. The two groups, however, cannot be sharply distinguished, for both qualitative and quantitative changes are found from the beginning to the end of some diseases of the blood and during certain phases of other diseases. With this reservation we may tabulate the more common diseases of the blood as follows:

\section{Group 1:}

(a) Protopathic or primary anæmia: Chlorosis, pernicious anæmia (generally), leukæmia, pseudoleukæmia.

(b) Deuteropathic, symptomatic, or secondary anæmia.

\section{Group 2:}

(a) Anæmia in which all the blood constituents are reduced proportionately (typical form: acute anæmia from hemorrhage).

(b) True plethora, a condition in which all the blood constituents are proportionately increased.

\section{THERAPY OF CHLOROSIS.}

All cases of false chlorosis will be excluded from the discussion; e.g., those arising from congenital smallness of the heart or vessels, from an early stenosis of the mitral orifice, or from Bright's disease.

When we are confronted with a true case of chlorosis, or anæmia during puberty, of known or unknown origin, our therapy must be both hygienic and medicinal.

Hygienic and Dietetic Therapy.-The hygienic therapy must begin with the diet. Here we must be ruled by the circumstances of the case. Sometimes the patient will have a good appetite, and very slight, if any, digestive disturbances. In other cases, there are present typical symptoms of dyspepsia (anorexia, sensation of pressure or heaviness after eating, cardiac distress). In such cases we must make the diet and the auxiliary medication suit the condition diagnosed as the cause of the dyspepsia. 
Hypochlorhydria.-In many cases of chlorosis this condition is one of simple hypochlorhydria. Here hydrochloric acid may be given, somewhat as follows:

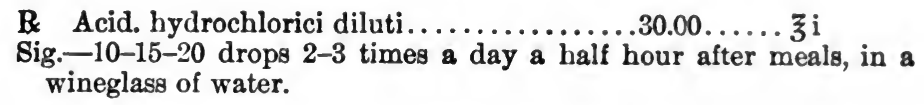

Hyperchlorhydria.- Sometimes exactly the opposite condition is the cause of the dyspepsia,-hyperchlorhydria. In that case, rather large quantities of alkalies should be given several times a day. (For a fuller discussion, see p. 417, etc.) We may prescribe 0.3-2.0 G. (5-30 gr.) per dose of bicarbonate of soda in powder or tablets, or as soda-mint tablets, or lime water diluted with equal parts of plain water, broth, or milk.

Alkaline mineral waters are also available. We may give $50-70$ c.c. (6-12 oz.) of warm Carlsbad Mühlbrunnen on an empty stomach often warm, but if so indicated, cold. [There seems to be a more or less common impression among many physicians that Carlsbad water is very laxative and depleting. At Carlsbad I found to my surprise that the physicians there were obliged to order cathartics for many patients, in addition to the colder waters. One or 2 glasses of the hotter springs is but very slightly laxative to ordinary people.-Ed.]

Anorexia.-If loss of appetite is the principal symptom, 1-2 glasses (6-12 oz.) of warm Carlsbad Mühlbrunnen on an empty stomach often proves beneficial. We may also employ stomachics and hydrotherapy (see p. 156).

Gastric and Intestinal Atony.-Atony of the intestinal musculature is quite often at the root of the digestive disturbances. This hardly needs any other therapy than the choice of such foods as will arouse peristalsis or encourage the flow of liquid into the intestine (fresh fruit, green vegetubles, graham bread, honey).

If atony of the stomach is present, the food should be so chosen as not to overload the stomach, and the extract or tincture of nux vomica should be given (see p. 420).

In case of hyperæsthesia of the mucous membrane of the stomach, belladonna, chloroform water, or cocaine hydrochlorate may be given internally (see p. 430).

Pica Chlorotica.-We often see cases of chlorotic girls who have an abnormal longing for some particular article of diet, as acid, sweet, or salty foods. It is not best to refuse entirely the gratification of these tastes; for, in the first place, other food is taken more willingly if accompanied by the favorite food, and, in the second place, it may well be in many cases that this peculiar longing is not a senseless whim, but an instinctive effort of nature to correct some abnormal condition of the digestive organs. For example, may not the desire for sweets and acids (salad) be perhaps caused by intestinal atony, the desire for salty food by lack of hydrochloric acid, the desire for chalk by hyperacidity, and 
the desire to chew or suck coffee beans by a decreased secretion of saliva or by the need of a cardiovascular tonic? The longing for wine may also be due to nature's need of a heart stimulant. Its gratification would be more rational therapeutics than is the giving of wine, as is often done, from the mistaken notion that red wine will make red blood. The author, therefore, believes in giving way to these individual longings for certain foods in so far as their gratification is not directly contraindicated by the disease itself. [I do not believe in prescribing acid for hypoacidity and alkalies for hyperacidity in any such routine fashion to chlorotics with symptoms of dyspepsia, or in promptly attacking symptoms, such as hyperæsthesia of the gastric mucous membrane, by this or that drug. We have a much more important indication to work upon, the diseased condition at fault, which can be improved only by careful general measures. Nor can I agree with the author in humoring the abnormal tastes these patients so often exhibit, upon the assumption that they may be dictated by the organism's need for the food craved. I do find two indications for direct symptomatic treatment based upon the blood coagulability. Thrombosis and hemorrhage are frequent enough in chlorosis to warrant a very careful study of the coagulability of the blood in every case, and a prompt administration of 10-20 gr. of calcium lactate 3 times a day, to prevent hemorrhage, or, on the contrary, of the juice of 1 or 2 lemons a day when the blood coagulates very rapidly. During the administration of calcium salts, the coagulability should always be studied, as large doses or prolonged administration sometimes decrease the coagulability (Nat. Dispensatory).-Ed.]

If no marked symptoms of dyspepsia are present, so that it is not necessary to restrict the food either in quality or in quantity, a generous diet should be given, based on the following principles (von Noorden) :

Diet.-It is well known that proteids are quickly absorbed when administered upon an empty stomach and enter into the circulation at once, while carbohydrates, although they are absorbed just as quickly, are deposited for some time in the liver. Fats, on the contrary, are absorbed very slowly. In consideration of these facts, it is evident that breakfast should be rich in proteids.

Before rising, the patient should take 2 glasses of milk, sipping it slowly during 15-30 minutes. This may, however, be omitted, especially if the physician finds that it interferes with the taking of the later breakfast. The patient should remain in bed half an hour after the milk is finished, then rise, take a dry rub-down or sponge-bath, dress, and have breakfast. This should consist of a small cup of tea with cream, 1 or 2 slices of buttered toast or dried bread, and as much meat as possible (at least 50-100 G.-11/2-3 oz.). This may be hot, cold, boiled, or roasted, or scraped raw meat, or easily digestible fish. It is highly important to get as great a variety as possible. Caviar or sardines may often be advantageously given first as an appetizer. [They would not stimulate the appetite of most American girls in the early morning.-Ed.] It is 
well, especially if the patient is thin, to give a glass of sherry, or, better, ale, porter, or Pilsener beer, along with the breakfast [or some malt preparation, beef and wine, or panapeptone-Ed.]. About $2^{1} / 2$ hours later the patient should have some bread and butter with 2 soft-boiled eggs, followed soon by a glass of milk. For the rest of the meals, more may be left to individual choice. In general, however, the mid-day meal should consist principally of meat with easily digestible vegetables, the afternoon lunch of bread or zwieback with fresh or cooked fruit or cocoa or tea, and the evening meal of simple food (eggs in some form, cheese, butter, meat, broth, or beer). Juicy, raw meat, unless the patient has an aversion to it, is a rational article of diet for a chlorotic, on account of the large amount of hæmoglobin, and consequently of iron, contained in its abundant blood content. Raw beef, ham, and game, are, therefore, exceedingly suitable for chlorotic patients.

Under one condition the above arrangement of meals may be changed. If the oversensitive stomach cannot stand so much food at a time, lighter meals may be given every hour, consisting alternately of liquids and of solids, chosen from the foregoing list. But if marked œdema has developed, the amount of fluids must be cut down. [I have recently cut down the amount of salt in the diet if œdema is present, and if at the same time the blood coagulates slowly, I prescribe calcium lactate.-Ed.]

Rest.-Although it used to be the practice to advise chlorotics to take a great deal of exercise, especially in the open air, it is now well known that what they need is rest (Oppolzer). The reason for this is evident when we remember that every bit of muscular work means the breaking up of red corpuscles. This is the explanation of the frequent occurrence of urobilin in the urine of chlorotic patients after exercise, and its disappearance when they are kept in bed. The urobilin is merely a proof of the destruction of hæmoglobin in the organism. The almost universal complaint on the part of chlorotic girls of weariness and of excessive fatigue after even a slight exertion is also to the point. In a light case, therefore, the patient should never exercise to the point of fatigue and should rest the greater part of the day; she may drive with advantage. In a severe case (noticeable dilatation of the heart-chambers, visible stasis in the cervical veins, pronounced œema) we must insist on absolute rest for $3-\mathbf{5}$ weeks. Of course, the diet must be somewhat cut down from that mentioned above. For the first week the patient should be in bed, and for the next 2 or 3 weeks, on a rolling couch or a hammock in the open air if the climate permits. If it is an unfavorable time of year for this, it is a good plan, though not absolutely necessary, to send the patient to a southern, inland climate, provided this can be easily and comfortably done [Sonthern California, Carolina, Georgia. Good medical care should be assured-Ed.]. In case the nervous symptoms predominate, the author especially advises against the Mediterranean on account of the winds, and against high altitudes on account of their changes in temperature. If a seashore climate is desired, especially 
during the summer, it should be on the Baltic, [coast of Massachusetts and Maine during July and August-Ed.,] not only in nervous but also in all severe cases of chlorosis. If the case is a mild one, or if improvement has begun, or if dyspeptic symptoms are present, there is no objection to the Mediterranean or the mountains, especially as the change may improve the appetite.

HYDROTHERAPY.- In light cases, or during the stage of decided improvement, hydrotherapy is very beneficial (cold sponge-baths, rubdowns with wet towels, Scotch douches (not too cold), shower-baths with the water somewhat tempered). These procedures stimulate metabolism, and are a sedative to the nervous system. The patient should, however, be closely watched, and if any unfavorable symptoms follow (chill, shortness of breath, palpitation of the heart, etc.), all hydrotherapy must be given up for a time.

Wet Rubs.- The simplest and most useful method seems to be a wet, cool rub-off, followed by a vigorous rubbing down, as soon as the patient gets out of bed, while the body is still warm. A half hour or so before rising, the patient should have taken a glass of hot milk or tea. The temperature of the water should be about $78^{\circ}$, reduced afterward to $68^{\circ}$. A coarse towel, $2 \mathrm{r} / 2$ by 2 yds., is well wet and wrung out by the nurse or assistant. Meanwhile the patient gets out of bed and stands naked with arms folded on the trunk and a cold compress on the head. The nurse places one corner of the towel on the patient's shoulder and then brings the wide side over the breast and passes it around the back and forward to the other shoulder. A better method is the following one, described by Winternitz: The nurse folds the towel up so that it will just reach from hand to hand when the arms are outstretched. She then takes it in her left hand by the upper edge and with her right hand places the free corner of the upper edge in the left armpit of the patient, who stands opposite with the arms raised. The attendant, using both hands, then rubs the patient up and down through the towel, until the skin feels warm everywhere. The wet towel is then removed, a dry one is substituted, and the body is rubbed as before. The whole procedure should take only 1 minute at first, and later be extended to 2, and again to 4 . The patient should then return to bed. When the treatment has been carried out for 1-2 weeks, the stimulative effect may be increased by using salt water $(1: 1000)$.

Dry Rubs.-If, for any of the reasons already given, this procedure is contraindicated, the patient may be rubbed down with a bath towel directly after rising; or, after improvement has begun, may have partial baths (often with alcohol or salt water).

Sweat Baths.-Sweat baths are recommended by some authors (Scholz) as a depletory process which stimulates the formation of new hæmoglobin. The author's personal experience extends only to 1 case, in which recurrent chlorosis appeared to be more benefited if hot-air baths were added to the iron treatment. Sweat baths might be of use 
in cases of chlorosis with symptoms of autointoxication, the toxins being eliminated through the sweat glands. [I think other methods would be preferable. The explanation is faulty.-Ed.]

Marriage.-The practical question often comes up: Should a chlorotic person marry? Presupposing it to be a case of true chlorosis, the author is inclined to allow marriage, provided the person has first recovered from the discase. In ease of recurring chlorosis, he would, so far as the disposition of the patient will permit, advise against marriage, on account of the danger in pregnancy. It should not, however, be forgotten that an obstinate case of chlorosis, which resists all hygiene and medication, is sometimes cured after marriage.

Specific Medication: Iron.-So far as medication is concerned, there is, of course, a specific remedy for chlorosis,-iron. Although it has been used so long for this purpose, we still have no absolutely clear conception of its exact action. Almost all authors agree that, if it is given in a compound analogous to hæmoglobin, it is absorbed from the mucous membrane of the digestive tract and is used up in other parts of the body. But if it is given in other compounds (either organic or inorganic) some authors (Quincke, Woltering) hold that it is absorbed from the stomach and intestines, and others (Kobert) that its undoubtedly good effects are due to its local action upon the stomach and intestines. They hold that numerous series of experiments show that preparations of iron cause active hyperæmia of the mucous membrane of the stomach and intestines, thus stimulating digestion and making the mucous membrane better able to absorb the iron contained in the food and to render it capable of being utilized by the whole organism. Some time ago Binz formulated a similar theory, which has recently been advocated by Liebreich. According to this, there are, beside "nutritive" preparations of iron, others which act as astringents on the anæmic mucous membrane of the digestive tract and so increase the absorption of the products of digestion. This increased amount of nourishment makes the blood richer in nutritive elements. These preparations of iron, which Liebreich calls tonics, consist of the compounds of iron which are absorbed only with difficulty, particularly the iron salts. The ferrous compounds are preferable in this respect to the ferric. Whether or not iron has a local effect, however, the latest investigations apparently prove that every preparation of iron organic or inorganic, is absorbed, and that the principal effect of iron is general, no matter in what form it is given. This is the most generally accepted modern theory, in which the author also concurs. [Clinical experience certainly confirms this. I sometimes obtain even better results with iron hypodermically.-Ed.]

Difficulties.-The choice of the preparation and the dosage depend upon the effect of the separate compounds, their differing contents of metallic iron, and their digestibility. Difficulties of digestion due to chlorosis alone and not to gastric ulcer (much rarer in the author's opinion [and mine-Ed.] than is generally supposed), gastritis, etc., are 
no contraindication to iron, although in severe dyspepsia it is better in many cases to precede the iron therapy by medication intended simply to mitigate the digestive symptoms. For this purpose, we may (see p. 418) use hydrochloric acid, alkalies, or, in atony of the stomach and intestines, rhubarb, nux vomica, etc., and mechanical methods.

Gastralgia.-Severe gastralgia, which is made worse by iron, may hinder us from the routine iron treatment. In this case, it is best to combat the hyperæsthesia of the stomach by means of an exclusively milk diet, hot cloths on the abdomen, and the administration of belladonna or silver nitrate:

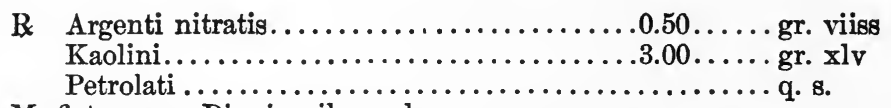

M. fiat mass. Div. in pil. no. 1.

Sig. $-2-3$ pills a day, before meals.

Menthol in small doses $(0.05-0.10$ G. (3/4-11/2 gr.) a day), with or without alkalies, is also often good. [A preliminary rest cure in bed often accomplishes better results with fewer drugs.-Ed.]

After 2 weeks or so of such treatment, the stomach is ready for iron. If hyperacidity is demonstrable, the iron is better given before rather than after meals.

Daily Dose.-It is best in general to begin with moderate doses, $0.05-0.10 \mathrm{G}$. $(3 / 4-1 \mathrm{I} / 2 \mathrm{gr}$.), or at most $0.20 \mathrm{G}$. (3 gr.) of metallic iron, or a corresponding amount of the preferred iron preparations. But the author's personal experience would lead him to believe, with such authors as Niemeyer, Immermann, and Litten, that large doses are sometimes needed, although he believes in always beginning with small ones. [I agree with von Noorden in recommending a gradual increase of the dose at the beginning and a gradual decrease at the end of a course of iron medication. At the Massachusetts General Hospital the routine treatment of chlorosis was 1 Blaud's pill 3 times a day after meals during the first week, 2 during the second, 3 during the third, 2 during the fourth, and 1 during the fifth, and usually a powder or tablet 3 times a day before meals, containing:

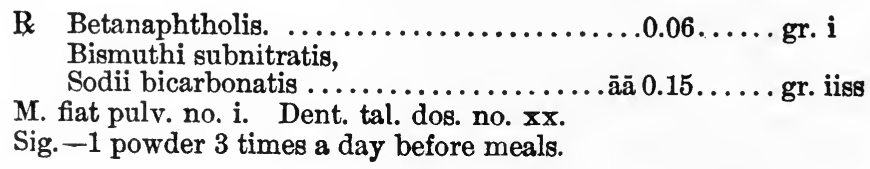

Preparations of Iron.-The following discussion of the preparations of iron is based on the author's personal experience. [I have summarized his account for convenient reference and have added a few suggestions.-Ed.] 


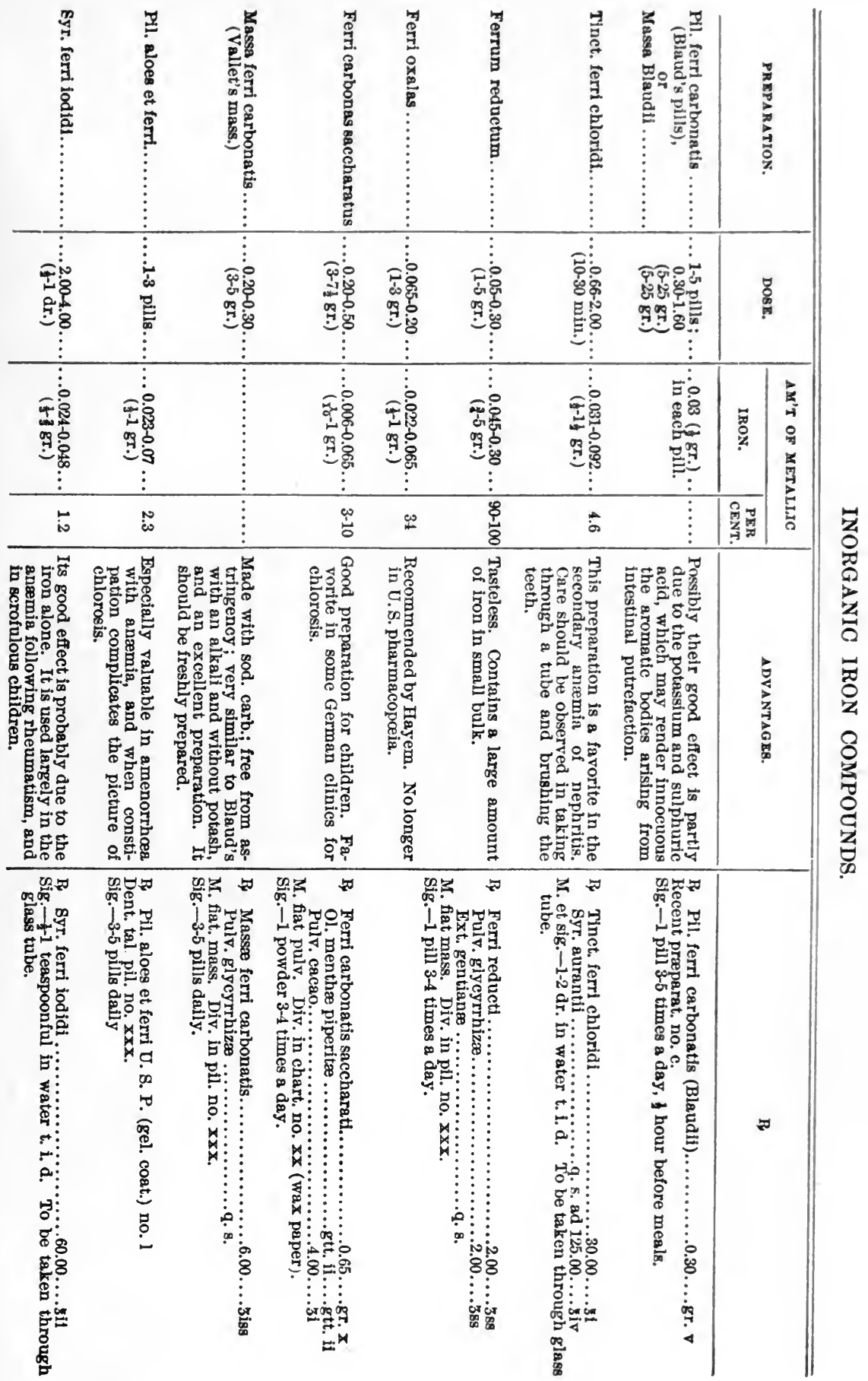




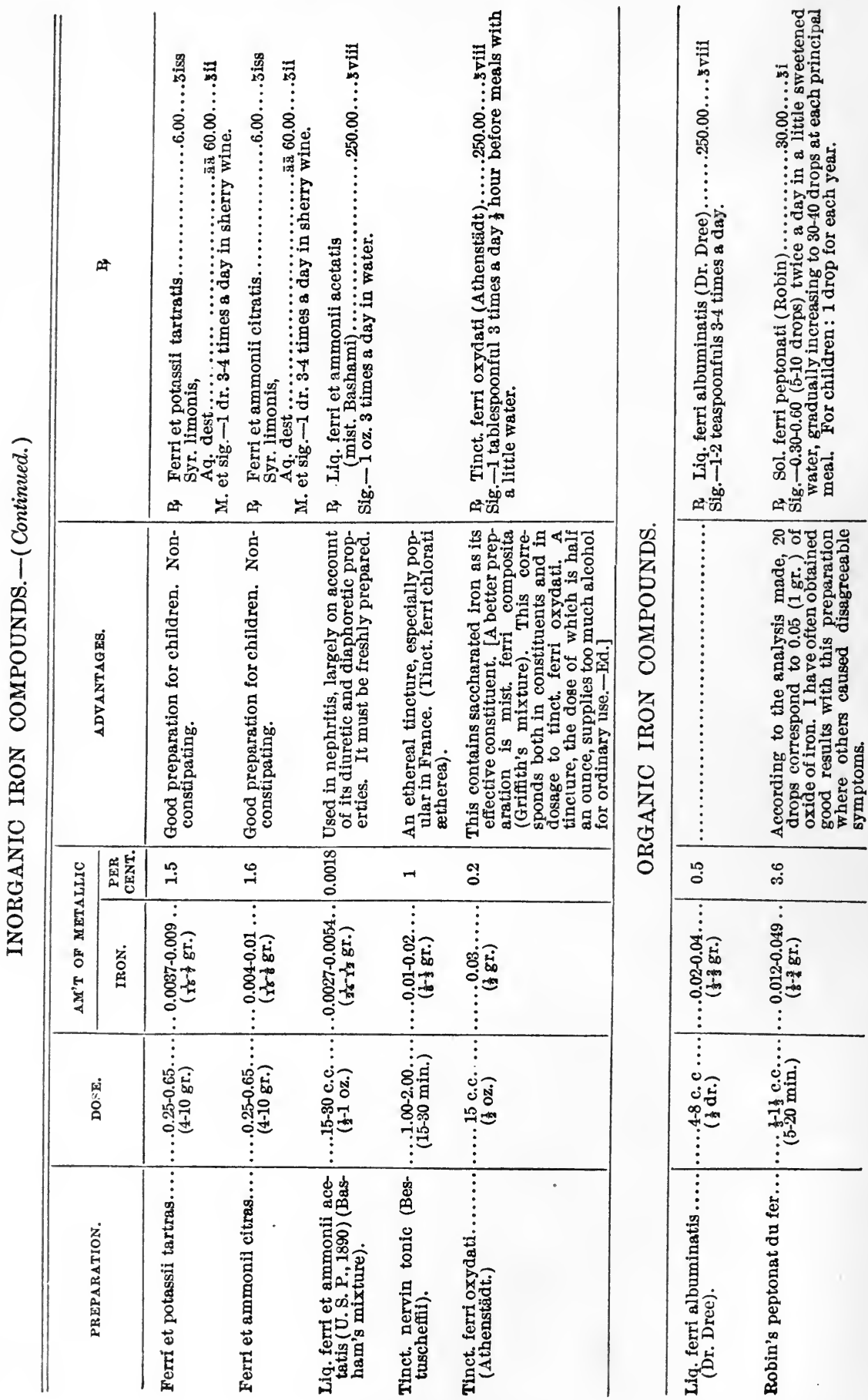




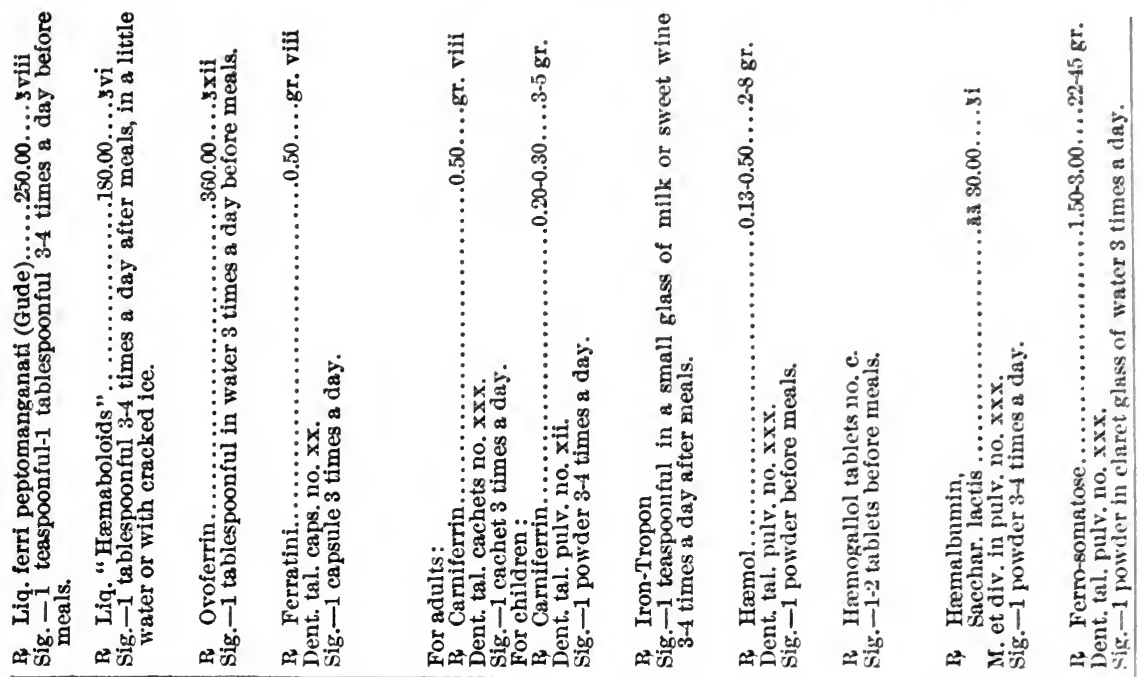

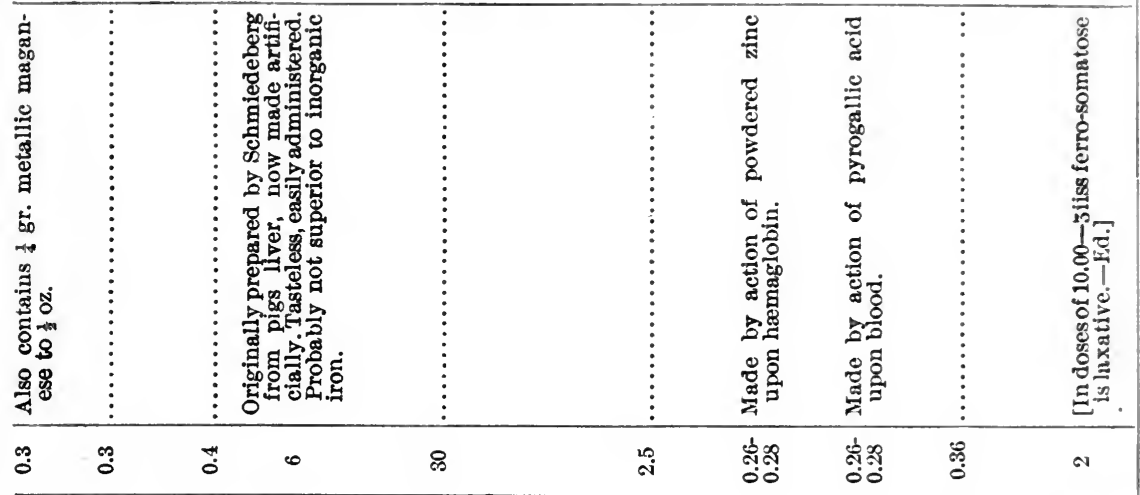

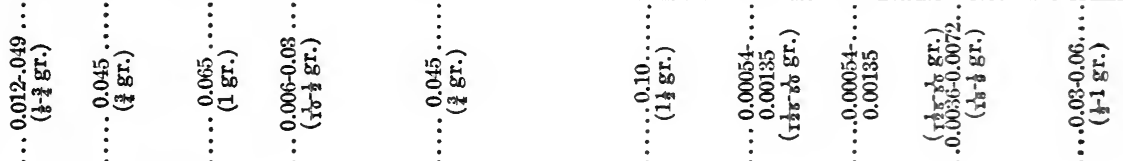

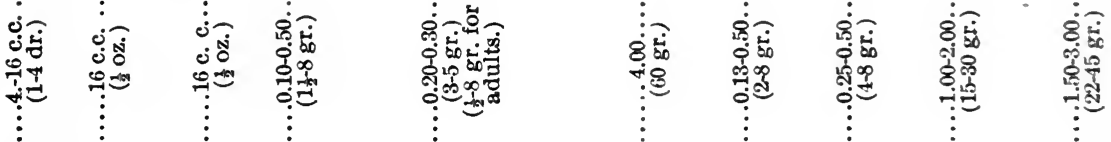

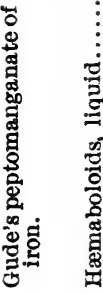


The author has never found any better effect from Krewel's "blood pills" than from any other preparation of iron, although other authors (Litten) attribute to them great efficacy in many cases. They consist of 10 per cent. hæmoglobin, 40 per cent. natural blood salts in an easily soluble form, and 44 per cent. freshly peptonized meat albumin. "Fersan " and " alboferrin" may be used (2-3 teaspoonfuls or more a day mixed with cold water and then added to hot milk, tea, cocoa, or soup). Both come in pastilles ( $2-4$ pastilles 3 times a day), and are useful for foods as well.

The author, believing that all preparations of iron are absorbed from the stomach and intestines and that the action is the same whether an organic or an inorganic preparation is employed, generally uses either oxalate of iron or Blaud's pills, and occasionally iron somatose, fersan, or alboferrin, when it is desirable to improve as much as possible the poor state of nutrition.

If it is found that, in any given case, iron does not agree with the stomach, it should be given in pills, either keratin-coated or enclosed in Sahli's glutoid capsules (grade II of hardness, see p. 428). This will insure their not being dissolved until they reach the small intestine.

Duration of Iron Treatment.-The treatment with iron should be kept up for 4-6 weeks, and then, if the chlorosis has not disappeared, be repeated after an intermission of 3-4 weeks. Indeed, even if the disease appears wholly conquered, this second administration is advisable. It is not necessary to prescribe any particular diet because the patient is on iron, although fats, fruits, and acids should not be given at or near the same time as the iron, and individual tolerance for them should be taken into careful consideration.

Mineral Waters Containing Iron.-It is often desirable to send a patient to one of the iron springs, in case the chlorosis has not yielded to treatment at home or the circumstances of the case prevent proper hygienic measures from being carried out. These iron springs may be divided into two classes: (1) Alkaline-iron springs, in which the iron is in combination with carbon dioxide, appearing as ferrous carbonate, as at Pyrmont (Waldeck), Cudowa and Flinsberg (Prussian Silesia), Schwalbach (Hesse-Nassau), Koenigswart (Bohemia), Alexanderbad (in the Fichtelgebirge, Upper Franconia), St. Moritz (Switzerland), and Spa (Belgium); (2) Alkaline-saline iron waters, in which the iron appears as ferrous sulphate, as at Pyrawarth (Lower Austria), Franzensbad (Bohemia), Elster (Bohemian frontier of Saxony), and Tarasp (Switzerland). The effect of the latter class is due not only to their content of iron, but also to their content of sulphuric acid or sulphates. French investigations have shown that saline mineral waters should be beneficial in chlorosis, where the excretion of nitrogen is almost always small, and metabolism and oxidation are diminished.

Baths Containing Iron.-In both the above-mentioned classes of spas, baths may also be taken, sometimes in the iron water itself, some- 
times as iron mud bath. The mud baths are too relaxing to be recommended as a routine in cases of simple chlorosis, but are particularly suited to cases complicated by nervous symptoms. But the alkaline-iron baths have a stimulating effect on the whole system, as the free carbon dioxide causes active cutaneous hyperæmia, reddening the skin and giving a pleasant sensation of tingling. As examples of such springs rich in carbon dioxide, may be mentioned St. Moritz, Tarasp, Koenigswart, Schwalbach, and particularly Szliacs in Upper Hungary. The springs of the last have the peculiar advantage, so far as the baths are concerned, of a somewhat high temperature $\left(77^{\circ}-89^{\circ}\right)$. The warmth of the water is also sometimes advantageous for internal use, as cold water containing iron is often disturbing to the stomach of a chlorotic person. The amount to be taken is, to begin with, 600 c.c. (a pint) and more a day, 1 glass in the morning on an empty stomach (if this agrees with the patient), 1 just after the principal meal, and 1 in the afternoon. The water, if cold, should first be warmed in a double boiler. The amount may often afterward be increased by the addition of 1 glass in the morning just after the bath, and another in the evening. The cure should last from 4 to 6 weeks.

If the cure must be carried out at home, it is best to use the alkalinesaline iron waters, as the composition of the carbonate of iron waters is altered by the precipitation of the iron after bottling, first as simple ferrous carbonate and afterward as ferrous hydroxid.

Alkaline and Alkaline Chloride Springs and Indifferent Hot Springs.-A word may be added concerning the employment of other kinds of mineral waters in chlorosis. They are of use in combating the various forms of dyspepsia which so often accompany chlorosis. In atony of the stomach, we may give alkaline or alkaline chloride waters (Ems, Neuenahr, Homburg, Kissingen); in severe constipation, these and also Carlsbad or Vichy (taken cold); in hyperacidity and motor irritability, where a sedative effect is needed, indifferent hot springs (Plombières in the Vosges, Wildbad in the Black Forest, Gastein (particularly good), Roemerbad in Styria, Ragatz-Pfäffers in Canton St. Gall, Switzerland, and especially Johannisbad in Bohemia). At the last named place there is also an alkaline-saline iron spring (Kaiserquelle, $50^{\circ}$ ).

Iron and Manganese.-In spite of all precautions and the choice of the most efficacious preparations, we occasionally encounter a case of chlorosis which refuses to yield to the iron therapy. It has been proposed to treat such cases with a mixture of manganese and iron, and a liq. ferri mangano-peptonate has been accordingly prepared and put on the market. The author has, however, never obtained any better success from it than from iron alone. [Nor have I.-Ed.]

Iron in Chlorosis with Dilatation of the Stomach (Autointoxication).- One particular class of cases in which iron does no good is that pointed out by Neusser and Pick, where the distinguishing characteristic 


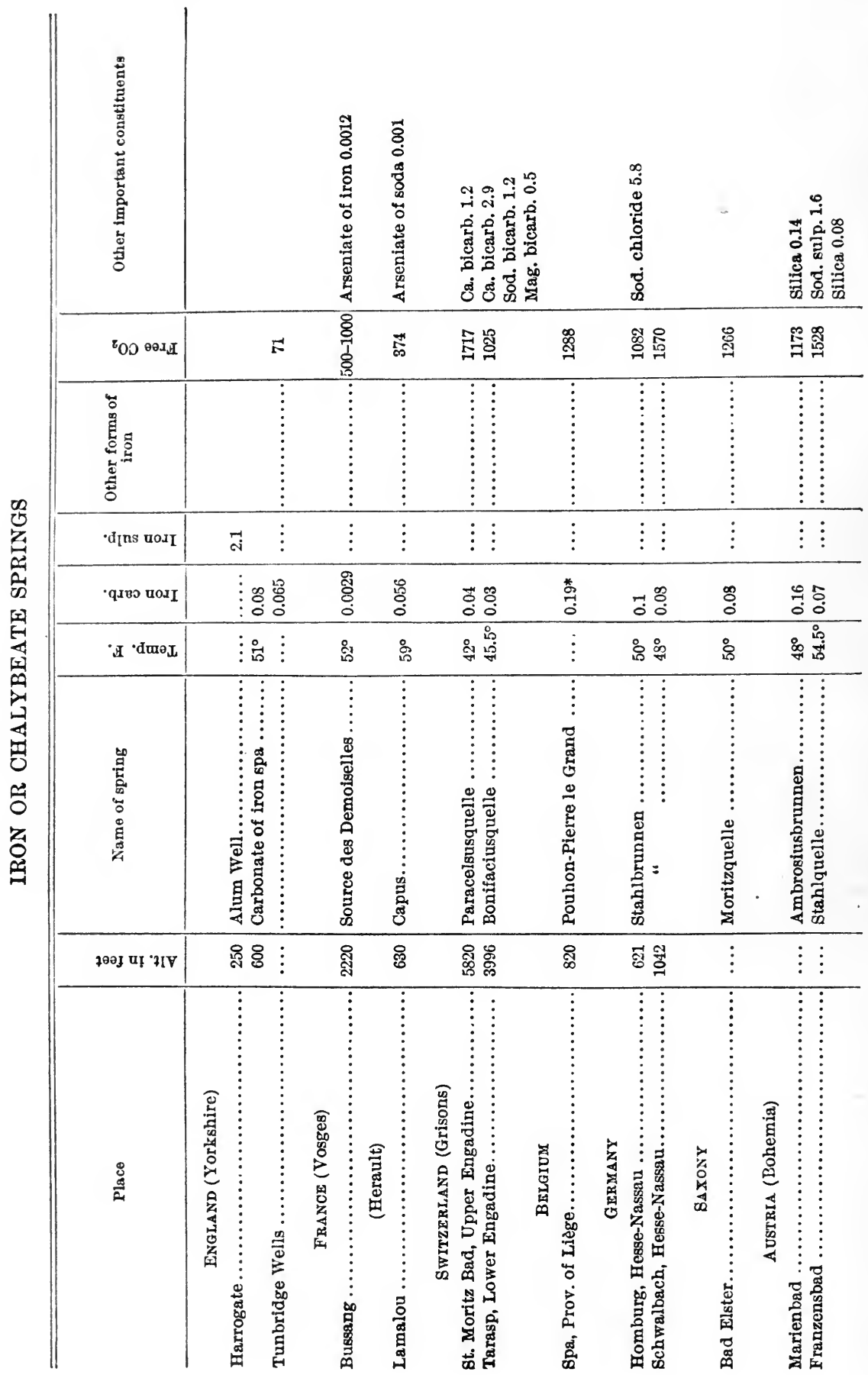




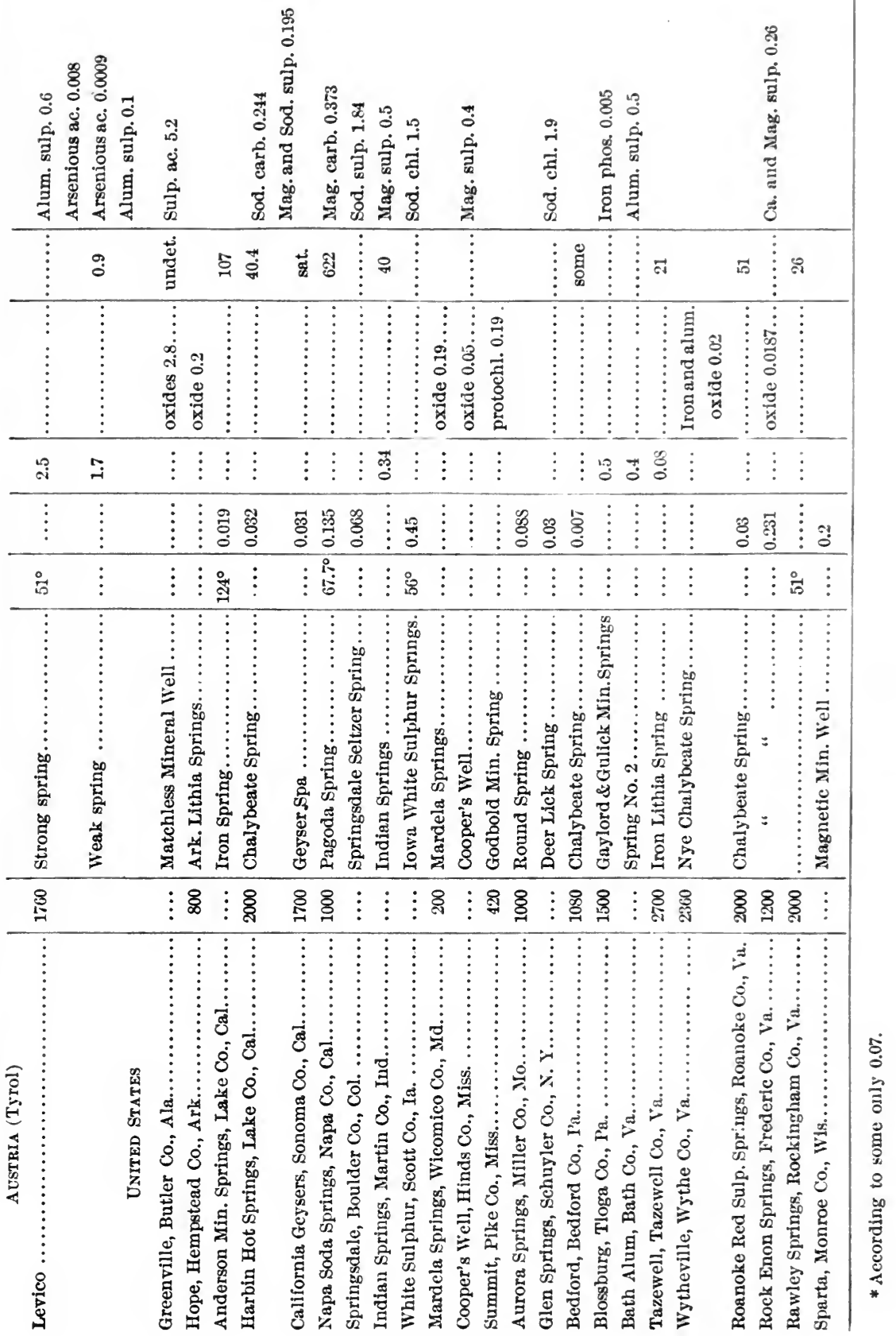


is the severity of the stomach symptoms, both subjective and objective (pressure, belching, nausea, vomiting,--otherwise rare in chlorosis,severe atony, and often true dilatation). The stomach contents, if removed in the morning, show remains of food taken the evening before or even earlier. The breath has a fetid odor, which cannot be traced either to diseases of the mouth or to the condition of the lungs. These are probably cases of autointoxication, in which the chlorosis is secondary to the dilatation of the stomach. This theory is supported by the fact that, although iron has no effect, lavage is most successful. Pick found that 3-4 weeks of the latter treatment would effect a cure where a month's administration of iron had produced no effect. Good results may also be obtained by the internal use of creosote, thus avoiding the disagreeable features of lavage:

B Creosoti (beechwood) ..................0.06....mi

Ol. olivæ.......................... mi

M. fiat caps. no. i. Dent. tal. caps. molles no. 1 .

Sig. -1 capsule 3 times a day directly after each meal.

Resorcin or betanaphthol may be similarly used (see p. 361).

Iron in Chlorosis with Obstinate Constipation.-There are other cases of chlorosis which apparently must be attributed to autointoxication, but of intestinal rather than of gastric origin. They are distinguished by severe constipation, accompanied by a not otherwise explainable fetid breath. In such cases a cure at Carlsbad, Homburg, or Kissingen is advisable. The waters at the last two places are all common salt waters, two special springs at Homburg (the Louisa and the Stahlbrunnen) containing also bicarbonate of iron, a smaller quantity of which is also found in the Rakoczy spring at Kissingen. Neusser recommends a strongly vegetarian diet, the reason for which is self-evident when we remember that the proteids give rise to the formation in the intestine of products of decomposition, which are absorbed. The carbohydrates, on the contrary, in splitting up give off organic acids (lactic, butyric, acetic), which tend to destroy the bacterial activity that causes the putrefaction of the proteids. It is obvious, at any rate, that in every case of chlorosis complicated by constipation, a combination of iron with some cathartic is advisable. For this we may employ, for example, pil. aloes et ferri, 3-5 a day, each pill containing $0.024 \mathrm{G}$. ( $1 / 3 \mathrm{gr}$.) of iron.

Iron in Chlorosis with Nervous Symptoms.-If chlorosis is accompanied by nervous symptoms, Johannisbad (Bohemia), which also contains an iron spring, would be the author's first choice, then Roemerbad (Styria), Neuhaus (not far from Cilli in Styria), which also has an iron spring, and Schlangenbad (Hesse-Nassau). If the patient has only slight chlorosis, she may do well at a high altitude, the best resort for that purpose being St. Moritz, as it combines a mountain climate with an iron spring. If such cases are to be treated at home, the author recommends lecithin-perdynamin (up to 3 liqueur glassfuls daily). 
Other Medication : Arsenic.-Two drugs besides iron are often helpful in chlorosis, - quinine and (especially) arsenic. The latter, according to Nothnagel-Rossbach, is contraindicated in all cases of chlorosis complicated with dyspepsia. Since the majority of authors agree that, even in medicinal doses, arsenic diminishes metabolism, reducing the amount of nitrogen (in urea) excreted from the kidneys, and of carbon dioxide excreted from the lungs, it seems rational to the author to add arsenic to iron in eases of chlorosis where chemical investigation proves that increased oxidation is present. But this condition is very rare, occurring, according to the author's experience, only in those exceedingly severe cases of chlorosis which tend to pass over into pernicious anæmia. [I do not believe that such transitions occur. In the severe cases of chlorosis which I have seen the blood picture still preserved its character of chloranxmia.-Ed.]

Combination of Iron and Arsenic.-Aside from mineral waters containing both iron and arsenic (see p. 170), two combinations of these drugs may be recommended, the well-known prescription that follows:

B Ferri et ammonii citratis...............30.00.... $3 \mathrm{i}$

Liq. potassii arsenitis................ 19.50... $3 \mathrm{v}$

Aq. menthæ piperitæ.............. s. ad $180.00 \ldots \ldots$.

\section{M. fiat mist.}

Sig. $-\frac{1}{2}-2 \frac{1}{2}$ dr., increasing and decreasing, after dinner and supper.

Or : $\left(\frac{1}{2} \mathrm{dr} .=3 \mathrm{~min}\right.$. Fowler's solution and $5 \mathrm{gr}$. iron salts. $)$

R Ferratini ............................... 3 iiss

Liq. potassii arsenitis............................. 3 i $\mathrm{m}$ xlv

M. fiat caps. no. $\mathrm{c}$.

Sig. -3 capsules 3 times a day after meals.

Or we may use " arsenferratose," the syrup of ferratinum arseniatum (0.3 per cent. iron, 0.003 per cent. arsenic), 3-4 tablespoonfuls daily.

Quinine.-The most suitable cases for the use of quinine combined with iron are those in which the chlorosis is complicated by nervous asthenia, for quinine must, without doubt, be looked upon as a cerebral tonic. The following is a good prescription:

B Ferri et quininæ citratis solubil.................gr. ii

Sacchar. lactis............................ vii

M. fiat cachet no. l. Dent. tal. cachets no. xx.

Sig. -1 cachet every other day.

Quinine combined with chloride of iron is also of use in chlorosis. although the latter is employed chiefly as a styptic in cases of internal hemorrhage. It is sometimes given as the solution of quinine and chloride of iron (Dr. Kersch), (10 drops in water or white wine every 3 hours,) sometimes in the form of the following powder:

B Quininæ ferri chloridi (Kersch) .....0.05-0.10.....gr. i-gr. ii

Sacchar. lactis........................ vii

M. fiat cachet no. i. Dent. tal. cachets no. xx.

Sig. -1 cachet 3 times a day after meals. 
Sulphur.-There remain several other methods of treatment to be mentioned. Schulz and Strübing recommend the internal use of sulphur in those cases in which iron has no effect. They prescribe:

B Sulphuris sublimati $\ldots \ldots \ldots \ldots \ldots \ldots \ldots . \ldots \ldots \ldots \ldots \ldots$ iiss

Sacchar. lactis..................... $30.00 \ldots$

M. fiat cachets no. c.

Sig. - 1 cachet 3 times a day.

The author considers sulphur indicated where severe constipation and increased intestinal decomposition are present.

Oxygen.--He believes that another method of treatment suggested, the inhalation of oxygen (15-25 gal. a day), is more efficacious, especially in case of vomiting and persistent anorexia.

VENESECTION.-It should be remembered that chlorosis is one of the indications for venesection (see p. 63). This should, however, be resorted to only when all other methods fail. Only small amounts of blood (50-100 c.e.) should be taken, and the process should not be repeated until after 4-8 weeks. [I have never tried this method.-Ed.]

\section{THERAPY OF PROGRESSIVE PERNICIOUS AN AMIA.}

In discussing the therapy of pernicious anæmia, the term will be confined to true primary anæmia, the cause of which at present is wholly unknown. In other cases, where the anæmia is secondary, it is hardly correct to speak of a " progressive pernicious anæmia," as in such cases the successful treatment of the underlying cause often causes the secondary condition of the blood to disappear, so that one general characteristic of pernicious anæmia-the fatal outeome-is not present. This underlying cause may be atrophy of the glands of the stomach and intestine; intestinal parasites (Bothriocephalus latus, Tcenia solium, Anchylostoma duodenale, Trichocephalus dispar, Oxyuris vermicularis, and Ascaris lumbricoides) ; blood parasites (Filaria sanguinis, Distomum homatobium); syphilis $^{1}$ (rarely); and pregnancy. In the last case, it is to be noted that premature delivery is of no avail. Anæmia is, of course, a contraindication for maternal nursing.

"Gasterine" ${ }^{2}$ in Secondary Anæmia.--In reference to the first of these causes, atony of the glands of the stomach and intestines, various authors report great improvement in the blood from the use of "gasterine," a new preparation from the gastric juice of a dog operated upon according to Pawlow's method (see p.423). The author himself has seen a case of very severe anæmia-though not strictly pernicious anæmiawonderfully improved by a two months' treatment ( 3 teaspoonfuls a day). The diarrhœa, anorexia, œdema of the lower extremities, and the abnormal temperature all disappeared, the pains in the joints became much better, the body weight of the emaciated patient markedly increased,

${ }^{1}$ [ $\mathrm{I}$ believe syphilis is a commoner cause of a severe anæmia than most authorities grant.-Ed.]

"[For sale under the name of "Hepptine."-Ed.] 
and the blood findings improved. It is only natural that gasterine should be beneficial in atony of the glands of the stomach and intestine, as it consists of the pure pepsin and hydrochloric acid from a dog.

Primary Pernicious Anremia.-In case of true primary pernicious anæmia we base our treatment to-day on Grawitz's theory that pernicious anæmia is due to autointoxication from the digestive tract. We therefore lay great weight on keeping the mouth clean and giving the patient only vegetable food in purée form (mashed potatoes, rice, spinach, farina, etc.). The usual nutrient enemas (of milk, egg, salt, sugar) may be used and the stomach may be washed out daily with tepid normal salt solution. [Herter's recent investigations show that high irrigation of the colon is of even greater importance than is gastric lavage. The prompt improvement in the symptoms and blood picture after instituting high colon irrigation with normal salt solution is very striking.-Ed.] If the condition improves, we may proceed to medication, particularly the administration of arsenic.

Arsenic.-It may be given as follows :

$\mathrm{R} \quad$ Liq. potassii arsenitis................ $15.00 \ldots \ldots \mathrm{ss}$

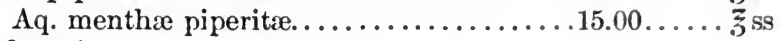

M. fiat mist.

Sig. $-6-15$ drops in water twice a day, directly after meals.

The minimum dose is increased by 2 drops a day until it reaches the maximum, after which it is decreased in the same way. The treatment should be intermitted after 2-4 weeks, and then renewed a second or third time. Arsenic may also be given in pills (Asiatic pills, see p. 10); in tablets (each containing 1 drop of Fowler's solution); and in gelatin tablets (gelatina acidi arsenicosi, with 0.001-0.002 G. (3/200-3/100 gr.) of arsenious acid). The author believes in giving much larger doses of arsenic than these conventional ones in pernicious anæmia, leukæmia, and pseudoleukæmia. He has found the treatment much more successful if the daily dose is increased to 30 or even 60 drops of Fowler's solution and the treatment is kept up for several weeks. [Authorities differ as to the amount of arsenic which should be given to accomplish the best results. I rarely give arsenic uninterruptedly for more than 4 weeks, and generally begin with a moderate dose, 3-5 min. of Fowler's solution, increase rapidly to near tolerance, persist with this dose for 2 weeks, and then gradually diminish again. I believe that corresponding doses of Pearson's solution (liq. sodii arsenatis N. F., 1-8 min.) are more apt to cause symptoms of arsenical intoxication. Since I have tried Murray's dosage in chorea, I have used larger doses of arsenic in the anæmias and but rarely have poisoned my patient.-Ed.]

Arsenical Poisoning.-The dyspeptic disturbances so often accompanying pernicious anæmia, particularly diarrhœa, frequently oblige us 
to give up arsenic, although it may sometimes be borne by beginning with small doses. Again, after a somewhat prolonged use of arsenic, we may be warned to discontinue it by the appearance of symptoms of arsenical intoxication (burning in the mouth, thirst, belching, epigastric pains).

Arsenic Waters.-Sometimes a patient who cannot take arsenic in any other form is tolerant of mineral waters containing it. [The most representative of such waters I have grouped in a table for convenient reference.-Ed.]

ARSENICAL WATERS

ALKALINE WATERS CONTAINING ARSENIC

\begin{tabular}{|c|c|c|c|c|}
\hline Place & Alt. in feet & Name of spring & $\begin{array}{c}\text { Parts per } \\
1000 \\
\text { Sod. arsen. }\end{array}$ & Dose \\
\hline La Bourboule, Auvergne & 2770 & Source Perriere......... & 0.028 & $\begin{array}{l}\text { 3-20 oz. daily in } 3 \text { portions } \\
\text { at about } 104^{\circ}\end{array}$ \\
\hline Royat, Auvergne......... & 1460 & Saint-Victor $\ldots \ldots \ldots \ldots \ldots$ & 0.004 & 1-3 pts. daily \\
\hline Vichy, Allier............ & 850 & $\begin{array}{l}\text { Grand Grille, Hôpital, } \\
\text { Celestines. }\end{array}$ & 0.002 & 1-3 pts. daily \\
\hline Mont Dore, Puy de Dôme & 3400 & La Madeline............. & 0.001 & 1-3 pts. daily \\
\hline
\end{tabular}

IRON WATERS CONTAINING ARSENIC SULPHATE OF IRON WATERS

\begin{tabular}{|c|c|c|c|c|c|c|c|}
\hline \multirow{2}{*}{ Place } & \multirow{2}{*}{$\begin{array}{l}\text { Alt. } \\
\text { in feet }\end{array}$} & \multirow[b]{2}{*}{ Name of spring } & \multicolumn{4}{|c|}{ Parts per thousand } & \multirow[b]{2}{*}{ Dose } \\
\hline & & & 竞 & 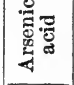 & 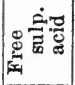 & $\begin{array}{c}\text { Other } \\
\text { constituents }\end{array}$ & \\
\hline Vals, Ardèche......... & 800 & St. Louis....... & 0.39 & 0.001 & & & \\
\hline Roncegno, Tyrol ....... & 1750 & $\ldots \cdots \cdots \cdots \cdots$ & 3.0 & 0.15 & & $\underset{1.39}{\text { Alum. sulp. }}$ & $\begin{array}{l}1 / 2-11 / 2 \text { ozs. well dil. } \\
\text { with water after } \\
\text { meals }\end{array}$ \\
\hline \multirow[t]{2}{*}{ Levico, Tyrol............ } & 1760 & Strong Spring. & 4.6 & 0.006 & 1.6 & $\begin{array}{l}\text { Alum. sulp. } \\
0.2\end{array}$ & $\begin{array}{l}1 / 2-2 \text { ozs. daily, dli. } \\
\text { with water after } \\
\text { meals }\end{array}$ \\
\hline & & Weak Spring. . & 0.37 & traces & $\cdots \cdots$ & & $\begin{array}{l}\text { Often mixed with } \\
\text { with wine or beer } \\
\text { and drunk at } \\
\text { meals }\end{array}$ \\
\hline Siebrenica, Bosnia .... & 1200 & Guberquelle. . & 0.37 & 0.006 & 0.008 & $\underset{0.22}{\text { Alum. sulp. }}$ & $1 \mathrm{oz}$ \\
\hline
\end{tabular}

CARBONATE OF IRON WATERS

\begin{tabular}{|c|c|c|c|c|c|c|c|}
\hline \multirow{2}{*}{ Place } & \multirow{2}{*}{$\begin{array}{c}\text { Alt. } \\
\text { in feet }\end{array}$} & \multirow{2}{*}{ Name of spring } & \multicolumn{4}{|c|}{ Parts per thousand } & \multirow{2}{*}{ Dose } \\
\hline & & & $\begin{array}{c}\text { Sod. } \\
\text { bicarb. }\end{array}$ & $\begin{array}{c}\text { Iron } \\
\text { bicarb. }\end{array}$ & $\begin{array}{c}\text { Iron } \\
\text { Arsen. }\end{array}$ & $\underset{\text { ccm. }}{\mathrm{CO}_{2}}$ & \\
\hline Sylvanes, A veyron ...... & 1312 & & & 0.02 & 0.016 & & \\
\hline Bussang, Vosges.......... & ......... & Source des Demoiselles. & ........ & 0.0029 & 0.0012 & $500-1000$ & \\
\hline Cudowa, Silesia ......... & & Engenquelle ........... & 1.2 & 0.063 & 0.0026 & 1217 & . \\
\hline
\end{tabular}


Arsenic Subcutaneously.-Finally, we may give arsenic subcutaneously. Pearson's solution (liq. sodii arsenatis ${ }^{3}$ (see p. 175), [sodium arsenate $0.001 \mathrm{G} .\left(1 /{ }_{60}\right.$ gr.) - Ed.,] or atoxyl (meta-arsenic-acid anilid), or we may replace arsenic by cacodylate therapy. The latter owes its effect to its giving off of arsenic in the organism and seems to be no more effective than arsenic itself. The author uses it only exceptionally (see p. 348). Atoxyl, however, is the best fitted of the three for injection, as it is wholly without reaction. We use a 15 or 20 per cent. solution. In the latter case the solution must be warmed to be sure that all the atoxyl is dissolved. We begin by using $0.40 \mathrm{G}$. ( $6 \mathrm{~min}$.) of the solution, increasing by $0.40 \mathrm{G}$. ( $6 \mathrm{~min}$.) every day until we reach $0.3 \mathrm{G}$. ( $5 \mathrm{gr}$.) atoxyl, continuing this for 4 weeks, then reducing the number of injections to 2 a week, then to 1 a week, then intermitting the treatment altogether for $6-8$ weeks.

Bone Marrow.-Several authors have strongly recommended bone marrow, attributing brilliant success to its use, but the author has seen no effect from it. [Nor have I.-Ed.] The marrow from beef or veal bones may be salted and spread thickly over bread (2-3 slices a day) or may be compressed into tablets ( 0.25 G. (4 gr.) tablets, 3-15 daily).

Neither has iron apparently any good effect; indeed, it is generally badly borne.

[Hunter suggests (1) antisepsis of mouth, (2) of gastro-intestinal tract, (3) arsenic, (4) antistreptococcus serum. Despite his careful work, I do not believe that most cases of primary pernicious anæmia depend upon oral sepsis as a cause, although many are accompanied by such a condition. Lazarus recommends, as soon as the patient's strength and general condition (gastro-intestinal tract) permit it, $2-4$ G. of aspidium, even in cases in which neither eggs nor segments have been found. R. C. Cabot, reviewing 110 cases, concludes that neither bone marrow, arsenic, nor oxygen inhalations have any effect upon the disease. Grawitz commends the use of gastric and intestinal lavage.-Ed.]

Salt and Carbonated Baths.-In the early stages of the disease, salt and artificial carbonated baths are desirable. For the salt baths we use rock-salt or sea-salt in a 1-3 per cent. solution. In the author's opinion, a 5 per cent. solution is better; that is, $15 \mathrm{~kg}$. (37 lb.) in 300 litres ( 75 gal.) of water. He usually uses 500 litres ( 125 gal.) for a full bath, adding $25 \mathrm{~kg}$. (62 lb.) of rock-salt. If a 1-3 per cent. solution is employed, the author advises adding to the bath 1-10 qt. of mother-lye (Mutterlauge), - the brown liquid left in salt vats after the erystallizing out of the salt.

A southern climate in winter is, of course, desirable.

Transfusion and Infusion.-If the disease has reached an advanced stage and the anæmia has become extreme, there is one method left to be tried, which, as reported by many observers, may effect an improve-

${ }^{3}$ [This solution should not be confounded with liq. sodii arsenatis U. S. P., which is 10 times as strong.-Ed.] 
ment or even a cure; i.e., a transfusion of blood, or an infusion of salt solution.

There are four methods commonly employed: direct intravenous blood transfusion, indirect intravenous blood transfusion, intravenous infusion of a salt solution, and subcutaneous injection of a salt solution. They are all used chiefly in anæmia from hemorrhage (the last one also in cholera and septic diseases), but the description of their technic may be given here.

Intravenous Blood Transfusion.-In direct blood transfusion, the blood is transferred unchanged from vein to vein (Ziemssen's method) ; in indirect blood transfusion, it is first defibrinated.

In direct transfusion, the arm of the patient and that of the person from whom the blood is to be taken, are each bound by a ligature until the veins stand out. The skin over the veins selected is then thoroughly scrubbed and disinfected. An aspirating needle with a rubber tube attached is next thrust into the vein of the well person and about 50 c.e. $(1 \mathrm{I} / 2 \mathrm{oz}$.) of blood slowly withdrawn by means of a syringe. The instrument must first be warmed in hot sterile water. A similar needle is then thrust into the vein of the patient. As soon as the blood begins to flow out through the needle, the rubber tube is compressed with the finger and the ligature is removed. The syringe full of blood drawn from the other person is then fastened to the rubber tube and the blood slowly forced into the patient's vein. Meanwhile, the second syringeful is being drawn from the other person. The process is repeated until 200-300 c.c. (6-10 oz.) of blood have been transferred. It may be necessary to use the other arm of the person furnishing the blood, or even to employ a second person, already prepared. If enough syringes are not at hand, the syringes already employed may be used a second time, provided they are very carefully cleansed with sterile water to prevent any coagulated blood from being forced into the vein of the patient. Whether, as Ziemssen held, there is no danger of the blood's clotting in the patient's veins if this preeaution is observed, must be decided by collating the results of a large number of blood transfusions carried out by this method.

Indirect Transfusion.-Indirect transfusion is a much more common method. There are needed, beside the ordinary instruments for venesection and ligation of the vessels, several good-sized glass beakers to receive the blood, a stout glass rod for whipping it, filter funnels and fine linen or several layers of sterile gauze to filter it through, and, finally, the transfusion apparatus proper, which is composed most simply and best of a small glass funnel, a rubber tube, and a small glass cannula, bent at an obtuse angle. The technic is as follows: Rather more blood than is needed is drawn from the arm of the healthy person by veneseetion. This is placed in the glass beaker, set inside of another one containing water at $100^{\circ}-105^{\circ}$, and beaten with the glass rod until no more elotted blood adheres to the rod. It is then filtered through fine linen into a warmed glass beaker. Meanwhile, the veins of the patient's arm are made to 
stand out by compression, one of them is laid bare, and two catgut or silk threads are passed under it. The peripheral end of the vein is now ligated, and the glass cannula, tube, and funnel are filled with the filtered blood, the tube being closed by a stop-cock above the cannula. The patient's vein is now raised with a pair of pincers, a small cut is made in it, and the cannula is inserted in the central direction and bound to the vein so that it cannot slip out during the rest of the procedure. The stopeock is now opened and 200-300 c.c. $(6-10 \mathrm{oz}$.) of blood is allowed to flow slowly in. The cannula is then withdrawn, the central end of the vein is also ligated, the vein is cut through between the two ligatures, and the surface wound is sewed up and bound with an antiseptic bandage.

Consequences of Transfusion.-After such a transfusion, many patients exhibit symptoms of a ferment intoxication, such as severe chills and rise of temperature; sometimes there are much more severe symptoms, as tenesmus, bloody stools, and even sudden death by asphyxiation. If during the process marked dyspnca and cyanosis appear, the procedure should be stopped, at least until the symptoms have disappeared.

It may be mentioned that some investigators claim that it is not necessary to keep the blood at the body temperature. They report that no bad results follow the injection of blood at the temperature of the room. Moreover, blood at that temperature can be more thoroughly defibrinated.

Intravenous Infusion of Salt Solution.-The technic of the intravenous infusion of salt solution is very like that of indirect transfusion, just described. The same apparatus may be used or a glass irrigator of 1 quart capacity. One to 2 qt. of a sterile 0.6 per cent. salt solution is infused.

Hypodermic Injection of Salt Solution.-For the final procedure, hypodermic injection of salt solution, the apparatus is the same, except that the cannula is replaced by one or two aspirating needles, sloped off at the end and without openings on the side. If two are used, the simple rubber tube must be replaced by one ending in a $\mathrm{Y}$. The puncture is best made in the thigh, abdomen, or breast [very painful in women-Ed.]. One to $2 \mathrm{qt}$. of a physiological salt solution is allowed to flow in, and distributed by means of massage during and for a quarter of an hour after the process. The procedure is decidedly painful, so that chloroform narcosis is often necessary, an obvious disadvantage. [Five hundred to 1000 c.c. is as much as I have ever given at one time in infusion or hypodermoclysis.-Ed.]

Of these four procedures the author prefers intravenous infusion of salt solution, not only in pernicious anæmia, but whenever one of the procedures is indicated. It is rather more difficult to carry out than a hypodermic infusion, but it is less painful and more direct. If a suitable person is on hand to furnish the blood, he also recommends indirect blood transfusion. He has twice seen it save life, not in pernicious anæmia, but (1) in a case of severe post-hemorrhagic anæmia, and (2) in a case of apparent morbus maculosus Werlhofii of the worst kind. In general, 
however, the subcutaneous injection of salt solution is preferred, as being the simplest procedure.

Symptomatic Therapy.-Finally, in the treatment of pernicious anæmia we often come to the point where our therapy must be merely symptomatic. For instance, for collapse or severe cerebral anæmia we use stimulants, particularly wine and camphor. The head should be lowered and the legs wrapped in tight bandages to force their supply of blood to the brain. Where there are severe external hemorrhages (from the nose, bladder, rectum, genitalia), styptics [or adrenalin solution-Ed.] must be applied locally; ${ }^{4}$ if there are signs of severe internal hemorrhage, styptics must be given subcutaneously or internally. Not infrequently profuse diarrhœa is present. This may be treated by giving $0.50 \mathrm{G}$. $(7 \mathrm{I} / 2 \mathrm{gr}$.) of tannigen 3 times a day, or $1 \mathrm{G}$. (15 gr.) of tannalbin every $2-3$ hours. If these fail, we have recourse to opiates, alone or in combination with the above-mentioned drugs or others.

\section{THERAPY OF LEUK AEMIA AND PSEUDOLEUK FMIA.}

Leukæmia and pseudoleukæmia are undoubtedly separate diseases clinically, but therapeutically there is no object in considering them apart, for the same agents are efficacious in both.

Arsenic.-The most powerful of all these agents is arsenic, either in the form of arsenious acid or in that of Fowler's solution. It must be given in relatively large doses (Billroth-Winiwarter). In using Fowler's solution, we begin with 5 drops a day in water or wine, and increase by 1-3 drops daily until we have reached 50 or even $60-100$ drops. The daily amount should be divided into 3 doses. If symptoms of intoxication appear, we are obliged to stop the increase of the dose or even to decrease it. If the intoxication shows itself only in a moderate diarrhœa, we may overcome it by giving opium along with the arsenic. In fact, it is better to obviate this complication by giving 10-15 drops of the tincture of opium with Fowler's solution up to 60 drops. As another preventive measure against intoxication, we give a mild laxative from time to time or large quantities of diuretic drinks, in order to free the body from arsenic and the possible products of cell decomposition caused by it. For this purpose we may employ 3-4 teaspoonfuls of the tincture of rhubarb. It has been recently stated that the taking of a good deal of fat (butter) will prevent arsenic poisoning.

Arsenious Acid.-Black pepper should always be administered with arsenious acid, in order to stimulate the mucous membrane of the stomach and so insure as quick an absorption of the arsenic as possible, thus avoiding any injury to the mucous membrane. The Asiatic pills furnish a convenient combination. Each pill contains 0.005 G. (1/12 gr.) of

- [I have succeeded, I believe, in preventing a recurrence of nasal hemorrhages in such cases by the internal administration of chloride or lactate of calcium in $10 \mathrm{gr}$. doses 3-4 times a day.-Ed.] 
arsenious acid, so that we need 1-3 pills to furnish the usual maximum dose (that is, of course, if no symptoms of intoxication appear), and we give as many as 8 pills a day $(0.04 \mathrm{G}$. $(2 / 3 \mathrm{gr}$. $)=60$ drops Fowler's solution).

We may also use tablets (see p. 169), giving 10-15 of those containing 0.0006 G. (1/100 gr.) of arsenious acid.

Naturally, we may also use mineral waters containing arsenic, provided much more than the usual quantity be taken, in order to get the requisite amount of arsenic.

Injections of Arsenic.-Arsenic may also be injected intraparenchymatously or subcutaneously in either leukæmia or pseudoleukæmia. But the injection into the spleen in pseudoleukæmia the author believes to be exceedingly dangerous, because (1) a case is recorded in which a fatal hemorrhage into the peritoneal cavity followed a simple exploratory puncture; (2) in Kussmaul's clinic, sudden death occurred after the injection of $0.10 \mathrm{G}$. (11/2 gr.) of sclerotic acid into the spleen; and (3) a case was personally known to the author where the post-mortem examination revealed a necrotic lesion at the spot where an exploratory puncture had been made in typhoid fever. So far as leukæmia is concerned, injections have no advantage over the internal use of arsenic and therefore should not be preferred. But in pseudoleukxmia and sarcoma of accessible lymph-glands, parenchymatous injections are of great value. They tend to diminish the swelling of the glands (or spleen), to reduce the anæmia, and to inerease the bodily strength. For this purpose, we use equal parts of Fowler's solution and distilled water with $1 / 2$ per cent. carbolic acid or 5 per cent. glycerin added to increase the permanency of the solution. At times we suceeed better by beginning with a weaker solution ( 1 part. of Fowler's solution to 2 of water). We may begin with 2-5 min. (5-10 min. in case we use the weaker solution) and increase to 10-20 min., ehanging the lymph-gland selected each time. For 3-4 hours after such an injection there may be severe neuralgic pains in that region and a rise of temperature. If we wish to inject into the spleen, an ice-bag should be laid over it an hour beforehand, in order to contract it and diminish the blood content.

Subcutaneous injection may be carried out in the same way, and does not produce symptoms of poisoning (burning in the throat, dyspeptic disturbances, diarrhœa, colic, exanthem of the skin) nearly as soon as does the internal administration. It is better to use Pearson's rather than Fowler's solution, as the sodium salt is much less of a local irritant than the potassium salt. We may prescribe as follows:

\footnotetext{
B Liq. sodii arsenatis (Pearson) $\ldots \ldots \ldots \ldots . . .10 .00 \ldots \ldots 3$ iiss

Aq. dest. ......................... $20.00 \ldots \mathbf{z} \mathrm{v}$

M. et sig. $-10-30 \mathrm{~min}$. for hypodermic injection.
}

The best place for subcutaneous injection is in skin well supplied with blood vessels. Katzenstein and Ziemssen have recently used such injec- 
tions with great success in pseudoleukæmia. Ziemssen, who lays great stress upon large doses of arsenic, begins with 0.25 G. (33/4 min.) of the solution daily, increasing to twice a day, until gradually he gives $0.02 \mathrm{G}$. $(3 / 10$ gr. $)$ sod. arsenate a day. The author has himself tried this subcutaneous injection in a number of cases. In several it was followed by a reduction of the swollen glands, and generally by an improvement in body weight and general condition, once with the entire disappearance of the intermittent fever which had earlier characterized the case. In 2 cases, which came to post-mortem, no alteration of the skin or subcutaneous tissue was found at the sites of injection. In general, then, the subcutaneous injection of Pearson's solution is well worth trying. If it is used for injection into the glands, the maximum dose must be only half the maximum dose for subcutaneous injection. The author, as has been said, uses atoxyl much more frequently, however.

Cacodylic Acid-Cacodylate of Sodium.-French authors have recently recommended the use of arsenic in another form,-cacodylic acid or sodium cacodylate (ascodyle),-maintaining that much larger doses of this than of arsenious acid can be given without danger of intoxication. For a more detailed discussion of this procedure, see p. 348. Its use is also mentioned in the chapter on pernicious anæmia. It is, to repeat, as good as arsenic, but no better, since its relatively large doses are no more effective than smaller doses of arsenic.

Quinine-Phosphorus.-Besides arsenic, both quinine and phosphorus are used in leukæmia and pseudoleukæmia, the former being usually combined with arsenic. Quinine is given as quinine hydrochlorate, 2 or 3 times daily up to $0.50 \mathrm{G}$. $(7 \mathrm{I} / 2 \mathrm{gr}$.) ; phosphorus is best dissolved in fats or ethereal oils; e.g.:

B Sol. phosphori (Thompson) $\ldots \ldots \ldots \ldots \ldots 30.00 \ldots \ldots$. $\mathrm{i}$

Ol. morrhuæ ................... s. ad $240.00 \ldots \ldots$. ว viii

Acaciæ,

Aq. dest...............................āa q. s.

Ol. menth. piperitæ................. $1.00 \ldots \ldots$ m $\mathrm{xv}$

M. fiat emuls.

Sig. $-\frac{1}{2}$ oz. 3 times a day. (Each $\frac{1}{2}$ oz. $=\frac{1}{48}$ gr. phosphorus.)

Inhalation of Oxygen.-The author also believes in inhalations of oxygen (20-40 gal. daily). In his own experience he has seen it produce, especially in leukæmia, reduction of the dyspnœa and anæmia, with an improvement of the strength and appetite.

Organotherapy.-Too few results are as yet at hand to judge of the efficiency of organotherapy. The author has himself used tablets made of bone, lymph-glands, and spleen in cases of splenomyelogenous leukæmia, apparently without effect, or at best with only transitory results.

Iron.-The author cannot recommend iron, iodides, or eucalyptus, the latter so highly advocated by Mosler in leukæmia.

Tuberculin.-The recent suggestion that leukæmia be treated by the injection of tuberculin does not appear to be at all justifiable. It is 
based on the well-known fact that if a patient with leukæmia is attacked by an acute infectious disease or tuberculosis, not only do the spleen and the lymph-glands grow smaller, but the leucocytes decrease in absolute number, and the specific leucocytes decrease relatively to the simple polynuclear ones. But even if similar phenomena do follow the injection of tuberculin, there does not seem to be any real improvement, to say nothing of the fact that it may have a detrimental effect from the beginning.

Roentgen Rays.-The newest method of treating leukæmia is by subjecting the spleen, bones, and lymph-glands to the Roentgen rays, generally every day. [Dangerously often.-Ed.] Although this treatment cannot cure a case of leukæmia, still, as long as it is kept up, a great improvement is manifest; the spleen and glands return to their normal size, the blood picture loses its leukæmic character, and the patient feels better. In pseudoleukæmia no success has been obtained so far by this method. [Dock reviewed the reports of 29 cases of leukæmia in which the Roentgen rays had been employed, and concluded that under this treatment some cases undergo marked improvement. In no case had the treatment been continued long enough to be called a cure. The rays probably act upon the tissues which produce the pathological leucocytes, either directly or through the production or setting free of substances that influence cell formation, degeneration, or chemotaxis, or all of these processes. The treatment should be followed with eareful metabolism investigations and very brief exposures allowed. Many of the eases in which this method had been tried, died with symptoms of acute general poisoning, as if the economy had been suddenly overwhelmed with enormous doses of disintegrated proteids.-Ed.]

Diet.-The diet in leukæmia and pseudoleukæmia should be as nourishing as possible and at the same time easily digestible, on account of the frequent occurrence of disturbances of the stomach and intestines. The following list may be recommended: shaved raw ham or beef, tender white meat (boiled ealves' brains, sweetbread, fowl, or squabs), jellies, rare roast beef, some game (venison, quail), such fish as trout and pike, caviar; milk, kefir, custards, eggs, and artificial meat preparations; spinach, carrots, asparagus, artichokes, green peas, mashed potatoes; wheat bread, rolls, dried bread, zwieback, erackers; apple sance, pear compote, orange compote, stewed plums; cocoa. chocolate; good wine and beer; bouillon, beef tea. (See p. 372.) Butter is generally the only fat permissible.

Mental and Physical Rest-Warm Climate-Baths.-As in all other diseases of the blood, mental and physical rest is imperative, and a residence in a warm climate, particularly in cold weather, is highly desirable. Sea-bathing is beneficial in the earliest stages, before the anæmia and cachexia are at all marked. Warm baths, fresh and salt, of about 10 minutes' duration, are to be permitted, unless they seem to be weakening. In their place douches of only a few seconds' duration 
may be used. These may be either cold or tepid, depending upon the grade of anæmia and the constitution of the patient, and should be followed by vigorous rubbing. If the patient is too weak to stand douches, dry rubs may be given as in chlorosis (p. 156), or the patient may be rubbed morning and night with some stimulating liquid; e.g.:

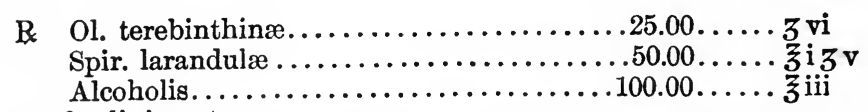

M. fiat linimentum.

Or :

Sig.-External use only. Rub in until the skin is slightly red.

B Chloroformi ......................... $15.00 \ldots \ldots$ ss

Tinct. capsici........................ $8.00 \ldots$ ii

Alcoholis,

Spir. odorati (N. F.)............ āā ad $120.00 \ldots \ldots$ z iv

M. fiat linimentum.

Sig. - For external use only.

We may also use cologne, wine vinegar, or brandy, sometimes diluted with water.

\section{THERAPY OF SECONDARY AN FMIA.}

In treating secondary anæmia, our first task must naturally be to remove the disturbing cause, of which the anæmia is merely a symptom.

Symptomatic Therapy.-We are sometimes obliged, however, to treat the anæmia as such, for (1) it may continue after the cause has been removed, (after intestinal parasites, after severe vomiting and diarrhœa, after internal hemorrhage, and after an infectious disease); and (2) it may be such a prominent feature of the whole symptom complex as to require special treatment (anæmia in interstitial nephritis, scrofula, and pulmonary tuberculosis).

Diet.-The diet in all secondary anæmias must be chosen with a view to repairing the lost tissue. Since, so far as we know, the proteids are the only tissue-building materials, while the fats and carbohydrates produce heat and spare the proteids, it is obvious that the diet must consist principally, but not exclusively, of animal food. The details of such a diet have already been given in the discussion of leukæmia and pseudoleukæmia (see p. 177). Naturally, however, this diet must be modified if the cause of the secondary anæmia is one which has also severely injured the digestive tract (severe vomiting, gastric or intestinal ulcers, etc.). Here we must begin with food which, while nourishing, is very easily digested, and only very gradually work up to the full diet. In the first stages of convalescence, we may give milk, albumin water, and soups made from tapioca, sago, etc., sometimes with meat preparations (somatose, 1 teaspoonful 3 times a day), or with hygiama, nutrose, eucasin, or sanatogen added. We may then gradually introduce raw scraped meat, meat jellies, brains, sweetbreads, bouillon, cold veal, fowls, dried bread, zwieback, crackers, stale white bread, and apple or plum sauce. In the 
third stage, we may allow by slow degrees earbohydrates (rice, noodles, farina, etc., in small quantities, other farinaceous foods), vegetables, some fat, and wine, beer, coffee, and tea. If the stomach will not retain any food, we must employ nutrient enemas (see p. 394).

Medication.-The medication must differ with the cause of the anæmia. Iron is contraindicated where there have been inflammatory or ulcerative processes in the stomach or intestine, and where fever is present. It should also be avoided in pulmonary tuberculosis. Although the author has not seen it produce hæmoptysis in such a case (Trousseau), he has, in common with other observers, noticed an undoubted exacerbation of the disease from its use. In a woman with obvious anæmia and only suspected apical tuberculosis, the administration of iron improved the condition of the blood but slightly, while the lesions at the apex made such strides that the patient lost rapidly and the mischief was repaired only by the use of creosote.

Arsenic appears to be contraindicated in inflammations of the digestive tract and in severe kidney lesions.

In case iron and arsenic are not contraindicated, they may be given under the same circumstances and in the same way as in primary anæmia. In a good many cases of secondary anæmia, (in convalescence, in latent tuberculosis without demonstrable apical lesion, in kidney diseases. in neurasthenia, and after hemorrhage,) fersan, an acid albuminate containing iron and phosphorus, is to be recommended. Since its phosphorus is in the form of a paranuclein compound, it is free from alloxuric bases and extractives, and is therefore available in the anæmia of gout and Bright's disease. In case of severe vomiting, it can, as Dr. Brunner (Triest) has suggested, be given in the form of a "fersan ice" (15 G. ( $\mathrm{I} / 2 \mathrm{oz}$.) of fersan, $10 \mathrm{G}$. ( $\mathrm{I} / 3 \mathrm{oz}$.) of sugar, $15 \mathrm{G}$. ( $\mathrm{I} / 2 \mathrm{oz}$.) of milk, vanilla to flavor, mixed and frozen). This is of especial use when the anæmia is caused by ulcer or cancer of the stomach, or by chronic gastritis. Alboferrin may be similarly used.

Anæmia caused by scrofulous glands is frequently treated with codliver oil, or with malt extract (a teaspoonful to a tablespoonful 1-3 times a day, either alone or in milk, beer, or bouillon).

Treatment at Spas.- The treatment at health resorts depends upon the cause of the anæmia:

1. Post-hemorrhagic Ancmia.-In post-hemorrhagic anæmia chalybeate waters (St. Moritz, Schwalbach, Cudowa, Koenigswart, see p. 163) are to be recommended in case the hemorrhage was external. If it was a uterine hemorrhage, Franzensbad or Elster or saline baths (Ebensee, Gmunden, Ischl, Sooden, Hall (Tyrol), Neuhaus .(Styria), Pyrmont. (Waldeck) are to be preferred. If the hemorrhage was from the stomach and intestines, Carlsbad or Vichy [or Hot Springs (Virginia), Saratoga -Ed.] is indicated.

2. Arcemia From Loss of Body Fluids.-In anæmia from loss of body fluids our common salt springs (Kreuznach, Nauheim, Kissingen, 
Homburg, Ischl), sea baths, or hydropathic sanatoria are indicated.

3. Ancmia After Overwork.-In anæmia from overwork, the patient should go to a mountain resort (St. Moritz, ) or to common salt springs, or to hot springs (Gastein), [Hot Springs, Virginia-Ed.].

4. Ancemia Caused by Nervous Conditions.-In this type, particularly in nervous dyspepsia in hysteria, the patient is best treated in a quiet sanatorium.

5. Post-Infectious Ancmias.-The treatment of post-infectious anæmias depends upon the nature of the infection.

(a) Ancemia Following Malaria.-In anæmia following malaria, the arsenic springs are of use (see p. 170). If there are coincident alterations in the liver, or symptoms of an abdominal plethora, Carlsbad or Vichy, Elster, Franzensbad, or Rippoldsau, [Mt. Clemens, French Lick Springs-Ed.,] are the proper places, or we may choose the earthy springs containing sulphates at Lippspringe (Westphalia), which contain 0.019 G. of carbonate of iron per litre.

(b) Ancmia Following Articular Rheumatism.-In anæmia following articular rheumatism, iron alone or with arsenic is indicated. The author does not believe in the use of manganese, which some authorities recommend (p. 165), but the liq. ferri-mangano-peptonate may be used. If there are no indications of rheumatism remaining, treatment at iron springs is beneficial, as in all other post-infectious anæmias. Suitable resorts are: Alexanderbad (Fichtelgebirge), Flinsberg (Prussian Silesia), Cudowa (Silesia), Driburg (Westphalia), Elster (Saxony), Franzensbad and Koenigswart (Bohemia), Kellberg (near Passau), Schwalbach (Wiesbaden), St. Moritz (Switzerland), Pyrmont (Waldeck), Vellach (Kaernten, the Austrian St. Moritz), Pyrawarth (Lower Austria), and Szliacs (Hungary).

(c) Ancemia Accompanying Tuberculosis.-In anæmia accompanying tuberculosis, iron, as has already been said (p. 179), is contraindicated. A high mountain or seaside climate is also not desirable, since they both make too great demands on the hæmatopoietic organs, which are not in condition to effect the necessary blood regeneration. Moderate altitudes and a warm climate are, therefore, indicated (see pp. 337-338).

(d) Ancmia Caused by Scrofula.-In anæmia caused by scrofula, the simple salt waters are very beneficial (see p. 412). Examples are: Kissingen (Bavaria), Soden (in the Taunus), Berchtesgaden (Bavaria), Hall (Tyrol), [Saratoga, Poland-Ed.]; the springs containing iodides and bromides, such as Aussee (Styria), Hallein (near Salzburg), Ischl (Upper Austria), Kreuznach (in the Rhine province), and Reichenhall (Bavaria); and the springs containing iodine, as Hall (Upper Austria), Csiz (Upper Hungary), Darkau (Silesia), Toelz Sanatorium (Upper Bavaria), and Lipik (Slavonia).

(e) Ancemia Caused by Syphilis.-In anæmia caused by syphilis (hereditary or acquired), the springs just mentioned and the hot sulphur springs may be used. Examples of the latter are: Aix-la-Chapelle 
(Prussian Rhine province), Baden (near Vienna), Herculesbad (near Nehadia in Hungary), St. Lucasbad (Buda-Pesth), Baden (near Zurich), Kaiserbad (Buda-Pesth), Pistyan (Upper Hungary), Schinznach (Canton Aargau in Switzerland), Trencsin-Teplitz (in the Carpathians), and Warasdin-'Toeplitz (Croatia).

[The table on page 182 gives the constituents of the more important sulphur waters of Europe and the United States.-Ed.]

6. Ancmia From Metal Poisoning.-These sulphur springs are also beneficial in secondary anæmia from chronic lead or mercury poisoning.

7. Ancemia From Diseases of the Genitals.-In anæmia from diseases of the genitalia (particularly in women), we sometimes use iron waters, sometimes hot or cold sulphur waters, sometimes salt waters or salt or mud baths, sometimes bitter waters.

8. Ancemia From Bright's Disease.-Chronic Bright's disease, even if it is not of a hemorrhagic nature, may give rise to severe secondary anæmia,-the clinical picture of pseudochlorosis. Here alkaline-iron springs are indicated; e.g., Franzensbad, Cudowa, Elster, Koenigswart, Pyrawarth, and Marienbad (Ambrosiusbrunnen). (See p. 121.)

\section{THERAPY OF ACUTE HEMORRHAGIC AN EMIA.}

In discussing the therapy of acute hemorrhagic anæmia, it will be presupposed that the bleeding has been stopped. If it has left behind it such an extreme anæmia that the life of the patient is threatened, we must have recourse to rectal, or, better, subcutaneous, infusion of salt solution, or to transfusion of blood (see p. 172). In less severe cases, autotransfusion (Schoenborn), a mild form of transfusion, may be employed. The patient's legs are raised, bound with elastic bandages wound from the periphery toward the centre, and kept in a position higher than the head. 'The bandages are left on $1 \frac{1 / 2-2}{2}$ hours, when the patient usually begins to complain of their painful pressure. They serve the purpose of driving as much blood as possible during the critical time to the brain, medulla, and heart.

At all events, the patient should be flat on his back, or even with his head a little lower than his body [elevating the foot of the bed-Ed.]. He should be kept warm by hot cloths and hot-water bags, and be given strong wine, brandy, or coffee with cognac, to stimulate the heart. If he cannot swallow, we may give alcohol in enemas or inject ether or camphor. If the heart action is threateningly weak, [infusion-Ed.,] renewed transfusion, or at least autoinfusion should be resorted to, the extremities should be wrapped in hot cloths, and the soles of the feet should be rubbed or brushed. When the danger of immediate death has passed, we must treat the post-hemorrhagic anæmia. Unless the hemorrhage came from the digestive tract, the diet may be the same as in leukæmia (see p. 177), and iron may be given, as in chlorosis (see p. 157). [In this form of anæmia and in many others it is well to remember that some patients prove more susceptible to drug treatment, so that when 
SULPHUR WATERS

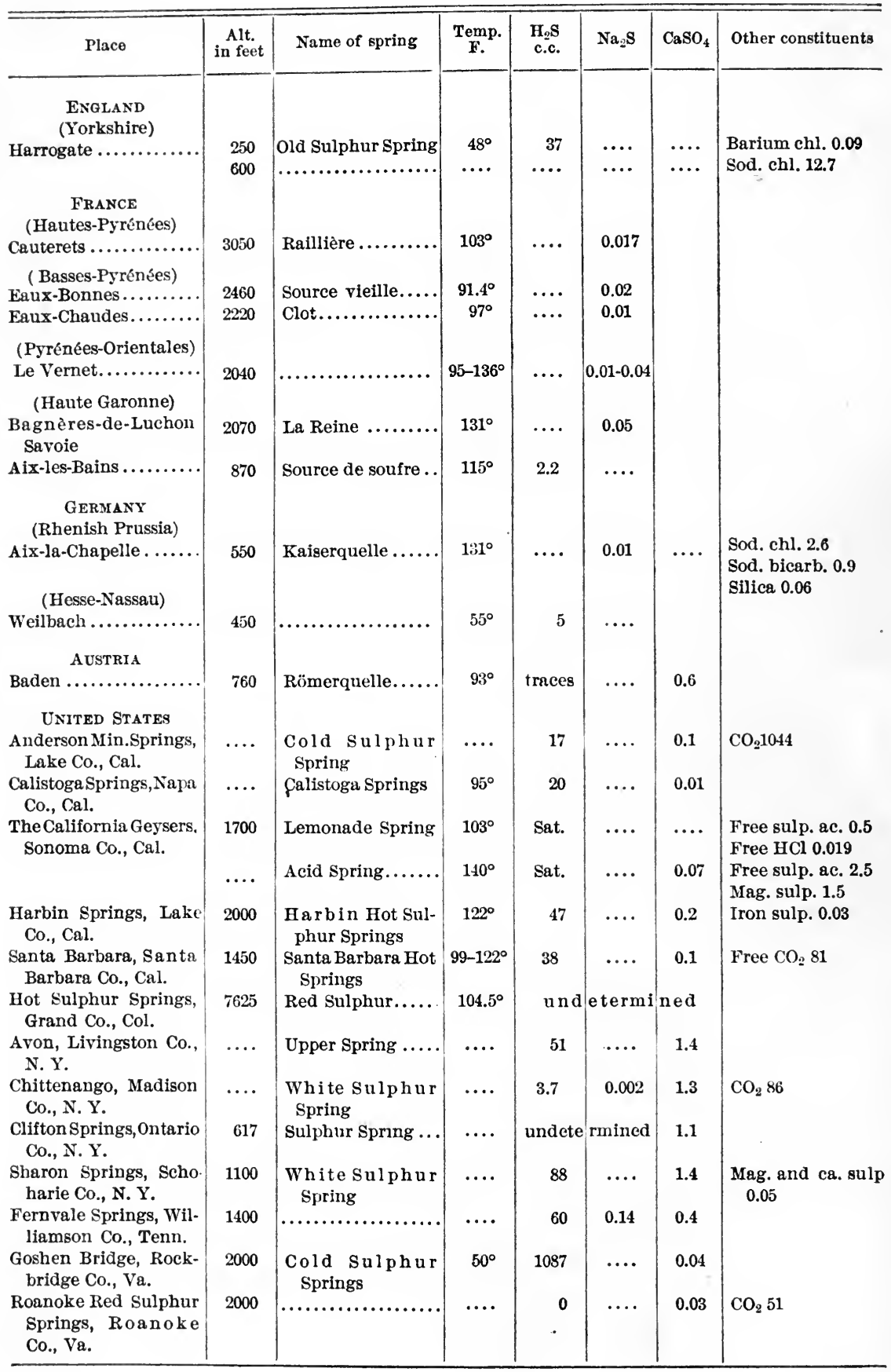


treating symptoms in these conditions it is advisable to begin with a small dose.-Ed.]

\section{THERAPY OF TRUE PLETHORA.}

True plethora, or-as a symptom-polyæmia, occurs in two forms: first, in a transitory or intermittent form in those individuals who are subject to regular recurrent hemorrhages, such as the menstrual flow, or nose-bleed which takes its place; secondly, in a permanent form when former constant hemorrhages (e.g., from hemorrhoids) have permanently ceased. It is also found in individuals leading very luxurious lives with little or no exercise.

The therapy of both these forms consists in the withdrawal of blood.

Venesection.-From his own experience, the author has found that venesection undeniably affords great relief. In many cases, however, this is merely temporary, so that repeated venesection is necessary.

Leeches.-The best method of local blood-letting is by means of leeches. If, as is so often the case, the patient complains of cerebral symptoms, such as pressure and throbbing of the veins in the head, giddiness, noise in the ears, spots before the eyes, etc., 3 leeches may be placed behind the ear or on the neck. Sacharjin advises 5 leeches at the end of the coccyx near the anus, to draw the blood away from the head, which process may be repeated in a week or two. The author has tried this method in a few cases and has found it uniformly successful.

Plethora with Stasis in the Portal Veins.-If true plethora is complicated by symptoms of stasis in the portal veins, particularly constipation, the latter must be treated by an appropriate diet and by laxative drugs or mineral waters. The diet should consist of meat and fish (not fat), with vegetables, salads, milk, eggs, and, especially, raw and cooked fruit. Farinaceous foods, sugar, sweets, peas, beans, and potatoes should be forbidden, and all carbohydrates and fat restricted as much as possible. Water, cider, grape juice, and suitable mineral waters are permissible; beer, wine, and all carbonated drinks are forbidden. A grape-cure or a whey-cure is advisable, as is the use of cream of tartar or tamarind water, as all of these are laxatives.

Mineral Waters.-For mineral waters we may use the cold alkalinesaline springs (Marienbad, Franzensbad, Tarasp, the cold springs at Vichy, Rohitsch (in Styria), [Mount Clemens, French Lick SpringsEd.,] although the latter contain a good deal of carbon dioxide). The warm waters at Vichy have a constipating effect, on account of their temperature; consequently, they must be taken with great caution unless they are first cooled. The bitter waters are also good (Friedrichshall, Hunyadi János, Franz Josef, A penta, Püllna (Bohemia), and Saidschitz).

Exercise.-A good deal of exercise should be taken, but it must be chosen very carefully and increased very slowly if with the plethora a hypertrophy of the left heart has developed. Finally, if a marked development of fat is combined with the polyæmia, the treatment is the same as that for obesity (see p. 234). 
To this list of diseases of the blood or of the blood-producing organs, there will be added a few others, one of which is properly a constitutional disease, while the correct classification of the others is not wholly clear. They will be treated here, because clinically and therapeutically they have much in common with the diseases of the blood. They are (1) hæmophilia, and (2) purpura, which may be subdivided into (a) scurvy, (b) morbus maculosus Werlhofii, (c) purpura rheumatica, and (d) purpura simplex. So little do we know about their proper therapy that the discussion will occupy but a few pages.

\section{THERAPY OF HAMOPHILIA AND OF PURPURA HEMOR- RHAGICA.}

Therapy of HamophiLIA.-Aside from prophylactic measures (avoidance of trauma), the therapy of hæmophilia must consider (1) the stage of actual bleeding, and (2) the periods between hemorrhages. In the first case, absolute rest and applications of cold are indicated, as well as styptics in case of internal hemorrhage (gelatin, adrenalin, ergot, ferrum sesquichloratum, hydrastis, hamamelis, lead acetate, and opium), and surgical aid in case of external hemorrhage. In the latent periods we must keep in mind three aims: (1) to strengthen the body, (2) to economize the strength, and (3) to lessen the danger of a threatening hemorrhage, which is frequently preceded by congestive manifestations. For the latter purpose, we may use hot foot-baths, or, especially, repeated doses of mild cathartics. Absolute rest in bed during menstruation is necessary. The diet should be a mixed and easily digested one; many authorities recommend green vegetables, though without any very well founded reason. Milk is exceedingly valuable. Anything which excites the heart should be avoided; e.g., alcohol, tea, and coffee. Careful hydropathic measures, such as dry rubs or the baths described under chlorosis (p. 156), or sea bathing in summer, help to strengthen the organism. It is best to send the patient for the winter, or, better, for the entire year, to a southern climate (the Riviera, Corsica, Egypt, [the Carolinas, Florida, Southern California-Ed.],) as the disease is much less common in warm than in cold countries. The strength may also be increased by suitable tonics (cod-liver oil, quinine, arsenic, kola). Many authorities also recommend iron. It has recently been claimed that the use of chemically pure calcium chloride will increase the coagulability of the blood and so decrease the danger of hemorrhage. It is given in powder form (up to 3 G. ( 45 gr.) or even 5-6 G. (75-90 gr. a day), or, better, to avoid any corrosive effect, in some such a mixture as follows:

$\mathrm{B} \quad$ Calcii chloridi................... $2.00-6.00 \ldots \ldots, \mathrm{zss}-3$ iss

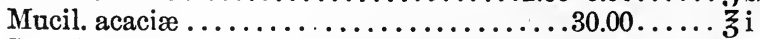

Syr. limonis...........................15.00.... $3 \mathrm{ss}$

M. fiat emuls

Aq. dest. ...........................

Sig.-To be taken in tablespoonful doses throughout the day, until all is taken. 
[Following Wright's investigations, calcium lactate ${ }^{5}$ or chloride should be administered internally in doses of 10-20 grains 3 to 6 times a day.

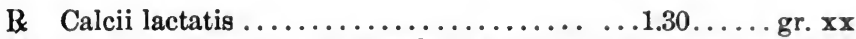

M. fiat. Chart. no. i. Dent. tal. dos. no. xii (in waxed paper).

Sig. -1 powder 3-6 times a day.

Calcium lactate seems to be absorbed somewhat more rapidly but not invariably better than calcium chloride. Until we know the etiology of hæmophilia, we are not apt to find any specific remedy either for the disease itself or for its local manifestations (Sahli). A general improvement of the nutrition should be aimed at. Special food stuffs, such as vegetables, probably aid in attaining this aim. Sahli does not believe that the internal administration of gelatin is based upon rational premises, because the gelatin does not reach the blood as such. He considers the internal or subcutaneous administration of adrenalin as dangerous; but advises its use as an external hæmostatic. For the same purpose, he recommends the local application of cotton soaked in a 1 or 2 per cent. solution of calcium chloride. Weil claims to have accomplished good results in one case of hæmophilia by repeated intravenous injections of 10-15 c.c. of healthy human serum. Bovine serum was not so efficacious. The dangers of the method would seem sufficient objection to its employment.-Ed.]

Therapy of Purpura Hemorrhagica.-The treatment of a hemorrhagic diathesis, with the exception of scurvy, is almost entirely symptomatic. Styptics are used in morbus maculosus Werlhofii, in purpura simplex, and purpura rheumatica. The following are examples:

B Ext. ergotæ fluidi ..............

Syr. limonis...........................

Aq. dest. ......................... $150.00 \ldots$

M. fiat mist.

Sig. - 1 tablespoonful every 2 hours.

Or :

B Ext. hydrasti fluidi $\ldots \ldots \ldots \ldots \ldots \ldots \ldots 24.00 \ldots \ldots z$ vi

Vin. xerici....................... s. ad $180.00 \ldots \ldots$ วิ vi

M. fiat mist.

Sig. - 1 tablespoonful every 3 hours.

Or :

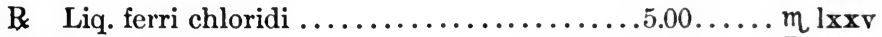

Vin. xerici $\ldots \ldots \ldots \ldots \ldots \ldots \ldots$. . s. ad $180.00 \ldots \ldots$. $\tilde{\tilde{z}}$ vi

M. fiat mist.

Sig. $-\frac{1}{2}-1$ teaspoonful 3 times a day.

Or :

B Liq. ferri chloridi .................... $1.00 \ldots \ldots \mathrm{m}_{\mathrm{xv}}$

Aq. cinnamomi.................. s. ad $180.00 \ldots \ldots$. $\tilde{z}$ vi

M. fiat mist.

Sig. -1 tablespoonful every 2 hours.

${ }^{5}$ [It is well to specify either Merck's or Squibb's soluble calcium lactate.-Ed.] 
If there is severe pain and swelling in the joints (peliosis rheumatica), antirheumatics should be given (sodium salicylate, aspirin, acetopyrine, antipyrine, salipyrin). The extremities should be raised, supported, and wrapped in light cotton compresses, or surrounded by hot bandages wrung out in vinegar.

Calcium chloride is recommended in purpura hemorrhagica, as well as in hæmophilia. The author has used it in severe cases of purpura, continuing the treatment for some time. In most cases it had no effect, not preventing renewed hemorrhages. In 1 case, however, with obstinate hemorrhages from the stomach and kidneys, it was the only drug that would prevent recurrence of the bleeding. [To obtain the best effects from calcium chloride or lactate, the diet must be very carefully regulated. All acids, acid fruits, strawberries, crabs, lobsters, other shellfish, and egg-albumin (Wright's decalcifying agents) must be avoided. Moderate catharsis should be secured and 0.6-2 G. (10-30 gr.) of one of the above salts administered. If these details are regarded, successful results in controlling hemorrhage will generally be attained.-Ed.]

In another case of morbus maculosus Werlhofii with very severe metrorrhagia, the latter was stopped immediately by one injection of gelatin ( 0.50 G.: 50 c.c. of water ( $7 \mathrm{gr} .: 11 / 2$ oz.) ; see p. 82$)$, and equally good results were obtained in a case of severe hemorrhagic diathesis consequent upon icterus. The author would, therefore, in all cases of hemorrhagic diathesis and severe hemorrhage, recommend gelatin injections where the object is to stop the bleeding, and calcium chloride when we wish to prevent the recurrence of hemorrhage.

\section{THERAPY OF SCURVY.}

Dietetic Therapy.-The old theory that scurvy is due to a lack of potassium salts in the food, is disputed. Nevertheless, the diet that experience has proved useful takes the first place in the therapy of the disease. It consists of green vegetables (spinach, cresses, cabbage, sorrel, lettuce, etc.), and of fresh ripe fruit, particularly fruits rich in acids (lemons, oranges, melons, gooseberries, and grapes). English authors especially recommend potatoes as being very rich in potassium and poor in sodium. The scorbutic patient should also eat a good deal of meatparticularly scraped raw beef-and milk in all forms. The Russians recommend an exclusively milk diet for from 1 to 3 weeks, claiming that this will effect a complete cure. A recent theory is that scurvy is due to a lack of fats rather than of potassium salts. Keeping this possibility in mind, we should give butter, and, if the state of the digestive organs will allow, other fats. All authorities agree in attributing peculiar virtue to lemonade. Since 1854 it has been served in English merchant ships every 5 days. Beer, wine, and cider may be given also.

The patient should, of course, have plenty of fresh air and sunshine. Exposure to cold and dampness is often among the causes of the disease. 
Medication.-There is no well grounded scientific medication for scurvy. At one time herba cochleariæ (scurvy-grass, similar in action to horse-radish) was given as a matter of course, and later potassium salts were considered the rational remedy. Again, acids were strongly advocated (citric, sulphuric, hydrochloric). Brewers' yeast is still often given, perhaps on account of its potassium (200-300 f. (6-10 oz.) a day, pure or in sweetened water).

Digestive Tract.-Especial care must be taken to keep the digestive tract in good condition. We may use bitters and aromatic tonics (quinine, cascarilla, calumba, absinthe, gentian, calamus), and mild laxatives (castor oil, cascara sagrada, tamarinds, frangula).

Stomatitis.-Care must also be taken of the gums. All carious teeth or stumps should at once be removed. If stomatitis is already present, the mouth may be washed out with potassium chlorate; e.g.:

B Potassii chloratis..................... $8.00 \ldots \ldots$ ii

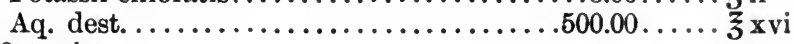

M. fiat mist.

Sig.-To be used as gargle during the day.

The affected spots should be carefully painted with astringents (see p. 271).

In case of painful infiltration of the blood into the muscles, most frequently into those of the lower extremities, cloths wrung out in 1 part of vinegar to 2 parts of hot water may be applied.

Styptics.-If hemorrhage occurs from the mucous membranes or into the internal organs, surgical and medical styptics are indicated.

Douches.-If the state of the patient and of his blood permits, very short cold douches are beneficial; if not, tepid baths. Not infrequently. the patient may faint away during the latter procedure.

\section{THERAPY OF SCROFULA.}

Relations Between Scrofula, Glandular Tuberculosis, and PseuDOLEUKAMIA.-Although scrofula is usually classed either among chronic infections or among constitutional diseases, it will here be appended to diseases of the blood, because (1) internal medicine has to do with scrofula chiefly in the form of swollen glands (external lymph-glands, bronchial or mesenteric glands), in which form the disease has to be differentiated-and sometimes only with difficulty-from pseudoleukæmia; and (2) scrofula and pseudoleukæmia have similar relations to tuberculosis. Cases of febrile pseudoleukæmia have recently been recorded where the post-mortem examination has shown partial central cheesy degeneration of the lymph-glands, and a few tubercle bacilli,often so few that they could be demonstrated only by inoculating animals with the material. Whether this form, which is still called pseudoleukæmia, is always a primary tuberculosis of the lymph-glands, or whether the appearance of the tubercle bacillus is sometimes merely 
secondary, is an open question. The same difference of opinion prevails in regard to scrofula. Some authorities consider it simply a form of tuberculosis; others believe that it is a separate constitutional anomaly, although one particularly favorable for the development of tubercle bacilli.

The discussion of the therapy of scrofula will be limited to the scrofulous-lymphatic diathesis, and the scrofulous affections of the lymphglands, the questions of prophylaxis and diet in scrofulous infants, and of the local therapy of the different scrofulous affections of the skin, nose, eye, ear, joints, and bones being left to the specialists.

DIET.- The diet must be very carefully chosen so as to be easily digestible, rich in proteids, and relatively rich in fats. Starches must be taken in only small quantities. Food containing much cellulose or sugar must be reduced to a minimum, while sugar itself must be absolutely prohibited. For, although it is certain that the scrofulous tendency may be congenital, it is equally true that the actual process may be awakened, if not caused, by eating too much coarse bread, farinaceous foods, potatoes, and sweets of all kinds. The diet should, therefore, consist largely of animal foods ; e.g., roast beef, veal, mutton, lamb, ham, birds, and game. Cooked fruit, rice, macaroni, noodles, etc., may be given with the meat. Toasted bread and zwieback, and raw fruit in moderation, are permissible. Potatoes should be mashed and softened with milk, and even then should not be served very often. Peas, beans, lentils, etc., may be used in purée form, or in the fine, dextrinized leguminous meals (Hartenstein, Knorr, Timpe, Maggi, Liebe, and Liebig) made into gruel or added to broth. Milk, eggs, cream, and butter are particularly suitable, on account of their fat content. For breakfast café au lait or, if diarrhœa is present, acorn coffee or acorn cocoa is allowed. Broths, wine, and good beer are all useful as stimulants to the sluggish digestion and delayed metabolism so often found in these cases.

In an Erethic Diathesis-In a Torpid Diathesis.-Among these articles of diet, the choice must depend upon which type of the scrofulous diathesis we are dealing with, the erethic or the torpid. Both exhibit symptoms of anæmia, poor nutrition, and a slight febrile tendency, particularly if the disease has assumed any local form (affections of the skin, mucous membranes, nose, ears, joints, or bones, or ulcerated upper lip). But the erethic type is distinguished from the torpid by a delicate, slight frame, mental alertness, and nervous excitability, and large eyes with a remarkably bluish shining sclera. On the other hand, the torpid type, especially in children, is marked by a thickset frame, flabby, pasty skin and subcutaneous fat, physical and mental slowness, sluggish digestion, and a large abdomen. In erethic cases we should give milk in all forms and abundant fat, restricting the amount of meat and forbidding wine; in torpid cases, lean meat, soup, a little fat, eggs, rice, fruit, and wine. Both types of patients must always have plenty of fresh air and proper attention to the hygiene of the skin. 
Medication: Oleum Morrhuæ.-One of the favorite preseriptions for a serofulous diathesis is cod-liver oil. Its good effect is due simply to the fact that it furnishes a form of fat that is readily absorbed. It is suited to the erethic but not to the torpid diathesis. The favorite form is oleum morrhuæ; the brown varieties have the advantage over the lightcolored of containing morrhuic acid and peculiar organic bases (penthylamine, amylamine, hexylamine, aselline, jecorine, ete.), all of which stimulate metabolism. Cod-liver oil is given in rather large doses and is best enclosed in gelatin capsules, on account of its unpleasant and often nauseating taste.

R Ol. morrhuæ.............1.00-3.00-5.00.... $m x v-m v l-\eta \mid x x v$ Fiat caps. no. i. Dent. tal. caps. molles no. 1.

Or: Sig. - 4-5 caps. a day.

R Ol. morrhuæ...................200.00.... zi vi

Sig. $-\frac{1}{2}-1$ tablespoonful 3 times a day (for small children 1 teaspoonful 3 times a day).

Cod-liver oil is given for from 3 to 4 weeks and then omitted for a week. On account of the readiness with which it is decomposed, it is better to omit it during warm weather. It should be given half an hour after eating, a peppermint drop or a bit of orange peel being taken directly before, and coffee, sugar, rum, or bread directly afterward. It may also be taken in soup or milk, or beaten up. with the foam on beer. Some authorities-particularly the French-recommend still larger doses, at least $100 \mathrm{G}$. ( 3 oz.) a day.

On account of its rather better taste, the oleum morrhuæ aromaticum (Standke) may be preferred to ordinary cod-liver oil, if expense does not have to be considered.

A substitute for cod-liver oil is von Mehring's lipanin, fine olive oil with 6 per cent. of oleic acid. It is prescribed as follows :

R Lipanini........................ $300.00 \ldots \ldots$

Sig. $-3-5$ tablespoonfuls a day for adults; $1-4$ for children.

To give iodine with the cod-liver oil-a rather old-fashioned procedure-we may use oleum morrhuæ iodatum (1 per cent. iodine), or oleum morrhuæ ferro-iodatum, if iron is also indicated. The dose of either is 2-3 tablespoonfuls a day. The syrup of ferrous iodide may also be employed (see Iron Preparations, p. 159).

Another substitute for cod-liver oil, particularly useful when the latter brings on diarrhœa, is malt extract. It may be prepared with iron, iodine, or ealcium (where scrofulous diseases of the bones are present). The dose is a teaspoonful to a tablespoonful, alone or with milk, etc. Equal parts of malt and of cod-liver oil is a favorite prescription.

Creosote.-Creosote is another drug which is useful in a scrofulous diathesis (Sommerbrodt). It may be given undiluted, or diluted with 
compound tincture of gentian (1:4). We may also use the creosotecarbonate (creosotal), solveol (see p. 347), the oleum morrhuæ creosotale (1-2 G. (15-30 min.) creosote : 100-150 G. (3-5 oz.) of oleum morrhuæ), the dose being 1-2 tablespoonfuls a day. Or creosote and codliver oil may be given together' in gelatin capsules.

R Creosoti ............................ ii

Ol. morrhuæ............... $0.50-1.00-2.00 \ldots \ldots m_{v i i-} m_{x v}-m_{x x x}$

Dent. tal. caps. no. c.

Sig.-Up to 20 caps. a day. (Creosote $=0.20)$ gtt. iii (pro dosi; 1.00 ) gtt. xv (pro die.)

A Russian author, Dr. Eck, has recently come out very strongly in behalf of creosote in scrofulous diathesis, claiming that it relieves the dyspeptic disturbances, increases the appetite, reduces the swelling of the glands and the affections of the eye, and even has a favorable influence on the pathological processes in the bones and joints.

Tuberculin.-A modern remedy, which is claimed to be a specifie, must be mentioned-tuberculin. Such an able and critical observer as Biedert maintains that it arouses the torpid cell infiltration, stimulates healing, and dries up permanently the softened or purulent glands. We begin by injecting subcutaneously $0.10 \mathrm{mg}$., and if there is no febrile reaction, increase this every day, at first by only $0.10 \mathrm{mg}$., until $0.50 \mathrm{mg}$. are given, then by $0.50 \mathrm{mg}$. or even by 1,2 , or $3 \mathrm{mg}$. If a reaction occurs the dose is not increased beyond the point where the reaction appeared, until this quantity has been borne with no reaction 2 to 4 times on 2 to 4 successive days. Scrofulous processes in the bones, eyes, skin, and mucous membranes, he asserts, are helped by this method, even when they have resisted many other forms of treatment.

Rubbing with Soft Soap.-Rubbing with soft soap has proved itself of undeniable use, not from any specific action, but from its general stimulating effect, first on the skin, and then on the circulation and metabolism. The improved circulation in and around the torpid cells, be they in the skin, joints, bones, or glands, prepares the way for their absorption. The technic, as given by the originator of the method, Dr. Kapesser, is as follows: For 10 minutes in the evening the back is rubbed with a tablespoonful of soft soap diluted with water; half an hour later the soap is washed off with warm water. If the back is red to begin with, from previous treatments, the chest may be rubbed (particularly if the bronchial glands are affected) or the abdomen (if the mesenteric glands are affected). In the case of a child, the soap should be rubbed into the backs of the thighs and the buttocks as well as the back, and should be allowed to remain on only 10 minutes. The process should be repeated every day, or every other day, or 3 times a week, according to the degree of reaction obtained.

Climate: Choice of Health Resort.-When the patient is to be sent to a health resort, the choice must depend upon (1) whether it is an 
erethic or a torpid case, (2) whether it is a question merely of a scrofulous diathesis or of definite local lesions, and (3) whether the latter are slight or extended. In case of a general scrofulous diathesis, we must choose such a place as will stimulate metabolism and increase the patient's resistance; in case of a local lesion, one that will favor the absorption of the products of the pathological processes.

In the first instance, the climate is the most important factor. For poor children various colonies have been organized where they can have the benefit of a suitable climate (Schloss Thernberg near Scheiblingkirchen, Altenmarkt, Schönau near Leobersdorf, Giesshübl near Brunn, Ischl in the Salzkammergut). Here, in a few weeks, the children gain enough to be equal in bodily development to the normal children of their age, whom they have been a year or so behind (Schmid-Monard). If the circumstances of the patient permit a free choice, he may be sent in summer to Grado, Ischl, Aussee, Baden-Baden, or Wiesbaden; in spring and fall to Grado, Tyrol (Bozen-Gries and Arco), Ragusa, Cattaro, the Lake of Geneva, or the upper Italian Lakes; and in winter to the two latter, to Tyrol, and particularly to the Riviera, South Italy, Sicily, Abbazia in Istria, and the islands of the Mediterranean.

A pronounced erethic case may remain all the year round at the winter resorts.

The bathing-places on the Baltic may also be used for summer resorts, even if the sea baths are not utilized-Ahlbeck, Swinemünde and Heringsdorf (all on the island of Usedom), Ahrendsee (Mecklenburg), Bellevue near Kiel, Binz and Breege (on the island of Rügen), Glücksburg (Schleswig-Holstein), Kohlberg (with a special sea bath for scrofulous children), Misdroy (on the island of Wollin), Travemünde, Warnemünde, the port of Rostock, and Zoppot near Danzig.

Effect of Sea Air.-The sea-coast produces its good effect on account of the constant breeze, off the shore during the night, off the water during the day. This takes more heat from the body than normal, thus causing increased oxidation in the tissues, improved appetite, and better metabolism. The dampness and salt in the air prevent the steady wind from being unpleasant.

Baths ANd Mineral Waters: Sea Baths.-In general, the patient at these, as well as at all seaside resorts, should add to the advantages to be obtained from the climate alone, those of sea bathing. Salt water has an undeniably good effect on a person with a scrofulous diathesis, stimulating the organism and favoring absorption. Sea bathing is, therefore, especially indicated for the second class of patients, those in whom pronounced local lesions are present. Caution is, however, necessary in the selection of the place. Erethic individuals should be sent to the Mediterranean, Adriatic, or Baltic; while torpid patients may be sent to the North Sea or the Atlantic Ocean, where the colder temperature and rougher waves demand a tolerably good power of resistance. At certain places, sea bathing is possible all the year round (Dollfuss on the Ri- 
viera, several Adriatic resorts, Glücksburg on the Baltic). The length of the bath should depend on the temperature and roughness of the water, but should not exceed a quarter of an hour.

Several sanatoria for scrofulous children have been established at the seashore, where they can have the advantages of sea air and sea bathing and yet can be sheltered during bad weather.

Natural Salt Baths.-Baths in natural salt spring waters are also beneficial. Their effect is due to their content of chlorides (particularly common salt) and, in many cases, of carbon dioxide. The salts penetrate by endosmosis through the epidermis to the upper layers of the cutis, where they cause a larger amount of water to be given up by the deeper portions of the skin. This loss of water from the tissues stimulates the cutaneous nerve-endings. The stimulation passes to the central nervous system and thence, reflexly, to the different centres, particularly the vasomotor and cardiac centres. In this way, metabolism is stimulated and conditions more favorable for the absorption of pathological cell products are brought about. Naturally, the temperature of the water is an important factor. Biedert attributes to " the combined actions of the salts and of the cold, a hardening and strengthening of the skin, a stimulation of the entire nervous system, and, in connection with drinking the waters, an improvement in the state of nutrition and an absorption of pathologic exudates and deposits, brought about by the improvement in general strength and the increase of metabolism." The colder springs and those richer in salts are suited to torpid cases; the warmer, and those less rich in salts, to erethic cases. The greater the amount of carbon dioxide, the lower may be the temperature. The temperature varies from $80^{\circ}$ to $95^{\circ}$; the percentage of salts from 1 to 10 per cent. and over; the duration of the bath from 10 to 45 minutes. For a list of salt baths, see p. 180. Generally, the baths are combined with a course of mineral water from the weakest of the salt springs. Sometimes on this account salt springs containing iodine and bromine are preferred, particularly Bad Hall, in Upper Austria. (For a list, see p. 180.) These are not, however, to be recommended where there is a tendency to obstinate recurrent bronchial catarrh as one of the symptoms of the scrofulous diathesis. In that case, the simple saline waters or the weakest of the salt springs, are preferable. Of the common saline waters used for drinking, examples are those at Homburg, Kissingen, Salzschlirf (HesseNassau), and Soden in the Taunus; of the weaker salt springs, which may be diluted so that they can be drunk, Berchtesgaden (Bavaria), Hall (Tyrol), Koesen (Merseburg), and especially Aussee, Gmunden, Ebensee, Ischl, and Reichenhall. Ischl has also two regular saline springs used for drinking, the Klebelsberg and the Maria Louisa springs.

Salt Baths at Home.-If it is impossible for the patient to take salt baths at the seashore or at salt springs, they may be given at home. For details, see p. 171 . The temperature should vary between $89^{\circ}$ and $95^{\circ}$, the higher temperature being chosen when the content of salt is small 
(1-7 per cent.), or when the patient is particularly anæmic and delicate. The bath should at first last only 10 minutes, but may afterward be gradually extended to 30 or even 45 minutes. The number of baths in one course of treatment should be 15-20 for small children, and 2530-50 for older ones. Torpid cases require the baths every day; erethic cases only every other day at first, and afterward three days out of four.

Alkaline and Alkaline-Chloride Waters.-The simple alkaline and alkaline-chloride waters are suited for cases with bronchial eatarrh. Examples of the first are, Bilin (Bohemia), Giesshübl (near Carlsbad), Preblau (Carinthia), and Salzbrunn (Silesia); examples of the second, Ems (on the Lahn), Gleichenberg (Styria), Luhatschowitz (Moravia), and Selters (Hesse-Nassau). If these waters are to be taken at home, we prescribe $1 / 2-1$ pint during the day.

One possibility remains to be mentioned: we may meet cases of very obstinate glandular swellings which refuse to yield to salt baths or sea bathing. In such instances, a year's residence on the North Sea or at one of the high Alpine resorts should be tried. Gossensass and Brenner, Toblach in the Pusterthal, Semmering, and particularly St. Moritz, Klosters, and Davos in Switzerland are examples of the latter; Borkum, Dangast (opposite Wilhelmshaven), Fanö, Helgoland, Norderney (Hanover), Ostend (Belgium), Scheveningen (Holland), Sylt (SchleswigHolstein), Westerland, Wenningstedt, Wyk on the island of Föhr, and Wangerooge, are examples of the former.

Local Treatment of Scrofulous Glands.-Scrofulous glands may be rubbed with mercurial ointment; dressed with a Priessnitz bandage with a 0.02 per cent. solution of bichloride of mercury, or a 0.50 per cent. solution of solveol or iodine water; painted once a day with tincture of iodine (or with this and tinctura gallarum in equal parts); or rubbed with iodine-potassium iodide ointment (iodine 1 , potassium iodide 2, vaseline and lanolin āā 15), with iodinevasogen (6 or 10 per cent.), or with ichthyol ( 1 part of ichthyol to $5-1$ parts of vaseline or glycerin). Applications of ice may be tried if there is local inflammation. In chronic cases, rubbing with soft soap, as described above (p. 190), is especially good. Biedert, from his own experience, recommends Lebert's ointment (red biniodide of mercury, 0.25: 20 fat). As this may induce dermatitis, it should be used only for a short time once a day.

[I do not differentiate tuberculous glands from scrofula. The latter I regard as an old name alone. Modern investigations still induce me to separate pseudoleukæmia from tuberculous glands, although I acknowledge that cases do occur in which it is impossible to make a distinction during life. I cordially approve the author's dietetic, balneological, and climatic therapy, and usually attempt to distinguish cases requiring an excess of proteids and fats from those improving most when milk and the carbohydrates compose the bulk of the dietary. So many individual peculiarities, however, require attention that no sharp line can be drawn 
between the two groups. For many years, indolent glandular and bone tuberculosis in children has exhibited striking improvement at the seashore during the summer months, but it is only recently that the attention of the medical profession has been called to equally favorable results obtained during the winter. Our Atlantic seaboard from Cape Ann to Cape Hatteras offers numerous favorable locations. Selection will depend upon proximity or the individual peculiarities of the case. On our Pacific coast sea bathing is feasible the year round. For a brief mention of some of the most desirable climatic resorts in America, see pp. 333-340.

Under medicinal therapy, a few additional preparations of cod-liver oil, favorites in America, may be mentioned:

Emulsio Phosphatica (Nat. Form.).

$\mathrm{B} \quad$ Ol. morrhuæ.....................250.00... $\zeta$ viii $z$ iiss

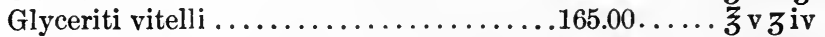

Acid. phosphorici diluti ...............50.00.... $3^{\mathrm{i}} \mathrm{z}_{\mathrm{v}}$

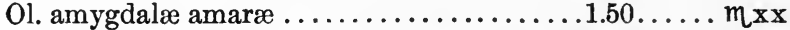

$\operatorname{Rum}^{6}$. ........................250.00.... $\xi$ viii $z$ iiss

Aq. aurantii flor. ............... s.ad $1000.00 \ldots \ldots$. 3 xxxii

M. fiat emuls.

Sig. $-\frac{1}{2}$ oz. 3 times a day.

Emulsum Olei Morriete cum Hypophosphatibus (U. S. P.).

B Ol. morrhuæ....................500.00.... $3 \times$ xvi

Acaciæ .......................... $125.00 \ldots \ldots$ iv 3 i

Calcii hypophosph..................10.00.... 3 iiss

Potassii hypophosph.................5.00.... 3 i gr. xv

Syr. simplicis .....................100.00.... 3 iii 3 iiss

Ol. gaultheriæ......................... $4.00 \ldots \ldots z \mathrm{z}$

Aq. dest. .................... s. ad $1000.00 \ldots \ldots$. 3 xxxii

M. fiat emuls.

Sig. - $\frac{1}{2}$ oz. 3 times a day.

Emulsto Olei Morrhue cem Calcir Lactophosphate (Nat. Form.).

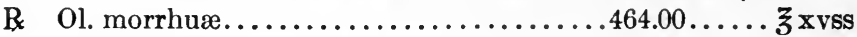

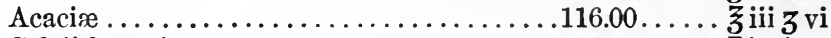

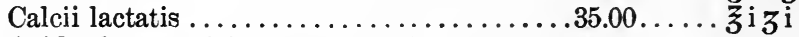

Acid. phosphorici ( 85 per cent.)........20.00.... $3 \mathrm{v}$

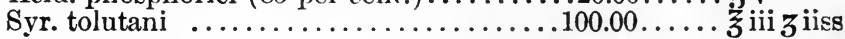

Ol. gaultheriæ ..........................

Aq. dest....................... s. ad $1000.00 \ldots \ldots$ J $3 \times x i i$

M. fiat emuls.

Sig. $-\frac{1}{2}$ oz. 3 times a day.

Emulsio Olei Morreude cum Extracto Malti (Nat. Form.).

B Ol. morrhuæ....................500.00.... $3 \times$ vi

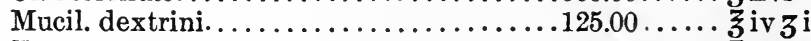

Ext. malti.......................375.00.... 3 xiiss

M. fiat emuls.

Sig.-As directed (usually $\frac{1}{2}$ oz. t. i. d.). of rum.

${ }^{6} \mathrm{~A}$ much more palatable preparation is obtained by the use of brandy instead 
Emulsio Olei Morreu a cum Syrdpo Ferri Iodidi.

B Ol. morrhuæ.....................250.00.... 3 viii 3 iiss

Acaciæ........................... $125.00 \ldots$ iv $3^{i}$

Syr. ferri iodidi.................... $125.00 \ldots \ldots$. ${ }^{i}{ }^{i}$

Aq. dest. ...................... s. ad $1000.00 \ldots \ldots$. 3 xxxii

M. fiat emuls.

Sig. $-\frac{1}{2}$ oz. t. i.d. p. c. (each $\frac{1}{2}$ oz $=\frac{1}{2}$ dr. syr. fer. iod. U. S. P.).

Should some patients be unable to take the oil in emulsion, the following capsules are often prescribed:

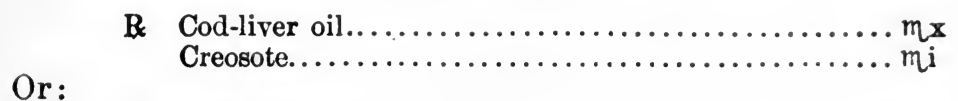

Or :

Or :

R Cod-liver oil..............................m $m_{x}$

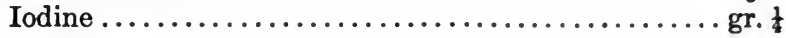

B Cod-liver oil ..............................m $m_{x}$

Iron iodide.......................... $\frac{1}{2}$

All these should be dispensed in soluble elastic capsules.

Fowler's or Pearson's solution of arsenious acid in rather large doses over a long period has given excellent results in my hands. Whenever I use arsenic I interrupt it frequently for 1 or 2 weeks.

The Roentgen ray treatment has warm advocates. Bullit collected 226 cases of glandular tuberculosis in 1905, showing 35 per cent. cured, 40 per cent. improved, and 25 per cent. unimproved. George C. Johnston favors this treatment, and summarizes its advantages thus: (1) relative freedom from danger, (2) absence of pain during or after treatment, (3) relief of pain if present, and (4) avoidance of scars, paralysis, deformity, hemorrhage, or shock; and its disadvantages: (1) prolonged treatment, 3 months, (2) necessity of expert and expense, and (3) question as to the permanency of cure. To these I should add the danger of X-ray exposure, so pertinently emphasized by Edsall before the American Medical Association, June, 1906.

Paterson recently reports success from injecting sterile caseous matter; but the use of tuberculin seems to me much more accurate and scientific. Very encouraging results have recently been reported from the use of minute doses of tuberculin in various forms of tuberculosis. Wright regards glandular tuberculosis as an especially hopeful variety for this treatment. He inoculates a patient exhibiting a low tuberculoopsonic index with a dose of tuberculin just sufficient to avoid a marked negative phase. His second inoculation is undertaken toward the end of the positive phase resulting from the first inoculation. He increases the doses very gradually, always avoiding a marked negative phase. It is impossible to decide as yet whether the painstaking and laborious blood examinations must be carried out in each case, as Wright suggests, or whether very careful observation of the clinical symptoms will suffice, as Trudeau believes. Continued observation must determine this point. Bullock, among others, has confirmed Wright's results. He has so treated 
2 cases of genito-urinary tuberculosis, 7 cases of lupus, and 2 cases of glandular tuberculosis, with uniformly favorable results, 1 case of lupus in which the injections apparently could not be tolerated, and 1 case of abdominal tuberculosis without benefit. Pogue reports the favorable action of small doses of tuberculin (0.01-0.50 mg.) in 167 cases; 9 of these had cervical tuberculo-adenitis and all nine recovered. Leube reports excellent results with small doses.

Trudeau compared 135 patients treated with tuberculin and 619 untreated. Of the incipient cases that were treated 18 per cent. more are living to-day than of the untreated, and of the advanced cases 25 per cent. more. He is inclined to believe that the most efficient tuberculins in use are those in the preparation of which no heat has been applied. Koch's "bacillen emulsion" and the filtrate of ripe cultures as used by Denys meet this requirement. The former contains all the toxins and endotoxins contained in the body of the crushed tubercle bacilli; the latter, the toxins and any other substances which may be produced by the growth of the bacilli or dissolved from their disintegrating bodies in a liquid medium. Koch, in his last paper on "bacillen emulsion," says : " One increases the dose very rapidly in order to obtain reactions with elevations of temperature from $1.5^{\circ}$ to $2^{\circ} \mathrm{C}$. As soon as a strong enough reaction is obtained, the intervals must be lengthened to from 6 to 8 days or more." Trudeau continually makes every effort to avoid such reactions, and so does Wright in administering tuberculin according to the estimation of the phagocytic power of the patient's blood-serum. Both use very minute doses. In cases with fever, Trudeau begins with $0.0000001 \mathrm{G}$. or even $0.00000005 \mathrm{G}$. ( $B F$ or $B E$ liquid measure); in apyretic cases, 0.0000001 is the usual dose. This is unnecessarily small for many cases, but susceptibility varies so greatly in different patients that the only safe way is to begin low down in the scale. He has seen in one case a rise of temperature from $0.0000005 \mathrm{G}$. He increases the dose so gradually and at such intervals as to carry the patient to full doses with but occasional and moderate reactions. At the slightest evidence of intolerance, as manifested not only by the patient's temperature but by his symptoms, by marked irritation at the point of injection, or by depreciation of his general condition, he lengthens the intervals and diminishes the dose for a time. He never injects after a reaction till all effects of the previous reaction upon both the patient's temperature and his general condition have disappeared. The patient should exhibit no depression in his general health, and no fever above his usual range, except occasionally for the first 48 hours following an increase in the dose. If other undesirable conditions result, he discontinues the inoculation until the patient has reached his normal state. If full doses, i.e., 1 c.c. of "old tuberculin" or 0.005 G. (that is, $5 \mathrm{mg}$.) solid substance, can be reached without violent reaction and depreciation of the general health, but not otherwise, the injections should be continued until 1 full dose has been given; but the interval should be lengthened gradually as 
the high doses are reached, until a week or two intervenes between the last 2 or 3 inoculations. Trudeau emphasizes the paramount necessity of the individualization of patients in the use of tuberculin. He agrees with Denys that the numerous faults in the treatment, which may lead to failure or even seriously endanger the patient's chances of recovery, are: too large doses in the beginning, raising the dose too rapidly, administration at too short intervals, reinoculation before all effects of the previous reaction, both constitutional and local, have passed away, increasing the dose after a reaction has occurred, neglecting clinical evidence of the patient's limit of tolerance (i.e., malaise, headache, insomnia, anorexia, and increase of cough). A proper series of injections requires at least 6 months; in most cases a year or more would be better. He believes that immunity is very slowly produced and does not last indefinitely, and he counsels a second course of treatment in 4 to 6 months if any symptoms reappear.

Dowd has summarized the surgical treatment of tubereular cervical lymph-nodes from a study of 100 cases submitted to operation: (1) 86 per cent. are apparently due to infection from the fauces, pharynx, or nasal mucous membrane. (2) The disease shows a tendency to extend to the lungs and other internal organs. Statisties show that such extension occurs in one-quarter to one-half of the cases from whom the nodes are not removed. (3) The disease is very tedious, causes great discomfort and disability, and leaves disfiguring scars. (4) The thorough removal of the diseased nodes by operation has given better results than has any other method which the author finds recorded. (5) The records of operations justify the following conclusions: (a) In favorable cases: safety of operation; a scar hardly to be seen; probable confinement to bed of 2 or 3 days; the wearing of a bandage or dressing from $1 \frac{1}{2}$ to 3 weeks; freedom from recurrence in about 75 per cent. of the cases, and ultimate recovery in about 90 per cent. (b) In the less favorable cases: safety from operation; less disfigurement from scars than discharging sinuses will cause; freedom from recurrence in 50 to 55 per cent., and ultimate cure in 70 to 75 per cent. of the cases.-Ed.]

\section{THERAPY OF MYXEDEMA, CRETINISM, AND EXOPHTHAL- MIC GOITRE.}

The next diseases to be discussed will be myxœedema, including infantile cretinism, and exophthalmic goitre, although they have no connection with diseases of the blood, one of them being certainly, and the other probably, a disease of a special organ.

\section{THERAPY OF MYXEDEMA AND SPORADIC CREIIINISM.}

Etiological Therapy.-The therapy of these diseases may be dismissed with a few words. Thanks to modern investigation, we have a specific, causal treatment. As these diseases arise from an insufficiency 
or a total lack in the functioning of the thyroid gland, we endeavor to make up for the loss by giving the fresh thyroid glands of sheep, or pharmaceutical preparations made from them. If the gland itself is used, we may begin with $1 / 8$ of a gland a day, increasing the dose gradually to 2 glands, spread on bread or given with rice or milk. The most important pharmaceutical preparation is the tablet prepared from the dry extract. In Vienna one of these tablets contains $0.25 \mathrm{G}$. (4 gr.) of the gland; in England, 0.33 G. (5 gr.). [In America we use tablets of 0.32 G. (5 gr.).-Ed.] If there is any difficulty in taking these tablets, they may be given in milk, wine, or soup. We begin with 1-2 grains, increasing to $15-40 \mathrm{gr}$., as the patient becomes used to the medication. A carboglycerin extract may also be injected subcutaneously, 10-12 drops once, twice, or three times a week, according to the kind of reaction. The complete reaction, the so-called "thyroidism," comprises a number of symptoms, which may also be observed when the drug is given internally. These symptoms are tachycardia, palpitation, dyspnœa, weakness or faintness, nausea, vomiting, anorexia, loss of flesh, nervous excitement, exhibiting itself in headache, tremor, sleeplessness, restlessness, and fear, and severe cardiac and cerebral attacks like the first attacks of angina pectoris. With a coexistent myocardial or endocardial affection, these attacks may even be fatal. The possibility of such threatening symptoms of intoxication warns us to be cautious in giving large doses. It is sometimes better to give arsenic at the same time (see p. 201).

As, thanks particularly to Baumann, we know the essential principle of the thyroid gland, we may employ this instead of the gland itself:

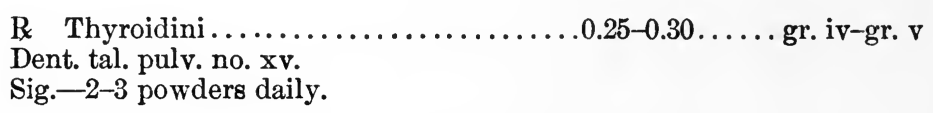

Even after an apparent cure, the treatment must be continued, with smaller doses; otherwise, as experience has shown, single symptoms at least of the disease will regularly reappear. [Forchheimer's rules in infantile myxœdema or cretinism are as follows: Calculating the adult dose at 5 gr., the dose for an infant is obtained by multiplying this dose by a compound fraction whose numerator is the age in months divided by 12 and whose denominator is 20 ; thus, for a child of 3 months of age,

$$
\frac{\frac{3}{12}}{20}=\frac{\frac{1}{4}}{20}=\frac{1}{80} ; \frac{1}{80} \times 5 \text { gr. }=\frac{1}{16} \mathrm{gr} .
$$

He cautions against even so small a dose, and at first employs it only once a day, later twice, and finally three times. Untoward symptoms in children are restlessness, sleeping badly, loss of appetite, and irritability, any one of which presents a striking contrast to the previous condition of the child. A rapid pulse, vomiting, or collapse never occurs if the infant is carefully watched and the drug is interrupted upon the appearance of one of the preceding symptoms.-Ed.] 
Diet.-One other point must be kept in mind in the treatment of myxœdema, i.e., the regulation of the diet. The author is personally aequainted with one ease in Billroth's clinic, in which Eiselsberg had extirpated the thyroid gland on account of struma. The patient suffered from tetania strumipriva. He instinctively avoided all meat, having found out from experience that its use was apt to bring on tetanic attacks. Other surgeons have had the same experience. Von Lanz's experiments on dogs proved that after the thyroid gland had been removed, tetanic attaeks followed a meat diet even if the dogs had been given the thyroid extract treatment; whereas the attacks did not occur if the animals were fed on milk. These facts would indicate that a patient with myxœdema should avoid meat, and live on milk, eggs, butter, cereals, and vegetables.

\section{THERAPY OF EXOPHTHALMIC GOITRE.}

(Basedow's, Graves's, or Parry's Disease.)

The treatment of exophthalmic goitre is by no means so satisfactory as is that of myxœdema. 'The former disease is to a certain degree the opposite of the latter, as it arises partly from an increased secretion of the thyroid gland. The author, however, does not believe that this is the only etiological factor. The histological findings in the central nervous system, especially in the restiform bodies, would indicate that the nervous system also shares in the etiology. Our therapy, then, in so far as it is not general, must be directed toward the thyroid gland and the nervous system.

General Therapy: Rest.-The first requisite of the general therapy is mental and bodily rest. All strenuous exercise must be given up. In some cases, rest in bed is indicated, even if the disease is not accompanied by symptoms of cardiac insufficiency. The patient will frequently do better away from home and business, at a health resort or even at any quiet place in the country.

Resorts.-Opinions differ as to the choice of resorts. The author inclines to a rather high altitude, provided the patient will abstain from any difficult mountain walks. At first he may experience a good deal of distress, particularly palpitation of the heart, but such symptoms will wholly disappear, especially if he moves about but little. Examples of suitable places are Semmering (3000 ft.), Toblach in the Pusterthal (3700 ft.), Neu-Schmecks in the high Tátra (3000 ft.), Zakopane in the Polish Tátra in Galicia (almost $3000 \mathrm{ft}$.), Brenner in the Brenner Pass (4000 ft.), Churwalden (3800 ft.), and Engelberg in the Canton Unterwalden (3000 ft.). [See pp. 334-337 for resorts at similar altitudes in America. I think a quiet country place, where the patient ean remain as many hours as possible in the fresh air with a maximum of sunshine, is the main object. The altitude is quite a matter of individual peculiarity. Over $3000 \mathrm{ft}$. should generally be avoided.-Ed.] 
The seashore or a sea voyage usually does no good. It will be remembered that in the discussion of the therapy of chlorosis (see p. 152) it was stated that chlorotic patients, especially those with nervous symptoms, almost always do badly at the seashore. Patients with exophthalmic goitre are almost always chlorotic and are decidedly nervous, so that we should expect the same thing to hold true for them. To be sure, if these symptoms are not prominent, that is, if the disease is in a latent stage, sea air may be beneficial by stimulating metabolism. In that case we should choose a mild climate, preferably that of the Baltic or the Mediterranean.

Mineral waters are seldom used, although there is often no objection to iron or arsenic waters.

Diet.-The diet should be abundant and mixed, but the amount of animal food should be somewhat restricted. Alcohol, coffee, tea, chocolate, and condiments are forbidden. The meals should be frequent and relatively small in amount.

Hydrotherapy.-Hydrotherapy and electrotherapy also play an important part in the treatment of the disease. The hydrotherapy should consist of ordinary baths at home or at a hydropathic establishment, or of the use of mineral waters for baths at the several spas. The particular method is not very important, the main consideration to be kept in mind being that all violent stimulation must be avoided (e.g., hot or too cold baths, hard shower-baths, cold sponges, cold douches, cold rubs). From the mild procedures we choose those fitted to the special case, taking into consideration the condition of the nervous system, the blood, the heart, and the digestive tract. We begin with tepid sponge-baths, gradually using cooler water, or rub the body down with towels wet in water at $79^{\circ}-68^{\circ}$, to which salt may be added (see p. 156). Wet packs at the temperature of the room are available in some cases, but by no means in all. A blanket is thrown over the bed, a dry sheet on top of that, and then a wet sheet. The patient, entirely naked, is then laid on the wet sheet and the coverings wrapped closely about him. A wet compress should be placed on his head, and, in the cases under consideration, his arms should be left free, as experience has shown that all nervous patients undergo so much discomfort from being entirely enclosed in a wet pack (palpitation of the heart, restlessness, etc.) that it is often necessary to unwrap them immediately. The patient should remain in the pack until the skin is thoroughly warm, which will take anywhere from a quarter of an hour to an hour or more. He should never be allowed to stay long enough to get into a sweat, as in that case the effect would be stimulating rather than sedative. If it is desirable for the patient to remain in the pack all night, for the sake of overcoming the sleeplessness so common in this disease, the blanket should be replaced by some light-weight coverlet. During the entire duration of the wet pack the compress on the head should be frequently changed. When the patient has been taken out, he should be quickly dried off. 
For palpitation of the heart and tachycardia, cold applications to the neck and vertebral column, or, as in heart disease, ice-bags or a Leiter's coil over the cardiac region, are employed. In many cases, hot applications on the neck also have a good effect (Heller).

Treatment at Carbonated or Salt Springs.-A course of baths at a spa is much less often ordered than is the simple hydropathic treatment at home just described. The hot salt springs (Baden-Baden), particularly those containing carbon dioxide (Nauheim, Franzensbad, Oeynhausen-Rehme), are generally chosen, Nauheim enjoying a special reputation in this connection. [These patients generally do better nearer home.-Ed.]

Electrotherapy.-The electrotherapy of exophthalmic goitre consists chiefly in subaural galvanization, which affects the various nerves of that part of the neck (vagus nerve, carotid plexus, etc.), and indirectly the brain and spinal cord. The author's technic is as follows: The small round electrode $\left(2.5-4 \mathrm{~cm} .\left(1-1 \frac{1}{2} \mathrm{in}\right.\right.$.) in diameter) is held with moderate pressure under the angle of the lower jaw, pressed inward and upward; a large concave flat electrode is placed on the neck at the height of the lowest cervical vertebra (corresponding to the ciliospinal ganglion). The first electrode is connected with the anode; the second, with the cathode. By gradually decreasing the resistance in the rheostat the current is raised to $0.5-4$ milliamperes, and is then gradually decreased again by increasing the resistance. After 2-3 minutes. the apparatus is changed to the other side of the neck for the same length of time. Many authors prefer to change the direction of the current during the procedure (Meyer, Stintzing). Some physicians galvanize the goitre and the bulbi, while Charcot's pupil, Vigouroux, uses the galvanic current and recommends general faradization. [So do Horsley and G. R. Murray. Thoostek taught us to apply the anode at the sternal notch, the cathode at the angle of the jaw.-Ed.] The latter procedure, however, has few supporters. Galvanization of the neck, on the contrary, has undeniably good effects in reducing the nervous irritability, sleeplessness, asthenia, and tremor, in slowing the heart action, in making the pulse normal, and in diminishing palpitation.

Medication: Iron and Arsenic-Potassium Bromide.-Medical treatment aims to combat the nervous or anæmic symptoms. If a chlorotic or anæmic symptom complex is present, we employ iron and arsenic. For marked nervous symptoms, we use potassium bromide, ${ }^{7}$ best in doses of $2-3 \mathrm{G}$. (30-45 gr.) in $100 \mathrm{G}$. (3 oz.) of an alkaline mineral water (2-5 G. (30-75 gr.) a day), or in the form of aqua bromata (Erlenmeyer) (1-3 glasses daily), or in the form of Sandow's effervescent bromides ( $1 / 2$ capsule in $\mathrm{I} / 2$ glass).

Camphor Monobromate.-The author has found camphor monobromate worth trying.

${ }^{7}$ Sodium and strontium bromide are less liable to produce unpleasant symptoms.-Ed. 
B Camphoræ monobromatæ ........ . $0.30-0.50 \ldots \ldots$ gr. v-gr. viii Sacchar. lactis ...................... iii

Dent. tal. pulv. (chart. cerat.) no. xv.

Sig. -3 powders daily.

Quinine, Antipyrine, etc.-Quinine, antipyrine, arsenic, opium, belladonna, and cannabis indica are recommended by one or more authors, but rejected by others as useless or even harmful. Seguin particularly recommends aconitine for strong palpitation or marked tachycardia.

B Aconitini .......................... $0.003 \ldots \ldots$ gr. $\frac{1}{25}$

Ext. glycyrrhizæ .................... s.

M. fiat mass. Div. in pil. no. $x \times x$.

Sig. -3 pills a day. Increase to 6 or even 8 .

The treatment may be kept up for weeks at a time with an occasional intermission for a few days ; the pulse often sinks from 140-160 to 80100 , and the exophthalmus and the goitre improve.

Sodium Phosphate.-Moebius, following the example of others, has tried sodium phosphate, $2-10$ G. $(1 / 2-21 / 2 \mathrm{dr}$.) a day, a constituent of the artificial serum of the French, which should serve the same purpose as spermin. Like Kocher and Sahli, he observed an improvement in the patient's sleep and nervousness. These two observers reported a slowing of the heart contractions and a lessening of the palpitation of the heart, and maintained that many patients prefer sodium phosphate to potassium bromide. The author in several cases has confirmed its sedative effect on the heart. [G. R. Murray has obtained relief from the cardiac symptoms by the use of the tincture of convallaria and of belladonna.Ed.] From his own experience the author recommends dilute sulphuric acid (10 drops, 3 times a day after meals) for the vasomotor disturbances so often occurring in exophthalmic goitre. Quinine sometimes seems to have a similar effect. Both are nerve tonics.

Digitalis-Iodine.-Two more drugs remain to be discussed, digitalis and iodine.

Some authors claim remarkably good effects from the use of digitalis; others consider it dangerous. The author believes that it should be used only when there are indubitable symptoms of cardiac insufficieney, never merely to moderate a tachycardia or to influence palpitation of the heart.

A similar difference of opinion prevails in regard to the use of iodine. The author maintains most emphatically that it is to be strictly avoided, unless the disease is secondary to syphilis. Even when used only externally, he has frequently seen it aggravate the symptoms of the disease. In three cases of apparently simple struma, an acute exophthalmic goitre was brought on, in two cases by an intraparenchymatous injection of tincture of iodine, and in the third by the mere external use of an iodine ointment. The acute condition disappeared fully and in a relatively short time, only to reappear and become chronic, leading, in the third 
case, to death in about a year. Such observations make the author agree with Moebius and Trousseau that there are latent cases of exophthalmic goitre, marked only by strurna, which may be made acute by the use of iodine, and, without any complication, may even come to a fatal termination.

Thyroid Extract-Thymus Gland.-Thyroid extract and preparations of the thymus gland are sometimes given. The former the author from his own experience considers useless or even absolutely injurious. It should be remembered, however, that reliable observers have reported that the unpleasant or harmful effects from its use may be obviated by giving arsenic along with it (3-6 drops of Fowler's solution daily).

Treatment with the thymus gland (3-20 tablets $(0.25$ G. $-4 \mathrm{gr}$. each) daily, or 10-25 G. ( $1 / 3-3 / 4$ oz. $)$ of chopped thymus gland of a sheep spread on bread has apparently in some cases a distinctly favorable effect.

Rodagen.-But this has been supplanted by a more recent treatment, the administration of (1) the milk, (2) the milk powder, or (3) the blood serum, obtained from animals from whom the thyroid gland has previously been extirpated. Practically, only the last two are available. The milk powder, called " rodagen," is given in daily amounts of 5-10 G. $\left(1 \mathrm{I} / 4^{-2} \mathrm{r} / 2 \mathrm{dr}\right.$.). The blood serum, on the market in the form of "antithyroid serum Moebius," is much more efficacious. It is given 3 times daily, beginning with a dose of 5 drops and increasing by 5 drops each time to even 120 drops, or, if necessary, to 150-200 drops a day. In most of the author's cases, though not in all, it caused a retrogression of the nervous symptoms (palpitation, tremor, nervousness, and especially sleeplessness), increase of weight, diminution of the amount of perspiration, reduction of the goitre, and even of the exophthalmus, while the tachycardia was but little influenced. Even with continued large doses (120 drops), no symptoms of myxœdema (intellectual sluggishness, trophic cutaneous changes) appeared to make the reduction of the dose necessary. The author tried giving sodium sulphanilate as an iodine antidote $(10 \mathrm{G}$. ( $1 / 3 \mathrm{oz}$.) a day), along with rodagen, but got no satisfactory results. Naturally, neither rodagen nor the antithyroid serum produces any effect upon already developed anatomical lesions, particularly those of the heart.

Surgery.-The author has seen death, apparently from acute dilatation or insufficiency of the heart, follow soon after operation in two cases of partial extirpation of the struma. Although a few similar cases have been reported from other sources, and although there are also cases on record in which a fatal result from paralysis of the heart has quickly followed extirpation, there are, nevertheless, instances of primary exophthalmic goitre where this operation (resection of the struma, or, more rarely, tying of the arteries of the thyroid gland) has resulted in decided improvement or even in a nominal cure. Still, the number of deaths is so great that operation should not be resorted to until all other 
means have failed, unless we are forced to it by tracheal stenosis or by a giant struma.

Another operation has recently been advocated, the entire resection of the sympathetic, first on one side and then on the other. Theoretically, this would appear a rational procedure, since there is no doubt that the nerves of the vessels play a great part in the etiology of the disease. some authors (Marie, Abadie) even attributing to the sympathetic the primary lesion. The author had the opportunity of seeing Prof. Jonnescu in Bucharest perform this operation in a masterly way, and was astonished to see the unilateral resection of the sympathetic followed by an immediate unilateral disappearance of the exophthalmus. Still, the operation is dangerous, and is sometimes followed by serious after-effects (psychoses), while success is very uncertain at best, so that it has never been very favorably received, especially in Germany.

In isolated cases, the removal of the hypertrophied nasal muscles or the extirpation of the ovaries has proved beneficial.

Radiotherapy.-Radiotherapy, especially the treatment of the thyroid gland with the Roentgen rays, has so far apparently met with no success. [Pfahler and Thrush have noted a favorable influence in a number of cases. Dock has not, and depends upon rest, feeding, and symptomatic treatment. Osler and Tyson advocate the same plan. W. $\mathrm{H}$. Thompson has called especial attention to the use of laxatives, intestinal antiseptics, and a non-purin diet in Graves's disease. Forchheimer, a warm advocate of general medical treatment, has for the past twenty years employed hydrobromate of quinine, with or without ergotin, in doses of $0.30 \mathrm{G}$. ( $5 \mathrm{gr}$.), in gelatin coated pills, 4 times daily; to each pill is added ergotin $0.065 \mathrm{G}$. ( $1 \mathrm{gr}$.), when quinine alone does not improve the symptoms in 48 hours. He claims favorable results from this treatment in from 70 to 90 per cent. of all cases. The first change occurs within 48 hours, and consists generally in an improvement, followed by a disappearance of the tachycardia, then a diminution in the size of the thyroid gland, and finally disappearance of the tremor and exophthalmus. In fully developed cases he accomplishes this result in as short a time as 4 months, but it may take as long a time as 3 years. He continues the medication until all symptoms have disappeared. Forchheimer's method has been followed at the Massachusetts General Hospital with remarkable success. They use a neutral salt. Beebe and Rogers have reported remarkably favorable results with a cytotoxic serum, the use of which is based upon the theory that exophthalmic goitre is an intoxication due to thyroglobulin. They obtained the most efficacious serum from animals injected with pathological glands. The patients were inoculated every 48 hours. In a few cases, improvement was rapid and striking within the first 48 hours; in most, however, much more gradual. Some of the virtue of the serum may possibly be due to its cytolytic properties. They believe that 23 out of 90 patients were cured of all symptoms of thyroidism, 52 were more or less improved, 11 failed to 
improve, and 4 died. Even the slightest improvement in any one subjective or objective symptom during the first 2 weeks of the treatment strongly suggests the prospect of a cure. When no response to the treatment occurred, different grades of serum were tried, and then sera from different species of animals. By "normal" rabbit, dog, or sheep serum they mean an antitoxic or antithyroidal serum obtained by inoculating these animals with eombined nucleoproteid and thy roglobulin separated from the normal human gland. "Pathological serum" is obtained by inoculating the same substances from thyroid glands extirpated from patients with exophthalmic goitre.

Ewing has recently discussed the status of serum therapy in exophthalmic goitre. He believes that the action of the serum can be safely judged only in early and uncomplicated cases. Many considerations point to extensive limitations in the use of antitoxic serum in all stages of the disease, even assuming that there is a sound theoretical basis for the employment of antitoxic serum directed against the thyroglobulin. Such considerations are: (1) We are not sure that the thyroid gland is the sole primary cause of the disturbances met with in the disease. The relations between the parathyroids and the thyroid are not definitely understood. (3) The relations of the hypophysis to Graves's disease have not yet been settled. (4) In advaneed stages of the disease, the anatomical state of the thyroid itself would constitute a serious limitation to serum therapy. The cellular hyperplasia with only a trace of colloid, the diffuse conneetive tissue growth obliterating capillaries and lymph vessels, sclerosed arteries with hyaline degeneration of the stroma and eyst formation, are examples in point. The results of the treatment of advanced cases, as reported by Beebe and Rogers, seem to prove that their antitoxic serum becomes less potent as the disease advances. Other organs show anatomical changes in the late stages of the disease; i.e., fibrosis or even atrophy of the cervieal sympathetic, and of the vagus and cardiac nerves, atrophy of the restiform body, hypertrophy and dilatation of the heart, interstitial myoearditis, and coronary arteritis. -Ed.] 


\section{IV.}

\section{THERAPY OF DISEASES OF METABOLISM}

UNDER this head will be included (1) diabetes (mellitus and insipidus), (2) obesity, (3) emaciation, and (4) uric acid diathesis.

\section{THERAPY OF DIABETES MELLITUS.}

InTroduction.-Before discussing the therapy of diabetes, it is necessary to take up briefly the essential nature of the disease and the distinction between its various manifestations, which, from a therapeutic standpoint, need to be carefully discriminated; for a thorough understanding of the pathogenesis of diabetes mellitus is the key to successful treatment.

To begin with, a few words concerning the disposition of carbohydrates and the sources of the formation of sugar in the normal human body, are in place. The earbohydrates which are not already in the form of grape sugar, milk sugar, or fruit sugar-that is, the starches and the cane sugar-are changed, partly in the mouth by the action of the saliva. but principally in the small intestine by the action of the pancreatic and intestinal secretions, passing through a series of intermediate products into grape sugar; or, in the case of cane sugar, into a mixture of grape and fruit sugar, the so-called "inverted" sugar. The carbohydrates are absorbed from the intestine, then, in the form of grape sugar, milk sugar, fruit sugar, or inverted sugar. Only a very small portion passes into the thoracic duct; the main part is carried off to the liver by the portal veins. The liver stores up the sugar, converting it into glycogen. By the help of a diastatic ferment in the blood, some of this glycogen is changed back again into grape sugar, to be supplied by the blood to the different parts of the organism whenever the latter are in need of sugar. The amount thus given back by the liver is used in two ways: (1) It is burnt up in the muscles in order to furnish energy, being changed into water and carbon dioxide; and (2) it is stored up in the muscles as reserve material in the form of glycogen. The liver and the muscles, therefore, both serve as storehouses for sugar. A smaller portion of the sugar is changed in the organism into fat, if the glycogen depots are already full. A very small amount is secreted unchanged by the kidneys, the normal urine containing a trace of grape sugar.

Under only one condition does the non-diabetic urine contain considerable amounts of sugar: when the blood is suddenly flooded with so much sugar that it cannot all be stored at once. This occurs in the 
so-called "alimentary glycosuria," when-generally intentionally-we give the organism so much sugar at once that it cannot all find room in the glycogen depots, and the residue cannot all be disposed of by muscular work or by conversion into fat. In that ease, some must be excreted by the kidneys. A similar process apparently occurs in those cases of glycosuria which, in consequence of nervous diseases, follow the celebrated Bernard experiment. The liver (owing to nervous disturbances) is unable to retain its glycogen, so that the blood is suddenly overwhelmed with sugar.

When the organism is in need of sugar, it draws first from the glycogen in the liver, and then, when that is exhausted, from the reserves of glycogen in the muscles.

The sugar in the organism is not formed exclusively from the glycogen derived from the carbohydrates, however, but also from the glycogen derived from proteid and probably from fat. In other words, "the sugar changed from glycogen owes its formation not only to the carbohydrates, but also to the proteids, and apparently to the fats," a most important fact to keep in mind in understanding severe cases of diabetes.

The sugar content of normal blood can vary but little from $0.09-0.10$ per cent., reaching at the most only 0.18 per cent.

Essential Nature of Diabetes Mellitus.-Diabetes mellitus, then, is a disease characterized by a permanent surplus of sugar in the blood (hyperglycæmia) and by a permanent increase of sugar in the urine (glycosuria). This oversupply is due to a decreased ability of the organism to consume sugar, though to what this inability itself is due, is by no means wholly elear. In some, but certainly not in all cases of diabetes, it arises, as experiments and experience have proved, from a lack of the "internal" pancreatic secretion.

Essentials of a Rational Dietetic Therapy.-It follows that a rational therapy must aim at giving the organism as little sugar as possible to consume until the cells have regained their power to dispose of it. when its ingestion may be very eautiously tried again. This means that the carbohydrates must be strictly limited in amount or temporarily prohibited absolutely. But as in some cases, particularly the severe ones, the production of sugar from the proteids plays an important part, and as many diabetics are great eaters, it is evidently necessary that the amount of proteids allowed be also carefully calculated.

Before entering into the details of the treatment, however, it seems best to digress somewhat, in order to take up briefly the general subject of nutrition and of the chemical constitution of our common foods.

\section{Principles Underiying the Scifnce of Human Nutrition.}

All substances used as food may be divided into (1) the group of fats and carbohydrates, which are composed of carbon, hydrogen, and oxygen, and (2) the group of proteids, which contain, in addition to these 
three constituents, nitrogen and sulphur. To call alcohol an additional form of food seems at least questionable, so far as the healthy organism is concerned. But in the diseased and especially the fevered organism, alcohol, apparently at least, is an indirect food, whose use in moderate amounts and appropriate forms is positively beneficial.

Disposition of the Foods in the Body.-If we follow any one of these food constituents through its course in the body, we find that a certain part of it is unused, being eliminated from the body in the feces. The rest is absorbed from the intestine, and oxidized in the tissues, the complex compounds being broken down into simple ones and the useless end-products being eliminated as carbon dioxide, hydrogen, and urea. As a result of this oxidizing process, and partly also as a result of the splitting up of the molecules of the food-stuffs, the potential energy is transformed into kinetic energy, exhibiting itself in the form of heat and muscular work.

Numerical Expression of the Amount of Heat Produced-Heat Value of the Separate Food Elements.-The amount of energy obtained from any food-stuff by its transformation in the organism is expressed numerically in terms of calories, a (large) calorie being the amount of heat necessary to raise the temperature of $1 \mathrm{~kg}$. of water through $1^{\circ}$ Centigrade. Exact experiments have shown that $1 \mathrm{G}$. of carbohydrate burned in the body produces 4.1 calories, and $1 \mathrm{G}$. of fat about 9.3 calories. The proteids when oxidized in the body are not broken down, like the carbohydrates and the fats, into simple components that cannot be oxidized farther; on the contrary, the substances excreted from the body as the end-products of the proteids have a certain caloric value. This value subtracted from the total caloric value of the proteids gives the actual caloric value of the proteids for the human organism, and this corrected caloric value is found to be just the same as that of the carbohydrates, -4.1 calories for each gram of proteid. One hundred G. of proteid, 100 G. of carbohydrates, or 44.1 G. of fat,-each produces, then, 410 calories when burnt in the body. Or, carbohydrates, proteids, and fats, so far as their heat values are concerned, stand in the ratio of $1: 1: 2$. The heat value of alcohol stands between that of the fat and that of the other two, $1 \mathrm{G}$. of alcohol, when oxidized in the body, giving off 7 calories.

Amount of Energy Needed by the Organism-Metabolic Equilibrium. - The need for food and the exact amount of that need depend -aside from what is required for the growth of the organism-upon the extent of the oxidization going on in the body. A man of average weight, at rest or doing only moderate work, uses on an average 2500 calories a day; or, expressed more exactly, 14-17 calories for every pound of weight. The same number of calories must be supplied daily by the food; that is, the organism must be kept in the so-called "metabolic equilibrium." Whether these calories shall be supplied by proteids, carbohydrates, or fats, remains up to a certain point indifferent, for 
within certain limits all three, as well as alcohol, may be used interchangeably, so far as their caloric value is concerned. The reasons why this is true only within certain limits are as follows: (1) The capacity of the digestive tract is different for each group; for instance, should we wish to use only a carbohydrate, potatoes for example, such a large mass would be required that the digestive organs would soon be unable to handle it. (2) The feeling of satiety comes more quickly with some kinds of food than with others; for instance, it would prevent the ingestion of enough fat to furnish alone the required amount of nourishment. (3) A certain minimum of proteid (either animal or vegetable) seems to be absolutely needed in order to preserve physiologic metabolic equilibrium. This amount is not so large as was formerly supposed. We can no longer believe that the most nourishing diet demands an overwhelming proportion of proteid, particularly animal proteids; but a certain moderate amount does appear to be absolutely necessary. This absolute minimum may be put at $40-50 \mathrm{G}$. (11/2-13/4 oz.) per day; the practical amount for a person at rest or doing only light work, an amount which, under physiological conditions, should not be exceeded, may be put at $100 \mathrm{G}$. ( $3 \mathrm{x} / 2$ oz.).

Components of the Regular Normal Diet.-The amount of food needed by a man of average weight, who is doing only very light work, should be distributed about as follows : $100 \mathrm{G}$. (31/2 oz.) of proteid (400 calories), 50-60 G. (13/4-2 oz.) of fat (500 calories), 400-450 G. (14-16 oz.) of carbohydrates (1600 calories), making roughly 2500 calories in all. The smaller numbers represent the amounts needed if the person is at absolute rest. This gives an average of 38 gross calories and 34 net calories for every kilogram of weight. By gross calories we mean the absolute number of calories furnished to the body by the food; by net calories, the number of calories which are made utilizable in the body (see p. 210). These should all be reduced by one-fifth to compute the corresponding amounts for a woman.

A man of average weight doing a moderate amount of work needs $110 \mathrm{G}$. (33/4 oz.) of proteid, $500 \mathrm{G}$. (17 oz.) of carbohydrate, and $60 \mathrm{G}$. (2 $o z$.) of fat; or the carbohydrate may be left as before and the required increase obtained by giving more fat or adding alcohol. A man of average weight doing very hard work needs $120-130 \mathrm{G}$. (4-41/2 oz.) of proteid, 500 G. ( 17 oz.) of carbohydrate, and $100 \mathrm{G}$. ( $3 \mathrm{r} / 2 \mathrm{oz}$.) of fat, or 50 gross calories per kilogram. In order to calculate these quantities for an individual of any given weight, it is convenient to know the amount of each kind of food needed per kilogram of weight. For a man doing a moderate amount of work, we get $1.7 \mathrm{G}$. of proteid, $0.8 \mathrm{G}$. of fat, and 7.1 G. of earbohydrate per kilo.

Amount of Loss in the Different Food Components from NonAbsorption.-All these amounts include the part of the special foodstuff which is not absorbed in the alimentary eanal, but passes out, unutilized, with the feces. In other words, the amounts are all calculated 
in gross, not net, calories. The unabsorbed portion is roughly one-tenth of the whole, provided a mixed diet is used. More exactly, on an average:

1 G. proteid.. ..........gives 4.1 gross calories and 3.2 net calories

1 G. fat............... gives 9.3 gross calories and 8.4 net calories

1 G. carbohydrate........ gives 4.1 gross calories and 3.8 net calories

The exact figures depend somewhat, however, upon the particular form of proteid or carbohydrate used. In meat, flour, bread, etc., 10 per cent. is lost; in rye-bread, vegetables, etc., 13-17 per cent. In a purely vegetable diet this per cent., especially for the proteids, becomes still larger.

Source of the Proteids.-Experience has taught us the following additional facts: The daily amount of proteid needed may be divided between animal and vegetable proteid. Voit selects one-third animal and two-thirds vegetable, but it is not necessary to preserve this ratio. People with a uric acid diathesis, or those who cannot afford much meat, or those so situated that they cannot obtain it, frequently obtain almost all their proteid from vegetables. Again, the ratio given between the amount of fat and the amount of carbohydrate is not necessarily a fixed one. In some countries proportionally more fat is ingested, a custom which is good in so far as the digestive tract is thus given smaller masses to handle. It is also true that the richer classes in general use more fat-butter, cream, salad oil-than do the poorer.

Number of Meals.-The author approves of 3 meals a day, the heaviest at mid-day. One-sixth of the total food for the day should be taken at breakfast, three-sixths at dinner, and two-sixths at supper.

Significance of Mineral Salts.-Two more substances necessary for human nutrition remain to be mentioned,-mineral salts and water. Ordinary food contains a sufficient quantity of all the mineral salts except common salt, which must be furnished separately. A greater quantity is needed with a vegetable than with an animal diet.

Significance and Amount of Water.-An adult man excretes daily $2200 \mathrm{G}$. (41/2 pt.) of water if at rest, $2700 \mathrm{G}$. (51/2 pt.) if working. Twothirds of this is excreted by the kidneys; the rest, by the skin, lungs, and intestines. Only about five-sixths of this needs to be ingested daily, the other one-sixth being formed in the body by the oxidization of the hydrogen of the organic compounds (of all of the fat and of a part of the proteids). The amount to be ingested is, then, about $1825 \mathrm{G}$. (33/4 pt.) at rest and 2250 G. (43/4 pt.) at work. Practically, more water than this is nearly always furnished by the ordinary amount of food and drink (on an average, 2200-3500 G. (41/2-7 pt.). This extra supply merely washes out the tissues more thoroughly and is excreted by the kidneys in a few hours. Sometimes this increase of water in the tissues causes an increased excretion of urea and therefore an increased demand for proteid.

Quantitative Analysis of Ordinary Articles of Food.-To this short account of the principles underlying human nutrition, which it seemed 
necessary to discuss before taking up the dietetic therapy of diabetes mellitus, is appended a table giving the quantitative analysis of our common articles of food (see pages 212, 213, and 214). For a good therapeutist must be able to tell his patient exactly what particular foods and exactly what quantities of them he may take, not contenting himself with broad, general directions. In no disease is this detailed instruction more necessary than in diabetes mellitus.

In this table only round numbers are given, representing average values. Variations up to 10 or even 20 per cent. occur in analyzing different specimens of the same kind of food. The only object in analyzing the drinks is to show their content of alcohol. Practically, they are taken as stimulants, the quantities consumed being generally too small to make their food values of any account.

Full Diet: Daily Amount in Hospital.-The following is a specimen daily dietary for a patient in the hospital, who is able to make bodily movements and whose digestive apparatus is intact:

100 G. beef ( $3 \mathrm{oz}$, or 1 medium large slice)

100 G. $\left(3 \mathrm{oz}\right.$.) roast veal, or $100-150 \mathrm{G}$. $\left(3-4 \frac{1}{2} \mathrm{oz}\right.$.) sausage; sometimes (exceptionally) $100 \mathrm{G}$. ( $3 \mathrm{oz}$.) cheese

20 G. butter ( $\frac{2}{3} \mathrm{oz}$., or a piece $1 \frac{1}{2}$ by $1 \frac{1}{2}$ by 1 inch)

$\frac{1}{2}$ L. (1 pt.) milk

300 G. (10 oz.) vegetables (peas, beans, and lentils excepted)

300 G. potatoes ( $10 \mathrm{oz}$., or 2 medium potatoes, or 1 large cupful, when mashed)

100 G. rice (3 oz., or 6 tablespoonfuls, uncooked)

350 G. bread ( $12 \mathrm{oz}$., or $9-11$ slices or $\frac{1}{2}$ loaf)

$50 \mathrm{G}$. roll ( $1 \frac{3}{4} \mathrm{oz} .$, or 1 roll)

20 G. sugar (in milk or café au lait) ( $\frac{2}{3}$ oz., or $1 \frac{1}{3}$ tablespoonfuls, or 5 lumps)

$\frac{1}{2}$ L. ( 1 pt.) beer

The nutritive value of this dietary is shown in the following table:

\begin{tabular}{|c|c|c|c|}
\hline FOODS. & Proteid. & Fat. & $\begin{array}{l}\text { Carbo- } \\
\text { hydrates. }\end{array}$ \\
\hline 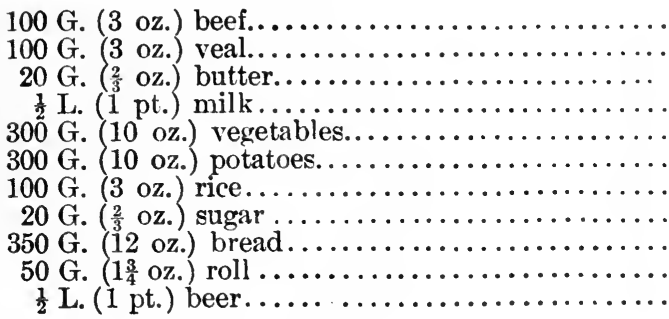 & $\begin{array}{r}21.0 \\
19.0 \\
17.0 \\
6.0 \\
6.0 \\
6.0 \\
0.1 \\
24.0 \\
4.0 \\
3.0\end{array}$ & $\begin{array}{r}5.5 \\
7.5 \\
15.0 \\
20.0 \\
1.5 \\
0.6 \\
1.0 \\
\dddot{1.8} \\
0.5 \\
\cdots\end{array}$ & $\begin{array}{l}\ldots \\
\cdots \\
\ldots \\
25.0 \\
20.0 \\
62.0 \\
78.5 \\
19.0 \\
70.0 \\
29.1 \\
25.0\end{array}$ \\
\hline 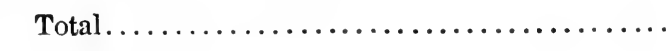 & 106.6 & 53.4 & $42 S .5$ \\
\hline
\end{tabular}




\begin{tabular}{|c|c|c|c|c|}
\hline \multirow{2}{*}{ FOODS. } & \multicolumn{4}{|c|}{ AVERAGE PER CENT. OF } \\
\hline & Proteid. & Fat. & $\begin{array}{c}\text { Carbo- } \\
\text { hydrate. }\end{array}$ & Alcohol. \\
\hline 1. Animal : & & & & \\
\hline 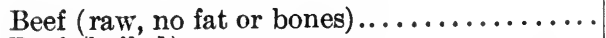 & 20.5 & 1.5 & $\cdots$ & \\
\hline 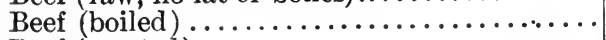 & 38.0 & 9.15 & $\ldots$ & $\ldots$ \\
\hline 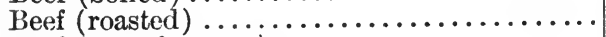 & 32.0 & 8.0 & $\ldots$ & $\ldots$ \\
\hline Beef $($ very fat, raw $) \ldots \ldots \ldots \ldots \ldots \ldots \ldots$ & 17.0 & 29.5 & $\ldots$ & $\ldots$ \\
\hline 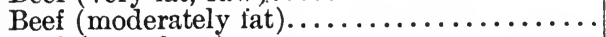 & 21.0 & 5.5 & $\ldots$ & $\ldots$ \\
\hline 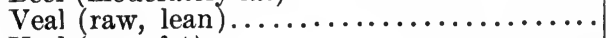 & 20.0 & 1.5 & $\ldots$ & $\ldots$ \\
\hline 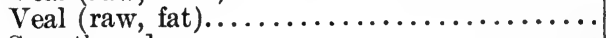 & 19.0 & 7.5 & $\cdots$ & ... \\
\hline 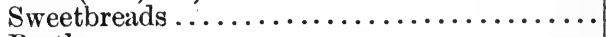 & 28.0 & 0.4 & $\cdots$ & $\cdots$ \\
\hline 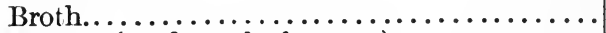 & 0.4 & 0.6 & ... & $\cdots$ \\
\hline 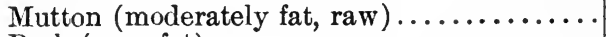 & 17.0 & 6.0 & .... & $\ldots$ \\
\hline 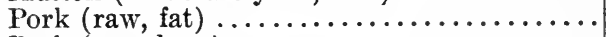 & 14.5 & 37.5 & $\ldots$ & $\ldots$ \\
\hline 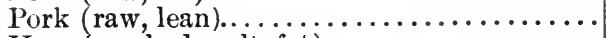 & 20.0 & 7.0 & ... & $\ldots$ \\
\hline 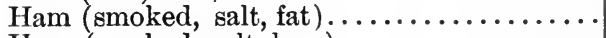 & 21.0 & 36.0 & $\cdots$ & $\cdots$ \\
\hline 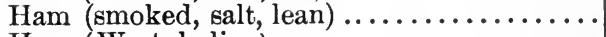 & 24.0 & 8.0 & $\cdots$ & ... \\
\hline Ham (Westphalian) $\ldots \ldots \ldots \ldots \ldots \ldots \ldots \ldots$ & 25.0 & 35.0 & .... & $\ldots$ \\
\hline 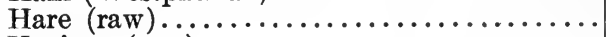 & 23.34 & 1.13 & 0.19 & $\ldots$ \\
\hline 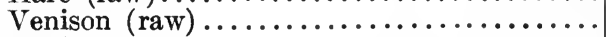 & 20.0 & 1.5 & 1.0 & $\ldots$ \\
\hline 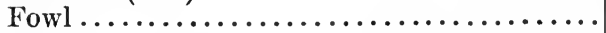 & 21.0 & 2.0 & 2.0 & $\ldots$ \\
\hline 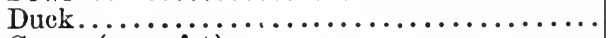 & 22.0 & 3.0 & 2.0 & .... \\
\hline 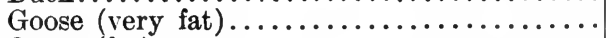 & 16.0 & 45.1 & $\ldots$ & $\ldots$ \\
\hline Goose (fat) $\ldots \ldots \ldots \ldots \ldots \ldots \ldots \ldots \ldots \ldots \ldots$ & 16.0 & 30.0 & & $\ldots$ \\
\hline 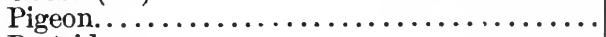 & 22.0 & 1.0 & 0.76 & $\ldots$ \\
\hline Partridge..$\ldots \ldots \ldots \ldots \ldots \ldots \ldots \ldots$ & 25.0 & 1.4 & & $\ldots$ \\
\hline 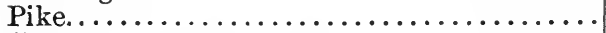 & 18.5 & 0.5 & 0.6 & $\ldots$ \\
\hline 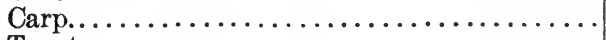 & 21.0 & 1.0 & $\cdots$ & ... \\
\hline 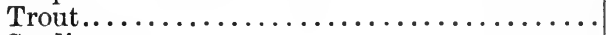 & 19.0 & 2.0 & .... & $\ldots$ \\
\hline Sardines $\ldots \ldots \ldots \ldots \ldots \ldots \ldots \ldots \ldots \ldots \ldots$ & 23.0 & 2.0 & .... & $\ldots$ \\
\hline Salmon $($ fresh $) \ldots \ldots \ldots \ldots \ldots \ldots \ldots \ldots$ & 21.0 & 12.5 & $\ldots$ & $\ldots$ \\
\hline Salmon (smoked) $\ldots \ldots \ldots \ldots \ldots \ldots \ldots \ldots$ & 24.0 & 12.0 & $\ldots$ & $\ldots$ \\
\hline Eels $\ldots \ldots \ldots \ldots \ldots \ldots \ldots \ldots \ldots \ldots, \ldots$ & 13.0 & 28.5 & $\ldots$ & $\ldots$ \\
\hline Shell-fish $($ fresh $) \ldots \ldots \ldots \ldots \ldots \ldots \ldots \ldots$ & $\mathbf{1 7 . 0}$ & 0.35 & $\ldots$ & $\ldots$ \\
\hline 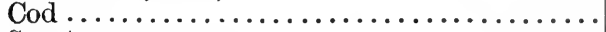 & 82.0 & 0.5 & $\ldots$ & $\ldots$ \\
\hline Sprats $\ldots \ldots, \ldots, \ldots \ldots \ldots \ldots \ldots, \ldots \ldots \ldots$ & 23.0 & 16.0 & $\ldots$ & $\ldots$ \\
\hline Herring (pickled) $\ldots \ldots \ldots \ldots \ldots \ldots \ldots$ & 19.0 & 17.0 & 1.6 & $\ldots$ \\
\hline 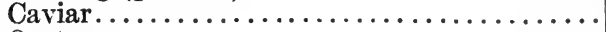 & 32.0 & 15.5 & 2.0 & $\ldots$ \\
\hline Oysters $\ldots \ldots \ldots \ldots \ldots \ldots \ldots \ldots \ldots \ldots \ldots \ldots$ & 8.0 & 1.5 & 2.6 & $\ldots$ \\
\hline$\quad\lceil$ Lungs ............ & 15.0 & 2.5 & 1.0 & .... \\
\hline Average of those from Heart............ & 17.5 & 9.5 & 0.6 & $\ldots$ \\
\hline all the domestic ani- $\{$ Kidneys......... & 18.0 & 5.0 & 0.15 & $\ldots$ \\
\hline Liver........... & 19.5 & 4.5 & 3.28 & $\ldots$ \\
\hline (Tongue.......... & 15.0 & 17.5 & 0.5 & $\ldots$ \\
\hline 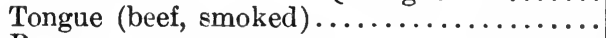 & 24.5 & 31.5 & $\ldots$ & $\ldots$ \\
\hline 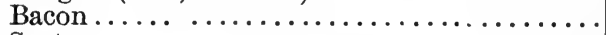 & 9.5 & 76.0 & $\ldots$ & .... \\
\hline Suet.$\ldots \ldots \ldots \ldots \ldots \ldots \ldots \ldots \ldots \ldots \ldots \ldots \ldots \ldots$ & 0.5 & 98.0 & $\ldots$ & $\ldots$ \\
\hline 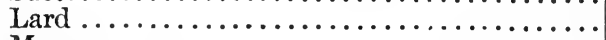 & 0.5 & 99.0 & ... & .... \\
\hline 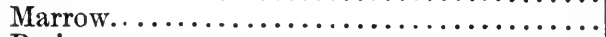 & 1.3 & 93.0 & $\ldots$ & $\ldots$ \\
\hline Brain sausage $\ldots \ldots \ldots \ldots \ldots \ldots \ldots \ldots \ldots$ & 17.5 & 40.0 & & $\ldots$ \\
\hline 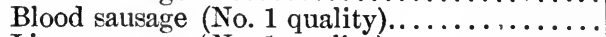 & 12.0 & 11.5 & 25.0 & .... \\
\hline Liver sausage (No. 1 quality) $\ldots \ldots \ldots \ldots \ldots$ & 16.0 & 26.5 & 6.5 & $\ldots$ \\
\hline 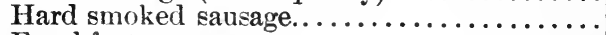 & 23.0 & 11.0 & & ... \\
\hline Frankfurter sausage.................. & 12.0 & 40.0 & 2.25 & $\ldots$ \\
\hline Egg (with shell) $\ldots \ldots \ldots \ldots \ldots \ldots \ldots \ldots \ldots$ & 12.5 & 12.0 & 0.5 & $\ldots$ \\
\hline 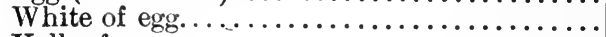 & 12.7 & 0.25 & 0.7 & $\ldots$ \\
\hline Yolk of $\operatorname{egg} \ldots \ldots \ldots \ldots \ldots \ldots \ldots \ldots \ldots$ & 16.0 & 32.0 & 0.1 & $\ldots$ \\
\hline Cow's milk $\ldots \ldots \ldots \ldots \ldots \ldots \ldots \ldots \ldots \ldots$ & 3.5 & 4.0 & 4.9 & $\ldots$ \\
\hline 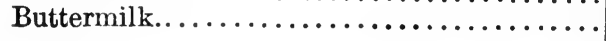 & 4.0 & 0.9 & 3.7 & .... \\
\hline
\end{tabular}




\begin{tabular}{|c|c|c|c|c|}
\hline \multirow{2}{*}{ FOODS. } & \multicolumn{4}{|c|}{ AVERAGE PER CENT. OF } \\
\hline & Proteid. & Fat. & $\begin{array}{c}\text { Carbo- } \\
\text { hydrate. }\end{array}$ & Alcohol. \\
\hline 1. Animal:-Continued. & & & & \\
\hline Skim milk...................... & 3.0 & 0.7 & 4.75 & \\
\hline 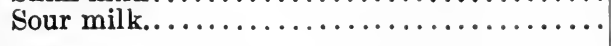 & 8.4 & 3.6 & 3.5 & $\begin{array}{l}\text { about } 2 \% \\
\text { lactic acid }\end{array}$ \\
\hline 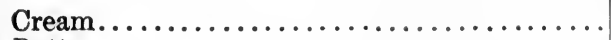 & 3.6 & 25.8 & 3.5 & .... \\
\hline 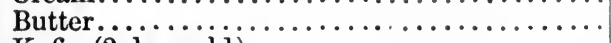 & 0.7 & 84.0 & 0.06 & \\
\hline Kefir $(2$ days old $) \ldots \ldots \ldots \ldots \ldots \ldots \ldots \ldots \ldots \ldots \ldots \ldots \ldots \ldots$ & 3.3 & 2.6 & 1.9 & $\begin{array}{l}(0.5 \text { alcohol }) \\
\text { (0.4 lactic } \\
\text { acid) }\end{array}$ \\
\hline $\int$ lean $\ldots \ldots \ldots \ldots \ldots \ldots \ldots \ldots \ldots \ldots \ldots \ldots \ldots$ & 34.0 & 11.0 & 3.5 & $\ldots$ \\
\hline Cheese $\left\{\begin{array}{l}\text { half fat. } \ldots \ldots \ldots \ldots \ldots \ldots \ldots \ldots \ldots \\
\text { fat } \ldots \ldots \ldots \ldots \ldots\end{array}\right.$ & 29.5 & 24.0 & 2.0 & $\cdots$ \\
\hline Neufchâtel $]^{\cdots \cdots \cdots \cdots \cdots \cdots \cdots \cdots \cdots \cdots \cdots \cdots}$ & 25.0 & 30.5 & 1.5 & $\cdots$ \\
\hline Cheese $\left\{\begin{array}{l}\text { Stracchino } \\
\text { Imperial.. } \\
\text { Gervais... }\end{array}\right\} \ldots \ldots \ldots \ldots \ldots \ldots \ldots$ & 19.0 & 41.0 & 1.0 & $\cdots$ \\
\hline 2. Vegetable: & & & & \\
\hline Flour (fine) $\ldots \ldots \ldots \ldots \ldots \ldots \ldots \ldots \ldots$ & 10.0 & 1.0 & 72.0 & $\ldots$ \\
\hline 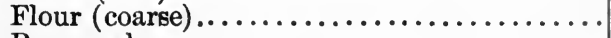 & 12.0 & 1.5 & 70.0 & $\ldots$ \\
\hline 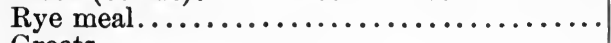 & 11.5 & 2.0 & 69.5 & $\cdots$ \\
\hline 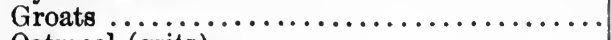 & 7.5 & 1.0 & 76.0 & ... \\
\hline 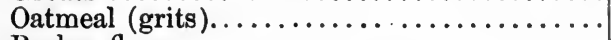 & 13.5 & 6.0 & 67.0 & $\ldots$ \\
\hline 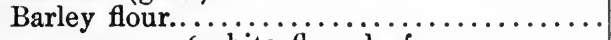 & 11.0 & 1.5 & 71.5 & ... \\
\hline & 7.0 & 0.5 & 52.0 & $\cdots$ \\
\hline Rye bread $\ldots \ldots \ldots \ldots \ldots$ & 9.0 & 1.0 & 59.5 & $\cdots$ \\
\hline 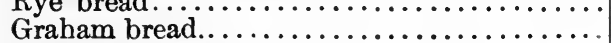 & $\begin{array}{l}6.0 \\
9.0\end{array}$ & $\begin{array}{l}0.0 \\
1.0\end{array}$ & $\begin{array}{l}47.0 \\
50.0\end{array}$ & $\cdots$ \\
\hline Zwieback (fine) $\ldots \ldots \ldots \ldots \ldots \ldots \ldots \ldots \ldots \ldots \ldots \ldots$ & 13.0 & 3.0 & 80.0 & $\cdots$ \\
\hline 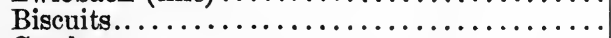 & 12.0 & 7.5 & 68.0 & $\ldots$ \\
\hline 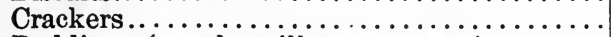 & 10.0 & 5.0 & 65.0 & $\ldots$ \\
\hline Puddings (starch, milk, eggs, sugar)....... & 4.25 & 4.0 & 23.0 & $\ldots$ \\
\hline Dumplings (whole wheat flour rolls, eggs, & 24 & 40 & 70 & \\
\hline 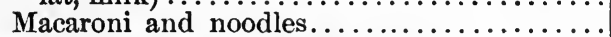 & $\begin{array}{l}2.4 \\
9.0\end{array}$ & $\begin{array}{l}4.0 \\
0.5\end{array}$ & $\begin{array}{r}7.0 \\
77.0\end{array}$ & $\cdots$ \\
\hline Souffle (farina, sugar, eggs, milk).......... & 7.0 & 5.0 & 13.5 & $\cdots$ \\
\hline Fritters (flour, milk, eggs, fat)........... & 9.0 & 16.0 & 31.0 & $\ldots$ \\
\hline 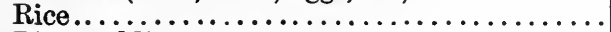 & 6.5 & 1.0 & 78.5 & $\ldots$ \\
\hline 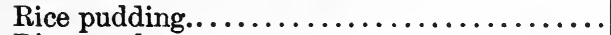 & 9.1 & 3.5 & 29.0 & $\ldots$ \\
\hline 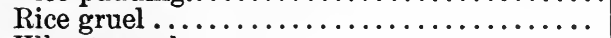 & 4.0 & 3.0 & 13.0 & $\ldots$ \\
\hline 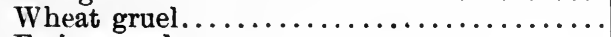 & 5.0 & 4.0 & 9.0 & ... \\
\hline Farina gruel $\ldots \ldots \ldots \ldots \ldots \ldots \ldots \ldots \ldots$ & 3.0 & 2.0 & 7.0 & $\cdots$ \\
\hline Potatoes........................ & 2.0 & 0.2 & 20.5 & $\ldots$ \\
\hline 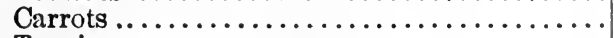 & 1.0 & 0.2 & 8.0 & $\ldots$ \\
\hline 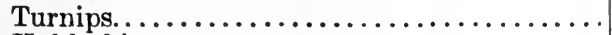 & 1.0 & 0.2 & 6.0 & $\ldots$ \\
\hline 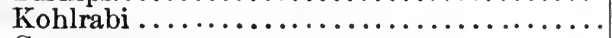 & 3.0 & 0.2 & 8.0 & ... \\
\hline 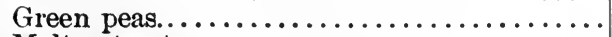 & 6.0 & 0.5 & 11.0 & $\ldots$ \\
\hline Malt extract $\ldots \ldots \ldots \ldots \ldots \ldots \ldots \ldots \ldots \ldots \ldots \ldots$ & 5.0 & & 65.0 & $\ldots$ \\
\hline 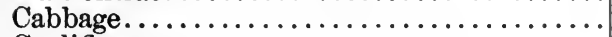 & 2.5 & 0.5 & 6.5 & $\ldots$ \\
\hline Cauliflower $\ldots \ldots \ldots \ldots \ldots \ldots \ldots \ldots \ldots$ & 2.5 & 0.3 & 4.5 & $\ldots$ \\
\hline Sauerkraut................... & 1.0 & 0.2 & 4.5 & ... \\
\hline 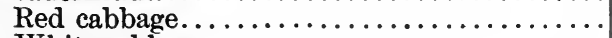 & 2.0 & 0.2 & 6.0 & $\ldots$ \\
\hline 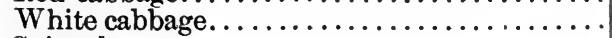 & 2.0 & 0.2 & 5.0 & $\ldots$ \\
\hline 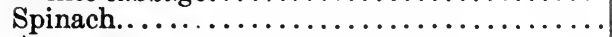 & 3.0 & 0.5 & 5.0 & $\ldots$ \\
\hline Asparagus $\ldots \ldots \ldots \ldots \ldots \ldots \ldots \ldots \ldots \ldots$ & 2.5 & 0.3 & 2.5 & $\ldots$ \\
\hline 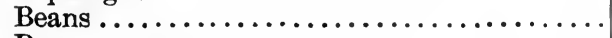 & 24.5 & 2.0 & 52.0 & $\cdots$ \\
\hline 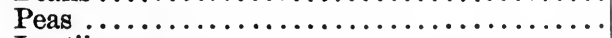 & 23.0 & 2.0 & 52.5 & ... \\
\hline 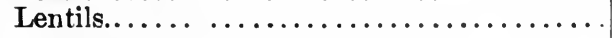 & 26.0 & 2.0 & 53.0 & ... \\
\hline
\end{tabular}




\begin{tabular}{|c|c|c|c|c|}
\hline \multirow{2}{*}{ FOODS. } & \multicolumn{4}{|c|}{ AVERAGE PER CENT. OF } \\
\hline & Proteid. & Fat. & $\begin{array}{l}\text { Carbo- } \\
\text { hydrate. }\end{array}$ & Alcohol. \\
\hline 2. Vegetable :-Continued. & & & & \\
\hline Radishes (small) $\ldots \ldots \ldots \ldots \ldots \ldots \ldots \ldots$ & 1.2 & 0.1 & 3.7 & $\ldots$ \\
\hline Lettuce $\ldots \ldots \ldots \ldots \ldots \ldots \ldots \ldots \ldots \ldots \ldots$ & 1.4 & 0.3 & 2.2 & ... \\
\hline Green salads $\ldots \ldots \ldots \ldots \ldots \ldots \ldots \ldots \ldots$ & 1.3 & 0.5 & 3.5 & $\ldots$ \\
\hline Endives $\ldots \ldots \ldots \ldots \ldots \ldots \ldots \ldots \ldots \ldots$ & 1.8 & 0.1 & 3.5 & $\ldots$ \\
\hline Greens $\ldots \ldots \ldots \ldots \ldots \ldots \ldots \ldots \ldots \ldots$ & 2.0 & 0.4 & 2.7 & $\ldots$ \\
\hline 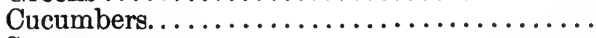 & 1.0 & 0.09 & 2.2 & ... \\
\hline $\operatorname{Sugar} \ldots \ldots \ldots \ldots \ldots \ldots \ldots \ldots \ldots \ldots \ldots$ & 0.5 & & 96.5 & $\ldots$ \\
\hline$\ldots \ldots \ldots \ldots \ldots \ldots \ldots$, & & 95.0 & & $\ldots$ \\
\hline 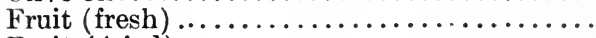 & 0.5 & & 10.0 & $\ldots$ \\
\hline Fruit $($ dried $) \ldots \ldots \ldots \ldots \ldots \ldots \ldots \ldots \ldots \ldots$ & 2.5 & 1.0 & 55.0 & ... \\
\hline Mushrooms (champignons) $\ldots \ldots \ldots \ldots \ldots$ & 2.5 & 0.1 & 4.8 & $\cdots$ \\
\hline Drinks : & & & & \\
\hline Beer.................... & 4.3 & … & $\begin{array}{c}0.8 \\
\text { and extract }\end{array}$ & 4.0 \\
\hline Light white wine (Rhine or Moselle)....... & 0.25 & ... & 1.6 & 10.0 \\
\hline Red wine (Bordeaux, Burgundy)........... & 0.2 & $\ldots$ & 11.0 & 10.0 \\
\hline Hungarian wine $\ldots \ldots \ldots \ldots \ldots \ldots \ldots \ldots \ldots \ldots \ldots \ldots \ldots \ldots$ & 0.2 & $\ldots$ & 3.6 & 12.2 \\
\hline 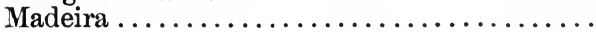 & 0.2 & $\ldots$ & 3.0 & 20.2 \\
\hline 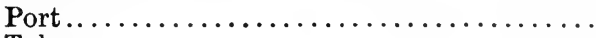 & 0.18 & $\ldots$ & 5.8 & 18.0 \\
\hline Tokay $\ldots \ldots \ldots \ldots \ldots \ldots \ldots \ldots \ldots \ldots \ldots$ & & $\ldots$ & 12.0 & 17.0 \\
\hline Sherry.$\ldots \ldots \ldots \ldots \ldots \ldots \ldots \ldots \ldots \ldots$ & 0.2 & $\ldots$ & 5.0 & 17.0 \\
\hline 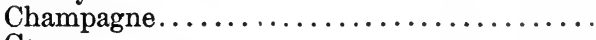 & 0.2 & $\ldots$ & 12.0 & 11.0 \\
\hline $\operatorname{Cognac} \ldots \ldots \ldots \ldots \ldots \ldots \ldots \ldots \ldots \ldots \ldots$ & $\ldots$ & $\ldots$ & & 70.0 \\
\hline 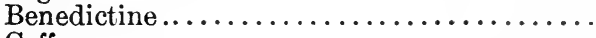 & $\cdots$ & $\cdots$ & 33.0 & 52.0 \\
\hline Coffee & 0.16 & 0.5 & 1.4 & $\ldots$ \\
\hline 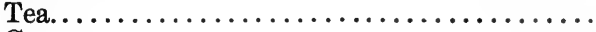 & 0.3 & & 0.6 & \\
\hline 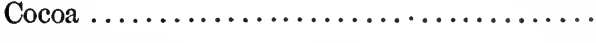 & 14.0 & 47.0 & 18.5 & $\begin{array}{l}1.6 \\
\text { theobromine }\end{array}$ \\
\hline Cocoa (fat removed in part).............. & 21.5 & 27.0 & 34.0 & 1.8 \\
\hline 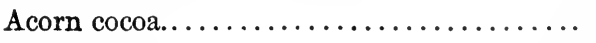 & 8.2 & 14.4 & 48.5 & 2.0 \\
\hline Chocolate.................. & 5.0 & 15.0 & 75.0 & .... \\
\hline "Kraftschocolade" .............. & 4.0 & 21.0 & 73.0 & .... \\
\hline
\end{tabular}

[It is well constantly to bear in mind that bread and cereals contain 60 per cent. of carbohydrates, potatoes and bananas 20 per cent., grape-fruit 4.5 per cent., oranges 10 per cent., and cream and milk 3 to 5 per cent. - Ed.]

The fat total, which is rather low, may be raised to any desired amount by giving fatter meat. Instead of milk, café au lait may often be given (10 per cent. infusion of coffee added to milk) and 250-300 G. (9-10 oz.) of soup at noon.

Distribution: Specimen Daily Dietary.-The foregoing list may be divided between the different meals as follows: First breakfast: 1 glass milk (or coffee), roll. Second breakfast:1 One-half or all the butter, half the bread. Dinner: Soup, beef, vegetables, potatoes, 1 glass of beer. Afternoon lunch: 1 glass of milk (or coffee), one-quarter the bread, the rest of the butter (if any was left). Supper: Veal, sausage, or cheese, rice, one-quarter the bread, 1 glass beer. 


\section{Dietetic Therapy of Diabetes Mellitus.}

Principles.-The two following general principles apply to the dietetic therapy of all cases of diabetes: First, we must aim to preserve, in the end, the normal body weight, or if this has fallen, to bring it up again; and second, we must entirely eliminate the carbohydrates from the food, or if this is unnecessary or, for reasons soon to be explained, impossible, we must restrict the amount allowed. This naturally means an increased amount of fat and proteid.

We have spoken of the "normal" body weight, for it is evident that many individuals with diabetes of a light grade may be so fat that they very advantageously lose a good deal in weight when the excess of carbohydrate (beer, puddings, etc.), is cut down. And we have expressly specified that this weight must be maintained "in the end," for it is sometimes necessary to put up with a temporary loss of a few pounds in order to secure other advantages.

It is essential to recognize that too much proteid cannot be allowed, for (1) it may cause indigestion; (2) one group of diabetics cannot assimilate the sugar formed from proteid; (3) in severe cases unlimited meat may bring on diabetic coma and (4) many diabetics, up to the time of the discovery of their condition, have been in the habit of taking altogether too much proteid (meat). Naunyn put the daily amount of proteid at $625 \mathrm{G}$. ( $1 \mathrm{r} / 4 \mathrm{lb}$.) of raw, or $500 \mathrm{G}$. ( $1 \mathrm{lb} ., 5$ or 6 medium slices) of cooked meat, recommending that part of this be replaced by eggs. The author wishes to emphasize particularly the desirability of giving a good deal of fat. It is as easy for the patient to get too little fat as it is for him to get too much proteid.

It is often better to send a diabetic to a sanatorium, in order that the desired dietetic therapy may be strictly enforced.

Before the exact diet can be properly determined, it is advisable to divide all cases of diabetes mellitus into three classes; i.e., the light, the severe, and the moderately severe, this classification being based principally upon the tolerance of each individual case for carbohydrates, and secondarily upon eertain accompanying chemical and clinical characteristics.

To determine the tolerance for carbohydrates the patient is fed for 3 days on a test diet, in which the amount of carbohydrates can be as exactly as possible determined. On the third day the 24-hour urine is collected and the total amount of sugar is estimated. By a comparison of this amount with the amount of carbohydrates ingested, it is determined how much sugar the patient can oxidize and how much is excreted in the form of dextrose in the urine.

Test Diet.-This test diet should consist of a varying quantity of meat broth and some form of meat free from carbohydrates (beef, veal, and bacon), or oil, and carefully weighed amounts of green vegetables, such as cabbage or asparagus (never potatoes, peas, beans, or turnips, 
on account of their high percentage of carbohydrate), milk, and bread or rolls. The total amount of carbohydrates can now be easily reckoned, the green vegetables containing 4.5 per cent., the milk 4 per cent., and the bread 60 per cent., while the meat and broth may be disregarded. If flour and eggs are used in the preparation of the food, their carbohydrates must be included in the total (flour 70 per cent., eggs 0.50 per cent.). For instance, if the patient receives, beside his broth and sometimes very weak tea, $300 \mathrm{G}$. (10 oz.) of meat, $200 \mathrm{G}$. ( $7 \mathrm{oz}$.) of the selected vegetables, $1 \mathrm{I} / 2 \mathrm{~L}$. ( $3 \mathrm{pt}$.) of milk, $2 \mathrm{eggs}$, and 2 rolls, the total amount of carbohydrates ingested is, in round numbers, $130 \mathrm{G}$. (41/2 oz.)

If now during the third day he excretes $3 \mathrm{~L}$. (3 qt.) of urine with 1.5 per cent. of sugar, he excretes $45 \mathrm{G}$. (11/2 oz.) of sugar, having burned 85 G. (3 oz.). His tolerance for carbohydrates is, therefore, not bad. Another patient on the same diet may excrete the same amount of urine, but with a 4 per cent. sugar content. This means that he has burned only 15 G. ( $1 / 2$ oz.) of the total 130 G. $\left(4 \frac{1}{2}\right.$ oz.) of sugar ingested, a much greater intolerance, but still not indicating a hopeless case. If, however, his urine should contain 5 per cent. of sugar, his condition would be much more serious, as he would then excrete $150 \mathrm{G}$. (5 oz.) of sugar against the $130 \mathrm{G}$. $(4 \mathrm{r} / 2 \mathrm{oz}$.) of the carbohydrates ingested.

These different grades of cases naturally react differently to a reduction of the carbohydrates in the diet. In some cases, the sugar content of the urine may be quickly made to disappear entirely, in others a reduction of even 1 per cent. cannot be accomplished, and between these two extremes lie all possible grades.

The Three Grades of Diabetes.-Light cases of diabetes may be defined as those in which a reduction of the carbohydrates quickly produces a total disappearance of sugar from the urine, which remains normal even after the amount of carbohydrates is increased. The patient may lose flesh in the process, but suffers no distress from it. The tolerance for carbohydrates is never bad. Such patients are usually over 40 years old, generally between 50 and 60, of good nutrition (often too stout) and appearance, with no acetone or diacetic or oxybutyric acid in the urine, and in general only $2-3$ per cent., at most, of sugar.

The severe cases are mostly young individuals, under 40 years, exceedingly thin (about $100 \mathrm{lb}$.), and weak. Polyuria is usually present and the urine has a high specific gravity (1.025-1.040 or even more), with 4-10 per cent. of sugar, and some acetone and diacetic and oxybutyric acids. The carbohydrate tolerance is very poor, sugar being made from the proteids in the food.

Between these two extremes come all transitional grades,- the "moderately severe" cases. Clinically, they present the typical picture of diabetes: noticeable thinness (about $120 \mathrm{lb}$.), moderate polyuria, sugar content generally moderate (though sometimes as high as 8 per cent. or over), but without acidosis upon an ordinary diet. 
After determining the grade of the ease, our next task is to reduce the carbohydrates. This should not be done too suddenly, for fear of inducing marked loss of weight, dyspeptic disturbances, or signs of acidosis. Furthermore, the diet should always furnish the required number of calories (2500 a day). In practice it is so difficult to get an aecurate calculation of the amount of calories furnished, that it is usually sufficient to see to it that the weight and strength are amply maintained.

\section{DIETETIC TREATMENT OF THE MILD FORM OF DIABETES MELLITUS.}

Initial Test Diet.- The patient is first put on a test diet, to determine his tolerance for carbohydrates. If this diet causes the sugar to disappear or fall to 0.10 per cent. or less, he is kept on the same amount of carbohydrates for $2-3$ weeks. If, however, the sugar content remains above 0.10 per cent., a further reduction of carbohydrates is indicated. In 3-4 weeks, our aim will usually be accomplished. The patient must then remain 2-3 weeks longer on the final carbohydrate ration. In either case, after the 2-3 weeks are up, the amount of carbohydrates may be gradually increased, until the patient is getting a normal ration (quantitatively) of the forms already mentioned (e.g., bread or milk). The amount of proteids may also be increased, should it seem desirable.

Foods Forbidden.-The list of foods permanently forbidden is as follows: Sugar, honey, preserves, jellies, cake, ice cream, sweet ices, all sweets, rice and all farinaceous foods, sweet wine, and generally also beer.

Foods Allowed in Moderation.-The following may be allowed in limited quantities, to be increased only with great caution: Milk, bread, potatoes, and sauces and gravies containing flour or starch. Some patients of this type can take a small amount of beer, $1 / 4-1 / 2 \mathrm{~L} .(\mathrm{I} / 2-1 \mathrm{pt}$.).

Foods Permitted.-All foods which contain no carbohydrates, or almost none, may be permitted. The following is von Noorden's table:

Fresh Meat: Beef, veal, mutton, lamb, pork, game, and poultry, roasted or boiled, with dish gravy or mayonnaise, hot or cold; tongue, heart, lungs, brains, sweetbreads, kidneys, marrow, prepared with sauces in which no flour or starch is used.

Salt, Smoked, and Pickled Meats : Dried beef, smoked meats, smoked or salted tongue, ham, corned beef, canned meats.

Fresh Fish: All kinds of fresh fish, boiled or broiled, served with melted butter or any kind of sauce containing no flour or starch.

Dried, Salt, and Smoked Fish: Dried codfish, salt and smoked fish of all kinds, sardines, anchovies, etc., caviar, cod-liver oil.

Shell-fish : Oysters, mussels, crabs, lobsters, turtles.

Meat Extracts : Meat peptones of all kinds, somatose.

Eggs : Prepared in any way, provided no flour or starch is used. 
Fats : Every kind.

Fresh Vegetables: Lettuce, endive, cress, spinach, chicory, cucumbers, onions, asparagus, leeks, cabbage, cauliflower, sorrel, string beans, parsley. Butter, salt, stock or meat extracts, mayonnaise or French salad dressing may be used in preparing them.

Pickles : Mixed pickles, pickled beans and cucumbers, olives, sauerkraut.

Condiments: Salt, pepper, cayenne pepper, paprika, curry powder, cinnamon, nutmeg, mace, saffron, mustard, anise, citron, carawayseed, garlic, dill, borage, bay leaves, capers. (Carbohydrate content is too small to be considered.)

Soups: All soups made from stock, with nothing added but eggs, marrow balls, or vegetables (selected from among those allowed above).

Cheese: Stracchino, Neufchâtel, old Camembert, Gorgonzola.

Drinks: All kinds of chalybeate mineral waters and artificial Seltzer, either plain or with the addition of lemon juice and saccharin or glycerin, or with a little rum, brandy, whiskey, cherry brandy, etc. Light Moselle or Rhine wine and the like; Bordeaux, Burgundy, and "saccharin champagne" as prescribed by the physician. Coffee with or without cream, but with no sugar (saccharin if needed). Tea with cream, lemon, or rum.

The diet should be varied as much as possible, but the amount of substituted carbohydrates must always be kept down to a strict equivalent of the amount of carbohydrates (milk, bread, potatoes) allowed. (For certain exceptions, see page 219.)

Maximum Quantity of Carbohydrates Permitted.-In general, such patients can take $1 \mathrm{~L}$. (1 qt.) of milk and $60-90 \mathrm{G}$. of bread (2-3 oz., or 2-3 slices), or $100 \mathrm{G}$. ( $3 \mathrm{~T} / 2 \mathrm{oz}$.) of bread and $150 \mathrm{G}$. of potatoes ( $5 \mathrm{oz}$., or 1 medium large potato) daily without resulting glycosuria.

Substitution of Other Carbohydrates.-To decide how much of other carbohydrate foods are equivalent in each case to the quantities just cited, we must take into consideration (1) the content of carbohydrate, and (2) the chemical composition of the particular carbohydrates in question. For we know that in diabetes some carbohydrates are oxidized much more easily than are others. Fruit sugar (levulose) and inulin are disposed of most easily; grape sugar and starch only half as easily, while cane sugar and milk sugar come half-way between the two. From these data, the equivalents of $50 \mathrm{G}$. (13/4 oz.) of white bread (with about 30 per cent. of starch) may be calculated (von Noorden).

50 G. of white bread ( $1 \frac{3}{4} \mathrm{oz}$, or 2 slices) is equal to :

2 qt. Gaertner's " fat-milk"

1 qt. milk (sweet, sour, buttermilk)

$1 \frac{1}{2}$ qt. kefir (at least 2 days old, if no sugar has been added)

1 qt. cream

60 G. (2 oz.) graham bread, rye bread, or pumpernickel 


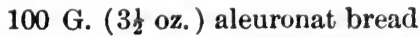

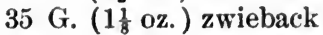

50 G. (1 $1 \frac{3}{4} \mathrm{oz}$.) Rademann's diabetic rods

30 G. (1 oz.) English biscuit

30 G. (1 oz.) acorn cocoa (Stollwerck)

$50 \mathrm{G}$. (13 oz.) chocolate

40 G. (1 $\left.\frac{1}{4} \mathrm{Oz}.\right)$ French chocolate

40 G. ( $1 \frac{1}{4} \mathrm{oz}$.) chestnuts (weighed with shells off)

$60 \mathrm{G}$. ( $2 \mathrm{oz}$.) chestnuts (weighed with shells on)

35 G. ( $1 \frac{1}{8} \mathrm{oz}$.) white or brown sugar

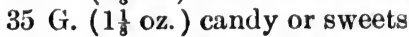

$40 \mathrm{G}$. (1 oz.) fruit sugar

40 G. (1 oz.) milk sugar

50 G. (193 oz.) jam

40 G. ( $1 \frac{1}{4}$ oz.) meals made from wheat, rye, barley, buckwheat, corn, or rice

$45 \mathrm{G}$. (15 oz.) meals made from peas, beans, or lentils

35 G. ( $1 \frac{1}{8}$ oz. $)$ fine, sifted meals-tapioca, rice meal, sago meal, maizena

35 G. $\left(1 \frac{1}{8}\right.$ oz. $)$ rice

35 G. (1 $1 \frac{1}{8}$ oz.) noodles, macaroni, pearl barley, or oatmeal

50-60 G. (13-2 oz. ) dried peas, beans, or lentils

100 G. ( $3 \frac{1}{2}$ oz.) green peas

$180 \mathrm{G}$. $(6 \mathrm{oz}$.) new potatoes

$140 \mathrm{G}$. ( $4 \frac{1}{2} \mathrm{oz}$.) old potatoes

120 G. (4 oz.) fresh apples, pears, plums, grapes, apricots, or cherries

200 G. (7 oz.) strawberries, gooseberries, mulberries, raspberries, huckleberries, cranberries, or blackberries

3 peaches

50 G. (1 $\left.\frac{3}{4} \mathrm{oz}.\right)$ figs

3 bananas

A handful of hazelnuts, almonds, walnuts, or Brazil nuts $1 \frac{1}{2} \mathrm{pt}$. of any kind of beer

$\frac{1}{3} \mathrm{pt}$. of sweet wine

The author agrees with Naunyn in recommending from the above list even for more severe cases, fruit and nuts, especially almonds, mandarins, oranges, ${ }^{2}$ cranberries, gooseberries, and strawberries. Grapes and dried fruit are generally best avoided.

List of Extra Carbohydrates Permitted.-Certain foods containing carbohydrates may be allowed in limited amounts as extras, without reducing the quantities of the standard carbohydrates given. Such foods either have such a low per cent. of carbohydrates, or else are taken in such small quantities absolutely that the amount of carbohydrates is negligible. Examples are:

Calves' Liver or Poultry Liver: Up to 100 G. ( $3 \frac{1}{2}$ oz.).

Sausages : Liver sausage of the best quality(fat), blood sausage, $60 \mathrm{G}$. ( 2 oz. ), meat sausage, 80 G. ( $2 \frac{3}{4}$ oz. ), frankfurters, brawn, calves' head, 100 G. ( $3 \frac{1}{2}$ oz.).

\footnotetext{
2 [Sweet and sour oranges, according to Overlander, contain respectively 9 and 10 per cent. of sugar, and grape-fruit 4.5 per cent. - Ed.]
} 
Potted and Fresh Meats: Strassburg pâté de foie gras, potted beef, tongue, salmon, and lobster, anchovy paste, 1-2 tablespoonfuls.

English Sauces : Worcestershire, anchovy, soy (India and China), lobster and shrimp sauces, 1 teaspoonful.

Cream: 4-6 tablespoonfuls daily.

Cocoa (without sugar): 25 G. ( $\frac{3}{4}$ oz.).

Cheese: Stilton, Holland, Gruyére, Brie, Gervais, 50 G. ( $1 \frac{3}{4}$ oz.); Emmenthal, Romadour, 60 G. (2 oz.); Edam, Cheddar, Gloucester, Roquefort, Parmesan, 30 G. (1 oz.); Cheshire, 25 G. $\left(\frac{3}{4}\right.$ oz.).

Vegetables (prepared without sugar or flour): Squash, kohlrabi, celery root, green peas, beans, and carrots, $1 \frac{1}{2}$ artichokes, 1 truffle, 5 champignons of medium size, 1 tablespoonful of morchella and other edible mushrooms.

Raw Vegetables : 8 little radishes, 2 stalks of celery, 2 medium sized tomatoes.

Nuts, etc.: 2 walnuts, 6 hazelnuts, 5 almonds, 1 thin slice of cocoanut, 8 Brazil nuts.

Fruit: 1 small slice of melon, 1 small sour apple, $1 \frac{1}{2}$ peaches, 1 tablespoonful of raspberries or strawberries, 4 tablespoonfuls of currants, 12 cherries, half of a pear.

Only from two to four of the above articles to be used on the same day.

Milk.-The average amount of milk, if given (see Table on p. 218), is 1 quart, but this may often be greatly increased. In case glycosuria or digestive disturbances appear, we may try sour milk or kefir, where a part of the milk sugar has been fermented. Gärtner's "fat-milk" is particularly good, and is prepared very easily, as follows: Ordinary milk, warm from the cow, is diluted with an equal quantity of warm water and centrifuged. One tube is connected with the part of the apparatus where the cream collects; another, with that containing the diluted skimmed milk. By drawing the proper amount from each tube into the same vessel, a milk of any desired composition can be obtained. We can, for instance, get a milk containing 5 per cent. fat, 2 per cent. casein, and 1 per cent. sugar. If the patient wishes, this can be sweetened by the addition of saccharin. One quart of this "fat-milk" gives 388 calories. As von Noorden says, by using it we can get all the advantages of a milk diet, without the disadvantage of too much milk sugar. [Gärtner's diabetic milk was prepared at von Noorden's instigation and is sold in sterilized bottles, each containing .3 litre. Numerous analyses show that it contains 5-6 per cent. fat and only .9-1 per cent. milk sugar. It is well to remember in applying the author's method of preparing Gärtner's fat-milk that the percentage of sugar in eream varies inversely with the percentage of fat:

16 per cent. cream, about...............4 per cent. milk sugar

20 per cent. cream, $\ldots \ldots \ldots \ldots \ldots \ldots \ldots \ldots 2-3$ per cent. milk sugar

40 per cent. cream, about..............2 per cent. milk sugar 
Robert Hutchinson recommends the following sugar-free milk for diabetes: "Take 1 litre of skim milk, heat to a temperature of $38^{\circ} \mathrm{C}$., and add 10 c.c. of glacial acetic acid, diluted with 100 c.c. of water. Mix, and allow the mixture to stand for about 15 minutes. Collect the separated casein, and let it drain on very fine muslin, using no pressure. Remove the casein to a mortar, rub to a smooth paste, add $1 / 2$ litre of distilled water, and strain as before. Repeat this washing of the casein twice. Transfer to a mortar, rub until quite smooth, and add $2.5 \mathrm{G}$. of potassium hydrate dissolved in 100 c.c. of water (or as much of the KOH as is necessary to make the product just alkaline to phenolphthalein). Add $100 \mathrm{G}$. of ordinary Devonshire clotted cream, $5 \mathrm{G}$. of gelatin previously dissolved, $0.06 \mathrm{G}$. of saccharin, and water, at about $38^{\circ}$ C., up to 1 litre. I astly, strain through fine muslin. The product tastes very much like ordinary cow's milk, and can be taken either plain or with some effervescing water, or it can be added to tea or coffee or made into custards with eggs. In some cases as much as $5 \mathrm{pt}$. of it have been taken in one day, and it does not seem to have any appreciable effect in increasing the output of sugar."-Ed.]

Provision for Sufficient Proteid and Fat.-When we have determined the exact amount of carbohydrates allowed a patient with the light form of diabetes, the only other dietetic requisite is the provision of enough proteids and fats to give the required total of calories (about 2500 a day, but according to Naunyn this can often be reduced without the patient's suffering loss of weight or of energy). Thirty to $100 \mathrm{G}$. $(1-31 / 2 \mathrm{oz}$.) of alcohol may be given daily in order to avoid the necessity of too much fat. The latter must be taken regularly, however, in rather large quantities, as it has to take the place of the carbohydrates. Von Noorden gives it as an "iron-clad rule" that the patient should receive a prescribed amount of fat each day; e.g.:

60 G. (2 oz.) butter.......................... 480 calories

2 eggs..................................... 150 calories

10 G. ( $\frac{1}{3}$ oz. $)$ olive oil............................ 90 calories

30 G. (1 oz.) fat cheese........................115 calories

To this we may add 1 qt. of milk (590 calories) and $30 \mathrm{G}$. ( 1 oz.) of alcohol (210 calories), making 1600 calories in this part of the food alone.

Importance of Fresh Vegetables and Fruit.-As has been said before, a patient with the light form of diabetes can eat certain fresh vegetables and fruit (p. 219). These the author gives as soon and in as large quantities as possible, for not only are they an agreeable change for the patient, but their fruit sugar (levulose), when oxidized in the body, is broken down into carbonates, thus increasing the alkalinity of the blood. Moreover, with the exception of grapes and cherries, they are not very rich in sugar, and a half of what sugar they do contain is levulose. Cranberries, oranges, and currants are especially desirable on account of 
their large percentage of fruit sugar in relation to the other sugars (Naunyn).

In general it is not necessary to calculate the amount of animal proteid required; enough will usually be taken spontaneously. Indeed, the patient has frequently been in the habit of eating too much, so that a reduction of the proteids and fats is desirable.

Should 0.10-0.50 per cent. of sugar reappear in the urine, the whole treatment must be repeated.

\section{DIETETIC THERAPY OF THE MODERATELY SEVERE FORM OF DIABETES MELLITUS.}

Reduction of Carbohydrates.-Here, too, we begin by reducing the carbohydrates, so that the bread ration is 50-60 G. (2 oz.). The sugar content of the urine then usually falls considerably, but not to 0.50 per cent. or below, so that it becomes necessary to reduce the milk and bread still further. If after 3-4 weeks the sugar has not yet practically disappeared, all carbohydrates whatever (fruit, vegetables) must be withdrawn, and the amount of meat also be reduced. The author agrees with Naunyn in the advantages of inserting here a day of fasting (only tea and bouillon allowed), as frequently the patient can afterward take food containing a little carbohydrate, which before the fast caused glycosuria. Under this treatment the patient usually loses a pound or so the first week. If the loss amounts to $2 \mathrm{lb}$. or more by the second week and continues into the third week, then, but not sooner, must carbohydrates be given again. Meanwhile, the ferric chloride reaction for diacetic acid must be carefully tried. If it is positive, the author gives $15-20$ G. $(1 / 2-3 / 4$ oz. $)$ of sodium bicarbonate daily. But if within 3 or 4 days it becomes constantly more marked, until a dark brown color is obtained, he follows Naunyn's advice and gives $250-500$ c.c. ( $1 / 2-1$ pt.) of milk. The sugar content is hardly noticeably increased by this, while the ferric chloride reaction diminishes, so that one can reduce the amount of bread, still continuing the milk, until it too, if necessary, can be withdrawn.

We have so far assumed that it has been possible to cause the disappearance of the glycosuria. But there are cases in which this is not possible; the patient cannot stand the necessary reduction in the amount of food. We must then be contented to keep the sugar as low as possible, consistently with maintaining the body weight. If this can be done, the case may still be considered as one of medium intensity; but if the patient constantly loses weight, then the case belongs to the severe type, although the first tests for tolerance would properly place it in the moderately severe class. The treatment then will be identical with that described in the next section.

When once the glycosuria has disappeared, the patient must keep to the same diet for $2-3$ weeks, even if it involves a total abstinence from carbohydrates. We then commence gradually to introduce them again, 
beginning with milk and fruit rather than bread. We start with 50 c.c. $(13 / 4$ oz.) of milk, increasing daily by $50-100$ c.c. $(13 / 4-31 / 2$ oz.) until the reappearance of sugar (even if under 0.50 per cent.) shows us that we have passed the bounds of tolerance. We then reduce the milk and begin again until we have ascertained aceurately the limits of tolerance for milk. When this has been determined, we may begin to substitute bread for milk (e.g., $30 \mathrm{G}$. ( $1 \mathrm{oz}$.) of bread for 200 c.c. $(7 \mathrm{oz}$.$) of milk),$ and after waiting a few days, reduce the milk still farther, replacing it by more bread, vegetables, or potatoes. Naunyn strongly recommends that on one day of the week only meat, fat, and the permitted vegetables be allowed. By following out this procedure carefully, we often obtain permanently good results. Sometimes, however, the patient will grow worse, and the case passes over into the severe type.

Fats.-Naturally, in the moderately severe cases, fat must play an even more important rôle than in the light cases, the chief forms in which it is given being butter, bacon, olive oil, egg yolks, marrow, cream, cream cheese, and fat meat.

At the risk of repetition, the following table is given of foods particularly rich in fat and proteid and poor in carbohydrates. In reckoning the total nutritive values, that of the carbohydrates is omitted, since they are probably not utilized:

\begin{tabular}{|c|c|c|c|c|}
\hline FOODS. & Proteid. & Fat. & $\begin{array}{c}\text { Carbo- } \\
\text { hydrate. }\end{array}$ & $\begin{array}{l}\text { Calories in } \\
100 \mathrm{G} \text {. of the } \\
\text { substance. }\end{array}$ \\
\hline 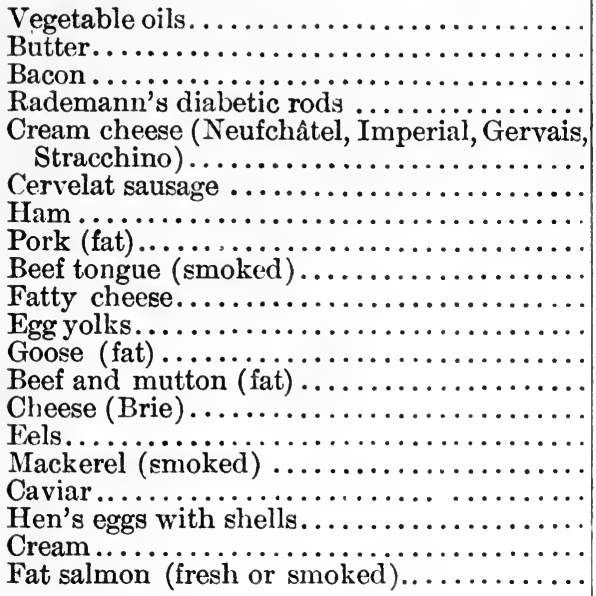 & $\begin{array}{r}1 \\
10 \\
22 \\
19 \\
18 \\
21 \\
14 \\
24 \\
25 \\
16 \\
16 \\
17 \\
19 \\
13 \\
19 \\
32 \\
12 \\
4 \\
22\end{array}$ & $\begin{array}{r}100 \\
84 \\
76 \\
48 \\
\\
41 \\
40 \\
36 \\
37 \\
32 \\
30 \\
32 \\
30 \\
29 \\
26 \\
28 \\
22 \\
16 \\
12 \\
25 \\
13\end{array}$ & $\begin{array}{c}0.5 \\
\dddot{22} \\
1 \\
\ldots \\
\ldots \\
\cdots \\
\cdots \\
1.5 \\
0.1 \\
\ldots \\
\dddot{1} \\
\ldots \\
\ldots \\
\cdots \\
0.5 \\
4 \\
\ldots .\end{array}$ & $\begin{array}{l}459 \\
446 \\
421 \\
401 \\
396 \\
381 \\
363 \\
345 \\
337 \\
321 \\
312 \\
282 \\
280 \\
261 \\
248 \\
210\end{array}$ \\
\hline
\end{tabular}

In this mixed diet, the fats should amount to $160-200 \mathrm{G}$. $(51 / 2-7 \mathrm{oz}$.), of which $40-50$ G. (11/4-13/4 oz.) should consist of butter, and the rest of bacon, olive oil, bone marrow, cream, yolks of eggs, or of butter used in preparing the other foods. 
Alcohol.-If a half bottle (somewhat less than a pint) of wine also be taken, or a glass of brandy, after particularly fat food, these $40 \mathrm{G}$. $(1 \mathrm{I} / 4 \mathrm{oz}$.$) of alcohol will furnish 280$ calories, bringing the nutritive value of fats and alcohol together up to 1700-2000 calories.

\section{DIETETIC THERAPY OF THE SEVERE TYPE OF DIABETES MELLITUS.}

In these cases we again begin by reducing the carbohydrates as far as possible. Great caution must be observed, however, for not only does the patient lose weight rapidly, but a fat and proteid diet involves great danger of intoxication and diabetic coma. We therefore give $20 \mathrm{G}$. ( $5 \mathrm{dr}$.) of sodium bicarbonate daily as soon as diacetic acid is demonstrated. If in the next 3-4 days the amount of diacetic acid greatly increases and oxybutyric acid appears in increasing quantities, then we are obliged to abandon our strict regimen and give milk, fruit, and vegetables, trying to keep up the body weight as much as possible and at the same time to reduce the glycosuria. In some cases (though, according to the author's experience, rather rarely), a fast-day is beneficial, but only when we have been able to reduce the sugar content beforehand to 1 per cent. or less.

The strict fat and proteid diet must always be abandoned if it leads to digestive disturbances, as in severe diabetes these involve serious danger.

\section{Other General Principles of the Dietetic Therapy.}

Amount of Liquid to be Ingested.-Another question remains to be answered: How much liquid should be given? Too much does harm by burdening the stomach and hastening metabolism; but too little is quite as injurious. [Too much also makes excessive demands upon the vascular system.-Ed.] The amount should depend on the quantity of urea to be excreted-that is, on the amount of proteid ingested. On an average, a patient on the diet given above will need $2-2 \frac{1}{2} \mathrm{qt}$. a day, or enough to keep the specific gravity of the urine at about 1.025 (Naunyn).

Substitutes for Bread and for Sugar: Aleuronat Bread, Almond Bread.-Are there no substitutes for common bread and for sugar that will be better fitted to the diabetic patient and yet serve the same end in his dietary? The most common form of bread made for diabetes is aleuronat bread; but, as this contains one-half as much carbohydrates as does common bread, it is evidently not to be used with impunity. Pavy's "almond bread," made of almond meal with butter, eggs, and salt, is better, but contains some sugar. Von Noorden advises Pokorny's diabetic bread, which is the poorest in carbohydrates. Roborat bread is also used. But, no matter what kind is selected, the carbohydrate content must always be taken into account.

Saccharin-Crystallose-Dulcin.-For sugar we substitute saccharin (gluside or benzoyl sulphonimide), which is 300 times as sweet. It may 
be used to sweeten tea, coffee, liqueurs, lemonade, or cooked fruit, and even in the manufacture of sparkling wine (Kohlstadt, Frankfort-amMain). Crystallose is another satisfactory substitute. It is a refined crystallized form of saccharin, easily soluble in water, and 400 times as sweet as sugar. The daily amount of saccharin and of crystallose may reach $0.10 \mathrm{G}$. ( $1 \frac{1}{1} / 2 \mathrm{gr}$.). Dulcin (para-phenetolcarbamide) has recently been recommended, but apparently cannot be taken without danger. Aldehoff. found that it induced icterus.

\section{Hygienic, Etiological and Medicinal Therapy.}

Muscular Work.-Next in importance to the diet in the therapy of diabetes comes muscular work. Experiments (Külz, Zimmer, von Mehring, etc.) have shown that in the light cases and those of medium severity, as well as in some of the severe type, exercise increases the power of the muscies to oxidize carbohydrates, so that the glycosuria is reduced or sometimes stopped entirely, and the organism can dispose of increased quantities of carbohydrates. This exercise may take the form of walking, mountain climbing, gardening, rowing, riding, bicycling, bowling, playing tennis, etc., or may consist in part of gymnastics. It should, if possible, be vigorous; mild, gentle movements do not accomplish as good results. Of course, this presupposes a normal heart and arteries, and a good state of nutrition and of muscular strength. Moreover, the urine should be constantly tested, to be sure that this muscular work is not causing an increased excretion of urea instead of the desired decreasei.e., that it is not being done at the expense of the proteid rather than of the carbohydrate molecule. In fact, the amount of exercise must be gauged by the excretion of urea, which should remain unaltered. If possible, the exercise should be taken as soon as possible after the ingestion of carbohydrate and should last about two hours (von Mehring).

If the state of the patient will not permit such vigorous exertion, he may begin with light exercise and gradually increase it, a close watch being kept on the urine and precaution taken to avoid all overfatigue. In severe cases it is usually impossible for the patient to exercise; he must rest and spare himself as much as possible. Exercise should also be intermitted during the periods of a proteid-fat diet in the moderately severe cases.

Massage.-If the state of the muscles and the general condition of nutrition are poor, or if the heart and vessels are diseased, or there are other complications (lung, kidney), all exercise must be given up, and general massage for 20 minutes once or twice a day be employed instead (Zimmer). Under its influence the glycosuria is decreased, even if the patient is taking some carbohydrates, the thirst is diminished, the general condition is improved, and the body weight is increased.

Mental Rest.-It is exceedingly important that all diabetics, especially those in whom the disease is of nervous origin, should be protected 
from mental excitement. With such patients, any psychical disturbance is often followed by an increase of glycosuria.

The Care of the Skin.-The skin should be intelligently cared for, in order to avoid all danger of the skin diseases that are so liable to complicate diabetes, from furunculosis to erysipelas and gangrene. We may employ daily cool or tepid sponge baths, tepid baths, carbonated baths, weak salt baths, or baths to which the extract of pine needles has been added (100-200 G. (31/2-7 oz.) to a full bath). Strong salt or sea baths do not usually agree with these patients [unless mild cases-Ed.], and dry rubs should be used only with great caution, on account of the vulnerability of the skin. [Thorough washing with an alcoholic soap is useful.-Ed.]

Clothing.-Woollen underwear, changed frequently, should be recommended to most diabetics, as a protection against sudden changes of temperature. [I consider woollen underwear best for winter, but think that linen mesh, or silk, or silk and wool are quite as good for spring, summer, and fall.-Ed.]

Climate.-The summer may be spent at a mild seashore resort, or, better, in a moderately high mountain region; the winter in a southern climate. [Change of scene two or three times a year, the maximum amount of out-door life, congenial surroundings, situations where the dietetic demands can be readily and accurately satisfied, are all important and essential.-Ed.]

Etiological Therapy: Nervous Diabetes.-If the cause of the diabetes is apparently mental overwork or mental shock, certain hydropathic procedures (hot baths or baths at indifferent hot springs) are of undoubted advantage, affecting, as they do, the cause of the disease. [Arsenic, bromides, valerian, and antipyrine are available. Other general rules and drugs will be found in the chapter upon neurasthenia.-Ed.]

Syphilitic Diabetes. - When the disease arises from syphilis of the pancreas (interstitial pancreatitis), mercury, iodides, or both together often bring about a great improvement or even a cure. [Care should be urged in such a plan. I have seen considerable harm result: salivation, stomatitis, and loss of weight and strength.-Ed.]

Pancreatic Diabetes without Syphilis.-When the disease is due to an affection of the pancreas without syphilis,---perhaps an atheroma of the pancreatic arteries, perhaps a pancreolithiasis, or perhaps some obscure affection of the gland,--both the pancreas itself and the various preparations from it have been given with expectations of great success. This therapy is based on the theory, supported by experiment, that such cases of diabetes are due to a deficient production of the internal pancreatic secretion. Pancreas is given either raw, or as an aqueous, alcoholic, or glycerin extract, either by the mouth, by the rectum, or subcutaneously. Pancreatin has also been given, subcutaneously. Although improvement has been noted several times, the results have not generally been satisfactory. The author has succeeded with pancreatin in only 2 cases. One 
was improved, but left the hospital with permanent glycosuria, while the other died of pulmonary tuberculosis. He knows no record of a permanent improvement, to say nothing of a cure. Not enough observations have been made to decide the question properly, but the outlook is apparently not promising.

Judgment must also be reserved on two new remedies proposed by the French, extract of pig's liver and yeast. Of the former, $150 \mathrm{G}$. (5 oz.) (fresh) should be given daily by the rectum. The yeast may be given in suspension or in extract, by mouth, by rectum, subcutaneously, or intravenously. The author has not been successful with either of these. [Yeast is of undoubted value in treating a complicating furunculosis.-Ed.]

Medication: Opium.-Opium has an assured place in the medical treatment of diabetes. Most authors agree that it produces a diminution of the polyuria, a decrease or even the entire disappearance of the glycosuria, diminution of the thirst, and the disappearance or easy satisfaction of the feeling of hunger; in some cases, it appears to effect a general improvement. Von Mehring has observed that opium has more effect in suppressing the sugar formed in severe cases from the albuminates, than that formed in light cases from the carbohydrates. [Von Noorden considers that the drug is indicated in severe and moderately severe cases when a rigid dietary does not cause all the sugar to disappear from the urine. Under such circumstances its effect is very striking, but it has very little value when the patient is still ingesting carbohydrates.-Ed.] The author uses the extract of opium, $0.10-0.50 \mathrm{G}$. (11/2-71/2 gr.) daily in pills or powders :

Ix Ext. opii........................... xv

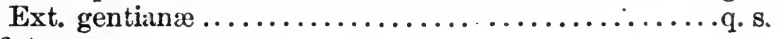

M. fiat mass.

Div. in pil. no. $\mathrm{xxx}$.

Sig. $-3-12$ pills daily. ( 1 pill $=0.03$ ( $\frac{1}{2}$ gr.) extract of opium.)

Even better success follows the use of opium combined with belladonna (Villemin) :

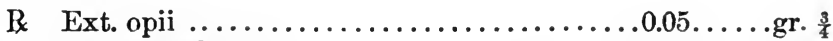

Ext. belladonnæ........................ ss

Sachar. lactis....................... $v$

M. fiat pulv. Dent. tal. dos. no. $\mathbf{x x v}$.

Sig. -3 powders daily.

The author has sometimes given much larger doses, even up to $1 \mathrm{G}$. (15 gr.) of the extract of opium daily, without any symptoms of intoxication. He decidedly prefers opium to codeine or morphine. [Von Noorden, Pavy, and others consider codeine just as efficacious and less constipating.-Ed.]

Alkalies.-As important as opium in the treatment of diabetes are the alkalies and alkaline mineral waters. Large doses of sodium bicar- 
bonate, $30-40$ G. ( $8-10 \mathrm{dr}$.) daily, should be given in every case with plain signs of acid intoxication of the organism (diacetic acid, oxybutyric acid in the urine). Even if these symptoms are not present, the alkalies, especially the alkaline mineral waters, are important. Until recently we have had no plausible explanation for the action of the alkalies in diabetes. But Gans showed experimentally some years ago that glycogen is changed into sugar more slowly if bicarbonate or carbonate of soda is present, although it is apparently unaffected by sodium chloride or Glauber's salts. Perhaps this may indicate that in the living organism also, bicarbonate of soda retards the change of glycogen into sugar and therefore gives the diabetic organism more time to oxidize its sugar. $\mathrm{Be}$ that as it may, the beneficial effect of alkaline mineral waters has been proved by long experience.

Alkaline-Saline Springs. - If the diabetes is complicated by a uric acid diathesis, obesity, or intestinal disturbances, particularly by chronic constipation, or symptoms of a congested, enlarged liver, we choose Carlsbad, Vichy, Tarasp, or the milder Marienbad. In cases of weakness, anæmia, dyspepsia, and gastro-intestinal atony, the common salt springs, such as Homburg and Kissingen, are more suitable. If there are severe nervous symptoms, especially nervous excitability with or without a uric acid diathesis, the simple alkaline springs are most suitable; e.g., Neuenahr, Preblau, Giesshübl, Bilin, Salzbrunn, or Tarasp. [Forchheimer cautions against the danger of reducing the patient too much at Carlsbad. I agree heartily that patients in the later stages of diabetes should not be sent there. They are much better off at home than anywhere else. I may be pardoned for emphasizing again that a cure at Carlsbad will not necessarily reduce a patient. I gained 10 pounds there in less than 6 weeks and saw another person gain 20 pounds in a still shorter time. The effect of the treatment depends largely upon the intelligence of the physician selected there and the eare with which the patient follows his instructions. Many diabetics are sent to Waukesha and Saratoga. The difficulty in both places is that the dietetic regulations are imperfectly carried out.-Ed.]

Arsenic.-Arsenical springs are recommended by many authorities, but arsenic has not yet proved itself of any certain advantage. [Arsenic is one of the most useful drugs which we possess, especially in the severe form of diabetes. Large doses, up to tolerance, are necessary. Diet cannot, however, be neglected even when employing this drug. I do not believe that it raises the assimilative power for carbohydrates, but patients taking it improve. There is a list of arsenical waters on p. 170.-Ed.]

Other drugs which have been recommended for diabetes, but which do not seem to have accomplished what was expected of them from the claims of their respective advocates, are salicylic acid, carbolic acid, lactic acid, eugenia jambolana, pilulæ myrtilli (Jasper), benzosol, sodium selenite, and antipyrine. With the possible exception of antipyrine, these can no longer be looked upon as of direct help in combating the disease as such. Sodium salicylate is often useful, however, in treating 
certain symptoms of diabetes (see p. 231). [Von Noorden recommends salicylic acid, or, better, aspirin, in light cases while carbohydrates are being allowed. He considers that many such patients tolerate more carbohydrates during the administration of some one of these derivatives. He regards them as rather better than antipyrine, though the latter acts in a similar way. He cautions against their prolonged use (not more than a few weeks in a year); against the reaction of the urine to ferric chloride which occurs during the administration of either of these drugs, and which may thus produce the mistaken impression that diacetic acid is present; against the combination of antipyrine with glycuronic acid, in passing through the body, by which the plane of polarized light is turned to the left; and, finally, prohibits the use of salicylic acid or its allies in cases of diabetes with renal complications and of either salicylic acid or antipyrine in gastric disorders. As a result of many experiments, he also recommends the use of jambul preparations occasionally for $3-4$ weeks at a time. Under their influence he has found that he can gradually relax a little from a strict diet and create for a short time, at least in many cases, a higher tolerating power for carbohydrates. He uses 5-8 dr. of the dried powder from the fruits macerated in the cold. His directions follow: Two hundred G. (6 oz.) of last year's dried fruits with seeds are finely powdered, macerated at body temperature for 24 hours in 2 litres (2 qt.) of water with $0.60 \mathrm{G}$. $(10 \mathrm{gr}$.) of salt. The mixture is frequently stirred. One hundred c.c. $(3 \mathrm{oz}$.) of the mixture are ordered cold every morning before breakfast and in the evening before going to bed. Forchheimer has employed urotropine, $0.3 \mathrm{G}$. (5 gr.) 3 or 4 times daily, for the past two years in patients whom he could not trust as to diet, with good effect upon sugar excretion. Fraenkel reports a marked improvement in 22 cases out of 29 following the employment of the salts of Trunecek's serum and eserine, the former to control the early arteriosclerotic manifestations, the latter to improve the hepatic blood supply by its action upon the vagus. Abram has recently suggested an acid extract of the duodenal mucous membrane.-Ed.]

Calcium.-Grube (Neuenahr) has recently advocated the use of calcium, not as a specific to reduce the glycosuria, but as a means of increasing the body weight and improving the general condition. The author's experience confirms this view. The calcium is preseribed as follows :

R Calcii carbonatis..................... $\mathrm{z}$ ii

Calcii phosphatis.......................... $\mathrm{z}_{\mathrm{i}} \mathrm{gr} . \mathrm{xv}$

M. et sig. $-1-3$ teaspoonfuls daily.

\section{THERAPY OF THE COMPLICATIONS AND THE DIFFERENT FORMS OF DIABETES MELLITUS.}

Therapy of Complications: Indication for Alteration of the Diet.-The essentials in the therapy of diabetes, especially the dietetic measures, are not altered, but rather more urgently demanded, by the complications of the disease. If these complications are obstinate and 
will not yield to the usual treatment (e.g., incurable eczema, constantly recurring furuncles, wounds which will not heal, torturing cutaneous itching, neuralgia, neuritis, or diseases of the eyes), it is often better to put even a light case on the diet appropriate to the moderately severe cases.

The special measures to be taken against the manifold complications of diabetes are sometimes prophylactic, and sometimes are directed against the actual presence of such complications.

The Mouth.-As the mouth is exceedingly likely to get into a bad condition, every diabetic patient should have impressed upon him from the beginning, the necessity for using a mouth wash. A 3 por cent. bicarbonate of soda solution or an alkaline mineral water may be used; to a wineglass of either of these may be added a teaspoonful of the official antiseptic solution, which is made up as follows:

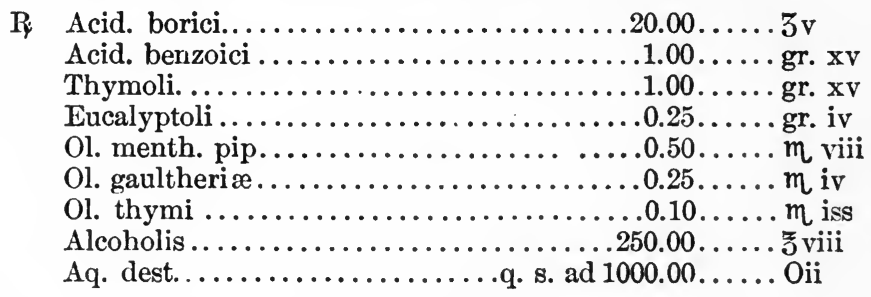

Caries of the Teeth and Fetid Breath.-If the teeth have already begun to be carious and the breath fetid, the following may be recommended :

R Beta naphtholis...................... $0.20 \ldots \ldots$ gr. ii

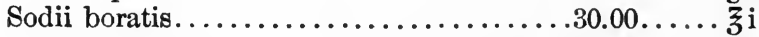

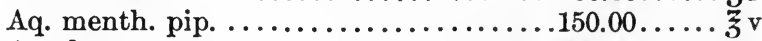

Aq. dest...................... s. ad $1000.00 \ldots \ldots$. Oii

M. fiat sol.

Sig. - To be used as a mouth wash several times a day.

A better combination is the following:

B Formalini .........................10.00.... 3 iiss

Mentholis .............................

Tinct. krameriæ.....................5.00.... 3 i $\eta_{\mathrm{xv}}$

M. fiat sol.

Alcoholis ..................... s. ad $100.00 \ldots \ldots$.. 3 iii 3 ii

Sig. $-\frac{1}{2}-1$ teaspoonful in a quarter of a glass of water.

Stomatitis.-In acute painful inflammation of the gums and mucous membrane of the tongue and mouth, the following gargle is useful:

R Tinct. opii.......................... $4 . \ldots 3^{\text {i }}$

Potassii chloratis,

Sodii boratis ................... āa $10.00 \ldots \ldots z$ iiss

Aq. aurantii flor....................... $30.00 \ldots \ldots$, 3

Aq. dest...................... s. ad $1000.00 \ldots \ldots$. Oii

M. fiat mist.

Sig.-Make lukewarm and use as a gargle. 
Diseases of the Stomach and Intestines.-Acute dyspepsia or acute catarrh of the stomach or intestines demands very careful, special treatment, as the well-being of the diabetic patient depends upon the proper functioning of his stomach. He should be given nothing for a day or two but $1-1 \frac{1}{2}$ L. (2-3 pt.) of red wine, mixed with water, tea, or brandy, to quench the thirst. (Cigar smoking helps to dissipate the abnormal thirst that usually accompanies diabetes.) In many other cases, even the severe ones, the exclusive use of milk or of milk and oatmeal gruel for several days, sometimes accompanied by small doses of opium, will often be successful. When the symptoms have been improved by either of these two methods, the further treatment follows the usual rules for acute digestive disturbances, due regard being of course paid to maintaining an antidiabetic diet.

Heart, Kidney, or Liver Diseases.-Heart, liver, and kidney complications should receive their usual treatment. If, however, a diabetic patient acquires Bright's disease, the latter is more important therapeutically than is the diabetes.

Pulmonary Tuberculosis.-Tuberculosis is the most frequent pulmonary disease complicating diabetes. The usual therapy should be carried out, but great emphasis must be laid upon giving as much fat as possible. Cod-liver oil may be given internally, although it is of questionable use, and also creosote and its derivatives, as in primary pulmonary tuberculosis. [W. H. Thompson warmly advocates cod-liver oil in all cases of diabetes. If patients stand it well I have found it a very useful fat.-Ed.]

Neuralgia.-Many diabetics suffer exceedingly from neuralgias (sciatica, neuralgia in the arm); here pyramidon is often useful (0.50 G. ( $7 \mathrm{I} / 2 \mathrm{gr}$.) 2 or 3 times daily, dissolved in at least 2 tablespoonfuls of water), or the following combination of quinine and opium:

R Quininæ hydrobromatis ............... 0.30....gr. v

Ext. opii ........................... $0.01 \ldots \ldots$ gr. $\frac{1}{6}$

Pulv. glycyrrhiz............................. s.

M. fiat pil. no. i. Dent. tal. pil. no. xxx.

Sig. -1 pill 3 times a day.

Salicylate of Soda in Nervous Disturbances.-Sodium salicylate [or aspirin-Ed.] also is useful in diabetic neuralgia, and also, as Litten has pointed out, in many other nervous disturbances often accompanying the disease; e.g., cutaneous itching (which may be treated as in icterus, q. v.), thirst, desire to urinate, and a ravenous appetite. Litten gives a tablespoonful of a solution of $8 \mathrm{G}$. (2 dr.) of sodium salicylate in $200 \mathrm{G}$. (6 oz.) of water every 2 hours.

Gangrene.-A frequent complication of diabetes is gangrene, generally in the extremities. The extremity should be dressed antiseptically and the line of demareation be awaited, unless the patient shows symptoms of a general sepsis. In that case, primary amputation is indicated. 
Local incisions are of use only where there is local suppuration without any general infection.

Coma.-There remains for discussion the treatment of diabetic coma. By this is meant not the coma which may occur suddenly as the result of heart weakness, but that which occurs with an evident diacetic acid and oxybutyric acid reaction in the urine. When diabetic coma in this narrow sense of the word can be foreseen from the premonitory symptoms,-i.e., abnormal cerebral action (inclination to apathy, to sleep, or to cerebral excitement), digestive disturbances, characteristic alterations in the breathing, an odor of acetone in the breath, and an increase of acetone and diacetic acid in the urine,- the diet should immediately be changed. A strict proteid diet must immediately be modified by the addition of bread, milk, and potatoes. Von Noorden also advises exactly the opposite change if the diet already contains a good deal of carbohydrates, i.e., these should be reduced and considerable quantities of meat and fat be given. Large doses of alcohol, distributed in small quantities over the day, are also indicated. Any medication which has been previously prescribed, especially the use of antipyrine, opium, or bromine as nerve depressors, should be immediately discontinued, and a saline laxative be given-15 G. ( $1 / 2 \mathrm{oz}$.) of sodium sulphate and each day $30-40$ G. (1-11/4 oz.), or even 100 G. (3 oz.) of sodium bicarbonate, sometimes dissolved in an alkaline mineral water, of which 1-2 bottles should be taken during the day. If the heart is weak, digitalis or caffeine may be given. By this alkaline therapy it is sometimes possible to avert an incipient coma; two such cases have fallen under the author's observation. [Diabetic coma is often precipitated by rapid changes in diet. When the urine shows the ferric chloride reaction, all dietetic changes should proceed slowly. Sears (Boston Medical and Surgical Journal, Nov. 30, 1905) reports that 17 cases of recovery from diabetic coma have been recorded in the literature.-Ed.]

If the coma is already definitely present, we must take refuge in intravenous injections of alkalies, although at best their effect is only transitory. One L. ( 1 qt.) ( 250 c.c. ( $1 / 2$ pt.) every 3 hours) of a 0.60 per cent. sterile salt solution in which $50 \mathrm{G}$. (11/2 oz.) of sodium bicarbonate has been dissolved, is injected. We are also often obliged to use strong stimulants and inhalations of oxygen.

Special Therapy of Clinically Different Forms of Diabetes: Diabetes and Gout.-Diabetes is frequently combined with a uric acid diathesis (diabetes uraticus). These cases are usually light "uratic glycosuria." The uric acid diathesis determines the principal points of the therapy; but foods very rich in carbohydrates (sugar, sweets) should be entirely prohibited, and sufficient attention should be paid to the glycosuria. If it does not disappear, all carbohydrates must be suitably reduced.

Diabetes and Obesity.-If diabetes is combined with obesity, the former must be adequately treated (see p. 241). 
Finally, if the patient is very weak, tonics (iron, arsenic, sodium phosphate) are in place; mineral waters, especially Carlsbad, are not indicated.

[The author's treatment of diabetes is so thorough that few additions seem necessary. Our aim in every case of diabetes should be, first, to determine the patient's tolerance for carbohydrates; next, to increase this tolerance. One principle guides us in the latter effort: if the urine can be rendered free from sugar for a few days, the tolerance promptly increases. Various expedients may be resorted to in severe cases where such reduction of sugar is difficult: (1) The total quantity of food should be reduced until the patient begins to lose weight, because it is easier to reduce the sugar excretion upon a diet of low than of high caloric value. (2) If still unsuccessful, the total quantity of proteids should be further reduced. (3) The interpolation of a day's feeding with scarcely any proteids or carbohydrates (e.g., fat, and green vegetables). (4) A day's starvation, allowing only water, tea, coffee, alcohol, beef-tea, and broths. Further, it is well to remember that a single variety of carbohydrate is usually tolerated better than the same amount of several varieties; hence the efficacy of Donkin's pure milk cure, of von Noorden's oatmeal cure, and of Mossé's potato cure. ${ }^{3}-\mathrm{Ed}$.]

\section{THERAPY OF DIABETES INSIPIDUS.}

This disease resembles diabetes mellitus only in a certain number of symptoms common to both, and is undoubtedly due, as investigations from Müller's clinic have shown, to a functional lesion of the kidneys, which renders them incapable of urine concentration. The therapy is very unsatisfactory.

Reduction of Liquid.-If we cannot make use of a causal therapy (cerebral injuries, cerebral syphilis, hysteria), the author has advised as great a reduction of liquids as possible, in order to combat the principal symptoms, polyuria and polydipsia, believing that he has seen good results obtained in this way, when polydipsia was not the primary symptom. In the light of the investigations mentioned above in regard to the origin of the disease, however, he admits that he is quite possibly mistaken. In that case he would advise against this treatment, as an unnecessary discomfort for the patient.

The thirst may be combated by the use of acid drinks, pieces of ice, cigar smoking, and sometimes by the cautious use of small doses of opium. The diet should be in general abundant and mixed.

Medication.- The drug almost exclusively used is valerian in large doses (Trousseau). We may prescribe:

B Ext. valerianæ................. $0.10-0.20 \ldots \ldots$ gr. iss-gr. iii

Fiat caps. no. i. Dent. tal. dos. no. xxx.

Sig. -1-2 capsules 3 times a day.

8 [Futcher confirms the value of Mosse's potato cure from the course of several cases in which this was tried at Johns Hopkins Hospital.-Ed.] 
Antipyrine (2-3 G. (30-45 gr.) a day) also has a certain reputation, and ergot, arsenic, sodium salicylate, aspirin, and other drugs have proved successful in single cases. The author himself has never obtained good results from them or from suprarenal extract, which has recently been recommended because in one case it was successful. It proved useless in one of the author's cases and of doubtful advantage in another. Galvanization of the cervical portion of the cord is also said to be of value. The care of the skin is exceedingly important, and baths and rubbing are therefore advisable. The author once cured a case of what he believed to be undoubted diabetes insipidus in the course of a few weeks by cold douches on the neck, the application of Chapman's ice-bag, and a systematic reduction of liquids. R. Schmidt recommends systematic purging (podophyllin) and sweating.

\section{THERAPY OF OBESITY.}

Classification of Cases of OBesity.-In discussing the therapy of obesity, we must carefully distinguish between (1) those cases in which the obesity is due to excessive eating and drinking or too little exercise, or to both; and (2) those in which it occurs in spite of a moderate or even a relatively scanty diet, and is due to an hereditary tendency or to other pathological conditions. The dietetic regime often ordered for every case of obesity applies only to the first class.

DieteTic TherAPY: Reduction of Fats and Carbohydrates.-In discussing this class, we must investigate the influence of each of the three food elements. The ingestion of a large amount of proteids does not cause the deposition of a large amount of fat within the body. Not so with the fats and carbohydrates. Taken into the body in abnormally large amounts, they cause an abnormal deposition of fat. Whether the carbohydrates do this directly, by being changed into fats, or indirectly, by keeping the fats already in the body from being oxidized, makes no essential difference. The author himself holds to the former view. To reduce the amount of fat in the organism, then, the amount of fats and carbohydrates must be reduced. This reduction should affect the carbohydrates more than the fats, since the latter are usually taken in relatively small quantities, a sense of satiety occurring more quickly from their use than from that of most of the carbohydrates. Some individuals, however, can bear a reduction of fat much more easily than a reduction of carbohydrates.

Reduction of Total Amount of Food.-Before limiting the supply of either, it is well (Pfeiffer) to try the effect of reducing the total amount of food to the normal amount for a person of the weight which would correspond properly to the height and age of the patient. That is, the patient should get $1.7 \mathrm{G}$. of proteid, $0.80 \mathrm{G}$. of fat, and $7.1 \mathrm{G}$. of carbohydrates per kilo (or $12 \mathrm{gr}$., $5 \mathrm{~L} / 2 \mathrm{gr}$., and $48 \mathrm{gr}$. respectively per pound) of the weight normal to his actual height and age (compare the table page 235). Practical experience shows that this reduction will 
often, although not always, have a great effect. The following tables from Quetelet show the proper weights for the different ages and heights.

The author considers these values too low and prefers Landois's tables, page 236, which, unfortunately, however, do not give the heights.

By referring to these tables the normal weight of the person ean be obtained and from this the proper amount of food ealeulated. By such a reduction in diet many obese persons will lose fat without any injury to their nitrogen equilibrium. In order to be sure that such is the fact,

\begin{tabular}{|c|c|c|c|c|c|c|c|c|c|}
\hline \multicolumn{5}{|c|}{ MALES. } & \multicolumn{5}{|c|}{ FEMALES. } \\
\hline $\begin{array}{c}\text { Age in } \\
\text { years. }\end{array}$ & $\begin{array}{c}\mathrm{He} \\
\text { feet }\end{array}$ & $\begin{array}{l}\text { ght in } \\
\text { inches. }\end{array}$ & $\begin{array}{l}\text { Weight in } \\
\text { kilos. }\end{array}$ & $\begin{array}{l}\text { Weight in } \\
\text { pounds. }\end{array}$ & $\begin{array}{l}\text { Age in } \\
\text { years. }\end{array}$ & $\begin{array}{r}\mathrm{He} \\
\text { feet }\end{array}$ & $\begin{array}{l}\text { ght in } \\
\text { inches. }\end{array}$ & Weight in & Weight in \\
\hline 0 & 1 & $7 \frac{1}{2}$ & 3.20 & 7.0 & 0 & 1 & 7 & 2.91 & 6.4 \\
\hline 1 & 2 & 3 & 9.45 & 20.7 & 1 & 2 & $2 \frac{1}{2}$ & 8.79 & 19.2 \\
\hline 2 & 2 & 6 & 11.34 & 24.8 & 2 & 2 & $6 \frac{1}{2}$ & 10.67 & 22.3 \\
\hline 3 & 2 & 10 & 12.47 & 27.2 & 3 & 2 & 91 & 11.79 & 25.8 \\
\hline 4 & 3 & 1 & 14.23 & 31.2 & 4 & 3 & $1^{2}$ & 13.00 & 28.6 \\
\hline 5 & 3 & 3 & 15.77 & 34.5 & 5 & 3 & $2 \frac{1}{2}$ & 14.36 & 31.5 \\
\hline 6 & 3 & 5 & 17.24 & 37.8 & 6 & 3 & $7^{4}$ & 16.01 & 35.2 \\
\hline 7 & 3 & $7 \frac{1}{2}$ & 19.10 & 42.0 & 7 & 3 & 9 & 17.54 & 38.5 \\
\hline 8 & 3 & $9 \frac{1}{2}$ & 20.76 & 48.5 & 8 & 3 & 10 & 19.08 & 41.8 \\
\hline 9 & 4 & 0 & 22.65 & 49.7 & 9 & 3 & $10 \frac{1}{2}$ & 21.36 & 46.9 \\
\hline 10 & 4 & 2 & 24.52 & 52.8 & 10 & 4 & 1 & 23.52 & 51.7 \\
\hline 11 & 4 & $4 \frac{1}{2}$ & 27.10 & 59.6 & 11 & 4 & 3 & 25.70 & 56.5 \\
\hline 12 & 4 & $6 \frac{1}{2}$ & 29.80 & 65.5 & 12 & 4 & 5 & 29.80 & 65.5 \\
\hline 13 & 4 & $8 \frac{1}{2}$ & 34.40 & 75.6 & 13 & 4 & 7 & 32.90 & 72.3 \\
\hline 14 & 4 & $10 \frac{1}{2}$ & 38.80 & 85.3 & 14 & 4 & 9 & 36.70 & 80.7 \\
\hline 15 & 5 & 1 & 43.60 & 95.9 & 15 & 4 & $10 \frac{1}{2}$ & 40.40 & .88 .9 \\
\hline 16 & 5 & $2 \frac{1}{2}$ & 49.70 & 109.3 & 16 & 5 & $\frac{1}{2}$ & 43.60 & 95.9 \\
\hline 17 & 5 & 4 & 52.90 & 116.3 & 17 & 5 & $1 \frac{1}{2}$ & 47.30 & 104.0 \\
\hline 18 & 5 & 5 & 57.90 & 127.3 & 18 & 5 & $1 \frac{1}{2}$ & 51.00 & 112.2 \\
\hline 20 & 5 & $5 \frac{1}{2}$ & 60.10 & 132.3 & 20 & 5 & 2 & 52.30 & 115.0 \\
\hline 25 & 5 & 6 & 62.90 & 138.3 & 25 & 5 & $2 \frac{1}{2}$ & 53.30 & 117.2 \\
\hline 30 & 5 & 6 & 63.70 & 140.1 & 30 & 5 & $2 \frac{1}{2}$ & 54.30 & 119.4 \\
\hline 40 & 5 & 6 & 63.70 & 140.1 & 40 & 5 & $2 \frac{1}{2}$ & 55.20 & 121.4 \\
\hline 50 & 5 & $5 \frac{1}{2}$ & 63.50 & 139.7 & 50 & 5 & $\frac{1}{2}$ & 56.20 & 123.6 \\
\hline 60 & 5 & 4 & 62.90 & 138.3 & 60 & 5 & 0 & 54.30 & 119.4 \\
\hline 70 & 5 & $3 \frac{1}{2}$ & 59.50 & 138.9 & 70 & 5 & 0 & 51.30 & 112.8 \\
\hline
\end{tabular}

the amount of nitrogen in the urine and feces should be calculated from time to time, for no treatment to reduce the fat should also reduce the output of urea. If such a reduction does occur,- -and this can almost be diagnosed from clinical signs,-the amount of proteid should be increased.

Alteration in the Proportionate Amounts of the Proteid, Fat, and Carbohydrate, the Absolute Total Being Kept Constant.-If this simple decrease in the total amount of food does not reduce the patient's weight, the relative amounts of the different food elements must be changed, more proteid being given and less fat and carbohydrate. This reduction should, however, be very gradual, and each case should be 
treated individually. As will appear from this statement, the author does not believe in starting with the Banting, Ebstein, or Oertel treatment, which seem to him to be of more historical than practical value, and which, therefore, will not be described in detail.

Restriction of Liquid.-In regard to the restriction of the amount of water ingested, which is the main point in Oertel's treatment, the author believes in eutting down the total to $1000-1500$ c.c. ( $2-3$ pt.) a day, provided a comparison of the amount of water ingested with that excreted

\begin{tabular}{|c|c|c|c|c|c|}
\hline \multicolumn{3}{|c|}{ MALES. } & \multicolumn{3}{|c|}{ FEMALES. } \\
\hline Years. & $\begin{array}{l}\text { Weight in } \\
\text { kilos. }\end{array}$ & $\begin{array}{l}\text { Weight in } \\
\text { pounds. }\end{array}$ & Years. & $\begin{array}{l}\text { Weight in } \\
\text { kilos. }\end{array}$ & $\begin{array}{l}\text { Weight in } \\
\text { pounds. }\end{array}$ \\
\hline $\begin{array}{r}0 \\
1 \\
2 \\
3 \\
4 \\
5 \\
6 \\
7 \\
8 \\
9 \\
10 \\
11 \\
12 \\
13 \\
14 \\
15 \\
16 \\
17 \\
18 \\
19 \\
20 \\
25 \\
30 \\
40 \\
50 \\
60 \\
70 \\
80 \\
90\end{array}$ & $\begin{array}{r}3.20 \\
10.00 \\
12.00 \\
13.21 \\
15.07 \\
16.70 \\
18.04 \\
20.60 \\
22.26 \\
24.09 \\
26.12 \\
27.85 \\
31.00 \\
35.32 \\
40.50 \\
46.41 \\
52.39 \\
57.40 \\
61.26 \\
63.32 \\
65.00 \\
68.29 \\
68.90 \\
68.81 \\
67.45 \\
65.50 \\
63.03 \\
61.22 \\
57.83\end{array}$ & $\begin{array}{r}7.0 \\
22.0 \\
26.2 \\
29.0 \\
33.0 \\
36.7 \\
39.6 \\
44.0 \\
48.4 \\
52.8 \\
57.4 \\
61.1 \\
68.2 \\
77.6 \\
89.1 \\
102.0 \\
115.0 \\
126.0 \\
134.6 \\
139.2 \\
143.0 \\
150.1 \\
151.5 \\
151.3 \\
148.2 \\
144.1 \\
138.6 \\
134.6 \\
127.1\end{array}$ & $\begin{array}{r}0 \\
1 \\
2 \\
3 \\
4 \\
5 \\
6 \\
7 \\
8 \\
9 \\
10 \\
11 \\
12 \\
13 \\
14 \\
15 \\
16 \\
17 \\
18 \\
19 \\
20 \\
25 \\
30 \\
40 \\
50 \\
60 \\
70 \\
80 \\
90\end{array}$ & $\begin{array}{r}2.91 \\
9.30 \\
11.40 \\
12.45 \\
14.18 \\
15.50 \\
16.74 \\
18.45 \\
19.82 \\
22.44 \\
24.24 \\
26.25 \\
30.54 \\
34.65 \\
34.65 \\
41.30 \\
44.44 \\
49.08 \\
53.10 \\
\dddot{2} . .9 \\
54.46 \\
55.14 \\
56.14 \\
56.65 \\
58.45 \\
56.73 \\
53.72 \\
51.52 \\
49.34\end{array}$ & $\begin{array}{r}6.4 \\
20.4 \\
25.0 \\
27.3 \\
31.0 \\
34.1 \\
36.7 \\
40.5 \\
43.5 \\
49.2 \\
53.2 \\
57.6 \\
67.1 \\
76.1 \\
76.1 \\
90.8 \\
97.6 \\
107.8 \\
116.8 \\
113.6 \\
119.6 \\
123.4 \\
124.5 \\
128.4 \\
123.6 \\
118.1 \\
113.3 \\
108.4\end{array}$ \\
\hline
\end{tabular}

shows that water is being retained in the body,- that is, that hydræmia is present. This minimum quantity is necessary to avoid the danger of irritating the kidneys. But even if water is not retained, it may still be well to restrict the amount of water, simply because in that case the patient will probably eat less. For the same reason, no liquid should be taken with meals. Other liquids beside water must, of course, be limited in quantity. If we permit beer, sweet wine, milk, coffee, or cocoa, their nutritive values must be taken into account in calculating the daily amount of food allowed. 
[The table below and on page 238, in use at the Adirondack Cottage Sanatorium, has been more serviceable in my hands.-Ed.]

Reduction Below the Normal Total.-If, in any given case of obesity, successful results are not obtained by increasing the proteids and decreasing the fats and carbohydrates within the normal total amount,

\section{WEIGHT TABLE (Male.)}

(Showing average weight for each height and age. Based on "Nylie Graphic Table." Correct to one pound.)

\begin{tabular}{|c|c|c|c|c|c|c|c|c|c|c|c|c|c|c|c|c|c|}
\hline Age. & $\begin{array}{c}57 \\
\text { Ins. }\end{array}$ & $\begin{array}{c}58 \\
\text { Ins. }\end{array}$ & $\begin{array}{c}59 \\
\text { Ins. }\end{array}$ & $\begin{array}{c}60 \\
\text { Ins. }\end{array}$ & $\begin{array}{c}61 \\
\text { Ins. }\end{array}$ & $\begin{array}{c}62 \\
\text { Ins. }\end{array}$ & $\begin{array}{c}63 \\
\text { Ins. }\end{array}$ & $\begin{array}{c}64 \\
\text { Ins. }\end{array}$ & $\begin{array}{c}65 \\
\text { Ins. }\end{array}$ & $\begin{array}{c}66 \\
\text { Ins. }\end{array}$ & $\begin{array}{c}67 \\
\text { Ins. }\end{array}$ & $\begin{array}{c}68 \\
\text { Ins. }\end{array}$ & $\begin{array}{c}69 \\
\text { Ins. }\end{array}$ & $\begin{array}{c}70 \\
\text { Ins. }\end{array}$ & $\begin{array}{l}71 \\
\text { Ins. }\end{array}$ & $\begin{array}{c}72 \\
\text { Ins. }\end{array}$ & $\begin{array}{r}73 \\
\text { Ins. }\end{array}$ \\
\hline 20 & 104 & 108 & 111 & 114 & 117 & 121 & 125 & 128 & 132 & 136 & 140 & 144 & 149 & 153 & 158 & 163 & 167 \\
\hline 21 & 105 & 108 & 111 & 115 & 118 & 122 & 125 & 129 & 133 & 137 & 141 & 145 & 150 & 154 & 159 & 164 & 168 \\
\hline 22 & 106 & 109 & 112 & 116 & 119 & 123 & 126 & 130 & 134 & 138 & 142 & 146 & 151 & 155 & 160 & 165 & 169 \\
\hline 23 & 106 & 109 & 113 & 116 & 119 & 123 & 127 & 130 & 135 & 138 & 143 & 147 & 152 & 156 & 161 & 166 & 170 \\
\hline 24 & 107 & 110 & 114 & 117 & 120 & 124 & 128 & 131 & 136 & 139 & 144 & 148 & 153 & 157 & 162 & 167 & 171 \\
\hline 25 & 108 & 111 & 114 & 118 & 121 & 125 & 128 & 132 & 136 & 140 & 144 & 149 & 154 & 158 & 163 & 168 & 172 \\
\hline 26 & 108 & 111 & 115 & 118 & 122 & 126 & 129 & 133 & 137 & 141 & 145 & 150 & 154 & 159 & 164 & 169 & 173 \\
\hline 27 & 109 & 112 & 116 & 119 & 122 & 127 & 130 & 134 & 138 & 142 & 146 & 150 & 155 & 160 & 165 & 170 & 174 \\
\hline 28 & 109 & 112 & 116 & 120 & 123 & 127 & 130 & 134 & 138 & 142 & 147 & 151 & 156 & 161 & 166 & 170 & 175 \\
\hline 29 & 110 & 113 & 117 & 120 & 124 & 127 & 131 & 135 & 139 & 143 & 148 & 152 & 157 & 162 & 167 & 171 & 176 \\
\hline 30 & 110 & 114 & 117 & 121 & 124 & 128 & 132 & 136 & 140 & 144 & 148 & 152 & 157 & 162 & 167 & 172 & 177 \\
\hline 31 & 111 & 114 & 118 & 121 & 125 & 129 & 132 & 136 & 140 & 145 & 149 & 153 & 158 & 163 & 168 & 173 & 178 \\
\hline 32 & 111 & 115 & 118 & 122 & 125 & 129 & 133 & 137 & 141 & 145 & 150 & 154 & 159 & 164 & 169 & 173 & 179 \\
\hline 33 & 112 & 115 & 119 & 122 & 126 & 130 & 133 & 138 & 142 & 146 & 150 & 155 & 159 & 164 & 170 & 174 & 179 \\
\hline 34 & 112 & 116 & 119 & 123 & 126 & 130 & 134 & 138 & 142 & 147 & 151 & 155 & 160 & 165 & 170 & 175 & 180 \\
\hline 35 & 112 & 116 & 120 & 123 & 127 & 131 & 134 & 139 & 143 & 147 & 152 & 156 & 161 & 166 & 171 & 175 & 181 \\
\hline 36 & 113 & 117 & 120 & 124 & 127 & 131 & 135 & 139 & 143 & 148 & 152 & 156 & 161 & 166 & 172 & 176 & 181 \\
\hline 37 & 113 & 117 & 120 & 124 & 128 & 131 & 135 & 140 & 144 & 148 & 153 & 157 & 162 & 167 & 172 & 177 & 182 \\
\hline 38 & 113 & 117 & 121 & 124 & 128 & 132 & 136 & 140 & 144 & 149 & 153 & 158 & 162 & 167 & 173 & 177 & 183 \\
\hline 39 & 114 & 118 & 121 & 125 & 129 & 132 & 136 & 141 & 145 & 149 & 154 & 158 & 163 & 168 & 173 & 178 & 183 \\
\hline 40 & 114 & 118 & 122 & 125 & 129 & 133 & 136 & 141 & 145 & 149 & 154 & 158 & 163 & 168 & 173 & 178 & 184 \\
\hline 41 & 114 & 118 & 122 & 125 & 129 & 133 & 137 & 141 & 146 & 150 & 154 & 159 & 164 & 168 & 174 & 179 & 184 \\
\hline 42 & 115 & 118 & 122 & 126 & 130 & 133 & 137 & 142 & 146 & 150 & 155 & 159 & 164 & 169 & 174 & 179 & 185 \\
\hline 43 & 115 & 119 & 123 & 126 & 130 & 134 & 138 & 142 & 146 & 151 & 155 & 161 & 165 & 170 & 175 & 180 & 185 \\
\hline 4 & 115 & 119 & 123 & 126 & 130 & 134 & 138 & 143 & 147 & 151 & 155 & 160 & 165 & 170 & 175 & 180 & 186 \\
\hline 45 & 116 & 119 & 123 & 126 & 131 & 134 & 138 & 143 & 147 & 151 & 156 & 161 & 165 & 170 & 176 & 181 & 186 \\
\hline 46 & 116 & 119 & 123 & 127 & 131 & 135 & 139 & 143 & 147 & 152 & 156 & 161 & 166 & 170 & 176 & 181 & 186 \\
\hline 47 & 116 & 120 & 124 & 127 & 131 & 135 & 139 & 144 & 148 & 152 & 157 & 161 & 166 & 171 & 176 & 181 & 187 \\
\hline 48 & 116 & 120 & 124 & 127 & 131 & 135 & 139 & 144 & 148 & 152 & 157 & 161 & 166 & 171 & & 182 & 187 \\
\hline 49 & 117 & 120 & 124 & 127 & 131 & 135 & 139 & 144 & 148 & 153 & 157 & 162 & 167 & 171 & 177 & 182 & 187 \\
\hline 50 & 117 & 120 & 124 & 127 & 132 & 136 & 140 & 144 & 148 & 153 & 157 & 162 & 167 & 172 & 177 & 182 & 188 \\
\hline 51 & 117 & 120 & 124 & 128 & 132 & 136 & 140 & 145 & 149 & 153 & & 162 & 16 & & & 182 & \\
\hline 52 & 117 & 121 & 125 & 128 & 132 & 136 & 140 & 145 & 149 & 153 & 158 & 162 & 167 & 172 & 178 & 183 & 188 \\
\hline 5 & 117 & 121 & 125 & 128 & 132 & 136 & 140 & 145 & 149 & 154 & 158 & ] 63 & 168 & 172 & 178 & 183 & 188 \\
\hline 5 & 118 & 121 & 125 & 128 & 132 & 136 & 140 & 145 & 149 & 154 & 15 & 16 & 16 & 173 & & 18 & 188 \\
\hline 55 & 118 & 121 & 125 & 128 & 132 & 136 & 140 & 145 & 149 & 154 & 158 & 163 & 168 & 173 & 178 & 183 & 188 \\
\hline
\end{tabular}

we may try reducing the fats and carbohydrates without increasing the proteids; that is, we may try a "hunger cure;" e.g., Banting's, Ebstein's, or Oertel's. By reducing the carbohydrates 50-100 G. (11/2-3 oz.) each week, they may be reduced to as low as $100 \mathrm{G}$. ( $3 \mathrm{oz}$.). Care must, however, be taken to see that the nitrogen equilibrium is maintained. Since in practice we cannot always determine the urea output or avail ourselves 
of the sphygmomanometer or dynamometer, we must carefully watch for any clinical symptoms which would indicate that we are carrying the treatment too far; e.g., tones suggestive of a relaxed heart, a weak pulse with low tension, muscular weakness, weariness, giddiness, digestive disturbances, sleeplessness, depression-symptoms which demand an immediate increase of proteids.

\section{WEIGHT TABLE (Female.)}

(Showing average weight for each height and age. Based on "Nylic Graphic Table.")

\begin{tabular}{|c|c|c|c|c|c|c|c|c|c|c|c|c|c|c|c|c|c|}
\hline Age. & $\begin{array}{c}57 \\
\text { Ins. }\end{array}$ & $\begin{array}{c}58 \\
\text { Ins. }\end{array}$ & $\begin{array}{c}59 \\
\text { Ins. }\end{array}$ & $\begin{array}{c}60 \\
\text { Ins. }\end{array}$ & $\begin{array}{c}61 \\
\text { Ins. }\end{array}$ & $\begin{array}{c}62 \\
\text { Ins. }\end{array}$ & $\begin{array}{c}63 \\
\text { Ins. }\end{array}$ & $\begin{array}{c}64 \\
\text { Ins. }\end{array}$ & $\begin{array}{c}65 \\
\text { Ins. }\end{array}$ & $\begin{array}{c}66 \\
\text { Ins. }\end{array}$ & $\begin{array}{c}67 \\
\text { Ins. }\end{array}$ & $\begin{array}{c}68 \\
\text { Ins. }\end{array}$ & $\begin{array}{c}69 \\
\text { Ins. }\end{array}$ & $\begin{array}{c}70 \\
\text { Ins. }\end{array}$ & $\begin{array}{c}71 \\
\text { Ins. }\end{array}$ & $\begin{array}{c}72 \\
\text { Ins. }\end{array}$ & $\begin{array}{l}73 \\
\text { Ins. }\end{array}$ \\
\hline 20 & 100 & 103 & 106 & 109 & 113 & 116 & 120 & 123 & 127 & 130 & 134 & 138 & 142 & 147 & 152 & 156 & 161 \\
\hline 21 & 101 & 104 & 107 & 110 & 114 & 117 & 120 & 124 & 127 & 131 & 135 & 139 & 143 & 148 & 152 & 157 & 162 \\
\hline 22 & 101 & 105 & 107 & 110 & 114 & 118 & 121 & 124 & 128 & 132 & 136 & 140 & 144 & 149 & 153 & 158 & 162 \\
\hline 23 & 102 & 105 & 108 & 111 & 115 & 118 & 122 & 125 & 128 & 132 & 137 & 140 & 145 & 149 & 154 & 158 & 163 \\
\hline 24 & 102 & 106 & 108 & 111 & 115 & 119 & 122 & 126 & 129 & 133 & 137 & 141 & 145 & 150 & 155 & 159 & 164 \\
\hline 25 & 103 & 106 & 109 & 112 & 116 & 119 & 123 & 126 & 130 & 134 & 138 & 142 & 146 & 151 & 155 & 160 & 165 \\
\hline 26 & 103 & 107 & 110 & 113 & 117 & 120 & 124 & 127 & 131 & 134 & 139 & 143 & 147 & 151 & 156 & 161 & 166 \\
\hline 27 & 104 & 107 & 110 & 113 & 117 & 121 & 124 & 128 & 131 & 135 & 139 & 144 & 148 & 152 & 157 & 162 & 166 \\
\hline 28 & 104 & 108 & 111 & 114 & 118 & 121 & 125 & 128 & 132 & 136 & 140 & 144 & 149 & 153 & 158 & 162 & 167 \\
\hline 29 & 105 & 108 & 111 & 114 & 118 & 122 & 126 & 129 & 133 & 136 & 141 & 145 & 149 & 154 & 158 & 163 & 168 \\
\hline 30 & 105 & 109 & 112 & 115 & 119 & 123 & 126 & 129 & 133 & 137 & 141 & 146 & 150 & 154 & 159 & 164 & 169 \\
\hline 31 & 106 & 109 & 112 & 116 & 119 & 123 & 127 & 130 & 134 & 138 & 142 & 146 & 151 & 155 & 160 & 165 & 170 \\
\hline 32 & 106 & 110 & 113 & 116 & 120 & 124 & 127 & 131 & 135 & 138 & 143 & 147 & 151 & 156 & 161 & 166 & 170 \\
\hline 33 & 107 & 110 & 113 & 117 & 120 & 124 & 128 & 131 & 135 & 139 & 143 & 148 & 152 & 156 & 162 & 166 & 171 \\
\hline 34 & 107 & 110 & 114 & 117 & 121 & 125 & 128 & 132 & 136 & 140 & 144 & 149 & 153 & 157 & 162 & 167 & 172 \\
\hline 35 & 108 & 111 & 115 & 118 & 122 & 125 & 129 & 133 & 137 & 140 & 145 & 150 & 154 & 158 & 163 & 168 & 173 \\
\hline 36 & 108 & 112 & 115 & 119 & 122 & 126 & 130 & 133 & 137 & 141 & 146 & 150 & 154 & 159 & 164 & 169 & 174 \\
\hline 37 & 109 & 112 & 116 & 119 & 123 & 126 & 130 & 134 & 138 & 142 & 146 & 151 & 155 & 160 & 165 & 170 & 175 \\
\hline 38 & 109 & 113 & 116 & 120 & 123 & 127 & 131 & 135 & 139 & 142 & 147 & 152 & 156 & 161 & 166 & 170 & 175 \\
\hline 39 & 110 & 113 & 117 & 120 & 124 & 128 & 131 & 135 & 139 & 143 & 148 & 153 & 157 & 161 & 166 & 171 & 176 \\
\hline 40 & 110 & 114 & 117 & 121 & 124 & 128 & 132 & 135 & 140 & 144 & 148 & 153 & 157 & 162 & 167 & 172 & 177 \\
\hline 41 & 111 & 114 & 118 & 121 & 125 & 129 & 132 & 136 & 140 & 145 & 149 & 154 & 158 & 163 & 168 & 173 & 178 \\
\hline 42 & 111 & 115 & 118 & 122 & 125 & 129 & 133 & 137 & 141 & 145 & 150 & 155 & 159 & 163 & 169 & 173 & 179 \\
\hline 43 & 112 & 115 & 119 & 122 & $126^{\circ}$ & 130 & 134 & 138 & 142 & 146 & 150 & 156 & 159 & 164 & 169 & 174 & 179 \\
\hline 44 & 112 & 116 & 119 & 123 & 127 & 130 & 134 & 138 & 142 & 147 & 151 & 156 & 160 & 165 & 170 & 175 & 180 \\
\hline 45 & 113 & 116 & 120 & 123 & 127 & 131 & 135 & 139 & 143 & 147 & 152 & 157 & 161 & 166 & 171 & 175 & 181 \\
\hline 46 & 113 & 117 & 120 & 124 & 128 & 131 & 136 & 139 & 143 & 148 & 152 & 157 & 162 & 166 & 171 & 176 & 182 \\
\hline 47 & 114 & 117 & 121 & 124 & 128 & 132 & 136 & 140 & 144 & 149 & 153 & 158 & 162 & 167 & 172 & 177 & 18 \\
\hline 48 & 114 & 118 & 121 & 125 & 129 & 133 & 137 & 141 & 144 & 149 & 154 & 159 & 163 & 168 & 173 & 178 & 183 \\
\hline 49 & 115 & 118 & 122 & 125 & 129 & 133 & 138 & 141 & 145 & 150 & 154 & 159 & 164 & 168 & 174 & 179 & 184 \\
\hline 50 & 115 & 119 & 122 & 126 & 130 & 134 & 138 & 142 & 146 & 150 & 155 & 160 & 164 & 169 & 174 & 179 & \\
\hline 51 & 116 & 119 & 123 & 126 & 130 & 134 & 139 & 143 & 147 & 151 & 156 & 161 & 165 & 170 & 175 & 18 & \\
\hline 52 & 116 & 120 & 123 & 127 & 131 & 135 & 139 & 143 & 147 & 152 & 156 & 161 & 166 & 170 & 176 & 181 & 186 \\
\hline 53 & 117 & 120 & 124 & 127 & 131 & 135 & 140 & 144 & 148 & 153 & 157 & 162 & 166 & 171 & 177 & 182 & \\
\hline 54 & 117 & 120 & 124 & 128 & 132 & 136 & 140 & 144 & 148 & 153 & 158 & 162 & 167 & 172 & 177 & 182 & 18 \\
\hline 55 & 118 & 121 & 125 & 128 & 132 & 136 & 140 & 145 & 149 & 154 & 158 & 163 & 168 & 173 & 178 & 183 & 188 \\
\hline
\end{tabular}

If such a reduction of the carbohydrates is unsuccessful or only temporarily successful, the fats must be restricted and the proteids be raised again. In this way a satisfactory antifat treatment may be carried out without any danger to the patient. It is often better attempted in a sanatorium than at the patient's home. 
Carbohydrates Specially Forbidden.-The foods which should be forbidden first are all those rich in carbohydrates, i.e., sugar, sweets, cake, sweet puddings, beets, potatoes, bread in large amounts (aleuronat or roborat bread may be allowed in larger quantities), milk, beer, liqueurs, and wine, with the exception of some light Austrian, Moselle, or Bordeaux wine. Small amounts of stronger wines or of brandy are useful when fatty degeneration has caused symptoms of cardiac insufficiency. The vegetables (peas, beans, and lentils excepted) need not be forbidden, although they belong to the carbohydrates; for (1) only a very inconsiderable part of them is absorbed from the intestine, (2) they increase the work of the digestive tract, and (3) on account of their bulk they bring about quickly the desired sense of satiety. All kinds of green vegetables - cucumbers, cabbage, lettuce, asparagus, spinach, etc.-are therefore very desirable.

Dietary.-The following dietary is given by Debove and has been repeatedly used with success by the author, modified, however, to suit individual peculiarities. Those foods which are bulky but not nutritive (skimmed milk, green vegetables, fruit) are given the preference. The supply of water is not restricted. Breakfast: A cup of tea with milk. Dinner: One or 2 slices of lean meat, a small amount of green vegetables or salad, 100-150 G. (3-5 oz.) of bread, a little cheese, fruit ad libitum, and a cup of black coffee without sugar. Supper: One pint of hot sugared milk, 30-50 G. (1-11/2 07.) of bread, fruit as before. Before each meal he gives 10 drops of the tincture of aloes. So far as the author has been able to judge, the patient bears this dietary well, and loses a good deal of weight without becoming weak.

Hygiene: Exercise.-Exercise plays a part only secondary to diet; in fact, lack of exercise is often a secondary and occasionally the primary cause of the condition. If the heart is sound, every kind of exercise may be taken in gradually increasing amounts. Unfortunately, outdoor sports, such as riding, sometimes increase the patient's appetite, so that it is hard to keep him down to the prescribed amount of food. Mountain climbing, rowing, and bicycling are better than riding. If fatty degeneration of the heart is present, the amount of exereise permitted cannot be exactly laid down. In some eases of this kind we may use massage or passive movements; in others, Oertel's "terraincur," or walking, measured by a pedometer.

Sleep.-The patient should be allowed only 7-8 hours of sleep at the most, and should take a walk (not a nap) after meals.

Baths.-Baths (especially hot air and steam baths) play an important part in the therapy, a sound heart and vessels being presupposed. Electric light baths (though more efficacious in theory than in practice), cold douches, and tub baths are also useful.

Medication: Mineral Waters.-Not much need be said concerning medication. For 4-6 weeks, alkaline-saline mineral waters may be taken at the springs themselves. The principal benefit lies, not so much in the 
waters, as in the dietetic and hygienic (gymnastic) regulations, as well as, in some cases, in the use of the baths (carbonated, salt, iron, steam, and electric light). The best waters are the cold Glauber's salts springs, such as Tarasp and Marienbad. Warm waters are, in general, contraindicated. An exception will be mentioned later (see p. 241). If the obesity is only of moderate grade, common salt springs may be used, particularly Homburg and Kissingen. Kisch, who has had great experience in treating obesity in this way, generally gives $700-1000$ c.c. $(1 \mathrm{r} / 2-2$ pt.) of the Marienbad Kreuz- or Ferdinands-brunnen in the morning on an empty stomach, the amount being divided into 3-4 glasses with a pause of 12-20 minutes between. Then comes a 1-2 hours' walk before breakfast. After the treatment at Marienbad is finished, he recommends a high mountain climate, in order that the lungs may get more oxygen and metabolism may be stimulated, and also the use of sea bathing and cold baths, in order that more fat may be consumed by the quickened metabolism. If it is impossible for the patient to go to the springs, Marienbad water, or a teaspoonful of the Marienbad salt in a pint of water, may be given at home. [At Mount Clemens, French Lick Springs, and the Hot Springs of Virginia, obesity cures are given with good results. There is no doubt that better success is attained abroad.-Ed.]

Cathartics.-For cathartics we give mineral waters and salts, because they are less drastic and weakening than are many of the vegetable purges, and because they draw water from the system into the lumen of the intestine.

Thyroid Extract.-Thyroid extract is another remedy particularly suited to cases of obesity from too much food. By increasing oxidation, it brings about a loss of flesh in a surprisingly short time. The method of giving it has already been discussed (see p. 198). It goes without saying that a careful watch must be kept on the nervous and circulatory systems for symptoms of intoxication. The author has himself seen 2 cases in which the use of thyroid extract gave rise to the severest symptoms of cardiac insufficiency and to symptoms of acute Graves's disease. Glycosuria and even permanent diabetes have also been observed to follow its use. In view of all these facts, the author is personally very loath to employ it.

Complications of This Type of Obesity: Heart Weakness: Arteriosclerosis.-All the therapy so far described applies particularly to simple cases of obesity from over-eating, where the patient has a flushed, bloated face, a thick neck, and a full pulse,-i.e., the symptoms of true plethora. If this clinical picture is complicated by symptoms of cardiac weakness, we must be very cautious in allowing exercise. We may begin with the shortest of walks and then try Oertel's "terraincur," with the restriction of the amount of water ingested, provided it exceeds the amount excreted. In pronounced arteriosclerosis the same precautions regarding exercise should be observed, and small doses of potassium or sodium iodide be given for a prolonged period. 
Uric Acid Diathesis.-If, as happens relatively often, the obesity is complicated by a uric acid diathesis, alkaline or alkaline-saline mineral waters (particularly Carlsbad) are indicated. The diet need not be essentially changed, except that there should be no attempt to reduce the amount of water ingested, for fear of favoring the formation of urinary calculi.

Diabetes.-Obesity is rather frequently combined with diabetes. In fact von Noorden advances the exceedingly attractive theory that one form of obesity is simply a masked form of diabetes, a premonitory stage, when the organism is still able to make fat from carbohydrates, but cannot wholly oxidize its sugar. If there is the least suspicion of such a condition, we should proceed very cautiously in cutting down the food. A reduction of the fats should not be thought of; even the foods very rich in carbohydrates (sugar, sweets, cakes, farinaceous foods, sweet wine, liqueurs, sweet preserves, and beer) should be reduced only slowly and gradually; much exercise should be taken; and an alkaline-saline water, especially Carlsbad, Marienbad, or Vichy, be given. The same treatment should be carried out in a case of manifest diabetes. In other words, the therapy of the diabetes takes decided precedence over that of the obesity. For example, in spite of obesity, the author would not deprive such a patient of light wine, in case he had been accustomed to it for a long time. It is, under any circumstances, better for the patient to remain fat than to lose weight too rapidly (100 G. (3 oz.) a week or over). Beside the mineral waters above mentioned, alkalies and arsenic should be given; e.g., for $3-4$ weeks a teaspoonful of lithium carbonate or the effervescing citrate, or $0.50 \mathrm{G}$. ( $7 \mathrm{I} / 2 \mathrm{gr}$.) of the simple salts dissolved in a glass of water, with 8-10 drops of Fowler's solution added. Exercise is important in these cases.

Abdominal Plethora.-A final combination worth mentioning is that of obesity with abdominal plethora. This is particularly common with women, and is accompanied by marked meteorism, constipation, hemorrhoids, and enlarged liver. In these cases the usual dietetic therapy for obesity should be carried out and cold alkaline-saline waters be given (Carlsbad, Marienbad).

Obesity in Consequence of Pathological Conditions: Anæmia.Beside the ordinary obesity caused by over-indulgence, two other forms may be briefly mentioned: that caused by anæmia, and that due to an hereditary predisposition. The first occurs in (1) individuals with a scrofulous diathesis, (2) chlorotic girls, and (3) women after several childbirths or after the menopause. In these cases, we never reduce the food, but rather try to build up the system in every way,-by giving nourishing food, especially raw meat, by mild exercise, by cautious hydropathic procedures (tepid baths, cool packs in the morning. salt baths), and by tonics (iron, arsenic, quinine, cod-liver oil). Suitable mineral waters are Elster or Franzensbad, and the Ambrosius-quelle at Marienbad (all iron and alkaline-saline waters), or Kissingen (common salt springs). 
An Hereditary Tendency.-Finally, there are the cases of the second class, in which the only cause for obesity seems to be an hereditary predisposition. This may occur even when the amount of food taken has always been moderate or even noticeably small. Here it is of course absurd to put the patient on a restricted diet and alkaline-sulphur waters. Exercise and hydrotherapy are our only resources.

Tuberculosis.-In conclusion, the author never advises trying to reduce the diet in cases where the obesity has developed in consequence of a diet chosen on account of pulmonary tuberculosis. In such a case, exercise alone remains to us.

\section{THERAPY OF EMACIATION.}

Etrology.-Excessive emaciation may be due to any one of many pathological conditions. Sometimes it is the consequence of "wasting" diseases; e.g., carcinoma, tuberculosis, some acute infectious disease, or diseases of the digestive tract, the circulatory system, or the kidneys. In other cases, it is the result of nervous disturbances, particularly nervous dyspepsia, which cause a loss of appetite. In some cases, it forms what may be called a disease in itself, although even in some of these instances, definite causes for the loss of flesh can be assigned. Here may be included cases of excessive emaciation after some severe constitutional disease (e.g., typhoid), and those arising from abnormally great physical or mental exertion, from persistent late hours, from sexual excesses, from pregnancy, from lactation, and from voluntary abstinence from sufficient nourishment.

Therapy of the So-Called Idiopathic Form: Dietetic Therapy.This discussion will treat only the latter, the so-called idiopathic form. Naturally, if the patient is to gain flesh, all the above mentioned etiological factors which impose an abnormal demand on the organism and make the necessary amount of nourishment impossible, must be removed. It is also obvious that increased nourishment must be the first and most important part of the therapy. We must gradually work up to the amount of food suitable for a person of the weight corresponding normally to the patient's age and height (see table on p. 235). The additional food should be given in small quantities frequently during the day, and should consist first of the most easily taken forms of carbohydrate. We may begin with soup thickened by farinaceous food-stuffs, such as barley, rice, farina, sago, oatmeal, noodles, etc., or made of dried peas or beans or the prepared infants' foods (Nestlé's, Timpe's, etc.). We may also give bread and foods baked with a good deal of sugar, sweet puddings, sugar added to water, wine, or milk, and honey or syrup on bread.

If increasing the carbohydrates up to the amount tabulated in the standard diet will not raise the total number of calories to the requisite amount, we may also increase the fats. They are theoretically, as has 
already been said, twice as valuable as the carbohydrates for the production of heat, but practically they fall back to the second place, because (1) they are often hard to digest and (2) they bring about too quickly a feeling of saticty. For the first of these reasons we must naturally give only the most easily digestible forms of fat (butter, cream, bacon, cocoa, chocolate, olive oil, cotton-seed oil, lipanin, and particularly von Mehring's "Kraftschocolade"). Cod-liver oil deserves special mention. In spite of its unpleasant taste, some individuals like it and can take it in considerable quantities; the aromatic form is preferable. In practice we do not, of course, sharply separate the carbohydrates and fats. For instance, we give butter, or sometimes another fat, with bread, potatoes, asparagus, etc. Beer or any good form of alcohol is useful, not only as a food in itself, but also as a means of increasing the appetite and helping in the digestion of fats. We may give $1 / 2-1$ bottle of light table wine or a liqueur glass of brandy after eating, or a small glass of sherry or port before eating. It must not be forgotten that experiments on animals apparently prove that increasing the carbohydrates may cause "fatty heart," while increasing the fats will not. Should this result be confirmed, it is evident that Ebstein is right in demanding primarily an increase of the fats.

If the increase of carbohydrates and fats does not effect the desired end, it must be followed by an increase of the proteids, in the most easily digestible forms, of course; e.g., eggs (cooked soft, or in milk, soup, or broth), and preparations of meat and meat peptones (Cibil's, Kemmerich's, Denayer's, or Koch's meat extracts, Leube-Rosenthal's meat solution, Dr. Scholl's meat juice "Puro" (both a stimulant and a food), somatose (2-4 teaspoonfuls daily in soup, milk, or coffee), Brandt's "Essence of Beef," and Corneli's "Peptone"). Brandt's Essence and Puro the author knows to be good and to be well borne even when taken for a considerable length of time. We may also try Ziemssen's famous "Succus carnis rec. parat." If the stomach permits, we generally allow raw beef or ham. Preparations of milk are also of course available; e.g., eucasin, sanatogen, nutrose, or milk somatose (2-8 teaspoonfuls daily in milk).

In many cases, the restriction to a particular diet often proves more efficacious (milk, kefir, koumiss, grapes).

Hygiene.-The dietetic therapy just described must be supplemented by physical and mental rest. Very weak patients must remain perfeetly quiet. Others may be helped by short walks, drives, and fresh air, provided the appetite is thereby increased. A good deal of sleep should be taken, particularly directly after meals, and, if possible, in the open air. Weir Mitchell's "rest cure" is also usually beneficial (see p. 387).

For the use of stomachics, see the Therapy of Diseases of the Stomach (p. 418).

Daily tepid baths or douches are also useful.

Those forms of emaciation which are due, not merely to a general 
poor state of nutrition, but to some definite disease, will be discussed at greater length elsewhere.

\section{THERAPY OF GOUT.}

We have, strictly, no exact scientific knowledge as to the essential nature of gout. Even the newest theories are far from convincing. Pfeiffer attributes it to a qualitative difference in the uric acid; Kolisch to an alloxuric rather than a uric acid diathesis. But neither of these hypotheses is wholly satisfactory, and the latter has been almost completely refuted. Our therapy must, then, be practically empirical, founded upon what has been found helpful at the bedside. We may, however, with Minkowski, lay down these general rules: we should endeavor (1) to insure the least possible formation of uric acid in the body; (2) to effect as complete an oxidation of it as possible; and (3) to favor its solution in the blood and tissues and its excretion in the urine.

It is necessary to distinguish between (1) the treatment of an acute attack of gout, and (2) the therapy of the chronic gouty alteration of metabolism, the "uric acid diathesis."

\section{THERAPY OF CHRONIC URIC ACID DIATHESIS AND ITS MANIFESTATIONS.}

Prophylactic Therapy.-In the therapy of a gouty diathesis, prophylactic measures are of prime importance. If there is any good ground for suspecting even in childhood the beginning of this condition, or even its purely latent stage (e.g., from inheritance, or from an irrational mode of life that might lead to such a condition), the same dietetic, hygienic, and medicinal therapy should be carried out as in cases where a gouty diathesis has been fully demonstrated by an attack of gout, or by the blood or urine findings (increase of uric acid and alloxuric bases, insufficient solvent power for the uric acid, signs of abnormally great intestinal putrefaction, etc.).

DieteTic TherAPY.- In view of the fact that most, but by no means all, persons with a gouty diathesis have been luxurious livers, the first task of the dietetic therapy must be to cut down the amount of food to approximately the quantity absolutely necessary for maintaining a good state of nutrition (see p. 234). It must be expressly stated, however, that a reduction of food is contraindicated in debilitated individuals, and in asthenic gout, or in cases where the development of the latter is to be feared. On the contrary, more than the normal amount of food should often be given in such cases.

Just what foods are to be given and in what proportions to make up the total amount decided upon, are points upon which authorities differ. The author will repeat his opinions, already stated in the discussion of the therapy of nephrolithiasis urica (see p. 130). 
Vegetables and Fruit.-The patient should take a good many fresh green vegetables and fruits (grapes and peaches excepted, as being too rich in sugar). Their vegetable acids and salts are converted in the organism into carbon dioxide or carbonates, and excreted as such, thus effecting a binding of the uric acid by increasing the alkalinity of the blood. Again, fruit and vegetables tend to the production of hippuric and benzoic acid and also have a diuretic effect. Especially suitable are all salads (small amounts of vinegar do no harm, but lemon juice is better), cucumbers (provided the stomach can digest them), cauliflower, sprouts, spinach, beet tops. Sorrel and tomatoes should be sparingly used, on account of their content of oxalic acid, as oxaluria often goes hand in hand with uraturia. Onions, radishes, and garlic should be forbidden, on account of their effect as renal irritants, and all kinds of cabbage should be strictly limited in quantity.

Meats.-The patient should not confine himself to fruit and vegetables, however. The old theory that meat must be greatly reduced or forbidden, appears to the author without foundation, and its practice seems sometimes even dangerous. Both white and red meats are to be allowed, in spite of the greater amount of extractives in red meat. Game and sausages are forbidden; the former, because it contains too many toxins to be introduced into the digestive tract of a person already showing signs of abnormal proteid decomposition; the latter, because they contain substances which might injure the kidneys, always vulnerable in a gouty diathesis. Oysters are permissible ; crabs and lobsters, forbidden on account of their indigestibility. Beef tea, beef broth, meat extracts, and highly flavored sauces, as well as brains, liver, kidneys, and sweetbreads, are forbidden, on account of the nuclein they contain, which leads to the formation of uric acid. So far as the mode of preparation is concerned, boiling is better than broiling or roasting, since boiled meat is poorer in extractives. Dish-gravy must be entirely avoided, as it contains the extractives in a very concentrated form. Pickled meat is not to be recommended, but moderate amounts of ham or any smoked meat may be given. Mushrooms contain so much nitrogen that they should be eaten only in small quantities, if at all.

Carbohydrates.-The abundant use of foods very rich in carbohydrates should also be checked, especially in.obstinate cases. Sugar, sweets, and honey the author entirely forbids, and decidedly diminishes the quantity of unsweetened farinaceous foods (noodles, macaroni, rice, dried peas and beans [the last two being rather rich in nucleins-Ed.], potatoes, and bread [here also better replaced by aleuronat bread-Ed.]). He lays great stress on the use of easily digestible forms of fat (cream, butter, bacon, olive oil, eggs, and fat cheeses, such as Neufchâtel, Imperial, and Gervais) unless they are contraindicated by a coincident obesity. The sharp cheeses (Gorgonzola, Liptauer, and Swiss) are forbidden. Foods rich in gelatin are useful. 
No sharp condiments or piquant sauces must be used in the preparation of any food, as they are irritating to the intestines and the kidneys.

Milk.-Authorities differ as to whether milk in generous quantities is advantageous or not. The author has always strongly recommended it, sound digestive organs being presupposed, and has obtained only good results. Other authors agree with him. But Pfeiffer, without doubt one of the best authorities on gout, warns us against a copious use of milk, maintaining that its lactic acid is apt to start up gouty pains. The author also finds sanatogen, eucasin, and nutrose (2-3 tablespoonfuls) useful.

Alcohol.-All authorities agree in prohibiting alcohol in large quantities, unless indicated by cardiac weakness. Pfeiffer even goes so far as to prohibit it entirely. The author allows half a pint (exceptionally 1 pint) daily of a light wine diluted with water or mineral water, or one small glass of Pilsener beer; stronger wines or beer (Munich beer and ale) are strictly forbidden. Beer is worse than wine, on account of its xanthin bases. The degree to which the restriction of alcohol should be carried is best determined by the condition of the individual. Absolute abstinence is advisable if the patient is young and strong, or elderly and plethoric.

Water.-Weak tea and coffee, cocoa, and chocolate may be taken in moderation. Large quantities of water (2-3 at. daily) are necessary to keep the system well flushed out and the products of decomposition disposed of as far as possible. The author strongly recommends 300 c.c. (1 large glassful) of hot water on an empty stomach in the morning, another some time during the day, and a third on retiring. Water should be taken at each meal, and an extra quantity at supper, to help dispose of the products of metabolism that have accumulated during the day.

Number of Meals.-Only 3 meals a day should be allowed, for fear of overworking the stomach in disposing of the strongly vegetarian diet, and so causing digestive disturbances, which would bring on an exacerbation of the gouty symptoms. The reason for this is evident, when we take into consideration the fact that by abnormal acid fermentation in the stomach the carbonates and neutral phosphates contained in the food are neutralized or changed to acid salts while they are still in the stomach, so that the blood is no longer supplied with the salts necessary for entering into combination with the uric acid (Pfeiffer).

Hygrene: Exercise.-The proper dietetic measures must be reinforced by systematic exercise, unless contraindicated by some special gouty organic disease, such as myocarditis uratica or nephritis uratica. The exercise must, however, never be carried to the point of fatigue. It may consist of walking, riding, rowing, swimming, fencing, bicycling, tennis, bowling, billiards, etc., all being carried on as far as possible in the open air. If bad weather necessitates it, work in the gymnasium may be substituted. If one of the above contraindications, or any other, is present, ordinary exercise must be replaced by Oertel's "terraincur" or 
by massage. A sponge bath with water at $64^{\circ}-75^{\circ}$ should be taken every morning, with one or two warm baths $\left(89^{\circ}-95^{\circ}\right)$ a week.

Medication: Alkaline Mineral Waters.-The regular medication in cases of a gouty diathesis has always been the administration of alkalies to keep the uric acid dissolved in the urine. Any of the alkalies or the earthy carbonates may be used interchangeably, but the best and most common method is to give alkaline mineral waters. They should be taken in equal portions distributed over the day, the first in the morning, the last at night. This treatment should be kept up for a long time, often indefinitely. The amount should never be large enough to cause an alkaline reaction of the urine, but enough to furnish 3-4 G. (45-60 gr.) daily of sodium bicarbonate; e.g., $1 \mathrm{~L}$. (1 qt.) of Fachingen or Preblau water; $3 / 4 \mathrm{~L}$. ( $1 \mathrm{t} / 2 \mathrm{pt}$.) of Bilin water; $1 \mathrm{~L}$. ( $1 \mathrm{qt}$.) of the Obersalzbrunn Oberbrunn; $3 / 4$ L. (1 $1 / 2$ pt.) of Wiesbaden; or $3 / 4$ L. (11/2 pt.) of Vichy. (See p. 132.)

Alkalies in Other Forms.-If such treatment is not possible, we may prescribe alkalies in other forms,--sodium and potassium carbonates, vegetable salts of sodium, potassium, and calcium, and salts of magnesium and lithium. The salts of calcium, according to von Noorden and Strauss, lessen the monosodium phosphate in the urine, because more phosphoric acid combines with calcium and leaves the body by the intestine. Any of the following, for example, may be used, dissolved in $3 / 4 \mathrm{~L}$. (1/2 pt.) of water :

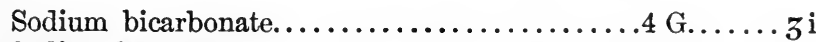

Sodium benzoate............................. $\mathbf{3}^{\mathbf{i}}$

Lithium carbonate............................. viiss

Lithium salicylate $\ldots \ldots \ldots \ldots \ldots \ldots \ldots \ldots \ldots 1-2$ G........... $x v-$ gr. $x x x$

Lithium citrate.....................

The mode of administration is the same as that for mineral waters. Of calcium carbonate we give $2-4 \mathrm{G}$. (30-60 gr.), sometimes necessarily raised to $10 \mathrm{G}$. ( $2 \mathrm{1} / 2 \mathrm{dr}$.), in order to drive the monosodium phosphate from the urine. Artificial mixtures of alkalies may also be used; e.g.:

B Sodii bicarbonatis............................ viiss

Lithii carbonatis.......................... iv

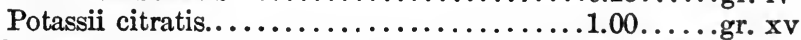

M. fiat pulv. no. i. Dent. tal. dos. no. xx in chart. cerat.

Sig. -1 powder in Seltzer water morning and evening.

Strohschein's new preparation, uricedin, has the same object. The dose is $1 / 2-1$ small teaspoonful, dissolved in a glass of warm water, 1-3 times a day (see p. 134).

Piperazine does not seem reliable, though the author has often used it in connection with phenocoll in the following combination:

R Piperazini hydrochloratis....................... $x \mathrm{xv}$

Dent. tal. dos. no. $x x$ in chart. cerat. 
B Phenocollis hydrochloridis.............. $1.00 \ldots . \mathrm{gr} . \mathrm{xv}$

Dent. tal. dos. no. $x \mathbf{x}$ in chart. cerat.

Sig. -1 of each of the powders dissolved separately in 1 pint of mineral water, and the two solutions to be poured one into the other, stirring briskly all the time. The whole quantity to be drunk during the day.

Other Preparations.-Recently, several preparations of quinic acidurosin (lithium quinate), sidonal (piperazine quinate), chinotropin (quinic acid with urotropine), and urol (quinic acid with urea)-have been recommended for acute as well as chronic gout. The first three, the only ones used so far by the author, are given in $0.50 \mathrm{G}$. ( $7 \mathrm{r} / 2 \mathrm{gr}$.) doses up to 4-5 G. (60-75 gr.) per day, in powders or tablets. The author has seen 2 cases of uretic sciatica of several weeks' duration, on which no other therapy had had any effect, gradually disappear after 1 week's treatment with urosin, until there was scarcely a trace left; 1 case of true acute podagra yielded to 3 days' treatment with sidonal, although the patient's earlier attacks had lasted longer. So, although authorities differ in regard to the merits of sidonal and urosin, the author would provisionally recommend them for use in both acute and chronic cases.

Another preparation, citarin, promises more. It is a compound of formaldehyde with citric acid (anhydromethylene citric acid sodium). The dose is $8-10$ G. (2-2 $1 / 2 \mathrm{dr}$.) the first day, and $4 \mathrm{G}$. ( $1 \mathrm{dr}$.) for the next 3 or 4 days. It is used in acute gout and in acute exacerbations of chronic gout.

Treatment at the Spas.-It is an old custom to send a patient with a gouty diathesis for a month every year to Carlsbad or Vichy, or sometimes to Marienbad. The author, however, considers Carlsbad suited only to well nourished patients, with a good body weight, or to those who have complicating affections of the digestive tract or liver, or chronic constipation. For cachectic individuals, Carlsbad is positively harmful, the alkaline waters mentioned above (Preblau, Bilin, Fachingen, Neuenahr) being preferable. The use of the common salt springs (Homburg, Kissingen, Ischl) is only conditionally advisable, since too much sodium chloride apparently diminishes the solvent power of the urine for uric acid. [One quart of Pluto water at French Lick Springs contains 2.4 G. (36 gr.) of common salt.-Ed.] The hot springs are of great use in the treatment of chronic gout. Three or 4 weeks every spring, with sometimes a shorter stay in the fall, may prevent recurrent eases of acute gout. Suitable resorts are Badenweiler, Gastein, Johannisbad, Krapina-Töplitz (Croatia), Ragatz-Pfäffers (Switzerland), Römerbad (Styria), Teplitz-Schönau (Bohemia), Bad Tüffer (Styria), Vöslau, Warmbad Villach, Warmbrunn (Silesia), Wildbad (Würtemberg), and the hot salt springs at Baden-Baden and Wiesbaden.

The use of tepid baths several times a week has been already mentioned, and other gentle hydropathic measures are beneficial. Steam baths, cold baths, sea bathing, or any other vigorous hydropathic procedures are, on the contrary, too severe. 
The Gouty Symptoms: Tophi.-The therapy of the pathological symptoms of chronic gout is chiefly that of the disease itself. In the author's opinion, the tophi should not be touched except in the case of the ear muscles, when they may be scratched off. It is especially undesirable to resort to surgical aid, as the extent of their surface often obliges either a large incision, or the abandonment of the operation. If after such a procedure or after a spontaneous rupture of a gouty concre-

\section{THERMAL WATERS}

\begin{tabular}{|c|c|c|c|}
\hline Place & Alt. in Ft. & Name of Spring & Temp. F. \\
\hline $\begin{array}{l}\text { ENGLAND } \\
\ldots \ldots \ldots \ldots \ldots \ldots \ldots \ldots \ldots\end{array}$ & 1000 & & $82^{\circ}$ \\
\hline $\begin{array}{c}\text { France } \\
\text { Plombieres } \ldots \ldots \ldots \ldots \ldots \ldots \ldots \ldots\end{array}$ & 1470 & & $54-156^{\circ}$ \\
\hline 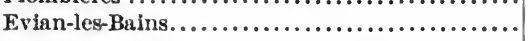 & 1230 & & $53^{\circ}$ \\
\hline 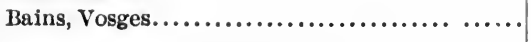 & 1000 & ............. & $90-122^{\circ}$ \\
\hline 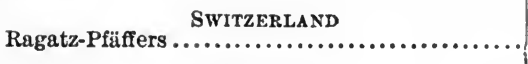 & $1700-2250$ & & $99^{\circ}$ \\
\hline 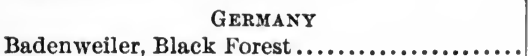 & 1380 & & \\
\hline Wildbad, wurtemberg ....................... & 1400 & & $\begin{array}{l}80^{\circ} \\
95^{\circ}\end{array}$ \\
\hline Schlangenbad, Hesse-Nassau.................. & 1020 & ............................ & $82-90^{\circ}$ \\
\hline 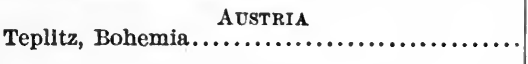 & 750 & 年 & $82-120^{\circ}$ \\
\hline 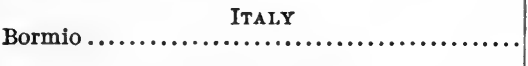 & 4700 & & $105^{\circ}$ \\
\hline $\begin{array}{c}\text { United States } \\
\text { Hot Springs, Garland Co., Ark. ................ }\end{array}$ & $700-800$ & & $76-157^{\circ}$ \\
\hline Arrow-head Springs, San Bernardino Co., Cal. . & 2000 & ................................ & $193^{\circ}$ \\
\hline Paraiso Springs, Monterey Co., Cal. .............. & $\ldots$. & Great Paraiso Hot Soda Spring & $118^{\circ}$ \\
\hline Warm Spring, Meriwether Co., Ga. . ............. & 1200 & Warm Spring ................. & $90^{\circ}$ \\
\hline Springdale, Gallatin Co., Mont. ................ & 4480 & Hunter's Hot Springs......... & $148-168^{\circ}$ \\
\hline Geneva, Douglas Co., Nev. .................... & 4000 & Walley's Hot Springs .......... & $136-160^{\circ}$ \\
\hline Las Vegas Hot Springs, San Miguel Co., N. M. .. & 6767 & & $110-140^{\circ}$ \\
\hline Lebanon Springs, Columbia Co., N. Y............ & .... & Lebanon Thermal Spring..... & $75^{\circ}$ \\
\hline Hot Springs, Madison Co., N. C. ..... . . . . . . . & $1700-1800$ & N. C. Hot Springs.............. & $96-104^{\circ}$ \\
\hline Belkuap Springs, Lam. Co., Ore. ........... & 2000 & Belknap Hot Springs .......... & $188^{\circ}$ \\
\hline Hot Springs, Fall River Co., S. D. ............. & 3400 & Minnekahta Spring............ & $98^{\circ}$ \\
\hline Hot Springs, Bath Co., Va. ...................... & 2300 & Boiler (Bath) Spring.......... & $108^{\circ}$ \\
\hline
\end{tabular}

tion, fistulous processes and ulcers are left, the hot salt springs (see p. 248) are of great benefit in promoting absorption. These springs also appear useful in residual joint affections or functional limitations, as do massage, iodide internally, salt mud baths (Marienbad, Franzensbad), and hot sulphur springs. The same measures are useful in eombating neuritis or its consequences, and neuralgia (e.g., sciatica).

Digestive Disturbances.-In ease of atony of the digestive tract, Kissingen, Homburg, Wiesbaden, Vichy, and particularly Marienbad and Carlsbad are to be recommended, the last being likewise indicated in case of liver eomplieations, chronic constipation, or abdominal plethora. 
In case of obstinate constipation, if the patient cannot be sent to Carlsbad, we may employ our ordinary catharties (podophyllin, rhubarb, sodium sulphate, sulphate of magnesia, or Carlsbad salts).

In dyspepsia, stomachies are indicated (quinine, nux vomica, hydrochloric acid). The last two are combined as follows :

R Acid. hydrochlorici diluti...............5.5.... $3^{\text {i }}$

Strychninæ sulphatis................... $0.02 \ldots \ldots$ gr. $\frac{1}{3}$

Syr. aurantii.................... $100.00 \ldots \ldots$ iii

M. fiat mist.

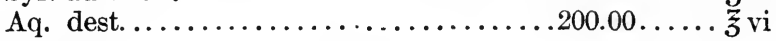

Sig.-1 tablespoonful w ell diluted with water at each meal.

For slight atony of the stomach and intestines, we use gentle laxatives (rhubarb, magnesia, washed sulphur).

Catarrh.-The common salt springs are also used for the chronic catarrh of the throat and respiratory tract which so often accompanies gout. Weilbach, thanks to its lithium spring and its good inhalation apparatus, is particularly noted for its treatment of such cases.

Arteriosclerosis.-In case of arteriosclerosis, so often caused by a gouty diathesis, we are obliged to take refuge in a long continued use of potassium, sodium, or lithium iodide (see p. 75).

Anæmia-Cachexia.-If anæmia and cachexia are prominent symptoms, the patient may be sent to iron or arsenic springs or be given ferratin-iron pastilles, "arsen in ferratese" (see pp. 163 and 170).

Internal Gout.-At times in the course of the disease, one or more acute attacks of gout in the joints are followed by pathological symptoms referable to the inner organs, sometimes sudden, sometimes very gradual in their development. These are known as "the inner metastases" of gout. In such cases, generally the first thing to be done is to bring the gout back into the joint by hot foot-baths, by rubbing the instep and especially the large toe with alcoholic and ammoniacal mixtures or turpentine, or by bandages or hot compresses. If there are cerebral symptoms (giddiness, headache, deafness, somnolence, or the danger of an epileptic or apoplectic attack), we use venesection, drastics, and an icebag on the head. If the stomach is the organ affected, we give tepid water or ipecac to induce vomiting, and apply leeches or cupping to the epigastrium. If eardiac symptoms with an inclination to collapse are present, we use camphor, ether, caffeine, or applications of mustard to the precordial region.

Uræmic symptoms may also arise, and may be treated in the usual manner.

The same principle applies to all the other different symptoms of a gouty diathesis, whether these occur in the nervous system (migraine, headache, giddiness, insomnia), the respiratory tract (bronchitis, asthma), the skin, or the digestive tract.

Complications of Gout: Nephritis.-The combination of gout and nephritis needs special mention. Here we must be exceedingly cautious 
in the use of opium, sodium salicylate, and colchicum. The diet must be chosen so as to avoid any possible injury to the kidneys. Residence in a warm climate (Algiers [Southern California-Ed.]) is advisable.

Obesity.-The subject of gout and obesity has already been treated at sufficient length (see p. 241).

Diabetes.-If diabetes is present, with or without obesity, the alkaline or alkaline-saline waters are doubly indicated (Carlsbad, Vichy, Neuenahr). The French authors give arsenic and the alkalies together for 3-4 weeks, 3 times a day after meals. The dose is 1 teaspoonful of lithium carbonate or effervescing citrate, or $0.50 \mathrm{G}$. $(7 \mathrm{1} / 2 \mathrm{gr}$.) of the simple salt, dissolved in a glass of water, with 8-10 drops of Fowler's solution added. At the same time, sugar must be prohibited. Under this medication, the thirst should subside, the strength increase, and the glycosuria decrease or disappear. In that case, no further attention to the diet is necessary. If marked glycosuria persists, we must regulate the diet according to the rules for uncomplicated diabetes. These are, however, the cases in which the glycosuria is not so important and in which, sugars being wholly forbidden, we give the patients bread and potatoes freely, the urine being constantly watched.

\section{THERAPY OF AN ACUTE ATTACK OF GOUT.}

Relief of the Pain by Applications.-In any acute attack of gout, the first indication is to relieve the pain. Generally, the physician can do little more than lessen it, fearing to make matters worse for the future. Cold applications, especially ice-bags and ice compresses, should not be used on the diseased joint, for they only injure the local circulation and favor the formation of necroses, of deposits of uric acid, and of tophi. Extreme heat is also bad. The joint should at first be kept absolutely quiet, and be bound up with cotton or with a compress wrung out in water or some simple decoction at the room temperature, and then be covered with cotton. The bandages may also be soaked in some soothing solution; e.g., a mixture of equal parts of chloroform and olive oil or oil of gaultheria, sometimes with the addition of opium (1 part of opium or the tincture of opium to 10 parts of the solution), or a mixture of chloroform and belladonna liniment. Or the part may be rubbed gently with these or with a mixture of opium and oil of gaultheria in the ratio of $1: 10$, or of oil of gaultheria and chloroform in equal parts, or with the following salve :

\footnotetext{
R Ext. belladonnæ............................... gr. xlv

Ext. opii...................... $0.30-1.00 \ldots \ldots$ gr. v-gr. xv

Ungt. ichthyolis 10 per cent............... $30.00 \ldots \ldots \tilde{\tilde{z}} \mathrm{i}$

M. fiat ungt.

Sig.-Apply once or twice a day.
}

Finally, there is the French Laudanum Rousseau, a tincture 4 parts of which correspond to 1 part of opium, [and which is therefore $2 \frac{1}{2}$ 
times as strong as the U. S. P. tincture, which latter we prescribe as follows :

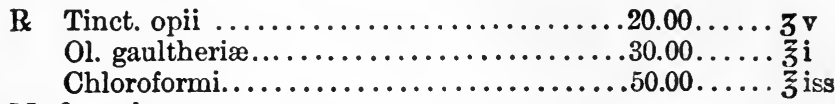

M. fiat sol.

Sig.-Paint on several times a day. Shake well before using.

Mesotan (see p. 546) is also worth a trial.

Massage.-Massage, though it may have a temporary good effect, is apt to encourage necrotic processes in the joint, and so favor the formation of deposits of urates and tophi. For this reason it is contraindicated, even if the pain is not in itself prohibitive.

Medication: Sodium Salicylate.-A favorite internal remedy is sodium salicylate. The author prefers large doses, $6-10 \mathrm{G}$. $(1 \mathrm{1} / 2-2 \mathrm{t} / 2 \mathrm{dr}$.) a day in $1 \mathrm{G}$. (15 gr.) doses every 1 or 2 hours. [So large a dose is very apt to produce toxic symptoms. I use the drug very guardedly in this condition, on account of the kidneys.-Ed.] If it has no effect, or if, as often happens, the effect wears off after 2 or 3 days, it may be replaced by phenacetin (up to 3 G. -45 gr.), antipyrine, salophen, salipyrin, aspirin, acetopyrine, or phenocoll, up to $4 \mathrm{G}$. ( $1 \mathrm{dr}$.) daily.

Colchicum.-The author, however, considers the old-fashioned remedy, colchicum, the best of all drugs for acute articular gout. He has used it often and with marked success. It is best given after the attack has fairly begun, on the second day. He prescribes either 20 drops of the tincture of colchicum seed 4-5 times a day (occasionally with the tincture of opium), or else the wine of colchicum seed in equally large doses, 1-1.3 G. (15-20 drops) 3 times a day.

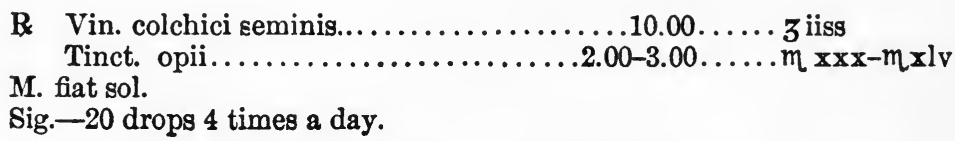

The purpose of the addition of opium is to mitigate the pain, but the author considers its use inadvisable, preferring to give a mild laxative. If the latter is to be given with the colchicum, we may use senna once a day or $1 \mathrm{G}$. ( $15 \mathrm{gr}$.) of carbonate of magnesia with each dose of colchicum; if it is to follow the colchicum, we may use calomel or colocynth.

Other Remedies.- "Liqueur Laville" deserves its excellent reputation. Its efficacy is due to 0.10 per cent. of colchicine. Like the tincture of colchicum, it is given in daily quantities of 4-7 G. (1-13/4 dr.), or even as much as $8-12$ G. ( $2-3 \mathrm{dr}$.). ["Colchi-sal" corresponds rather closely to the "Liqueur Laville." I have obtained excellent results with it in doses of 1-2 capsules every 2-4 hours. It is apt to derange the digestion. As in chronic gout, I prefer colchicine and employ it in doses of $0.0006-0.0012 \mathrm{G}$. (1/100-1/50 gr.) every hour or two until the 
pain is relieved or symptoms of poisoning (diarrhœa, nausea, etc.) supervene. The addition of codeine phosphate, 0.008-0.016 G. ( $1 / 8-1 / 4 \mathrm{gr}$.) permits larger and more frequent doses.-Ed.]

For the use of eitarin and the recent preparations of quinic acid, see p. 248.

Pfeiffer considers that hydrochloric acid alleviates the pain in many individuals, and gives $5 \mathrm{G}$. (75 min.) of dilute hydrochloric acid; on the other hand, many authorities, including Duckworth, strongly advocate alkalies (salts of sodium, potassium, and lithium). If the joint pains continue for a long time, even though reduced in intensity, iodide of potassium, best eombined with colchicum, is recommended. Pfeiffer prescribes it as follows:

R Potassii iodidi...................... 5.00.....gr. $1 \times x v$

Tinet. colchici seminis................ $10.00 \ldots \ldots$ iiss

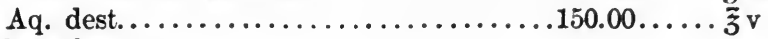

M. fiat sol.

Sig.-1 tablespoonful well diluted with water 3 times a day after meals.

If only mere traces of the inflammation are left, these will as a rule subside under the influence of hot springs or hot baths at home.

Exercise.-The patient with acute gout of the lower extremities should get up from his bed just as soon as possible and try to walk, even though the movement causes exacerbations of the pain; in not a few cases this produees recession of the inflammatory symptoms. If the gout is in the upper extremities, he should use these joints in gymnastic exercises as soon as possible.

Dietetic Therapy.-The diet during an acute attack must be quite different from that in chronic gout. From his own experience, the author recommends a simple liquid diet, of milk and soups made without stock. The patient may drink large quantities of water or a very weak alkaline mineral water; e.g., Seltzer, Krondorf, or Apollinaris. The stronger alkaline waters (Fachingen, Vichy, Obersalzbrunn) are forbidden. All fruit acids and juices are permitted. Alcohol often tends to increase the local inflammation, and should be given, if at all, only very tentatively and in prolonged attacks.

Prevention of Recurrence.-To prevent the recurrence of an attack, Trousseau recommends smoking the sound joints, never those that are still inflamed, with tobacco smoke. Tobacco leaves are burnt on a coal fire and the joint is exposed to the smoke from them as hot as ean be borne. The smoke may be kept close to the leg by making a little tent of a large stocking or a blanket. The process should be repeated once every week.

If acute gout manifests itself, not as a joint affection, but as a disease of another organ (bronchitis, some affection of the eye, etc.), the therapy must be (1) that of a gouty diathesis in general, and (2) that of the particular disease in question. 


\section{THERAPY OF PHOSPHATURIA.}

Etiology.-To the discussion of the therapy of the diseases which are clinically very clearly due to derangements of metabolism, will be appended a short sketch of the therapy of phosphaturia. This condition is (1) primary; or (2) secondary, due to $(a)$ local diseases of the genitourinary tract, particularly of the bladder, $(b)$ various nervous affections, (c) dyspepsia and dilatation of the stomach, $(d)$ incipient tuberculosis, and $(e)$ a coincident or incipient diabetes mellitus (phosphatic diabetes). In the second group of cases, i.e., in secondary phosphaturia, our therapy should be mainly causal.

Dietetic Therapy.- When the phosphaturia is primary or is a symptom of some disease of the genito-urinary tract which cannot be removed at the time, a special dietetic therapy should be adopted. Unlike gout, in which the neutral phosphates are present in the urine in too small quantities relatively to the uric acid, phosphaturia is characterized by the absence of acid phosphates from the urine, and the presence of the neutral and alkaline phosphates exclusively. To supply the acid phosphates, we allow a moderate use of meat soups and a practically unlimited use of meats and eggs (only very rarely does the large phosphate content of meat and eggs lead to increased excretion of phosphates), with large quantities of milk, bread, carbohydrates of every kind, and fat. Wine, beer, and liqueurs are allowed. Green vegetables are restricted as far as possible, and potatoes, root vegetables, fruit, vinegar, and sour foods of all kinds are forbidden, on account of their content of vegetable salts. Naturally, all akaline mineral waters are prohibited.

Medication.-The object of medication must be to furnish the body with mineral acids, particularly hydrochloric acid, in large doses. Drugs which change the alkaline reaction of the urine to an acid one (urotropine, salol) are also very useful. Pfeiffer recommends hot springs (especially Wiesbaden); other authorities claim that warm baths decrease the acidity of the urine. The author himself has had no personal experience in this regard.

For the therapy of oxaluria, which is also clinically an evident anomaly of metabolism, see p. 137.

\section{THERAPY OF RICKETS.}

Although rickets properly belongs to the domain of pediatrics, it will be briefly discussed here.

Prophylactic Therapy.-Rickets is almost exclusively a disease of infancy and early childhood and must be combated in its very beginning. To protect a child against it, proper feeding is of the first importance. Next to maternal nursing, which must always occupy the first place in infant feeding, comes wet-nursing, then feeding with modified cow's milk, and lastly feeding with various substitutes for milk. Plenty of air and sunshine is the second prime requisite in the care of an infant. 
Hardening Process.-Of especial importance in the prophylaxis of rickets is the rational "hardening" of a child by systematic hydrotherapy. From birth the infant should be bathed daily in tepid water $\left(91^{\circ}-93^{\circ}\right)$, and then carefully dried, the whole process taking from 6 to 10 minutes. After the first 2 or 3 weeks, this tepid bath should be followed by a quick sponge off with water at $82^{\circ}-84^{\circ}$. This temperature should be reduced $2^{\circ}-3^{\circ}$ every 3 weeks, until it has reached the temperature of the room, about $68^{\circ}$. At $3-4$ months, the time of the bath may be reduced to 5 minutes, and after the first year, it may be given only every other day. In the case of strong, healthy children, the number of tepid baths may be gradually reduced during the second year to two a week. In their place we gradually introduce daily morning sponge baths at the temperature of the room $\left(65^{\circ}\right)$ followed by a rub down and exercise, and cold sponge baths before going to bed. With delicate children the temperature of the bath water should not go below $75^{\circ}$, and they need not be subjected to cold sponges until they are 6-7 years old. At this age, the temperature of the morning sponge bath for healthy children may be gradually reduced to the minimum of $59^{\circ}$. Naturally, the colder the water, the shorter the bath. We should be even more energetic in carrying out all these prophylactic measures, if we have to do with a child who is inclined to rickets.

Ther 4 py of Developed Rickets: Diet.-If the disease has already appeared, the first thing to be cared for is proper nutrition. If we are obliged to adopt artificial feeding, we must use the best milk available or some of its best substitutes. Malt extract and Mellin's Food are both good, on account of their content of malt. At the end of the first half year, some veal broth and later beef broth (on account of its content of salts) may be added to the milk. In preparing the broth some spinach and carrots should be cooked with the meat. With the beginning of the second year, meat and eggs may be allowed, and some light vegetable, such as spinach or carrots.

Rest.-The little patient, who instinctively keeps very quiet, should never be forced to sit or stand, as this only tends to bring on spinal curvature or bow-legs. It is important that he be not carried always on the same arm of the nurse, for fear of lateral curvature. When he does begin to sit up voluntarily, his back should be supported by a pillow. He should, of course, be kept out of doors and in the sun as much-as possible.

Climate.-Beside the mild form of hydrotherapy discussed above, salt baths are generally useful. The method of giving them at home or at salt springs has already been discussed on pp. 192 and 193. In winter especially, but also in the spring and fall, the child may well be sent to a warm seaside resort (Grado, San Pelagio, Abbazia, Porto Rose near Pirano, the Lido at Venice, all on the Adriatic, also the resorts on the Riviera, [Florida, Southern California.-Ed.]). Here he should enjoy the sea air, sit in the warm sand, and move about on the beach, or he may 
also take sea baths. In warm weather, rachitic children do best in a rather high altitude (1200-2700 ft.). Suitable resorts are Gmunden (1400 ft.), Aussee, Innsbruck, Interlaken, Partenkirchen (2200 ft.), Constance and Ueberlingen on Lake Constance, Sonnenberg on the Seelisberg, Axenstein, Bürgenstock, and particularly Gersau on Lake Lucerne. [For similar resorts in America, see p. 336.-Ed.]

Medication: Cod-liver Oil.-The drug which has always enjoyed the greatest reputation in the treatment of rickets is cod-liver oil. Its value lies simply in its nutritive worth as an easily emulsified fat. It is best to begin giving it in winter, in daily doses of only $2 \mathrm{G}$. ( $\mathrm{I} / 2 \mathrm{dr}$.). Unfortunately, with many rachitic children, it brings on digestive disturbances, in which case we may try lipanin, or Mehring's "Kraftschocolade."

Phosphorus.-Many authorities claim that phosphorus is a specific in the treatment of rickets; others deny that it has any good effect whatever. The majority of pediatrists regard it as having a good influence on the nervous disturbances, particularly the laryngeal spasms, which so often accompany rickets, and as aiding in the hardening of the bones. It is necessary to give at least $0.0005 \mathrm{G}$. (3/400 gr.) daily for 4 weeks. Kassowitz prescribes it as follows :

R Ol. morrhuæ....................100.00.... そ iii

M. fiat sol.

Phosphori.........................0.01....gr. $\frac{1}{6}$

Sig. - 1 teaspoonful a day. $(5.00 \mathrm{G}$. $(75 \mathrm{~min}$. $)$ of the cod-liver oil contains 0.0005 G. ( $\frac{3}{40}$ gr. ) of phosphorus.)

If cod-liver oil cannot be borne, Kassowitz recommends the following prescription :

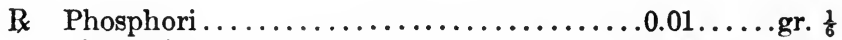

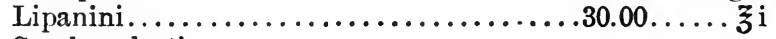

Sacchar. lactis,

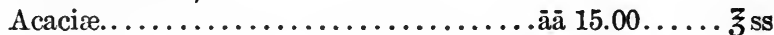

Aq. dest......................... $40.00 \ldots$

M. fiat emuls.

Sig. -1 teaspoonful a day.

According to Kassowitz, this emulsion is always well taken. [Jacobi employs elixir phosphori, $4 \mathrm{G}$. (1 dr.) of which represents $0.001 \mathrm{G}$. $(1 / 65 \mathrm{gr}$.) of phosphorus.-Ed.] The easiest and pleasantest way to give phosphorus, however, is in the form of Gärtner's phosphorus chocolate tablets, each of which contains $0.00025 \mathrm{G}$. (3/800 gr.) of phosphorus.

Calcium.-A third drug often recommended is calcium. Vierordt considers it indicated in cases of relapsing rickets, when, as often happens with older children, milk in considerable quantities is not well taken and the diet consists principally of cereals, meat, broths, and vegetables. Calcium carbonate in doses of $0.50 \mathrm{G}$. ( 7 gr.) several times a day may then be given. The author recommends the simple method of dissolving 
1-2 pinches of calcium phosphate or calcium carbonate in soup. The following syrup is also useful in such cases:

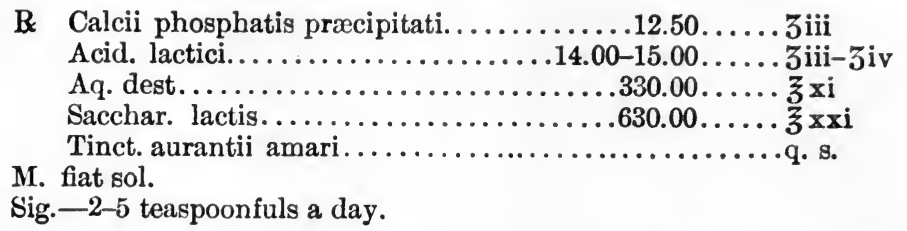

Bone Marrow.-Bone marrow may prove a useful way of giving fat, provided cod-liver oil cannot be taken.

Heubner's experiments in giving thyroiodin for rickets, on account of the connection between the thyroid gland and the growth of the bones, have led to no positive results.

It may be worth while to try the effect of the inhalation of condensed air, with the object of preventing the development of thoracic deformities.

Symptomatic Therapy.-Beside all these general methods of treatment, it is often necessary to treat symptomatically coincident disturbances of digestion, and affections of the respiratory tract and of the nervous system. Surgery must also be frequently brought into requisition.

[Forchheimer, without offering any satisfactory reason, encourages the use of salt in all children disposed to rickets. He emphasizes the detailed eare of the child to prevent later deformities, the use of firm hair mattresses and pillows, changing its position carefully and frequently, avoiding lifting it up under the arms, not allowing the child to bear its weight upon its legs until after the bones are firm, and the necessity of fresh air and sunshine. Holt considers early treatment most important; from the sixth month to the fifteenth much can be done. He is not a warm advocate of phosphorus.-Ed.]

\section{THERAPY OF OSTEOMALACIA.}

Medication.-As the essential nature of osteomalacia is very like that of rickets, -in both a lack of calcium in the bones,- the treatment is, naturally, very similar. We use cod-liver oil, bone marrow, phosphorus, and especially the combination "phosphor-codliver oil." Larger doses are necessary than in infantile rickets, of course. Sternberg, the youngest and one of the most ardent champions of the phosphorus therapy in osteomalacia, gradually works up to 0.005 G. (1/12 gr.) daily and continues this dose for weeks and months. If the combination of phosphorus and cod-liver oil cannot be borne, phosphorus may be given alone in the form of pills (to adults) :

R Phosphori........................... $0.10 \ldots . .$. gr. iss

Ext. glycyrrhiz .......................... 8.

M. fiat mass. Div. in pil. no. c.

Sig. $-2-3-5$ pills a day. 
Winkel recommends the combination of cod-liver oil with colchicum, 1-2-3 tablespoonfuls of the former with 30-40 drops of wine of colchicum seed added, at morning and night.

As in rickets, ealcium is useful in cases which are relapsing and where the food is apparently poor in calcium salts. Calcium phosphate or carbonate, $0.50 \mathrm{G}$. ( $7 \mathrm{gr}$.) $3-4$ times a day, may be given; or both, in the following combination:

R Calcii carbonatis præcipitat.,

Calcii phosphatis....................āā 1.00.....gr. xv

Ferri carbonatis saccharat................ $0.50 \ldots \ldots$ gr. viiss

M. fiat pulv. no. i. Dent. tal. dos. no. $x$.

Sig. -1 powder twice a day.

Malt extract may also be given, with or without the addition of milk or calcium phosphate ( 1 teaspoonful to 1 tablespoonful several times a day).

The use of chloral or chloroform, 2 G. (30 gr.) per day in solution internally for 3 weeks, has been recommended by the Italians, but seems to have found no supporters.

The author has tried atropine for such cases of osteomalacia as have occurred in his hospital service. It had a very satisfactory effect in the 4 or 5 cases of puerperal osteomalacia in which it was tried; but it must be confessed that they were of a slight or only moderately severe typee.g., the women could move about, though with great fatigue-and the rest and care at the hospital were undoubtedly weighty factors in bringing about the improvement. Atropine was continued until symptoms of intoxication appeared, and in some cases was renewed after such symptoms had vanished. Under this treatment the pains improved and disappeared, the bony skeleton became firm and free from pain, and the patient could walk freely again. A similar good result was also obtained in a case of severe puerperal osteomalacia in which several months' treatment with phosphorus had had no noticeable effect.

Hydrotherapy.-Hydropathic procedures are also of value in the treatment of osteomalacia. Simple tepid baths $\left(91^{\circ}-95^{\circ}\right)$ may be used, or the so-called "aromatic baths." These are prepared by adding to a warm bath either a decoction made by boiling 500-1000 G. (1-2 lb.) of aromatic herbs in $10 \mathrm{~L}$. (10 qt.) of water, or $250-500 \mathrm{G}$. ( $1 / 2-1 \mathrm{lb}$.) of pine needles or the buds popularly called balm of Gilead buds, with $1 / 2-2$ small teaspoonfuls of the corresponding ethereal oil. Or, we may add to the bath water a decoction made by boiling a bag containing $1 / 2-1 / 2 \mathrm{~L}$. (1-3 pt.) of bran in 4-10 L. (4-10 qt.) of water for a quarter of an hour. Malt baths, hot sand baths, and salt baths are also used. The malt baths are prepared by adding to the bath $250-500 \mathrm{G}$. ( $1 / 2-1$ pt.) of malt extract or the decoction obtained by digesting 1-3 kg. (2-6 lb.) of ground air-dried malt in 5 times its volume of water for several hours. For salt baths, which, as in rickets, are the most common form of baths employed, see p. 192. 
Schmidt, of Neusser's clinic, has recently recommended daily hot air baths (phènix à l'air chaud, see p. 43), on the practical ground of having obtained good results from them, and on the theoretical ground that they relax the vessels of the bones and hasten a cure.

Surgery.-As in rickets, surgery should sometimes be resorted to. In a severe case of osteomalacia, if an operation is not contraindicated by complications or by the weakness of the patient, ovariotomy or Porro's supravaginal amputation of the uterus may be undertaken, with remarkably successful results.

Hygiene.-It is hardly necessary to urge the importance of air, light, and good food. According to some authorities, the latter should be vegetable rather than animal.

\section{THERAPY OF CHRONIC RHEUMATISM.}

Definition.-Under chronic rheumatism will be included both chronic muscular rheumatism and chronic articular rheumatism, since (1) the therapy is the same, (2) both are manifestations of a constitutional anomaly, and (3) the two are clinically so often found together that the distinction between them is a merely artificial one. Chronic rheumatism, in this sense, then, is a constitutional disease whose seat is partly in the joints, partly in the tendons and fasciæ, and less often in the muscles proper. In only a small number of cases has it any connection with acute rheumatism, which is without doubt an infectious disease. In the majority of cases it must be placed, from a therapeutic standpoint, along with the diseases of metabolism. [Many of these conditions, both muscular and articular, included by the author under the heading "chronic rheumatism," are, in my judgment, forms of irregular gout, and yield to corresponding treatment. I object seriously to the term "chronic rheumatism," and feel that less confusion, both in diagnosis and in therapy, would result if we limited the word "rheumatism" to the acute articular varicty, or to that which is, without doubt, microbic in origin.-Ed.]

External Therapy.-Almost the only way of securing any results in the treatment of chronic rheumatism is by external therapy, which may be subdivided into (1) baths (in the widest sense), (2) massage, and (3) electricity.

Hot springs should be selected for the treatment of chronic rheumatism, since the heat reduces the irritability and sensitiveness of the diseased parts, and, by increasing capillary circulation, promotes the absorption of the pathological products of exudation. Various kinds of hot springs may be selected. First come the simple hot springs; e.g.: Wildbad-Gastein (Salzburg), $109^{\circ}-118^{\circ}$; Hof-Gastein (Salzburg), $95^{\circ}-104^{\circ}$; Krapina-Töplitz (Croatia), $99^{\circ}-111^{\circ}$; Neuhaus (Styria), up to $99^{\circ}$; Ragatz-Pfäffers (Switzerland), $100^{\circ}$; Römerbad (Styria), $101^{\circ}$; Teplitz-Schönau (Bohemia), up to $115^{\circ}$; Kaiser Franz Josefs-bad Tüffer (Styria), $95^{\circ}-102^{\circ}$; Warmbrunn (Silesia), $77^{\circ}-104^{\circ}$; Wild- 
bad (Würtemberg), $91^{\circ}-104^{\circ}$; Bormio (Italy), $90^{\circ}-106^{\circ}$. Some of these (Teplitz, Warmbrunn, Neuhaus, etc.) also have some springs of lower temperature.

Next come the simple tepid springs: Johannisbad (Bohemia), $84^{\circ}$; Badenweiler (protected, subalpine climate), $79^{\circ}$; Schlangenbad (Wiesbaden), $82^{\circ}-90^{\circ}$; Wiesenbad (Saxony), $73^{\circ}$.

The baths should of course be adapted by the resident physician to each individual case, but on an average they should be taken in water at $91^{\circ}-97^{\circ}$ and should last at first 10 minutes, the time being increased by 5 minutes each bath until it reaches 30 minutes. As in all hot baths, cold compresses should be kept on the head. Five baths a week is the usual number, unless a contraindication occurs, in the shape of nausea, etc. Sometimes, after the fourth or sixth bath, acute pains in the joints appear during bathing, but disappear again after the tenth or twelfth bath.

Hot salt springs have the additional effect, produced by their content of salt, of stimulating the skin and hence indirectly the circulation, respiration, and metabolism. They are represented by Baden-Baden $\left(156^{\circ}\right)$ and Wiesbaden $\left(156^{\circ}\right)$.

The carbonated hot springs are even more useful, combining as they do the effect of heat and of salt with that of carbon dioxide. Among them are Nauheim (Hesse), Oeynhausen (Minden), and Kamm and Koenigsborn (Westphalia). Nauheim has a hot carbonated salt spring, two sprudel springs $\left(88^{\circ}-95^{\circ}\right)$ and a salt bath free from gas. If a carbonated bath is contraindicated by extreme nervousness, Oeynhausen has an ordinary salt spring and one with a temperature of $90^{\circ}$.

Iron-mud and sulphur-mud baths are also of great help in chronic rheumatism. The former are prepared by mixing the mud with water in a bath-tub and heating it by steam to the desired temperature. This is usually $95^{\circ}$ to begin with, gradually raised during the bath to $99^{\circ}-104^{\circ}$, if symptoms of cerebral congestion do not appear as a contraindication. The time should be at least 20 minutes, and generally half to three-quarters of an hour. The patient is then rinsed off with warm water, put in a warm bath, and then carefully dried. After a half hour's rest, he is given a cooler rub down. At the beginning of the treatment, the baths should be taken every other day; during the second week, every third day; and still less frequently during the third week. The total number of baths in any one treatment may be 15-20, and occasionally 30 . The sulphur-mud baths are given in the same way. Five a week is the usual number, with simple salt or sulphur baths on the other two days. The most famous iron-mud baths are, Franzensbad, Marienbad, Carlsbad, Pyrmont, Anna Moorbad Belohrad (Northeastern Bohemia), Cudowa, Karlsbrunn (Silesia), Elster and Reinerz (Glatz), and Nenndorf (near Hanover); the most famous sulphur-mud baths, Pistyan, Driburg (Westphalia), and Eilsen (Schamburg-Lippe). The mud baths produce their effect by (1) the high temperature, (2) 
the cutaneous stimulation produced, and (3) the gentle pressure on the body, somewhat similar to very mild massage. They have the advantage over water baths of retaining the heat longer and more uniformly. In case the rheumatism is local, they may be utilized as partial baths for the upper or the lower extremities alone.

Ordinary sulphur baths, either naturally hot or artificially heated, do not have so strong a general effeet as do the mud baths, and for that reason are often preferable, especially in cases where the nervous irritability is strongly marked and there are slight lesions of the heart and vessels. Among the natural hot sulphur springs are Baden (near Vienna), Trenesin-Teplitz (Upper Hungary), Warasdin-Töplitz (Croatia), Hereulesbad (near Mehadia), Kaiserbad and St. Lucasbad (Budapest, especially as winter resorts), Aix-la-Chapelle, and Baden (near Zurich). Among the cold sulphur springs, Kainzenbad (near Partenkirehen), Eilsen (in the Bavarian mountains), Langensalza (Thuringia), Leuk (Canton Bern), Nenndorf, Vals (Canton Graubündten) and Weilbach (Nassau).

One bath in particular has proved successful in some of the author's cases of joint rheumatism and even arthritis deformans, after many others had been tried in vain,-Porto Rose, not far from Pirano. It has a strong salt bath, and a shore bath which in summer may reach a temperature of $86^{\circ}$.

[Climate has a decided influence upon rheumatic patients, but I know no resorts generally applicable to such patients, except that all do better in a warm as well as in a dry climate. The selection of a suitable health resort depends more upon the efficiency of the bathing appliances and of the medical care than upon the composition of the waters. Hot saline waters, with a more stimulating action upon the skin, especially when charged with carbonic acid, allow the employment of higher temperatures than the indifferent waters. Among the best resorts in England and America for such cases may be mentioned the following:

Buxton, in Derbyshire, at an elevation of $1000 \mathrm{ft}$., is a simple thermal spring and is comparable to Ragatz or Wildbad. Temperature of the water is $82^{\circ}$, and it contains a large amount of nitrogen gas. Both natural and hot baths are prescribed.

Bath, in Somersetshire, is a picturesque and attractively situated city with a mild equable climate. The surrounding hills furnish protection against severe winds. Its waters are classed among the simple indifferent thermal springs, but possess sufficient earthy, calcareous constituents to resemble Contrexéville. There are three hot springs for bathing, with a temperature of $104^{\circ}-117^{\circ}$, and $120^{\circ}$.

Harrogate, in Yorkshire, is a cold sulphur spring, with a very large number of different mineral springs. The strongest contain a large amount of salt. The new Montpelier Baths contain every modern appliance. The weaker sulphur waters and chalybeate waters are used for drinking. The climate is dry and bracing. 
Richfield Springs, on Lake Canaderaga, Otsego County, N. Y., at an altitude of $1800 \mathrm{ft}$., in a beautiful rolling country, has been popular since the early part of the last century. Its waters are heavily charged with sulphuretted hydrogen, and contain $112 \mathrm{gr}$. of calcium sulphate in the gallon. There are also iron and magnesium springs. The bath house has been recently modernized. The hotels and boarding houses are fair.

Hot Springs, Virginia, with an elevation of $2300 \mathrm{ft}$., is perhaps the best equipped "spa" in America, and is deservedly popular among the well-to-do. The hotels are well kept, the bath establishments are furnished with every modern device, and the medical attendance is excellent. The seasons are from May 1 to June 15, and September 1 to November 1 . The warm springs, at $96^{\circ}$, are gaseous, earthy, sulphuretted waters. The "Healing Springs," which resemble those of Schlangenbad and Ems, are gaseous, earthy, alkaline, and sulphuretted.

Hot Springs, Arkansas, in the Ozark Mountains, lies in a valley at an elevation of $425 \mathrm{ft}$. This resort is owned by the United States Government. There are 70 springs, with a temperature ranging from $76^{\circ}$ to $148^{\circ}$. The solid constituents are chiefly calcium carbonate and silica. The springs also contain considerable quantities of free carbonic gas. They are perhaps even more celebrated in the treatment of syphilis than of rheumatism. Enormous doses of mercury and iodide of potash are borne by such patients, even in the late cachectic stages.-Ed.]

Sun baths are often beneficial. The patient, with as few clothes on as possible, should be exposed to the rays of the sun out of doors or in a glass room, the face and head being shielded from the direct sunlight.

Baths of various kinds may also be given at home. First come simple warm baths, $95^{\circ}-100^{\circ}$, lasting from 15 to 30 minutes. The head should be kept cool, the temperature of the room should be at least $68^{\circ}$, and after the bath the patient should go at once to a warm room or to bed. A hot drink directly after the bath is advisable. During the first week, two baths should be given; during the second, three; and during the third, one every other day. Iron-mud baths may also be prepared at home; they are unsatisfactory, however, because being made from the salt or brine obtained from the mud, they lack the mechanical effect of the mud itself. Steam baths, hot air baths (phénix à l'air chaud), which may also be used for partial baths, steam cabinets, and Roman-Irish baths are all indicated, provided there is no cardiac weakness or atheroma of the vessels. The face and head should first. be sponged off with cold water and should be kept cool during the bath by a cold compress or an ice-bag. The duration of the steam and Roman-Irish baths should be at first 15-30 minutes. A steam bath may be directly followed by a hot air bath (one room at $104^{\circ}$, one at $122^{\circ}$ ). At the end of any of these baths, the body should be completely cooled off by a cool shower.

Hot Compresses.-If the patient cannot move easily, or if he has arteriosclerosis or a weak heart, or if for any reason it is impossible 
to prepare the baths at home, iron-mud or sulphur-mud may be applied locally. This may be done also if only a few joints are affected. The author can also most warmly recommend from personal experience the so-called steam compresses. The diseased joint is bound with a compress wrung out in boiling water and placed between two layers of flannel. As soon as the patient feels that the compress is growing cool, it should be renewed, the process being kept up for 2 hours (or for a whole day), at the end of which time the part is washed off with cool water.

Hot Sand Baths.-Hot sand baths deserve especial mention. The patient, wearing a thin bath-robe, lies on a 5-inch layer of sand (temperature $107^{\circ}-122^{\circ}$ ) in the bottom of a wooden bathtub. His head and neck being left free, he is covered up to the shoulders for a full bath, or up to the navel for a partial bath, with a 4-inch layer of sand of the same temperature. A profuse sweat is soon induced, the weight of the sand acting meanwhile like a mild massage. After $1 / 2-1$ hour, the sand is washed off in a tepid bath. Arteriosclerosis or cardiac weakness is in general a contraindication. There are establishments for giving such baths at various sand-dunes; e.g., at Köstritz (Thuringia), Neu-Wittelsbach (Neuhausen near Munich), and Berka on the Ilve.

Fango Baths.-Fango baths, that is, applications of the volcanic Euganean mud known as Fango, are used with great success both in private practice and in special institutes. The bed is spread with a blanket, then with a rubber sheet, and then with a sheet covered with an inch deep layer of the mud, which has previously been heated to $113^{\circ}-122^{\circ}$ by immersing the vessel containing it in hot water. The patient is then laid on the mud, his breast, abdomen, and extremities are covered with more of it, and the sheet and blanket are wrapped closely around him, the neck and head being left free. He remains in the baths $1 / 2-3 / 4$ of an hour and then is given a tepid cleansing bath. Several cases so treated in the author's private practice have received great benefit, as well as have most of those treated in the Fango Institute at Vienna. Equally good results are obtained from the Pistyan mud. 'The Fango Institute in New York also gives these baths.-Ed.] Warm " ichthyol mud" has also proved beneficial in the author's practice, particularly for local application.

Pine-needle baths (see p. 258) do not appear to be particularly successful in the treatment of chronic rheumatism.

Baking.-Finally, the process of "baking" the affected extremities by means of Tallermann's (Sheffield) or some similar apparatus has proved of remarkable value. The Tallermann apparatus consists of a cylindrical copper chamber in which the extremities, and if necessary the pelvis, may be enclosed. The air inside the chamber is brought by means of a gas flame to a temperature of $150^{\circ}$, which may gradually be raised to $300^{\circ}$ or even $340^{\circ}$ after the limb is enclosed. The part is prevented from burning by being supported on an asbestos frame. There are other similar pieces of apparatus, in some of which the source of 
heat is electricity instead of gas. They are all, naturally, more suited for use in properly equipped sanatoria than at home.

Cold Applications.-The effects of cold remain to be mentioned. It is applied sometimes in the form of cold sprays on the affected region, sometimes in that of short showers, sometimes as Scotch douches (quickly alternating applications of hot and of cold water), and sometimes as cold compresses alternating with hot. All of these, especially if followed by rubbing, are very beneficial. They appear, however, to be indicated especially in the stages of remission in the progress of the disease, when the object is to harden the body gently and gradually against a fresh attack. As soon as possible after the cessation of a local attack, the spot affected should be rubbed with cold water, be given cold sprays, or have cold water poured over it, and then be scrubbed with a soft brush. All these measures tend to eliminate the place of least resistance, by inducing a more active circulation and a better state of nutrition in the region affected.

Massage.-Hydrotherapy may well be supplemented by massage and electricity, especially the former. Massage may be used as an independent therapeutic measure, or it may follow directly after some form of bath. The movements, provided the fasciæ, aponeuroses, and muscles are the parts affected, may consist partly of stroking and circular rubbing, partly of kneading and vibratory pressure. The preference should, however, be given first of all to chopping movements with the edge of the palms, which may be followed by passive and active movements of the muscle. If it is the joints that are chiefly affected, the soft parts above and below them may be strongly manipulated, particularly in the region of the great vessels. After this the region of the joint itself may be rubbed spirally from the periphery to the centre. If the pain is not too severe, "chopping" massage and passive movements (later also active) may be added, the whole ending with a more prolonged "effleurage." The treatments should, in general, be given daily and last $1 / 4-1 / 2$ hour.

Electricity.-Electricity is not so markedly beneficial. The faradic current may be employed in both muscular and articular rheumatism, by stroking the skin over the affected part with the so-called faradic pencil or brush. The galvanic current is, however, preferable. In muscular rheumatism we treat the affected parts with wet electrodes, using a moderate current, or place the anode on some painful point. In joint rheumatism we galvanize the joint directly with broad plate electrodes. A desirable combination of massage and electricity, especially in articular rheumatism, may be obtained by means of the massage roller, either kind of current being employed.

Gymnastics, either with or without apparatus (particularly Zander's), may be employed to improve the impaired motility of the joints (see p. 5). 
Medichtion : External Applications.-It is hardly necessary to add that, so far as medication is concerned, external applications play the most important part. We may use alcohol, chloroform liniment, liniment containing opiates, gray ointment, tincture of iodine, iodine vasogen, ichthyol ointment (10 per cent.), or the following mixture of chloroform and veratrin :

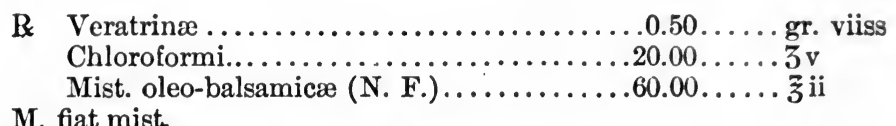

M. fiat mist.

Sig.-For external use, only on unbroken skin.

In using iodine on an affected joint, a strip through the middle, an inch or two wide, should be left unpainted. The painting may be continued until the outer epidermis peels off, unless the irritation of the skin obliges us to stop for a few days.

The author has obtained good results (improved mobility, decreased pain) with guaiacol, internally and externally. The expense is, however, an objection. Internally, the dose is $0.50 \mathrm{G}$. $(7 \mathrm{gr}$.) 3 times a day of guaiacol carbonate. Externally, the following prescription may be used:
R Guaiacolis,
Ol. olivarum..................... à $\bar{a} 100.00 \ldots \ldots z$ ziii
M. S.-To be painted on the affected joint once a day.

Internal Medication.-Only a few drugs are useful internally. Among them are, iodide of potassium, ichthyol, in $2 \mathrm{gr}$. pills, about 1.26 G. (18 gr.) being given throughout the day; the tincture or wine of colchicum; the preparations of lithium, and similar mineral waters (in true articular rheumatism); Fowler's solution; and, in acute exacerbations of the chronic process, the various preparations of salicylic acid. [Intestinal autointoxication is doubtless an important etiological factor in many of these conditions. Appropriate treatment to combat such a poisoning, as high colon irrigation, is often effective.-Ed.]

Hygiene.-Unless a uric acid diathesis is present, no particular attention need be paid to the diet, except to see that it is nourishing and combines animal and vegetable foods. The physician should, of course, be sure that the patient is protected from dampness and has plenty of light and air. [Appropriate clothing is very essential for the proper care of patients with chronic rheumatism. In general, such patients keep in better condition and have less rheumatic disturbances when wearing thin flannel or a thin mixture of silk and wool. Some of them, especially those who perspire freely upon only moderate exertion, have, in my opinion, been benefited by wearing linen-mesh garments, so popular in America just now. It is wise to caution against overheated houses, as a temperature which promotes perspiration is apt to predispose 
these patients toward taking cold and toward exacerbations of their trouble. Although a very guarded attempt should be made to harden them, they should not be allowed to feel cold, and should be urged to select carefully their outer garments each day, so that the very pronounced variations of temperature in our climate do not affect them unduly.-Ed.] 


\section{THERAPY OF DISEASES OF THE MOUTH}

The diseases of the mouth belong in part to the domain of the specialist rather than to that of the general practitioner. But as too little attention is generally paid by the physician to the physiological and pathological conditions of the mouth, a brief discussion of such conditions, and of the most important diseases of the mouth, does not seem out of place here.

\section{CARE OF THE MOUTH.}

Under Physiological Conditions.-An exception to the general plan of omitting from this book all prophylactic measures will be made in the case of the mouth; for a rational care of this organ is of great importance, involving, as it does, the prophylaxis of a great number of diseases, not only of the mouth itself, but also of the other organs, particularly the stomach, the intestines, and the respiratory tract.

Care of the Teeth.-Every person should cleanse the teeth and rinse out the mouth directly after each meal if possible, but at any rate every morning and night. The tooth-brush for an adult should contain four rows of stiff bristles; for a child, two rows of medium soft bristles. Care should be taken to select a brush in which the bristles are firmly fixed, as cases have been known in which a bristle, working loose, has been swallowed and has caused appendicitis.

For cleansing the teeth there are available various kinds of soaps, tooth-powders, or pastes made by combining soap, syrups, and alcohol, or soap and alcohol alone. The soaps, whose reactions should be either neutral or weakly alkaline, are preferable to the powders for steady use, for three reasons: (1) the soaps permit possibly an easier entrance into the carious cavities of the teeth; (2) the powders, especially those containing pumice, carbon, or calcium carbonate, wear away the enamel; and (3) the powders containing carbon are apt to cause the incorporation of disfiguring black specks in the gums. It is best to avoid altogether toothpowders containing carbon. Those containing very finely pulverized chalk or pumice-stone may be used once a month to advantage (ScheffPaschkis). [Powdered cuttle-fish bone (os sepiæ) is better than pumicestone, as it is alkaline, and its wearing effect on the enamel of the teeth is so slight as to be scarcely perceptible, even after long continued usage. -Ed.] The following is a prescription that may be used daily with impunity : 
B Calcii carbon. præcip.............. $80.00 \ldots \ldots$ ii $\mathbf{3} \mathrm{v}$

Magnesii carbon.................. 3 iiss

Saponis pulv.,

Sodii bicarbon $\ldots \ldots \ldots \ldots \ldots \ldots \ldots \ldots . \bar{a} \bar{a} 50.00 \ldots \ldots \bar{\zeta}^{\mathrm{i}} \mathrm{z} \mathrm{v}$

Ol. menth. pip.,

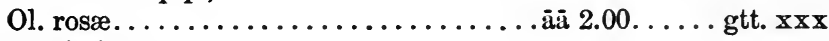

M. exactissime.

Sig.-Use daily.

As an example of a good soft soap may be given :

B Saponis pulv...................15.00.... $3_{\text {ss }}$

Iridis pulv $\ldots \ldots \ldots \ldots \ldots \ldots \ldots \ldots . . .10 .00 \ldots \ldots$ iiss

Spir. ol. caryoph (10 per cent.)..........4.00.... $3^{i}$

Aq. dest............................... s.

M. fiat pasta satis compacta. Dispens. in tubes.

[The use of iridis pulvis (orris root) should be discouraged, as it is very apt to accumulate in the carious teeth, and, being organic, decomposes rapidly, thereby causing the teeth to decay more quickly. Nearly all the newer tooth-powders contain the perborates or the peroxides and are ideal preparations for the mouth. They contain no organic material, and because of the ease with which they give up their available oxygen, not only serve as thorough disinfectants for the oral cavity, but also bleach the teeth. While all of these salts, calcium, sodium, magnesium, and strontium perborate, will act in an acid medium and liberate their oxygen, strontium perborate deserves special mention, as it acts in an alkaline medium. Since there are times, the early morning for instance, when the buccal secretions are alkaline, the immense value of this salt becomes apparent at once. The following tooth-powder is highly recommended for daily use :

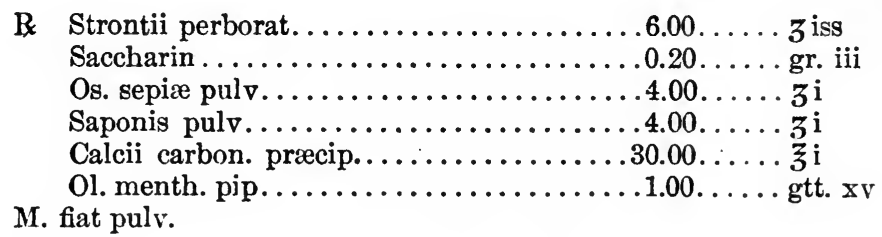

The soap known as Odontine is composed as follows:

B Saponis pulv., Magnesii carbon..............āā 15.00.... 3 iv

Sacchar. pulv.,

Acaciæ pulv................. āā 7.50.... z ii

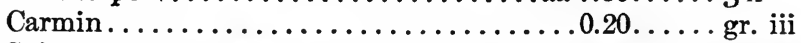

Spir. menth pip............................ $8 \nabla$

M. fiat pasta. Dispens. in porcelain boxes. 
A combination recently recommended is as follows:

B Thymol................................ iv \&

Ext. kramer. fluidi........................ $m$ xv

Glycerini............................. 3 iss

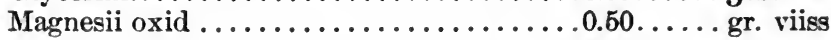

Sodii boratis........................00.... $3^{\mathrm{i}}$

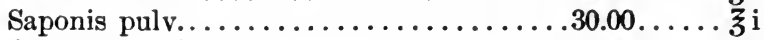

Ol. menth. pip....................

M. fiat pasta. Dispens. in tubes.

Mouth Washes.-The teeth may also be brushed with clear tepid or cold water, or, better, with the wash used at the same time for rinsing the mouth. As an example of a suitable mouth-wash may be given the one first mentioned on p. 230, or the following prescription of Miller, the taste of which is unfortunately not particularly pleasant:

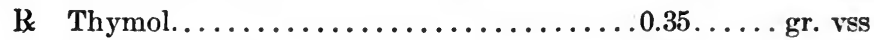

Acid. benzoici...................... $3.00 \ldots \ldots$ gr. xlv

Ol. eucalypti...................... $3.00 \ldots \ldots$ xlv

Alcoholis...................... $100.00 \ldots \ldots$ zii $z$ ii

M. fiat solut.

Ol. menth. pip.................gtt. xx..... gtt. $x x$

Sig.-A dessertspoonful in half a wineglassful of water (i.e., enough to make the water distinctly turbid).

It is well to add to this mouth-wash $0.8 \mathrm{G}$. (12 gr.) bichloride of mercury, or to replace the thymol by it, provided, of course, that the individual has not an intolerance for mercury.

Or we may choose the following combination, in which the author has added saccharin to another's prescription:

B Saccharin.,

Saloli........................ āa $1.00 \ldots \ldots$ gr. xv

Spir. menth. pip...................... 3 ii

Tinct. catechu....................

M. fiat mist.

Sig. $-\frac{1}{2}-1$ teaspoonful in half a glassful of tepid water.

A similar prescription, which the author has found to be good, although rather unpleasant to the taste, is as follows:

B Thymol.,

Saccharin.................. āa $2.00 \ldots \ldots$ ss

Tinct. catechu or myrrhæ,

Tinct. vanillæ................... āa $5.00 \ldots \ldots 3^{\mathrm{i}} \eta \mathrm{x} \times$

Ol. menth. pip...................... $3.00 \ldots \ldots m$ xlv

Ol. cinnamon........................... $m \times x v$

M. fiat mist.

Alcoholis..........................

Sig. $-\frac{1}{2}-1$ teaspoonful in a glassful of tepid water.

A good cheap mouth-wash, given by Seifert, may be made by dissolving 1 small teaspoonful of sodium bicarbonate in a pint of water, and adding 1 tablespoonful of spir. meliss. composit., G.P., or spirits of peppermint, or cologne, or simply a piece of camphor or a tablespoonful of 
spirits of camphor. A small amount of this mixture is diluted with water, half and half, each time. The disadvantage of this combination is its strongly alkaline reaction.

Finally, there may be mentioned Eau de Pierre, Eau de Botot, eucalyptus mouth-wash, and the new preparation, Odol, the antiseptic properties of which are, however, doubtful.

By the beginning of the third year every child's teeth should be brushed carefully at least once a day with soap or paste and his mouth be rinsed out. By the time he is six or seven, he should be able to brush his teeth himself and to gargle. Before he has learned to gargle he may chew pastilles or tablets containing disinfectant substances and spit them out again. Suitable tablets are the pastill. anginos. aseptic (see p. 277), or Hartmann's tablets, composed as follows:

B Sachar........................

Saccharin.................. 0.30-0.35.....gr. iv-gr. $\mathbf{v}$

Alcoholis,

Thymol......................āā $0.20 \ldots$. gr. iii

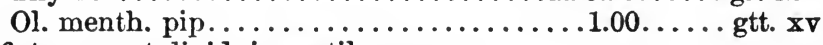

M. fiat mass. et divid. in pastil. no. c.

Sig.-Several tablets to be chewed daily.

Szana uses a pleasant form of saccharin, $0.1 \mathrm{G}$. (11/2 gr.), with an equal amount of resina guaiaci, worked up with sugar into little bonbons, which may be held in the mouth until dissolved. Dr. Labouschin-Jenner's pastilles are similarly composed.

It need hardly be mentioned that a carious tooth, even in a child, should receive prompt and competent attention.

Under Pathological Conditions.- - It is even more important to keep the mouth clean under pathological than under physiological conditions. If this is neglected, serious or even fatal complications may spread from the mouth to other organs. This is especially true in the case of unconscious cachectic patients, or those with high fever (especially typhoid). The danger of pathological processes in such cases is due to diminished mastication, reduced salivary secretion, and favorable conditions for local irritation (bad teeth, harboring bacteria).

Cleansing the Mouth.-Another important reason for caring for the mouth is the fact that if it is kept clean, fever patients take nourishment much more readily. The mouths of all such patients should be washed out with a wet swab every 2 or 3 hours, and sufficient pressure be exerted on the surface of the tongue to give rise to choking movements, which will take the place of the ordinary movements of mastication. In severe fevers (e.g., typhoid), and also in other diseases (e.g., cirrhosis of the liver), where the tongue begins to get dry and there is danger of fissures or of a fuliginous deposit, the tongue should be painted every 2 hours, or even every hour, with pure glycerin or with a 1:5 solution of glycerite of boro-glycerin or syrup of hypophosphites, so much being used that part will be forced by swallowing movements from the surface 
of the tongue to the entrance of the larynx and pharynx. Such patients should be given water, weak lemonade, or milk at least every hour. If voluntary swallowing is impossible, repeated efforts should be made to induce involuntary swallowing movements by pressing down the tongue with a spoon.

If caries of the teeth is already present, Miller's mouth-wash with the addition of bichloride (p. 269), or the second prescription given on p. 230 , may be recommended.

Cracked Lips.-For cracked lips, especially in fever, lanolin, cold cream, or the white lip pomade (unguentum aquæ rosæ or ceratum cetacei) may be employed. Glycerin, although frequently used, has the disadvantage of drying up the tissues. If these precautions are taken, even the severest case of typhoid will be spared a fissured tongue, catarrhal stomatitis, or sores in the mouth. [Henna has recently called my attention to the efficacy of chewing gum as an aid in the care of typhoid patients' mouths.-Ed.]

\section{THERAPY OF STOMATITIS.}

Therapy of Catarrhal Stomatitis.-The treatment of catarrhal stomatitis is relatively simple. If possible, the first thing to be done is to eliminate the cause (too hot, too cold, or strongly spiced food, sharp or decayed teeth, badly fitted false teeth, and poisoning by mercury, iodine, bromine, arsenic, lead, chlorine, or air saturated with sulphuric acid).

If the stomatitis is simply a local symptom of a general infection, as in measles, scarlet fever, influenza, or smallpox, or if it is caused wholly by some other disease, as in typhoid, meningitis, or catarrh of the gastrointestinal tract, our first care must be to cleanse and disinfect the mouth. Every 2 hours it should be rinsed out with pure water, or with an alkaline, astringent, or antiseptic solution (e.g., a 1-2 per cent. solution of potassium chlorate, a 3 per cent. solution of sodium bicarbonate, or a 1-4 per cent. solution of boric acid). For antiseptic mouth-washes see p. 230 .

Therapy of Ulcerative Stomatitis.-In ulcerative stomatitis the therapy is the same, except that we also employ local treatment, such as cauterization by means of a lunar caustic or chloride of zinc pencil, by thermocautery, or by painting with glycerite of boro-glycerin, potassium chlorate glycerin $(1: 2)$, or salicylic acid and cocaine, as in the following prescription :

R Acid. salicylici.....................2.00... $3^{\text {ss }}$

Alcoholis................................. q. s. ad. sol.

Cocainæ hydrochloratis. . . . . . . . . . . $0.30 \ldots \ldots$ gr. ivss

Aq. dest.,

Glycerini ....................āa $15.00 \ldots \ldots \bar{z}$ ss

M. fiat mist.

Sig.-For painting. 
Therapy of Aphthous Stomatitis and of Herpes Buccalis.Aphthous stomatitis and herpes buccalis receive similar treatment, especially burning with lunar caustic and painting with boric or salicylic acid glycerin, or a 1-15 per cent. borax solution. In herpes a $1 /{ }_{20}-1 / 10$ per cent. solution of potassium permanganate may also be used. In aphthous stomatitis potassium chlorate is painful and must be avoided; a good mouth-wash in such cases is simple tepid water or hot tea made from camomilla, althæa, or malva, sometimes with a few drops of the tincture of opium added, or from malva or camomilla with an equal quantity of coca leaves.

Therapy of Mercurial Stomatitis: Prevention.-Mercurial stomatitis deserves especial mention. To avoid it we must take the very greatest care of the mouth during the administration of mercury. The teeth should be brushed 2 or 3 times a day with soap or paste, and the mouth should be washed out every hour, particularly with an astringent solution to harden the gums. The following prescription is useful:

R Ext. krameriæ fluidi..................4.00... $z^{\mathbf{i}}$

Tinct. myrrhæ.......................

Alcoholis.......................... 30.00 iii

Sig. $-\frac{1}{2}$ teaspoonful in a cupful of water.

Before beginning any mercurial treatment, carious teeth should be removed, irritating sharp points of teeth be smoothed off, and any lesion in the mouth allowed to heal. During the treatment, smoking and the use of hot or highly spiced food must be wholly avoided.

Treatment.-In case mercurial stomatitis is actually present, we have recourse to two drugs, potassium chlorate and hydrogen peroxide, the latter being often quickly effective where the former apparently is of no use. Potassium chlorate as a mouth-wash is used in a 2-3 per cent. solution. [An excellent mouth-wash in use at the New York Hospital in mercurial stomatitis is the following:

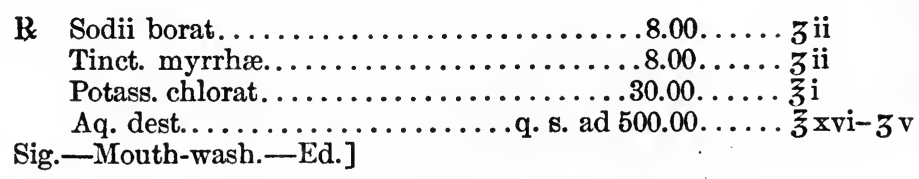

Unna recommends a 50 per cent. potassium chlorate tooth paste, put up by Beiersdorf \& Company, or pure potassium chlorate. If the latter is put dry upon the tooth-brush, a paste is soon formed by the increased secretion of saliva and mucus induced. The mouth should be rinsed afterwards, but even then a salt taste will remain for a considerable time. The fetid odor vanishes immediately. The author often prefers hydrogen peroxide [or perborate of calcium, 2 per cent.-Ed.] at least as a mouth-wash. The peroxide may be used in the ordinary 10 per cent. solution in which it usually comes, but it is generally diluted to a 2 per 
cent. solution. Its effect is due to its extraordinary antiseptic properties. Even a $1: 20,000$ solution of peroxide will check the growth of the bacteria of fermentation.

Treatment of Ulcers in Mercurial Stomatitis.-If local ulcers develop in mercurial stomatitis, they should, after being treated with cocaine, be touched with the tincture of iodine or with lunar caustic, or should be wiped off with bits of cotton saturated with pure hydrobromic acid, or with a concentrated aqueous solution of chromic acid. [I prefer the last named.-Ed.]

Therapy of Thrush: Conditions Under Which It Arises.-We have already mentioned the necessity for taking proper prophylactic precautions against thrush in children, especially if the digestion is disordered, and in adults with severe fevers, cachexia, or a disturbed sensorium. If the condition arises we use a local application of borax (5-10 per cent. solution), potassium permanganate ( 1 per cent. solution), or potassium chlorate (1-3 per cent. solution), without sugar or syrup. A cloth wet in one of these is wrapped around the finger and each spot of thrush is carefully rubbed off. Any slight bleeding which results may be neglected. Whenever a new patch is seen, the process is repeated. A mouth-wash of a 5-10 per cent. solution of sodium borate is advisable. Many authors recommend painting the patches with bichloride of mercury $(1: 10,000)$. If the thrush has spread to the œsophagus, we give resorcin ( $1 / 2-1$ per cent.) internally ( 1 small teaspoonful every hourBaginsky). Aufrecht recommends for adults the internal use of a 3 per cent. solution of sodium borate. [The routine treatment at the Sloane Maternity Hospital is to wash out the baby's mouth with 20 grains of bicarbonate of soda to the ounce of water before and after nursing, and once a day with a 2 per cent. solution of nitrate of silver. Holt recommends a 1 per cent. solution of formalin.-Ed.]

\section{THERAPY OF DISEASES OF THE NOSE AND THROAT.}

Almost all diseases of the nose and throat fall to the care of the specialist, not to that of the general practitioner. There are, however, two conditions which the latter is so often called upon to treat that they will be discussed here, acute rhinitis, and simple catarrhal pharyngitis and follicular tonsillitis.

\section{THERAPY OF ACUTE RHINITIS.}

Etiology.--Rhinitis may be caused by (1) taking cold, (2) toxic influences, (3) a purely mechanical irritation, (4) nervous causes (coryza vasomotoria). If we are able to attack the disease within a few hours, especially if it is caused by catching cold, we try to avert the coryza; if not, our therapy is merely symptomatic.

Averting the Coryza.- To avert the coryza, we use hot foot-baths, particularly mustard baths, $50-100 \mathrm{G}$. $\left(1 \frac{1}{2}-3 \mathrm{oz}\right.$.) of mustard flour in a 
foot-bath, and administer internally some diaphoretic. A favorite prescription is composed of equal parts of linden flowers and elder flowers in the form of a tea (a teaspoonful to a cup of water). Many authors recommend sodium salicylate, salophen, aspirin, or salipyrin. Mustard plasters may also be used on the neck, the patient going to bed as soon as they are applied. As a local measure we may use the following:

B Menthol................... $0.50-1.00 \ldots \ldots$ gr. viiss $-\mathrm{gr} . \mathbf{x \nabla}$

Chloroform. or $æ$ ther..............

M. fiat solut.

Sig. $-4-6$ drops to be rubbed on palm of hand, and the resulting vapors to be inhaled repeatedly.

This process is to be repeated 2-3 times in the first 12 hours. A favorite remedy is Hager-Brandt's prescription, as follows:

B. Phenol liquefact.,

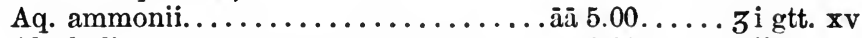

Alcoholis.........................

Aq. dest........................ $15.00 \ldots \mathrm{ss}$

M. fiat mist.

Sig.-4-5 drops on blotting-paper, inhaled for a few seconds every hour or half hour, or the open bottle to be sniffed every hour.

Or the following powder may be snuffed every hour. This is oftener used after the development of the coryza:

B Menthol............................ $0.30 \ldots$ gr. ivss

Cocainæ hydrochlor................. $0.40 \ldots \ldots$ gr. vi

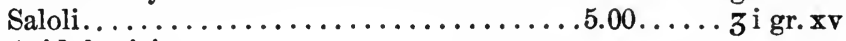

Acid. borici,

Coffeæ tostæ..................āä 10.... 3 iiss

Misce subtil. pulv.

Sig.-To be snuffed up nose.

In the same way we may snuff up calomel or the following mixture:

B Cocainæ hydrochlor................ $0.30 \ldots \ldots$ gr. ivss

Iodol.,

Glucose....................āa $2.50 \ldots \ldots$ gr. $x \times x x$

M. fiat pulv.

Formalin, as formalin cotton, is worth a trial. [ $\mathrm{I}$ regard the use of formalin as injurious for the eyes.-Ed.] Five to ten drops of the tincture of opium (sometimes repeated), potassium bromide, atropine, and alcohol in large quantities internally, have all been recommended, but the author puts no great faith in any of these remedies for averting coryza, unless the rhinitis be of a vasomotor origin. The old method of giving very little liquid he believes to be helpful. [Lactate of calcium in $0.65-1.30 \mathrm{G}$. (10-20 gr.) doses is useful in vasomotor coryza.-Ed.] 
Symptomatic Therapy.-If the cold has lasted at least a day, we treat it symptomatically. Cocaine will almost immediately relieve the congestion of the veins of the nasal membrane, allow the free passage of air again, and mitigate coincident headache. A 1-2 per cent. solution may be sprayed with an atomizer or inhaled, or cocaine (in the same concentration) may be snuffed as a powder. In the latter case, it may be mixed with gum arabic, or the following prescription may be used:

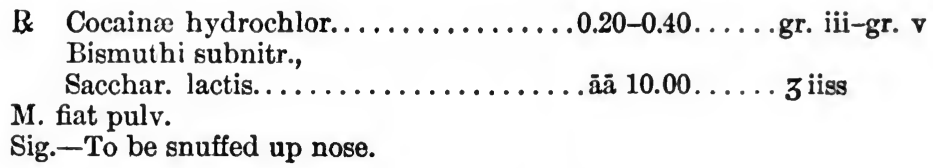

Adrenalin $(1: 1000)$ has an effect similar to that of cocaine and is usually given with it. Quinine is also recommended (every 2-3 hours or three times a day when the symptoms have subsided). It may be used in an atomizer, snuffed up, or dropped into the nose.

In case of extreme dryness of the inflamed mucous membrane of the nose and nasal passages, inhalation of steam, with or without the addition of salt to the water, brings great relief. The congestion may also be helped by the use of cathartics.

In case of neuralgic headaches from involvement of the trigeminus, we may use $0.50-1 \mathrm{G}$. (7-15 gr.) of phenacetin 1-3 times a day, or sometimes antipyrine, alone or with cocaine hydrochlorate, as in the following prescription :

B Antipyrin......................... vii

Cocainæ hydrochlor..............0.02-0.03.....gr. $\frac{1}{3}-$ gr. $\frac{1}{2}$

M. fiat pulv. no. i. Dent. tal. dos. no. iii.

Sig. -3 powders daily.

In case of overabundant secretion, the stoppage of the nose may be relieved by applying Pollitzer's nasal air douche to one nostril, the other being kept open. Syringing of the nose should be avoided, on account of the danger of carrying infectious material to the ear and setting up an otitis.

Soreness of the nostrils and upper lip should be guarded against by using vaseline as soon as the cold begins; vaseline or lanolin, with or without the addition of cocaine (eucaine), should also be applied to the interior of the nose to allay the inflammation.

Coryza Vasomotoria.-In coryza vasomotoria the treatment of the nasal cavities with 1-2 per cent. cocaine solution followed by a snuff of $0.20-0.50$ G. (3-7 gr.) of menthol to $10 \mathrm{G}$. (21/2 dr.) of dermatol, has been recommended. This must be accompanied, of course, by all the usual methods of treatment for the nervous condition. The author has known of one case of recurring vasomotor coryza in which the nasal secretion showed a great abundance of eosinophilic cells, which was made to disappear by the use of atropine internally. 


\section{THERAPY OF ACUTE CATARRHAL PHARYNGITIS AND TONSILLITIS.}

Therapy of Pharyngitis.-A simple primary catarrhal pharyngitis demands only very simple treatment. The throat should be wrapped in a cloth wrung out in boiling water (Priessnitz's application). This may be covered with oiled silk, rubber, or oiled paper, with a bandage to keep it in place; or it may simply be covered with a piece of flannel, in which case it must be taken off and wet again as soon as it becomes dry.

The throat should be gargled with some mild alkaline solution; e.g., a 1 per cent. solution of potassium chlorate or alum (1 teaspoonful to a pint of water), limewater pure or diluted, or a 1 per cent. solution of sodium bicarbonate. The latter may also be used for inhalation. If there is great difficulty in swallowing, $2 \mathrm{G}$. (30 min.) of laudanum may be added to a pint of the gargle. Avellis recommends the following mixture of cocaine and antipyrine:

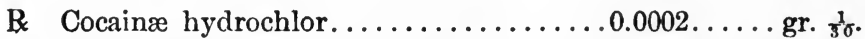

Antipyrinæ................. . iii

Sacchar. lactis.

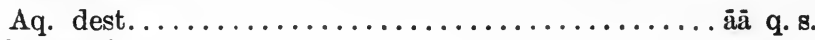

M. fiat pastil.

Dent. tal. dos. no. xl.

Sig.-3-4 pastilles twice a day, to be held in the mouth until dissolved.

Difficulty in swallowing may also be helped by cold applications and by lumps of ice held in the mouth until dissolved.

If there are indications of a peritonsillar abscess, B. Fränkel recommends the internal use of guaiac. It should also be employed in tonsillitis, under similar conditions, to check the purulent processes. It is prescribed as follows:

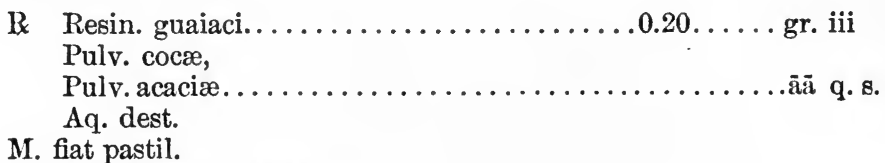

Dent. tal. dos. no. $\mathbf{x x x}$.

Sig. -1 pastille every 2 hours.

In such cases, ice and cold gargles usually increase the pain in the throat. Hot gargles (e.g., sage or mallow tea, sometimes with the addition of coca leaves) are more grateful. The diet must be light, smoking must be given up, as in rhinitis, the bowels must be kept open, and if the attack is at all severe the patient must be kept in bed.

If the pharyngitis runs a protracted course, it is well to use a gargle made of equal parts of tepid milk and water, or of milk and a tea made by adding to a cup of water a tablespoonful of a mixture of sage and 
mallow, to which may be added $1 \mathrm{G}$. (15 min.) of laudanum, in case there is considerable pain on swallowing.

Therapy of Tonsillitis.-The general therapy of tonsillitis consists usually in the administration of quinine. Fränkel recommends at least 1 G. (15 gr.) of quininæ hydrochloras in 24 hours. In most cases the discase ends after 1-3 days with a crisis, characterized by profuse sweating. Locally we may use gargles and compresses, or, if the attack is of a severe type, a daily intratonsillar injection of 10-20 min. of a warm 2-3 per cent. carbolic acid solution. The instrument used is a Taube syringe, differing from a Pravaz syringe merely in having a ring around the cannula $1 / 4-1 / 2 \mathrm{~cm}$. from the end, to prevent its penetrating farther into the tonsil. The process is brought to a standstill after 1-3 injections. This procedure is strongly recommended by Ziemssen, and the author has had an extensive and successful experience with it.

Other authorities paint the parts with $a^{1 / 10}$ per cent. solution of corrosive sublimate:

B Hydrarg. chlor. corros..................... iv

Acid. tartarici $\ldots \ldots \ldots \ldots \ldots \ldots \ldots \ldots, 0.05 \ldots \ldots$ gr. $\frac{3}{4}$

M. fiat mist.

Aq. dest....................... $250.00 \ldots$ viii 3 iiss

Sig.-Poison-To be used only by the physician.

Dr. Bergmann's pastillæ anginosæ asepticæ appear to be of some use in both pharyngitis and tonsillitis. Each one contains $0.002 \mathrm{G}$. $(1 / 30 \mathrm{gr}$.) of thymol, $0.02 \mathrm{G}$. ( $1 / 3 \mathrm{gr}$.) of sodium benzoate, and $0.015 \mathrm{G}$. ( $1 / 4$ gr.) of saccharin, with an indifferent mass. They are chewed for 10-15 minutes, until their taste has disappeared, and then spit out. Three to 6 pastilles a day may be used. The abundant saliva secreted on account of the prolonged chewing, carries the effective principles of the pastilles to every part of the tonsils and mucous membrane of the throat. 


\section{THERAPY OF DISEASES OF THE LARYNX}

DISEASES of the larynx, like those of the nose and throat, do not, with a few exceptions, belong to the domain of internal medicine. These exceptions, which alone will be treated here, are the following: Acute laryngitis, pseudocroup, and, up to a certain grade, tuberculosis of the larynx. To these may be added laryngeal spasm, especially as the attack demands symptomatic treatment, which is not affected by the varied etiology of the disease.

\section{THERAPY OF ACUTE LARYNGITIS.}

Avoidance of All Irritation to the Larynx.-In acute laryngitis, the larynx must be spared all irritation that it is possible to avoid. Talking, smoking, and alcoholic drinks must be wholly given up. The air breathed must be pure, at an even, moderate temperature $\left(70^{\circ}-73^{\circ}\right)$, and be kept moist by steam from boiling water or by water vapor from cloths wrung out in hot water and hung up.

Sweating.-If the patient has caught cold, he should be given some sort of hot tea or other diaphoretic and be put immediately to bed, in order to bring on a good sweat. This procedure is indicated in every case accompanied by general constitutional symptoms.

Severe Cough: Narcotics Internally.-A severe cough demands the use of narcotics (morphine, codeine, opium), in frequent small doses following an initial larger dose; e.g. :

R Morphinæ hydrochlor...................... iss

Aq. amygdal. amaræ................. 3 iiss

M. fiat solut.

Sig.-At first 20-30 drops, then 5-10 drops every other hour.

Or :

R Codeinæ phosphatis........................... ii

Aq. amygdal. amaræ................. 3 iiss

M. fiat solut.

Sig.-At first 20-30 drops, then 10-15 drops every hour.

Or :

B Pulv.ipecac. et opii....................... iss

Sacchar. lactis . . . . . . . . . . . . . . . $0.20 \ldots \ldots$

M. dent. tal. dos. no. xxv.

Sig. -1 powder in wafer every 2 hours. 
We may also give hourly doses of 2-3 drops of the tincture of opium, peronine, heroine, or dionine (see p. 285).

Inhalation of Sedatives.-The cough, pain in swallowing, and painful sensations in the larynx may also be relieved by the inhalation of sedatives (see p. 281).

Dryness and Burning in the Larynx.-In case of dryness and burning in the larynx, with difficulty in swallowing, the inhalation of an alkaline solution is beneficial (a 1-2 per cent. solution of sodium bicarbonate or common salt, or an alkaline mineral water, Seltzer, or, especially, Ems). If severe pain is present, 10-20 drops of laudanum may be added before each inhalation. The effect of the inhalation combines that of hot steam with that of bicarbonate of soda, which, at least according to most authorities, dissolves the collected mucus, and, according to Virchow, increases the activity of the ciliated epithelium, thus forcing up the mucus.

Excessive Secretion.-If the laryngitis is accompanied by an abundant secretion and shows a tendency to become chronic, the ethereal oils are indicated. They may be inhaled by means of a Bulling's (not a Siegle's) apparatus, [I usually recommend a Maw's inhaler or an ordinary pitcher.-Ed.] or 5 drops of oil of turpentine, oil of juniper, oil of eucalyptus, or a mixture of any two of them, may be dropped into a basin of boiling water, and the steam be inhaled, the patient's head and the basin being covered with a towel. The amount of oil may be increased a drop a day until it reaches 20 drops if necessary. Its effect is due to its decreasing the secretion (see p. 300). We may prescribe as follows :

B Ol. terebinth. rect.

Ol. juniperi. āā $15.00 \ldots . .3$ iv

M. fiat mist.

Sig.-For inbalation.

Or :

B Ol. terebinth. rect.,

Terebinth. canadens............ āā 15.00.... 3 iv

M. fiat mist.

Sig.-For inhalation.

Or :

B Terebinth. canadens...................

Ol. eucalypti..................... $1.00-1.50 \ldots \ldots$ gtt. $\mathbf{x} \nabla-g t t . \times x$

M. fiat mist.

Sig.-For inhalation.

A teaspoonful of the tincture of benzoin may be used in the same way. Externally we may apply hot compresses, and internally try potassium iodide to liquefy the mucous secretion. It is not necessary to treat the larynx locally.

Protection from Recurrence.-To protect against constantly recurring laryngitis, all irritation (dust, chemically irritating gases and 
vapors, changes from warm to cold air) must be as far as possible avoided. If these are not the causes, the laryngitis returning whenever the patient takes a slight cold, a hardening process is in order (see p. 255).

\section{THERAPY OF PSEUDOCROUP.}

Therapy of a Single Attack.-Pseudocroup is essentially a disease of infancy and childhood, but is occasionally observed in adults. The treatment of an attack is very simple. At the first sign of choking, the patient is given a hot drink (tea, milk, or alkaline mineral water, or Ems), and is made to inhale a 1-2 per cent. salt solution, a sodium bicarbonate solution, or Ems water. A mustard plaster is placed on the neck or the laryngeal region. If the cough is very distressing, codeine or heroine is given. In case the attack is prolonged and a good deal of mucus has collected in the upper air passages, an emetic is indicated; e.g., apomorphine, or according to Liebermeister :

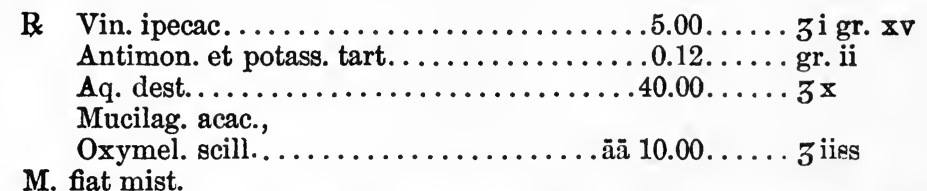

Sig. -1 teaspoonful every 5 minutes, followed by hot tea till vomiting occurs.

Therapy Between Attacks.-Between attacks the continued use of inhalations and the internal administration of our so-called resolvent expectorants (ipecacuanha, ammonium chlorate, apomorphine, liquor ammonii anisati) are indicated. [Forchheimer attributes spasmodic or false croup to diminished inhibition. For children he prescribes large doses of sodium or potassium bromide during the attack and advises its continuance in smaller doses the following day to prevent a recurrence. For adults he usually adds chloral. Holt recommends a tablet triturate of antimony and ipecac $0.0006 \mathrm{G}$. $(1 / 100 \mathrm{gr}$.) each. To a child of 2 years 1 tablet may be given every 10 or 15 minutes, until free vomiting occurs. -Ed.]

\section{THERAPY OF TUBERCULOSIS OF THE LARYNX.}

The local treatment of tuberculosis of the larnyx belongs to the laryngologist. But the general practitioner is sometimes obliged to treat cases of laryngeal tuberculosis accompanying severe pulmonary tuberculosis, which have reached such an advanced stage that direct local treatment is impossible. In such cases, where, in general, tubercular abscesses are present in the larynx, we use inhalations of ethereal oils (see p. 279), tincture of benzoin, tincture of myrrh (10-15 drops), balsam of Peru, $10 \mathrm{G}$. (21/2 dr.), with $5 \mathrm{G}$. (1 $1 / 4 \mathrm{dr}$.) spirits of wine (10-20 drops 3 times a day), 1-5 per cent. carbolic acid, 1 per cent. lysol or 
creolin, or 2-4 per cent. boric acid. If there is severe cough, or pain on swallowing, we may add 10-20 drops of the tincture of opium, or use the following prescription:

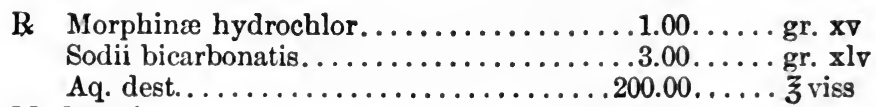

M. fiat mist.

Sig.-For inhalation several times a day.

Pain in swallowing may also often be relieved by sucking bits of ice or by the insufflation of the following powder by means of an atomizer:

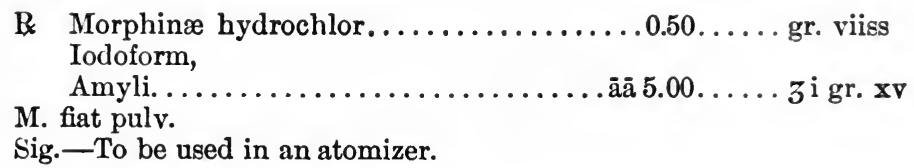

\section{THERAPY OF THE LARYNGEAL SPASM.}

Varied Etiology.-Laryngeal spasms occur much more frequently in children than in adults. They may be caused by (1) a local inflammation of the larynx, catarrhal or otherwise, (2) an organic lesion of the central nervous system or the peripheral vagus, (3) a functional disturbance of the central nervous system, (4) reflex action arising from the nose, ears, digestive tract, or genitals, (5) rickets, or (6) especially craniotabes.

Therapy During an Attack.-The rapidity with which an attack comes and goes makes possible only external measures that can be instantly applied. The windows should be opened, the clothing be loosened, a mustard plaster be put on the neck or breast and epigastrium, a sponge squeezed out in hot water applied to the neck, a vinegar enema be given (1-2 tablespoonfuls to $60 \mathrm{G}$. (2 oz.) for a child, 2-5 tablespoonfuls to $200 \mathrm{G}$. (6 oz.) for an adult), and the body be sprinkled with cold water. The patient should breathe wholly through the nose, his mouth being held shut if necessary. If ether is used it should be inhaled through the nose. If the attack is hysterical we may try compressing the phrenic nerve in the neck between the insertions of the sternocleidomastoid muscle.

In case of a second attack, or a series of attacks, the author has used chloroform narcosis, or, much oftener, an enema of chloral hydrate and potassium bromide, prescribed for children as follows :

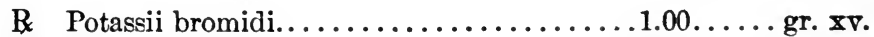

Chloral. hydrat.................... $0.50 \ldots \ldots$ gr. viiss

Mucilag. acac...................50.00.... 3 iss

M. fiat mist.

Sig.-For an enema. 
Therapy Between Attacks.-In the intervals between attacks, potassium bromide (up to $2 \mathrm{G}$. ( $30 \mathrm{gr}$.) daily for children, $10 \mathrm{G}$. (21/2 dr.) for adults), the tincture of valerian, the tinctura castorei, codeine, morphine, belladonna, arsenic, and sometimes chloral may be employed to lessen the abnormal reflex irritability and so to avert the recurrence of an attack. 


\section{VII.}

\section{THERAPY OF DISEASES OF THE BRONCHI}

Tracheitis, the only disease of the trachea which belongs to the domain of internal medicine, can be discussed at the same time as bronchitis, so that we may pass directly from diseases of the larynx to those of the bronchi.

\section{THERAPY OF ACUTE BRONCHITIS.}

Acute inflammatory affections of the bronchi may be classified as follows :

1. Acute inflammation of the large bronchi (almost always associated with inflammation of the trachea): Acute tracheobronchitis.

2. Acute inflammation of the small bronchi :

(a) Of the smaller bronchi : Acute deep bronchitis.

(b) Of the smallest bronchi : Acute capillary bronchitis.

\section{THERAPY OF ACUTE TRACHEOBRONCHITIS.}

EnERgetic Diaphoresis.-If the attack is already under way, we resort first to diaphoretic measures, in the hope of breaking it up. The patient should be put to bed and be given large quantities of some hot drink. Many practitioners, the author included, use alcoholic drinks, believing that they cause an active flow of blood to the skin, which aids an abundant secretion of sweat. We may give two cups of Russian tea with a good deal of rum or brandy, or 2-3 glasses of mulled wine (red wine diluted and heated with sugar and cinnamon), or punch (hot water, brandy, sugar, and a slice of lemon). Other physicians employ special herb-teas which have been supposed to be peculiarly diaphoretic in their action, but whose effect, according to modern pharmacology, is probably due only to the hot water used in their preparation; e.g., elderberry flowers, linden flowers, orange flowers, alone or in combination (1 tablespoonful to 2 cups of tea). Still other authorities prefer hot milk with an equal quantity of an alkaline-chloride water (Gleichenberg, Ems, or Seltzer). The internal use of diaphoretics (e.g., sodium salicylate, phenacetin, folia jaborandi, or pilocarpine subcutaneously) is not so effective. A steam bath may be tried, provided the patient has no fever or constitutional disturbances. The greatest precautions must be taken to prevent his catching cold afterward. As soon as the sweating is over, he should be rubbed dry without being uncovered and then transferred to another bed, previously warmed. If this is impracticable, the under 
sheet should be replaced by a fresh, warmed one, the night-shirt be changed, and the patient be covered with a warm blanket. A cathartic (castor oil, magnesia), or an enema is also employed at the beginning of the sweating process.

Symptomatic Therapy: Inhalations.-If these measures do not succeed in breaking up the attack, we must depend upon symptomatic treatment. If. the mucous membrane is very dry and swollen, the air should be kept moist and the patient should inhale steam directly. The author uses Siegle's apparatus (or, better, Bulling's) 3 or 4 times a day, adding to the water salt, bicarbonate of soda, or an alkaline mineral water (Ems or Seltzer). The cough may be combated at the same time by adding to the liquid for inhalation some sedative (e.g., the tincture of opium (10-20 drops), morphine $0.10-0.30 \mathrm{G}$. ( $1 \mathrm{I} / 2-4 \mathrm{~L} / 2$ gr.), cocaine or eucaine $0.50-1 \mathrm{G}$. (7-15 gr.), or the extract of belladonna $0.30-1 \mathrm{G}$. (5-15 gr.).

Internal Use of Mineral Waters.-Mineral waters are also used internally, a half glass being added to the same amount of hot milk and taken 3-4 times a day. Their exact action is not clearly understood. Neither the theory that a small amount is excreted through the bronchial mucous membrane, thus liquifying the thick mucus, nor the theory that the bicarbonate of soda increases the vibratory action of the ciliated epithelial cells, appears well founded.

Treatment of the Cough: Narcotics.-It is exceedingly important in dry bronchitis to quiet the cough as much as possible. A number of sedatives are available. We may use opium, morphine, codeine, or the morphine derivatives, peronine, heroine, and dionine; or belladonna, hyoscyamus, or stramonium. The effect of the first group is due to their sedative influence on the central nervous system and in part to their lowering of its reflex irritability. They also, especially opium, appear to decrease the secretion of the bronchial mucous membrane. The second group, however, produce a greater effect on the secretion and also decrease the reflex irritability. It is well, therefore, to combine opium, or one of its derivatives, with belladonna, hyoscyamus, or stramonium. In giving the dosage for all the following sedatives, it is presupposed that the treatment is to be kept up all night.

We prescribe morphine or codeine as in acute laryngitis (see p. 278), or :

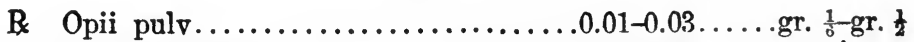

Sacchar. lactis..........................0.40....gr. vi

Dent. tal. dos. no. xx.

Or:

Sig. -1 powder every 2 hours.

R Opii pulv., or

Ext. opii........................... $0.30 \ldots \ldots$ gr. ivss

Pulv. ext. glycyrrh .............................. s.

M. fiat pil. no. XXx. Dust with lycopodium.

Sig. -1 pill every 2 hours. 
Or: B Morphinæ hydrochloratis.........0.003-0.005.....gr. $\frac{1}{20}-\mathrm{gr} . \frac{1}{12}$

Sacchar. lactis, or

Pulv. acac. comp. (N. F.) $0.40 \ldots . . . g r$. vi Dent. tal. dos. no. xxx.

Sig.-Take up to 12 powders a day.

Or :

B. Morphinæ hydrochloratis............0.02-0.05.....gr. 1-gr.

Aq. dest........................180.00.... 3 vi

Syr. acaciæ ....................... $20.00 \ldots \ldots \mathrm{v}$

M. fiat. mist.

Or :

Sig. -1 tablespoonful every 2 hours.

B Codeinæ phosphatis................0.05-0.02.....gr. $\frac{3}{4}$-gr. $\frac{1}{3}$

Emuls. amygdal...................180.00.... 3 vi

Or:

Sig. -1 tablespoonful every 2 hours. (Easily decomposed.)

B Codeinæ phosphatis or hydrochloratis.......0.02....gr. $\frac{1}{3}$

Sacchar. lactis.......................0.50.....gr. viiss

Dent. tal. dos. no. $\mathbf{x x x}$.

Or :

Sig. -1 powder every $2-3$ hours.

B Codeinæ phosphatis....................0.30.....gr. ivss

Ext. gentianæ $\ldots \ldots \ldots \ldots \ldots \ldots \ldots \ldots \ldots \ldots \ldots \ldots$. s.

M. fiat mass. Div. in pil. no. xxx.

Sig. -1 pill every 2 hours. tion:

We may also use fresh solutions of codeine for subcutaneous injec-

Ix Codeinæ phosphatis .................. 0.50....gr. viiss

Aq. dest.......................... $30.00 \ldots$ iiss

Sig. -5-20 min. subcutaneously.

Peronine may be given as follows:

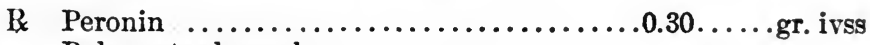

Pulv. ext. glycyrrh.......................... s.

M. fiat. mass. Div. in pil. no. lx.

Or :

Sig. $-6-12$ pills daily.

R Peronin..........................0.04-0.05.....gr. $\frac{2}{3}$-gr. $\frac{5}{6}$

Alcoholis...........................5.00..... 3 i $m \times v$

Aq. dest......................180.00.... हु vi

Syr. pulv. acac. co. (N. F.) ..............15.00.... 3 iv

I. fiat mist.

Sig. -1 tablespoonful every 2 hours.

Heroine (the diacetic acid ester of morphine, soluble in water only after the addition of an acid), heroine hydrochlorate (soluble in water), and dionine (hydrochlorate morphine-ethyl-ester), are used much more than is peronine. They are prescribed as follows:

R Heroin.......................... $0.005 \ldots \ldots$ gr. $\frac{1}{12}$

Pulv. acac. comp......................... 0.40 . vi

M. fiat pulv. Dent. tal. dos. no. xxx.

Sig. -1 powder every 3 hours (up to 0.02 G. (gr. $\frac{1}{3}$ ) a day). 
Or: $\quad$ R Heroin. hydrochloratis.............. 0.10-0.15..... gr. iss-gr. iiss

Aq. amygdal. amar................... $10.00 \ldots \ldots 3$ iiss

Or :

Sig. $-10-15$ drops 3 to 4 times a day.

B Dionin...........................0.03-0.04.....gr. 光-gr. $\frac{7}{8}$

Aq. dest.....................................

Syr. acac. comp. (N. F.)...............15.00.....

Or :

Sig. -1 tablespoonful every 2 hours.

Or :

R Dionin ............................. 0.30.....gr. ivss

Aq. amygdal. amar....................10.00.... 3 iiss

Sig. -10 drops 3 times a day.

R Dionin.............................. 0.30....gr. ivss

Pulv. ext. glycyrrh.............................. s.

Or:

M. fiat pil. no. Xxx.

Sig. $-3-4$ pills daily.

B Dionin............................. $0.30 \ldots \ldots$. gr. ivss

Aq. dest. ........................... $10.00 \ldots \ldots$ iiss

Sig. -20 min. subcutaneously.

Heroine and dionine appear to be about equal to codeine in their effect, being superior to peronine and inferior to morphine. Both lessen the cough, slow the respiration, and act as sedatives rather than as strong narcotics like morphine.

If we wish to use one of the second group of narcotics, as we do especially when the bronchitis is accompanied by abundant secretion and torturing cough, we may prescribe as follows:

Belladonna: 1. Powdered leaves, 0.20 G. ( 3 gr.) per dose, $0.60 \mathrm{G}$. (9 gr.) per day.

2. Powdered root, 0.07 G. ( $1 \mathrm{gr}$.$) per dose, 0.30$ G. ( 5 gr.) per day.

3. Extract of the leaves, 0.05 G. ( $\frac{3}{4}$ gr. $)$ per dose, 0.20 G. ( 3 gr.) per day. (Much oftener used. The only practical form when belladonna is to be given in a fluid mixture.)

Hyoscyamus: 1. Extract of the leaves, maximum dose, $0.10 \mathrm{G}$. (1 $1 \frac{1}{2}$ gr.), 0.50 G. ( 7 gr.) a day.

Stramonium Leaves : 1. Fluidextract, maximum dose, 00.5 G. ( $\left.\frac{3}{4} \mathrm{gr}.\right)$, 0.15 G. (21 gr.) a day.

2. Dry or duplex extract, maximum dose, $0.025 \mathrm{G}$. ( $\left(\frac{8}{8} \mathrm{gr}\right.$.), 0.075 G. (1t gr.) a day.

The French are fond of the following prescription:

B Ext. opii ........................... $0.50 \ldots \ldots$ gr. viiss

Ext. stramon....................... $0.25 \ldots \ldots$ gr. iv

M. fiat pil. no. 1 .

Sig.-Up to $10-11$ pills daily.

The camphorated tincture of opium (paregoric), alone or added to an expectorant mixture, is particularly indicated where a deep seated 
catarrh is accompanied by severe cough and a bad or stagnating expectoration, since the benzoic acid acts as an expectorant, while the opium soothes the cough. The dose is $20-60$ drops a day.

An old-fashioned remedy is lactucarium, which, although opium and morphine have supplanted it as a sedative for cough, still deserves some consideration, especially where it does not seem wise to give opium on account of its constipating effect or of the advanced age of the patient; $0.05-0.10$ G. $\left(3 / 4-1 \frac{1}{2}\right.$ gr. $)$ may be given $3-4$ times a day; e.g.:

\section{B Lactucarii,}

Pulv. althex.
M. fiat pil. no. 1 .

Sig. $-3-5$ pills a day.

Since the cough is apt to be worse at night, it is well to give 2-3 single doses of any of the above prescriptions just before the patient goes to sleep, adding a single dose whenever he awakes, until $2 / 3$ of the daily amount has been given, and then to distribute the remaining $1 / 3$ over the rest of the 24 hours. There is a practical advantage in ordering smaller single doses, to be taken 12 times in the 24 hours.

Cough from the Throat.-A frequent source of cough in tracheobronchitis is the throat. In such cases an oily or mucilaginous drink, by spreading a protective layer over the mucous membrane, will often bring relief. We may employ syrup of acacia, emulsion of almonds, or the emulsio oleosa, 150-200 G. (5-6 oz.) of each a day; or a tea made from the leaves and root of marshmallow, or from species pectorales (N.F.) or a decoction made from $200 \mathrm{G}$. (6 oz.) of each of these, with the syrup of sweet almonds or of acacia added. In such cases inhalation and the internal use of mineral waters are particularly helpful. The most suitable waters for this purpose are those containing carbon dioxide, which has a local sedative effect on the mucous membrane of the throat (Apollinaris, Bilin, Giesshübl, Krondorf, Preblau, Rohitsch, Seltzer, or the Römerquelle in Carinthia).

Retrosternal Pain.-Many patients with acute tracheitis complain of sharp retrosternal pain. This may be relieved by inhalations (often with 1 teaspoonful of the tincture of benzoin added), hot drinks, narcotics, or, as the author has personally proved, by the use of a drug which is comparatively little used, ledum palustre:

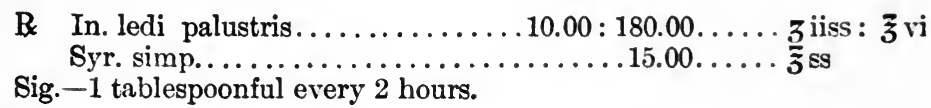

Stimulating Compresses.-In every kind of bronchitis where we have not been able to break up the attack, Priessnitz's compress should be used on the chest. This consists of a cloth wrung out in water at the room temperature, with a hot cloth bound over it. As soon as the inside 
compress is dry or begins to get cool, it should be renewed; after it is finally removed, the chest should be thoroughly rubbed, sometimes after having first been washed off with cold water. The action of this compress is not exactly understood, but appears to be somewhat as follows: The cold application at first causes an active contraction of the cutaneous vessels. As soon as the cold bandage is warmed by the hot one bound over it, this contraction gives way to an active vasodilatation combined with a sustained tone of the vessels, which persists for some time and causes an active hyperæmia of the skin. Later, as the compress becomes dry, this dilatation of the vessels leads to a slowing of the blood current, the skin grows cool, and the individual begins to feel chilly. The compress is now renewed and the whole process is repeated, or else the compress is removed and the skin is rubbed dry. By the initial contraction of the cutaneous vessels, the nervous centre is reflexly stimulated, the breathing is momentarily deepened, and the nervous tone of the vessels is increased. This transitory effect is followed by the beneficial relaxation of the cutaneous vessels, which causes a freer circulation in the skin, and, indirectly, in the heart and lungs. In consequence of this dilatation, blood, if only in small amounts, is drawn away from the hyperæmic mucous membrane of the trachea and bronchi. The skin being kept damp and evenly warm, loses some of its irritability, so that the nervous centre is spared many stimulations which would otherwise reach it from the periphery. The patient is quieted, and his shortness of breath, and often his cough, is relieved. When the compress is changed, a new stimulation follows the sedative effect. By this alternating effect of heat and cold, the innervation of the inner organs, especially those beneath the compress, is repeatedly stimulated in a reflex manner, and the secretion of the diseased mucous membrane, and the circulation in the diseased organs, are favorably affected. Roehring also attributes to the use of the compress the penetration of water vapor into the organs by a kind of transfusion; while Rhoden maintains that the secretion is thinned, a certain amount of water which would naturally be excreted from the lungs remaining in the body, on account of the fact that the air immediately surrounding the thorax contains more water than usual.

From various causes (anæmia, cachexia, constitutional diseases, hyperæmia of the organ beneath the compress) it sometimes happens that the supply of blood to the skin, or the tone of the cutaneous vessels, is so far below the normal that the cold compress remains cold even after it is covered with the hot bandage, the patient experiencing an unpleasant feeling of chilliness until the compress is removed. In that case, instead of using flannel or linen for the outer bandage, we may employ an impermeable material (rubber, oiled silk, etc.), or a double or treble fold of the ordinary cloth. But, though we may in this way avoid the chill, the vessels are not dilated as they should be. It is better, therefore, to increase the tone of the capillary vessels by wringing out the inner compress in colder water than usual (at $57^{\circ}, 54^{\circ}$, or even $50^{\circ}$, instead of 
$\left.63^{\circ}-67^{\circ}\right)$. The colder the water, the greater the stimulation; the stronger the initial contraction, the more energetic and prompt the reaction, i.e., the active dilatation of the vessels, provided, of course, that the cold is not so intense as to bring on direct passive vasodilatation (paralysis of the vessels), a contingency practically impossible under the circumstances. A simpler and surer way of reaching the same end is to wash the chest with cold water before putting on the compress.

Instead of Priessnitz's application, the so-called "cross-binder" may be used. A linen bandage $1 \frac{1}{2}-3$ yards long and 6-16 inches wide is wrung out in water as before and is adjusted in the following manner: One end is placed in the right axilla, the binder is then brought forward obliquely over the thorax to the left shoulder, and over this and across the back to the right axilla again. It is then passed forward to the left axilla, under the left arm, and obliquely across the back to the right shoulder. It is finally carried over the right shoulder to its starting point or to the sternum. The outer bandage is put on in exactly the same way. It is usually only $3-5$ hours before the ordinary cool compress begins to dry, but the cross-binder keeps wet much longer, so that it often is left on all night. This is not advisable, however, unless we can be sure that it will not begin to dry until shortly before the patient would naturally waken. Instead of the cross-binder two sleeveless cotton jackets may be used.

Abundant Viscid Secretion.-If the above measures have started abundant secretion or if the latter has been present from the beginning, our next task must be to get rid of the secreted mucus. If this is still viscid and hard to raise, means must be taken to thin it. The mildest method is to give alkaline and muriatic mineral waters, either alone or with hot milk. Ipecacuanha also may be employed. Although this increases the secretion and thins the mucus, its chief effect is that of an expectorant and an emetic. It is, therefore, admirably suited to cases of viscid secretion in the bronchi with insufficient expectoration. It is most frequently given in the infusion:

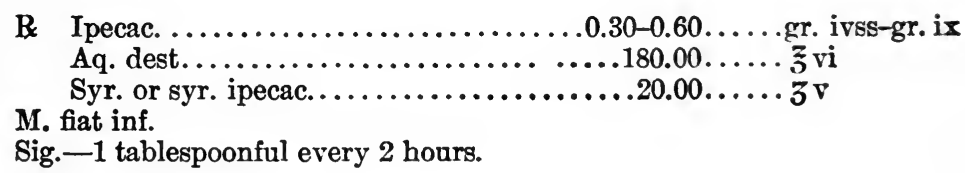

But the powder may also be employed:

B Pulv. ipecac....................0.005-0.05.....gr. $\frac{1}{13}-g r . \frac{5}{6}$

Sacchar. lactis..................... $0.40 \ldots .$. gr. vi

M. fiat pulv. Dent. tal. dos. no. xxx.

Sig. -1 powder every other hour during the day (or less frequently).

We may also use the troches (composed of the infusion and sugar, or of the powder, sugar, and mucilage of tragacanth, with the addition of 
aqua aurant. flor.), each containing $0.01 \mathrm{G}$. ( $1 / 6 \mathrm{gr}$.) of the root; or the wine ( 1 part ipecac to 10 parts sherry wine), 15-20 drops 3-4 times a day. The tincture is best employed as an addition to other expectorants. It is often a good plan to combine opium or morphine with the infusion or powder. The well-known Dover's powder, for instance, is composed of $1 \mathrm{G}$. (15 gr.) each of powdered opium and ipecacuanha, with $8 \mathrm{G}$. ( $2 \mathrm{dr}$.) of sugar of milk. We prescribe:

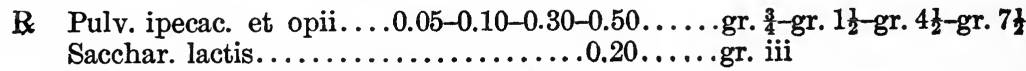 M. fiat pulv. Dent. tal. dos. no. xxv.
Sig. -1 powder every 2 hours.

The ammonium salts also have a solvent action on the mucus. We give ammonium chloride, generally as an addition to other expectorants, as the root of ipecacuanha, and frequently with the addition of extract of licorice. For example:
B Ipecac........................ 0.30-0.50.....gr. ivss-gr. viiss Aq. dest.......................... 180.00.... $\mathrm{z}$ vi

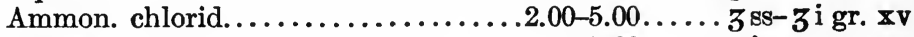
Ext. glycyrrh.....................15.00.... $3_{\text {iv }}$
M. fiat inf.

Sig. -1 tablespoonful every 2 hours.

The mistura solvens (Nat. Form.) contains also opium, and fennel water is used in place of distilled water, according to the following prescription :

R Ammon. chlorid....................5.00.... $3^{\mathrm{i} g r . x v}$

Ext. opii ....................... $0.20-0.30 \ldots \ldots$ gr. iii-ivss

Aq. fœeniculi.......................185.00.... $\mathrm{z}$ vi

Ext. glycyrrh......................... 3 iiss

M. fiat mist.

Sig. -1 tablespoonful every $2-3$ hours.

Abundant Thin Secretion.-In case of abundant thin secretion stagnating in the bronchi, insufficient expectoration, and a good heart, apomorphine is indicated $(0.01-0.05 \mathrm{G} .-\mathrm{T} / 6-3 / 4 \mathrm{gr}$.). Its action as an emetic is much more rapid than is that of ipecacuanha. It is the only emetic that acts directly on the vomiting centre. In small doses, however, like all emetics, it acts as an expectorant. For this purpose it is best given internally:

B Apomorphin. hydrochlor .............0.02-0.04.....gr. $\frac{1}{3}$ gr. $\frac{2}{3}$

Acid. hydrochlor. dil................. $0.50 \ldots \ldots$ gtt. viiss

Aq. dest.......................... $150.00 \ldots \mathrm{z}$

$\left(0.02-0.03\right.$ G. $\left(\frac{1}{3}-\frac{1}{2}\right.$ gr. $)$ of hydrochlorate of morphine is often added.)

Sig. - 1 tablespoonful every 2 hours.

As a substitute for apomorphine the author can strongly recommend euporphine (the brommethylate of apomorphine). It is as powerful an 
emetic, but is not so injurious to the heart nor so apt to induce vomiting. It may be given in the aqueous solution, up to $0.01-0.04 \mathrm{G}$. ( $1 / 6-2 / 3 \mathrm{gr}$.) a day, or in tablets, to which morphine is generally added; e.g.:

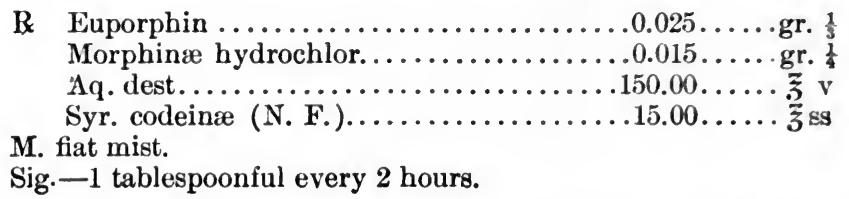

Although the preparations of antimony have justly fallen rather into disuse, the author oceasionally employs one of them, antimonium sulphuratum, to assist other expectorants (dose, $0.03 \mathrm{G}$. ( $1 / 2 \mathrm{gr}$.), up to $0.02 \mathrm{G}$. (3 gr.) a day). Liq. ammonii acetatis, $4-15$ G. (1-4 dr.) daily, is also used as an expectorant, as well as the liq. ammonii anisati and the liq. ammonii succinici. All of these, especially the first two, are particularly indicated when a stimulative effect on the heart is also desired, and are, therefore, chiefly of use in the case of infants and aged persons. With these we generally employ the violent expectorants, like senega or benzoic acid, or, when the bronchi are dangerously clogged with the stagnating mucus, an injection of apomorphine. [The author has eited a number of drugs, all of them useful for some eases of acute tracheobronchitis; but I deem it well to emphasize my cpinion that most of such cases get well as promptly and completely if medicines are left alone and we rely upon hydrotherapy, carefully applied inhalations of medieated steam, a fluid and nourishing diet, and rest in bed. If these measures do not suffice, the drugs mentioned in this section may be resorted to.-Ed.]

\section{THERAPY OF ACUTE DEEP BRONCHITIS AND CAPILLARY BRONCHITIS.}

HydrotherdPy.-Acute deep bronchitis, particularly of the capillary type, is always a serious disease, especially in children and old people. It is generally accompanied by fever, and always involves the possibility of the lumen of the bronchi becoming so much eontracted that the air is unable to reach the alveoli of the lungs. This danger is best combated by suitable hydropathic measures, which at the same time are the most rational means for redueing the fever. It is very important to keep the temperature down, in order to spare the abnormally taxed heart and the respiratory muscles (Jürgensen).

Tepid Baths.-The best form of hydrotherapy is tepid baths $\left(75^{\circ}-82^{\circ}\right.$, sometimes $\left.86^{\circ}\right)$, lasting $10-15$ minutes and followed by cool water poured over the patient. While the patient sits in the tub, his whole body is splashed with the water and vigorously rubbed; then, at the end of the bath, 3-5 quarts (sometimes as much as 7 gallons) of water at the room temperature $\left(54^{\circ}-68^{\circ}\right)$ is poured over his neck, breast, and back, and he is quickly rubbed down. The action of this procedure is apparently as follows: At first the tepid water, cold in 
comparison with the temperature of the skin, produces a contraction of the cutaneous vessels, and a stimulation of the skin, which reflexly leads to forced, deeper inspirations and to a sudden increase of the supply of air to the lungs. This primary contraction is soon followed by an active vasodilatation, which is arrested by the energetic rubbing. The hyperæmia of the bronchial mucous membrane is therefore relieved, the cardiac action is made easier, and the breathing is deeper and quieter. The cold water at the end causes cutaneous stimulation, and reflexly several deep breaths and repeated coughs. Three such baths a day are generally given, but there is no objection to giving them oftener, provided the heart action is thereby strengthened. They also have a diuretic effect. Occasionally the heart cannot overcome the primary contraction of the cutaneous vessels, and collapse ensues, demanding the immediate use of heart stimulants. It is therefore advisable always to have brandy close at hand.

Cold Packs.-If it is impracticable to give the baths, recourse may be had to cold packs. The arms should be left free for the sake of ease in breathing. In case of poor cutaneous circulation, cyanosis, and chilliness of the peripheral parts of the body, it is best to enclose only the trunk in the pack. The temperature of the water varies with the amount of the fever (generally $57^{\circ}-64^{\circ}$ ). The higher the fever, the colder and wetter should be the sheet. This is placed on top of a dry blanket, and is then folded rather loosely around the patient, who is then wrapped in the blanket. Whether such a pack should be followed immediately by another one already prepared in another bed, or even by several others, is dependent upon the temperature and upon whether or not the breathing is deepened. As soon as the feverish patient feels his skin growing hot again, in ten, five, or even three minutes, the pack is renewed, until the temperature has been reduced to the desired minimum. If it rises again to any considerable height $\left(102^{\circ}-103^{\circ}\right)$, the whole process must be repeated.

Hydrotherapy in Capillary Bronchitis, with Stasis of the Peripheral Circulation.-It not infrequently happens that we encounter cases characterized by cyanosis, coldness of the surface of the body, particularly of the distal portions, deficient oxidation, and therefore a subnormal temperature. Under these circumstances we cannot use any of the hydropathic measures already described, since they all withdraw heat from the body. The first thing to be done is to procure deeper, more vigorous breathing, and there is no better way of accomplishing it than to spray the back of the head and neck (corresponding to the medulla, therefore below the occipital protuberance), with a stream of cold, even ice-cold, water $1 / 2$ inch in diameter. This procedure should be kept up for several seconds, and be repeated 5-10 times at intervals of one second. Another, but by no means as good a method, is to give the patient a short hot pack (water at $104^{\circ}$ ), and then rub him energetically to stimulate the cutaneous circulation and to get rid of the 
passive hyperæmia, finally giving him a cold rub ( $1 / 2-1$ minute) to induce an active hyperæmia and, reflexly, deep breathing and coughing.

Hot Baths.-Hot baths (advocated by the Japanese) are most efficacious. The head is washed with cold water and covered with an ice compress, an ice-bag being also placed over the precordium. The patient is then put into a bath of $102^{\circ}-104^{\circ}$, which is quickly raised to $111^{\circ}$ and kept there. After 15-20 minutes he is put to bed and rubbed dry to increase the cutaneous circulation already stimulated by the hot bath. On account of the hinderance to the respiration, it is not advisable to induce subsequent sweating.

The essential effect of these hot baths lies in their tonic action (Baelz), and in their raising of the blood pressure (not in all cases, according to the author's experience). They are especially indicated in acute capillary bronchitis, bronchopneumonia, and croupous pneumonia, since they draw the blood away from the congested lungs, and relieve the pulmonary circulation. They also induce diaphoresis, thus eliminating bacterial toxins and possibly bacteria themselves, and they have an eminently sedative effect on all kinds of pleuritic pain. For more than one reason, then, they deepen and relieve the respiration, especially as at the first contact with the hot water the breathing centre is reflexly stimulated. To increase this effect and eause a permanent stimulation of the skin, $300 \mathrm{G}$. ( $1 / 2 \mathrm{lb}$.) of mustard may be added to the water. It is best enclosed in a cloth bag, and then slowly pressed out under water. The author's experience (over 60 cases in different diseases) shows that such baths produce at first a slight rise of temperature (generally

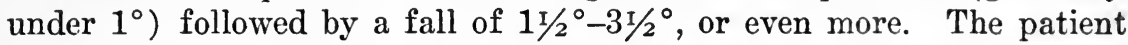
is soothed and usually sleeps for 2 or 3 hours. The baths should be given at least 2 or 3 times within the 24 hours, and repeated whenever the temperature goes above $103^{\circ}$. If it is impossible to lower the temperature, they may even be given every 3 hours. They are contraindicated, of course, in cardiac or renal insufficiency and in arteriosclerosis.

Mustard Plasters and Packs.-The author has recently used a mustard plaster surrounding the entire thorax in similar cases of suffocative bronchitis or of inflammatory œdema in pneumonia, leaving it on until the skin is red. Heubner recommends a mustard water pack for children : 1 pint of mustard is added to 3 pints of tepid water and compresses are wrung out in it as soon as the irritating oil of mustard begins to rise. The pack is left on till the child becomes restless, and is followed by a tepid bath or pack for $1 \frac{1}{2}$ hours, and then by a tepid bath or a quick cold rub-down.

DIET.-The diet must be nourishing-eggs, milk, broth and beef-tea, both with the addition of puro, sanatogen, somatose, or some similar preparation ; and even, in spite of fever, minced or roast chicken, scraped and chopped raw beef, occasionally cooked or raw fruit, caviar, sardines, or similar appetizers, jellies and aspic, and very easily digested carbohy- 
drates, such as soufflés and crackers. The disease is so prolonged that we must try from the start to keep up the patient's strength.

Alcohol.-As both of the forms of bronchitis under consideration subject the heart to a long strain, it is better to give alcohol from the beginning-grog, wine in tea, brandy, strong wine (sherry, champagne, Bordeaux, Hungarian, or Spanish wines), or Stokes's mixture:

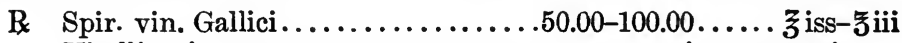

Vitelli ovi..........................no. i...... no. i

Aq. cinnamon......................150.00...5 v

M. fiat mist.

Sig. -1 tablespoonful every 2 hours.

If there are any signs of heart weakness, alcohol is indispensable.

Hygiene: Position.-The patient should be kept in bed, with his head and back raised, the position being changed frequently to minimize the danger of hypostasis or of the development of lobular pneumonia.

Damp Air in the Room.-The air in the sick-room, especially around the bed, must be kept damp, in order to relieve the distressing cough and the unpleasant feeling of dryness in the throat and larynx. This may be accomplished by (1) hanging sheets wrung out in hot water around the bed; (2) keeping vessels of steaming water near the bed; (3) plunging a red-hot iron in a tub of hot water from time to time; and (4) by heating a large inhalation apparatus. The last two methods are the least desirable. The best way is to make a tent over the bed by means of a screen and sheets, and to introduce steam by a tube from a kettle.

Medication: Narcotics.-On account of the danger that the air passages may become clogged with secretion and narrowed from the swelling of the mucous membrane, the object of medication is rather to promote expectoration than to suppress the cough. Narcotics are indicated only when the distress from the cough is so great that the patient loses sleep, and when at the same time the general condition, and particularly that of the heart, are below normal (e.g., when the heart is noticeably irregular at the end of an attack of coughing). Even then the author gives only a small dose, and, if possible, only one. The tincture of opium and Dover's powders are particularly suitable.

Expectorants.-In general, we proceed at once to the use of strong expectorants (ipecacuanha, senega, apomorphine subcutaneously), provided the heart action is good and we are sure that we have a fresh preparation:

R Senegæ................... $0.60-10.00 \ldots \ldots . g r$. ix -3 iiss

Liq. ammon. anisat............1.00-2.00....m xv-3 ss

Syr. ipecac.................... $15.00 \ldots \ldots$ zs

Aq. dest....................... 180.00.... 3 vi

M. fiat inf.

Sig. -1 tablespoonful every 2 hours.

If symptoms of heart weakness follow the injection of apomorphine, 
a hypodermic of sulphuric ether should at once be administered. Euporphine is now very often used internally as a substitute for apomorphine.

If from the beginning there are symptoms of cardiac insufficiency, even of a slight grade, we have recourse to heart stimulants. The early use of digitalis and strophanthus in small doses (e.g., 2-4 drops of the tincture of strophanthus every 2 hours) apparently hastens the favorable conclusion of the disease.

In case of dyspnœa, cyanosis, marked diminution of the respiratory surface, and coincident weakness of the heart, the above-mentioned hydropathic measures may be supplemented by the inhalation of oxygen. If 25 gallons at least are inhaled daily, good results often appear, although we may not be able to explain them theoretically. The breathing and heart action are slowed, and the subjective symptoms are much improved, showing that, owing to the inhalation of oxygen, more oxygen has passed into the blood and the tissues. Michaelis, of Leyden's clinic, has recently given confirmatory reports in this connection.

Treatment of the Pain.- The patient may complain of severe pain in the chest, which is sometimes to be referred to myalgia, sometimes to a concurrent, dry pleurisy. To relieve it, we may use dry cupping, mustard plasters, mixtures, salves, [strapping-Ed.] (see p. 364, dry pleurisy), or, if necessary, a local hypodermic of morphine, especially where the pain hinders the respiration. Hot baths are also useful.

Prophylaxis.-In determining the proper prophylactic therapy, we must take into consideration the cause of the bronchitis. If it arises from Bright's disease, heart disease, pulmonary tuberculosis, emphysema, or bronchial catarrh, protection against the recurrence of an attack can be afforded only by treating the primary condition. But in some cases the feeble resistance of the individual to catching cold is the only etiological factor. The nerves of the cutaneous vessels are unable to bring about the proper vaso contraction, demanded by sudden changes of temperature, drafts, etc. The lumen of the capillary vessels remains abnormally wide and the loss of water and of heat from the skin abnormally great, and the patient "catches cold." Naturally, this occurs the more easily the more blood there is in the skin, the greater the secretion of sweat, and the more intense the muscular work that may have just preceded the exposure to the cold.

Hardening Process.-In such cases our therapy must consist of a "hardening" process. The suitable hydropathic measures in the case of a child have already been discussed under rachitis (see p. 255). An adult patient should be rubbed down every morning as soon as he gets up, with a towel wet in water at $68^{\circ}$ and well wrung out, and then be given a dry rub. Very delicate individuals should go to bed again for a half hour during the first days of the treatment; others should take a quarter of an hour's walk. The next step is to have the towel wetter, and then to reduce the temperature of the water to $62^{\circ}-59^{\circ}$, and later even to $54^{\circ}-50^{\circ}$. The patient should now wash and rub himself and afterwards 
exercise actively. We then proceed to the use of douches, the patient beginning by giving himself a douche at $75^{\circ}$ for 15 seconds, and gradually reducing the temperature, increasing the time to half a minute and longer. The douche should always be followed by a dry rub and a half hour's walk.

In all these hardening measures the cold water and the rubbing which follows cause an active contraction and an active relaxation of the cutaneous vessels, and so train the nerves supplying these vessels to accommodate themselves to changes of temperature, their tonus being preserved. All these procedures, when once the patient has become used to them, may be kept up during a slight recurrence of the disease.

There are some individuals who cannot stand such a cold water treatment, as it leads to a high degree of excitement of the nervous system. For them a suitable course of air baths (10-60 minutes) may be substituted, for in an air bath the patient can keep himself warm by all sorts of gymnastic movements. Moreover, water conducts heat from the body twenty-five times as quickly as does air. Furthermore, the air bath, with its variations in temperature, humidity, and air currents, is much more like the actual conditions of every-day life to which we wish to harden the patient (Keller). To accomplish its purpose, the air bath should cause only a few minutes' chill, followed by a reaction.

Out-door bathing, in either salt or fresh water, should not generally be allowed until the individual is considerably hardened. Even then, he should at first take only a single dip, which may afterward be prolonged to a 2 or 3 minutes' bath. Five minutes or over may finally be permitted, if he keeps warm by swimming.

The Hardening Process in (a) Bronchitis from Abnormal Perspiration, (b) Reflex Bronchitis.-There are two classes of patients with whom this hardening is not successful: (1) those who perspire abnormally easily and eatch cold in that way; and (2) those neurasthenic or arthritic individuals in whom the slightest action of cold on the hypersensitive skin brings on a reflex attack of bronchitis (e.g., from washing the head with cold water). In such cases, the whole body is rubbed with a dry sheep-skin glove (with the wool on), and then with a flannel wet in an alcoholic mixture; e.g. (Renant) :

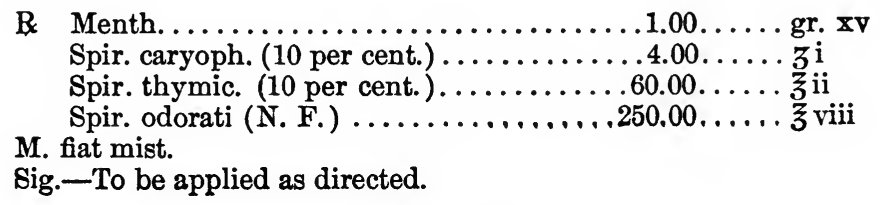

Renant also recommends for "reflex" bronchitis 0.30 G. (gr.) of ergotine in a suppository for 3 consecutive evenings, followed for 3 days by $0.015 \mathrm{G}$. ( $1 / 4 \mathrm{gr}$.) of extract of stramonium in 3 pills, one in the morning, one 2 hours after eating, and the third just before going to bed. 
Clothing.-The patient should wear wool next his body, because wool prevents the rapid evaporation of the perspiration and minimizes the loss of heat to the outer air. The latter effect is due to the fact that the rough surface of wool prevents the garment's lying close to the skin and insures an intermediate layer of air, which is a very poor conductor of heat compared with cloth (1:90). Several layers of thin goods instead of one of thick are for the same reason advantageous. Especial care must be taken to keep the feet warm, as they always have a tendency to be cold on account of their distance from the heart, their frequently profuse perspiration, and their relatively large surface in proportion to their volume. Wet feet frequently cause an attack of bronchitis.

Too much clothing is a great mistake. The perspiration thereby induced soaks the underwear, making it a better conductor of heat from the body. Moreover, the overheated patient is often tempted to loosen the clothing and let the cold air strike his wet body, thus increasing the chances of his catching cold. Wet clothes are another frequent source of an attack. Rumpel has shown that they conduct heat from the body three times as fast as would the air, were the individual entirely naked.

Climate.-Persons with a tendency to bronchitis do best in a mild climate, with air free from dust, no great changes in temperature, and much sunshine. The winter should be spent in the south, preferably at the seashore, the Riviera, or the islands of the Mediterranean in winter; the Italian lakes, Lake of Geneva, and South Tyrol, in spring and autumn, [Southern California, Bermuda, Nassau, The Carolinas, Georgia, Florida.-Ed.].

\section{THERAPY OF CHRONIC BRONCHITIS.}

We shall consider here only cases of primary chronic bronchitis, since those in which the condition of the bronchi is only one symptom of some other disease (diseases of the nose, emphysema, pulmonary tuberculosis, heart lesions, obesity, or a uric acid diathesis) demand, at first at least, a causal therapy. Primary bronchitis may be subdivided into:

1. Dry :

(a) Catarrhal.

2. Secretory :

(a) Catarrhal.

(b) Purulent.

(c) Putrid.

(d) Fibrinous.

THERAPY OF DRY CATARRHAL BRONCHITIS.

Medication.-The treatment is very similar to that of acute, dry bronchitis. The inhalation of steam is very important (p. 284), and the cough is treated as in the acute form. But, because of the long duration of the disease, the mode of administration of the sedatives 
should be varied in order to avoid gastric disturbances. The following is a suitable form of suppository:

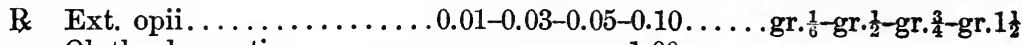
Ol. theobromatis .................. $1.00 \ldots \ldots$ gr. xv

M. fiat suppos. anal. Dent. tal. suppos. no. $x$.

Sig.-1-2 suppositories a day, especially in the evening.

To such a suppository, we may, if desirable, add the extract of belladonna, $0.02-0.05$ G. $(1 / 3-3 / 4$ gr. $)$, or the extract of stramonium, 0.01 G. ( $1 / 6$ gr.) per dose. The same dose may be given in 60-70 G. $(2-2 \mathrm{I} / 2 \mathrm{oz}$.) of tepid water as an enema, preferably in the evening, after a previous cleansing irrigation. The dose of morphine in suppositories or enemas is $0.01-0.03 \mathrm{G}$. ( $1 / 6-1 / 2$ gr.). In this way opiates may be given for several weeks without danger of any bad results.

As a variation in the narcotics, we may make use of a tea made from the species pectorales (Nat. Form.), or prescribe as follows :

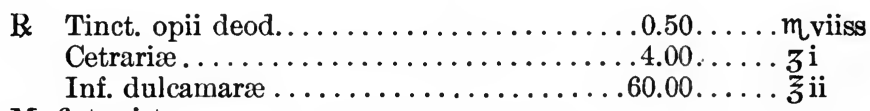

M. fiat mist.

Sig. - 1 tablespoonful in water twice a day.

A chronic bronchitis frequently causes a secondary involvement of the right heart. In that case, a heart tonic (digitalis, strophanthus) should be added to the narcotics.

The author cannot agree with certain authors in ascribing satisfactory results to the use of potassium iodide in dry catarrhal bronchitis. He has never found it successful, even when the cough was of a paroxysmal character, and believes that those cases in which others have reported success were not simple chronic bronchial catarrh, but rather the so-called "catarrhal asthma."

Priessnitz Compress-Cross-Binder.-Hydropathic measures are useful, particularly Priessnitz's compress and the cross-binder. An essential part in the therapy is also played by the climate and by treatment with mineral waters.

Climate.-The elimate should be equable, warm, and humid. The most famous winter resorts for bronchitis patients are Madeira, Algiers, Mogador in Morocco, Pisa, Palermo, and Ajaccio. For spring and fall, the Riviera, the Italian lakes, Lake Geneva, and South Tyrol are suitable; for summer, the resorts on the North Sea, provided the patient is not thin and run down, or in the mountains (Partenkirchen, Davos, Ischl, Görbersdorf), or often any country place, provided it is sheltered from winds.

If it is impossible to send the patient away in winter, he must take great care of himself at home, going out only in fine weather, or sometimes not at all. For proper clothing, see p. 297. 
Treatment at Spas.-Alkaline and alkaline-chloride waters are the most useful in the treatment of chronic dry catarrh, but earthy and sulphur springs are also efficacious:

Alikaline and Alkaline Chloride.

(In case of strong spasmodic cough, and shortness of breath. From June to September.)

Ems (Kränchen, Kesselbrunn, Wilhelms-Augustaquellen)

\section{Selters}

Bilin

Gleichenberg (Constantinsquelle)

Luhatschowitz

Neuenahr (in the Rhine Provinces)

Salzbrunn (in Silesia)

Wiesbaden (Kochbrunnen)
EArtiry.

Driburg (in Westphalia)

Lippspringe (in Westphalia)

Wildungen (in Waldeck)

Weissenburg (in the Bernese Oberland)

Rajeczfürdő (in Upper Hungary)

\section{Sulphur Springs.}

(For torpid cases with scanty and difficult expectoration.)

Eilsen (in Schaumburg-Lippe) Heustrich (in the Bernese Oberland)

Kainzenbad (near Partenkirchen in Upper Bavaria)

Langensalza (in Thuringia)

Leuk (in Canton Bern)

Nenndorf (in Prussia)

Weilbach (in Wiesbaden)

[Harrogate (in England).-ED.]

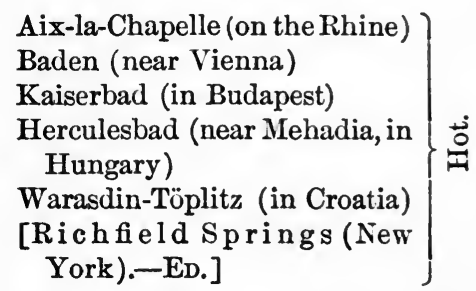

The sulphur waters are used both internally and for inhalation. They are particularly suited to cases where symptoms of hyperæmia of the portal veins are found in a patient otherwise in a good general condition. Their action is due to a small part of the sulphur being given off through the bronchial mucous membrane as sulphuretted hydrogen, and so causing active hyperæmia in the atonic membrane, which is accordingly better nourished. The sulphuretted hydrogen also works as an antiseptic.

Treatment with Mineral Waters at Home.-If the patient cannot be sent to the springs, mineral water may be given at home (750-1000 c.c. (11 $12-2$ pt.) daily of the alkaline and alkaline-chloride waters, either in milk or alone; 1000 c.e. ( 1 qt.) daily of the sulphur waters, but only $100-150$ c.e. ( $1 / 2$ glass) on an empty stomach in the morning, generally mixed with milk). We may use the water from the Kainzenquelle in 
Kainzenbad, the sulphur springs at Weilbach, the Kaiserbrunnen at Aix-la-Chapelle, or the Kaiserbad at Budapest.

Sulphur internally is much less efficacious ( $I / 2$ teaspoonful of the flowers of sulphur in milk in the morning).

Pneumatic Chambers.-Pneumatic chambers (see p. 71 for the method of using them) are also useful and strikingly successful, as they make the patient breathe slowly and deeply, a habit which to some extent will remain with him later. They are found at many resorts; e.g., Reichenhall, Baden-Baden, Aix-la-Chapelle, Herculesbad (near Mehadia), Schinznach in Canton Aargau, Gleichenberg, Ems, Kissingen, Meran, and in most large cities. Simple portable chambers are found at most of the spas.

\section{THERAPY OF CHRONIC CATARRHAL BRONCHITIS WITH A PURULENT SECRETION.}

Medication.-The same expectorants are indicated here as in acute bronchitis with abundant secretion (see p. 289). To them may be added the ethereal oils, especially where the catarrh is markedly purulent.

Oil of Turpentine.-First comes the oil of turpentine (10-20 drops in a tablespoonful of milk followed by half a glass of milk; 40-50 drops a day). It may also be given in gelatin capsules:

$\mathrm{B} \quad$ Ol. terebinth. rect..............1.00-1.50.....m $\mathrm{xv}$ - $\mathrm{m}_{\mathrm{x} x \mathrm{xii}}$

Dent. tal. caps. molles no. 1 .

Sig.-1-2 capsules daily with milk and followed by milk.

The milk is to prevent the irritating effect of the turpentine on the stomach. If the patient cannot take milk, 5-6 drops of turpentine may be put between two slices of bread and butter or on a little sugar. In case of abundant secretion, turpentine may be inhaled as well as taken internally. It sometimes has an irritating effect on the kidneys; the author has seen one patient in whom the continued use of turpentine inhalations brought on a remarkable exacerbation of existent Bright's disease, and another in whom it started an acute nephritis.

The oil of turpentine has been partly replaced by certain of its derivatives. Among them the author uses terpin hydrate, 0.20-0.50 G. (3-7 gr.) a day, as follows:

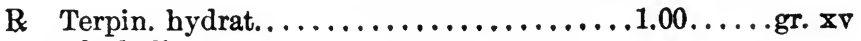

Alcoholis,

Aq. dest.,

Syr. rubi. idæi...............āã $50.00 \ldots \ldots$ żs

M. fiat sol.

Sig. - 1 tablespoonful 5 times a day.

It may also be given in pills and powder form.

Terpinol is another good preparation. It is a colorless liquid, 
smelling like hyacinths, and is best given in pills or gelatin capsules (maximum daily quantity, 1 G. -15 gr.) :

B Terpinolis .................. $0.10-0.20 \ldots \ldots$ miss $-m$ iii

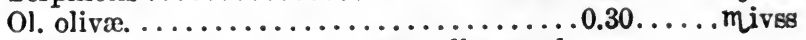

M. fiat cap. no. i. Dent. tal. caps. molles no. 1.

Sig. - - -10 capsules a day.

Both of these thin the bronchial secretion, facilitate expectoration, and then diminish the secretion; they also act as disinfectants of the whole bronchial tract.

Balsams.-The balsams, such as the balsam of Peru and balsam of tolu, are not nearly so available. The first, a dark brown viscid liquid, is usually given internally in an emulsion:

R Balsam. Peruvian........................... $\mathbf{x \nabla}$

Emuls. amygdalæ................. 180.00.... $\mathrm{zi}$

M fiat emuls.

Or:

Sig. -1 tablespoonful every 2 hours.

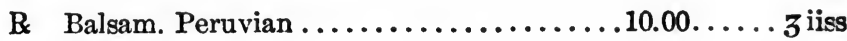
Acaciæ,

Ext. glycyrrhizæ........................ ā̄ q. 8.

M. fiat mass. Div. in pil. no. 1.

Sig. -1 pill 5 times a day.

The balsam of tolu is administered in the same way, but is much less often used. The author gives it in the form of a syrup added to other expectorants; e.g.:

B Vin. ipecacuanhæ.................15.00.... $3 \mathrm{ss}$

Syr. tolutan..................... $30.00 \ldots$

Aq. dest.................... s. ad $180.00 \ldots \ldots$. 3 vi

M. fiat mist.

Sig.-1 tablespoonful every 2 hours.

The balsam of copaiba is a yellowish brown liquid with a peculiar aromatic odor and a sharply bitter taste. It is best given in an emulsion, 4-5.5 G. (60-80 drops) a day. It may be prescribed as follows:

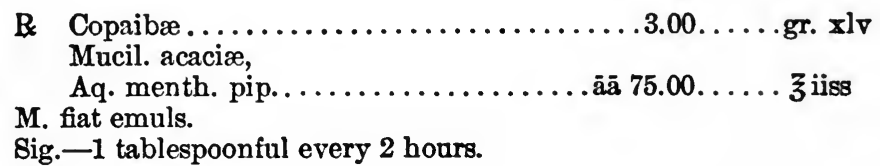

In using any of these three balsams, particularly the last, we must continually watch for gastric disturbances, for they all have a tendency to irritate the mucous membrane of the stomach. In case of any such condition, antecedent or acquired, their use must be immediately stopped. 
Other Expectorants.-Benzoic acid, yellowish flakes or white needlelike crystals, is not so frequently used nowadays as is sodium benzoate, an amorphous powder. The first, which also acts as an irritant on the stomach, is usually employed in connection with other expectorants, especially where we want a stimulating effect, 1 G. (15 gr.) a day; e.g.:

R Acid. benzoici..................... $0.30 \ldots \ldots$ gr. ivss

Pulv. ipecacuanhæ .................. $0.05 \ldots \ldots$ gr. $\frac{3}{4}$

Ol. menth. pip...................... miss

Sacchar. lactis ..................... $0.20 \ldots \ldots$ gr. ii

M. fiat pulv. no. i. Dent. tal. dos. no. xx.

Sig. -1 powder 3 times a day.

Sodium benzoate is generally given in an aqueous solution, the dosage being 6-10 times as great as that of benzoic acid, 1-4 G. (15-60 gr.) a day.

The author has frequently obtained good results by the use of pyrenol (benzoyl-thymol sodium-benzoyl-oxybenzoate), a white, soluble powder, with a sweet taste and a slightly aromatic smell, 3-4 G. (45-60 gr.) daily in a mixture or in tablets.

The fluidextract of hydrastis (20-25 drops 3 times a day) has also proved of undoubted use in several of the author's cases of chronic catarrhal bronchitis. Its good effect, perhaps, is due to its causing a vasoconstriction of the bronchial vessels and so reducing the congestion.

No Narcotics.-So far as possible, no narcotics should be given, for fear of the stagnation and possible decomposition of the mucus in the bronchi, particularly if it be purulent to begin with.

Lime water may be given in daily quantities of 150-200 c.e. (5-6 oz.), 1 tablespoonful every 2 hours (Biermer).

Mineral Waters.-The earthy mineral waters, especially. Weissenburg, are much more often used in catarrhal bronchitis with severe cough and relatively slight expectoration, than in dry bronchitis.

If there is abundant though difficult expectoration, we use the common salt springs. The simple common salt springs (e.g., Homburg, Kissingen, Baden-Baden, Cannstatt and Berg near Cannstatt in Würtemburg, Hall in Tyrol, Salzschlirf (Hesse-Nassau), Soden in the Taunus, and Wiesbaden) are indicated when the patient is poorly nourished and exhibits symptoms of venous congestion in the abdominal organs. They are also suitable for old persons with chronic bronchitis. Soden is the favorite resort for individuals with a scrofulous diathesis combined with chronic bronchitis.

The alkaline-saline springs are indicated in those cases of chronic bronchitis which are complicated by obesity, and, therefore, by a sluggish pulmonary circulation, accompanied sometimes by venous hyperæmia of the abdominal organs; i.e., in cases of chronic catarrh caused by overeating and overdrinking, and especially by alcoholism. Examples are Carlsbad, Marienbad, Rohitsch, Tarasp, Salzerbad (Lower Austria), and Salzbrunn (Silesia). Salzbrunn, as well as the other alkaline and alkaline-chloride springs (Ems, Luhatschowitz, Gleichenberg), is also 
suitable for patients with chronic bronchitis and a uric acid diathesis. In choosing a health resort, the climate as well as the springs must be taken into account.

Climate.-In general, the climate should be mild and dry. In winter, Egypt is preëminently suitable, especially Helouan near Cairo, as it has warm sulphur springs; the Riviera is the next choice, from Nervi to Hyères, also Mentone, San Remo, Nice, and Pegli. For the spring and fall we may choose Abbazia, Meran, and Arco in Austria, Baden-Baden or Wiesbaden in Germany, Bex, Lugano, Montreux, or Vevey in Switzerland, or Lake Garda in Italy. Naturally, the most decisive factor in the selection of a health resort for any case of bronchitis, dry or catarrhal, is the underlying cause of the condition. If this is a uric acid diathesis, we may choose Carlsbad; if weakness of the heart muscle, Nauheim, Marienbad, or Franzensbad; if Bright's disease, Egypt.

Milk-Cure-Whey-Cure-Grape-Cure--Many of these resorts offer facilities for a milk-cure or whey-cure; e.g., Aussee, Gleichenberg, Reichenhall, Meran, Bozen, Interlaken, etc. This treatment benefits those patients who are much run down or anæmic, or have a scrofulous tendency or an abnormally sensitive mucous membrane, so that there is a constant tendency toward an acute exacerbation of the catarrh. An exclusive milk diet is not employed, but 2000 c.c. (2 qt.) of milk a day, varied as much as possible (sweet, sour, hot, cold), is given along with other light foods. Whey, as it is not so nutritious as milk, has rather gone out of favor.

A grape-cure is often given after the conclusion of a course of mineral waters. It is particularly suitable, either as a subsidiary cure or as an independent mode of treatment, in chronic, dry, bronchial catarrh, or in bronchial catarrh with abundant secretion, especially in those individuals who suffer at the same time from abdominal plethora and chronic constipation. The patient's general diet should be light, the quantity of food being earefully measured. He should begin with $2 \mathrm{lb}$. of grapes a day, gradually increasing to 4-5-6 lb., one-third being taken on an empty stomach in the morning. The treatment is usually kept up for 4 weeks. Digestive disturbances often manifest themselves, but soon disappear. The bowels usually move 2-4 times a day. Such grape-cures may be carried out at Baden near Vienna, Meran, Bozen-Gries, Rüdesheim, Wiesbaden, Montreux. Vevey, etc.

Mechanical Compression of the Thorax.-Finally, expectoration is often aided by mechanical compression of the thorax during expiration.

\section{THERAPY OF PUTRID BRONCHITIS AND BRONCHIECTASIS.}

Therapeutic Indications.-The following section on the therapy of putrid bronchitis may properly include that of bronchiectasis, since if the secretion be fetid, the therapy of the two conditions is identical; 
while if it is not fetid, the proper treatment of the bronchiectasis is that already discussed under chronic bronchitis with secretion.

The therapeutic indications in putrid bronchitis are (1) the alteration of the bronchial secretion, and, especially, the antisepsis of the bronchial mucous membrane; (2) the expulsion of the expectorate; (3) sometimes the combating of single symptoms, generally due to the bronchitis; (4) the strengthening of the whole system.

The Antisepsis of the Bronchi.-The antisepsis of the bronchi may be accomplished in two ways: (1) by the direct application of antiseptics to the bronchial mucous membrane by inhalation and insufflation; and (2) by the internal use of disinfectants, which are excreted, in part at any rate, through the bronchial mucous membrane. The latter method is by far the more effective.

Internal Antiseptics.-The best disinfectant is the rectified oil of turpentine (the same dosage as in bronchitis with secretion, see p. 300). Even after the sputum shows no sign of decomposition, 10-20 drops should be given daily for a long time. It is not certain whether the oil of turpentine is directly antiseptic, or whether it owes its effect to the fact that it increases the supply of oxygen to the part. Terpin hydrate, $1 \mathrm{G}$. (15 gr.) a day in mixture, appears to be as effective as oil of turpentine, while terpinol is inferior to both.

None of the balsams mentioned on p. 301, nor sodium benzoate, is equal to the oil of turpentine. Leyden's prescription for carbolic acid internally appears to be practically given up. He prescribes as follows:

B Phenolis liquefact............. 0.50-1.00....m $v$ viiss $-m_{x v}$

Syr. simp....................... $15.00 \ldots$ ss

Aq. menth. pip................ s. ad $180.00 \ldots \ldots$. 3 vi

M. fiat sol.

Sig. - 1 tablespoonful every 3 hours.

If the urine begins to show a green color, the carbolic acid must be immediately abandoned.

Myrtol, an oily liquid, is generally helpful. The author uses it with oil of turpentine. Even if administered alone, it immediately gives the breath an aromatic odor, although it does not always deodorize the fetid sputum. Eichhorst gives the following prescription:

R Myrtol................................ mii

Dent. tal. cap. molles no. 1 .

Sig. $-3-6$ capsules a day.

The French recommend eucalyptus, in either the tincture or the oil:

R Tinct. eucalypti $\ldots \ldots \ldots \ldots \ldots \ldots \ldots 2.00-4.00 \ldots \ldots 3$ ss $-3^{\mathrm{i}}$

Syr. aurantii...................... $15.00 \ldots$ iv

Aq. dest........................ $180.00 \ldots \ldots$ vi

M. fiat mist.

Sig.-1 tablespoonful every 2 hours. 
Or:

R Ol. eucalypti.

Dent. tal. cap. molles no. 1 .

Sig. -1 capsule 6 times a day.

They also, especially Lancereaux, strongly recommend sodium sulphite, a colorless, bitter, salty powder, which is weakly disinfectant; $4-4.5 \mathrm{G}$. (60-65 gr.) are given either in mixture (e.g., with the syrup of eucalyptus) or in powder form.

Local Antiseptics.-The foregoing antiseptics may also be utilized locally, i.e., for inhalation. For this purpose, the oil of turpentine, oil of pine (pumilio), and the oil of eucalyptus are the more frequently used; carbolic acid and thymol less often. The oil of eucalyptus may be prescribed as follows:

B Ol. eucalypti.......................

M. fiat mist.

Ol. lavandulæ..........................m $\mathrm{mv}$

Sig. -10-20 drops in boiling water for inhalation.

Carbolic acid, according to Leyden, should be inhaled 3 times a day in a 1-4 per cent. solution; thymol, in a 0.05 per cent. concentration. Formol is also inhaled, by means of a Hamaide's inhalation apparatus. The author has tried soaking sheets in turpentine and keeping them hung around the bed, but with no effect. [Forchheimer recommends dipping strips of muslin in Platt's chlorides and hanging them about the bed, or sprinkling the solution upon the bed clothing.-Ed.]

In purulent bronchitis the author practically always uses balsams internally and oil of turpentine for inhalation.

The Expulsion of the Sputum.-The second indication, the expulsion of the putrid sputum, is best accomplished by the use of ipecacuanha, sometimes with the addition of similar expectorants (p. 289). Compression of the thorax may also be tried.

In bronchiectasis with a single definitely localizable and isolated dilatation, it is advisable to place the patient from time to time in such a position that the secretion will flow from the dilatation into the discharging bronchus, and so cause him to cough hard and force up the fetid sputum. Furthermore, the inhalation of antiseptics is apparently often more efficacious with the patient in this position. Those patients whose cough is confined to several hours in the morning are often advantageously kept flat in bed without any pillows for about 2 hours at that time. After a few days the foot of the bed may be raised every morning, in order to place the head and thorax relatively lower. The secretion which has collected during the night in the dilatation will then make its way toward the healthy bronchial membrane, provoke a cough, and be expelled. So far as possible no narcoties should be given, for the more we relieve the cough, the more we increase the danger of stagnation and decomposition of the sputum. 
Surgical Interference.-A single demonstrable dilatation may sometimes be opened up from the outside. The author has seen a complete cure from such an operation (performed by Hofmokl), on a dilatation in the upper lobe. The author has always avoided the intratracheal injection of antiseptics, but the following combination has been used: menthol 10 G. ( $2 \mathrm{r} / 2 \mathrm{dr}$.), guaiacol 2 G. (30 gr.), and olive oil $88 \mathrm{G}$. $(23 / 4 \mathrm{oz}$.) (Stewart).

\section{THERAPY OF FIBRINOUS BRONCHITIS.}

Acute Fibrinous Bronchitis.-It is fortunate that this is a rare disease, for the therapy is most unsatisfactory. In acute cases, whether primary or secondary (particularly from pulmonary tuberculosis), all that can generally be done is to administer expectorants and to compress the thorax in order to prevent the bronchi from being clogged by a cast. Apomorphine or euporphine is especially indicated. No other therapy is apparently possible, the author not being able to concur in the energetic mercurial treatment proposed by Biermer.

Chronic Fibrinous Bronchitis.--In chronic and often in recurrent cases, usually primary, potassium iodide is generally used. The author has tried it in a few cases, with no effect. In two instances, however, he has obtained good results from the use of the extract of belladonna or atropine. In both of these cases, which were distinguished by an abnormal number of eosinophile cells in the sputum, the coagulation of the mucus in the bronchi ceased, and the patients left the hospital apparently sound, with no subjective symptoms and no bronchitis. One, however, returned a month later with a recurrence of the disease, while the other was lost sight of.

One case has been reported from France in which, the condition being apparently caused by streptococci, the antistreptococcus serum was used with good results. This treatment would not, however, be applicable in all cases, as chronic fibrinous bronchitis is not always due to streptococci. The author has seen one case in which the pseudomembrane gave a pure culture of the pneumococcus.

Inhalations of such substances as lime water, lactic acid, and papayotin are useful on account of the action of the warm steam. To conclude: the therapy of fibrinous bronchitis is purely symptomatic, and is, therefore, in general, analogous to that of ordinary bronchitis.

\section{THERAPY OF BRONCHIAL ASTHMA.}

Etiological Therapy.- The therapy of bronchial asthma must be primarily a causal one whenever the primary condition to which the attacks are secondary can be discovered. This etiological factor may be: a disease of the nose or throat, a specific exudative bronchitis, a disturbance of digestion, tape-worm, a genito-urinary disease, a direct or indirect affection of the vagus nerve, or a constitutional disease; e.g., diabetes, nephritis (uræmic), uric acid diathesis, or lead poisoning. 
Therapy of Primary Bronchinl Asthma.-If a cause is absent or cannot be determined, so that the asthma is apparently a primary affection, the aim of our therapy must be two-fold: (1) to avert a present or threatening attack, and (2) to prevent recurrence. In either case the physician should never prevent the patient from employing any means which he has already proved useful in preventing or stopping his individual attacks, no matter how eontrary such means may be to the physician's own experience, for so paradoxical is the nature of the disease that what helps one patient may do no good or even harm to another.

Treatment of an Attack: Morphine-Chloral.-To stop or to render tolerable an attack already begun, two means are at our command: morphine and ehloral. On account of its more rapid action, the former is preferable, provided it ean be given hypodermically, $0.01 \mathrm{up}$ to 0.015 or $0.02 \mathrm{G}$. ( $\mathrm{t} / 6$ up to $1 / 4$ or $1 / 3$ gr.). As this is not always practicable, we must often have recourse to the internal use of chloral. If we do not know the patient, we begin with a dose of $1 \mathrm{G}$. (15 gr.), increasing, if necessary, to 2-3 G. (30-45 gr.). The system does not become habituated to chloral so easily as to morphine, so that it is not as necessary to inerease the dosage and run the risk of chronic intoxication. But chloral has the disadvantage of affecting the heart, and is, therefore, contraindicated in any case of heart weakness.

Leaves of Stramonium, Belladonna, or Hyoscyamus.-In the majority of instances the leaves of stramonium, belladonna, or hyoscyamus afford relief. They may be made into cigarettes by being soaked in a saltpetre solution and then dried, or they may be placed on a plate and burned, the patient inhaling the smoke. In the latter case they may be also prepared with saltpetre, or be cut up and mixed with an equal quantity of potassium nitrate. Cigarettes may also be made from a mixture of all of these or of similar plants of the nightshade family. Trousseau's Cigarettes d'Espic, for example, are composed of leaves of stramonium, belladonna, hyoscyamus, and phellandrus, soaked in a solution of the extract of opium in cherry laurel water.

Grindelia.-It is impossible to predict which of these preparations will help the individual patient, but on the whole stramonium is the most generally efficacious. In ease they all fail, we are thrown back on our reserve material. Cigarettes made from grindelia, or sometimes the fluidextract of the same plant, 1-3 G. (15-45 gr.), may sometimes prove of value; e.g.:

B Ext. grindeliæ fl............... $1.00-300 \ldots \ldots$ gr. xv .xlv

Vin xerici................ $50.00 \ldots \ldots$ iss

M. fiat mist.

Sig. - To be taken at one time. 
Cannabis Indica.-Cannabis indica may also be smoked or burned, or the extract or tincture be added to the other asthma cigarettes. For example:

B Ext. cannabis indic......................... $x \nabla$

Ext. opii........................ iss

Aq. amygdal. amar.................... s.

Belladonnæ foliæ,

Stramonii..................... àā 2.00.....gr. xxx

Fœniculi........................... viiss

Minutim dissecta et contusa. M. fiat cigaretæ no. $\mathbf{x}$.

Or :

Sig.-One or two a day to be smoked.

B Potass. nitratis,

Stramonii.................. āā 20.00..... $\mathrm{z}_{\mathrm{v}}$

Belladonnæ foliæ,

Cannabis indic............... āā 10.00.... $z$ iiss

M. fiat misc.

Sig.-To be burned and the fumes inhaled.

Almost all the proprietary remedies on the market contain one or more of these drugs. Among them are the Zematone-cigarettes (Nos. $i$ and ii, stronger and weaker), the Zematone "smoke-powder," Neumayer's "smoke-powder" (a favorite with the author), and Exibard's "Abyssinian Remedy" (Paris).

Other Remedies. - The author puts little faith in the old-fashioned remedy, lobelia. It is generally used in the tincture; e.g.:

R Tinct. lobeliæ .....................5.00.....gtt. lxxv

Aq. amygdal. amar...................

M. fiat mist.

Sig.-10-15 drops every half-hour for 3 or 4 successive doses.

Pyridine is even less desirable, on account of its lack of reliability, its highly unpleasant odor, and its frequent effects on the stomach and the heart. 5-8 G. (11/4-2 dr.) are poured into a plate and the vapor inhaled.

Two new drugs, chloralamid and hyocine hydroiodide, deserve a trial. Chloralamid (chloral formamid) is used not only as a narcotic, but also as an antispasmodic. It consists of colorless, odorless crystals with a slightly bitter taste, soluble in water. $3 \mathrm{G}$. (45 gr.) correspond to $2 \mathrm{G}$. (30 gr.) of chloralhydrate. It may be given either in the powder or dissolved in beer or hot water. Hyoscine hydroiodide is recommended by Edlefsen (a hypodermic of $1 / 4-1 / 3 \mathrm{mg}$. $(1 / 250-1 / 200 \mathrm{gr}$.) in an aqueous solution).

The author tried scopolamine hydrobromate (hyoscine) in the same way in a very severe idiopathic nervous asthma, which resisted all other remedies, but with negative results.

B Scopolamin. hydrobrom.............0.01....gr. $\frac{1}{6}$

Aq. dest.......................

M. fiat sol.

Sig. $-10 \mathrm{~min}$. to be injected during an attack $\left(10 \mathrm{~m}=\mathrm{gr} \cdot \frac{1}{90}\right.$ (about) ). 
A temporary effect, for several weeks, was produced by the use of diphtheria antitoxin (800 units a day, after three days only 200 units, Paltauf No. ii).

In case the ordinary remedies fail, amyl nitrate may be tried, apparently with success in many cases. [Hoffmann's Anodyne is sometimes efficient in relieving an attack. Solis-Cohen recommends adrenalin chloride especially for the vasomotor type.-Ed.]

Potassium nitrate may be used in substance, or its smoke inhaled. Arsenic is also inhaled.

Some patients are helped by snuffing ammonia fumes, either from a bottle or from a dish containing a tablespoonful and held a foot or two from the nose. In the latter case the nostrils should be stopped with cotton.

Action of these Drugs.-Some of these drugs have a narcotic action, stopping or mitigating the reflex spasm of the bronchial muscles, others perhaps a solvent action; but with many of them the effect is at least partly due to a purely mechanical action, as is proved by the fact that smoking an ordinary cigarette is often efficacious.

Kraus recommends for cases where the spasmodic rather than the catarrhal element is uppermost, the use of the following combination:

R Caffeinæ sodii salicyl................ $0.20 \ldots \ldots$ gr. iii

Antipyrini $\ldots \ldots \ldots \ldots \ldots \ldots \ldots \ldots \ldots, 0.80 \ldots \ldots$ gr. xii

M. fiat cap. no. i. Dent. tal. cap. no. $\mathbf{x}$.

Sig. -1 capsule as necessary.

Many patients are helped by a hot mustard foot-bath and by filling the room as rapidly as possible with steam.

Therapy Between Attacks.-The most efficacious drugs to be administered between attacks with the view of preventing a recurrence, are three: (1) potassium iodide taken for months or even for years, (2) arsenic, and (3) atropine.

Potassium Iodide.-The first may be prescribed as follows:

R Potass. iodidi.................. $10.00 \ldots \ldots z$ iiss

Sodii bicarbon.....................

Aq. dest..................... $150.00 \ldots \ldots$ z $\mathrm{v}$

M. fiat sol.

Sig. -1 tablespoonful in water or milk, twice a day after meals.

The rather large daily dosage, $2 \mathrm{G}$. (30 gr.), is highly advisable, unless the patient can bear only smaller amounts, in which case a smaller dose, $0.5 \mathrm{G}$. ( $7 \mathrm{gr}$.), to be taken in the morning may be substituted. The treatment should be stopped after ten days, or should be intermitted for a week every three weeks. The action of potassium iodide seems to be due to the fact that it is partly excreted through the bronchial mucous membrane, thereby causing local hyperæmia and increased secretion of more liquid mucus. In asthma, as in other conditions, some authorities 
prefer sodium iodide to potassium iodide. [I always select the former. -Ed.] The dose is $0.3-1.0$ G. (5-15 gr.), preferably in solution and administered well diluted.

Arsenic.-Arsenic may be given as arsenious acid in tablet triturates, gelatine capsules, or pills, $0.002 \mathrm{G}$. (1/30 gr.) up to $0.006 \mathrm{G}$. (1/10 gr.) and more a day, after eating. The treatment should be kept up for several months, being suspended for 8-10 days each month. Arsenic may also be given in the form of Fowler's solution, or of mineral waters containing arsenic (see p. 170), or of cacodylate of soda or atoxyl subcutaneously.

Whether potassium iodide or arsenic is more likely to be successful in any given case cannot be decided with any certainty. But, in general, the former is better in cases of chronic bronchial asthma with an hereditary uric acid diathesis, evident emphysema, or arteriosclerosis; and the latter, in young persons where the bronchial spasm is the chief symptom, accompanied by only slight, if any, bronchial asthma.

Atropine.-Atropine was first made famous by Trousseau. Some other authors do not report such success as he experienced from its use, but the author, basing his judgment on only about ten cases, has found it exceedingly useful in primary asthma. He gives three pills, each containing 0.0005 G. (1/120 gr.), a day, in some eases even doubling the dose if no symptoms of intoxication appear. In fact the effect appears to depend on quickly increasing to large doses, sometimes more than 0.0025 G. (1/25 gr.) a day. Von Noorden also recommends atropine for the time between attacks while Riegel uses it hypodermically during attacks. [Murray among others pushes the drug to tolerance or even toxæmia.-Ed.] The action of atropine appears to be due to its paralyzing the ends of the vagus and thus suppressing the spasm.

The author has had success in the use of pyrenol, $3-4$ G. (45-60 gr.), in several attacks.

Eumydrin.-In place of atropine its far less toxic compound eumydrin (methyl-atropine-nitrate, a white, odorless powder, soluble in water) may be used, in single doses of $0.001-0.002 \mathrm{G}$. $\left(1 /{ }_{60} 0^{-1 / 30} \mathrm{gr}\right.$.). The author himself has not tried it in asthma. For further details concerning its use, see p. 352.

[Between attacks anæmia, if present, should be combated by the use of iron combined with laxatives, or arsenic (as above) or, especially in children, cod-liver oil. If constipation or a gastro-intestinal intoxication is a factor, appropriate remedies will often diminish the frequency and severity of the attacks.-Ed.]

Hydrotherapy.-Various forms of hydrotherapy, chosen with regard to the individual cases, are often useful (baths, douches, cold sponges). Foelkel recommends hot packs for use during the attacks, particularly in catarrhal asthma. The patient, with the head and shoulders raised, is wrapped up to his neck in a sheet wrung out in water as hot as possible, and then covered with a blanket. After an hour the pack is removed 
and he is rubbed first with a wet and then with a dry towel. By this process, the dyspnoea is materially relieved and the catarrhal symptoms reduced.

Compressed Air.-The author would not recommend the use of compressed air in bronehial asthma, believing it indicated only in case of a coincident true emphysema.

Choice of Residence.-The question as to the choice of residence must be answered by experiment in each individual case. One patient, for example, does best in the mountains, another at the seashore, a third at a sulphur spring, and a fourth in a large city. In general it may be said that a somewhat cold climate is usually preferable. 


\section{VIII.}

\section{THERAPY OF DISEASES OF THE LUNGS}

\section{THERAPY OF CROUPOUS PNEUMONIA.}

Protective Serum.-As we know that croupous pneumonia is an infectious disease caused by definite micro-organisms, the ideal therapy would consist in the administration of a protective serum obtained from men and animals immune to, or immunized against, the disease. Pässler has recently obtained hopeful results from the use of Römer's pneumococcus serum (Merck). He injected 10-30 c.c. (1/2-1 oz.) in severe cases, from one to four times, and reports an unmistakably good effect on the progress of the disease. If these results are confirmed, much will be gained in the treatment of pneumonia. ${ }^{1}$

Till then we must be content with knowing that three-fourths of all the cases run a short typical course, and recover without any interference on our part. For the remaining one-fourth we must use, aside from the serum treatment, which has not yet been sufficiently tested, what therapeutic means are at our command. [I have found no convincing evidence to show that any method of treatment, medicinal or otherwise, has the slightest influence in aborting or shortening the disease.-Ed.]

Therapy of Typical Pneumonia.-In general, if the patient is otherwise sound and healthy, no active therapy is needed during the regular clinical course of the disease.

If at the onset the patient complains of a severe stitch in the side, we may give a hypodermic of morphine $0.008-0.015$ G. (gr. $1 / 8-1 / 4$ ), and if this is insufficient, apply a leech, an ice-bag, or dry cups locally. A mustard plaster on the chest is not advisable, as it restricts the patient's movements and hinders his sleeping. [I occasionally employ flaxseed and mustard in poultices $(4: 1)$ where local cold does not relieve the pain, and often with very good results.-Ed.]

The author strongly opposes the rather common practice of giving large doses of digitalis, believing that such treatment is useless and even dangerous to the heart. Our treatment must be partly expectant, partly symptomatic. During the first days of a typical case we may give dilute hydrochloric acid 1 G.: 180 c.c. of water (15 min.: 6 oz.), or the tincture of quinine (Br.), one teaspoonful in 180 c.c. $(6 \mathrm{oz}$.

${ }^{1}$ [It is hardly necessary to add that the author refers only to cases of pneumonia in which the pneumococcus is plainly the cause, as made evident by the sputum examination. In numerous cases of streptococcus pneumonia a streptococcus serum has been reported of assistance in combating the disease.-Ed.] 
of water. Large quantities of alcohol are not in place, though 500-750 G. (1-1 $1 / 2$ pt.) of light wine a day may be allowed. [Liquor ammonix acetatis 15 G. $(1 / 2$ oz.) every 4 hours may be administered as a mild stimulant and as a diuretic (Loomis).-Ed.]

Delirium.-The ordinary delirium of fever is quite frequently met with, especially when the temperature takes a considerable rise suddenly. In such cases, if the temperature is very high, over $105.8^{\circ}$, we give a cold bath $\left(57^{\circ}\right)$ and splash the neck with water as cold as we can get it. If necessary, these baths may be repeated every two hours, an ice-bag being kept on the head during the intervals. If the temperature is not quite so high, it will be enough to pour cold water over the patient, using the ice-bag as before. If the delirium is of a purely toxic character, the bath should be tepid, and the water poured over the patient less cold than in the other cases. If it arises from inanition, we endeavor to furnish a better blood supply to the brain by employing heart stimulants (ether, camphor, alcohol) ; while if it is due to meningitis, we are practically helpless, although we may try cold applications to the head, lumbar puncture, and injections of morphine. [In mild delirium, Aufrecht recommends the hypodermic use of hydrochlorate of quinine, 0.5 G. $(71 / 2$ gr. $)$. Such a dose requires half an ounce of water for its solution. One such dose, or if necessary repeated at the end of 24 or 48 hours, is usually sufficient.-Ed.]

Sleeplessness.-Sleeplessness is often present at the beginning of the disease, due simply to the high fever. If this can be reduced, the sleeplessness will disappear. Cold applications to the head may be tried, and, in extreme cases, very small doses of narcotics (trional, veronal, or morphine).

Pleural Pain.-Pleural pain, often of a severe type, and the resulting dyspnœa are indications for small injections of morphine, $0.005 \mathrm{G}$. $(1 / 13$ gr.) not over $0.02 \mathrm{G}$. ( $1 / 3 \mathrm{gr}$.) a day. This may be injected in one or more painful spots in the thorax. Hot or cold applications may follow. Leeches and dry cupping are not so effective, while mustard plasters and cantharides blisters should be avoided (see p. 312). [Strapping the chest carefully for a day or two will frequently relieve the pain and so prevent the use of drugs.-Ed.]

The Hastening of the Absorption of the Exudate.-We have no specific means of hastening the absorption of the exudate. Hot, wet compresses on the chest do more good than anything else. The patient should also, several times a day, take 20 deep breaths in succession, lying on his sound side. As a prophylactic measure against the decomposition of the exudate remaining in the lung, 10-20 drops of oil of turpentine daily may be given in milk.

The Crisis.-With the critical fall of temperature, symptoms of heart weakness often appear, on account of the depressing effect of the fever on the heart. Although such symptoms disappear of their own accord, yet the administration of stimulants is advisable (heavy wine, Hun- 
garian, Tokay, port, sherry, mulled wine, champagne, whisky, brandy or rum with hot water or tea, and black coffee).

The fall of the temperature does not mean the end of the pathological process. Five days or more are generally needed for complete resolution, the disappearance of resonant râles, and the return to normal conditions as shown by percussion and auscultation.

Expectorants.- To aid in getting rid of the secretion in the lungs and bronchi, some expectorant, such as antimony, is sometimes given a day before the expected crisis (on the fourth or sixth day). The author has never found this efficacious, and in some cases he considers preparations of antimony directly injurious to the heart and digestive tract. Sometimes, even if an expectorant is used, the pneumonic exudate is absorbed with little or no expectoration. Further, without the use of an expectorant, the patient coughs up great masses of a muco-purulent sputum. Again, the infiltrate may remain in the lung and a chronic pneumonia develop, even though an expectorant is assiduously administered. It, therefore, seems useless to prescribe an expectorant either before, during, or after the crisis. It is naturally irrational to give one during the height of the infiltration process, when the exudate is solid. [The employment of expectorants during the course of a pneumonia is, in my judgment, very poor treatment. Except in cases of so-called senile pneumonia accompanied by a bronchitis with considerable secretion, which the patient is unable to raise, I never use them.-Ed.]

Hygiene.-Not much remains to be done in a typical case of pneumonia. As in all fevers, the mouth should be carefully cleansed and kept clean (p. 321). The room should be freely ventilated, even if a direct current of air is necessary. As long as the fever keeps up, the patient cannot eatch cold. As soon as convalescence begins, however, he is exceedingly sensitive to drafts and must be earefully protected. [Cold fresh air in the treatment of pneumonia has recently been very strongly urged especially for children. It is only necessary to try its effects a few times to become convinced of its value in reducing many of the distressing symptoms, especially the rate of respiration. It is well to caution the nurses about their own clothing, for I have several times failed to continue the cold air treatment because it was too severe for the nurses. Most old people and some adults, however, feel better and do better in a room with warmer $\left(70^{\circ}\right.$ F.) moist air.-Ed.]

Diet.-The diet should from the beginning be as nutritious as possible. In most cases the appetite is so poor that the patient will only take milk (sometimes with hygiama, sanatogen, or eucasin), broths (perhaps with Puro or an egg), [jellies and gruels. Most alcoholics require large quantities of liquid nourishment. It helps their thirst.-Ed.] But if he wishes to eat, there is no reason for feeding him differently from a patient with acute bronchitis of the fine tubes (see p. 293).

In short, typical pneumonia requires but little treament; the atypical cases, presenting at least a doubtful prognosis, however, do require our attention. 
Therapy of Atypical Pneumonias. - A pneumonia may prove fatal from various causes: (1) The heart may give out either from (a) the increased mechanical work required by the contraction of the pulmonary circulation on account of pulmonary infiltration, or from $(b)$ the injury done the heart muscle by the high fever; (2) the blood may fail to receive enough oxygen on account of the reduction of the normal pulmonary area, or on account of heart-weakness; (3) the patient may, so to speak, have a hemorrhage into the lungs on account of the rapidly accumulating infiltration and the heart may be almost pumped dry; (4) the general intoxication may reach a fatal intensity; (5) a fatal complication may occur (meningitis, endocarditis); (6) some peculiar condition of the individual may cause a lethal ending. Each of these possibilities must be taken into account in a concrete case.

Cardiac Insufficiency: Stimulants.-The heart is the organ that most frequently requires our attention. As a stimulant we may use large amounts of concentrated alcohol, 100-150 G. (3-5 oz.) of whisky, brandy or rum for men, 50-60 G. ( $1 / 2-2$ oz.) for women. Digitalis (as the infusion or dialysed or digalen), caffeine, or the two in combination, sometimes with the addition of ammonia ; or strychnine hypodermically, are all available stimulants for the right heart. Strychnine internally, as far as the author's experience goes, is generally not so serviceable as a hypodermic; $0.005-0.006 \mathrm{G}$. $\left(1 / 1^{-1} /{ }^{-10} \mathrm{gr}\right.$.) of the nitrate of strychnine may be given daily in 2-6 doses. [I agree with the author in relying only upon subcutaneous administration when I administer strychnine in pneumonia ; but I employ it less as a routine stimulant than formerly. Cabot's observations go to show that it does not increase the blood pressure. I am convinced that it increases the patient's restlessness and respiratory rate in some cases.-Ed.]

Digitalis should be given $(a)$ from the beginning in every pneumonia when the heart muscle cannot be trusted, and $(b)$ should be begun at any point in the course of a pneumonia when any signs of cardiac weakness are noted, or $(c)$ even when there are manifest only abnormal claims upon the heart (abnormally full, hard pulse, excited heart action, strong precordial pulsation, severe dyspnœa), for it is better to anticipate cardiac weakness than it is to wait for its appearance. [Fränkel administers 3-4 G. (45-60 gr.) of digitalis daily for the first three days of the disease. When $I$ have employed digitalis on account of some one of the above indications I have given large doses.--Ed.]

In cases of acute heart failure, we may try repeated hypodermics of camphor or of ether, wrap the extremities in hot cloths, rub them with hot alcohol, and apply hot water bottles, with the aim of improving the peripheral circulation.

Venesection.-On the contrary, if the patient is strong and full blooded, with full bounding pulse, throbbing carotids, livid, bloated face, marked dyspnœa, and rapidly progressing pulmonary involvement (acute pulmonary œdema), the heart must be relieved by reducing the 
amount of blood. In such comparatively rare cases, venesection is certainly indicated. It should, however, not be attempted if at the same time the patient is suffering from repletion of the pulmonary circulation, and shows decided cyanosis, with the cervical veins markedly distended and the arterial pulse small. Even if venesection should relieve such a condition, the relief would only be temporary, and repeated venesection would be absolutely contraindicated.

Antipyresis.-A previously sound heart may be injured by continued high fever, [perhaps by the accompanying toxæmia-Ed.,] so that measures should always be taken against hyperpyrexia (over $104^{\circ}$ or even, if long continued, over $103^{\circ}$ ).

Drugs.-Among medicinal agents the author gets the best results from the use of quinine, in doses for adults ranging from 1-2 G. (15-30 gr.) a day. For repeated administration it can be given in enemas or suppositories whenever the temperature reaches $104^{\circ}$, usually best in the evening corresponding to the maximum. The temperature drops gradually and stays at the low level for 15-20 hours.

B Quininæ hydrochloratis................ $1.00 \ldots$. gr. xv

Ext. belladonnæ..................0.04-0.05.....gr. $\frac{2}{3}-\frac{3}{4}$

Ol. theobromatis........................

M. fiat suppos. Dent. tal. suppos. no. iii.

Or :

Sig.-As directed.

B Quininæ hydrochloratis............1.00-2.00.....gr. $\mathrm{xv- \textrm {xxx }}$

Tinct. opii...................60 $0.60 \ldots \ldots \mathrm{m}-\mathrm{xv}$

Aq. dest.,

Mucil. acaciæ................āā $50.00 \ldots \ldots$ xiv

M. et sig.-As an enema.

Quinine is the safest antipyretic in this condition, and possesses the added advantage of stimulating the heart. [Aufrecht warmly advocates the routine hypodermic administration of $0.5 \mathrm{G}$. $(7 \mathrm{gr}$.) of hydrochlorate of quinine. He injects it in the late afternoon, corresponding to the maximum temperature, rarely finds it necessary to repeat the dose within 48 hours, and generally requires it but two or three times. He believes that it acts more as an antiseptic than as an antipyretic. His statistics are hardly over a sufficiently long period to be convincing.-Ed.] The author has given up the use of antifebrin, having seen a case of heart failure result from the administration of $0.25 \mathrm{G}$. ( $4 \mathrm{gr}$.) of the drug in a patient sixty years old. Of the other antipyretics (see p. 350), antipyrine, phenacetine or pyramidon may be used with comparative safety. [If in doubt combine them with caffeine or better omit them.-Ed.] Lactophenin, on the other hand, is not a good antipyretic in pneumonia, as it has a deleterious influence on the hæmoglobin.

Cold and Tepid Baths.-Much more important than any of these drugs, however, is the use of [tepid-Ed.,] cool, and cold baths, the principles underlying which have been especially well formulated by 
Jürgensen. With their aid we can reduce the fever, stimulate the respiration, lighten the work of the heart, and produce a favorable effect on the symptoms of severe intoxication, should they be present.

Whenever the temperature goes above $103^{\circ}$ or $104^{\circ}$ the patient is put in a bath of $81^{\circ}-75^{\circ}$, which is then reduced to $72^{\circ}$ or lower. The patient remains in the bath 20-30 minutes (unless a severe chill occurs), the water being continually splashed over him. He is then put back into bed, wet or only slightly dried. If he does not get warm of his own accord, a hot-water bottle may be used. Such baths are rather too severe for old people. For them the water may be tepid $\left(82^{\circ}-86^{\circ}\right)$ and the duration of the bath only 15-20 minutes. They should generally be given in the morning, but if necessary may be repeated 3-4 times a day. In general, however, all procedures which tend to draw heat from the body are contraindicated in old persons. Finkler uses baths of $73^{\circ}$, one at 5-6 A.M. and one at 5 P.M., the resulting vaso-paralysis of the cutaneous vessels producing a marked fall of temperature and a feeling of comfort. For half an hour afterwards the patient is wrapped in a blanket.

If the temperature mounts very high, and the individual is young and strong, a really cold bath (even as low as $45^{\circ}$ ) is preferable; it should not last more than 10 minutes.

If our particular aim is to relieve dyspnœa or severe symptoms of general toxæmia, moderately tepid baths $\left(80^{\circ}\right)$, followed by cold water poured over the body, are the most desirable.

Contraindications for Baths.-If the cardiac insufficiency is pronounced, cold baths are contraindicated, as the sudden demand upon the weak heart made by the rise of pressure in the peripheral vessels may be followed by collapse. The better supply of blood to the heart, as a result of the contraction of the peripheral vessels and the greater amount of blood remaining in the aorta and coronary arteries, is of no avail when once the muscle is badly degenerated. A heart lesion or sclerosis of the coronaries is also a contraindication for cold baths.

Substitutes for Cold Baths.-If it is impracticable to give a cold bath, other hydropathic procedures may be substituted, as already enumerated in the chapter on acute bronchitis (see p. 291). Either four or five sponge baths during the 24 hours with cold water, the body not being rubbed vigorously afterwards, or cold compresses to the chest (renewed every 3-10 minutes until the temperature drops to at least $102^{\circ}$ ) seem particularly efficacious. [Except for temperatures above $105 \mathrm{r} / 2^{\circ}$ I prescribe only cold sponging or cold packs; and frequently I obtain equally good results by sponging with tepid water. I do not regard fever alone as an indication for us to interfere and attempt to reduce the temperature. I do not feel certain that the ordinary temperature of pneumonia is not a healthy and natural reaction of the body to the disease. The restlessness, delirium or stupor, and the rapid respiration often improve after sponging with tepid or cool water or after cold 
packs. A full bath necessitates considerable movement and more exertion, or at least nervous unrest, than I like for patients with pneumonia. Before any of these procedures it is often advisable to administer a hot alcoholic drink.-Ed.]

Hot Baths.-Hot baths also may often be used with great advantage. For a discussion of their effect and the method of giving them, see p. 293. The copious sweating thus produced aids in eliminating the toxins, while the undue concentration of the blood is prevented by giving large amounts of water. In extremely severe cases of toxæmia in pneumonia the injection of salt solution should also be tried.

The lack of oxygen for the tissues is helped by the use of tepid baths and also by the inhalation of oxygen, a procedure which has given the author very satisfactory results.

Infusions and Transfusions.-If we have a case of rapid and extensive involvement, in which we suspect ischæmia of the heart as a result of the withdrawal of great quantities of serum to form the exudate, the only procedures possible are blood transfusion, an intravenous injection of salt solution, and the subcutaneous injection of one of the so-called "artificial serums," which are in great favor in France. The following is an example:

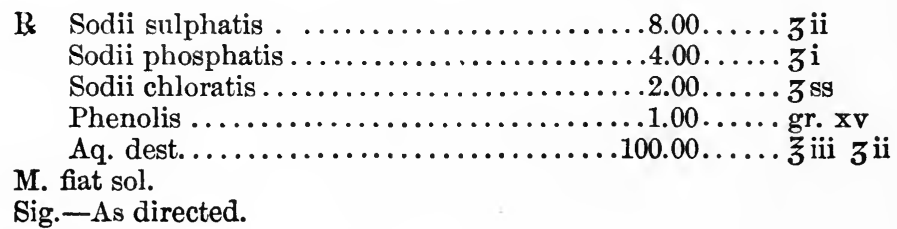

About 10 c.c. $\left(2 \frac{1}{2}\right.$ dr.) at $100^{\circ}-104^{\circ}$ is injected two or three times a day. Its effect is due to its stimulative action rather than to its supplying fluid to the body tissues. Sometimes $0.001-0.002$ G. (1/60-1/30 gr.) of strychnine is added (e.g., 1 c.c. of a 0.01:10 solution). Another combination would be:

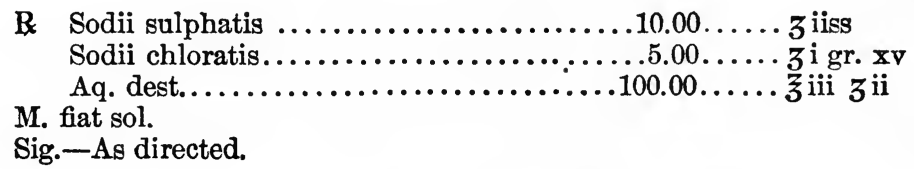

$10-50$ c.c. $(2 \mathrm{r} / 2 \mathrm{dr} .-1 \mathrm{r} / 2$ oz. $)$ at $100^{\circ}-104^{\circ}$ may be used for an injection. The author has repeatedly tried $200-300$ c.c. $(6-10 \mathrm{oz}$.) of a 0.6 per cent. salt solution, injected under the skin on the chest, with the purpose of attacking severe intoxication, of raising the tension of the peripheral pulse, of increasing diuresis, and of strengthening the nervous system. 
In a number of cases he has tried fluoroformol (as recommended by Stepp) but without any good results.

Therapy of the Complications of Pneumonia.-The complications of pneumonia demand speedy treatment. An extensive acute deep bronchitis, endocarditis of the aortic, mitral, or tricuspid valve, and meningitis must all be handled as if they were independent affections. The last, it must be confessed, is even more unsatisfactory to treat than the epidemic form. An acute, often hemorrhagic nephritis does not constitute a contraindieation for baths, although in general these will be hot rather than eold. Many pneumonias are aceompanied by obstinate symptoms of disturbances of the digestive tract, particularly, by frequently painful diarrhœa, slight meteorism, and marked splashing in the intestine and a thickly coated tongue. It is generally not advisable to check the diarrhœa, even if it is annoying and painful, as it is evidence that toxins are being excreted through the intestine. If it is necessary to take any measures against it, the author uses only intestinal antiseptics, such as bismuth. In case of pronounced gastric symptoms (anorexia, coated tongue, icteric coloring without septic icterus) the old practice was to induce vomiting by giving $1.5-2.0$ G. $(20-30 \mathrm{gr}$.) of ipecae, divided into three doses, within 10 minutes. The author has never been obliged to proceed against the dyspepsia directly, although it may become necessary to treat it symptomatically with chloroform water, alkaline mineral waters, dilute hydrochlorie acid, ete. It goes without saying that in extreme metastases (inflammation or suppuration of the cellular tissue, lymph glands, and salivary glands) recourse must be had to surgical interference.

Conditions Pre-existent Influencing Course of Disease: Heart Disease.-Individual constitutional. peculiarities or preëxistent diseases may demand a special therapy. For instance, a heart lesion, either valvular or muscular, a kyphoseholiosis, or a bilateral obliterative pleurisy may have so weakened the heart that it is incapable of performing the additional work required of it in pneumonia. In consequence of the stagnation of the blood in the pulmonary circulation, the right heart is threatened with paralysis. With such a patient cardiac stimulants are indicated from the beginning (digitalis, caffeine, strong alcohol). Inhalation of oxygen is also useful, especially in kyphoseholiosis.

A single dose of calomel $0.5 \mathrm{G}$. (7 gr.) is advisable at the beginning of the disease, sometimes repeated, divided into two doses, the next day, in order to relieve the circulation in the liver and to stimulate the kidneys.

Obesity.-In the case of an obese individual, we employ similar measures, except that we substitute for digitalis some other stimulant. such as strophanthus, strychnine, sparteine, or, if the cardiac weakness is severe, caffeine or theobromine.

Diabetes or Bright's Disease.-If the patient is suffering from diabetes or Bright's disease, the ordinary treatment for all severe cases 
of pneumonia need not be modified, except that alcohol must be given with caution in nephritis. The author, personally, is inclined to believe, however, in subcutaneous injections of salt solution or of the artificial serum, as well as in the intravenous infusion of salt solution, and the inhalation of oxygen.

Alcoholism.-In treating alcoholies, the quantities of alcohol given must be greatly increased. Instead of 100-150 G. (3-5 oz.) we give 150-200 G. (5-7 oz.) of rum, brandy, or whisky, or a tablespoonful every hour. If wine is used instead, the quantity must of course be increased. The patient must be kept as well nourished as possible, with milk, soup with the yolk of egg added, meat preparations, beef juice and beef tea. Camphor, digitalis, and hypodermics of strychnine are often needed. Cold baths are generally considered contraindicated.

Delirium Tremens.-In case the pneumonia induces delirium tremens, we give opium or morphine in full doses, chloral having a certain, though slight, weakening effect upon the heart. We may give a daily dose of $0.01-0.04 \mathrm{G}$. ( $1 / 6-2 / 3 \mathrm{gr}$.) of the extract of opium, preferably in some such mixture as the following:

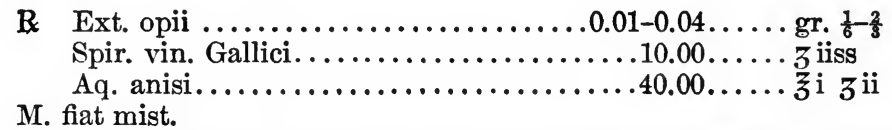

Sig.-To be taken at one time and repeated, if necessary, 2-3 times a day.

Morphine is given hypodermically, $0.02-0.04 \mathrm{G}$. ( $1 / 3-2 / 3$ gr.) a day. Sometimes still larger doses are needed. [It is my practice in treating pneumonia in alcoholics to begin immediately the administration of alcohol in the form of whisky well diluted with water and sometimes with milk or eggnog. With this method I believe that delirium tremens is sometimes avoided. At the first sign of wandering I order a cool or cold pack, or even a cold bath. Extra feeding, especially with a large proportion of liquid food including beef juice and hot broths with egg and well seasoned with cayenne pepper, is of assistance. I have never been impressed with the value of ergot hypodermically. Hyoscine in my hands has proved almost as dangerous as helpful, so that for drug treatment I generally rely upon chloral in 1-2 G. (15-30 gr.) doses every hour or two. Strychnine hypodermically is a wise addition when employing chloral. The bromides seem to act so slowly that recently I have not used them much. Paraldehyde, 4-8 G. (1-2 dr.) every hour or two, either alone or combined with whisky, often succeeds where chloral fails. With any plan of treatment, success often depends upon beginning as soon as the first signs of restlessness or wandering are observed.-Ed.]

Protection Against Recurrence.-As one attack of pneumonia predisposes a person to another, every precaution should be taken to guard against it. In particular the mouth should be carefully and frequently 
disinfected with thymol, peroxide of hydrogen, or some such preparation. [An excellent mouth-wash and gargle is the following copied from Wadsworth:

R Sodii chloridi...................... $8.00 \ldots \ldots$ ii

Sodii bicarbonatis..................... $3.00 \ldots \ldots$ gr. xl

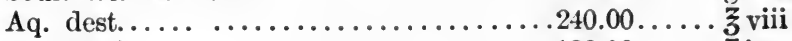

Glycerini........................... $120.00 \ldots \ldots$.

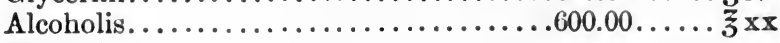

Thymol.,

Mentholis....................... āi $0.30 \ldots . .$. gr. iv.

Ol. gaultherix........................ $0.80 \ldots$ xii

Ol. cinnamomi.......................50....m m viii

Ol. eucalypti.............................m xx

Tinct. cudbear....................24.00.... 3 vi

Tinct. krameriæ.................... $8.00 \ldots \ldots$ ii

M. fiat mist.

Sig.-To be used as a mouth-wash.-Ev.]

\section{THERAPY OF BRONCHOPNEUMONIA.}

ACUTE Bronchopneumonia.-As acute bronchopneumonia is almost always due to a preceding bronchitis, it is evident that its therapy is, in a great measure, identical with that of the latter disease, while in other respects it is like that of lobar pneumonia. It is such an exceedingly dangerous affection in young children and in the aged that it requires the most careful treatment.

Hydrotherapy.-Similar hydropathic measures to those already described in the therapy of bronchitis and of lobar pneumonia are in order: simple tepid baths in the aged, tepid baths followed by cold water poured over the body, douches on the neck, and simple compresses on the chest. All these are designed not so much to reduce the fever, as to assist in combating the pathological process going on in the lungs by aiding the pulmonary circulation. Henoch has particularly recommended one procedure that has not yet been described; e.g., a cold compress on the chest. A cloth wrung out in water at $50^{\circ}-60^{\circ}$ and folded several times is wrapped around the thorax, covered with dry muslin, and held in place by a binder. As soon as it begins to become warm (20-30 minutes on an average) it is replaced by a fresh cold one. In this way the blood is drawn from the thoracic organs to the skin and the work of the heart lightened, the respiration naturally becoming deeper and more rapid. These cold compresses are indicated rather for children than for the aged, on account of the danger to the heart and arteries in the latter, a danger which in general contraindicates all applications of cold.

Heart Stimulants.-Such great demands are made upon the heart that one of the most important indications, particularly in old people, is to preserve good cardiac action. As in a typical lobar pneumonia. we may give alcohol from the very beginning. A pleasant form in which 
to administer alcohol to children is the following, recommended by Comby :

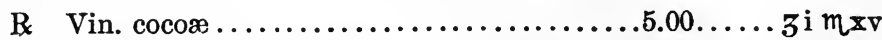

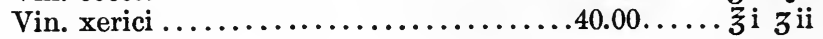

Aq. dest.

Syr. aurant ..................āā $20.00 \ldots \ldots 3 \mathbf{v}$

M. fiat mist.

Sig.-One teaspoonful every hour.

Signs of heart failure may oblige us to use heart stimulants, as in lobar pneumonia (see p. 315).

Inhalations of oxygen are also advisable, especially in the aged, as well as the saturation of the air in the sick-room with steam.

Antipyresis.-High fever may be kept down by hydrotherapy, or by the use of quinine or antipyrine.

Hygiene.-As in capillary bronchitis, the diet should be as nutritious as possible, although generally no solids can be permitted, on account of the fever. The sick-room should be large and kept well ventilated. The position of the patient must be frequently changed. An infant may be carried about the room in the nurse's arms. [The same indications for the cold air treatment exist with limitations as in lobar pneumonia. -Ed.]

Medication.-The medication is similar to that in acute bronchitis with secretion. Narcotics are to be given only when the cough becomes so severe as to deprive the patient of sleep or to bring on considerable vomiting. Expectorants are indicated, particularly ipecac or euporphine.

In case of pleural pain, we may employ local cold applications, cupping, mustard plasters, or local applications of analgesics (see p. 363).

CHRONIC BRONCHOPNEUMONIA.-Occasionally an acute attack of bronchopneumonia leaves behind it a chronic form of the affection. This must be treated in very much the same way as chronic bronchitis with secretion. The balsams or ethereal oils should be given internally, and the patient should be kept as well nourished as possible. Cod-liver oil or artificial food preparations are sometimes useful. The use of the sulphur springs, or of sulphur water at home, $1 / 2-1$ glass in the morning, is to be advised. Whatever climate proves favorable for the bronchitis may be recommended.

\section{THERAPY OF INTERSTITIAL PNEUMONIA (PULMONARY SCLEROSIS).}

Interstitial pneumonia arises (1) from mechanical lesions in the lungs caused by the inhalation of injurious articles, or (2) as a consequence of capillary bronchitis, of an acute pneumonic infiltration of the lungs, or of pleurisy. As prophylactic measures, then, we must guard against the inhalation of injurious dust, and must do everything possible 
to promote the absorption of the exudate in lobar or bronchopneumonia ( see p. 313). The treatment of pleuritis will be discussed a little later.

Care of the Heart.-When once the disease has been established, the heart must be carefully watched and at the proper time strengthened in every possible way to enable it to meet the abnormal demands upon it.

Therapy of the Accompanying Bronchitis, Bronchiectasis, and Emphysema.-The pulmonary conditions themselves cannot be helped, but we must combat the concurrent chronic bronchitis, frequently purulent, the occasional bronchiectasis, and the pulmonary emphysema. For the first two the treatment has already been given,-balsams, alkaline mineral waters, sulphur waters, above all oil of turpentine (10-30 drops daily for a considerable period). For emphysema see p. 328.

Some authorities recommend intratracheal injections, usually of menthol :

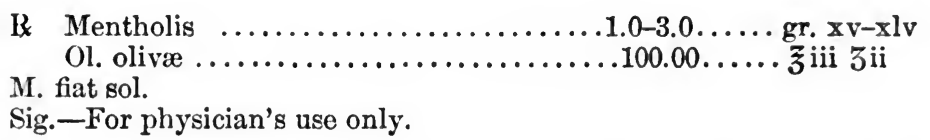

Hemorrhage.-Hemorrhages may be frequent, and often very severe and even fatal. For a full account of the methods of treating see the chapter on tuberculosis (p. 344). In interstitial pneumonia they are due to the rupture of the dilated arteries, which, on account of the stiff connective tissue in which they are embedded, cannot contract. For this reason the formation of a thrombus is hindered, so that the only way in which the bleeding can be stopped is by the stagnation of the blood in the bronchial tree between the lungs, and the consequent direct pressure upon the ruptured vessels. Measures should, therefore, be taken to prevent as far as possible the expulsion of the blood (Jürgensen). For this purpose large injections of morphine may be given, 0.02-0.03 G. $(1 / 3-1 / 2$ gr. ) several times a day; later a daily amount of $0.03-0.05 \mathrm{G}$. $(\mathrm{I} / 3-3 / 4 \mathrm{gr}$.$) . [Although I agree with the therapy recommended, I can$ not concur in the author's explanation of its action.-Ed.]

\section{THERAPY OF HEMORRHAGIC INFARCT, OF ABSCESS, AND OF GANGRENE, IN THE LUNGS.}

Therapy of Hemorrhagic Infarct: Etiology.-The treatment of a hemorrhagic infarct in the lungs depends upon its cause. Emboli of the pulmonary arteries are caused (1) by thrombi of the peripheral veins, or (2) by thrombi of the right heart, generally in consequence of cardiac weakness, less often from endocarditis in the right heart. A thrombus of the pulmonary arteries or veins is developed in consequence of cardias weakness, either primary or secondary. Among the thrombi of the peripheral veins, those due to phlebitis-phlebitis of veins of uterus, of those of the lower extremities, of the hemorrhoidal veins, and of the cerebral 
sinuses, and phlebitis accompanying fractures-are much more apt to produce emboli than the so-called "marantic" thrombi, whose slow development makes their position more secure.

Prophylactic Therapy.-Therefore, our first prophylactic measure must aim at a thorough organizing of the thrombus, particularly in the case of phlebitic thrombi. Absolute rest and immobility of the part affected are indicated. All massage must be omitted, the extremities kept in a horizontal position, and all energetic movements of the abdomen avoided, the bowels kept open, and any severe coughing stopped. If it is an extremity that is affected, there must be no sudden quick movement of the whole body. These precautions must be kept up until all symptoms of obstruction have disappeared-cyanosis, œdema, and cold in the extremities. This will take on an average about a month.

When once a hemorrhagic infarct is present, the treatment indicated is directed against (1) the dangerous attacks of syncope so often occurring, and (2) the pulmonary symptoms.

Attack of Syncope.-The treatment of syncope consists in placing the person flat, in rubbing the skin if possible with alcohol, in the injection of ether and camphor together or in immediate succession, and in artificial respiration.

Pain in the Chest-Dyspnoa-Cough.-For the frequently severe pain in the chest, the marked dyspnœa, and the severe cough, a hypodermic of morphine $0.01-0.02$ G. ( $1 / 6-1 / 3$ gr.) may be used. For the dyspnœa we may also employ local revulsives, mustard plasters, dry cupping, and sometimes venesection and removal of 200-300 c.c. (6-10 oz.) of blood, in case the person is young and strong and the infarct is not extensive, particularly if there is danger of an acute pulmonary œdema. Inhalation of oxygen is also useful.

For extensive hæmoptysis, see p. 60.

If the infarct of the pulmonary arteries is caused by thrombosis of the right heart, generally of the auricular appendage, digitalis is indicated, provided cardiac weakness is present.

Therapy of Abscess in the Lung.-If the embolic material comes from a phlebitic thrombus in the radicles of either vena cava, or from a bacterial endocarditis of the right heart, the hemorrhagic infarct usually develops into a pulmonary abscess. This may also be caused by suppurative processes in pneumonia, by a general pyæmia, by trauma, or by aspiration of a foreign body. The physician is here almost helpless. The best that he can do is to order inhalations of disinfectants (oil of turpentine, eucalyptol), spraying of the room with antiseptics (2 per cent. carbolic acid solution or 0.1 per cent. thymol solution), the internal use of balsams, and the maintaining of the patient's strength by tonies and suitable food. In case there is but one abscess and that exactly localizable, the foreign body may be removed through the trachea, or pneumotomy be undertaken.

Therapy of Gangrene in the Lung.-Surgical aid is all that can be 
of real use in gangrene. For the medication, see the chapter on putrid bronchitis (p. 303).

\section{THERAPY OF GEDEMA OF THE LUNGS.}

Classification and Etiology.-Pulmonary œdema may be (1) acute and active, caused by sudden paralysis of the musculature of the arterioles of the lung, or (2) chronic and passive, caused by hinderances to the flow of blood from the pulmonary veins into the left auricle. It is true, however, that some passive cases may arise very quickly and sometimes recede as quickly (nocturnal pulmonary œdema in fatty heart (Stoffella), in acute dilatation of the heart from excessive work, and in chronic myocarditis). Also some acute cases run a subacute course. There are three causes of acute pulmonary œdema: (1) the passage of bacterial toxins from neighboring foci of inflammation through local lesions which they have eaused in the vessel wall into the plasma and red corpuscles (inflammatory cedema); (2) the paralysis of the nerves of the vessels, $(a)$ directly, (acute pulmonary œdema in hysteria and in all possible diseases of the aorta, perhaps also angioneurotic odema), (b) indirectly, by auto-intoxication or intoxication of the organism, in which anatomical alterations of the vessel walls and abnormalities in the constitution of the blood play a part, (true, toxic-uræmic pulmonary œdema, acute pulmonary œedema in uric acid diathesis and in various infectious diseases, such as acute articular rheumatism, typhoid, measles, infiuenza, diffuse infectious bronchitis, acute alcohol poisoning, and experimental iodine poisoning) ; (3) a purely mechanical action, the sudden cessation of a pressure to which the pulmonary vessels have been subjected and the consequent rush of blood. into vessels which have not been supplied with sufficient blood to keep them in good condition, and whose walls, therefore, cannot hold their content, (acute pulmonary œdema after aspiration of the thorax).

Therapy of an Active Pulmonary CEdema.-In case of an attack of acute active œdema, no matter from which of these canses it arises, measures for relief must be taken immediately, the most efficacious being to withdraw $200-300$ c.c. $(6-10$ oz. $)$ of blood by venesection. It is an interesting fact that this condition may be greatly relieved by a coincident hemorrhoidal bleeding.

We also employ our quickest heart stimulants (ether, camphor, strong alcohol) and dry cupping or extensive mustard plasters at different points on the lower extremities or on the thorax. To strengthen the weak vessel walls so that their contents may not transude, ergot may be given :

R Ext. ergotæ fld. ........................ $m$ xlv

Spir. etheris......................... $\mathrm{i}$ i $\mathrm{xv}$

Aq. cinnamomi.................. 150.00...

M. fiat mist.

Sig.-A tablespoonful every hour or half-hour. 
More frequently we use lead acetate:

R Plumbi acetatis $\ldots \ldots \ldots \ldots \ldots \ldots \ldots \ldots .0 .02-0.03 \ldots \ldots$ gr. $\frac{1}{3}-\frac{1}{2}$ Sacchar. lactis ...................... $0.30 \ldots \ldots$ gr. ivss

M. fiat pulv. no. i. Dent. tal. pulv. no. xx.

Sig.-One powder every two hours.

If with the symptoms of cedema, large bubbling râles are also present, indicating a collection of fluid in the larger bronchi, expectorants are indicated. If the acute odema is caused by bronchial catarrh and the heart is normal, a single large dose of ipecac, repeated if necessary in a quarter of an hour, may be given as an emetic, 1.0-1.5 G. (15-20 gr.) of the powder.

Therapy of Chronic Pulmonary Edema.-In case a subacute or chronic œdema can be traced to some of the causes already given for acute œdema, (e.g., true uræmic œdema in parenchymatous Bright's disease), it is to be treated by the methods already indicated. But the majority of chronic cases are due to heart weakness (e.g., subacute or chronic œdema in Bright's disease in consequence of the insufficiency of the hypertrophied and dilated heart), so that our therapy must in general be aimed at strengthening the heart (digitalis, strophanthus, caffeine, alcohol, inhalations of large quantities of oxygen, dry cups on the chest, purges).

Sometimes an immediate puncture of the abdomen, in case ascites is present, will prove very successful. The author agrees with Pribram in believing it probable that by the removal of the peritoneal transudate, the intra-abdominal veins are so relieved that they can accommodate greater amounts of blood and so afford relief to the heart and pulmonary circulation.

The administration of iodides or bromides in passive œdema of the lungs is contraindicated, since they increase secretion and augment the fluid which it is desired to remove.

\section{THERAPY OF EMPHYSEMA OF THE LUNGS.}

When we consider that emphysema is due to the rarefaction of the elastic lung tissue and the destruction of numberless lung capillaries, it is evident that we can never expect to achieve a veritable cure. Socalled specifics (quebracho and quillaja) are useless.

Assistance of Respiration.-In an uncomplicated case, considerable improvement may, however, be obtained by lightening the work of respiration. This may be accomplished by at least two or three months' use of the pneumatic chamber or of a portable apparatus which insures inspiration of condensed air, and expiration into rarefied air. Expiration into rarefied air, however, though formerly considered of prime importance, has been proved not to be an essential factor, since little more air is expired than under normal conditions. The pneumatic chamber has 
a very favorable effect both on the emphysema itself, and on the bronchial catarrh that almost always accompanies it. Under the increased pressure the permanently hyperæmic bronchial mucous membrane becomes relatively ischæmic, and the atonic catarrh less marked. The good effect on the emphysema itself is due to the fact that expiration into the condensed air leaves the lungs somewhat more expanded than usual at the beginning of inspiration, so that the air during inspiration penetrates more easily and deeper into the lungs. The relief thus afforded the respiration becomes permanent if the pneumatic chamber is used for weeks at a time, as the elasticity of the lungs, in spite of the deepened breathing, suffers no loss. The treatment should be carried on for about two hours, provided the pressure is raised half an atmosphere.

Respiration may also be aided by mechanically expelling the residual air that would otherwise be left in the lungs. The simplest method is to press on the thorax with the hands (Gerhardt). But this procedure, if energetically carried out, may induce slight hæmoptysis, giddiness, and muscular tremor. The use of a "Breathing Chair" or a "Breathing Belt" is better. The first consists essentially of a mechanical contrivance by which the movement of the patient's arms causes the compression of the thorax during expiration; the second, of a belt around the thorax, which during expiration is filled with condensed air.

Health Resorts in Uncomplicated Emphysema.-In choosing a health resort for an uncomplicated case, we must select one which affords pneumatic chambers or the portable pneumatic apparatus, as well as one sheltered and at a high altitude, so that the air shall be as rarefied as possible (Ems, Gleichenberg, Ischl, Reichenhall). Other places may sometimes be chosen for the sake of their mineral waters, which are used for the purpose of stimulating the intestine and increasing its blood supply, and so favoring the lesser circulation, (alkaline-saline (Marienbad, Tarasp, etc.), common salt (Homburg), and bitter waters). Or the mineral waters may be taken at home or at some mountain resort.

Therapy of the Accompanying Bronchial Catarrh.-The remaining treatment in emphysema is purely symptomatic. One of our first tasks is to combat the almost inevitable bronchial-catarrh. If this is a simple catarrh, either primary or secondary to the emphysema, it should be handled like any other chronic catarrh. It often takes the form of a true catarrhal asthma (abundance of eosinophiles in the sputum); in that case it is treated like nervous asthma, the chief remedy being potassium iodide. Here also belong apparently the considerable number of cases of pulmonary emphysema which are helped by the internal use of arsenic. In such cases and in ordinary bronchial catarrh with emphysema, where a tenacious secretion is accompanied by a severe cough. the author has found the bi-iodide of codeine, $0.02-0.04 \mathrm{G}$. $(\mathrm{r} / 3-2 / 3 \mathrm{gr}$.) a dose, up to 0.15 G. (2 $1 / 4$ gr.) a day, successful. Pyrenol may also be tentatively given. The bronchial catarrh may be due to a weak heart, in 
which case we use heart stimulants or diuretics, as also in all cases of emphysema with cardiac insufficiency, whether or not bronchitis is present.

It is important in each case to decide whether the emphysema or the bronchial catarrh is the more important condition. For the emphysema as such and for most cases where the accompanying catarrh is of the nervous asthmatic type, a high altitude is indicated. But where the catarrh is more prominent than the emphysema and is of a simple bronchial type, a mild climate must be chosen, particularly the south in winter. Alkaline-saline, common salt, and sometimes sulphur springs are also beneficial.

Dyspnœa.-The dyspnœa often accompanying emphysema must also receive an etiological treatment, although occasionally we are forced to the symptomatic use of narcotics. If it is due to the emphysema itself, pneumatic therapy and breathing exercises are indicated; if it is paroxysmal, an antispasmodic therapy is needed. Often it is due to the catarrh or to a weak heart, and sometimes it is of nervous origin induced by aortitis and periaortitis, which, in such a case, we must consider as the cause of the emphysema. Each case must receive its appropriate causal therapy.

Oxycamphor has been warmly recommended from several quarters for use in dyspnœa caused by emphysema, as well as by cardiac insufficiency, tuberculosis, and bronchitis. It is on the market in the form of the 50 per cent. alcoholic solution oxaphor. The dose of oxaphor is 0.5-1.0 G. (7-15 gr.), 1.5-4 G. (20-60 gr.) a day, given either in sweetened water or milk, or on sugar, or in a mixture. The following is a representative mixture:

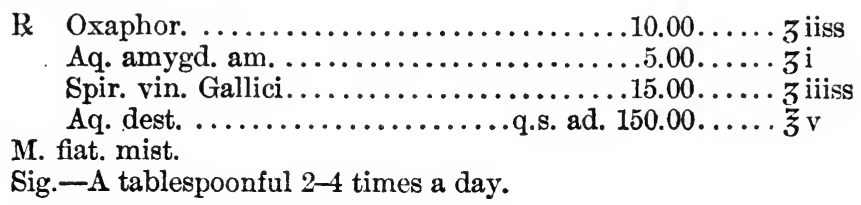

In the author's hands it has never produced more than a slight and temporary effect.

Hygiene.-No special diet is needed. The food should be strengthening and the meals moderate in amount and near together, in order to avoid any overloading of the stomach, high position of the diaphragm, or abnormal distribution of the blood. Only a light meal should be taken in the evening. A satisfactory movement of the bowels should be obtained daily.

Emphysema and Obesity.-When, as not infrequently happens, the emphysema is accompanied by obesity, the treatment demanded by the latter will often affect the pulmonary affection favorably. The loss of intra-abdominal fat allows a greater excursion of the diaphragm, and the gradual removal of fat from the heart increases its contractile power. 
Protection from Bronchitis.-The patient with emphysema must be carefully protected from an attack of acute bronchitis. The same rules for clothing, etc., apply as in protection against recurring bronchitis, but the energetic hydrotherapy designed as a hardening process must usually be omitted, especially considering the ordinary age of emphysema patients.

\section{THERAPY OF PULMONARY TUBERCULOSIS.}

Pulmonary tuberculosis is a curable disease. To effect a cure, two conditions are primarily necessary: the patient must receive the best possible nourishment, and must be placed under the best climatic conditions.

DieT.-The one essential in the diet is that it be as nutritious as possible. To effect this such foods must be selected as shall (1) please the palate of the individual patient, and (2) be, in themselves, strengthening and heat-producing.

With the former of these ends in view we must choose such foods as the patient naturally likes, in as great a variety as possible, and see that they are well cooked and daintily served. Small quantities of brandy or wine may be taken before or during the meal as an appetizer. Five meals a day should be given. A sound digestive tract is, of course, hypothecated.

In order that the diet may be as nutritious as possible, it should be a mixed one. (The chronic cases with little or no fever are those at present under consideration.) Meat must, however, be given the preference, on the basis of the fact, proved experimentally, that meat eaters are less susceptible to tubercular infection than vegetarians. In all cases where the individual has lost the subcutaneous fat, abundant amounts of fat [and carbohydrates-Ed.] should be ingested. The total quantity of food should be large enough to afford more than the number of calories normally needed.

Proteids.-All kinds of meat (preferably with some fat), fish, shellfish, and gelatinous foods may be allowed.

Eggs are exceedingly nutritious, but many individuals are apt to tire of them quickly, so that it is generally wiser to use them abundantly in the preparation of other foods rather than to serve them separately (e.g., in salads, soups, mayonnaise dressings, soufflés, custards, omelettes, etc.).

The vegetables containing a relatively large amount of proteid are especially to be recommended (peas, beans, lentils, rice, etc.).

Fats.-Easily absorbable fats should be chosen (butter, cream, oil, bacon, cream cheese, fatty cheese).

Milk.-Two to 3 pints of milk should also be taken during the day, a glassful twice in the morning, once in the middle of the afternoon, and once just before going to bed. It may be varied by koumyss or kefir (a wineglassful to a cupful 4 times a day). These may be given every 
hour or every half hour at the start, in tablespoonful quantities. Some individuals have an intolerance for milk. Others have to take it at first in very small amounts. Others, usually with atonic stomachs, feel such a sense of repletion after taking a quart a day that they can take nothing else. In the latter case we should abandon the milk. If it induces obstinate constipation, kefir two days old should be substituted.

Alcohol.-Alcohol should in general be prescribed on account of its beneficial effect upon the appetite and the digestive tract, to say nothing of its nutritive value. Contraindications to its use are: marked tachycardia, appearance of tachycardia when alcohol is used, hæmoptysis, increase of cough or laryngeal pain in tuberculosis of the larynx, organic lesions of the digestive tract, inflammation of the kidneys. It is best given in the form of 1-2 wineglasses of table wine during dinner, and 1-2 glasses of good beer during supper. Stronger alcoholic drinks (rum, brandy) are not indicated, unless for the suppression of night sweats (see p. 351) or for the sake of making milk more acceptable. If the individual is habitually dependent upon their use, however, he may be allowed a small glass of cognac at lunch. Beer is to be preferred to any other alcoholic drink, as it contains not only alcohol but also 5-6 per cent. of carbohydrates. Taken in the evening, it often helps in inducing sleep. [As a drug to improve the appetite when that flags, to help nutrition when impoverished by fever, or as a beverage for patients addicted to its use, and in far advanced cases where it gives comfort, I heartily approve of the use of alcohol; otherwise only for special indications and certainly very rarely to promote sleep.-Ed.]

Broths, tea, coffee, and, better, chocolate and cocoa are all permissible in moderate quantities, while it is often advantageous to make use of beef juice, beef tea, meat extracts, peptones, eucasin, sanatogen, and hygiama, [plasmon-Ed.].

Stuffing the patient with milk, raw eggs, and meat powders through a stomach tube is indicated only where an absolute unwillingness to eat, in spite of fresh air and stomachics, is changing a mild case with a favorable prognosis to a serious one. [Supernutrition has, I believe, been overdone in tuberculosis, and too much stress laid upon an excessive proportion of proteids. Without sufficient data for very definite rules an equally balanced, mixed diet daintily served, thoroughly masticated, with a careful individualization of amount and character in each case, will meet the indications best.-Ed.]

Fresh Air Treatment.-The second requisite of successful treatment, the best climatic conditions, must be brought about by giving the patient as much fresh, pure air as possible. In just what way fresh air is so potent in the cure of pulmonary tuberculosis is not wholly clear, but its effect is doubtless partly due to its freedom from bacteria (tubercle bacilli and other bacteria, especially those which cause inflammation and suppuration and so are liable to give rise to a mixed infection), and also from chemical irritants, as well perhaps as to its content 
of ozone. The air indoors, on the contrary, contains less oxygen and more carbon dioxide and other toxic gases, and therefore is distinctly injurious.

Fresh air is needed not only in warm pleasant weather, but summer and winter, day and night, rain or shine. Very strong winds, and-for some patients-mists and fog may, however, make it necessary to keep the patient temporarily indoors.

In general the patient must take his fresh air lying down or sitting. Rest in bed is absolutely indicated where there are present fresh inflammatory processes in the lungs or pleura, generally accompanied by fever. Only when these have disappeared, and when the temperature has remained normal for a week and there is demonstrable no acute exacerbation of the local process, may the patient be allowed mild exercise.

In a Sanatorium.-Naturally this fresh air cure can be best carried on in properly equipped sanatoria. These are usually provided with solaria open on one side to the south, west, or east, and provided with protection against the direct rays of the sun. Here the patient spends most of his time in a reclining chair. At first this exposure is apt to induce sleeplessness, giddiness, general discomfort, and increased cough, so that, at first, the time must be short. If the weather is cold, the patient must of course be well wrapped up and if necessary provided with hot water bottles or hot drinks. In suitably situated institutions this treatment may be kept up the year round.

At Home.-If it is impossible to send the patient to a sanatorium, we must do our best to carry out the treatment at home. A balcony or veranda on the south side of the house may be utilized, or the patient may be kept out in the garden from the time the sun has warmed the air in the morning until sunset. If this is not possible, the windows should at least be thrown wide open, unless there is a violent wind, and the patient, well wrapped up, be exposed to the air. In winter the windows must be kept at least partly open day and night, the patient's bed, if necessary, being protected by a screen.

It must be acknowledged that now and then individuals are found who cannot stand a consistent fresh-air treatment. They cannot lie with an open window at night or in raw weather without acute laryngitis and severe cough. In such cases the patient must be subjected to out-ofdoor air at first only during the day and in pleasant weather; later he may become tolerant of cooler temperatures.

Exercise.-Unless complications (e.g., hæmoptysis) prevent, the patient should be given moderate exercise in the lighter cases when there is no acute exacerbation of the local process, and especially where several months have elapsed since such processes were healed. The amount of exercise should be graded strictly according to the patient's strength and reduced in case it causes any rise of temperature. At first ten minutes' walk on level ground in the morning is enough. As the patient grows stronger this may gradually be increased, and the walk chosen be 
over more hilly ground, until a strong patient may be allowed three hours, half in the morning and half in the afternoon. Under no conditions should he be allowed to exercise hard enough to bring on shortness of breath, palpitation, giddiness, or perspiration. It is well for him consciously to breathe regularly and deeply through the nose, one respiration to each step.

Climate: Classification.-From a therapeutic standpoint, climates in general may be classified as follows:
1. Inland. a. at high altitudes
$b$. at low altitudes
2. Sea.
a. at sea
b. on the coast or islands

Mountain Climate in Pulmonary Tuberculosis.-No climate has a specific effect in bringing about a cure in any disease, but in pulmonary tuberculosis the climate of high altitudes undoubtedly is the most favorable. By a high altitude we mean, in general, one of 3500 feet or over. It must be kept in mind, however, that altitude is a relative term, therapeutically considered, for the effect of a locality depends not so much on its absolute height in feet, as on its relative height compared with that of the surrounding country and with that to which the patient has been accustomed.

The characteristics of a climate at a high altitude, or a mountain climate, are as follows: rarer air, less oxygen, lower pressure, increased effect of the sun's rays (the ground relatively warmer), lower temperature, less change of temperature during the year, dryness of the air with a good deal of rain and snow (except above a certain height), strong winds in summer, less in winter (especially if there is snow), winds down from the summit in the evening and up from the valleys in the morning, purity of the air, freedom from dust, and a greater content of ozone. Of all these characteristics the most important are (1) the rarefaction of the air, as this demands increased activity on the part of the respiratory apparatus and stimulates the circulation, effects which are beneficial to the whole organism and particularly to the diseased lungs [(2) the low humidity, (3) the wind and (4) the freedom from dust-Ed.]. In addition the mountain climate increases the appetite, stimulates the skin, improves the patient's sleep (usually after a period of disturbed sleep), and helps the whole state of nutrition and possibly the blood regeneration. In short, a mountain climate is, after proper food, the most important factor in the cure of pulmonary tuberculosis.

Contraindications to a Mountain Climate.-Naturally, however, it is not suited to every case. Among its contraindications are: a-high grade of anæmia, (the organism not being in condition to satisfy the demands of a high altitude on the blood-forming organs), advanced stages of the pulmonary complications, (emphysema, pneumothorax, empyema, etc.), 
severe intestinal and laryngeal ulcers, and concurrent diseases of the heart (pericardial adhesions, muscular and valvular lesions, etc.). Generally, though not always, the following may be looked upon as contraindications: slight but obstinate fever even where the local process is not extensive [and especially where there is a degree or two of fever in the morning as well as an evening exacerbation-Ed.], tendency to hemorrhages [particularly from cavities-Ed.], slight laryngeal or intestinal ulcers.

Choice of Seasons.- The best season for a residence at a high altitude is during the winter, as the comparative freedom from wind, the dryness of the air, and the intense sunshine make the cold less felt than at a lower altitude. The autumn and particularly the spring (with its melting snows) are not so favorable. The patient sent from home during the spring had best go to a sheltered resort at a lower level (1200-2000 ft.). Although the summer is not so advantageous as the winter, still the patient may be sent to the mountains in the summer and stay there even through the autumn in order to get used to the place before winter sets in. In fact, provided he begins his stay in the summer, [the fall-Ed.] or the winter months, he may remain all the year round in the mountains with great benefit.

\section{Winter Resorts at High Altitudes for Mild and Stationary Cases.}

Switzerland: The Upper Engadin; Pontresina, 5997 ft., 1 hour's drive from St. Moritz; The Lower Engadin; Tarasp, $3950 \mathrm{ft}$., excellent laxative waters, May to October; Rigi-Kulm, $5400 \mathrm{ft}$; Rigi-Scheideck and Rigi-Staffel, $4800 \mathrm{ft}$; Rigi-Kaltbad, over $4200 \mathrm{ft}$.; Samaden, over $5100 \mathrm{ft}$; Klosters, $3600 \mathrm{ft}$. The Tyrol: Sulden, $6000 \mathrm{ft}$; Madonna di Campiglio, $4500 \mathrm{ft}$; Innichen, over $3000 \mathrm{ft}$; Cortina d'Ampezzo, 3600 ft. ; Sandro and Schluderbach, $4200 \mathrm{ft}$.

\section{Winter and Summer Resorts at High Altitudes.}

\section{European.}

Davos-Platz, $5350 \mathrm{ft}$., [much dust and wind in summer, good sanitary arrangements, sports in winter, large English colony $(26 \mathrm{hrs}$. from London)-Ed.].

Arosa, $5740 \mathrm{ft}$., [hotels surrounded by hemlocks, $6 \mathrm{hrs}$. by carriage from Chur. Both these resorts are in Canton Graubünden, Switzerland. -Ed.].

Leysin, $4757 \mathrm{ft}$., [near Montreux, 1 hour by rail from Aigle (Canton Waadt)-Ed.].

St. Moritz, $5804 \mathrm{ft}$., [in the Upper Engadin, beautifully situated, iron springs, a popular resort for English and Americans. Excellent winter sports. Colder and rather more wind than at Davos. Fewer seriously ill patients, more society-Ed.]. 


\title{
New Mexico:
}

\author{
[AMERICAN, ETC. (3500 FT. AND OVER).
}

Santa Fé, $7000 \mathrm{ft}$., 75 per cent. of possible sunshine. Mean temperature $50.5^{\circ}$, extremes $7^{\circ}$ and $89^{\circ}$. Twelve to 16 inches rain. High winds in March and April. Fair hotels. St. Vincent's Sanatorium; Montezuma Sanatorium, capacity 200, \$15 and upward a week, Dr. J. W. Coon.

Las Vegas, $6418 \mathrm{ft}$., good hotel at the springs. St. Anthony's Sanatorium at East I as Vegas, capacity $35, \$ 9$ to $\$ 15$ a week.

Albuquerque, $5200 \mathrm{ft}$. Even less rain than in above places.

Silver City, $5800 \mathrm{ft}$. Sandy soil, pine forests, excellent place for out of door life. Average rain fall 12.3 inches. Average number of cloudy days to the year 37. Nine miles from Fort Bayard, which was chosen by the United States authorities as offering the greatest climatic advantages for the treatment of tuberculosis. St. Joseph's Sanatorium, capacity $50, \$ 25$ a week, Dr. E. S. Bullock.

Roswell, $3570 \mathrm{ft}$.

$E d d y, 4000$ ft. Good hotel.

Alamogordo, $4400 \mathrm{ft}$. All three rather hot in summer.

Las Cruces, $3800 \mathrm{ft}$, excellent winter climate with marked diurnal variations in temperature; but the hotel is poor. Las Cruces Sanatorium, capacity $40, \$ 15$ to $\$ 20$ a week.

\section{ARIZONA :}

Arizona with a similar climate to New Mexico offers fewer resorts with sufficient comforts for delicate invalids. Very marked diurnal variations of temperature, pronounced dryness, two rainy seasons, during the summer and during the winter, intensely hot summers, mild, sunny, and favorable winters, and annoying alkali dust are the important general features.

Prescott, $5456 \mathrm{ft}$. Fair accommodations, excellent climate from September to February.

Flagstaff, $6800 \mathrm{ft}$., near pine forests.

\section{Colorado:}

The plateau of Colorado, from $4000 \mathrm{ft}$. to $6000 \mathrm{ft}$., possesses in general a mountain climate. The Rocky Mountains running from north to south still further diminish the degree of humidity by causing a precipitation of the moisture of the winds from the Pacific Ocean. Hence it offers a much lower degree of humidity, both relative and absolute, than would be accounted for by altitude alone. It is also protected from the western winds, but not from the winds from the north and east. Health resorts of Colorado named below as well as others with similar climatic conditions, are suited to patients with a considerable reserve of vitality. Those with feeble circulation and poor assimilation will nearly 
always do better at lower altitudes, in a warmer, moister climate. The same rule is generally applicable to the resorts in New Mexico and Arizona.

Denver, 5196 ft., population 138,859 (1900), in an arid open plain, 15 miles east of the foot-hills and 30 miles east of the main ridge of the Rocky Mountains. Large amount of sunshine, very little rain, a hot summer, cool winter, very dry, good deal of wind and dust. Arrested cases, of robust constitution, who must live in a large town to make their living, will do well, although it is difficult to find work. Agnes Memorial Sanatorium, capacity $100, \$ 7$ to $\$ 10$ a week, Dr. G. W. Holden; Association Health Farm, capacity 45, \$25 a month, Dr. J. Wethered; The Home, capacity 150, $\$ 25$ a week, under the Episcopal Church; National Jewish Hospital for Consumptives, capacity 88, free, Dr. Moses Collins.

Colorado Springs, 5992 ft., 10 miles east of Pike's Peak, which is $14,000 \mathrm{ft}$., population 21,085 (1900), is nearer the mountains, more winds, alkali dust. Patients require a good deal of vitality to do well. A rather social city for the better class. Nordrach Ranch, capacity 35, $\$ 60$ a month, Dr. J. E. White; Glockner Sanatorium, capacity 50, $\$ 8$ to $\$ 40$ weekly.

Glenwood, $5600 \mathrm{ft}$., 160 miles west of Denver, in a protected valley in the foot-hills, less wind and less dust than either of the above; thermal springs.

Estes Park, $7500 \mathrm{ft}$., for summer especially, an excellent hotel.

\section{Mexico:}

City of Mexico, $7400 \mathrm{ft}$., upon the central plateau of Mexico, is famous as possessing one of the favorable all the year round climates. Accommodations are said to be inferior.

\section{SOUTH AMERICA :}

The Andes in South America furnish resorts in Peru, Ecuador, Colombia, 1880 to $12,000 \mathrm{ft}$. The excellent results obtained by sending phthisical patients from Peru into the high valleys of the Andes were largely responsible for the selection of a mountain climate for pulmonary tuberculosis in Europe and North America.

\section{AFRICA :}

In the north the Great Karoo, 2800 to $6000 \mathrm{ft}$., with a long winter from April to September, offers a winter climate during our summer. The accommodations are said to be inferior.-Ed.]

Resorts at Lower Mountain Altitudes: For Spring and Falt.

Switzerland: Montreux; Vevey. Tyrol: Bozen; Gries; Meran. 


\section{For Summer and Sometimes Winter.}

Mendelpass, over $4200 \mathrm{ft}$; Brennerbad, $3900 \mathrm{ft}$; Gossensass, over 3000 ft.; Semmering, over 3000 ft.; Neu-Schmecks; Zakopane; Neumarkt; Aussee. [Les Avants, 3232 ft., hot in summer, excellent hotel open in winter. Milder winter than at Davos. Protected by mountains from north and east winds.-Ed.] Any place in the country with plenty of trees. Interlaken, $1600 \mathrm{ft}$. ; Gleichenberg, $900 \mathrm{ft}$. ; Reichenhall, $1400 \mathrm{ft}$; Velden and Pörtschach and Millstadt, $1800 \mathrm{ft}$.; Swiss Lakes; Bavarian Lakes; Bavarian and Thuringian Forests.

\section{[ARIZONA :}

Fort Phonix, $1080 \mathrm{ft}$; sanatoria: Palm Lodge, eapacity 55, $\$ 10$ to $\$ 35$ a week, Dr. H. H. Stone; Mercy Hospital, capacity 20, \$15 a week.

Tucson, $2400 \mathrm{ft}$. Very hot summers, dust showers in winter, beautiful scenery. St. Mary's Hospital, capacity 30, \$15 a week.

\section{CALifornia :}

Redlands, $1350 \mathrm{ft}$., hot, dry in summer, noted for its orange groves. Mentone Sanatorium, capacity 50, \$15 a week, Dr. F. J. Koepke.

\section{South Carolina:}

Ashwell.

\section{NEW YORK:}

The Adirondacks. The Adirondack Cottage Sanatorium, Saranac Lake, $1650 \mathrm{ft}$., a worthy monument to the discernment, intelligence, and industry of Dr. Trudeau, is largely responsible for the popularity of the Adirondack range in the treatment of pulmonary tuberculosis. It is planned for patients who cannot afford to pay more than $\$ 5$ a week, and who are in the very early stages of pulmonary tuberculosis or are at least favorable types ; its capacity in summer is 100, 112 by use of tents, Dr. E. L. Trudeau. Other sanatoria: Sanatorium Gabriel, Paul Smith's, $2000 \mathrm{ft}$., capacity 70, $\$ 10$ to $\$ 18$ a week, Dr. R. L. Strong; Stony Wold Sanatorium, capacity 70, $\$ 7$ a week, Lake Kushaqua, 9 miles from Paul Smith's ; Raybrook, State Hospital, capacity 120, Dr. J. H. Pryor. Without much sunshine, with slightly sandy soil, and cold dry winters, the Adirondacks owe almost as much of their fame as a resort for tuberculosis patients to the advantages resulting from sanatorium treatment as to the climate itself. The summers are apt to be hot, the nights usually cold. Camp life, moderately good fishing and some hunting add considerable attraction.

Sullivan County. The establishment of the Loomis Sanatorium at Liberty emphasized the advantages of the dry air on the southern slope of a range of hills in Sullivan County; $2300 \mathrm{ft}$., capacity 140, $\$ 10$ to $\$ 35_{\text {r }}$ Dr. H. M. King. 
Maine. New Hampshire. Vermont :

Moosehead Lake, The Rangley Lakes, $1900 \mathrm{ft}$., the White and the Green Mountains offer especially favorable climatic features for patients with pulmonary tuberculosis who are in good enough condition to do well with active out-door exercise, who do not require sanatorium treatment or who for some reason may be unduly depressed by association with persons sufiering from their own malady. In some of the smaller towns very comfortable accommodations can be obtained at low rates. The disadvantages are the difficulty in obtaining proper medical supervision, and the lack of sanatorium treatment.

\section{Masínachusetts:}

The Berkshire Hills have been selected for many patients who do not stand so rigorous a winter as that of the Adirondacks. The accommodations, especially at Lenox, are excellent. It is too warm, there is too much moisture, and the soil is too full of clay to make the climate ideal during the summer months.

The Sharon Sanatorium, at Sharon, $350 \mathrm{ft}$., capacity 21, $\$ 5$ a week, Dr. V. Y. Bowditch, has demonstrated the effect of sanatorium treatment in a climate only moderately favorable. It is sheltered on the north and north-west by thick woods. Massachusetts State Hospital at Rutland, $1000 \mathrm{ft}$., capacity 250, \$4, Dr. W. J. Marclay, was the first state sanatorium to be established in the United States. The buildings are on a southern slope, protected from the north-west by wooded hills.

\section{AFrica :}

The Karoo, 2000 to $3000 \mathrm{ft}$.-Ed.]

Climate of Low Altitudes.-The second subdivision of inland climates, the climate of the lowlands, has in general no especial effect on the human organism. Still there are quite noticeable differences between the climates of different localities at the same altitude, so that a further subdivision may be made into a damp and a dry climate. The former has a somewhat sedative, and the latter a stimulative effect. The damp climate is suited for cases of erethic tuberculosis where fever is very easily brought on, particularly if the disease has already reached an advanced stage. Such resorts are suited for spring and autumn, especially for patients who have passed the winter in the south and who wish an intermediate climate for April and May before going home, or for patients whose disease has made its appearance in the winter. Patients who have passed the winter in the high mountains will not find the lowland climate stimulating enough, but should pass the spring in some lower mountain resort.

\section{EuropenN.}

Damp.-Lake of Garda, Arco, Lake Como, Lake Maggiore, Goritz, North Shore of Lake of Lucerne. 
Dry.-Egypt, Biskra.

Moderately Damp.-Lake Lugano.

\section{[AMERICAN.}

Damp.-Florida and Gulf States, Shores of the Great Lakes. Dry.-Arizona, New Mexico.

Moderately Damp.-Georgia: Summerville, and Thomasville, $330 \mathrm{ft}$; South Carolina: Aiken, $565 \mathrm{ft}$., and Camden, $200 \mathrm{ft}$. These four have become favorite winter resorts for people of means. The winters are mild, the soil is sandy, pine trees about. There are excellent accommodations, pleasant society, encouragement for out-door life. Lakewood, New Jersey, in a sandy pine belt, is especially favorable for consumptives who cannot be sent far from New York. The place has become so favorite a fashionable resort during the fall, winter and spring, that the hotel accommodations are particularly good.

Moderately Dry.-Texas, Inland Southern California, Minnesota, Dakota, Nebraska, Winnipeg.-Ed.]

Sea Climate.-Sea climates may be subdivided into (1) the climate of islands and the seacoast, and (2) the climate of the high seas. The characteristics of the climate of the seacoast and islands are: purity of the air, salt in the air, abundant oxygen, small amount of carbon dioxide, slight temperature changes, high relative humidity and slight relative changes in humidity, frequent rain but little snow, high atmospheric pressure, and strong, steady winds. To the last two is due the greater part of the effect on the human organism. The cutaneous nerves are stimulated, the heart action is slowed and strengthened, and the appetite and sleep generally improved.

Sea Voyages.-For the effect on the appetite and sleep a sea voyage is perhaps preferable, in so far as the air at sea is particularly pure, and the patient is given the greatest possible opportunities for rest and fresh air. The ship should be comfortable, and the food good. With these provisos, a sea voyage in winter in southern seas may be recommended for (1) cases where the pathological process is at a standstill and the individual still has resistant power; and (2) cases where the individual shows a tendency to tuberculosis but where the disease has not yet appeared. [I rarely recommend a sea voyage for a patient with tuberculosis. Almost the same climate can be obtained nearer, on our own coast: Maine, Cape Cod, Buzzard's Bay, Newport, Long Island, Martha's Vineyard, Nantucket, Block Island, Nassau, the Bermudas. Unless a good sailor he is often unable to eat, often obliged to be content with only moderately good fare without fresh milk, cream, and eggs, sometimes confined to a stuffy cabin, and when below decks subject to drafts, and to changes of weather even more striking than on the coast.-Ed.]

Coast and Island Climate.-The climate on the coast and islands may be subdivided into: (1) damp and cold, (2) dry and cold, (3) damp 
and warm, (4) dry and warm, (5) moderately warm and damp, and (6) moderately cold and damp. The first two may for our purposes be disregarded.

Damp and Warm.-The damp and warm coast climate is marked by: relatively high humidity, high mean temperature, and slight daily changes in temperature. In general it is sedative in its action, with a healing effect on the diseased respiratory mucous membrane, sometimes, however, inducing loss of appetite and diarrhœe. Examples are: Madeira, the Canary Islands, and the Azores. They furnish a favorable residence for tubercular patients from October to May. [During three months in the year, January, February, and March, Jacksonville and St. Augustine in the north, and Palm Beach and Tampa in the south, offer a very mild, humid coast climate with a considerable amount of sunshine. Accommodations are excellent. Although Florida is no longer a favorite selection for cases with pulmonary tuberculosis, the amount of sunshine and number of hours per day which a patient can spend in the open air during the three months mentioned above, make it a suitable choice for some cases with feeble circulation, poor assimilative powers, and for elderly invalids, especially for those to whom a long journey, such as an ocean crossing, is undesirable. Other similar island or coast climates are to be found at The Bahamas, West Indies, Hawaiian Islands, and the Philippine Islands.-Ed.]

Dry and Warm.-The dry and warm coast climate is very stimulating on account of the dry air. Examples are: The Riviera (San Remo, Mentone, Monte Carlo, Nice, Cannes, Hyères, etc.) ; the region around Naples and Palermo (Sorrento, Amalfi, the Islands of Capri, Ischia, ete.). Malta, the Balearic Islands, and Malaga stand midway between the places with a damp and warm and those with a dry and warm climate. The resorts mentioned are suitable from Oetober to April, especially for patients with abundant expectoration. [With the Pacific Ocean and its great current on one side and a range of mountains succeeded by high plateaus on the other, California, or perhaps more accurately southern California, is noted for its mild winters, cool summers and abundance of sunshine, high humidity, and summer fogs. Individual variations depend upon the latitude, proximity to the coast, and situation with respect to the ranges of mountains running parallel with the coast line. Below Pt. Conception, half way between San Franeiseo and San Diego, the climate is distinctly milder than above it, the mountain ranges shutting out the northerly winds. The climate of California is especially favorable for people with moderate vitality. They find an opportunity to interest themselves in out of door occupations, such as fruit growing. The objections for consumptives are, in summer, the dryness, dust, and wind; in winter, the high degree of humidity, severe cold winds, and the difference between sunshine and shade.

San Diego and Coronado Beach, in the extreme south-west corner of California, have the mildest climate, and most equable temperature in 
the state, milder and with less humidity than either Cannes or Mentone. There is an excellent hotel at Coronado Beach.

Los Angeles, $283 \mathrm{ft}$., 14 miles from the coast, with a good deal of clay in the soil, is warmer in summer, and colder in winter, with less humidity, fogs, and winds than the two above. It is more or less of a business centre. The best season is from November to May. Barlow's Sanatorium, eapacity 15, $\$ 5$ to $\$ 7$ a week, Dr. W. J. Barlow; Pottenger Sanatorium, capacity 40, $\$ 25$ a week, Dr. F. M. Pottenger, 16 miles east of Los Angeles.

Pasadena, 1000 ft., 20 miles from the coast, is like Los Angeles, though with less fogs, and a more inland climate, sandy soil, excellent hotels; Esperanza Sanatorium, 1800 ft., capacity 50, \$25 a week, Dr. F. C. Melton. Santa Barbara, 50 miles south of Pt. Conception, 1 mile from shore, is protected from the north winds, and suitable for summer and winter; the climate is similar to that of San Diego. There are two excellent hotels, and very capable physicians.-Ed.]

Moderately Warm and Damp.-Examples of the moderately warm and damp coast climate are: Algiers, Cadiz, Alicante, Malaga in Spain, Palermo, Catania, all of which have a very even climate; the Ligurian coast (Nervi, Spezia, Rapallo, Viareggio, and Santa Margherita); Pegli on the Riviera; the island of Lussin; the Gulf of Quarnero (Abbazia); Lovrana, Corfu, Ragusa, and the region around Venice; [BermudaEd.]. The resorts on the Adriatic are not, however, suited for winter residence, on account of the cold, dry, wind coming down from the high mountains. They are suitable for the spring and autumn, and even for summer, but in January and February have an irritating effect on the respiratory mucous membrane. The others are too relaxing in summer, but are suitable for many patients all through the winter.

Moderately Cool and Damp.-The moderately cool and damp coast climate is found on the islands and coasts washed by the Gulf Stream: England, France, Norway, etc. The resorts on the North Sea and, to some extent, on the Baltic, come under this head, but none of them are important, as far as the treatment of pulmonary tuberculosis is concerned.

[Monterey, 80 miles from San Francisco, on Bay of Monterey, a winter resort for Easterners, and a summer resort for San Franciscans, is fairly protected from the winds, and offers a large, well-kept hotel.

Nantucket, Block Island, Cape Cod are excellent examples of our best island climates. Some patients with tuberculosis, notably children with joint and glandular tuberculosis, do extremely well there, especially during the summer.-Ed.]

Summary.-To sum up the climatology of pulmonary tuberculosis: In general, a mountain climate is the best. The patient may go to a high altitude (4000 ft. or more) in the winter or in the summer, staying there during the following autumn and winter, but should not begin his residence during the spring or fall, a lower and warmer climate (still in 
the mountains, however,) being more suitable for these seasons. When once he has become accustomed to the higher altitude, he may spend the whole year there, if desired, or simply the winter, coming lower down for the rest of the year. If a mountain climate is contraindieated, the patient should be sent to a sea climate, suited to his condition and to the time of year.

To whatever place he is sent, it is exceedingly important that he be under the constant care of a physician. This is more easily accomplished in high mountain stations than in the large Southern resorts with all their various allurements. It is accomplished best of all in sanatoria for pulmonary troubles where every detail of the patient's life may be regulated. After the proper routine has been ingrained in the patient's memory and habits at a sanatorium, he may get equal benefit at an ordinary resort. Before he is sent anywhere, the physician should have him under careful observation for at least a week, to determine whether the prognosis is favorable enough to justify his leaving home. He should be warned to take plenty of warm clothing for the autumn and winter. It will be needed not only at the mountains but even at the Mediterranean resorts during cold, damp days in winter in the insufficiently heated houses.

[In recent years countless strikingly good results in the treatment of tuberculosis have been obtained under relatively poor climatic conditions. The reports of sanatorium treatment seem to show that nearly equal benefit is derived from such care without ideal climatic conditions. Some patients do well in any reasonably favorable climate or at home even in an unfavorable climate provided they follow carefully the most advantageous mode of life. Others do badly in the climate best suited for that disease despite a judicious mode of life. No single climate is best for all cases. Hence to-day we lay much less stress upon the selection of the climate. Formerly the selection of a climate depended upon the presence or absence of a cavity, or upon the duration of the disease. More logical principles now guide our choice. Of these the most important is the influence of the climate upon metabolism, which influence depends upon the climate's power of heat abstraction. The latter in turn depends upon the temperature combined with the relative and absolute humidity of the air and with the amount of wind. In warm climates the amount of heat abstraction depends largely upon the relative humidity, in cold climates upon the amount of wind. Climates equable in temperature are necessarily rather humid and hence are among the most irregular and variable in their heat demands. Dry climates, though never equable in temperature, are more apt to be regular in their heat demands.

In my judgment much of the success of climatic treatment depends upon the stimulation of cold dry air upon the patient's metabolism. Whether a climate is depressing, relaxing, or bracing to patients depends upon their general vigor or power to resist cold. The best climate is where the individual's nutrition is at its maximum, where the demand for the production of heat best coincides with his response. 
Young people usually respond well to a climate which demands from them a high rate of heat production. Old people usually do not.

The cold dry winter climate of the Alpine resorts, of the Adirondacks, of Colorado, or of the northern Karoo in South Africa during their winter, suits the robust and those whose organs of nutrition and oxidation are functionally capable of responding to a demand for much greater work than at home, and this without reference to the extent of lung damage. In the Adirondacks, for example, nearly all patients who are able to react to the cold do well during the fall and winter months, eat well, sleep well, and gain weight. In late spring a few mild, warm, enervating days produce striking effects; the patients' appetites fail, they lose weight and often require a change. Those on the contrary with feeble circulation, with poor digestion and assimilation, and with small lung capacity, even if the pulmonary mischief is slight, fail to react and are extremely depressed by such cold climates.

In early cases the value of the psychical, hygienic, and disciplinary factors of sanatorium life, outweighs most other features. In more advanced patients with chronic or progressing disease an appropriately regulated life at home is oftentimes more suitable unless the patient's financial condition warrants a large expense. The anatomical features of the disease are here less important than social and personal conditions.

The duration of a patient's residence in a sanatorium is generally decided by the physicians there. In general no rules apply. Most do not remain long. enough. See editorial note on the after treatment in tuberculosis. A change of residence from one resort to another at different seasons is sometimes indicated. In the best places the sanatorium physicians are keenly alive to this need.-Ed.]

HyGIENE: Clothing.-The patient should wear woolen underclothing in the winter, and a light weight woolen or merino in summer. None of the clothing should be tight and no corsets should be worn, in order that the breathing may be quite unrestricted.

Care of the Skin.-Many tubercular patients are continually catching cold, because the cutaneous nerves are not able to regulate the vessels and so compensate for the changes in the outer atmosphere. Many suffer also from an almost continuous and abundant perspiration. These drawbacks suggest a careful treatment of the skin. If the skin is pale and the perspiration excessive, we employ dry rubbing until the skin is reddened, while the patient lies under the bed-covers wrapped up in a sheet. Gradually we proceed to rubbing with alcohol and then with alcohol and water, using a wet bath-mitten. Next we use tepid water, then cold water, gradually working up to short cold packs. Douches must be very cautiously employed and only where the individual is strong and the local process stationary, or where we are dealing with a patient who merely has a predisposition to the disease. Swimming also may be permitted the latter class of cases, but like every other outdoor sport, riding perhaps excepted, can hardly be allowed to a patient in 
whom the disease has once been demonstrated, even if he be strong and the pathological process stationary.

There is no objection to two 10 minute tepid baths a week for cleansing purposes, provided the patient goes to bed immediately afterwards or after dressing remains at least 10 minutes in an adjoining warm room. The morning is the best time for all these hydropathic measures; but in case of heavy night sweats the dry rub may be given both night and morning.

Individunl Prophylaxis.-The physician is often confronted with the problem of how to prevent the actual appearance of tuberculosis in an individual who apparently is predisposed to that disease (heredity, phthisical diathesis, severe psychical trouble, weakness from overwork or previous disease, etc.).

The first prophylactic measure is to insure plenty of fresh air, the patient being kept away from tuberculous individuals or from close rooms with other people (schools, etc). A hardening hydrotherapy also plays an important part. Cold sponge baths and showers $\left(59^{\circ}\right.$ or even $53^{\circ}$ ) from the age of one year, moderately warm but not too heavy clothing, a temperature of $63^{\circ}-66^{\circ}$ in the living rooms, and cold sleeping rooms, all help to harden the patient. Exercise is also a most essential part of the prophylaxis. The child should swim, play out-door games, and do gymnastic work, the young man or woman adding rowing, riding, skating, snowshoeing, etc. Daily breathing exercises should be insisted upon, taken preferably $1 / 2-1$ hour before a meal, or if this is not possible, one hour at least after breakfast, lunch, or supper, or two hours after dinner. They should consist of lung exercises and groups of arm and leg exercises, with a pause between each set. The following are examples of proper movements: (1) Arms: raising the extended arms over the head from one side, raising the arms over the head from in front, swinging the arms forward, up, and backward, swinging the arms in a sagittal plane; (2) Legs: raising the leg forward, sideways, backward, spreading the legs, bending the knees; (3) Trunk: bending at the waist, raising oneself from a horizontal position, twisting the trunk, etc.; (4) Lungs : slow breathing with the hands on the hips, holding the breath, forced inspiration, deep breathing with arms stretched backward. The following is an example of a suitable combination of movements :

Raising the arms from the sides.......... 10

Slow breathing, hands on hips........... 10

Spreading the legs.................... 6

Rolling the shoulders................. 10

Holding the breath ( 30 seconds) $\ldots \ldots \ldots \ldots \ldots 2$

Slight bending of knees............... 8

Swinging the arms forward, over, and back... 12

Forced inspiration..................... 4

Twisting the trunk................... 10

Swinging the arms in the sagittal plane...... 6

Expanding the chest................... 2

Deep bending of knees................. 6

$\begin{array}{rrr}20 & 30 & \text { times } \\ 15 & 20 & \text { “ } \\ 8 & 10 & \text { “ } \\ 15 & 20 & \text { "6 } \\ 2 & 2 & \text { “ } \\ 16 & 24 & \text { “ } \\ 18 & 24 & \text { “ } \\ 6 & 8 & \text { “6 } \\ 15 & 20 & \text { “ } \\ 10 & 16 & \text { “ } \\ 3 & 4 & \text { “ } \\ 9 & 12 & \text { “ }\end{array}$


It may be said in passing that breathing exercises are contraindicated when once tuberculosis has appeared in the lung unless it occurs in the form of an undoubtedly old and solidly healed nodule. Inflammatory or necrotic processes are only intensified by breathing exercises, the leucocyte infiltration is increased, and the tissue degeneration hastened.

Two more points in the prophylaxis remain to be noted. The diet should be nourishing with a good deal of meat, and the occupation, if the individual is obliged to work, should be one to keep him as much as possible in the open air and yet at the same time not expose him to severe bodily strain. He may for example be a ship's surgeon, forester, country hotelkeeper, gardener, gamekeeper, boatman, overseer in the country, or letter-carrier in the country.

\section{MEDICAL THERAPY OF PULMONARY TUBERCULOSIS.}

Creosote.-No specific for pulmonary tuberculosis has so far been discovered. Creosote, especially in Germany, was formerly held to have a specific action, and it and its substitutes, still widely used, are of great value. Creosote, a yellowish liquid with a strong smoky smell and a sickish taste, is the distillation product of beechwood tar and consists of different benzol derivatives, among them creosol, cresol, and especially guaiacol. It has no specific effect on the tubercle bacillus although possibly, taken into the blood, it has a slight antizymotic action. In many cases its good effect is due to its increasing the appetite, improving the digestion, and relieving the pain in the stomach so frequent in this disease; at the same time it has a favorable effect on the pulmonary condition itself by lessening the cough. It often relieves wholly or in part the night sweats and fever. Its use is indicated in all cases with slight fever and no complications, but contraindicated where there is high fever or where a trial of it is found to decrease the appetite instead of increasing it. For there are a minority of cases in which creosote at once diminishes the appetite and sometimes brings on digestive disturbances, such as burning, belching, vomiting, pain, and colic (the last two alone, however, do not contraindicate its use). Sometimes it even induces severe symptoms of intoxication (gastro-enteritis, profuse sweat, etc.). Some individuals are absolutely intolerant of it.

It is generally given by the mouth, $1 \mathrm{G}$. (15 min.) a day. Twice this amount may be used and Sommerbrodt even goes as high as $4 \mathrm{G}$. (60 min.). It is best given in gelatine capsules:

R Creosoti (Beechwood) ...........0.05-0.01....m $m^{\frac{3}{4}-\text { iss }}$

Olei olivæ .................. $0.20-0.30 \ldots \ldots m$ ii-v

M. fiat caps. no. i. Dent. tal. caps. molles no. 1.

Sig. - 3-7 capsules 3 times a day, not to be taken on an empty stomach. [The same dose of cod-liver oil or balsam of tolu may be substituted for the olive oil in the above.-ED.] 
It may also be given in pills, or in wine (Bouchardat and Gimbert) :

B Creosoti (Beechwood) .................. 3 iv

Tinct. gentianæ comp. .................30.00...

Alcoholis........................... $350.00 \ldots$ viii 3 ii

Vin. xerici.................... ad. $1000.00 \ldots \ldots$ Oii

M. fiat sol.

Sig. -2-3-6 tablespoonfuls in a glass of milk, after meals. 1 tablespoonful contains $0.2 \mathrm{G}$. ( $3 \mathrm{gr}$.) of creosote.

The cheapest but the least pleasant preparation is the tincture (creosote 1.0 G. (15 gr.), tincture of gentian $4.0 \mathrm{G}$. $(1 \mathrm{dr}$.$) ). We begin$ with 7-20 drops daily and increase to 50 drops, always given in milk.

Two other methods of administration are as follows:

B Creosoti (Beechwood) ...........2.50-5.00.... $3 \mathrm{ss}-3^{i}$

Olei morrhuæ ............................

Acaciæ,

Aq. menth. pip. āā q.s.

M. fiat emuls.

Sig. -1 tablespoonful $2-4$ times a day. Each tablespoonful contains $0.2-0.4$ G. (3-6 gr.) of creosote.

Or :

B Creosoti (Beechwood) $\ldots \ldots \ldots \ldots \ldots \ldots \ldots .00 \ldots \ldots z^{i}$

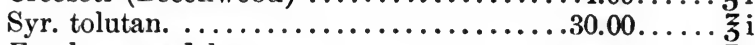

Emuls. amygdal. ...............180.00.... 3 vi

M. fiat emuls.

Sig. -2-6-12 tablespoonfuls a day.

Creosote may also be given in tepid enemas, prepared by emulsifying $1 \mathrm{G}$. ( 30 drops) of creosote with 30 c.c. ( $1 \mathrm{oz}$.) of milk, and adding 250 c.c. $(8 \mathrm{oz}$.$) of water and sometimes a little tincture of opium. Three or$ 4 of these enemas may be given daily.

It may also be combined with calcium, as follows:

R Creosoti (Beechwood)......................

Calcii phosphatis .......................... $\mathbf{z}^{\mathrm{i}} \mathbf{z}_{\mathrm{ii}}$

M. fiat mass. Div. in caps. aeq. no. xl.

Sig. -1 capsule to be taken during each meal.

Another method of giving creosote, or its substitute guaiacol, has been advocated,-5-20 per cent. creosote, or 20 per cent. guaiacol vasogen. Vasogens are oxygenated vaselines; i.e., hydrocarbons saturated with oxygen and oxygen carriers which facilitate the complete solution of various chemical substances. $50-200$ drops of either of these are given daily in a glass of water or milk. ${ }^{2}$

Pure Guaiacol.- The unpleasant properties of ereosote may be avoided by giving its essential principle, guaiacol, or the carbonate of creosote.

${ }^{2}$ [This explanation and the claim of superior oxygen carrying property of vasogen are very likely unfounded. "There does not seem to be any reason for supposing that vasogen ointments are really superior to ointments made with vaseline." (U. S. Dispensatory 1907).-Ed.] 
It is now possible to obtain pure guaiacol in the form of colorless crystals. The author has tried it subcutaneously but has been obliged to give it up on account of the local irritation, and, in one case, contrary to the experience of others, the rise of temperature. He has also tried it internally, but prefers the carbonate and salicylate. The benzoate (benzodol), cinnamylate, valerianate, and phosphate may also be used. The author gives the carbonate and salicylate in powders $1 \mathrm{G}$. (15 gr.) a dose, increasing gradually to 5-6 G. (75-90 gr.) :

B Guaiacolis carbonatis or salicylatis .........0.50..... gr. vii

Dent. tal. cap. no. c.

Sig. - 2-12 capsules daily.

These preparations are usually well borne, and in a great number of cases, not all of the lightest grade, they increase the appetite, lower the temperature, and relieve the cough. For night sweats the carbonate is preferable to the salicylate, as in a good many cases the latter preparation apparently even increases diaphoresis.

Creosotal.-The best of all substitutes for creosote, however, is, in the author's opinion, its carbonate, the so-called " creosotal," an oily yellow liquid, with only a slight creosote odor. Even if given in large doses, it shows no signs of irritating the mucous membrane of the stomach, and no general toxic qualities. In large doses it increases the appetite and body-weight, reduces the expectoration and cough, puts an end to the fever, and improves the pulmonary symptoms, if they are not too far advanced. Creosotal may be given as follows:

B Creosotalis ......................... 3 iii $z$ ii

Sig. -1 teaspoonful $3-4$ times a day in milk during meals. [A large dose.-ED.]

Or in gelatine capsules:

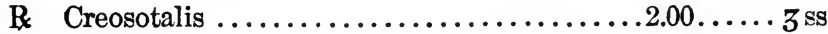

Dent. tal. caps. molles no. c.

Sig.-Begin with 1 capsule 3 times a day and increase to 9 a day.

R Creosotalis .........................20.00.... $\mathrm{z} \mathrm{v}$

Olei morrhuæ ......................100.00..... $\xi_{\text {iii }} z^{\text {ii }}$

Acaciæ,

Aq. menth. pip.............āā q.s. ad. $180.00 \ldots \ldots$ z vi

M. fiat emuls.

Sig.-One tablespoonful 3-6 times a day. Each tablespoonful contains $10 \mathrm{G}$. $\left(2 \frac{1}{2} \mathrm{dr}\right.$.) of cod-liver oil, and $2 \mathrm{G}$. ( $\left(\frac{1}{2} \mathrm{dr}\right.$.) creosotal.

If the patient shows an intolerance for the drug, manifested by gastric disturbances, it may be given in a mixture; e.g.:

B Creosotalis ....................10.00-15.00.... 3 iiss $-z$ iv

Vitelli ovi ................................. 1

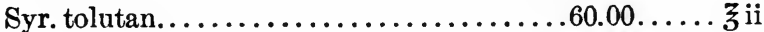

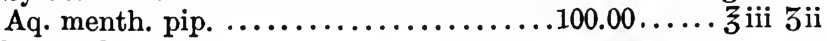

M. fiat emuls.

Sig. - A teaspoonful 3 times a day during meals. 
Creosotal may also be given by the rectum twice a day (night and morning) after first cleansing the bowel with warm water or salt solution.

Five-10 G. (11/4-2 $1 / 2 \mathrm{dr}$.) are first mixed with the yolk of an egg and then added to 150-300 G. (5-10 oz.) of tepid milk or water with a few drops of the tincture of opium.

Thiocol-Sorisin-Sirolin.-Thiocol, the tasteless and relatively innocuous potassium guaiacol sulphonate, sorisin, a 10 per cent. solution of sodium guaiacol sulphonate in syrup of orange, and sirolin, a 10 per cent. solution of thiocol with a pleasant taste, have recently come into use, and apparently are as efficacious as creosotal. The first is given in powders, generally 3-4 G. (45-60 gr.) daily; the other two sometimes undiluted, sometimes mixed with wine, water, or milk, 3-4 small teaspoonfuls daily.

Solveol.--Some patients who cannot take creosote get on well with solveol, (a neutral solution of cresol with sodium cresotate, containing about 25 per cent. of free cresol), a yellowish liquid which apparently increases the appetite and body weight and lessens the cough. It is prescribed as follows:

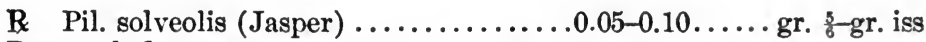

Dent. tal. dos. no. c.

Sig. $-3-10$ pills a day, gradually increased by $2-3$ pills until 7 pills 3 times a day are being taken.

Creosotal, or creosote in any form, also guaiacol or guaiacol carbonate may be given in kefir. The additional nourishment contained in the kefir is of course an advantage.

Cinnamic Acid.-Besides creosote and its derivatives, numerous other drugs have been recommended for the tubercular process as such. Only a very few are worth considering, and the effect of even these cannot as yet be definitely stated. Landerer recommends an intravenous injection of the following mixture:

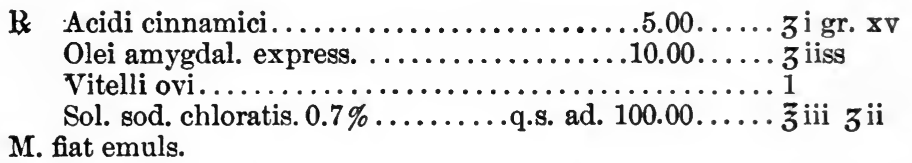

[He emphasizes the importance of properly preparing this emulsion, so that the acid is very finely divided and no large erystals of it remain. For each subcutaneous injection 1 c.c. of this emulsion is made alkaline by about 5 drops of a $71 / 2$ per cent. solution of sodium hydroxide shortly before use. It is well to test this 10 or 15 minutes after preparation to be sure that it has remained alkaline. The initial dose is generally $1 \mathrm{mg}$. If the patients are very much emaciated and running a high temperature, he employs even smaller doses. Rarely, when the patients are in exceptionally good condition, well nourished and withont fever, he begins with larger doses but never over $2 \mathrm{mg}$. Before inoculating he makes a prac- 
tice of having each patient's temperature estimated 2-6 times per day for 2-4 days. He generally inoculates every other day and usually increases the dose about $1 \mathrm{mg}$. In very advanced cases he increases at the rate of $0.5 \mathrm{mg}$. and inoculates but once a week. He never gives more than $50 \mathrm{mg}$. as a maximum, and rarely over $25 \mathrm{mg}$. In uncomplicated cases his maximum is reached in 15-20 injections.-Ed.] ${ }^{3}$

Hetol-Heterocresol.-Hetol (sodium cinnamate) and heterocresol (meta cresol cinnamic acid ester), used by Landerer, have the advantage over injections of cinnamic acid in being more easily measured and injected. Opinions so far differ as to their efficacy.

Copper.-Copper is recommended by French authorities and recently by Kobert, who uses hemol cuprated or cuprohemol (hæmoglobin combined with cupric oxide salts) :

B Cuprohemolis

Dent. tal. cachet no. xii.

Sig. -1 cachet 3 times a day, taken with chocolate.

Tuberculin.-The use of tuberculin is still in the experimental stage. The author would advise only very small doses, which must be still further reduced if after an injection the slightest symptoms of a marked reaction occur (languor, pain in the limbs, fever, headache, local symptoms in the diseased lung). We may begin with $1 / 10 \mathrm{mg}$. and increase very cautiously up to $0.02 \mathrm{G}$. ( $\mathrm{r} / 3 \mathrm{gr}$.) as the final doses. In this way no harm will be done, and some cases, especially the less advanced ones, may be helped. A better preparation is, however, needed before a final judgment can be given. [It is far safer to begin with doses of $1 / 1000$ mg. or less.-Ed.]

Klebs' tuberculocidin, Koch's new tuberculin, and Maragliano's serum are all disappointing.

Experiments with "bovine tuberculin" have been carried on for some time in sanatoria for tuberculosis, but no estimate of its value can yet be given. [Consult the note upon the use of tuberculin in the section on scrofula (p. 195).-Ed.]

To this list of remedies recommended for tuberculosis will be appended a few drugs to be used partly to keep up nutrition, partly as substitutes for certain substances excreted in abnormally large amounts in pulmonary tuberculosis.

Arsenic.-The first of these is arsenic, given to stimulate nutrition. It may be administered in the form of pills, gelatine tablets, Fowler's solution, atoxyl, or mineral waters containing arsenic (Guber, Levico, and Roncegno-Quelle, Mitterbad, La Bourbole). Asycodyle (sodium cacodylate) has recently been recommended instead of arsenic. It is best given

${ }^{3}$ The German was not clear as to dosage and method of administration, so this note taken from Landerer's original article has been inserted.-Ed. 
subcutaneously, in doses of 0.05 G. $(3 / 4$ gr.) daily; but may also be administered by the rectum or internally. In the latter case we prescribe :

R Sodii cacodylatis....................2.00....gr. $\mathrm{xxx}$

Spir. vini Gallici,

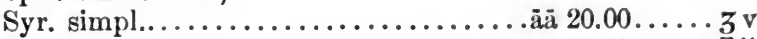

Aq. menth. pip ...........................60.00.... zु

M. fiat sol.

Sig. -1 teaspoonful a day.

This method of administration apparently has no advantage over the older ones.

Cod-liver Oil.-Cod-liver oil or one of its substitutes (e.g., lipanin, v. Mehring's "Kraftschocolade," and olive oil) is of the greatest value in keeping up nutrition. [For the different preparations see the section on scrofula, p. 194. Unless suspicious of hyperacidity, I prescribe codliver oil $1 / 2-2$ hours after eating in order to avoid inhibiting the gastric secretion.-Ed.]

Salts.-Salts should be given to supply those excreted in abnormal quantities in the sputum and urine. The patient should be instructed to take large quantities of table salt, and should also be given phosphates. $30-80 \mathrm{G}$. (1-3 oz.) of calcium phosphate in milk may be taken during the day, or 1-3 G. (15-45 gr.) of calcium hypophosphite (soluble in water) or calcium phosphate (made soluble in water by the addition of hydrochloric or lactic acid). Daremberg, for example, prescribes the latter as follows:

B Calcii phosphatis ..................10.00.... 3 ii gr. $\mathbf{x x x}$

Acid. hydrochlorici or lactici ................00.... $m$ xlv

Aq. dest.........................3 $300.00 \ldots \ldots$

M. fiat sol.

Sig. -1 tablespoonful 3 times a day after meals.

[Two excellent preparations in our own pharmacopœia are the syrup of hypophosphites and the syrup of calcium lactophosphate in doses of 4 G. ( 1 dr.) three times a day.-Ed.]

Recently the glycero phosphates, introduced by Robin, have been used especially by the French; e.g.:

B Calcii glycerophosphatis............... 0.50....gr. vii

Dent. tal. cachets no. $\mathrm{xxx}$.

Sig. -1 or 2 cachets 3 times a day.

SYMPTOMATIC THERAPY OF PULMONARY TUBERCULOSIS.

Fever: Indications for Antipyresis.-The fever in pulmonary tuberculosis should not be treated symptomatically until rest in bed and fresh air have proved powerless to reduce it. In such a case the indications for antipyretic measures, medicinal or hydropathic, are: (1) con- 
stant and considerable rise of temperature, with the danger of a steady breaking down of proteids, and the difficulty of giving enough nourishment at proper intervals; (2) severe, unpleasant subjective symptoms, such as intense chills, headache, giddiness, prostration, sweats, nausea, neuralgic pains, etc.

Diet During Fever.-During a severe, acute attack of fever, the patient must generally be limited for a time to a liquid diet. But otherwise the fever is no contraindication to a nourishing and abundant diet, which should, however, be especially easy to digest (soup, white meat, milk, eggs, cooked fruit, easily digested vegetables). Alcohol is valuable, as it combines an antipyretic with a stimulative effect. During a chill the patient may be given a warm alcoholic drink; he may have strong wine with his meals and add brandy to his milk (1-2 small teaspoonfuls of brandy to a glass of milk).

If antipyretic measures are indicated, as above, we may use either medication or hydrotherapy.

Antipyretic Medication: Antipyrine-Antifebrin-PhenacetineLactophenine.-The drugs most commonly used are antipyrine, antifebrin, phenacetine, or lactophenine. It is best to give them 2-3 hours before the temperature is expected to rise, repeating, if necessary, within the limits, whenever it threatens to go up. In this way, the ingestion of food is not interfered with. The maximum doses are:

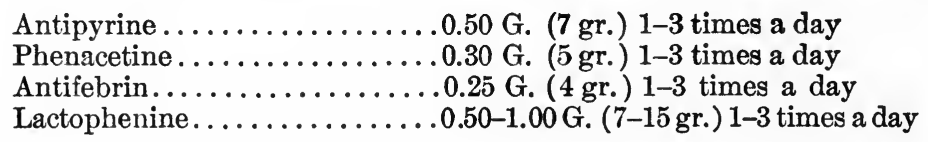

If these antipyretics, especially antipyrine or phenacetine, appear to increase an already marked tendency to diaphoresis, it is best to give them more frequently in smaller doses (phenacetine or antifebrin, one $0.05 \mathrm{G}$. ( $3 / 4$ gr.) pill [or a one grain tablet-Ed.] every hour; antipyrine in a 2-3:150 solution, one tablespoonful every other hour). Lactophenine apparently has no such effect, a fact, which, together with its sedative action on the nervous system, gives it a certain advantage over the others.

Pyramidon.-Pyramidon, an antipyrine derivative, (dimethylamido antipyrine) is the best antipyretic in pulmonary tuberculosis, producing an effect in smaller doses than even antipyrine. The author gives a single dose of $0.30-0.50$ G. (5-7 gr.) or even 1 G. (15 gr.) dissolved in $30 \mathrm{G}$. (1 oz.) of water. The dose may be repeated if necessary up to 3 G. (45 gr.) a day without the slightest bad effects. On an average the temperature becomes normal or subnormal within three hours. It may be equally well used to anticipate an expected rise of temperature. [I have found doses of 0.065-0.2 G. (1-3 gr.) effectual.-Ed.]

To avoid producing sweating we give pyramidon in the form of pyramidon camphorate, dose $0.5-1.0$ G. (7-15 gr.). Of all antipyretics in pulmonary tuberculosis this is the author's favorite. 
Maretin.-Maretin, methylated acetanilide (the so-called "non-toxic" antifebrin), a white almost tasteless powder, is similar to pyramidon, but apparently less reliable (dose $0.5 \mathrm{G}$. ( $7 \mathrm{gr}$.) once or twice a day). Like pyramidon, it often causes a profuse sweat.

Quinine-Eupyrine.-Quinine is nowadays used much less often than the above antipyretics. In pulmonary tuberculosis it appears to be not nearly so effective. It is, however, to be preferred to the others when there are symptoms of a weak heart. The dose is $0.30-0.50 \mathrm{G}$. (5-7 gr.) of quinine hydrochlorate.

The author also uses eupyrine (vanillin antipyrine), dose $0.50 \mathrm{G}$. ( $7 \mathrm{gr}$.), which on account of its vanillin radicle has a slightly stimulative effect on the heart.

Hydropathic Antipyresis.-Although hydropathic measures are not used so much in pulmonary tuberculosis as in other infectious diseases, the author personally is in favor of sponging and packs to relieve the fever and its accompanying subjective symptoms. In sponging, the whole body may be kept under cover or each part may be successively exposed, the face, neck, chest, back, upper extremities, abdomen, etc., being sponged in turn. If a washcloth is used, it is best to wrap it wet around the part being bathed and then rub vigorously, following this with a rub with a dry washcloth. We may follow the sponge bath by a rub off with alcohol. For the method of giving a pack, see p. 292.

Baths are employed only exceptionally, when the patient is strong and the rise of temperature is acute. Cold applications, ice-bags or cloths. may also be used on the head, over the heart, and over those portions of the lung where the inflammatory processes are known to exist.

NIgHT Sweats: Sponging.-To prevent the exhausting night sweats, the body may be sponged off just before going to bed, and if necessary during the night or in the morning. For this purpose, equal parts of water and alcohol or aromatic vinegar may be used. Brandy in water or milk ( 1 teaspoonful to 1 tablespoonful in a cup of hot or cold milk) may also be given at bedtime. If neither of these measures meets with success, we must have recourse to drugs. [Porter believes night sweats are evidence of exhaustion. He has the patient awakened a short time before the expected sweat and administers an ounce of whisky.-Ed.]

Medication: Potassium and Sodium Tcllurate.-For this purpose the use of sodium (or potassium) tellurate, introduced by Neusser, is to be recommended. Unfortunately this gives an odor like that of garlic to the breath. It is prescribed as follows:

$\mathrm{R}$ Sodii or potass. tellurati........... $0.40-0.60 \ldots$. gr. vi-gr. $x$

Pulv. acaciæ .............................

M. fiat mass. Div. in pil. no. $x x$.

Sig. $-1-3$ pills 3 times a day.

We begin with 1 pill taken 2-3 hours before the sweating is expected, increasing to 2-3 pills, if, as often happens, the patient acquires such a tolerance for the drug as to make it necessary. 
Atropine.-Atropine may also be used. It often becomes necessary to give this also in large doses. It may be prescribed as follows :

R Tab. atropinæ sulphatis............0.00027.....gr. $\frac{1}{2} \frac{1}{50}$

Sig. - 1-4 tablets (or if necessary more, cautiously used) 2 hours apart, in the evening.

Eumydrin-Agaricin.-Eumydrin is also very satisfactory in doses of $0.001-0.004$ G. (1/60-1/16 gr.), and is less toxic than atropine. Agaricin, a white powder, barely soluble in water, is another possibility. It is contraindicated in coincident digestive disturbances, particularly an inclination to diarrhœa, as it has a tendency to make the bowels loose. It is generally combined with a Dover's powder:

B Agaricini............................. ii

Pulv. ipecacuanhæ et opii............ 2.00....gr. $x \times x$

Altheæ,

Mucil. acaciæ .......................... s.

M. fiat mass. Div. in pil. no. $x x$.

Sig. -1 or 2 pills 5-6 hours before the expected appearance of the night sweat.

Camphoric Acid.-Camphoric acid has the advantage of producing a quick effect (in $1 / 2$ hour), which lasts for $6-8$ hours. It is prescribed as follows:

R Acid. camphorici..........................gr. xv

Dent. tal. cap. no. xv.

Sig.-1-2 capsules in the evening.

Hydrastis Canadensis-Ergot.-The fluidextract of hydrastis (30 drops at bedtime), ergot, veronal, and sulphonal are also available.

The author has never seen as undoubtedly good results from the recently recommended tincture of sage.

Guacamphol.-Guacamphol, the camphoric acid ester of guaiacol, is sometimes used. So far the author has not found its effects certain, whether given in the dose preseribed by its advocate, 0.50-1.00 G. (7-15 gr.), or in larger amounts, $2-3$ G. (30-45 gr.).

Formalin.-Formalin or formol (40 per cent. aqueous solution of formaldehyde) may be painted on the skin, first on one arm and the back, then on the lower extremities, then on the other arm and chest. Its effect lasts for several nights. The suffocating effect of the formaldehyde may be prevented by placing the patient near an open window and having him hold in front of his mouth and nose a sponge saturated with oil of turpentine. [I am inclined to think the remedy would be worse than the symptom.-Ed.]

Tannoform.-The best and simplest of all remedies, however, in the author's opinion, is tannoform. Powdered on thickly it promptly prevents all sweating wherever it covers the skin. It must be put on every evening. 
It may be said in passing that it is equally useful in the abnormal production of sweat from other causes than tuberculosis (sepsis, leukæmia, uric acid diathesis, after infectious diseases).

Salicylate Powder.-Finally when the sweating is of only slight grade, it is often enough simply to rub the skin and dust it with salicylate powder. The face should be covered while the powder is being applied, as it is apt to induce severe coughing:

R Acid. salicylici.....................1.00....gr. $x v$

Talci purificati................. $100.00 \ldots$ iii 3 ii

M. fiat pulv.

Sig.-For external use.

[Zinc peroxide is an excellent dusting powder and may be prescribed as follows:

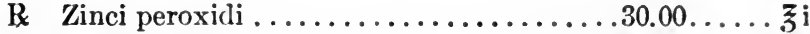
Talci purificati................... $45.00 \ldots \ldots$ iss
M. fiat pulv.
Sig. -To be dusted on the skin.-ED.]

Of course whatever causes the retrogression of the disease as such, causes the retrogression of the night sweats (open air treatment).

CoUGH AND EXPECTORATION.-The cough in pulmonary tuberculosis may be roughly classified into (1) a cough resulting in the expectoration of sputum, and therefore, in that respect, useful; (2) a cough without sputum, which is useless and often injurious. Even the cough with sputum is frequently dangerous, since new specific centers of infection may be set up by aspiration, emphysema of the still healthy portion of the lungs may be caused, and, finally, hemorrhage or a pneumothorax may be induced. It is, therefore, self-evident that our therapy must be aimed at reducing the cough much more often than at indueing expectoration.

The patient is frequently tortured by a cough which has its origin in the upper air passages (throat, larynx, trachea). Minor surgical measures, the application of certain drugs to the seat of the cough, sedative inhalations, or the internal use of similar remedies, are of good service.

Many patients can be trained to suppress a cough at its very beginning by mere force of will. This is often more easily accomplished if a small piece of ice or a sip of cold water is taken immediately. [Patients should be taught how to cough so as to raise sputum by a deep slow inspiration followed by a short quick expiration. Unless productive of expectoration the desire to cough should, if possible, be controlled.-Ed.]

Narcotics.-If none of these measures succeed, narcotics are indicated. They are indispensable in case the cough keeps the patient from sleeping or eating, or brings on vomiting. In the former instance we give frequent, small doses of some narcotic during the evening and night; 
in the latter, we give a hypodermic of morphine or a somewhat larger dose internally before meals. The author prescribes a hypodermic injection as follows:

R Sol. morphinæ magendie............15.00.... 3 iv

Sig.-For hypodermic use as directed by physician. $\left(7 \mathrm{~min} .=\mathrm{gr} . \frac{1}{4}.\right)$

A combination of morphine and apomorphine hydrochlorate is often used either in powder or tablet form, or in the following (Rossbach's) mixture :

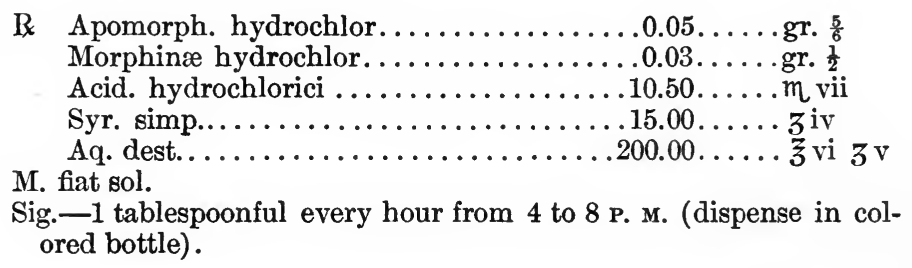

The author now prefers to this mixture, one consisting of morphine and euporphine. [I object to advising morphine except as a last resort when some of the derivatives (codeine, heroine, or dionine) have failed to relieve. With me codeine is a favorite. Sometimes local treatment of the nose, pharynx, or larynx will prevent the cough.-Ed.]

Position in Bed.- It is well to have the patient, on going to bed, lie at first on whichever side induces the coughing. In this way the lung cavities will soon be emptied and the patient can rest, at least until they fill up again.

Expectorants.-If we are convinced that a cavity or the whole bronchial tree is full of secretion, we may have recourse to an expectorant (ipecac, senega, apomorphine, euporphine). In case the secretion is purulent, the same balsams are indicated as in purulent bronchitis.

Cross-binder.-In all forms of cough and secretion, the cross-binder (see p. 289) is useful. It may be said in passing that it also relieves the pain in bronchitis, dry pleurisy, and in painful affections of the thoracic muscles and the intercostal nerves.

Smoking.-The patient may be allowed two or three mild cigars a day, provided they do not bring on coughing. [I have seen patients whose cough was benefited by smoking but I nearly always condemn smoking for any one with active tuberculosis.-Ed.]

HжмортYSIS.-In pulmonary tuberculosis hemorrhage is frequently the symptom which summons the physician for the first time because it is so alarming to the patient and his friends. The physician's first duty is to quiet their immediate fear and urge the necessity of calmness as a means of stopping the bleeding.

Rest-Local Cold.-Bodily rest must also be insisted upon, the patient being put to bed in a sitting or a half sitting position to aid 
expectoration. It is sometimes advisable to put an ice-bag over the part of the lungs from which the hemorrhage is supposed to come, partly for the effect of the cold, but more for the sake of making the patient keep absolutely still. In case the heart action is markedly excited, the bag may be placed alternately over the chest, and then over the precordia, for $1-1 \frac{1}{2}$ hours.

If the patient is threatened with coughing, cracked ice may advantageously be held in the mouth.

Narcotics.-If these measures do not succeed, medication may be resorted to. The author, in fact, usually employs it from the beginning.

Hypodermics of morphine, heroine, or codeine are most effective. If their injection excites the patient, any of these may be given by the mouth, or, in the case of morphine, by the rectum $(0.02 \mathrm{G}$. ( $1 / 3 \mathrm{gr}$.) in an enema or suppository). The dose will sometimes need to be repeated one or more times.

Hæmostatics: Ergot.-The hæmostatics are next to be considered. The one most frequently used is ergot (see p. 60). The author usually combines an injection of ergot with one of a 2 per cent. solution of morphine, filling the syringe half with one and half with the other. Ergotin may also be given:

R Pil. ergotini

Sig. -1 pill $3-4$ times a day.

. $0.10-0.15 \ldots .$. gr. iss-gr. ii

A very reliable preparation is ergotole, 20 drops $3-5$ times a day, or 1-2 syringefuls in a hypodermic. This is the pleasantest preparation for internal use.

An astringent, e.g., krameria, may often be added to a mixture containing ergot:

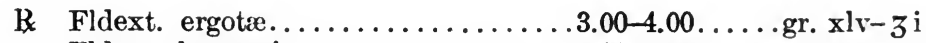

Fldext. krameriæ..............2.00-3.00.....gr. xxx-gr. xlv

M. fiat mist.

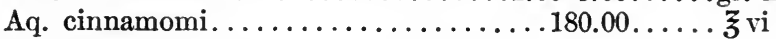

Sig. - 1 tablespoonful every hour at first, then every 2 hours.

Instead of the extract of krameria, the syrup, 15-40 G. ( $1 / 2-1 / 2$ oz.) may be used.

The mode of action of ergot is not fully known. It undoubtedly contracts the smooth muscle fibres of the arteries. But this would increase the blood pressure and so certainly not stop the hemorrhage. It must. therefore, have a second action. It may possibly diminish the force of the heart's contractions more than enough to counteract the arterial contractions, or it may, according to Filehne's theory, induce the formation of hyaline thrombi, which close the bleeding vessels. [I think it is useless to theorize upon its mode of action because authorities differ so decidedly and the preparations vary a great deal. I rarely use it for hæmoptysis.-Ed.] 
Lead Acetate.-The second hæmostatic is lead acetate, although it apparently is not always to be relied upon. Its action is due to its contraction of the blood vessels. It is best not given when fever is present, and is contraindicated in digestive disturbances. Large doses are essential. The author generally combines with it morphine or opium, in order to combat the cough:

R Plumbi acetatis $\ldots \ldots \ldots \ldots \ldots \ldots \ldots .0 .03-0.05 \ldots \ldots$.gr. $\frac{1}{2}-$ gr. $\frac{3}{4}$

Ext. opii...................... $0.02 \ldots \ldots$ gr. $\frac{1}{3}$

Sachar. lact........................... v

M. fiat cachet no. i. Dent. tal. dos. no. xxx.

Sig. -1 cachet every 2 hours until the maximum dose, 0.5 G. ( $7 \mathrm{gr}$.$) ,$ has been given.

[I never use this drug for such a purpose; nor do I believe it has any such action upon the blood vessels. Cf. Cushny.-Ed.]

Hydrastis.-If the hemorrhage is of moderate intensity, though prolonged, the fluidextract or glycerite of hydrastis is useful. It appears to be tolerably reliable in hemorrhage from the lungs, as well as the uterus, although its action is slower than that of ergot. Its action is not wholly understood, but appears to be due to a decrease of the blood pressure. [Cushny says that it increases blood pressure. Its mode of action is uncertain. I never employ it for this purpose.-Ed.]

R. Fldext. hydrastis, Vin. xerici..................āă $20.00 \ldots \ldots z^{v}$

M. fiat mist.

Sig. - 30-40 drops 3 times a day.

Witch Hazel.-The author has often obtained good results from the use of witch hazel:

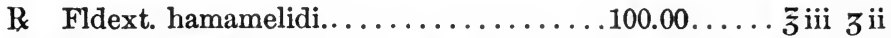

Sig. $-30-40$ drops (or even 1 teaspoonful) 5 times a day.

Shepherd's Purse.-Pribram especially recommends the fluidextract of shepherd's purse. The author has tried it on several cases, in most of which it had no stronger action than the other hæmostatics. In one case, however, a hemorrhage which had continued uninterruptedly for 3 days in spite of the administration of ergot and opium, was stopped after one day's use of the following prescription:

B Fldext. bursæ pastoris,

Vin. xerici................... āă $60.00 \ldots$ ii

M. fiat mist.

Sig. -1 teaspoonful $3-4$ times a day.

Hydrastis, witch hazel, or shepherd's purse may also be used in the form of a tea (1-2 teaspoonfuls added to a cup of tea; several such cups taken during the day). 
Quinine-Oil of Turpentine.-The author has tried quinine, 1.50-2.00 G. (22-30 gr.) a day, and the oil of turpentine. The former seemed to be entirely unreliable, but the latter had a surprisingly prompt and rapid action in most of the cases in which it was tried ( 5 drops every 2 hours until the bleeding stopped). A combination of quinine and ergot often proves successful:

B Quininæ hydrochlor........................ $z^{\text {i }}$

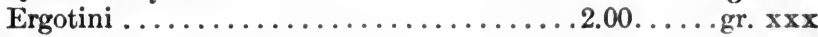

M. fiat mass. Div. in pil. no. xl.

Sig. -2 pills 3 times a day.

Gelatine.-Gelatine, given internally, proves very satisfactory (daily dose $10-20 \mathrm{G}$. ( $1 / 3-2 / 3 \mathrm{oz}$.) of gelatine, $1.20 \mathrm{G}$. (18 gr.) of salt, $200 \mathrm{G}$. ( $6 \mathrm{oz}$.) of distilled water, $1 / 4$ of the tepid mixture to be given immediately, followed by a tablespoonful every hour). Although other authorities (Curschmann) report excellent success from hypodermics of gelatine, the author does not believe in giving them except in emergencies, as they are apt to induce a rise of temperature above $102^{\circ}$, mental excitement and pain.

$A$ Concentrated Solution of Salt.-In case we are called upon to treat a case of hemorrhage at once and none of the above mentioned drugs are at hand, we may resort to the popular remedy of salt and water ( 1 tablespoonful of salt in a little water). Its effects may be due to its stimulating the mucous membrane of the digestive organs and so causing a reflex contraction of the pulmonary vessels. [This old-fashioned remedy still has a place in modern treatment of hæmoptysis although the explanation of its action is not clear.-Ed.]

Expectorants.-If the hemorrhage is profuse enough to flood the whole bronchial tree with blood so that asphyxia threatens the patient, an expectorant or an emetic is indicated. Jaccoud gives $0.10 \mathrm{G}$. (11/2 gr.) of powdered ipecac every quarter of an hour until nausea, but not vomiting, is induced. Champagne is also good in such cases.

Binding the Extremities.-The amount of blood supplied to the lungs may be restricted by binding the upper arms and thighs tightly at the middle, and so causing a venous stasis. The bandages must be removed gradually one at a time, generally after a quarter of an hour, although they may be left on longer if the patient does not experience discomfort from them. [This method of altering the distribution of blood probably also raises a dangerously low blood pressure. In serious cases it may save life.-Ed.]

Duration of Rest in Bed.-The patient should stay in bed until every trace of blood has disappeared from the sputum; i.e., for from 3 or 4 days to a week after the hemorrhage. He should be given plenty of fresh air. During the first few days he must be very careful about using his voice. 
Diet.-While the hemorrhage continues, the diet must of course be liquid and exclude tea, coffee, alcohol, and charged waters. After the bleeding has been stopped for twenty-four hours, solids may be very gradually allowed again (raw or soft boiled eggs, somatose and similar preparations, jellies, bread and crackers soaked in milk or broth, cereals, rice pudding, scraped beef and ham, tender chicken, easily digested vegetables).

Stools.-During the first few days of a severe hemorrhage the bowels should be kept from moving, if necessary by direct medication (opium), for fear that the intrapulmonary blood pressure may be raised by the effort of evacuation. When once the hemorrhage is over, however, we must, naturally, be more than usually assiduous in seeing to it that the bowels are properly emptied.

[Many of the drugs mentioned by the author have, in my judgment, little or no value in controlling pulmonary hæmoptysis. Physical rest and mental quiet are the principal indications. The patient should, therefore, be in bed in the position most favorable for breathing and expectoration, usually propped up with pillows or a bed-rest. The second indication, mental quiet, is often difficult to obtain. The physician's command of psychical therapy will frequently be severely taxed. $\mathrm{He}$ may, however, quiet the patient's anxiety by assuring him of the very slight danger, and by alluding to the hemorrhages of menstruation, of childbirth, or epistaxis. The patient should not be allowed to talk. No physical examination of the chest should be undertaken unless a very serious doubt as to the origin of the hemorrhage exists. I rely almost wholly upon morphine administered hypodermically, in doses of 0.008 $0.02 \mathrm{G}$. ( $1 / 8-1 / 3 \mathrm{gr}$.) ; it quiets the breathing and the cough, and, what is far more important, the patient. An ice-bag over the precordium will assist in quieting an overacting heart. Lawraison Brown advises diminishing the patient's blood pressure to 110-115 millimeters of mercury, by administering nitro-glycerin or sodium nitrate hypodermically. Porter of St. Louis attempts fixation of the area of the chest from which the hemorrhage arises, by means of a pad held in place by a bandage. The value of this would be more striking if we could be sure of locating the bleeding spot. Calcium lactate in $2 \mathrm{G}$. ( $\mathrm{I} / 2 \mathrm{dr}$.) doses three times a day for two days may stop repeated small hemorrhages. Large profuse hemorrhages with collapse necessitate the use of adrenalin hypodermically, of salt solution, and of bandaging the extremities.-Ed.]

Insomnia : Causal Therapy.-In treating sleeplessness in pulmonary tuberculosis we must primarily endeavor to remove its cause (cough, high fever, pain in the chest, dyspnœa, etc.). If the pain in the chest is due to a slight pleurisy, it is best relieved by leeches, the actual cautery, occasionally a mustard plaster, hot or cold applications, analgesic ointments (see p. 363), and the internal use of antipyrine, sodium salicylate, phenacetine, pyramidon, or exalgine, the latter being also indicated where the pain is due to an intercostal neuralgia or rheumatism of the 
intercostal muscles. A particularly good antineuralgic, especially suited for sleeplessness resulting from neuralgic or rheumatic pains, is trigemin, a chemical compound of pyramidon and butylchloralhydrate, white needles with a mild taste and an aromatic odor, easily soluble in water. The dose is $0.5-1.0 \mathrm{G}$. (7-15 gr.) once or twice in 24 hours. If none of these are successful, we may give a hypodermic of morphine at the spot where the greatest pain is located. [Codeine phosphate, $0.03 \mathrm{G}$. ( $1 / 2$ gr.) is worth trying before resorting to morphine.-Ed.] In case it proves impossible to remove the cause of the sleeplessness, we are obliged to resort to symptomatic therapy, already sufficiently discussed (see p. 47).

Disturbances of the Digestive Organs.-Digestive disturbances, especially of the stomach and intestines, are exceedingly common in pulmonary tuberculosis, and must be treated with great care, as on the condition of the digestive functions depends, in great part, the fate of the patient.

In case digestive disturbances exist, a careful examination should first be made to determine their exact nature. The stomach contents should be examined, and the size and motility of the stomach tested. Frequently we find a purely nervous dyspepsia, sometimes a true neuritis of the vagus, and again a chronic or acute gastric catarrh, atony, dilatation, or, possibly, an amyloid degeneration of the mucous membrane. The treatment of each of these conditions will be taken up more fully in the chapter on diseases of the stomach. Here only a few of the most essential points will be given.

Nervous Dyspepsia.-In cases of nervous dyspepsia the patient should be allowed all possible freedom in indulging his individual preferences in regard to his diet. Frequently he will take highly spiced dishes, salads, pickled meat and fish, etc., when he cannot eat plainer food. In case he can himself suggest nothing to tempt his appetite, it is sometimes advantageous to prescribe experimentally one limited form of diet; e.g., (1) milk; (2) raw meat and vegetables; (3) a preponderance of vegetables, especially peas and beans.

Anorexia.-It is exceedingly important to combat anorexia. In case a high fever is responsible for the loss of appetite, we must endeavor to reduce it, and must give food, as far as possible, at the times when the temperature is down. If cough or pain in swallowing is responsible, narcotics may be given before meals. If general prostration is the only assignable cause, the fresh air treatment and inhalations of oxygen are indicated. Stomachic bitters and appetizers are useful only in a fraction of such cases. Generally such patients need a fluid or semi-fluid diet. Raw meat may be used to advantage in severe cases of anorexia. A piece of tenderloin (300 G.-10 oz.) should be cut into small pieces and rubbed through a fine sieve; about $150 \mathrm{G}$. $(5 \mathrm{oz}$.) will be thus obtained. This may be given in bouillon (with the fat first removed) or mixed with mashed potatoes. Some patients like lemon juice or wine added to it. We may begin with $50 \mathrm{G}$. (11/2 oz.) a day increasing rapidly to the neces- 
sary minimum of $200 \mathrm{G}$. (6 oz.). [Change of climate often succeeds where drugs fail.-Ed.]

Stomachics.-The most useful stomachics are condurango (wine, or peptonized extract, a liquor glass before each meal), strychnine, quinine, gentian, and herb teas. For example:

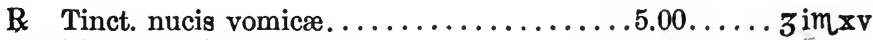
Tinct. gentianæ comp.,
Tinct. cinchonæ comp............ āa $7.50 \ldots \ldots z$ ii
M. fiat mist.
Sig. - 15 drops 3 times a day before meals, in water.

In case of abnormal fermentation in the stomach, one of the last two tinctures is best replaced by tincture of anise or peppermint.

Or :

Or :

$\mathrm{R} \quad$ Inf. quassiæ................ $300.00 \ldots$ vi 3 ii

Sig.-A liqueurglassful before each meal.

B Fldext.gentianæ......................... 3 im $\times \mathbf{x v}$

Aq. cinnamomi.................... 3 iii 3 ii

Sig.-A small teaspoonful before each meal.

If, finally, the loss of appetite is caused by constipation, as not infrequently happens, the bowels must of course receive the proper attention.

Other Gastric Disturbances: Pepsin and Hydrochloric Acid.-The discussion of other disturbances of the stomach must be deferred to a later chapter. It may be said, however, that in some cases where it is difficult to obtain the opportunity for exact examination, the combined use of pepsin and hydrochloric acid often hits the mark; e.g.:

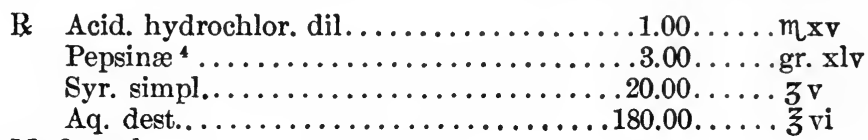

M. fiat sol.

Syr. simpl.....................20.00.... 3v

Sig. - 1 tablespoonful every 2 hours.

Mineral Waters.-Mineral waters are often available, especially when the gastric symptoms are more prominent than any others, even than the pulmonary symptoms. We may prescribe treatment at the earthy alkaline springs (Lippspringe) ; at the common salt springs (Homburg, Kissingen, Wiesbaden, Reichenhall); at the alkaline-chloride springs (Ems, Luhatschowitz); and, but only very rarely, at the alkaline-saline springs (Carlsbad, Vichy). All of these waters may also be used at home, often in small frequent doses. In case of hypochlorhydria and

${ }^{4}$ The Nat. Form. gives an essence of pepsin which is an excellent preparation, and corresponds in action to the prescription here given. It, however, as well as all the well-known proprietary preparations, contains rennet, which in some cases might be undesirable.-Ed. 
atony, it is well to give some alkali (magnesia, chalk, sodium bicarbonate, $100-200$ c.c. ( $1 / 2$ glass) of Carlsbad or Vichy) directly before eating, and hydrochloric acid directly after.

Intestinal Disturbances: Antifermentatives.-Abnormal fermentation in the stomach or intestine is best combated by ereosote or resorcin, the latter generally in a mixture, but also in powder form, 1.00-5.00 G. (15-75 gr.) or even more a day. For example:

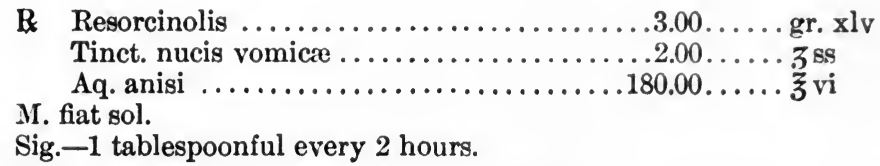

Charcoal may also be given, $1.00-2.00 \mathrm{G}$. (15-30 gr.) in single doses of $0.50 \mathrm{G}$. ( $7 \mathrm{gr}$.). On account of its hygroseopic properties, it should always be enclosed in capsules:

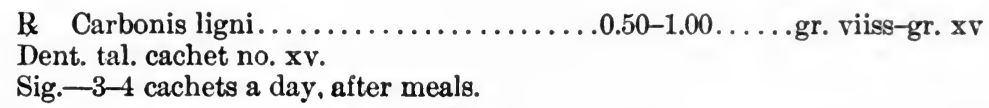

A feeling of pressure after eating is generally relieved by the use of bismuth (the salicylate or subnitrate, or dermatol).

For the treatment of obstinate diarrhœa, with or without ulcerative processes, see p. 502 .

After-Treatment.-When the tubercular process has been checked or even apparently healed, the physician must still see to it that the patient continues to follow out as far as possible the general hygienic measures of abundant food and fresh air. He should choose an occupation which keeps him out of doors; in fact it should be his aim to live as much as possible in the fresh air. [Occupation, and by that I mean useful work with some definite purpose and at least a possibility of accomplishment, should be advised as soon as possible. I am convinced that many physicians neglect this most important psychical therapeutic measure. Stevenson's life has often stimulated patients to work. I am inclined to discourage prolonged residence at sanatoria or in climatic resorts where no appropriate occupation can be secured.-Ed.] If the tubercular process has healed leaving only a contracted apex, the patient can gradually indulge in out of door sports; but if there have been severe infiltration processes and cavity formation, resulting in extended contracture of lung parenchyma, all bodily strain is forbidden, for fear of a wider dispersion of the tubercular poison, hemorrhage, or heart strain. Such a patient is best off permanently in a suitable climatic resort (Davos).

[Osler states, "Benefit is usually a matter of months, complete arrest a matter of years, absolute cure a matter of many years." 
Of 569 patients admitted to the Adirondack Cottage Sanitarium as advanced cases, and who had been away from it from two to eighteen years, Brown reported 28.6 per cent. as well and 52 per cent. dead.

Of 100 cases, two-thirds of whom were originally classed as incipient cases, who averaged about eight months' treatment and rest before returning to work or home, and who were at least quiescent for one to two years, Wilder reports that 87 per cent. are either quiescent or cured and able to work, 13 per cent. have relapsed and recovered, 8 per cent. have relapsed and died, 4 per cent. are chronic invalids. Of the 87 per cent. quiescent or cured more than half continued to live in the place where they recovered, or in one with a similar climate.

Such figures emphasize the chronicity of the disease, the liability to relapse, the necessity for the patient's giving up sufficient time for thorough arrest of the process, and for his continuing the same careful hygiene after returning to work.-Ed.]

The physician is often called upon to give his advice concerning the marriage of a tubercular person. In general, as long as there is the slightest sign of an active process, marriage should be forbidden.

If, however, the disease has been absolutely stationary for at least two years, permission may be given, comfortable circumstances being presupposed, especially if the patient be a man. For a woman there is always danger that child bearing may cause the acute reappearance of the latent disease. After parturition she should be sent to the most favorable climate and in no case should she be allowed to nurse her child. 
IX.

\section{THERAPY OF DISEASES OF THE PLEURA}

\section{THERAPY OF PLEURISY.}

Pleurisy may be divided into three varieties: (1) fibrinous, (2) exudative, and (3) fibrous. The exudative form in its early stages, however, and frequently throughout its whole course, is almost always accompanied by a plastic exudate and, if it is of short duration, is treated like the fibrinous form.

\section{THERAPY OF ACUTE, FIBRINOUS PLEURISY.}

A patient with fibrinous pleurisy usually consults a physician on account of two symptoms, pain in the side and shortness of breath. He may also be distressed by a painful cough. To relieve these subjective symptoms and to allay the inflammation are the two aims of a proper therapy.

Rest in Bed.-The patient should be kept quiet, usually in bed. $\mathrm{He}$ should be allowed to lie in whatever position he finds most comfortable. Generally he will instinctively lie on his sound side; but occasionally he lies on the painful one in order to prevent or lessen its movement during respiration.

Withdrawal of Blood.-The blood should be drawn from the painful part of the thorax by wet cupping (or dry, if the patient is weak or rundown) or by 5-10 leeches. Mustard plasters or turpentine ointment are much less efficacious.

Analgesic Applications.-The thorax may also be rubbed with various applications to relieve the pain ; e.g., chloroform and oil of wintergreen in equal parts, or other ointments of various kinds, or the following ointment containing chloral and camphor:

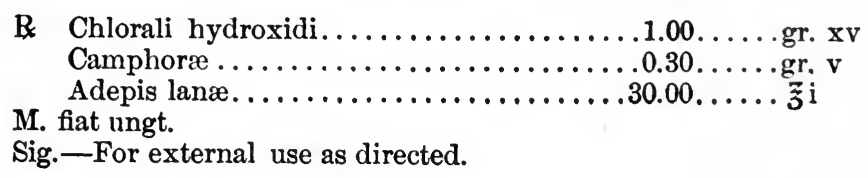

Or this, containing menthol and cocaine:

R Mentholis .................. 0.50-1.00.....gr. viiss-gr. $x v$

Cocainæ hydrochlor................. $0.30 \ldots \ldots$ gr. v

M. fiat ungt.

Adepis lanæ...................... $30.00 \ldots \ldots \tilde{\tilde{z}}$

Sig.-For external use as directed. 
Or, finally, camphor-vasogen or camphor-chloroform-vasogen, which last the author strongly recommends.

The skin may also be painted every day with pure chloroform and the painted part covered with rubber tissue, [or painted with a light coat of iodine-Ed.].

A binder may be passed around the diseased half of the thorax to aid in keeping it quiet. The patient stands in front of the physician with the painful side turned away from him. The middle point of a strip of adhesive plaster three to four inches wide and long enough to reach from the sternum to the middle of the back, is then pressed down on the axilla and the two ends pulled as tightly as possible toward the physician and fastened respectively on the sternum and the vertebral column. Several other smaller strips may be similarly put on at the same time. Some patients are much relieved by these bandages, but others find they are only made more uncomfortable, so that the straps must either be removed entirely or put on tighter.

Hydrotherapy.-Hot and cold applications are both efficacious, some patients being helped more by the former, others by the latter.

Morphine.-If the pain or cough is very severe, we may resort to a hypodermic of morphine. It is generally injected at the most painful part, the location of course for the sake of suggestion. An ice-bag should also be used.

Diaphoresis.- Sweating is often helpful to a pure fibrinous pleurisy. The most popular method of inducing it is by the use of hot teas, but a hot bath $\left(99^{\circ}-104^{\circ}\right.$ of $1 / 2-1$ hour's duration) followed by a hot, dry pack is more effective, as are also steam- and "Roman-Irish" baths. Jaborandi leaves or the essential principle, pilocarpine, 0.01-0.02 G. $(1 / 6-1 / 3 \mathrm{gr}$.) hypodermically, are very seldom used on account of their bad effects in other directions.

Sodium Salicylate.-The use of sodium salicylate will be discussed in the next section.

Breathing Exercises.-As soon as the inflammation has subsided and the pain gone, the patient should take regular breathing exercises for the sake, if possible, of preventing adhesions. Lying upon his sound side he should take 20-30 deep breaths, repeating this exercise a good many times a day (Traube). Or he may go through the same exercise standing, at the same time raising and letting fall his arms.

\section{THERAPY OF PLEURISY WITH EFFUSION.}

Derivatives.-In its primary stages a pleurisy with effusion cannot be distinguished from a fibrinous pleurisy and receives the same treatment. It is impossible to foretell whether in a fibrinous pleurisy exudation will or will not appear. When once it has been clearly demonstrated, the author, in spite of the opposition of many authorities, believes in a single application of a soft cantharidial plaster. $\mathrm{He}$ has 
seen it cause the quick disappearance of several pleural exudates. It should be 10-15 cm. (4-6 in.) long and 10-12 cm. (4-5 in.) wide and should be left on not longer than 9-10 hours. The blister should then be pricked with a sterile instrument, the fluid withdrawn, and an antiseptic dressing applied.

Sodium Salicylate.-In every case of rheumatic or of doubtful origin, we give sodium salicylate in rather large doses, 6-10 G. (11/2-21/2 dr.) a day, or more often salophen or aspirin. In rheumatic cases, they apparently have a specific effect; in other cases, the author has found them useless.

Diaphoresis.-Diuresis.-In non-rheumatic cases, generally of tubercular origin, after a few days' trial of sodium salicylate and its derivatives, we proceed to diuretic or diaphoretic methods. The latter are advisable only when the patient is in good general condition and without fever. If he is in bed, the hot air bath (phénix à l'air chaud) is the most convenient; if he is up and about, any form of hot bath. Diuretics sometimes assist in getting rid of the effusion. We may use digitalis, generally with potassium acetate or squills; the decoction of ononis spinosa with squills; calomel; caffeine or theobromine; diuretine alone or as an addition to digitalis, morphine, agurin, or theocine; and urea. The last the author has found effective in certain cases of pleurisy with effusion caused by tuberculosis or carcinoma. He prescribes it as follows :

R Ureæ.................... 10.00-20.00.... 3 iiss $-\mathbf{z} \mathrm{v}$

Aq. menth. pip.,

Spir. vin. Gallici...............āā $75.00 \ldots \ldots$ ろ̌ iiss

M. fiat mist.

Sig.-1 tablespoonful ice-cold every 2 hours. Pieces of ice to be swallowed directly afterwards.

In some other cases, parsley water has had an effect. It is given as a decoction in tablespoonful doses repeated every 2 hours.

Diuretic teas are not suitable because they contain too much liquid.

Iodine.-The author puts no reliance upon iodine, internally or externally, as an aid in promoting absorption.

Diet.-The diet should not contain large amounts of any liquid except milk. If the patient suffers from thirst, he may be given a tablespoonful of lemonade with the addition of hydrochloric or phosphoric acid every hour or two, or may suck pieces of ice or chew peppermint lozenges. If he has sufficient appetite he may have easily digestible forms of meat, jellies, milk-pudding, custards, etc. If meat is not well borne, preparations of meat may be substituted. One half to one glassful of light wine as a heart tonic is allowed. Stronger wines are indicated only where there are symptoms of cardiac insufficiency, in which case other strong heart stimulants are also indicated, unless thoracocentesis is resorted to. 
Schroth's Cure.-This consists in forbidding all liquid and feeding the patient entirely on stale rolls. In one such case, the author saw it cause the absorption of an exudate which had recurred even after puncture.

Stools.-The bowels must be kept open. If the patient is strong and has no fever, mild cathartics may be used for a few days.

Puncture: Indications.-If in spite of the foregoing measures, the effusion either remains stationary or increases, there are only two alternatives left: (1) to wait until absorption takes place naturally, which may not be for 5-6 weeks or even longer, or (2) to proceed to puncture.

Thoracocentesis is indicated when an exudate of moderate extent, to the fourth or, at any rate, the third rib in front, to the middle of the scapula behind, shows no sign of being absorbed by the end of the third week. In setting this time as the limit, we have to balance the dangers of puncture (emboli of the cerebral and pulmonary arteries, sudden paralysis of the heart, cerebral anæmia, albuminous expectoration, etc.) against the greater danger involved in too long a duration of the exudate; i.e., the possibility that the lungs may, under too long a pressure, lose their elasticity, so that when the pressure has finally disappeared, connective tissue formation and atrophic processes will have taken place (chronic interstitial pneumonia with dilatation of the bronchi and bronchiectasis). This danger of permanent injury is much more serious than that of the very rare accidents above enumerated. Fever is no contraindication for operation, although, of course, a normal temperature is preferable.

Again, thoracocentesis is indicated when the exudate is very extensive, especially if it is bilateral, as in that case it is exceedingly unlikely that it will be spontaneously absorbed.

A third indication is the danger of a fatal termination, shown by intense dyspnœa, pronounced symptoms of pressure on the mediastinum, particularly on the heart, marked cyanosis, congestion of the cervical veins, heart weakness, or cerebral anæmia.

A fourth and last indication is the existence of severe subjective distress caused by the fluid in addition to its original cause (carcinoma, tuberculosis, lymphosarcoma of the thorax and especially of the mediastinum), a distress which may be caused by a small amount of effusion but which can be relieved only by puncture.

On the contrary, if the effusion is hemorrhagic, we should wait as long as possible before aspirating, as in such cases the exudate usually collects again rapidly, and so the puncture means an increased loss of blood.

In case of a tuberculous sero-hemorrhagic effusion it is also best to defer puncture, as often better care and nourishment, with the administration of tonics and hæmostatics, may turn it into a pure serous effusion, which will be absorbed spontaneously. 
Pure hemorrhages into the pleural cavity should also be given all possible chance for spontaneous absorption.

Technic.-The technic of thoracocentesis is as follows: The patient should be in a half-sitting position, propped up firmly in bed. The spot selected is first thoroughly disinfected and then anæsthetized with ethyl chloride. An exploratory puncture is first made with a hypodermic syringe. If fluid is found, a trochar with lateral openings is thrust quickly through the chest wall. A sudden lack of resistance shows that the instrument has entered the free pleural cavity; Potain's aspirating apparatus is then attached to the trochar and the fluid withdrawn. If the fluid contains air bubbles there is generally some leak in the apparatus. If the flow suddenly ceases, the opening has been closed either by the diaphragm, or by a clot of fibrin. By withdrawing the trochar slightly the first difficulty may be overcome; the second, by pushing the needle carefully in again. At the end of tapping, the skin should be pressed close to the trochar and the latter withdrawn as quickly as possible to prevent the entrance of air.

Although the foregoing method of aspiration affords the least possible risk of injecting air into the pleural cavity, such detailed care is not absolutely necessary in a fresh effusion (pleuritis acutissima). Here a simple apparatus, based on the principle of the siphon, will suffice. A hollow needle is connected with a small funnel by means of about a yard of rubber tube, which can be closed close to the needle by a stop cock, [or an artery clamp-Ed.]. The tube and funnel are filled with a 4 per cent. boric acid, 0.1 per cent. salicylic acid solution, or a sterile physiological salt solution. The stop-cock is first closed, the needle is then thrust in, the funnel slowly lowered and plunged into a receiving vessel filled with an antiseptic fluid, and the stop-cock opened. The solution in the tube flows out followed by the pleural effusion.

A hypodermic of morphine, 0.01 G. ( $1 / 6 \mathrm{gr}$.), before commencing either operation, will quiet the patient, prevent coughing after the tapping, and with it the possible danger of an albuminous expectoration. Heart stimulants should always be at hand in case of syncope.

With a free pleural effusion we, generally speaking, select a point for puncture as low as possible. To prevent the possibility of injuring the diaphragm, its position may be marked on the skin of the sound side as a guide. If we puncture in the anterior axillary line or midway between this and the mamillary line, the point will usually lie in the 5th or 6 th intercostal space. The reasons for not choosing a spot on the back, although more of the effusion is usually collected there, are these: (1) the muscular walls are thicker posteriorly; (2) the intercostal spaces are narrower; and (3) there is a thicker sedimentation of fibrin. [None of these deter me. I always select the back unless adhesions, peculiar location of fluid, or some special reason become contraindications.-Ed.]

The quantity of fluid withdrawn naturally depends upon the extent of the effusion. On an average 1500-1600 c.c. ( 3 pints), may be 
aspirated with safety. It should be withdrawn slowly. Too rapid aspiration or the withdrawal of too much fluid, apparently increases the danger of subsequent pulmonary œdema.

Diuretics after Puncture.-After aspiration we should renew the use of diuretics and local applications. If the effusion continues to increase, a second puncture is indicated.

If, a week after aspiration, the effusion has definitely disappeared, the patient feels no subjective distress, and the appetite has returned, he may get out of bed for a short time. He never should be allowed to get up, however, until his temperature remains normal, even though absorption has taken place spontaneously.

Breathing Exercises.-As soon as the symptoms of acute inflammation have disappeared, the patient must begin systematic breathing exercises, so as to strengthen the weak side, expand the compressed lung, and so prevent extensive pleural adhesions. Fven before he gets up, he may begin with 26-30 deep, slow respirations during the day, gradually making them deeper and deeper. Later he may lie on his sound side in order to exercise the weak one as much as possible. For the same purpose he may raise the arm of the diseased side during inspiration, as soon as he is taking his exercises standing up. When he is perfectly well, the use of a pneumatic chamber and moderate mountain climbing are often of great use in increasing the breathing capacity of the lungs.

Unabsorbed Residuum.-Occasionally, both where puncture has been resorted to and where partial absorption has taken place without it, a small residual amount of exudate remains unabsorbed. In such cases, we may try the different sweat-cures and hydropathic procedures (sunbaths, Priessnitz's bandage, cross-binder, packs, salt-baths); we may, for example, send the patient for treatment to Ems, Kissingen, Ischl, Gmunden, or Reichenhall.

Climate.-Unless the pleurisy is undoubtedly due to rheumatism, tuberculosis may always be suspected. The patient should therefore be carefully guarded from catching cold, and should be sent to an equable southern climate in winter and to the mountains or seashore in summer.

\section{THERAPY OF FIBROUS PLEURISY.}

After some attacks of pleurisy with effusion, extensive, often very thick, fibrous adhesions are formed. These cause various subjective symptoms (pain in the side during deep inspiration or when lying upon the affected side). These should be treated by breathing exercises and by the use of pneumatic chambers, with the view of stretching the pseudomembrane. Various analgesic ointments, local massage, and the local and general use of heat are also efficacious. The author places 4-5 thicknesses of paper over the sore spot and irons it with a hot iron until 
the patient can stand no more heat. This procedure once or twice a day for several days often causes a rapid disappearance of the pleuritic pains.

\section{THERAPY OF SUPPURATIVE AND PURULENT PLEURISY.}

All that can be said of the therapy of this condition is that surgical interference is demanded as early as possible. Fither Bülau's siphon drainage is employed, or thoracotomy is resorted to, with or without resection of the ribs. There are only two conditions under which thoracocentesis is indicated: (1) where there is no time or no opportunity for surgical interference, the patient's life being threatened, and (2) where the patient is so weak that the radical operation, with its rapid change of the intrathoracic pressure relations, is dangerous, even if it is performed under local anæsthesia. In the latter case, we temporarily relieve the patient by puncture in order that his strength may be built up for thoracotomy. The latter operation is indicated in all other cases, with the exception of empyema in pulmonary tuberculosis. Here, in spite of Ziemssen, the author takes a conservative position and advises operation only when absolutely necessary. He has, to be sure, seen one case of pneumothorax in pulmonary tuberculosis cured by repeated thoracotomy, except for a residual pleural fistula.

\section{THERAPY OF PNEUMOTHORAX.}

The pain and orthopnca due to a fresh pneumothorax, are best made bearable by hypodermics of morphine, $0.01-0.02 \mathrm{G}$. ( $1 / 6-1 / 3 \mathrm{gr}$.) several times a day. These are also necessary later on, provided dry cupping, mustard plasters, etc., are powerless to allay the pain. With symptoms of much increased intrathoracic pressure, the thorax should be punctured by means of a hollow needle connected with a long rubber tube, whose end is submerged in a sterile physiological salt solution. As long as the intrathoracic pressure is greater than that of the atmosphere, bubbles of air will pass out through the tube. In case of a valve pneumothorax, the procedure must, of course, be repeated.

Symptoms of heart weakness appear oftener during the formation of a pneumothorax than after its full development. They should receive the usual treatment. In case a purulent effusion is joined with the pneumothorax, thoracotomy or resection of a rib should be undertaken, unless it is contraindicated by: (1) a tuberculous or carcinomatous etiology, or (2) extreme weakness, especially bad heart action. In such cases aspiration may be resorted to. 


\section{GENERAL THERAPY OF DISEASES OF THE STOMACH AND INTESTINES}

IN order to avoid unnecessary repetition, a general discussion will precede the special treatment of the different diseases of the stomach and intestines.

\section{GENERAL PRINCIPLES UNDERLYING THE THERAPY OF DISEASES OF THE STOMACH AND INTESTINES.}

\section{Dietetics.}

The Digestibility of Different Articles of Food.-It is clear that in the therapy of diseases of the digestive tract, the diet plays the most important part. The quality of the food, the combination of different foods, and the times at which food is to be given, should all be carefully regulated. In most cases, though not in all, an easily digested diet is necessary; we begin, therefore, with a brief discussion of the digestibility of the different articles of food. This discussion should, however, be prefaced by the aphorism that " what is one man's meat is another man's poison "; i.e., the digestibility of a food is a purely subjective matter. Yet some general principles may be laid down which hold good for the majority of people, and these will be taken up in the following paragraphs.

Meat.-The digestibility of .meat depends upon different factors. As far as the kind of meat is concerned, the following list shows the order of digestibility: Fowls and pigeons, capons and chickens (not too fat), partridges and pheasants, ducks and geese, veal (the most easily digestible form being boiled calves' brains), fish (particularly [fresh codfish-Ed.] trout, pike, and carp), beef, pork, and mutton.

A second important factor in the digestibility of meat is its fat content. Other things being equal, the fatter the meat, the longer it stays in the stomach, for the fat, which cannot itself be acted upon by the gastric juice, surrounds the muscle fibres of the meat and makes it harder for the gastric juice to get at them. Koenig gives the following table for the average fat-content of the common kinds of meat and fish:

Calves' brains, veal, ham, shell-fish, pike....Fat: 0.4-1.0 per cent.

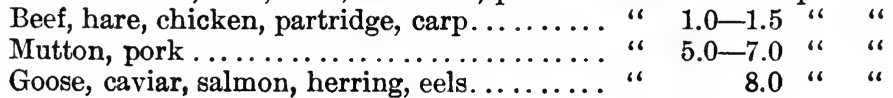


Its large fat-content makes tongue a very indigestible food, aside from the fact that its tip is composed of very tough muscle fibres. Attention should be called to the fact that oysters are not so easily digested as was formerly believed. ["Three medium sized oysters are entirely disposed of by the stomach in one and three-quarters hours."-Hutchison. The American oysters are more easily digested than the European.-Ed.]

The third important factor in the digestibility of meat is its mode of preparation. Pounding the meat crushes the connective tissue fibres and thus separates the muscle fibres, leaving the latter more accessible to the pepsin and hydrochloric acid. The division of the meat into small pieces (scraping, grinding, chopping, mincing) also lessens the work of the stomach at the outset. Experience shows that raw meat is more digestible than meat cooked in any way. The mode of preparation used in the kitchen varies between broiling, roasting, boiling, and steaming. In boiling the result depends upon whether the meat is put immediately into boiling water, or into cold water and then brought to a boil. In the first case the heat of the water at once coagulates the albumins of the surface, thus preventing any further escape of extractives and salts from the meat into the water; this process results in good meat and bad soup. In the second case we get good bouillon, but meat which is cooked to pieces and tasteless. In roasting, the results are also affected by the exact mode of procedure. If the surface of the roast is rubbed with a little fat and then quickly exposed to great heat, and if the meat is repeatedly basted with the juice which comes out during the roasting, the surface is quickly coagulated, and the escape of the extractives and salts from the interior again prevented. In general there is no especial difference to be noted in the digestibility of roasted, boiled, and steamed meat of the same kind and quality. In the case of birds, however, the author agrees with Leube in giving to boiling the preference over roasting. An underdone roast, hot or cold, is more easily digested than one well done. In smoked meats (ham and smoked fish) the creosote produced prevents putrefaction but delays digestion; while in pickled meats, the abnormally large amount of salt hinders the secretion of hydrochloric acid and so makes digestion more difficult. Hams are more digestible if mildly cured, and may then be eaten raw if one is sure of the absence of trichinæ.

The digestibility of meat is next affected by the part of the carcass from which it comes and the time that has elapsed since the death of the animal. Fresh meat (i.e., that which is still in rigor mortis, or myosin coagulation resulting from the development of lactic acid) is tougher and harder to digest than that which had been hung several days. In the latter case, the connective tissue fibres are broken up by the formation of lactic acid after death, and made more easily soluble, while the myosin coagulation, in consequence of the action of bacteria, is ended and compensated for. Meat must, of course, be kept only a limited time and then only under favorable conditions,-in a cold place free from insects, 
so that no process of decay may begin. Birds are usually cooked at once before the myosin coagulation has begun to take place. Fish, too, should be used either absolutely fresh or suitably preserved.

The digestibility of meat is further affected by the age of the animal, the fibres of a young animal being finer and more tender than those of an old one. Finally, it is somewhat influenced by the presence of various other substances used in cooking; for example, a small quantity of condiments, such as pepper and mustard, apparently makes digestion easier.

Meat Soups or Broths-Meat Juices-Meat Extracts.-The nutritive value of these meat derivatives is slight; but they furnish energy to the body as exhibited in osmotic pressure, they facilitate the absorption of other foods, and, most important of all, they arouse the appetite. Unless especially contraindicated (e.g., on account of the inadvisability of diluting the gastric juice) a small cup of soup may be taken at the beginning of each hearty meal. To increase its nutritive value without at the same time taxing the digestion, we may add an egg, well cooked cereal, or meat, such as calves' brains or chicken finely minced and passed through a sieve. [Clear soups contain meat extracts, ${ }^{1}$ the flavoring, and from 1 to 5 per cent. solids, chiefly fat and gelatin, so that a large soup plateful equals less than $1 / 3$ ounce of nutriment. Thick soups can be made as nutritious as desired by adding grated cheese, macaroni, barley, rice, peas, or lentils. Beef tea when properly prepared contains less than 2 per cent. proteid, in addition to the salts and extractives contained in raw beef juice. In the latter the proteids are soluble, while in beef tea they have been coagulated by heat, but in such fine flakes that they are probably no less digestible than the soluble proteids. Beef tea is, therefore, valuable largely for stimulating the appetite, and refreshing and making a pleasant change for the patient. Natural raw beef juice prepared at home contains from 3 to 7 per cent. coagulable proteid and from 2 to 4 per cent. extractives. Among the preparations sold largely for invalid consumption, the following are worth mentioning: "Vigoral," a suspension of powdered beef in beef extract, contains 68 per cent. albuminoid. It must be used fresh. "Puro" contains 29 per cent. coagulable proteid. It is palatable. "Bovinine" contains between 14 and 17 per cent. of coagulable proteid. Its taste is unpleasant and much of the proteid is probably derived from blood. Wyeth's and Brand's beef juices, Bovril beef juice (containing meat fibre), "Taurine," and "Liquor carnis," contain considerable unaltered blood, and are therefore properly regarded as uncooked juices. They contain between $1 / 2$ and 5 per cent. coagulable proteid.-Ed.] Ziemssen's beef essence contains 7 per cent. proteid. To obviate its disagreeable taste which may nauseate the patient, it may be taken in meat soups or flavored with cognac or vanilla, and frozen to a frappé. [Valentine's meat juice contains still less proteid. The objection to all these meat juices is that

${ }^{1}$ Creatin, creatinin, carnin, xanthin, sarcin, osmazome.-Ed. 
in proportion to the amount of nutriment they contain they are expensive, and that large amounts cause thirst and diarrhœa. It is well to remember that the white of one egg diluted with 3 ounces of water contains as much coagulable proteid as the same amount of ordinary commercial beef juice.-Ed.] The same objections apply to the use of meat extracts. They have still less value as foods. Liebig's, Kemmerich's and Cibil's are among the best known of the semi-solid preparations. A teaspoonful added to a plate of soup will stimulate the appetite, and perhaps improve the soup's flavor.

Meat Peptones.-These preparations were designed to furnish predigested proteid in the form of albumoses or peptones. To-day they are not rated so highly as foods as they were formerly. The meat proteids are transformed into albumoses by the action of pepsin, papayotin, or superheated steam. When the proteids of ordinary food cannot be given in sufficient quantities, the author is convinced that these preparations may be substituted for a certain length of time, and doubtless they possess the important property of mechanically stimulating the mucous membrane of the stomach. In obstinate anorexia or when the patient refuses food which he usually likes, because he believes that he cannot digest it, these artificial foods can be given until the natural food can be again resorted to. They are of more decided use in hypochlorhydria when the intestinal secretion is also deficient, because, as we know, if there is a deficiency of gastric juice, unchanged proteid will be transformed in the intestines provided it reaches there soon enough. Riegel, from special investigations upon somatose, found that these preparations have more use when the other food is deficient in nitrogen. Their use has been objected to on the ground that their taste is disagreeable, [that the stomach may eventually become incapable of carrying on its usual transforming function, largely theoretical-Ed.], and third, that they tend to induce diarrhœa. We may conclude, therefore, that these predigested forms of meat are of limited value for a short time.

Among the most useful of these preparations are: Somatose, made from meat, a grayish powder, readily soluble in water, tasteless and odorless, containing 80 per cent. albumoses. [Tankard's analysis estimates the albumoses at 34 and the peptones at 3 per cent.-Ed.] Several [three or four-Ed.] teaspoonfuls may be given daily in soup, milk, coffee, or chocolate. Other forms are somatose biscuit, somatose chocolate, somatose cocoa. The author has found that somatose is willingly taken and well tolerated by patients and rarely causes diarrhœa. [Mosquera beef meal, a powder made from beef, partially digested by the proteolytic ferment from the juice of pine-apples, has a high nutritive value, contains 77 per cent. proteids, 13 per cent. fat, 11 per cent. soluble albumoses, and 18 per cent. peptones (Chittenden). It is easily digested, well absorbed, and especially valuable in gastric atrophy associated with intestinal disease. Pemmican and Charque are similar preparations.-Ed.] Antweiler's peptone, a powder, [contains 60 per cent. peptones, 14.5 albumoses- 
Ed.], Kemmerich's meat peptone (dry) [contains 32.5 per cent. peptones, 14.5 albumoses-Ed.], Maggi's, Bolero's, and Merck's are similar solid preparations. The dose of these preparations is one teaspoonful to a cup of some liquid several times a day; or the preparation may be used alone or spread on toast. Among the liquid and semi-solid preparations are Denayer's peptone [12 per cent. soluble proteids, chiefly albumoses; Fairchild's panopepton containing sherry, 3 per cent. soluble proteids (chiefly albumoses), 15 per cent. extractives and organic matter (the last largely sugar.)-Ed.]; and Leube-Rosenthal's meat solution, a meat preparation partially digested with hydrochloric acid under the action of steam [containing 6.5 per cent. peptones, 11 per cent. albumoses, and 6 per cent. fat-Ed.]. Ross's "Kraft-bier" contains 38 per cent. albumoses. It has a disagreeable taste and no more nutrition than any other good beer.

Gelatin.-One food which is useful in stomach diseases as well as under many other circumstances is gelatin prepared [from isinglassEd.] from commercial gelatin or from calves' feet, pigs' feet, calves' heads, ox tail, or ordinary veal or chicken. It may be served in various forms of jellies and aspic. Large quantities are apt to bring on diarrhœa. [Gelatin is very easily digested but its chief value as a food is as a proteid-sparer; it also favors the secretion of gastric juice and combines with a good deal of its acid, hence its utility in cases of hypersecretion. Most jellies used for invalids contain less than 7 per cent. of gelatin and a large amount of sugar.-Ed.]

Eggs.-Eggs are a most nutritious food, since they contain both proteid and fat; but their digestibility depends upon the way in which they are cooked. They are most easily digested when soft boiled. The best method is to put them into cold water, and bring the water to a boil, removing them a few seconds later, or to let them cook for 3 minutes in boiling water. Raw eggs carefully stirred into hot soup are as good as soft-boiled ones, but if they are taken quite raw they are considerably more difficult to digest, while hard-boiled eggs are invariably indigestible. [The nutritive value of an egg equals that of one-half tumblerful of good milk, of $1 \frac{1}{2}$ ounces of fat meat, or about 70 calories. Raw egg, especially if beaten to insure breaking up the cell walls enclosing the proteid of the white, remains in the stomach perhaps one-half hour longer than if lightly boiled. This is probably because the former is so bland as to excite neither the secretion of the gastric juice nor the movements of the stomach. Its digestion is really performed in the intestines alone. Hard-boiled eggs, if finely subdivided, are probably just as easily digested as soft boiled (Hutchison).-Ed.] An important distinction must also be made between the yolk and the white, the former being much less easily digested on account of its high fat-content. A food which may be given in the most serious disturbances of the digestive tract, cholera for example, is albumin water prepared by slowly stirring into the white of an egg about 200 c.c. (a teacupful) of cold water and then adding sugar 
or, especially in cases of vomiting, wine or brandy [or the juice of half a lemon or orange-Ed.].

Milk.-In general the most satisfactory of all foods in stomach troubles is milk. As Pawlow's investigations have shown, it requires very little gastric juice and almost no " psychical secretion." At the same time we must recognize the fact that it cannot for any great length of time fully satisfy the need for nourishment even if the patient rest in bed, and also that for many people it is not easy, but hard to digest, bringing on marked meteorism or obstinate diarrhœa. It may be made more digestible by various additions ; e.g., salt, brandy, lime water (especially), burnt magnesia or bicarbonate of soda [dilution, aeration, or a thin gruel-Ed.] If none of these attempts succeed in an individual case, the use of milk must be abandoned. Its digestibility does not depend upon whether it be taken raw or cooked, sweet or sour, but is affected by its fat-content; the more cream it contains, the more difficult it is to digest. [In addition to its digestibility, milk possesses other advantages as a food for individuals with gastro-intestinal affections. In virtue of its fat-content it has a restraining influence upon the amount of the gastric juice, it neutralizes a considerable quantity of acid, in proportion to its nitrogen content it requires less strength in the gastric juice, it acts as a diuretic. and it diminishes intestinal putrefaction.Ed.]

Substitutes for Milk.-Among the preparations which may be used in place of milk in cases of stomach or intestinal disease, are [koumissEd.] kefir (contraindicated in hyperacidity), condensed milk, and Gärtner's fat-milk (see p. 100). Condensed milk is made by evaporating milk in a rarefied air-chamber, generally with the addition of cane sugar; Loefflund's, however, has no sugar, so that for gastric diseases it is preferable. In Gärtner's fat-milk the reduced casein-content contributes to its digestibility (as far as the author's rather limited experiments go), and it is perhaps to be recommended, especially in those cases in which there is a natural tendency to, or in which the use of milk causes, constipation. [Pure casein can be obtained in the form of protene flour. Sanose contains 80 per cent. of pure casein and 20 per cent. of albumose from egg white. Nutrose is a similar compound whose solubility is ensured by the presence of an alkali. Eucasein is an ammonia casein. Plasmon is the latest and cheapest of these preparations. In it the milk proteids are made soluble by being combined with bicarbonate of soda.Ed.] Then there are the numerous preparations used especially for infants' food, Liebig's malted milk, Biedert's, [Horlick's, Borden's,-Ed.] etc. Finally there is Lahmann's "vegetable milk," a thick extract made from almonds and other nuts. One part of this added to five parts of water makes the so-called "plant-milk." This, mixed with one or two parts of cow's milk, gives a combination very like mother's milk in its fat and proteid contents. Milk somatose is made from milk; and contains 5 per cent. of tannin in organic combination. It may be used to alleviate 
an existing diarrhœa or when we wish to avoid every possible irritation to the intestine. Three or four teaspoonfuls may be given daily in any liquid.

Farinaceous Foods.-Milk cannot for any considerable period of time furmish enough heat for the body. It is therefore best from the beginning not to depend on an exclusively milk diet, even when it is necessary to spare the stomach, but to supplement the milk as soon as possible with preparations of the various kinds of meals and cereals. Common flour can be given, creamed with butter and used to thicken soup, or boiled barley, oatmeal, rice, [hominy, farina-Ed.], or Indian meal in various preparations. There are also a number of finely ground meals in which the starch has been dextrinized, among which are Hartenstein's, Liebe's, Liebig's, Maggi's or Knorr's Legume-meal, Timpe's or Weibezahn's " soup meal" and Theinhardt's hygiama [Avenacia, Horlick's foodEd.]. The various infants' foods are also sometimes available. They are in general mixtures of condensed milk and some meal, with the insoluble changed into soluble starch. Among the best known are Nestlé's and Kufeke's [and Eskay's-Ed.]. When the digestive tract can be given a little more work, the patient may often take noodles or macaroni.

Vegetables.-Peas, beans, and lentils are also available in the form of meal, but, although they are very nutritious owing to their large carbohydrate and proteid content, they can only exceptionally be used in gastric affections. Prepared as purées, however, they are not so difficult to digest as is commonly supposed.

The same thing holds true of potatoes, the most widely used of all our root and tuber vegetables. Made into soup, they are incomparably more digestible than if boiled in the ordinary way; but the best of all methods of serving them is to mash and whip them until they are light and mealy. Next to potatoes, the most useful of the common root vegetables for such patients as we are considering, are young carrots. But of far greater worth are two foreign products which come under this same head: sago, from the pith of several varieties of palm, and tapioca from the tubers of a Brazilian manchot. Poor in proteid, they are very rich in starch (over 80 per cent.), and can be given in a very easily digestible form in soup or pudding.

But few of the other vegetables can be recommended in these cases. The green vegetables, for example, are rich in cellulose, which contains but little nutriment and is hard to digest. According to Penzoldt, the order of their digestibility is cauliflower, asparagus, kohlrabi, carrots, spinach, and string beans. To these may be added young green peas. Ordinary salad is made still more indigestible by the oil, vinegar, and condiments employed in its preparation.

Fruit.-Much the same may be said of fruit. Not particularly indigestible in itself, it often has a deleterious effect on the stomach on account of its large amount of fruit acid and, with ripe, sweet fruit, on account of its sugar, which may produce an abnormal fermentation in 
the alimentary canal. Moreover, fruit is often the bearer of many bacteria. This suggests the necessity of carefully washing or peeling fruit, cutting out all decayed portions, and being careful to sterilize it thoroughly when canning or preserving it. As fruits with stones contain more acid than those with seeds, the latter are to be preferred, apples, for instance, and particularly soft pears, which contain the least acid. They are better served cooked than raw. We can also allow preserved cherries, as they are not acid. Plums are useful in chronic constipation but should be given only when the stomach is intact. Berries are also too acid. Grapes, as is well known, have a peculiar medicinal value, particularly in abdominal plethora and sluggishness of the intestine. A grape cure will cure ptyalism.

Nuts.-On account of their richness in proteid, fat, and sugar, and their small amount of prussic acid, nuts, particularly almonds in the form of a fresh emulsion, may be employed in inflammatory affections of the alimentary canal. Acorns (acorn cocoa or coffee) in virtue of their tannic acid are valuable in diarrhœe.

Bread.-Ordinary bread, on account of the yeast employed, is very rarely advisable for patients with weak stomachs. Stale bread is better than fresh, and the crust better than the soft inside, for in the crust more of the starch has been dextrinized. Zwieback, toast, and crackers are still more digestible. Soaking toast or zwieback does not make it easier to digest, for " the diastatic action of the saliva is stronger with the prolonged mastication which the dry crust demands, than with the briefer mastication needed after the crust has been softened" (Penzoldt). Breads which are made of coarsely ground cereals (e.g., graham bread, pumpernickel) demand strong digestive secretions. They are of therapeutic value when it is desired to augment insufficient peristalsis by increasing the contents of the intestine and so affording a stronger stimulation of the intestinal wall.

Aleuronat bread, already mentioned in the section upon diabetes mellitus, is sometimes valuable for patients with stomach disease. It may be also obtained in the form of "Kraftzwieback" and "Kraftbrot," with about 30 per cent. proteid. Roborat bread, prepared from vegetable proteid meal and rice meal, fulfils a similar purpose.

The importance of thorough mastication must be insisted on, especially if the diet is rich in carbohydrates.

Sugar.-Sugar is introduced into the stomach in different forms, as cane sugar, grape sugar, fruit sugar, and milk sugar. The first is contained in sugar-cane and sugar beets, the second and third in fruit and honey, and the last in milk. Cane sugar in the intestines is first inverted, but grape and fruit sugar are absorbed directly. Patients with gastric or intestinal troubles should be careful not to ingest too much sugar lest its absorption be delayed and so an abnormal fermentation be produced in the stomach, which, spreading to the intestine, may cause diarrhœa. It is worth noting, also, that too great quantities of cane sugar may 
reduce the digestive power of the stomach, lessen the flow of hydrochloric acid, and increase the time required for digestion. On the contrary, as Strauss has shown, grape sugar unites with the hydrochloric acid, an important fact for the therapy of hypersecretion.

Honey.-Honey and malt extract are distinguished by their high content of carbohydrates. In view of the fact that a teaspoonful of honey contains more calories than an egg, scarcely sufficient attention seems to be paid to honey as a carbohydrate food. [Honey is an invert sugar, and contains, if pure, about 40 per cent. of lævulose, 35 per cent. of dextrose, and 3 per cent. of cane sugar. An equal amount contains about 25 per cent. more calories than malt extract, so that it is one of the most valuable forms of sugar for patients with digestive troubles. The diastasic power in malt extracts has probably been exaggerated.Ed.]

Fats.-Fat is popularly supposed to be unsuitable for people with weak stomachs. This is not true of fresh butter, which is very easily digested. Almost all such patients will obtain only benefit from 50 to 100 G. (1 $1 / 2-3$ oz.) of butter daily with crackers, zwieback, or fine wheat bread. Cocoa, oat-cocoa made by adding oatmeal to cocoa, eucaseincocoa, von Mehring's "Kraftschocolade," sanatogen chocolate, all deserve mention here. In general it may be said that the lower the melting point of a fat, the more easily it can be absorbed and digested. The fats of butter, fat cheese, and milk, and the vegetable fats, such as olive oil and sesame oil, must therefore be regarded as easily digested. Fats enclosed in animal cells, such as bacon and fat meat, are much more difficult to digest.

Condiments.-Among the condiments, salt holds the first place. Its effect on the secretion of gastric juice is not yet satisfactorily understood. Whether in small quantities it increases the secretion is very doubtful, but it is probable that, taken into the stomach after eating, it diminishes the secretion, and it is certain that in the intestine it is easily carried off, on account of the attraction from the blood serum into the lumen of the intestine. The other salts required for nutrition, phosphoric acid alkalies, earthy alkalies, and iron as well, are ingested in sufficient quantities with the food. The use of condiments other than salt for patients with digestive troubles is very seldom advisable, and then only when it is desired to stimulate the walls of the stomach and intestines, as for example in atony of the digestive tract. In everyday life their use is to be avoided, or at any rate decidedly curtailed, on account of their action on the nervous system in general and on the nerves of the stomach in particular. Vanilla, caraway-seed, anise, cloves, and cinnamon are less harmful than pepper, paprika, and mustard.

Liquids.-Water is absorbed from the stomach only in very small quantities [if at all-Ed.], the greater part being soon emptied into the intestine. Large quantities of water or of any liquid are detrimental to the stomach in two ways: by overloading it and by retarding diges- 
tion. Care must be taken never to drink too much of any liquid, particularly before or during a meal. Even a pint is enough to delay gastric digestion. [Hot water reaches the intestines much more rapidly than cold; the heat stimulates the motor activity and relaxes the pylorus.Ed.]

Alcohol.-Alcohol can destroy pepsin, but in small quantities it can stimulate the motor function of the stomach, and in many cases increase or arouse the appetite. Moreover it is highly probable that it promotes the passage of fats from the stomach; [and protects them to some extent from oxidation-Ed.]. Hence the author advises the use of a small amount of alcohol for those patients whose appetite must be aroused and whose stomachs must be made functionally more active. For this purpose either a liqueurglass of cognac or half a wineglass of heavy wine (sherry or port) may be ordered about a quarter of an hour before meals, or one or two wineglasses of light white or red wine towards the end of the meal. On account of its relatively great amount of tannic acid, the Greek wine known as Camarite has the same effect in many intestinal diseases as huckleberry-wine, which has taken its place. [Blackberry brandy has a similar effect.-Ed.] Haut Sauterne enjoys a popular reputation because it forms no acid in the digestive tract. Cider cannot generally be recommended, on account of its large amount of pyromalic acid; still in some diseases of the intestine it often has a salutary effect. Sparkling wines and champagne must frequently be used as stimulants in various acute diseases of the digestive tract. They owe their strongly stimulative power to the fact that they contain both alcohol and carbon dioxide, and the absorption of the alcohol is greatly hastened by the presence of the carbon dioxide. In severe cases of vomiting, champagne cooled on ice is a valuable remedy on account of the anæsthetizing effect of the carbon dioxide and of the cold on the mucous membrane of the stomach. Without such special indications, however, people with weak stomachs should not use sparkling wines and champagne, for the carbon dioxide, by distending the stomach and overstimulating peristalsis, disturbs gastric digestion. Beer is a form of alcohol which should almost never be permitted to the patients under consideration. Its detrimental effect is due, first to the fact that it is almost always taken in too great quantities, thereby distending the stomach and diluting the gastric juice, and second to the danger that with it great quantities of lees and fungus may be taken into the alimentary canal. To be sure, pasteurization prevents this and makes beer less harmful; but beer is of real use only in exceptional eases, those with simple hyperacidity, and then only in small quantities. Irritation and distention of the stomach (especially as a consequence of pyloric stenosis) is an absolute contraindication for the use of any kind of alcohol; for, even if the alcohol is absorbed from the stomach, its presence there certainly increases the secretion of water and therefore the dilatation. When 
alcohol must be used as a stimulant in such cases, it should be given by the rectum.

Tea and Coffee.-Tea and coffee, as far as experiment shows, have no perceptible influence on the time required for digestion. Practical experience seems to prove, however, that fresh coffee infusion, especially if taken on an empty stomach, increases peristalsis with many individuals; while tea, on the contrary, by reason of its tannic acid, tends to check intestinal activity. If one wishes to minimize this last effect, he should use a weak solution of tea that contains as small a quantity of tannic acid as possible, pekoetea. ${ }^{2}$ In general tea is to be preferred to coffee in all cases of gastric irritation. We must not, however, overlook the fact that with many people coffee and tea both have a detrimental stimulative effect on the nerves supplying the muscles of the stomach, and so may cause feelings of flatulency, increased peristalsis, diarrhœa, and, if their use is continued, intestinal atony. Moreover, they often stimulate abnormally the nerves supplying the heart, a second reason why their use is obviously prohibited in individual cases.

Mineral Waters.-A final word, on the use of charged waters. There can be no doubt that, on account of their carbon dioxide, they stimulate the appetite and peristalsis and perhaps also influence the gastric juice (earlier and more profuse secretion), and that they are refreshing and quench thirst. Nevertheless they are often taken too frequently and in too great quantities at a time. The excessive amount of carbon dioxide thus introduced into the stomach brings on feelings of pressure and flatulency and may lead to dilatation, especially if too much of the gas is freed relatively quickly. In all inflammatory conditions of the intestinal tract and peritoneum, drinks containing carbon dioxide appear to be directly contraindicated. [Natural are probably more valuable than artificial mineral waters, perhaps on account of double salts or traces of salts present in the former and absent in the latter, perhaps on account of radioactivity. Certainly in the natural waters the carbon dioxide gas is not in excess, is present combined, and is evolved more slowly, so that there is less likelihood of their causing a sudden gastric dilatation.Ed.]

We have now considered all the principal articles of food with regard to their digestibility and their availability in diseases of the digestive tract in general. As in a former section (see p. 209) it has been shown how foods must be combined quantitatively and qualitatively in order to meet the demands of nutrition, the discussion of that question may be omitted here.

Number and Times of Meals.-In regard to the number of meals and the proper times for them, the above scheme (see p. 210) must often be totally disregarded in diseases of the stomach and intestine. If the condition is severe and acute, it is often best to spare the diseased organ

${ }^{2}$ [A superior black tea whose leaves are picked young.-Ed.] 
as much as possible by witholding food entirely for a day or more. To a less degree we may spare the stomach and intestines by giving food often in small quantities. This is true from purely mechanical causes as well as on account of the fact shown by Pawlow that the gastric juice secreted at the beginning of a meal, the "appetitsaft" (psychical secretion), has a much stronger digestive power than that secreted later. On the other hand, it is often our duty in atony of the digestive tract to give an abundant supply of food at long intervals, only three times a day, and so excite a corresponding peristalsis. Again in other cases (nervous dyspepsia) we may accomplish our end by giving an abnormally large amount of food in connection with a rest-cure.

Temperature of Food.-Too hot food is as bad for the stomach as too cold. The frequent occurrence of gastric ulcer among cooks should teach us the detrimental effect of too high temperature on the stomach. [If the heat of the food is an etiological factor it is certainly only one of several.-Ed.] On the other hand, as Wegele has pointed out, the effects of cold are shown by the number of cases of severe dyspepsia in America induced by the constant use of ice-cold drinks. Too hot or too cold food indirectly retards digestion. Ice-cold drinks containing carbon dioxide (soda water, mineral waters, champagne, or beer) are particularly injurious because the carbon dioxide held in solution while the water is cold, is suddenly set free in the stomach, and may, as has been pointed out, produce a rapid dilatation. Exceptionally, the author believes in the use of a small quantity of ice-cold champagne immediately or shortly after an abnormally full meal, for, on account of its content of carbon dioxide, it stimulates the peristalsis of the stomach and hastens the food more quickly into the intestine, an advantage that may, however, be attended with the disadvantage of having the food but partially prepared in the stomach for the intestine. Medium temperatures are, in general, the only correct ones for food and drink. For the first the temperature should be for the most part about blood-heat $\left(98^{\circ}-100^{\circ}-\right.$ $101^{\circ}$ ); the latter we enjoy more at a much lower temperature, which, however, except for particular reasons, should never fall below $50^{\circ}$.

Conduct After Eating.-There is great divergence of opinion concerning what the patient should do directly after eating. The author holds that he should lie down for at least an hour, flat on his back or half turned on his right side, for rest allows the blood, which would otherwise be distributed to different parts of the body, to be put at the disposal of the alimentary canal and so to hasten as much as possible the work of the stomach; whereas, if the body were in motion, the stomach would be loaded abnormally long and atonic disturbances of digestion would result. If the case be one of weakened digestive function of any kind, of irritation in the stomach, or of impoverished nutrition, the dorsal decubitus facilitates the passage of the contents of the stomach to the intestine. This advantage can also be obtained to a less degree, by the half upright position. Lying on the left side and slow walking delay 
digestion. For nervous dyspeptics, however, rest after eating cannot be always advised, as in these cases one must be guided more by direct experiment in each case and by the state of nutrition, than by their subjective feelings. The experiments of Penzoldt and others seem to show that driving after eating can be expected to hasten gastric digestion. Riding, on the contrary, must be absolutely prohibited, except to some nervous dyspeptics. When the patient has rested for one or two hours after a meal he may for the next hour or so take a long walk, if his bodily condition permits. The evening meal should not approach too near the time of going to bed; because, as experiment and experience have shown, digestion is retarded during sleep; and the stomach would be kept loaded abnormally long so that its muscles would gradually lose their tone and become incapable of doing their work. A light evening meal should be taken three or at least two hours before going to sleep. Dinner at about 6 P.M. and no nourishment afterwards, except possibly a cup of tea or soup, seems the best plan.

SMokIN.G.-Cigar-smoking should never be permitted where there is any acute stomach disease, and for chronic cases should be in general forbidden on account of its effect in reducing the appetite. Still, in some cases, it may be advisable to remove the prohibition if the patient is an habitual smoker, for, besides its psychical effect, smoking is of use in stimulating intestinal peristalsis. But a patient should never be permitted to smoke on an empty stomach, and should be limited to two or three light cigars a day directly after a meal, preferably dinner. A cigar holder should be used and the smoke should never be inhaled.

In the dietetic section of the general therapy of diseases of the stomach and intestines, there still remain two important questions which require our attention,- - first, the selection of the different articles of diet appropriate to a diseased stomach and the minute details for gradually increasing the choice as the condition at fault improves; and second, the selection suitable to such individual disturbances as depend upon the chemistry, the motility, or the power of absorption of the stomach, with an especial effort to avoid foods which would be contraindicated by the known pathological condition.

Time Required For the Digestion of Different Foods.-In the first task, the determination of what foods are easy, what are difficult for weak stomachs to digest, is simplified by knowing how long each food must remain in the stomach. Penzoldt ranks the digestibility of a food on this basis as follows:

Time in Stomach : 1-2 Hours Inclusive.

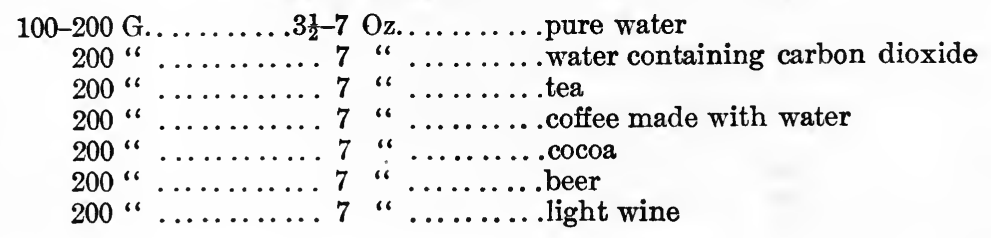




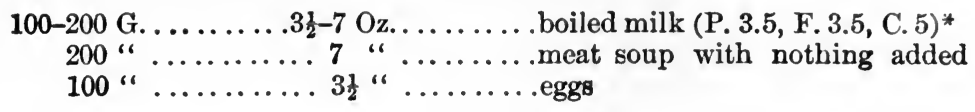

2-3 Hours.

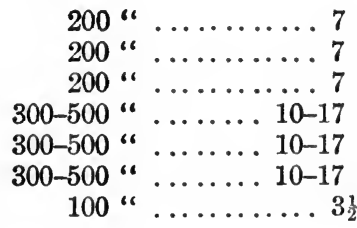

“ “...........coffee with cream

“..........Malaga wine

“.........water

“........ beer

........ boiled milk

........raw eggs, hard boiled eggs, or omelet (P. 12, F. 12)

100 “ ........... 3r “

250 " $\ldots \ldots \ldots \ldots 9$.....

250 “ $\ldots \ldots \ldots \ldots . .9$

beef sausage, raw

72 " $\ldots \ldots \ldots \ldots 22$

200 " $\ldots \ldots \ldots \ldots 7$

200 “ .......... 7

200 “ .......... 7

200 " .......... 7

150 “ $\ldots \ldots \ldots \ldots 55^{\frac{1}{2}}$ “

150 " $\ldots \ldots \ldots \ldots .5 . \ldots 5$.

150 “ .......... 5 $5 \frac{1}{2} "$ "

150 “. .......... 5. $5 \frac{1}{2}$ “

150 “. .......... 5 5

150 " .......... 5 $5 \frac{1}{2} "$

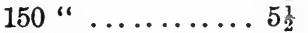

70 " $\ldots \ldots \ldots \ldots 2 \frac{1}{2}$ "

70 “ $\ldots \ldots \ldots \ldots .2 \frac{1}{2}$ “

70 “ $\ldots \ldots \ldots \ldots .2 \frac{1}{2} "$,

80 “.......... 3 .boiled calves' brains

............boiled sweetbreads

.......... boiled carp

“ ..........boiled pike (P. 18, F. 0.5)

“ ......... boiled shellfish (P. 17, F. 0.5)

......... boiled codfish (P. 80, F. 1)

......... boiled cauliflower (P. 2, C. 4)

“.........cauliflower salad

........ boiled asparagus (P. 2, C. 2)

"..........cabbage soup

.........mashed potatoes (P. 2, C. 20)

“.........cherry compôte

.......... raw cherries with tead, fresh or old, or with tea (P. 7, C. 52)

.........zwieback, fresh or old, dry or with tea "..........cracknels

.......... Albert biscuits

230 “ $\ldots \ldots \ldots . . .8$

$220-260$ “ $\ldots \ldots . . .88-9$

195 “ $\ldots \ldots \ldots \ldots 7$

230 " $\ldots \ldots \ldots \ldots 8$

$220-230$ “ .........7 $\frac{7}{2}-8$

3-4 Hours.

250 “ $\ldots \ldots \ldots \ldots 9$

250 “ $\ldots \ldots \ldots \ldots . . .9$

“ ........ boiled chicken (P. 20, F. 4)

" "......... boiled pigeon

" "......... roast pigeon

“ ......... roast partridge

“ ......... roast chicken

“ ........ raw or cooked beef (lean) (P. 21, F. 1.5)

160 “ $\ldots \ldots \ldots \ldots .5 \frac{1}{2} "$ ".

......... raw or cooked ham (P. 24, F. 36)

100 “......... 3. $3 \frac{1}{2}$ “

100 “ ............ $3 \frac{1}{2}$ “

"......... hot or cold roast veal (lean, P. 20, F. 15)

100 " ............. $3 \frac{1}{2}$ “"

100 “ $\ldots \ldots \ldots \ldots . . \ldots 3 \frac{1}{2}$ “.

“........ hot or cold broiled beefsteak

“........ raw or scraped beefsteak

“........ sirloin roast

“........salt caviar (P. 31, F. 16)

200 “ ........... 7 "

150 “ $\ldots \ldots \ldots \ldots 5$

150 “ $\ldots \ldots \ldots \ldots .5$

$100-150$ “ $\ldots \ldots \ldots . .3 \frac{31}{2}-5$

" . ........ pickled lamprey, smoked herring

" . ......... rye bread

" ......... white bread

150 “......... 5

150 “ .........5 5

“ ...........Albert biscuit

" "..........cabbage

“ ......... boiled rice (P. 3, C. 76)

150 “ $\ldots \ldots \ldots \ldots .5$ “ $\ldots \ldots \ldots$. boiled kohlrabi (P. 3, C. 8)

$* \mathrm{P} .=$ proteids, $\mathrm{F} .=$ fats, $\mathrm{C} .=$ carbohydrates. The amount is expressed in percentages. 


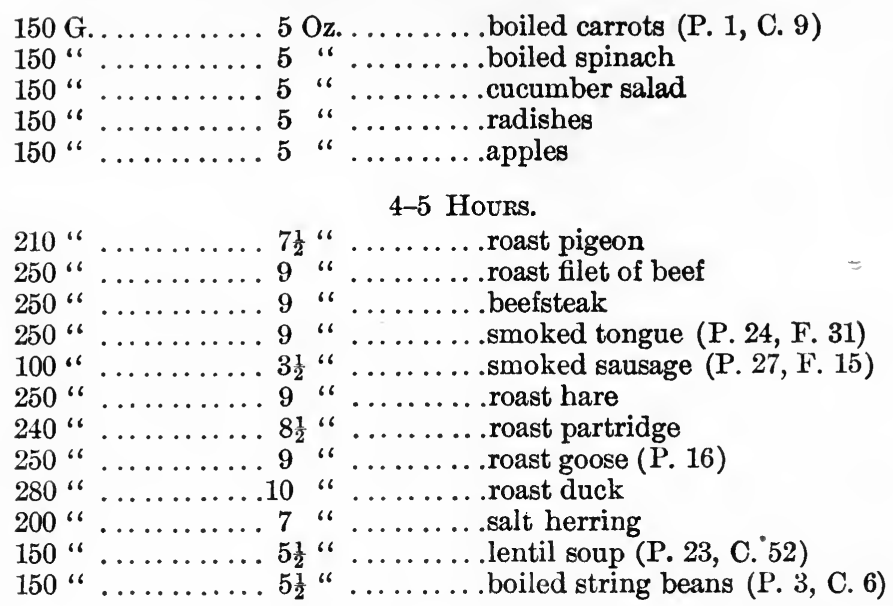

From these tables a diet can easily be selected which will give the stomach greater or less work to do; but it must be remembered that the digestibility of a food does not always correspond wholly to the time it must remain in the stomach. A food may be easy for the stomach to digest and yet be indigestible for other reasons. Radishes, for example, remain in the stomach only from 3 to 4 hours, and yet, on account of their sharp oil, they may irritate the intestine and even the kidneys. Cucumbers furnish another example. Both often cause disturbances of digestion which would not be expected from the result of experiment.

[Other facts influence the digestibility of food. Hemmeter defines a digestible food as "one that makes relatively small demands upon the secretory and motor functions of the stomach, which is readily absorbed and produces no subjective complaints or feeling of discomfort." To be of easy digestibility he considers that "food substances must-

1. Offer only slight resistance to the digestive juices-i.e., they must be of easy solubility.

2. They must not impede or accelerate peristalsis.

3. They must not excessively irritate the digestive organs, either mechanically or chemically.

4. They must not increase the processes of fermentation or putrefaction.

5. The greater portions of the substance must be absorbable either in the stomach or intestines."

A food which is easy for a normal stomach to digest is not necessarily so for a pathological organ nor equally so for different types of disease. Hence these rules and diet lists must be accepted with a certain amount of reserve.-Ed.]

The following table of Penzoldt may serve as a guide. It was drawn up as a practical, well tested diet scheme, exemplifying the gradual change from a very light diet to a fuller one (Leube's so-called "ulcer cure"). 


\section{PENZOLDT'S DIET TABLE}

First Diet (about 10 days)

\begin{tabular}{|c|c|c|c|c|}
\hline Food or drink & $\begin{array}{c}\text { Maximum quantity } \\
\text { at once }\end{array}$ & Preparation & Condition & Method of taking \\
\hline Meat broth $\ldots \ldots \ldots \ldots$ & 250 G. $(9$ oz. $) \ldots \ldots$. & From beef ........ & $\begin{array}{l}\text { No fat, little or no } \\
\text { salt }\end{array}$ & Slowly ............ \\
\hline Cow's milk ............ & 250 G. $(9$ oz. $)$....... & Boiled or sterilized & $\begin{array}{l}\text { Pure milk or with } \\
1 / 3 \text { lime water }\end{array}$ & Or with a little tea \\
\hline Eggs $\ldots \ldots \ldots \ldots \ldots \ldots$ & One or two ......... & $\begin{array}{l}\text { Soft boiled, just } \\
\text { warmed or raw }\end{array}$ & Fresh ............. & $\begin{array}{l}\text { If raw, stirred well } \\
\text { into warm not } \\
\text { boiling }\left(105^{\circ} \text { - }\right. \\
\left.112^{\circ}\right) \text { broth }\end{array}$ \\
\hline $\begin{array}{l}\text { Leube and Rosenthal's } \\
\text { meat solution }\end{array}$ & $30-40$ G. $(1-11 / 2$ oz. $)$ & n................... & $\begin{array}{l}\text { With only a weak } \\
\text { meat savor }\end{array}$ & $\begin{array}{l}\text { By the teaspoonful } \\
\text { or stirred into } \\
\text { broth }\end{array}$ \\
\hline $\begin{array}{l}\text { Biscuits (Albert bis- } \\
\text { cuits) }\end{array}$ & Six $\ldots \ldots \ldots \ldots \ldots \ldots$ & (n................... & Without sugar.... & $\begin{array}{l}\text { Not moistened but } \\
\text { thoroughly mas- } \\
\text { ticated }\end{array}$ \\
\hline Water $. . . \ldots \ldots \ldots \ldots . . .$. & $\begin{array}{l}125 \mathrm{G} .(1 / 2 \text { tumbler- } \\
\text { ful) }\end{array}$ & $\ldots \ldots \ldots \ldots \ldots \ldots \ldots \ldots$ & $\begin{array}{l}\text { Either ordinary } \\
\text { water or that } \\
\text { naturally charg- } \\
\text { ed with a small } \\
\text { amount of car- } \\
\text { bon dioxide } \\
\text { (Seltzer) }\end{array}$ & Not too cold ...... \\
\hline
\end{tabular}

Second Diet (about 10 days)

\begin{tabular}{|c|c|c|c|c|}
\hline Food or drink & $\begin{array}{l}\text { Maximnm quantity } \\
\text { at once }\end{array}$ & Preparation & Condition & Method of taking \\
\hline Calves' brains .......... & 100 G. $\left(3 \frac{1}{2}\right.$ oz. $) \ldots$. & Boiled ........... & $\begin{array}{l}\text { Free from all } \\
\text { membrane }\end{array}$ & Best in meat broth \\
\hline $\begin{array}{l}\text { Calves' sweetbreads... } \\
\text { (Thymus gland) }\end{array}$ & 100 G. $(31 / 2$ oz. $) \ldots$. & Boiled ............ & $\begin{array}{r}\text { Freed carefully } \\
\text { from its capsule }\end{array}$ & Best in meat broth \\
\hline Pigeon................ & One $\ldots \ldots \ldots \ldots \ldots$ & Boiled ........... & $\begin{array}{l}\text { Young, without } \\
\text { skin, tendons, } \\
\text { etc. }\end{array}$ & Best in meat broth \\
\hline Chicken .............. & One $\ldots . . . . . . . . . .$. & Boiled ........... & $\begin{array}{l}\text { Young, without } \\
\text { skin, tendons, } \\
\text { etc. (Not fat- } \\
\text { tened) }\end{array}$ & Best in meat broth \\
\hline Raw beef $\ldots \ldots \ldots \ldots \ldots$ & 100 G. $(31 / 2$ oz. $) \ldots$. & $\begin{array}{l}\text { Finely minced or } \\
\text { scraped with a } \\
\text { little salt }\end{array}$ & $\begin{array}{l}\text { Taken from the } \\
\text { filet }\end{array}$ & $\begin{array}{l}\text { Eaten with crack- } \\
\text { ers }\end{array}$ \\
\hline $\begin{array}{l}\text { Raw beef sausage } \ldots \ldots \\
\text { Tapioca } \ldots \ldots \ldots \ldots \ldots\end{array}$ & $\begin{array}{l}100 \mathrm{G} .\left(3 \frac{1}{2} \text { oz. }\right) \ldots \ldots \\
30 \mathrm{G} .(1 \mathrm{oz} .) \ldots \ldots\end{array}$ & $\begin{array}{l}\text { Without spices... } \\
\text { Cooked with milk } \\
\text { to make a cus- } \\
\text { tard }\end{array}$ & Slightly smoked.. & $\begin{array}{l}\text { Eaten with crack- } \\
\text { ers }\end{array}$ \\
\hline
\end{tabular}


Third Diet (about 8 days)

\begin{tabular}{|c|c|c|c|c|}
\hline Food or drink & $\begin{array}{c}\text { Maximum quantity } \\
\text { at once }\end{array}$ & Preparation & Condition & Method of taking \\
\hline Pigeon...$\ldots \ldots \ldots \ldots$ & One $\ldots \ldots \ldots \ldots$ & $\begin{array}{l}\text { Roasted with fresh } \\
\text { butter }\end{array}$ & As in second diet & Without sauce.... \\
\hline Chicken .............. & One $\ldots \ldots \ldots \ldots \ldots$ & $\begin{array}{l}\text { Roasted with fresh } \\
\text { butter }\end{array}$ & As in second diet & Without sauce.... \\
\hline Beefsteak.............. & 100 G. $(31 / 2$ oz. $) .. .$. & $\begin{array}{l}\text { Raw with fresh } \\
\text { butter }\end{array}$ & $\begin{array}{l}\text { Taken from filet } \\
\text { well pounded }\end{array}$ & Without sauce.... \\
\hline $\operatorname{Ham} \ldots \ldots \ldots \ldots \ldots$ & 100 G. $\left(3 \frac{1}{2}\right.$ oz. $) . . .$. & $\begin{array}{l}\text { Raw, finely shav- } \\
\text { ed }\end{array}$ & $\begin{array}{l}\text { Slightly smoked } \\
\text { without bones }\end{array}$ & With white bread. \\
\hline $\begin{array}{l}\text { Bread made with milk } \\
\text { or } z \text { wieback }\end{array}$ & 50 G. $(13 / 4$ oz. $) \ldots$. & & $\begin{array}{l}\text { Stale rolls [pulled } \\
\text { bread.-Ed.] }\end{array}$ & $\begin{array}{l}\text { Thoroughly masti- } \\
\text { cated }\end{array}$ \\
\hline Potatoes....$\ldots \ldots \ldots$ & 50 G. $(13 / 4$ oz. $) . \cdots$ & $\begin{array}{l}\text { A. Potato soup .... } \\
\text { B. Mashed or riced }\end{array}$ & Mealy............. & $\begin{array}{l}\text { Thoroughly masti- } \\
\text { cated }\end{array}$ \\
\hline Cauliflower............ & 50 G. $(13 / 4$ oz. $) ..$. & $\begin{array}{l}\text { Boiled in salted } \\
\text { water }\end{array}$ & $\begin{array}{l}\text { Only the flowerets } \\
\text { to be eaten }\end{array}$ & \\
\hline
\end{tabular}

\section{Fourth Diet (8-14 days)}

\begin{tabular}{|c|c|c|c|c|}
\hline Food or drink & $\begin{array}{c}\text { Maximum quantity } \\
\text { at once }\end{array}$ & Preparation & Condition & Method of taking \\
\hline Venison.....$\ldots \ldots \ldots$ & $100 \mathrm{G} .(31 / 2$ oz. $) \ldots .$. & Roasted........... & $\begin{array}{l}\text { The back, hung } \\
\text { until tender but } \\
\text { not "high" }\end{array}$ & \\
\hline Partridge.............. & One...$\ldots \ldots \ldots$ & $\begin{array}{l}\text { Roasted without } \\
\text { lard or bacon }\end{array}$ & $\begin{array}{l}\text { Young, without } \\
\text { skin, s i news, } \\
\text { etc. Well hung }\end{array}$ & \\
\hline $\left.\begin{array}{l}\text { Roast beef......... } \\
\text { Filet } . . . \ldots \ldots \ldots \ldots\end{array}\right\}$ & 100 G. $(31 / 2$ oz. $) \ldots .$. & Rare .............. & $\begin{array}{l}\text { From a well fat- } \\
\text { tened beast } \\
\text { Pounded }\end{array}$ & Hot or cold .... \\
\hline 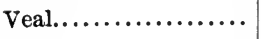 & $100 \mathrm{G} \cdot(31 / 2$ oz. $) \ldots$. & Roasted............ & Back or leg ....... & Hot or cold ...... \\
\hline 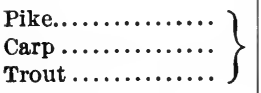 & 100 G. $(31 / 2$ oz. $) \ldots$ & $\begin{array}{l}\text { Boiled in salted } \\
\text { water without } \\
\text { condiments }\end{array}$ & $\begin{array}{l}\text { Carefully freed } \\
\text { from bones }\end{array}$ & With fish sauce... \\
\hline Caviar................ & $50 \mathrm{G} .(13 / 4 \mathrm{oz}.) \ldots$. & Raw .............. & $\begin{array}{l}\text { Slightly salted, } \\
\text { Russian }\end{array}$ & \\
\hline Rice...$\ldots \ldots \ldots \ldots$ & 50 G. $(13 / 4$ oz. $) \ldots$. & In a soft pudding & $\begin{array}{l}\text { The rice to be } \\
\text { cooked soft }\end{array}$ & \\
\hline Asparagus ............. & 50 G. $(13 / 4$ oz. $) \ldots$. & Boiled ............ & Only the soft ends & With a little melt- \\
\hline Scrambled eggs........ & Two $\ldots \ldots \ldots \ldots \ldots$ & $\begin{array}{c}\text { With a little fresh } \\
\text { butter and salt }\end{array}$ & & \\
\hline Soufflé................ & Two eggs ........... & $\begin{array}{l}\text { With about } 20 \mathrm{G} \text {. } \\
(2 / 8 \mathrm{oz} .) \text { sugar }\end{array}$ & Must be light..... & $\begin{array}{l}\text { To be eaten im } \\
\text { mediately }\end{array}$ \\
\hline Fresh fruit sauce ...... & 50 G. $(13 / 4$ oz. $) \ldots$. & $\begin{array}{l}\text { Freshly stewed, } \\
\text { strained }\end{array}$ & $\begin{array}{l}\text { Without skins or } \\
\text { seeds }\end{array}$ & \\
\hline Red wine............. & 100 G. $(31 / 2$ oz. $) .. .$. & $\begin{array}{l}\text { Light, pure Bor- } \\
\text { deaux }\end{array}$ & $\begin{array}{l}\text { Or a correspond- } \\
\text { ingly unadulter- } \\
\text { ated red wine }\end{array}$ & Warmed ........ \\
\hline
\end{tabular}


The Rest CuRe.--In this connection may be discussed a form of treatment often to be considered in cases of nervous dyspepsia, particularly nervous anorexia, hysteria, and neurasthenia,- the Weir Mitchell rest-cure. The patient, in a sanatorium, isolated from his relatives and friends, is given easily digested food in greater quantities than usual and about every two hours; meanwhile he is kept absolutely quiet and is given massage and perhaps appropriate hydropathic treatment. Weir Mitchell originally used only milk in his rest-cure, but to-day it seems much more rational to enlarge the diet. The following is Binswanger's menu :

First Meal, 7 A. M.

250 G. ( 1 tumblerful) boiled milk, or cocoa made with half milk and

half water, or oat-cocoa (strongly to be recommended)

2-3 biscuits or zwieback

A cup of bouillon

Second Meal, 9 A. M.

20 G. ( $\frac{2}{3}$ oz.) meat

$30 \mathrm{G}$. (1 oz.) of graham bread or toast

10 G. ( $\frac{1}{3} \mathrm{oz}$.) of butter

Tirird Meal, 11 a. M.

$125-175$ G. ( $4 \frac{1}{2}-6$ oz.) milk with a tablespoonful of condensed malt or the yolk of an egg

Fourth Meal, 1 P. M.

80-100 G. ( $2 \frac{1}{2}-3 \frac{1}{2}$ oz.) of soup with oatmeal, barley, or rice

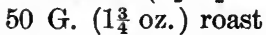

$10 \mathrm{G}$. ( $\frac{1}{3} \mathrm{Oz}$.) potatoes

7-10 G. ( $\left(\frac{1}{4}-\frac{1}{3}\right.$ oz. $)$ vegetables

20 G. ( $\frac{2}{3}$ oz. ) sweet rice pudding

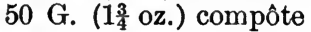

Fifth Meal, 4 P. M.

125 G. ( $4 \frac{1}{2}$ oz.) weak tea, cocoa, or malted milk

2 biscuits

Sixth Meal, 6 P. M.

20 G. ( $\frac{2}{3}$ oz.) meat (hot or cold roast, scraped raw meat, tongue, ham, etc.)

10 G. ( $\frac{1}{3}$ oz.) graham bread or toast

5 G. ( $\frac{1}{6}$ oz. ) butter

Seventh Meal, 8 P. M.

125 G. ( $4 \frac{1}{2}$ oz.) soup, cooked with $10 \mathrm{G}$. ( $\frac{1}{3} \mathrm{oz}$.) of butter and the yolk of an egg, oatmeal, barley, etc.

Eighth Meal, 9:30 P. M.

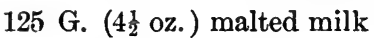

These quantities are gradually increased, so that at the end of a fortnight, the milk, cocoa, and soup are doubled, and the meat, bread, and butter trebled. Richer compôtes are allowed, and small quantities of fresh vegetables, with simple puddings. 
The simplest form of this cure merely adds milk to the usual food, a cupful being taken about every two hours in place of the ordinary water, soup, tea, or coffee. In every form of rest-cure it is very important to increase the daily supply of fat by taking more butter, cocoa, oil, etc.

If the patient objects seriously to so much butter and milk, one or more teaspoonfuls of lipanin may be substituted. Binswanger gives alcohol only when it is impossible for the patient to take minced meat without the addition of a few drops of port or sherry.

If at the beginning of the rest-cure the patient complains of digestive disorders, sensations of pressure and fulness, or a sense of pressure in the head, this should not be considered sufficient ground for interrupting the treatment. On the contrary it should be persevered in, and the symptoms will disappear of their own accord. For the most part they may be prevented by placing a hot-water bag or a thermophor over the stomach before the principal meal and letting it remain there until one or two hours after eating. Occasionally, especially if there be any persistent organic disease of the stomach, a patient contracts acute gastric catarrh, in which case the treatment must of course be immediately stopped. If constipation is induced, or, as more frequently happens, a former constipation persists or increases, the amount of fat and fruit should be increased, and if necessary an enema or a mild laxative given, (castor oil, cascara, etc.). The appearance of diarrhœa demands an immediate change in the diet until the intestine is normal again.

The patient should be strictly confined to bed for a week or two, especially when there is any degree of emaciation. If he can bear the strict cure, he should then be allowed merely to get up while the bed is made, then to sit up for awhile, and finally to undertake the care of his own person, which last has up to this time been the nurse's duty. The mental activity permitted him is also decreased to the minimum; he must neither read, write, nor be entertained for any great length of time.

It should be noted, however, that there are some patients who cannot stand such a complete rest. They may be wheeled out of doors on a reclining chair or taken to drive each day, and be allowed as much mental activity as apparently does them good. During this stage of the treatment massage must be substituted for exercise; the method here recommended is chosen from different authors.

General Massage.-There are four principal kinds of massage,effleurage or stroking, petrissage or kneading, friction or rubbing, and tapotement or slapping. In stroking, the hand of the masseur is laid with the thumb on one side of the muscles to be massaged and the fingers on the other side, and the muscle stroked centripetally. In kneading, the hand is laid on in a similar way and the muscles pressed between the fingers. Rubbing is done either with both hands, or with both index and middle fingers moving in circles or ellipses within small areas. In slapping, the muscles to be treated are hit either with the tips of the 
fingers or with the palm of one or both hands. If the fingers are used, the palm of one hand is turned down, and of the other up. Massage has both local and general effects. The circulation, particularly of the venous blood, is increased, the flow of lymph is made more active, the skin and muscle fibres stimulated, the nervous system reflexly influenced, and metabolism and assimilation aided. The brain undoubtedly is much soothed by mild stroking of the whole surface of the body, without pressing or kneading the muscles that lie beneath.

In the use of massage in connection with a rest-cure, most authors lay great stress on strong kneading, but some, Binswanger for example, put greater faith in slow, moderately strong stroking with its reflex effect on the central nervous system. General massage treats first the extremities, then the back, abdomen, chest, and neck. The duration of manipulation varies with the method chosen and its effect, general massage being usually given for about fifteen minutes once a day. If the patient is very thin and anæmic only a part of the body should be massaged at once. Directly after the massage, especially if fat or vaseline has been used, the whole body should either be washed off with warm water or rubbed off with tincture of calamus. To increase the sedative effect the patient may be covered with a blanket and then left for half an hour to an hour after the treatment. In some cases besides massage general faradization may be used in the form of electrical baths or the usual faradic stimulation of the muscles.

A rest-cure properly lasts 6 weeks. For the first week or two the patient is given a suitable diet and made to take complete rest; for the next fortnight he is allowed only the most unavoidable movements, already enumerated; and during all this time he is given massage or faradization. After the month is up, he is given passive gymnastics for the next fortnight, as a preparation for the active movements, which are gradually introduced and gradually increased. These passive movements, carried out partly with and partly without resistance, are at first applied only to the lower extremities, which are no longer massaged. After the first four days, the upper extremities are included in the treatment. When the six weeks of the cure proper are over, the patient begins active muscle-movements, partly in walking, partly in simple gymnastics, or again in different manual occupations, such as woor carving, sawing and splitting wood, book binding, gardening, or exercise at rowing machines. [We cannot overestimate the value of a well planned "rest-cure", in starting a sufferer from a gastric or an intestinal affection towards health; nor can we exaggerate the debt we owe Weir Mitchell for his pioneer work along this line. The "cure" outlined above by the author is an excellent pattern for us to copy; but there is, I believe, no class of patients and no form of treatment which require more intelligent individualization. (See section upon neurasthenia.) - Ed.] 
Other Cures Depending on a Particular Diet.-Other cures depending on some peculiar diet hardly need mention in connection with diseases of the stomach and intestine. The whey-cure formerly much used is properly losing its prestige, although it cannot be denied that in some cases the use of a certain amount of whey is beneficial. The grape-cure, which is unquestionably of use in many cases of chronic constipation, presupposes a good stomach, strong digestion, and a good constitution. As far as diseases of the alimentary canal are concerned, its field is, therefore, a very limited one.

Special Diets.-The second question propounded above (p. 382)how the diet may be best suited to special disturbances affecting the stomach-can be decided best by contrasting the diseases of the stomach accompanying hyperacidity with those accompanying hypoacidity or anacidity, and the diseases accompanying hypermotility with those accompanying atony or dilatation of the stomach (motor insufficiency of the first and second grades).

Diet in Hyperacidity.-Hyperacidity occurs either as simple hyperacidity or as continual hypersecretion. In both, though to a greater extent in the latter, the digestion of carbohydrates is impaired, for the hyperacidity suddenly interrupts the salivary digestion of the carbohydrates, and also hinders their digestion in the intestine, for the food is overacidified when it reaches the intestine, and so the bile, pancreatic, and intestinal secretions lose something of their digestive power. Carbohydrates, therefore, should be used only in strictly limited quantities and should be given in their most easily digested forms, preferably those in which the starch has been dextrinized beforehand. The dextrinized meals are useful here (Knorr's, Kufeke's, Timpe's, Maggi's, etc.). For the same reason we should replace cane sugar, whose inversion is delayed by hyperacidity, by gràpe or fruit sugar (honey), both of which are absorbed directly. Cane sugar, moreover, like the other starches stimulates the secretion of hydrochloric acid, so that limited quantities of solution of grape sugar seem advisable, except with gastric atony. In the latter event even grape sugar must be withheld so as to avoid abnormal fermentation in the stomach. It is further evident that with hyperacidity or hypersecretion, all vegetables that contain much cellulose, and all foods the greater bulk of which passes through the rectum unused, thus vigorously stimulating the intestinal wall (cabbage, turnips, brown bread, rye bread), must be entirely avoided.

Naturally the degree to which earbohydrates influence the gastric secretion depends also greatly upon their mode of preparation. For example, peas, beans, and cabbage are less irritating if they are made into soup or purée.

All sharp condiments must be forbidden because they stimulate the gastric juice. For this reason the author does not approve of the use of smoked meat or of salt, smoked, or pickled fish. 
If, as has already been stated, digestion in the intestine suffers on account of the abnormal amount of hydrochloric acid in the chyme passed from the stomach to the duodenum, fatty foods are theoretically unsuited to a condition of hyperacidity or hypersecretion. Experience teaches, however, that good fat, particularly butter, cream, oil, and fat cheese, agrees well with such patients; and experiments on animals show that the ingestion of fat diminishes the gastric secretion. Besides fat, grape sugar, and honey, a normal amount of proteid should be given. Fasily digested forms of meat, usually boiled, should be chosen, especially if there is gastric atony.

Most peptone preparations use up so little hydrochloric acid and proportionally excite the secretion so much, that they certainly do not diminish, but rather increase the hyperacidity.

In all cases of hypersecretion it is best to give the food often and in relatively small quantities. In hyperacidity, on the contrary, only three meals a day are advisable. For in the former ease, we must aim at neutralizing as much of the superfluous acid as possible; while in the latter case the object is to avoid exciting oftener than necessary the abnormally sensitive secretory apparatus.

Alcohol, strong tea and coffee, and tobacco should be forbidden on account of the stimulative effect of all of them, alcohol in particular, on the stomach. Weak tea and coffee with a good deal of milk are allowable, but milk itself, in not too large quantities, is the best drink.

None of these rules apply to one particular kind of hyperacidity, however, the symptomatic hyperacidity of gastric ulcer, the special dietetic therapy of which will be given later.

[A striking difference of opinion exists in regard to the type of diet in hyperacidity. Some authorities advocate excessive proteids so as to combine with all the acid possible and limit carbohydrates strictly to prepared carbohydrates, because the salivary digestion of ordinary carbohydrates is too quickly inhibited by the abundant acid; whereas others cut down all foods which stimulate the secretion of the gastric juice, such as meats and other proteids, and urge the selection of carbohydrates and fats. This difference alone emphasizes the necessity of individualization in each case. I have obtained excellent results in following the general principles cited by Robin. He orders such patients at first a purely milk diet for 2 or 3 weeks, prescribing 1 to 2 tumblerfuls of warm milk without much cream every three hours from 7 o'clock in the morning until 100 'clock at night, requires them to be one half hour in swallowing this quantity, gradually increasing the amount until the patient is taking between $3 \mathrm{r} / 2$ and 4 quarts per day, and continues the regimen as long as it does not disagree with the patient. As soon as the latter stops gaining weight or the milk disagrees, Robin adds eggs, soups made with milk and thickened with some carbohydrate, or purées, a small amount of crisped bread or zwieback, and stewed fruit. After 10 to 20 days of such a routine, he adds small quantities of boiled soft fish, raw or lightly broiled 
minced meat, and then withdraws the milk. The next addition consists of oysters, finely divided meat, which must be slowly and carefully masti. cated, without sauce, purées of the green vegetables, baked potatoes, rice, fresh butter, oil, bread, and simple desserts. The best results are obtained when such a regimen is combined with a rest-cure modified to suit the individual requirements. The future dietetic rules for such patients vary for each case. In general they do better, in my experience, upon a low proteid, high fat, and moderate carbohydrate ration. Regularity of meal times, avoidance of fatigue, excitement, or nervous influences just before and after eating, slow, careful mastication, and careful attention to all the psychical aids of digestion, will do more than any rigid diet. -Ed.]

Diet in Hypoacidity or Anacidity.-Where the opposite condition occurs, i.e., hypoacidity or anacidity, a distinction must be made, as before, between the diet appropriate to gastric atony and that to be employed when the motor activity of the stomach is normal. In the latter case, in order to avoid injuring the normal motility, so important for the patient, food is given oftener than usual and in smaller quantities. Meat can be used because, as has already been stated, if there is not enough pepsin in the stomach, the intestine undertakes the digestion of the proteid. It should be served only in its more digestible forms : sweetbreads, brains, tender white meat, particularly fowl from which all the indigestible parts, such as skin and gristle, have been carefully removed, and which has been either cooked almost to pieces in a steamer, or else finely minced, shaved, or grated. Eggs also should be given only in their most easily digested forms, (soft-boiled or scrambled). The same is true of all forms of food which are rich in vegetable proteid. Peas and beans, for instance, should be made into soup, and even then not allowed often or in large quantities. The digestion of starches, of course, does not suffer, so that they may be used, as well as the more easily digested vegetables, such as spinach, cauliflower, and asparagus. Many patients turn to a mild vegetarianism, which the author does not consider as good as a diet containing an appropriate amount of meat.

The fats deserve an important place on account of their high nutritive value, but care must be taken lest too free a use of them bring on irritation of the stomach or intestines. For many such patients condiments are desirable, as well as occasionally other foods that stimulate the flow of gastric juice (meat extracts, peptone preparations, caviar, and salt fish). The meat peptones are useful, especially when the small intestine is not doing its work properly. In addition, small quantities of good alcohol may well be given.

The food should be appetizingly prepared and served, and should be very thoroughly chewed, as the action of chewing may increase gastric secretion. If, however, there is joined to hypoacidity motor insufficiency of the stomach, the latter must be the determining factor in the regulation of the diet. 
Diet in Motor Insufficiency.-The diet in motor insufficiency of the stomach depends upon whether this is due to atony (better hypotony or myasthenia) or to true dilatation. A stomach is atonic when it cannot empty its contents into the intestine in the normal time, but, if longer time be given, can completely unload itself. Such a stomach, given food in the evening, is empty the next morning and of normal size. But in true dilatation, the stomach has lost the power of completely emptying itself so that, given food in the evening, some of it is present in the stomach the next morning, and the organ is permanently enlarged. Dilatation may arise gradually from atony, or as a consequence of mechanical obstruction or of pyloric stenosis.

In both of these conditions, especially for dilatation, it is first of all necessary to limit as far as possible the amount of liquid ingested; because liquids are not absorbed by the stomach nor, in this case, passed on. The total quantity of liquid ingested daily should not exceed 1500 c.c. (3 pts.), and should be taken in small amounts. Thin soups and much liquid at meals must therefore be prohibited. The patient should take light meals about every two hours throughout the day, so that the stomach may never be heavily loaded. For the same reason the food should be of the most digestible kind and prepared in the most digestible way.

Just what should constitute the diet depends, however, on whether the case is one of atony or of dilatation. In atony, proteids are possible, especially if the secretion of the gastric juice is sufficient. Naturally, coarse meat in indigestible form is never given. Carbohydrates should have the starch already dextrinized, and only the easily digested vegetables should be employed. If hyperacidity and hypersecretion are present, the quantity of carbohydrates must be even more reduced. There is no objection to the use of good fat. Whether large quantities of milk may be given or not depends on the individual case. Some patients bear it well, others badly.

Even in dilatation of the stomach, provided there is good secretion, proteids in the most easily digestible form may be used. Carbohydrates, however, if not wholly forbidden, must be greatly reduced in quantity; because the dilated stomach ensures stagnation of its contents, with abnormal decomposition and putrefactive processes, and because carbohydrates are decomposed much more quickly and easily than proteids.

But if a high degree of motor insufficiency of the stomach is accompanied by a reduced secretory power, we must resort to easily digested carbohydrates and only the very simplest forms of meat, such as meat preparations, meat powder, or, tentatively, meat in finely divided form. Fats too can only be given conditionally and not without interruption. There are certain times in dilatation of the stomach, when an exclusive meat diet seems indicated.

Finally, especially in severe cases, if direct recourse is not had to surgery, nutrient enemas are necessary as a last resort. 


\section{Nutrient and Cleansing Enemas.}

Indications.-Nutrient enemas are indicated not only in severe dilatation of the stomach, but also in many other diseases of the digestive tract; e.g., in stenosis of the cesophagus or of the cardiac orifice, in hemorrhage from the stomach, in inflammatory or phlegmonous processes of the stomach, in severe vomiting, and in acute inflammation of the intestine. They cannot, however, be used for any protracted treatment, as (1) the digestive power of the intestinal secretion is, on the whole, very weak, and (2) the large intestine absorbs nutriment much less readily than the small intestine [c.f. Boyd and Robertson's investigations, below.-Ed.].

Technic.-240 c.c. ( $1 / 2$ pt.) of the appropriate nutrient enema (at $95^{\circ}$ ) should be given every 8 to 12 hours, the bowel having been cleansed at least an hour before by irrigation with one quart of water at the body temperature. The enema should be introduced into the intestine as high up as possible by means of a long, flexible rubber tube, to one end of which is attached a small funnel. A syringe should never be employed. The patient should lie on his left side or back with the hips raised, and should keep perfectly quiet for an hour afterwards. If there is difficulty in retaining the enema, 10-15 drops of the tincture of opium may be added, or an opium suppository may be given just after the enema or just after the evacuation of the eleansing irrigation. [Hemmeter agrees with the author in advising that each nutrient be preceded by a cleansing enema. I rarely employ but one cleansing enema daily, and that nearly always in the early morning, so as to remove the fecal accumulation of the preceding 24 hours. If the action of this is not satisfactory, I may administer another in the late afternoon or early evening. I generally use plain water at $99^{\circ}$, but sometimes add salt or soapsuds and glycerin.-Ed.]

Composition.-No one admixture of food elements has been universally agreed upon as that best fitted for rectal feeding; but one fact appears to be well established-any combination is absorbed better if salt is added to it. A satisfactory explanation for this has not yet been given. It may be that the salt causes anti-peristaltic movements, and so carries the food into the upper part of the intestines or possibly even into the stomach (Gruetzner). There is a second noticeable fact, which the author has often confirmed-that peptones taken by the rectum very soon induce irritation of the intestine, and often an inclination to painful diarrhœa attended by tenesmus. They are, therefore, contraindicated in any protracted use of rectal feeding. [Peptonized milk is still employed for rectal feeding by many practitioners. I prefer pancreatized milk or ordinary milk.-Ed.]

A favorite mixture consists of a cup ( $250 \mathrm{G}$.) of milk, the yolks of two eggs, two teaspoonfuls of red wine, and a pinch of salt, well stirred together. Singer recommends a similar combination-125 G. (4 oz.) of 
milk, 125 G. ( 4 oz.) of wine, the yolks of one or two eggs, a pinch of salt, and a teaspoonful of peptone. As grape sugar is undoubtedly easily absorbed from the rectum, it may be utilized for nutrient enemas. especially in practice among the poor. $15 \mathrm{G}$., one tablespoonful, may be added to either of the enemas already mentioned or to any other preferred; or 150 c.c. $(5$ oz.) of a 15-20 per cent. solution of grape sugar may be combined with $3-5$ eggs. Rosenheim recommends one to two teaspoonfuls of peptone, $15 \mathrm{G}$. ( $1 / 2 \mathrm{oz}$.) of grape sugar, $30-40 \mathrm{G}$. (1-11/2 oz.) of an emulsion of cod liver oil with a few tablespoonfuls of a 0.3 per cent. soda solution, and warm water up to 200-250 G. (8 oz.) ; the peptone may be replaced by a well beaten raw egg. Boas employs the following excellent combination: $250 \mathrm{G}$. $(8 \mathrm{oz}$.) of milk, the yolks of two eggs, a tablespoonful of red wine, a teaspoonful of starch, and a pinch of salt. If eggs are used, Leube advises cleaning the rectum after each enema has been absorbed, in order to prevent the decomposition of any egg residue. [The value of rectal feeding, especially in gastric ulcer, is usually accepted without discussion. Umber has, however, clearly demonstrated upon a patient with a gastrotomy wound that the administration of rectal enemas stimulates gastric secretion, (free hydrochloric acid $=20$, total acidity $=30$ ). This explains the frequent occurrence of gastric pain, of which many patients with gastric ulcer complain after the introduction of an enema, and may, of course, delay cicatrization of the ulcer. Whenever nutrient enemas are indicated, I usually begin with $250-300 \mathrm{G}$. (8-10 oz.), and if this is retained without discomfort I increase the bulk to $375-500 \mathrm{G}$. (12-16 oz.), in fact up to tolerance. The larger the amount, the more the patient's thirst is relieved, and the greater is the possibility that part of the enema may pass through the ileocæcal valve and the percentage of absorption be thus increased. Gruetzner has even claimed that starch granules can be washed from the stomach 4-6 hours after the rectal administration of a starch emulsion in normal saline. Unless contraindicated for some particular reason, I always include 5-15 drops of laudanum in each enema, because in my experience the patients then stand the treatment better, retain the enema longer, absorb more, and are more comfortable. Until recently I have usually selected some one of the enemas mentioned above. Since reading Boyd and Robertson's "Contribution to the Value of Rectal Feeding," (Scottish Medical and Surgical Journal, March, 1906), I have adopted their choice:

The yolks of two eggs

$30 \mathrm{G}$. (1 oz.) pure dextrose

$0.5 \mathrm{G}$. ( $7 \mathrm{gr}$.) common salt

Pancreatized milk to 300 c.c. $(9 \mathrm{oz}$.)

To this I add the laudanum as mentioned above, and increase all the ingredients proportionally up to the patient's tolerance. These author's believe that less than one half the caloric value of this enema is absorbed 
under the most favorable circumstances. In other words, from rectal feeding is absorbed about one quarter of the nourishment required to sustain equilibrium. The reason they advise so low a proportion of proteid is because their researches showed that about one sixth of the albumin given was absorbed, about one third of the fat, and nearly nine tenths of the sugar. Hemmeter advises that the entire amount should never exceed $8 \mathrm{oz}$., that a hot towel should be held firmly against the anus for 15-20 minutes after its introduction, that the tube should be introduced 12-18 inches, the higher the better, and that the temperature of the mixture should be that of the body.-Ed.]

Other Fluids by the Rectum.-In gastric diseases we are often obliged to give fluids by the rectum for various purposes other than that of nourishment. For instance, in dilatation and all other diseases in which the motility of the stomach is seriously impaired (e.g., cancer), the necessary supply of water cannot be ingested in the ordinary way; for, even if the dilatation is only moderately marked, stagnation of the stomach contents, including all the liquid introduced through the mouth, must follow. Therefore, since water is not absorbed from the stomach, torturing thirst soon appears, increased by the fact that the introduction of some foods even causes the transmission of water from the circulation into the stomach. In such cases the simplest and best way to supply the body with water is through the rectum. For this purpose we inject 2 or 3 times a day $250-500$ c.c. ( $1 / 2-1$ pt.) of warm normal saline solution, adding, if a stimulating effect is desired, 1-3 teaspoonfuls of brandy. Simple bouillon enemas may also be chosen, or Fleiner's combination of two parts broth to three parts sweet white wine. The introduction of water by the rectum is also recommended in acute infectious diseases, e.g., typhoid. The author has often used it, however, without seeing any influence on the issue of the disease in question. [Nor have I. Typhoid patients crave water by the mouth and their stomachs generally take care of large amounts.-Ed.]

Colon Irrigation.-For a colon irrigation one needs an irrigator or Hegar's funnel, a connecting tube with a stop-cock or clamp, and a flexible rectal tube. The last should be about $15-20 \mathrm{~cm}$. (6-8 in.) long with an opening of $1 \mathrm{~cm}$. ( $\mathrm{r} / 2 \mathrm{in}$.) ; if high irrigation is desired, a tube $30-40 \mathrm{~cm}$. (12-16 in.) should be used, or a stomach tube. Before the tube is inserted, the air must always be expelled by opening the stopcock or loosening the clamp, and letting some liquid flow through the whole apparatus. The tube, well greased with vaseline or oil, is then slowly inserted by a cautious, slightly twisting movement and pushed upward, while the patient lies on his left side, or, if high irrigation is desired, in the knee-chest posture or in the more comfortable dorsal position. It should always be inserted for at least $15 \mathrm{~cm}$. (6 in.) beyond the sphincter tertius, and usually much farther. One to three litres (1-3 qt.) of liquid is then allowed to flow in very slowly, so that the intestine may accustom itself to its unusual content instead of quickly 
rejecting it all. If the sphincter does not contract strongly enough to prevent the liquid's flowing out during the injection, the anus must be closed by pressing the nates together. If the liquid refuses to flow, the tube should be slightly withdrawn, and then, if the stream does not start again, should be taken out and cleaned, for unless bent near the end it has probably been stopped by feces. The liquid selected for injection depends upon the purpose for which it is used. Sometimes it is pure water; sometimes water with glycerin, soap, or salt; sometimes oil; sometimes a laxative, e.g., aqua laxativa; sometimes an astringent or antiseptic solution. [A soft double tube (Kemp) is a favorite tube for high colon irrigation, especially to stimulate peristalsis or to overcome obstinate tympanites. I usually obtain quite as good results with an ordinary long rectal tube, attached by means of a $\mathrm{T}$ shaped glass connection to a.large irrigating reservoir capable of holding 1 or 2 gals. of fluid. To the other limb of the $\mathrm{T}$ tube is attached a plain rubber tube long enough to reach to the bottom of a receiving vessel on the floor. With two simple clamps one nurse alone can then carry on a continuous irrigation, allowing the bowel to be filled as completely as possible before the fluid is allowed to escape.-Ed.]

\section{Gastric Lavage and Douches.}

Technic.-Gastric lavage is used for both diagnostic and therapeutic purposes. A simple syphon apparatus is generally employed. This consists of a large glass funnel or irrigator holding about a litre (1 qt.) and attached by a rubber tube $1 \mathrm{M}$. ( 1 yd.) long to a short glass connection, which in turn is connected with a stomach tube. The latter should be $6-12 \mathrm{~mm}$. ( $1 / 4-1 / 2$ in.) in diameter and $3 \mathrm{~mm}$. ( $1 / 8$ in.) thick, with two openings (longest diameter $2.5 \mathrm{~cm} .-1$ in.) near the rounded end. There is sometimes an opening at the end itself, but this is not so good an arrangement. After being moistened thoroughly with warm water the tube is inserted into the mouth of the patient, who should sit facing the physician, with slightly bowed head. It is first directed back over the tongue to the posterior wall of the pharynx; the patient is then made to bow his head deeper and to swallow, until the tube, pushed along the wall of the throat, reaches the cricoid cartilage. After a slight pause another swallow will carry it behind the larynx into the œsophagus. It may then be passed quickly down into the stomach. If the patient breathes deeply and rather rapidly during the whole procedure, the tendency to choke will be lessened.

A rubber bulb may conveniently replace the connecting glass tube. If this bulb is compressed while the tube is pinched between the free end and the bulb, the contents of the stomach tube will be forced hack into the stomach, thus getting rid of any bits of food which are stopping up the tube. But if the bulb is first compressed, the tube then pinched, and the bulb then allowed to expand again, the contents of the stomach 
and the stomach tube will be sucked up into the bulb. Then if the stomach tube is pinched, the other tube released, and the bulb compressed, the contents of the bulb will be forced out into the funnel. [A simpler method is to use a bulb furnished with a valve.-Ed.]

In washing out the stomach, the tube is first pinched at the patient's mouth, and the glass funnel or irrigator held upright and filled with $500-1000$ c.c. (1-2 pt.) of liquid, usually water at about $90^{\circ}$; the funnel is then hung up somewhat above the level of the patient's head. The tube is then released and nearly all the liquid allowed to flow into the stomach. At this point the funnel is slowly lowered and inverted into a large receiving vessel. In this way the tube is kept filled with liquid, and the water introduced into the stomach, together with the stomach contents. flows back into the receiving vessel by syphonage. At least as much liquid should flow back as has been introduced. It is convenient to employ a tube which has a mark to indicate the distance from the teeth to the cardia. If the stomach is enlarged or lies deeper than the normal position, the tube must of course be pushed in farther.

Duration of Lavage.-If the patient's condition permits, lavage should be continued until the water comes out clear. Even then one cannot be sure that the stomach is empty, for, when the patient is sitting up, only the fundus of the stomach, the region of the greater curvature, the pyloric region, and a part of the anterior wall are reached. The rest of the stomach can be washed out only by having the patient lie down. In this position most patients experience so much discomfort at first that the process is successful only after several attempts. In time, however, many individuals may be trained to help in distributing the water throughout the stomach by proper movements of the trunk during the process. Again the fluid may return clear and yet the stomach not be clean, because the mucous membrane is covered with a coat insoluble in water. In such an event it is better to use alkaline solutions. Finally if, after a thorough washing until the water is clear, water with stomach contents suddenly follows, the diagnosis of an hour-glass stomach is suggested.

Accidents During Lavage.-Water is often vomited outside the tube during lavage. This is due to one of two causes. Either the water has been poured in too rapidly or the wall of a very sensitive stomach has been touched by the tube. In the first case, the pressure should be lessened by lowering the funnel or irrigator and only a little water should be allowed to flow in; in the second case, the tube should be withdrawn $1.5 \mathrm{~cm}$. ( $1 / 2$ in.) or so.

The tube should never be removed from the stomach empty, for fear that the mucous membrane may be sucked up into the openings of the tube. To avoid this the funnel should be filled with water at the close of the operation and raised. At the moment when the water begins to flow from the funnel, the tube should be slowly withdrawn. When above the height of the cardia it should be pinched together to prevent the 
liquid's flowing back during the passage of the tube over the larynx. A stomach pump often injures the mucous membrane and hence should not be employed.

Should a fresh hemorrhage, even a small one, occur during lavage, a day or two should be allowed to elapse before the process is repeated, and only cool, liquid food be given the patient. If the hemorrhage is copious, the tube should not be withdrawn until the stomach is completely emptied, in order that the latter may contract as forcibly as possible, thus bringing the edges of the bleeding surface together and checking the hemorrhage. The occurrence of lumps or old streaks of blood, such as are frequently observed in cases of old ulcers and especially of ulcerated cancer of the stomach, is no indication for interrupting the procedure.

Best Time for Lavage.-The author believes that, unless there is some special contraindication, the best time for lavage is in the morning before the patient has eaten. The stomach contents are then so much softened and disintegrated that they pass through the tube easily. Moreover they are already so decomposed as to be more or less toxic. This rule cannot, however, be followed in all cases. Evening lavage is indicated in the case of many patients with marked dilatation, stagnation, and decomposition of the stomach contents, who complain of great distress at night which prevents their sleeping. In some cases lavage is necessary after each principal meal or at least twice a day, once 6-7 hours after the midday meal if the stomach still contains food, and once again the same evening or better the next morning.

Contraindications.-Contraindications for lavage are: (1) a fresh hemorrhage from the stomach, (2) severe irritation or inflammation of the stomach, or (3) inflammation of the peritoneum. Advanced arteriosclerosis, heart failure, aortic aneurism, ulcerated or hemmorrhagic processes (e.g., in the cesophagus), varices of the œsophagus, and, finally, great general weakness, should make us proceed with caution.

Fluids Used.-Sometimes for special reasons liquids other than water are used; e.g., alkaline solutions for cleansing the mucous membrane (artificial Ems salts (a heaping teaspoonful dissolved in the water), or alkaline mineral waters) ; or antiseptic solutions, among them a $0.2 \mathrm{per}$ cent. solution of hydrochloric acid. They may be either immediately removed or allowed to remain in the stomach one-half to five minutes and then withdrawn and followed by a thorough irrigation with water. Germicidal solutions must be weak enough not to irritate the mucous membrane or to cause any pain, and must be employed only in such quantities as might be totally absorbed with impunity (Penzoldt). They should not be left in the stomach over half an hour until after repeated trials of the procedure, when the time may be somewhat prolonged or the concentration of the solution carefully increased. Penzoldt prefers boracic acid or a tannin solution. 
The stomach tube may also be used for artificial feeding or for the introduction of liquids with a disagreeable taste.

Nasal Feeding.- In many cases, (diseases of the mouth, trismus, mental diseases), food may be given by a tube passed through the nose. A well oiled rubber catheter is introduced through the lower nasal passage and slowly passed down the pharynx into the œsophagus. To make sure that it does not enter into the larynx, the patient should be directed to say "ah;" 750 c.e. ( $1 \mathrm{I} / 2$ pt.) of milk, two eggs, and $100 \mathrm{G}$. (3 oz.) of grapesugar may be given thus twice a day.

Gastric Douches.-The stomach douche is useful in treating severe cardialgia and in stimulating gastric peristalsis and secretion. Fleiner has recently noted a stomachic effect from the use of bitter solutions; e.g., quassia or hops. The author employs for stomach douches an ordinary stomach tube with a large number of side-holes (1-2 mm. (1/24-1/12 in.) in diameter) at the stomach end.

He uses chloroform water as an anodyne; a 0.4 per cent. salt solution for increasing the secretion of gastric juice; a 0.1-0.2 per cent. solution of silver nitrate for diminishing it; and warm water for stimulating peristalsis. Such medicinal fluids should not be allowed to remain in the stomach more than one half to two minutes; and their use should be preceded and followed by irrigating with plain water. The most effective time for their employment is early in the morning when the stomach is empty or, if this is not possible, three or four hours after breakfast. Irrigating the stomach directly before meals produced an excellent effect in one of the author's patients with a cicatrized gastric ulcer who complained of severe cardialgia directly after eating and sometimes of vomiting as well.

\section{Massage of the Stomach and Abdomen.}

MASSAGE OF THE STOMACH.-Massage of the stomach is evidently most useful when the stomach muscles have lost some of their contractile power,-especially, therefore, in simple atony and atonic dilatation, and also in some cases of dilatation from pyloric stenosis. However, in the latter instance there is danger of pushing on into the intestine material already decomposing in the stomach and so injuring the intestinal function. This deters the author from recommending massage in cases of dilatation of the stomach from pyloric stenosis except under very exceptional circumstances. He avoids it particularly when there is a good deal of gas resulting from fermentation, for fear of forcing the gas on into the intestine. It is also, of course, contraindicated in acute inflammation, ulcers, hemorrhage from the stomach, or inflammation of the peritoneum.

Time for Massage.-In simple atony of the stomach, the object of massage is merely to strengthen the feeble muscles. It is, therefore, best given in the morning when the stomach is empty. But in atonic dilatation, or (very rarely) in dilatation from pyloric stenosis, the object 
is to accelerate the passage of the food from the stomach into the intestine, so that, as a rule, the best time is about six or seven hours after the principal meal, when the food will have been churned by the stomach into particles of as nearly the same size as possible, and can be passed on into the intestine with the minimum of exertion. [This selection pre-supposes that the patient is eating the ordinary three meals a day. -Ed.] Massage is often supposed to stimulate gastric secretion, although this has not been proved, and so is recommended in cases of impaired gastric secretion, and in nervous dyspepsia.

Technic.-For the technic of gastric massage the reader is referred to special works on the subject; only a few points will be mentioned here. Different methods are used. The author usually employs movements from the fundus to the pylorus, made by the outspread fingers of the right hand. The left thumb presses on the back of the right hand, while the rest of the left hand surrounds the right hypochondrium. To push the chyle out of the stomach into the intestine, we grasp the fundus region between the thumb and fingers of the right hand, and by backward movements, pressing down, try to throw the contents of the stomach towards the pylorus. Kneading and tapping the abdomen is also useful. Massage should be given every day for 2-3, or later 5-10 minutes. The patient should lie down, with the head slightly raised, and the legs bent at the knees and drawn up toward the abdomen in order to relax the abdominal muscles.

Abdominal Massage: Indications and Contraindications.-On account of the close relation between diseases of the stomach and those of the intestine the entire abdomen, as well as the gastric region, should usually be massaged. The chief indication for abdominal massage is atony of the intestinal muscle and a resulting constipation, whether these are, for example, symptoms accompanying enteroptosis or abdominal plethora, or have developed as a consequence of a pendulous abdomen or general obesity. Massage is, of course, contraindicated in all inflammatory and ulcerative diseases of the intestine, in inflammation of the peritoneum, in the different forms of enterostenosis, and in hemorrhage from the intestine. When arteriosclerosis exists, massage can be employed only with great caution.

Technic.-Abdominal massage should be given only by especially trained persons. The patient lies flat with his head somewhat raised, the mouth slightly opened, and the knees and hips bent. The stomach, bladder, and, if possible, the rectum should be empty. The hands of the masseur are then laid flat on the abdomen, with overlapping fingers, and light, stroking movements made in lessening circles from the symphysis in the direction of the hands of a watch; this relaxes the abdominal muscles. Then comes the so-called deep "petrissage," in which both hands laid on each other seize the whole abdomen, grasp the intestines and make zigzag movements; this loosens the fecal masses. In connection with this procedure, "effleurage" of the large intestine is employed. 
The upper arm is raised and rotated inward, the forearm bent at a right angle at the elbow and strongly pronated, the fingers of the right hand bent back as far as possible and laid on the ileocæcal region, with the finger tips of the left hand pressing the right hand as deeply as possible. The hand is then moved, keeping up the pressure, upwards along the ascending colon, and then across the abdomen, following the course of the transverse, and then that of the descending colon. This process is repeated several times. In the next movement, "rotating petrissage," the course of the colon from the ileocæcal region is also followed. Both hands are placed together, the finger tips pressed in as deeply as possible, and the large intestine massaged with rotatory movements, whose extent corresponds to the diameter of the bowel; this forces the contents of the intestine onward. The patient meanwhile should breathe as deeply as possible. In addition to these more important movements two others, beating and shaking, are used. In the former, the abdominal wall is hit with the overlapping finger tips, or the dorsal surface of the phalanges of the bent fingers; in the latter, the right hand with outstretched fingers shakes the abdomen vigorously. This stimulates the abdominal muscle. In order to try to stimulate the nerve plexuses, so-called "vibrations" are used. Between the ensiform process and navel (the cœliac plexus), and between the navel and symphysis (the splanchnic plexus) a finger is pressed slowly and strongly inward, and trembling movements made toward the spinal column (Hoffa).

Duration of Massage.-Massage of this kind should not in general last more than a quarter of an hour. The morning is the best time, for before breakfast the digestive tract is empty, and the hour an opportune one for a stool, should it follow the massage. The treatment must be kept up for different lengths of time according to its success, generally for at least a month, often for several months.

Auto Massage.-Ordinary massage may be supplemented by auto massage. For a quarter of an hour the patient should roll on his abdomen from right to left, following the direction of the colon, a wooden ball about $2 \mathrm{Kg}$. ( $4 \mathrm{lb}$.) in weight or a hollow massage ball filled with shot, so that the weight may be altered at will. In either case the ball is preferably covered with flannel. At the end of this process he should let the ball fall from a height of about $10 \mathrm{~cm}$. (4 in.) upon different parts of his abdomen, again following the course of the colon.

Gymnastics fOR the AbDominal Muscles.-Massage may also be supplemented by gymnastic exercises directed towards the strengthening of the abdominal and intestinal muscles, the general improvement of the circulation, and the toning up of the whole system. They should consist of exercises without apparatus (bending and stretching the legs, twisting and turning the trunk, quickly drawing up the legs to the abdomen while the patient is lying down, bending the knees, etc.), or of exercises with apparatus, particularly the horizontal bar. For the general im- 
provement of the system, outdoor sports may be added, such as riding, rowing, skating, swimming, and bicycling.

\section{Electrotherapy.}

In Diseases of the Stomach.-Like massage, electrotherapy undoubtedly has an excellent effect in diseases of the stomach although no very satisfactory explanations of its action can be given. From experiments upon animals and men, it is more than probable that it increases the secretion, the motor activity, and the power of absorption of the stomach. Practical experience, at any rate, shows unmistakably that it has a favorable effect on a large number of gastric neuroses, and on atony of the stomach and intestine. Among the former should be mentioned particularly sensory neuroses, such as nervous anorexia and nervous vomiting, which seem to receive the greatest benefit from electricity, and also all kinds of gastralgia, the purely nervous and often the gastralgia due to lesions of the stomach or of neighboring organs.

Technic.-In applying electricity to the stomach we possess a choice of two kinds of currents, faradic and galvanic, and of two methods of application, cutaneous and intraventricular. Static electricity is also of service in a few instances. To influence the motor activity, the author applies the faradic current cutaneously; to treat gastric neuroses or gastralgia, he selects the galvanic and employs it intraventricularly. In the first case two large, slightly curved plate electrodes of about 500 or 600 sq. cm. surface (9 sq. in.) are used. According to Ziemssen's method, the larger is applied to the anterior abdominal wall over the pyloric region, and the smaller, not more than $1 \mathrm{~cm}$. distant, is applied from the pylorus toward the spinal column. Both should be well moistened. The current must be strong enough to produce visible twitchings of the abdominal muscles without substantial pain. The application must not last over 10 minutes. To treat the stomach from the inside, a special gastric electrode is employed, consisting essentially of a wire ending in a metal knob, both of which can be fitted to a stomach tube and so inserted into the stomach. The end of the wire is connected with one pole, usually the anode, and to the other pole is attached a large plateelectrode applied to the epigastrium. There are several kinds of such gastric electrodes; e.g., those of Einhorn, Ewald, and Wegele. The current should not be turned on until the stomach is half or two-thirds full of water so as to avoid burning the mucous membrane. The faradic current may also be used with this same arrangement.

If intraventricular galvanization is not practicable, cutaneous galvanization can be used instead, with two plate electrodes applied as in the faradic process.

In Diseases of the Intestine.-Electricity may be used to increase the activity of the intestine as well as that of the stomach. Except for the rectum, the author employs only cutaneous faradization, applying two 
large plate electrodes to the sides of the abdomen for about ten minutes. For the rectum internal faradization is readily carried out by inserting a metallic rectal sound or probe, insulated to its free end by a covering bougie or catheter or a rectal cannula. Before employing the galvanic current salt solution must first be injécted in order not to burn the mucous membrane.

Electromassage.-Massage may often be combined with electrical treatment by making use of the electrical abdominal roller. This method is useful in atonic conditions of the stomach and intestine.

\section{Hydrotherapy.}

BatHS: Tub Baths.-The hydropathic and thermal procedures suitable for treating diseases of the alimentary canal, may be divided into two classes. The first aim wholly at the local treatment of the stomach and intestine; the second affect the whole system. To the latter belong ordinary tub baths. If the water is hot, they certainly have a sedative effect, especially in painful conditions of the digestive tract. The way in which tepid or cool tub baths, full or half baths, sitz baths, sponge baths, whipping with wet towels, wet packs, and douches may be employed in nervous dyspepsia, will be discussed in the section on the special therapy of that disease.

Sitz Baths.- Sitz baths are often used, especially in intestinal diseases. If the water is cool, they immediately affect the rectum, withdrawing the blood and allaying inflammation; their influence also extends to the whole intestine by shifting the blood from the abdomen to those parts of the body that have not been cooled. Soon, however, after the vascular contraction the blood flows back to the abdomen, the organs become hyperæmic, and the patient feels warm inside. This secondary return of the blood comes in 4-6 minutes or sooner, if the temperature of the water is $64^{\circ}-52^{\circ}$. If the bath is stopped after 5 or 6 minutes, the condition of the intestine remains the same for some time. Sitz baths of this temperature and duration are indicated when it is desired to improve the circulation of the abdominal organs, to hasten metabolism in them, to strengthen the alimentary canal, and to stimulate peristalsis. Sitz baths $\left(59^{\circ}-52^{\circ}\right)$ of only $2-3$ minutes duration are often of use in hemorrhoids, especially when they are accompanied by pain. If the baths are continued for half an hour or an hour, the long, cooling process brings on a secondary contraction of the abdominal blood vessels -a condition of anæmia, which is advantageous in treating diarrhœa or chronic catarrh of the stomach and intestine. Tepid sitz baths $\left(86^{\circ}-68^{\circ}\right)$ lasting 5-15 minutes have a more general application. Their effect may briefly be described as stimulating all the abdominal organs. They may be used in all weak conditions of the digestive organs, or in hemorrhoids, etc.

Douches.-Local douches are often helpful in stimulating the intestine, since they have both a thermal and a mechanical effect. In addi- 
tion to their reflex action through the nervous system, they act like thermal massage. Their stimulating effect varies with the amount of water, its temperature, the height from which it is let fall, and the frequency with which it strikes the abdomen. To accomplish its purpose, the water must be $50^{\circ}$ or even colder. If the patient is sensitive to cold water, we begin with $68^{\circ}$ and gradually accustom him to the lower temperature. The so-called Scotch douche deserves its high reputation for stimulating intestinal peristalsis. The warm $\left(95^{\circ}\right)$ and cold $\left(58^{\circ}-50^{\circ}\right)$ showers alternating every $10-20$ seconds, for $2-3$ minutes produce a powerful effect because the stimulation of the nerves and vessels by the cold is increased by the previous warm applications.

Applications of Heat and Cold.-Applications of heat and cold are of wide use in the therapy of stomach and intestinal diseases. Sometimes the applications are as cold as possible (ice-bags, frozen or cold compresses, or Leiter's cooling apparatus. The last is particularly useful in hemorrhage from the digestive tract and inflammation of the peritoneum). Hot applications may be either wet (e.g., flaxseed poultices, fomentations of chamomile-tea, cloths or sponges dipped in hot water, wrung out, and laid on the abdomen) or less preferably dry (e.g., simple hot compresses, a hot cover, plate or brick wrapped in a cloth, or the socalled Japanese hand-stoves, [hot water bottle or bag, electric pad -Ed.]).

For the same purpose, the thermophore is certainly the best contrivance, as it keeps up a steady heat for from four to five hours. The indication for hot applications is the presence of severe, cramp-like pains in the gastro-intestinal tract (gastralgia and enteralgia from various causes).

Winternitz's Procedure.-For the same purpose and also for severe vomiting (as in the gastric crises of tabes), for nervous dyspepsia, and for organic disease of the stomach or intestine (e.g., chronic catarrh in either organ), the author has employed for some time a process suggested by Winternitz, which has proved of great value. First a cloth wrung out in water at the temperature of the room is laid over the pit of the stomach or on the abdomen; on that is placed a Leiter's coil and over the whole is bound a dry compress. Hot water $\left(104^{\circ}-140^{\circ}\right)$ is then allowed to flow through the tubes. The application is generally left on from half an hour to an hour; the coil is then removed and the wet compress a half hour later. The essential principle is the combination of a stimulating fomentation with the heat. Its effect is principally that of the stimulating fomentation. This effect (the expansion of the vessels and a corresponding warming of the skin), which is often obtained very slowly and with difficnlty, especially in anæmic individuals, is aided by the hot water sent through the Leiter's coil, so that the patient gets a pleasant feeling of warmth after the combined application has been on only a short time. 
Priessnitz's Applications.-The sedative effect which Priessnitz's applications undoubtedly have upon pain, has not as yet been clearly explained. They are employed for gastro-intestinal conditions according to the method described in the section upon diseases of the thoracic organs (see p. 287).

\section{Mineral Waters in Diseases of the Stomach and Intestines.}

General Principles.-There is no doubt that there are several factors to account for the good effect produced by "taking the waters" at the various spas. There is the chemical constitution of the water itself, its temperature, the ingestion of so much more water than usual, as well as the regular life, the separation from business, the change of residence and surroundings, and, most important of all, the strictly regulated diet. The latter should be primarily and almost exclusively determined by the nature of the disease in each case. In the author's opinion, the use of mineral water in and of itself imposes no peculiar diet, no exclusion of certain foods such as acids or uncooked fruit, although the latter are naturally avoided in case they and the mineral water taken at the same time would overload the intestinal tract. To drink Carlsbad water in the morning and take salad at dinner or fruit for supper, would never do any harm, if an already established disease did not forbid salad and fruit. But to take salad or fruit only a little while after Carlsbad water, might give any one intestinal colic.

Temperature.-The temperature is an important factor in the effect of every kind of mineral water. A naturally or artificially high temperature has a sedative influence on the mucous membrane of the alimentary canal, reduces abdominal irritation, and therefore enables the stomach to receive again, without any detrimental effect, foods which before have noticeably irritated it or the intestine. This brings about an increase in body-weight, an improvement in general condition, and a renewal of appetite. For the practical choice of a mineral water or for the decision as to whether a given water should be taken hot or cold, it is well, then, to keep the following principle in mind. Where there is present abnormal irritation of the stomach or intestine, a tendency to pain or to diarrhœa, naturally warm waters, or those artificially heated, should be preferred to cold; but where it is desired to stimulate the digestive tract-e.g., to increase peristalsis of the intestine and overcome constipation - then cold or cooled waters should be chosen.

Classification of Mineral Waters: Simple Carbonated Waters.There are various kinds of mineral waters which may be used in diseases of the digestive tract. Simple carbonated [or acidulous-Ed.] water, containing a great deal of carbon dioxide, is generally of use rather as a table than as a medicinal water. Its carbon dioxide gas induces belching, perhaps a benefit, for with the eructation are also expelled gases developed during the process of digestion. It also seems probable that carbon dioxide may assist peristalsis, arouse the appetite, and perhaps 
also slightly increase the secretion of gastric juice. Certainly such waters quench the thirst. As representatives of this class may be mentioned: Krondorf, Giesshübl, and Apollinaris (Sauerbrunnen).

Alkaline and Alkaline Chloride Waters.-A second group owes its effect to carbon dioxide on the one hand, and to alkalies, particularly bicarbonate of soda, on the other,- - simple alkaline and alkaline chloride waters. How the alkalies act on the whole digestive tract, and particularly on the gastric function, will be discussed at greater length later (see p. 417); suffice it to say here that alkaline waters combine with the free hydrochloric acid, increase peristalsis somewhat, and apparently serve to dissolve the masses of mucus lining the mucous membrane in gastric catarrh. It is questionable whether they also increase the secretion of hydrochloric acid and bile.

The composition of simple alkaline waters suggests their preference in case of hypersecretion or hyperacidity of the stomach. Since these pathological conditions accompany abnormal irritation and eructation, warm waters should be chosen, cr cold ones should be heated. As an example of naturally warm, simple alkaline waters may be given Vichy; as an example of cold: Preblau, Bilin, Fachingen, and Obersalzbrunn. The alkaline chloride springs, containing sodium chloride, are represented by Wiesbaden (Kochbrunnen), Luhatschowitz (Vincentbrunnen), Gleichenberg (Constantinsquelle), Ems, Seltzers, and Szczawnicza. Ems, especially, has several warm springs. By practical experience it has been found that the alkaline chloride springs are most useful in atonic catarrh of the digestive tract, and secondary dyspepsia and catarrh, as in the initial stages of pulmonary tuberculosis, or of other diseases of the lungs, such as chronic bronchitis or emphysema. [The table on p. 408 gives the composition of the more important alkaline chloride waters of Europe and the United States.-Ed.]

Alkaline Saline Waters.-The most important of all mineral waters, as far as their use in diseases of the digestive tract is concerned, are the sulphated alkaline or alkaline saline waters. Their chief constituent is sodium sulphate, besides which they contain varying quantities of sodium carbonate, sodium chloride, and free hydrochloric acid. Here, too, we have warm and cold springs. [The table on p. 409 includes the important alkaline saline waters of Europe and the United States.-Ed.]

Effect of Carlsbad Water.-Although a series of experiments has been made upon the special effects on the stomach of the most important one of all the waters mentioned-Carlsbad-we still have no new knowledge on the subject. We can say only that Carlsbad water neutralizes acid and arouses peristalsis. The latter action, however, depends upon the spring and the amount of water taken; small quantities of warm Carlsbad waters are constipating. It is also more than probable that Carlsbad water may increase the motor power of the stomach. But it is a question whether it can also stimulate the secretion of hydrochloric acid in the stomach and of bile in the liver. Its practical use in abdominal 


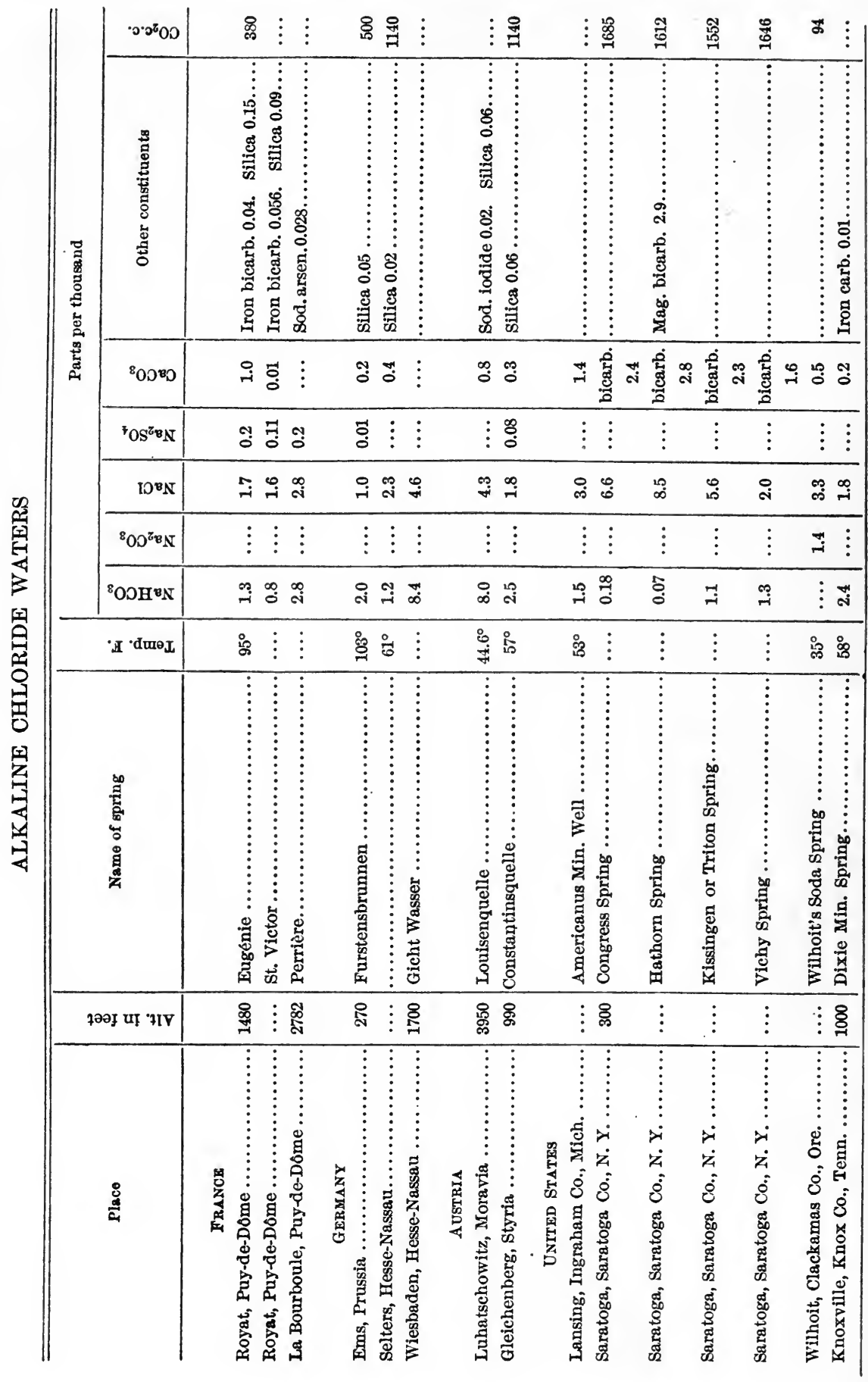




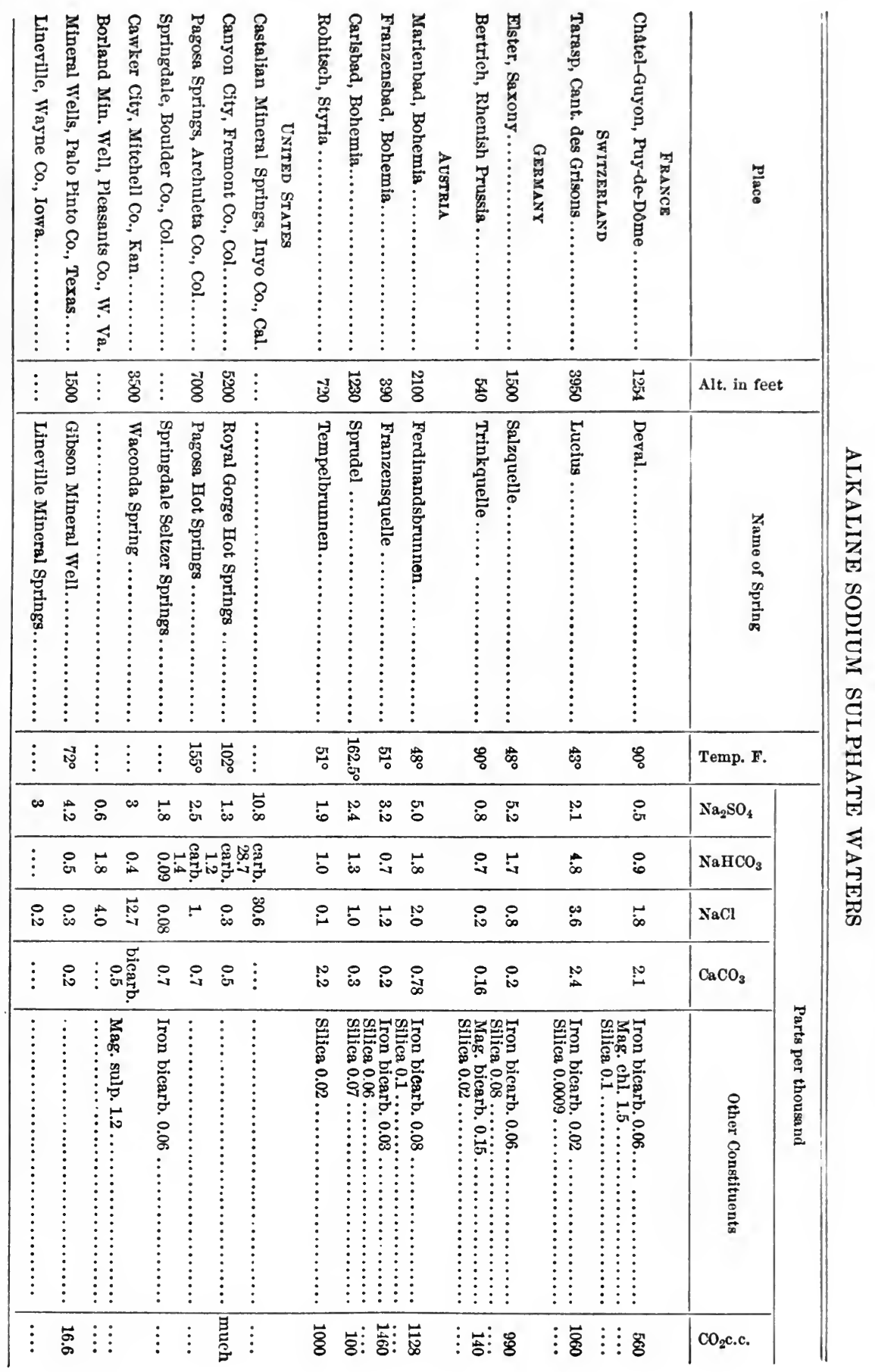


plethora and hyperæmia of the liver shows that if it is taken in appropriate quantities and at an appropriate temperature, it has a laxative effect. To this is due at the same time an improvement of the appetite and a possibly better absorption of food. Finally, one thing is fully proved by Jaworski's fundamental investigations-prolonged use of the Carlsbad cure is undoubtedly detrimental. The author also opposes a too frequent, aimless repetition of the treatment.

Indications for Carlsbad Water.-The chief indications for Carlsbad water are: (1) all forms of dyspepsia accompanied by abnormal formation of hydrochloric acid, except purely nervous cases; $(2)$ the so-called "acid" catarrh of the stomach, especially when accompanied by an abnormal amount of mucus; (3) chronic gastric and duodenal ulcers, especially with hyperacidity; (4) the mildest cases of atony of the digestive tract, (such as arise from a sedentary life, unsuitable food, or constipation) ; finally, (5) dyspepsia accompanied by a very slight reduction of the digestive power of the stomach. In the last case its use should be only tentative. The author does not approve of employing it (1) in cases where the nutrition of the patient is already very poor or, where, for example, malignant tumors are suspected; (2) in any form of purely nervous dyspepsia, or of organic affection of the digestive tract which develops in purely nervous subjects, (3) or in any form of dyspepsia accompanied by serious and obstinate constipation.

Amount of Carlsbad Water.-If hyperacidity or hypersecretion is being treated by the Carlsbad-cure, the patient should usually drink about 1-3 L. (1-3 qt.) a day, of one spring or of combined springs. In other cases he should take only 2-3 glassfuls. These should sometimes be taken one after the other on an empty stomach (only a swallow at a time with an interval of a half to three-quarters of an hour between each glass); sometimes a glassful at a time at different times during the day; sometimes in medicinal doses every $2-3$ hours. If his strength permit it is best to take a brisk walk after drinking the water in the morning. In general the springs of medium temperature are preferable.

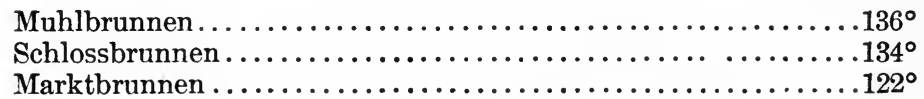

Those of higher temperature are mostly reserved for the treatment of chronic catarrhal affections of the large and small intestines.

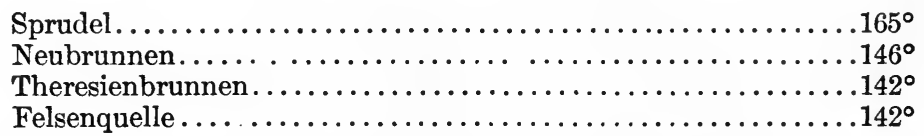

Marienbad Water.-Among the cold alkaline salt springs the best known and most visited is Marienbad. Aside from the temperature of the water, this is distinguished particularly by its large amount of sodium 
sulphate, double that of the Carlsbad springs. Its particular province is the treatment of severe constipation. Elster stands next to it in this respect. If constipation is not overcome by the use of the spring water alone, 1-2 teaspoonfuls of Marienbad salt may be added to the water, or Marienbad water may be taken in combination with some bitter water. For the employment of either of these waters or of one of the milder ones which contain sodium sulphate, the indications are the same as for Carlsbad. In cases of irritation the water should be warm $\left(95^{\circ}-131^{\circ}\right)$, or as hot as it can be taken. The richer the water is in carbonic acid (e.g., Marienbad, Elster, and Rohitsch) the more advantageous it is. In ease of nervous complications or of purely nervous dyspepsia, the author can recommend Tarasp, with its particularly good climatic conditions.

Common Salt Springs.-Common salt springs are distinguished by their relatively high content of sodium chloride which, however, should not be above 1 per cent. or at most 1.5 per cent. Practical experience, although it is not wholly in accord with many experimental investigations, seems to indicate that they increase the secretion of gastric juice, improve the appetite, and stimulate the stomach to increased motor activity. They are, therefore, suited especially to recent gastric catarrh, with decreased secretion of hydrochloric acid and abnormal formation of mucus. They are also often helpful in: (1) chronic catarrh; (2) moderate atony, with or without accompanying catarrh; (3) cicatrized ulcers with diminished secretion; (4) gastric carcinoma, by increasing the appetite and promoting the patient's nutrition; (5) those cases in which the patient is badly nourished or has a naturally weak constitution. With the exception of a few isolated cases they are contraindicated in hyperacidity and in chronic advanced gastric insufficiency. In small doses (1-1 $1 / 2$ glassfuls taken at different times during the day) they are laxative and relieve flatulency. [The table on pp. $412-413$ gives the constituents and temperature of the important sodium chloride waters of Europe and the United States.-Ed.]

The cold waters may be warmed before drinking. This is particularly advisable in the case of the Kissingen Rakoczybrunnen, which contains a relatively large amount of carbonic acid.

Bitter Waters.-One more group of mineral waters is often used in diseases of the alimentary canal,-bitter waters. They are almost never employed when the stomach alone is at fault, but rather in chronic and severe constipation, whether accompanied by gastric dyspepsia or not. They may also be of use in hemorrhoidal and abdominal plethora.

In chronic intestinal catarrh with constipation, the author has obtained successful results in a few cases from small quantities of bitter waters several times a day (60-90 c.c. (2-3 oz.) every $2-3$ hours). He has also obtained good results by using a glassful as an enema, especially in chronic constipation due to the large intestine.

They are contraindicated in all cases of gastric or duodenal ulcer on 


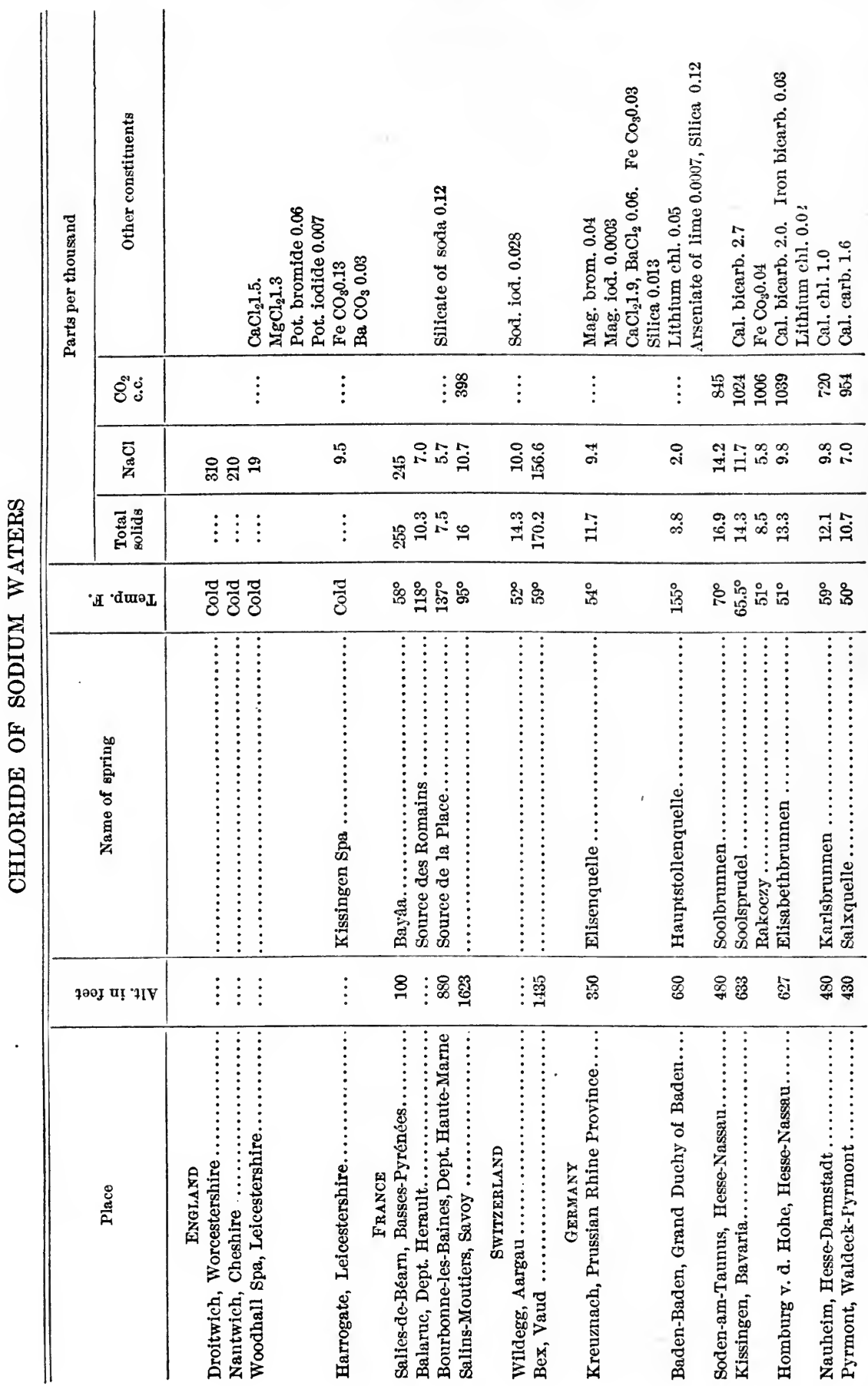




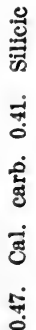

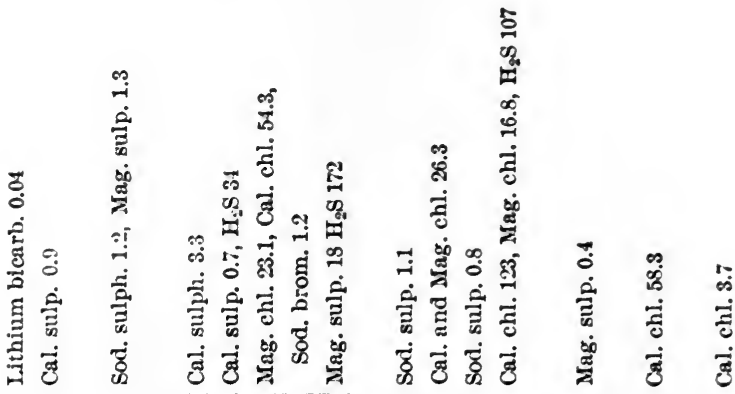

8

성

뉵 \&

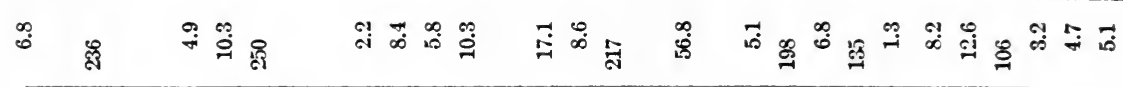

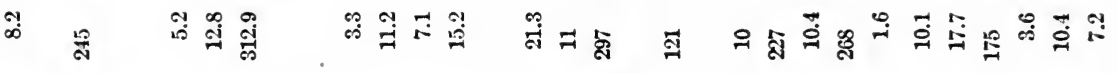

兽 品 \&

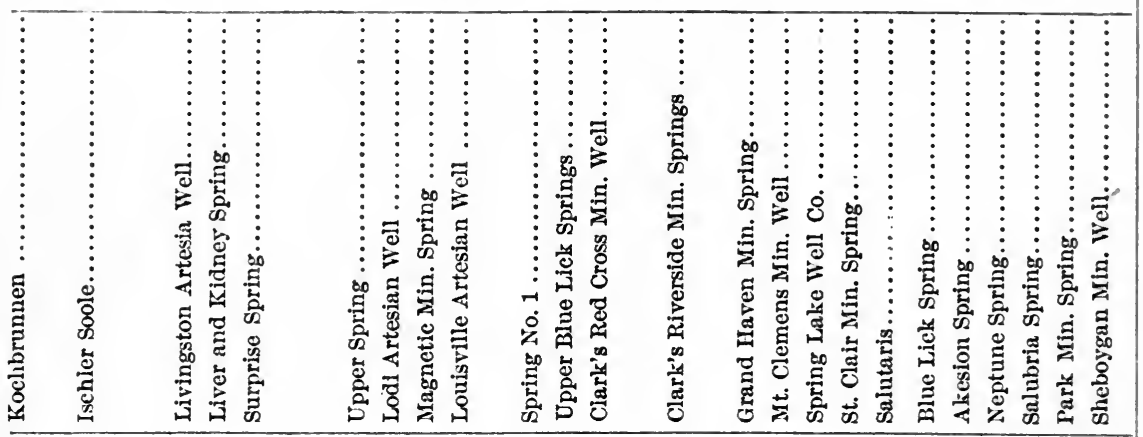

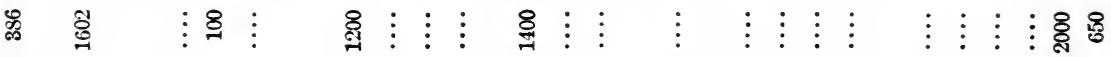

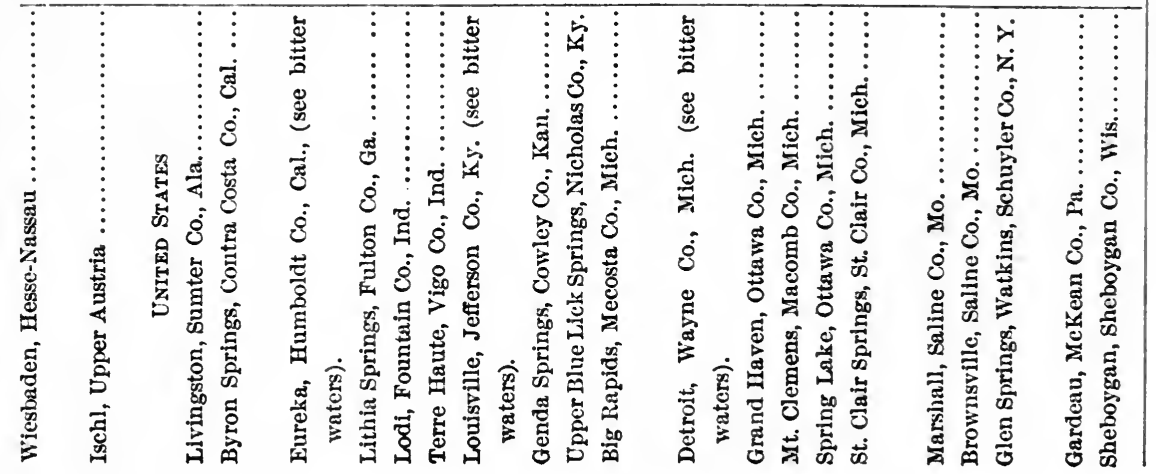


account of the danger of perforation. The author considers them contraindicated also in any extensive inflammation of the intestine or peritoneum, for example in chronic perityphlitis. They are almost always taken cold, usually one or two glassfuls just before going to bed, or in the morning on an empty stomach. Among these waters may be mentioned: the Ofen bitter waters (Hunyadi János, Franz Josef, Apenta, Lajos), the Püllna, Saidschitz, Friedrichshall, and Saratica. [The constituents of the chief bitter waters of Europe are given in the following table.-Ed.]

BITTER WATERS

\begin{tabular}{|c|c|c|c|c|c|c|}
\hline \multirow{2}{*}{ Place } & \multicolumn{6}{|c|}{ Parts per thousand } \\
\hline & $\mathrm{MgSO}_{4}$ & $\mathrm{Na}_{2} \mathrm{SO}_{4}$ & $\mathrm{CaSO}_{4}$ & $\mathrm{NaCl}$ & $\mathrm{MgCl}_{2}$ & Other constituents \\
\hline EUROPE & & & & & & \\
\hline Franz-Josephquelle, Budapest .............. & 24.8 & 23.1 & 1.3 & $\cdots$ & 1.7 & \\
\hline 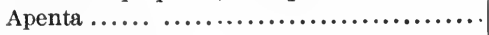 & 24.4 & 15.4 & ... & 1.8 & & \\
\hline Hunyadi-János...$\ldots \ldots \ldots \ldots \ldots \ldots \ldots \ldots \ldots \ldots$ & 22.0 & 22.5 & .... & 1.7 & & \\
\hline Birmensdorf, Switzerland .................. & 22.0 & 7.0 & ... & & & \\
\hline 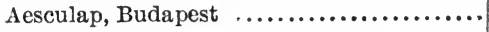 & 17.2 & 13.9 & .... & 2.9 & & \\
\hline Sedlitz, Bohemia ......................... & 13.5 & $\ldots$. & 1.0 & $\ldots$. & 0.3 & \\
\hline Saidschitz $\ldots \ldots \ldots \ldots \ldots \ldots \ldots \ldots \ldots \ldots \ldots \ldots \ldots \ldots \ldots \ldots$ & 10.9 & 6.0 & 1.3 & $\ldots$ & & \\
\hline Püllna, Bohemia.......................... & 10.8 & 9.6 & 1.5 & 2.5 & & \\
\hline Friedrichshall, Saxe-Meiningen, Germany.. & 5.1 & 6.0 & 1.3 & 7.9 & 3.9 & \\
\hline Kissingen, Germany (Bitterwasser) .......... & 5.1 & 6.0 & 1.3 & 7.9 & 3.9 & \\
\hline Rubinat, Spain $\ldots \ldots \ldots \ldots \ldots \ldots \ldots \ldots \ldots \ldots \ldots \ldots$ & 3.2 & 96.0 & $2.1^{2}$ & 2.0 & & \\
\hline UNITED STATES & & & & & & \\
\hline Eureka Spring, Humboldt Co., Cal. .......... & 3.5 & .... & 0.7 & 23.4 & 1.7 & Saturated $\mathrm{H}_{2} \mathrm{~S}$ \\
\hline Harrodsburg Springs, Mercer Co. Ky. & & & & & & \\
\hline Greenville Spring ....................... & 2.1 & .... & 1.4 & trace & & \\
\hline Saloon Spring $\ldots \ldots \ldots \ldots \ldots \ldots \ldots \ldots \ldots$ & 3.7 & .... & 1.3 & 0.6 & & \\
\hline Crab Orchard Springs, Lincoln Co., Ky. & & & & & & \\
\hline 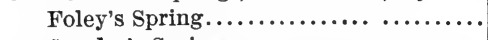 & 3.4 & 1.0 & 0.18 & 0.3 & & \\
\hline Sowder's Spring. ........................ & 2.9 & 0.39 & 1.5 & 1.0 & & \\
\hline $\begin{array}{l}\text { American Carlsbad Springs, Nashville, Wash- } \\
\text { ington Co., Ill. }\end{array}$ & 1.7 & 0.9 & 1.1 & 0.17 & & \\
\hline Gypsum Spring, Ariz. .................... & 1.6 & 0.5 & 1.2 & 3.9 & & \\
\hline Louisville Artesian Well, Louisville, Ky. ... & 1.3 & 1.2 & 0.49 & 10.3 & 0.24 & $\mathrm{H}_{2} \mathrm{~S} 7.2$ \\
\hline Alleghany Springs, Montgomery Co., Va. .. & 0.85 & 0.03 & 1.9 & 0.003 & & \\
\hline Blue Ridge Springs, Botetourt Co., Va. ...... & 0.8 & 0.006 & 1.7 & 0.02 & $\cdots$ & $\begin{array}{l}\mathrm{CO}_{2} 137 \\
\mathrm{H}_{2} \mathrm{~S} 10.8\end{array}$ \\
\hline $\begin{array}{l}\text { Mineral Park Bitter Spring, Ariz. ......... } \\
\text { French Lick Springs, Orange Co., Ind. }\end{array}$ & 0.6 & trace & 1.1 & $\cdots$ & 0.05 & \\
\hline Proserpine spring...................... & 0.5 & 0.6 & 2.3 & 1.5 & 0.1 & $\begin{array}{l}\mathrm{CO}_{2} 36 \\
\mathrm{H}_{2} \mathrm{~S} 51\end{array}$ \\
\hline Pluto's Well........................... & 0.3 & 0.34 & 1.0 & 2.3 & & \\
\hline Concentrated Pluto.................. & 24.0 & 36.0 & 8.4 & 14.9 & & \\
\hline Bowling Green, Pike Co., Mo. ............... & 11.1 & 1.0 & 1.3 & $\ldots$ & .... & Album. sulp. 0.3 \\
\hline
\end{tabular}

2 Sulphate of lime and potash.

Calcium Waters.-As the bitter waters are particularly suited to chronic constipation, so waters containing calcium are suited to intestinal catarrh with a tendency to diarrhœa. Under such conditions they are 
often used as table waters, or else taken at intervals during the day up to $1 \mathrm{~L}$. (1 qt.).

As representatives of these springs may be mentioned: Wildungen (Königsquelle), Driburg (Hersterquelle), Lippspringe (Arminiusquelle), Rappoltsweiler (Carolaquelle).

Waters Containing Iron.-In conclusion it may be noted that there are a number of forms of anæmia combined with dyspepsia, and usually chronic constipation, either as original causes or accompanying symptoms. In such cases the Franzensquelle at Franzensbad, the Salzquelle at Elster, and Reinerz, Rippolsau and Cudowa are often of great help. The Kissingen and Homburg waters and the Ambrosiusbrunnen at Marienbad also contain iron, though not so much as the springs just mentioned.

\section{Climatic Therapy.}

As for climatic therapy in diseases of the stomach, not much can be stated positively. In many cases of dyspepsia with nervous alterations but with no organic lesion of the stomach or intestine, any change of climate has a good effect. After patients have finished a course of mineral water, as at Carlsbad, they are often sent to health resorts to complete the cure. Sea air and sea bathing are generally best suited to those dyspepties who are run down physically, depressed mentally, and suffering from loss of appetite and constipation; while a mountain climate is better for thin, nervous, excitable patients who are suffering from sleeplessness.

Care in a Sanatorium.-It need hardly be said in conclusion that many diseases of the stomach, both anatomical and nervous, are best treated in a sanatorium, where a suitable diet can be provided, or some individual treatment, dietetic or otherwise, can be carried out. Such sanatoria are found in Carlsbad, Kissingen, Wiesbaden, Baden-Baden, Lausanne, Meran, and Hocheppan near Bozen. [Such institutions in America are generally more expensive and inferior to those in Europe. Among the best for this class of patients, if they have ample means, might be mentioned "Miradero" at Santa Barbara, Cal., "Waldheim" at Katonah, N. Y., "Cromwell Hall" at Cromwell, Conn. In most of the larger institutions with which I am familiar there is not sufficient care applied to the dietetic requirements.-Ed.]

\section{MEDICATION IN DISEASES OF THE STOMACH AND INTESTINES.}

Hydrochloric AcID.-Recent investigations of the gastric contents have taught us to recognize whole groups of diseases characterized by the partial or total failure of the hydrochloric acid secretion. In such cases we instinctively, so to speak, try to make up for what the stomach is no longer able to supply by giving hydrochloric acid. All experiments have gone to show, however, that it is not possible to give a quantity 
sufficient to leave some free acid in the stomach in spite of the digestion of proteids, as under normal conditions. ${ }^{3}$ Hence it follows that we must give the hydrochloric acid in large and frequent doses, generally 10-30 drops of dilute hydrochloric acid in $100 \mathrm{G}$. $(3 \mathrm{oz}$.) of water, the first dose to be taken directly after eating, and then a similar dose every hour for $3-4$ hours after the principal meal, and for 2 hours after other meals. Or it may be taken 3 or 4 times at intervals of a quarter or half hour after each meal. Concentrated hydrochloric acid, if used at all, should be given in doses of 5-10 drops very well diluted. For the sake of the teeth hydrochloric acid, even if well diluted, should be taken through a glass tube. So much water after eating is an obvious but unavoidable disadvantage. As has just been stated we cannot supply sufficient acid to replace the quantity which is lacking in hypo- or anacidity; nor can we consider it as proved, although so stated by many authors, that the addition of hydrochloric acid stimulates its secretion. If this were the case, success could probably be obtained with relatively small doses.

Hydrochloric acid should serve us not only as an aid to digestion but as an undeniable antizymotic. There can be no doubt that, under normal conditions of the gastric contents, it has an antifermentative action. It seems questionable, however, whether a constant abnormal amount of fermentation can be mitigated or stopped by the small amounts of hydrochloric acid that we can introduce. Moreover we know that certain abnormal fermentative processes often develop in the stomach despite an abundant secretion of hydrochloric acid,-for instance, in a cicatrization of the pylorus following gastric ulcer and in dilatation of the stomach.

The best results from hydrochloric acid seem to be obtained by using it for a third purpose,-as a stomachic. From many trials the author considers it proved that 20 drops of dilute hydrochloric acid taken with water a quarter of an hour before each meal, has a marked effect in arousing the appetite. In addition, unlike the other mineral acids, it is useful in diarrhœa on account of its tonic properties. One author, Perraud, maintains that it increases the pancreatic secretion. It need hardly be said that it is contraindicated in all cases of hypersecretion.

Summing up, the author maintains: (1) that hydrochloric acid can be used as a substitute for the acid lacking in deficient secretion only by giving large and frequently repeated doses after meals, and then only with uncertain success; (2) that small quantities of hydrochloric acid introduced into the stomach may possibly excite secretion and that, therefore, there is some reason for giving even small quantities; (3) that its antifermentative effect, if abnormal fermentation be present, is very questionable; (4) that taken before meals it is a good stomachic.

${ }^{3}[21 / 2$ litres of 0.2 per cent. $\mathrm{HCl}$ are needed to combine with a daily consumption of $100 \mathrm{G}$. proteid (Chittenden). Without pepsin $\mathrm{HCl}$ possesses no digestive value. The addition of pepsin is valueless because we cannot administer enough $\mathrm{HCl}$ to leave free acid.-Ed.] 
ALKalies: Indications.-The alkalies have an extended use in diseases of the stomach and intestine, especially of the former. Although we do not fully understand all their effects, it is obvious that they can neutralize an excess of hydrochloric acid in the stomach. They are, therefore, indicated in all cases of hyperacidity and hypersecretion; but in the author's experience they must be given in large doses. In hyperacidity they should of course be given after meals, preferably shortly before the time at which the patient expects the disagreeable sensations arising from the hyperacidity, such as pyrosis and heartburn. In hypersecretion they should be taken on a fasting stomach, which will contain hydrochloric acid already secreted. They may also be used to neutralize organic acids abnormally formed in the stomach. But in such cases they should be given only when there is acute and transitory pain, never when there is chronic dyspepsia. In the latter case it is much better to wash out the stomach.

Effect.-Although contradicted by the results of recent experiments and certainly as yet unproved, still practical experience makes it probable that the alkalies, and especially sodium bicarbonate in small doses, 1-2 G. (15-30 gr.), increase the secretion of gastric juice and arouse the appetite. Sodium bicarbonate or small doses of Carlsbad water (2-4 tablespoonfuls), or Carlsbad salts (1-2 G. (15-30 gr.), dissolved in water), have often given relief in the author's cases of severe anorexia. On the other hand, he has seen cases of hyperacidity in which small doses of sodium bicarbonate directly increased the difficulty.

The Most Useful Alkalies.-It seems rather probable that the alkaline carbonates also dissolve the mucus from the mucous membrane, a reason for their frequent use in chronic gastric catarrh. Sodium bicarbonate, burnt magnesia, and the ammonium phosphate of magnesia (Boas) are usually preferred to sodium bicarbonate, which does not dissolve the mucus as easily as do the others. Burnt magnesia neutralizes almost 4 times as much hydrochloric acid as does bicarbonate of soda, the ammonium phosphate of magnesia about 3 times as much. For moderate hyperacidity we administer 8-12 G. (2-3 dr.) of bicarbonate of soda, 2-3 G. (30-45 gr.) of burnt magnesia, 4-6 G. (1-1 I/2 dr.) of ammonium phosphate of magnesia ; but if there is over 0.3 per cent. of hydrochloric acid present we have to use 12,5 , and $7.5 \mathrm{G}$. $(3,1 \mathrm{r} / 4,13 / 4 \mathrm{dr}$.) respectively. With coincident flatulency burnt magnesia, on account of its power of absorbing gases, is particularly indicated, and sodium bicarbonate is contraindicated, because the carbon dioxide so rapidly evolved, increases the amount of gas in the stomach. Burnt magnesia is also, as are the other alkalies, a mild laxative, a fact often important therapeutically. We often give a teaspoonful an hour after eating of a mixture of sodium bicarbonate with burnt magnesia or sometimes with the ammonium phosphate of magnesia. This or half such a dose may be repeated once or even twice. We must remember in prescribing burnt magnesia that it is extraordinarily light in weight; 1 teaspoonful equals 
0.3 G. ( $4 \frac{1}{2}$ gr.). To avoid the rapid development of carbon dioxide, similar doses of magnesium carbonate may. be substituted for sodium bicarbonate. If we wish to obtain a strong action on the mucous membrane of the stomach to dissolve the mucus, sodium bicarbonate is preferable to all the other salts. All these alkalies are best prescribed in tablet form so that they may be slowly dissolved in the stomach (Liebreich).

Carlsbad Salts.-Carlsbad salts, natural or artificial, may be used for the same purpose as the other alkalies; and it is an excellent selection especially to counteract the constipation that so often accompanies hyperchlorhydria. Prof. E. Ludwig gives the composition of natural Carlsbad salt as follows: sodium sulphate 45 per cent., sodium bicarbonate 36 per cent., sodium chloride 18 per cent.; and that of the artificial salt is very similar. We need not adhere rigidly to these proportions; but may vary the ingredients to suit the particular circumstances, adding more sodium bicarbonate if the object is to neutralize the hydrochloric acid, or more sodium sulphate if a stronger laxative is needed.

Carlsbad salt, natural or artificial, or any similar mixture of salts as a substitute, is used in the following way. One teaspoonful of the salt is dissolved in a cup of ordinary water, Carlsbad water, or any kind of mineral water chosen for the purpose (Vichy, Seltzer, Bilin, Giesshübl, etc.). The solution is usually taken tepid $\left(86^{\circ}-95^{\circ}\right)$, or warm $\left(122^{\circ}-131^{\circ}\right)$, in two doses with a half or three-quarters of an hour between. It should be taken cold only to produce a stronger laxative effect, and then when there are no indications of irritation in the digestive tract.

In small doses before meals, bicarbonate of soda increases the gastric secretion and arouses the appetite. This property, together with the power of increasing the motor activity of the stomach, may also be ascribed to a considerable number of other drugs, which we class together under the name of stomachics or bitters.

Stomachics.-From a practical standpoint it is hardly possible to distinguish between these two groups, and it is not very important to do so, especially as we do not clearly understand the exact action of any of them. We know only that these drugs increase the gastric secretion reflexly by their action on the nerves of smell and taste, that they often prove of use in cases of anorexia resulting from hypoacidity, and that they are contraindicated in hyperacidity. Reichmann proved by experiments on patients that bitters increased the gastric secretion only after the drug itself had left the stomach. From this fact follows the practical rule that bitters or stomachies should be taken a half hour before eating.

The list of these drugs is very long and they are often given in combination. Of the newer ones may be mentioned condurango and orexin; of the familiar old ones, gentian (tinctura gentianæ composita, $1 / 2-1$ teaspoonful several times daily), quassia, wormwood (absinthium), 
salicin, cinchona (tinctura cinchonæ composita), menyanthes (Nat. Form.), rhubarb, cascarilla, quinine, and strychnine. The last is in very frequent use and increases both the gastric secretion and motility. The following prescriptions may be given here:

B Tinct. gentianæ comp. .............. $30.00 \ldots \ldots$

Tinct. cinchon $x$ comp. . ....................

M. fiat mist.

Or :

Sig. -A teaspoonful or less 3 times a day, before meals.

B Vini ferri amari..................... $60.00 \ldots$ ii

Sig.-A liqueurglassful before each meal.

Or :

B Quassiæ....................................

Or :

Sig.-A teaspoonful in 2 cups of tea.

R Salicini........................... $\mathrm{v}$

M. fiat tabella.

Dent. tal. tabellæ no. xii.

Or:

Sig. -1 tablet 3 times a day, before meals.

R Tinct. gentianæ comp..................30.00.... $3 \mathrm{i}$

Tinct. nucis vomice.................5.00.... $3^{\mathrm{i}} \eta_{\mathrm{xv}}$

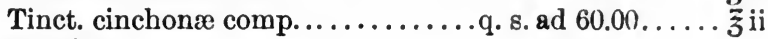

M. fiat mist.

Or :

Sig. - A teaspoonful in water before each meal.

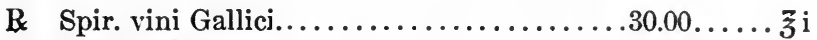

Tinct. calumbæ $\ldots \ldots \ldots \ldots \ldots \ldots \ldots \ldots, 20.00 \ldots \ldots, 3 \mathrm{v}$

Syr. aurantii......................

M. fiat mist.

Or :

Sig.-A teaspoonful to a liqueurglassful 3 times a day before meals.

R Strychninæ sulphatis.................. $0.001 \ldots \ldots$ gr. $\frac{1}{60}$

M. fiat tabella.

Dent. tal. tabellæ no. xx.

Or :

Sig. -1 tablet 3 times a day before meals.

B Quininæ sulphatis.................... $0.20 \ldots \ldots$ gr. ii

M. fiat cap.

Dent. tal. cap. no. $x x$.

Sig. -1 capsule 3 times a day before meals.

Or :

B Elixiri ferri quininæ et strychninæ phosphat........................... $60.00 \ldots$ ii

Sig. -1 teaspoonful 3 times a day before meals.

Or:

(Each drachm contains quinine $\frac{1}{2}$ gr., iron $1 \mathrm{gr}$., strychnine $\frac{1}{6 !} \mathrm{gr}$.)

B Quininæ hydrochloratis . ............... $0.10 \ldots \ldots$ gr. iss

Strychninæ nitratis..................0.0065......gr. I I

Ext. gentianæ....................... $0.15 \ldots \ldots$ gr. ii

M. fiat pilul. no $\mathrm{i}$.

Dent. tal. dos. no. xx.

Sig. $-1-2$ pills before each meal. 
Or: $\quad$ R Tinct. rhei,

Tinct. calami...................āā 6.5..... $3^{i}$ m.xl

Tinct. capsici...........................

or

Tinct. zingiberis

M. fiat mist.

Aq. dest................. s. ad. $180.00 \ldots \ldots$. . vi

Sig.-A tablespoonful in $\frac{1}{2}$ glass of beer or wine $\frac{1}{2}$ hour before meals.

Or :

B Tinct. nucis vomicæ.............60.00.... 3 ii

Sig. -5-15 drops 3 times a day $\frac{1}{2}$ hour before meals.

Rosenheim recommends the tinctures (tincturæ calami, absinthi, gentianæ, quassiæ, rhei), giving at least 30 drops a quarter to a half hour before meals in red wine or heavy southern wine (sherry, port, malaga).

Cortex Condurango.-Cortex condurango, one of our best stomachics, is administered in one of several forms: (1) as a macerated decoction, 1 tablespoonful twice a day a half hour before eating; (2) with 1-2 G. (15-30 min.) of hydrochloric acid added to the decoction if there is no free hydrochloric acid in the stomach; (3) as vinum condurango (a small teaspoonful to 2 liqueurglassfuls) before meals; (4) in the fluid extract, 15-20 drops before meals; (5) as the elixir condurango peptonatum (Immermann).

[The wine of condurango, a P. G. preparation, is in common use here. It is much the best preparation because all the active constituents remain in solution. In the decoction or the fluidextract the principles which are soluble in alcohol are precipitated by the water and according to some authorities may produce irritation.-Ed.]

Orexin.-Penzoldt recommends orexin, which is a phenyldi hydrochinazolin quinolin. To avoid unpleasant gastric symptoms (burning, etc.) it is better given as orexin basicum than as orexin hydrochlorate. The author's results with the former have varied considerably; sometimes no apparent effect was produced, sometimes the appetite was remarkably improved after only a few days. Orexin undoubtedly has a mildly stimulative effect on the stomach, increasing secretion and, perhaps, the power of digestion. It is prescribed as follows (Penzoldt) :

B Orexin............................. $0.30 \ldots \ldots$

M. fiat cap. no. i.

Dent. tal. dos. no. $x$.

Sig. -1 capsule at 10 o'clock in the morning, followed by a cup of soup.

To avoid the possibility of disagreeable symptoms such as burning or vomiting, we may begin with $0.1-0.2 \mathrm{G}$. (11/2-3 gr.), increasing $0.1 \mathrm{G}$. $(1 \mathrm{I} / 2 \mathrm{gr}$.$) on the next day and ordinarily continuing the medication for$ 5 days. Sometimes the appetite is improved in a shorter time and remains good even when the drug is no longer given. If, however, no 
result appears or if the appetite sinks as soon as medication is suspended, orexin should be begun again after about a week, the dose being increased to $0.4-0.5 \mathrm{G}$. (6-7 gr.) or to 2 doses of $0.3 \mathrm{G}$. ( $5 \mathrm{gr}$.). [Orexin is now used exclusively as the name for the tannate. Orexin hydrochlorate, as mentioned above, has been found to be irritating to the stomach and hence its use discontinued.-Ed.]

Quinine.-Nanning's fluidextract of quinine is a stomachic recently put on the market, which contains all the effective constituents of the red quinine bark without the ineffective ones. 10-20 drops in water or wine may be given 2 or 3 times a day. The extremely bitter taste, which often persists for a long time, is very unpleasant to the patient.

Fleiner claims that the best way to take some of these bitters, in case of anorexia, is through a stomach tube. For instance, a funnelful of a decoction of quassia or gentian, or a teaspoonful of wine of condurango in warm water, may be introduced before a meal, allowed to remain several minutes in the stomach, and then withdrawn. ${ }^{4}$

Alcohol-Condiments.-Alcohol in small quantities and many of our condiments, such as pepper and mustard, also act as stomachics. The condiments should not, however, be employed constantly.

Digestive Ferments: Pepsin.-Although pepsin has been extensively employed in the past and is still being employed, it is seldom really indicated, for even where hydrochloric acid is lacking, there is only very exceptionally a lack of pepsin or pepsinogen. Moreover, the preparations on the market are so far from satisfactory that we are still further limited in our use of it. According to Penzoldt's tests of four different pepsin preparations, Langenbeck and Jensen's Concentrated Pepsin is the best with Finzelberg's next. A single dose of 0.2-0.5 G. (3-7 gr.), is enough, given with hydrochloric acid. [Very little pepsin is taken up by alcohol; and alcohol itself impairs the action of pepsin, so that wines and essences are poor preparations. The officinal pepsin in $0.3-0.6 \mathrm{G}$. (5-10 gr.) doses is more potent. I do not consider the use of pepsin rational. Hemmeter no longer uses it.-Ed.]

Papayotin-Papain.-Nothing better can be said for papayotin or its derivative papain, powders made from the milky juice of carica papaya. It is true that both of these products digest proteids in an acid, neutral, or alkaline solution. This fact might give them the preference over pepsin did they not, according to investigations in Riegel's clinic, require more time to accomplish the proteid digestion. They are indicated in all cases of hypochlorhydria, more especially if this deficiency be chronic, as in chronic gastric catarrh, atrophy of the glands of the stomach, gastric carcinoma. dilatation, and nervous anacidity and apepsia. Papain, a white, odorless, and tasteless powder, is the more frequently employed. It is given in $0.3-0.5$ G. (4-7 gr.) powders several times directly after eating, with a quarter or a half hour between

4 [Pawlow found that bitters placed in the stomach without being swallowed produced no effect upon the secretion of acids.-Ed.] 
doses, in tablets containing the same dose, or in a thin paste made by stirring up the same quantity with water directly before taking (Sittmann). [Chittenden found a stronger digestive power for proteids and especially for starches in "caroid," a highly concentrated extract of carica papaya.-Ed.]

Pancreatin.-Pancreatin seems to the author to deserve a higher recommendation than pepsin and papayotin. It is a dry extract made from the pancreas of the ox. As it digests proteids in an alkaline solution, it is of course useful in all cases where the gastric juice is lacking. But if the gastric juice is merely being secreted in subnormal amountsand this is generally the case when we use pancreatin-an alkali should be added to secure neutralization of the free hydrochloric acid present. In both cases all we are trying to do is to anticipate in the stomach the digestion of proteids by the pancreatic secretion. The following is Boas's prescription:
B Pancreatini,
Sodii bicarbonatis ..............āā $0.50 \ldots \ldots$ gr. vii
M. fiat caps. no. $i$.
Dent. tal. caps. no. xxx.
Sig. $-3-4$ capsules a day, $\frac{1}{4}$ of an hour after eating.

Of useful preparations of pancreatin, may be mentioned Engesser's, Merck's, Witte's, and Schering's, [Fairchild's-Ed.], of any of which the dose is $0.3-0.5$ G. (4-7 gr.) several times daily after eating.

Pancreon.-All these preparations are surpassed by pancreon, a preparation obtained by treating pancreatin with tannin. It is a gray, tasteless, odorless powder, and has the advantage of resisting the gastric juice for five hours or so, and of possessing a remarkable power of digesting proteids. The author has used it successfully where he suspected a decreased secretion of pancreatic juice. $0.5 \mathrm{G}$. $(7 \mathrm{gr}$.) stirred into 100 c.c. (3 oz.) of water, is given a half hour before each meal, except in hypoacidity, when it is preferably used during or after meals. For anacidity he has used it much more frequently $(0.5$ G. $(7$ gr. $)$ before meals). He considers it the best ferment we have during the alkaline stage of digestion.

Malt Diastase Ptyalin.-It is only in exceedingly exceptional cases that a lack of the salivary ferment obliges us to find a substitute. But much more frequently we find it necessary to reinforce the saliva with some similar artificial ferment in hyperacidity or hypersecretion, as in such cases the digestive power of the saliva is quickly suppressed by the overabundant hydrochloric acid in the stomach. Boas recommends for this purpose Merck's ptyalin, rather than malt-diastase, in single doses of $0.5-1.0$ G. (7-15 gr.) about half an hour after eating. Takadiastase has also been recently advocated. It is a hygroscopic, almost tasteless powder, of strong sacchariferous properties, made from aspergillus oryzæ in the process of manufacturing Japanese rice-wine. The 
dose is $0.1-0.3$ G. ( $1 \frac{1}{2}-41 / 2$ gr.) dissolved in water and taken during meals. In prescribing either of these ferments, alkalies (e.g., sodium bicarbonate) should also be given, to neutralize the excess of acid.

Other Enzymes.-There are many other enzymes that digest proteid, analogous to papayotin and like it derived from plants; e.g., ananaspain (bromelin) made from pineapples, vegetable pepsin made from different plants (e.g., drosera), and cradine from ficus carica, ${ }^{5}$ all of which are employed like papain and usually in combinations with it or with pepsin or pancreatin. 'They have, however, but slight value.

Nutrol is a peculiar mixture of dextrose, maltose, dextrin, hydrochloric acid, pepsin, and bromelin, in the form of a golden, syrupy, acid liquid. In the author's experience patients do not take it well for any great length of time. The dose is a teaspoonful in a half glass of water, several times a day.

Gasterine.-Finally gasterine, the new drug already mentioned in the section upon pernicious anæmia (p. 168), is prepared from the gastric juice obtained from the stomach of a dog [or pig-Ed.] by inserting a fistula into the œsophagus, closing the œsophagus below the fistula, and inserting another fistula into the stomach. When food is taken into the animal's mouth, gastric juice is secreted by the stomach and collected. Gasterine is apparently useful where hydrochloric acid and pepsin are deficient on account of atrophy of the stomach, but hardly enough investigations are at hand to prove the fact conclusively, and its use must be necessarily limited on account of the difficulty in procuring it. [Patients for whom I have prescribed gasterine have not been benefited as much as when they used it on the Continent. Whether the preparation is not sufficiently fresh or not, I do not know.-Ed.]

Stomach AnTiseptics.-We have already discussed hydrochloric acid as an antiseptic agent in the stomach, and its questionable value. Its effect is similar to that of the numerous other gastric antiseptics. In addition it retards the peptonization of proteids in the stomach. The use of such drugs-e.g., menthol, $\beta$-naphthol, or salicylic acid-can be recommended only in those cases where the stomach can never functionate normally again, as in carcinoma. Here also belong peroxide of magnesia and Merck's permanganese. The latter, also called hopogan or biogen, should be rationally used only when there is free hydrochloric acid in the stomach. For by this it is split up into chloride of magnesia, and peroxide of hydrogen, which again is separated into water and oxygen. ${ }^{6}$ The oxygen is the effective component. The author gives hopogan or biogen in powders of $0.25-0.5 \mathrm{G}$. (4-7 gr.) after meals, either enclosed in capsules or taken with $60-90 \mathrm{G}$. (2-3 oz.) of water.

${ }^{5}$ [Rusby in Nat. Dispens. says that there is no ferment cradine in ficus carica.Ed.]

[According to the following reaction:

$2 \mathrm{Mg} \mathrm{O}+4 \mathrm{HCl}=2 \mathrm{MgCl}_{2}+2 \mathrm{H}_{2} \mathrm{O}_{2}$

$2 \mathrm{H}_{2} \mathrm{O}_{2}=2 \mathrm{H}_{2} \mathrm{O}+\mathrm{O}_{2}$. - Ed.] 
The best way to disinfect the stomach, however, is to wash it out or to give an emetic. The latter, however, should be avoided as much as possible in gastric disease, no matter for what reasons it may be indicated; for all emetics given by the mouth (ipecac, copper sulphate, antimony, and potassium tartrate) produce varying degrees of irritation or inflammation of the gastric mucous membrane. The general effect of antimony and potassium tartrate, alone might contraindicate their use. If vomiting must be induced it is better to give a subcutaneous injection of apomorphine hydrochloride.

Intestinal Antiseptics: Calomel-Creosote.-Intestinal antiseptics are much more frequently employed, although to avoid injuring the mucous membrane of the stomach and intestine, we must dilute them so much that we obtain only partial service from them. Among those used are calomel in small doses $(0.05 \mathrm{G}$. ( $3 / 4 \mathrm{gr}$.), three times a day, for some length of time) and creosote, which Klemperer maintains also stimulates the motor activity of the stomach, and the use of which has already been spoken of in sufficient detail in the section upon pulmonary tuberculosis (p. 344). Rosenheim gives the following prescription for it:
B Creosoti .............................2.00....m $\mathrm{mxx}$
Alcoholis .......................20.00... $3 \mathrm{v}$
Tr. rhei........................ $30.00 \ldots$ iiss
M. fiat sol.
Sig. -30 drops 3-5 times a day in milk.

Or :

B Creosoti .................................... $m \times x$

Spir. vini Gallici ....................100.00.... そ iii

M. fiat sol.

Sig.-A teaspoonful in a glass of milk after each meal.

Or :

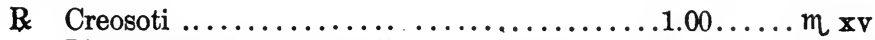

Tinct. gentianæ comp........................ m $\mathbf{x x x}$

Vini xerici.........................

M. fiat sol.

Sig.-A teaspoonful in sherry wine twice a day.

[Francine recommends the administration of creosote shortly before eating, 1-15 drops in as many tablespoonfuls of very hot water.-Ed.]

Bismuth Compounds.-Here too belong the bismuth compounds; e.g., bismuth salicylate, which splits first in the intestine, the subgallate of bismuth or dermatol, the soluble phosphate of bismuth, and eudoxin (already almost forgotten), the bismuth salt of nosophen (the tetraiodide of phenolphthalin) :

B Bismuthi salicylatis............0.50-1.00-2.00.....gr.vii-gr.xv-gr.xxx Fiat pulv. no. i.

Dent. tal. pulv. no. xxx.

Sig.-A powder every hour or every 2 or 3 hours (up to $6 \mathrm{G}$. (1 $\frac{1}{2} \mathrm{dr}$.)

or over may be given). 
The same doses of dermatol are given in the same way; of eudoxin from $0.3 \mathrm{G}$. $(4 \mathrm{r} / 2 \mathrm{gr}$.) at first, to $0.5 \mathrm{G}$. ( $7 \mathrm{r} / 2 \mathrm{gr}$.) three times a day, also in powder form; the phosphate of bismuth, as follows :

B Bismuthi phosphatis solubil.........2.00-4.00.....gr. $x x x-3^{i}$

Sacchari lactis........................... q. s.

M. fiat pulv. no. i.

Dent. tal. pulv. no. xii.

Sig. -1 powder every 2 hours.

All these bismuth compounds, of which the salicylate is the author's favorite, will be mentioned again several times, particularly in the therapy of intestinal catarrh (see p. 473).

Compounds of Salicylic Acid.-The reason why bismuth salicylate or bismuth benzoate is better for this purpose than the others is that the salicylic acid and the benzoic acid act as additional antiseptics. Salicylic and benzoic acids themselves were formerly used as intestinal antiseptics and now their various compounds are so employed. For instance, salol (phenyl-salicylate) splits up only in the intestine under the influence of the pancreatic juice into salicylic acid and carbolic acid; in the same way sodium benzoate gives up its benzoic acid. The use of salol, however, seems to be attended with some danger. We also employ salacetol (salicylic acidester of acetone, belonging to the acetone alcohols), which has been manufactured in the endeavor to obtain something compounded with salicylic acid which shall be less powerful than the carbolic acid set free when salol is split up.

R Acidi salicylici..................1.00-2.00.....gr. $\mathrm{xv}$-gr. $\mathbf{x x x}$

Emuls. amygdal...................180.00.... 3 vi

Mucil. acaciæ,

Syr. aurantii..................āa $10.00 \ldots \ldots z$ iiss

M. fiat emuls.

Sig. - A tablespoonful every 2 hours.

Salicylic acid may be given in capsules $(0.2-0.5$ (3-71/2 gr. )) up to 10 capsules daily. The author, however, prefers the above prescription.

R Salolis............................0.50....gr. viiss

Fiat caps. no. i.

Dent. tal. caps. no. xii.

Sig. -1 capsule 3-4 times a day.

R Salacetol. $.0 .50-1.00 \ldots . .$. gr. viiss-gr. $\mathrm{xV}$

Fiat pulv. no. i.

Dent. tal. dos. no. $x \times x$.

Sig.-A powder every hour or two (sometimes in castor oil).

Naphthol Compounds.-To these antiseptics may be added naphthalene, $0.1-0.5$ G. ( $11 / 2-71 / 2$ gr.) up to $5 \mathrm{G}$. (11/4 dr.) a day in wafers. The author has found it quite harmless but not of much service. Phenol, with the ereosote first removed, is preferable. There are also $a$ - and 
$\beta$-naphthol, and various naphthol compounds, particularly $\beta$-naphtholbismuth or orphol, benzo-naphthol, and naphthalol or betol. $\alpha$ - and $\beta$-naphthol are both colorless substances with a faint odor of phenol, almost insoluble in cold water. The first occurs in needle-like crystals, the second in flakes. $\beta$-naphthol-bismuth or orphol is a bright brown powder insoluble in water. The dose is $0.2-0.5 \mathrm{G}$. (3-71/2 gr.) from 3 times a day up to every 2 hours. The author has found it very good in both acute and chronic intestinal catarrh. Benzo-naphthol (the ester of benzoic acid naphthol), white odorless crystals not very soluble in water, is given in powders up to $0.5 \mathrm{G}$. ( $7 \mathrm{I} / 2 \mathrm{gr}$.) each, and $5 \mathrm{G}$. ( $75 \mathrm{gr}$.) a day. Betol (naphthosalol or salinaphthol, the salicylic acid-naphtholester) comes in the form of colorless, almost odorless flakes, insoluble in water. It is given up to $3 \mathrm{G}$. (45 gr.) a day, in doses of $0.5 \mathrm{G}$. ( $7 \mathrm{I} / 2 \mathrm{gr}$.) each. Its action appears to be uncertain, as the pancreatic juice does not split it up uniformly or easily.

Among these drugs, the first place should certainly be given to benzo-naphthol. It may be used with sodium bicarbonate, with bismuth salicylate, or with both together.

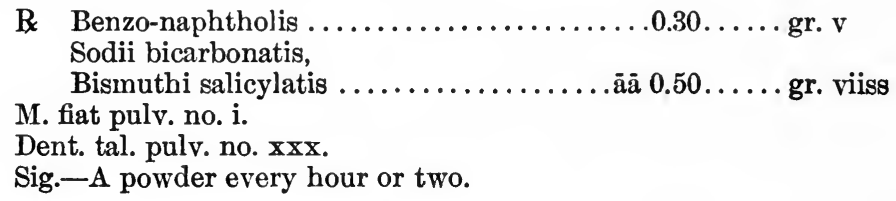

$\beta$-naphthol may be given in the following combination:

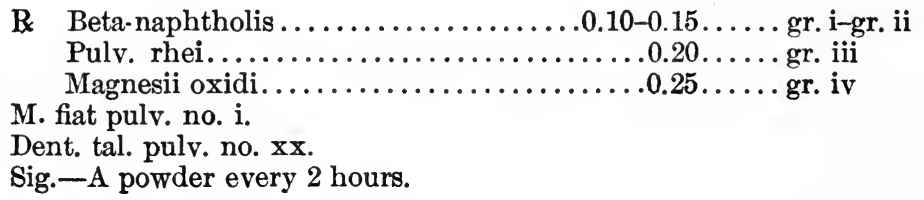

Or, according to Bouchard:
R Beta-naphtholis...................15.00.... 3 ss
Bismuthi salicylatis,
Sodii bicarbonatis ............... āa $7.50 \ldots \ldots z$ ii
M. fiat pulv.
Div. in dos. aeq. no. xxx.
Sig.-A powder after each meal.

[J. D. Steele found that bismuth salicylate and $\beta$-naphthol reduced the amount of bacteria in the feces of normal individuals, but that they increased the amount of bacteria in some gastrointestinal cases.-Ed.]

Resorcin-Bismuth Resorcinate-Ichthalbin-Menthol, etc.-Next come four more antiseptics: resorcin, bismuth resorcinate, ichthalbin, and menthol. Resorcin, sweet, colorless crystals, easily soluble in water, 
seems to the author to have a certain, though limited, value as a gastric and intestinal antiseptic. Bismuth resorcinate, its compound with bismuth, is given in powders of $1 \mathrm{G}$. (15 gr.) or less, up to $3 \mathrm{G}$. (45 gr.) a day.

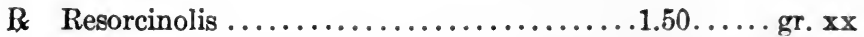

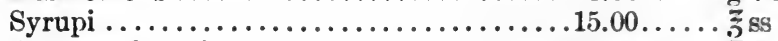

Aq. menthæ pip.................... $150.00 \ldots$

M. fiat sol.

Sig. - A tablespoonful every 2 hours. (Dispense in dark bottle.)

Or, according to Boas :

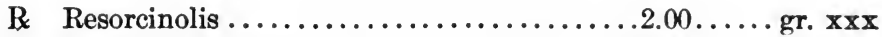

Aq. chloroformi...................

M. fiat sol.

Sig. -1 tablespoonful 3 times a day after meals. (Dispense in dark bottle.)

Ichthalbin is a fine, golden-brown, odorless, and almost tasteless powder, which in the intestine slowly splits up, forming ichthyol. The author gives it either in chocolate $0.30 \mathrm{G}$. ( $5 \mathrm{gr}$.) tablets up to $6.0-8.0 \mathrm{G}$. $\left(1 \frac{1}{2}-2 \mathrm{dr}\right.$.) a day, or else according to the following prescription:

B Ichthalbini $.1 .00-2.00$. gr. $x v-g r . x x x$

Dent. tal. cachets no. 1 .

Sig. $-2-3$ cachets a day.

Ichthoform, a compound of formaldehyde and ichthyol, should also be mentioned. It is a dark brown, almost odorless and tasteless powder, insoluble in water. Taken in quantities up to $2-3 \mathrm{G}$. (30-45 gr.) daily, it proves as good an intestinal antiseptic as the drugs just enumerated, although it is no better than bismuth. It probably owes its antiseptic action to the formaldehyde set free. In diarrhœa resulting from intestinal tubercular ulcers, it is of no marked benefit, even if 6-12 G. (1 $1 / 2-3 \mathrm{dr}$.) a day be given. Even such large doses, however, produce no bad effects.

Among the more reliable intestinal disinfectants is cerevisiæ fermentum (yeast), $12-16$ G. (3-4 teaspoonfuls) daily. Its antiseptic power is due to the fact that it is itself a representative of the lower organisms and so can counteract bacterial decomposition. The author has had very satisfactory results from its use in chronic furunculosis. [I usually employ 8-15 G. (2-4 dr.) of brewer's yeast or one half as much compressed yeast shortly before each meal three times daily, and can heartily commend its use.-Ed.]

Menthol is the drug the author most frequently employs to obtain as high a degree of intestinal antisepsis as possible, in many cases apparently with absolute success. Menthol comes in crystalline form. It is insoluble in water but soluble in alcohol and in oil, and has a characteristic odor. 
R Mentholis .

Olei amygdal. express.

$0.10-0.25 \ldots \ldots$ gr. iss-gr. iiiss

or

Olei olivæ

$.0 .30 \ldots \ldots m$ ivss

M. fiat caps. no. $i$.

Dent. tal. caps. molles no. xxx.

Sig.-One capsule 3-5 times a day after meals.

A similar dose of the following mixture may be employed:

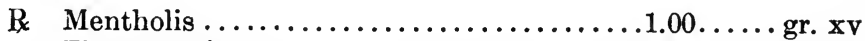

Tinct. gentianæ...................

- M. fiat sol.

Sig. $-10-15$ drops 3 times a day after meals.

Method of Administration: Pills Covered with Keratin or Salol.To ensure the passage of any of these disinfectants unchanged through the stomach into the intestine, it is often a good plan to give them in pills covered with keratin or liquid salol; e.g.:

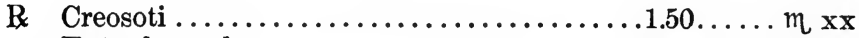

Ext. glycyrrh.......................... $3^{\mathrm{i}}$

M. fiat mass.

Div. in pil. no. $x x x$, (coat with keratin or salol).

Sig. -1 or 2 pills 3 times a day after meals.
}

Glutoid Capsules.-Another valuable method of administration is by means of the glutoid capsules, recommended by Sahli. These are gelatine capsules hardened with formalin, and come in three different grades of hardness; Grade II, the one most frequently used, can be depended on to pass through the stomach and be easily dissolved in the intestine. The following is a representative prescription:

B Mentholis,

Olei amygdal. express...............aa $0.125 \ldots \ldots$ gr. ii

M. dent. tal. dos. no. l, (dispense in glutoid caps. grade ii).

Sig.-One capsule 3 times a day after meals.

Carminatives.-Menthol immediately suggests another class of drugs especially useful in intestinal diseases, - the carminatives. Although we are almost entirely ignorant of their mode of action, we employ them for flatulency in the digestive tract.

The most common are: mentha piperita, mentha crispæ, anisum, and anthemis. They are used in the form of an infusion or tea, a tablespoonful every 2 hours being given. The ethereal oils of these plants may also be employed, 5-10 drops a day added to mixtures.

Three other drugs, used to aid the absorption of an abnormal amount of gas formed in the stomach and intestine, may be mentionedasafœitida, charcoal, and burnt magnesia. 
Brunton considers asafotida the best carminative for gas in the intestine, but unfortunately its very disagreeable odor often prevents its use. The dose is a teaspoonful of the tincture in a tumbler of warm water daily.

Carbo ligni, formerly frequently employed, is now seldom used, for, although charcoal undoubtedly absorbs gas easily, it loses this power as soon as it comes in contact with liquids, and therefore has little effect in absorbing gases in the intestine. French authorities claim that it has a peculiar attraction for alkaloids and so is particularly suitable in cases of ptomaine poisoning. The dose is $0.5-2.0 \mathrm{G}$. (71/2-30 gr.) 4-6 times a day, in powders or, better, tablets, capsules, or biscuits.

Magnesium oxidum also absorbs gases, at least carbon dioxide. Free hydrochloric acid in the stomach will, however, unite with the magnesia and form chloride of magnesia, leaving no magnesia available for intestinal gases. Burnt magnesia (magnesium oxide) is a light, white powder almost insoluble in water. $2-4 \mathrm{G}$. $(1 / 2-1 \mathrm{dr}$.) may be given daily. In case an antiseptic, slightly laxative carminative is indicated, the author employs magnesium salicylate, generally in powders, up to 3-6 G. (45-90 gr.) a day.

The valuable drug, calomel, is also a mild purgative and absorbs gases. As an antiseptic it is rather weak, for even small quantities of it, when brought in contact with the intestinal secretion, apparently split up into the bichloride of mercury, which in that dilution has but a slight antiseptic action. The author gives it in powders, sometimes in single doses up to $0.5 \mathrm{G}$. ( $71 / 2 \mathrm{gr}$.), sometimes in repeated doses of $0.1-0.3 \mathrm{G}$. $\left(1 \mathrm{I} / 2-4 \frac{1}{2} \mathrm{gr}\right.$.) with another cathartic accompanying or following it:

R Hydrargyri chloridi mitis,

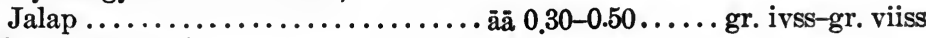
M. fiat chart. no. i.

Sig. - To be taken as directed.

Catrhartics.- - These two drugs bring us to a final group constantly employed in diseases of the stomach and the intestine,- - the cathartics. The more detailed discussion of cathartics will be taken up in the section on the therapy of constipation. Here it will be enough to say that recent investigations divide them into two groups. The first produces its effect by chemical and mechanical action and by increased peristalsis ; the second, by increasing the amount of fluid in the intestine by drawing it from the blood through the intestinal wall by osmosis. To the first class belong all the vegetable and alkaloid cathartics; to the second, the saline cathartics.

Classification.-According to their strength, cathartics may be divided into mild laxatives, medium purges, and drastic purges. They may be arranged in order as follows: mild laxatives: substances containing sugar (e.g., honey and molasses), those containing sugar and fruit acid (e.g., plums, figs, and tamarinds), sulphur, magnesium oxide, fatty 
oils (particularly castor oil); medium purges: the saline cathartics (especially Glauber's and Epsom salts), calomel, rhubarb, frangula, cascara sagrada, aloes, and senna; drastic purges: jalap, gamboge, podophyllin, colocynth, and croton oil. According to experiments, Penzoldt's in particular, all of these drugs, in so far as they are really cathartic in action, delay digestion in the stomach.

For clinical and practical purposes, it would often be convenient if we could divide eathartics into those which act primarily on the large intestine and those which act primarily on the small, but at present such a classification is impossible. We do know, however, certain individual drugs that experiment shows produce their chief effect upon the large intestine, viz: senna, aloes, and colocynth.

In prescribing an intestinal disinfectant, it is well to add one of the mild purges, especially if the antiseptic chosen be one of the bismuths, which have a slightly astringent effect on the intestine. The author often makes use of the following prescription:

B Bismuthi salicylatis.................. $0.50 \ldots \ldots$ gr. viiss

Pulv. rhei...................... $0.10-0.30 \ldots \ldots$ gr. iss-gr. iv

M. fiat pulv. no. i.

Dent. tal. pulv. no. $\mathrm{xxx}$.

Sig. -1 powder every 2 hours.

SEDatives-NARCotics.-The final group of drugs used in diseases of the stomach and intestine is the sedatives or narcotics. They are employed in cases of vomiting as a symptom of an abnormal irritability of the stomach, and in cardialgia and enteralgia. Opium, morphine, and codeine are the principal sedatives. Belladonna may be added in many cases of clonic or tonic contractions of the intestinal muscles with resulting pain, for opiates and belladonna paralyze the muscles of the intestines. They are given in various forms: opium as a powder, extract, or tincture; belladonna as a tincture (dose 10-20 drops), or an extract (0.015-0.03 G. ( $1 / 4-1 / 2$ gr.) in powder or solution) several times daily. For vomiting, chloroform (5-10 drops on sugar), aqua chloroformi, ether, cocaine hydrochlorate (0.02 G. ( $1 / 3$ gr.) a dose), menthol (probably a local anæsthetic), or silver nitrate are also used. In cardialgia and especially enteralgia, anthemis (chamomile) as a tincture or infusion is often useful, its sedative action being due to its ethereal oil.

Instead of menthol, validol (valerianic-acid menthol) may be used as a sedative in cardialgia or nervous vomiting, though in the author's experience it works no better than menthol alone. It is given 1-3 times a day, 5-15 drops on sugar, in coffee, in 5 drop chocolate tablets, or in soft capsules.

Morphine and opium are given sometimes alone, sometimes in simple mixtures. Morphine is used more frequently for the stomach; opium, for the intestine. Morphine increases the gastric secretion, and therefore is contraindicated when the pain is due to hyperacidity. In that case 
atropine, belladonna, or eumydrin (atropine methyl nitrate) should be employed. It is important to remember that opiates decrease the motor activity of the stomach, so that, whenever possible, their use should be avoided. For instance in cardialgia, as a result of gastric ulcer, bismuth should be used instead. The continued use of opium or morphine can be justified only in gastric carcinoma which is beyond the reach of operation.

Silver nitrate is indicated when the pain is due to hypersecretion. It is best given in pills.

Simple chamomile tea is often used in enteralgia ( $\mathrm{L} / 2$ teaspoonful to a cup of water), or the infusion of chamomile. If we wish to combine this with the tincture or extract of opium, the following is a good prescription:

B Tinct. anthemidis,

Tinct. opii....................āa $15.00 \ldots \ldots z \mathrm{zs}$

M. fiat sol.

Sig. $-15-20$ drops 3 times a day. 


\section{SPECIAL THERAPY OF DISEASES OF THE S'TOMACH AND INTESTINES}

\section{SPECIAL THERAPY OF DISEASES OF THE STOMACH.}

\section{THERAPY OF ACUTE GASTRITIS.}

Fasting.-Any acute disease demands that the organ or organs affected shall be rested as far as possible. In acute gastritis, therefore, the patient must consent to fast for the first 24-48 hours.

Methods of Relieving Thirst.-Generally he suffers from intense thirst. This may be mitigated by repeatedly rinsing the mouth with an alkaline solution or carbonated water, by lumps of ice held in the mouth without swallowing the ice water, by spoonfuls of cold unsweetened Russian tea, to which, in cases of collapse, brandy or rum may be added, by drinks of lemonade, made from fresh fruit or from phosphoric, hydrochloric, or sulphuric acid, or by small quantities of mineral waters containing hydrochloric acid or carbon dioxide. With the carbonated waters the anæsthetizing effect of the gas may be taken into account.

Diet.-After this fast the patient is at first limited to a liquid diet, taken in small quantities at frequent intervals. Gradually other foods may be allowed, following the order of the subjoined table:

(1) Soups thickened with sago or tapioca.

(2) Gruels with an egg yolk stirred in.

Cold boiled milk $45-100$ G. (1 $1 / 2-31 / 2$ oz. $)$.

(3) Meat jelly (after the first sensations of hunger).

Milk prepared in various ways.

(4) Bouillon (sometimes with the addition of a meat preparation).

(5) Meat and carbohydrates in easily digested forms, (when the patient becomes hungry enough to ask for them), such as:

Meat: Boiled fowl, chicken, or pigeon (without skin), veal, scraped beef, shaved ham, calves' sweetbreads (in soup or boiled in bouillon), boiled calves' brains.

Carbohydrates: Arrowroot gruel, tapioca pudding, potato soup flavored with a little butter.

(6) Roast meat, apple sauce, rice, zwieback, crackers, or toast.

If the appetite remains poor after all the other symptoms of the malady have disappeared, a stomachic may be given (wine of condurango, 
tinctura amara N.F., tincture of rhubarb, or, in case of hypochlorhydria, 10 drops of dilute hydrochloric acid in a wineglassful of water before eating). Appetizers may also be tried; e.g., mild caviar, sardines, or fine herrings.

Medication.-In general, drugs should not be given in acute gastritis, for the inflamed stomach needs most to be left alone. In the comparatively exceptional cases where nature fails to effect the cure, the substances causing the trouble in the intestinal tract should be removed by inducing vomiting or by eatharsis. Emesis is indicated whenever the patient feels nauseated without being able to vomit. Emetics should not be used on account of their irritating effect on the stomach. It is better to give tepid water, to tickle the throat, or in extreme cases to inject apomorphine hypodermically. Lavage is better than vomiting induced by any method. [Robin acknowledges the theoretical value of lavage but objects to its use in practice. He prefers a combination of ipecac and antimony followed a day or two after the emesis by a saline. In adults Forchheimer properly limits the employment of the tube to patients accustomed to its use, and in others accomplishes the same result with repeated copious draughts of lukewarm water. In infants he employs gastric lavage without hesitation.-Ed.]

Catharsis.-Where the accumulation of gas, intestinal colic, and abnormally fetid diarrhœa indicate that the undigested masses have passed from the stomach into the intestine and set up decomposition, a mild cathartic may be employed. Castor oil is perhaps the best, as its action is very mild and its odor is particularly apt to excite vomiting. Calomel, too, is often serviceable, especially in severe cases of acute gastro-enteritis in children, and when repeated vomiting has not relieved the symptoms, for experience shows that it has a favorable effect on vomiting. For adults a single dose of $0.2-0.3 \mathrm{G}$. (3-5 gr.) should be used, followed 3 hours later by a tablespoonful of castor oil if no stool has resulted, or 3 doses of $0.1-0.2$ G. (1 $1 / 2-3 \mathrm{gr}$.) may be given from half an hour to an hour apart. [Repeated doses of 0.006-0.015 G. $1 / 10^{-1 / 4}$ gr.) every 15 minutes up to $0.12-0.20 \mathrm{G}$. (2-3 gr.) will often relieve the vomiting and secure better results, especially if followed 6-10 hours later by some simple saline.-Ed.]

Castor oil and mild saline cathartics, sometimes combined with vegetable laxatives, may be used if constipation complicates the condition. The following prescription may be useful:

R Sodii bicarbonatis,

Sodii chloratis ...............āā 20.00...3v

Pulv. rhei ....................10.00.... 3iiss

M. fiat pulv.

Sig.-A teaspoonful, followed by a glass of water.

In private practice Fleiner employs a laxative lemonade prepared from magnesium oxide, citric acid, and sugared water with a few drops 
of lemon oil [our effervescing citrate of magnesia-Ed.]. The simple saline mineral waters are, however, quite good enough. Before using any cathartic, however, it is best to stimulate the intestine by irrigating the colon with water to which, if necessary, soap, glycerine, honey, or vinegar has been added. When a satisfactory movement has been obtained in any of these ways, no further attempt should be made for 3 or 4 days.

Applications-Narcotics.-Occasionally we have to treat special symptoms. The patient complains frequently of pain or pressure in the region of the stomach. This is best relieved by hot applications, or by Priessnitz's bandage upon the abdomen, or sometimes by the Winternitz process. If the necessity arises, we may employ a subcutaneous injection of morphine or codeine, or sedative suppositories containing the same drugs with or without the addition of belladonna; e.g.:

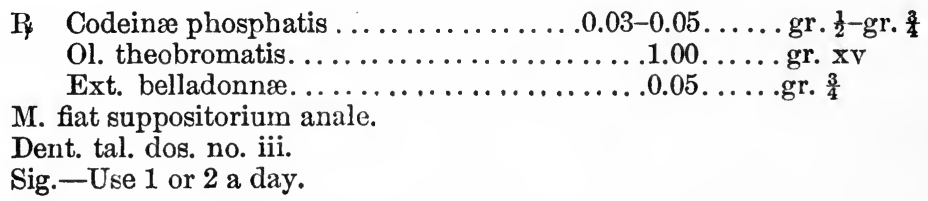

If the patient is distressed by severe vomiting after the stomach contents have been removed, he may be given lumps of ice, iced champagne, or champagne and mineral water frozen to a frappé. If these measures fail, it is sometimes necessary to inject morphine subcutaneously, or to gives doses of chloroform water or of cocaine; e.g.:
P Cocainæ hydrochloratis $\ldots \ldots \ldots \ldots \ldots \ldots . . . .0 .10 \ldots$ gr. $1 \frac{1}{2}$
Syr. aurantii ..................... $100.00 \ldots \ldots$.

M. fiat sol.

Sig. -1 teaspoonful each hour until the vomiting is relieved. $\left(3 \mathrm{i}=0.004 \mathrm{G}\right.$. (gr. $\left.\frac{1}{16}\right)$ cocaine.)

The author considers the morphine injection preferable. It should be remembered that there are a good many cases in which morphine increases vomiting, and also that hot drinks sometimes stop obstinate vomiting beter than cold ones.

Chloral hydrate also has a very good effect in many cases of obstinate vomiting, not only in acute gastritis but in various other diseases of the stomach or liver. The prescription is as follows:
R Chlorali hydrati $\ldots \ldots \ldots \ldots \ldots \ldots \ldots \ldots . . .1 .00 \ldots \ldots$ gr. $\mathbf{x v}$
Aq. dest......................... $5.00 \ldots$ iss
M. fiat sol.
Sig. $-10-20$ drops on shaved ice, every 2 hours.

Alkalies.-In case of severe heartburn we use alkalies, to be more fully discussed later (see p. 463). 
Styptics.-A styptic is to be used for diarrhœa only when the latter persists after we are sure that anything that could cause the disturbance has already passed out of the intestine. One cannot be sure of this until after the first two days of an attack. Opium is then generally the choice, preferably in suppositories.

\section{THERAPY OF CHRONIC GASTRITIS.}

Secondary Gastritis.-When chronic gastritis is secondary to some other disease, the therapy is of course directed against the primary lesion, be it in the lungs, heart, or liver. If the gastritis is secondary to tuberculosis of the lungs, the most important remedy, besides those suited to primary chronic gastritis, is the alkaline chloride mineral waters, which produce a good effect on both lungs and stomach.

Therapeutic Indications in Primary Chronic Gastritis.-The therapy of primary chronic gastritis must treat several conditions: (1) The lessened secretion that results from catarrh, or in rare cases the increased secretion of gastritis acida or of ordinary gastritis at the onset; (2) the abnormal formation of mucus in the stomach; (3) the weakness of the stomach muscle caused by chronic inflammation; and (4) the abnormal processes of decomposition and putrefaction resulting from the other conditions.

Lavage.-An abnormal secretion of mucus and abnormal decomposition oblige us: (1) To get rid of the mucus, which keeps the food from coming in contact with the secretory epithelial glands and, (2), to remove the decomposing masses. The one efficient method of accomplishing both these ends is lavage. When there is only a relatively small secretion of mucus, water at $90^{\circ}-95^{\circ}$ may be used, or, if stimulation of the motor activity of the stomach is also desired, the temperature may be reduced to $86^{\circ}$. But if there is a good deal of mucus, it should be dissolved by employing an alkaline solution; e.g., artificial Ems salts (a teaspoonful to 2-3 quarts of water), with the addition of 1-5 teaspoonfuls of sodium bicarbonate or common salt, or 4-5 teaspoonfuls of lime water to a quart; or by selecting one of the alkaline mineral waters, Carlsbad, Ems, or Vichy. The alkaline solutions also neutralize the abnormal amount of organic acids formed in the stomach. The washing should always be continued until the water returns clear. If the mucus adheres closely to the wall of the stomach, more pressure should be used. One more end may be attempted by the process,- the neutralization of the substance that is causing the fermentation. By adding $1 \%$ salicylic acid, $1 \%$ boric acid, or $0.8-1 \%$ hydrochloric acid to the liquid used, we come as near as we can to accomplishing this purpose. The best time of day for washing out the stomach depends on the individual case, and particularly on the extent of atony produced by the inflammation, and on the amount of decomposition.' If these have not gone far, the author waskes out the stomach in the morning when it is empty. In more severe 
cases, it is better to do it about six hours after the principal meal, and then to give only a light supper. Now and then there are cases which need lavage twice a day, at least temporarily. The length of time during which the treatment should be employed depends upon the severity of the case and the progress of the patient. In the milder cases when the disease has not run too long, a few days is enough to bring about relief of the subjective symptoms and better digestion in the stomach, in proportion to the increase in the hydrochloric acid secretion. In other cases, lavage must be kept up for weeks or months.

Mineral Waters.-A course of certain mineral waters, while of some use, is indisputably less effective than lavage. By the former the stomach can be only partially washed out, and the mucus and undigested masses are not removed but only forced on into the intestine. A number of waters may be used. Alkaline and alkaline-chloride waters drive the mucus from the stomach and enter into combination with the organic acids present. Saline waters stimulate the secretion of hydrochloric acid. Alkaline-sulphate waters are suited to cases with a tendency toward constipation. Bitter waters are contraindicated, as they are a powerful stimulant to the gastro-intestinal tract. A suitable temperature for the water is important. With constipation, weak motility, or decreased secretion, the water should be cold; if there is inflammation in stomach or intestine, or a tendency to diarrhœa, warm springs, such as Carlsbad or the Wiesbaden Kochbrunnen, should be selected, or the waters from the cold springs should be warmed. We should also remember that the choice of the springs depends not wholly on the local affection but also on the condition of the patient. Anæmic, nervous, or run-down individuals should never be given the Carlsbad or Marienbad cure, either at home or at the springs themselves. Such gastritis patients may be sent to Tarasp, where they can have the benefit of a high altitude, or may be given iron-chloride or saline waters. One fact deserves particular attention: the mineral water cures are not usually suited to cases with a pronounced degree of motor insufficiency. The author would emphatically denounce the use, or rather misuse, of large quantities of mineral water drunk quickly (3 glasses or even more). Even slight atony or the mere probability of its occurring if the disease lasts a long time, makes it impossible to give large quantities of mineral waters at a time. A glassful should be sipped slowly and about three-quarters of an hour afterward another taken in the same way, with sometimes a third after the same interval. This is in case the usual custom of taking the water on an empty stomach is followed. Sometimes part is taken early and part later in the day, a glass a half an hour or so before a meal. This is the method, for instance, in case of heartburn or a burning in the stomach resulting from an excess of organic acids.

Diet.-Next in importance to lavage - the most efficacious of all therapeutic measures in chronic gastritis-and to the occasional use of mineral waters, comes a suitable diet. This must of course be chosen with refer- 
ence to the lessened secretion of hydrochloric acid, the reduced motility of the stomach muscle, and the abnormal fermentative processes in the stomach. We should naturally give the preference to the carbohydrates, as they are digested chiefly in the mouth and intestine, and do not demand hydrochloric acid in the stomach. But the digestive powers of the mouth and intestine are not always intact, and in any event we are prevented from giving carbohydrates the first place because of the ease with which they are broken up in the stomach and the resulting increase of fermentation. The diet, then, must be a mixed one, composed of proteids and carbohydrates, to which, in most cases, may be added easily digestible fat. Every food, however, must be so prepared that it shall give the stomach as little work as possible.

In severe cases we limit ourselves to milk, rice or farina pudding, farinaceous soups, eggs, light puddings made from rice, farina, or maizena with milk and eggs, toast, zwieback, and, instead of tea or coffee, maltcocoa, oat-cocoa, or in diarrhœa acorn-cocoa. In some cases malt extract may be added to the milk-soups. For meat, we can give meat-preparations, boiled calves' brains and sweetbreads, boiled chicken without skin, raw chopped beefsteak, raw finely chopped lean ham, all kinds of meat jellies, game, filet, and sometimes, as a temporary variety, boiled fish, such as trout, pike, or carp. Any of these should be allowed only once a day, and should be accompanied by a farinaceous food, such as noodles, rice, macaroni, mashed potatoes, and, in some cases, spinach or carrots. With constipation, cooked fruit may be ordered once or twice a day at the end of the meal (e.g., apple-sauce, prunes with the skins removed, or marmalade). If, on the other hand, diarrhœa is complicating the disease, huckleberry sauce is useful. Between meals the patient may take half a glassful of milk, sometimes sweet, sometimes sour. Sour milk is better if there is a tendency toward constipation, but, as it is not always taken easily, Fleiner recommends that a special kind be prepared from boiled milk. "To a pint of boiled milk which has been allowed to cool, is added 2 tablespoonfuls of sour cream. The mixture is thoroughly stirred and set away in a clean warm place. In winter it will be thick in two days, in summer in one. Every day a new portion is prepared by taking 2 tablespoonfuls of that already soured and repeating the process." Other drinks should be given only in small quantities frequently repeated. Water and simple carbonated waters may be used, sometimes with red wine added; stronger alcoholic drinks are forbidden.

Medication.-There is but little use for any medication except a purely symptomatic one. The principal drug used is dilute hydrochloric acid. With hypochlorhydria 8 or 10 drops of this in a wineglassful of water should be taken through a glass tube after every meal which includes meat. When atrophy of the intestinal glands is a sequel of chronic gastritis, pancreon or gasterine is indicated (pp. 422-423). If the stomach is abnormally sensitive, so that increased peristalsis is present and the stomach contents are passed so quickly into the intestines that 
eating is followed almost immediately by diarrhœa or by a normal stool, the following prescription of Fleiner's is particularly good:

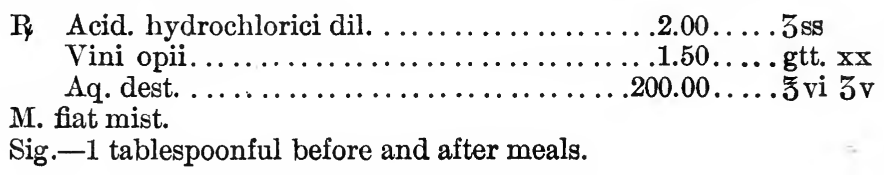

In case of anorexia we give some stomachic,-never orexin, however. Condiments should also be avoided on account of their irritating effect upon the mucous membrane.

For heartburn, alkalies are indicated; e.g., carbonate of magnesia, sodium bicarbonate, or, particularly if there is much gas, burnt magnesia (magnesium oxide).

To relieve pain we can use ordinary hot compresses or Priessnitz's application, giving internally chloroform water or cocaine, or, if necessity requires, morphine or opium, the latter better by the rectum.

In case of an accompanying constipation, the diet should be carefully selected, and some mineral water given, to which may sometimes be added half a teaspoonful to a tablespoonful of Carlsbad salt. The more salt, the greater must be the amount of ordinary or mineral water, in order that the solution may not be too concentrated. We may also treat constipation by suppositories or colon irrigations.

In case of diarrhœa, it is important to determine whether it is a consequence (1) of too long a time having passed without a movement, or (2) of hypermotility of the stomach, or (3) of intestinal catarrh. (See p. 468).

Should hyperacidity accompany the gastritis our therapy must be essentially altered, an exceedingly easily digested diet and alkalies being then indicated.

Baths-Massage.-Finally it should be noted that simple hydropathic measures are useful; e.g., cold sponge baths, douches on the abdomen, and short, cool sitz baths. They are particularly beneficial when the intestine is sluggish, in which case gymnasties and massage may also be employed.

Surgery.-Durgery has been as yet of no especial use in the therapy of chronic gastritis. A gastro-enterostomy would be justified in case of atrophy of the gastric glands and cirrhosis of the stomach resulting in a relative pyloric stenosis, where the imperfectly digested stomach contents are left in such large pieces that they can only imperfectly and partially pass the pylorus.

[For therapeutic convenience Robin divides chronic gastritis into three stages:

1. The period of hyperfunction with $\mathrm{HCl}$ in a normal or increased proportion. This stage demands general hygiene, a non-irritating diet, and gastric sedatives. He orders a rigid milk diet; begins with and 
continues for as long a time as possible, 250 c.c. ( $1 / 2$ pint) of warm milk every 3 hours from 7 A.M. to 10 P.M., to be sipped very slowly, and gradually increases up to patient's tolerance or to $3-4$ litres (quarts) per day. He neutralizes the excessively acid juice with powders of the alkaline earths and for indirect antisepsis prescribes fluoride of ammonia in daily doses of $0.20-0.50 \mathrm{G}$. (3-7 gr.) dissolved in water to diminish the lactic acid fermentation, or the double iodide of bismuth and cinchonidine, as follows :

P Bismuthi et cinchonidini iodidi .....0.02-0.10 ....gr. $\frac{1}{3}-\mathrm{gr} .1 \frac{1}{2}$

Calcii fluoridi...................0. $02-0.10 \ldots \ldots$ gr. $\frac{1}{3}-$ gr. $1 \frac{1}{2}$

Cretæ prepar. ............................ $1 \frac{1}{2}$

M. fiat pulv. Dent. tal. dos. no. xii. Dispense in cachets.

Sig. -1 cachet immediately after each meal.

2. The period of first acid and subsequent mucous catarrh, with $\mathrm{HCl}$ decidedly diminished or absent, and with more or less excessive amounts of mucus. During the early stages of this period he selects a sedative, during the mucous catarrh, a stimulating diet. After a preliminary milk diet, he gives thickened soups, tender well-cooked meats and fowl (minced sometimes), meat powders, soft lean fish, gelatinous foods, soft eggs, the Italian pastes, vegetable purées, and cooked fruits. During the later (mucous) stage, he adds an animal diet with abundant salt, prescribing such stimulating drugs as quassia, strychnine, and condurango, and such aperitives as the phosphate and sulphate of soda. Gasterine in his hands has proved of use in several cases. He rarely employs gastric lavage during this period and almost never in the hypersthenic period. $\mathrm{He}$ practically limits lavage to cases in which the stomach is not empty 6 or 7 hours after eating.

3. The period of atrophic catarrh without any $\mathrm{HCl}$ and with or without the lab-ferment. He begins again, if possible, with a milk diet and later adds foods which contain the maximum amount of nutriment and which require the minimum amount of work for digestion. He uses the various acids, digestive ferments, and Frémont's gasterine up to 500 c.c. (1 pt.) in 24 hours.-Ed.]

\section{THERAPY OF ULCER OF THE STOMACH.}

An ulcer in any organ of the body should always be spared every possible irritation. This principle, in the case of ulceration of the stomach, determines us to forbid the patient for some time any food by the mouth. Such a fast also favors the contraction of the stomach, thus bringing the edges of the ulcer as near together as possible and promoting healing.

Fasting.-A fast of 4 or 5 days or more at the beginning (unless the patient is very much run down) is therefore urgently indicated, especially if the ulcer has induced a hemorrhage or is believed to have penetrated deeply toward the serosa. Thirst should be quenched by rinsing the mouth with cold or carbonated water (p. 432), or by colon irri- 
gations. To relieve the sense of dryness pieces of ice may be held in the mouth, but the water should not be swallowed. Nourishment should be given by nutrient enemas, to which, in case of symptoms of collapse, half a tumbler or less of white wine may be added. [It is usually advisable to delay rectal alimentation until 24-36 hours after a hemorrhage. See p. 395 for Umber's explanation of the gastric pain produced by the introduction of enemas.-Ed.] Four or five days after a hemorrhage, food may be given again in the form least irritating to the stomach. It should at first consist wholly of milk. In many cases of recent ulcer, in fact in nearly all of the large number the author has treated, it has been possible to give milk from the beginning without insisting on a preliminary fast. This has not aggravated the local lesion nor delayed the healing process, and has been much better for the general condition of the patient. But in other cases, particularly when a chronic ulcer will not heal in spite of all other measures, fasting sometimes even for a week accomplishes the desired result. Very run-down or nervous individuals rarely endure fasting well. On account of the lack of saliva while the patient is not eating, eare must be taken to keep the mouth clean.

DIET.-Whether milk is allowed immediately or only after a preliminary fast, it should be given at first in small quantities, slowly increased. We begin with a wineglassful of milk, preferably boiled, or buttermilk, at the temperature of the room, or, if hemorrhage has. preceded, at a lower temperature. This is given every 2 hours; then, if it is well taken, every hour. If vomiting ensues, we discontinue it for several hours, and then begin again with a smaller amount, -50 c.c. ( 1 1 $/ 2$ oz.) every 2 hours, increased gradually to 200 c.c. $(6 \mathrm{oz}$.). Later we give more, until the patient is getting from $3-4 \mathrm{~L}$. (3-4 qt.) every 24 hours. To make the milk more nourishing, we may add sanatogen, hygiama, milk-somatose, eucasin, or a teaspoonful of condensed milk [or cream-Ed.].

Some patients cannot take milk. Similar amounts of tea diluted with considerable milk, or of kefir, which agrees with many patients in spite of its carbon dioxide, should be tried. If this is not satisfactory, we may allow tapioca, rice, wheat, or cornmeal gruel made with milk, or even meat jelly. The latter is prepared as follows: Boil a fowl or some beef and a calf's foot for several hours. Add salt freely. Then clear the broth by boiling it up once or twice with the addition of a well-beaten egg and its shell. Strain and cool. A clear, gelatinous mass should be the result. A teaspoonful of this may be taken every few hours. Some patients may take raw or soft boiled eggs even at this early stage.

Even if the patient has begun with milk only, the author always gives meat jelly and eggnog by the end of the second week. In the third week we may add some milk gruel made with fine meals, such as arrowroot, wheat, cornmeal, hygiama, or oat-cocoa [strained-Ed.]. Oat gruel made with water may also be allowed, or meat broth with rice, barley, or [strained-Ed.] oatmeal. No solid meat preparations should be added, however, for they always irritate the stomach somewhat. 200- 
300 G. (7-10 oz.) of these broths may be given once or twice daily, alternating with pure milk or milk gruels. Some patients take zwieback softened in milk very readily. In the fourth week we may begin to introduce meat itself, at first chicken, brains, or sweetbreads in soup, then small quantities of boiled white meat, game, fowl, brains, young veal, fish (not fat), or raw scraped beef or ham. With this may be served mashed potatoes, boiled rice, noodles, macaroni, sago or barley cooked in soup, and finally a light soufflé. In the fifth week come roast beef, game, and beefsteak with tender vegetables, such as green peas, spinach and carrot purée, cauliflower, and asparagus tips. The patient continues to take milk in the middle of the forenoon and afternoon. For all this matter of diet, the reader is referred to the table of Leube's ulcer eure, already given on p. $384 .^{1}$

${ }^{1}$ [Directions for making Koumiss, copied from Dr. S. W. Dana.

Take five heaping teaspoonfuls of granulated sugar and one cake of Fleischmann's yeast and diffuse them evenly through five quarts of selected milk at $70^{\circ} \mathrm{F}$. After one-half hour, stir thoroughly and bottle. Leave the bottles in a temperature of $70^{\circ}$ for three hours, shaking them twice, then transfer to a temperature of $64^{\circ}$ or between $60^{\circ}$ and $64^{\circ}$, and keep them there for a week. They should then be put on ice.

If the temperature gets a little above $64^{\circ}$, or a little below $60^{\circ}$, it does not appear to matter; but if kept constantly at $68^{\circ}$ curds form.

Commercial Weissbier bottles are convenient on account of their fastenings, strength and size-holding between ten and eleven ounces. They can be obtained from Rudde and Westermann, 50 Vesey Street. Five quarts require 15 bottles.

In opening the bottles some care is required. If the bottle is reversed and held with its neck inside a pint goblet and the lever slowly raised the koumiss will come out in fine streams and none will be lost. Contents of bottle (10 ounces) will expand to thirty ounces and fill two pint goblets.

The bottles should be shaken twice a day for a week, and once a day afterwards until used.

Directions for making Matzoon or Zoolak, (German Hospital, New York City).

Take forty-five pints of milk, boil thoroughly, cream two or three times; that is, until all the cream is removed. When the milk is still quite warm, add two bottles of prepared bottled Zoolak. Mix thoroughly. Bottle quickly in pint bottles, not entirely full. Cork tightly immediately, and put in a warm place until the liquid shows creamy through the bottles. Then place and keep in a cold place.

N. B. If chilled before it is thick, it remains thin and the flavor is spoiled. If not kept very cold after it is made, the fermentation is carried too far.

Directions for making Gruels.

If gruels are made from well-cooked cereals, such as boiled rice, farina, hominy, or oatmeal, they are less apt to produce tympanites; and in my experience, they are usually easily digested. I have found these two recipes useful:

Oatmeal gruel (Miss Corbett, Dietitian, Dept. of Charities, New York City).

$$
\begin{aligned}
& 1 / 3 \text { cup coarse oatmeal } \\
& 11 / 2 \text { cups cold water } \\
& 1 / 2 \text { teaspoonful salt } \\
& \text { Milk or cream }
\end{aligned}
$$

Pound oatmeal in mortar or roll on a molding board. Add to it in bowl $1 / 3$ of the cold water, let settle, stir occasionally, pour off mealy water. Boil mealy water thirty minutes, then add salt, milk or cream.

Zwieback or Dextrinized Cracker Gruel, (Miss Corbett).

11/4 tablespoonfuls browned cracker or zwieback rolled and sifted

$3 / 4$ cup milk

$1 / 8$ teaspoonful salt

Scald milk. Add cracker. Cook over hot water 5 minutes, then add salt.-Ed.] 
Lenhartz's Treatment.-Lenhartz has recently advocated increasing the diet much more rapidly than in the usual treatment. The author has found that this change, though sometimes successful, is as often the cause of pain and a feeling of pressure, and would therefore advocate trying it only in those cases where the patient is in an exceedingly rundown condition to begin with. [Lenhartz originally tried this more liberal diet with chlorotic girls who had gastric ulcer but had had no hemorrhage. They did so well that he essayed the same plan with more severe cases. He began with 200 c.c. (6 oz.) of milk and one to three eggs within 24 hours of the hemorrhage and added 100 c.c. $(3 \mathrm{oz}$.) of milk and one egg daily until the patient was taking 8 eggs and 1000 c.c. ( 1 qt.) of milk in a day. Sugar was given early and increased from $20 \mathrm{G}$. ( $5 \mathrm{dr}$.) at the start to $50 \mathrm{G}$. $(1 \mathrm{I} / 2 \mathrm{oz}$.) at the end of one week. Meat was then allowed, 35-70. G. (11/6-2 $1 / 3 \mathrm{oz}$.), and at the end of two weeks, rice, zwieback, raw ham, and butter. His detailed schedule follows:

\begin{tabular}{|c|c|c|c|c|c|c|c|c|c|c|c|c|c|c|}
\hline & \multicolumn{14}{|c|}{ Days after last hæmorrhage } \\
\hline & 1 & 2 & 3 & 4 & 5 & 6 & 7 & 8 & $\begin{array}{llllllll}9 & & & & & \end{array}$ & 10 & 11 & 12 & 13 & 14 \\
\hline Eggs, no ... & 2 & 3 & 4 & 5 & 6 & 7 & 8 & 8 & 8 & 8 & 8 & 8 & 8 & 8 \\
\hline Sugar, G. .............. & $\ldots$. & $\ldots$. & 20 & 20 & 30 & 30 & 40 & 40 & 50 & 50 & 50 & 50 & 50 & 50 \\
\hline Milk, c. c.............. & 200 & 300 & 400 & 500 & 600 & 700 & 800 & 900 & 1000 & 1000 & 1000 & 1000 & 1000 & 1000 \\
\hline Raw meat, G........... & $\ldots$ & $\ldots$. & $\ldots$ & $\ldots$. & $\ldots$. & 35 & 70 & 70 & 70 & 70 & 70 & 70 & 70 & 70 \\
\hline Milk rice, G. ....... & $\ldots$. & $\ldots$ & $\ldots$ & $\ldots$ & $\ldots$. & $\ldots$ & 100 & 100 & 200 & 200 & 300 & 300 & 300 & 300 \\
\hline Zwieback, G........... & $\ldots$. & $\ldots$. & $\ldots$. & $\ldots$. & $\ldots$. & $\ldots$. & $\ldots$. & 20 & 40 & 40 & 60 & 60 & 80 & 100 \\
\hline Raw ham, G..... & $\ldots$ & $\ldots$. & $\ldots$ & $\ldots$ & $\ldots$ & $\ldots$ & $\ldots$. & $\ldots$ & $\ldots$. & $\ldots$ & 50 & 50 & 50 & 50 \\
\hline Butter, G....... & $\ldots$. & $\ldots$. & $\ldots$. & $\ldots$. & $\ldots$. & $\ldots$ & $\ldots$. & $\ldots$ & $\ldots$ & $\ldots$ & 20 & 40 & 40 & 40 \\
\hline Calories ... & 280 & 420 & 637 & 777 & 955 & 1135 & 1588 & 1721 & 2138 & 2478 & 2941 & 2941 & 3007 & 3073 \\
\hline
\end{tabular}

S. W. Lambert reports uniformly good results with Lenhartz's treatment at the New York Hospital during the past year. Wirsing reports 42 cases of gastric ulcer successfully treated by Lenhartz's method. The severe cases did the best. Minkowski reports satisfactory results in 30 patients with a modification of Lenhartz's plan. He begins 2-3 days after the hemorrhage. He advocates cream and butter and specifies the avoidance of salt. Senator prescribes in fresh cases of hemorrhage a solution of pure white gelatin, $15-20$ G. $(1 / 2-2 / 3$ oz. $)$ to $150-200$ c.c. (5-6 oz.) of water flavored with lemon juice, a dessertspoonful every 1 or 2 hours, or even every quarter of an hour, and butter up to $30 \mathrm{G}$. ( $1 \mathrm{oz}$.$) and cream to at least 250$ c.c. $(8 \mathrm{oz}$.) per day. The butter may be given frozen in pill form, the cream iced and beaten up with sugar. $\mathrm{He}$ begins milk earlier and increases the amount and adds shaved meat. $\mathrm{He}$ has not used rectal feeding in late years.-Ed.]

REST IN BED.-The stomach is saved work not only by choosing a suitable diet, but by resting the entire body. It is therefore advisable that the patient should remain in bed and absolutely necessary that he 
should do so whenever the ulcer has eaused hemorrhage or peritoneal inflammation. The author keeps his patients in bed for a fortnight, cautioning them to avoid any quick movements; the third or fourth week they get up for a little while and later take short walks. After every principal meal they lie down for at least an hour. This precaution should be kept up for some months after healing has taken place, as the stomach empties itself more rapidly in that position. [I do not agree with Forchheimer in allowing patients with a mild gastric ulcer to be up and about, but believe that the diagnosis of an ulcer of the stomach carries with it the necessity of a rest and dietetic cure, the duration and stringency of which will depend upon the severity of the symptoms. I do not, however, enforce the same rigid rules for resting and isolation in gastric ulcer as in neurasthenia, but usually permit alcohol sponges, light massage of the extremities (avoiding the back and abdomen), reading, dictating or even writing letters.-Ed.] To keep the stomach well contracted and so give the uleer the most favorable conditions for healing, an ice-bag should be applied to the epigastrium several times daily for an hour or two at a time, and especially whenever any signs of meteorism manifest themselves.

Medication : Alkalies.-An important condition that militates against the healing process is the hyperacidity present in almost all cases. As milk alone is not sufficiently alkaline to counterbalance this, the administration of some alkali is indicated. The author almost always gives lime water, a dessertspoonful to each wineglassful of milk up to 200-250 G. (6-8 oz.) daily, or the same quantity of an alkaline mineral water; e.g., Carlsbad or Vichy. ${ }^{2}$ More mineral water (100-150 c.c. (3-5 oz.) after the principal meal) should be taken as soon as the patient begins a semisolid diet, particularly after he begins to eat meat. The same amount of the mineral water (tepid) or a corresponding dose of artificial Carlsbad salt in tepid water should also be taken on an empty stomach a half hour before breakfast, as soon as the patient has begun to substitute gruel for pure milk at that meal. This will serve both to cleanse the stomach and to neutralize any acid that may be present.

Belladonna.-Belladonna is an excellent drug to diminish hyperacidity. Its use will be discussed later at greater length (see p. 446). 0.03 G. ( $1 / 2 \mathrm{gr}$.) of the extract may be given three times a day, usually with alkalies. Atropine is also used, in subcutaneous doses of $0.001 \mathrm{G}$. ( $1 / \mathrm{g}_{\mathbf{6}}$ gr.) ; and eumydrin internally, 0.001-0.002 G. $(1 / 64-1 / 32 \mathrm{gr}$.$) three times$ a day.

Bismuth.-To accomplish the healing of a recent ulcer, the use of large amounts of bismuth is, in a great many eases, exceedingly advantageous. - The author generally gives it by mouth, only very seldom introducing it, as Fleiner advises, by means of a stomach tube. The dose is

"[Vichy means the French Celestin Vichy. The ordinary siphon vichy sold throughout our cities is rarely a good diluent for milk. It is usually too highly charged with carbon dioxide, and not uniform in its saline constituents.-Ed.] 
8-12 G. (2-3 dr.) of the subnitrate of bismuth in 100-500 G. (3-16 oz.) of water to be taken on an empty stomach. If the ulcer can be localized with any degree of probability, the patient should assume a position such that the drug suspension shall be in contact with the ulcer. For instance, if it is at the pyloric end, the patient should lie on his right side; if at the lesser curvature, on his back with the hips raised. Less frequently the ulcer may be so situated that he should lie on his left side, on his stomach, sit up, or take the knee-chest posture. He should remain in the appropriate position for at least a quarter of an hour, if possible an hour. If the ulcer cannot be localized, he should lie first on one side, then on the other side, and then on the stomach, making a complete turn every quarter of an hour for an hour. If bismuth is to be given through the stomach-tube, the stomach should first be washed out and then a suspension of subnitrate of bismuth in water be injected. The tube should be clamped outside the mouth, allowed to remain from 5-10 minutes, and then withdrawn. ${ }^{3}$ This procedure is, however, contraindicated (1) in an ulcer that bleeds easily, (2) in the early stages of any ulcer, especially one that began with hæmatemesis, (3) in severe pain in the stomach, and (4) in an ulcer at the cardia. The bismuth acts mechanically. It forms a coating over the ulceration, protecting it from the gastric juice and from food, lessening the danger of hemorrhage, mitigating the pain greatly, and hastening the healing process. Bismuth should be begun as soon as the diagnosis is determined, even if hæmatemesis is present, for the author has repeatedly found that it also curtails the hemorrhage. At first it should be given daily, but after the inflammation has subsided, only every other day. The author has continued the treatment for several weeks (3-6), and only in one instance has he seen severe stomatitis ensue, and then in a case of coincident duodenal ulcer. [During the administration of bismuth especial care of the mouth and teeth should be enjoined.-Ed.] The bismuth therapy should not be limited to recent ulcers but is particularly suited to old ones that have healed only around the edges.

Silver Nitrate.-Another remedy for the ulcerative process as such is silver nitrate. It is suited to cases of obstinate long-standing ulcer accompanied by hyperacidity. Its action is due, as Gerhardt has shown, to its union with hydrochloric acid, forming silver chloride. According to Boas, one should begin with a solution of $0.25 \mathrm{G}$. (4 gr.) silver nitrate to $120 \mathrm{G}$. (4 oz.) of distilled water (about 0.2 per cent.), giving a tablespoon-

${ }^{8}$ [1. Fleiner's method: Every morning, fasting, gastric lavage is carried out until the return flow is clear and free from acid reaction. An even suspension of subnitrate of bismuth $\left(10-20 \mathrm{G}\right.$. $\left(2 \frac{1}{2}-5 \mathrm{dr}\right.$.) in $200 \mathrm{G}$. $(6 \mathrm{oz}$.$\left.) of water \right)$ is then introduced through the tube, after 2 or 3 minutes most of the supernatant water siphoned off, and the tube then withdrawn. The patient then assumes the position requisite to keep the bismuth in contact with the supposed site of the ulcer. This posture is retained for half an hour, when breakfast is given. He begins this cure as soon as gastric feeding is commenced and continues it for 2 weeks. Ewald has employed calcium carbonate and Fariser a mixture of chalk, tolane, and magnesia instead of the bismuth.-Ed.] 
ful three times a day; then increase to one or two bottles of a $0.3: 120 \mathrm{G}$. ( $4 \mathrm{1} / 2 \mathrm{gr}$. : $4 \mathrm{oz}$.) solution ( 0.25 per cent.) taken in the same way, to be followed if necessary by one or two of a $0.4: 120 \mathrm{G}$. (6 gr.: $4 \mathrm{oz}$.) solution. The bad taste is best disguised by a few drops of peppermint. A simpler method is to give from the beginning 3 tablespoonfuls daily of a $1: 1000$ (0.1 per cent.) solution. The diarrhœa which is at first often caused by this treatment is never severe and almost always disappears of its own accord. [Robin discourages this use of nitrate of silver because with it he has never obtained any especially striking results.-Ed.]

Orthoform and Anæsthesin.-Orthoform (m-amido-p-methylester of oxybenzoic acid), which the author has repeatedly tried, sometimes in that form, sometimes in that of orthoform-hydrochlorate, has proved inferior to bismuth. The dose is $4-5 \mathrm{G}$. (1-1//4 dr.) in $100 \mathrm{G}$. (3 oz.) of water as a suspension like the bismuth, [or 5 to 7 grains 6 to 8 times a day-Ed.]. Anæsthesin (the para-amido-ethylester of benzoic acid) is similar to orthoform and is at least as good a local analgesic. It is a tasteless powder, barely soluble in water, and therefore best given in powder form, $0.2-0.5$ G. (3-7 gr.) 3-6 times a day before nourishment. [These two drugs are useful only to control pain. Neither of them acts mechanically as bismuth does.-Ed.]

Oil Treatment.-In many cases of old gastric ulcer and frequent dilatation, spasm of the pylorus muscle is the chief cause of the retention of food in the stomach. In such cases $50 \mathrm{G}$. (11/2 oz.) of olive oil, 3 times a day an hour before each meal, or $150 \mathrm{G}$. (5 oz.) in the morning on an empty stomach, is sometimes recommended. This treatment is often helpful where the ulcer is also accompanied by hyperacidity.

Symptomatic Therapy : Pain.-Any other medical therapy in gastrie ulcer is purely symptomatic. Often we are obliged to relieve severe and constant pain. This may be caused (1) by the erosion of a nerve in the region of the ulcer, (2) by simple hyperæsthesia of the gastric mucous membrane, (3) by the hyperacidity accompanying the ulcer, or (4)-in old and cicatrized ulcers-by perigastric adhesions.

Fibrolysin.-In the last case surgical interference is necessary. The treatment by means of subcutaneous or intramuscular injections of thiosinamin or of fibrolysin-a chemical compound of the former with sodium salicylate-the author has so far found worthless. However, he is in favor of giving it a further trial, and would prefer fibrolysin to thiosinamin, since the latter causes severe pain and inflammation at the site of injection. Fibrolysin comes in sterilized glass tubes containing 2.3 c.c. (corresponding to $0.2 \mathrm{G}$. ( $3 \mathrm{gr}$.) of thiosinamin). One of these should be injected each time. Thiosinamin is injected 2 or 3 times a week ( 1.5 c.c. ( $23 \mathrm{~min}$.) of the 15 per cent. alcoholic or 20 per cent. glycerin and water solution; ;ie., $0.15-0.20 \mathrm{G}$. (2-3 gr.) of thiosinamin). [I have had no experience with either of the drugs.-Ed.]

Belladonna-Atropine-Eumydrin.-In most cases the pain is caused by hyperacidity. If this cannot be controlled by a suitable diet and the 
administration of alkalies, then belladonna, atropine hypodermically, 0.001 G. $(1 / 64$ gr.) not more than 3 times a day, eumydrin, or silver nitrate may be given to decrease the secretion. Belladonna may also be combined with an alkali; e.g.:

R Magnesii oxidi...................... $0.50 \ldots \ldots$ gr. viiss

Ext. belladonnæ................... $0.03 \ldots \ldots$ gr. $\frac{1}{2}$

M. fiat pulv.

Dent. tal. dos. no. xv.

Sig. -1 powder 3 times a day after eating.

Or bismuth may also be added:

R Ext. belladonnæ.................. $0.50 \ldots \ldots$ gr. viiss

Bismuthi subnitratis,

Magnesii oxidi,

Sodii bicarbonatis .............āā 10.00.... 3 iiss

M. fiat pulv.

Sig. - A good pinch 3 times a day after eating.

[Binet believes that sodium bicarbonate, although it neutralizes but little acid, relieves the pain which develops a considerable time after eating, more efficiently than magnesium and calcium, which neutralize more acid. He considers that such delayed pain is due to pyloric spasm and that bicarbonate of soda stimulates the motor function and so tends to empty the stomach.-Ed.] We may also try chloroform water, 150200 G. (5-7 oz.) daily, 1 tablespoonful every 2 hours, alone or with subnitrate of bismuth $3 \mathrm{G}$. ( $45 \mathrm{gr}$.).

In such conditions opium and morphine are contraindicated because they increase the acidity, but where the pain is due to simple hyperæsthesia of the mucous membrane they may be resorted to.

Finally when the erosion of a nerve is the cause of the pain, we may use bismuth, orthoform, or anæsthesin.

Cold and Heat.-External applications of ice or Leiter's cooling apparatus are often useful; sometimes, however, hot applications are more efficient. The choice between them is to be determined by the individual tolerance in each case. The author has also found hot applications successful in cases of gastric ulcer which have.already begun to heal, that is, about two weeks after the onset. For this purpose he prefers hot flaxseed poultices frequently changed. The steady heat seems to improve the circulation in the territory around the ulcer, and facilitates the healing process. Leube uses these poultices from the start in all cases of gastric ulcer where there has been no hæmatemesis. They are changed every 10-15 minutes during the day and replaced by Priessnitz's applications at night. After an average of ten days they are replaced entirely by the latter. Recently the author has followed Leube and has employed in every gastric ulcer applications hot enough to make little blisters on the abdomen, which has previously been greased 
with vaseline. In case of hemorrhage, this treatment is not begun for at least a fortnight afterward. The results have been good,-rapid disappearance of the pain and generally prompt healing. [It was my good fortune some two years ago to see Prof. Leube's method in application at Marzburg. A linen cloth smeared with a preparation of wax is laid upon the patient's epigastrium and upon this cloth a very thin hot flaxseed poultice. An extra poultice is constantly in readiness and kept very hot over a water bath at the head of the bed. During the day the poultices are frequently alternated and the skin is always kept very hot. If blisters develop, they are treated aseptically and no bad results ensue.-Ed.]

Hæmatemesis.-Hæmatemesis necessitates the withholding of all food, even lumps of ice being given only when absolutely necessary; the patient must keep absolutely still, and an ice-bag [or coil-Ed.] be placed upon the epigastrium. All our familiar styptics come into play; e.g., extract of ergot or ergotin or ergotole subcutaneously, a $0.05 \mathrm{G}$. ( $3 / 4 \mathrm{gr}$.) powder of lead acetate every 2 hours; or $5-15$ drops of tincture of chloride of iron. In some cases this last drug has seemed to do the most good. In severe vomiting it may be necessary to give morphine or opium subcutaneously or in suppositories. The author has repeatedly used a 5-10 per cent. solution of gelatine (p. 82) and remembers especially one case of exceedingly severe hemorrhage from gastric ulcer in which the bleeding was completely stopped after twice using about $200 \mathrm{G}$. (6 oz.) of a 5 per cent. solution. He now employs exclusively the sterilized 10 per cent. Merck gelatine (40 c.c. (10 dr.) hypodermically). As long as blood appears either in the stools or in the vomitus [macroscopically-Ed.], nourishment should be given only by the rectum. In severe and long continued hemorrhage, no food should be given until several days after the bleeding has stopped, when cold milk may be begun very cautiously. Of course in abundant hemorrhage one may be obliged to use autotransfusion or direct saline infusion. [I have never been able to convince myself that any of these styptics are useful; sometimes they do positive harm by causing vomiting. Ewald sprays the throat with a weak cocaine solution and then washes the stomach with ice water with apparent advantage in eases of severe hemorrhage. He first inserts the tube but a short distance into the stomach. After the ice water has been poured in, the tube can be pushed farther in without harm. He strongly recommends this procedure before resorting to operative interference. Hemorrhage if slight requires no special treatment beyond the usual routine; if more marked, the extension of the initial period of starvation to 2 or 3 days; if severe, absolute quiet in bed aided by morphine or opium, and a light ice-bag or ice-coil to the epigastrium; and if repeated, gastric lavage with ice water, introduction of a solution of adrenalin chloride, and hypodermoclysis of normal saline if the pulse is very weak. Operation is indicated according to Leube in repeated small, persistent hemorrhages which are not controlled by medical treat- 
ment; in profuse hemorrhages if repeated, provided the patient's condition is good.-Ed.]

Care of the Bowels.-Another thing to be carefully watched in gastric ulcer is the action of the bowels. The author allows no movement of the bowels for several days after a hæmatemesis. But when the hæmatemesis is over or when it has been absent from the beginning, a gentle movement must be brought about by a simple enema or by the use of Carlsbad salts, magnesium oxide, or powdered magnesia with rhubarb. Straining at stool must be avoided.

After-Treatment.-Although many physicians favor a systematic Carlsbad cure from the start, the author considers it at least superfluous if alkalies are being given. It seems to be indicated rather in those cases in which, after the ulcer is apparently healed (on an average about 6 weeks), the patient continues to suffer from some abnormality of the clinical functions of the stomach, particularly hyperacidity, which carries with it the danger of a new ulcer or the return of the old one. For these cases the use of alkaline salt springs (e.g., Carlsbad) is very beneficial. The water should be taken lukewarm-never hot-generally 1 or 2 glassfuls on an empty stomach in the morning, another about a half hour before dinner, and sometimes a third or a fourth at the time when the digestive process is at its height. According to the author's experience, it is well in cases of hypersecretion to use frequent small doses, about $60 \mathrm{G}$. (2 oz.) of Carlsbad Mühlbrunnen every 2 hours. For a year after the ulcer is healed, the patient should refrain from eating anything particularly irritating, as the tender newly-healed scar needs to be carefully guarded. For these reasons he should avoid the skin or gristle of meat, the crusts of bread or zwieback, the skin, rind, and seeds of fruits, the woody parts of vegetables, all alcoholic drinks, all those containing carbon dioxide, and all the sharper condiments. [Bismuth should be persisted in for several months after all symptoms have disappeared, (F. C. Shattuck). Care in diet should be continued for 2 or 3 years, and such patients should never risk traveling or living where a proper diet is difficult to obtain. Many chronic cases would not relapse if they were under careful medical supervision for several years after the cure. -Ed.]

Recurrent Ulcer.-Occasionally we encounter a recent gastric ulcer which keeps recurring in spite of all therapeutic measures. Sometimes severe pain is felt after even a very little milk; sometimes an insufferable feeling of fulness; sometimes an unexpected hemorrhage. In such cases of recurring ulcer, we give food by the rectum alone for 2-3 weeks, relieving thirst by lumps of ice held in the mouth or by washing out the mouth with mineral water or a soda solution. Unless contraindicated by hæmatemesis, hot poultices are kept on the abdomen.

Partially Healed Ulcer.-In the case of an old ulcer which is only partially healed and which still causes a good deal of distress, the bismuth or silver nitrate treatment is often useful. In such cases, which 
are seldom accompanied by hemorrhage, regular lavage is indicated on account of the hyperacidity of the gastric secretion, unless this can be suppressed by belladonna with alkalies, or by subcutaneous doses of 0.001 G. $\left(1 /{ }_{64} \mathrm{gr}.\right)$ of atropine.

SURGERY.-Certain types of gastric ulcer demand operative interference: (1) those which, in spite of all medical treatment, continue to cause the patient severe pain, preventing him from attending to his business; (2) those which weaken the patient by constantly recurring though slight hemorrhages; (3) those which continue to cause extensive hemorrhage; (4) those which have obstructed the stomach by an extensive, strongly contracted scar, sometimes even producing an hour-glass stomach; (5) those with complications like perigastric abscesses or perigastric adhesions which distort the pylorus; (6) those which threaten perforation. The operation may consist of excision of the ulcer, of gastro-enterostomy, or of removal of the perigastric adhesions. [It is not always easy to correctly differentiate an acute from a chronic ulcer of the stomach, but for therapeutic purposes such a differentiation should be attempted in every case. Unless complicated the former always belong to the physician, while many of the latter which resist careful medical treatment, should come to operation and much earlier than is usually the case. Billings properly emphasizes the necessity of proving the accomplishment of a cure in chronic cases. For acute ulcers a reasonably definite routine treatment may be prescribed in nearly all cases, with, of course, modification in the amount of rest, the character of the diet, the drugs, etc., according as they are severe or mild, accompanied by hemorrhage or not. The author's plan outlined above is a good general guide for such cases, and sufficiently definite and rigid. For chronic ulcers I protest vigorously against adopting any routine. Each case must be studied carefully in regard to anæmia, nutrition, chronicity, situation of the ulcer, previous attempts at treatment, psychical conditions, etc., and a plan of treatment outlined to suit the individual and not the disease. Where the patient's hæmoglobin is low, either from loss of blood or from more general causes, e.g., following dyspepsia, I give iron hypodermically with excellent results as far as the hæmoglobin percentage is concerned. I also believe the improved blood assists healing of the ulcer.-Ed.]

\section{THERAPY OF CANCER OF THE STOMACH.}

Surgery.-The greater the progress made towards an early diagnosis of gastric carcinoma, the oftener can the physician treat the case in the only really efficacious way,-by referring the patient as soon as possible to the surgeon. The only therapy that can have any lasting result is the resection of the diseased part. If this is no longer advisable, gastroenterostomy is often beneficial for a time. The patient recovers quickly and surprisingly well from the operation, and is helped for some months, a half year or even longer. In carcinoma at the cardia gastrostomy 
offers a last resort. According to the author's experience it is often simply impossible to tell with any certainty before laparotomy whether or not a radical operation-resection of the pylorus-can be carried through successfully. The cases of cancer of the pylorus which are best suited to pyloric resection are those in which the tumor is movable on all sides, the strength of the patient has not been much reduced, and metastases of other organs have not manifested themselves. But who can be sure that metastases are not present, e.g., in the great omentum or the liver, without our having any suspicion of them before the abdomen is opened? If they are disclosed by the operation we may perform gastro-enterostomy instead of resection. If a radical operation is out of the question, gastro-enterostomy is indicated when symptoms of pyloric obstruction with stagnation of the stomach contents make it impossible to nourish the patient properly.

Inoperable Cases.-There are always some inoperable cases: (1) where resection is impossible, while gastro-enterostomy does not seem necessary; (2) where it is too late for any operation, i.e., in extreme cachexia or recognizable metastases of other organs; (3) where operation is refused; (4) where, after gastro-enterostomy and the temporary recovery following it, a new deposit has appeared with new gastric symptoms.

Diet.-Especially in the first and last of these groups, the diet plays an important part, unless, of course, it is merely a question of euthanasia.

Most patients with gastric carcinoma suffer from hypo- or anacidity, and frequently exhibit a distaste for meat; hence it is well to reduce the quantity, to select only the most easily digestible forms of meat, and to depend largely upon a vegetable diet. In the choice of particular foods, we must take into account the fact that even without stenosis, stagnation appears early in the disease. Motor insufficiency, then, is the principal factor in the selection of the diet. All indigestible foods or those that induce fermentation must be avoided,-coarse vegetables, hulls, skins, seeds, gristle, tendons, black bread, and cabbage, for instance. However, in every incurable disease, insistence upon any particular diet is often out of place. To a certain degree it is best to indulge the patients' whims, and give them the delicacies they ask for, even caviar, oysters, beer, tea, or fruit.

Of meats the most digestible are the white meats, and of fish the soft and dry, e.g., trout, pike, and carp. Fowl, pigeon, partridge, roast veal, calves' brains, and sweetbreads, sometimes beefsteak, roast beef, and game may be selected. To aid in the digestion of meat, hydrochloric acid should be given, 8-10 drops in a wineglassful of water after each meal. Sometimes it is better to distribute this throughout the day, giving a tablespoonful of 1-2 G. (15-30 min.) of dilute hydrochloric acid in 180 G. (6 oz.) of water every 2 hours. Fleiner recommends a sauce prepared by adding 10 or more drops of dilute acid to a half teaspoonful of Liebig's or Valentine's extract and 3 tablespoonfuls of 
warm water. This is to be poured over finely chopped meat. The taste of the acid is almost concealcd, but the meat treated with the sauce is very quickly broken up. Meat jellies may also be recommended. Meat preparations may be used in soup, but their use should not be continued too long. Somatose can be taken longest and most easily.

A vegetable diet in the broad sense of the term includes milk (often with the addition of sanatogen, hygiama, or eucasin), buttermilk or kefir, rice, barley, tapioea, sago, or oatmeal gruel, milk soup, eggs, and light farinaceous foods, such as noodles, macaroni, and mashed potatoes. Only small quantities of milk should be given if marked stagnation is present, for it will stay in the stomach broken up into large flakes, or be vomited in this form. This diet also includes all available vegetables, such as spinach, carrots, eauliflower, artichokes, beans, peas, etc. Easily digestible fats may be allowed at least tentatively (butter, Mehring's "Kraftschocolade"). Patients with gastric disorders digest them more readily than is generally supposed. Port or Hungarian wine or brandy and water may be permitted unless motor insufficiency or pyloric stenosis is present, for in that case the alcoholic liquid not only remains in the stomach but draws out a considerable amount of water from the mucous membrane. Thirst may under these circumstances be mitigated best by giving water by the rectum,--a pint of warm water morning and evening, sometimes with the addition of a pinch of salt.

If the patient is on a vegetable diet-and many run-down cases increase in weight when put on such a diet-at least five meals a day should be given. If meat is added to any meal, at least 4 hours must elapse before the next.

Mineral Waters.-A quarter to a half hour before each meal 100-150 G. (3-5 oz.) of a mineral water containing alkalies or common salt, but not carbon dioxide, may be taken to stimulate gastric secretion.

Ferments.-In many cases artificial ferments may be taken along with the food; e.g.:

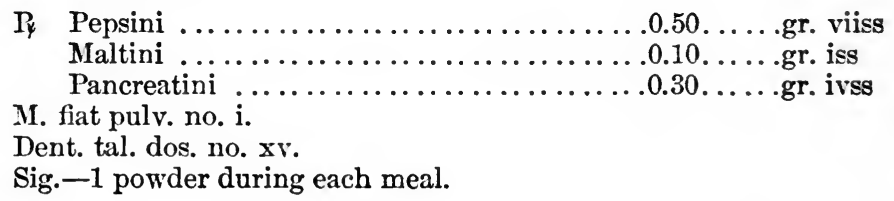

Frequently a loss of appetite prevents the patient's taking enough food. To offset this we may try stomachics or bitters, though it must be confessed that they are often of no use. [Condurango is a favorite with Ewald, Boas, Einhorn, and others. Robin recommends the hydroehlorate of quinine in doses of at least $1 \mathrm{G}$. (gr. xv) daily. He usually administers it hypodermically for one week, then by the mouth and rectum, giving half the dose upon an empty stomach and the second half in a suppository at night for another week and so on. He claims that the drug acts as a palliative.-Ed.] 
Lavage.-The best stomachic is, after all, constant lavage, which also accomplishes the even more important purpose of relieving the stomach of its stagnating and decomposing contents. The best time is in the morning; but if, as often happens, the patient is kept awake at night by a sense of pressure in the stomach, nausea, belching, etc., the stomach should be washed a second time late in the evening. Many keep up lavage almost to the very end, as they obtain more relief from it than from anything else. The liquid used may be pure water or an antiseptic solution, sometimes with the addition of stomachics or bitters. If the procedure makes the patient faint, it may be followed by an enema of water and wine, or, (Fleiner), 50-100 c.c. ( $1 \mathrm{1} / 2-3 \mathrm{oz}$.) of white wine in $200 \mathrm{G}$. (6 oz.) of meat broth. Of course if there is a tendency to hemorrhage, lavage must either be given up, or carried out with great caution. It may be temporarily abandoned in those cases in which pyloric stenosis in consequence of cancer has been relieved by ulcerative decomposition of the neoplasm.

Symptomatic Treatment.-The only other way in which we can help patients with inoperable cancer, is by treating the symptoms.

Hamatemesis-Pain.-Hemorrhages may be handled in the same way as in gastric ulcer, though in carcinoma they are seldom so abundant. Pain, too, may be combated in the same way with the one difference that in cancer morphine holds the first place. Cocaine, codeine, or dionin may also be tried, or the following prescription:

R Bismuthi subnitratis ...............10.00....3iiss

Phenolis........................gtt. xv .....gtt. xv

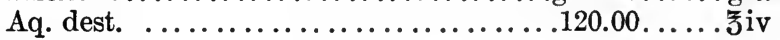

M. fiat mist.

Sig.-A teaspoonful every hour until the pain is relieved.

[Einhorn recommends methylene blue, $0.20 \mathrm{G}$. (gr. iii) in gelatine capsules once or twice daily. He believes this drug relieves pain and sometimes prevents loss of weight and enlargement of the tumor.-Ed.]

Vomiting.-Vomiting, as far as it is not relieved, may be mitigated by narcotics such as cocaine, chloral hydrate, chloroform, or chloroform water.

Constipation.-Constipation is best treated by water enemas containing different laxatives, by oil or glycerine enemas, or by glycerin suppositories. If these prove insufficient, vegetable laxatives may be tried, e.g., compound cathartic pills, or compound laxative pills U. S. P.

Saline cathartics cannot be used, as they doubtless weaken the patient. For the same reason we are cut off from a systematic treatment with alkaline saline mineral waters.

\section{THERAPY OF MECHANICAL INSUFFICIENCY OF THE STOMACH (ATONY AND CHRONIC DILATATION OF THE STOMACH).}

Definition.-Although many authors regard atony of the stomach as sharply differentiated from chronic dilatation, the author is supported by many others in maintaining that, as far as their therapy is concerned, 
the two processes should be treated under one head, the difference between them being one merely of degree. An atonic stomach may be defined as one which takes an abnormally long time to get rid of its contents, but which eventually empties itself completely or nearly so, so that decomposition very rarely occurs, and whose size, when it is not full, does not exceed the size of a normal stomach. After a test supper of two cups of tea, cold meat, and bread, the atonic stomach in the morning is free from any residue of food, or at the most has but a very little left, and that not at all decomposed. If $500-1000$ c.c. $(1 / 2-1$ qt.) of water is introduced, the lower border of the stomach reaches no farther than the umbilicus, any dislocation of the organ, of course, being excluded. After a similar test supper the dilated stomach on the contrary always contains a residue of food in the morning and this generally decomposed, and the organ itself is permanently enlarged, with the greater curvature reaching below the umbilicus. Some cases of dilatation are simply cases of aggravated atony-atonic dilatation; others are due to a mechanical contraction of the pylorus--sometimes benign, sometimes malignant-or to habitual overfeeding, particularly with liquids. Atony of the stomach may be caused by (1) a general muscular weakness arising from a constitutional anomaly, from chronic diseases, or as a sequel of severe infectious diseases; (2) an excessive mental or physical strain; (3) venereal excesses of any kind; (4) insufficient or irrational feeding, e.g., too heavy meals; (5) irrational ways of life, such as lack of exercise; (6) nervous affections; (7) the diminution of intra-abdominal pressure, e.g., following childbirth; (8) tight lacing, which increases the work of, the stomach by altering its position; or finally (9) any kind of dyspepsia which involves the over-stimulation of the stomach wall and the consequent relaxation of the muscle.

From the etiology cited above it is evident that in gastric atony the physician can frequently direct his effort toward the removal of the cause. But this generally affords only partial relief. Our therapy must go farther, and include dietetic and mechanical remedies.

Diet.-When treating either atony or dilatation, we must always bear in mind that we are contending with motor insufficiency. Hence our constant aim should be to lessen as much as possible the work of the stomach. The diet should, therefore, consist of such articles of food as are most quickly and easily propelled into the intestines, such as are (in gastric dilatation) least likely to decompose, and such as contain the maximum amount of nourishment for the organism. The diet should in general be a mixed one, but its exact composition should depend upon the gastric analysis in each patient. In every case of gastric dilatation the author strongly advises the passage of a stomach tube six or seven hours after a full and mixed meal in order to determine (by microscopic examination of the residue) which of the foods have already passed into the intestines, and the selection of such articles as the main part of the patient's dietary. With this reservation the author recommends semi- 
solid food, since this passes most easily through the pylorus. For this reason he does not approve of Schroth's "dry cure," which used to be so popular in the treatment of dilatation. A suitable dietary may be selected from the following list: milk in all forms, thick soups without stock, arrowroot and hygiama gruels, light farinaceous puddings, tapioca custard or pudding, eggs in various forms, mashed potatoes, crackers soaked in milk, milk cocoa, jellies, a moderate amount of meat broths used chiefly as a vehicle for other foods such as eggs or noodles, chopped or scraped rare or smoked meat, hash, thick meat soups, boiled white meat, and finally, if it agrees with the individual patient, easily digestible fats. It need hardly be said that, as in gastric ulcer, all very indigestible foods or those that cause fermentation, such as black bread, whole potatoes, and green vegetables must be absolutely forbidden.

Reduction of Liquids.-In every form of motor insufficiency of the stomach the amount of liquid must be reduced to $1000-1500$ c.c. (2-3 pt.) in 24 hours, for liquids load the stomach much more than solids. Milk may, however, be given in greater quantities in those cases of atony resulting from a poor state of nutrition (e.g., anæmia, convalescence from severe, prolonged illness, tuberculosis, etc). Charged waters are naturally forbidden, because the escaping gas distends the stomach. Beer should be forbidden, but about $200 \mathrm{G}$. (6 oz.) of wine or 15-20 G. (a tablespoonful) of brandy a day may be allowed, unless contraindicated by some other gastric affection. Alcohol should be used very cautiously, however, because, as mentioned above, it withdraws water from the tissues into the stomach, although it may improve gastric motility. Peptones, salts, and sugar should be avoided for the same reason. The patient suffers in any case from torturing thirst, because too little water is being absorbed. Such discomfort may be relieved by liquids given by the rectum (salt solution or enemas containing bouillon, either alone or with wine).

Meal Times.-Food should be given frequently and in small amounts, but, as meat remains longer in the stomach than eggs or milk, the time between meals which consist principally of meat must be longer than when milk and eggs are used-4 hours instead of 2 or 3. [The institution of 5 or 6 meals in some individuals does more harm than good. Hemmeter's plan of two meals at 8 A.M. and 3 P.M. will often suit such cases far better.-Ed.]

Lavage.-Next in importance to the diet in the therapy of motor insufficiency of the stomach come the various forms of mechanical treatment. The first of these is lavage. In atony the stomach will be emptied of its contents, though abnormally slowly, and decomposition will not ensue. Therefore, the object of lavage in this condition is not to evacuate the stomach but to increase the tone of the weakened muscle by rinsing the emptied organ. For this purpose the author uses water at $61^{\circ}-79^{\circ}$ under high pressure. In dilatation, on the contrary, the object is to remove the stomach contents before decomposition begins, 
and lavage becomes by far the most important of all therapeutic measures.

Technic.-The most favorable time for the process must be determined by the conditions of each individual case. Sometimes it is best to wash out the stomach in the evening, 6 or 7 hours after the principal meal, and give only a small amount of food afterwards; sometimes the fasting stomach should be washed in the morning; and sometimes it becomes necessary to do it twice a day.

To accomplish any particular good, lavage must be carried out very carefully and thoroughly. It is not enough to have the water run out clear while the patient is sitting up; he must be accustomed early to undergo the process while lying down, turning first on his right, then his left side. The pressure must be high, and the tube withdrawn occasionally and then pushed farther in. In this way many parts of the stomach otherwise inaccessible, will be reached. Sometimes pure water is used; sometimes antiseptic fluids, e.g., boric acid (2-3 per cent.), salicylic acid (3 per cent.), sodium salicylate (2-4 per cent.), hydrochloric acid (0.2 per cent.), sodium bicarbonate (2-4 per cent.), resorein (1-3 per cent.), and quinine ( 0.1 per cent.). If there is any abnormal formation of organic acids or of mucus, the stomach, after having been emptied, may be washed out with alkalies; e.g., Ems, Carlsbad, or Vichy water, or Carlsbad salt ( 4 G. ( 1 dr.) to 1000 c.c. ( 1 qt.) of water).

Electrotherapy -Massage-Posture-Bandage. - Electrotherapy, massage, and hydrotherapy may all be employed as subsidiary methods of treatment.

After every meal the patient should lie down for an hour or two, trying to keep on his right side, to facilitate the passage of food into the intestine. Patients with ulceration at the pylorus cannot, of course, retain this position.

Many authors recommend a rubber bandage to support the abdominal viscera, particularly the stomach, when the abdominal wall is relaxed.

Medication.-Medication aids very little in increasing the motility of the stomach or checking abnormal fermentation. For the first purpose, the tincture or extract of nux vomica is employed:

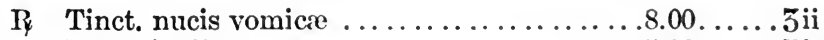

Resorcinolis .....................5.00.... $3 \mathrm{igr.} \mathrm{xv}$

Aq. dest. q. s. ..................ad $60.00 \ldots \ldots$. $\mathrm{n}$ ii

M. fiat mist.

Sig. - A teaspoonful 3 times a day after meals.

[When employing strychnine or nux vomica in these conditions, I prefer very large doses, such as $0.008 \mathrm{G}$. ( $1 / 8 \mathrm{gr}$.) of strychnine or about $4 \mathrm{G}$. ( $1 \mathrm{dr}$.) of the tincture of nux daily.-Ed.] 100-150 c.c. (3-5 oz.) of Carlsbad water, either natural or artificial, may be taken during eating.

To inhibit fermentation, creosote, ichthyol, menthol, resorcin, and sodium salicylate are recommended, but all of them together are not so efficacious as lavage. Of these drugs the author prefers sodium salicylate 
(1-2 G. (15-30 gr.) several times a day). Dilute hydrochloric acid (10-20 drops in water several times a day) may be of service, especially if the secretion of hydrochloric acid in the stomach is greatly reduced and lactic acid fermentation is present. Zinc sulphate $(0.2$ G. ( 3 gr.) to 150 c.c. $(5 \mathrm{oz}$.) of water) or silver nitrate $(0.1 \mathrm{G}$. $(1 \mathrm{I} / 2 \mathrm{gr}$. $)$ to 150 c.c. $(5 \mathrm{oz})$. of water) may also be used, 3 tablespoonfuls a day. [I prefer administering silver nitrate in pill form.-Ed.]

Olive Oil.-Cohnheim has introduced a special method of treatment for dilatation caused by spastic contraction of the pylorus or by organic stenosis. For a considerable length of time the patient takes 150 c.c. ( $5 \mathrm{oz}$. .) of olive oil a day, by the mouth or by the stomach tube, either in 3 doses an hour before eating or else in one dose early in the morning on an empty stomach. In the latter case it is warmed to the body temperature. The theory of this treatment is that the oil mitigates the pyloric spasm which accompanies pyloric stenosis and so makes it easier for the food to pass through. The author has had success with it in a case of pyloric stenosis after gastric ulcer; in other cases it has failed.

Mineral Waters.-It may be expressly repeated here that all treatment with mineral waters should be forbidden, as the amount of liquid must be carefully restricted.

Symptomatic Treatment.-Vomiting, hæmatemesis, or constipation accompanying motor insufficiency should of course receive appropriate symptomatic treatment.

Surgery.-If in spite of all these therapeutic measures, the patient loses weight noticeably, it is time to employ surgery before the anæmia and cachexia have proceeded too far. For dilatation pyloroplasty is sometimes used, more often gastro-enterostomy. The latter is almost the only operation for cases of severe atonic dilatation. Bircher's method of sewing up a fold in the stomach is rarely recommended.

[The author has not sufficiently emphasized the importance of early operation (gastro-enterostomy) in cases of gastric dilatation. I believe that all not benefited materially by a $3-4$ weeks' carefully conducted rest-cure are operable; all cases whose nutrition fails as soon as active treatment is interrupted and work is resumed; all cases who continually relapse soon after resuming work; all cases with evident pyloric stenosis ; many cases of gastric atony as soon as it becomes evident that they are overstepping the boundary line, so that the fasting stomach on a fluid diet is not always empty but sometimes contains food remains. There may be, of course, individual exceptions to these indications, such as very profound neurasthenia necessitating a more prolonged rest-cure or so pronounced malnutrition as to preclude the possibility of an operation. -Ed.]

\section{THERAPY OF GASTROPTOSIS.}

Gastroptosis is much more frequent among women than among men, since its most frequent causes are unsuitable corsets, irrational clothing, and particularly childbirth. It may, however, arise from other causes, 
e.g., rapid loss of fat. It usually accompanies enteroptosis. The first thing to do, especially if the patient looks thin and anæmie, is to insure rest in bed for 2 weeks or longer, followed by a period in which most of the time is spent lying on a couch. Later, lying down for an hour's rest after each meal will be sufficient. The effectiveness of this treatment is shown by the fact that gastroptosis has often been cured spontaneously when patients have been obliged by some other disease to stay in bed for a considerable length of time. The best position is on the back or right side, as then the stomach empties itself most easily. The difference in height between the greater curvature and the pyloric portion, which is appreciable in the erect posture and which causes a kink in the stomach, disappears when the patient lies down.

Diet.-The diet should be nourishing and, especially, fattening, for the stomach is supported by deposition of fat in the omentum and mesentery. With this in view, we choose a mixed diet, with carbohydrates predominating, it being taken for granted, of course, that the motility and the secretory function of the stomach are normal. The food should be given in the most easily digested form, in order to prevent the development of atony. The following is a suitable dietary: White meat, beefsteak, game, fish, smoked fish; milk with eggs; milk with arrowroot or hygiama; eggs in all forms; oatmeal; cereals (rice, farina, barley, corn, arrowroot) ; potato, pea, and bean soup ; macaroni, noodles, dumplings; crackers, zwieback, or white bread; a good deal of butter (if the patient digests it easily); spinach, earrots, peas, asparagus, cauliflower, artichokes; coffee and cocoa with milk.

There should be 3 principal meals and 2 lunches between. If the patient is very thin, a rest-cure is often beneficial until the requisite normal weight has been regained (see p. 387). The diet should also be directed to counteract the constipation which almost always accompanies gastroptosis. We therefore give fruit (cooked and raw), the mildly laxative kinds of kefir, cider, graham bread, and milk-sugar (50 G. $\left(1 \frac{1}{2}\right.$ oz.) a day in milk, tea, etc.).

The patient should wear a linen, flannel, or rubber bandage passing over the symphysis and pressing the abdominal viscera upward and backward towards their natural position.

Massage is also beneficial and, in case of necessity, may even be given by the patient himself. It may be supplemented by cold, and alternating cold and warm douches on the upper part of the abdomen, by sitz baths lasting only a minute or two in water of from $86^{\circ}-68^{\circ}$ followed by quickly rinsing the body, by sponge baths, or by fresh water or sea bathing. If the patients are not too weak, electricity may also be utilized.

Lavage.-With symptoms of beginning atony, it is well to irrigate the stomach in the morning. Even when atony is not present, lavage strengthens the stomach and improves the appetite. If kept up for some time, it also increases the abnormally low secretion of hydrochloric acid. For this purpose it is better to use common salt mineral waters 
(for example, the Kissingen Rakoczy spring or the Wiesbaden Kochbrunnen [Saratoga Kissingen-Ed.], or a $1 / 2$ per cent. salt solution).

Medication.-Medication is of very little use. Almost all that we can accomplish by drugs is to relieve the constipation which usually accompanies the condition. Even this is best done by irrigating the colon, although vegetable laxatives may be employed, or a mixture of a vegetable and a saline cathartic; e.g., rhubarb and powdered magnesia, or rhubarb, sodium sulphate, and sodium bicarbonate, (see p. 496). Another almost universal prescription is strychnine, or nux vomica, generally combined with an intestinal antiseptic. The author prefers for the latter resorcin, benzo-naphthol, or salicylate of bismuth; e.g.:

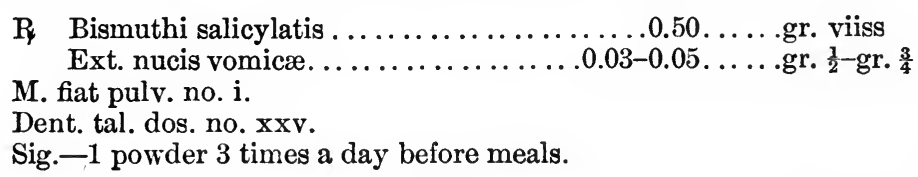

If the secretion of hydrochloric acid is below normal, hydrochloric acid may be given, or a sauce containing it may be used on meat (see p. 450). There is no need, however, of cutting down the amount of meat, for, provided the motility of the stomach is normal, the digestion of proteids will be quickly completed in the intestine. [Patients with gastroptosis are so commonly affected with a general splanchnoptosis and with pronounced neurasthenic symptoms that the section upon the treatment of neurasthenia should be consulted.-Ed.]

\section{THERAPY OF THE GASTRIC NEUROSES.}

Under the head of the neuroses of the stomach, are included a variety of diseases of that organ which can be accounted for by no anatomical lesion. Some of these affect the sensory, some the motor, some the secretory functions. Their treatment is, of course, by the very nature of the disease, often psychical. We make use of mountain or seashore climate, life in the open air, "occupation cures," various kinds of hydrotherapy directed toward toning up the general nervous system including the affected part, and electrical treatment.

Hydrotherapy.- The author has touched upon hydrotherapy and electrical treatment in several sections. For instance there have been discussed: (1) the use of partial or whole sponge baths to stimulate the entire body and particularly the whole nervous system; (2) the similar but rather stronger effect produced by sponging the body off with cold water and then rubbing down (see Chlorosis, p. 156); (3) the sedative effect of wrapping the entire body, or only the lower extremities, in a sheet wrung out in hot water, then covering the patient with a blanket and letting him stay quiet till the sheet is dry, even if it takes all night, and then rubbing him down; (4) the sedative effects of tepid full baths at an indifferent temperature and lasting from 5-10 minutes and 
followed by a dry rub; (5) the stimulating effect of cold or Scotch douches. There is one more hydropathic procedure, however, which may be used to good advantage in many forms of neurasthenia, and is useful in nervous dyspepsia, i.e., the half-bath followed by a dry rub or a cold douche. The patient sits for 5 or 6 minutes in a tub with tepid water $\left(80^{\circ}-89^{\circ}\right)$ up to his navel. He wears a cold compress around his head. The attendant takes up the water in a pail and splashes it over the patient from the neck down, rubbing his back and sides with the other hand, while the patient himself washes and rubs the front of his body. After the time is up, he is either rubbed dry or first has water about $18^{\circ}$ colder than the bath poured over him while he either sits or stands. The temperature of the bath may be reduced $4^{\circ}-7^{\circ}$ by letting in cold water for the last minute or two. The tonic effect is self-evident, and can be regulated by changing the temperature of the bath-water and of that poured over the patient.

Symptomatic Treatment.-Besides hydrotherapy there is little for us to depend upon in treating patients with the various gastric neuroses except what is directed toward combating individual symptoms. The text-books contain practically all the therapeutic resources, so that the author will discuss only those neuroses concerning which he has something new to contribute.

\section{THERAPY OF GASTRALGIA.}

Causal Therapy.-It is clear that the therapy of gastralgia must be directed primarily against the cause of the condition. This may be: (1) organic diseases, such as ulcer and cancer; (2) anomalies of gastric secretion, such as continuous secretion; (3) diseases of parts in close relation to the stomach, such as perigastritis, hernia of the linea alba, neurosis of the solar plexus, and affections of the abdominal aorta; (4) a reflex from the genital tract; (5) infectious, toxic, or constitutional diseases, such as tobacco or lead poisoning, tuberculosis, Addison's disease, malaria, chlorosis, and gout; (6) organic lesions of the spinal cord and brain, as in the gastric crises of tabes, myelitis, and multiple sclerosis; (7) neurasthenia and hysteria ; and (8) simply an empty stomach (Boas). In the last case, the possibility of which should not be forgotten, the proper therapy is very evident; some simple food, such as milk, chocolate, or crackers, should be given between meals. If none of these causes can be diagnosed or if the cause or causes assigned cannot be eliminated immediately or at all, the treatment must be purely symptomatic, in so far as the mitigation or cessation of the attacks themselves is concerned. Between the attacks we must combat, if possible, the causes of the disease. For instance, in the gastric crises of cerebro-spinal syphilis we may try anti-syphilitic treatment and electrotherapy, and in gastralgia arising from neurasthenia or hysteria, we must place the patient in a sanatorium. The diet must also, of course, be suited to the special cause of the disease, except that the increased sensitiveness of the 
stomach forbids, in practically every case, abnormally large quantities of food of any kind, indigestible food, or that which quickly ferments. It is usually best to give 5 meals a day.

Symptomatic Therapy : Hydrotherapy-Electrotherapy.-To relieve an attack we first try hot applications, wet or dry; Winternitz's process, in which warm water is run through Leiter's tubes, is effectual in many of the less severe cases. So is the galvanic current applied either intraventricularly with a stomach electrode for the anode, or extraventricularly with the anode on the epigastrium and the cathode as the indifferent pole either on the sternum or spine. The current should be applied for 5-10 minutes and should not be very strong. [Hemmeter strongly advocates galvanism.-Ed.] According to some authorities (Oser) the faradic current has practically the same effect. In Kussmaul's clinic stomach douches are used. Sometimes simple warm water $\left(99^{\circ}-111^{\circ}\right)$ is employed, sometimes water from a siphon, chloroform water, a saturated solution of chloroform, or a 1 to 1000 solution of silver nitrate.

Medication: Narcotics and Anodynes.-For internal use in severe attacks, particularly gastric crises, we can have recourse to morphine, given in hypodermic injections or suppositories, or to opium, with or without belladonna in suppositories:

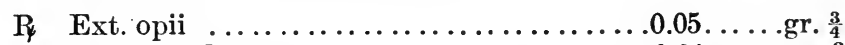

Ext. belladonnæ .....................0.04....gr. $\frac{2}{3}$

Olei theobromatis ...................1.00.....gr, xv

M. fiat suppos. no. i.

Dent. tal. suppos. no. $\mathbf{x}$.

Sig. -1 or 2 suppositories during the attack of pain.

When the use of a narcotic must be kept up for some time, codeine given subcutaneously may be temporarily substituted for morphine.

R Codeinæ phosphatis .................0.03....gr. $\frac{1}{2}$

Aq. dest. ......................gtt. $x \ldots \ldots$ gtt. $x$

M. fiat sol.

Sig.-For one injection.

In less severe attacks we may give internally choloroform, chloroform water, cocaine hydrochlorate, orthoform or better anæsthesin, sulphuric ether, spirits of ether (10-20 drops on sugar), 0.0005 G. $(1 / 125 \mathrm{gr}$.) of atropine 2-3 times a day in pills, or some of our antineuralgies; e.g., antipyrine 0.5 G. (7 gr.), exalgin, or pyramidon. Exalgin (methylacetanilid) is prescribed as follows :

R Exalgini $.0 .25 \ldots . .$. gr. iv

Dent. tal. pulv. no. $x x$.

Sig. -1 or 2 powders during the attack.

It may be said parenthetically that the author has found exalgin of great use in many different kinds of neuralgia; e.g., in intercostal neuralgia or the lancinating pains of tabes dorsalis or sciatica. For many patients it is decidedly better than antipyrine. 
Pyramidon (dimethylamido antipyrine) is eminently useful, not only as an antipyretic in pulmonary tuberculosis, but also in various neuralgias, particularly in tabes dorsalis. In a large number of cases the author has seen it stop the laneinating pains in a few minutes. In fact the pains could always be stopped if pyramidon were given at the beginning of each attack. In the author's opinion-and he has tried it in many cases-it is a remarkably useful addition to our pharmacopceia. The prescription is as follows: ${ }^{*}$

R Pyramidi $.0 .50-1.00 \ldots$. gr. viiss-gr. $\mathrm{xv}$

Dent. tal. dos. no. xxv.

Sig. -1 powder 4 times a day until $2 \mathrm{G}$. (30 gr.) have been taken, never over $4 \mathrm{G}$. ( $1 \mathrm{dr}$.) to be taken in one day.

The author has recently tried bromoform internally, particularly in the gastric crises of tabes, and has had some success with it. In one case of the severest kind of gastrie crisis in a morphine habitué, it stopped at once the only attack in which it was tried. The author prescribes $150-200$ G. $(5-61 / 2$ oz.) daily of a 0.1 per cent. solution of bromoform. The mixture should always be well shaken before using. [Einhorn recommends trying 10-20 drops of Hoffman's anodyne, or 15-20 drops of tincture of valerian. I have sometimes succeeded with 5-10 drops of validol; and I always exhaust my therapeutic resources before I take refuge in codeine or especially in morphine hypodermically. Prevention of another attack is after all the point of most interest to physician and patient. Some definite cause can almost always be found and so avoided. but no condition requires greater patience and more persistent psychical treatment than this. Where everything else has failed, success with psycho-therapy is often brilliant.-Ed.]

\section{THERAPY OF NERVOUS ERUCTATION AND PNEUMATOSIS.}

If these conditions do not arise from any evident cause-e.g., reflex action or tapeworm-but simply from neurasthenia or hysteria, psychical treatment has the best effect so far as eructation is concerned. In such cases this condition usually is brought about by the patient's continually swallowing air both during eating and between meals, and apparently also by the entrance of air into the digestive tract without any act of swallowing. Penzoldt advises that the habit be broken up by ordering the patient to hold his mouth open for a half hour or so several times a day. The stomach tube may also be used, and in pneumatosis is our best recourse.

Of medicinal preparations, the bromine compounds are the most efficacious, although belladonna or atropine may also be used with good effect.

- [I might mention again that I have succeeded with much smaller doses of pyramidon.-Ed.] 
In a few cases of hysterical eructation, the author has had remarkably good success from the use of iodide of sulphur:

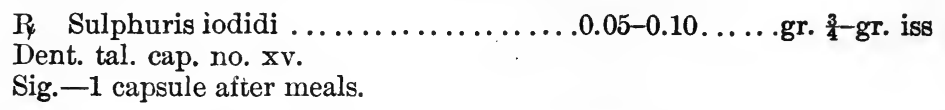

In pneumatosis Ewald advises a subcutaneous injection of morphine, and Boas recommends the following pills:

$\mathrm{R}_{4} \quad$ Ext. physostigmatis.................. $0.50 \ldots \ldots$ gr. viiss

Ext. nucis vomicæ ..................... $1.00 \ldots \ldots$ gr. $x v$

Ext. glycyrrhizæ $\ldots \ldots \ldots \ldots \ldots \ldots \ldots . . .10 .00 \ldots \ldots$.

M. fiat pil. no. l.

Sig. -1 pill 3 times a day. (Each pill contains ext. physostig. 0.01 G. (gr. $\frac{1}{6}$ ), ext. nucis vom. 0.02 G. (gr. $\frac{1}{3}$ ).

The diet must be nourishing and easily digested, with no food that will set up fermentation, no charged water, and limited liquids.

\section{THERAPY OF NERVOUS VOMITING.}

The therapy of strictly idiopathic nervous vomiting is the same as that of gastralgia as far as drugs are concerned, except that, of course, the antineuralgics are not employed. We may use bitter almond water, morphine, opium, belladonna, cocaine, chloroform, bromine, validol, and menthol; e.g.:

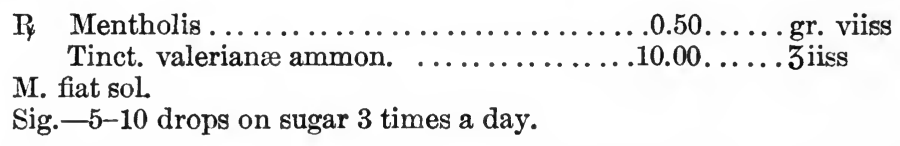

Chloralhydrate the author has found very useful (10-20 drops of a 20 per cent. solution on ice every 2 hours). The bromides should be given only in a very weak solution, for, if too concentrated, they will, like every salt solution, induce vomiting.

The patient must of course remain in bed and during the attack take nothing into the stomach except iced milk, champagne, or tea, in very small quantities, or tiny lumps of ice if they can be kept down. Nourishment is given by the rectum. Between the attacks the patient should be put on a special diet, be given hydropathic treatment, and sent to the mountains or seashore, or else treated at an appropriate sanatorium.

\section{THERAPY OF PYROSIS.}

Pyrosis is a frequent complaint in a number of diseases of the stomach. If it is not a symptom of some one of these, it is caused by the presence of some special food or foods, which differ with different individuals but which generally have the common property of either containing organic acid themselves or setting it free by fermentation in the stomach; e.g., 
wine, beer, vinegar, fruit, sweets, bad fat, etc. To overcome pyrosis the cause must be removed, although the condition may be treated symptomatically by lavage or alkalies. Sodium bicarbonate is the alkali usually recommended, but on account of the large amount of carbon dioxide that it sets free in the stomach the author does not consider it as good as oxide or carbonate of magnesia. None of these drugs should be given with much sugar, for the latter may itself bring on the burning again. The dose of oxide of magnesia is $0.5 \mathrm{G}$. $(7 \mathrm{~L} / 2 \mathrm{gr}.) .^{5}$ The following is Penzoldt's prescription:

P Magnesii oxidi...................22.50.... 3vi

Sodii boratis.........................5.00.... $3 \mathrm{i}$ gr. $x \mathrm{vv}$

Acaciæ............................2.50....3ss

Olei limonis ......................gtt. x.....gtt. x

Glycerini ...........................

M. fiat mass.

Aq. dest. ...............................

Div. in pastill. no. $\mathrm{lx}$.

Sig. -1 or more pastilles a day.

Pastilles prepared from spring waters, such as Vichy or Bilin, are contraindicated on account of their content of sugar.

\section{THERAPY OF HYPERSECRETION OF THE GASTRIC JUICE.}

Under the title of hypersecretion of the gastric juice will be included two different conditions: (1) hyperacidity, occurring only periodically; and (2) a constant hypersecretion of the gastric juice.

Diet.-The therapy of hypersecretion is concerned principally with the diet (see p. 390), which must be so chosen as to avoid everything that might increase the secretion of hydrochloric acid. For this reason we forbid all indigestible parts of foods (skin, seeds, shells, peels, woody fibres), all condiments (pepper, paprika, and mustard), radishes, all sour foods and drinks, all liquors that contain much alcohol, game, sausage, smoked meat, mushrooms, peptone preparations, pickled meat, sharp sauces, and coffee. The digestion of carbohydrates suffers slightly when the first of the two conditions, hyperacidity, is present; but much more severely in case of the second, true hypersecretion. The carbohydrates should therefore be cut down in quantity and confined to easily digestible forms where the starch has been converted into dextrin; e.g., toast, zwieback, crackers, the crusts of white bread, and infant foods. Sugar must be limited to small amounts. All vegetables must be cooked soft and passed through a sieve. Easily digestible fats, e.g., cream, butter, and oil, agree well with the patient and may be warmly recommended. Aside from these, the food must consist principally of milk, eggs, and white meat (preferably chopped or shaved thin), occasionally red meat,

${ }^{5}$ [Kinnicutt uses drachm doses of equal parts of magnesium carbonate and bismuth subcarbonate.-Ed.] 
fish, bean or pea soup, oatmeal, and aleuronat bread. All these use up hydrochloric acid without over-stimulating secretion. As a matter of fact, a milk and egg diet often puts an end to all the disturbing symptoms attendant on hypersecretion. It is well to add to the milk from one fourth to one third its volume of lime water, which breaks up the casein into finer curds and combines with the superfluous hydrochloric acid.

If no gastric atony exists with the hypersecretion, the addition of the heavier kinds of meat (game, beef, and mutton) to the lighter dietary just given produces good results (see p. 391). We may also try fine meal gruels-arrowroot, for instance-with hygiama or sanatogen added. It is apparently better never to let the stomach become quite empty. Boiled water may be given, or simple alkaline mineral waters from which the greater part of the carbon dioxide has been allowed to escape. Small quantities of well fermented beer or light wine may be added to the mineral waters. Both liquids and solids must be taken at a medium temperature. Tobacco should be used only very moderately if at all.

Lavage. - Next in importance to a proper diet is lavage, preferably given late in the evening. Often it is best to use an alkaline liquid (Vichy, Carlsbad, or a solution of Carlsbad salt and sodium carbonate). Many authors have reported good results from a 1:1000 solution of silver nitrate. The stomach is first washed out several times with water, then twice with the silver nitrate solution, which is kept in the stomach half a minute each time, then rinsed out again with warm water. Penzoldt recommends 800 c.c. ( $1 \mathrm{I} / 2$ pt.) of a 1 per cent. boric acid solution, to be kept in the stomach 5 minutes. The stomach must, of course, be thoroughly washed out both before and afterwards. He reports a subsidence of distressing persistent symptoms as a result of five or ten repetitions of this treatment; and the good effect has frequently lasted for many months. Fleiner recommends the similar use of large doses of bismuth. The author has also found this well worth trying.

Medication: Alkalies.-But the principal drugs to be used are the alkalies and the earthy alkalies, to neutralize the hydrochloric acid. The author greatly prefers the earthy alkalies (e.g., magnesium oxide) to sodium bicarbonate or carbonate, but he warns against the long continued and uninterrupted use of these alkalies, for while they unite with the hydrochloric acid, they also tend to stimulate its secretion. The best results are obtained by using alkaline mineral waters, freed from carbon dioxide (Bilin, Fachingen, Vichy, Neuenahr). In mild cases especially, a regular course of treatment at one of the spas, Carlsbad by choice, is doubtless valuable. A pinch of sodium borate is warmly recommended by many authorities.

The author has used Jaworski's "effervescent alkaline water,"-the stronger solution made up of $8 \mathrm{G}$. ( $2 \mathrm{dr}$.) of sodium bicarbonate, $2.5 \mathrm{G}$. (38 gr.) of sodium salicylate, and $2 \mathrm{G}$. (30 gr.) of sodium borate; the 
weaker, of $5 \mathrm{G}$. ( $75 \mathrm{gr}$.) of sodium bicarbonate, $2 \mathrm{G}$. ( $30 \mathrm{gr}$.) of sodium salicylate, and $1 \mathrm{G}$. (15 gr.) of sodium borate, to 1000 c.c. (1 qt.) of charged water. The dose of the former is $1 / 2-1$ glassful, generally on an empty stomach; of the latter, any amount up to 3 glassfuls a day, before meals. This water is almost always agreeable to the patient and is good, not only for hypersecretion and hyperacidity, but also for acid fermentation in the stomach.

Belladonna-Atropine-Eumydrin.-There remains to be mentioned one other group of drugs which have been known to reduce gastric secretion,--belladonna, atropine, and eumydrin. They may be prescribed as follows :

R Ext. belladonnæ....................0.03....gr. $\frac{1}{2} 6$

Magnesii oxidi $\ldots \ldots \ldots \ldots \ldots \ldots \ldots \ldots, 0.50 \ldots \ldots$. gr. viiss

M. fiat cachet no. $i$.

Dent. tal. cachets no. $x \mathrm{v}$.

Sig.-One cachet after meals.

If this does not have the desired effect, the amount of belladonna may be increased; or, if necessary, sulphate of atropine in $0.001 \mathrm{G} .(1 / 60 \mathrm{gr}$.) doses may be given subcutaneously up to $0.003 \mathrm{G}$. $(1 / 20 \mathrm{gr}$.) a day. Eumydrin is given in $0.001-0.002$ G. $\left(1 /{ }_{60}-^{1} /{ }_{30}\right.$ gr. $)$ powders $3-4$ times a day.

As has already been stated, silver nitrate ( 3 tablespoonfuls daily of a 1: 1000 solution) and olive oil are also useful in this connection.

The constipation which is generally present in a marked degree may be relieved by means of oil enemas (see p. 494) and the occasional use of a mild cathartic. This treatment alone has in some cases relieved the hyperacidity (Ebstein), proving that the abnormal condition originated in the intestine rather than the stomach.

\section{THERAPY OF HYPOSECRETION OF THE GASTRIC JUICE.}

This section contains simply a summary of what has already been said on the subject. The regulation of the diet is the most important of all therapeutic measures in hyposecretion of hydrochloric acid, whether it is an independent neurosis or an accompanying symptom of a general nervous condition like neurasthenia or hysteria, or of various anatomical lesions of the stomach.

Diet.-Only the very digestible forms of meat should be allowed (see page 392). Eggs in any form are available, but milk should not be given in very large quantities. There is no reason for not permitting a good deal of easily digested fat. As the digestion of carbohydrates does not depend on hydrochloric acid, they form the most suitable food; still even they should be prepared in the most easily digested way. The occasional use of food that stimulates the wall of the stomach and excites secretion,

${ }^{6}$ [One half or even one third this dose often induces toxic symptoms after a few days.-Ed.] 
such as caviar, salt meat, and meat peptones, may be allowed, as well as small quantities of wine or brandy and water. The meals must be light and frequent in order to avoid overloading the stomach and inducing atony.

Medication: Hydrochloric Acid.-The principal drug indicated is dilute hydrochloric acid. It may be given (1) after meals; (2) at intervals throughout the day; (3) as acidol tablets ( 1 tablet is equivalent to 5 drops of hydrochloric acid); (4) added to sauce to be eaten with meat (see p. 450). As already stated the author does not place very much reliance on the general effect of hydrochloric acid. We have, according to his opinion, as little right to consider it an efficient substitute for the hydrochloric acid which the stomach fails to secrete, as we have to-day to assume that even in small doses it increases the secretion of acid.

Next in importance comes pancreon, which enables the stomach to anticipate some of the work of the intestine, particularly the digestion of proteids.

The use of saline mineral waters or of orexin also stimulates secretion. Small doses of alkalies or alkaline mineral waters may also be tried (100-150 c.c. ( $3-5$ oz.) of Carlsbad or Vichy, a quarter of an hour before eating).

Gasterine the author believes is better worth a trial than any other drug (see p. 423).

Lavage.-Lavage with pure water or with a saline solution frequently increases secretion. Fleiner maintains that it can restore the secretion of hydrochloric acid even after this has failed for months, so that in every case we should give it a trial of at least $2-4$ weeks.

Hydrotherapy.-Hydrotherapy often brings relief. The author recommends either Priessnitz's applications, or particularly Scotch douches on the epigastrium, or douches all over the body. We may start with the water at $75^{\circ}$ and lower it $4^{\circ}$ daily until we get it down to $57^{\circ}$ even $50^{\circ}$.

A change of climate, or sea or mountain air is often beneficial.

\section{THERAPY OF NERVOUS DYSPEPSIA.}

Etiology.-Nervous dyspepsia may be defined as a symptom complex in which disturbances of digestion arise without anatomical or functional anomalies in the digestive tract to account for them. It should, however, be stated that anatomical diseases of the stomach or other organs (e.g., movable kidney, tuberculosis, diseases of the genitals, tapeworm, etc.) may cause nervous dyspepsia, though it more commonly arises from: (1) a general neurosis, such as neurasthenia or hysteria, (2) mental excitement or strain, (3) chronic poisoning (tobacco, alcohol, morphine, lead), and (4) chronic constipation. Even malaria may impose itself upon us as nervous dyspepsia. 
The therapy of nervous dyspepsia must always be directed against the cause of the disease: (1) the etiological lesion of the stomach or other organs, or (2) the general neurosis. In the latter case it is preferable by far to send the patient to a sanatorium. If this cannot be done, hydrotherapy, massage, and electrotherapy, the three most important means at our disposal in the treatment of general neuroses, may be employed at home.

Hydrotherapy.-As the object is to quiet the nervous system, only the milder hydropathic measures should be employed (cold rubs or slappings, tepid sitz baths and tub baths, sometimes warmer baths in fresh or salt water).

Climatology.-The patient should also have a change of climate. If he is strong, he should be sent to the high mountains; if run down and weak, to a moderately high altitude or to the sea. Many patients are especially depressed. A popular resort, offering various distractions, is to be preferred for them to a quieter and more retired one. Open air treatment often produces good results, as does very moderate out-of-door exercise.

Diet.-As for diet, no general rule holds good for all cases. What food suits each patient best, must be discovered by repeated trials. But as the patient is usually badly nourished, it is generally safe to say that he should be given a mixed diet relatively rich in fat and carbohydrates. Milk, or its substitutes, may be advantageously used in large quantities, if the patient can take it.

Many patients are helped by a Weir Mitchell rest-cure. Others are benefited most quickly by a diet containing a relatively large amount of green vegetables; others, again, by suddenly abandoning a very carefully chosen diet and eating ordinary, rather heavy meals. The food must be as varied as possible, be rather rich in condiments, and comply with the patient's whims. If constipation is present, we may resort to cooked fruit, honey, and graham bread.

The patient may drink milk, beer, wine, and often a little black coffee after eating. Smoking should generally be forbidden, but not in all cases. Inveterate smokers who lose their appetites and become mentally depressed when they stop smoking, are better off with it than without it. It is generally best for the patient to lie down after meals but not to sleep. Some nervous dyspeptics suffer so for hours after taking any liquid that they should be given liquid by the rectum, just as in cancer, ulcer, or dilatation.

Lavage.-Lavage works well in nervous dyspepsia, principally by suggestion. Washing out the stomach is perhaps even better than simply removing its contents.

Medication.-The drugs indicated may include stomachics, tonics, sedatives, and hypnotics. The best stomachics are orexin, condurango, strychnine, rhubarb, and gentian; the best tonics are preparations of iron and of arsenic, and quinine; the best sedatives are the bromides and 
validol, the latter being given in doses of 5-10 drops in syrup 3 times a day. Fellows' syrup of hypophosphites (a teaspoonful twice a day), and Scholz's hypophosphite pastilles (2 daily) should also be mentioned.

One bromide preparation is as follows:

R. Potassii bromidi,

Sodii bromidi,

Ammonii bromidi, ...............āā 1.50.....gr. xxii

M. fiat. pulv.

Dent. tal. pulv. no. xxv.

Sig.-1-2 powders daily in solution.

Maximowitsch and Boas both recommend the following combination:

R Ferri bromidi,

Quininæ hydrobromidi .............ää 2.00.....gr. xxx

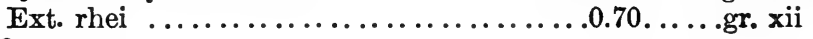

M. fiat. mass.

Div. in pil. no. lx.

Sig.-2 pills twice a day. (Each pill contains ferri brom., quin. hydrobrom. āa $0.03 \mathrm{G} .=$ gr. $\frac{1}{2}$.)

The hypnotics have already been discussed (p. 54).

Mineral waters may also prove efficacious. Iron waters (Franzensbad, Elster, Pyrmont, Cudowa) are the most useful; ordinary salt springs are of doubtful advantage; and alkaline salt waters should be wholly interdicted on account of the weakened nervous condition.

Constipation is frequently present and is best treated by enemas. Even mild vegetable laxatives should be given only in cases of necessity.

\section{SPECIAL THERAPY OF INTESTINAL DISEASES.}

\section{THERAPY OF ACUTE INTESTINAL CATARRH.}

Causal Therapy.-The etiological therapy of acute intestinal catarrh varies according to the exact cause of the condition. If the eatarrh is caused by eatching cold, we give internal sudorifies or external applications to bring on sweating; if by malaria, we give quinine; if by a chemical poison that can be neutralized, we give the antidote; if by a chemical poison that cannot be neutralized, by a toxin, or by some mechanical irritation, such as too coarse food or impacted feces brought on by constipation, we try to get rid of the irritating substance.

Cathartics.-In the majority of cases, then, the indication is to expel the irritating agent, whether it has been brought into the intestine from outside, or from the blood, as in uræmia or septic diarrhœa. This may be accomplished by catharsis or by colon irrigation. On account of the inflammatory alterations in the intestine, only the mild laxatives can be used. In fact there are only two practically available-castor oil 
and calomel. As both of these will be discussed at considerable length in the section on constipation, all that need be said here is that in acute intestinal catarrh castor oil is best given in a single dose of about $15 \mathrm{G}$. $(1 / 2$ oz. $)$ in 1,2 , or 5 G. $(15,30,75 \mathrm{~min}$.) capsules, or in a suitable quantity of peppermint water or peppermint tea. [If castor oil is apt to cause griping, a few drops of laudanum may be added. Other methods of administering castor oil are in sarsaparilla, well foamed and drunk quickly at a soda fountain, in strong black coffee, between layers of port, sherry, or lemon juice. Holding the breath and pinching the nose while drinking so that no odor can be noticed will often alone do away with any disagreeable effects.-Ed.] If castor oil produces nausea or vomiting, calomel may be given in a single large dose (0.3-0.5 G. $-41 / 2-71 / 2 \mathrm{gr}$.). Smaller doses, which produce little or no diarrhœa, may easily cause inflammatory alterations of the mucous membrane of the intestine.

Colon Irrigation.-The intestine may also be cleaned out by irrigating the colon with $1 \mathrm{~L}$. (1 qt.) of warm water (occasionally with the addition of about 20 drops of tincture of opium to retain the liquid longer in the intestine), or with oil, soap, or glycerin enemas.

Diet.-The most important factor, however, in the therapy of acute intestinal catarrh, is the regulation of the diet with the view of relieving the work of the intestine as much as possible. The author generally insists on complete starvation for the first twenty-four hours, relieving thirst by spoonfuls of tea at the room temperature.

Only in cases of necessity he gives sterilized water or a little brandy. In mild cases food may be allowed even on the first day; otherwise it is best put off until the second. It should consist at first wholly of suitable gruels (barley and rice rather than oatmeal, which is too laxative), of preparations of arrowroot or sago (one or two tablespoonfuls every hour), and of albumin water. For liquids we may give tepid or hot tea, herb tea (anise, chamomile, peppermint, or fennel), red wine diluted with 3 parts of water and 4 parts of warm tea, mulled wine, or brandy diluted with boiled water. If the diarrhœa has decreased by the next day, we may give broths with the yolk of an egg, or cocoa (especially Michaëlis' acorn-cocoa), zwieback, crackers, and bread crusts. After the diarrhœa has quite subsided, we may allow chicken broth, pea and bean soup, tapioca and rice puddings, boiled calves' brains and sweetbreads, chopped meat (especially chicken), the meat of fowl without skin or tendon, aspic, meat jelly, scraped beefsteak, raw scraped ham, sterilized milk, and chocolate. Gradually we add mashed potatoes, macaroni, rice and farina, asparagus tips, cauliflower, spinach, string beans, carrots, etc., and finally work up to a full diet, although for a long time the patient should avoid fruit, coarse vegetables, heavy puddings, graham bread, foods containing an abnormal amount of fat or acid, condiments, fruit ices, and beer. During the entire attack all food should be given only in small quantities and neither very hot nor very cold; even water had better be taken at the temperature of the room. Milk should be tried by 
giving a tepid teaspoonful or tablespoonful every hour, sometimes with the addition of brandy, lime water, or red wine. With many patients it increases the diarrhœa, and should be sterilized, replaced by kefir, or given up entirely.

Medication: Narcotics.-In many cases the clearing out of the intestine and the regulation of the diet are all that is needed. But where diarrhœa persists after these measures have been taken, and where there is intense pain which cannot be relieved by hot or cold applications, we are justified in giving a narcotic, such as opium, morphine, or belladonna. These, especially the first, apparently have a double action: they lower the sensibility of the intestinal ganglia so that they do not respond to stimulations arousing peristalsis, and they decrease peristalsis by stimulating the inhibitory nerves of the intestines. If the stomach is only slightly affected sympathetically, opium in powder form or oftener the extract, may be given by rectum, usually in suppositories, but sometimes added to starch enemas (1-2 small teaspoonfuls of starch added to a cup of warm water). In either case the extract of belladonna may be added to the opium. If the stomach is seriously involved so that vomiting complicates the profuse diarrhœa, hypodermics of morphine should replace the opium. No attention need be paid to temporary constipation from the use of morphine or opium, nor to that frequently following the cessation of the catarrhal diarrhœa.

Intestinal Antiseptics.-To control the bacterial growth many authors recommend the use of an intestinal antiseptic after the bowel has been cleaned out. The author does not place implicit confidence in the efficacy of this treatment; still there is no harm in trying one of the antiseptics already discussed (p. 424). [I can recommend soft capsules of castor oil 0.6-1.3 G. (10-20 min.) with salol 0.3 G. (5 gr.) administered every 2-4 hours and gradually withdrawn.-Ed.]

Intestinal Astringents. - It is almost uniformly superfluous to use an astringent unless the diarrhœa has lasted an abnormally long time (see p. 472).

Stimulants.-In exceptionally severe cases, collapse may occur. In such an event we give heavy wine, mulled wine, and hot tea with brandy, rub the extremities and wrap them or the entire body in hot cloths, and give hot mustard baths and subcutaneous injections of ether or camphor. Under these circumstances we should not be so rigorous in the matter of diet, but begin immediately with broth and milk.

\section{THERAPY OF CHRONIC INTESTINAL CATARRH.}

DIET.-The most important factor in the treatment of chronic intestinal catarrh, as in that of so many of the diseases of the intestinal tract, is the regulation of the diet. When accompanied by chronic constipation, the diet need not be as strictly regulated as when accompanied by diarrhœa or alternating diarrhœa and constipation. The aim is to build up the patient's strength by a nourishing diet and at the same time to 
avoid irritating the intestine. The suitable kinds of animal food are white meat and fish, scraped beef, calves' brains and sweetbreads, broths, chopped meat, and meat jellies. To these may be added eggs,- - raw, soft boiled, scrambled, or in omelettes. Farinaceous soups are less liable to irritate the intestine than meat soups, but a small cup of broth without vegetables or meat preparations may be given without doing any harm. For the most part, vegetables should be served only in purées (potatoes, peas, beans, or lentils). Asparagus tips, eauliflower, spinach, and thoroughly boiled macaroni or noodles may be served with meat. Rice, farina, sago, and arrowroot are all good. They should preferably be cooked with milk, which of itself is so valuable a food for almost all eases of chronic intestinal eatarrh that 1-2 L. (1-2 qt.) in some form should be taken daily. Many patients, however, especially if the small intestine is the seat of the trouble, find that milk only increases the intestinal disturbances. This difficulty may be avoided by adding lime water to the milk, by sterilizing it, or by replacing it by kefir. The kind of kefir should depend upon whether the tendency is towards diarrhœa or towards constipation. No. 1, which has fermented 24 hours, is slightly laxative; No. 2 is indifferent; No. 3, which has fermented 3 days, is constipating. 1-2 L. (1-2 qt.) may be taken during the day. If the trouble is in the large intestine, milk is generally well borne, while meat disagrees with the patient on account of decomposition of its proteids. Only the lightest kinds of puddings, such as souffés, may be allowed, and they must be eaten without much sugar. Crackers, zwieback, and crusty bread are permissible. Fresh fruit must be entirely forbidden. Apple-sauce may be given tentatively. Fat in general is to be avoided, but there is no objection to good butter. Salads or strongly spiced or peppery foods of any kind must be abandoned.

The patient may drink: (1) good red wine, (2) Greek wine, preferably Camarite, (3) huckleberry wine, which is constipating, (4) brandy and water, (5) tea, (6) alkaline mineral waters from which most of the carbon dioxide has been allowed to escape, (7) fresh or boiled water. If diarrhœa is present, cocoa (particularly acorn-cocoa) is much safer than coffee. Beer is so cold and contains so many bacteria that may produce fermentation, that it should be tried very cautiously or better left alone entirely. Hot water or hot tea, sipped slowly, is often beneficial.

Food should be taken five times a day. It should always be prepared in the most digestible way, should be served neither very hot nor very cold, and should be thoroughly masticated.

Often the symptoms will not disappear until the patient is put on an exclusive milk or kefir diet. In the latter case, the treatment cannot well be kept up for more than a few days, as $3-4 \mathrm{~L}$. (3-4 qt.) of kefir must be taken daily. To keep the patient well nourished, raw meat can generally be given with the milk or kefir.

Finally, to make sure that the best diet for the individual patient has been ehosen, the stools should be examined to determine what foods have 
been well digested, what only half digested, and what have gone through without being digested at all.

Cathartics-Colon Irrigation.-Besides feeding the patient properly, we must make sure that all detrimental substances are expelled from the intestine. In case of chronic or temporary constipation, therefore, mild laxatives are indicated. We may give castor oil (1 tablespoonful in the morning, or the same quantity in gelatine capsules), or calomel. If the large intestine is the part principally affected, we can make use of colon irrigations, using (1) warm water alone, (2) a saline solution, (3) chamomile tea, or (4) olive oil. In case of diarrhœea disinfectant or astringent solutions are indicated. They are also useful in constipation after the intestine has been washed out. The following are those most commonly employed:

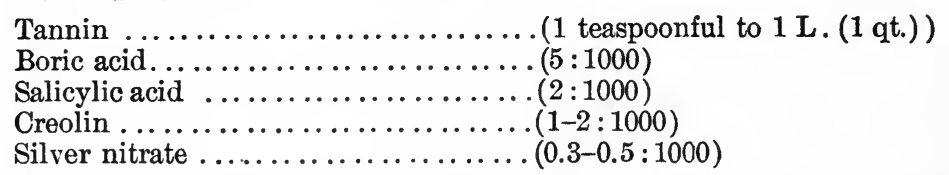

These irrigations must be kept up daily for weeks or even months. If the intestine shows considerable irritation, the temperature of the irrigation should be raised to $95^{\circ}-104^{\circ}$. Oil enemas may also be used (see p. 494). In deepseated catarrh especially, the author has recently used tannalbin to good advantage ( $3 \mathrm{G}$. (45 gr.) added to a daily starch enema of from 200-300 G. (6-10 oz.)).

Medication: Astringents.-If diarrhœa is a chronic symptom, astringents may well be given also by the mouth. Most of them owe their effect to the antiseptic properties of their content of tannic acid. Among them are: tannin itself, cascarilla bark, krameria, and gambir. These are often combined with opium (see p. 474).

Tannigen and Tannalbin.-Better than any of these old astringents, however, are the two newer preparations - tannigen and tannalbin. They are so compounded as to be free from two of the great disadvantages of tannin itself, i.e., its unfavorable effect on the stomach and its absorption in the upper part of the intestine before it has had a chance to produce much effect upon the intestine as a whole. The author after a wide experience with both gives the preference to tannalbin, considering it one of the very best preparations at our command. It is a golden brown, tasteless powder, containing 50 per cent. tannic acid in combination with albumin. By prolonged heating at about $250^{\circ}$, it has been rendered capable of resisting the digestive action of pepsin, but is gradually attacked and split up by the alkaline intestinal juices. The daily dose is usually 4-6 G. (60-90 gr.). A result generally appears by the third or fourth day, especially in the subsidence of the diarrhœa. It is prescribed as follows:

R Tannalbini ......................... $1.00 \ldots \ldots$ gr. $\mathbf{x v}$

Dent. tal. cap. no. xxx.

Sig. $-4-6$ capsules daily. 
Tannigen (diacetyl tannic acid) is a yellow gray, tasteless powder, not as constant in its effect as tannalbin.

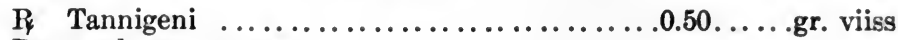

Dent. tal. cap. no. $\mathbf{x x}$.

Sig. - 1 capsule every 2 hours.

Alum-Lead Acetate-Silver Nitrate.-Besides these drugs, which all owe their action to their content of tannic acid, there are several others which undoubtedly have a good effect on the diarrhœa of chronic intestinal catarrh. First, two which the author seldom uses,-alum, a 0.1-0.25 G. (1 $1 / 2-4$ gr.) powder every two hours, and lead acetate, a 0.05-0.1 G. $(3 / 4-11 / 2$ gr. $)$ powder every two hours. Next, two metals which deserve consideration. The first is silver nitrate, which the author has found of little service in this connection, apparently because it is changed in the stomach into silver chloride. It is best prescribed in pills (see p. 456). [To insure a local action upon the intestinal mucous membrane, it is advisable to administer any of these drugs in gelatine capsules hardened with formalin according to Sahli's directions. Those of Nos. 2 and 3 hardness are to be preferred for this purpose.-Ed.]

Bismuth.-The second metal is bismuth, which is very widely used and rightly, although the reason for its effect is not clearly understood, and although in the intestines it enters into combination with sulphur, which causes black stools. The author lays great stress upon its being given in large doses, 3-5 G. (45-75 gr.) a day. Both the subnitrate and salicylate, white powders insoluble in water, are used.

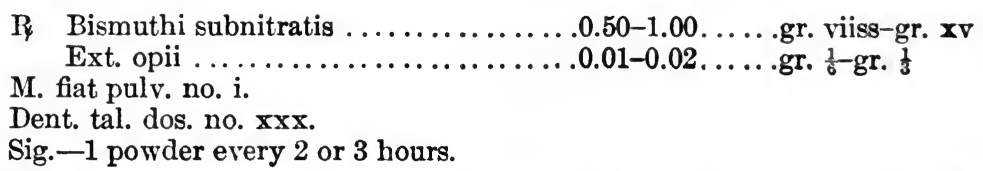

Possibly the salicylate has a slight antiseptic action on account of its salicylic acid. [I prefer the subnitrate and use it in still larger amounts, 2-4 G. (30-60 gr.) at a dose; or combine it with the subgallate as in the following prescription:

P Bismuthi subnitratis .................2.00....gr. xxx

Bismuthi subgallatis $\ldots \ldots \ldots \ldots \ldots \ldots \ldots . . .6 .70 \ldots \ldots$ gr. $x$

M. fiat chart. no. $\mathrm{i}$.

Dent. tal. chart. no. $\mathrm{x}$.

Sig. -1 powder every $2-3$ hours.-Ed.]

A third preparation of bismuth is dermatol, bismuth subgallate, a fine saffron-colored, tasteless, and insoluble powder, which the author has often used for diarrhœa, not only in intestinal catarrh but in other conditions. It apparently does not work so quickly or so well as the other preparations. It is prescribed as follows:

Py Dermatolis .........................50....gr. viiss

Dent. tal. pulv. no. xxv.

Sig. -1 powder every 2 hours as necessary. 
In using bismuth, tannalbin, or tannigen, the author insists on small doses at frequent intervals.

Eudoxin-Fortoin-Ichthoform.-Another available drug is eudoxin, already discussed on p. 424. The author has tried it in several cases of chronic intestinal catarrh with diarrhœa, but has not found it any better than the subnitrate and the salicylate of bismuth. On the contrary, some patients complained that it caused disagreeable belching and pressure in the stomach, while upon others it seemed to be almost inert. He has also tried fortoin (cotoin-formaldehyde) in many cases of chronic intestinal catarrh and in tuberculous ulceration of the intestine, but has not been satisfied with the effect. It is certainly inferior to either tannalbin or bismuth. The dose is $0.5 \mathrm{G}$. $(7 \mathrm{I} / 2 \mathrm{gr}$.) in capsules three or four times a day. Ichthoform (5-10 G. (1 $1 / 2-2 \frac{1}{2}$ dr.) a day) has not proved any more successful in his hands.

Astringent Teas.-Huckleberry wine, as we have said, is a good astringent on account of its tannic acid. Winternitz also suggests the use of huckleberry tea, made from the dried berries, two cups daily. [Blackberry brandy, either alone or with equal parts of cognac, acts in a similar way.-Ed.] In other cases of chronic profuse diarrhœa, absinthe tea, made by adding a tablespoonful of the leaves to a cup of tea, is useful.

Opium-Belladonna.-Opium is often combined with astringents. The author contends, however, that its use should be much restricted, as it undoubtedly has the disadvantage of decreasing peristalsis and so causing stagnation of the intestinal contents, and increasing any existing inflammation from abnormal fermentation. The author employs it only when, in spite of proper diet and the use of other drugs, the diarrhœa has not been lessened, and the patient is suffering from intense abdominal pains which are not relieved by hot applications or Priessnitz's bandages. If it must be given, it is best administered by the rectum, the colon being washed out at the same time to get rid of the contents of the intestine. The same difficulties lie in the way of the use of belladonna, which may be employed either alone or with opium in both acute and chronic cases.

MINERAL WATERS.- There remains to be discussed a very important class of remedies in chronic intestinal catarrh-the mineral waters. At their head come the alkaline salt waters, (1) the naturally warm or artificially heated, and (2) the cold. The first are not laxative; in fact they are often slightly constipating on account of their high temperature and possibly of their relatively small amount of free carbon dioxide. The naturally warm or warmed Carlsbad water or warmed Tarasp and Rohitsch, are therefore suited to those cases of chronic intestinal catarrh which are complicated with diarrhœa. If the patient takes the waters at home, he should use only small quantities, sipping perhaps two glasses a day, one in the morning and the other at about 11 A.M. or 4 P.M. if the midday meal be taken at noon. It is often advisable, as Nothnagel has recommended, to divide this amount into even smaller quantities, say 
30-60 c.c. (1-2 oz.), to be taken from three to five times during the day. In many cases the author departs from the rather general custom of giving Carlsbad on an empty stomach, preferring to put off the first dose till an hour or an hour and a half after breakfast, in order to reduce to a minimum any possible irritation.

It is important not to confine the Carlsbad treatment to a single month in the course of the year. As Nothnagel justly maintains, it should be taken four times a year, a month at a time. "A chronic process demands a chronic treatment." The patient may take his course of waters at the springs themselves, or stay one month there and then use the same waters at home for the other three treatments during the year.

In those cases in which the large intestine is chiefly affected, the author uses Carlsbad water in enemas (250-500 c.c. ( $1 / 4-1 / 2$ qt.) daily at the body temperature).

All that has been said about the internal use of Carlsbad water applies equally well to warmed Tarasp or Rohitsch.

Next in importance to Carlsbad in the treatment of cases attended by diarrhœa comes Vichy water, then the salt springs, Kissingen, Homburg, and particularly the Wiesbaden Kochbrunnen. Generally two or three glasses carefully heated are given daily, two in the early morning with a suitable time between, and the third in the forenoon or afternoon.

Whether Kissingen is to be preferred in special cases to Carlsbad or Vichy depends on the patient's state of nutrition. If he is badly run down, Vichy or one of the common salt springs is advisable. They also seem suited to those cases in which diarrhœa alternates with constipation, unless we prefer the mild, cold Glauber's salt springs, like Tarasp and Rohitsch, in order to keep the intestine free from all obstruction by mild but constant laxatives.

Where constipation is present instead of diarrhœa, the mild Tarasp and Rohitsch are the appropriate waters, except in severe cases, when the Marienbad Kreuz and Ferdinand springs are more efficacious. The daily amount should depend on how much is necessary for bringing on a movement. On an average $400-600$ c.c. (13-19 oz.) is enough. Some patients with abdominal pain as well as constipation, complain that the pain is aggravated by taking Marienbad water on an empty stomach. This may be avoided by waiting until an hour to an hour and a half after breakfast. [In America, Saratoga Congress and Hawthorne waters, or Rockbridge Alum waters may be tried.-Ed.]

No matter in which form the catarrh manifests itself, diarrhœa or constipation, it is a good plan to follow up the course of mineral waters by sending the patient to a stimulating mountain climate.

HYDROTHERAPY.-Hydrotherapy and massage also play an important part in this stage of convalescence. For either diarrhœa or constipation, or for an alternation of the two, a Priessnitz application is useful. The other available procedures differ with the form taken by the catarrh. If 
it is accompanied by diarrhœa, short sitz baths in cool water, long ones in tepid water, or Winternitz's process of passing warm water through Leiter's tubes are often very beneficial. If it is accompanied by constipation, there are a number of stimulating procedures at our command; e.g., packs, rubbing, Scottish douches, sitz baths of $54^{\circ}-59^{\circ}$, and massage.

In conclusion, the sufferer from chronic intestinal catarrh should be carefully protected from taking cold. For this purpose he should wear woolen underwear [or a flannel abdominal band-Ed.] and warm stockings, a precaution which is also useful in preventing repeated attacks of acute intestinal catarrh.

\section{THERAPY OF MEMBRANOUS ENTERITIS.}

[Dismissing the treatment of this baffling condition with the author's "few words" seems so inadequate that I have discarded the translation and substitute the following discussion of the more useful therapeutic measures at our command. They may be directed toward (1) the acute attack, and (2) the chronic condition.

(1) An acute attack, if severe, should be controlled at once by an opiate combined with an antispasmodic, preferably a hypodermic of 0.01-0.02 G. ( $1 / 6-1 / 3$ gr.) morphine with $0.0014-0.0005$ G. $\left(1 / 50^{-1 / 150}\right.$ gr.) atropine or, if this is contraindicated, a suppository containing double the dose of these two alkaloids or corresponding amounts of opium and belladonna. Despite the danger of employing morphine in so chronic an affection and especially in one complicated with so unstable and depressed a nervous system, my experience has convinced me that no other measures can relieve a severe attack. A hot bath, hot compresses, or some one of the other hot appliances to the abdomen will assist the opiate in subduing the spasms of pain, or if the attack is mild, will relieve it without resorting to morphine. The bowel should then be irrigated with warm normal saline solution to assist in expelling the mucus which has become detached from the wall of the gut. This may be followed by an injection as high as possible of $250-500$ c.c. $(\mathrm{r} / 2-1 \mathrm{pt}$.) of warm olive or cotton-seed oil. The patient should retain the oil all night if possible. The opiate will induce a quiet long sleep, and in the morning the oil will usually produce a copious evacuation and carry off most of the mucus associated with this attack. If the attack was severe enough to require an opiate the patient should be kept in bed for 2-3 days, the hypersensitive nervous system quieted by full doses of bromide, and the diet limited to light, nutritious food in small amounts every $2-3$ hours.

(2) The underlying chronic condition is controlled with more difficulty. Permanent success depends largely upon the choice of diet and the physician's skill and ingenuity in properly modifying a rest-cure to suit the individual invalid. According to my own experience, it is almost impossible to obtain a lasting cure without instituting such a routine, and better, of course, in an appropriate sanatorium. The choice of diet 
has been much disputed. Whether we follow von Noorden at one extreme or Combe at the other, the divergence of opinion suggests the two factors. The disease is a neurosis and its cure must depend largely upon psychical measures. It is perfectly evident to me that the masterful personality of such a man as von Noorden, controlling every detail of the care of an exhausted nervous invalid in a well equipped sanatorium, would succeed in curing a large proportion of intractable cases whether he attempted to train the intestinal tract, suddenly increasing its work by his very coarse diet, or insisted upon Combe's bland, non-irritating, nutritious diet. The second factor suggested to me by these opposed views, is that some cases require one type of diet, others the exact opposite. Einhorn states that a large proportion of his cases were accompanied by an achylia. Von Noorden found superacidity particularly common. Until quite recently I have tried to follow von Noorden's plan, and in several instances have failed signally, and succeeded only when I changed to a bland diet. Without journeying further into this disputed territory and without even attempting a discussion of the question as to whether a real inflammation of the intestine exists or not, I will briefly outline von Noorden's abrupt dietetic exercise treatment, and contrast the main features of the bland diet extolled by Combe.

Von Noorden recommends half a pound of coarse bread daily, containing plenty of bran; all vegetables rich in cellulose; all fruits with skins and small seeds, such as cranberries, currants, gooseberries, grapes, raspberries; fruit juices, marmalade, dried prunes, sugar of milk, honey; $200-250 \mathrm{G}$. (6-8 oz.) of pure fat in the form of milk, cream, butter, or bacon. The first few days are spent in bed, often with hot compresses to the abdomen. On the first and third evening an oil clyster. is usually given and sometimes repeated later if necessary. He orders a tumblerful of Kissingen, Rakoczy, or Homburg Elizabeth water, not as a laxative but as a stomachic, upon an empty stomach or one hour after the first tumbler of milk and cream mixture. During the first 8-10 days he employs daily massage of the sigmoid and lower bowel despite the condition of spastic constipation upon which he hinges his plan of treatment. The treatment lasts from 4-6 weeks and the patient is gradually brought back to his ordinary diet. Einhorn and Hemmeter follow von Noorden's principle, but accustom the patient first to a full nutritious diet. The patients are usually starved victims of some dietetic fad like beef-tea, beefsteak, roast beef, and hot water. After their nutrition has been improved, provided constipation still exists, a change is made towards the coarse foods which leave a large amount of residue.

Starting with the assumption that an infection of the gut coexists with or causes the neuropathic condition, Combe in an attempt to modify the culture medium, i.e., the contents of the large intestine, selects foods which are theoretically injurious to and avoids those favoring bacterial growth. Among the former he places first the farinaceous foods such as rice, macaroni, noodles, the Italian pastes, cereals, etc. He saturates 
the patient with these by prescribing them in small amounts every two or three hours. He alternates a liquid meal with one composed of solids. Although the milk products (milk, fermented milk, kefir, fresh cheese) contain fermentable casein, they also contain lactose, which combats fermentation, and so he allows them combined with the farinaceous foods. $\mathrm{He}$ also permits rather moderate amounts of fresh butter and cream. $\mathrm{He}$ prohibits meat, meat soups, broths and essences, fish, game, and the white of eggs, on account of their content of fermentable proteid. He begins by disinfecting the intestinal tract with calomel followed the next morning by castor oil and repeats this combination at the end of the first and third weeks or when necessary. He also employs high colon irrigations of warm normal salt solution every day. These then are the two extremes. The study of each patient will determine which course or what combination of the two shall be followed.

In nearly all cases $I$ have found it useful to follow Kussmaul and Fleiner's plan of giving $250-500$ c.c. $(1 / 2-1$ pt.) warm oil to be retained all night, and washing out the bowel with a cleansing enema of salt solution in the early morning until the patient has established a regular time for a morning evacuation, when the cleansing enema can be dispensed with. I continue the oil every night for 3 or 4 weeks, then every other night for the same period, then twice a week, and finally once a week for several months. If the oil is not well tolerated at first, we may add a few drops of laudanum.

The general principles underlying the treatment of all neuroses should be carefully applied to these patients, rest followed by appropriate occupation, hydrotherapy, general massage, electricity, an inland climate at a moderate elevation, and especially training them to expend their energies less wastefully, and to learn to be content at least for some years with moderation in all things. As far as drugs are concerned, arsenic and the glycerophosphate of soda have accomplished the best results at my hands.-Ed.]

\section{THERAPY OF INTESTINAL ULCER.}

Specific Treatment.-In consideration of the varied etiology of intestinal ulcer and the severe pain and danger which may accompany it, the means at our command for treating the condition are most inadequate. The only cases in which a specific therapy is possible are those in which the ulcer (1) is of syphilitic origin, (2) can be excised from the rectum, or (3) is stercoral, in which third case the use of mild laxatives, preferably castor oil, softens the fecal masses and so prevents the formation of the ulcers.

Possibly the use of bismuth in duodenal ulcer might also be called specific, although its effect is not nearly so marked as in the case of gastric ulcer. The author has, however, used it in the few instances that have recently come under his observation, prescribing it in pills coated with salol, which is not acted on by the gastric juice. 
P Bismuthi subnitratis................ $0.50 \ldots \ldots$ gr. viiss

Glyceriti tragacanthi....................... q. 8.

M. fiat pil.

Dent. tal. pil. no. c. (coat with salol).

Sig. -1 or 2 pills every 2 hours.

[The advisability of a gastro-enterostomy to promote healing by relieving the duodenal ulcer from the irritation of the food should always be entertained. With profuse hemorrhage it sometimes saves life.-Ed.]

Symptomatic Treatment.-In all other cases of intestinal ulcer, the treatment must be purely symptomatic, directed against the frequent diarrhœa, the pain, and hemorrhage, and insuring the greatest possible rest for the intestine.

Diet.-It is obvious that the diet must be similar to that laid down for chronic intestinal catarrh. It is important, especially in beginning phthisis, that it should be as nourishing as possible, but in general it must be mainly liquid.

Medication: Opium.-The use of opium is certainly indicated. It is the best intestinal anodyne, and by paralyzing the intestinal muscles, keeps the intestine quiet and quickly checks diarrhœa. If the stomach is sympathetically affected it must be given by the rectum; otherwise by the rectum or by the mouth.

It must, however, be admitted that, not only in stercoral ulcers but also in those of other origin (e.g., tuberculosis) castor oil is even more efficacious than opium, especially in checking the diarrhœa, the essential cause of which is stagnating fecal masses. Calomel had better be entirely avoided.

Bismuth-Tannalbin.-Bismuth (the subnitrate or the salicylate) can be given with the opium (see p. 473). Considerable benefit may also be obtained from the use of tannalbin, especially in obstinate tuberculous ulcers. There are cases in which even tannalbin cannot overcome the profuse symptomatic diarrhœa, but, on the whole, it is the best drug next to opium. Tannigen, which can also be given with opium, has proved useful in single cases of tuberculous ulcers, but in general is less effective than tannalbin. Lactic acid (3-4 G. (1 dr.) a day), and methylene blue (0.1-0.15 G. (11/2-2 $1 / 4$ gr.) with saccharine or powdered nutmeg once to several times a day, are also sometimes helpful in similar cases.

Local Treatment.-If the ulcer is situated in the lower part of the large intestine, local therapy should always be employed, the colon being washed out with an antiseptic or astringent solution, tannin and tannalbin, particularly, giving good results. The greatest care should be taken not to rupture the intestinal wall. If the patient's pain increases considerably during the irrigation, the latter should be stopped at once or continued at a very moderate pressure, only about a pint of water being introduced.

Colostomy.-In very obstinate cases of chronic ulcerative colitis, the author advocates making a cæcal fistula, in order to treat the lesion more 
effectively with silver nitrate ( 0.1 per cent.) or astringent or disinfectant solutions. The fistula must not be closed until all traces of blood or pus have disappeared from the stools. In one such case the author was unable to effect a cure until he resorted to this method.

Applications of Heat.-The pain from intestinal ulcers may be alleviated by hot compresses, hot water bags, poultices, or a thermophor.

Treatment of Intestinal Hemorrhage.-There is one more symptom which must often be combated,-profuse hemorrhage. The diet should be the same as in hemorrhage from gastric ulcer. The patient must, of course, stay in bed. The most suitable drug is opium, which keeps the intestine quiet and so gives every possible chance for the natural cessation of the hemorrhage. If the stomach is upset, the opium may be given by the rectum or a hypodermic of morphine may be employed. If the hemorrhage can be located, a light ice-bag should be placed over the spot. Without doubt the cold penetrates slightly to the ulcer; but the principal advantage lies in the fact that, in order to keep the ice-bag where it belongs, the patient is obliged to lie perfectly still. In order to make sure of this, strict orders are given to keep the bag continuously on the exact spot chosen. In practice, however, it has been the author's experience that this cannot always be done. There are many patients who cannot bear the ice-bag more than an hour or so, without becoming chilled.

Finally the author recommends a trial of the styptics in every form of hemorrhage, although his experience with them has not been very encouraging. Among those which may be employed are: ergotole subcutaneously or internally up to $3 \mathrm{G}$. (45 min.), the fluidextract of hydrastis, the fluidextract of witch hazel, lead acetate, or gelatine, administered exactly as in hæmatemesis, or hæmoptysis.

If the hemorrhage is from the lower half of the large intestine, we may use hot irrigations ( $1 \mathrm{~L}$. ( $1 \mathrm{qt}$.) of water at $113^{\circ}-118^{\circ}$ ) provided the bleeding is profuse enough to warrant our overlooking the amount of irritation necessarily produced by such a procedure. Four G. (1 dr.) of calcium chloride may be added to the quart of water in order to increase the blood coagulability. Such hot irrigations are also useful in hemorrhage from the stomach or the lungs. A gelatine solution may be used if the hemorrhage is from the rectum.

\section{THERAPY OF INTESTINAL STRICTURE AND. OBSTRUCTION.}

Chronic Stenosis.-All diseases which cause intestinal stricture or obstruction may be divided into two classes: (1) those that cause a chronic stenosis of the intestine, and (2) those that cause an acute obstruction or ileus. A chronic stenosis of slow growth may, however, change quickly into an acute obstruction, passing from group 1 to group 2.

Causal Therapy: Surgery.-In the case of a chronic stenosis, the therapy must depend on the cause of the stricture. If the cause is chronic constipation, the removal of the constipation will relieve the stenosis. Such cases belong to the sphere of internal medicine, whereas 
almost all the others belong to that of surgery. Among the latter are included intestinal tumors, strictures with the formation of dense connective tissue, and peritoneal adhesions outside the intestine. Surgery is not, however, always necessary where the stenosis is caused (1) by the malposition of intra-abdominal organs, (2) by pressure exerted upon the intestine by the inflamed and swollen neighboring organs, or (3) by the thickening of the intestinal wall, particularly of the serous coat, arising from inflammation or ulceration of the mucous membrane. In the first of these cases we use proper belts or bandages to keep the organs back in their normal positions; in the second, we try to reduce the inflammation; and in the third, together with proper attention to the diet and evacuation of the bowels, we try massage, gymnastics, and local hydrotherapy (applications, sitz baths). The author has treated several cases of perisigmoiditis and chronic appendicitis in the last way. But in many cases, especially those of the last two groups, surgery alone can bring any radical relief.

Diet.-In all nonoperable cases, except those caused by constipation, unless we are prevented by intercurrent attacks of acute colic, we must put the patient on an easily digested diet and one which will diminish the contents of the intestine. We must, therefore, avoid graham bread, fruit, fish, vegetables, and rice (unless it has been cooked very soft). Potatoes, peas, and beans should be given only in purées. All meat must be freed from skin and gristle. White meat prepared in the most easily digestible form, broth, eggs, and milk may be given. The meals should be light and frequent. If the stenosis is severe, the patient must be put on a semi-solid or liquid diet.

Cathartics-Enemas.-Constipation must not be permitted under any condition. An abundant movement must be secured every day, if necessary by giving an enema of tepid water either alone, or with the addition of salt, glycerin, soap, infusion of senna, or oil, or by the use of cathartics (bitter waters, Epsom salts, castor oil, cascara sagrada, frangula, senna, or even, if necessary, colocynth or croton oil). If the condition is acutely aggravated and particularly if we suspect a transient weakness of the intestine above the stenosis, we may try the salicylate of physostigmine either in pills, or better hypodermically in doses of 0.0005 G. $(1 / 120$ gr. $)$ up to a maximum dose of $0.001 \mathrm{G}$. $(1 / 64$ gr.) 3 times a day. It is an intense stimulant to the intestinal musculature.

We must prohibit all nourishment by the mouth in the presence of an attack of colic caused by (1) a temporary stasis of the contents of the intestine above the stricture, and the resulting periodic blocking of the intestine, or by (2) the much rarer condition of a temporary weakness of the part of the intestine above the stricture (in which case hypodermics of physostigmine should be tried). If the stenosis is situated in the small intestine, saline laxatives, preparations of senna, and enemas should be tried in order to make the intestine move at the place of stricture. If the stenosis is in the large intestine, only enemas are practicable. 
Even in case of stenosis of the small intestine, however, the author would employ laxatives only conditionally. Where peristalsis is plainly to be seen and felt above the stricture, laxatives are not only not indicated, but positively harmful. The intestine is already putting forth all its strength of its own accord. In this case, as in acute obstruction, the author believes in relying upon opium and enemas only.

If a chronic stenosis suddenly becomes an acute obstruction, the same therapy is indicated as in any other kind of acute obstruction, except that perhaps it is not quite so urgent.

ACUTE Ileus.-In passing to the discussion of acute obstruction, we approach a subject which lies on the border line between internal medicine and surgery. Surgical treatment is undeniably the more effective, but in many cases medical treatment is also of great benefit.

Ileus Caused by Coprostasis: Cathartics.-To speak first of nonoperative therapy, there is one subdivision of acute ileus which practically never demands the aid of surgery, i.e., coprostasis. If we are sure of this diagnosis-in actual practice often a very difficult one to make with absolute certainty-we should promptly attack the cause, and, at least in part, employ measures quite contrary to those used in any other kind of obstruction, i.e., we should at once give cathartics. Still even here we should proceed with some caution if we have any reason to suppose that the intestinal musculature is weakened or paralyzed; for in that case eathartics may do harm by forcing the intestinal contents down as far as the obstruction, without being able to get them past that point. This would result only in an increased distention of the intestine just above the stricture. A previous hypodermic of physostigmine is therefore doubly useful.

Enemas.-Rectal enemas should also be used to stretch the part of the intestine below the point of obstruction and to soften the impacted feces. If the water is cold, peristaltic action is also stimulated; but the author has never used cold water, and considers it strongly contraindicated if there is any sign of weakness or collapse. Tepid or warm water is generally chosen, although it produces no peristaltic action. In that case we should be sure to use enough (2-5 L. $(2-5$ qt.)), but still should be careful not to overstretch the intestine. A strong saline solutioneven as strong as 8 per cent.-is preferable to pure water. It stimulates the intestinal wall, and, even if only a small quantity (500 c.c. ( 1 pt.)) is used, penetrates into the lower ileum on account of the antiperistalsis it sets up (Grützner). The author also employs compound infusion of senna (about $200 \mathrm{G}$. (6 oz.) added to water or oftener to a saline solution). The saline solution, either alone or combined with the senna, has the further advantage of being so small in necessary bulk (1-2 L. (1-2 qt.)) as not to increase the distention of the intestine. Oil enemas $(1 / 2-1 \mathrm{~L}$. ( $1 / 2-1 \mathrm{qt}$.) of tepid olive or cotton-seed oil) have the additional advantage of lubricating the intestinal wall. 
All these enemas or irrigations should be given with the patient either in the knee-chest position or on his left side or on his back with his hips well elevated. They should be repeated, if necessary, every second or third hour, until the bowel is completely emptied. Of course, a fresh enema must not be given before the last has been discharged, or the intestine will be overstretched. The substitution of charged water or of air for the ordinary enema, will be discussed later on.

Mechanical Removal of Feces.-If there are impacted fecal masses in the lower part of the rectum, which prevent the enemas from passing into the intestine, they must be broken up and removed from the rectum by the hand or some blunt instrument. Before doing this, however, we may in suitable cases try the method that Ebstein has recently suggested of pressing the contents of the rectum out by means of stroking moveinents from the coccyx to the left side of the anus.

Massage:-Intestinal massage is also useful in this kind of obstruction. Its effect is twofold: it helps peristalsis, and it tends to break up and push along the fecal masses. It may well be preceded by oil enemas. But in cases which exhibit any marked degree of inflammation, it should seldom be employed, and then only with great caution.

The last method of internal treatment is lavage, but this is suited to other kinds of obstruction as well as to that caused by impacted fecal masses, and will therefore be discussed a little later.

Ileus Caused by a Gallstone or Other Foreign Bodies in the Intestine.-Before taking up this general discussion, a relatively rare kind of obstruction may be mentioned which, at first, at least, may be treated in the same way as obstruction from impacted feces: that is an obstruction caused by the lodging of a gallstone or other foreign body in the intestine. In many cases nothing can be accomplished by laxatives, irrigation, massage, and lavage, and we must proceed to operation. But this is by no means so often the case as in the other kinds of acute obstruction, such as strangulation, kinking of the gut, intussusception, and volvulus of the intestine.

Other Kinds of Acute Ileus: Medical vs. Surgical Treatment.-In all these other cases the decision as to whether internal measures should be first tried or surgery be immediately resorted to can hardly depend on the exact cause of the occlusion, for it very seldom happens that this can be determined during life with any certainty. It must. rather, be decided by the effect of the local lesion upon the general condition of the patient, and particularly on the state of the heart, and by the duration rather than the intensity of the symptoms of obstruction.

If the heart is in good condition and the pulse normal in rate, tension. and fulness, and there are no symptoms of collapse (e.g., cold and cyanotic extremities, and cold sweat) internal measures are indicated. But if they-particularly opium, lavage, and a warm bath-do not bring about a movement of the bowels within at most two days, the patient must be handed over to the surgeon. Naunyn's statisties have shown 
that the prognosis is twice as favorable if the operation is undertaken within the first two days. If at any time symptoms of heart weakness intervene, if the pulse becomes quickened and the arteries soft and less full, or if collapse symptoms appear, not a moment should be lost before operation, no matter how short a time has elapsed since the first symptoms of obstruction appeared. The operation may be tried in any case except when the obstruction has already lasted for some time, and pronounced collapse or complicating peritonitis is present. If, however, the pronounced collapse symptoms have appeared at the very beginning of the attack-this is particularly the case in strangulation-a hasty operation may be advised, for in it lies the only hope of recovery. [The acute onset of symptoms of collapse would be more apt to deter me from recommending immediate operation until after an attempt to improve the patient's condition by hypodermoclysis, and hypodermics of caffeine and adrenalin.-Ed.]

The effect of the local lesion, then, on the heart and the rest of the organism, is the chief factor in the determination of the advisability of an operation. But if it is possible during life to diagnose the special anatomical nature of the obstruction, this also should influence our choice of the time for operation. The most dangerous of all the kinds of obstruction, especially with reference to the speed with which collapse appears and the futility of medical treatment, is strangulation and, next to that, volvulus; kinking and invagination offer a better prognosis. In the first two cases, then, we should be particularly cautious in trying internal therapy, and from the very beginning prepare for operation. It should be undertaken immediately in all cases in which a positive diagnosis of strangulation can be made from the distention of a definite segment of the gut, the consequent severe steady pain from the onset of obstruction, and, less positively, peristalsis in the definite segment and early development of a free sero-hemorrhagic discharge into the peritoneum (Naunyn).

Lavage.-In those cases in which we employ internal therapy the two cardinal measures are lavage and opium.

The principal advantage of lavage is that it relieves the stomach from the mass of food which often has collected in it. Moreover, as Oser has pointed out, the pressure on the stomach is so lessened that often a reflux stream sets in from the intestine, where the pressure is much greater. This accounts for the fact that two or three hours after the stomach has been emptied, it may be found quite full again. The second benefit of lavage is the elimination from the organism of a great quantity of toxic substances which would lead to continued auto-intoxication.

But the effect of lavage is not only palliative but curative. By relieving the stomach and intestine from the abnormal amount of liquid which is distending them, it lowers the intra-abdominal pressure, relieves the distention and the tension of the abdomen, and puts an end to fecal vomiting and violent peristalsis. Moreover by the reduction of the intra- 
abdominal pressure-i.e., the pressure exerted by the stomach and intestine upon each other and the pressure between the different coils of the intestine-it may and often does happen that the obstruction is removed. However, if this result is not accomplished within 24-48 hours, we must resort to surgery. Lavage requires frequent repetition, for many cases every two or three hours. Collapse is of course a contraindication. It is also self-evident that lavage should be abandoned in all cases in which no fecal matter or bile is brought up. It should be noted, however, that fecal matter is frequently raised again after the water has once come up clear, a proof of the rapid passage of the intestinal contents into the stomach. [Lavage is especially successful and so indicated when the obstruction is high up in the small intestines and when vomiting is an early and obstinate symptom.-Ed.]

Opium.-Opium is as useful as lavage. The whole nervous system is quieted, the intense symptoms of collapse induced by the abnormal stimulation of the nerves of the stomach and intestine are considerably relieved, the temperature of collapse disappears, the pulse improves, the cold sweat vanishes, the pains in the abdomen are lessened, the nausea and vomiting subside, and, most important of all, the excessive intestinal peristalsis is checked. This peristalsis pushes forward all the contents of the intestine above the point of obstruction, so that the intestine at that point becomes more and more congested and distended, and intestinal paralysis arises. Opium militates against this danger by quieting intestinal peristalsis. And as this often is the cause of the obstruction or increases it if it has already begun, it is evident that opium in many cases strikes directly at the root of the disease.

In the author's judgment opium should be given early and in large doses. The most convenient form is in suppositories, containing the extract or powder $(0.5-1.5 \mathrm{G}$. ( $7 \mathrm{r} / 2-22 \mathrm{gr}$.) of either in twenty-four hours, divided into hourly or two hour doses). If this is not possible, opium or preferably morphine may be given hypodermically. If opium is chosen, $0.05-0.15 \mathrm{G}$. $(3 / 4-2 \mathrm{r} / 4 \mathrm{gr}$.) of the aqueous extract in a 10-20 per cent. solution is injected at a time. The prescription is as follows :

P Fldéxt. opii ............... $0.50-1.50 \ldots \ldots$ mvii-mxxii

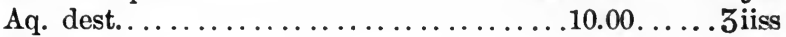

M. fiat sol.

Sig.-10 min. hypodermically to be repeated as directed. If $0.5 \mathrm{G}$.

(gr. viiss) be used, $m x=m \frac{1}{2}$ fluid ext. opium.

[If opium is used, a preliminary hypodermic of $0.032 \mathrm{G}$. ( $1 / 2 \mathrm{gr}$.) is preferable, followed every $2-3$ hours by a suppository of $0.032-0.065 \mathrm{G}$. $(1 / 2-1 \mathrm{gr}$.) of opium, just enough being given to keep the patient free from pain and to calm the effort at peristalsis.-Ed.]

Opiates should be used only with the greatest caution in the presence of the later symptoms of collapse (weak heart action, quickened or irregular pulse, cold extremities). 
When there is no longer any possibility of operation, opium or morphine may be used freely for the sake of euthanasia. Otherwise, if there is such severe pain that morphine absolutely must be employed, it should be given hypodermically together with heart stimulants, such as camphor or ether.

Atropine.-Medical therapy ean offer nothing else comparable in efficacy to lavage and opium. There are, however, a few more means to be mentioned which are sometimes helpful. A very useful drug, which the author has seen cure a case which he considered fatal, is atropine. It is given subcutaneously, $0.002-0.003 \mathrm{G}$. $\left(1 /{ }_{32}{ }^{1} /{ }_{20} \mathrm{gr}\right.$. $)$ in a daily injection, or even as much as $0.005 \mathrm{G}$. $\left(1 / 12 \mathrm{gr}\right.$.) given three times in two days. ${ }^{7}$ If the smaller dose is used and there is no sign of intoxication, it may be given even twice a day. Generally, if it has any effect at all, two or three injections will clear the passage. It is not yet known how atropine accomplishes its effect. Some maintain that it is by increasing peristalsis and reducing the inhibitory power of the splanchnic; others, by checking secretion in the parts of the intestine above the stenosis. It is also unfortunately not clear whether atropine succeeds only in cases of obstruction from coprostasis, or whether it is applicable to other forms as well. The author believes that it often succeeds in complete obstruction in which constipation has produced an anatomical stenosis. But as it is exceedingly difficult to be sure of these fine points of diagnosis, especially when the symptoms of obstruction come on relatively quickly, it seems best, practically, to give two or three injections of atropine at the very outset in all cases not demanding immediate operation. If this does not succeed we may proceed to lavage, opium, and the other remedial measures about to be discussed.

Colon Irrigation.-The first of these is irrigation of the colon. This should be tried in all cases, but particularly in those caused by constipation or gallstones. It is of some value in many cases, at least where the obstruction is in the deeper parts of the large intestine-especially in intestinal invagination,- - or where an abnormally large sigmoid flexure has become kinked and so obstructs the intestine. Even if the obstruction is in the small intestine, irrigation may be of use to the patient by arousing peristalsis, and in general it will free the colon at least from any fecal masses. [Both Jonathan Hutchinson and Treves recommend light anæsthesia during the colon irrigation.-Ed.]

For irrigation we use (1) tepid water, or oftener (2) a saline solution, frequently with the addition of a compound infusion of senna, every two hours. Oil enemas are sometimes used once a day between the others. It is evidently useless to continue giving an enema if the liquid runs out immediately, and obviously injurious to give another until the last has been expelled, for fear of unduly increasing the pressure in the intestine.

${ }^{7}$ [Murray employed even larger doses than this maximum.-Ed.] 
Inflation of Intestine.-Instead of irrigating the colon it may be expanded with air by connecting an intestinal tube with a bulb. To make it possible to withdraw the air at will, Curschmann puts a forked glass tube between the bulb and the intestinal tube. One branch connects the two, the other is connected with a tube which can be closed with a stopcock. Through this the air collected in the intestine can be let out. This process has no apparent advantages over irrigation.

The same thing is true of enemas containing carbon dioxide. They are administered by connecting the intestinal tube with a siphon and letting the contents of the latter flow slowly into the intestine. Irrigation of the colon, and injections of air or carbon dioxide are both contraindicated in case there is any danger of intestinal gangrene or of peritonitis.

F'asting.-It should be expressly stated that as long as there is any hope in the success of intestinal medicine, no nourishment [nor liquidEd.] of any kind is to be given by the mouth. Milk, wine, or champagne only load still more the overburdened digestive tract and cannot be absorbed to any appreciable extent. If the obstruction is in the upper part of the small intestine and has already persisted a considerable time, nutrient enemas must be considered. Thirst may be mitigated-aside from opium-by the use of pieces of ice wet in brandy and held in the mouth. The melted water must not be swallowed. Peppermint lozenges may be chewed, or the mouth washed out frequently with a cold cloth or rinsed with an alkaline solution. If the obstruction is in the small intestine, lavage and enemas are also of assistance.

Abdominal Puncture.-In case the abdomen is very much distended with gas, many authorities recommend that the intestine be punctured with a very small trochar the size of a cannula of a Pravaz syringe. The process seems attended with some danger, and should be used only when surgical or other efficacious treatment is contraindicated.

Surgery.-A large number of cases, after all, can be treated only surgically. As for the exact operation, it may be laparotomy and removal of the obstruction, laparotomy and entero-anastomosis, or the construction of an artificial anus. Often the surgeon cannot decide until the actual operation, which of these is the most advisable. [The author makes a stronger plea for medical treatment in acute ileus than I can accede to. In my judgment it is a surgical disease, and if treated tentatively it should be followed by a surgeon as well as a physician. Impacted gallstones, foreign bodies, or feces, occasionally a volvulus of the sigmoid and cases of uncertain diagnosis running a mild course, merit an attempt at medical treatment for 24-36 hours. If not influenced by then, they, too, as well as all other conditions of acute intestinal obstruction and those simulating such a diagnosis, belong to the surgeon and require immediate operation. "There is one measure for the treatment of acute intestinal obstruction, and that is by means of laparotomy. The 
operation should be performed at the earliest possible moment, as soon, indeed, as the diagnosis is reasonably clear. In case of acute abdominal trouble in which the diagnosis is not clear, the better and safer course is to operate. . . . The earlier the operation the less the need for enterostomy. Laparotomy should be performed at an early enough period to render an opening into the bowel unnecessary" (Treves).-Ed.]

\section{THERAPY OF CHRONIC CONSTIPATION.}

In treating a patient with so-called chronic constipation, we must first determine whether or not it is a true case, for there are people who physiologically have a movement every second or only every third day. If true constipation exists, the next thing to be determined is whether it arises from some primary abnormality of the intestine, or is secondary to some other pathological condition, for instance to some disease of the central nervous system (particularly neurasthenia) or of the circulatory system, or to hyperacidity. If it is secondary, it will yield when the disease causing it is relieved. The third question to be answered is: Is the amount of fecal matter actually too small or is the amount normal, but the time between each movement abnormally long? If the former, the diet is at fault. It contains too little or no material for residue to form stools. The therapy is self-evident. We must give the patient along with other food a good deal of material which cannot be absorbed, but will collect in the intestines and so set up peristalsis-e.g., cabbage, cucumbers, green salads, potatoes, peas, string beans, turnips, mushrooms, dumplings, brown, graham, and rye bread, and fruit. In these cases a vegetarian diet is often efficacious. The author can also confirm A. Schmidt's recommendation of regulin (agar with the fluidextract of cascara sagrada, 3 teaspoonfuls to 2 tablespoonfuls, in apple sauce or mashed potatoes).

If, however, the movements are too frequent, or if, although one stool is passed every day, it is too small and hard, we must first decide whether it is a case of spastic contraction or a case of insufficiency of the intestinal muscles with some diminution of secretion and with or without an alteration in the position of the intestine. The first, spastic constipation, is less common than the second, the so-called intestinal atony. [In practice the distinction between these two types of constipation is difficult, especially in neurasthenic or hysterical individuals with some degree of enteroptosis and a colon contracted down upon fecal masses. In some cases, I feel sure, both types are combined. Where the differentiation is not perfectly clear, the administration of opium or belladonna, one or both, may settle the diagnosis. If the condition is relieved, the constipation is due to spastic contraction nearly always. I believe that spastic constipation is especially common in American women and often is accompanied by a mucous colitis. The cause must, of course, 
be treated, but unless the diagnosis of the type of constipation is first accurately made out, therapeutic measures will not succeed, because purgatives are particularly pernicious in just this class of eases.-Ed.]

Spastic Constipation.- Spastic constipation occurs in lead poisoning, in diseases of the brain and spinal cord, in neurosis in the region of the coliac plexus, and particularly in neurasthenia and hysteria. In the first three of these, a movement may be induced by the use of opium or belladonna, and sometimes by extractum physostigmatis prescribed as follows :

R Ext. physostigmatis ................. $0.05 \ldots \ldots$ gr. $\frac{3}{4}$

Glycerini .............................

M. et sig. -6 drops in water every 3 hours.

But when neurasthenia or hysteria is the etiological factor, the therapy should treat the eause, and should be partly psychic and partly hydropathic. Rest, change, short journeys, mountain air, are all useful. The hydrotherapy may include tepid douches at low pressure on the abdomen, prolonged tepid sitz baths, hot applieations during the night,in short, such measures as will act sedatively upon the abnormally irritated sensory and motor nerves of the intestine. Oil, water, or chamomile tea enemas may be employed symptomatically, but laxatives should be given only in very exceptional cases, and massage and mineral waters should be wholly forbidden.

Atonic Constipation.-In the atonic form, unless it is purely symptomatic in anæmia, in cachexia, or after acute infectious diseases, a greater variety of therapeutic measures is at our disposal-dietetic, mechanical, and medicinal.

Diet.-The diet must forbid all foods which delay intestinal activity and supply those which hasten it. Among the latter may be classed cold or carbonated drinks. A glass of cold water, or, with some people, hot water, taken in the morning on an empty stomach, will often bring on a movement. A glass of cold champagne on an empty stomach with many people brings on a quick, copious movement. This explains why simple carbonated waters are so popular as table waters. They are not only refreshing, but they tend to keep the bowels open.

Foods which are useful in constipation on account of their rich content of acids and salts include raw and cooked fruits (plums, peaches, cherries, strawberries, raspberries, grapes, and, in a less degree; apples and pears), fruit juices, lemonade, cider, and all kinds of fruit wines. The carbonates, when acted on by bacteria in the intestine, give off a large amount of carbonic acid, which helps to bring on a movement. Fresh wine from grapes is especially useful because it contains not only acid, but a large amount of sugar. Sugar plays an important part, especially in syrups, honey, marmalade, candy, jellies, grapes, and in a lesser degree in all the fruits mentioned. Its effect is due to two facts: it 
hinders the absorption of water from the intestine and it is split up in the intestine partly into carbon dioxide and marsh gas. Milk sugar is laxative and is therefore often used in the preparation of food and on the table, in cases of chronic constipation. Several factors, mechanical and chemical, make other fruits, figs for example, good laxatives. Other foods owe their laxative action to their content of ferment; e.g.: beer, sour milk, and kefir (2 days old at most). Still others, such as melons and juicy plums and apples, owe theirs to their large percentage of water. Others again, such as salt fish, to the quantity of salt they contain. Finally, there is one food which acts in a peculiar way. Fat facilitates the movements by lubricating the intestinal walls, so that the feces move more easily. Oil, butter, and cream are therefore effective, and fatty sauces should often be served with meat. With many people, coffee is another peculiar laxative, which acts by its content of caffeine or empyreutic caffeine. Abundant quantities of cold water, or of mineral water, also help to keep the bowels open.

Finally those substances which have already been enumerated (p. 488) as favoring regular stools, also excite peristalsis by irritating the intestinal wall. But it is clear that, if in any individual case they do not counteract the constipation, they must themselves stagnate in the intestines and so do harm.

The same thing is true of carbonated drinks, and of food containing much ferment or much sugar, which tend to set up an irritation of the intestine. If, in any concrete case, they do not move the bowels, they will increase the constipation. They should all be given tentatively.

The diet in chronic constipation, then, must be suited to each patient. With some individuals milk brings on constipation; with others, diarrhœa. In general, however, it may be laid down that the patient should have a mixed diet with an ample supply of vegetables and fruits. In practice, we prescribe a glass of cold water before breakfast, bread and honey and coffee and milk for breakfast, and a varied meal for lunch and dinner, avoiding certain foods to be mentioned later, and followed by fruit or compôte.

All foods which decrease the activity of the intestines must be forbidden. This is the case with tea, red wine, and huckleberry wine, on account of their tannic acid. Cocoa and chocolate, particularly acorn cocoa, come under the same head. To these must be added some cereal foods, such as sago, farina, and rice. Their mucilaginous consistency thickens the fluid contents of the intestine and so delays the progress of the feces.

Regular Time for Stool.-Much depends on training the patient to go to stool at a fixed time each day, best directly after breakfast. Even if this does not prove a success at first, it usually will after a longer trial. Sometimes when the regulation of the diet for at least a month and the establishment of a regular time for going to stool have failed to bring 
about success, the smoking of a cigar or cigarette, especially after breakfast, may stimulate a regular movement. ${ }^{8}$

Massage-Hydrotherapy-Gymnastics.-If all of these fail, we must begin to use in addition the mechanical remedies: massage, hydrotherapy, and gymnastics. Massage has already been discussed. The hydrotherapy should consist principally of cold or Scotch douches, or, if it is the lower part of the intestine which we wish particularly to affect, of cold sitz baths, at $54^{\circ}-57^{\circ}$. These are best taken in the afternoon or shortly before the evening meal. The patient should stay in 5-10 minutes, with a cold compress on his head, and should rub the abdomen with his hands. Gymnastics may be carried out either with or without apparatus. The first should consist of trunk movements in both the standing and the sitting position, and of leg movements with the patient flat on his back. The legs should be raised quickly towards the breast, and then spread apart as far as possible. The same end is, of course, furthered by out-door exercise, such as riding, walking, rowing, or tennis. It must be confessed, however, that chronic constipation is often found among those whose occupations keep them in active bodily motion much of the time.

Electricity.-Rectal faradization, too, often helps intestinal peristalsis, and particularly in the colon.

Manipulation.-All these mechanical measures may be aided by two

${ }^{8}$ [The following note quoted from Du Bois' " Psychic Treatment of Nervous Disorders," is so pregnant with helpful suggestions for patients with constipation, due to bad habits, prolonged use of laxatives, or neurasthenia, that $I$ recommend its careful perusal to all physicians: "I [Du Bois] have seen persons whose evacuations were formerly very regular, bring obstinate constipation upon themselves by a poor choice of time devoted to this function. It was at an hour when the person was occupied and sometimes could not obey the call. The next day the need was felt at the same hour, and again the person resisted it. Later the intestine, whose warnings were neglected, felt the need less. The bonds of habit had been broken and constipation established. I therefore thought of using this tendency of habit and of concentrating in the morning hours all the stimuli which could act upon the intestine.

"I choose the morning because it is the time when we are freer to attend to those hygienic cares, and because, normally, during the long night, the slow movement of the intestine has brought to the rectum all the waste product of our food. There is therefore an early invitation to go to the toilet which arises from the very accumulation of material.

"The act of waking itself constitutes a second stimulus. I know a number of persons for whom the awakening of peristaltic movements follows the waking of their person. It is inconvenient, for they are obliged to obey immediately, and to jump out of the bed in which they were so comfortable.

"The act of getting up, with the movements of the body which are caused by one's toilet, the movement of putting on one's stockings and of getting into one's trousers, has an effect like massage, which is so efficacious that I have had some people complain that they cannot forego the need after having laced their first boot.

"Here are three invitations which follow one after the other, and which become habitual, especially if the time for rising follows the waking at a fixed time.

"To take a glass of water on getting up is a measure which has often been recommended. Entering a stomach which has been empty sinee the evening before, the water stimulates the movement of the stomach, and the contraction extends throughout the intestine; this is a fourth stimulus. If the patient has noticed that 
processes which aim at the direct removal of the impacted feces, if they have collected in the ampulla of the rectum. They may be removed directly either manually or by Ebstein's method already described (p. 483).

Colon Irrigation.-Much oftener, however, intestinal irrigation and enemas must be resorted to. All of these produce their effect by stimulating peristalsis, and the colder the liquid used the stronger the stimulation. Ice water, however, should be employed only under very exceptional circumstances (see p. 521). The peristaltic effect can be increased by adding some substance that stimulates the intestine chemically. For this purpose the author occasionally uses vinegar enemas (water and vinegar in equal parts, about $100 \mathrm{G}$. (3 oz.)), but oftener soap enemas ( $20 \mathrm{G}$. ( $5 \mathrm{dr}$.) of green soap dissolved in 200 c.c. $(6$ oz. $)$ of water), salt solutions (2-3 tablespoonfuls in 200 c.c. (6 oz.) of water), a solution of Glauber's salts ( 1 tablespoonful to 200 c.c. $(6 \mathrm{oz})$.$) , or 200$ c.c. $(6 \mathrm{oz}$.) of some bitter water). A tablespoonful of castor oil may be made into an emulsion with the white of one egg, and 200 c.c. ( 6 oz.) of water. In exceedingly obstinate constipation it may be necessary to add to the first enema one or two drops of croton oil. Glycerin in small doses (1-5 G.-15-75 min.) may be injected, or a glycerin suppository may be used. It stimulates the intestinal wall by attracting enough water into the intestine to induce a movement. For

hot or warm water succeeds better, I do not insist on the cold water. If the patient is a smoker who has felt the good effects of a cigarette, I permit him to use it.

"The eating of breakfast, especially if it is quite hearty, and consists in part of bread and butter, particularly whole wheat or graham bread, also stimulates peristaltic movements. Marmalade and honey may be a useful adjunct when a person can take it.

"At this point one must establish the formation of the habit of going at a fixed hour by prescribing regular training. Let the patient go to the toilet one hour after the beginning of his breakfast and you will thus have caused six successive invitations to act upon the intestine, any one of which would have been enough for a subject who is not constipated, but a regular succession can alone overcome the intestinal apathy of your patient. The will has, in fact, considerable effect upon defecation. The voluntary bringing into play of the abdominal pressure displaces the fecal masses, irritates the anal region, and induces rectal peristaltic movement.

"In short, the patient must be coaxed to take a hearty diet, and reminded of the adage: Large eaters are never constipated. His attention may be called also to the laxative effects of a vegetable diet and fruits (the herbivora are scarcely ever subject to constipation), and one will have instituted a physiological treatment of constipation which will be found to be much more efficacious than all artificial means.

"From the point of view of diet, the peasant and the workman, when they are not actually poverty-stricken, are better nourished than the people of the upper classes. Obliged to be satisfied with vegetables of little nutritive value, they are led to eat more in order to be nourished and have a satisfied feeling.

"Try to go to the toilet at a fixed time. Do not go at any other hour, and refuse to do so, saying to your intestine: 'You will not move at nine o'clock, now you can wait until to-morrow!' Use a copious diet, giving the preference to vegetable foods.

"Micturition and defecation are usually spontaneous. Therefore, when by an awkward effort the will intervenes in an act which is normally automatic, it disturbs the functioning of the organ."-Ed.] 
very sensitive individuals it may be diluted with an equal quantity of water or added to chamomile tea. A small syringe is usually used, with either a curved tube $10 \mathrm{~cm}$. (4 in.), or a drainage tube $1 / 2 \mathrm{M}$. (20 in.) long attached for inserting into the rectum.

Glycerin usually acts very quickly; often within five minutes it is followed by a stool, sometimes loose, sometimes formed. With many individuals, however, its efficacy wears off with use. To increase the effect some other cathartic may be added to a glycerin suppository; e.g.:

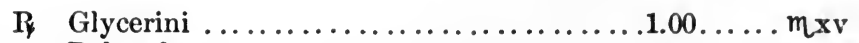

Pulv. aloæ.........................

Ext. belladonnæ ....................0.015 ....gr. \&

Olei theobromatis ............................ s.

M. fiat suppos. no. i.

Dent. tal. suppos. no. v.

Sig. -1 as directed.

Technic.-In all the enemas mentioned so far, the small quantity of liquid is noticeable. We use only about $200-250$ c.c. (6-8 oz.) when we wish to empty the lower part of the large intestine. As the amount of liquid is so small, we usually employ a tube with a tip only $3-4 \mathrm{~cm}$. (11/4$11 / 2$ in.) long. In order that the liquid may remain longer in the bowel, its temperature, unless changed for some particular purpose, is from about $77^{\circ}$ to the body temperature. Glycerin is used at the ordinary room temperature.

To reach the upper part of the colon the amount of liquid must be increased to 500-1000 e.c. (1-2 pt.) or more. It is injected by a Hegar's apparatus ${ }^{9}$ under a strong enough pressure to prevent the liquid from escaping even more rapidly than it has been forced in. The peristaltic effect can be increased by adding laxatives or oil, or lowering the temperature of the liquid; but tepid water is generally more satisfactory. It remains longer in the intestine and besides arousing peristalsis, also serves to soften the feces. Such tepid irrigations should not be used every day for fear of stretching the lower part of the large intestine until it becomes so relaxed that it no longer responds. The temperature should also be reduced as soon as possible from tepid to cold (down to $54^{\circ}$ ).

If the intestine responds neither to a small enema of cold water, nor to a large one of warm, the following process is often beneficial: $500-1000$ c.c. (1-2 pt.) of lukewarm water under a low pressure is slowly injected; the reservoir is then lowered, and the water allowed to escape; it is then raised and more water injected (see ed. note, p. 478). This process is repeated for 10-15 minutes, until the water expelled shows no sign of fecal matter. Then the warm water is allowed to flow out entirely, and a glassful of water at the room temperature is injected and retained as long as possible. If no stool follows the first attempt, the process should

- [A douche bag or better a douche can with a glass window so arranged as to show the level of the liquid, is the most convenient reservoir.-Ed.] 
be repeated the next day until an abundant movement has been secured. The day after this has once been accomplished we employ only the glassful of water at the room temperature.

It is important that all enemas or irrigations should be given at the same time of day, preferably after breakfast.

Oil Enemas.-Oil enemas are also to be highly recommended. Kussmaul and Fleiner have recently given them renewed popularity. The apparatus is the usual irrigator connected by a rubber tube with a cannula, generally of hard rubber, about $10 \mathrm{~cm}$. (4 in.) long and at least $0.3 \mathrm{~cm}$. ( $1 / 8 \mathrm{in}$.) in diameter. $400-500$ c.c. $(13-16 \mathrm{oz}$.) of pure olive or cotton-seed oil at the body temperature is injected under a very low pressure, so that the process takes about a quarter of an hour. The most favorable posture is with the hips well elevated or upon the left side. Rubber sheeting under the patient will prevent staining the bedding with oil.

The first movement usually comes in a few hours, but often not for twelve, and sometimes not until after a water enema has been given for uneasiness and rumbling in the bowel. The oil acts in several ways: (1) It detaches the fecal masses adhering to the intestinal walls; (2) if inflammation is present, it reduces the pain and so helps induce a movement; (3) it checks the reabsorption of water; and (4) if it remains a considerable time in the intestine, it excites peristalsis by virtue of the oily acids split off by the bile and pancreatic juice. It apparently does not soften the feces. Oil enemas that reach as far as the ileocæcal valve are as useful in spastic as in atonic constipation. As it generally takes an entire day for the oil to be wholly eliminated from the intestine, such enemas should be given only twice a week and stopped entirely as soon as a permanent passing of loose stools has been accomplished. But it is possible to give 300 c.c. (10 oz.) daily even for weeks (Ebstein's oil cure).

The author has had good success in obstinate constipation from the use of a combination of oil enemas and water irrigations. He first gives 3-4 tablespoonfuls of tepid oil, sometimes alone, but generally with the yolk of an egg and half a glass of water, directing the patient to hold the enema. A half hour later an irrigation of 1000 c.c. (1 qt.) of barely tepid water is given. The contents of the intestine have been already somewhat softened and the intestinal wall slightly stimulated by the first enema, so that the second one induces a movement.

Cathartics.-If, in any case of chronic constipation, all of these dietetic and mechanical measures fail, we are unwillingly forced to resort finally to eathartics. Two principles concerning their use in general should be kept constantly in mind: First, that very great differences occur in the effect of one and the same drug on different persons, so that in each individual case, first one cathartic and then another should be tried until the most efficacious for the particular patient is determined; secondly, that a cathartic should be used no oftener than absolutely necessary, and, if possible, in decreasing doses. It is even better not to 
repeat the use of any one drug, but to alternate our cathartic with an enema or with another cathartic, or to employ a mixture of two catharties.

Cascara Sagrada.-If a laxative must be given every day, cascara sagrada, the bark of rhamnus purshiana, is to be recommended, either in the pure fluidextract, or mixed with an equal quantity of syrup of orange peel $(2-4 \mathrm{G}$. $(1 / 2-1 \mathrm{dr}$.) or even more daily). It may also be given as the wine of cascara sagrada (Liebe's for example, $1 / 2-1$ liqueur glassful), or as pills containing $0.1 \mathrm{G}$. ( $1 \frac{1}{2} \mathrm{gr}$.) of the dry extract (1-2 or more pills a day). Pastilles may also be recommended (0.5-1.0 G. (7-15 gr.) of cascara sagrada; or $0.1 \mathrm{G}$. ( $1 \frac{1}{2} \mathrm{gr}$.) of the dry extract). The action of cascara is mild and not disagreeable. As the movement often does not come for twenty-four hours, it is best to give it in the evening. [I prefer the fluidextract of eascara in doses of 5-30 drops three times a day before meals. When the patient has once determined his proper dose, he should begin to decrease the amount a drop per day or per dose.-Ed.]

Leprince has extracted the active principle of cascara sagrada, which he has named cascarine. Pills containing $0.1 \mathrm{G}$. ( $11 / 2 \mathrm{gr}$.) prove to be a very mild laxative, generally taking twelve hours to produce their effect, and are therefore best given in the evening.

Frangula.-The author can also recommend frangula as a good, mild laxative, its only drawback being its unpleasant taste. He uses the fluid extract (3-4 tablespoonfuls daily), or 1-3 tablets (each corresponding to $15 \mathrm{G}$. ( $4 \mathrm{dr}$.) of the fluidextract). [The dose given in the U. S. P. is $20-40 \mathrm{~min}$. twice daily.-Ed.]

Rhubarb.-The author frequently combines the dry extract of cascara sagrada with rhubarb. This drug, made from the root of the officinal rhubarb by cutting it in pieces and scraping off the bark, deserves the reputation it has long enjoyed of being one of the best and least injurious of all cathartics. It has, however, one disadvantage, common to all the others; it causes loose movements, which soon make the constipation twice as bad. This looseness is apparently due simply to the fatigue of the intestinal muscles brought on by the stimulation of the drug. In many cases therefore it is necessary to increase the dose, or better, to combine the rhubarb with some other laxative.

If rhubarb is to be given alone we have a choice of several methods of administration. For example, the simple powdered root may be given (a pinch to a teaspoonful), or the $0.5 \mathrm{G}$. ( $7 \mathrm{gr}$.) pastilles ( $1 / 2-4$ pastilles), or we may use the following prescription:

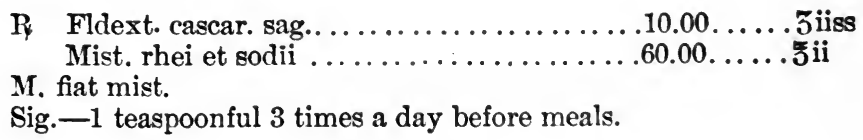

[The author gives several other prescriptions which are no longer used with us.-Ed.] 
Rhubarb is slow in its action, the movement generally not coming until the following day. If the total daily amount is to be given in one dose, it should, of course, be taken in the evening.

For continued use, a combination of rhubarb with some other laxative is preferable, in order to avert the danger of increasing the constipation. A good combination is that of rhubarb with a neutral salt. For instance:
R Pulv. rhei
$.20 .00 \ldots . .3 \mathrm{v}$
Sodii sulphati
$10.00 \ldots . .3$ iiss
Sodii bicarbonatis
$.5 .00 \ldots . .3 \mathrm{sigr.} \mathrm{xv}$
M. fiat pulv.
Div. in pulv. no. $\mathbf{x v}$.
Sig. - 1 powder at night in a glass of warm water.

Or :

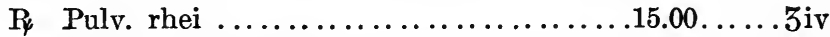
Magnesii carbonatis................60.00....ร
Olei menth. pip..................... $0.20 \ldots \ldots$ m.iii
Glycerini .......................... $10.00 \ldots \ldots$ Ziiss
Aq. dest.................... s. ad $120.00 \ldots \ldots$.

M. fiat mist.

Or :

Sig. -1 teaspoonful $2-4$ times a day.

R Ext. cascar. sag.,

Ext. rhei $\ldots \ldots \ldots \ldots \ldots \ldots \ldots \ldots \ldots \ldots$ a āa $2.00 \ldots \ldots$ gr. $\mathbf{x x x}$

Ext. glycyrrhizæ ...................4.00....3i

M. fiat mass.

Div. in pil. no. $\mathrm{xx}$.

Or :

Sig. -1 or 2 pills morning and evening.

R Pulv. rhei,

Sodii bicarb. ....................āã $0.25 \ldots \ldots$ gr. iv

Magnesii oxidi ...................... $0.20 \ldots \ldots$ gr. iii

Ext. nucis vomicæ $\ldots \ldots \ldots \ldots \ldots \ldots \ldots . . .0 .02 \ldots \ldots$ gr. $\frac{1}{3}$

M. fiat pulv. Dent. tal. dos. no. xxx.

Sig. -1 powder before each meal.

Compound Licorice Powder-Sulphur.-For repeated use in chronic eonstipation, the following compound is effective:

R Pulv. glycyrrhizæ comp.

$.30 .00 \ldots . .5 \mathrm{~s}$

Sig. - $\frac{1}{2}$ to 1 teaspoonful at night.

In spite of its content of senna this combination is suitable for repeated use. It is particularly helpful when chronic constipation is complicated with hemorrhoids or marked flatulency.

It is suitable in the former case on account of its content of sulphur, which, contrary to a rather general opinion, the author has found to be an efficacious and mild laxative. In the lower part of the small intestine and in the large intestine, the sulphur is gradually changed into sulphur alkalies, particularly sodium sulphide, which easily arouse peristalsis and stimulate the intestinal secretion. If there is a good deal of carbon 
dioxide in the intestine, sulphuretted hydrogen is also formed from the sulphur alkalies, and this gas also helps to increase peristalsis.

The author seldom gives sulphur alone; but he uses teaspoonful doses of precipitated sulphur (lac sulphuris) or sublimed sulphur (the former is probably more efficacious because finer). He also uses the following combination :

P Sulphuris præcip.,

Pulv. rhei,

Elæosacch. carui ...............āa 10.00.... 3iiss

Or :

M. et sig. -1 teaspoonful morning and night.

R Sulphuris loti,

Sodii tartratis ................ āa 10.00.... 3iiss

Sennæ pulv. ...................5.00....3iss

Syr. frangulæ ........................ 8.

M. fiat electuar.

Sig. -1 teaspoonful morning and evening.

The effect of compound licorice powder in cases of flatulency is due to the fennel which it contains. Flatulency may also be helped by adding to each dose of the cathartic selected (e.g., rhubarb or sulphur) 1-3 drops of oil of fennel, oil of caraway seed, oil of anise, or a suitable quantity of sugar of milk.

Podophyllin.-Another drug suitable for chronic constipation and often proving useful when every other cathartic fails, is podophyllin. The author prescribes it as follows :

R Podophylli................................ $\mathbf{x v}$

Ext. belladonnæ ......................... ii

Ext. frangulæ (G. P.) ..................... s.

M. fiat mass. Div. in pil. no. xl.

Sig. -1-2 pills at night.

The rhamnus frangula in this prescription acts as a laxative, while the belladonna is added to check the irritation, and particularly the colic, often caused by podophyllin.

Podophyllin in doses up to 0.05 G. $(3 / 4$ gr.) usually takes about twelve hours to produce an effect.

Kleewein's Pills.-Another mixture similar to those already given is found in Kleewein's aperient pills, which may often be used with marked success in chronic constipation. They are compounded as follows :

R Ext. rhei,

Ext. cascar. sag. .................āā $3.00 \ldots \ldots$ gr. xlv

Podophylli .....................50..... gr. viiss

Ext. belladonnæ.................. $0.50 \ldots \ldots$ gr. viiss

M. fiat mass.

Pulv. glycyrrhizæ ..................... q. s.

Div. in dos. no. 1.

Sig. -1 or 2 pills to be taken at night. 
Purgen.-The author considers purgen (phenolphthalein) generally reliable as a cathartic, but one attended with some danger. $\mathrm{He}$ has abandoned it himself, after having seen tolerably severe symptoms of collapse with vomiting and diarrhœa follow its use in three cases. Laxan and laxatol are similar drugs.

Tamarind.-A very mild laxative, often too mild to have any effect on chronic constipation, is the tamarind. It owes its cathartic properties to its content of potassium bitartrate, malic and citric acid, sugar, and pectin. It is given either pure, one or more teaspoonfuls at night, or more pleasantly in the form of pastilles; e.g., pastilles tamarindien Grillon or pastilli tamarindi Hell ( $1 / 2-2$ pastilles at night). Taken at night they usually do not act until the next morning. [The U. S. P. confection of senna contains tamarinds.-Ed.]

Carlsbad Salt.-Another class of laxatives which are available even for prolonged use, are Carlsbad salt and the natural mineral waters. The first is given as sal Carolinum naturale or factitium (N.F.), a teaspoonful to a tablespoonful in a glassful of warm water [upon risingEd.]. Its laxative effect ensues within an hour or two and is due to its content of sodium sulphate. The latter may also be used as a laxative (a tablespoonful [or less-Ed.] in a glass of water in the morning).

Seidlitz Powder.-The famous Seidlitz powder, or pulvis effervescens compositus, is made up of two different powders, one composed of potassium sodium tartrate (10 G. $-2 \mathrm{r} / 2 \mathrm{dr}$.) and bicarbonate of soda ( 3 G. -45 gr.) enclosed in a colored paper, and the other of tartaric acid ( 3 G. -45 gr.) in a white paper. The two are dissolved, one after the other, in a half glass of sweetened water, and taken in the morning. In mild constipation a movement usually follows in a few hours, but in the more obstinate cases not infrequently no result occurs.

Castor Oil.-Castor oil stands ,half way between those cathartics which can be used every day and those that cannot. Many patients cannot take it on account of nausea. If this is not the case, it may be given daily in gelatine capsules:

$\mathrm{P}$ Olei ricini

Dent. tal. dos. in caps. molles no. xxx.

Sig. -2 or 3 capsales.

(Or a corresponding number of smaller capsules each containing 1-3 G. (15-45 min.) of castor oil.)

Or as an emulsion:

$\mathrm{R}$ Olei ricini $\ldots \ldots \ldots \ldots \ldots \ldots \ldots .20 .00-30.00 \ldots \ldots .3 \mathrm{v}-\eta^{\mathrm{i}} \mathrm{i}$

Olei amygdal. amar. ................. $0.10 \ldots \ldots$ miss

Emuls. amygdal. .............. s. ad $250.00 \ldots \ldots$. viii $^{-3}$ ii

M. fiat emuls.

Sig.-Take half at one dose, following with the second half in 2 or 3 hours.

In taking castor oil, it is a good plan to hold the nose, for the nausea is more a consequence of unpleasant smell than of unpleasant taste. The 
common emulsion of castor oil is not so efficacious, on account of the constipating effect of its contained acacia. [A proprictary remedy, laxol, has given excellent results in my hands. I use rather larger doses of it than of castor oil. It has no taste nor smell.-Ed.] Brunton recommends putting the oil into a glass containing some water or peppermint water, and then covering the oil with brandy. The oil will keep its position as a middle layer, and the whole may be taken easily. The same author maintains that 50-60 drops every morning on an empty stomach will often overcome chronic constipation. With most patients, however, castor oil should be used only occasionally.

Saline Cathartics.-The same is true of the purges, and particularly of the saline eathartics, e.g., sulphate of soda, sulphate, oxide, or citrate of magnesia, and potassium and sodium tartrate. The last is much oftener used in combination with some other laxative (as in a Seidlitz powder). Sulphate of magnesia is given in the morning, as it acts in an hour or so. The dose is a teaspoonful to a tablespoonful in a glass of warm water, if necessary with the addition of $10-30 \mathrm{G}$. ( $1 / 3-1 \mathrm{oz}$.) of syrup of lemon.

The dose of oxide of magnesia for adults is about $10 \mathrm{G}$. $\left(2 \frac{1}{2} \mathrm{dr}\right.$.). Such a large volume makes the use of the drug undesirable. Citrate of magnesia, particularly in the effervescent form, is suited to practice among the better classes. A tablespoonful is dissolved in a glassful of water and taken while effervescing. If the desired effect is not produced, the dose may be repeated until as many as four glasses have been taken. [The officinal preparation, effervescent solution of magnesium citrate, may be given in $240 \mathrm{G}$. ( $8 \mathrm{oz}$.) doses, which may be repeated in 3 hours unless the desired effect is produced.-Ed.]

Calomel.-A good drug to relieve temporary constipation is calomel. It is apparently particularly efficacious in cases where there is nausea, or where vomiting must be avoided. The author always combines with it some other cathartic. A combination which he often uses, but which may have a drastic effect, is :

R Hydrargyri chloridi mitıs,

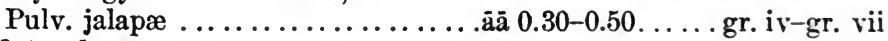
M. fiat pulv.

Sig.-To be taken at one time.

Senna-Aloes.-Two other drugs, senna leaves and aloes, are on the boundary between the milder cathartics and the drastic purges. They should not be repeated often; in fact they are usually used but once. Both of them, as well as the preparations of magnesia, are contraindicated in all inflammatory conditions of the intestine and peritoneum; aloes is also forbidden in hyperæmia or inflammation of the pelvic organs or kidneys, in diminished biliary secretion, and in pregnancy.

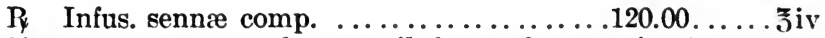

Sig. -2 ounces every hour until thorough purgation has taken place. 
Considerable use is made of the so-called St. Germain tea, which owes its cathartic action to the senna leaves it contains. One or two small teaspoonfuls are added to a cup of water. Chambard tea is an analogous preparation. Like all preparations of senna leaves, both of these are liable to produce intestinal colic and loose stools in two or three hours.

Aloes may be prescribed in various ways; but generally in combination with another drug, as in the officinal compound rhubarb pills, which may be taken every hour until the bowels move, or one or two pills may be taken at night. The officinal pills of aloes and myrrh (2-5 pills at night) may be given, or the officinal tincture of aloes and myrrh $(1 / 2-1$ small teaspoonful once or twice a day). [The latter is a noxious dose. -Ed.]

Drastic Purges.-All the above mentioned cathartics may be used more or less constantly; i.e., if not every day, at least once a week. It will be noticed that the least harmful for repeated use were discussed first, and this order has been followed up to this point, when we come to the true purges. These should be given but once, and then only in very obstinate cases which cannot be relieved in any other way.

The methods of giving colocynth, gamboge, and jalap have already been discussed (see pp. 36-37), so that there is only one drastic purge left to be spoken of-croton oil, the strongest of them all. This and every other drastic purge is to be employed only when there is absolutely no sign of intestinal or peritoneal inflammation. It should always be given in combination with castor oil, as follows:

R Olei tiglii $\ldots \ldots \ldots \ldots \ldots \ldots \ldots \ldots .0 .05-0.10 \ldots \ldots m^{\frac{3}{4}}-m_{\text {iss }}$

Olei ricini $\ldots \ldots \ldots \ldots \ldots \ldots \ldots \ldots \ldots . . .15 .00 \ldots \ldots$ 3iv

M. fiat mist.

Sig.-To be taken at one dose. Prepare as most agreeable to the patient.

Mineral Waters.-There remain to be discussed the mineral waters. They are used in both chronic and acute constipation of all kinds, and may be ranked according to their utility as follows : bitter waters, alkaline waters, common salt waters, and sulphur waters.

Bitter Waters.-The bitter waters are distinguished by their content of sulphate of magnesia and sodium sulphate, and by their almost complete lack of carbon dioxide. They have a strongly stimulating effect on the intestinal secretion, and make the intestinal contents more liquid; hence, taken in small quantities, they are mildly laxative without irritating the intestine. In larger quantities, however, they act as drastic purges and irritate the intestine. Naturally any inflammation of the gastro-intestinal tract is a contraindication. They are also contraindicated in case of rundown or markedly anæmic patients, for, if used for any considerable length of time, they reduce the content of fat already present and check the deposit of new fat. The amount prescribed varies, of course, with the content of sulphates, but, if employed daily, it must 
always be small, generally a scant wineglassful morning and evening, (100-150 c.c. $-3-5$ oz.) to be followed by a glass of cold water. It must be remembered that the Bohemian and Hungarian bitter waters are much stronger than the German. [For list of European and American bitter waters and their constituents, see table p. 414.-Ed.]

To induce one copious movement, we give a single dose of 300 c.c. (10 oz.), or more, if necessary, of some one of the more generally used Hungarian waters. Many cases of chronic constipation require but 2-3 tablespoonfuls of a bitter water on rising, followed by a glass of cold water.

Alkaline-Saline-C'ommon Salt Waters.-The bitter waters are generally taken at home; the cold alkaline-saline, or-less frequently-the common salt waters, are usually taken for chronic constipation at the various springs. [See table of alkaline-saline waters p. 409, and of common salt waters p. 412.-Ed.]

The daily quantity is generally one or two glasses, seldom as many as six. It is almost always taken on an empty stomach, never during or directly after a meal for fear of neutralizing the acid of the gastric juice. If it is desirable to get rid of the carbon dioxide before the water is taken - as for example in case of accompanying atony of the stomach-this may be accomplished by opening the bottle an hour before it is wanted, by shaking the glassful of water several times, by stirring it violently, or by heating it. If it is heated, it must be cooled again, for neither the naturally warm alkaline-saline waters nor the cold ones artificially heated are suitable for chronic constipation. They retard rather than stimulate peristalsis.

Sulphur Waters.-If the chronic constipation is complicated by abdominal plethora, or particularly, by hemorrhoids, the sulphur waters are to be recommended. The preference should be given to the following: The Elisenbrunnen at Aix-la-Chapelle, Neudorf, Schinznach, and Baden near Zurich; then the numerous French springs, such as Aix-lesBains, and Amélie les Bains. The daily quantity varies, in different cases, from about 200 c.c. $(6 \mathrm{oz}$.) to 1000 c.c. $(1 \mathrm{qt}$.). The best time to take them is in the morning. Occasionally milk, whey, saline cathartics, or bitter waters are added. [See table of sulphur waters p. 182.-Ed.]

Iron and Arsenic Waters.-If chronic atonic constipation is only one sign of a general muscular weakness, the cause must, of course, be treated; in many cases therefore we may advantageously use other mineral waters besides the distinctively laxative ones; e.g., waters containing iron and arsenic.

Hypodermic Injections of Cathartics.-All the cathartics mentioned so far are given either by the rectum or by the mouth. Sometimes we find the first method impossible (intestinal atony) and at the same time do not wish constantly to give a cathartic internally. A cathartic is needed that can be given hypodermically. So far, however, none can be absolutely recommended. Either the injection induces pain, as in the 
case of aloin or cetrullin, and is therefore impracticable, or the action is not constant. The latter objection applies to apocodeine, which the author has recently tried in hospital practice. It sometimes works very well, from 5 to 12 hours after the injection, but sometimes has no effect at all. The dose is $0.02-0.03 \mathrm{G}$. ( $1 / 3-1 / 2 \mathrm{gr}$.) or sometimes $0.04 \mathrm{G}$. ( $2 / 3 \mathrm{gr}$.), when the smaller quantity has had no effect.

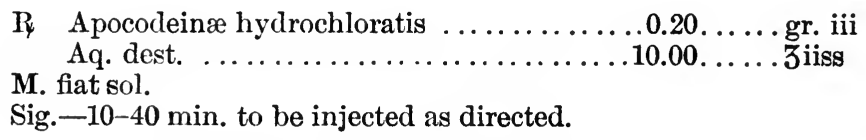

The author has never seen any unpleasant local or general effects from it, and would therefore give it a trial in all cases of necessity.

\section{THERAPY OF DIARRHCEA.}

Classification.-The therapy of diarrhœa may be dismissed with fewer words than that of constipation. It is, of course, necessary to diseover the etiology of the attack before determining its therapy. It may be caused by an anatomical lesion of the intestine, as acute or chronic intestinal catarrh or ulcers, in which case the therapy must be directed primarily against the lesion. In the absence of a lesion, the excessive irritation of the intestine which induces the diarrhœa may arise from (1) the contents of the intestine, (2) the condition of the nervous system, or (3) the condition of the blood. Under the latter head are included uræmic and septic diarrhœa. And it may be repeated that it is possible, though not satisfactorily so in practice, to bring on diarrhœa through the blood, by the subcutaneous injection of eertain drugs; e.g., aloin or apocodeine.

Diarrhœa Caused by Intestinal Contents.-In the first class of cases, those in which the excessive stimulation is caused by the intestinal contents, we may have to contend with a simple dyspeptic diarrhœa. Its therapy will then correspond to that of acute intestinal catarrh, of which it is merely an initial stage. If caused by (1) one of the different enterozoa, we must get rid of the parasite; if caused primarily by (2) gastric disturbances, such as hypersecretion, or by the passage of decomposed food masses from the stomach into the intestines, the appropriate causal treatment should be instituted; e.g., diet, hydrotherapy, or dilute hydrochloric acid, sometimes with the addition of opium, antiseptics, and lavage; if caused (3) by partial stagnation and thickening of the intestinal contents (stercoral diarrhœa), the intestines must be emptied and we must endeavor to prevent the recurrence of such fecal accumulation. Enemas may be used, but mild laxatives are preferable, particularly castor oil, which may be given several times in correspondingly small quantities, even after the intestine has apparently been completely cleaned out. For this purpose 5-10 G. $\left(1 \frac{1}{4}-2 \frac{1}{2}\right.$ dr.) is often enough, 
best given in the morning. The patient should not, as he almost always does, decidedly limit his diet. The diet planned for chronic constipation is much more rational, or, if he has already very strictly limited his diet, he should increase this slowly and gradually by the addition of new foods (see p. 489). Very cold food and any that causes abnormal fermentation must be avoided. Naturally all the mechanical measures useful in chronic constipation may be employed to advantage in stercoral diarrhoea-massage, hydrotherapy, colon irrigation, and gymnastics.

Nervous Diarrhœa: Diet.-In the second class of diarrhœas, those caused by the nervous system, the diet plays a much less important rôle than in any other kind of diarrhœa, or any other intestinal disease so far considered. There is such a great difference between individual cases that the proper diet for any one patient can be determined only by noting what suits him personally. If he himself has no clear ideas gained from experience on this point, it is best, as a rule, to depart gradually from a strictly limited diet, and tentatively increase the variety of foods allowed. In many cases the exact opposite to the former dietary accomplishes the desired result most quickly. In other cases too much attention should not be paid to the diarrhœea, but a light mixed diet should be selected. In many cases of nervous diarrhœa some meals are followed without any regularity by several violent watery movements accompanied by nausea and faintness. The author has obtained the remarkable result of stopping such a diarrhœea and its accompanying distress by ordering a glass of brandy just after eating, or even during the meal.

Hygiene.-In nervous diarrhœa it is well to quiet the overstimulated intestine by external application of heat, and to use a flannel band in order to prevent the possibility of the intestine's being irritated by reflex action from the skin of the abdomen. Some individuals have a sudden attack of diarrhœa-obviously reflex-as soon as they touch the foot to the floor in the morning. A piece of carpet alongside the bed, if it has not been there before, may be sufficient remedy. Reflex nervous diarrhœa may arise also from diseases of the genital organs, of the nose, or of the skin, from hernia of the linea alba, and finally in enteroptosis from a stretching of the nerves. Here a causal or hardening therapy is in place. If the diarrhœa is simply a symptom of neurasthenia or hysteria, the proper treatment of these diseases (hydrotherapy, etc.) will also tend to stop the diarrhœa.

Medication.-In the author's opinion there is no reliable medication for nervous diarrhœa. In most cases, to be sure, opium is of use, but nervous diarrhœa usually persists so long that opium must be employed very cautiously. And in other cases it is not effective, and we must have recourse to other drugs; e.g., tannalbin, salicylate of bismuth, some preparation of belladonna, an intestinal antiseptic, cannabis indica, bromides or arsenic. A tea made from absinthe leaves, taken several times daily, 
is also recommended. Usually we are forced to try therapeutic experiments. Often diarrhœa is a consequence of too much nicotine and the patient's smoking must be cut down. [The sufferer should train himself to resist the call for a stool except at the appropriate time. Persistent effort will first check the frequency of the desire and later regulate the number of stools. - Ed.]

Two Especial Forms of Nervous Diarrhcea.-In conclusion, two forms of nervous diarrhœa often met with in practice may be mentioned. In one of these the patient. complains of the diarrhœa's coming on directly or almost directly after the midday meal. In that case, all irritating substances must be avoided, and the combination of hydrochloric acid and opium already mentioned (p. 438) may be used; or arsenic (1/2-1 drop of Fowler's solution directly before eating). The patient should lie down for an hour after the meal, sometimes with a hot application upon the abdomen.

In the second form, the patient has one or more violent attacks of diarrhœa at 4 or 5 o'clock in the morning, or as soon as he wakes up, and then has no further impulse to go to stool. In such cases he should have only a little roast meat in the evening with soft steamed rice and should take no liquid after about six o'clock. He should wear a flannel bandage at night, to guard against any possibility of taking cold. If he has hyperacidity, he should take a teaspoonful of bicarbonate of soda in the evening.

\section{THERAPY OF FLATULENCY (METEORISMUS).}

We are often confronted with the task of relieving a patient who is suffering from an abnormal amount of gas in the intestine. If this is a result of stenosis, of atony, or of complete paralysis of the intestine, or of inflammation of the peritoneum, then a successful causal therapy will relieve the meteorism; e.g., the use of salicylate of physostigmine to stimulate the intestinal musculature. But, if the flatulency is due to the formation of an abnormally large quantity of gas in the intestine, or to an abnormally small absorption of intestinal gases, then, if we cannot check the gas formation itself, we must attempt to get the gas out of the intestine.

Carminatives-Colon Irrigation-Cathartics.-The best therapeutic measures are the administration of laxatives, and colon irrigations. The so-called carminatives are widely used, but their efficacy is much less than that of cathartics, because, although they produce some antizymotic effect, they have only a slight laxative action, and, in the author's opinion, do not increase the absorption of intestinal gases. The carminatives are usually prescribed either as waters (e.g., peppermint water may be given, a tablespoonful every two hours up to 150-200 G. (5-6 oz.) a day), or else as one of their essential ethereal oils or of their oleosaccharates, or finally as a tea, alone or with a cathartic. 
Such carminatives are: anise, cloves, caraway, myrtle seed, fennel, peppermint, and thyme, to which may be added enemas containing asafœtida water.

The so-called "flatulin pills" contain a useful combination of carminatives with laxatives, viz: sodium bicarbonate, magnesium carbonate, rhubarb, oil of fennel, oil of mint, and oil of earaway (2-3 pills, 3 times a day).

Charcoal and some salts of magnesia are also sometimes believed to act as gas-absorbing media. In the author's opinion they are of very little use. The same is true of bismuth. Although it may unite with the sulphuretted hydrogen of the intestine to form bismuth sulphide, it possesses no especial virtue against flatulency.

Rectal Tube-Puncture of the Intestine.-When catharties and enemas accomplish nothing, many cases of severe flatulency are relieved by the introduction of a long, thick rectal tube as high up as possible into the large intestine. It is allowed to stay there, and in some cases the gas is aspirated from it by a syringe. In desperate cases of meteorism, the intestine may be punctured with a small trochar or a somewhat thicker cannula with a hypodermic syringe. The author does not recommend this procedure.

Diet.-Whenever flatulency or even a tendency towards it exists, the diet should be modified first to counteract the cause (atony of the intestine, chronic constipation, etc.), and next all foods which directly tend to produce meteorismus must be excluded. Among the latter are the fermenting liquids (new beer), and those containing carbon dioxide (carbonated mineral waters, charged waters, beer, champagne, and kefir or koumiss). Among the solid foods which must be prohibited because they are too difficult to digest and ferment too easily, are fresh fruit, cabbage, peas, beans, and potatoes (except in purées), all vegetables rich in cellulose, black bread, sweets, and fatty foods. In general, the starches and fats must be reduced as much as possible.

THERAPY OF NEOPLASMS OF THE INTESTINAL WALL, ENTEROPTOSIS, AND INTESTINAL COLIC.

There remains to be discussed the therapy of: (1) neoplasms of the intestinal wall, (2) abnormal positions of the intestine, particularly enteroptosis, and (3) intestinal colic. The first of these belongs entirely to the field of surgery, unless an operation is impossible, in which case all we can do is to treat the symptoms, e.g., diarrhœa or stenosis. The second demands the same therapy as gastroptosis (massage, gymnastics, hydrotherapy, and bandages), see p. 456. The third must be treated either etiologically, in which case the therapy has already been discussed under the treatment of constipation and of flatulency, or symptomatically, in which case we may prescribe opium, morphine, or belladonna internally or in enemas, use hot applications, and give hot drinks. 


\section{THERAPY OF INTESTINAL PARASITIC DISEASES.}

Only the more common parasitic diseases will be discussed and those only briefly. The most important of them all is tapeworm.

Tapeworm (Tænia, Bothriocephalus).-If the presence of proglottides or a microscopic examination of the stools has proved the existence of tapeworm in the intestine, the regular treatment for expelling it should be at once begun, unless there are positive contraindications. Such contraindications are: (1) diseases of the stomach, intestine, or peritoneum, present either at the time, or very shortly before, (2) severe affections of the heart or kidneys, (3) severe anæmia or cachexia, (4) an inclination to hæmoptysis, (5) the status lymphaticus with hypoplasia of the vessels, (6) pregnancy, the puerperum, lactation, or menstruation. Much caution must always be exercised in treating children, old people, or patients with weak hearts.

Tapeworm Cure.-In the absence of any contraindications, we may proceed directly to the tapeworm cure. It should always be carried out under the supervision of the physician. The day before attempting to expel the tapeworm the patient must be forbidden food which will produce abundant feces, as they would make it difficult for the drug, which is to be given the next day, to act upon the head of the tapeworm. After his usual midday meal he must have that night only broth, herring, cucumber pickles, and black coffee. Secondly-and this is the most important point-his intestine must be cleaned out as thoroughly as possible. For this purpose, a cathartic is given, generally $0.3-0.5 \mathrm{G}$. (4 $1 / 2-$ $7 \mathrm{I} / 2 \mathrm{gr}$.) of calomel with or without the same quantity of jalap. If this does not act thoroughly, it should be followed in the evening by a strong enema. The next morning, the patient is given tea or coffee with rum or brandy, and half an hour to an hour later, the specific tapeworm drug. The most efficacious is male fern, aspidium, in the form of the fresh ethereal extract. The dose is 8-15 G. $(2-4 \mathrm{dr}$.), generally about $12 \mathrm{G}$. (3 dr.) :

R Oleoresin, aspidii ........................... $\mathbf{x v}$

Dent. tal. dos. in cap. molles no. $x i i-x v$.

Sig.-To be taken as directed, all within a half hour.

Particular stress must be laid on the total amount's being taken within a half hour. The first dose stupefies, the others continue this effect upon the tapeworm until it has been expelled from the intestine. To ensure its expulsion while still in this condition a powerful cathartic should be given an hour after the last dose of the male fern,--never castor oil, ${ }^{10}$ but compound infusion of senna $(10: 150)$ or the infusion combined with rochelle salts, $100-150$ c.c. $(3-5$ oz. $)$ to be taken in a single dose. If no

${ }^{10}$ [The oil probably renders the toxic principle soluble and so should be avoided. 
result follows in an hour or two, the colon should be washed out with a saline solution. This usually achieves the desired end, and the head of the tapeworm or several heads are found in the stools. Otherwise the tapeworm has apparently been divided at the neck and the head left in the intestine. To be sure to get rid of the latter a high injection should be resorted to. Unless found then, we must wait about two months and see whether eggs or proglottides appear in the stools before we can be certain that the first trial miscarried and that the process must be repeated.

It should be noted that the treatment is best carried out with the patient lying upon his back. To prevent nausea or to combat it, if present, lemons and iced tea or coffee may be given. It is, of course, not necessary to prescribe the drug in gelatine capsules. The next best way is as follows:

R Oleoresin. aspidii. ...........8.00-12.00....5ii-3iii

Emuls. amygdal. .............q. s. ad $60.00 \ldots \ldots$...

M. fiat emuls.

Sig. - To be taken in two doses, 15 minutes apart.

None of the other drugs for tapeworm are at all comparable to male fern. Perhaps the next best is pomegranate, prescribed as usual :

P Cortic. radic. granat. .........40.00-60.00....

Macer. $\bar{c}$ aqua per horas xii

Deinde coque ad remanent .........200.00....5 vii

Adde :

Syr. menth. pip. ...............20.00...3v

M. et sig.-To be taken in the morning in three or four doses, a half hour apart.

After about two hours a cathartic is given, followed if necessary by an enema, as before. But pomegranate is not so reliable in its effect as male fern, because the preparation used is not so good. The fresh bark of the root belongs to the almost uniformly reliable vermifuges, but the decoction not infrequently induces unpleasant gastro-intestinal and cerebral symptoms. [The tannate or sulphate of its active principle, pelleterine, is prescribed in doses of $0.2-0.3 \mathrm{G}$. (3-5 gr.).-Ed.]

There is practically no other drug to be considered. Fresh kousso flowers may be tried experimentally, either 150-200 G. (5-6 oz.) of the powder, or better the fluidextract, the dose for an adult being $16 \mathrm{G}$. ( $1 / 2$ oz. $)$.

Round Worm (Ascaris Lumbricoides): Santonin.-After tapeworm, the next most important parasite to be considered is the round worm (ascaris lumbricoides). Relatively speaking, we are not often called upon to expel round worms from the intestine, but, if we are, we first clean out the intestine by a cathartic and then almost without exception give santonin, which is obviously preferable to santonica. The 
dose of santonin is $0.05-0.1$ G. $(3 / 4-11 / 2$ gr.) (for children, 0.01-0.05 G. $(1 / 6-3 / 4$ gr. $))$, two or three times daily for several days. After it has been administered, the intestine should be emptied completely by a cathartic or enema. To decide whether the treatment has succeeded, or whether it must be repeated, the stools are examined for ascarides eggs. Santonin may be prescribed in various ways:

R Santonini $\ldots \ldots \ldots \ldots \ldots \ldots \ldots \ldots \ldots . .0 .05-0.10 \ldots \ldots$ gr. $\frac{3}{4}$-gr. iss

Sacchar. lactis $\ldots \ldots \ldots \ldots \ldots \ldots \ldots \ldots \ldots . . .6 \ldots \ldots$ gr. vi

M. fiat pulv. no. i.

Dent. tal. dos. no. vi.

Sig.-A powder every morning and night for three days.

R Trochisci santonini.............. $0.025-0.05 \ldots \ldots$ gr. $\frac{3}{8}$ gr. $\frac{3}{4}$

Dent. tal. dos. no. xx.

Sig. $-1-2$ troches every morning and night for three days.

Or, combined with a cathartic:

R Santonini $\ldots \ldots \ldots \ldots \ldots \ldots \ldots \ldots \ldots \ldots, \ldots \ldots \ldots$ gr. iii

Olei ricini. ..............................

M. fiat mist.

Sig. -1-2 tablespoonfuls for two or three days.

$\mathrm{P}_{\psi} \quad$ Santonini $\ldots \ldots \ldots \ldots \ldots \ldots \ldots \ldots \ldots . . .0 .025-0.05 \ldots \ldots$ gr. $\frac{\frac{8}{8}}{8}$ gr. $\frac{3}{4}$

Hydrarg. chlorid. mitis . ............... $0.05 \ldots \ldots$ gr. $\frac{3}{4}$

Sacchar. lactis $\ldots \ldots \ldots \ldots \ldots \ldots \ldots \ldots \ldots .0 .50 \ldots \ldots$ gr. viii

M. fiat pulv.

Dent. tal. dos. no. vi.

Sig.-A powder twice a day for three days.

To be sure of avoiding toxic effects, santonin should never be given on an empty stomach. [Forchheimer properly emphasizes the dangers of santonin medication. The drug should not be given, unless a recent examination of the feces has disclosed the existence of the characteristic ovathe prescription should not call for more than three doses, and not more than $0.065 \mathrm{G}$. ( $1 \mathrm{gr}$.) for children, $0.3 \mathrm{G}$. (5. gr.) for adults, in the 24 hours. He always combines it with calomel.-Ed.]

Oxyuris Vermiformis.-The next parasite to be discussed is oxyuris vermiformis. As this parasite is found both in the small intestine and in the large, particularly in the rectum, and generally in masses, it often is difficult to get rid of it completely. Therefore, any therapy designed to accomplish this result, must act upon both the small and the large intestine. This is accomplished by prescribing a vermifuge-almost always santonin with a cathartic, as in case of round worms-and at the same time ordering certain enemas, which must be kept up every evening, or even twice a day for one or more weeks, until the examination of the stools for parasites gives a negative result.

For these enemas, about 1000 c.c. ( 1 qt.) of water is used, generally with the addition of 4 tablespoonfuls of salt, or 1-2 tablespoonfuls of 
vinegar, or $5-10 \mathrm{G}$. (1 $1 / 4-21 / 2 \mathrm{dr}$.) of soap. When increased itching, more pronounced tenesmus, and abnormal restlessness at night make it probable that a large number of the parasites are massed in the rectum, we substitute enemas containing some drug. A popular remedy is garlic; two or three bulbs are chopped up and boiled in 1000 c.c. $(1$ qt.) of water or milk. 'The liquid is then strained and divided into three enemas, to be used on successive days. The smell that lingers so long about the patient is rather a drawback to this treatment. Instead we may employ, e.g.: thymol (1:100 olive oil), ether (4-8:80-100 distilled water), naphthaline (0.5-1:50-100 cod-liver oil), benzoin (1.2 G. (18 gr.) with the yolk of an egg and 120 c.c. (4 oz.) of distilled water for two enemas), or finally a weak bichloride solution (1-3:10000), or 1-2 tablespoonfuls of chlorine water in 1000 c.c. ( 1 qt.) of water.

To relieve the painful itching of the anus, it may be smeared once in the evening with a small quantity of mercurial ointment (dilute), as much as possible of the rectal mucous membrane being reached, or a suppository made from this salve may be used :

P Ungt. hydrargyri dil. ........... $0.30-0.50 \ldots \ldots$ gr. iv-gr. vii Olei theobromatis......................... gr. xv

M. fiat suppos.

Dent. tal. dos. no. vi.

Sig. - A suppository in the evening.

It is obvious that the greatest cleanliness must be insisted upon. The hands should be washed several times a day, the nails cut and scrubbed with a stiff brush, and the buttocks and anus washed after every movement of the bowels, for infection generally occurs by the direct transference of the eggs by the fingers from the anus to the mouth. [Cleanliness and perseverance will obviate the use of santonin and the medication of the enemas. Persistent thorough flushing of the colon with saline enemas and prevention of reinfection, especially cleaning the hands and nails, will cure even the most obstinate case.-Ed.]

Ankylostomiasis.-Finally the therapy of ankylostomiasis may be briefly discussed, although the author has personally had very little experience with it. The patient is prepared the day before exactly as in the case of tapeworm. On the next day after breakfast he is given male fern-again as in tapeworm-or thymol, which the author found successful in the few cases that have come under his personal observation. The dose is 1-2 G. (15-30 gr.) in a gelatine capsule. Either 15-20 G. $(4-5 \mathrm{dr}$.) are given on one day, or else the quantity is spread over several days, $2 \mathrm{G}$. ( $\mathrm{r} / 2 \mathrm{dr}$.) being given two hours apart. If the examination of the stools shows any trace of eggs, the treatment must be repeated, sometimes more than once. [Stiles agrees with Sandwith in the choice of thymol and administers 2 G. (30 gr.) at 8 A.M., again at 10 A.M., and castor oil or magnesia at 12 noon. The day before and two days after the treatment, the patient is placed on a milk and soup diet. Contrary 
to Sandwith he advises against the use of alcoholic stimulants during the administration of thymol (because the drug is soluble in half its weight of alcohol), but is inclined to rely upon hypodermic stimulation to counteract a state of exhaustion or debility. He believes that our American hookworm is less difficult to expel than the old world species. The vegetable cathartics or calomel would seem to me safer than either oil or magnesia, because thymol is freely soluble in oils and forms soluble compounds with alkalies. For the same reason I prefer to avoid the cream and milk he advises in the diet and too much fluid. For we are employing thymol for its local action and require larger and more concentrated doses than are quite safe. Attention to these details will nearly always prevent poisoning. If symptoms of poisoning do develop, such as dizziness or discoloration of the urine, the drug must be stopped, and hypodermic stimulation, eatharsis, and irrigation of the colon promptly instituted.-Ed.] The symptoms (anæmia) which remain after the parasites are expelled, will almost always disappear of themselves, though they may of course be benefited by the appropriate therapy. 


\section{XII.}

\section{THERAPY OF DISEASES OF THE PERITONEUM}

\section{THERAPY OF PERITYPHLITIS [APPENDICITIS.-Ed.].}

Definition.-Under the term perityphlitis are here included all inflammatory and suppurative processes that start from the vermiform appendix or-very rarely-from cxeal uleers, and affect the subperitoneal tissue around the cxeum. Therefore neither simple eatarrhal appendicitis nor appendicular colic are included; but, since their therapy is identical with the medical treatment of perityphlitis, they scarcely demand special discussion.

Medical Treatment.-The medical treatment of perityphlitis depends upon the universal law that an inflamed organ must be given all possible rest.

Rest in Bed.-The patient should lie quietly with the lower extremities, or at least the right lower extremity, slightly flexed and abducted at the hip joint and flexed almost to a right angle at the knee. The rest of the body should be somewhat raised. This is the position which most patients naturally take, but they should be encouraged to retain it permanently by having a pillow folded double and placed under the knee. The patient must be strictly forbidden to turn over, sit up, or rise. When the bed pan is used, he must be lifted on to it without any exertion on his own part. This absolute rest must be kept up for at least a week after the disappearance of all acute symptoms, general and local.

Rest for the Intestine.-Especial eare must also be taken to keep the intestine as quiet as possible, in order to prevent the spread of the inflammatory process or a perforation of the abscess. Even after the inflammation has begun to subside, the intestine must still be kept quiet to prevent the possibility of fresh extensions of the lesion, or of the tearing apart of the new peritoneal adhesions. Everything which even slightly stimulates peristalsis, must therefore be avoided.

Diet.-A patient with perityphlitis must be kept upon a liquid diet until the acute stage has passed, the local pain and tenderness have wholly vanished, and the temperature has been normal for several days. Milk is the principal food, given in small quantities-several tablespoonfuls to a wineglassful every half hour to an hour. Broth, with or without the beaten white of an egg, may also be given. To either milk or broth some milk preparation (milk somatose, sanatogen, encasin) may be added later. However, in the presence of severe or repeated vomiting, par- 
ticularly at the beginning of the disease, an absolute fast for 24-36 hours, or even for several days or a week, is indicated; for the vomiting (with the accompanying muscular contractions) may lead to an increase of the inflammation or to a perforation into the peritoneal cavity. The patient may be allowed swallows of cold water, or, to quench the torturing thirst, may hold pieces of ice in his mouth, without swallowing the water if severe nausea is present. His mouth should also be washed out several times a day with a cloth wet in cold water, or rinsed with an alkaline solution. [For several excellent solutions see pp. 269, 272.Ed.] Enemas, $250-500$ c.c. ( $1 / 2-1$ pt.) of water several times a day, are sometimes used, but the author considers them contraindicated on account of the necessity of keeping the intestine quiet. When all vomiting and nausea have subsided, ice-cold milk or bouillon may be given in small quantities every hour. The transition to solid food should be exceedingly gradual (see p. 440). The author very rarely uses nutrient enemas, as he believes that absolute rest on the part of the patient is more important than nourishment, as long ás the disease is in the acute stage.

Medication: Cathartics. - No cathartics should be given, for although, in the author's opinion, cases of perityphlitis due to impacted feces do occur, yet they are very difficult to distinguish clinically from others. Simple irrigations may be used if necessary, as soon as the inflammatory process has ceased to spread, (200-500 c.c. (8-17 oz.) of tepid water). These will often cause a rapid diminution of the ileocæcal tumor, a quick disappearance of the fever, and a prompt improvement of the meteorism, gastric symptoms, and subjective distress. Occasionally in this stage the author has given 10-15 G. (1 tablespoonful) of castor oil, which has often proved successful and never harmful. Generally the first spontaneous movement of the bowels comes towards the end of the first or the beginning of the second week, unless opium has been given.

Opium-Morphine.-The author formerly considered opium and morphine indispensable, since by them the intestine may be kept still, the nausea and vomiting suppressed, the torturing thirst to some extent quenched, and the pain relieved. The doses, as he then gave them, were graduated according to the intensity of the pain, just enough being given to keep the patient almost free from pain, and particularly from colicky exacerbations. In spite of these advantages he has now given up opium entirely, since its use prevents an accurate diagnosis by masking some of the symptoms, while he uses morphine only when the patient is suffering most excruciating pain, and generally even then only a single hypodermic. He prefers belladonna to other drugs, but usually confines himself to local treatment. [Einhorn still employs opium in sufficient amount to allay the pain, and follows Sahli's method of administration: 10-15 drops of laudanum are at first given every hour until there is a subsidence of the pain; then 5 or 6 drops are given every 2 hours until the pain disappears. Unless an exacerbation of pain requires another large dose, no more opium is given. If nausea or vomiting result from 
its use, a suppository may be substituted, to which extract of belladonna may be added. He does not consider morphine so satisfactory since it has but a slight effect in diminishing peristalsis. Forchheimer uses $1 / 3-1 / 4$ the medicinal dose of opium or corresponding amounts of morphine.Ed.]

Local Therapy: Leeches-Heat, Cold.-Opinions differ as to the advisability and the methods of local therapy. The author has never employed leeehes, but believes that their use at the beginning might afford temporary relief to the subjective symptoms. The choice between hot and cold applications may be left to the preference of the patient. If there is no choice, the author is inclined to use an ice-bag during the acute stage of the disease (first 4-8 days). The patient ean generally bear this continuously; if not, a simple Priessnitz application may be used, replaced after 24 hours by a compress wrung out in hot water or by a hot poultice, which helps the absorption of the exudate. This sequence, however, is of no especial importance. In case of a simple appendicular colic, a hot compress is preferable. In severe forms of perityphlitis, the author can strongly recommend the use of an alcohol dressing (5-10 folds of gauze soaked in 50 per cent. alcohol and changed once in 12 hours). Its effect is doubtless due to its causing intense hyperæmia, which helps to destroy the bacteria. [Forchheimer warns against masking of the symptoms by the use of an ice-bag,- - especially slowing the pulse.-Ed.]

The local application of iodine appears almost useless in promoting absorption, but salt or mud baths, or even simple sitz baths at home, are worth trying after the subsidence of the acute symptoms.

Therapy of Convalesence.-On account of the natural tendency of perityphlitis to recovery, the majority of cases may be improved or cured by the above therapy. Treatment should, however, be carefully continued in order, if possible, to prevent recurrence and to get rid of any residual processes which may cause subjective symptoms (thickened. inflamed appendix, adhesions of the appendix, ete.). To avert recurrence we should take great pains to secure regular movements of the bowels, employing for months the baths above described, and intestinal irrigations or mild laxatives (castor oil, rhubarb, cascara). Abdominal massage, even in its gentlest form, is contraindicated, on account of the danger of its arousing the latent pathological process. The diet must be carefully chosen, especial care being taken to avoid any hard bodies incapable of digestion (seeds, gristle, fruit-stones, etc.). Contrary to many authorities, the author forbids all outdoor sports, at least until all local tenderness has vanished and there is absolutely no trace of pain when the patient walks or goes up stairs. Even then he prefers to substitute gymnastics carefully directed by the physician. The patient should not be sent to a spa for treatment until a considerable time after the attack. If the digestion is normal and constipation obstinate, he may then be sent to Marienbad; if there are symptoms of anæmia, to Franzensbad; and, in case of dyspepsia, to Carlsbad or some other alka- 
line-saline spring or sometimes to a common salt spring, especially if diarrhœa is the most prominent symptom.

INDICATIONS FOR SURGICAL INTERFERENCE.-Although about two-thirds of the cases of perityphlitis can be satisfactorily handled without surgery, an operation is indicated in : 1 . Those chronic cases in which local inflammatory processes remain, causing the patient frequent recurrence of severe pain, and interrupting his business and pleasure to such an extent that he himself desires radical relief. 2. Cases of recurring acute perityphlitis, especially if the attacks come frequently, indicating to the author an evident incapacity for eventual spontaneous recovery. 3. Certain types of a single acute attack, whether or not it be the first one. A distinction must here be made between: I. Cases seen on the first or second day. II. Cases seen on the third day or later. The first of these groups (I) may be still further subdivided into:

$a$. Cases with several attacks of colic, generally at first in the epigastrium, then in the ileocæcal region and around the navel; normal or subfebrile temperature; pulse not essentially quickened; tongue moist; no cyanosis or icterus; local muscular rigidity and tenderness to touch or pressure in the ileocæcal region, the rest of the abdomen generally somewhat tender; possibly a local resistance. Here we may rely upon rest, diet, and, if necessary, opium.

$b$. Severe, repeated vomiting; severe colic; the whole abdomen distended and tender; no definite local signs determinable by palpation; only slight dulness in the ileocæcal region; marked local muscular rigidity; considerable fever; pulse generally much quickened. Immediate operation is here indicated. If, when operation is delayed, the pulse becomes even more rapid, small, and fluttering, or if this is the primary condition, and if to all the other symptoms are added quickened respiration, slight icterus, cold extremities, sunken countenance, profuse sweating, and dry tongue, we get the typical picture of a severe septic peritonitis. Here we usually avoid an operation. In general it is of as little use as medical treatment. Possibly, as a last resort, it may be attempted.

$c$. Severe violent onset as in $b$; high temperature; severe pain; no definite local signs; the whole abdomen tender; only slight local muscular rigidity; no indications of sepsis (pulse rapid (over 100) but good, no icterus, tongue moist, no subnormal temperature). In such cases we wait before operating, to see if medical treatment will prove effectual.

The second group (II) may be subdivided into those cases presenting:

$a$. The picture of a diffuse peritonitis without demonstrable effusion (pain, diffuse muscular rigidity, meteorism, no dulness, rapid pulse, high temperature). Here an operation has little chance of success, since many circumscribed exudative lesions are generally found between the folds of the intestine. Local cold or heat or alcohol dressings and opium offer more hope. If an abscess presents itself immediately beneath the abdominal wall, it should be opened. 
b. The picture of a diffuse suppurative peritonitis. This should always be operated on.

$c$. The picture of a common perityphlitis with demonstrable exudate (by palpation, sometimes only by percussion) in the ileocxeal region. In this very frequent condition, the diagnosis must decide whether the exudate is fibrinous, plastic, or suppurative. This decision can usually be reached only after observing the patient carefully for some length of time. The diagnostic signs of a suppurative exudate are: no recession or even an extension of the local tumor by the fifth day; marked rigidity; high temperature and relatively greater pulse frequency at the same stage; constant pain; œdema and redness of the abdomen over the tumor; marked polynuclear leucocytosis (over 25,000 leucocytes); peptonuria; and (not, in the author's opinion, a reliable sign) increased pain on the application of a hot compress. In such a case operation should be resorted to by the seventh day in spite of the fact that even then we cannot always make a positive diagnosis and that suppurative cases occasionally recover without operation. The diagnostic signs of a fibrinousplastic exudate are: diminished exudate, and general improvement by the fifth or sixth day. These cases, fortunately the most numerous of all, do not demand operation.

In spite of the strong preference of surgeons for an early operation (within 48 hours), the author cannot endorse such a procedure. He has never seen a case where the preceding directions were accurately followed, and yet operation was deferred until too late, while he has known of two cases where operation showed a normal appendix, and the condition proved to be typhoid disguised by appendicitis symptoms. In one of these cases a permanent ventral hernia was the result. Neither can he agree with the axiom of a Berlin clinician: "Better operate once too often than once too seldom." If this could be taken literally, it might be true, but the facts of the case, if early operation is carried out uniformly, oblige us to read instead of "once too often," "several times too often," or "once fatally." The author has seen such a fatal case in spite of the most correct surgery.

The conclusion of the whole matter may be summarized as follows: Operate when it is necessary, and discriminate cases of gangrenous and perforative appendicitis from simple appendicitis by the following symptoms: local painful muscular rigidity (most important); pulse over 100 (but a quiet pulse is no contraindication); repeated vomiting; dry tongue; severe pain; quickened, thoracic breathing. The temperature, blood picture, and general condition are of less importance. If even only one of these unfavorable symptoms is present, be prepared for operation.

[I cannot subscribe to the author's views upon surgical intervention. Appendicitis, and by this name I include all the conditions mentioned by the author under the title of perityphlitis as well as all forms of true 
appendicitis, is a surgical disease and should have at least the benefit of a surgeon's advice as soon as the diagnosis is made.

Keen expresses the American point of view in saying, "The first indication in appendicitis is to call in a surgeon."

Maurice Richardson, after an experience of more than 1000 cases, cited the following indications for operation in acute appendicitis:

1. In all severe cases seen early, unless there are contraindications to operation in other organs or the patient's general condition.

2. In all severe cases which when first seen are at a standstill or increasing in severity.

3 . In all severe cases, unless they are unmistakably improving.

4. In severe cases in which the symptoms are well marked and well localized and in which the appendix is easily accessible, i.e., in the right iliac fossa or in the lumbar region.

5. In all cases in which it is clear that the disease is limited to the appendix itself and in which it is presumably certain that the abdomen can be closed without drainage.

In reviewing 2000 operations for appendicitis, Murphy divides the time of operation into 4 classes :

1. Within the first 48 hours.

2. In the active increasing inflammatory processes from the second to the fifth day.

3 . In the subsiding inflammatory processes from the fifth to the seventh day on.

4. In the intermediate stage between attacks.

He considers the first period the time of election and that the danger is scarcely more than that of an exploratory laparotomy. In the second stage he also urges operation but emphasizes the necessity of rapidity and cautions against manipulation. He simply opens and inserts an efficient drain, and, in cases where the constitutional reaction is mild and the appendix readily accessible, he also removes the latter. In the third stage operation is not so urgent, but the presence of pus he considers always an element of danger and so operates wherever he is certain that pus exists. In the fourth stage he advises operation upon every patient who has been through an acute attack of appendicitis.

Kelly summarizes the advantages of early operation as follows: In the first class the operation is safest, is more easily performed, the duration and intensity of the patient's suffering lessened, the liability of recurrent attacks and of hernia is obviated.

In the second class of cases he advises operation upon cases which manifestly are getting worse and those which are not distinctly improving, and cases which are undoubtedly on the mend, unless they are very 
favorably situated for careful continued observation and prompt skilful intervention, (such as in a hospital).

In the fourth class Kelly urges a delay of at least several (4-6) weeks after the subsidence of the acute severe attack.-Ed.]

\section{THERAPY OF ACUTE, DIFFUSE PERITONITIS.}

Aside from the exceedingly severe septic forms of peritonitis mentioned above, in which practically no therapy is of any avail, acute diffuse peritonitis presents itself under two forms: (1) acute perforative peritonitis and (2) acute fibrinous-suppurative peritonitis or chronic inflammation.

Perforative Peritonitis.-In the first class of cases, immediate operation should always be performed, unless contraindicated by protracted shock. In the latter case we must do our best with morphine or opium and heart stimulants (camphor, ether, heat to the abdomen and the extremities). If the collapse is less pronounced, we must treat the peritonitis by all the non-surgical means at our command.

Fibrinous-Suppurative Peritonitis: Laparotomy.-In the second class of cases (acute fibrinous-suppurative peritonitis), unless perforation into the peritoneal cavity takes place rapidly, operation should be resorted to only as a last resort. For on the one hand a fibrinous-suppurative peritonitis may eause many peritoneal adhesions possibly leading to a multiple encapsulation of the exudate, while on the other hand laparotomy exposes the whole peritoneum to infection; hence operation appears to be an added danger rather than a means of relief.

Internal Therapy.-The internal therapy of a fibrinous-suppurative peritonitis is almost exactly identical with that of perityphlitis, which is merely a circumseribed form of the same condition.

Diet.-The patient must take nothing by the mouth for the first few days or, if necessary, the first week of the disease. He may relieve his thirst by letting pieces of ice dissolve in his mouth without swallowing the water. Only after disappearance of the vomiting, singultus (hic. coughs), and marked meteorism, can we begin on a very careful diet, supplemented by non-irritant nutrient enemas.

Stools.-No enemas should be given at first to combat constipation and meteorism. Later, small and afterwards larger ones may be used, but cathartics must be entirely avoided.

Opium.-Opium is the most valuable of all remedies, having, besides the effect spoken of in the discussion of perityphlitis, the additional good results of hindering the spread of infection from one part of the intestine to another by contact, of favoring peritoneal adhesions, and often of combating collapse and shock.

The author prefers the powder or extract, preferably by the mouth, though, if necessary, in the form of suppositories. The tincture is less useful. They are all given every hour or two in divided doses. We begin 
with one-half the maximum daily amount, increasing if necessary to the full maximum doses. As soon as the pain has been relieved, the dose is reduced to just the amount needed to deaden the pain and then increased again, if necessary. For example, 4 drops of the tincture every hour or 7-8 drops every two hours at first; then 3-4 drops every two hours; then possibly the original larger doses again, or even a single dose of 20 drops. If we use the powder we may begin with $0.02 \mathrm{G}$. (1/3 gr.) every hour or $0.04 \mathrm{G}$. ( $2 / 3 \mathrm{gr}$.) every two hours. If vomiting makes it necessary to use suppositories, three or four $0.1 \mathrm{G}$. (1 $1 / 2 \mathrm{gr}$.) suppositories may be given. If this method of administration also is impossible, we may use a hypodermic of morphine (0.03-0.12 G. $-1 / 2-1^{4} / 5$ gr.) [a very large doseEd.].

Rest in Bed.-The patient should be kept absolutely still in bed, if possible, but there are some cases where the pain is so intense that not even opium can insure quiet.

Local Heat or Cold.-Whether hot or cold applications should be used on the abdomen may well be decided by the patient's own preference. Leiter's tubes, alcohol dressings, hot poultices, Priessnitz binders, and hot compresses are all available.

Symptomatic Therapy.-For vomiting we give morphine or chloroform water, sometimes cocaine and pieces of ice. The frequently troublesome singultus (hiccough) may be relieved by the same means, or (Penzoldt) by atropine $\left(0.0005-0.001 \mathrm{G} .\left(1 /{ }_{120} 0^{-1 / 60} \mathrm{gr}.\right)\right.$ per dose, 0.003 G. $\left(1 /{ }_{20}\right.$ gr. $)$ per day). Severe meteorism, bringing with it the danger of asphyxia, is best treated by the insertion of a very soft rectal tube, which may be left in for hours at a time. For collapse we use hypodermics of salt solution, ether and camphor, wine, vinegar enemas, hot cloths, hot water bottles, etc.

\section{THERAPY OF CHRONIC TUBERCULOUS PERITONITIS.}

Rest in Bed.-Tuberculous peritonitis is also on the border line between medicine and surgery. The physician seeks to aid nature's efforts at healing by the best possible nourishment, by strict rest, if possible in bed and in the fresh air (fresh air cure), or at least in a well ventilated room.

Abdominal Bandages.-A rather tight abdominal bandage is, also, of great aid in keeping the abdomen quiet.

Potassium Soap.-In stimulating the absorption of the exudate, the author has found alcohol dressings and the treatment with green soap most efficacious. The latter is carried out as follows: One tablespoonful of soap is dissolved in warm water to the consistency of a soft paste and then rubbed into the skin of the abdomen and allowed to stay on for half an hour. If this treatment causes eczema, the skin of the back or buttocks may be rubbed instead, until that of the abdomen is well again. The author has had marked success with this method, sometimes even securing 
a complete and rapid disappearance of the exudate, which, however, leaves behind peritoneal adhesions or a thickened great omentum. Alcohol dressings, if used at the same time, should be confined to the abdomen, while the soap may be used on the rest of the trunk. Cod-liver oil or unguentum cinereum G. P. (2-4 G. (1/2-1 dr.) daily) similarly used, has proved less successful. [Forchheimer recommends creosote vasogen.-Ed.]

Diuretics.-Occasionally a diuretic apparently produces the desired effect,-urea, for instance, prescribed as follows :

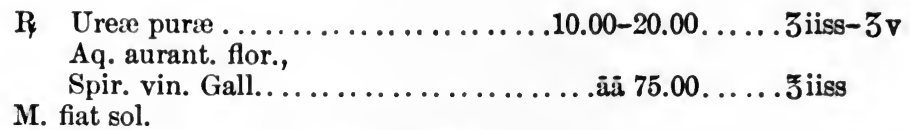

Sig.-A tablespoonful every 2 hours.

Or parsley water (150 G. (5 oz.), a tablespoonful every two hours). It is, however, exceedingly difficult to judge of the actual worth of any of these remedies, since the condition is capable of spontaneous improvement.

Symptomatic Therapy.-Symptomatically we may be obliged to treat the pain, constipation and diarrhœa, or fever.

When the fever has gone and the size of the abdomen has become diminished, the patient may be given cod-liver oil, lipanin, or von Mehring's Kraftschocolade.

Surgery.-If all other measures fail, we may resort to laparotomy, or, less often, to paracentesis and a tight bandage. By the first method many cases are healed, although peritoneal thickenings and growths are often left behind. An exploratory incision is indicated only in the case of an abundant, serous exudate. [Spontaneous healing occurs often enough in this disease to warrant a preliminary attempt at careful and thorough medical treatment, at least in every case seen early. Rotch, who advocates operative interference as the first measure in this disease, has obtained exceptionally good results. Tuberculin has not been used extensively enough to merit judgment upon its efficacy in this form of tuberculosis.-Ed.] 


\section{XIII.}

\section{THERAPY OF DISEASES OF THE LIVER AND}

\section{GALL PASSAGES}

\section{THERAPY OF CATARRHAL JAUNDICE.}

Some individuals with catarrhal jaundice are not sufficiently ill to stop their usual work and would not realize their condition except for the color of their skin and conjunctivæ; others are sick enough to be confined to bed.

Cathartics.-As the condition is produced by catarrh of the stomach and intestines, it is obvious that its initial symptoms and its treatment, at least in the earlier stages, are identical with those of gastroduodenitis. Before the appearance of the jaundice, however, several days have almost always elapsed after the initial indigestion; hence cathartics are indicated rather than emetics or lavage. We may use calomel, castor oil, bitter waters, or saline cathartics, bearing in mind that some cathartics, like podophyllin, rhubarb, and scammony, are useless when there is no bile in the intestine.

When once the symptoms of an acute gastroduodenitis have subsided, we direct our therapy against the condition due to the obstruction of the bile passages and the resulting lack of bile in the intestine.

Diet.-In all acute cases of biliary obstruction, and generally in chronic cases, we avoid the ingestion of fat, since, as the abnormal amount of fat in the feces shows, its absorption is hindered by the lack of bile. We also know that the splitting up of the fats by the pancreatic secretion is much disturbed. In some cases, especially those accompanied by extreme prostration, emaciation, or a rapid cachexia suggesting carcinoma, there is reason to suspect a coincident obstruction of the pancreatic duct. If this is confirmed by a microscopic examination of the stools and urine (large numbers of undigested meat fibres, starch granules and fat, glycosuria, sometimes maltosuria, lack of indicanuria in spite of a proteid diet), there is, of course, added reason for excluding fats from the diet. Some chronic cases, however, can take butter.

Unless proteids are contraindicated by abnormal proteid decomposition in the intestine or by the obstruction of the pancreatic duct, easily digestible proteids and carbohydrates may be given as soon as the gastroduodenitis has subsided.

Two litres (2 qt.) of milk should be taken daily, and the carbohydrates that contain the least cellulose; e.g., gruels made of arrowroot or 
other fine meals, toast, zwieback, crackers, eucasin or sanatogen biscuit, mashed potatoes, souffles, grape or milk sugar, and malt extract. For proteids we may select easily digestible forms of meat, eggs, broths, pea or bean soup, and aleuronat bread. Although meat undoubtedly contains more ptomaines than vegetable food, and although these ptomaines need the action of the bile to render them harmless, yet it seems unnecessary to insist on giving the vegetable portion of the proteid diet great predominance over the animal. The patient may drink water, milk, cocoa, café au lait, tea, and the common table waters; alcohol is prohibited from the beginning by the primary gastric and intestinal catarrh.

Alkaline-saline Waters.-To reduce this primary catarrh and icterus. we give alkalies internally as a routine measure, in the form of alkalinechloride or, better, alkaline-saline waters or their salts. Carlsbad, Vichy, and Ems have a world-wide reputation in this connection. We are not, however, clear about the exact action of these alkalies. It may be that they are passed through the liver and biliary passages and in the process have a sedative effect on the swollen and inflamed mucous membrane. But it is more probable that the carbon dioxide and the sodium sulphate in these mineral waters directly stimulate the flow of bile, making it thinner and less concentrated. Or it may be that they simply have a favorable effect on the duodenum and so hasten the opening of the obstructed bileduct. Or, finally, their effect may be due merely to the large amount of liquid ingested, which helps to thin the bile. For this reason it seems well to order large quantities of liquid, as far as the state of the digestion will permit. Carlsbad or Vichy should be given at the body temperature, one glass the first day, two the next, and after that three a day, 25 minutes apart. The water should be sipped slowly. It is well to continue the treatment for some time after the jaundice has entirely disappeared. A teaspoonful to a tablespoonful of Carlsbad salts added to $250-500$ c.c. $\left(1 / 2-1\right.$ pt.) of water at $98^{\circ}-104^{\circ}$ may be substituted for the natural water. [Instead of one of these waters I frequently employ a teaspoonful of phosphate of soda in a tumbler of hot water to be drunk slowly upon an empty stomach 2-4 times a day.-Ed.]

Krull's Irrigations.-In case a proper diet and the use of mineral waters do not have the desired effect, we may try the so-called "Krull's irrigations," that is, a daily quick colon irrigation with a litre (1 qt.) of water at $59^{\circ}-68^{\circ}$ or even ice-cold. This cold irrigation apparently causes violent peristalsis but with it strong contractions of the musculature of the biliary passages, which start a renewed flow of bile into the intestine. Possibly this contraction is also helped by a slight irritation of the bile duct, caused by the stretching of the colon on account of the amount of liquid. Like other irrigations, they also help the functioning of the liver by quickening the circulation in the portal veins and possibly by affecting the hepatic nerves. In many cases the author has observed a rapid retrogression of obstinate jaundice, and a surprisingly quick recovery and return of appetite. 
Exercise.-If the jaundice lasts for some time, outdoor sports, as far as the patient's strength will permit, abdominal massage, and breathing exercises are all efficacious in helping the flow of bile into the intestine. [Lauder Brunton recommends inspiratory exercises and the various sports to increase the expression of the bile by the more powerful excursions of the diaphragm.-Ed.]

Cholagogues.-For the same purpose the cholagogues formerly much used are sometimes employed to-day. The author considers euonymin and podophyllin quite useless, and puts the most faith in sodium salicylate (0.5-1.0 G. (7-15 gr.) several times by the mouth or rectum), and the bile salts, although he does not by any means feel sure that either of these has the desired effect. The bile salts are given as follows :

R4 Fellis bovis purificati,

Pulv. rhei comp......

M. fiat caps. no. xxx.

Or :

Sig. - 1 or 2 capsules 3 times a day.

R. Sodii glycocholatis, Pulv, rhei comp. ................āā 4.00.....3i

M. fiat caps. no. xii.

Sig. -1 or 2 capsules 3 times a day.

[Pulv. rhei comp., which contains oxide of magnesia, has been substituted for the author's selection of plain rhubarb. The magnesia will help to counteract the nausea.-Ed.]

Eunatrol (sodium oleate) seems to have an equally questionable effect. It is on the market only in tablet form, $0.25 \mathrm{G}$. (4 gr.), and is best given in doses of 4 tablets twice a day.

Itching.-When catarrhal jaundice persists for some time, we often have to combat the intense itching, a condition which still oftener accompanies chronic jaundice in other diseases of the liver and biliary passages. One remedy helps one patient; another, another. It frequently happens that the effect of any one wears off in a few days, so that the patient requires a change. The author most frequently uses tepid baths, sometimes putting 100-120 G. (3-4 oz.) of sodium bicarbonate in the water. Besnier's "clover baths" he also finds useful. Many patients are relieved by cold sponging, especially with diluted alcohol, or by rubbing the skin with lemon-peel or smearing it with bacon fat; others, by cold baths or packs. Sometimes the spirits of menthol, or, if the itching is circumscribed, menthol ointment is efficacious :

P Mentholis ...................... $1.00-2.00 \ldots \ldots$ gr. $\mathrm{xv}-\mathrm{gr} . \mathrm{xxx}$

. Alcoholis dil. .................80.00.... .

M. fiat sol.

Or :

Sig. - To be rubbed into the skin.

P Mentholis........................2.00....gr. $\mathbf{x x x}$

Ol. olivæ $\ldots \ldots \ldots \ldots \ldots \ldots \ldots \ldots \ldots . . .6 .00 \ldots \ldots$.

Lanolini ..........................50.00.... Jiss

M. fiat ungt.

Sig.-As an ointment, for external use. 
Or: R Mentholis,

Zinci oxidi .äā 5.00......3i gr. xv

Amyli,

Talci .āå 30.00 .

M. fiat pulv.

Sig. - To be powdered on the skin.

A 2 per cent. solution of carbolic acid is good in many cases, but must be used with great caution where there are any considerable excoriations on the skin. Toxic absorption must also be carefully watched for.

Again, some patients are helped by salicylic acid used either as a powder in combination with talcum, or as follows in alcoholic solution :

R Acid. salicylici .....................4.00....3i

Alcoholis dil....................200.00,.... ₹viss

M. fiat sol.

Sig.-External use.

In other cases ichthyol proves efficacious:

R Ichthyolis $\ldots \ldots \ldots \ldots \ldots \ldots \ldots \ldots \ldots . \ldots \ldots$. $10.00 \ldots \ldots$ iiss

Alcoholis,

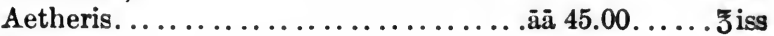

M. fiat mist.

Sig. - To be rubbed on the skin.

Or an infusion of coca leaves:

R Cocoæ ...........................15.00.....

Aq. dest...........................1000.00..... Oii

M. fiat infus.

Sig. - To be rubbed on the skin.

Bamberger especially recommends amygdalin, which the author has found sometimes useful, sometimes not.

R Amygdalini $\ldots \ldots \ldots \ldots \ldots \ldots \ldots \ldots \ldots .2 .00 \ldots \ldots$ ss

M. fiat sol.

$200.00 \ldots . . .5$ viss

Sig. - To be rubbed on the skin.

The author has, however, obtained the best results from two new preparations, bromocoll, (bromine, tannin, and gelatin), and anæsthesin (the ethylester of para-amido benzoic acid) both in the form of ointment. [Bromocoll is marketed as a 20 per cent. ointment, having a special base, which increases the value of the preparation.-Ed.] Anæsthesin is best dispensed according to the following:

R Anæsthesini ....................... 10.00....

Petrolati,

Lanolini......................āä 50.00..... ₹ iss

M. fiat ungt.

Sig.-External use. 


\section{THERAPY OF CHOLELITHIASIS.}

The therapy of cholelithiasis may be divided into: (1) the treatment of an acute attack of gallstone colic; (2) the treatment between attacks; and (3) the treatment of the complicating inflammation of the biliary passages.

Therapy of an Attack of Gallstone Colic: Narcotics.-In treating an attack of gallstone colic the most important indication is the mitigation of the often overpowering pain. There is no better remedy than morphine, which not only relieves the colicky pain but, by relaxing the spasmodically contracted muscles of the biliary passages, favors fresh peristaltic movements, which force the stone farther toward the intestine. If the stone becomes lodged again, another injection of morphine must be given. At first we inject $0.02 \mathrm{G}$. ( $1 / 3 \mathrm{gr}$.) ; afterwards smaller amounts, graduated according to the nature of the attack. In less severe attacks opium may be given by the mouth or by the rectum.

Belladonna is not so powerful, even in comparatively mild attacks. It may be given in several forms:

P Belladonnæ fol. ................. 0.50-1.50.....gr. viii-gr. xxii Aq. dest..................... 150.00... . $\mathrm{v}$

M. fiat infus.

Or :

Sig. - 1 tablespoonful every half hour or every hour (Sticker).

I $\quad$ Ext. belladonnæ fol. ..................1.00.....gr. xv

Aq. amygdal. amar..................10.00.... 3iiss

M. fiat sol.

Sig.-10 drops every hour; shake before using (Leichtenstern).

[I prefer atropine sulphate hypodermically in doses of 0.0016 G. $(1 / 40$ gr. $)$ or of one half to one quarter this amount. If the second dose has not relieved the condition, the danger of poisoning is too great for further repetition.-Ed.]

The author has not been successful with chloroform (4-5 drops on cracked ice), chloroform water (1 tablespoonful every hour), or chloral. Some authorities recommend antipyrine ( $0.5 \mathrm{G}$. ( $7 \mathrm{gr}$.) every hour) or sodium salicylate (first dose, $2-3$ G. (30-45 gr.)), provided it is given at the very beginning of the attack. The author has no faith in this treatment, although he has not tried it himself.

Heat-Cold.--Local heat in all forms and often a protracted hot bath $\left(104^{\circ}-122^{\circ}\right)$ or hot drinks help to relieve the pain. Occasionally heat cannot be borne, but local cold is effective. Possibly these are cases where the biliary passages are infected, so that the simple attack is complicated by inflammation.

Stimulants.-Occasionally the pain is so severe as to cause a more or less serious state of collapse, demanding the use of morphine or, if the individual appears incapable of resistance, of strong stimulants. [My usual course of treatment when called to relieve an acute attack is 
promptly to immerse the patient in a very hot bath and to keep him there for one-half to three-quarters of an hour at least. At the same time I administer atropine as above. If this method is not successful within an hour, I usually repeat the atropine or give a few whiffs of chloroform and a hypodermic of $0.015-0.03 \mathrm{G}$. ( $1 / 4-1 / 2 \mathrm{gr}$.) of morphine.-Ed.]

Therapy Between Attacks: Mineral Waters.-The therapy of an uncomplicated cholelithiasis between attacks is confined to the administration of alkaline, alkaline-chloride. or most frequently alkaline-saline waters. Three to six glassfuls a day should be taken, as hot as possible $\left(122^{\circ}-140^{\circ}\right)$. Or a cooler spring (Schlossbrunnen) may be chosen for the first fortnight. The spas most frequently used for this purpose are: Carlsbad, Vichy (Grande Grille, Céléstine, L'hôpital,), Ems (Kesselbrunnen and Kränchen), Neuenahr, and Bertrich. The hot common salt springs may also be used; e.g., the Kochbrunnen at Wiesbaden, especially if there is a tendency towards diarrhoa, Marienbad in case of obesity and abdominal plethora, and Tarasp if there are concomitant nervous symptoms. If the Carlsbad waters are given at home, 3 glassfuls at $104^{\circ}-122^{\circ}$ should be taken in the morning on an empty stomach, and sometimes three more glassfuls in the afternoon, or two on rising, two an hour before the midday meal, and two four hours afterward. The patient should not exercise for $1 / 4-1 / 2$ hour after drinking. It is highly improbable that these waters have any direct solvent action upon the stone; their effect is probably due to their influence upon the secretion of bile and upon the catarrh of the biliary passages and intestine, and to the hygienic life led at the springs. The treatment should last a month and should be taken once a year at the springs and three times at home, even if the patient is suffering no trouble. [ $I$ believe with Naunyn, Forchheimer, and others that the results of a Carlsbad cure at the spa are convincing. and advise this course wherever it is feasible for the patient to go there.-Ed.]

Olive Oil.-Beyond the use of mineral waters there is almost nothing to be done between attacks. Some authors warmly recommend olive oil; others reject it entirely. [The method was first advocated by an American, Kennedy.-Ed.]

Rosenberg prescribes it as follows:

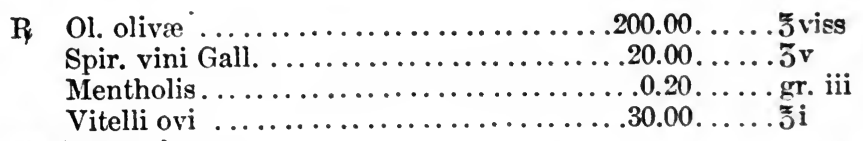

M. fiat emuls.

Sig. - To be taken within an hour, $\frac{1}{2}$ at a time. The disagreeable taste left in the mouth may be removed by orange or lemon juice.

It may be given every day for a fortnight, or its use may be interrupted during that time. It is possible that it has a direct forcing action on the stone. [It is more probable that the oil acts as an emetic or laxative.-Ed.] 
Butter.-For the same purpose a large amount of butter, $20 \mathrm{G}$. $(2 / 3 \mathrm{oz}$.), on a little bread or crackers every morning, is sometimes prescribed.

Eunatrol, etc.-The author has had no success with eunatrol, with chologen (a combination of mercury with vegetable cathartics), with cholagogues (podophyllin), or with antispasmodics (camphor, etc.).

Glycerin.-Glycerin (30 G. (1 oz.) a day with lemon juice), although not wholly inert, is by no means so efficacious as in renal stone. It and oil are apparently indicated only when the stone has lodged in the hepatic or bile duct and cannot be quickly expelled.

Diet.-The proper diet depends upon whether or not chronic jaundice is present. If it is, fat must be excluded, as in catarrhal jaundice, and only easily digestible proteids and carbohydrates given. If, however, the flow of bile is normal, especially if the stone is in the gallbladder or the cystic duct, an abundant diet is indicated in order to help maintain the flow of bile and prevent sedimentation and the formation of concretions. For this purpose five meals during the day and one during the night (Kehr) may be given (a glass of milk and zwieback or crackers after midnight) for at least a half year after all the symptoms have disappeared. Bread and butter may be given in the evening for the sake of the action of the fat. The patient should drink a good deal of water and alkaline mineral waters. Good wine or liquor need not be entirely forbidden, as Weintraud has shown that alcohol does not enter the bile. [The explanation of the utility of large amounts of fluid or of alkaline waters is not certain. Experimental evidence seems to show that the latter do not influence the alkalinity nor does the excessive fluid dilute the bile. It is more than probable that their effect is due to their beneficial action upon any coincident catarrh of the stomach or intestines, and to their stimulating peristalsis and serving as a laxative. Coarse foods, fresh fruits, salads, legumins, excessive starches, sugars, fats, and alcohol should be avoided. At the same time the diet should not be too uniform. Frequent meals, according to Frerichs, keep the bile from stagnating in the bile passages or gallbladder.-Ed.]

Surgery.-If in spite of the foregoing treatment a gallstone remains lodged in the common duct, surgery must be resorted to. Laparotomy is indicated: ${ }^{1}$ 1. In chronic gallstone icterus. Here it should be undertaken within 2-3 months, as an icterus of long standing weakens the patient for the operation and favors hemorrhage. 2. In case of frequently recurring attacks of colic, provided the stone is not passed, especially if the patient's strength or, reflexly, the muscular power of the heart, is being reduced. It should be done in case the patient is much disturbed in his business, but may be avoided in case he has time and opportunity to undergo the proper treatment at some spa.

${ }^{1}$ [Operation, if merely an exploratory one, is always indicated in the presence of symptoms which threaten life.-Ed.] 
In case the cholelithiasis is accompanied by an infectious cholecystitis with cholangitis, we first try symptomatic therapy (local cold or heat, Carlsbad water). If in a few weeks this has not lessened the symptoms, we next put the patient on a strict diet and antiseptic-cholagogue medication. This course should also be followed if, often at irregular intervals, attacks of colic occur (generally with fever, with or without icterus, and with digestive disturbances as a symptom complex of an infection of the biliary passages), although it may not be possible to decide whether stones are present or whether the difficulty is due simply to cicatricial alterations in or around the biliary passages. In such cases the author agrees with Chauffard in giving 3 or 4 times a day a powder which contains $0.5 \mathrm{G}$. ( $7 \mathrm{gr}$.) each of sodium salicylate and sodium benzoate (sometimes with the addition of 1-2 G. (15-30 gr.) of Carlsbad salt), ${ }^{2}$ and with this twice a week $0.10 \mathrm{G}$. (1 $1 / 2 \mathrm{~min}$.) of Oleum Haarlemense in gelatin capsules. This medication should be continued for 20 days at a time for several months; then, if the patient is in good condition, for 15 days at a time, and finally for 10 days, the treatment being kept up in all for a year or more. For the first week an exclusive milk diet should be insisted upon; in the second, vegetables may be given; and in the third, meat. In one case which was subject to repeated attacks of this kind, in spite of frequent treatment at Carlsbad, the above medication has relieved the patient entirely for seven years; in other similar cases it has produced a great improvement. In case it does not succeed, however, reeourse must be had to laparotomy. Laparotomy is also indicated: In case hydrops of the gallbladder, often occurring as an independent affection, causes unpleasant subjective symptoms; in case the stone has caused rupture of the gallbladder or biliary passages with consequent perforative peritonitis; in case the peritoneal inflammation has caused adhesions of the gallbladder with the neighboring organs (stomach, colon, duodenum), resulting in serious disturbances (gastrectasis, biliary colie, etc.).

[The indications for operation are, according to Osler:

"(a) Repeated attacks of gallstone colic. The operation is now attended with such slight risk that the patient is much safer in the hands of a surgeon than when left to nature, with the feeble assistance of drugs and mineral waters. (b) The presence of a distended gallbladder, associated with attacks of pain or with fever. (c) When a gallstone is permanently lodged in the common duct, and the group of symptoms above described are present, the question, then, of advising operation depends largely upon the personal methods and success of the surgeon who is available.

In 1,000 consecutive operations for gallstone disease the brothers Mayo, of Rochester, Minn., had 50 deaths, 5 per cent. In 673 cases of

2 [I am a warm advocate of phosphate of soda, see p. 521.-Ed.] 
cholecystotomy the mortality was only 2.4 per cent. In 186 cholecystectomies the mortality was 4.3 per cent. In 137 operations for stone in the common duct the mortality was 11 per cent."'-Ed.]

\section{THERAPY OF CIRRHOSIS OF THE LIVER.}

Therapy of a Hypertrophic Biliary Cirrhosis and of the Early Stages of aN Atrophic CirRhosis.-Clinically we distinguish between a hypertrophic biliary and an atrophic cirrhosis. But the therapy of the two is the same as far as the first stages of the latter are concerned (preatrophic, or atrophic without ascites).

Diet.-The diet must be so chosen as to be nourishing and yet to spare the liver cells as far as possible, and must also take into consideration the gastric and intestinal catarrh. Meat should be restricted and eggs or gelatinous foods substituted. Milk, farinaceous foods, vegetables, cooked fruit, and carbohydrates in general may be given. Sharp condiments must be avoided, and alcohol, also, unless the patient is so addicted to the latter that its entire withdrawal would mean the danger of collapse. In that case we may give a little in the form of kefir, cider, or wine. Whether fat (butter) may be allowed should depend entirely on the individual tolerance of the patient.

Mineral Waters.-Medication usually consists of the administration of mineral waters, most frequently Carlsbad water either at the springs or at home. The treatment should be repeated every year. We may also use Ems, Vichy, Neuenahr, or Marienbad in case of plethora and obesity, and Wiesbaden (Kochbrunnen), Kissingen, or Homburg in case of digestive disturbances. For the details of their administration, see p. 475. Their effect in hypertrophic cirrhosis is to be explained in the same way as in chronic obstruction of the biliary passages. In atrophic cirrhosis before the stage of ascites, it is due to their influence on the coincident catarrh of the stomach and intestine, and to their stimulative effect on peristalsis with the consequent improvement of the portal circulation.

Laxatives: Calomel.-Other laxatives are also given. In hypertrophic cirrhosis calomel is often recommended ( 0.06 G. (3/4 gr.), 3-6 times a day for a month or so). The author has tried it in several cases without any decided influence on the course of the disease. [The danger of salivation and stomatitis would deter me from so long a course.-Ed.]

Cream of Tartar, Etc.-In both forms of cirrhosis complicated by meteorism or constipation, any of the mild laxatives may be employed (rhubarb, bitter salts, aloes, cascara, bitter waters, Carlsbad water, or Marienbad salts). The author has repeatedly followed Sasaki's recommendation and given cream of tartar for atrophic cirrhosis, but without any success.

Stomachics.-Stomachics may be used symptomatically.

Therapy of the Later Stage of Atrophic Cirrhosis: Heart Stimulants. - When atrophic cirrhosis is accompanied by ascites, the first 
requisite is to make sure whether the latter is due simply to the diseased liver alone or also, as is not infrequently the case, to cardiac insufficiency. In the latter case digitalis or other cardiac stimulants are indicated. though the heart muscle in most cases is generally too much degenerated for success.

Diuretics.-If, however, the ascites is due to the liver alone, diureties are our only resource, and their effect is only temporary. The author prefers, besides calomel, theobromine and its double salts, sometimes also employing the double salts of caffeine, and old-fashioned diuretic teas. In judging of the effect of any of these, it must be kept in mind that even a severe ascites in atrophic cirrhosis sometimes improves spontaneously, perhaps in consequence of the opening of new collateral venous channels. Two such cases have been known to the author. [A pocynum cannabinum has been used recently by a number of observers with great success. It slows and steadies the cardiac action, increases blood-pressure, stimulates the kidneys (probably by dilating their arterioles), and seems to have a tonic effect on the general capillary system, thus lessening the transudation of serum. Lloyd's tincture is generally used in doses of $0.12-0.18$ G. (2-3 min.) every 3 or 4 hours. The drug is not, however, free from dangerous qualities (Packard).-Ed.]

Abdominal Puncture.-In the majority of cases, however, diuretics are of so little avail that abdominal puncture is the only ultimate resort. This should be undertaken when the collection of fluid is so great as to interfere with the heart and respiration. Severe and painful œedema of the lower extremities, severe digestive disturbances or diarrhœa caused by the pressure of the fluid, intestinal hemorrhages, and marked oliguria in spite of diuretics, generally accompany severe ascites and are therefore indications for puncture. An earlier operation is contraindicated as, after the abdomen has once been tapped, a second and a third puncture are usually necessary. Occasionally, however, after one or more punctures, the ascites remains stationary, apparently on account of the formation of new collaterals. Each repeated puncture and re-collection of fluid cause a certain loss of proteid to the organism. Another reason for putting off the operation as long as possible is the danger of a consequent tuberculous peritonitis. [A very insufficient reason.-Ed.]

Surgery.-Another operation is sometimes undertaken. The abdomen is opened up, the fluid removed, and the great omentum or spleen sewed to the abdominal wall in order to furnish a number of collateral venous channels for the blood of the portal vein. This procedure is justifiable (1) in desperate cases of atrophic cirrhosis, when the patient is in tolerably good condition and the liver is not too much atrophied; (2) in cases of gummatous hepatitis with an atrophic liver; or (3) in case of an adherent pericardium with hepatitis.

Final Stage.-In the final stage, heart stimulants are often indicated. Alcohol need not then be denied the patient. [An early hæmatemesis, or other sufficiently suggestive symptoms to establish the diagnosis of hepatic 
cirrhosis, gives such a patient an excellent chance for recovery to comparative health provided he excludes alcohol entirely, eats simply, and lives carefully. In my experience no form of treatment avails much late in the disease. I believe in early tapping, as soon as diuretics, cardiac tonies, rest, and diet have proved that the ascites cannot be influenced by these measures, and a course of potassium iodide has been equally unsuccessful. The latter should be tried in every case whether syphilis is suspected or not. I have not been so favorably impressed with the results of omentopexy,-stitching the omentum to the abdominal wall with the idea of establishing a collateral flow between the portal and the systemic circulations; but most of the cases I have followed have been from the very chronic material at the New York City Hospital and operated upon as a very last resort. Osler gives the following figures: "In 224 cases there were 84 deaths and 129 recoveries; 11 cases doubtful. Among the 129 successful cases, in 25 the ascites recurred; 70 appeared to have completely recovered." Greenough found an improvement after ten years in only 9 out of 105 cases. If the operation is to be tried at all, it should be performed early.-Ed.] 


\section{THERAPY OF ACUTE INFECTIOUS DISEASES}

\section{THERAPY OF TYPHOID FEVER.}

In view of the fact that we have no specific serum-treatment for typhoid fever, we stand to-day just where we have stood for years in the therapeutics of this disease: we must place our chief reliance upon diet and hydrotherapy [and especially careful nursing-Ed.].

DiET.-During the fever and for some time afterwards the diet must be exclusively liquid. We give milk, eafé au lait, milk soups (sometimes with the addition of sanatogen, eucasin, or hygiama); broths (sometimes with somatose, puro, or the beaten yolk of an egg); gruels made from oatmeal, barley, or arrowroot, salted, (and sometimes with meat extract, broth, or the yolk of an egg added to improve the taste); soups made from stock or meat extract with the addition of the leguminous or maltoleguminous meals; and beef juice ( 2 tablespoonfuls added to a cup of broth or 1 tablespoonful by itself). The beef juice should be expressed fresh each day and kept on ice. Ziemssen places less reliance on Liebig's beef tea. Another kind of beef tea may be prepared at home by putting chopped meat in a casserole and heating it to the boiling point without adding water. The juice extracted will form a jelly on cooling. This when heated and added to ordinary broth will improve the flavor, though not the nutritive value. If it is prepared from fresh veal, however, the meat gelatine is of value for taking the place of proteids. The patient should not be stinted in the amount of liquid food during the fever. In fact the more he drinks, the better is the diuresis and the consequent elimination of toxins. An exception must be made, however, in the case of patients who cannot easily take milk. They should be given it at first only in frequent small doses (100 G. (3 oz.) every hour or two, 500700 G. (1-1 $1 / 2$ pt.) a day), which may be increased gradually to the desired amount of $2000-3500$ (2-31/2 qt.) a day, (100-250 G. (3-8 oz.) every $2-4$ hours). To test the truth of the objections urged by some authorities against the use of milk the author has tried giving it up entirely and substituting adequate amounts of other liquid foods. The patients apparently did neither better nor worse than those on a milk diet. Milk must, of course, be abandoned in those individual cases which show an intolerance for it. [See p. 375 for directions for modifying its administration.-Ed.] It may also be added that a milk diet increases the chances of a secondary mouth infection, unless the most careful attention to the cleanliness of the mouth is carried out. 
Not until a week after the end of the fever should the patient be started on a light solid diet, as exemplified in the following dietary: eighth day after the fever, chicken soup, chopped ham (2 teaspoonfuls every two hours), a small piece of delicate fish, wine jelly, cocoa, tea with milk, zwieback; ninth day, increase in quantity; tenth day, chopped chicken or veal, steamed rice, potato soup, crackers; fourteenth day, game, bread without crust, buttered toast, a soft boiled egg; to these may gradually be added finely minced roast meat, spinach, macaroni, apple sauce, or plum sauce. All these should be served frequently and in small quantities. In three weeks a return may be made to the ordinary diet.

[In the choice of their diet, many of the ablest clinicians still treat the disease rather than the patient. Osler and McCrea limit the diet to $120-180$ G. (4-6 oz.) of milk, diluted with $60 \mathrm{G}$. ( 2 oz.) of lime water, every four hours, and $120 \mathrm{G}$. (4 oz.) of albumin water, made from the white of one or two eggs, every four hours. McCrea says, "More patients are overfed than underfed." Osler says, "It is possible that we give too much food. Of late years the disease has been treated by what has been called therapeutic fasting-little or no food, only water." The simplicity of a diet composed of milk alternating with albumin water has an advantage in a very active and large typhoid service, where, as is usually the case, the nurses are overworked. Whenever milk is used for a diet, and especially with typhoid patients, it is well to be sure that the milk is pure. Edsall recently traced several outbreaks of intestinal trouble among typhoid patients to the milk supply.

Curschmann and Forchheimer among others give a much more varied diet and especially recognize the importance of carbohydrates. The latter favors strained oatmeal jelly. The former uses mucilaginous soups prepared from rice, wheat, green corn, maize, tapioca, palm sago, aleuronat, or other carbohydrates. For the last 15 years F. C. Shattuck has prescribed a much more liberal diet, which I quote from Forchheimer :

"(a) Milk: hot or cold, with or without salt, diluted with lime water, soda water, Apollinaris, or Vichy; peptonized milk; cream and water (i.e., less albumin) ; milk with white of egg, buttermilk, koumiss, matzoon, milk whey, milk with tea, coffee, cocoa.

(b) Soups : beef, veal, chicken, tomato, potato, oyster, mutton, pea, bean, squash; carefully strained and thickened with rice (powdered), arrowroot, flour, milk or cream, egg, barley.

(c) Horlick's food, Mellin's food, malted milk.

(d) Beef juice.

(e) Gruels : strained cornmeal, crackers, flour, barley water, toast water, albumin water with lemon juice.

$(f)$ Ice cream.

(g) Egg, soft boiled or raw; eggnog. 
(h) Finally, minced lean meat, scraped beef, the soft part of raw oysters, soft crackers with milk or water, soft puddings without raisins, soft toast without the erust, blancmange, wine jelly, apple sauce, and macaroni."

In a series of 288 eases from 1892 to 1904 , there were 14 per cent. relapses, 8.3 per cent. hemorrhages from the intestine, 3.1 per cent. perforation, and 10.7 per cent. mortality. Any such diet as this necessitates daily individualization and sometimes the orders must be changed more than once a day. It adds considerably to the work of the physician and especially to that of the nurse. With his variety and amount of food the patient is generally in much better nutrition at the end of his fever than if he is limited to milk and albumin water, and convalescence is hastened.

Kinnicutt, who has recently followed Shattuck's example, has contributed an interesting paper upon the "Principles of Feeding in Typhoid and in Other Fevers," 1 from which I have taken the following.

In mild cases without contraindications, Marsden in the Monsell Fever IIospital, England, allows bread and butter, milk with custard, fish with mashed potato, ehicken and minced meat. He considers only the individual and his condition and does not necessarily restrict the diet for slight exacerbations of fever or occasional irregularity of the bowels. His cases are too few (100) and his statistics too incomplete (mortality not given) to warrant any conclusions.

Bushuyev, an enthusiast for a liberal diet in typhoid fever, employs the following at the Kief Military Hospital, Russia:

" 7 A.M. Tea with a roll.

8 4.м. 400 e.c. (13 oz.) of soft (liquid) oatmeal, barley or wheat porridge, with butter.

9 A.M. One or two boiled eggs, soft or hard as the patient desires.

10 to 11 A.M. A glass $(200-220$ c.c. $-61 / 2-71 / 2$ oz.) of milk with a roll, one-half a cutlet, and a bit of boiled meat (160-168 G. (51/2 oz.)).

12 to 12.30 P.M. A plate ( 220 c.c. ( $71 / 2$ oz.) ) of chicken soup or a bowl of ordinary soup, sometimes with a bit of chicken from the soup, and a small cup of 'kisel' (a sort of sour jelly); rarely a little preserved fruit.

3 P.M. Tea with a roll.

6 P.M. A cup of chicken or beef soup; semolina pudding or milk; a bit of chicken.

8 P.M. Milk with a roll.

During the night. Coffee or tea, with milk, two to four times; coffee with cognac. (The milk is usually boiled; occasionally it is given in the form of junket.)

In addition, the patients receive 1-3 ounces of wine in the morning, and every two hours half an ounce of Stokes' mixture. (Stokes' mixture : 2 egg yolks, 50 c.c. of brandy, 120 c.c. of aqua aurantii florum, sugar or syrup sufficient to sweeten.)"

\footnotetext{
${ }^{1}$ The Transactions of the Association of American Physicians, 1906.
} 
Bushuyev says, "Under this regimen the general condition of patients is incomparably better than when kept upon an exclusively fluid diet. The common complaints are scarcely ever heard. At breakfast, dinner, and supper, the patients are uncommonly wide awake. Even those who are very ill sit up in bed, beg for food, and eat with much satisfaction; only a few have to be fed by nurses. If one observes the patients at mealtimes, he wholly forgets that these individuals are seriously ill with temperatures above $39^{\circ} \mathrm{C}$. During the first hours in the ward the patient lies in a motionless condition, failing to answer questions and refusing food. But, if one succeeds in some way or another in persuading him to eat a bit of meat or cutlet, or an egg, he immediately begins to show some interest in his surroundings.'”

He emphasizes not the proper relation between proteid, fat, and carbohydrate, but the careful preparation of the food so as to stimulate and cater to the patient's appetite. In 398 cases treated between 1895 and 1897 , hemorrhage occurred in four (1.005 per cent.). Three of these four cases were confined to a liquid diet. Perforation occurred once ( 0.31 per cent.) and the mortality was 8.2 per cent. The loss of weight was slight, and the general condition at the beginning of convalescence seems to have been almost incredibly good. The mortality for the ten previous years in the same hospital on a fluid diet was 12.4 per cent. (2887 cases).

What evidence we have from the results of metabolism studies of different diets in typhoid fever seems to favor a more generous regimen than is usually practised; but the whole subject requires revision.

Abrupt changes in diet are unwise. Whether to awaken a patient during the night for feeding must also be decided in each case. It is, of course, just as absurd to omit regular feeding by night as well as day for a dull typically typhoidal patient who sleeps quite as much during the day as during the night, as it is unnecessary to waken a patient in good nutrition who takes and digests a large amount of nourishment during the day and who sleeps only at night. With almost any patient the nurse has an opportunity once or twice during the night to get the patient to take some liquid food and that without annoyance or loss of sleep. -Ed.]

Care must be taken that the patient drink abundantly during the entire fever and for some days afterwards. Besides his liquid food he should have plenty of water and about $500 \mathrm{G}$. (1 pt.) a day of light wine diluted with water, or, in place of wine, punch, brandy (40-50 G. $(1 \mathrm{I} / 4$ oz. $))$ or tea with rum in it. Brandy may be given in Stokes' mixture as follows:

R Spir. vin. Gallici $\ldots \ldots \ldots \ldots \ldots \ldots \ldots . .50 .00 \ldots \ldots$. $3 \mathrm{i} 3 \mathrm{v}$

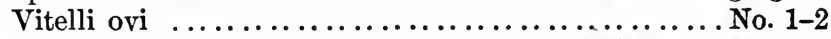

Aq. cinnamon $\ldots \ldots \ldots \ldots \ldots \ldots \ldots \ldots \ldots \ldots \ldots .150 .00 \ldots \ldots .5 \mathrm{v}$

M. fiat mist.

Sig. -1 tablespoonful every 2 hours. 
Mulled red wine, $250-500 \mathrm{G}$. ( $1 / 2-1 \mathrm{pt}$.), is suitable when meteorism or diarrhoa is present; champagne or grog, when a heart or nerve tonie is needed. The amounts of alcohol here indicated must be varied to suit the individual ease.

Lemonade refreshes the patient and excites a flow of saliva, which helps to keep the mouth from becoming dry. For the same purpose the mouth may be rinsed with oatmeal water. Even if the patient does not ask for it, he should be given some drink every half hour while he is awake. [Unless a patient has myocarditis, pronounced arteriosclerosis. or some failing cardiac lesion, the amount of water to be taken during 24 hours is almost unlimited. MeCrea urges 3 litres $(3$ qt.) as a minimum and much better 5 or 6 litres (5-6 qt.). Delirious or toxic patients are certainly improved by ingesting large quantities of water.-Ed.]

In typhoid, as in all severe sickness, when we wish to support the circulatory system, aleohol or stimulants, if they are being used, should be given during the night, espeeially after midnight, when the patient's vitality is lowest. Especial eare should be taken to keep the room at the proper temperature during those hours. ["Aleohol is unnecessary in a great majority of the eases. Of late years I have used it mueh less freely; but, when the heart is feeble and the toxic symptoms are severe, 240-360 G. (8-12 oz.) of whisky may be given in the 24 hours." (Osler). An ounce of whisky is the equivalent of 100 calories. When patients are unable to take sufficient food a little alcohol helps the nutrition; but, when they are eapable of digesting Shattuek's generous diet, they rarely need alcohol. If patients who are being tubbed require whisky, it is a good plan to administer it just before or just after the baths, aecording to their reaction to the bathing. - Ed.]

HYDROTHERAPY.- Next in importance to the diet in the treatment of typhoid fever is hydrotherapy. The fate of the typhoid patient may depend upon its persistent use, and it is the physician's duty to insist strictly upon the proper proeedures even at the risk of being thought hard-hearted.

Baths.-The appropriate hydrotherapy consists ehiefly in the use of cold water, the object of which is to reduce the body temperature and so mitigate the bad effect of the fever on the parenchymatous organs, and to affect favorably the secondary nervous affections and the whole course of the disease. Cool baths are always preferable, but, when they are not practicable. cold packs may be substituted. The initial temperature of the baths is usually $79^{\circ}$, reduced gradually to $68^{\circ}$. [I usually prefer $85^{\circ}$ for the first bath.-Ed.] If the patient is anæmic, weak, and sensitive to cold, the temperature may be $7^{\circ}-11^{\circ}$ higher. With a new patient whose reaction to cold we do not know, it is often well to begin with $86^{\circ}-90^{\circ}$ reduced to $75^{\circ}$ during the bath, and then gradually lower the temperature of the succeeding baths. The duration of the bath should be 15-20 minutes, unless the patient begins to have a severe chill. [ 5 or 10 minutes is sufficient for the first bath.- 
Ed.]. The whole surface of the body should be continuously rubbed [excepting, of course, the abdomen-Ed.]. At the conclusion of the bath, cold water should be poured over the head, neck, and trunk, provided that: (1) a diffuse bronchitis or pneumonia or a true bronchotyphoid makes it desirable to insure a sudden good ventilation of the lung, or (2) the nervous system is not functioning properly. After the bath the patient is wrapped immediately in a sheet and put to bed, not being dried until he has had some rest. If it is found that he feels chilly for some time after the bath, he should be wrapped at once in a warm sheet. In general, a reduction of $2.2^{\circ}-4.5^{\circ}$ in temperature is expected from the cold bath, but, if the patient is delicate so that there seems to be some danger of collapse, he may be taken out after the temperature has fallen $2^{\circ}$.

[Brand's bath treatment does reduce the temperature in most instances; but often not at all or not more than a degree during the height of the fever when the effects of the bath are most marked upon the other symptoms of the disease. It lessens the delirium and the tremor and diminishes the toxic features, stimulates the kidneys, improves the general circulation, slows the pulse, raises the blood pressure, benefits the initial bronchitis and probably increases oxidation by stimulating deeper breathing, cleans the skin, and improves the cutaneous circulation so that bedsores are less liable to appear. In addition to all of which, it has lessened the mortality of the disease approximately 50 per cent.-Ed.]

Cold Packs.-In applying the cold pack, the patient is wrapped in a sheet wrung out in water at $50^{\circ}-54^{\circ}$, the head and feet being left free. After 6-10 minutes he is transferred to another wet sheet, and the process repeated 2-3 times, the duration of the last pack being increased to $1 / 4-1 / 2$ hour. This procedure is less efficacious and less pleasant for the patient than a cold bath, but is preferable in case of severe prostration.

Contraindications for Cold Baths or Packs.-Contraindications for the use of either cold baths or cold packs are: intestinal hemorrhages, perforation of the intestine and peritonitis, and extreme collapse. [Old and very young patients, patients with acute or serious chronic cardiac or cardiovascular changes, with phlebitis, with nephritis, some cases with pneumonia, advanced pregnancy, and the very obese, anæmic, or alcoholic.-Ed.]

Other Hydropathic Procedures.-Other hydropathic procedures are : colder baths $\left(59^{\circ}-64^{\circ}\right)$ for $5-6$ minutes; cold water poured over the patient while he is in a tepid bath or an empty bathtub; prolonged tepid baths ( 2 each day, of more than 2 hours duration, at $88^{\circ}-95^{\circ}$ ). The author does not employ any of these means, but, if the temperature is only of moderate degree, he orders a cool sponge every day with water or alcohol. The patient is only partly dried, in order that there may be a slight antipyretic action produced by the evaporation of the moisture left on the skin, in addition to the general refreshing and invigorating effect. 
In general, measures to reduce the temperature are not indicated in light or moderately severe cases, but only when the temperature remains for a considerable time at $103^{\circ}$ or over, and severe toxic symptoms appear (cerebral, cardiac, respiratory). When these indications are present antipyretic hydrotherapy is indicated. Usually $2-4$ baths given during the day and before midnight are enough. After midnight the patient should be left undisturbed. [McCrea emphasizes the importance of giving the baths early and systematically if they are given at all. "To use the baths only in severe attacks, or to wait until the patient is desperately ill before beginning them, is fair neither to the method nor to the patient." I prefer to use them symptomatically and, when possible, personally to order the baths for each day. As a general rule, I begin and continue them once every four hours whenever the rectal temperature reaches $103^{\circ}$, if the patient is fairly sick; but I often order them given when the temperature is lower than this, if there is much intoxication. It is not possible to give definite rules about bathing at night. I order tubs through the night the same as during the day for very toxic and stupid cases; but omit them at night, whenever possible. Similar reasons guide my decision as in feeding; each case must be studied carefully to obtain the best results. I usually try to have the hot feedings come soon after the baths are over, the cold feedings at the two-hour interval between the baths.-Ed.]

Medication : Antipyretics.-Medication is by no means so important as hydrotherapy in reducing the fever, but still antipyretics are sometimes employed, generally quinine, lactophenin, antipyrin, or pyramidon. With the exception of a certain form of pyramidon treatment, the author uses them only when hydrotherapy is contraindicated, or when the patient is so strongly averse to it that it is advisable to lessen the number of baths or packs. For the latter purpose, especially, he prefers the hydrochlorate of quinine ( $0.5 \mathrm{G}$. ( $7 \mathrm{gr}$.) on three successive evenings), from which a reduction of $4^{\circ}-51 / 2^{\circ}$ in 12 hours may be expected.

Instead of quinine, euquinine or aristoquin may be used (see p. 553). The author considers lactophenin, antipyrin, and phenacetin inferior to quinine, although in a few eases he has used lactophenin alone (without baths) and found that it promptly reduced the temperature, quieted the nervous system, and often produced pleasant sleep and a relatively comfortable feeling. Antipyrin and phenacetin, although they lower the temperature and usually have no unpleasant secondary effects, are very seldom used by the author. [I never employ any of the above. Forchheimer states that "quinine is no longer used for the purpose of reducing temperature in typhoid."-Ed.]

Pyramidon.-There is, however, one method of antipyretic medication in typhoid which the author employs in all severe cases, especially when the proper hydrotherapy cannot be carried out, and that is the continuous use of pyramidon $(0.2-0.25 \mathrm{G}$. ( $3-4 \mathrm{gr}$.), sometimes even $0.4 \mathrm{G}$. ( $6 \mathrm{gr}$.) at the beginning, every 2 hours day and night, as long as 
there is any fever). After the fever has ended, it may be kept from returning by further suitable doses. This treatment probably does not shorten the course of the disease, but it does keep the patient conscious and comfortable, and makes it easier to feed him. In short, the "typhoidal" character of the disease is wholly eliminated. In one case only has the author had to give up the steady use of pyramidon on account of a resulting heartburn. He has never seen any injurious effects from its use. If desired, $0.2-0.3 \mathrm{G}$. $(3-4 \mathrm{~T} / 2 \mathrm{gr}$.) of the sodium benzoate of caffeine may be given with it to protect the heart.

[I have had no personal experience with pyramidon in typhoid fever. E. Libman showed me a few cases in whom they employed the drug at Mt. Sinai Hospital. He tells me the following in regard to their experience: "We have usually begun in adults with two grains of pyramidon and increased it to three or four, if necessary, generally combining it with caffeine. We have not attempted to keep the temperature low all the time, but have used the pyramidon when the temperature was high. In some cases we use pyramidon alone, in some, pyramidon and hydrotherapy, and in some, hydrotherapy alone. We do not like to use the drug after the third week has begun, because the drops in temperature produced by it are at times rather sharp, and might confuse the clinical picture at any time when hemorrhages or perforation were suspected. We have had no bad results from the use of the drug and have had no depression. Neither the systolic nor diastolic pressure, tested with the Erlanger apparatus, has been depressed. At times the temperature does not reach its height after the use of pyramidon for seven or nine hours. If the patient complains of feeling weak, or if there is too much perspiration after the exhibition of the drug, it is not tried again.'-Ed.]

A few facts remain to be told concerning further medication.

Calomel.-If the disease is in the first week, we give calomel $0.5 \mathrm{G}$. ( 7 gr.) either in a single dose or in divided doses (2-3 in an hour). The nearer to the first day of the disease the calomel can be given, the better, as its early use apparently mitigates the severity of the general infection and particularly of the local intestinal symptoms. [I never use calomel, except symptomaticlly for nausea and vomiting. I do not believe its early use mitigates the severity of the disease.-Ed.]

Intestinal Antiseptics.-The various intestinal antiseptics are utterly worthless in influencing the course of the disease. The author has tried the fermentum cerevisiæ (3 teaspoonfuls daily in milk) generally without success, but in one case it noticeably improved the general condition, especially by stopping the profuse diarrhœa. The yeast apparently drives out the injurious bacteria from the intestine.

Genersl Hygiene: Cleansing the Mouth.-The cleansing of the mouth is exceedingly important. It is the fault of the physician or the nurse, if, in cases of typhoid which recover, there is any secondary infection from the mouth, for example of the lymph-glands in the neck or of the parotid glands. The method of keeping the mouth clean has already 
been discussed (p. 270). [The teeth, mouth, and tongue should be carefully cleaned after each feeding, and, if the patient's condition warrants, he should rinse his mouth with a mild antiseptic gargle. Henna recommended chewing gum to one of my patients. Forchheimer recommends chewing gum to quiet the excessive appetite of convalescence. In seriously ill toxic patients the nurse will have a great deal of difficulty in keeping the back of the tongue and throat clean, but perseverance and eare will generally accomplish this.-Ed.]

Avoidance of Bedsores.-To avoid the danger of bedsores the patient's position should be frequently changed, and, if possible, a waterbed used. When once a sore has developed, the place should be carefully disinfected and then covered with [an appropriate ointment or powder, zinc oxide ointment, aristol-Ed.]. The water-bed then becomes absolutely necessary. [The bath treatment renders patients much less liable to bedsores. In addition it is advisable at least once a day to cleanse the buttocks, thighs, and lower back with a weak bichloride solution and then rub with alcohol, dry, and finally dust on a simple powder.-Ed.]

Symptomatic Therapy : Therapy of Cerebral Symptoms.-Cerebral symptoms often demand attention. If the patient becomes stuporous or comatose, he may be put in a tepid bath, and cold water may be poured over him. If he is sleepless and exeited or delirious, tepid baths at night, an ice-bag to the head, and the administration of lactophenin are useful. If these are not sufficient, we may give trional or veronal, and morphine either hypodermically or internally, but combined with stimulants (e.g., ethereal tincture of valerian or eamphor in sweet oil). The continued use of pyramidon is, however, decidedly the best form of treatment. [I prefer small doses of morphine, opium, or codeine to other drugs for delirium, restlessness, excessive nervousness, or insomnia ; but before resorting to internal medication I always try to counteract these symptoms by modifying the hydrotherapy.-Ed.]

Therapy of Intestinal Symptoms: Diarrhoea.-Diarrhœa demands treatment in case the number of daily movements exceeds 5-6. [Substituting cold tea for the water will sometimes help.-Ed.] The subnitrate or the salicylate of bismuth ( $0.5 \mathrm{G}$. ( $7 \mathrm{gr}$.) every hour or two) may be given, or the fermentum cerevisia. If these are not suecessful, we may give opium, preferably by the rectum, 10-15 drops of the tineture being added to starch enemas, $2-3$ of which are given daily. [For intractable diarrhœa S. W. Lambert recommends the following prescription :

R Pulv. opii $\ldots \ldots \ldots \ldots \ldots \ldots \ldots \ldots \ldots . ., 0.016 \ldots \ldots$ gr. $\neq$

Pulv. camphoræ $\ldots \ldots \ldots \ldots \ldots \ldots \ldots \ldots . ., 0.065 \ldots \ldots$ gr. i

Plumbi acetatis.................... $0.20 \ldots \ldots$ gr. iii

Bismuthi subnitrat. .................2.00 …..gr. xxx

M. fiat pulv. no. i.

Dent. tal. pulv. no. xii.

Sig. -1 powder every 4 hours. 
Prolonged colon irrigations with warm normal saline are sometimes efficient. They supply fluid, promote diuresis, relieve the toxæmia, stimulate the heart, and reduce tympanites.-Ed.]

Meteorism.-In case of severe meteorism, calomel (0.05 G. (3/4 gr.) three times a day) may be given. It is also worth while to try Leiter's cooling tubes or an ice-bag on the abdomen, or hot compresses or linseed poultices. [Tympanites should be watched for and combated promptly. The nurse should have a standing order to insert a large soft catheter (a very soft flexible rectal tube) whenever it begins. A turpentine stupe carefully and promptly applied is often very useful. Eserine, 0.0013 G. $\left(1 / 5_{0} \mathrm{gr}\right.$.), may be tried. Often the diet is at fault and should be changed, gruels omitted, milk peptonized or omitted.-Ed.]

Intestinal Hemorrhages, etc.-Intestinal hemorrhage, peritonitis, and heart weakness are treated according to their special indications, and as if typhoid fever were not an element in the case. [For hemorrhage a very light ice-bag or coil to the abdomen, stopping all stimulation, and usually all food, except, perhaps, cold albumin water, a small dose of opium by the mouth or morphine hypodermically, if the patient is very restless, and the calcium salts in large doses, are the indications. Saline solutions under the skin or in a vein, only if the patient is in extremis. For perforation, a rapid operation, often under cocaine, if the diagnosis is certain or probable, is the only indication.-Ed.]

Therapy of Complications and Sequelde.-Complications and sequelæ should also receive the usual treatment. In ease of broncho- or lobar pneumonia we may use mild expectorants, in spite of a typhoid infection of the intestine.

Relapses.-If a relapse occurs, and the disease takes on a protracted character, the patient's strength must be carefully kept up by alcohol. But, in general, a relapse demands exactly the same treatment as the original attack, the therapy being carefully suited to the severity of the condition.

Convalescence.-A convalescent patient should not be allowed to get up until his strength has fully returned. Usually he may be allowed to get out of bed for a very short time 10-14 days after the fever has gone, but should not return to his business until 1-2 months after convalescence has begun. [Convalescence in typhoid fever is a slow, tedious process. A change of scene is imperative for every person that can afford it. Six months is soon enough for a child to return to school or an adult to resume his duties. This detail must be worked out carefully in each case.-Ed.]

\section{THERAPY OF DYSENTERY.}

Acute Dysentery: Diet.-The first measure to be taken in a case of dysentery is to put the patient on a suitable diet. If possible $2 \mathrm{~L}$. (2 qt.) of milk should be given daily, preferably 1 tablespoonful every 
15 minutes. It may be made more acceptable to the patient by the addition of bicarbonate of soda, lime water, an alkaline mineral water, salt, tea, or coffee. In addition to the milk, we may give barley gruel by the spoonful or, if the local symptoms are not of the severest type, acorn cocoa, soups made from tapioca or the leguminous meals, or broths pure or added to rice, barley, or oatmeal gruel. If the patient can stand it, an egg, raw or cooked, may be added to the soup or milk. Albumin water is also suitable (the whites of 20 eggs added to 3 cups of water and a tablespoonful of milk sugar, to be taken within 24 hours).

For the mitigation of thirst, gruels and cold Russian tea may be given, also, in the lighter cases, huckleberry wine and occasionally carbonated waters and possibly lemonade. The sucking of pieces of ice is not advisable, as it apparently sometimes induces peristalsis.

When the acute symptoms (colic, tenesmus, and frequent diarrhøa) have disappeared, the transition may be made to a light solid diet, as already discussed in the sections on typhoid and on acute intestinal catarrh. Lemon souffle with crackers or zwieback is particularly suitable. Such a diet may be given from the start if the local symptoms are mild and there is not much fever.

Medication: Calomel-Tannin Irrigations.-Calomel should be given from the beginning as an intestinal disinfectant $(0.05 \mathrm{G}$. $(3 / 4 \mathrm{gr}$.) every hour during the day, if necessary). This treatment, however, is by no means always efficacious, colon irrigations usually proving much more successful. The author, following Kartulis's recommendation, uses $11 / 2-$ $21 / 2$ L. $(11 / 2-21 / 2$ qt.) of a $1 / 2$ per cent. aqueous solution of tannin, giving 3 irrigations daily with a soft rectal tube, which must be inserted at least $8 \mathrm{~cm}$. (4 in.).

Ipecac.-He has also tried ipecac in a few cases, but without success. Kartulis cites the following method as that recommended for its administration by the English physicians in India. The patient is given a few drops of the tincture of opium, or a morphine injection, a mustard or turpentine plaster being placed upon the abdomen. A half hour later $1 \mathrm{G}$. (15 gr.) of ipecac is administered, repeated, if necessary, 1-3 times at intervals of an hour. All liquids are forbidden, but ice is allowed. In this way considerable quantities of ipecac may be given without inducing vomiting.

Silver Nitrate-Quinine-Castor Oil.-The author has also tried irrigations with silver nitrate and quinine, but with no satisfactory result. In fact, no other drug of the various ones recommended by different authorities seems to him efficacious except castor oil, which is indicated in case there is reason to suspect a partial stagnation of the intestinal contents, or in case we wish to obviate such a possibility. He is, in fact, growing more and more inclined to give it daily from the beginning in an emulsion or in capsules (15-20 G. (4-5 dr.) a day), abandoning all other medication and even tannin irrigations. Although its use may aggra- 
vate the local condition for a few days, it will eventually shorten the attack surprisingly. The author uses the following prescription:

$\mathrm{P}$ Ol. ricini..................15.00-20.00..... 3iv-3v

Acaciæ ........................5.00....3i gr. xv

Syr. simp. .............................

Aq. dest. .......................150.00.... $5 \mathrm{v}$

M. fiat emuls.

Sig. -1 tablespoonful every 2 hours.

Opium.-Opium is, of course, sometimes necessary on account of the severity of the pain.

Antitoxin Serum.-In case of Shiga's dysentery, Kruse's serum should be injected (25-50 c.c. repeated several times in the same or a lessened amount). The results are reported as being encouraging.

Chronic Dysentery: Medication.-The therapy of chronic dysentery is similar to that of acute, tannin irrigations and repeated doses of castor oil being the most successful form of medication. Intestinal antiseptics (salicylate of bismuth, salol, benzo-naphthol) are also in place. A small teaspoonful, night and morning, of an infusion of quassia in $50-75$ G. $\left(1 \frac{1}{2}-21 / 2\right.$ oz.) of warm water is also efficacious.

Diet.-The diet should be the same as in the convalescent stage of acute dysentery.

Symptomatic Therapy.-In both acute and chronic dysentery, we are often obliged to treat symptomatically intestinal hemorrhage, peritonitis, intestinal perforation, etc. After dysentery thickened areas are often left in the intestinal wall, which are best treated by massage and cool sitz baths.

[The author has not emphasized sufficiently variations in treatment depending upon whether the dysentery is caused by the amœba or by the dysentery bacillus. He has not insisted upon absolute rest during the acute and subacute stages of both types, nor upon the value of heat, nor upon the detrimental action of cold even in food and drink. During the acute stage neither ice nor cold liquids should be swallowed, although there is no objection to, and, in fact, much relief may be obtained from the thirst by rinsing the mouth frequently with cold water or cold alkaline waters. External applications of heat, light poultices, turpentine stupes, hot fomentations are grateful to the patient and reduce the number of stools. During the early and most acute stage thin chicken or mutton broth, thin barley or rice water, or albumin water are usually better borne than milk. Opium or morphine is an essential during the acute stages. The constipation induced by its use may be counteracted by the appropriate selection of laxatives or lavage. It should not be used in doses sufficiently large to narcotize the patient. Calomel, castor oil, and saline laxatives, all three, have warm advocates. W. J. Buchanan has treated 855 cases of bacillary dysentery by the saline treatment with only 9 deaths. He advises a teaspoonful of sodium sulphate, 6-8 times a day until all blood and mucus have disappeared from the stools-usually 2 or 3 days. Shiga agrees with most authors in recommending calomel 
in the beginning of the disease to clear out the bowel, remove the infectious material, and hinder the extension of the disease upward. He gives $0.5-0.8 \mathrm{G}$. (8-12 $\mathrm{gr}$.) of calomel once or twice, either alone or followed 1 or 2 hours later by 10-15 G. (3-4 dr.) of castor oil. Castor oil, especially for children, is recommended as the best laxative by Forchheimer. He gives it in the beginning of every attack and repeats it, if scybala persist in the stools or there is evidence of fecal aceumulation in the sigmoid or the flexures of the colon, as shown by physical examination. Ipecac is still recommended by many physicians in India, Africa, and Brazil. Shiga in bacillary dysentery gives it "a very poor place as a dysenteric remedy." Forchheimer advises its use early in the disease and quotes Sodré's (Rio Janeiro) method of administration as follows: "Take $4 \mathrm{G}$. (1 dr.) of powdered ipecac and $200 \mathrm{G}$. $(6 \mathrm{oz}$.) of boiling water, and let it steep for 12 hours, at the end of this time pour off the liquid, which is to be administered to the patient; then keep the powder, pouring $200 \mathrm{G}$. (6 oz.) more of boiling water on it and leaving it to steep for 12 hours, after which pour off the liquid as before, which is to be administered to the patient; from the same powder make a third infusion in the same manner, and use it in the same way. The liquid resulting from each infusion and maceration is administered in the course of the day either all at once or in broken doses." By giving it in broken doses, adding cinnamon and small doses of morphine, this author obviates the emetic results of the drug. Strong's experience in the Philippines with amœbic dysentery made him believe that ipeeac probably had no curative effect beyond that of the opium with which it was combined. Berther gives methylene blue internally in doses of 0.10.2 G. (1 $1 / 2-3 \mathrm{gr}$.), combined with enemata of $0.1-0.2 \mathrm{G}$. (1 $1 / 2-3 \mathrm{gr}$.) of the drug to $1 / 2-1 \mathrm{I}$. ( $1 / 2-1$ qt.) of water both in amœbic and bacillary dysentery. Other irrigations are recommended by various authorities. Osler lauds large injections of quinine solution for amœbic dysentery. He begins with 1 to 5000 and increases to 1 to 1000 using 1-2 L. (1-2 qt.) at each irrigation. Strong increases the strength more rapidly, within a few days to 1 to 500 . Tuttle has recently obtained very favorable results with ice-water irrigations. Harris obtained no benefit in any of his cases with this vigorous treatment. Shiga in acute bacillary dysentery recommends saline irrigations, but in the chronic forms only uses silver nitrate 1 to 500-1000, tannin 1 to $200-400$, thymol 1 to 500-1000, resorcin 1 to 50-100, ereolin 1 to 50-100, and lysol 1 to 100 . Shiga reports a reduction of one third in the mortality of bacillary dysentery under the use of serum and much better results since 1906 with a polyvalent serum. Other authors are hardly so encouraging.-Ed.]

\section{THERAPY OF INFLUENZA.}

In spite of the large number of cases of influenza treated since the pandemic of 1889, little is vet known about its therapy. As there is no known specific treatment, our therapy must be purely symptomatic. 
Diet.-Except in very light cases, the patient should be put to bed and given a fluid diet (ordinary fever diet with the addition of drinks to quench the thirst, and alcoholic drinks, if the patient does not object).

Diaphoresis.-The author believes in inducing diaphoresis at the onset of the disease, ordering, as in acute bronchitis, a hot bath or hot drinks.

Antipyretics-Antineuralgics.-As a secondary measure antipyretics or antineuralgics may be given to improve the general condition and particularly to mitigate the special symptoms of severe headache, muscular pains, restlessness, torturing cough (salophen), and the neuralgias accompanying or following the disease. Salicylate of soda, phenacetin, antipyrin, lactophenin, salipyrine, and aspyrin are all serviceable, although the author prefers the last two, $(0.5 \mathrm{G}$. ( $7 \mathrm{gr}$.) every other hour, up to 3-4 G. (45-60 gr.) a day). The dose of antipyrin and lactophenin is the same; that of phenacetin, one-half as much.

Antipyresis.-Antipyresis is indicated only when the fever is high and protracted, with an evident effect upon the central nervous system and the patient's general condition. The antipyretics are preferable to cold baths.

Hydrotherapy.-Hydrotherapy, with the exception of the application of cold to the head to relieve the headache, is of no great use. Sometimes baths even increase the patient's discomfort. In general, heat is preferable to cold.

Cathartics.-A cathartic (calomel or castor oil) may be given once, in case of constipation.

Symptomatic Therapy.-The manifold complications and sequelæ of influenza must be treated by the usual methods (bronchitis, bronchopneumonia, pleurisy, diarrhœa).

\section{THERAPY OF ACUTE ARTICULAR RHEUMATISM.}

Medication.-In acute articular rheumatism, a specific remedy is fortunately at our command,-salicylic acid and its preparations.

Salicylic Acid: Physiological Action.-Salicylic acid occurs in the form of needles or a white, soft, crystalline powder, with no odor, but an exceedingly acid taste. Applied locally, it has an irritating or even a somewhat corrosive effect upon the mucous membranes. According to Kolbe's experiments upon himself, small doses (1-1.5 G.-15-20 gr.) internally have no effect, while larger ones (3-6 G. $-45-90$ gr.) quicken the pulse, dull the senses of sight and hearing, cause congestion in the head, bring on a ringing in the ears lasting for several hours and often accompanied by giddiness, and induce nausea and sometimes vomitingsymptoms similar to those caused by quinine. Much less often, particularly if given in a single large dose, it causes headache, sweating, delirium, unconsciousness, collapse, and slowing of the pulse and respiration. Sodium salicylate has the same action, but in a less degree. 
Therapeutic Effect.-We have no well substantiated theory concerning the therapeutic action of the salicylates, but, perhaps, they are best regarded as analgesies for the nerve-endings in the joints, with possibly a specific anti-bacterial or antitoxic action in addition. In most cases which are of long standing or exceptional severity, they cause the disappearance of the pains and redness and the reduction of the swelling in 8-18 hours after the first large dose, while the temperature goes down gradually, 2-5 days or even a week elapsing before it becomes normal. There is no essential difference between salicylic acid and its most important preparation, sodium salicylate, as far as their specific action is coneerned. But the former is somewhat quicker in its effect, and is, therefore, more irritating, so that the acid cannot be given in as large doses as the salt.

Administration.-Salicylic acid is generally prescribed in capsules:

R Acid. salicylici gr. vii

M. fiat cap. no. i.

Dent. tal. cap. no. $x x$.

Sig. - 1 capsule every hour, followed by a glass of milk or wine.

Pills are necessarily so large that their use is unpleasant. [Tablets are less certain and sometimes so hard as to be insoluble.-Ed.] Pastilles, however, are available :

P Acid. salicylici .....................

Mass. cacao ............................. s.

M. fiat pastillæ no. xx.

Sig. - 2 pastilles every hour.

It should never be given in solution, as it is not sufficiently soluble in water, and in alcohol has too corrosive an action on the upper part of the intestinal tract.

Four to eight G. (1-2 dr.) according to the severity of the attack and the condition of the patient, should be given on the first day of the disease.

Sodium Salicylate.-Sodium salicylate is a pure white crystalline powder, with a very unpleasant taste, and soluble in an equal quantity of water. Five to ten G. $\left(1 / \frac{1}{4}-2 \frac{1}{2}\right.$ dr.) a day should be given either in substance or in solution. It is preferable to salicylic acid, particularly if we desire to give large doses (3-6 G.-45-90 gr.). Such a large dose. in the author's opinion, however, is desirable only in the evening for the sake of obviating the necessity of disturbing the patient's rest. The following are the ordinary methods of administration:

R Sod. salicylatis $.10 .00 \ldots . .3$ iiss

Div. in caps. no. $x$.

Sig. - 1 capsule every two hours.

[Better divided into 20 or 30 capsules and given 2 or 3 at a dose.-Ed.] 
R Sod. salicylatis $.12 .00-18.00 \ldots . .3$ 3ii -3 ivss

Div. in pulv. no. vi.

Sig. -3 powders daily in a glass of Vichy or Preblau water.

R Sod. salicylatis $.12 .00 \ldots .3$ iii

Aq. aurant. flor.,

Spir. vin. Gallici . $\bar{a} a \bar{a} 40.00$. .3 i 3ii

M. fiat sol.

Sig. -1 teaspoonful every 2 hours.

For children under 1 year the daily amount is $1 \mathrm{G}$. (15 gr.) or less; for those of $2-5$ years, $2-3 \mathrm{G}$. (30-45 gr.) ; for those of 5-12 years, $3-5 \mathrm{G}$. (45-75 gr.).

We may also give sodium salicylate (or salicylic acid) by the rectum or as an inunction. Both drugs are readily absorbed by the rectum; but the salicylate is preferable to the acid on account of the latter's corrosive action. The author's prescription for an enema is as follows:

R Sod. salicylatis............... 2.00-10.00.... 3ss-3iiss Tinct. opii.................. $0.70-1.00 \ldots \ldots m . x-m \times v$ Aq. dest. . .................... $30.00-60.00 \ldots \ldots$.

M. fiat mist.

Sig.-For an enema.

As an inunction we may use the following:

R Acid. salicylici, Ol. terebinth..................āa 5.00.... 3i gr. xv

Adipis lanæ hydros.,

Ol. olivæ...................... àā 10.00.....3iiiss

M. fiat ungt.

Sig.-To be rubbed into the skin.

Or :

R Sod. salicylatis ...............15.00-20.00....3iv-3v

Ol. terebinth.....................5.00....3i gtt. $x v$

Ol. olivæ,

Adipis lanæhydros., ............. āā $15.00 \ldots \ldots .{ }_{\mathrm{ss}}$

M. fiat ungt.

Sig.-To be rubbed into the skin.

The following preparation, however, appears to be the best:

P Acid. salicylici ....................10.00.... 3iiss

M. fiat ungt.

Vasogenini spiss......................100.00..... Jiii

Sig.-To be rubbed into the skin.

Mesotan.-Still pleasanter to apply is mesotan, methyloxymethylester of salicylic acid, an oily, slightly aromatic fluid, which has a less irritant and quite as good an effect as salicyl-vasogen. It is prescribed as follows :

R Mesotani..........................95.00..... .

Ol. olivæ ...............................5.00.....

M. fiat mist.

Sig.-For external use. 
The diseased joint should be painted twice a day with 1-2 teaspoonfuls of this mixture and then covered with gauze, never with an impermeable bandage. [Mesotan has been introduced as a substitute for oil of wintergreen in external treatment, but has no advantages of any consequence over the older drug (Cushny).-Ed.] In this way a complete cure may be effected by salicyl-vasogen or mesotan alone, a great advantage, when we consider the frequent digestive disturbances induced by the internal administration of the salicylates. Practically the author usually combines with the external treatment small doses (4 G. (60 gr.) a day) of sodium salicylate or one of the substitutes.

Other Preparations for Salicylic Acid.-The second method of getting rid of the unpleasant taste of sodium salicylate is to substitute for it one of the numerous modern preparations on the market whose active principle is salicylic acid. Among them are: (1) salophen (acetylparaamido salol) soluble, white, tasteless and odorless crystals, which is given in powder form in doses of $0.5-1.0 \mathrm{G}$. (7-15 gr.) up to $6-8 \mathrm{G}$. (11/2-2 dr.) a day; (2) salacetol (acetol-salicylic-acidester) in similar doses; saligenin (an ortho-oxy-benzyl alcohol) in similar doses; (4) malakin (salicyl-para-phenetidin), clear, yellow, insoluble crystals; and (5) agathin (a salicyl-alpha-methyl-phenyl-hydrazone), greenish-white, tasteless and odorless flakes, with the same dosage as above.

All of these are weaker in their effect than sodium salicylate, but have the advantage of producing fewer unpleasant secondary effects.

Aspirin.-There is one other preparation, aspirin (acetylsalicylic acid) which is as powerful as sodium salicylate and yet has almost none of its unpleasant results. It does not disturb digestion or cause ringing in the ears, and can be given in the presence of renal inflammation. [It is decomposed in the intestines into salicylic acid.-Ed.] It is prescribed as follows :

Ry Aspirini

Dent. tal. cap. no. $\mathbf{x x x}$.

Sig. - 8-10 (or 4-5) capsules daily (4-5 G. (1-11 dr.) or more daily).

[Aspirin is prescribed in capsules so that the alkaline saliva shall not decompose it into salicylic acid. It should not be combined with alkalies. -Ed.]

Maretin.-In the author's hands maretin (a white powder insoluble in water, $0.5 \mathrm{G}$. ( $7 \mathrm{gr}$.) several times a day) has not proved so successful as aspirin. It is a carbaminic acid meta-tolyl-hydrazid.

Oil of Wintergreen.-Finally we may use the methylester of salicylic acid in the form of the well-known oil of wintergreen:

R Ol. gaultheriæ, Adipis lanæ hydros..............āa $10.00 \ldots \ldots$ ziiss

M. fiat ungt.

Sig. - To be used as an ointment. 
It should be rubbed first on the affected joints and then, as a prophylactic measure, on the other joints. [Kinnicutt recommends the internal administration of oil of gaultheria, 20 drops in capsules or in milk every 2 hours.-Ed.]

Contraindications for Salicylic Acid and Its Preparations.-Salicylic acid, sodium salicylate, and, in a less degree, the other preparations, should be used cautiously in case of coincident obesity with congestion, organic heart disease, and possibly severe brain symptoms. They are contraindicated in acute kidney disease, unless this is due to the rheumatic infection, in which case some of the various substitutes for sodium salicylate should be employed.

In regard to the unpleasant secondary effects of both the acid and sodium salicylate, it should be stated that, for the sake of affecting a cure, these drugs have to be given in rather large doses and, if possible, for at least 2-3 days. Fortunately by the time the first toxic symptoms appear, i.e., after the ariministration of 5-6 G. (1 $1 / 4-1 \mathrm{r} / 2 \mathrm{dr}$.) of salicylic acid or 8-10 G. (2-2 $1 / 2 \mathrm{dr}$.) of sodium salicylate, the acute symptoms of rheumatism have usually subsided. Although a cure cannot be said to have been completed, much has been accomplished towards it. But, if we cease to give the drugs, the symptoms often reappear, and sometimes very quickly. To avoid such a relapse and at the same time to avert any symptoms of intoxication, we should abandon the stronger drugs, and try some one of the milder preparations already mentioned, or some one of the substitutes for the salicylates to be considered later. Or, if the swelling or pain has entirely disappeared, we may stop medication and have recourse to an appropriate hydrotherapy. In case of a relapse, we must then return to large doses of either salicylic acid or sodium salicylate. Under one other condition the salicylates may well be abandoned: that is, in the subacnte and almost chronic cases, in which the salicylates fail in their effect, and the pains have become more or less fixed in one or more joints. [Osler recommends salicin in doses of $1.3 \mathrm{G}$. ( 20 gr.) every hour or two in acute cases until the pain is relieved. He believes that it is less depressing than the salicylate of soda, and considers it, perhaps, the best drug for children. Following Ransom, I have used it in such doses every hour for more than two days in several cases without any particularly disagreeable effects. It is a glucoside found in many species of willow and poplar, is decomposed into salicylic alcohol, which is oxidized to salicylic acid in the body, so that its action after absorption is similar to that of the acid. When administered by the mouth it is excreted in the urine partly as salicin, partly as salicylic alcohol, and partly as salicylic and salicyluric acids (Cushny). If this drug does not relieve the condition within 48 hours, I shift to salicylic acid, sodium salicylate, aspirin, or methyl salicylate, always selecting the natural rather than the synthetic preparation. I usually combine the salicylic with the alkaline treatment, in the hope of thus rendering 
cardiac complications less liable to occur. Fuller gave $6 \mathrm{G}$. $(11 / 2 \mathrm{dr}$.) of bicarbonate of soda with $4 \mathrm{G}$. ( $1 \mathrm{dr}$.) of acetate of potash in $100 \mathrm{G}$. ( $3 \mathrm{oz}$.) of water, and made this solution effervescent by adding $2 \mathrm{G}$. $(1 / 2 \mathrm{dr}$.) of citric acid or $30 \mathrm{G}$. ( $1 \mathrm{oz}$.) of lemon juice. As soon as the urine becomes alkaline, this amount should be diminished. This alkaline treatment, in addition to the other, sometimes proves too much for the patient's digestion and has to be decidedly curtailed or abandoned. -Ed.]

Substitutes for the Salicylates.-All the substitutes for salicylic acid and its derivatives belong, with the one exception of potassium iodide, to the aromatic series, and are all antinervines, antineuralgies, or antiseptics. The explanation of their effect is, in general, the same as that which we have tried to give for salicylic acid.

Amygdophenin.-One of the best is amygdophenin, (phenetidin mandelate), a white crystalline salt, barely soluble in water. In many cases it is of great benefit, while in others, which can be helped by the salicylates or lactophenin, it is powerless. In acute eases it is given in large daily doses :

R Amygdophenin. $.10 .00 \ldots . .5$ iiss

Div. in cap. no. $x x$.

Sig. - 1 capsule every hour for 16 hours (8 G. -2 dr.).

Lactophenin.-Another is lactophenin (the phenetidin of lactic acid), which is similar to amygdophenin in its physical properties and is prescribed in the same way. In case of slight pains the dose may be decreased. It often produces marked cyanosis, and twice the author has observed a manifest, though moderate, icterus.

Phenacetin.-Phenacetin (para-acet-phenetidin) up to $5 \mathrm{G}$. (11/4 dr.) [a larger amount than I dare give-Ed.] a day is equally effective.

Phenocoll.-Phenocoll (amido-acet-para-phenetidin) may be given in the form of a salt combined with either hydrochloric acid or salicylic acid; but its effeet is much less than that of the salicylates.

Antipyrin.-The French recommend antipyrin, especially for the more chronic cases. It is a good substitute for the salicylates, though less effective. It is given in solution or in capsules :

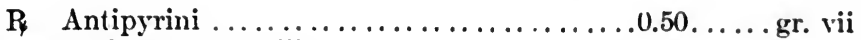

Dent. tal. cap. no. xviii.

Sig. -6-8 capsules daily.

Or :

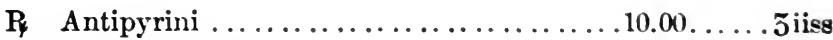

Aq. menth pip.,

Spir. vin. Gallici,

Syr. simp. .................. a $40.00 \ldots \ldots$ zi 3 iiss

M. fiat sol.

Sig. -1 teaspoonful every $1-2$ hours. 
Antipyrin unfortunately sometimes has unpleasant, although not dangerous, secondary effects.

Salipyrin.-Salipyrin, a salicylate compound of antipyrin, is as good as antipyrin and perhaps better, on account of its content of salicylic acid.

Acetopyrin.-A combination of antipyrin and acetylsalicylic acid, acetopyrin, has recently come into use. Occasionally, as in every form of salicylic therapy, the ordinary dose (4-6 G. $-1-11 / 2 \mathrm{dr}$. in capsules or sweetened water) is not sufficient, so that we are forced to give large doses (8-12 G.-2-3 dr.) and even then may leave some joint unaffected. Occasionally these large doses induce burning in the stomach, nausea, and even vomiting.

Analgen.-Analgen, a quinine compound, (0.5-1.0 G. (7-15 gr.) of the powder daily) has proved fairly satisfactory in many cases.

Colchicum.-The greater number of other drugs used in acute joint rheumatism have only historical interest. One old remedy, however, particularly for the protracted form of the disease, deserves mention, i.e., colchicum, either as the tincture or the wine, or prescribed as follows:

R Tinct. or vin. colchici sem. ..........10.00.... 3iiss

Vin. xerici.....................300.00....5

M. fiat mist.

Sig -1 tablespoonful every 3 hours up to 6 tablespoonfuls daily.

[If an acute polyarthritis does not respond to the salicylates, I try colchicine, $0.0006 \mathrm{G}$. $(1 / 100 \mathrm{gr}$.) in tablets every hour, until the condition is relieved or toxic symptoms from the colchicine supervene.-Ed.]

Local Therapy : Injections of Carbolic Acid.-Aside from the local use of the salicylates, already described, local medication is practically limited to iodine externally (and internally as potassium iodide) in some gonorrheal cases, and to periarticular injections of carbolic acid in others (2-4 hypodermics of a 1-2 per cent. solution daily). Apparently, however, these injections do not relieve the pain as in muscular rheumatism. In many of these gonorrheal cases, Bier's treatment with elastic bandages to produce passive congestion will gradually decrease the pain and swelling. The bandages are left on for an hour the first day; two hours the second; and so on up to twelve hours. They must be so adjusted that the pulse will always be palpable. [Torrey's serum has proved efficient in a number of cases. Cole has reported some success with therapeutic inoculations of bacterial vaccines according to Wright's method. In every case a careful examination of the deep urethra, prostate, and seminal vesicles is absolutely necessary, and if any inflammation remains, it should be treated locally (etching with silver nitrate). I have seen several obstinate cases cured by this means alone.-Ed.]

HYDROTHERAPY: Baths-Compresses.-Full baths are contraindicated on account of the difficulty the patient experiences in moving, and also often on account of a concomitant affection of the circulatory system. 
After the process has beeome subacute, warm baths, combined with careful massage, are often helpful in removing the stiffness of the joints. If some single joint remains affected after the rest of the body has been relieved, local hot baths (water, steam, hot air, sand) are sometimes very beneficial. [The best counter-irritant is the Paquelin cautery lightly and carefully applied. A 25-50 per cent. ichthyol ointment is often helpful in chronic cases, especially when combined with gentle massage and 3 or 4 times weekly the use of superheated dry air.-Ed.] Cold compresses or ice-bags are used to relieve obstinate pain with extensive swelling or inflammation, particularly in gonorrheal joint affections. Hot compresses are useful in protracted processes of a more or less chronic nature.

Alcohol Bandages.-The inflammation and pain can often be wonderfully relieved by an alcohol bandage, made by soaking at least nine layers of gauze in absolute alcohol. It must be covered with rubber tissue and secured firmly in place. It should be changed when the alcohol has evaporated (12-24 hours).

Hygiene: Position.-The position of the patient should, of course, be that which is least painful to him. He may often be relieved by the proper support of some joint or limb. [Absolute fixation of the joint by means of splints or plaster of Paris or starch bandages lessens the pain. The heavy cardboard, which can be cut in any shape, softened in boiling water, and then moulded to the part, forms a very convenient splint, especially for the shoulder joint. All splints should be carefully padded, and the joint proper should be left accessible. Cold (an ice coil or ice-bag on top of a light bandage) often relieves the agonizing pain. Heat is sometimes more effective. Fuller's lotion (carbonate of soda $24 \mathrm{G}$. ( $6 \mathrm{dr}$.), tincture of opium $30 \mathrm{G}$. (1 oz.), glycerin $60 \mathrm{G}$. (2 oz.), water 270 G. $(9$ oz. $)$ ) applied upon hot cloths often relieves the pain. Equal parts of guaiacol and olive oil or glycerin are often helpful.-Ed.]

Diet.-During the acute stage the diet should be very light. consisting of milk, rice cooked in milk, and a moderate amount of zwieback, bread, or crackers, etc., as such food furnishes the organism little or no material for the formation of uric acid. Later the patient may have raw or cooked fruit and easily digestible foods. Not until the disappearance of all the symptoms should eggs, meat, or broth be allowed, as. given too soon, they frequently cause a relapse or an exacerbation of the process. If the case becomes more or less chronic, we may be obliged to depart from this strict regulation; but we should keep the amount of meat down as much as possible.

Temperature of the Room-Rest in Bed.-The patient must be kept from cold, damp, and draughts in a room at $63^{\circ}-64^{\circ}$, and should remain in bed, even if the pain is not very severe. Even light bed-coverings are often so unbearable that they must be supported by a cradle or some other device. He should not be allowed to get up for at least a week after the cessation of the subjective symptoms, for fear of relapse or 
cardiac complications. [If possible, the patient should wear a flannel nightgown open in the back and easily put on and taken off. If he sweats much, it is better, in addition, for him to lie between thin woollen blankets. Extra precaution should be urged for quiet and repose. Even the shutting of a door will cause exquisite pain to a patient in the acute stage. It is very easy to furnish too much nursing. As much as possible he should be let alone, and, when moved, sufficiently strong assistance should be available.-Ed.]

RELAPSES.-After an apparently complete cure, we are sometimes surprised by a sudden and often considerable rise of temperature, lasting for several days, for which the closest investigation can find no cause. Kahler has pointed out that this fever may precede a fresh attack. The author would add that the administration of aspirin or sodium salicylate not only reduces the fever, but also prevents the threatened outbreak. [A course at one of the sulphur springs, after convalescence is established, is serviceable in many chronic cases, especially where there is a good deal of digestive disturbances, and anæmia. Mt. Clemens, Salt Sulphur Springs at Martinsville and many others are available; see p. 182. Most patients need iron and arsenic after an acute attack.-Ed.]

\section{THERAPY OF MALARIA.}

Quinine: Action.-The treatment of malaria consists almost entirely in the administration of quinine or its salts. In small doses the physiological action of quinine is very similar to that of the bitters; in large doses (0.5-5.0 G.- $7-75$ gr.) it often produces nausea, vomiting, ringing in the ears, deafness, disturbances of taste and sight, and occasionally amaurosis, headache, giddiness, confusion, and drowsiness. Even lethal cases have been observed. Its therapeutic value is due to its specific effect in malarial infection. This effect was empirically discovered and has not yet been satisfactorily explained. The experiments of Bing, by which he proves that quinine, like strychnine, is a specific protoplasmic poison, would seem to indicate that it kills the malarial plasmodia. That, in medicinal doses, it does not harm the human organism may be attributed to: (1) the relatively small dose, and (2) the greater resistance of human protoplasm.

Administration.-The first steps in the treatment of malaria are to relieve the gastro-intestinal symptoms, if present (vomiting, diarrhœa, constipation), and to determine the type of infection by microscopic examination of the parasites in the blood, or, if this is not possible, by allowing the disease to run unhindered through one or two attacks of fever. This may usually be done without any detriment to the patient, except, of course, in pernicious malaria with its severe nervous and gastrointestinal symptoms, where the next attack may be fatal. Fortunately such cases are rare. 
When the type of the fever has been determined, we prescribe as follows :

P Quininx hydrochlor. or bisulph........ 0.50,...gr. vii

Dent. tal. cap. no. iv.

Sig. -3 capsules on the evening of the day withont fever, and 1 on the morning of the attack; or 1 capsule 3,2 , and 1 houn before the beginning of the expected attack.

Or in pill form:

If Quininæ hydrochlor. or. bisulph. ......2.00....gr. $x \times x$

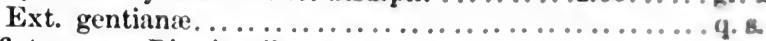

M. fiat mass. Div. in pil. no. $x x$.

Sig.-During the evening before the attack, 2 pills every 30 minutes up to 14 pills. In the morning of the attack the rest of the pills.

If the fever is quotidian, we begin the quinine direetly after or during the outbreak of the perspiration.

The hydrochlorate of quinine is to be preferred to the sulphate on account of its 8-9 per cent. greater content of quinine. [It is also very much more soluble; hydrochlorate 1:35, valerianate 1:100, sulphate 1:800 parts by weight of water. The addition of acid renders them all much more soluble.-Ed.] If we desire a slightly sedative effect, the hydrobromate or valerianate may be tised.

By this treatment the next attack is usually averted, or at least made much less severe. The repetition of the treatment is generally enough to prevent a return.

To prevent relapse, we either give $0.5 \mathrm{G}$. ( $7 \mathrm{gr}$.) a day for a week, or omit quinine entirely for a week, and then give as large a dose as at the beginning.

In case severe gastric disturbances prevent the administration of quinine by the mouth, it may be given by the rectum. a very effective method, since quinine is readily absorbed by the intestinal mueous mem. brane; e.g. :

R Tinct. opii................................. $m \times x$

Quininæ hydrochlor..............2.00-3.00.....gr. xxx-gr. xls

Mucil. acaciæ..................... $40.00 \ldots$ jii

M. fiat emuls.

Sig. - As an enema. [It is liable to cause irritation and be rapidly rejected (Cushny).-Ed.].

Or :

R Quininze hydrochlor................6.6...... $x$

Ext. belladon........................... gr.

Ol. theobromatis............................. $\mathrm{x} x \mathrm{x}$

M. fiat suppos. no. i. Dent. tal. suppos. no. v.

Sig. - 1-3 suppositories the day before an attack; 1-2 the morning of the attack.

Pernicious cases with severe gastro-intestinal symptoms sometimes force us to subcutaneous or intravenous injections.

Euquinine-Aristoquin.-Euquinine (quinine carbonie ester) and aristoquin (diquinine carbonic ester) have recently come into use, especially 
in malaria, as a substitute for the quinine salts. They have all the medicinal effects of quinine without such strong toxic properties. Euquinine is best given in capsules, the dose being half as large again as that of quinine. The dose of aristoquin is $0.5 \mathrm{G}$. ( $7 \mathrm{gr}$.) per dose, up to $1.5 \mathrm{G}$. (22 gr.) per day. The author puts great reliance upon the latter, preferring it to euquinine.

Methylene Blue.-Another drug used in malaria is methylene blue (methyl thionine hydrochloride), a dark brown bronze-like powder, easily soluble. It occasionally causes dysuria, which may be relieved or averted by the use of powdered nutmeg. It is best prescribed in capsules as follows :

R Methylthioninæ hydrochlor.,

Pulv. myristicæ................ āā $0.10 \ldots \ldots$ gr. iss

M. fiat cap. no. i. Dent. tal. dos. no. xx.

Sig. $-5-6$ capsules daily.

It is not so effective as quinine or aristoquin, and, to overcome the malaria completely, must be given again 8-10 days after the last attack. It is also not so sure as quinine in preventing a return. It is possible, however, that it is to be preferred to quinine in the æstivo-autumnal form of malaria. [In pregnancy it may be selected.-Ed.]

Arsenic.-Methylene blue and arsenic are the remedies usually used when there is an idiosyncrasy against quinine. Arsenic is particularly suited to inveterate cases of malarial cachexia and nervous disturbances (intermittent cramps, neuralgias). It is also used prophylactically.

Eucalyptus.-Of all the other drugs recommended for malaria, eucalyptus is the only one worth mentioning, particularly for prophylactic use. It is given in the tincture, alone or with arsenic :

Or :

R Tinct. eucalypt. (G. P.) ...............30.00..... $\mathrm{z}$ i

Sig. - 20-40 drops 3 times a day.

R Tinct. eucalypt.....................30.00....气 $\mathrm{i}$

Sod. arsenatis..................... $0.025 \ldots \ldots$ gr. $\frac{1}{3}$

Acaciæ,

Aq. gaultheriæ................ āā q.s. $60.00 \ldots \ldots .5$ ii

M. fiat emuls.

Sig. - 1 teaspoonful 3 times a day, increased to 9 times a day.

[Each teaspoonful contains 15 drops tinct. eucalyptus and $1 / 60$ gr. sod. arsenate. An emulsion is the only method of dispensing the above.-Ed.]

Diet.-During the treatment the diet should be restricted, except in the case of a cachectic individual, who must receive as much nourishing food as the state of his digestive tract will permit.

After-Treatment.-The after-treatment should be regulated according to the condition of the spleen. If this is still enlarged, we must repeat our specific for some days or use one of its substitutes. Most of the tumors of the spleen may thus be caused to disappear. Sometimes, however, one will be found so obstinate as to demand a mild Carlsbad or Marienbad cure, either at home or at the springs. 
[I always endeavor to give $0.2-0.6 \mathrm{G}$. (3-10 $\mathrm{gr}$.) of calomel and a brisk saline before I begin the use of quinine. The object of quinine administration is to mass as much quinine as possible in the circulation at the time of, and shortly before the paroxysms, because segmentation occurs then, and the parasites are most readily destroyed when they are free in the circulation, i.e., during and just after segmentation. "Quinine appears in the urine within a short time $(30$ minutes $)$ after its exhibition by the mouth, and about one-half of the quantity absorbed is excreted in six hours"' (Cushny). Osler prescribes 1.3-2.0 G. (20-30 gr.) daily for the first three days, and then continues smaller doses of the remedy for the next two or three weeks. In restivo-autumnal fever doses of $2-3 \mathrm{G}$. (30-45 gr.) daily may be necessary. In cases with pernicious symptoms he advises $1-1.3 \mathrm{G}$. (15-20 gr.) of the bisulphate every 2 or 3 hours hypodermically, or similar doses of the muriate of quinine and urea. Forchheimer advises giving the drug in one large dose, preferably in wafers or solution, 4 hours before the expected chill or better 6 hours before the maximum temperature. In the grave pernicious forms Baccelli recommends the whole of the following for intravenous use: quinine hydrochloride $1 \mathrm{G}$. (15 gr.), sodium chloride $0.75 \mathrm{G}$. (12 gr.), distilled water to make 10 e.c. $\left(2 \frac{1}{2} \mathrm{dr}\right.$.). In the severe forms all our therapeutic skill is required, hydrotherapy, eardiac stimulants, nervous sedatives, extra feeding, very careful nursing, etc. Craig agrees with Dock in giving one large dose during the decline of the temperature in tertian and quartan infections. The former prefers it in solution with dilute hydrochloric or sulphuric acid, one drop of the acid for each grain of quinine. Whenever quinine is used either hypodermically or for intravenous injections, the greatest eare should be taken with the instruments and the skin as regards disinfection. For hypodermics the buttock is generally selected and the injection made very deep.-Ed.]

\section{THERAPY OF TETANUS.}

Tetanus Antitoxin: Prophylactic Use.-Although the use of a serum has not thus far proved so suceessful in tetanus as in diphtheria, it is an efficient prophylactic measure. Given directly after the infection, it prevents the attack; while, even if the treatment is delayed for some time, it decidedly mitigates the severity of the disease. Experiments on both animals and men confirm this view, and so far no detrimental effects have been noted to militate against preventive inoculation.

[Punctured wounds, especially those produced by stepping upon rusty nails, wounds produced by toy pistols, blank cartridges, and giant firecrackers, crushed wounds with a good deal of dirt ground into them and wounds inflicted near and about stables are the most common causes of tetanus. A patient with such a wound should be ansesthetized, the wound opened widely, thoroughly curetted, cleaned. and freely drained. By the time this procedure has been completed the antitoxin, which should have been sent for immediately, will be at hand and a prophylactic dose 
of 1500 units $^{2}$ is injected into the subcutaneous tissues just above the wound. Scherck of St. Louis quotes the following figures to show the probable value of the antitoxin:

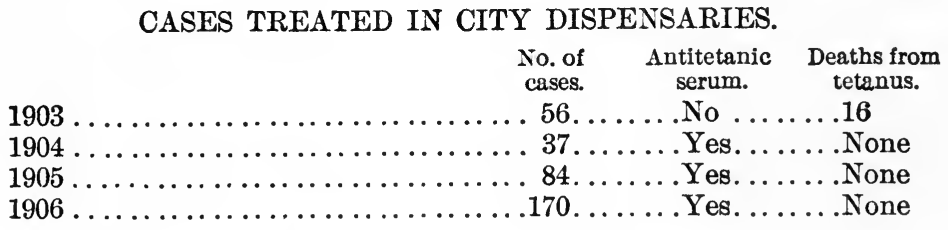

Newspaper aid is probably responsible for the striking increase of the cases in 1906.

Curative Use.-In the acute form of tetanus which develops a few days after the injury, no treatment seems to be of much avail after the symptoms have commenced; but, in the more chronic type in which the period of incubation is much longer, active treatment is often successful even after the onset of symptoms. Haste is, of course, the important factor. Three thousand to five thousand units should be immediately injected intravenously and, if possible, a similar dose into the trunks of the nerves from the infected part, because the toxin probably ascends by means of the lymphatics of the nerve. The dose should be repeated every 6 to 24 hours according to the severity of the symptoms.-Ed.]

The antitoxin may be regarded as neutralizing the tetanus toxin in the circulation. Where this toxin has already entered the ganglion cells of the central nervous system, the antitoxin is of no avail, except possibly immediately after the toxin has combined with the cells. This explains the necessity for an early administration, and also the fact that the disappearance of the symptoms is only very gradual. It is also evident that, when once the disease has manifested itself, and especially if some time has elapsed since the onset, we should not be content with the administration of the antitoxin, but should use every other known means for combating the disease.

Local Treatment.-The first and most important of these is the local treatment of the wound by thorough cauterization. If the exact spot of the entrance of the toxin is not known, we should cauterize every open wound, even the slightest, and every freshly healed one, since they, too, may contain tetanus spores.

Where cauterization is not possible, the wound should be thoroughly cleaned and then enveloped with a 2 per cent. [ $1 / 2$ to 1 per cent. solution is safer-Ed.] carbolic acid dressing; if carbolic intoxication appears, a $21 / 2$ per cent. solution of salicylic acid or a $1 / 10$ per cent. solution of thymol should be used. In some cases it is best to amputate the wounded extremity as quickly as possible.

${ }^{2}$ [The unit which has been adopted in the United States equals the amount which neutralizes 1000 fatal doses of tetanus toxin. This is about ten times the diphtheria unit.--Ed.] 
Other Medication.--To reduce the reflex irritability, we may use chloral (6-12 G. (1 $1 / 2-3 \mathrm{dr}$.) in 2-3 doses during the day), hypodermics of morphine repeated several times if necessary $(0.02-0.03 \mathrm{G} .(1 / 3-1 / 2 \mathrm{gr}$. $)$ each), or chloroform narcosis until the convulsions are controlled. Jaksch recommends urethane $(8-10 \mathrm{G}$. $(2-21 / 2 \mathrm{dr}$.) during the day); Strumpell. sodium salicylate $(0.5 \mathrm{G}$. ( $7 \mathrm{gr}$.) every hour); and Ziemssen, antipyrine (0.5-1.0 G. (7-15 gr.)) in case of fever.

General Measures: Absolute Quiet.-Prolonged, tepid baths (lasting several hours) and diaphoretic procedures (phénix à l'air chaud) may be tried, but only if the attempt does not induce an attack. The strictest quiet is, as a rule, imperative. The sick-room should be in as isolated a position as possible, it should be darkened, and all sounds and talking prohibited. Even the physician's visits should be short. Large quantities of fluid (milk, soup, wine, etc.) should be given to flush the organism. If trismus prevents their being taken by the mouth, enemas may be employed.

[The feeding of a patient with tetanus, especially in a prolonged case, is very important. If trismus is marked, liquid food can be introduced into the stomach by means of a catheter passed through the nose or by removing a tooth. The attendant should be given latitude and watch the patient's muscular contractions, so as to select the most favorable moment for feeding or for other details of nursing which necessitate the patient's moving.

Meltzer's discovery of the value of magnesium salts in the production of anæsthesia has already proved of considerable value in the treatment of tetanus. He employed intraspinal injections of a 25 per cent. solution of magnesium sulphate upon 8 monkeys and 3 human subjects with tetanus. The monkeys received $0.06-0.07 \mathrm{G}$. $(1-1 \mathrm{r} / 6 \mathrm{gr}$.) and the patients 0.02 G. ( $\mathrm{r} / 3 \mathrm{gr}$.) per kilo of body weight. All the monkeys died, but those treated lived 24-4S hours longer than the controls. One patient recovered. This treatment has been recently tried in a number of cases with success in several of them. The therapeutic value of the injection lies in the possibility of tiding over the patient by completely abolishing all tonic and clonic muscular contractions, until the newly formed antitoxin can overtake the balanee of free toxin and the metabolic processes of the body have mastered the toxins fixed by the nerve cells.-Ed.]

\section{THERAPY OF THE MOST COMMON ACUTE POISONINGS.}

General Principles of the Treatment of Acute Poisoning.According to their most marked effects, poisons, in general, may be divided into:

1. Strongly corrosive and colliquative substances:

Elements : chlorine, bromine, iodine.

Alkalies: sodium, potassium, and ammonium hydrate.

Acids : sulphuric, hydrochloric, phosphoric. 
2. Corrosive substances injuring the organs which retain or excrete them; e.g., phosphorus, arsenic, salts of antimony and mercury, cantharidin, and vegetable substances like croton oil.

3. Substances poisoning definite organs, slightly or not at all corrosive:

Blood-, Nerve-, Heart-poisons :

a. Poisonous gases : carbon dioxide, carbon monoxide, hydrogen sulphide.

b. Some inorganic salts: potassium bromide, potassium chloride, potassium cyanide.

c. Many organic drugs and alkaloids.

In this discussion we shall enumerate only the most necessary measures to be taken in cases of acute poisoning. The following quotation from Lewin lays down concisely the principles upon which our therapy must be based:

"In any acute poisoning all remedial measures must be directed towards :

1. Eliminating the poison from the body quickly and thoroughly.

2. Altering the poison chemically, as much as possible, so that it will temporarily or permanently lose its toxic qualities.

3. Restoring to their normal condition all the functions disturbed by the poison (symptomatic therapy).

To accomplish these aims we employ mechanical, purely chemical, and pharmacological measures. Knowledge of toxicology enables a physician to decide quickly which of these is suited to a given stage of any given poison."

Elimination of the Poison.-The first indication is to get the poison out of the stomach by inducing vomiting or by lavage. This should be done, not only when we have reason to believe that all of the poison still remains in the stomach, but also in many cases where manifest symptoms of general poisoning are already present, since for some poisons the stomach acts as an excretory organ (e.g., iodine, potassium iodide, morphine (given hypodermically)).

Lavage.-Lavage must always be tried first, and thoroughly enough to insure a complete emptying of the stomach. It is best accomplished by means of a soft stomach tube and funnel. If trismus, however, is present, a siphon tube is introduced through the nose, a procedure which may easily produce dyspnœa. In no case, especially in acid and caustic poisoning, should a stiff stomach tube be employed.

Emesis.--If lavage is impossible, vomiting must be induced. This is always an unsatisfactory method, since the stomach is never completely emptied, particles of the poison (especially resinous substances) remaining adherent to the walls or embedded in the mucus. Fatty or oily emetics should not be employed, as they spread the poison and hasten its absorption (e.g., phosphorus and the resinous poisons are soluble in oil). The only emetics recommended by Lewin are: (1) mustard powder (1-2 
small teaspoonfuls in a cup of lukewarm water, repeated, if necessary); and (2) 1.G. (15 gr.) of copper sulphate:

P Cupri sulphatis .................0.5-1.00....gr. viiss-gr. xv

Syr. simp. .............................. $20.00 \ldots \ldots \mathrm{v}$

M. fiat sol.

Aq. dest..................... s. adde $60.00 \ldots \ldots$.

Sig. - One-half to be taken at once, followed in minutes by the other half if necessary.

Apomorphine may also be given, $0.02 \mathrm{G} .(3 / 10 \mathrm{gr}$.$) , hypodermically,$ especially if on account of the swelling of the pharynx or cesophagus swallowing is difficult, or in the presence of severe convulsions.

All these emetics act within 3-5 minutes. They may be assisted by tickling the throat, exerting moderate pressure upon the epigastrium, and bending the body. When vomiting occurs, it is necessary, especially if the patient is unconscious, to make sure that the head is bent to one side, in order to avoid the entrance of the vomitus into the larynx and upper air passages, and a subsequent aspiration pneumonia.

Unfortunately neither lavage nor emesis is advisable, or at least should be employed only with great caution, in case of corrosive poisons, on account of the danger of perforating or tearing the stomach, injuring the large vessels of the corroded œsophagus, forming a false passage, etc. It is hard to limit definitely the interval after the entrance of the poison during which the use of the stomach tube is allowable. The time depends upon the quantity and strength of the poison that has entered the stomach. It may, of course, be used immediately or very soon after the poison has been taken; if a longer time has elapsed, our decision must be based upon the extent of the injuries in the mouth and throat.

Although many of the poisons of the second and third groups are somewhat corrosive, their action is not intense enough to contraindicate the use of the stomach tube or emetics. Most of these poisons themselves induce spontaneous vomiting. For the sake of cleaning out the stomach more completely, however, a stomach tube or emetics should also be used. Naturally this does not apply to poisons which in the stomach become converted into gases.

Catharsis.-We must give a cathartic immediately in case the poison has reached the intestine, especially if it is a substance more soluble in the intestine than in the stomach (as for instance the active principles contained in the plants of the euphorbiacer, such as castor oil and croton oil, the former of which contains ricin and the latter, croton ricin with tiglic acid. The resinous drastics are contraindicated on account of their irritating action, and the oily purges on account of their power of dissolving the poisons. Large doses of the saline hydrogogues are always preferable, and frequently also act as antidotes to the poison (sodium sulphate in lead poisoning). 
Diuresis.-Diuresis may also be brought into play to aid in the elimination of the poison, unless the latter is of such a nature as to have injured the kidneys and prevented their functioning (potassium chlorate, cantharadin). The best diuretics for this purpose are wine well diluted with plain or carbonated water, and milk. The use of the latter, however, must be limited on account of its fat content. [Buttermilk or skimmed milk may be substituted.-Ed.] Diuresis is, of course, helpful for those poisons which are excreted by the kidneys, to which class most of the aromatic compounds and alkaloids belong (quinine, strychnine).

Neutralization of the Poison.-This cannot be accomplished unless the substance in question is still wholly, or for the most part, in the stomach.

Poisons of the First Class: Halogen Poisoning-Acid Poisoning.If overcome by chlorine or bromine gases, which exert a strong corrosive action upon the upper respiratory passages, the patient should be quickly carried into the fresh air and given inhalations of steam. If chlorine, e.g., chlorine water, has been taken internally, the treatment is similar to that in concentrated acid poisoning. Large quantities of water must be given for purposes of dilution. Bromine and iodine are only slightly soluble in water, so that, to counteract them, as in the case of all acids, we give solutions and mixtures of alkalies and the alkaline earths. It must be remembered, however, that the carbon dioxide liberated from the carbonates is liable to distend and tear the corroded wall of the stomach. If unslaked lime (which should be slaked before using) is not at hand, we may use a very dilute milk of lime, which may be strained through a cloth. We may also, if necessary, use a mixture of burnt magnesia, very dilute lye, and a very dilute solution of carbonates, care being taken to give only small quantities at a time and to remove them again from the stomach quickly. This procedure should be repeated until the material removed no longer has an acid reaction. Finally solutions of egg white may be used in all cases of acid poisoning, since acids readily combine with the albumin. Starch is a special antidote for both bromine and iodine, forming bromine and iodine starches. Another for bromine is a $1 / 2$ per cent. solution of carbolic acid, forming a tribromphenol. All these compounds are broken up again in the intestine, so that it is necessary to remove them from the stomach.

If the damage to the œsophagus or stomach is too extensive (e.g., fatal perforation) to make the direct procedure against the poison effective, we must resort to symptomatic therapy.

Alkali Poisoning.--In alkali poisoning it isessential to know how much time has elapsed since ingestion of the poison. On account of the strongly colliquative effect of the alkalies, the administration of their antidotes is indicated only within the first hour (Wollner). The most effective antidotes are diluted vegetable acids; e.g., diluted vinegar, lemon juice, a pinch of cream of tartar in a pint of water. After an hour's time 
they are of no more use, but, on the contrary, frequently do harm by irritating the injured mucous membrane of the stomach. In such cases the use of the stomach tube is superfluous or impossible. All we can do is to employ palliative measures. As cold liquids can be taken only with difficulty, if at all, we prescribe warm $\left(90^{\circ}-95^{\circ}\right)$ demulcents; $e . g$., $500 \mathrm{G}$. (1 pt.) of a decoction of mallow or flaxseed, $300 \mathrm{G}$. (10 oz.) of an emulsion of almonds, or mucilage of acacia or tragacanth may be given in tablespoonful doses every 15-30 minutes.

Poisons of the Second Class.-The administration of antidotes for poisons of the second and third groups is similar to that for those of the first group. The most important antidotes for the commonest of these poisons will be discussed in the following paragraphs.

Phosphorus Poisoning.-In recent phosphorus poisoning we first carefully wash out the stomach, and then administer either a copper salt (usually copper sulphate) or potassium permanganate. The former (1-2 G. (15-30 gr.) in substance or solution) coats the particles of phosphorus with a copper deposit, thus making them incapable of absorption. ${ }^{3}$ The latter changes the phosphorus to phosphoric acid. It may be added directly to the water used in washing out the stomach ( 0.1 G. $-11 / 2$ gr.), or it may be given afterwards $(500-2000 \mathrm{G}$. ( $1 / 2-2$ qt.) of a 0.1 per cent. aqueous solution), or the stomach may be washed out with the same amount of a 0.1 per cent. solution several times a day (5 gallons daily for several days), and a tablespoonful be given internally every 2 hours.

After we have followed out one of these procedures, or, if the use of the stomach tube is impracticable, have induced vomiting by copper sulphate, ozonized oil of turpentine may be given for several days. Its effect is due to the formation of turpentine-phosphoric acid, which is non-poisonous. It is usually prescribed as the emulsion, in teaspoonful doses every half hour for 8 doses and then every hour. [The ordinary American oil of turpentine appears to be of no antidotal value in phosphorus poisoning. Oil of turpentine was originally proposed by Audant, who was called to see an attempted suicide. The man had swallowed phosphorus paste, and then, to make it sure, had taken a drink of oil of turpentine. He made a good recovery with slight symptoms. Animal experiments showed that the turpentine either delayed or prevented the poisonous effects of phosphorus. The acid French oil of European commerce is efficient, if 100 parts are given to each part of phosphorus ingested.-Ed.]

In place of the oil of turpentine, copper carbonate may be used. It coats the particles of phosphorus, first with copper phosphate and then

${ }^{3}$ [The National Dispensatory states that most acute symptoms will be produced by the use of copper sulphate enough to coat the phosphorus. If emesis is induced, or the drug is introduced in solution by a stomach tube and promptly evacuated, this danger, I should think, would be avoided.-Ed.] 
with pure copper, thus preventing the solution and evaporation of the phosphorus.

R Cupri carbonatis................1.00-2.00.....gr. xv-gr. $\mathbf{x x x}$

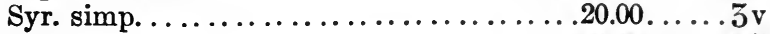

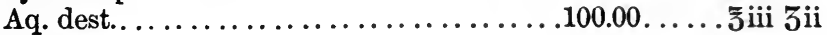

M. fiat mist.

Sig. - 1 tablespoonful every half hour.

With potassium permanganate or oil of turpentine we give drastic purges each time for several days (almost always an infusion of senna). [With severe symptoms large doses of alkalies should be administered, preferably by intravenous injection just as in diabetic coma. Such an attempt may counteract the acid intoxication.-Ed.]

Arsenic Poisoning.-Of the arsenic compounds two may be regarded as primarily poisonous-arsenous acid and arseniuretted hydrogen. Most of the other preparations owe their toxic properties to their content or to the formation of arsenous acid; some, to the formation of arseniuretted hydrogen.

Scarcely any other poison demands such speed in the administration of its antidote as does arsenous acid. Fortunately its antidote, iron and magnesium hydrate (ferri hydroxidum cum magnesii oxido) is remarkably effective. After washing out the stomach, we give it in ounce doses repeated every 15 minutes until there is purging and cessation of the acute symptoms, then every $1 / 2-1$ hour. This antidote changes the arsenous acid into its insoluble compounds, ferrous arsenate and magnesium arsenate, setting free at the same time magnesium sulphate, which has a somewhat powerful cathartic effect, aided by the excess of magnesium oxide. [The U. S. P. of 1890 recognized this combination, so that it should always be quickly available at any pharmacist's. Twelve or more times the amount of arsenic ingested should be employed. It is harmless, so that it is well to be upon the safe side. If some of the drug has been absorbed, opium may be given for the pain, stimulants for collapse, and mild diuretics and diaphoretics to assist in elimination.-Ed.]

Metal Poisoning.- The next groups of poisons to be considered are the earthy and the heavy metals, including antimony. Here, as usual, lavage or emesis must first be tried. If neither of these can be carried out, the most available antidotes, milk and white of egg, must be given in large quantities. These form more or less insoluble albuminates, which break up after a time in the stomach and intestine, and so they, too, must be removed by lavage or vomiting. It is unnecessary to enumerate the exact chemical antidote for each metal. In general, those should be chosen which are harmless themselves and which form with the given poison the least soluble compounds; e.g., for silver metals, a 1 per cent. sodium chloride solution; for barium or lead salts, sodium sulphate.

Poisons of the Third Group.-When we come to consider the poisons of the third group, we are met at once by the impossibility of giving an 
antidote for that class taken in by the lungs and carried in the blood stream. As for the others (e.g., sodium bromide, potassium chlorate, and the poisons of the aromatic group-phenol, etc.), they must be treated by the stomach tube and rapid elimination through the kidneys and intestines, with the accompanying appropriate symptomatic therapy. [The action of alcohol in neutralizing the effect of carbolic acid upon the skin is so suggestive that it is worth while administering 2-4 ounces of whisky after the usual lavage and administration of Epsom salts or sodium sulphate.-Ed.]

An antidote may be given for poisoning by cyanides, although their action is so rapid that little good can usually be accomplished. As the antidote forming insoluble iron salts is hard to give and uncertain in its action, potassium permanganate, which converts the hydrocyanic acid into oxamide, is usually employed:

R Potass. permangan..................6.00.... 3iss

Aq. dest. .......................2000.00.... Oiv

M. fiat sol.

Sig.-For lavage.

One pint of this 0.3 per cent. solution is allowed to flow into the stomach and removed again in 1-2 minutes, the process being then repeated. If no stomach tube is at hand, the patient must drink the solution. If it is not possible to get the antidote, the stomach should be washed out with water. ${ }^{4}$

In poisoning with alkaloids, the stomach should be washed out with a solution of tannin or potassium iodide, which should be removed immediately. We may prescribe as follows:

Or :

R Acidi tannici....................... $5.00 \ldots .5 \mathrm{igr} . \mathrm{xv}$

Aq. dest. . ......................... Oii

M. fiat sol.

Sig.-For lavage.

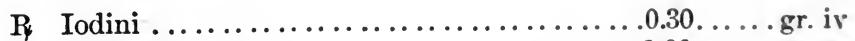

Potass. iod................................... xv

Aq. dest. ........................ 1000.00 . . .

M. fiat sol.

Sig.-For lavage.

Symptomatic Therapy.-Everything possible having been done to get the poison out of the body and to neutralize it in the body, the third. and perhaps the hardest, task remains, - to avert or overcome the

4 [Martin and O'Brian recommend the following to be on hand at all reduction works: (1) 30 c.c. ( 1 oz.) of 23 per cent. solution of ferrous sulphate, (2) 30 c.c. ( $1 \mathrm{oz}$.) of 5 per cent. solution of potassium hydroxide, both in hermetically sealed bottles, (3) $2 \mathrm{G}$. ( $30 \mathrm{gr}$.) powdered magnesium oxide, (4) metal receptacle capable of holding a pint. Break (1) and (2) into the receptacle with the magnesia, add a half pint of water, and swallow at once.-Ed.] 
threatening symptoms manifested by individual organs or by the whole organism. Such symptoms are:

1. Pronounced cardiac weakness.

2. Disturbance of respiration.

3. Incipient cerebral paralysis.

4. Severe convulsions.

5. Considerable alterations in the red blood corpuscles or the hæmoglobin.

These symptoms must be combated, in order to maintain life until the poison has ceased to work or has been excreted. Many of the following remedial measures may be used simultaneously.

Cardiac Insufficiency: Heat.-Heart failure and paralysis of the respiratory centre are generally the immediate cause of death in acute poisoning. Every energy must be bent to combat collapse. The chief indication is the application of heat, not only for the sake of bringing the temperature up to the normal, but also on account of its direct action upon the heart and indirect action upon the respiratory and cardiac centres. We must, therefore, endeavor to keep the temperature from becoming sub-normal or bring it back to the normal, if it has fallen below. The room should be kept at $64^{\circ}-68^{\circ}$, and the patient covered with blankets and rubbed and massaged vigorously through them. Hot cloths and hot bottles should also be used, a hot compress being laid on the præcordial region. It is, furthermore, advisable to administer hot drinks, if possible.

Heart Stimulants. - Not much can be expected from the use of heart stimulants. If given by the mouth, they are not absorbed quickly enough, especially if the motility of the stomach has been affected by the poison; while, if given hypodermically, their efficiency is diminished by the impaired activity of the viscera. It is best, on the whole, to give them by the rectum, as that portion of the intestine has a high power of absorption, and is also available during convulsions, trismus, ete. But it is possible to give them in any or all of the three ways as seems best in the individual case.

Digitalis is contraindicated on account of its toxic effect on the heart. One of its substitutes must, therefore, be chosen; e.g., caffeine, coffee, tea, strychnine, camphor, ether, musk, and possibly ammonia and the ammonium salts. For their effects, dosage, etc., see the chapter on diseases of the heart. There will be given here only such details as differ somewhat from the usual rules.

Caffeine is best given not in the alkaloid, but in the form of very strong black coffee, 150-200 G. (5-6 oz.) (10-15 per cent.) ; in this way we save time and also get the advantage of a warm drink and of the aromatic substances contained in the coffee. The same quantity of tea may be given in a 2-10 per cent. infusion, and here we get, in addition, the possible effect of the tannic acid as an antidote. Both coffee and tea should be taken without sugar or milk. They may also be given by the rectum; but, 
in case it seems advisable to use a hypodermic, the double salts of caffeine must be employed. (See p. 22.)

Strychnine the author would consider indicated only under peculiar circumstances. A hypodermic of $0.005 \mathrm{G}$. $(1 / 12 \mathrm{gr}$.) may be followed by one of $0.003 \mathrm{G}$. $(1 / 20 \mathrm{gr}$.$) .$

Camphor and ether [ 10 per cent. solution of camphor in ether-Ed.] are much more frequently used. They are usually given hypodermically, but may be used in enemas, (1-3 small teaspoonfuls in 60-100 G. (2-3 oz.) of water or starch solution, repeated several times).

If musk is employed, it should be given in the tincture, 3-4 G. (45-60 min.) internally or 4-6 G. (1-1/2 dr.) by the rectum.

Caution must be used in giving the patient ammonia to inhale for the purpose of stimulating the brain and the nervous centres. The vapor, with its irritating and corrosive action on the mucous membrane, may easily cause a reflex closure of the glottis with very serious effects on the already depressed respiration. Ammonia, if given at all, is best added to an enema (30 drops) or administered as described on p. 25.

Ammonium chloride is given hypodermically. Its effect is short, as compared with that of the solution of camphor either in oil or in ether.

Respiratory Weakness.-Another symptom in acute poisoning as common as heart failure, is enfeebled respiration, indicated by the symptoms of dyspnœa and asphyxia. To combat this, a large number of methods are available.

Two hindrances to normal breathing are usually present: first, mechanical obstructions, and, second, paralysis of certain nerve centres. The mechanical obstructions must first be overcome, or, if this cannot be accomplished, it must be made possible for the patient to breathe in spite of them.

Removal of Obstruction to Breathing.-Frequently masses of vomitus or mucus are lodged in the throat and entrance to the larynx. These should be removed immediately with the finger wrapped in a bit of cotton, or else rapidly sucked out with an elastic eatheter. This must be done with the greatest haste, in order not to delay the return of the respiration and not to induce fresh vomiting.

In case the entrance to the larynx has been so injured that the resulting œdema threatens asphyxia, tracheotomy must be resorted to. This operation is also indicated in case of trismus with accompanying unconsciousness. In such a case the tongue falls back against the posterior wall of the throat, choking the entrance to the larynx, while the usual methods of raising the epiglottis are impracticable on account of the trismus.

These ordinary methods, when the throat is accessible, consist of : (1) pulling forward the tongue, (2) pulling forward and raising the lower jaw, and (3) keeping the throat raised while the head and neck are allowed to fall back. All three are very practicable, and are easily combined with artificial respiration. 
The first is accomplished by pulling the tongue vigorously forward by means of forceps, suture, or cloth wound around it. The epiglottis is pulled forward by the glosso-epiglottic ligament and the entrance to the larynx cleared. The same effect may be brought about by energetically pulling the hyoid bone forward with the hand or a hook caught around it. In case of necessity, we try to reach the epiglottis with the index finger and raise it.

The second method consists in grasping the angle of the lower jaw and pulling it forward and upward. By means of the musculature of the hyoid bone and the lower jaw, and the hyo-epiglottic ligament, this pull is communicated to the epiglottis.

In the third method the head is allowed to fall back, so that the tongue no longer lies on the posterior wall of the throat, but on the gums, thus permitting the free passage of air from the nose into the larynx. A small cushion or a rolled up blanket is placed under the patient's shoulders, and the arms are stretched out above the head, with the skull touching the ground. This method has the advantage of enabling the physician to carry on artificial respiration at the same time.

Artificial Respiration.-If these methods alone do not succeed, artificial respiration must be carried on simultaneously. There are three principal methods, the efficiency of all of them depending upon the regular rhythm with which the movements are executed. In the first method the operator's hands are laid on the lower portion of the lateral chest walls, and 15-20 expirations a minute caused by pressing downward and inward and then relaxing the pressure. In Schuller's method the thorax is seized with both hands under the lower costal margin and vigorously pulled up and then pressed down. The pull enlarges the thoracic aperture and flattens the diaphragm, i.e., causes inspiration; the pressure, of course, causes expiration. Silvestri's method is, in a way, a combination of the other two, since expiration is brought about by pressure on the lower part of the thorax, and inspiration, by pulling on the rib muscles and the accessory respiratory muscles which are connected with the shoulder girdle. The physician, standing at the head of the bed, presses the patient's arms, which are bent at the elbow, strongly against the sides of the throax and then pulls them energetically and quickly backwards, until they are stretched out at full length over the head of the patient, which should be turned somewhat to one side. All three methods give good results, and the choice must depend upon whether or not assistance is at hand. If the physician is alone, it is best to employ the first or second, in combination with the third method of raising the epiglottis, that is, by letting the head fall backward. Silvestri's method may also be used by putting the patient on an inclined table with his head hanging over the edge.

The first and the third methods of artificial respiration have the advantage of stimulating the heart and circulation by means of the rhythmical movement of the thoracic wall. The same principle is applied 
in the so-called "massage of the heart," when the precordial region is slapped rhythmically and frequently (up to 120 times) with the hand. This may well be combined with artificial respiration.

Von Duchenne recommends the faradization of the nerves of the diaphragm. Both electrodes, well moistened, are pressed inward upon the outer border of the sterno-cleido-mastoid over the lower portion of the scalenus anticus. More or less deep inspiration is eaused by the stimulation of the phrenic and neighboring nerves (vagus) and of the accessory muscles of respiration, the sterno-cleido-mastoid, pectoralis minor, serratus, their points of insertion (head and shoulder girdle) being held still by an assistant. Fxpiration is then induced, immediately after shutting off the current, by compression of the lower thoracic aperture.

Artificial respiration should, if necessary, be carried out persistently for a long time, even if there seems very little chance of saving the patient. The author has known cases when it was kept up for 2-5 hours. thereby giving the organism time to eliminate the poison. In several cases of chloroform asphyxia, 20 minutes' artificial respiration brought back cardiac and respiratory action, although life had seemed entirely extinct.

Reflex Stimulation of Respiration.-Before beginning artificial respiration, however, we should endeavor to start natural breathing by reflex action. The most common methods are: sprinkling the face with cold water, flecking the face or the region of the precordium with a wet cloth, pouring a stream of cold water from a considerable height upon the neck. The last may be advantageously combined with a bath at $95^{\circ}$ or over. But the longer unconsciousness has continued, the less may be expected from any of these procedures, since the sensibility of the skin is diminished by the same amount as the reflex irritability of the respiratory centres. If the skin is not sufficiently sensitive, we try the mucous mem. branes, avoiding, however, the use of ammonia, as this gas is so irritating that it may lead to closure of the glottis, which, when every breath is of such supreme importance, may easily prove fatal. We may try spraying the nose with cold water, taking care not to fill up the throat, or we may stimulate the nasal membrane with a powerful faradic current for 10-20 seconds. Respiration is frequently started by one or two such applications.

Atropine.-Atropine should always be tried in all nareotic intoxications with symptoms of cardiac insufficiency and paralysis of the respiratory centres. It is especially useful in morphine poisoning, morphine and atropine being each respectively the antidote of the other. In severe cases we may give the maximum dose of $0.002 \mathrm{G}$. (1/so gr.) hypodermically.

Cerebral Paralysis.-A further symptom to be combated is incipient cerebral paralysis. If this is due to anæmia, we should immediately lower the head and raise and bandage the extremities, in order to bring about 
autotransfusion. If, however, it is due to hyperæmia, we should employ strong stimulation of the skin-mustard plasters to the soles of the feet, and the neck, rubbing of the irritated skin with croton oil, inhalation of ammonia, irritating enemas (vinegar enemas), or even sometimes venesection. In some cases the patient must be forcibly supported and made to keep walking, in order to prevent the cessation of cerebral functioning.

Muscular Convulsions.-In case of muscular convulsions brought on by such poisons as strychnine and santonin, we are unfortunately not able to use the ordinary antispasmodics, since they are also heart and respiratory depressants. Direct heart poisons, such as chloral, should especially be avoided. Morphine is also contraindicated. Ether or chloroform anæsthesia may be employed, either to stop a single convulsion or to suppress the tendency to continued spasms, as for example in strychnine poisoning. Paraldehyde may also be employed (2-6 G. $-1 / 2-11 / 2$ dr.). This drug spares the patient's strength as far as possible and gives the physician an opportunity to try other remedies. For example, it frequently relieves the trismus in strychnine poisoning, thus making it possible to use the stomach pump, give nourishment, etc.

Alterations of the Blood.-Many poisons alter the constitution of the blood, partly by chemical changes in the hæmoglobin, partly by the destruction of the red corpuscles. Carbon monoxide, for example, forms a stable compound with the hæmoglobin (carboxy hæmoglobin), while others change the hæmoglobin into methæmoglobin (sulphuretted hydrogen, aniline, nitrobenzine, antifebrin, sulphonal, trional, potassium chlorate). In such cases abundance of fresh air, artificial respiration, and the inhalation of oxygen are indicated, while venesection will frequently restore the lost consciousness and strengthen the weak pulse. If practicable, it may be followed by a blood transfusion or salt infusion.

Pain.-Common humanity demands that the frequently torturing pain should be as far as possible relieved. It is furthermore necessary to relieve pain, in order to spare the patient's strength and to put him in such a condition that the proper therapeutic measures may be tried with a free hand. We may add cocaine to the water used for lavage, especially if the pains are in the stomach. But in most cases the most efficacious anodyne is a $0.02-0.03 \mathrm{G} .(1 / 3-1 / 2 \mathrm{gr}$.) injection of morphine. 


\section{XV. \\ THERAPY OF INFECTIOUS DISEASES}

By PROF. DR. FERDINAND FRÜHWALD.

INTRODUCTION.-In no other diseases must we trust so much to Nature's curative power as in the infectious diseases about to be diseussed. In most of them we are confronted with an unknown toxin, which mani. fests itself clinically by countless external and internal symptoms with the most varied complications, and for which, with the one exception of the diphtheria toxin, we have no specific remedy. The physician should keep constantly in mind the pregnant remark which Leyden made in the opening address at the Congress for Internal Medicine in Berlin: "We have not only the disease, but, primarily, the patient, to treat, and noth. ing, not even an apparent trifle, is indifferent where he is concerned."

Antipyretic Medication.-Most of the infectious diseases are accompanied by more or less fever, especially in the initial stages. As the high temperature may cause parenchymatous degeneration of individual organs and tissues, especially in children, all rational measures to reduce the fever should be utilized. Unfortunately the antipyreties in common use cannot be employed so freely in the infectious diseases, especially with children, as in other diseases. It would, therefore, seem best to discuss briefly the antipyretics available, and then to consider at greater length the various hydropathic procedures that may be employed.

Quinine.-To obtain an effective antipyretic action from quinine 1.5-3 G. (20-45 gr.), are necessary (Liebermeister), the dose varying, however, with the strength of the patient. It is best given in solution, either quinine sulphate in a weak sulphuric acid solution, or quinine hydrochlorate in water. For children quinine tannate is preferable. If the solution is taken with difficulty, the powder in eapsules may be used for adults, and the quinine-chocolate tablets for ehildren. If the patient objects to taking it, or if it induces vomiting, it may be given hypodermically, in an enema (with the addition of tincture of opium), or as suppositories. The effect, if given by the reetum, will be only a little less strong than if given by the mouth. Quinine is sometimes borne by children in relatively larger doses than by adults. Binz advises giving it early in the morning, late in the evening, and at night. But it should never be repeated within 24 and usually not within 48 hours. Hagenbach gives $0.7-1.0 \mathrm{G}$. (10-15 gr.) to children under two years; $1.0 \mathrm{G}$. (15 gr.) to those $3-5$ years old; $1.0-1.5$ G. $(15-20 \mathrm{gr}$.) to those $6-10$ years old; and 1.5-2.0 G. (20-30 gr.) to those 11-15 years old. 
Euquinine.-The preparations of euquinine introduced by von Noorden have, especially for children, certain advantages over quininethey produce no quinine intoxication and no disagreeable gastric symptoms, and have hardly any taste; they do, however, cause ringing in the ears. The author prefers euquinine hydrochlorate, which is easily soluble in water, and aristochin, which has practically no taste. ["Except in cases of malaria, quinine should not be employed for the reduction of temperature in children. The dose required is so large, the difficulty of administration is so great, and the tendency to upset the stomach is so uniform, that its use should be discouraged altogether; besides its effect is extremely uncertain" (Holt).-Ed.]

Sodium Salicylate.-Sodium salicylate and aspirin are as reliable antipyretics as quinine. The fall of temperature in the individual case depends not alone upon the size of the dose, but also upon the time of day chosen for administration, the nature, intensity, and stage of the disease, the grade and obstinacy of the fever, and individual characteristics. They usually take effect more quickly than quinine (1-2 hours). Liebermeister gives $4-6$ G. $(1-11 / 2 \mathrm{dr}$.) as the dose for an adult. For children under 1 year Hagenbach uses 1.0 G. (15 gr.) ; from 1-5 years, 1.5-3.0 G. (20-45 gr.) ; from 6-15 years, 3.5-5.5 G. (52-80 gr.). These amounts should not be taken all at once, but should be distributed over $1-11 / 2$ hours. Like quinine, both sodium salicylate and aspirin cause ringing in the ears, uncomfortable feelings, weakness and trembling of the extremities. These manifestations are, however, less severe than those caused by quinine. Symptoms of heart weakness contraindicate their use. [Holt's statement for quinine quoted above might properly include this use of either salicylate of soda or aspirin. The author includes calomel and digitalis as drugs sometimes used for their antipyretic effect. Such a purpose is never in my mind in using either of them.Ed.] The modern antipyretics may injure the cardiac muscle, if used in the acute infectious diseases; hence they are contraindicated and need not be discussed.

HYdROTHERAPY.-More important than any drug for reducing fever in infectious diseases are the various hydropathic measures. Which of them is chosen in any case depends upon the conveniences at hand, the patient's preference, his individuality, and his condition at the moment. Care should be taken to involve as little annoyance as possible for the patient and attendants.

The importance of hydrotherapy in this connection is so great as to warrant a systematic discussion of the various practical methods of applying it, especcially as they are not known as thoroughly as they deserve to be among the profession. The following description is taken in part from Barwinski's discussion (Anleitung zur hydropathischen Behandlung der acuten Infectionskrankheiten. Med. Bibliothek, 16-18). He classifies hydropathic procedures as follows:

1. Internal use of water. 


\section{External use of water (sponging, packs, baths).}

Internal.- Internal therapy consists in the methodical administration of large quantities of water, which by its cooling effect and also by its chemical action, diminishes the dulness of the sensorium and the dryness of the tongue, and causes diuresis.

Introduction of Water into the Stomach.-If it is difficult for the patient to take so much water on account of apathy or unconsciousness, or of paralysis or spasm of the muscles of swallowing, we may employ the stomach tube to introduce water into the stomach. In private practice, however, this is hardly a practical method. [Saline enemas or saline irrigations are more commonly employed.-Ed.]

Nasal Irrigation.-For the dryness of the mucous membranes, as for example in scarlet fever and diphtheria, it is often best to irrigate or spray out the nose and throat with a physiological salt solution or a weak antiseptic or astringent solution.

External: Sponging.-This is especially appropriate to the initial stages of infectious diseases, and is such a mild method that it obviates any prejudice against hydrotherapy on the part of the patient or his family. The patient, lying on a dry sheet, is sponged off quickly with a sponge or wash-cloth wet with water at $45^{\circ}-50^{\circ}$. He is then rubbed vigorously with a dry bath-towel and covered with a blanket. This procedure should be repeated after a longer or shorter time according to its effect [e.g., every four hours if the temperature is $104^{\circ}$ or over-Ed.]. The body is much better cooled off by wrapping him while still wet in a dry sheet and blanket, than by rubbing the body dry. Too rapid a fall of the cutaneous temperature suggests cardiac weakness and threatening collapse, and necessitates watehing the patient most carefully.

Cold Rubs.-Cold rubs are more stimulating. The patient's head, breast, and armpits are first washed and a cold compress put on his head. The body is then wrapped in a towel wrung out in water at $45^{\circ}-50^{\circ}$, and rubbed quickly for $\mathbf{2 - 5}$ minutes with long, strong strokes.

Wet Paclis.- -One of the most common and useful of hydropathic procedures is the wet pack. It is indicated in most cases of infectious disease, especially for children and for the moderate grades of fever, the cold baths being reserved for continued high fever and severe cerebral symptoms. The patient's head, neck, breast, and back are first wet as a protection against congestion. He is then laid on a wet sheet, which is brought up smoothly around his body and a laver tucked between any two parts of his body that are in contact. It must be fitted securely and evenly around the neck. With young children, the arms should be left free; with others they may be bound down to the sides. The feet should be warmed before being enclosed in the sheet, especially in children. It may sometimes be best to leave them free. A blanket which has previously been spread on the bed is then wrapped closely around the patient. If a diaphoretic action is desired, more covering may be laid on top, until a profuse sweat is produced. If a sedative effect is all that 
is wanted, the sheet should be taken off as soon as the perspiration and drowsiness begin. If, on the contrary, we desire to stimulate the patient, he should be removed from the pack at the moment when the wet sheet has been warmed to the body temperature, and then be given a cold rub or half-bath. If the primary effect desired is to reduce the fever, the patient should be left in the pack until he is thoroughly warmed again (10-30 minutes), and then changed to a fresh one, the process being repeated 10-12 times, or until the temperature has fallen. If during the process the patient has a chill, he should not be subjected to another pack, but should stay wrapped up 3/4-11/2 hours, and afterwards be given a short rub-down or a half-bath.

Cold packs may be advantageously used for their sedative effect at the onset of infectious diseases where, although the fever is relatively slight, there is an inclination towards excitement, sleeplessness, delirium, pain, and, in children, convulsions. One or two cold packs in the evening will usually insure a quiet night, especially with children. The procedure should be followed by a short rub-down or half-bath $\left(68^{\circ}\right)$ or, with children, by a sponge bath. In fact, one of these should always be employed after every cold pack, whether it has been used as a sedative, stimulating, antipyretic, or diaphoretic measure.

Cold packs are contraindicated in coma, stupor, and weak heart.

Compresses.-The technic of and indications for compresses will best be treated under the separate infectious diseases.

Baths.-Although it is often difficult to make use of baths, especially in private practice, certain conditions in infectious diseases necessitate their employment, and with the greatest possible attention to exact technic. The physician should be perfectly definite in his directions and, at the slightest sign of an unsatisfactory result, should try some other mode of procedure.

Baths may be divided into tub or "whole" baths, "half-baths," and "sitz baths;" and into "cold" $\left(59^{\circ}-77^{\circ}\right)$, "cool" $\left(77^{\circ}-86^{\circ}\right)$, and "tepid" baths $\left(86^{\circ}-95^{\circ}\right)$. The latter two usually last 15-20 minutes, but a "prolonged" tepid bath may take half an hour. They are less powerful in reducing fever than are cold baths, but are suitable for the initial stages of infectious diseases.

The duration of the cold bath depends upon the stage of the disease, the body temperature, the quality of the heart action, the state of the nervous system, and the general constitutional condition. In general, the more recent the illness, the higher the temperature, the more resistant the heart and nervous system, and the stronger the constitution, the colder, longer, and more frequent the baths. The head and breast should be first washed with cold water and the patient then immersed up to his shoulders, his head being wrapped in a towel. For 10-20 minutes the extremities, breast, and back should be rubbed, and the bath water (or if there are nervous symptoms still colder water) splashed over his head. At the end of that time the patient is put back in bed, wrapped in a 
sheet, and vigorously rubbed, especially those parts that feel cold. The condition of the patient must be most carefully watehed, and the tem. perature and frequency of succeeding baths be determined by the subjective symptoms, the temperature, and the reaction after the first bath.

Half-Baths.-The half-bath, preferred by Winternit\%, differs from the "whole" bath in that there are only 10 inches of water in the tub. the attendant splashing the water over the part of the body exposed. In other respects the technic, including the temperature, is like that of the cold bath. In severe headache, unconsciousness, and delirium, a strong stimu. lation is secured by pouring colder water over the patient. For the first bath the higher temperature (limit $77^{\circ}$ ) is chosen, unless a strong nervous stimulant is needed, in which case we begin with $68^{\circ}-65^{\circ}$. If the ferer continues to go up, the temperature may be reduced to $61^{\circ}$ or $57^{\circ}$, and in case of necessity may even be brought down to $54^{\circ}$ by pouring in colder water or adding ice. As soon as the fever begins to abate, the temperature of the bath is raised. The duration at the beginning should be 8-10 minutes; later, until the armpits, supra-clavicular fossa, etc., are no warmer than other parts of the body. If any signs of cyanosis appear, the patient must be quiekly taken from the bath and rubbed dry in bed. Compresses should then be put around the trunk and changed every half. hour as long as the temperature remains high. In severe affections of the nervous system, disturbanees of consciousness, or comatose conditions, Winternitz recommends the combination of a stimulative shower-bath with an antipyretic half-bath. Water colder than the bath water is let fall upon the head and upper part of the body, the height, duration, and force of the stream being varied to suit the individual case.

The Sick-Room.-The largest and best bedroom should always be selected for the patient, especially in the more protracted infectious diseases. All eurtains, rugs, carpets, upholstery, and unnecessary furniture should be removed. The bed must be free from hangings, and so placed that it can be reached from both sides, and that the patient does not face the light. Another bed, to which the patient can be changed from time to time, is very desirable. The temperature, especially if the patient has a high fever, should be kept at $66^{\circ}$ (no higher). The room must be well ventilated, but free from drafts. The air should be kept sufficiently moist, either by hanging wet eloths in the room or by an apparatus designed for this purpose.

Any disagreeable odor is best avoided by good ventilation, frequent changes of bed-linen, and proper care of the excreta.

The patient's mouth. face, hands. and genitalia should be kept scrupu. lously clean and the hair well brushed. The bed-linen and night clothes should be frequently changed, all soiled articles being immediately removed from the room, and soaked two hours in a disinfectant fluid before being washed. The floor of the sick-room and any other space used in connection with the patient should be wiped up at least once a day 
with cloths or mop wet with a disinfectant fluid. All bedpans, spittoons, etc., should be filled with such a fluid.

The nurse's clothes should be disinfected in the same way as the patient's. She should wash her hands frequently in an antiseptic solution, and before seeing other people, should change all her clothing. It is wise for her to wear a closely fitting cap, thoroughly covering her hair.

The choice of a disinfectant must take into consideration its expense and, in private practice, its odor and method of application. A disinfectant whose odor is disagreeable to the patient, or one which is difficult or dangerous to use, should be avoided. First on the list of disinfectants comes carbolic acid. In a 1-3 per cent. solution, it will quickly kill the most varied kinds of bacteria, especially if it be warmed to $100^{\circ}$. For rough disinfection or particularly deodorization, raw carbolic acid is used. Other powerful bactericides are: Pearson's creolin ( $1 / 2$ per cent. solution), lysol or lysitol (2-10 per cent.), solveol, solutol, saprol, and chinosol $1: 1000-3000$ ), the latter being the best.

The most powerful of all disinfectants, however, is undoubtedly bichloride of mercury (best prepared by dissolving a so-called antiseptic tablet in 1 to 5 pints of water, $1: 1000-5000)$. Unfortunately its toxic qualities hinder its universal use, so that under some circumstances chinosol is preferable.

Finally there is formaldehyde, sold in a 40 per cent. solution under the name of formalin. Its exceedingly diffusible vapor is a very strong disinfectant. For the disinfection of the sick-room, clothes, bed-linen, books, etc., in private practice, Schering's formalin lamps are very convenient; in them formalin tablets are vaporized. The vapor is exceedingly irritating. [Paraform, the solid polymer trioxymethylen, was selected for use in the Schering lamp so as to avoid the use of a liquid preparation. The equivalent of $500 \mathrm{G}$. (1 pt.) of formalin or about 60 pastilles for each thousand cubic feet is necessary.-Ed.]

Prausnitz recommends spraying the walls, floor, etc., with formaldehyde by means of a special apparatus in principle like a Papin's kettle. Clothes put in a closet that can be securely closed may be sprayed in the same way. It has been experimentally proved that bacteria wrapped in filter paper and placed in the pockets of thick clothes are killed by this method. Prausnitz maintains that the use of 7-8 c.c. formaldehyde with 40 c.c. of water for each cubic metre will destroy even the most resistant bacteria (not spores). Without including the cost of the apparatus, Prausnitz's method is only about one-sixth as expensive as that of the formalin lamp. Schlossmann and Walther have contrived an evaporating apparatus by which a most thorough disinfection may be accomplished with glycoformal (40 per cent. formaldehyde solution mixed with 10 per cent. glycerin). After the apparatus has been 10 minutes in a room containing 60 cubic metres, the formalin vapor is so thick that an electric light can no longer be seen.

[Schneider 
considers the addition of glycerin unneeessary and disadvantageous. Everything becomes slimy and sticky. The process is also very expensive. Evans and Russell (Board of Health of Maine) devised the following method. Powdered potassium permanganate is placed in a tin receptacle 10 inches in diameter at the bottom and 17 inches high; and formalin is poured upon it. The yield of gas is 81 per cent. and occurs very suddenly-four fifths are set free within 5 minutes. For each pint of formalin $61 / 2$ ounces of potassium permanganate are employed. The method is simple, avoids the use of a flame, and tests have shown that it is highly efficient. The New York Health Department adds formalin containing aluminum sulphate to unslaked lime in pans, 10 ounces to 1000 cubic feet, exposure $2-4$ hours. The simplest device, which is also quite efficacious, is to pour 12 ounces (for each 1000 cubic feet) of formalin upon folded sheets, pull them out, and stretch them upon lines in the room. We must not forget that formalin gas is merely a surface disinfectant. Its power of penetration cannot be relied upon. Wilson, for example, protected organisms by the folds of a blanket and they were not killed. The value of clearing the sick-room of all unnecessary articles is thus apparent.-Ed.]

Disinfection of the Sick-Room after the Patient.-For the disinfection of the sick-room itself, Gärtner gives some valuable hints. After shutting the windows, doors, ventilators, entrances to chimneys, etc., the bacteria are allowed to settle for 12 hours, at the end of which time they will all have been deposited upon the floor, walls, and furniture. The floor is then washed up and the walls and furniture wiped down with a disinfectant solution. The beds, mattresses, bedclothes, and clothes of all kinds should be enclosed in a sack wet with carbolic acid and sent off to be disinfected by steam.

The walls must be most carefully disinfected. All pictures, mirrors, etc., should have been previously removed. Tapestried walls should be rubbed off with bits of soft bread, the crumbs afterwards being collected and burnt. Painted or kalsomined walls should be washed with a disinfectant and then receive a fresh coat. The furniture of all kinds, pictures, mirrors, oil paintings and their frames, and all objects made of leather or rubber should be washed off with a 3 per cent. solution of carbolic acid and then rubbed dry. All books used in the rooms should be burnt or disinfected with a formalin lamp. [Books are extremely difficult to sterilize. Unbound books may be subjeeted to steam. Books of little value are best burned. If formalin is generated in a room. the most scrupulous care should be taken to seal cracks, ventilators, and all openings.-Ed.] The floor should be vigorously scrubbed with a 3 per cent. carbolic acid or a 5 per cent. lysol solution.

DiET.-The question of diet for all infectious diseases except searlet fever will be discussed briefly in the following paragraphs. Scarlet fever requires specific dietetic treatment. 
Since an abnormal number of cells are being broken down by the processes involved in fever, as well as by the action of the specific toxin, the problem of proper nourishment is a most important one. Bauer (Handbuch der allgem. Therapie, Bd. I, Th. I) points out that the patient's strength must be maintained; but that at the same time only such food should be given as is absorbed with little or no tax on the digestive organs, and which neither chemically nor mechanically irritates the digestive tract. It should not, however, be chosen from a one-sided point of view, nor from a priori reasons, but after a careful weighing of all the circumstances of the case.

In fever the proteid destruction is much more extensive than the oxidation of the fats; hence, we should prescribe as much proteid as the nature of the case will permit. The probable duration of the disease, the age and strength of the patient, and the condition of the digestive tract must all be carefully considered.

With young children and aged persons, whose strength must be maintained, it is important to be sure that a fixed amount of nutritious food is taken. With anorexia and febrile dyspepsia the diet must be restricted if the individual is sufficiently vigorous and strong. In other cases, unless contraindicated for some reason, the amount of nutritive food should be materially reduced, while suitable forms of foods that are principally stimulative (e.g., meat broth) should be allowed.

If the fever lasts for some time, the amount of food should be increased and, if possible, its proteid content raised. This may be done by adding to the soups strong calves-foot broth or any broth containing much gelatine, beef-tea, succus carnis, Leube's meat solution, peptone preparations, Brand's or Baker's Essence of Beef, Mosquera's or Valentine's meat essence, Carnigen, or Puro.

The most important forms of food for fever patients, however, are milk-the best combination of proteid, fat and carbohydrate-and eggs. They should be given in small amounts at frequent intervals. In case of a marked antipathy to milk, it may be replaced by cocoa, or bean or malt coffee.

Alcohol.-Alcohol may be indicated from the beginning in eases of severe infection with visible depression of the nervous system and the heart. As a mild stimulant, the lighter wines may be given; as a more powerful one, if necessary, the strong wines, brandy, etc.

Thirst.-To quench thirst, water should be given freely. Lemon juice or dilute hydrochloric or phosphoric acid may sometimes be added. If good water is not procurable, any alkaline mineral water may be substituted. If there is a tendency to diarrhœea, cold toast-water or ricewater may be used.

Diet during Convalescence.-The diet during convalescence must be very carefully watched and only very gradually increased. In each individual case we must be guided by the age, strength, and general condition of the patient, in the gradual transition from a liquid to a solid diet. 


\section{THERAPY OF SCARLET FEVER.}

Prophylaxis.-For prophylaxis the one important measure to be taken is the complete isolation of the patient, no one but the nurses and doctor having access to him. The proper methods of disinfection have already been discussed. ["The disease is not contagious during the period of incubation; little, if at all, during the period of invasion; most contagious during the period of eruption, and decidedly so during the period of desquamation", (Forchheimer).-Ed.]

Therapy of UnComplicated Cases: Hydrotherapy.-Our therapy can be only symptomatic and must be mainly antipyretic, the nethod selected depending upon the grade of fever. In the milder cases the patient should be kept lightly covered in a cool room $\left[65^{\circ}-68^{\circ}-\mathrm{kd}\right.$. $]$, and sponged off with cool water or vinegar and water several times a day. This will often relieve the annoying itching. Thomas and Schill recom. mend giving a daily bath. At the onset of the disease Thomas begins with a Ziemssen (gradually cooled) bath, raising the temperature daily as the fever decreases, so that during convalescence warm baths are given. If, however, instead of diminishing, the fever increases, or if the disease begins with an excessively high temperature and visible symptoms of intoxication, energetic, hydropathic measures must be immediately used, the choice of methods depending primarily on the age and strength of the patient, and secondarily on the ease with which the respective pro. cedures can be carried out.

In cerebral congestion the hydrotherapy should be supplemented by energetic local antiphlogistic measures (ice-bags to the head. Leiter's coil or cold compresses frequently changed). [Holt uses cold baths when the temperature is $104^{\circ}$ or over, especially in septic eases with typhoidal symptoms and in cases with marked cerebral symptoms. MeCollum always applies an ice-bag to the patient's head during any form of hydrotherapy. Koplik reserves full baths for patients whose temperature persists high (above $104^{\circ}$ ) for days. For others he employs sponging or packs. He considers that baths below $75^{\circ}$ are not well borne by patients with searlet fever, especially children; and that they are particularly contraindicated in the asthenic forms of sepsis. He does not, however, believe that kidney complications are caused by cold baths. -Ed.]

Medication.-Quinine may be given 2-3 times a day for 1-2 days. Richy recommends salophen or lactophenin. The rapid pulse may be controlled by digitalis. [McCollum states that digitalis should be given with great eare, as in certain eases it may do more harm than good. Koplik relies upon alcohol and digitalis and says that strychnine does not seem to act so well in cases with acute myocarditis.-Ed.]

Collapse must be met by stimulants; e.g., camphor, musk, ether aceticus or spirits of ether, liquor ammonii anisati, brandy, rum, and strong wine. Water may also be poured on the patient's head as he sits in a tub, which may be empty or half full or full of hot water. The 
symptoms of intoxication may be met by bactericides and antizymotics, among which Thomas recommends quinine in medium and large doses, carbolic acid used internally and hypodermically, sodium phenolsulphonate (1-41/4 G. (15-64 gr.) a day, according to the age of the patient), the hyposulphites, and the inhalation of ozone. Seibert, of New York, recommends rubbing the whole surface of the body with $30-90$ G. (1-3 oz.) of a 5-10 per cent. ichthyol-lanolin ointment, every 6-12 hours, according to the intensity of the rash. The process should take about half an hour, at the end of which time no redness should be anywhere visible. Marmorek's, Prof. Tavel's, Aronson's and Mosel's antistreptococci sera, Credé's ointment, and the internal use of collargol are also recommended.

Treatment of Sore Throat.-The ordinary sore throat that characterizes the disease requires no treatment beyond cold compresses, the frequent administration of bits of ice, and gargling with some disinfectant solution ( 2 per cent. boric acid, $1 / 2-2$ per cent. salicylic acid, ${ }^{2} /{ }_{10}$ per cent. potassium permanganate, 1-3 per cent. carbolic acid, $1 / 10$ per cent. bichloride of mercury, 1-2 per cent. quinine solution).

Complications and Sequele: Severe Angina: Local Treatment.If the sore throat becomes diphtheretic, its intensity increasing, a membrane appearing on the tonsils or pillars of the fauces, accompanied by a bloody mucous, and later a purulent, discharge from the nose, while the painful swelling of the tissues of the neck and of the submaxillary glands constantly increases, the most vigorous measures must be taken. To prevent the spread of the local infection, the mouth must be most carefully disinfected. It should not only be gargled, but, especially in the case of children, should also be irrigated. Dusting the mouth with iodoform or sulphur has been recommended. Ziemssen advocates removing the membrane with a wad of lint, and then painting the spot with a carbolic solution. Cauterization with 10 per cent. spirits of salicylic acid and silver nitrate has also been advised, as well as the internal use of quinine, mercury, and pilocarpine. Vierodt condemns the use of the latter as dangerous to the heart. Heubner recommends the parenchymatous injection of a 3 per cent. carbolic solution by means of a hypodermic syringe and a long needle. 0.5 c.c. should be injected daily into the tonsil, the pillars of the fauces, and the adjacent tissues, until all necrotic patches have vanished and the glandular swelling has disappeared.

Seitz carries out the following course of treatment. Since the so-called "scarlet diphtheria" has nothing to do with true diphtheria, the universal use of the antitoxin serum is not indicated. He lays great stress in every case upon regular rinsing and gargling of the mouth with indifferent solutions (salt, borax) from the onset, and especially in feverish and somnolent cases. If during the first day a membrane appears, it should be gently removed with a cotton swab and the spot touched with a 3-5 per cent. carbolic solution. A fetid discharge from the nose and 
mouth, indicating a serious affection of the nose and throat, should be treated immediately by irrigating the nose to clear the air-passages. [Nasal irrigation as ordinarily practiced increases the danger of otitis media and should be avoided. Koplik recommends for the obstruction to breathing caused by such a condition either (1) intubing the nares with a No. 10 or 12 soft rubber catheter running back to the naso-pharynx and then cautiously irrigating with salt solution, or (2) the instillation of 3-5 drops of a $1: 4000$ adrenalin solution in each nostril 3 or 4 times daily.-Ed.]

When the streptococci from their original location in the nose and throat have penetrated into the glands, an invasion of the whole body. and particularly of the vessels, is threatened. To avert it Heubner and Seitz recommend the carbolic injections of the tonsils and soft palate as already described. Undoubtedly a symptomatic improvement results in all cases. Swallowing becomes easier soon after the first injection and remains so for several hours. This is due to the anasthetizing effect of the carbolic acid. After the second injection, the fever, up to that time continuous, generally begins to fall, and there is often a surprising improvement in the swelling and sensitiveness of the glands at the angle of the jaw, as well as in the general condition. This treatment is not liable to cause nephritis. Seitz injects carbolic acid where nephritis is present and has noticed a daily recession of the albumin content in the urine. Carbolic acid may appear in the urine, but disappears with the cessation of the treatment. The injection causes no lasting pain and demands no other assistance than that of the mother or nurse. A syringe with a 6 $\mathrm{cm}$. cannula is used. A ring is fastened to the cannula $0.5 \mathrm{~cm}$. from the tip to prevent its entering too deeply. One c.c. of a 3 per cent. carbolic solution is injected daily, but not all in one place. The injections are continued until the temperature is nearly normal and the glandular swellings have subsided, generally for 3-8 days. The local throat lesions heal more slowly and sometimes require a further use of gargles and irrigations. The indication for injections is: high fever with concurrent swelling of the cervical lymph-glands at the end of the first week. Good results may also be obtained during the second week, provided inflammation of the lungs and of the serous membranes does not point to a general streptococcus invasion. [I have never tried this rather vigorous method; but neither its safety nor efficiency appeals to me.-Ed.]

The author himself does not use injections, preferring to apply to the necrotic spots a cotton swab soaked in a $1 / 10$ per cent. bichloride solution, in sozoidol of sodium, or in boric acid, or to use an insufflation of boric acid or sodium sozoiodolicum (Schwartz). [Löfler's iron toluol solution (see p. 596) is recommended by Forchheimer and McCollum.-Ed.] Baginsky recommends for this purpose a mixture of hydrarg. bichlorati corros. 0.05 , with ammonii sulpho ichthyolici $5: 100$. But a better com. bination seems to be a mixture of equal parts of sozoiodol of soda and sublimed sulphur, applied daily by means of a powder blower. If this 
induces vomiting, however, half a teaspoonful of sodium sozoiodol in solution may be given internally 3 times a day. This treatment should be continued until the throat is quite clear again (2-3 days).

Serum Treatment.-In severe cases of angina, it may perhaps do good and will certainly do no harm, to use diphtheria antitoxin. [True diphtheria occasionally complicates scarlet fever in private practice. It is a frequent complication in hospital practice where prophylactic doses of diphtheria antitoxin are not used as routine measures, see p. 591 . Unless a culture from the throat shows that the inflammation is caused by the Klebs Löffler bacillus, I should not advise therapeutic doses of antitoxin to scarlet fever patients with severe angina.-Ed.] It is to be hoped that in the future the antistreptococcus serum of Marmorek and Tavel may be found effective. Marmorek injects 10 c.c. at a time, 20 e.c. daily in severe cases until the fever subsides. Generally one or two injections suffice. If the swelling reappears, or slight albuminuria occurs, the treatment should be given again until these complications disappear. Since the effect of the injection is only temporary, while the complications may appear after a considerable length of time, the case should be very carefully watched. No bad effects have ever been observed from the use of the serum. Baginsky has tried it in 48 cases and sees no reason for giving up its use. [Marmorek's serum produces disagreeable rashes. It has been proved to be valueless in scarlet fever.-Ed.] Better success is reported from the use of Moser's serum. According to Moser and Escherich 100-200 c.c. should be injected as early as possible into the lateral abdominal wall, the wound being carefully closed immediately with iodoform collodion. The fact that no preservative is added to the serum makes it possible to employ such a large amount with safety. [The Vienna school all speak well of the use of Moser's serum. Charlton in Montreal reports similar favorable results in a few cases. The serum is prepared by injecting horses with the mixed cultures obtained from 6 to 12 different cases of scarlet fever.-Ed.]

Glandular Swellings. - The glandular swellings, especially in the submaxillary region, are frequently painful. The inflammation of the deep cervical tissues and these swollen glands may be treated by cold applications, ${ }^{1}$ by iodoform ointment, 6 per cent. iodine vasogen, 15-20 per cent. ichthyol ointment, Credé's ointment, or ung. hydrarg. dilutum. If the glands show signs of softening or of suppuration, hot applications of one part "acetic acid clay" to 3-5 parts of water may be used, or hot poultices may be employed to hasten suppuration. As soon as fluctuation can be plainly demonstrated in any gland, it should be opened. Enlarged glands often persist after the disease has run its course and

${ }^{1}$ [Forchheimer combines moderate pressure by repeatedly painting the swelling with flexible collodium ( 49 parts of collodium, 1 part of castor oil) and the continuous application of the ice-bag separated from the skin by a piece of flannel and kept closely applied night and day.-Ed.] 
may be treated with hot compresses, wet with acetic acid, lead, or iodine water. Internally tonies (iodide of iron) may be given.

Affections of the Ears and Conjunctiva.-The ears and the conjunctivæ are often affected during or after scarlet fever. Such conditions should be carefully watched for, and should be treated as soon as possible by a specialist.

Affections of the Heart and Lungs.-Fortunately the heart and lungs are seldom affeeted. [In 2000 cases of scarlet fever McCollum found endocarditis in 13, pericarditis in 13, a mitral systolic murmur in 619 , a gallop rhythm in 20 , and irregularity in 144 . Osler states that it is to be borne in mind that the cardiac complications of the disease are often latent.-Ed.] If so, the proper therapy will be found discussed under diphtheria and measles. There are left, then, only the two most important complications or sequele,--arthritis and nephritis.

Arthritis.-To obviate the arthritis it is usually suffieient to immobilize the joint and apply cold or Priessnitz's compresses. The salicylates should always be tried, although many observers have reported that they cannot be depended upon in these cases. Aspirin $(0.3-0.5 \mathrm{G}$. ( $41 / 2-$ $7 \mathrm{r} / 2 \mathrm{gr}$.) $3-4$ times a day in cold milk) is the best preparation, as it has no injurious effect on the heart or kidneys. [The salicylates have not proved of sufficient service in combating the arthritis of searlet fever to offset the injurious effects upon the kidneys which their use may produce. Von Noorden has very properly emphasized this danger in nephritis. Aspirin is just as irritating as the other salicylate compounds.-Ed.] Chronic joint swellings are combated by the external and internal use of iodides. If suppuration, occurs in the joint, surgical interference is indicated.

Nephritis: Prophylactic Medication-Baths.-The oceurrence of nephritis depends not so much on the intensity of the individual case as on the nature of the epidemic, and often cannot be forestalled. Widowitz recommends as a preventive measure, the use of urotropin $(0.05-0.5$ G. (3/4-7 gr.) according to the age of the patient) for 3 days at the beginning of the disease, and again at the beginning of the third week. Buttersack uses helmitol $(0.1-1.0 \mathrm{G}$. (11/2-15 gr.) daily). Baths at the body temperature should be given every day or every other day $(1 / 4-1 / 2$ hour), after which the patient should be carefully dried and put into a previously warmed bed.

Prophylactic Diet.-A very eareful prophylactic diet should always be insisted on; it may almost be called the specifie diet. In choosing this we must be guided by the fact that eloudy swelling of the kidneys exists from the beginning of the disease. This is due not so much to the ferer as to the toxin. This alone, aside from the possible eardiac weakness. would oblige us to keep the patient in bed for 3-4 weeks, to be careful that he does not eateh cold, particularly after his baths, and to select a diet that shall spare the kidneys as much as possible. Such a diet should consist of milk, to which may be added, if the patient dislikes the taste 
of milk, a small amount of cocoa or malt coffee. But besides this, as Jürgensen points out, abundant proteid ${ }^{2}$ must be given ; e.g.:

first and second weeks: eggs, chicken or veal broth, white bread.

third and fourth weeks: rice and farina puddings, fruit juices, white meat.

fifth week : red meat, ham, suitable green vegetables, rice, mashed potatoes, cooked fruit.

Abundance of water or of any light mineral water (Giesshübl, Preblau, Bilin, Fachingen, Vichy) should be taken to dilute the urine. [Forchheimer emphasizes and McCollum advises a milk diet for the first two or three weeks of the disease.-Ed.]

Treatment of Existent Nephritis.-The urine should be tested for albumin daily. If albuminuria occurs, a profuse diaphoresis should be induced by enveloping the patient after the usual warm bath in warm blankets. In this way severe nephritis and its consequences can often be avoided. If blood appears in the urine we should resort to the same simple device and administer internally lead acetate $(0.05$ G. (3/4 gr.) 3 times daily), the infusion of digitalis, ergot, alum (2 per cent.), liq. ferri chloridi $(0.5-1.0: 100)$, pyoktanin. cœrulei (0.1 G. (11/2 gr.) 3 times a day), 0.1 per cent. sol. adrenalin. hydrochlor. (5-10 drops 3-4 times a day), gelatin solutions internally or by the rectum.

If, however, the amount of albumin and blood in the urine increases rapidly while the total amount of urine decreases, or if symptoms of uræmia (vomiting, headache, unconsciousness, convulsions), or of increasing dropsy appear, we must proceed to much more energetic measures.

To begin with, unless contraindicated by diarrhœa, a cathartic should be given, the choice depending upon the age of the patient (castor oil, a bitter water, calomel).

Hot baths $\left(97^{\circ}, 15\right.$ minutes' duration) should also be used, followed by a hot pack, the patient being wrapped in a sheet wrung out in hot - water. He should remain in the pack 1-2 hours, taking hot drinks meanwhile. At the end of this time, provided there has been abundant sweating, he should be rubbed dry with warm towels, be put into warmed night clothes, and placed in bed again. This procedure must be repeated 2-3 times a day, the temperature of the water being raised from $102^{\circ}$ to $104^{\circ}$ or $106^{\circ}$, and the duration of the bath being increased from $1 / 4$ to $1 / 2-$ 1 hour. This treatment and the proper diet are continued until the dropsy has disappeared. [Such drug treatment is useless and may be harmful. Any such persistent use of hot packs is weakening and may be dangerous. Venesection in severe cases and simpler measures in

${ }^{2}$ [During convalescence from an acute nephritis, meat is usually prohibited until the albumin has disappeared for a week; and then given only tentatively. Some patients do better if meat and other articles (salt-free diet) are allowed much sooner and continued unless they increase the albuminuria, casts, or some unpleasant symotoms.-Ed.] 
milder cases will accomplish better results. See section upon acute nephritis, p. 98.-Ed.]

If the heart is rather weak, we must give wine, tea with brandy, etc. If the process is prolonged, the patient's strength must be kept up by meat and eggs. If there is little or no odema we give a $0.25-0.50 \mathrm{G}$. (4-7 gr.) quinine tannate powder 3 times a day, or one of the other drugs already mentioned. As a heart stimulant we may use an infusion of digitalis with or without potassium acetate, or 20-30 drops of the tincture of strophanthus in $70 \mathrm{G}$. $\left(2 \frac{1}{2} \mathrm{oz}\right.$.) of water, or $10 \mathrm{G} .\left(2 \frac{1}{2} \mathrm{dr}\right.$.) of the syrup of jaborandi.

Treatment of Urcmia.-If, in spite of the proper treatment, no abundant diuresis ean be secured and the symptoms of uramia appear, a protracted hot bath with a cold compress on the head may be tried. But if vomiting and severe convulsions with disturbances of consciousness appear, they must be combated by every known possible remedy. For the vomiting Jürgensen recommends small quantities of tincture of iodine ( 1 drop in 100 c.c. ( $3 \mathrm{oz}$.) of water).

For the convulsions, inhalations of chloroform may be eautiously used until the museles relax. To insure the permanence of this effect.

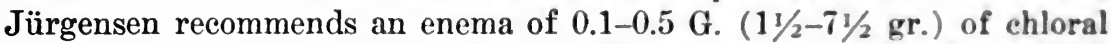
according to the age of the child ( $2 \mathrm{G}$. ( $30 \mathrm{gr}$.) maximum dose for adults) in 50-100 G. (1 $1 / 2-3$ oz.) of mallow tea or syr. or mucilag. aeacixe. If the heart and respiration are sympathetically affected, stimulants must be given; e.g., hypodermics of ether or camphor. In some cases of uræmia, life may be saved by a lumbar puncture.

Convalescence.-The treatment during convalescence must be suited to the conditions of the individual case, as judged by the patient's ap. pearance, strength, and general subjective symptoms. Every patient, however, should be given a bath every day or every other day from the second week on in the afternoon or evening, going to bed directly afterwards. To hasten desquamation and at the same time to aid in disin. fecting the skin, the whole body may be rubbed with soft soap directly before or during the bath, and 2-10 tablespoonfuls of lysol may be added to the water. The hair should be carefully washed. After 10-20 such baths, 1-2 G. (15-30 gr.) of bichloride of mercury may be used in the water. [Daily inunetions of cold cream. cacao butter, or lanoline are useful in facilitating desquamation and preventing the dissipation of the scales. The carbolic acid often combined with such ointments does not disinfect the skin and may irritate the kidneys.-Ed.] If the patient is anæmic, iron, arsenic. or the combination of the two in the arsenic-iron mineral waters (Guber, Roneegno, Levico. Mitterbad) may be given. The surroundings should be hygienic and invigorating, but the climate at first not too bracing. The weakness of the lower extremities may be helped by rubbing the joints with alcohol or by employing massage or electricity. 


\section{THERAPY OF MEASLES.}

As measles is a self-limiting disease, all that the physician has to do is to watch the patient carefully and ensure the best conditions for a normal course and the avoidance of complications.

Isolation.-The isolation of the patient is not nearly so efficacious in measles as in scarlet fever, for susceptibility to the former disease is much more general. Nevertheless isolation should be enforced, and individuals with a weak constitution or tuberculous tendencies should be most carefully guarded from infection.

HYGIENE OF THE SicK-ROOM.-The sick-room should be darkened, carefully ventilated, and kept free from dust or irritating disinfectants or perfumes. [If there is photophobia the room may be darkened or yellow curtains drawn; otherwise light is advisable.-Ed.] The air should be kept moist by means of steam from a kettle, frequent spraying, or wet sheets hung up and changed at frequent intervals. It is convenient to have a hydrometer in the room. [Unless indicated for some special purpose, such as a croupy cough, dyspnœa, or severe bronchitis, there is no necessity for steam or moisture in the sick-room.-Ed.]

DIET.-The patient should be kept in bed until the catarrhal symptoms have almost entirely disappeared. No limitation need be placed upon the amount of water allowed, but the quantity and kind of food must be regulated by the patient's strength and the grade of the fever. At first the diet should consist of milk and thin soups, except in the case of diarrhœe, when cocoa made with water and possibly a little milk, acorn coffee with milk, or Liebig's extract may be substituted. [Laxative foods should be avoided in the diet for fear of starting a troublesome diarrhœa. -Ed.]

Symptoms and Complications: Conjunctivitis and Rhinitis.-The simple catarrh which accompanies the disease needs no treatment except that the passages should be kept clear and washed out with a bit of cotton soaked in a 1-2 per cent. boric acid solution or a weak solution of potassium permanganate. [For severe conjunctivitis the eyelids should be smeared with vaseline (or yellow oxide of mercury $0.06 \mathrm{G}$. ( $1 \mathrm{gr}$.) in 16 G. (4 dr.) of vaseline), and the eyes covered with compresses wet with iced boric acid solution ( 2 per cent.).-Ed.] In case of very severe conjunctivitis or otitis media, a specialist should be consulted immediately.

Stomatitis and Angina Morbilosa.-The mouth should be kept scrupulously clean by means of antiseptic gargles and irrigations. If the child is too young to gargle, his mouth may be washed out with a piece of cotton, or irrigated 2-3 times a day with a 2 per cent. solution of boric acid, salicylic acid, or borax.

One of the most dangerous complications of measles is diphtheria, sometimes primary, sometimes secondary. In contradistinction to the severe angina of scarlet fever it is in many cases undoubtedly true diph- 
theria and should be treated as such (see p. 590). A preventive injection of diphtheria antitoxin is, therefore, advisable as a routine measure. [During epidemies of measles at the New York Foundling Hospital each case has received an immunizing dose of diphtheria antitoxin with strik. ing benefit (Holt).-Ed.] To facilitate breathing, which may be impeded by obstruction in the nares, the latter should be greased several times a day with 10 per cent. boric vaseline. In addition a warm mixture of equal parts of boiled milk and water, or a salt solution (a pinch of salt in a cup of boiled water, warmed) or a 1-2 per cent. boric acid or borax solution may be introduced into the nose.

Fever.-The chief attention, however, must be given to the fever and to the catarrh of the respiratory tract. If these persist abnormally long or return after they have once disappeared, they must be most carefully watched and treated, for fear of beginning complications. The fever can usually be sufficiently controlled by the use of mild hydrotherapy. such as cold sponge-baths or packs. But in the rare eases marked by extreme weakness, or in the so-called septic and hemorrhagic eases, with symptoms suggesting a weak heart, we are forced to bring into play all the hydropathie resources at our command. In such cases we use tepid baths, or, with very pronounced weakness, hot or mustard baths (Henoch). followed by cool water poured over the body. We may also administer tannate of quinine, alcohol and (Vierordt) hypodermics of a 1:10 solution of camphor in oil. The latter are especially advisable when in addition to the weakness and fever there appear from time to time attacks of extreme pallor, cyanosis, and failing pulse. They should be given every 2 hours or even every hour, $0.01-0.02 \mathrm{G}$. ( $1 / 6-1 / 3 \mathrm{gr}$.) of camphor for young children, $0.02-0.03 \mathrm{G}$. ( $1 / 3-1 / 2 \mathrm{gr}$.) for older ones.

Caffeine is sometimes useful, either in the form of black coffee or of caffeine-sodium benzoate $\left(0.1-0.2\right.$ G. $\left.\left(1 \frac{1}{2}-3 \mathrm{gr}.\right)\right)$ given either internall! or hypodermically. Hypodermoclysis may also be employed.

Catarrhal Affections: Cough.-Except for serious bronchitis or pneumonia the catarrhal affections of the respiratory tract demand only symptomatic treatment. The cough, which, especially in the initial stages of the disease, is often extremely annoying, should be relieved by aqua amygdalæ amara in minim doses, the extract of hyoseyamus, the extract of cannabis indica, codeine $(0.01-0.02 \mathrm{G} .-1 / 6-1 / 3 \mathrm{gr}$.) in tablet. powder. or solution, peronin $(0.01-0.02 \mathrm{G} .-1 / 6-1 / 3 \mathrm{gr}$.$) , the hydrochlorate of$ heroin $(0.005-0.01$ G. $-1 / 12-1 / 6$ gr. $) 3$ times a day for adults, or eren a dose of $0.0025 \mathrm{G} .(1 / 24 \mathrm{gr}$.) in the evening, for children of $2-7$ yens (0.001-0.002 G. $-1 /{ }_{80}-1 / 30$ gr.) 3 times a day, or dionin (same dose as codeine). In some circumstances a bitter almond water (10-20 drops 3-4 times a day) also proves a good sedative. [A few drops of paregoric are useful to control the cough. If the latter arises from the bronchial tubes in a very young child, any form of opium must be used with great caution. Forchheimer recommends a large dose of bromide of soda for a croupy cough.-Ed.] 
Laryngitis.-Laryngitis, if troublesome, may be relieved by inhalations of steam and the local application of compresses. For threatening laryngeal croup, the measures discussed in the section on diphtheria are appropriate (see p. 598).

Bronchitis.-In case the catarrh attacks the bronchi, hot applications to the chest, changed every 1-2 hours, or the cross-binder should be used. Expectorants are also indicated (ipecac 0.2-0.4:100 infusion, or an infusion of senna with $0.5-1.0 \mathrm{G}$. (7-15 min.) of liquor ammonii anisati added). In dry bronchitis with few râles and distressing cough, we may give in addition to the infusion of ipecac, potassium or, preferably, sodium iodide (0.5-1.0 G.-7-15 gr.), apomorphine (100 G. (3 oz.) of a 0.01-0.015:100 aq. dest., ammonium chloride (100 G. (3 oz.) of a 1-3 : 100 aq. dest. with $10 \mathrm{G}$. ( $2 \frac{\mathrm{r}}{2} \mathrm{dr}$.) of the extract of liquorice (contraindicated in diarrhœa)). If the fever is high and the general condition very poor, we prescribe $100 \mathrm{G}$. (3 oz.) of a decoction of quinine bark $(5-10: 100)$ with $0.5-1.0$ G. (7-15 min.) of liquor ammonii anisati, or 10-20 drops of tinc. ferri. citro. chloridi. For threatening heart failure, $0.5-1.0$ G. (7-15 gr.) of the citrated caffeine or $0.1-0.2$ G. (11/2-3 gr.) of camphor may be added to the first mixture. [I cannot recommend the author's generous choice of drugs for treating symptoms in the acute exanthemata. Many of them will prove valueless and some of them will be liable to cause digestive difficulties.-Ed.]

Severe Respiratory Complications.-If despite these therapeutic efforts the fever and catarrhal symptoms persist or reappear more intensely, a severe respiratory complication is evidently present and demands our special attention. If the catarrhal affection spreads to the mucous membrane of the finer bronchi or causes a lobular or lobar inflammation, the fever will mount still higher and nervous disturbances will complicate the clinical picture. The hydropathic measures already discussed will then be urgently indicated. If sponging and packs do not improve the functioning of the nervous system or the heart, full baths must be resorted to. The first bath should be at a temperature of $82^{\circ}$ and should last 5-10 minutes, the patient being taken out before he is at all chilly. The second bath may be cooler $\left(77^{\circ}-64^{\circ}\right)$. After each bath the patient should be covered up and fed. Whenever the temperature reaches $102.2^{\circ}$ the bath should be repeated. The intervention of collapse symptoms demands camphor, caffeine, or ether subcutaneously.

The patient should be nourished as well as possible and given plenty of water to drink. Coffee, and tea with brandy may be allowed, especially to help the reaction after the bath. Inhalations of oxygen may also be indicated, in case of cyanosis or a very rapid pulse. Plenty of fresh air should be secured. Infants and very young children should be carried around the room in the nurse's arms, thus changing their position.

Chronic Catarrh.-If the catarrh becomes chronic, we may use the above mentioned preparation of quinine bark, and in addition 10-20 drops of the rectified oil of turpentine. The latter may be sprinkled on 
a handkerchief and inhaled, or given in milk, 3-5 drops after each meal. In chronic bronchitis Quincke's treatment-laying the head low once or twice a day-may be recommended.

Complications of Heart or Kidneys.-Involvement of the heart, pericardium, or kidneys is rare. Nevertheless the urine should be ocea. sionally examined for albumin, especially in convalescence. The treatment of albuminuria has already been detailed in the section on scarlet fever.

Complications of Digestive Tract.-In some epidernies digestive complications are common. The diet should then be strictly regulated. In all intestinal conditions calomel should first be given, followed by some astringent, - a decoction of calumba root (5-8 G. (11/2-2 dr.) with the tincture of opium, a decoction of krameria, or hamatoxylon, $10: 100$, quinine tannate alone or with guarana, tannalbin, honthin, tannigen, and tannopin (0.3-1.0 G.-5-15 gr.) several times a day. We may also prescribe irrigations with $1 / 2-2$ per cent. sodium salievlate solution, boric acid, $1 / 2-1$ per cent. tannin solution, 1-2 per cent. alum solution or a weak solution of potassium permanganate. In case of severe tenesmus and hemorrhage a 33 per cent. solution of liquor Burowii (glycerinated alumi. num acetate 10 per cent.) or a $1 / 20$ per cent. solution of resorcin may be employed.

Convalescence.-Individuals with a tuberculous or a scrofulous diathesis, or those who are left with chronic bronchitis and swollen glands, require careful watching during the convalescent stage for fear of the development of tuberculosis or meningitis. All patients should be kept in bed until the catarrhal symptoms have disappeared. The time for going out of doors depends upon the weather and the pulmonary con. dition. If the patient does not recover his strength quickly and yet has no fever, he should be sent away to a favorable climate and given nourish. ing food and tonies; in fact, the treatment should be the same as that of incipient tuberculosis (see p. 343).

\section{THERAPY OF GERMAN MEASLES.}

German measles is the mildest of all acute exanthematous diseases. It is only in very exceptional cases that the fever is severe enough to demand any treatment beyond rest in bed with a suitable diet. The itching may be relieved by sponging with vinegar water or by powdering the skin with a mixture of equal parts of rice meal and talcum powder (or zinc peroxide-Ed.]. The catarrhal symptoms need only the simplest treatment. After the catarrh and rash have entirely disappeared, the patient should be given disinfectant baths for several days, being isolated mean. while. Isolation in the prodromal or acute stage is only very seldom successful, but is to be advised, especially if the children who would otherwise be exposed to the disease are delicate. 


\section{THERAPY OF CHICKEN-POX.}

Fever.-Chicken-pox is a more serious disease than German measles, especially for weak or tuberculous children, and isolation should therefore be enforced. The fever and accompanying discomfort may usually be relieved by cool sponge baths, cold compresses on the head, and cool drinks.

Efflorescence in the Mouth.-A much more troublesome symptom is the appearance of an aphthous-like eruption in the mouth and on the tongue. This is not only painful in itself, but it hinders the patient's eating. Hot drinks increase the pain and so must be avoided. If necessary, the separate pustules must be carefully touched, before eating, with a 2-4 per cent. solution of cocaine or aneson, the mouth being rinsed out immediately afterward, or an orthoform spray may be used. After eating, the mouth may be rinsed with some soothing tea (mallow tea) with a pinch of borax or boric acid added. Stronger disinfectants and gargles are contraindicated on account of the pain caused by their use.

Itching.- The eruption often brings on an intense itching, especially on the head, which disturbs the patient's rest and sleep. During the fever this may be relieved by sponging with vinegar water ( 1 part vinegar to $2-3$ parts water) or with a $1 / 4-1 / 2$ per cent. salicylic acid solution; after the fever has subsided, by the use of rice and talcum powder [or zinc peroxide-Ed.]. In some cases, particularly if the patient is of a nervous temperament, the itching is so intense as to demand the use of stronger sedatives. In such instances the author gives antipyrin or phenacetin internally in the usual doses (1-2 powders during one hour in the evening), or with young children, antipyrin in suppositories.

Suppurative Pustules.-If any pustule become enlarged, inflamed, and suppurating, it may be treated with poultices or with a 10 per cent. borlanolin, a 2-5 per cent. iodoform, a xeroform, or a dermatol ointment.

Catarrhal Symptoms.-The catarrhal symptoms need hardly any special treatment unless they drag on into the convalescent stage, when, if the patient is delicate or anæmic or has a scrofulous tendency, we may expect an exacerbation of the latent process. Such cases demand the same treatment as that laid down in the section on measles (see p. 586).

Albuminuria.-During and after the acute stage the urine should be tested for albumin, as the kidneys are involved after chicken-pox in some epidemics. If albuminuria is detected, the therapy and diet must be regulated as in a similar condition in scarlet fever (see p. 582).

Diet.-The diet must be regulated by the amount of fever and the general condition.

Normal Course of Disease.-The normal duration of the disease is a fortnight. As long as the fever persists and new pustules appear, the patient must remain in bed. When the fever and catarrh have disappeared, he may get up and be given warm baths to which lysol has been added. He should not be allowed to go out until he has had $3-4$ such baths and the skin is entirely clear again. 
Every disturbance of the normal course of the disease or delay of convalescence must be carefully heeded. As in similar eases after measles, the patient should be sent to the mountains or seashore and built up in every way possible.

\section{THERAPY OF MUMPS.}

Mumps is such an exquisitely contagious disease that isolation rarely prevents its spread. Still it is our duty to protect others from it as far as possible, especially those with a tendency towards tuberculous glands.

Fever-Pain and Difficulty in Swallowing.-The fever rarely de. mands any energetic treatment, but the swollen glands are so painful and so often prevent the ingestion of food that the pain must receive careful attention. It may usually be relieved by hot applications; and acetic acid. 1 part in 10 of water used on a Priessnitz bandage, which is first covered with cotton and then with oiled silk, is usually found to be very effective. In general it is necessary to change these bandages 3 or 4 times a day, according to the swelling or the idiosynerasy of the patient. A bandage which has been saturated with eamphorated oil, goose grease, or even boric acid ointment, and bound over the swollen glands often gives equally good results. If these do not suffice, the swelling may be rubbed gently several times a day with a mixture of equal parts of chloroform and olive oil (or oil of hyoscyamus), or with a 2 per cent. morphine or belladonna ointment. The use of naphthalene also affords great relief. It is spread thin on a cloth which is applied to the glands, covered with cotton, and then with an outer layer of oiled silk. This dressing should be changed twice a day. The pain is also relieved by carefully massaging the gland in the direetion of its duct, and thus pmmoting the discharge of the masses of mucus. After the fever has subsided the patient may get up, but he should be protected from cold and drafts, and the bandages should be retained until the swelling has entirely disappeared.

Diet.-The pain caused by swallowing frequently confines the patient to a liquid diet, especially at first; and even after the fever has disappeared he may not be able to take anything more solid than gruel.

Normal Course of the Disease.-The normal course of the disease is 1-2 weeks. If at the end of this time the swelling has not diminished. iodine may be applied (compresses of iodine water, painting with 6 per cent. iodinvasogen, or inunetions with potassium iodide ointment). If, especially in individuals with a serofulous tendeney, secondary swellings appear in the neighborhood of the parotid, they should receive the same treatment, together with a general anti-scrofulous therapy.

Inflammation of the Testicles.-Occasionally the testicles become swollen, either in addition to the parotid or vieariously. The patient must remain in bed, the testicles be supported, and suitable compresses applied. After the inflammation has subsided, a suspensory bandage should be worn temporarily. 
Suppuration of the Parotid.-Very rarely suppuration of the parotid occurs. This, as well as any complication involving the ear, demands the attention of a specialist. Another rare sequela of mumps is paralysis of the facial nerve. It is often eured with surprising rapidity by means of electricity.

\section{THERAPY OF DIPHTHERIA. ${ }^{3}$}

[In discussing the treatment of diphtheria our therapeutic efforts may be conveniently enumerated under the following heads:

1. Prophylaxis.

2. General Treatment.

3. Specific Treatment.

4. Local Treatment.

5. Treatment of Laryngeal Obstruction.

6. Prevention and Treatment of Sequelæ and Complications.-Ed.]

Prophylaxis: Hardening Against Taking Cold.-In this connection the importance of a systematic "hardening" treatment to prevent the child's catching cold or getting sore throat, must be impressed on all parents. [The general condition has no influence on susceptibility; the most robust are just as likely to contract the disease as the most delicate. (McCollum).-Ed.] The neck and chest should be washed regularly every morning with cool or cold water and then rubbed with alcohol. The parents should inspect the children's throats every day and see to it that before leaving home they rinse out the mouth and throat with cold water. They should be taught as early as possible to gargle, and then should use such gargles as have a tendency to prevent the development of the Löffler bacilli ( $1 / 2-1$ per cent. salicylic acid, $1 / 2-1$ per cent. benzoic acid, 2 per cent. boric acid, ${ }^{1} /{ }_{10}$ per cent. thymol, 2 per cent. tannic acid). Stronger disinfectants should not be habitually used, on account of the danger of absorption or of injury to the enamel of the teeth. Older children may chew Bergmann's antiseptic troches (thymol $0.002 \mathrm{G}$. $\left(1 /{ }_{30} \mathrm{gr}\right.$.), sodium benzoate 0.2 G. ( $3 \mathrm{gr}$.), saccharin $0.015 \mathrm{G}$. ( $1 / 4 \mathrm{gr}$.), with gutta percha as a vehicle). The act of chewing causes an abundant flow of saliva, which carries down with it these antiseptic substances. The hands should be carefully washed before each meal, particularly if the children are attending school. Any chronic catarrh should be properly treated, and enlarged tonsils or adenoids removed.

Quarantine.-The prophylaxis in case diphtheria has actually affected some member of the household demands the minutest attention to the rules for isolation and disinfection already laid down in the introduction to infectious diseases.

[Every suspected case should be promptly isolated until one or more cultures have proved negative. No case of diphtheria should

${ }^{3}$ [Whenever possible I have followed Prof. Frühwald's plan and treatment, but most of this section has been rewritten by me.-Ed.] 
be discharged from a strict quarantine until after two successive negative cultures from both the nose and throat have been obtained. These need not be commenced until seven days after the disappearance of the membrane from the throat or the discharge from the nose. The great danger of infection arises not from the patients who are seriously ill, but from those with the mild and unrecognized attacks of the disease, especially of the nose (McCollum). Physicians, nurses, and other persons coming in contact with diphtheria patients may acquire the disease or they may carry the infection to others. If possible the nurse should not be allowed to sleep in the sick-room, certainly not to eat there. It is a safe precaution to counsel the use of some simple gargle for those in attendance, although McCollum was unable to make a single positive culture from the throats of sixty healthy nurses on duty in the diphtheria wards at the Boston City Hospital.

Immunization by Antitoxin.-In hospital practice statistics show the advantage of a routine administration of antitoxin as an immunizing agent. At the Children's Hospital in Boston each child admitted is given 750 units if under 2 , and 1500 units if over 2 years of age. At the Infants' Hospital, Boston, they formerly used 300 but now use 500 units. At the Boston City Hospital for the past 5 years every patient in the scarlet fever wards has been immunized with 1000 to 2000 units according to his age. No ill effects have been noted in any of these institutions, although McCollum's influence in Boston makes the dosage larger than in most cities. Holt advises 600 to 800 units for a nurse in charge of a case of diphtheria; 100 to 600 units for children under one month; 500 units for children between 2 and 10 years. This immunity probably lasts three or four weeks, so that if exposure continues it is wise to repeat the dose at the end of three weeks. Forchheimer has practically discarded immunization in private practice. He believes the other members of the family when immunized become careless of exposure; and considers that the attending physician can detect an infection early enough to control the disease promptly with a therapeutic dose. Such a decision, in my opinion, must depend largely upon the family concerned. The results of immunization in hospitals (see above) are so striking that I am inclined to advise immunization in private practice wherever there are other children who might be exposed.-Ed.]

General Treatment: Hydrotherapy.-On account of the deleterious alterations produced in the various organs by the toxin, our antipyretic treatment must usually be confined to hydrotherapy, which is indicated not only in case of high fever, but especially when symptoms of a general intoxication become manifest in the beginning or during the course of the disease. The appropriate procedures have already been sufficiently described in the introduction and in the chapter on scarlet fever; all that need be mentioned particularly here is the danger of giving cool baths on account of possible collapse. [Only the milder hydropathic procedures should be used, and then only to counteract some 
especially serious symptom, such as hyperpyrexia or convulsions. The less exertion or movement the better for the patient. We should insist upon absolute rest in bed for at least one week after the membrane has disappeared. With any abnormality of the pulse or heart tones, the precautions to secure absolute rest should be redoubled and prolonged.

Diet.-The diet should be nourishing and concentrated liquids, generally better cold during the febrile stage. Feeding by the stomach or nasal tube or by the rectum may have to be resorted to. Nourishment is essential, but overzealousness to the point of distressing an already overtaxed patient is to be discouraged. Individualization is essential. The amount may be increased and the interval prolonged to 4 hours if there is a good deal of difficulty and distress in swallowing.

Medication.-Alcohol is valuable in adults and older children, and in the severer forms of the disease, especially the septic types. Holt and McCollum advocate early and free alcoholic stimulation. Strychnine, ${ }^{4}$ digitalis, nitroglycerine, and atropine may all be utilized, especially the first named. Jacobi recommends corrosive sublimate and McCollum considers it of advantage in small doses, $0.001 \mathrm{G}$. $\left(1 /{ }_{60} \mathrm{gr}\right.$.) for a child of 5 years and proportionate doses for younger children. The author gives a series of drugs and prescriptions recommended by various authorities, none of which I include, because we have no evidence that the disease is influenced by any drug except antitoxin.

Specific Treatment.-Diphtheria antitoxin is a specific. The following figures give a very good idea of the difference in mortality before and since its introduction. In an analysis of 250,000 cases reported from all parts of the world, Bayeux states that the mortality before antitoxin was employed was 55 per cent., and that since its general use this mortality has fallen to 16 per cent. At the Boston City Hospital McCollum cites a mortality of 43.20 per cent. before the introduction of antitoxin and 11.84 per cent. since. Such figures and others which could be cited are sufficient to justify our depending upon antitoxin as a specific in the treatment of diphtheria.

Indications for the Administration of Antitoxin.-Antitoxin should

${ }^{4}$ TABLE OF DOSES COPIED FROM ROTCH'S PEDIATRICS.

\begin{tabular}{|c|c|c|c|c|}
\hline Age & $\begin{array}{l}\text { Tinct. } \\
\text { digitalis }\end{array}$ & Strychnine & $\begin{array}{l}\text { Nitroglycer- } \\
\text { ine } 1 \text { per } \\
\text { cent. } \\
\text { solution }\end{array}$ & Atropine \\
\hline & Minim & Grain & Minim & Grain \\
\hline 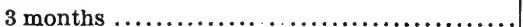 & $1 / 10$ to $1 / 2$ & $1 / 2000$ to $1 / 1000$ & 175 to $1 / 50$ & $1 / 3000$ to $1 / 1500$ \\
\hline 6 months. & $1 / 10$ to $3 / 4$ & $1 / 1500$ to $1 / 500$ & $1 / 25$ to $1 / 15$ & $1 / 2500$ to $1 / 1000$ \\
\hline 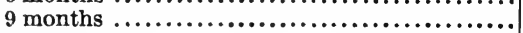 & $1 / 4$ to 1 & $1 / 1000$ to $1 / 300$ & $1 / 25$ to $1 / 10$ & $1 / 1500$ to $1 / 750$ \\
\hline 12 months $\ldots \ldots \ldots \ldots \ldots \ldots \ldots \ldots \ldots \ldots \ldots \ldots \ldots \ldots \ldots \ldots \ldots \ldots \ldots \ldots$ & $1 / 4$ to $11 / 2$ & $1 / 500$ to $1 / 250$ & $1 / 25$ to $1 / 5$ & $1 / 1000$ to $1 / 500$ \\
\hline 2 years...... & $1 / 2$ to 2 & $1 / 500$ to $1 / 150$ & $1 / 15$ to $1 / 3$ & $1 / 750$ to $1 / 250$ \\
\hline 3 years. & $1 / 2$ to 3 & $1 / 300$ to $1 / 100$ & $1 / 10$ to $1 / 2$ & $1 / 500$ to $1 / 200$ \\
\hline 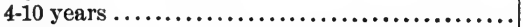 & 1 to 5 & $1 / 200$ to $1 / 60$ & $1 / 2$ to $3 / 4$ & $1 / 250$ to $1 / 150$ \\
\hline $10-12$ years $. . . . . \ldots \ldots .$. & 3 to 8 & $1 / 100$ to $1 / 40$ & $1 / 2$ to 1 & $1 / 200$ to $1 / 100$ \\
\hline
\end{tabular}


be given: 1. In adults (a) as soon as the clinical diagnosis is confirmed bacteriologically, unless the attack is exceedingly mild; (b) before the results of the bacteriological examination are known when the diag. nosis is doubtful, if the case is at all severe or if there is a definite membrane which shows a tendency to spread; (c) and finally whenever the physician is in doubt.

2. In young children, in every suspicious case without waiting for a confirmatory laboratory diagnosis.

3. In older children, whenever the elinical diagnosis of diphtheria is made with reasonable probability, and even when it is doubtful, provided the case is severe.

Bacteriological Diagnosis.-In the larger cities, the boards of health make a bacteriological examination in any suspected case free of charge. In New York, for example, if a practitioner sends an inoculated tube to the Health Department before 10 or 11 o'clock in the evening, he will receive a report by telephone before 11 the next morning. The culture tubes and a sterilized swab in another tube with a blank to be filled out by the physician are to be obtained at any of the numerous stations established throughout the eity. Much care should be observed in making a culture. No antiseptic or other gargle should be used within 2 hours before the culture is made. In tonsillar and pharyngeal diphtheria the swab should be rubbed carefully along the edge of the membrane and not in the centre. In nasal diphtheria it should be inserted well into the depths of the nares. In laryngeal diphtheria it should be rubbed against the posterior wall of the pharynx low down and the region of the epiglottis. The blood serum should be fresh and show some moisture in the bottom of the tube. The swab should be rubbed very firmly along the surface of the medium.

Smears may be made and examined immediately. A positive result is usually correct, but a negative result is not of much value.

Preparation and Dosage of Antitoxin.-The practitioner should not delay the administration of the initial dose, but should send for and inject it immediately. In all large cities it may be obtained for nothing or at cost from the Health Department or from one of the standard makers. With us serum manufacture is under federal control. The serum is put up in syringes ready for use by manufacturers or in vials by the state and city laboratories labelled with number of units; in 500 , $1000,2000,3000,4000$, and 5000 unit packages. The antitoxin globulins in solution are replacing the whole serum. The elimination of the other proteids in the serum allows of a greater concentration of antitoxin. so that 1000 units are usually eontained in 1 c.c. The rashes are less frequent after the concentrated antitoxin. An antitoxin unit is not a definite amount, but is what will neutralize a given quantity of diphtheria toxin of a definite strength. The dose should be large. MeCollum gives the drug until the full therapeutic action is apparent. He gives 4000 units at the outset if there is very marked congestion of the throat, even where 
there is no membrane or only a small patch, and he repeats this dose at the end of every 4-6 hours until the membrane has exfoliated and the congestion of the throat has disappeared. A very extensive membrane requires 8000-10,000 units per dose. "Except in very young children age should have no influence on the dose. No patient ill with diphtheria in the acute stage should be considered in a hopeless condition, but antitoxin should be given in large doses until he commences to improve or succumbs to the disease." He mentions as large a dose as 50,000 units in an almost hopeless case where the tube was constantly being clogged. He has given as high as 80,000 units in one case; Forchheimer, 64,000. The American Pediatric Society recommends as an initial dose 1500 units for a child under 2 years and 2000 units for one above that age. W. H. Park tells me that the dose is becoming greater, especially in the United States. $\mathrm{He}$ advises for mild cases 1500 units, for moderate cases 3000 units, for severe cases, including all croup cases, 5000-10,000 units.

Technic of Administration: Site of Inoculation.-The back near the angle of the scapula is selected by McCollum because the subcutaneous tissue there is lax and because if an abscess forms the pus does not burrow much.

Syringe Employed.-The simpler the better. They are furnished sterilized and filled ready for use by many of the prominent antitoxin manufacturers and by some health departments. The needle is often unnecessarily large. A Luer glass syringe or the ordinary glass and asbestos syringes are easily cleaned.

Precautions.-As much care should be taken in cleaning the skin about the point to be inoculated as for a surgical operation. Pinch the skin between thumb and forefinger and inject into the loose tissue slowly. Cover the point of entrance with cotton and collodion or a bit of adhesive dipped in bichloride solution. Do not rub or massage the tumor formed. Be careful not to inject any air.

Injurious Effects of Antitoxin.-Arthralgia follows the use of the serum in a certain proportion of cases. Immobilization and heat or cold are all that such joints require. McCollum has never seen a serious train of symptoms as a sequel. Rashes are frequent, though less so since the globulins alone have been used. Of the four types, erythematous, scarlatiniform, morbilliform and urticarial, the last is the commonest and causes the most discomfort, a severe itching, for which the usual dusting powders and lotions (see p. 522) may be ordered. The scarlatiniform often suggests a mistake in diagnosis for perhaps 24 hours. With any of them there may be fever, pains, albuminuria, and constitutional symptoms. None of them require any special treatment. I know of no contraindications to the administration of antitoxin unless a status lymphaticus might be so regarded.-Ed.]

Local Treatment: Antiseptics.-The destruction of the colonies of bacilli in the throat without any injurious secondary effects, may be accomplished by suitable and frequent local application of certain disin- 
fectants. [Park tells me that their experiments with disinfectants at the New York Health Department showed that it was impossible to free the mucous membranes of diphtheria bacilli. They employed peroxide of hydrogen and bichloride. Only the bacilli lying upon the surface and those free in the mucus were killed. Very probably, however, the local applications cited below by the author may be efficient in destroying bacteria which might cause secondary infections.-Ed.] The best of these, according to the results of many experiments, are 1-2-3 per cent. carbolic acid, $1 / 10$ per cent. bichloride, and $1 / 2-1$ per cent. chinosol, the latter being many times (30-50) more efficacious than earbolic acid and frequently more so than bichloride.

Methods of Local Application.-These include: rinsing, gargling, spraying, insufflation, and applications with a brush, a cotton swab, or a bit of sponge held in a pair of pincers. It need hardly be said that the swab or sponge should be immediately burned, and the brush be put into a disinfectant solution. For local treatment, the head should be bent forward and the infected places touched gently, eare being taken to avoid any injury to the surrounding mucous membrane. At the beginning of the attack applications should be made at least 4 times a day, the patient's sleep being left undisturbed.

For gargling, spraying, or irrigation we may use, in addition to the disinfectants mentioned just above, 2 per cent. solutions of boric, eitric, or salicylic acid; chinosol $1 / 2-1$ per cent. in rose water; one small teaspoonful of a 2 per cent. solution of actol in a glass of water; 2 tablespoonfuls of peroxide of hydrogen or 2-3 tablespoonfuls of liquor Burowii in a glass of water; a 1 per cent. boric acid solution at $56^{\circ}-95^{\circ}$ every 2-3 hours (Coneetti); or boiled water (Roux). Heller says: "The alpha and omega of most infectious diseases lies in a thorough cleansing of the nasal passages." Monti recommends rinsing the throat carefully every 3 hours with a solution of tribromate of iodine, 20-30 drops in 200 G. (6 oz.) of water.

E. Riegler treats diphtheretic angina, not complicated with croup. by alternate applications of a 3 per cent. peroxide solution and a mixture of 1 part iodic acid to 10 parts milk sugar. The former is sprayed upon the throat every 3 hours, followed $1 / 2$ hour later by the latter introduced by means of a powder blower. During the half-hour interval the following gargle is used:

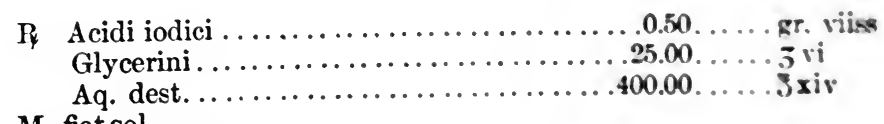

M. fiat sol.

Sig. -As gargle.

Whenever peroxide of hydrogen is employed, the lips of the child should be protected by vaseline. Great precaution must be used in ordering potassium chlorate internally or as a gargle. Lime water, though 
often used to dissolve the membrane, is not a very efficacious remedy. Other disinfectants, such as creolin, lysol, or lysitol, are ineffective in weak solutions, and, like carbolic acid or bichloride, are liable in strong solutions to produce toxic effects by being absorbed or swallowed.

For painting the throat we may use, besides the disinfectants already mentioned, a 5-10 per cent. solution of papayotin; a 10 per cent. solution of lactic acid; a $1 / 10$ per cent. bichloride in a normal salt solution (Behring); $\mathrm{a}^{1 / 50}$ per cent. bichloride solution; a tartaric acid bichloride solution (bichloride $1 \mathrm{G}$. ( $15 \mathrm{gr}$.), tartaric acid 5 G. ( $75 \mathrm{gr}$. ), water 1000 G. (1 qt.)). Strubing applies a cotton swab saturated with a carbolic solution every 4-8 hours (carbolic acid $3 \mathrm{G}$. (45 gr.), rectified oil of turpentine 40 G. (10 dr.), absolute alcohol 60 G. ( 2 oz. )). For it may be substituted a 3-5 per cent. aqueous solution in 30 per cent. alcohol, which, as well as the bichloride solutions, should be used 2-4 times a day, the throat being touched 2-3 (or according to Escherich even 8) times at each treatment.

A solution of the sesquichloride of iron is also highly recommended. For children over 2 years Ganghofner applies the undiluted liquor ferri chloridi two or three times a day on cotton. When the local process has begun to subside (generally in 2-3 days), the solution is diluted with 1-2 parts of water. With infants the 1:1 dilution should be used from the beginning. In nasal diphtheria a $1: 2-3$ dilution should be employed.

Löfler recommends the following mixture:

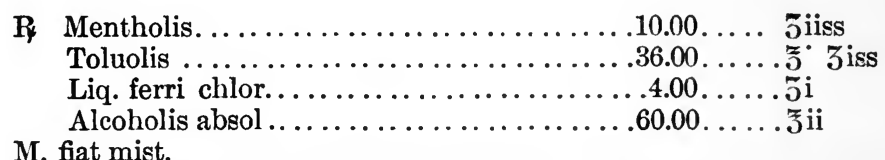

M. fiat mist.

Sig.-For a gargle, to be used as directed. Dispense in brown bottle.

In place of the liquor ferri chloridi Löfler found that creolin or metacresol 2 c.c. ( 30 min.) may be substituted with almost as good an effect. This mixture should be used energetically and frequently in the first days of the attack. After the spots have been cleansed from mucus, a cotton swab soaked in the mixture is held firmly against the membrane for 10 seconds. In severe cases it should be touched twice. This treatment must be repeated every $2-3$ hours until the membrane has entirely disappeared.

Wiemer employs prolonged irrigation of the entire nasal passages, mouth, and throat every 3 hours, or oftener if necessary. The head being bent forward $2 \mathrm{~L}$. ( $2 \mathrm{qt}$.) of sterilized water at $87^{\circ}$ is introduced, partly through the mouth, partly through the nose, and dermatol or iodoform then applied by means of a powder blower.

Monti uses, especially where there are local suppurative processes, 1 : 10 or 1 : 5 sodium bicarbonate $2-3$ times a day or painting with a solu- 
tion of 1 part iodoform to 10 parts glycerin. following for children from two to four years:

Schwarz prescribes the

R Sod. sozoiodol.,

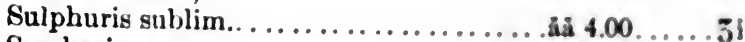

M. fiat pulv.

Saccharin . . . . . . . . . . . . . . .

Sig.-For insufllation as directed.

For children under two years:

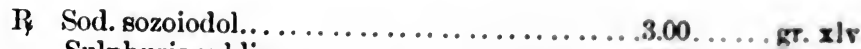

Sulphuris sublim. .................6.00.....

M. fiat pulv.

$1.00 \ldots . . . \mathrm{gr} . \times \mathrm{v}$

Sig.-For insufflation as directed.

For children over four years the sulphur is omitted.

Eka-iodoform, tannoform, thioform, europhen, itrol, iodoform, loretin, nosophen, lanoform, and xeroform may all be used for insuftlation as substitutes for iodoform. [Neither swabbing nor applicating is used at the New York Health Department Hospital; but only simple spraying or irrigating with normal salt solution. No loeal treatment is of sufficient value to compensate for the loss of strength induced by the child's struggling against the treatment. If resisted the attempt should be quickly abandoned. In septic cases irrigation of the pharynx from a fountain syringe is very useful. MeCollum has given up nasal irrigations on account of the danger of introducing suppurative germs into the middle ear.-Ed.]

Use of Cold.-The application of cold externally (compresses, icebags, Leiter's tubes), and internally (ice, ice water, ices) plays an important part in treatment. The ice water may have lemon juice added to it and be sucked through a tube. Very young children should have only a teaspoonful every 15 minutes. In this way we may mitigate the difficulty in swallowing, and the swelling of the lymph-glands.

Local Treatment of Nasal Passages.-As has already been mentioned, the nasal passages often require local treatment. The constant danger of a middle-ear infection must, however, be kept in mind. Insufflation of iodoform, dermatol, aristol 2 parts to milk sugar 18 parts, boral. and nosophen may be used; or disinfectant salves, e.g., 10-20 per cent. boric acid ointment, or 5-10 per cent. iodoform. dermatol, or airol ointment, which must be inserted as deeply as possible, care being taken to avoid injuries to the mucous membrane.

External Applications.-Mercurial ointment and Unguentum Credé have been recommended. 1-2 G. (15-30 gr.) of the former should be rubbed into the skin at some distance from the neck. $1-3 \mathrm{G}$. (15-45 gr.) of the latter should be rubbed in night and morning for about a half hour. 
Treatment of Laryngeal Obstruction.-Acute laryngeal croup, a most dangerous complication, occurs sometimes as a primary, sometimes as a secondary affection. It must be met with local, internal, and general treatment. Cold applications are useful, especially in the initial stages, as well as forcible rubbing with equal parts of mercurial and emollient ointments.

Local Treatment: Sweat Cure.-Pauli recommends Rauchfuss's continuous sweat cure until the membrane disappears. The patient is left three hours in a pack covering the whole body and fastened closely at the neck. He is then rubbed vigorously with cool water and covered with a blanket for an hour. For the next two hours wet compresses are applied to the neck and trunk, being changed every half or quarter of an hour. The treatment is then twice repeated, the whole procedure taking from 6 A.M. to 12 midnight, after which the patient is allowed to rest with only a compress on the neck, until 6 A.M. The intense thirst may be relieved by sweetened brandy and water or a $1 / 2-1$ per cent. solution of potassium chlorate.

Steam Baths.-Brunner-Weymarn recommend steam baths, the steam being introduced into a tent erected over the patient. The procedure is continued for 2-12 hours, with half hourly intermissions every two hours, during which the patient's head is released from the tent, the body continuing to sweat. The more severe the dyspnoa, the longer the process must be continued. Meanwhile stimulants are given internally.

The air should be kept very damp, Krukenberg going so far as to advocate a thick cloud of steam throughout the whole room. The patient is covered up to his neck with a blanket and a rubber sheet.

Inhalations.-For inhalation the most useful solutions are as follows: equal parts of water and lime water; a $2 \frac{1}{2}$ per cent. solution of lactic acid; a $0.005: 1000$ bichloride solution for 15 minutes every hour; acetic acid with $1 / 3-1 / 2$ water; a $2-3$ per cent. solution of boric acid, salicylic acid, or carbolic acid.

Internal Medication.-Internal medication is confined to stimulants and expectorants. They should be used with caution:

R Pilocarpinæ hydrochlor. ..................... gr. $\frac{1}{2}$

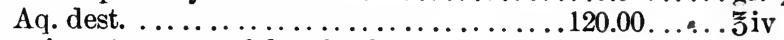

Or :

M. et sig. -1 teaspoonful to $\frac{1}{2}$ tablespoonful every hour.

P Pilocarpinæ hydrochlor................ $0.02 \ldots \ldots$ gr. $\frac{1}{3}$

Ammonii carbonatis $\ldots \ldots \ldots \ldots \ldots \ldots \ldots .2 .00 \ldots \ldots$ gr. $\mathbf{x x x}$

Potassii chloratis................................ gr. xlv

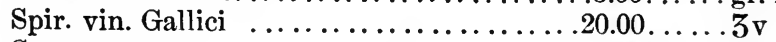

Syr. senegæ........................30.00..... $\mathrm{z}$

M. fiat mist.

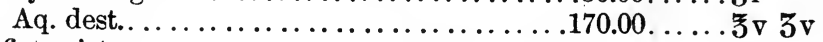

Sig. - 1 teaspoonful to a tablespoonful every hour until the patient begins to sweat. 
Or: $\quad$ R Vin.ipecacuanhr.

Spir. retheris

$8.00 \ldots . .3 \mathrm{sii}$

Syr. simp.,

Aq. dest.

I. fiat mist.

$0.5-1.00 \ldots . . . m v-m \times v$

Sig. - 1 teaspoonful every hour.

Or :

P Syr. senegre ...................90.00.... 3iii

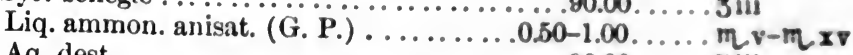

Aq. dest. ............................ J iii

Or :

M. et sig. - 1 tablespoonful every hour.

R Apomorphinæ hydrochlor. ..........0.01-0.03 ....gr. t-gr.

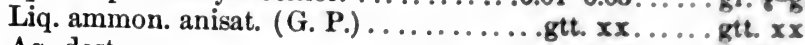

Aq. dest. ...................... $80.00 \ldots$ li $5 \mathrm{v}$

M. et sig. -1 teaspoonful every 2 hours. Shake well.

If there is abundant secretion in the respiratory passages, with loosening of the membrane or increasing dyspnoa, an emetic may be given once, provided the patient is muscularly strong:

P Antimonii et potassii tart. ..........0.05-0.10....gr. t-gr. If

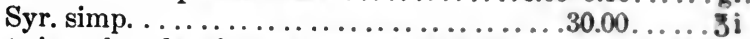

M. et sig. - $\frac{1}{2}$ to be given at once, and in 10 minutes 1 teaspoonful repeated at 10 minute intervals until the desired effect is obtained.

Or :

R Vin. ipecacuanhæ ....................

Ant. et pot. tart. . . . . . . . . . . . .

Oxymel. scill. (G. P.) ...............15.00..... 5iv

Aq. dest. ...........................

M. fiat mist.

Or:

Sig. - 1 teaspoonful every 10 minutes until emesis takes place.

R Syr. ipecacuanhæ ................90.00....

Or :

Sig. - 1 tablespoonful every 10 minutes until emesis takes place.

P Apomorphinæ hydrochlor.................... gr. $\frac{1}{8}$

Aq. dest. .......................... $x v$

M. et sig.-Hypo. solution. To be given at once and repeated in 15 minutes if necessary.

If there are attacks of suffocation 2-3 drops of tineture of belladonna, tincture of valerian, and tincture of aconite in equal parts are given 3 times a day.

Operative Procedures.-If, in spite of loeal and internal treatment. the croupous symptoms persist and the initial symptoms of carbon dioxide intoxication appear, intubation or tracheotomy is most emphatically indicated. Both must be performed by a specialist and need not be described in detail here.

[Antitoxin is by far the best measure we possess to counteract the laryngeal stenosis. The procedures mentioned above by the author are remnants of therapeutic attempts, often fruitless, before the days of 
antitoxin. A limited amount of steam is helpful, but McCollum properly warns against the debilitating effect of its constant use. He recommends saturation (not supersaturation) of the air of the tent with the following mixture :

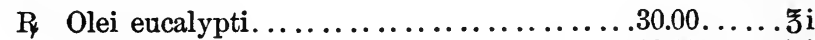

Phenolis liq.........................................

Olei terebinthinæ..................240.00....5viii

in the proportion of one ounce to a pint of water in a croup kettle. If prompt relief does not result, more antitoxin should be given or intubulation resorted to.-Ed.]

Prevention and Treatment of Sequel an and Complications: Difficulty in Swallowing.-The frequent difficulty in swallowing may be relieved by the local treatment already described and by swallowing bits of ice or ice water; the painful swelling of the submaxillary gland, by the local use of cold or of mercurial or Credé's ointment (see p. 597).

Cardiac Complications.-The pulse and heart are generally quickened, corresponding to the rise of temperature. As soon as the signs of general infection (weariness, indifference, somnolence) appear, the pulse is accelerated and out of proportion to the rise of temperature. Such a tachycardia, accompanied by arhythmia, appears generally in severe, septic cases. With the advent of coma and unconsciousness the pulse and heart action become weaker, slowed, and intermittent. In such cases, stimulants are indicated; (e.g., wine, brandy, black coffee, æther aceticus, moschus) or:

R Spir. ætheris,

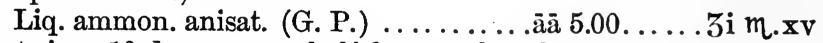

M. et sig. -10 drops every half hour or hourly as required.

Hypodermics of camphor (1 camphor : 10 oil of sweet almonds), 0.3-1.3 G. (5-20 min.), may also be used.

In the initial stages of paralysis of the heart muscle, the following injections are efficacious :

R Strychninæ nitratis ..............0.001-0.003.....gr. $\frac{1}{60}-$ gr. $\frac{1}{20}$ Aq. dest. .....................................

M. et sig.-Hypo. solution. To be given at once and repeated as directed.

Under some circumstances hydropathic measures such as have already been described in the introduction, are also indicated.

Respiratory Complications.-Diphtheria and occasionally laryngeal croup very commonly leave behind them distressing affections of the respiratory tract, which require treatment (see section on measles, p. 586 ).

Nephritis.- Nephritis very frequently accompanies or follows diphtheria. It seldom demands any special diet or treatment, although Ganghofner advises avoiding meat and strongly concentrated bouillon at the height of the disease. 
Paralysis.-The paralysis which often follows diphtheria either immediately or after a few weeks should be treated with electricity, either with the galvanic or the faradic current. In some cases hydrotherayy. combined with massage and gymnastics, is also efficacious. Paralysis of the diaphragm and respiratory muscles demands the almost constant use of electricity and artificial respiration.

Eyes, Ears, Etc.- The primary or secondary diphtheretic affections of the eyes, ears, or cervical lymph-glands, require a specialist's super. vision.

Relapse.-It sometimes happens that after the membrane has com. pletely disappeared a new one is formed. This often vanishes spontane. ously after a few days and needs no special treatment beyond the use of proper disinfectant gargles. [Another dose of antitoxin is indieated.

Persistence of Bacilli in Throat.-Where repeated cultures are posi. tive despite the fact that the patient otherwise shows no evidence of infee. tion, it is probable that the bacilli are lurking in the crypts of the tonsils, in the adenoid tissues, or in the accessory eavities of the nose. Holt recommends for this troublesome sequel gargling the throat and irri. gating the nose three or four times a day with a solution of 1 part of glycerin and 7 parts of bichloride $(1: 10,000)$. From the experiments at the New York Health Department, irrigating and gargling with a normal salt solution would seem quite as effieacious.

Convalescence.-Diphtheria requires more care after convalescence has set in than almost any of the other infectious diseases. A prolonged rest, tonics, extra feeding, careful selection of a suitable climate, moderation in exercise, and careful watching of the heart are all essential.-Ed.]

\section{THERAPY OF WHOOPING-COUGH.}

As we have no specific treatment of any kind for whooping-cough. our therapy must be symptomatic, and may be grouped under the following heads :

1. Measures directed against contagion (antiseptics).

2. Treatment of the paroxysms.

3. Treatment of the catarrh of the respiratory passages.

4. Treatment of the complications.

5. General hygienic measures.

Antiseptic Treatment.-Under this head we include external measures (inhalations and local applications) and internal measures (anti. septics).

Inhalations.-Benzoin, chloroform, ozone, or the gas of the cleansing rooms of a gas plant may be inhaled.

Schilling has the patient inhale 4 times a day the vapor from a croup kettle containing a tablespoonful of hot water and twice as many drops of chloroform as the child is years old.

Ozone may be inhaled for a quarter of an hour each day. [It is very expensive.-Ed.] 
Carbolic acid may be inhaled by means of a mask, or by holding in front of the patient a glass containing a piece of cotton wet in strong carbolic. Care must be taken not to burn the patient. The safest method is to hang about the room sheets wet in a 5 per cent. solution of carbolic acid.

By means of a special apparatus called a vaporizer, inhalations of phenol-kresol or the following prescription may be given:

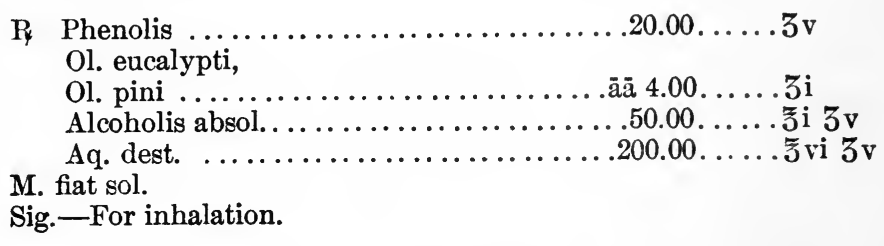

The author has also used naphthalin, evaporating $20 \mathrm{G}$. (5 dr.) on a plate over a flame.

Martin recommends inhalations of chinolin, claiming that they diminish the intensity of the paroxysm, and shorten the duration of the disease.

Lately lignosulphate, holzinol, and vaporin have been used. The patient should not bend over the apparatus to inhale the vapor from any of the above remedies.

The modified oil of turpentine may be evaporated in the room, or cloths wet with turpentine may be hung about the room.

For older children inhalations every 3 hours of the following mixture may be tried:

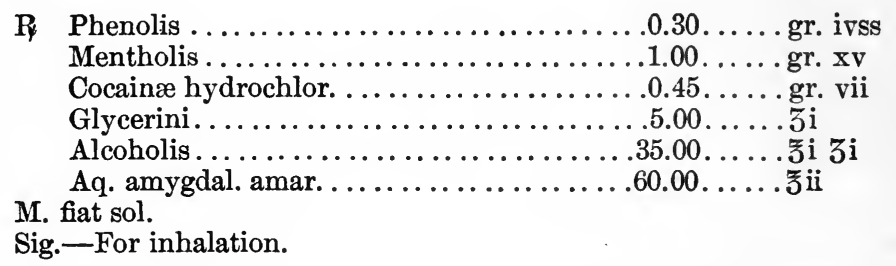

Or inhalations of sodium benzoate, and sodium salicylate, or spraying the throat with 3 per cent. solution of ichthyol in glycerin or 1:500 formalin solution may be employed to relieve the patient.

Local Treatment.-Local applications are too disagreeable to warrant the author's recommendation, and the use of sufficient force to control the child will generally induce a paroxysm of coughing. Various methods may, however, be mentioned:

Insufflations.-Michael recommends upon theoretical grounds the following powders for insufflation: talcum powder or magnesium oxide with 10 per cent. of benzoin, silver nitrate, or quinine. Cuerder advises boric acid mixed with finely pulverized roasted coffee, having the patient snuff it twice a day, while Guttmann prefers sodium sozoiodol. Before 
using any of the above insufflations the nostrils must be carefully freed from mucus. If some of the powder comes out of the other nostril we may be sure of the thoroughness of the procedure.

Painting the Throat.-The following solutions have been recommended by different authors for painting the throat: a solution of quinine, $1 / 2-2$ per cent. earbolic aeid, $1 / 3-1 / 2$ per cent. salicylic acid, equal parts of aqua picea (infus. of tar.) and distilled water, cocaine (with children the danger of intoxication is great), $1 / 10$ per cent. bichloride, and a solution of resorcin. Roskam has modified Moneorvo's use of the last. Every 4 hours during the day and if possible $2-3$ times during the night, he applies directly to the glottis a solution of resorcin, beginning with a 2 per cent. solution and later inereasing it to 3 per cent. when the patient is under 1 year old, and using a 3 per cent. solution from the start with older children. He continues the treatment for 10-12 days and resumes it after an intermission of 5-6 days if necessary. Most patients show marked improvement in all symptoms after about 5 days.

Other Forms of Local Treatment.-Other forms of local treatment recommended are: sprinkling the pillows of the bed with eypress oil or with iodoform powder; painting the neck with iod-vasogen; painting the pharnyx once a day with a 1-2 per cent. formalin solution; washing the chest with hot water, drying it, and then rubbing in "antitussin" (difluordiphenyl). The author does not recommend the latter method, although he has never had reason to attribute to it, as does P. Krause, the formation of cutaneous ulcers. ["I have never seen from any of the above methods (local applications to the larynx, insufflations into the nose) the beneficial results claimed, and I believe them to have been exaggerated." (Holt).-Ed.]

Internal Antiseptics: Quinine and its Substitutes.-One of the oldest antiseptics is quinine, first used for whooping-cough by Binz. Large doses are necessary. For children up to 4 years Unruh gives as many decigrams ( $11 / 2$ gr.) 3 times a day as the child is years old; with children over 4 years generally not more than $1.2 \mathrm{G}$. ( $18 \mathrm{gr}$.) a day in doses of $0.4 \mathrm{G}$. ( $6 \mathrm{gr}$.). If the child is strong, and the paroxysms severe, the dose may be increased to $0.5 \mathrm{G}$. ( $7 \mathrm{gr}$.) a dose, $1.5 \mathrm{G}$. (22 gr.) a day. With children under two years he gives, if necessary, relatively larger amounts, as a rule not more than as many centigrams (1/6 gr.) to a dose as the child is months old. Occasionally a strong child of 3 months may be given a dose of $0.05 \mathrm{G}$. $\left(3 / 4 \mathrm{gr}\right.$.) ; one of 6 months $0.1 \mathrm{G}$. $\left(1 \frac{1}{2} \mathrm{gr}\right.$.) ; one a year old, $0.12 \mathrm{G}$. $(14 / \mathrm{s} \mathrm{gr}$.) ; and one of $11 / 2$ years, $0.2 \mathrm{G}$. ( $3 \mathrm{gr}$.). These large doses should be given for a few (3-6) days only and as soon as improvement begins the single dose should be decreased and later the number should be reduced to two and then to one a day. With older children the dose should be given at first only twice, then once a day. But small doses should be continued for days after the cessation of the characteristic paroxysms. To older children quinine may be given in 
capsules; to smaller children in an aqueous solution with the addition of syr. rub. idæi and a few drops of dilute hydrochloric acid. Rectal injections and suppositories are not to be recommended. Euquinine and aristoquin are excellent substitutes for quinine with young children and are well borne; they may be taken in milk or sweetened water.

The author has found basicin very satisfactory. It is a mixture of quinine and caffeine, which is readily soluble in water. It produces no disagreeable secondary effects, and is best administered in capsules. The adult dose is 0.5 G. ( $7 \mathrm{gr}$.) up to $2 \mathrm{G}$. (30 gr.) a day. For children he orders the following prescription:

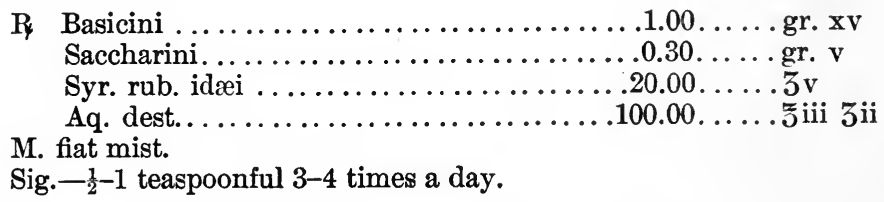

Antipyrin-Tussol.-Demuth, Windelband, and Sonnenberger all warmly recommend antipyrin, Sonnenberger calling it a specific for whooping-cough. He gives as many decigrams (11/2 gr.) as the child is years old, or as many centigrams ( $1 / 6 \mathrm{gr}$.) as the ehild is months old. With older children the doses may be relatively smaller; e.g., $0.5 \mathrm{G}$. ( $7 \mathrm{gr}$.) for a child of 8 years. It should be given 3 times a day after meals with sometimes an extra dose at night. Children take it readily in sugared water. In these small doses Sonnenberger gives it for weeks without any bad effects, believing that this prolonged treatment is necessary for a rapid cure, and that even after the paroxysms have ceased, it should still be given for one or two weeks, although in diminished quantity. With so protracted a use of antipyrin, the parents should be warned of the possibility of intoxication (brownish urine, etc.).

The mandelate of antipyrin (tussol) is also recommended in whooping cough, to diminish the number and intensity of the paroxysms, and to decrease the vomiting and improve the appetite.

Antipyrin is also given in combination with resorcin :

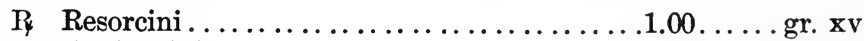

Antipyrini $\ldots \ldots \ldots \ldots \ldots \ldots \ldots \ldots \ldots . . . .6 .00 \ldots$ gr. $x l v$

Aq. menth. pip. .................... $10.00 \ldots \ldots$ Jiiss

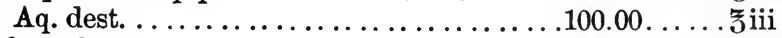

M. fiat sol.

Sig. -3 teaspoonfuls the first day; 4 teaspoonfuls the second day;

5 teaspoonfuls the third day; none the fourth day, and 3 teaspoonfuls the fifth day, increasing as before. follows :

If there are severe catarrhal symptoms, antipyrin may be ordered as

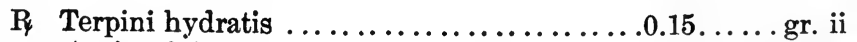

Antipyrini............................ iss

M. fiat caps. no. i. Dent. tal. dos. xx.

Sig. -1 every 3 hours for a 1-4 year old child. 
None of the drugs allied to antipyrin (antifebrin, phenacetin, etc.) are successful, but citrophen the author has used with generally satis. factory results and no bad effects of any kind $(0.5-4 \mathrm{G}$. (7-60 gr.) according to the age of the child, in $100 \mathrm{G}$. $(3 \mathrm{oz}$.) of water, $3-4$ tea. spoonfuls a day).

Other Antiseptics.-Other antiseptics recommended are:

If Sodii benz. .................1.00-2.00

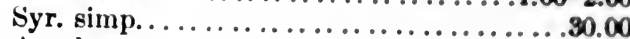

M. fiat sol.

Aq. dest. . . . . . . . . . . . . . . . .

gr. $x \mathrm{~V}-\mathrm{gr}, \mathrm{x} \times \mathrm{x}$

Sig. - 1 teaspoonful to a tablespoonful every 3 houm.

Or:

I. Thiolis

Dent. tal. caps. no. $\mathbf{x x}$.

Sig. - 1 every 3 hours.

R Chinolin tart.

M. et sig. -1 capsule every 3 hours.

gr. iii-gr. $x \mathrm{v}$ $0.30 \ldots . . .67$.

$.0 .20-1.00$

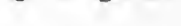

Also resorcin (0.2-1.5 G. (3-22 gr.) a day in solution) (Concetti); sodium salicylate 1-3:100 and its substitutes, asaprol (Moncorvo), and aspirin. Witthauer recommends aspirin in the same doses as antipyrin (not over 3-5 doses of $0.5 \mathrm{G}$. ( $7 \mathrm{gr}$.) a day).

Demme and the author have also recommended in whooping-cough "antispasmin," (1 molecule of narceine sodium with 3 molecules of sodium salicylate). Although its action is often attributed to its content of sodium salicylate, the addition of the chemically pure nareeine seems to have a favorable effect. The author has used it in over 500 cases, employing a 5 per cent. or a 10 per cent. solution in dilute bitter almond water. Of the 5 per cent. solution the doses are as follows:

\footnotetext{
For children under 6 months. . ......3-4 times a day, 3- 5 drops For children 6 months old $\ldots \ldots \ldots \ldots .3-4$ times a day, $5-8$ drope For children 1 year old ............3-4 times a day, 8-10 drops For children 2 years old ............3-4 times a day, 10-12 drope For children 3 years old . ...........3-4 times a day, 15-20 drope
}

For children over 3 years the author uses the 10 per cent. solution, beginning with 10 drops 3 times a day, and, if improvement does not soon appear, increasing to 10-15 drops 4 times a day, and with older children, or adults, even to 20-25 drops. As the preparation easily decomposes when exposed to the light and air, it should be kept in a blue bottle well corked. It may be given in a spoonful of sweetened water.

The remaining antiseptics used are benzol and creosote. Benzol is given in a syrup of acacia $(0.012 \mathrm{G}$. ( $1 / 5 \mathrm{gr}$.) for children of 6 months, 0.3 G. ( $4 \frac{1}{2} \mathrm{gr}$.) for adults, in capsules or solution). 
Creosote may be given in syrup ( $0.5 \mathrm{G}$. ( $7 \mathrm{~min}$.) creosote to $100 \mathrm{G}$. ( 3 oz.) syrup), 2-3 teaspoonfuls for children of 6 weeks to 2 months, and 6-8 teaspoonfuls for older children; or as follows :

R Creosoti (beechwood) $\ldots \ldots \ldots \ldots \ldots \ldots \ldots . . \ldots .25 \ldots \ldots$ gr. iv

Sulphonmethani ........................... iii

Syr. tolutan.,

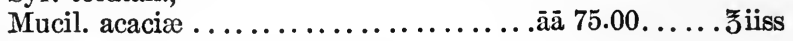

M. fiat mist.

Sig. -1 teaspoonful every 2 hours.

Francke gives 2-3 capsules daily of 0.05 G. ( $3 / 4$ min.) of creosote carefully mixed with $0.2 \mathrm{G}$. ( $3 \mathrm{gr}$.) of tamarind pulp, to be taken after meals. In case of severe cough, the number of capsules may be increased to 5 daily. For smaller children the doses are as follows:

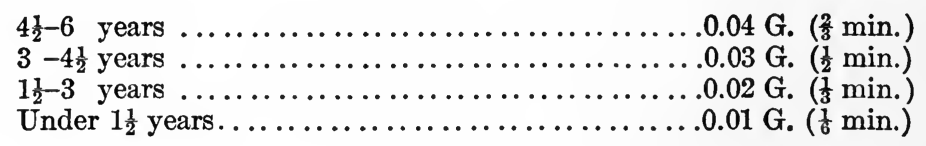

Creosotal is also recommended, 3-10 drops daily in milk or mucilage of acacia.

Maestro recommends ichthyol in pills $(0.05-0.1 \mathrm{G}$. (3/4-11/2 gr.) daily, according to the age of the child).

The following prescription has recently come into use :

P Pyrenolis .................. $0.30-0.50 \ldots \ldots$ gr. iv-gr. vii Thiocolis ..................... $0.50-0.70 \ldots \ldots$ gr. viiss - gr. $x$

M. fiat cap. no. i. Dent. tal. dos. no. xii.

Sig. - 1 capsule 3 or 4 times a day according to the age of the child.

Antispasmodic Treatment : Medication: Belladonna.-For diminishing the intensity of the paroxysms, narcotics or antispasmodies are indicated. One of the oldest preparations used for this purpose is belladonna. It is now less frequently employed and should never be given to infants or young children. For older children the dose of the powdered belladonna root is $0.003-0.005 \mathrm{G}$. $\left(1 /{ }_{20}-^{3} / 40 \mathrm{gr}\right.$.) for each year of the child's age. It may be prescribed as follows:

P Pulv. rad. belladon. ............. 0.10-0.20.....gr. iss-gr. iii Sacch. lact............................

M. fiat pulv. Div. in pulv. no. $\mathbf{x}$.

Or :

Sig. - 1 powder 3 times a day.

R Tinct. belladon. ................1.00-5.00....m $x \mathrm{v}-3 \mathrm{im} \times \mathrm{xv}$

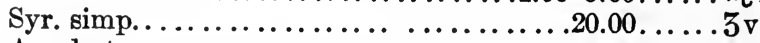

Aq. dest. ............................ $50.00 \ldots \ldots$ ii

Sig. -1 teaspoonful every 3 hours. 
Or: $\quad$ I Fldext. belladon.

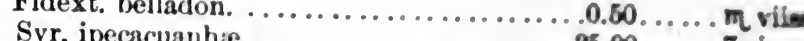

Syr. ipecacuanhie ...............

Vin. antimonii .....................

Aq. dest. $\ldots \cdots \cdots \cdots \cdots \cdots \cdots \cdots \cdots \cdots \cdots \cdots \cdots \cdots \cdots$
M. fiat mist.

Sig. -1 teaspoonful 2-3 times a day.

Trousseau, who first recommended belladonna and later atropine, gave of the latter $0.00025 \mathrm{G}$. $(1 / 250 \mathrm{gr}$.) in the morning, adding another dose of the same size every two or three days until the number of doses a day reached as many as 10 or 12 . In case of severe vomiting after the paroxysms, Trousseau gave $1 / 2-1$ drop of the tincture of opium.

Seifert uses a solution of $0.05 \mathrm{G}$. ( $3 / 4 \mathrm{gr}$.) extract of belladonna to $10 \mathrm{G}$. $(2 \mathrm{r} / 2 \mathrm{dr}$.) of bitter almond water, giving as many drops 3 times a day as the child is years old.

Mixtures of belladonna with aconite, opium. and quinine are also recommended; e.g.:

R Tinct. belladon.,

Tinct. aconiti . ................... $15.00 \ldots . .3 \%$

M. et sig. $-5-10$ drops daily for children under 2 years; $10-30$ dropa for older children.

Or :

R Tinct. belladon.,

Tinct. aconiti.,

Tinct. opii camphoratie. ........... ả 15.00.... 3ss

Or :

M. et sig. - 10-30 drops daily.

R Pulv. rad. belladon.,

Pulv. ipecacuanhre .............. ais $0.10 \ldots \ldots$ gr. is

Quininæ hydrochlor.................50..... gr. xxii

Oleosacch. fœniculi ..............5.00.... 3i gr. xv

M. fiat pulv. Div. in pulv. no. $x$.

Sig.-1-2 powders morning and evening.

Opium-Morphine.-Opium and morphine are also given, but their use demands great caution and careful observation of the patient. They are particularly suited to cases with severe paroxysms and disturbed sleep. The tincture or extract of opium may be given, or, more generally, Dover's powder. Suppositories or enemas are also available.

Codeine-Peronin, Etc.-Codeine phosphate, peronin, heroin, and dionin are less effective, but also less dangerous and free from any bad effects. The rules for dosage will be found in the section on measles.

Chloralhydrate.-Under some circumstances chloralhydrate is indicated. It may be given either internally (1 dessertspoonful every 2-3 hours of a $0.25-1.0: 100$ syrup of acacia) or in an enema (0.2-1.0:50 syrup of acacia, once or twice a day). 
Or, instead of chloralhydrate:

I Sulphonmethani ......................25....gr. iv

Creosoti (beechwood) $\ldots \ldots \ldots \ldots \ldots \ldots \ldots . .20 . \ldots \ldots$ gr. iii

Syr. tolutan........................ $30.00 \ldots \ldots .5 \mathrm{~s}$

Mucil. acaciæ.................... 120.00.... 万iv

M. fiat emuls.

Sig. -1 teaspoonful every 2 hours.

The various preparations of bromine are effective but are contraindicated in case of any tendency towards laryngeal or other form of spasms or convulsions.

Bromoform.-Bromoform is recommended by many authorities in spite of the fact that symptoms of intoxication have been noted after its use.

Ganghofner gives it in an aqueous solution to which some alcohol has been added, 5 drops of the solution corresponding to 1 drop of bromoform.

Cohn (Hamburg) prescribes it as follows:

R Bromoformi,

Alcoholis ..............āā 0.50-1.00-2.00..... gtt. xv-gtt. xxx-gtt. lx

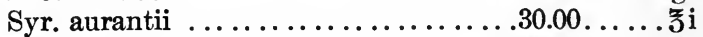

Mucil. acaciæ................90.00....5

M. fiat emuls.

Sig. -1 teaspoonful to 1 dessertspoonful every 2 hours. (Shake well before using.)

This emulsion will keep for days, works promptly, and does not produce symptoms of intoxication.

Gay's prescription is as follows:

R Bromoformi ................................. xlv

O1. amygdal. express..................15.00.....5

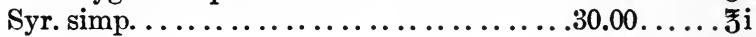

Pulv. acaciæ....................10.00.... 3iiss

Aq. menth. pip. ....................6. $65.00 \ldots \ldots$.

M. fiat emuls.

Sig. - For children under 2 years, $0.05-0.1$ G. ( $\left(\frac{3}{4}-1 \frac{1}{2} \mathrm{~min}\right.$.); for $2-4$ years, $0.1-0.15$ G. ( $1 \frac{1}{2}-2 \frac{1}{2} \min$.); for $4-8$ years, $0.15-0.3$ G. ( $2 \frac{1}{2}-4 \frac{1}{2} \min$.); for adults, $1.0-1.5$ G. (15-22 min.). (Shake well before using.)

Marfan gives it as follows:

P Bromoformi $\ldots \ldots \ldots \ldots \ldots \ldots \ldots \ldots \ldots . . .2 .30 \ldots \ldots$ gtt. lxiv

Ol. amygdal. express..................20.00.... $\mathrm{z} \mathrm{v}$

Pulv. tragacanthæ..........................

Pulv. acaciæ,

Aq. amygdal. amar.................. āā 4.00 ..... . $\mathrm{i}$

Aq. dest........................ s. ad. $120.00 \ldots \ldots .5 \mathrm{z}$ iv

M. fiat emuls.

Sig. -1 teaspoonful $=2$ drops bromoform. (Shake well before using.)

For children under 6 months, the dose is. . ............2-3 drops

For children $6-12$ months, the dose is....................

For children 1-5 years, the dose is 4 times as many drops as the child

is years old. 
The dose should never be more than 20 drops to begin with but may be doubled gradually by inereasing by $2-4$ drops daily. If symptoms of intoxication appear (cyanosis, cardiac or respiratory weaknew, narrowing of the pupils) the drug must be immediately stopped, and if necessary artificial respiration and injections of ether employed. The new com. binations of bromoform with extract of thyme, e.g., bromothymin and thymobromal, are useful (a teaspoonful to a dessertspoonful $3-4$ times a day).

Pasterin.-Paster's pasterin tablets have recently come into use. Pasterin is composed of 3 per cent. of codeine with sodium salicylate, sodium bromide, and extract of elecampane, and the tablets are made up as follows :

R Pasterini ............................. 15.00....

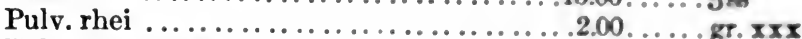

Pulv. glycyrrhiz................................ gr. $x \mid v$

Cacao,

Sacchar. lact.................... ha $5.00 \ldots$ gr. Ixxv

M. fiat mass. Div. in tab. no. c.

Sig.-For children under 2 years, $6-7$ tablets a day, 1 every 2 bours; for children over 2 years, 12-14 tablets a day, 2 every 2 hours; for adults, $18-24$ tablets a day, $3-4$ every 2 hours. The tablets may be dissolved in hot milk or taken in capsules.

The Dialysat Golaz (species thymie et pinguicula) is also used.

[The psychical treatment is very important. Children who have been well brought up can be made to inhibit mild paroxysms. By proper suggestion many attacks can be prevented.

Holt and Forchheimer recommend beginning belladonna with small doses and gradually increasing until full physiological effects are produced upon the pupils.

Holt has obtained most benefit from antipyrin, Forchheimer from quinine. "In a mild case, where the number of paroxysms does not exceed eight or ten during the day, where there is no vomiting and the general health is not affected, it is not usually advisable to continue the administration of any drugs throughout the disease. A single dose of antipyrin or codeine at night may be all that is necessary. All cases in infants must be watched with great care and the parents warned of the possible dangers which may supervene suddenly, even in the course of mild attacks. For severe cases antipyrin should be given to diminish the frequency and the severity of the paroxysms, and inhalations of creosote used if much eatarrh is present. All the fresh air possible should be allowed. For older children the same plan of treatment may be followed, or quinine or belladonna may be substituted for the antipyrin." (Holt.) Although some cases seem to be benefited by one and not by another drug, whether quinine, antipyrin, belladonna, or bromoform is used, the safest plan is to use too little and never too much medication. 
With seventy odd drugs recommended for whooping-cough in the U. S. Dispensatory, it is hardly necessary to caution the student and practitioner to devote most of his care and thought to the details of the external therapy and little to the drugs.-Ed.]

Manipulation.-If a paroxysm is accompanied by deep cyanosis, von Nägeli recommends the following procedure. The bent index and middle fingers are placed upon the ascending ramus of the lower jaw and the thumbs upon the chin, and the jaw then pulled gently forward and downward. As soon as the mouth is opened both index fingers are inserted in the region of the eye-teeth and the jaw held firmly. If the mouth is open at the start, the thumbs or index fingers are inserted as before and the other fingers placed on the lower jaw and the jaw pulled down. In moderate paroxysms it is often enough to pull the tongue forward with the finger or handle of a spoon, and blow energetically into the mouth. In deeper asphyxia artificial respiration, wet rubs, and flicking of the chest with a wet towel may be resorted to. The forcible removal of the mucus by means of a finger or towel during the paroxysm is always unnecessary and sometimes injurious. It is enough to bend the head down and wipe out the mouth after an attack.

[To overcome serious spasm of the glottis and to prevent asphyxia when the paroxysms are frequent and severe, O'Dwyer and Northrup use intubation.-Ed.]

Anti-Catarrhal Treatment.-The anti-catarrhal treatment has already been partly discussed in the section on measles (see p. 585). In mild uncomplicated cases of whooping-cough, alkaline mineral waters (Ems, Selters, Gleichenberg) with sugar or hot milk may be prescribed. In more or less extensive catarrh, expectorants may be given, and a cross binder applied during the night. Among the available drugs are ipecac, senega, preparations of antimony, benzoin (0.01-0.03 G. ( $1 / 6-1 / 2$ gr.) every two hours), and camphor (Stricker).

Liebermeister uses the following prescription:

R Antimonii sulphurati $\ldots \ldots \ldots \ldots \ldots \ldots \ldots .0 .50 \ldots \ldots$ gr. viiss

Syr. simp.,

Mucil. acaciæ................. āā 20.00.... 3v

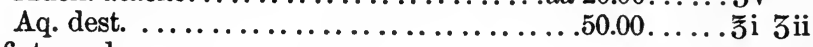

M. fiat emuls.

Sig.-1 teaspoonful every 1-2 hours.

To reduce the viscosity of the mucus, cochineal is also prescribed:

R Cocci $\ldots \ldots \ldots \ldots \ldots \ldots \ldots \ldots \ldots \ldots .0 .05-0.20 \ldots \ldots, g r . \frac{8}{4}-g r$. iii

Ammon. carbon.................1.00-2.00.....gr. xv-gr. xxx

Syr. simp. ...................................

Aq. dest...................... q. s. ad. $90.00 \ldots \ldots$.

M. fiat mist.

Sig. -1 teaspoonful every 2 hours. (Shake well before using.) 
The fluidextract of thyme added to syrupus sacchari $(10: 70)$ with a little potassium bromide, put on the market under the name of pertussin, is also used. The daily dose is 3-6 tablespoonfuls diluted with water.

Complications: Of the Respiratory Tract.-The most important complications of whooping-cough are affections of the respiratory tract. Any pathological process in the lungs or bronchi necessitates stopping any narcotic or antispasmodic. Expectorants with or without stimulants are indicated. At the slightest symptoms of cyanosis, inhalations of oxygen should be employed, as they always give the patient substantial relief.

The emphysema that sometimes accompanies whooping-cough seldom demands, at least with children, any treatment beyond systematic breath. ing exercises. In very severe cases the author has brought about a quick and complete cure by means of the treatment with increased air pressure in the pneumatic chamber.

Of the Nervous System.-The next most important complications are affections of the nervous system, particularly laryngeal spasms and convulsions. Rachitic or very nervous children must be carefully watched, especially at night, as such an attack too often ends fatally. Nerve seda. tives are indicated, especially chloral and the bromides internally or by the rectum. Simon's mixture is also valuable:

R Chloral. hydrati, Potassii bromidi .................åi 1.00..... gr. xv

Tinct. moschi $(10 \%)$,

Tinct. aconiti..................ä 10.00.... juiss

Aq. aurant. flor...................... 100.00 ....

M. fiat sol.

Sig. -1 teaspoonful every $2-3$ hours, or $\left\{-\frac{1}{2}\right.$ of the whole as an enema.

[This prescription calls for the maximum dose of aconite.-Ed.]

The irritability and nervousness often occurring with older children during or after the disease is met better by gentleness, patience, and the avoidance of any excitement than by drugs. During convalescence a change of air and hydrotherapy, particularly tepid half-baths, are often surprisingly helpful.

Of the Gastro-Intestinal Tract: Vomiting.-The romiting that so often accompanies whooping-cough is usually mechanical, though sometimes due to an idiosnycrasy against certain drugs. As it interferes with the child's nourishment, it must be combated by careful attention to the diet and to the number and times of the meals, as well as by appropriate medication. Escherich gives $1 / 2$ tablespoonful every 2 hours of a mixture composed of 3-10 drops of creosote :100 G. of water and $10 \mathrm{G}$. of syr. simp. Sometimes a few spoonfuls of black coffee or 2-5 drops of bitter almond water taken just before meals are of service. In some instances vomiting may be avoided if the patient takes his food lying down. See section on measles for treatment of intestinal symptoms, p. 557.

Mechanical Results of Severe Paroxysms.-In very severe paroxysms it sometimes happens that the capillaries are torn, and hemor- 
rhages occur from the lungs or nose, into the conjunctival sack, from the ears with rupture of the drum, into the skin in the form of petechiæ and ecchymoses, and, rarely, into the brain. The nasal hemorrhages may be stopped by the use of diluted vinegar, adrenalin chloride, or lemon juice or by inserting tampons soaked in chloride of iron. In every severe case plugging the posterior nares may be necessary.

Other mechanical consequences of severe paroxysms are involuntary urination and defecation, prolapse of the rectum, and umbilical and inguinal herniæ. Scrupulously clean linen and the application of an antiseptic ointment (boric, resorcin, or salicylic) will avert intertrigo and eczema. Prolapse of the rectum cannot be helped while the paroxysms continue; all that can be done is to replace the rectum in the usual way. The same is almost always true of hernix. No truss can be worn tight enough to do any good during a paroxysm. With infants the hand should be held on the hernia during an attack, and with older children the legs may be drawn up on the abdomen.

Sublingual Ulcer.-With infants sublingual ulcers are too often caused by injury to the frænum by the lower teeth. This often interferes with nursing. The pain may be relieved by painting the ulcer with aneson or dusting it with orthoform powder before meals, but a definite cure cannot be expected as long as the paroxysms continue.

Genernl Trentment.-As in every acute disease, care must be paid to the diet and hygiene during the course of the disease and convalescence.

Diet.-During the disease no especial diet is usually needed, although care must be taken to avoid overloading the stomach. If fever is present, the diet must be restricted but yet as nourishing as possible. In case of severe vomiting, food should be given in small quantities at frequent intervals soon after the attacks. Fluids are more easily vomited than solids, and dry or crumbly food tends to cause vomiting by the mechanical irritation of the throat. All sweet, sour, or irritating foods or drinks should be forbidden during the whole course of the disease. The refusal of food partly on account of the frequent vomiting, and with older children partly on account of psychical disturbances, must be met by gentle persuasion and the selection of favorite dishes.

Hydrotherapy.-Bergmann recommends a 15-20 minutes' hot bath morning and evening, followed by $3-4 \mathrm{~L}$. (3-4 qt.) of cold water poured from a considerable height over the breast, neck and back. The patient should not be rubbed dry for fear of exciting a paroxysm, but should be wrapped in a dry sheet and blanket and left to get dry and warm.

Hygiene.-The hygiene of the sick-room and of the patient's habits, mode of life, and surroundings is most important. So long as the patient is perfectly well except for the attacks of coughing, he need not be kept in bed, but all violent exercise (running, dancing, jumping) as well as weeping, shouting, and psychical excitement must be avoided. As soon 
as fever or bronchial disturbances appear, however, he must be kept in bed. Especial caution must be observed with delicate and rachitic children.

Fresh Air.-For the sake of disinfection and also for insuring the inhalation of as pure air as possible, Mohn recommends the following procedure: The patient clothed only in a sheet is earried out of the sick-room in the morning, everything which eannot be washed being left in the room, which is then disinfected by burning $25 \mathrm{G}$. (6 dr.) of sulphur for each cubic metre. After 5 hours, the room is thoroughly aired, and the patient brought back in the evening.

Jürgensen recommends moving the patient to a fresh room while the original sick-room is well aired out and washed down with a carbolic solution. The patient is not to be brought back until the attacks have diminished in number and intensity. The author has frequently proved the value of such an alternation of rooms, one being cleaned while the other is in use. Abundance of fresh air is of course always necessary.

This fact is emphasized in Wertheimber's fresh air treatment. By keeping the patients out of doors, the intervals between the paroxysms of coughing are made three times as long and their intensity diminished. This treatment is easy to carry out in summer or in a warm climate, but should be used only with great caution during cold or stormy weatber. Very young or delicate children should certainly not be exposed to winter weather and even older and stronger ones should be kept in except on sunny, calm days. The patient should be kept as quiet as possible while he is out of doors, often lying down in the open air from morning till night.

The following are Wertheimber's rules for the hygiene of the sick. room:

1. The patient should have two rooms at his disposal, the larger one being used to sleep in. During the day he should be changed from one to the other every three hours, the unoccupied one being meanwhile can fully aired.

2. The room should be kept at $66^{\circ}$ (for infants under a year, $68^{\circ}$ ) during the day and $55^{\circ}-60^{\circ}$ during the night, the child having on a shirt and long night-garment.

3. If the room is heated by a stove or fireplace, wood should be used in preference to coal, as it consumes only one-half as much oxygen.

4. If a kerosene lamp is used for lighting, it must be kept carefull! trimmed and scrupulously clean.

5. All carpets, heavy curtains, portieres, and upholstered furniture should be removed from the room. The emptier it is, the better.

6 . No one should be allowed in the sick-room except the patient and nurse.

7. The sputum should, if possible, be expectorated into vessels con. taining a 5 per cent. carbolic or a 0.1 per cent. bichloride solution. If 
any sputum gets on the floor, the spot should be immediately cleaned with the same disinfectant.

Change of Scene.-A change of air after the end of the third or fourth week of the convulsive stage is often advantageous. In an uncomplicated case a decided change of climate is very seldom necessary. It is often enough to get the child away from the noise and dust of the city into the country, where he can be better kept out of doors. But, as the danger of contagion is by no means over, the rules already given for the disinfection of the sputum and sick-room, must be rigorously carried out.

Clothing.-The clothing should be warm enough to prevent the patient's getting cold and yet so light that he will not get overheated. It must be comfortable and loose, and easy to open in case of a severe paroxysm.

Prophylaxis.-For 6-8 weeks, or as long as the cough continues, the patient must be isolated to prevent contagion. The clothing and sickroom should afterwards be carefully disinfected. Especially during an epidemic, all weak or delicate children, those predisposed to tuberculosis, and those in whom for any reason the results of whooping-cough are to be feared, should, if possible, be sent away, at any rate kept apart from other children and not allowed to go to schools or to public parks.

[Whooping-cough is too often considered a harmless disease and too little attempt made to prevent the spreading of the infection. The mortality in this country is almost as great as that of scarlet fever. Every patient should, therefore, be carefully isolated. Forchheimer says, "The infectiousness of the disease begins immediately with its onset, reaches its maximum in the paroxysmal stage, and then declines. It is impossible to determine accurately when infectiousness ceases. Filatow and Fischel assert that this continues as long as the patient coughs at all. I have always followed the following rules: No case of whooping-cough should be considered free from infection (1) unless six weeks from the onset of the disease shall have elapsed; (2) until the whoop shall have disappeared completely ; the whoop that may recur with ordinary infections of the respiratory tract is not taken into consideration; (3) until expectoration shall have practically ceased; a recrudescence of expectoration as the result of a secondary infection is not to be counted. It will be seen that this means a variable period of isolation-from six to ten weeks, and sometimes more. The patient should be kept away from all who have not had whooping-cough. One attack confers immunity. Children under two years of age or debilitated children should be most carefully guarded against infection; also subjects with a tuberculous predisposition or valvular cardiac trouble, or convalescents from any disease which has caused debility. A child with whooping-cough should not be allowed to sleep in the same room with healthy children, to go to school, or to any place where a number of people are confined in large or small rooms." -Ed.] 


\title{
XVI. \\ THERAPY OF NEURASTHENIA
}

\author{
By DR. NATHANIEL BOWDITCH POTTER.
}

A Physician's utility to his patients and professional success largely depend upon his ability to treat neurasthenia wisely. I purposely use the term neurasthenia in a general sense, somewhat at variance with modern psychological and neurological study, because I believe that my readers will find the plan outlined more helpful than specific methods aimed at the different subdivisions of the psycho-neuroses.

Prophylaxis.- All are more or less susceptible to neurasthenia. Im. pairment of nutrition, some general disease, or faults of temperament, increase this susceptibility. Neurasthenia is commoner to-day than it was a hundred years ago, and is increasing especially in our eities. This results from the lowering of the mortality in infancy and childhood by which less sturdy individuals survive; from the growth of wealth and luxurious habits; and from the complexity and strenuousness of modern life. To lessen the increase of so insidious and disabling a disease is as much the duty of physicians as its treatment.

In Childhood.-Prophylaxis begins in childhood or even in infancy. Special care is required in rearing frail or delicate children, those with any of the early nervous stigmata, or the children of mentally brilliant parents who have been subject to neurasthenia, to some other nervous affection or to gout. The mental development of such children should be secondary to physical, and their school life must not begin too early. They are benefited by a country life, with early hours, and without stimulating and exciting pleasures, such as children's parties and strenuous competitive games.

In Adolescence.-The period of puberty next requires most careful and intelligent supervision in order to prevent vicious influences acting upon the hypersensitive nervous system of an adolescent. The early awakening of the sexual instinct must be watched and intelligently ex. plained at the proper age; masturbation prevented or detected early, and promptly cured; a mysterious or an obscene conception of the sexual relations precluded; and such false modesty as leads to the delay of the natural functions of defecation and urination counteracted. American parents often shirk such a duty to their children. This is the very period, 
furthermore, when many children are away at boarding schools, where there is often a noticeable lack of medical supervision of athletics, of diet, and of the rules of hygiene, and where too little attention is paid to the individual.

In Adult Life.-In adult life, some young women develop neurasthenia, with or without a disturbed metabolism, shortly after an unfortunate love affair. Others exhibit their first symptoms after giving birth to several children in rapid succession, and others at the climacteric. Many such cases can be avoided by watchful, intelligent care at the critical times. With young men, sometimes the excessive work necessitated by an ambitious desire to enter college early, sometimes the strain of competition at college, sometimes the effort to be an athlete, a student, and a social success all at the same time, or sometimes unaccustomed application to a sedentary occupation after leaving college, produces a pernicious influence upon an unstable nervous system, and the symptoms of neurasthenia soon result.

After a Previous Attack.-After one attack of neurasthenia has been followed by recovery, every effort should be made both by the paticnt and by his physician to prevent another. No immunity will be conferred unless the physician's intelligence has influenced the patient's self control, reason, and common sense sufficiently to make him avoid instinctively even minor attributing causes.

TREATMENT.-The plan of treatment of neurasthenia is generally as simple as the exact determination of the etiological and attributing factors is difficult. Our intelligence in comprehending and properly balancing these factors will be mainly responsible for our success in curing or relieving the disease.

Physical and Psychical Examinations.-A neurasthenic patient should first receive a careful and deliberate physical and psychical examination. This may require a few days' observation at the patient's home or in an institution adapted for such a purpose. The patient experiences very great relief and reassurance if the examiner, after exhausting all modern methods of diagnosis, carefully explains that no organic fault exists. The psychical benefit of such a statement is tremendous. Subsequent examinations of general or special functions necessitated by symptomatic complaints may be required from time to time in very prolonged obstinate cases of neurasthenia, but, generally speaking, one examination should suffice, and one such reassurance should accomplish its purpose. If repeated examinations are demanded, the physician should search his armament for more efficient therapeutic influences or should transfer the patient to a more experienced colleague.

Psychical Treatment.-Any method of treatment exerts more or less of its influence in virtue of the psychical impression created upon the patient. "Psychic therapeutics is an adjuvant to the healing forces of nature and time; and a precious resource to the physician" (Edes). In America to-day, more especially in New England, psychic therapeutics 
is perhaps too prominently in the foreground for the good of the patient and the success of the physician. The impression produced and the cures effected by magnetism, mesmerism, hypnotism, Christian Science, and other psychic methods have, however, created so vivid an inpression upon the world at large that any thoughtful physician cannot fail to recognize the importance of psychic therapeutics in the treatment of neurasthenia.

The direction of patients with neurasthenia requires unusual mental and moral qualities in the physician in charge. He must inspire confidence, possess patience, comprehension, tact, and sympathy, and above all a thorough knowledge of human nature and a broad schooling in the ways of the world. All this presupposes an exceptional training. To counteract the patient's unfavorable auto-suggestions he must substitute contra or even hypnotic suggestions; he must free the patient from the sick feelings, the indecisions, and depressions of the morbid condition: he must awaken proper pride; strengthen the will power; ensure the cultivation of a purposeful attitude toward life, and especially inspire hope. Explanation, persuasion, and education, combined with a proper balance between isolation, rest, and occupation, are more serviceable in the treatment of neurasthenia than pure suggestion. In some patients it may be wise to commence with hypnotism, although most neurasthenies are so controlled by their auto-suggestions, that they are difficult to hypnotize. The late Dr. Russell Sturgis successfully employed what he called the slighter degrees of hypnosis. Janet compels the patient. under partial or complete hypnosis, to reproduce in memory the original scenes of noxious experiences and to suppress them. He then substitutes a different series of associations with a different outcome. Breuer and Freud, depending upon the relief which follows a full confession of perhaps some rather trivial affair, employ successive séances of enquiry and upturning of the subliminal consciousness to its lowest depths and afterwards substitute more normal impressions. Freud considers all cases of neurasthenia, psychasthenia, and hysteria as primarily due to the disorganizing effects of morbid sexual experiences or of experiences repressed for any cause; and believes that healthy impressions can be obtained only after catharsis. so-called. preferably under hypnosis. DuBois also employs psychoanalysis, but "side tracks" the patient 's morbid ideas and autohypnotic suggestions by a more rational series of associations and ideas. He endeavors to analyze the patient's character. to discern and eradicate temperamental faults, to reason with him, to re-educate and develop his mentality and self-control. "We preserve our temperament throughout our whole life just as we keep our physical blemishes; but we ean modify it greatly by educating ourselves. Our physical deformities are often definite: but our mentality is always malleable." The trainer Muldoon seized upon a similar principle and combined with it a very rigid training. His pupils have no time for auto-suggestions, and if their condition is sufficiently vigorous to endure 
the physical strain, the method is especially successful with business and professional men. Oswald dwells upon the fact that mental operations of a pleasurable sort directly favor nutrition and the normal flow of chemical energy, while those of a painful sort interfere with nutrition and hinder the flow of chemical energy. Grohmann teaches the curative principles of healthy work and a simple life in a small community. Hall has succeeded in helping many severe cases by employing occupation cures-grading the work and the hours of rest according to the patient's progress. Especially commendable are the results which Hallock has obtained at his admirable Health School for Invalids.

In estimating the possibility of a cure of a case of neurasthenia, its completeness, and its permanence, we must fully understand the laws of compensation just as is necessary in cardiac dilatation. Health is a stable equilibrium which the organism attempts to continue by mutual modification and adjustment of its interwoven energies. Any decided disturbances of this equilibrium, whether resulting in so-called organic disease or in neurasthenia, can rarely be completely repaired. Some excessive or abortive reactions or pernicious habits will always remain behind as evidences of instability or disease. It is the physician's province to balance accurately the extent of the mischief produced and the compensatory power in reserve, in order to instruct the patient in the ways and means of continuing his life upon the old or upon new lines. The patient must thoroughly comprehend his own resources and limitations. He must be taught the necessity of maintaining a maximum nutrition, be shown measures of relieving the minor discomforts, and be urged to reserve some measure of compensation for after demands.

Neurasthenics are continuously and painfully conscious of nervous weakness or of hypochondriacal depression. Their fatigues and faintheartedness depend at least partially upon what we might designate as a mental incoördination. An impaired general nutrition or some local ailment, one or both of which may be the partial cause or the result of the neurasthenic condition, must be attacked from the very beginning of the treatment; conditions for a relatively active life must be secured; the bodily nutrition must be improved in every possible respect; all reflex excitations must be quieted; sentiments of courage, confidence, determination must be imparted; the patient must be warned and then convinced of the dangers likely to impair his nutrition, increase reflex excitations, or induce morbid trains of thought; the development of a stoical attitude towards all minor unpleasant feelings must be insisted upon; the patient must be studied and his pride, reason, conscience, ambition, hope, fear, religion, or philosophy must be utilized for producing and continuing a proper mental attitude towards life in general.

Symptomatic Treatment.-Whether the treatment is commenced in a sanatorium or not, one of the first indications is to eradicate whatever factors we can determine either produce or contribute toward the exhausted nerve system. All the organs and their functions should be 
carefully studied. The first symptoms which appeared are expecially important, since they often disclose avoidable causes. If the neurasthenia exists merely as a complication, we apply to the underlying disease every known method of relief. Any complicating difficulty, such as gastric, intestinal, cardiac, pelvic, or nutritional disability, requires modern symptomatic therapy. The slight, even though transitory, well. being secured by improving an obstinate constipation, or penistent hyperchlorhydria, will be a tremendous stride towards securing the confi. dence and coöperation of a discouraged invalid. A physician's intelli. gence and cunning may be tried to their utmost in this direction. Some of these symptoms depend upon autosuggestions, the result of morbid introspection, and are better disregarded by the physician and patient. At first, however, we usually do well to listen to everything in detail, to map out a plan which promises success against some one, two, or three of the most troublesome symptoms; and later on to disregard minor symptoms and counsel a stoical attitude. Confidence ean be acquired at first only by some little success in relieving disagreeable symptoms.

Treatment in a Sanatorium.-Most patients with neurasthenia are benefited by isolation in a well equipped sanatorium. Hystero-neuras. thenic young women who are especially suggestible react better to this method than males. Many adequate reasons, however, may deter the physician from counselling this plan, or may lead him to delay so radical a measure until after the establishment of closer relations with bis patient. Generally speaking, a prompt change to the appropriate institution facilitates recovery more suecessfully than any other measure. Weir Mitchell's cure was very properly imitated throughout the world. In his hands isolation, rest, superalimentation, electricity, and massage worked wonders. As might have been expected, many physicians who copied the method in every detail failed to obtain the benefits that almost invariably resulted from the profound and lasting psychical impression created by so great a master. Dana, however, warns against too protracted sanatorium cures. I have seen many neurasthenics who are entirely dependent upon sanatoria and quite unable to withstand the drain of their ordinary routine unless every few months rejurenated by a rest cure. Besides the habit of dependence and the loss of will power which may result, another disadvantage is the eost. Good sanatoria are very expensive. Most of the large institutions, where people of moderate means can secure treatment, are commercial establishments not to be recommended for the majority of neurasthenies. In the latter, invalids are surrounded by invalids, and their entire horizon stretehes from their aches and pains to those of their fellows. Often some of the inmates are mental cases or patients on the borderland. The physicians in change are generally overworked and rarely of sufficient mental grasp or train. ing to individualize properly and direet the physical and psychical treatment. There is usually too much rest, too little occupation, and too much 
introspection. The diet often contains too much meat and is seldom properly outlined for each individual's needs.

Many neurasthenics are not sick enough to need sanatorium treatment and yet require measures adapted to bring their nutrition up to a maximum. Others whose health has been practically reëstablished by a few weeks' rest cure, need such measures to continue their well-being. Among such measures are the following:

Gymnastics-Diversions.-The main value of active exercise in neurasthenia is to improve the nutrition, to keep the patient from thinking too much about himself, and to fix his will power upon accomplishing something definite. All outdoor sports are beneficial. Walking, and especially walking alone a fixed period or a definite distance, is to be condemned. Most neurasthenies during such a walk think of nothing but their own unpleasant sensations. A walking trip with a congenial companion is another matter. Golf, tennis, sailing, fishing, paddling, rowing, swimming, riding, driving, climbing, shooting, gardening, forestry, building, motoring are all useful for some cases. Since motoring has become such a mania, a word of caution may be fitting in this place. Motoring as a means of stimulating the cutaneous circulation and nerves, of obtaining a maximum amount of oxygen and of securing diversion, change, and mild exercise is admirable, but the speed should not exceed 15 miles an hour and the time should be limited and only very gradually lengthened, otherwise the demands upon the nervous system will exceed its benefits. A purposeful piece of work such as building, tree-chopping, carpentry, with the necessary concentration for its accomplishment, is often of very great advantage to professional men, whose occupation is sedentary and one-sided. In such work we should avoid the necessity of too much decision, secure sufficient periods of rest, and avoid any possible overdoing.

Massage.-Massage is a valuable addition to other methods of treatment, especially during the early period of isolation so often necessary in commencing a cure. The patient's nutrition is generally improved, a soporific influence is often obtained, a troublesome insomnia relieved, and a period of complete physical and mental rest insured when the patient nearly always forgets or disregards his uncomfortable sensations and his pernicious auto-suggestions. As a rule, men do not stand massage so well or obtain so much benefit from it as women, although I have sometimes found that a more vigorous active massage, combined with active or passive movements under the direction of a trainer, will accomplish excellent results in them. Special types of massage for individual symptoms such as muscular and spinal pains, headaches, intestinal or gastric atony, need no particular discussion; their applicability and utility will occur to any physician as the occasion arises.

Electricity.-Electricity in the treatment of neurasthenia is, in my judgment, largely an adjuvant to psychic therapeutics. Much of its effect depends upon its suggestive influence. General faradization as 
employed by Weir Mitchell during the rest cure may promote nutrition; static electricity, galvanization, and high frequency currents may all act in some unknown way to favor nutrition, but I feel very atrongly that such methods are less trustworthy and certainly less undentood than the more definitely studied methods of hydrotherapy. Too much reliance upon such suggestive therapeuties will create habits of depend. ence and impair the value of the more logical methods of reasoning and control.

Hydrotherapy.- Hydrotherapy is one of the best physical agents in the treatment of almost any type of neurasthenia. Individualization is quite as necessary as in other diseases, but in general it may be stated that violent stimuli, as very cold baths or baths combined with inuch vibration, are usually contraindicated in the treatment of neurasthenia. The mildest measures are generally the most successful, and they must be employed for a long time before much benefit can be seen. Our choice will frequently be one of the following: Wet pack with friction from $80^{\circ}$ gradually lowered upon sucessive days to $60^{\circ}$, or a dripping wet pack without friction. The latter is less exciting and may be used as a preparation for the former. The half-bath with splashing, the tepid or neutral bath from $98^{\circ}$ down, and the temperate bath of $82^{\circ}$ are all sedative and tonic. A tepid bath at night lasting $30-40$ minutes often induces sleep. If followed by chilling of the surface. friction should be resorted to, and sufficient covering should be ensured after the bath. Many neurasthenies are rheumatic or arthritic and do not endure cold water treatment well. The tepid bath, the warm douche, or the Scotch douche are safer measures. A cold shower, if short, never to exceed 30 seconds, and if followed by drying and rubbing and moderate exercise, is still more active than the wet pack. Any of these hydropathic measures require continuance over a long period, several months. The morning hour immediately after wakening is the most favorable time.

For headache and vertigo a cold shower is directed against the lower half of the body only, or a Scotch douche followed later by a cold shower may prove effectual. For persistent insomnia the warm douche, $98^{\circ}-92^{\circ}$, duration 3-5 minutes, or the tepid bath, $88^{\circ}$, of brief duration, with cold compresses to the head is often advantageous. The damp pack will sometimes induce sleep when other methods have failed. Two woollen blankets are spread upon the bed, and upon them a sheet which has just been wrung out after being wet in water at $60^{\circ}$; the patient lies upon the sheet, is rapidly sprinkled with a few drops of cold water, is enveloped in the sheet, and the blankets are then wrapped about him. The duration is $15-20$ minutes.

Gastro-intestinal atony is often aided by an abdominal douche, abdominal wet packs, or mild applications of the Scoteh douche.

Among the other factors which require careful selection for the neurasthenic are the following: 
Climate.-The question of climate in neurasthenia is a complex one. The reason for the great diversity of opinion as to a suitable climate is perfectly apparent: different individuals require different climates to secure the maximum nutrition, whether they are sufferers from neurasthenia or from other diseases. A neurasthenic with a vigorous circulation will generally thrive best at a high altitude or a bracing seaside resort. A weakly neurasthenic with a feeble circulation will usually do better in a quiet inland country place. Cardiac, sexual, and arthritic neurasthenics rarely do well at an altitude much above 1000 feet. The important point to determine is the effect of the climate upon the patient's sleep and nutrition, the latter to include the appetite, digestion, and gain in weight. Wherever these factors are best provided for, is the place to select for the neurasthenic. No climatic advantage, however, can counteract such obvious dangers as poor food, poor cooking, lack of diversion, occupation, or repose. Further, we must always remember that nostalgia is with some people a real disease. No advantage to be gained from a change of climate can outweigh its injurious effect.

Diet.-The question of diet is almost as complex and individual as that of climate. Generally speaking, neurasthenics with an excessive amount of adipose tissue, especially anæmic women in young adult life or at the menopause, require thinning. Most neurasthenics, however, and especially all under-nourished individuals, improve much more rapidly as soon as they begin to put on weight. Arthritic or rheumatic cases usually improve more rapidly upon a diet low in purins, not necessarily low in proteids. Too much meat is a common fault with almost any dietary to which Americans or English are accustomed; but if we follow too closely in the footsteps of Chittenden we shall signally fail in the treatment of some types of neurasthenia. A vegetarian diet is often efficacious in cases with marked cerebral asthenia and headaches; combined with plenty of milk it is useful in arthritics and rheumatics. Alcohol should generally be tabooed; but I have often obtained marked benefit in cases with obstinate insomnia by ordering some malt liquor late in the evening or with the dinner. The patient's custom and his temperament are the most important factors in deciding abstinence or temperance in the use of wines. Coffee, tea, or tobacco in any appreciable amount are more harmful than beneficial unless, as in the case of alcohol, there is some especially good reason for their use.

Medication.--Drugs are of comparatively slight importance in the treatment of neurasthenia. When prescribed they are usually employed to fulfil one of two indications, as tonics or as sedatives.

Tonics.-Iron, arsenic, quinine, nux vomica, phosphorus, and zinc are all in common use. I have obtained better results with arsenic than with any other one drug, especially in neurasthenics with marked disturbances of the vasomotor system. The choice of the preparation is comparatively unimportant. I usually select arsenious acid in doses of $1 /{ }_{40} 0^{1 / 100}$ gr. three times a day. Hypodermic injections of cacodylate 
of soda have sometimes given me better results, but it is dificult to estimate how much depends upon the psychic impression produced by the daily visit for such treatment. I never employ iron in neurasthenia, except for the neurasthenic conditions occurring after acute infeetion, after hemorrhage, or after the puerperium where there is definite diminu. tion in the hamoglobin. A combination of bromide with iron has been recommended by many authors. Nux vomica or its alkaloid, strychnine, is particularly useful in neurasthenies with atonic conditions of the gastric or intestinal musculature. Small doses have in my hands proved quite as efficient as large ones. Hare and Fitz recommend very large doses of the tincture of nux vomica for neurasthenics with gastric atony. The prolonged use of large doses of strychnine should be condemned. I have seen many neurasthenics seriously injured by such treatment. The hypophosphites and the glycerophosphates, especially the glycerophosphate of soda, are useful. Though warmly recommended by many authors, phosphorus, zinc, and ergot have proved of little service in $\mathrm{my}$ hands.

Sedatives.-The best sedative in neurasthenia is some salt of bromine. I usually employ the sodium or the strontium salt in doses of 5-15 grains well diluted in water or milk three times a day upon a full stomach. Although bromides favor sleep-but only after at least $1 / 2-2$ hourslarger doses than this are necessary to induce much soporific effect. The addition of small doses of phenacetin is sometimes helpful, especially if the patient complains of severe pain at the base of the brain. For its hypnotic effect bromide should be employed only for a short time, with periodic interruptions. It counteracts psychical hyperasthesia, increased irritation and activity, especially in vasomotor, cardiac, and sexual neu. rasthenics, associated with much anxiety and fear. In hystero-neuras. thenia valerian is often advantageously combined with bromide. Where there is much cardiac or sexual irritation, valerian combined with monobromated camphor is highly recommended.

Hypnotics.-Veronal, sulphonal, and trional are all raluable for a brief period to break up a habit of insomnia. The combination of veronal $10 \mathrm{gr}$., trional $5 \mathrm{gr}$., or even half the dose has given me satisfaction. Where there is any danger of the patient's acquiring a habit, and such danger is constantly present in neurasthenia, I prefer amylene hydroxide, or paraldehyde in doses of $1-1 \frac{1}{2} \mathrm{dr}$. The disagreeable taste cannot be overcome, although it may be diminished by being combined with cognac or bitter orange syrup. Hamilton in 1544 recommended the fluidextract of piscidia erythrina. Von Krafft-Ebing commends it in doses of 2-3 teaspoonfuls with some syrup and considers that it acts as a psychic and cerebral analgesic. It should not, however, be used for any length of time. Extra-medicinal methods to secure sleep are in general much safer for neurasthenics than any drugs whatever.-Ed.] 


\section{INDEX}

Abclomen, puncture of

in Bright's disease, acute, 108

in cirrhosis of the liver, 529

in hydrops from broken compenation, 39 dangers, 41

Abclominal technic, to

massage, 401

puncture (see Abdomen, puncture of), 39 Abscess

peritonsillar, abortive treatment of, $27 t$ pulmonary, therapy of, 324

Absinthium, 418

Abyssinian remedy, Exibard's 308

Acacia, 287, 561

Acetopyrin, 252, 550

Acid

acetic (see Vinegar)

benzoic (see Benzoic acid)

boric (see Boric acid)

cacodylic, 176, 348

camphoric, 352

carbolic (see Carbolic acid)

chromic, 273

cinnamic, 347

citric, 136,187

hydrobromic, 273

hydrochloric (see Hydrochloric acid)

iodic, 595

lactic, 125, 257

phosphoric, 139

salicylic (see Salicylic acid)

sulphuric, 202

tannic (see Tannin)

Acid poisoning, 560

Aconite, 91, 607

Aconitine, 202

Acorn cocoa, 377, 469, 471

coffee, 377

Actol, 595

Acupuncture, 83

Acute articular rheumatism (see Rheumatism, acute articular), 544

Addison's pill, 32

Adonidine, 20

Adonis vernalis, 20

Adrenalin

in asthma, 309

in nasal hemorrhage, 174

in rhinitis, 275

in scarlet fever, 579

After-treatment

of gastric ulcer, 448

of measles, 587

of scarlet fever, 583

of tuberculosis, 361

of typhoid fever, 540

Agaricin, 352

Agathin, 547

Agurin, 29

Air baths, 296

Alboferrin, 162

Albumin water, $374,532,541$

Albuminuria

in Bright's disease, acute, 102

in chicken-pox, 588

in measles, 587

in scarlet fever, 582

Alcohol

in acute infectious diseases, 576

in Bright's disease, chronic, 112

in broken compensstion, 17

40
Alcolual

in bronehitis, capillary, 294

in bronchopneumonia, 3?

in diphtheris, 392

in kastro-intestinal dineasen, 379, 121

in infectious diveane, 876

in neursathenia, 622

in pneumonia. 315,310

in pulmonary tubereulotis, 300

in typhoid tever, 035

is uremia, 123

in urie seil diatbenis, 246

Aleobol dreswinge

in peritonitia 518

in perityphlitin, 513

in rlseumatiom, acute articular, 851

Aleoholism in pneumonis, 320

Aleurotiat breal. 221

Alkali poisoning, 500

Alkaliew

in Bright's disense, acute. 103 ehronic pareaehymatoun, 100

in diabetes mollitus, $2: 3$

in diseanes of the stomsch and istestisen 117 Carlsbad alt, 418 indications, $\$ 18$

in gastric ulcer, 443

in hypersecretion, 464

in intravenous injection, 232

in nephrolithiasis oxalurica, 135

in pyronis, 463 urica, 132

in uric acid diathesia, 247,251

Alkaline chloride waters

in bronchitis, chronio dry. 209

in diseases of the stomach and isten. tines 407

Alkaline saline waters

in constipation, 801

in diabetes, 228

in diseases of the stomach and in. teatines, $400^{\circ}$

in jaundice, 521

in oberity. 239

table of 409

Alkaline waters, nimple

in bronchitir chronie dry, 200

in dimeases of the stomach and is. testines $40^{\circ}$

in urio acid distberis $24 \%$

table of 133

Alkaloid poisoning, 563

Almond bread 224

Almonda emulsion of. 28:

Aloes, 36, 499

Aloin, 502

Alterations of the blood in seute primoning, sas

Althas, 28,

Altitude

in compensated ralvular lesions 6

Alum in tuberculoxis 332,340

in aortic aneurism. 82

in intestinal catarrh, 473

in pharyagitia 276

in pyelitis and pyelonephritia 14

Ammonia

in broken mmpenxation, 25. 3.8

in poisoning, acute 365

Ammonium chloride, KR, 290

Amabic dy rentery; 542 
Amygdalin, 523

Amygdophenin, 549

Amyl nitrite, 76, 78, 309

Amylene hydrate, 52

Anæmia

acute hemorrhagic, therapy of, 181

as a cause of obesity, 241

in chlorosis (see Chlorosis), 152

progressive pernicious (see Pernicious

anæmia, progressive), 168

secondary, therapy of, 178

accompanying tuberculosis, 180

after acute articular rheumatism, 180

after malaria, 180

balneotherapy in, 179

diet, 178

from Bright's disease, 181

from diseases of the genito-urinary tract, 181

from loss of body fluids, 179

from metal poisoning, 181

from nervous conditions, 180

from overwork, 180

from scrofula, 180

from syphilis, 180

from uric acid diathesis, 250

post-hemorrhagic, 179

Anæsthesin post-infectious, 180

in gastralgia, 460

in gastric ulcer, 445

in jaundice, catarrhal, 523

Analgen, 84, 550

Analgesic applications in pleurisy, 363

Aneurism of the aorta (see Aortic aneurism), 81

Angina

in laryngitis, 279

in pharyngitis, 276

in scarlet fever, 578

in tonsillitis, 277

Angina pectoris, therapy of, 77

causal and prophylactic therapy, 77 therapy of an attack, 77 antineuralgics, 80

chloral, 78

chloroform, 78

erythrol tetanitrate, 79

nitrites, 78

nitroglycerin, 78

revulsives, 81

theobromine, 80

Anise, 428

Ankylostomiasis, 509 thymol, 509

Anorexia

in chlorosis, 153

in tuberculosis, 359

Antidotes

for acids, 560

for alkalies, 560

for alkaloids, 563

for arsenic, 562

for carbolic acid 563

for cyanides, 563

for halogens, 560

bromine, 560

chlorine, 560

iodine, 560

for metals, 562

for phosphorus, 561

Antifebrin

in aortic aneurism, 84

in tuberculosis, 350

Antifermentatives

in lithiasis phosphaturica, 139

in tuberculosis, 361

Antimony, 291, 610

Antinervine, 80, 84

Antineuralgics

in angina pectoris, 80

in aortic aneurism 84

in influenza 544

in uræmia, 126

Antipyresis (see Antipyretics and Antipyretic hydrotherapy)
Antipyretic hydrotherapy

baths

in bronchitis, 291

in infectious diseases, 572

in pneumonia, $313,316,317$

in scarlet fever, 577

in typhoid fever, 535, 536

half baths

in infectious diseases, 573 packs

in bronchitis, 292

in infectious diseases, 571

in tuberculosis, 351

in typhoid fever, 536

rubs in infectious diseases, 571 spongings

in infectious diseases, 571

in pneumonia, 317

in scarlet fever, 577

in tuberculosis, 351

in typhoid fever, 536

Antipyretics

in infectious diseases, 569

in influenza, 544

in pneumonia, 316

in tuberculosis, 350

in typhoid fever 537

Antipyrin

in angina pectoris, 80

in aortic aneurism, 84

in diabetes insipidus, 234

in gout, acute, 252

in influenza, 544

in rheumatism, acute articular 549

in tuberculosis, 350

in whooping-cough, 604

Antisepsis of the mouth

under pathological conditions, 270

in diabetes, 230

in measles, 584

in scarlet fever, 578

under physiological conditions, 267

Antiseptics

in bronchitis, putrid, 304

in cystitis, chronic, 148

in diphtheria 594

in pyelitis and pyelonephritis, 142

in whooping-cough, 601

intestinal, 424

$\beta$-naphthol compounds, 426

bismuth, 424

calomel, 424

creosote, 424

ichthalbin, 427

ichthoform, 427

menthol, 427

naphthol compounds, 425

resorcin, 426

salicylic acid, 425

yeast, 427

stomach, 423

$\beta$-naphthol, 423

biogen, 423

hopogan, 423

hydrochloric acid, 423

menthol, 423

permanganese, 423

peroxide of magnesia 423

salicylic acid, 423

Antispasmin, 605

Antispasmodics in whooping-cough, 606

Antitoxin unit 593

Antitoxins

diphtheria (see Diphtheritic antitoxin), 592

Kruse's serum, 542

tetanus, 555

Antitussin, 603

Anus itching of, 509

Aortic aneurism, therapy of, 81

diet, 81

Tufnell's diet scheme, 82

local cold, 84

medication, 82

rest, 81

surgery, 83 


\section{INDEX}

Aortic aneurism, surgery

acupuncture, 83

compression, 83

galvanopuncture, 83

introduction of a foreign boily, 83

ligation of carotid and subelavian sympries, 84

symptomatic therapy, 84 dyspna.a, 8.5

Apocodeine, 502 pain, 84

Apocynum, 23, 529

A pomorphine

in bronchitis, acute deep and capillary, 294

in diphtheria, 599

in poisoning, acute, 559

in pulmonary stasis, 58

in tracheobronchitis, 290

Appendicitis (see Perityphlitis), 511

analgesic

in gout, acute, 25 !

in pleurisy, 363

in rheumatism, chronic, 26.5

cold (see also Ice-bag and Leiter's coils).

in aortic aneurism, 84

in arteriosclerosis, 77 in compensated symptomatic heart le-

in congestion of the liver, 65

in diseases of the stomach and intes-

in endo, 405

ditis, 89

in peritonitis, 518

in perityphlitis, 513

in rheumatism, chronic, 264

hot

in typhoid fever, 540

in arteriosclerosis, 77

in compensated symptomatic heart lesions, 72

in cystitis, 147

in diseases of the stomach and intestines, 405

in pericarditis, 90

in peritonitis, 518

in perityphlitis, 513

in renal pain, i40

Priessnitz's (see Priessnitz's application)

Aqua

bromata, 201

laurocerasi, 60

Arenaria in nephrolithiasis, 137

Aristoquin

in infectious diseases, 570

in malaria, 553

in whooping-cough, 604

Arsenic

administration, 169, 174

hypodermically, 171, 175

in arteriosclerosis, 76

in asthma, 310

in chlorosis, 167

in compensated valvular lesions, 10

in diabetes, 228

in endocarditis, 90

in leukremia and pseudoleukamia, 174

in neurasthenia, 622

in progressive pernicious antemia, 169

in scrofula, 195

in secondary anmmias, 179

in tuberculosis, 348

poisoning, 169, 562

preparations, 169, 174 310

waters, 170,501

table of, 170

with iron. 167

Arsenious acid, 174

Arteriosclerosis, therapy of, 73

baths, 74

causal therapy, 73

cerebral symptoms, 77

diet, 74

Arterioselesowin, t/serapy of hydrotherspy, 78 in obeeity. 240

in urie scil disthenis, 250

medieation, is

armenic, is

iodiules, is

nitrites, 76

Trupecek'- serum,

Arteritis

mineral waters is

elsronic, 73

syphilitie, therapy of, 81

iodides, 81

mereury, 8

Arthralgia following diphtheritle astitoxin, 294

Arthritis (nee Itheumation, acute artieular), ist

in scarlet fever, 381

cold, s\&!

immobilization, 881

Artificial valicylatea, \$81

mineral waters in sephrolithiavis urica, 13 respiration, 860

Analcrida, 429

Asaprol, 605

Ascaris lumbricoides, therapy of, 507

antonin, 308

Aspidium in tapeworm, 800

Aspirin an an antipyretic, 5\%0

in influen za. 344

in rheumatiwm, acute articular, 54

Asthma

bronchial. therapy of, 300

etiologieal therapy, 306

treatment between attacks, 300

arsenic, $\mathbf{3 1 0}$

atropine, 310

elimate, 311

potaveium iodide, 300

treatment of an attack. 30 ?

adrenalin efuloride, 300

belladonns, $30 \%$

cannabiv indica, 308

chloral, 30:

cigarettes 30 :

diphtheritie merum, 300

grindelia, 307

hot foot bath, 300

hot packs, 310

hyoseyamus 30 8

morphine, 307

uraemic, 125

stramonium, 307

Astringents

in cystitis, 149

in intestinal catarrh, 472

in pyelitis and pyelonephritis, 143

Asycodyle, 348

Atheroma, cerebral symptoms in. 77

Atony of the stomach, therapy of (Nee Masieal

Atropine insufficiency), 45 in chlorusis 153

in asthma, 310

in broken compenation, 17, 59

in cholelithiasia $\mathbf{5 2 4}$

in compensated. symptomatic beart lenions

in gastric ulces, ths

in leus, ncute

in osteomalacia, 258

in poisoning, acule, 567

in renal colic, $1+1$

in rhinitis 274,275

in tuberculoxis 35 ?

Automaerace, 402

Autotransfusion, 181

Bacteriolocieal diamnovis in diphtheria, 503

Baking in chronic rheumatism, 263 
Balneotherapy

in anæmia, secondary, 179

in bronchitis, chronic, dry, 299

in cirrhosis of the liver, 528

in diabetes mellitus, 228

in emphysema of the lungs, 327

in exophthalmic goitre, 201

in interstitial nephritis, chronic, 121

in leukæmia and pseudoleukæmia, 177

in obesity, 239

in osteomalacia, 258

in rheumatism, chronic, 259

in rickets, 255

in scrofula, 191

Balsam

in uric acid diathesis, 248

of copaiba, 149, 301

of Peru, 58, 280, 301

of tolu, 58, 301

Bandaging

in gastroptosis, 457

in mechanical insufficiency of the stomach, 455

in movable kidney, 147

in peritonitis, tuberculous, 518

in rheumatism, acute articular, 551

Banting's treatment of obesity, 237

Basedow's disease (see Exophthalmic goitre), 199

Basham's mixture, 160

Basicin, 604

Baths.

air, 296

aromatic, 258

bicarbonate of soda, 522

bran, 258

brine, 171

carbonated

in diabetes, 226

in hydrops in broken compensation 43

in pernicious anæmia, 171

in rheumatism, chronic, 260

clover, 522

cool

in infectious diseases, 572

cold

in typhoid fever, 535

in infectious diseases, 572

in pneumonia, 313,316

in scarlet fever, 577

in typhoid fever, 536

electric light, 107

Fango, 263

half

in gastric neuroses, 459

in infectious diseases, 573

hot

in neurasthenia, 621

in Bright's disease, acute, 106

chronic, 116

in hydrops in broken compensation, 42

in pneumonia, 318

in renal colic, 141

in rheumatism, chronic, 259, 261

hot air

n scarlet fever, 582

in Bright's disease, acute, 107

in hydrops in broken compensation. 43

in rheumatism, chronic, 262

in anæmia, 171, 179

in arteriosclerosis, 74

in Bright's disease, acute, 106

chronic, 116

in bronchitis, 291

in bronchopneumonia, 321

in chlorosis, 162

in compensated heart lesions, 7

in cystitis, 148

in diabetes, 226

in diseases of the stomach and intestines, 404 in hydrops in broken compensation, 42

in infectious diseases, 572

in interstitial nephritis, chronic, 121

in jaundice, catarrhal, 522
Baths

in leukæmia and pseudoleukæmia, 177

in measles, 586

in nephrolithiasis urica, 132

in neurasthenia, 621

in obesity, 240

in osteomalacia, 258

in pneumonia, $313,316,318$

in rheumatism, acute articular, 550 chronic, 259

in rickets, 255

in scarlet fever, $577,581,582$

in scrofula, 191

in typhoid fever, 535

in uric acid diathesis, 249

iron, 162, 260

malt, 258

mud, 165, 260

mustard, 273, 293

"mutterlauge," 171

pine-needles, 226,258

Roman-Irish, 262

salt

in anæmia, 171, 179

in diabetes, 226

in hydrops in broken compensation, 43

in osteomalacia, 258

in rheumatism, chronic, 260

in rickets, 255

in scrofula, 192

salt water, 191

sand, 258, 263

sea, 177,191

sitz, 404, 491

sponge

in infectious diseases, 571

in pneumonia, 317

in scarlet fever, 577

in tuberculosis, 351

in typhoid fever, 536

steam-cabinet

in Bright's disease, acute, 107 in diphtheria, 598

in hydrops in broken compensation, 42

sulphur, 26

in rheumatism, chronic, 262

sun, 262

sweat, 107

tepid

in Bright's disease acute, 106 chronic, 116

in bronchitis, 291

in cystitis, 148

in diabetes, 226

in jaundice, catarrhal, 522

in leukæmia and pseudoleukæmia, 177

in osteomalacia, 258

in pneumonia, 317

in rheumatism, chronic, 260, 262

Bedsores in typhoid fever, 539

Beef-tea, 372

Beer

fungi, 379

in Bright's disease, chronic, 112

in diseases of the stomach and intestines, 379

"Kraft bier," 374

malt, 112

Belching, nervous, therapy of (see Eructation, nervous), 461

Belladonna

in asthma, 307

in cholelithiasis, 524

in diabetes, 227

in diarrhœea, 503

in gastralgia, 460

in gastric ulcer, 443,445

in gout, acute, 251

in hypersecretion, 465

in intestinal catarrh, 474

in pulmonary stasis, 59

in renal pain, 140

in tracheobronchitis, 286

in whooping-cough, 606 


\section{INDEX}

Benzoic acid

as a mouth wash, 260

in bronchitir, purulent, $30^{\circ}$

in nephrolithiamis urica, 136

in pyelitis and pyelonephritin, 143

in tracheobronchitin, 291

Benzoin, tincture of

in laryngitis, 270

in tracheobronchitix, 28;

Benzol, 605

in tuberculosiy of the larynx, 280

Benzonaphthol, 426

Betol, 426

Bicarbonate of soda (see Sodium bicarbonate)

Bichloride of mercury

$$
\begin{aligned}
& \text { as a disinfectant, } 574 \\
& \text { in diphtheria, } 592,595 \\
& \text { in endocarditis, 88 } \\
& \text { in tonsillitis, } 277
\end{aligned}
$$

Bier's elastic bandages in rheumatism, acute articular, 5.50

Bile salts, 522

Biliary ducts, diseases of, therapy of (nee liver and gall passages), 520

Binding of extremities in hemorrhage in tuberculosis, 357

Biogen, 423

Birch leaves, 106

Bismuth oil of, 143
as an intestinal antiseptic, 424
in diarrhoea, 503
in gastric ulcer, 443
in intestinal catarrh, 473
in intestinal ulcer, 479
preparations, 424

Bitter waters

in congestion of the liver,

in constipation, 500

in diserses of the stomach and intestines, 411

Bitters, 418

table of, 414

Blackberry brandy, 474

Bladder

emptying of, by compression, 150

irrigation of, 150

paralysis of, 150

stone, 138

Blaud's pills, 159

Bleeders, 184

Blood, diseases of the, therapy of, 152 ansmia hemorrhagic acute, 181 pernicious, 168 secondary, 178

chlorosis, 152

hæmophilia, 184

leukamia and pseudoleuksmia, 174

purpura hemorrhagica, 185

scurvy, 186

Bloodletting.

plethora, 183

general, 6

local, 55

Blood-vessels, diseases of the, therapy of, 72 aneurism of the aorta, 81 arteritis, 73

chronic, 73

syphilitic, 81

arteriosclerosis, 73

B-naphthol, 426

Bone marrow

in osteomalacia, 257

in pernicious anæmia, 171

in rickets, 257

Borax, 273

Boric acid

in chicken-pox, 588

in cystitis, 150

in measles, 587

Borlanolin, 588

Bothriocephalus (see Tapeworm) 500

Bovinine, 372

Brandy in night-sweats, 351 is read

aleuronat, 224, 37\%

almond. 224

digeatibility of, 378

eraliaen, 183

roborat, 224

mubetitutes for, in disbetes 21

Breathing

artificial, 860

belt, 327

chair, 32 -

exereive

in pleuriay, 364, 300

in culvereulonia 343

reflex mimulation of s6:

Bright's dimease, therapy of

acute, 0 S

bathe and paeks, 100

causal therapy. os

diaphoresin 107 pilocarpise $10^{\circ}$

diet 100

kefir, 101

koumins. 101

thatsoon, 101

milk, 100

Cistnet's latemilk, 10

druks contraindicated, os

medication, 102

alkalies 103

diuretice 105

tannin, 102

mineral waters 104

oliksuria, 104

puneture, 108

purging 108

relief of pain, $\infty$

chronic. 108

alcohol, 112

bathy 116

diet, 100

eress 111

(a), 111

meat, 110

milk, 100

reduction of salt, 113

effect on therapy of pheumonia 319

hygiene, 116

medication, 117

recondary anstnia in, 181

symptomatic therapy. 118

Brine baths, 17

Broken cotmpensation, valvular diseaces is the

ntage of 11

applications of cold, 12

diet. 11

heart stimulants (Neart stimulasts) 12

hydrops and venous conkrstion, 26 congestion ( Congestion of the viscera) 47

hydrops (sellidrops) 27

morphine 68

rest. 11

Bromelin, 123

Bromidia, 54

Bromides

in exophthalmic goitre, 201

in insomnia, 48

in neurasthenia, 623

Siandow's, 201

Bromine poisoning. 560

Bromipin, 49

Bromocoli. 523

Bromoform

in gastralgia, 46!

in whooping-mugh, cos

Bronchi, diveases of the. therapy of. 25 acute bronchitia $2 \times 3$ acute deep bronchitis and capillary bronchitia 291 acute iracheobronchitia $2 \times 3$ bronchial anthma 306 chronic broschitis 29 ? 
Bronchi, diseases of the, therapy of

dry catarrhal, 297

in emphysema of the lungs, 327

fibrinous, 306

purulent, 300

putrid, 303

Bronchitis, therapy of, 283

acute deep and capillary, 29

alcohol, 294

diet, 293

expectorants, 294

apomorphine, 294

ipecac, 294

senega, 294

hydrotherapy, 291, 292

baths, hot, 293 mustard, 293 tepid, 291

packs, cold, 229 mustard, 293

position, 294

prophylaxis, 295

climate, 297

clothing, 297

hardening process, 295

in bronchitis, from abnormal perspiration, 296

in reflex bronchitis, 296

relief of pain, 295

steam in room, 294

chronic, 297

dry catarrhal, 297

climate, 298

compresses

crossbinder, 298

Priessnitz's, 298

inhalations of steam, 297

medication, 297

mineral waters, 299

at home, 299

at spas, 299

pneumatic chamber, 300

purulent, 300

balsams, 301

climate, 303

diet, 303

grape cure, 303

milk, 303

whey cure, 303

ethereal oils 300

expectorants, 302

mineral waters, 302

putrid and bronchiectasis, 303

expulsion of sputum, 305

internal antiseptics, 304 eucalyptus, $30-4$ myrtol, 304 turpentine, 304

local antiseptics, 305 oil of eucalyptus, 305

operation, 306 oil of pine, 305

fibrinous, 306

in measles, 586

in pulmonary stasis in broken compensation, 58

Broncho-pneumonia, 321

acute, 321

$$
\text { heart stimulants, } 321
$$

hydrotherapy, 321

baths, 321

cold compresses 321

hygiene, 322

medication, 322

chronic, 322

Broths 372

Butter in cholelithiasis, 526

Buttermilk, 100

Cacao l'avosie, 113

Cacodylic acid

in leukæmia, 176

in tuberculosis, 348
Cade, oil of, 143

Caffeine

in asthma, 309

in Bright's disease, acute, 105

in broken compensation, 21

administration, 21

toxic symptoms, 21

in hydrops in broken compensation, 34

in measles, 586

in poisoning, acute, 564

in uræmia, 123

Calcium

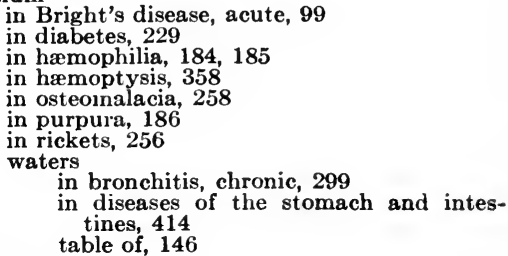
table of, 146

Calomel

as a carminative, 429

as an intestinal antiseptic, 424

in cirrhosis of liver, 528

in constipation, 499

in dysentery, 541, 543

in gastritis, acute, 433

in hydrops in broken compensation, 29 administration. 30 contraindications, 30

in intestinal catarrh, 469

in typhoid fever, 538

in uræmic vomiting, 124

Calories

number furnished by the different food elements, 208

number needed by body, 208

Calumba root, 587

Camphor

as a heart stimulant, $24,123,127,585$

in hydrops in broken compensation, 34

in pleurisy, 363

monobromate, 201

Camphor in ether, 565

Camphoric acid in tuberculosis, 352

Cancer of the stomach, 449

artificial ferments, 451

diet, 450

lavage, 452

mineral waters, 451

surgery, 449

symptomatic treatment, 452

constipation, 452

hæmatemesis, 452

pain, 452

Cannabis indica vomiting, 452

in asthma, 308

in insomnia, 54

Cantharides plaster, 364

Capsules, glutoid, 428

Carbohydrates

foods containing no, 217

foods containing small amounts of, 219

heat value, 208

in diabetes

reduction of, $218,222,224$

substitution values among, 218

in obesity, 234, 239

in uric acid diathesis, 245

Carbolic acid

as an antiseptic in whooping-cough, 602

as a disinfectant, 574 in diphtheria, 595

in scarlet fever, 578

dressings in tetanus 556 injections of

in rheumatism, acute articular, 550

in rhinitis, 274

in scarlet fever, 578,579

poisoning, 563 
Carbon (xec Charcoal)

Carbonated

baths

in diabetes, 226

in hydrops, 43

in pernicious anamia, $1: 1$

waters

in rheumatisn, chronic, 260

in constipation, 480

in diseases of the stomach and intewtines, 406

Carcinoma of the stomach (see Cancer of the stomach), 449

Cardiac insufficiency

in diphtheria, 600

in poisoning, acute, 564

Cardiac muscle, diseases of, therapy of, $x$; fatty heart, 85

idiopathic enlargement of the heart, 86

Carlsbad myocurditis, 87

salts

in constipation, 498

in diseases of the stomach and intentines, 418

water, 407

amount, 410

effect, 407

in cholelithiasis, 525

in nephrolithiasis urica, 132

indications for, 410

Carminatives

in flatulency, 504

in diseases of the stomach and intestines, 428

list of, 428

anise, 428

anthemis, 428

asafotida, 4:29

burnt magnesia, 429

calomel, 429

charcoal, 429

peppermint, 428

salicylate of magnesia, 429

Carniferrin, 16

Cascara sagrada, 495

Cascarilla, 419

Cascarine, 495

Castor oil

in constipation, 498

in diarrhœe, 502

in dysentery, 541,543

in gastritis, acute, 433

in intestinal catarrh, 469,470

in intestinal ulcer, 479

Catarrh with salol, 470

chronic, after measles, 586

in uric acid diathesis, 250

intestinal (see Intestinal catarrh), 468, 470

Catarrhal jaundice (see Jaundice, catsrhal), 520

Catechu, 144

Cathartics

classification of, 429

laxatives, 429

purges, 430

hypodermic injections of, 501

in broken compensation, 35

in constipation, 494

in flatulency, 504

in gastritis, acute, 433

in ileus, acute, 482

in intestinal catarrh acute, 468 chronic, $4{ }^{2}$

in intestinal stenosis, 481

in jaundice, catarrhal, 520

in obesity, 240

in poisoning, acute, 559

Catheterization, 150

Cautery (see Paquelin cautery)

Cereals, 101, 441

Cerebral

hyperæmia, 47
Cerebral oymotome

its arteruaclerusio, :-

in cotspensated os ruptcotnatue valvular Leavorn. 6

in congestion is brokes cuengertative 48

in polmating, neule, ill?

in typhoil fever, 830

in urarmia. 120

Cerium, 12t

Chambard teas s(y)

Chamornile, 431

Charcual

in diseramen of the oturnach ami intenotusene, 430 in tutnerculowe, 36 ]

Cluaruue, 373

Cliewing lozenges, 1:4, $2 \% 0$

Chickert-pux therapy of sas

allutuinuris, phas

efllorencence in the musth, owh

fever, ass

itching ant

Chinolin, $(2)^{\prime}$

Chinosol, 3it. 303

Chinotrupin, :-4.s

Chloral

as an hypnotic, 4.8

in angina pectoris, 78

in asthma, $30 \%$

in convulaions in scarlet fever, bes

in gastritis seute, 434

in larsngeal mpanti, 281

in pleurisy. 33

in pneumonia. 3:0

in renal colic. 141

in uramia, contraimlicatums to, 130

in whooping-coush, (d)

Chloralarnid

in authma, 309

in incornnia. 54

in pulmonary mearis, 60

Chloralous: 52

Chlorine poimoning. seo

Chloroforin

in angina pectoris, 78

in convulwons in searlet fever. $3 \mathrm{a}$

in diseases of the stomach and intertines 40 in gout, scute, 251

in laryngeal spasm, 281

in pleurisy, 363

in renal colic, 141

in rheumatism, chronic, 26.5

in tetanus, $35 \%$

in uremic convulsions, 128

in uremic vorniting. 124

in whooping-cough, 60

water

in compenated. symptomatic valvular leaions. 72

in dineases of the stomach and intestimes. 430

in uramic vomiting. 124

Chlorosis, therapy of, IS?

climate. 135

diet, 154

digentive diaturbancm $132-153$

hydrotherapy; ISE

baths containing iros, in?

rubs. 156

marriage, 157

medication

arsenic, 168

iron, 15:

alministration. 13:

mineral waters containing. IfS

preparationa 158

inoneanic. 130

oncanie. ica

quinine, $16 \%$

mineral water 163

pies chlorotica is3

rest, 135

venerection. 169

Chocolate

"Kraftochomlarle." 113, 243, 25a 378

Sanatogen, 375 
Cholagogues, 522

Cholelithiasis, therapy of, 524 acute attack, 524

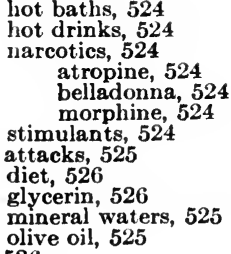

$$
\text { olive oil, } 525
$$

\section{Chromic acid, 273}

Chronic interstitial nephritis, therapy of (see Nephritis, chronic interstitial, therapy of), 118

Cider, 112

Cigarettes in asthma, 307

Cinchona, 419

Cinnamic acid in tuberculosis, 347

Circulatory system, diseases of, therapy of, 1 diseases of the blood vessels, 72 of the cardiac muscle, 85 endocarditis, 87

neuroses of the heart, 93 pericarditis, 90 valvular lesions, 1

Cirrhosis of the liver, therapy of (see Liver, cirrhosis of the, therapy of), 528

Citarin

in gout, acute, 253

in uric acid diathesis, 248

Citric acid, 136, 187

Citrophen, 605

Climate

classification, 332

inland, $\mathbf{3 3 2}$

high altitudes, 332

low altitudes, 337

moderately high altitudes, 335

sea, 338

on the coast and islands, 338

cold and damp, 338

cold and dry, 338

moderately cold and damp, 340

moderately warm and damp, 340

warm and damp, 339

warm and dry, 339

in asthma, 311

on ship-board, 338

in Bright's disease, chronic, 116

in bronchitis, acute, 297

chronic, dry, 298

purulent, 303

in chlorosis, 155

in cystitis, chronic, 148

in diabetes, 226

in exophthalmic goitre, 199

in hæmophilia, 184

in nephritis, chronic interstitial, 120

in nervous dyspepsia, 467

in neurasthenia, 622

in pernicious anæmia, 171

in pleurisy, 368

in pulmonary emphysema, 327

in rickets, 255

in scrofula, 190

in stomach and intestines, diseases of, 415

in tuberculosis, 332,340

in valvular lesions, compensated, 7

Clothing

in bronchitis, 297

in diabetes, 226

in intestinal catarrh, 476

in nephritis, 116, 120

in rheumatism, chronic, 265

in tuberculosis, 342

in whooping-cough, 614

Coca, 523
Cocaine

in aortic aneurism, 84

in compensated, symptomatic heart lesions, 72

in cystitis, 148

in gastritis, acute, 434

in pharyngitis, 276

in pleurisy, 363

in rhinitis, 275

Cochineal, 610

Cocoa

acorn, $377,469,471$

eucasin, 113, 378

oat, 378

plasmon, 113

Codeine

in diabetes, 227

in gastralgia, 460

in gastritis, acute, 434

in insomnia, 53

in laryngitis, 278

in measles, 585

in pulmonary congestion, 59

in tracheobronchitis, 285

in typhoid fever, 539

Cod-liver oil
American preparations of, 194
in osteomalacia, 257
in rickets, 256
in scrofula, 189
in tuberculosis, 349

Coffee, 380,564

bean, 576

malt, 576

Cohnheim's oil treatment in gastric ulcer, 456

Colchicine, 252

Colchicum

in gout, acute, 252

in rheumatism, acute, articular, 550

Colchi-sal, 252

Cold, local (see Local cold and Applications)

Colic

intestinal (see Intestinal colic), 505

renal (see Renal colic), 141

Collargol, 88

Colocynth

in constipation, 500

in diseases of the stomach and intestines, 430

in hydrops in broken compensation, 37

Colon irrigations
in constipation, 492
in dysentery, 541,543
in fiatulency, 504
in ileus, acute, 486
in intestinal catarrh acute, 469
chronic, 472

in intestinal ulcer, 479

in membranous enteritis, 478

in meteorism, 504

in perityphlitis, 512

in rheumatism, chronic, 265

in typhoid fever, 540

in uræmia, 127

technic of, 396, 493

Coma, diabetic, 232

Common salt (see Salt)

Compensated symptomatic valvular lesions, therapy of, 68 cerebral symptoms, 69 dyspepsia, painful, 71 palpitation, 71

respiratory symptoms, 69

Compensated valvular lesions, therapy of, 1 baths, 7

climate, 6

diet, 2

reduction of fluids, 3

salt-free diet, 4

exercise, 4

Oertel's terraincur, 5

Swedish movements, 5

Zander's gymnastics, 5

massage, 6

medication, 9 


\section{INDEX}

Compensated valvular lesions, therapy of tobacco, 4

Composite licorice powder, 490

Compresses (see A pplications)

Condiments, effect on digestion, 37x, 4:1

Condurango, 420

Congestion of the viscera in broken compenmation. therapy of, 47

brain (nee Passive cerebral hyperamia), 47

intestines, 67

kidneys, 67

liver, 64

causal therapy, is

diet, $6 t^{3}$

laxatives, 66

mineral water\%, 65

symptomatic therapy, the

lungs (seet Pulmonary mtasin), 87

Conjunctivitis in measles, 584

Constipation, therapy of, 488 atonic, 489

colon irrigations, 492
diet, 489
electrotherapy, 491
feces, removal of, 491

feces, removal of 491

gymnastics, 491

habits, 490

hydrotherapy, 49

massage, 491

medication

cathartics, 494

laxatives

aloes, 499

calomel, 499

Carlsbad salts, 498

cascara, 495

castor oil, 498 compound licorice powder,

frangula, 495

podophyllin, 497

purgen, 498

rhubarb, 495

salines, 499

Seidlitz powder, 498

senna, 499

sulphur, 496

tamarind, 498

purges, 500

colocynth, 500

croton oil, 500

gamboge, 500

jalap, 500

hypodermic, 501

apocodeine, 502

mineral waters, 500

alkaline saline, 501

bitter, 500

common salt, 501

iron and arsenic 501

sulphur, 501

oil enemas, 494

in cancer of the stomach, 452

in true plethora, 183

spastic, 489

Convallaria, 19

Convulsions

in poisoning, acute, 56.8

in scarlet fever, 583

in whooping-cough, 611

Cooling apparratus (see Ice-bag and Ieiter's coils)

Copper

carbonate, 56 i

in tuberculosis, 348

sulphate

as an antidote in phosplorus poisoning, as an emetic in acute poisoning, 5.59

Cordol, 53

Corrosive sublimate (see Bichloride of mercury:

Cortex condurango, 420

Coryza, therapy of (see Rhinitis), 273
Cough, therapy of

is bropehitio

acute, 298

etarubic :27

in Laryngitis, 278

in mesales, 685

in pulmonary stavis is breter ewespone. tvot, 88

in tracheobronetisin, 254, 2n:

in tuberculons, ars

Crucked lipe, 2:?

Cruline, 123

Creatn of tartar, 105

Creolin, 874

Creonotal, 346

Creusote

as an intestinal antineptir, 124

in serofula 180

in tubereulosia 34

in uramie vornisine. 12

in whooping-cough, 6006

Cretinism (ee Myxumlems). 197

Crisis in preumonia, 313

Crosmbinder

in bronchitis, elinonie dry, 295

in traebeubrusehitio, 259

in tuberculonis, 354

Cruton oil

in econstipation, 800

in diveares of the stomerh and inter. tines, 430

Croup. laryngeal, therapy of (oee lannerel spasm), 28

Crystallose, 224

Cuppine

in pleurisy, 363

in venous congestion is trokes comprose. tion, 5.5

technie

dry, 3.5

wet, 36

Cure

grape, 303, 390

lemon, 136

Ieube's ulcer, 354

milk, 303

ontmeal, 233

Oertel's terrain, 5 , s:

potato, 233

Sehroth's, 366

Weir Mitchell': reet, 14:, 243, 36

whey, 303, 390

Cyanide poisoning. 563

Cistitis, therapy of, 145

acute, 147

diet, 1t:

hot appliestions, 14:

local treatment, Its

mineral waters 145

nareotics, 148

chronie, 145

antimeptice, 148

astringents, 140

eatheterization, 130

diet, 148

irrigation of the bladiler, 1 :0

mineral water 148

paralywis of the hladiles, 130

silver nitmate, iso

Cywtoplegia, therapy of (nee Paralywis of the bladder), 150

Decaleifying agents, $1 \mathrm{sh}$

Decapsulation of the kilney in urstria, 125

Delinum, 313

Delirium iretnens, 320

Dermatol, 597

Dexirose. 33

Diabeter therapy of, 206

insipidur 233

medication, 233

miluction of fuinte 23

mellitus 206

as a complication of obesity. 241 
Diabetes, mellitus climate, 226

coma, 232

complications, 229

furunculosis, 226

gangrene, 231

gastro-intestinal disturbances, 231

gout, 232

heart, liver, and kidneys, affections of, 231

neuralgia, 231

obesity, 232

stomatitis, 230

tuberculosis, 231

dietetic therapy, 215

amount of liquids, 224

carbohydrates, tolerance for, 215 test diet, 215 three grades of, 216

mild form, 217

foods allowed, 217, 219

foods forbidden, 217

foods limited, 217

initial test diet, 217

maximum amount of carbohydrates, 218

milk, 220

substitution of different carbohydrates, 218

vegetables and fruit, 221

moderately severe, 222

fat, 223

reduction of carbohydrates, 222

severe, 224

substitutes for bread and sugar, 224

effect on therapy of pneumonia, 319

essential nature of, 207

etiological therapy, 226

nervous, 226

pancreatic without syphilis, 226

syphilitic, 226

exercise, 225

hygiene, 226

care of the mouth, 230

care of the skin, 226

clothing, 226

in uric acid diathesis 251

massage, 225

medication, 227

alkalies, 227

alkaline saline waters, 228

arsenic, 228

calcium, 229

codeine, 227

opium, 227

salicylic acid, 229, 231

mental rest, 225

Diabetic coma, 232

Diaphoresis

in Bright's disease, acute, 107

in broken compensation, 37

in diphtheria, 598

in influenza, 544

in interstitial nephritis, chronic, 122

in laryngitis, 278

in pleurisy

acute fibrinous, 364

with effusion, 365

in scarlet fever, 582

in tracheobronchitis, 283

in uræmia, 122

Diaphoretic teas, 283

Diarrhcea, therapy of, 502

caused by intestinal contents, 502 diet, 503

etiology 502

in typhoid fever, 539

in uræmia, 125

nervous, 503

diet, 503

hygiene, 503

Diathesis medication, 503

scrofulous, 187

uric acid, 244
Diet

general principles of

choice between fats, carbohydrates, and proteids, 208

digestibility of different foods, 370

transition from easily digestible

foods to those more difficult of digestion (Leube's ulcer cure), 385

number of meals, 210

principles underlying the science of human nutrition (see Nutrition, principles of), 207

qualitative analysis of different foods 210

quantity of food normally required 209 reduction in obesity, 237

rest after eating, 381

specimen daily diet, 214

in obesity, 239

temperature of food, 381

in anacidity, 392

in anæmia, secondary, 178

in angina pectoris, 81

in aortic aneurism, 81

in appendicitis, 511

in arteriosclerosis, 74

in atony and dilatation of the stomach, 393

in Bright's disease acute, 100 chronic, 109

in bronchitis, deep and capillary, 293

in cancer of the stomach, 450

in chlorosis, 154

in cholelithiasis, 526

in cirrhosis of the liver, 528

in congestion of the liver, 66

in constipation, atonic, 489

in cystitis

acute, 147

chronic, 148

in diabetes, 215

mild, 217

moderately severe, 222

severe, 224

test diet, 215

in diarrhœa, 503

in diphtheria, 592

in diseases of the stomach and intestines, 370

in dysentery, 540

in emaciation, 242

in exophthalmic goitre, 199

in fatty heart, 85

in flatulency, 505

in gastric ulcer, 440

in gastritis

acute, 432

chronic, 436

in gastroptosis, 457

in gout

acute, 253

chronic, 244

in hæmophilia, 184

in hæmoptysis, 358

in hyperacidity, 390, 463

in hyperæmia of the liver, 66

in hypoacidity, 392,465

in infectious diseases, $\mathbf{5 7 5}$

in influenza, 544

in interstitial nephritis, 119

in intestinal catarrh acute, 469 chronic, 470

in intestinal stenosis, chronic, 481

in intestinal ulcer, 479

in jaundice, catarrhal, 520

in leukæmia and pseudoleukæmia, 177

in lithiasis phosphaturica, 140

in malaria, 554

in measles, 584

in mechanical insufficiency of the stomach, 393,453

in membranous enteritis, 477

in mumps, 589

in myxœedema, 199 


\section{INDEX}

Diet

in nephrolithiaxis oxalurica, 13; urica, 130

in nervous dyspepsia, t6?

in neurasthenia, 62:

in obesity, 234

in peritonitix, 517

in perityphlitis, 311

in pernicious anamia, 100

in phosphaturia, 254

in pleurisy with effunion, 3 ths

in pneumonia, 314

in pulmonary emphysema, 328 tuberculonis, $3: 0$

in pyelitis and pyelonephritin, 14

in rheumatism, acute srticular, 351

in rickets, 255

in scarlet fever, 581

in scrofula, 188

in scurvy, 186

in typhoid fever, 531

in uraemia, 127

in urio acid diathesis, 244

in valvular heart lesions cornpensaterl, 2

uncompensaterl, II

in whooping-cougl, 612

Difluordiphenyl, 603

Digalen, 15

Digestibility of different foods, 3r0

alcohol, 379

bread, 377

condiments, 378

eggs, 374

farinaceous foods, 370

fats, 378

fruit, 376

gelatin, 374

liquids, 378

meat, 370

broths, 372

extracts, 372

juices, 372

peptones, 373

soup 372

milk, 375

mineral waters, 380

nuts, 377

sugar, 377

tea and coffee, 380

vegetables, 376

Digestion, time required for, 382

Digestive disturbances

as a symptom of congestion, 67

in pneumonia, 319

in tuberculosis, 359

anorexia, 359

intestinal disturbances. 36

nervous dyspepsia, 3.5

in uric acid diathesis, 240

Digestive ferments, 421

in cancer of the stomach, 451

list of

gasterine, 423

malt diastase piyalin, 422

pancreatin, $42^{\circ}$

pancreon, 422

papain, 421

papayot in 421

pepsin, 421

Digitalin, 15

Digitalis

administration, 13

contraindications, 16

cumulative and toxic effects of, 13

derivatives, 15

in Bright's disease, 108

in broken compensation. 12,27

in bronchitis, 295

in congestion of the stomach 69

in endocarditis, 88,89

in pneumonia. 315

in uræmia, 123
Dieitalis

istolerance for 14

subetitutee tor is

with sleohad, if

with diureticen, 34

Diejiralone, 18

Iheitaxin. is

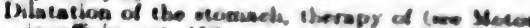
innuffelency). is:

thonise

in Larynation 270

in pulmonury atavin 20

in incheobropehitio. 2QV

Diphetheria, therapy of, ivis

as a cumplication

convalescence, COI

of meanies. 24

of mearlet tevet, eno

reberal treatment 39

diet. 202

hydrotherapy, 301

medication 892

aleotol ev

laryngeal obetruetion, tratusent of sou local treatment, sas

inhalations 20 .

miram bation, ela

nweat cure. 24 s

medieation. so:

expectoranta, 208

otimulanta, sos

operalive proendures, 200

local treatment. 394

antiapptica, 304

methoils of Joral applinatios. 205

external apolieatruas eg-

locel treatment of naval penages. 807

use of cold. 80 ?

prevention and treatment of notspliestione and erguelo. COO

cardiac complicationa, 600

eyes and ear. affections of COI

nephritis, 600

pain in swallowing. COO

paralywis, 601

peristence of bacilli, 60 !

relaper. 601

mopiratory cotnpliestiona roo

prophylaxis, 300

hardening proers 500

immunization by entitoxin, 391

quarantine, 500

specific treatment. 302

hacterioloriral disemomin sas

indieations for the atminiot rative of antitoxin, 502

injurious eteets of antitexin, 394

preparation and sunsere of antio taxin, sos

antitavin uait, 390

matistics meanting eteret of astjtoxin, 592

technic of administration of satj. taxin, 394 prorautiona 301

Diphtheritic antitoxin. 30

in axthma, 300

in diphtheria $\mathbf{5 9}$

prophylactic un. 501

therapeutic us, sor

dowark 50 s

indicationg 502

injurinus etterts of, 594

ntrparationa saj

technie, sos

in rearlet lever. ate

Ihiphtheritic paralyase pal

Divinfectance in infertion dieraura sis

bichloride of mercury, sit

rartmie acid. $5: 4$

chimont. S:1

formalin. sto

byont sit 
Disinfectants in infectious diseases paraform, 574 solveol, 574

Disinfection of gick-room after infectious diseases, 575

Dispnon, 60

Diuretics

classification of, 27

agurin, 29

calomel, 29

combinations of diuretics, 34

diuretic

tea, 32

wine, 35

diuretin, 28

heart stimulants, 27

milk, 33

potassium

acetate, 32

nitrate, 32

"sirop des cinque racines," 35

sodium nitrate, 32

squills, 31

theobromine, 28

theocine 29

theophylline, 29

urea, 34

uropherin, 29

in Bright's disease, acute, 105

in broken compensation, 27

in cirrhosis of liver, 529

in lithiasis phosphaturica, 139

in nephrolithiasis urica, 138

in pleurisy with effusion, 365

poisoning, acute, 560

Diuretic

\section{tea \\ in Bright's disease, acute, 106}

in hydrops in broken compensation, 32

wine in hydrops in broken compensation, 35

Diuretin, 28

Diversions in neurasthenia, 620

Dormiol, 52

Douches

in bronchitis, cold, on head and neck, 292

in diseases of the stomach and intestines, 404

in neurasthenia, 621

Scottish, 405, 491

stomach, 400

Dover's powder, 290

Drastic purges, 35

Drugs contraindicated

in Bright's disease, acute, 98

in insomnia in heart disease, 54

in interstitial nephritis, chronic, 119

Dulcin, 224

Dusting powders, 353, 523

Dysentery, therapy of, 540 acute

colon irrigations, 541, 543

diet, 54.0

heat, 542

medication, 541

calomel, 541,543

castor oil, 541, 543

ipecac, 541,543

Kruse's serum, 542

opium, 542

quinine, 541,543

saline cathartics, 542

silver nitrate, 541

tannin, 541

rest, 542

chronic, 542

castor oil, 542

diet, 542

irrigations, 542, 543

Dyspepsia

in compensated symptomatic heart lesions, 71

Dyspncea

nervous (see Nervous dyspensia), 466

in aortic aneurism, 85

in compensated symptomatic valvular lesions, 69
Dyspnoea

in emphysema of the lungs, 328

in pulmonary hemorrhagic infarct, 324

in uræmia, 125

in venous congestion in broken compensation, 60

Earthy waters, 146, 299

Ebstein's treatment of obesity, 237

Effervescent alkaline water, 464

Efflorescence in the mouth in chicken-pox, 588

E.ggs

digestibility of, 374

in Bright's disease, chronic, 111

in infectious diseases, 576

in tuberculosis, 329

Eka-iodoform, 597

Elaterin, 123

Elderberry flowers, 283

Electric pad, 405

Electromassage, 404

Electrotherapy

in constipation, 491

in diphtheria, 601

in diseases of the stomach and intestines, 403

in exophthalmic goitre, 201

in gast ralgia, 460

in mechanical insufficiency of the stomach, 455

in neurasthenia, 620

in rheumatism, chronic, 264

Flster water, 411

Emaciation, therapy of, 242

diet, 242

etiology, 242

hygiene, 243

Emetics in acute poisoning, 558

apomorphine, 559

copper sulphate, 559

mustard powder, 558

Emphysema, pulmonary, therapy of, 326 assistance of respiration, 326

breathing belt, 327

breathing chair, 327

pneumatic chamber, 326

health resorts, 327

in whooping-cough, 611

relation of obesity to, 328

treatment of

bronchitis in, 327

dyspnœa in, 328

Emulsio oleosa, 287

Emulsion of almonds, 287

Endocarditis, acute, therapy of, 87

cold applications, 89

collargol, 89

heart stimulants, 89

iron, 90

local therapy, 89

Enemas quinine, 88

alcohol, 181

benzoin, 509

bichloride of mercury, 509

carbon dioxide, 487

castor oil, 492

chlorine water, 509

cold water, 492

ether, 509

garlic, 509

glycerin, 481, 492

in constipation, 492

in ileus, acute, 482

in intestinal stenosis, chronic, 481

in membranous enteritis, 476,478

in oxyuris vermiformis, 508

naphthaline, 509

nutrient, 394

oil

in constipation, 494

in ileus, acute, 482,486

in intestinal stenosis, chronic, 481

in membranous enteritis, 476,478 
Enemas

maline

in constipation, 492

in dilatation of the stornach, 3Wi

in ileus, acute, 452, t\$o

in inteatinal ntenomis, chronic, is! senna

in ileus, acute, 486

in intestiual stenonis, elironic, 48?

in constipation, 492

ech in oxyuris vermiformin, 300

iechnic of, 493

thymol, 509

vinegar, 281, 402

wine, 396

Enteritis, membranous, therapy of acute, 476
chroule, 476
diet, 477

Enteroptosis, 505

Fpsom salts, 36

Ergot

in Bright's disease, acute, 103

in pulmonary cedema, 325

in pulmonary stasis, 60

in purpura, 185

in renal hemorrhage, 142

Ergotin

in tuberculosis, 352, 355

in renal hemorrhage, 142

in venous congestion in broken compensa. tion, 61

with digitalis, 17

Ergotinin, 61

Eructation (see Nervous eructation and pneuma. tosis), 461

Erythrol tetranitrate, 79

Eserine, 540

Eskay's food, 376

Ether

enemas, 509

in broken compensation, 25

in uramic asthma, 125

Ethereal oils

in bronchitis, purulent, 300

in laryngitis, 279

in tuberculosis of the larynx, 280

Ethyl chloride, 96

Eucalyptus

in bronchitis, 304

in malaria, 5.54

Eucalyptus oil

in bronchitis, putrid, 304, 305

Eucasin, 101

in laryngitis, 279

Eucasin cocoa, 113, 378

Eudoxin, 424, 474

Fumydrin

in asthma, 310

in gastric ulcer, 445

in hypersecretion, 465

in night sweats, 352

Funatrol

in cholelithiasis, 526

in jaundice, catarrhal, 522

Euporphin

in bronchitis, acute, 295

in tracheobronchitis, 290

Euquinine

in infectious diseases, 570

in malaria, 553

in whooping-cough, $60-4$

Furophen, 597

Exalgin

in angina pectoris, 80

in gastralgia, 460

Examinations in neurasthenia

physical, 616

psychical, 616

Fxercise

in chlorosis, 155

in compensated valvular lesions, 1

in diabetes, 225

\section{Fiverese}

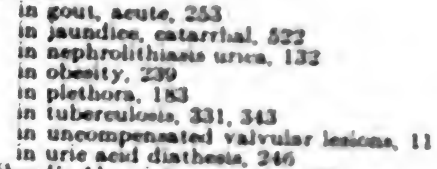

it cour. acule. 283

in isundice, calsrital, 83

in nephrolithians unera, 133

in obesity, 209

in plethors. I8]

in iuterculionis. 231, 34

in usecospensaled valvular lenienas 11

in urie acul diatbesia $2 t$

Fixibard'a Abymainian recties's, 30

Fiscophthalmie roitre. 100

elimatolozy, 190

diet, 200

electrotherspy, 201

hydrotherapy, 200

medieation 201

brotaides 201

rodapen, 203

quinine bydrotorutaste, 204

volium phoophate, sor:

thyenu aland. zod

thyrotel extract, sod

operation, 203

madiotherapy, 204

reat, 160

neruin irestment, 304

Expectorane

in bronehitis. 204. 302, as

in diphtheria, sos

in pneumonia. 314

is pulmonary stanie, 88

in incheobronchitis, 28

in tubereulowio, 351, 357

in whooping-cough, 610

Fixtract of pig's liver, 227

Fitractives, 110,131

Fainting (we Synempe), of

Farinaceous forml. dizestibility of, 3:6

Pasting
in gantric ulcer, 439
in gastritis, scute, 132
in tleus, acute, 457
in peritonitie, 517
in perityphlitin 512

Fat

digestibility of. 378

foods rich in, 223

heat value, 205

in Bright's diseave ehronic 111

in constipation, 400

in scurvy. 186

in tuberculoais, 329

reduction of in obesity, 24

Fat-milk (see Girtner's fat-milk), 100, 200

Fatty heart, therapy of, $\mathrm{R}$.

fatty dezeberation. 8

fatty overkrowth, 85

diet, 85

mineral watern 8 .

Oertel. cure \&s

Feces. removal of. $483,49 i$

Feeding

naval, 3.5\%

rectal, 394

Ferment cerevivia (Necest)

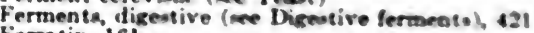

Ferratin, 161

Ferti carbonas saccharatue 159

et ammoni citrag 160

el polavii tartras 160

hylroxidum rum maknesii oxido in arsevie poimoning. 362

Ferro-sornatose, 16!

Ferwan, 179

Fever

in infectious dimasen scute, 500

in meavien ses

in scarlet lever, 575

in tuberculonias 349

in typhoid fever, 52s, 53:

Fibrolyxin, 445

Flatuleney, therapy of, 304 carminatives 504 
Flatulency, therapy of cathartics, 504 colon irrigations, 504 diet, 505 in peritonitis, 518 in typhoid, 540 puncture of intestine, 505 rectal tube, 505

Flowers of sulphur, 300

Food (see Diet)

Foods

digestibility of, 370

quantitative analysis of, 214

temperature of, 381

Formaldehyde, 574

Formalin lamp, 574

Formol, 352

Fortoin, 474

Frangula, 495

Fresh-air cure

in bronchopneumonia, 322

in croupous pneumonia 314

in scrofula, 191, 194

in tuberculosis, 330

in whooping-cough 613

Fruit

digestibility of, 376

in constipation, 489

in diabetes, 221

in nephrolithiasis urica, 131

in scurvy, 186

in uric acid diathesis, 245

Funnel drainage in hydrops in broken compensation, 39

Furunculosis in diabetes, 226

Gärtner's fat-milk

in Bright's disease, 100

in diabetes, 220

Gall-stones (see Cholelithiasis), 524

Galvanopuncture, 83

Gamboge

in constipation, 500

in diseases of the stomach and intestines 430

in hydrops in broken compensation, 37

Gangrene

in diabetes, 231

Gargles

pulmonary, therapy of, 325

in diphtheria, 590, 595

in pharyngitis and tonsillitis, 276

Gasterine

in anæmia from atony of the glands of the stomach and intestines, 168

in diseases of the stomach and intestines, 423 in gastritis, chronic, 437

in hyposecretion, 466

Gastralgia, therapy of, 459

causal therapy, 459

electrotherapy, 460

hydrotherapy, 460

Gastric

narcotics and anodynes, 460

hypersecretion, therapy of (see Hypersecretion, gastric), 463

hyposecretion (see Hyposecretion, gastric), 465

neuroses (see Stomach, neuroses of), 458

ulcer, therapy of (see Ulcer gastric), 439

Gastritis, therapy of

acute, 432

diet, 432

fasting, 432

methods of relieving thirst, 432

lavage, 433

medication, 433

cathartics, 433

calomel, 433

relief

castor oil, 433

of pain, 434

chronic, 435

of vomiting, 434

diet, 436

\section{Gastritis}

chronic

hydrotherapy, 438

lavage, 435

massage, 438

medication, 437

mineral waters, 436

surgery, 438

Gastroptosis, therapy of, 456

bandages, 457

diet, 457

hydrotherapy, 457

lavage, 457

medication, 458

rest, 457

Gavage in tetanus, 557

Gelatin

digestibility of, 374

in aortic aneurism, 82

in gastric ulcer, 447

in hæmophilia, 184

in purpura, 186

in tuberculosis, 357

Gelatinous foods digestibility of, 374

forms of, 374

Genito-urinary tract, diseases of therapy of, 98 cystitis, 147

kidney, diseases of the, 98

movable kidney, 145

nephrolithiasis, 129

Gentian, 419 pyelitis and pyelonephritis, 142

German measles, therapy of, 587

isolation, 587

relief of itching, 587

rest, 587

Glandular swellings

in mumps, 589

in scarlet fever, 580

in scrofula, 193

Glauber's salts, 492

Glottis, œdema of the, 126

Glutoid capsules, 428

Glycerin

enemas and suppositories in constipation, 492

in care of the mouth in fever, 270

in cholelithiasis, 526

in nephrolithiasis urica, 135

Goitre, exophthalmic (see Exophthalmic goitre), 199

Gout, therapy of, 244

acute, 251

applications, 251

diet, 253

exercise, 253

massage, 252

medication, 252

colchicum, 252

sodium salicylate, 252

prevention of recurrence, 253

chronic (see Uric acid diathesis), 244

Graham bread, 153

Grape cure

in bronchitis, chronic, 303

in diseases of the stomach and inteztines, 390

Graves's disease, 199

Griffith's mixture, 160

Grindelia in asthma, 307

Gruels, directions for making, 102, 441

Guacamphol, 352

Guaiac, 276

Guaicol

in rheumatism, chronic, 265

in tuberculosis, 345

Gums, sponginess of, 30

Gymnastics

abdominal, 402

as a prophylactic measure against tuberculosis, 343

in constipation, 491

in hydrops from broken compensation (Schott's movements), 45 
Gymnastics

in neurasthenia, 620

in rheumatism, chronic, 264

Swedish, 5

'Zander's, 5, 264

Haarlem oil

in chololithianis, 5227

in pyelitis and pyelonephritis, 143

Hamaboloids, 16!

Hiemalbumin, 101

Hamatemesin

in cancer of the stomach, 452

in gastric ulcer, 44\%

Hamogallol, 161

Haemol, 1 til

Hsemophilia, therapy of, 184 calcium, 18t

Hamoptysis gelatin, 184

in broken compensation, 60

in interstitial pueumonia, 323

in tuberculosis, 354

in whooping-cough, 612

Hamostatics

in hemorrhage in tuberculosiผ. 355

in intestinal hemorrhage, 480

Half baths

in gastric neuroses, 450

in infectious diseases, 5.3

in neurasthenia, 621

Halogen poisoning, 560

Hardening process

for adults, 280, 295, 343

Heart

compressors, 95

diseases of the

endocarditis, acute, 87

muscle, diseases of the cardiac (see Car-

diac muscle diseases of ), 85

neuroses (see Neuroses of the heart), 93 pericarditis, 90 valvular lesions (see Valvular lesions, therapy of ), 1

massage of the, 567

palpitation of the (see Palpitation of the heart), 93

stimulants

in acute poisoning. 564

in bronchopneumonia, 321

in cirrhosis of liver, 528

in diphtheria, 600

in endocarditis, acute, 89

in interstitial nephritis, chronic, 122

in pneumonia, 315

in uramia, 123

in valvular lesions, uncompensated, 12 dist of

adonis vernalis, 20

ammonia, 25

apocynum, 23

caffeine, 21

camphor, 24

convallaria, 19

digalen, 15

digitalin, 15

digitalis, 12

digitalone, 15

digitoxin, 15

ether, 25

kola, 22

moschus, 25

quinine, 23

spartein, 19

strophanthin, 18

strophanthus, 18

strychnine, 23

valerian, 26

supporters, 95

Hedonal, 51

Helmitol

in cystitis, 149

in pyelitis and pyelonephritis, 142

in scarlet fever, 581
Hetporrhage

castrie ( Hee Hamatersesio)

intewtinal (we Intestinal hemorthage)

nacal, 612

pulmonary (noe IIstroptyis)

retial, ItI

Hetnorrboid no witz bach in, tos

Herta arenarise, 137

Hernia from whooping-cough, 612

Heroin
in laryngitis, 279
in meanles, 885
in pulmonary stanis, 80
in iracheobronehitis, 285

Herpen buccalin, therapy of, 272

Heterucresol, 348

Hetol, 348

High altitudes, 332

in tuberculosin, 332, 34 ?

Hoflmann's drops, 25

Holzinol, 602

Honey, 378

Hookworm, 510

Hopogan, 423

llot

air bathw

in Bright's dikeare, scute, 107

in hydrop in broken compensation, 43

application:

in cystitis, 14:

in dysentery, 342

in eastric ulcer, 446

in gastro-intestinal digeares, 405

in intestinal ulcer. 480

in poisoning, acute, 564

baths

in rheurnatism, acute articular, 351

in Bright's diseare, acute, 106 chronic, 116

in bronchitis, 293

in cholelithiasis. 524

in hydrops in broken compensation, 42

in pneumonia, 318

in rensl colic, 141

in rheumatism. chronic, 259

in scarlet fever, 382

packs in asthma, 310

springs, table of 249

Huckleberry wine, 379,471

Hydragogin, 35

Hydragozues

in hydrops in broken compensation, 35

colocynth, 37

gambore, 37

jalap, 36

podophyllin, 37

in poisoning, acute, 559

Hydrastis

in bronchitis purulent, 302

in purpura, 185

in tuberculosis, 352, 356

Hydrated oxide of iron with maknesia in arsenic poisoning, 562

Hydrobromic acid, 273

Hydrochloric acid

in chlorosis 153

in dizestive disturbances in tubereur Josis, 360

in diseares of the stomach and inter tinea 415,423

in gastritis, ehronic, 437

in hyposecretion, 466

in lithiasis phosphaturica. 139

in urie acid diathesis, 250

Hydrogen peroxide

in diphtheria, 595

in hydrops in brokes compensation, 30

Hydrope

in stomatitis, 272

in Bright's disease.

acute, 104

chronic 118

in broken compensation. 27 cathartics and punges, 35 
Hydrops, in broken compensation colocynth, 37 gamboge, 37

jalap, 36 podophyllin, 37

diaphoretics, 37 pilocarpine, 37

diuretics, 27

agurin, 29

calomel, 29

combinations of, 34

diuretic

$$
\text { syrup, } 35
$$

wine, 35

diuretin, 28

hydrogogin, 35

milk, 33

potassium acetate, 32

potassium and sodium nitrate, 32

squills, 31

theobromine, 28

theocine, 29

theophylline, 29

urea, 34

uropherin, 29

mechanical and hydropathic ther-

apy, 42

baths

carbonated, 43

hot, 42

hot air, 43

salt, 43

gymnastics, 45

massage, 42

surgery, 38

extremities, of the, 38

funnel drainage, 39

incision, 38

puncture, 38

internal

abdomen, puncture of, 39

pericardium, puncture of, 41

Hydrotherapy

thorax, puncture of, 41

in arteriosclerosis, 7475

in asthma, 310

in Bright's disease

acute, 106

in bronchitis

chronic, 116

capillary, 292

deep, $29 \mathrm{i}$

dry catarrhal, 298

in bronchopneumonia, 321

in chlorosis, 156

in constipation, 491

in diabetes mellitus, 226

in diphtheria, 591

in dyspepsia, nervous, 467

in exophthalmic goitre, 200

in fever in tuberculosis, 351

in gastralgia, 460

in gastritis chronic, 438

in gastroptosis, 457

in hæmophilia, 184

in hydrops in broken compensation, 42

in hyposecretion of the gastric juice, 466

in infectious diseases, 570

in intestinal catarrh, chronic, 475

in leukæmia and pseudoleukæmia, 177

in measles, 585

in nephrolithiasis urica, 132

in neurasthenia, 621

in neuroses of the stomach, 458

in obesity, 239

in osteomalacia, 258

in palpitation of the heart, 95

in pleurisy, 364

in pneumonia, 313,316

in rheumatism

acute articular, 550

chronic, 259
Hydrotherapy

in scarlet fever, 577

in serofula, 191

in scurvy, 187

in stomach and intestines, diseases of, 404

in tuberculosis, pulmonary, 343,351

in typhoid fever, 535

in whooping-cough, 612

Ilygiene of the sick-room

in bronchitis, capillary, 294

in infectious diseases, 573

diphtheria, 591

measles, 584 whooping-cough, 613

Hyoscine, 308

Hyoscyamus

in asthma, bronchial, 307

in measles, 585

in pulmonary stasis, 59

in tracheobronchitis, 286

Hyperæmia, passive cerebral (see Passive cere-

bral hyperæmia), 47

Ilyperchlorhydria in chlorosis, 153

Hypersecretion, gastric, therapy of, 463 alkalies, 464

diet, 390,463

lavage, 464 medication, 464

Hypnal, 54

Hypnone, 54

Hypnotics, 54,623

Hypnotism in neurasthenia, 617

Hypochlorhydria in chlorosis, 153

$\mathrm{H}$ ypodermoclysis in measles, 585

Hypophosphites, syrup of, 349

Hyposecretion gastric therapy of, 465

diet, 392, 465

hydrotherapy, 466

lavage, 466

medication, 466

gasterine, 466

hydrochloric acid, 466

orexin, 466

Ice-bag

in aortic aneurism, 84

in arteriosclerosis, 77

in compensated symptomatic heart lesions, 71

in congestion of the liver, 65

in endocarditis, acute, 89

in hæmoptysis, 355

in intestinal ulcer, 480

in pericarditis, 90

in perityphlitis, 513

in typhoid fever, 540

Ichthoform, 427, 474

Ichthylalbin, 427

Ichthyol, 265, 523

$$
\text { ichthyol mud, } 263
$$

Icterus, therapy of (see Jaundice), 520

Idiopathic enlarged heart, 86

Ileus (see Intestinal obstruction), 482

Immunizing use of diphtheria antitoxin, 591

Incision of lower extremities for hydrops in broken compensation, 38

Indications for surgical interference

in intestinal obstruction, 487

in nephrolithiasis, 138

in perityphlitis, 514

in pyelitis and pyelotyplilitis, 145 in uræmia, 128

Infarct, hemorrhagic pulmonary, therapy of, 323 etiology, 323

prophylactic treatment, 324

pulmonary symptoms, 324

relief of pain, 324

syncope, 324

Infectious diseases, acute therapy of, 531, 569 articular rheumatism, acute, 544 chicken-pox, 588

diphtheria, 590

dysentery, 540

German measles, 587

influenza, 543 
Infectious diseases

introduction to, 568 antipyresis, 569 hydrotherapy, 570 diet, 575

disinfection, 573

hygiene, 573

malaria, 552

measles, 584

mumps, 589

scarlet fever, 577

tetanus, 555

typhoid fever, 531

Influenza, therapy of, 543

whooping-cougli, 601

antineuralgics, 544

antipyretics, 544

diaphoresis, 544

diet, 544

Infusion of a salt solution

in pernicious anxmia, 173

in pneumonia, 318

technic

int ravenous, 173

Inhalations subcutaneous, 173

in bronchiectasy, 305

in bronchitis, purulent, 305

in diphtheria, 598

in laryngitis, 279

in tracheobronchitis, 284

in tuberculosis of the larynx, 280

in whooping-cough, 601

of oxygen

in Bright's disease, chronic, 117

in bronchitis, 295

Injections in chlorosis, 168

in diphtheria, 600

in tonsillitis, 277

intratracheal, 300

of carbolic acid in rheumatism, acute articular, 550

of gelatin

in aortic aneurism, 82

in gastric ulcer, 447

in hæmophilia, 185

in purpura, 186

of sera (see Serum)

Inorganic iron compounds, 159

Insomnia, therapy of, 47

Insufflation powders

in diphtheria, 595, 596

in scarlet fever, 579

in whooping-cough, 602

Internal gout, 250

Interstitial nephritis, chronic (sce Nephritis, chronic interstitial), 118

Intestinal

antiseptics (see Antiseptics, intestinal), 424

astringents, 472

bismuth, 473

lead acetate, 473

silver nitrate, 473

tannalbin, 472

tannigen, 472

catarrh, therapy of

acute, 468

cathartics, 468

causal therapy, 468

colon irrigations, 469

diet, 469

medication, 470 castor oil and salol, 4\%

chronic, 470 narcotics, 470

astringents, 472

cathartics, 472

colon irrigations, 472

diet, 470

hydrotherapy, 475

medication, 472

belladonna, 477

bismuth, 473

\section{Intestinal catarrh, chronic, medication learl scetate, 473 \\ opiusn, 474 \\ wilver nitrate, 173 \\ tannalbin, 47"2 \\ tannigen, 472 \\ mineral waters 474}

colie, 505

disturbances

in meanlew, 58 ?

in tuberculowin, 361

in typhoid fever, 539

hemorrhage, therapy of

in inteatinal ulcer, 440

obetruction, 480

chronic stenonis, 480

cathartica, 481

diet, 481

enernas, 48 .

surzery, 480

ileus, acute 48

caused by coprontasin, 482

cathartics, 482

enemas, 482

masnage, 483

removal of feres, $4 \mathrm{ks}$

cauned by foreign bodies, 483

from other causes, 483

at ropine, 486

colon irrigations, 480

fasting, 487

indications for operation, 483

inflation of intestines. 487

lavage, 484

opium, 485

puncture of intestine, $437^{\circ}$

surgery; 487

parasites, therapy of, 506

ankylostomiasis, 509

tbymol, 509

ascaris lumbricoides, 50 santonin, 508

oxyuris vermiformis, 508

cleanlinexs, 300

enemas, 509

itching of anus, 509

santonin, 508

tapeworm, 506

male fern, 500

ulcer, therapy of, 478

diet, 479

hemorrhage, 480

local treatment, 479

medication, 479

bismuth, 479

castor oil, 479

opium, 479

tannalbin, 479

specific treatment, 478

sunzery, 479

Intestines, the

diseases of the therapy of, 468

constipation, 458

diarrhoea, 502

flatulency (meteorism). 504

intestinal

catarrh

scute, 468

chronic 470

ileus acute, $45^{\circ}$

obstruction, 480

parasites 506

stenosis, chronic, 4S0

ulcer, 478

membranous enteritis, 476

inflation of

in flatuleney, 505

in ileus, acrite, 487

puncture of, 487

Intoxications (see Poisonings), 5.37

Intrat racheal injections, 300

Iodic acid, 595 
Iodide, potassium

in aortic aneurism, 82

in arteriosclerosis, 75

in congestion of the kidney, 67

in endocarditis, 90

in gout, acute, 253

Iodides

in rheumatism, chronic, 265

in alkaloid poisoning, 563

in aortic aneurism, 82

in arteriosclerosis, 75

in asthma, 309

in Bright's disease, chronic, 118

in exophthalmic goitre, 202

in interstitial nephritis, chronic, 118

in syphilitic arteritis, 81

Iodine poisoning, 560

Iodoform, 596, 597

Iodol, 274

Iodvasogen, 580

Ipecac

in bronchitis

acute, 294

purulent, 301

in diphtheria, 599

in dysentery, 54

in measles, 586

in pseudocroup, 280

in pulmonary stasis, 58

Iron

in tracheobronchitis, 289

administration, 158

baths, 162

contraindications for, $158,165,179$ effects, 157

$$
\text { in chlorosis, } 157
$$

with constipation 160

with dilatation of stomach, 165

with nervous symptoms, 166

in endocarditis, acute, 90

in secondary anæmia, 179

mud baths, 165

preparations, $158-162$

inorganic, 159

organic, 160

tropon, 161

waters, 162

alkaline iron springs, 162

alkaline saline iron springs, 162

arsenic iron springs, 170,501

in chlorosis, 162

in stomach and intestines, diseases of the 415

Irrigation table of, 163,164

Krull's, 521

of the bladder, 150

of the colon (see Colon irrigations)

of the mouth, 578

of the nose, $\mathbf{5 7 1}, \mathbf{5 7 9}, 596$

of the pelvis of the kidney, 145

of the stomach, 397

of the throat, 595

Isopral, 53

Itching
in chicken-pox, 588
in German measles, 587
in jaundice, 522
baths, 522
ointments, 522
powders 523
of anus, 509

Jaborandi, 283

Jalap

in constipation, 500

in diseases of the stomach and intestines, 430

in hydrops in Bright's disease, 117

in hydrops in broken compensation, 36

Jaundice, catarrhal, therapy of, 520

alkaline saline waters, 521

cathartics, 520

cholagogues, 522

bile salts, 522
Jaundice, catarrhal, cholagogues sodium salicylate, 522

diet, 520

exercise, 522

itching, 522

baths, 522

ointments, 522

anæsthesin, 523

bromocoll 523

menthol, 522

powders, dusting, 523

Krull's irrigations, 521

Kefir

in Bright's disease, 101

in diseases of the stomach and intestines, 375

in gastric ulcer, 440

in intestinal catarrh, 471

Keratinized pills, 428

Kidneys

congestion of the, therapy of (see Congestion of the kidneys, therapy of ), 67

movable, therapy of (see Movable kidney therapy of), 145

stone (see Nephrolithiasis), 129

Kleewein's pills, 497

Kola

in Bright's disease, chronic, 117

Koumiss

compensation, 22

directions for making, 441

in Bright's disease, acute, 101

in diseases of the stomach and intestines, 375

Kousso flowers, 507

Kraftschocolade, 113, 243, 256, 378

Krameria, 144, 355

Krull's irrigations, 521

Kruse's serum, 542

Lactic acid, 125, 257

Lactophenin

in aortic aneurism, 84

in influenza, 544

in rheumatism, acute articular, 549

in tuberculosis, 350

Lactose, 33, 490

Lactucarium, 287

Lamp, formalin, 574

Landois's table of weights and heights, 236

Lanoform, 597

Laryngeal obstruction in diphtheria, 598

Laryngeal spasm, therapy of, 281

between attacks, 282

during an attack, 281

etiology, 281

in whooping-cough, 611

Laryngitis, acute, therapy of, 278

cough, 278

diaphoresis, 278

dryness and burning in larynx, 279

excessive secretion, 279

in measles, 586

in scarlet fever, 578

prophylaxis, 279

Larynx, diseases of the, therapy of, 278

laryngeal spasm, 281

laryngitis, acute, 278

pseudocroup, 280

tuberculosis of the larynx, 280

ethereal oils, 280

narcotics, 281

Laudanum, Rousseau's, 251

Lavage, 397

accidents during, 398

best time for, 399

contraindications, 399

gastric douches, 400

in cancer of the stomach, 452

in gastritis

acute, 433

chronic 435

in gastroptosis, 457

in hypersecretion, 464

in hyposecretion, 466 
Lavage

in ileus, acute, 481

in mechanical inmuffieieney of the stomach, 454

in nervous dyspepmia, 467

in poisoning, acute, jas

Laxative techinic, 397

in cirrlıomis of the liver, 528

in congestion of the liver, 60

in constipation, 495

in diseasen of the stomach and intentises, $1: 29$ Leal pills, 36

aceiate

in aortic ancurism, 82

in hemorrlinge in isterculonis, 350

in intentinul catarrh, 473

in pulmonary ardema, 320

in uramia, 126

poisoning, 56:

Lecithin, 82

Ledum palust re, 287

leeches

in congestion of liver, 65

in cystitis, acute, 148

in pleurisy, 363

in true plethora. 183

in venous congestion in broken compensation, 56

indications for, 57

method of application, 50

Leguminous vegetables, 376

Leiter's coils

in compensated symptomatic heart lesions, 71

in diseases of the stomach and intestines, 405

in pericarditis, 90

in typhoid fever, 540

Lemon cure in nephrolithiasis urica, 136

Lemonade

in scurvy, 186

in typhoid fever, 525

laxative, 433

Lenhartz's treatment of gastric ulcer, 442

Leube's ulcer cure, 384

Leukamia and pseudoleuksmia, therapy of 17 arsenic, 174 injections of, 175

internally, 17

cacodylic acid, 170

diet, 177

hydrotherapy, 177

organotherapy, 170

phosphorus, 176

quinine, 176

radiotherapy, 177

rest, 177

Licorice powder, compound, 496

Lignosulphate, 602

lime

water, 153, 302

waters, 146

Linden flowers, 283

Lipanin, 189, 256

Lips, cracked, 271

Liqueur Laville, 252

Liquids

in compensated valvular lesions, 3

in diabetes mellitus, 224

in diabetes insipidus, 233

in diseases of the stomach and intestines, 378

in infectious diseases, 576

in lithiasis phosphasurica, 139

in mechanical insufficiency of the stomach, 454

in nephrolithiasis urica, 131, 136

in obesity, 236

in pyelitis and pyelonephritis, 144

in typhoid fever, 534

in uramia, 123

in uric acid diathesis, 240

Liquor

ammonii acetatis, 291 liguor

ammonil aninat

is broken cumpenation, 25

in bronchitse, in

in diplstheria. 60

in perulocrung, 2no

in tracheobrutuelustia, 201

carnis, 372

Cerri albuminatis, 100

Id hiscin phosplacurica, 134

antifermentativen, 130

diet, 140

diuretics, 130

etiology, 138

inorkanic sciuls, 138

Lithium

urotropin, 130

in nephrolithineis urica, 134

preparations of, 134

benzonte, 134

carbonate, 134

citrate, 134

iodide, 134

water

in nephrolithines uries, 135

list of, 13.5

Liver

and gall pannages, dineanes of the, therapy of, 520

cholelithianis. 524

cirrhonis of the liver, 528

jaundiee, eatarthal, 820

cirrhosis of the therapy of 528

hypertrophic and early stakes of atrophie, $\mathbf{5 2 8}$

diet, 325

laxatives, 528

mineral waten, 328

late stages of atrophic, 528

diuretics, $5: 9$

heart stimulanta, 529

puncture of abulomen, 529

surgery, 5:0

congestion of (see Congestion of the liver), 64

Lobar pneumonia (see l'neumonia croupous). 312

Lobelia, 308

Local

cold

in aortic aneurism, 84

in bronchopneumonia, 321

in diphtheria, 597

in gastric ulcer, 416

in hemorrhage in tuberculonis, 354

in pericanditis 90

in rheumatism, chronic, 264

in uncompensated valvular leoiong, 12

treatment

of rheumatism, acute articular, $\mathbf{5 5 0}$

of tetanus, 556

of the throses

in diphtheria, 594

in larynzitix 279

in pharyngitis 27.5

in scarlet fever, 5 \%

in tracheobronchitis, 284

in whooping-cough cos

Sffler's mixture, $\mathbf{5 9 0}$

Loretin, 597

Lumbar puncture

in urrmia, 127

technic. 127

I.ungs diseases of the. therapy of, 312

bronchopneumonit 321

emphysems of the lungs 326

pneumonia

eroupous 312

interstitial, 32

pulmonary

abscese, 324

emphy ma. 326

cangrene 325

infaret, 323

adema, 325

tuberculosis 329 
Lysidin, 135

Lysitol, 574

Lysol, $\mathbf{5 7 4}$

\section{Magnesia}

ammonium phosphate of, 417

burnt

in constipation, 499

in diseases of the stomach and intestines, 417, 429

in pyrosis, 463

in ulcer of the stomach, 446

Magnesium

salicylate, 429

sulphate

in hydrops in broken compensation, injections in tetanus, 557

Malakin

in aortic aneurism, 84

in rheumatism, acute articular, 547

Malaria therapy of, 552

after-treatment, 554
diet, 554
medication
aristoquin, 553
arsenic, 554
eucalyptus, 554
euquinine, 553
methylene blue, 554
quinine, 552
administration, 552

Male fern in tapeworm, 506

Mallow, 561

Malt

diastase ptyalin, 422

extract of, 189, 255, 258

Manganese with iron, 165

Manipulation in whooping-cough, 610

Maretin

in angina pectoris, 80

in rheumatism acute

articular, 547

in tuberculosis, 35

Marienbad water, 410

Marmorek's serum, 580

Marriage

in chlorosis, 157

in tuberculosis, 362

Massage

abdominal, 401, 483

automassage, 402

contraindications, 401

technic, 401

general, 388

in arteriosclerosis, 75

in compensated valvular lesions, 6

in constipation, 491

in diabetes, 225

in enlarged or fatty heart, 87

in gastritis, chronic, 438

in hydrops in broken compensation, 42

in gout, acute, 252

in ileus, acute, 483

in mechanical insufficiency of the stomach, 455

in movable kidney, 147

in neurasthenia, 620

in rheumatism, chronic, 264

intestinal, 401

of the abdomen, 401, 483

of the heart, 567

of the stomach, 400

technic, 401

time for 400

Matzoon, 101, 441

Meals, 376

Measles, therapy of, 584

convalescence, 587

diet, 584

hygiene of sick-room, 584

isolation 584

symptoms and complications, 584
Measles, symptoms and complications catarrhal affections, 585

bronchitis, 586

catarrh, chronic, 586

cough, 585

laryngitis, 586

severe respiratory disturbances, 586

conjunctivitis and rhinitis, 584

diphtheria antitoxin, 585

fever, 585

hydrotherapy, 585

intestinal disturbances, 587

stomatitis, 584

antisepsis of mouth, 584

Meat

broths, 372

digestibility of, factors affecting, 370

extract, 372

beef tea, 372

in Bright's disease, chronic, 110

in uric acid diathesis, 245

juices, 372

peptones, 373

preparations, 373

soup, 372

Mechanical insufficiency of the stomach, therapy of, 452

Cohnheim's oil treatment, 456

diet, 393, 453

distinction between atony and dilatation, 452

electro therapy, 455

lavage, 454

massage, 455

medication, 455

mineral waters, 456

position, 455

reduction of liquids, 454

surgery, 456

Medication at night, 535

Mellin's food, 255

Membranous enteritis (see Enteritis, membranous), 476

Menthol

as an intestinal antiseptic, 427,430

as a stomach antiseptic, 423

in aortic aneurism, 84

in bronchitis, 296

in diabetes, 230

in diphtheria, 596

in gastralgia, 158, 462

in jaundice, catarrhal, 522

in pleurisy, 363

in rhinitis, 274

Mercury

in Bright's disease, 98

in syphilitic arteritis, 81

Mesotan, 252, 546

Metabolic equilibrium, 208

Metabolism, diseases of, therapy of, 206

diabetes insipidus, 23

diabetes mellitus, 206

emaciation, 242

obesity, 234

osteomalacia, 257

phosphaturia, 254

rheumatism, chronic, 259

rickets, 254

uric acid diathesis, 244

acute, 25

chronic, 244

Metal poisoning, 562

secondary anæmia in, 181

Meteorism (flatulency), therapy of, 504

Methyl violet, 103

Methylal, 54

Methylene blue

in Bright's disease, acute, 103

in malaria, 554

Milk

as a diuretic 100,560

condensed, 375

diet

in Bright's disease, 100, 109 
Milk diet

in bronchitis, chronic catarrhal, 303

in diabetes, 220

in dysentery, 540

in gastric ulcer, 440

in infectious diseases, 576

in scarlet fever, 581

in tuberculosis, 329

in typhoid fever, 531

in uncompensated heart lesjons, 33

in uric acid diathesis, 246

in Weir Mitchell's rest cure, 387

digestibility of, 375

modification of, 375,531

preparations from, 375

substitutes for, 375

condensed milk, 375

Gürtner's fat-milk, 100, 220, 375

infants' foods, 375

kefir, 101, 375, 440, 471

koumiss, 101, 375, 441

inilk somatose, 375

vegetable milk, 375

sugar as a diuretic, 33

Mineral waters

classification of, 406

alkaline, 299,407

chloride, 299, 407 table of, 408

saline, 407

arsenic, 170 table of, 409

table of, 170

arsenic and iron, 170

bitter, 411 table of, 170 table of, 414

calcium, 146, 414

table of, 146

carbonated, simple, 406

common salt, 411

table of, 412

earthy, 290

table of, 146

iron, $162,165,415$ table of, 163

sulphur, 182, 299 table of, 182

in Bright's disease, acute, $10-4$

in bronchitis

chronic, dry, 299

purulent, 302

in cancer of the stomach, 451

in chlorosis, 165

in cholelithiasis, 525

in cirrhosis of the liver, 528

in congestion of the liver, 65

in constipation, 500

in cystitis

acute, 148

chronic, 148

in digestive disturbances in tuberculosis, 360

in fatty overgrowth of the heart, 85

in gastric ulcer, 443

in gastritis, chronic, 436

in intestinal catarrh, chronic. 474

in mechanical insufficiency of the stom-

ach, 456

in nervous dyspepsia, 468

in obesity, 239

in pyelitis and pyelonephritis, 145

in serofula, 193

in secondary anxmia, 179

in stomach and intestines, diseases of, 380,406

in tracheobronchitis, 284

in true plethora, 183

temperature, 406

Mistura solvens, 290

Morphine

in aortic aneurism, 84

in asthma, bronchial, 307

in bronchitis, chronic dry, 298

in cholelithiasis, 524

\section{Morphine}

in compenasted symptornatic heart leaions, 69 in cystitis, 148

in diseases of the ntornach and intestines, 430

in rastritis, acute, 434

in beart discase, 68

in intestinal obstruction, 485

in laryngitis, 278

in membranous enteritis, 476

in peritonitis, 818

in perityphlitis, 312

in pleuriny, 304

in renal colic 141

in tetanus. 557

in tracheobronchitis, 285

in tuberculosis, 358

in typhoid fever, 530

in uriemia, 127

in whooping-cough, 607

intolerance for, 69

Moschus, 25, 565

Moser's nerum. 580

Mosquera's beef meal, 373, 576

Mountain resorts in tuberculosis, 332 at high altitudes, 333

Mouth at lower altitudes, 3.35

care of the

under pathological conditions, 270 cracked lips, 271

in diabetes, 230

in fever, 270

in measles, 584

in perityphlitis, 512

in scarlet fever, 578

in typhoid, $\mathbf{5 3 8}$

under physiological conditions, 267

mouth washes, 269

teeth, 267

diseases of the, therapy of herpes buccalis, 272 mercurial ulcers, 273 stomatitis

aphthous, 272

catarrhal, 271

mercurial, 272

ulcerative, 27

washes, 269

thrush, 273

Movable kidney, therapy of, 145

$$
\begin{aligned}
& \text { massage, } 147 \\
& \text { rest and support, } 145
\end{aligned}
$$$$
\text { rest cure, 14\% }
$$

Mud baths, 165, 260

Mumpe, therapy of, 589

inflammation of the testicle, 580 suppuration of the parotid, 590 swollen glands, 589

Munich heart, 86

Muscular convulsions

in poisoning, acute, 568

in scarlet fever, 583

in whooping-cough, 611

Musk (see Moschus)

Mustard

as an emetic, 558

baths

in capillary bronchitis, 293

in rhinitis, 273

packs in capillary bronchitis, 293

plasters

in capillary bronchitis, 293

in congestion of the liver, 65

in laryngeal spasm, 281

in adema of the lungs, 325

in rhinitis, 274

poultices, 65

Myocanditis, therapy of, 8 \%

Myrrh, tincture of, 31, 272, 280

Myrtol, 304

Myxodema and cretinism, therapy of, 197 diet, 199

organotherapy, 198

thyroid extract, 198

thyroidism, 198 
Nanning's extract of quinine, 421

Naphthaline, 425, 508

Naphthalol, 426

Naphthol, 425

Narcotics

in capillary bronchitis, 294

in gastralgia, 460

in heart disease, table of, 54

in stomach and intestines, diseases of, 430

Nasal in tracheobronchitis, 284

feeding, 400

irrigations

in diphtheria, 596

in infectious diseases, 571

in scarlet fever, 579

Nauheim baths, 9

Neoplasms of the intestinal wall, 505

Nephritis

chronic interstitial, therapy of, 118

balneotherapy, 121

care of the heart, 122

climatology, 120

clothing, 120

diet, 119

drugs contraindicated, 119

hygiene during latent period, 120

in diphtheria, 600

luetic treatment, 118

in measles, 587

in pneumonia, 319

in scarlet fever, 581 prophylaxis, 581

baths, 581

diet, 581

medication, 581

treatment, 582

baths, 582

cathartics, 582

diaphoresis, 582

venesection, 582

in uric acid diathesis, 250

Nephrolithiasis, therapy of, 129

complications, 140

renal colic, 141

renal hemorrhage, 141

renal pain, 140

oxalurica 137

alkalies, 138

diet, 137

diuresis, 138

etiology, 137

phosphaturica, 138

urica, 130

alkalies, 132

artificial mineral waters, 134

bicarbonate of soda, 132

Carlsbad salts, 132

benzoic acid, 136

diet, 130

exercise, 132

glycerin, 135

hydrotherapy, 132

increased ingestion of liquids, 131 . 136

lemon cure, 136

lithium, 134

lysidin, 135

mineral waters, 132

piperazin, 135

urea, 136

uricedin, 134

Nerium oleander, 23

Nervous

diabetes, 226

diarrhœa, 503

dyspepsia, 466

climate, 467

diet, 467

etiology, 466

hydrotherapy, 467

in tuberculosis, 359

lavage, 467

medication, 467

eructation and pneumatosis, 461
Nervous eructation psychical treatment, 461

vomiting, 462

Nestlé's food, 376

Neuralgia in diabetes, 231

Neurasthenia, 615

prophylaxis, 615

after a previous attack, 616

in adolescence, 615

in adult life, 616

in childhood, 615

treatment, 616

climate, 622

diet, 622

diversions, 620

electrotherapy, 620

gymnastics, 620

hydrotherapy, 621

baths, 621

douches, 621

half baths, 621

packs, 621

in a sanatorium, 619

advantages, 619

disadvantages, 619

massage, 620

medication, 622

hypnotics, 623

sedatives, 623

tonics, 622

occupations, 620

physical and psychical examination, 616

psychical treatment, 616

hypnotism, 617 influence of physician, 617

symptomatic treatment, 618

Neuronal, 50

Neuroses

of the heart, therapy of, 93

palpitation, 93

paroxysmal tachycardia, 95

syncope, 96

of the stomach, therapy of (see Stomach neuroses), 458

Niemeyer's pill, 32

Night sweats in tuberculosis, 351

medication (see Tuberculosis, pulmonary), 351

Nitrites sponging, 351

in angina pectoris, 78

in arteriosclerosis, 76

Nitroglycerin

in angina pectoris, 78

in arteriosclerosis, 76

Nose and throat, diseases of the, therapy of the, 273

catarrhal pharyngitis, 276

follicular tonsillitis, 277

Nosophen, 424, 597

rhinitis, acute, 273

Nutrient enemas, 394

composition, 394

indications, 394

technic, 394

Nutrition, principles underlying the science of, 207

components of normal diet, 209

disposition of foods in the body, 208

heat values of separate food elements, 208

metabolic equilibrium, 208

mineral salts, 210

number of calories needed by the body, 208

qualitative analysis of ordinary articles of food, 210

specimen daily diet, 214

water, significance and amount of,

Nutrol, 423 210

Nutrose, 101

Nuts, digestibility of, 377

Nux vomica

as a stomachic, 419,360 
Nux vomica

in cancer of the ntomach, 435

in neuranthenia, 623

Obesity, therapy of, 234

bath, 239

cathartics, 240

complications, 240

abdominal plethora, 241

arterioncleronim, 240

cliabetes, 234, 241

uric acid diathesin, 241, 251

iet, 234

duily dietary, 239

reduction of futs and carbohydrates, 234 of liquids, 236s

effect of, on therapy of total amount of food, 234

exercise, 239 of pneumonia, 319

in conseguence

of ansenia, 241

of hereditary tendencies, 242

minera! waters, 230

of tuberculosis, 242

sleep, 239

thyroid extract, 240

two types of, 234

weights, table of normal

Landois's, 236

Nylic graphic, 237, 238

Quetelet'8, 235

Odontine, 268

Edema of the lungs, therapy of, 325 acute, 325 venesection, 325

chronic, 326

etiology, 325

in uramia, 126

Oertel's terraincur

in compensated valvular lesions, 5

in fatty overgrowth of heart, 85

in obesity, 239

in uric acid diathesis, 246

Oil

castor (see Castor oil)

cod-liver (see Cod-liver oil)

croton (see Croton oil)

enemas

in constipation, 494

in hypersecretion. 465

in ileus, acute, 482,486

in membranous enteritis, 476, 478

eucalyptus

in bronchitis, putrid, 305

in laryngitis, 279

Haarlem

in cholelithiasis, 527

in pyelitis and pyelonephritis, 143

of anise, 428

of birch, 143

of cade, 143

of juniper, 143, 279

of pine, 305

of turpentine

in bronchitis

purulent, 300

putrid, 304

in cystitis, 149

in hemorrhage in tuberculosis, 357

in laryngitis, 279

in phosphorus poisoning. 561

in renal pain, 140

olive (see Olive oil)

treatment, Cohnheim's

in gastric ulcer, 445

in mechanical insufficiency of the stomach, 456

wint ergreen, 363,547

Oils, ethereal

in bronchitis, purulent, 300

in laryngitis, 279

in tuberculosis of larynx. 280
Ointments

aniesthenin, 523

analgewic, 363

boric acud, BSt

bromocoll. 823

menthol. 822

mercurial, 897

Olizuris
in Bright's disean acute, 104
chronic, 118

Olive oil

\author{
in cholelithiaria, 525 \\ in dilatation of the stotnach, 456 \\ in kastric uloer, 445 \\ in tnembranous enteritis, 478
}

Opium

in aortic aneurinm, 82

in appendicitis $\$ 12$

in bronchitis, chronic, 298

in compensated symptomatic heart lesions, 69

in diabetes mellitus, 227

in diseases of the stomach and intestines, 430

in dysentery, 542

in gastralgia, 400

in gastritis, acute, 435

in Ileus, acute, 485

in intestinal catarrh, 472

in laryngiter, 47

in peritonitis, 517

in perityphlitis, 512

in pulmonary stanis, 58

in renal colic, 141

in tracheobronchitix, 284

in typhoid fever, 339

in uramia, 125

Orexin

in whooping-cough, 607

as a stomachic, 420

in hyposecretion, 466

Organic iron compounds, 161

Organotherapy

in leuksemia, 176

in myxodema, 198

in pancreatic diabetes without syphilis 226

in uremia, 129

Orphol, 426

Orthocresol, 87

Orthoform

in gast ralgia, $\mathbf{4 6 0}$

in gastric ulcer, 445

Osteomalacia, therapy of, 257 baths, 258

medication, 257

Outdoor treatment (see Fresh-air treatment)

Overgrowth of the heart, idiopathic, therapy of, 86

Ovoferrin, 161

Oxalate stones (see Nephrolithiasis oxalurica), 137

Oxaluria, therapy of, 137

Oxaphor, 328

Ox-gall, 522

Oxygen, inhalations of (see Inhalations of oxygen)

Oxyuris vermiformis, 508

cleanliness, 509

enemas, 508

itching of anus, 509

santonin, 508

Ozone in whooping-cough, 601

Packs

in Bright's diseace, acute, 107

in bronchitis, 292 technic, 292

in infectious disenses, 571 technic, 571

in neurasthenia, 621

in pneumonis, 317

in typhoid fever, 536

mustard, 293 
Pain, relief of

in arteriosclerosis, 77

in Bright's disease, acute, 99

in bronchitis, acute, 295

in bronchopneumonia, 322

in cancer of the stomach, 452

in congestion of the liver, 65

in gastric ulcer, 445

in gastritis, acute, 434

in gout, acute, 251

in pneumonia, 313

in poisoning, acute, 568

in pulmonary infarct, 324

in tracheobronchitis, 287

in tuberculosis of the larynx, 281

Painting of the throat, 596,603

Palpitation of the heart, 71, 93

Pancreatin

in Bright's disease, chronic, 109

in cancer of the stomach, 451

in diseases of the stomach and intestines, 422

in pancreatic diabetes without syphilis, 226

Pancreon

in diseases of the stomach and intestines, 422

in hyposecretion, 466

Papain, 421

Papayotin, 421

Paquelin cautery in arteriosclerosis, 77

in endocarditis, 89

in rheumatism, acute articular, 551

Paraform, 574

Paraldehyde

in pneumonia, 320

in poisoning, acute, 568

Paralysis

in uncompensated heart lesions, 50

after diphtheria, 601

of the bladder, 150

Parasitic diseases, therapy of ascaris lumbricoides, 507 oxyuris vermiformis, 508

Paregoric, 585 tapeworm, 506

Parotid, suppuration of the, in mumps, 590

Parotitis (see Mumps), 589

Paroxysmal tachycardia (see Tachycardia, paroxysmal), 95

Parry's disease (see Exophthalmic goitre), 199

Parsley water, 365

Passive cerebral hyperæmia, 47

$$
\begin{aligned}
& \text { bleeding, } 55 \\
& \text { cupping, } 55 \\
& \text { leeches, } 56 \\
& \text { sleeplessness, } 47 \\
& \text { table of hypnotics, } 54 \\
& \text { symptoms, } 47
\end{aligned}
$$

Pasterin tablets, 609

Pastillæ anginosæ asepticæ, 270

Pearson's solution, 169

Pelleterine, 507

Pellotine, 53

Pemmicon, 373

Penzoldt's diet table, 385

Peppermint, 428, 504

Pepsin, 421

Peptomanganate of iron, 161

Peptones, meat, 373

Pericardiotomy, 92

Pericarditis, therapy of, 90

local cold, 90

puncture of the pericardium, 91

rest, 90

salicylates in rheumatic pericarditis, 91

Pericardium symptomatic medication, 91

adherent, therapy of, 93

puncture of, 41,91

Peritoneum, diseases of the, therapy of, 511 peritonitis

acute, 517

chronic tuberculous, 518

Peritonitis, therapy of

perityphlitis (appendicitis), 511

acute, 517
Peritonitis, acute

fibrinous suppurative, 517

internal therapy, 517

diet, 517

heat and cold, 518

opium, 517

surgery, 517

perforative, 517

surgery, 517

chronic tuberculous, 518

alcohol dressings, 518

bandages, 518

potassium soap, 518

rest, 518

surgery, 519

Perityphlitis, therapy of, 511

medical treatment, 511

cold, 513

colon irrigations, 512

convalescence, 513

diet, 511

heat, 513

morphine, 512

opium, 512

rest, 511

surgery, indications for, 514

Permanganese, 423

Pernicious anæmia, 168

arsenic, 169

administration of, 169

poisoning, 169

preparations, 169

waters, 170

bone marrow, 171

colon irrigations, 169

diet, 169

salt and carbonated baths, 171

symptomatic therapy, 174

transfusion and infusion, 171 infusion of saline intravenous, 173 subcutaneous, 173

transfusion

direct intravenous, 172

Peronin indirect intravenous, 172

in laryngitis, 279

in tracheobronchitis, 285

in whooping-cough, 607

Peroxide of magnesia, 423

Pertussin, 611

Pertussis, therapy of (see Whooping-cough), 601

Pharyngitis, acute catarrhal, therapy of, 276 applications, external, 276

gargles, 276

peritonsillitis, abortive treatment of, 276

Phenacetin

in angina pectoris, 80

in aortic aneurism, 84

in gout, acute, 252

in influenza, 544

in rheumatism, acute articular, 549

in tuberculosis, 350

Phenocoll

in gout, acute, 252

in rheumatism, acute articular, 549

Phenol-kresol, 602

Phosphate stone (see Lithiasis phosphaturica), 138

Phosphates in tuberculosis, 349

Phosphaturia, therapy of, 254

diet, 254

etiology, 254

medication, 254

inorganic acids, 254

Phosphoric acid, 139

Phosphorus

chocolate tablets, 256

in leukæmia, 176

in osteomalacia, 257

in rickets, 256

poisoning, 561

with cod-liver oil, 257 
Physostigmine, 481, 482

Pica chlorotica, 153

Pig'n liver, extract of, 227

Addison' $H, 32$

Axiatic, 174

Blaud' 's, 158, 159

Kleewein's, 497

Krewel's blood, 162

laxative, 36

Niemeyer's, 32

Trousseau's, 37

Vallet's, 150

Pilocarpine

in Bright's dinease, acute, 107

in diphtheria, 508

in hydrops in broken compensation, 37

pilula aloes et ferri, 130

Pine, oil of, 305

Piperazine

in nephrolithiasis urica, 135

in uric acid diathenis, 247

Pix liquida, 149

Plasmon, 101

Plethora

abdominal, 24!

true, therapy of, 183 constipation, 183

exercise, 183

leeches, 183

mineral waters, 183

venesection, 183

Pleura, diseases of the, therapy of, 363 pleurisy
acute fibrinous, 363

fibrous, 368

purulent and suppurative, 369

with effusion, 364

Pleurisy pneumothorax, 369

fibrinous, acute, 363

analgesic applications, 363

breathing exercises, 364

cupping, 363

diaphoresis, 364

hydrotherapy, 364

leeches, 363

morphine, 364

st rapping, 364

fibrous, 368

purulent and suppurative, 369

surgical interference, 369

with effusion, 364

breathing exercises, 368

climate, 368

derivatives, 364

diaphoresis, 365

diet, 365

Schroth's cure, 360

diuresis, 365

puncture, 366

indications for, 360

technic, 367

sodium salicylate, 365

unabsorbed residuum, 368

Pneumatic chambers

in bronchitis, chronic dry, 300

in emphysema of the lungs, 326

in pleurisy, fibrous, $36 \mathrm{~s}$

Pneumatosis (see Nervous eructation), 461

Pneumonia, therapy of, 312

croupous, 312

atypical, 315

cardiac weakness, 315

alcohol, 315

digitalis, 315

strychnine, 315

venesection, 315

high fever, 316

baths, 316

delirium 313

packs, 317

quinine, 316

sponge baths 317

infusions and transfusions, 318
Preumonia, therapy of, croupous complicasions in, 319

dizeative disturbances, 319 nephritis, 319

pre-exinting conditions infuencing counce of

alcoholism, 320

delirium tremens, 320

Bright's dimeave, 310

diabetes, 310

heart diveave, 319

obesity, 319

protection againut recurrence, 320

iypical, 312

crimis, 313

diet, 314

expectorants, 314

Iresh air treatment, 314

hygiene, 314

pain, relief of, 313

serum, 312

sleeplessners, 313

interstitial. 322

care of the heart, 323

hemorrhaze, 323

prophylaxis, 322

Pneumotherapy

in bronchitis, 300

in einphysema of the lungs, 326

in heart disease, 70

in pleurisy, fibrous, 368

in rickets, 257

Pneumothorax, therapy of, 360

cupping. 360

morphine, 360

mustard plasters, 309

Podophylin

puncture, 369

in constipation, 497

in hydrops in broken compensation, 37

Poisoning, acute, therapy of, 357

classification of poisons, 557

elimination of the poison, 5.55

catharsis, 559

diuresis, 560

emesis, 558

apomorphine, 55

copper sulphate, 5.59

mustand powder, 538

lavage, 558

neutralization of the poison, 560

acid poisoning, 560

alkali poisoning, 360

alkaloid poisoning, 563

arsenic poisoning. 562

carbolic acid poisoning, 563

cyanide poisoning. 363

halogen poisoning, 560

metal poisoning, 562

phosphorus poisoning. 561

symptomatic therapy, 563

alteration of the blood, 568

cardiac insufficiency, 364

heart stimulants, 564

heat, 564

cerebral paralyeis, 567

muscular convulsions, 568

pain, 568

respiratory weakness, 565

artificial respiration. 366

atropine, $56 \pi$

reflex stimulation of respiration, 567

removal of obstructions to breathing.

Poisons, claseification of, 557 565

Pomerranate, 50

Post-bemorrhagic anamia, 178

Post-infectious anamia, 1S0

Potassium

acetate

in Bright's disease, acute, 10.5

in hydrops in broken compensation, 32 
Potassium

bitartrate

in Bright's disease, chronic, 117

in diphtheria, 599

in pulmonary congestion, 58

bromide (see Bromides)

chlorate

as a mouth wash, $30,187,272$

in cystitis, 149

citrate, 247

iodide

in alkaloid poisoning, 563

in aortic aneurism, 82

in arteriosclerosis, 75

in asthma, bronchial, 309

in Bright's disease, chronic, 118

in congestion of the kidney, 67

in gout, acute, 253

nitrate, 32

permanganate, 561,563

salts in scurvy, 187

soap, 518

tartrate, 106, 499

tellurate, 351

Poultices

in gastric ulcer, 446

in hyperæmia of the liver, 65

in pericarditis, 91

Powder

composite licorice, 496

Dover's, 290

Seidlitz, 498

Powders

for dusting, 353, 523

for insufflation (see Insufflation powders) tooth, 268

Priessnitz's application

in Bright's disease, acute, 10

in bronchitis, chronic dry, 298

in diseases of the stomach and intestines, 406

in gastritis, acute, 434

in pericarditis, 91

in tracheobronchitis, 287

Prolapse of rectum in whooping-cough 612

Prophylactic therapy of

bronchitis, acute, 295

diphtheria, 590

hemorrhagic pulmonary infarct, 324

laryngitis, 279

nephritis in scarlet fever, 581

neurasthenia, 615

pneumonia, interstitial 322

rickets, 254

scarlet fever, 577

tuberculosis, 343

Proteids uric acid diathesis, 244

choice between animal and vegetable, 210

heat value of, 208

in pulmonary tuberculosis, 329

table of foods rich in, 233

Pruritus (see Itching)

Pseudocroup, therapy of, 280

between attacks, 280

single attacks, 280

Pseudoleukæmia (see Leukæmia), 174

Psychotherapy

in Bright's disease, 129

in gastralgia, 461

in neurasthenia, 616

in tuberculosis, 358

in whooping-cough, 609

Ptyalin, 422

Pulmonary

abscess, therapy of, 324

congestion (see Pulmonary stasis), therapy of, 57

emphysema, therapy of, 326

hemorrhagic infaret, therapy of (see Infarct,

hemorrhagic, pulmonary), 323

cedema, therapy of (see CEdema of the lungs), 325

sclerosis (see Pneumonia, interstitial), 322

stasis in broken compensation, 57
Pulmonary stasis in broken compensation cough, 58 belladonna, 59

codeine, 59

dionin, 59

heroin, 59

hyoscyamus, 59

opium and morphine, 59

dyspncea, 60

expectorants, 58

hæmoptysis, 60

ergotin, 60

ergotinin, 61

venesection, 61

revulsives, 58

symptoms, 57

tuberculosis (see Tuberculosis, pulmonary), 329

Puncture

of the abdomen

in atrophic cirrhosis of the liver, 529

in Bright's disease, acute, 108

in hydrops in broken compensation, 39

of the intestines

in flatulency, 505

in ileus, acute, 487

of the lower extremities, 38

of the pericardium

in hydrops in broken compensation, 41

in pericarditis, 91

of the thorax, 41 technic, 92

indications for, 366

technic, 367

Purgative lemonade, 433

Purgen, 498

Purges, 35, 430, 500

Purging

in Bright's disease acute, 108

chronic, 117

in hydrops in broken compensation 35

in poisoning, acute, 559

in uræmia, 123

Püro, 243, 372, 576

Purpura hemorrhagica, therapy of, 185

calcium chloride, 186

gelatin, 186 styptics, 185

Pyelitis and pyelonephritis, therapy of, 142

antiseptics, 142

astringents 143

diet, 145

etiology 142

indications for operation, 145

ingestion of liquid, 144

irrigation of pelvis of kidney, 145

mineral waters, 145

revulsives, 142

Pyramidon

in angina pectoris, 80

in aortic aneurism, 84

in gastralgia, 461

in tuberculosis, 350

in typhoid fever, 537

Pyrosis therapy of, 462

alkalies, 463

Quantitative analysis of ordinary articles of food, 214

Quarantine

in diphtheria, 590

in infectious diseases in general, 573

in measles, 584

in scarlet fever, 577

in whooping-cough, 614

Quassia, 419

Quebracho, 326

Quetelet's tables, 235

Quillaja, 326

Quinic acid compounds, 248 


\section{INDEX}

Quinine

us a stomachic, 419,421

bromate, 204

chloride of iron, 167

ferrocitrate, $16 \%$

hydruchlorate, $410,5.53$

iii broken conpersation, 23

III chloromin, 1 tiz

ut dyentery, 541

in endocarditin, acute, 88

in hemorrhage in tuberculowis, 357

its uffectious dimeanew in general. 560

in leukamia, 176

in inalaria, 552 mlministration, $55 \cdot 2$

in preumonia, 316

in scarlet fever, 377

in tonsillitin, 27\%

in typhoid fever, 337

in whooping-cough, 103

Nanning's extract of, 421

sulphate, 5.53

tannate, 385,587

valerianate, 72

Rachitis (see Rickets), 25-4

Radiotherapy

in exophthalmic goitre, 204

in leukmmia, 177

in scrofula, 195

Rashes following the adminimtration of diphtheritic antitoxin, 594

Rectal tube in flatulency, 505, 540

Rectum, introduction of liquids by the, 394, 396

Reduction of fluids

in compensated valvular lesions, 3 in obenity, 236

Reflex stimulation of respiration, $36 \%$

Removal of obstruction to breathing, 565

Renal

colic, therapy of, 141

at ropine, 141

chloroform, 141

hot baths, 141

hemorrhage, 141

morphine, 141

pain, 140

belladonna, 140

hot applications, 140

Resorcin turpentine liniment, 140

as an intestinal antiseptic, 139, 361, 426, 587

in thrush, 273

Respiration, artificial, 566

reflex stimulation of, 567

Respiratory symptoms

in broken compensation, 58

in compensated symptomatic valvular lesions, 69

in diphtheria, 600

in measles, 586

in poisoning, acute, 565

Rest

in whooping-cough 611

after eating, 381

cure (see Rest cure)

in aortic aneurism, 81

in Bright's disease, 99

in broken compensation, 11

in chlorosis, 15.5

in diphtheria, 592

in dysentery, 542

in exophthalmic goitre 199

in gastric ulcer, 443

in gast roptosis, 457

in leuksemia, 177

in movable kidney, 147

iu pericarditis, 90

in peritonitis acute, 518 chronic 518

in perityphlitis, 511

in pleurisy, 363

in rheumatism, acute articular, 551 llent

in rickets, 255

in tuberculosis, 33

Reot cure, Weir Mtiteliell's

in diseares of the stomsch and intestives, 387

in ermaciation, 243

in movable kudney; 14 ;

Hevulsiven technie of, 387

in ankina pectoris, 81

in purnonary menis, 88

in pyelitis and pyelonephritis 142

Khamnus, 495

Rlieumatisu, therspy of

acute urticular 54

diet, 551

hydrotherapy, 350

alcobol bandages, 851

baths, 550

comprenses, 550

hygiene. 5.51

position, 351

rent, 351

temperature of room, 551

local therapy, 550

Bier's elastic bandayge, 5.50

injections of cartolic acid. 550

Paquelin cautery, 5.51

relapses, 352

calicylates, 544

contraindications to, 548

list of

acetopyrin, 350

agathin. 5it

aspirin. 34

malakin, 547

maretin, 54

mesotan. 546

vil of wintergreen, 345

salicetol, 547

salicyl-varogen, 346

salicin 348

salicylic aciul, 544

saligenin, 347

salipyrin, 350

salophen, 547

sodium salicylate, 545

substitutes for, 549

amygdophenin, 549

analgen, 550

antipyrin, 549

colchicum, 550

lactophenin, 549

phenacetin 549

phenocoll, 549

secondary ansemia in 180

chronic, 259

applications

analgesic, 265

cold. 264

baking. 263

baths, 259

clothing. 265

electricity, 264

hot springs 259

massage, 26-4

medication, 265

Rhinitis therapy of 273

coryza
averting the, 273
mustand baths and plasters. 273,274

recomotoria 275

etiology, 273

in measles, 584

symptomatic therapy, 275

clearing out nasal pasages 275

adrenalin. 275

cocaine, 275

Rhubarb

neuralgic headaches 275

as a cathartic, 495

as a stomachic, 420 
Rickets, therapy of, 254

baths, 255

climate, 255

diet, 255

medication, 256

bone marrow, 257

calcium, 256

cod-liver oil, 256

phosphorus, 256

prophylaxis, 254 rest, 255

hardening process, 255

Rodagen, 203

Round worms (see Ascaris lumbricoides), 507

Rubs

in chlorosis, 156

in infectious diseases, 571

in leukæmia, 178

Saccharine, 224

Salacetol, 425,547

Salicin, 419, 548

Salicylates

in influenza, 544

in rheumatic pericarditis, 91

in rheumatism, acute articular, 544

Salicylic acid contraindications, 548

in diseases of stomach and intestines, 423,425

in jaundice, catarrhal, 523

in rheumatism, acute articular 544 chronic, 265

in stomatitis, 27

in tuberculosis, 353

Salicyl-vasogen, 546

Saligenin, 547

Salinaphthol, 426

Saline cathartics, 433,499

enemas (see Enemas, saline)

Salipyrin, 252, 550

Salol

in diseases of stomach and intestines 425

in intestinal catarrh 470

in lithiasis phosphaturica, 139

in phosphaturica, 254

Salophen, 544, 547

Salt

as a condiment, 378

baths (see Baths, salt)

free diet

choice of food in, 114

in Bright's disease, 113

in compensated valvular lesions, 4

in uncompensated valvular lesions, 12

infusions (see Infusion)

solution in hemorrhage in tuberculosis, 357

waters

in constipation, 501

table of 412

Salts, Carlsbad, 418, 498

Sambucus (see Elderberry)

Sanatogen 101

Sanatorium treatment

in diseases of the stomach and intestines, 415

in neurasthenia, 619

in tuberculosis, 331342

Sandow's bromides, 201

Santonin

in ascaris lumbricoides, 507

in oxyuris vermiformis, 508

Saprol, 574

Scarlet fever, therapy of, 577

complications and sequelæ, 578 affections of the ears and conjunctiv 581 of the heart and lungs, 581

arthritis, 581

cold, 581

immobilization, 581

salicylates, 581
Scarlet fever complications

glandular swellings, 580

nephritis, 581

prophylaxis, 581

baths, 581

diet, 581

medication, 581

treatment, 582

catharsis, 582

diaphoresis, 582

hot baths, 582

venesection, 582

severe sore throat, 578

local treatment, 578

adrenalin, 579

antisepsis of the mouth, 578

carbolic injections, 579

insufflations, 579

irrigations, $\mathbf{5 7 9}$

swabbing, 579

serum treatment, 580

diphtheritic, 580

Marmorek's, 580

Moser's, 580

uræmic convulsions, 583

chloroform, 583

convalescence, 583

prophylaxis, 577

uncomplicated cases, 577

hydrotherapy, 577

Schott movements, 45

medication, 577

Schroth's cure in mechanical insufficiency of the

in pleurisy with effusion, 366

Scopolamine, 308

Scrofula therapy of, 187

climatology, 190

diathesis, torpid or erythric, 188

diet, 188

hydrotherapy, 191

salt baths, 192

sea baths, 191

local treatment, 193

medication, 189

cod-liver oil, 189

American preparations, 194 creosote, 189

tuberculin, 190, 195

mineral waters 193

radiotherapy, 195

relations to pseudoleukæmia and tuberculosis, 187

secondary anæmia in, 180

soft soap treatment, 190

Scurvy, 186

surgical treatment 197

diet, 186

douches, 187

medication, 187

stomatitis, 187

Sea

styptics, 187

baths 191

climates and resorts, 338

voyages, 338

Secondary anæmia (see Anæmia, secondary), 178

Sedatives

in diseases of the stomach and intestines, 430

in neurasthenia, 623

Seidlitz powder, 498

Senega, 294

Senna, 499

Serum

antistreptococcus, 312578

artificial, 318

diphtheritic

in asthma, 309

in diphtheria 591592

in exophthalmic goitre, 204

in pneumonia, 312

in scarlet fever, 580 . 
Sierum

in tetanus, 555

Marmorek' $\%, 580$

Moser's, 580

Shepherd's purse, 350

Shigs's dymentery, 54:

sick chamber

disinfection of, 875

hygiene of

in infectious direares in general, 573 in measles, 584

in whooping-cough, 613

Sidonal, 248

vilver nitrate

in cystitis, 150

in dysentery, 541

in gastric ulcer, 444

in intestinal catarrh, 473

simple alkaline waters, $40^{-}$

sirolin, $\mathbf{3 4 7}$

Sirop des cinque racines, 35

Sitz baths, 404, 491

skin, care of

in diabetes mellitus, 220
in scrofula, 190

in tuberculosis, 34:

Sleep in obesity, 239

Sleeplessnexs

in hydrops in broken compensation, 47

anylene hydrate, 52

bromides, 48

chloralose, 5:

cordol, 53

dormiol, 52

hedonal, 51

isopral. 53

neuronal, 50

paraldehycle, 50

pellotin, 53

sulphonal, 49

tetronal, 50

trional, 50

urethane, 51

in pneumonia, 313

veronal, 50

table of hypnotics, 54

Smoke-powder, 308

Smoking

in compensated valvular lesions, 4

in constipation, 491

in diarrhcea, 504

in diseases of the stomach and intestines, 382

in tuberculosis, 354

Sodium

benzoste

in Bright's disease, acute, 103

in bronchitis, purulent, 302

in pyelitis and pyelonephritis, 143

in whooping-cough, 605

bicarbonate

in chlorosis, 153

in diabetes, 227

in diphtheria, 596

in diseases of the stomach and intestines, 417

in gastritis, acute, 433

in nephrolithiasis urica, 132

borate

in uric acid diathesis, 24

in nephrolithiasis phosphaturia, 13

in stomatitis, 272

in thrush, 273

cacodylate, 176,348

chloride (see Salt)

cinnamate, 348

jodide (see Iodides)

nitrite

in arteriosclerosis, 76

in hydrops in broken compensation, 32 phosphate

in exophthalmic goitre, 202

in jaundice, 521

in pneumonia. 318
Bodiurn

salicylate

as an antipyretic, $\mathbf{5 7 0}$

in cholelithisis, 527

in kout, acute, 252

in jaundice, catarrhal, 522

in pleurisy. 305

in rheurnatiam, acute articular, 545

in whooping-cuugh, 605

sozoiodol, 597

sulphate

in constipation. 496

in pneumonis, 318

sulphite, 305

tannate, 102

tartrate, 497

cellurate, 351

Soft soap, rubbing with

in scrofula 190

Solutol, 574

in tuberculous peritonitis, 518

Solveol, 347, 574

Somatose

meat, 373

milk, 375

Sorisin, 347

Spartein, 19

Spirits of ether, 25

Sponge baths

in exophthalmic goitre, 200

in infectious disesses, 37

in pneumonis, 317

in scarlet fever, 577

in tuberculosis, 351

in typhoid fever, 536

Spongy gums, 30

Spraying of the thiroat, 595

Squills, 31

Starch as an antidote in halogen poisoning. 5e0

Starvation (see Fasting)

Stasis (see Congestion), $4 i$

Steam

baths

in Bright's disease, acute, 10 -

in diphtheria, $\mathbf{5 9 8}$

in hydrops in broken compensation, 42

in tracheobronchitis, $2 \times 3$

in the sick-room, 294, 584, 598,600

intestinal (nee Intestinal obstruction), 4.(x)

laryngeal, in diphtheria, 598

Stimulants, heart (see Heart stimulants)

Stokes's mixture, 294, 533, 534

Stomach and intestines, diseases of the, zeneral therapy of, 370

abdominal exercive, 402

climate, 415

diet, 370

cures, 390

in anacidity, 392

in hyperacidity. 390

in hypoacidity, 392

in mechanical insuficiency, 393

digestibility of different foods. 370

Ieube's ulcer cure. 384

Weir Mitchell rest cure, 38\%

electro-massage, 404

elect rot herapy, 403

hydrotherapy, 404

applications, 405

Priessnitx's 406

baths, 404

douches, 400, 404

Winternitz's procedure, 405

irrigations

colon, 396

stomach, 399

lavage, 397

maseage, 400

automascage, 402

ceneral, 400

of the abdomen, 401

of the stomacl, 400

medication, $\$ 15$ 
Stomach and intestines, medication alkalies, 417 antiseptics, 423,424

carminatives, 428

cathartics, 429

digestive ferments, 421

hydrochloric acid, $\mathbf{4 1 5}$

narcotics, 430

sedatives, 430

stomachics, 418

mineral waters, 406

classification, 406

alkaline, 407

alkaline chloride, 407

alkaline saline, 407

bitter, 411

calcium, 414

carbonate, simple, 406

common salt, 411

nasal feeding, 400 ron, 415

nutrient enemas, 394

congestion of the, 67

diseases of the, therapy of the, 432

cancer of the stomach, 449

gastric neuroses, 458

gastric ulcer, 439

gastritis

acute, 432

chronic, 435

gastroptosis, 456

mechanical insufficiency, 452

douches, 400,404

neuroses of the, 458

gastralgia, 459

hypersecretion, 463

hyposecretion, 465

nervous

dyspepsia, 466

eructation and pneumatosis, 461

vomiting, 462

Stomachics, 418

pyrosis, 462

alcohol, 421

cinchona, 419

condiments, 421

cortex condurango, 420

gentian, 419

orexin, 420

quassia, 419

quinine, 419,421

rhubarb, 420

salicin, 419

strychnine, 419

Stomatitis, therapy of

aphthous, 272

catarrhal, 271

in diabetes, 230

in measles, 584

in scurvy, 187

in tuberculosis, 360

mercurial, 272

ulcerative, 271

Stone, kidney (see Nephrolithiasis), 129

Stool, regular time for, 491,504

Stramonium

in asthma, 307

in tracheobronchitis, 286

Strapping of chest

$$
\begin{aligned}
& \text { in bronchitis, acute, } 295 \\
& \text { in pleurisy, } 364 \\
& \text { in pneumonia, } 313
\end{aligned}
$$

Stricture, intestinal (see Intestinal obstruction), 480

Strophanthin, 18

Strophanthus, 18

Strontium, 103

Strychnine

as a stomachic, 419

in broken compensation, 23

in diphtheria, 592

in mechanical insufficiency of the stomach, 455

in pneumonia, 315

in poisoning, acute, 565
Styptics

in hæmophilia, 184

Styptics

n purpura hemorrhagica, 185

in scurvy, 187

in tuberculosis, 355

Sublingual ulcer in whooping-cough, 612

Sugar

digestibility of, 377

in constipation, 489

milk, as a diuretic, 33

substitutes for, 224 crystallose, 225

dulcin, 225

saccharine, 224

Sulphonal, 49

Sulphur

in chlorosis, 168

in constipation, 496

waters

in bronchitis, chronic, dry, 299

in constipation, 501

table of, 182

Sulphuric acid, 202

Sun baths, 262

Supports for the heart, 95

Surgery

in adherent pericardium, 93

in aortic aneurism, 83

in atrophic cirrhosis of the liver, 529

in bronchitis, putrid, 306

in cancer of the stomach, 449

in cholelithiasis, 526

in diphtheria, 599

in exoplithalmic goitre, 203

in gastric ulcer, 449

in gastritis, chronic, 438

in hydrops in broken compensation, 38

in ileus, acute, 487

in intestinal

$$
\text { stenosis, } 480
$$

ulcer, 479

in mechanical insufficiency of the stomach, 456

in osteomalacia, 259

in pericarditis, 91

in peritonitis

acute, 517

chronic tuberculous, 519

in perityphlitis, 514

in pleurisy, suppurative, 369

in pneumothorax, 369

in pyelitis and pyelonephritis, 145

in scrofula, 197

Sweat

in uræmia, 128

baths, 107

cure in diphtheria, 598

Sweating (see Diaphoresis)

Swedish movements, 5

Symptomatic compensated valvular lesions (see Valvular lesions, symptomatic compensated), 68

Syncope, therapy of, 96

Syncope in pulmonary infarct, 324

Syphilis, secondary anæmia in, 180

Syphilitic

arteritis, 81

diabetes, 226

Syrup of the iodides of iron, 159

Table

of diet for mild cases of diabetes, 217

of foods

containing no carbohydrates, 217 containing small amounts of carbohydrates, 219

rich in fat and proteid and poor in carbohydrates, 223

of hot springs, 249

of iron preparations, 159

of mineral waters alkaline chloride, 408

saline, 409

arsenic, 170 
Table

of mineral waters

hitter, 114

calcium, 146

colnmon salt, 412

iron, 163

iron and armenie, 170

sulphur, 182

of ordinary daily diee, 214

of Penzolde' diet lime, 385

of quantitative analysis of foods, 211

of resorts

at high altituden

for summer and winter, 333

for winter, 333

at low altituilew, 337

at lower mountain altitudes for wprimg and tall, 335

for muinmer and winter, 336

of substitution values amons carbohydrates. 218

of time requireal for digention of different forxl:s, 382

of weixhts and heights, normal 235236 , 237.238

Tachycardia, paroxymal, 95

Tienia (see Tapeworm), 306

Taka-diastase, 42:2

Tallermann's apparatus, 2ti3

Tasnarind, 498

Tamnalbin

in Bright's disease, scute, 103

in cliarrhoea, 503

in intestinal

$$
\text { catarrh, } 472
$$

Tannigen ulcer, 479

in intestinal catarrh, 472

in measles, 587

in uramic vomiting, 125

Tannin

in Bright's disease, acute, 102

in intestinal catarrh, 472

irrigations in dysentery, 541

Tannoform, 352, 597

Tannopin, 587

Tapeworn, therapy of, 506

$$
\text { tapeworin cure, } 506
$$

Tartar

$$
\text { male fern, } 506
$$

cream of, in Bright's disease, acute, 10i

emetic

in diphtheria, 599

Taurine, 37.2

in pseudocroup, 280

Tea

Chambard, 500

diaphoretic, 283

diuretic, 32, 106

in constipation, 490

in diseases of the stomach and intestines, 380

in poisoning, acute, 564

pekoe tea, 380

Russian, 541

St. Germain, 500

Teeth, care of the, 267 powders, 268 soaps, 268

Temperature of fool. 381

Tepid baths (see Baths, tepid)

Terpin hydrate, 300, 304

Terpinol, 300

Terrain cure, Oertel's (see Oertel's terrain cure)

Test diet in diabetes, 215

Testicles, inflammation of, in mumps, 359

Tetanus therapy of. 555 antitoxin 555

curative use, 556

prophylactic use, 555

carbolic acid, $\mathbf{5 5 6}$

cauterization, 556

feeding. 557

magnesium sulphate, injections of, $55 \%$ narcotics, 557

quiet, 5.57
Tetronal. 80

Theobromine

in angina pectorim, 80

in bydrops in bruken compenmation, 38

Theocine, 20

Theophylline, 29

Thermal watern table of, 249

'Thermophor, 65, 72405

Thiocol, 347

Thioform, 597

Thiol, 005

Thiosinamin, 443

Thirnt, methods of relieving, 432, 440,512

Thoracocentesis

in hydropa in broken compensation, 11

in pleurisy. 366 indications, 360

in pneumochnic, $30 \%$

Throat, diseases of the, therapy of (nee Nose and throat, diveaves of the, therapy of), 276

local treatmetst of (nee Local

Thrush, 273 treatment)

Thymol, 305, 309

Thymus gland, 203

Thyroid extract

in exophthalmic goitre, 203

in myxaedems and cretinism, 198

in obesity, 240

Thyroidin, 198

Thyroidism, 198

Time required for digestion of different foods, 382

Tinctura ferri oxidati, 160

Tineture of chloride of iron, 159

Tobacco (see Smoking)

Toluol, 596

Tonics in neurasthenia, 622

Tonsillitis, therapy of, 277

local applications, $27 i$

carbolic acid injections, 277

medication, quinine, 277

Tooth powders, pastes, and suaps, 268

Tophi, 249

Tracheobronchitis, acute, therapy of, 283 compresses, 287

crossbinder, 289

Priessnitz's, 28:

cough, 284

from the throat, 287

mucilaginous drinks, 287 narcotics, 284

narcotics
diaphoresis, 283
inhalations, 284

mineral waters, 284

pain, retrosternal, 287

secretion

abundant, thin, 290

apomorphine, 290 euporphine, 290

viscid. 289

ipecac, 289

Tragacanth, 561

Transfusion

in pernicious anamia, 171

in pneumonia, 318

technic of

direet, 172

indirect, 172

Trional, 50

Trousseau's pills. 37

Trudeau's method of using tuberculin, 196

Trunecek's serum, 76

Tuberculin

in serofula, 190

in tuberculosis, 348

method of administration, 195

Tuberculosis

of the larynx, therapy of, 280 cthereal oils, 280

pain, relief of, 281

pulmonary therapy of, 329

after treatment, 3 it

as a cause of obesity, 242

climate. 332 
Tuberculosis, pulmonary, climate

high mountain altitudes, 332

low altitudes, 337

lower mountain altitudes, 335

seashore, 338

sea voyages, 338

diet, 329

tummary of effect of 340

alcohol, 330

fats, 329

milk, 329

proteids, 329

exercise, 331

fresh-air treatment, 330

at home, 331

hygiene, 342

in a sanatorium, 331

clothing, 342

skin, care of the, 342

in diabetes, 231

marriage, 362

medication, 344

arsenic, 348

cinnamic acid, 347

cod-liver oil, 349

copper, 348

creosotal, 346

creosote, 344

guaiacol, 345

heterocresol, 348

hetol, 348

salts, 349

sirolin, 347

solveol, 347

sorisin, 347

thiocol, 347

tuberculin, 348

prophylaxis, 343

breathing exercises, 343

diet, 344

hydrotherapy, 343

secondary anæmia in, 180

symptomatic therapy

cough and expectoration, 353

crossbinder, 354

expectorants, 354

narcotics, 353

psychotherapy, 358

smoking, 354

digestive disturbances, 359

anorexia, 359 mineral waters, 360

stomachics, 360

dyspepsia, nervous, 359

intestinal disturbances, antifermen-

fever 349 tatives, 361

antipyretics, 350

antifebrine, 350

antipy rin, 350

maretin, 351

phenacetine, 350

diet, 350

yramidon, 350

hydrotherapy, 351

hæmoptysis, 354

binding of extremities, 357 diet, 358

expectorants, 357

hæmostatics, 355

ergot, 355

gelatin, 357

hydrastis, 356

lead acetate, 356

oil of turpentine, 357

quinine 357

salt solution, 357

shepherd's purse 356

witch hazel, 356

local cold, 354

narcotics, 355

rest, 354
Tuberculosis, pulmonary, therapy of, hæmoptysis stoo 18,358

insomnia, 358

night sweats, 351

medication, 351

agaricin, 352

atropine, 352

camphoric acid, 352

ergot, 352

eumydrin, 352

formalin, 352

guacamphor, 352

hydrastis, 352

potassium and so-

dium tellurate, 351

salicylate powder, $\mathbf{3 5 3}$

tannoform, 352

zinc peroxide, 353

sponging, 351

Tuberculous glands (see Scrofula)

Tuffnell's diet scheme, 81,82

Turpentine

in bronchitis, 300, 304

in cystitis, 149

in hemorrhage in tuberculosis, 357

in laryngitis, 279

in phosphorus poisoning, 561

in renal colic, 140

stupes in typhoid fever, 540

Tussol, 604

Tympanites in typhoid fever, 540

Typhoid fever, therapy of, 531

convalescence, 540

diet, 531

alcohol, 535

broths, 531

carbohydrates, 532

differences of opinion in regard to, 532

large amounts of liquid, 535

milk, 531

night feeding, 534

hydrotherapy, 535

baths

cold, $\mathbf{5 3 6}$

cool, 535

packs, cold, 536

hygiene, 538

bedsores, 539

mouth, care of the, 538

medication, 537

antipyretics, 537

pyramidon, 537

calomel, 538

relapses, 540

symptomatic therapy, 539

cerebral symptoms, 539

intestinal symptoms, 539

diarrhœe, 539

hemorrhages, 540

meteorism, 540

Ulcer

gastric, therapy of, 439

after-treatment, 448

diet, 440

Lenhartz's treatment, 442

fasting, 439

hæmatemesis, 447

medication, 443

alkalies, 443

belladonna, 443

bismuth, 443

mineral waters, 443

olive oil, 445

silver nitrate, 444

pain, relief of, 445

anxsthesin, 445

cold, 446

heat 446

orthoform, 445

recurrent ulcer 448

rest, 442

surgery, 449

intestinal (see Intestinal ulcer), 478 
Uncompensated valvular lesions (seo Broken compensation), 11

Uræma, therapy of, 122

asthusa, 125

cerubral symploms, 120

lumbar puncture. 127

decapsulation of kiduey, 128

diaphoresis, 122

diarrho'a, 125

diet, 127

ingestion of water, 123

heart stinulants, 123

in scarlet fever, $3 \times 3$

irrigations of the colon, 127

adema of the glottis, 120

organotherapy, 129

psychotherapy, 129

purging, 123

venesection with infusion, 124

vomiting, 124

Urate stones (see Nephrolithiasis urica), 130

Urea

in hydrops in broken compensation, 34

in nephrolithiasis urica, 136

in peritonitis, 519

Urethane, 51

Uric acid diathesis, chronic, therapy of, 244

balneotherapy, 248

complications

diabetes, 232, 251

nephritis, 250

obesity, 241, 251

diet, 244

alcohol, 246

carbohydrates, 245

fruit, 245

meat, 245

milk, 246

vegetables, 245

water, 246

exercise, 246

medication, 247

alkalies, 247

alkaline mineral waters, 247

citarin, 248

quinic acid compounds, 248

oymptomatic therapy, 249 ansemis, 250

arteriosclerosis, 250

catarrh, 250

digestive dist urbances, 240

internal gout, 250

Uricedin tophi, 249

in nephrolithiasis, 134

in uric acid diathesis, 247

Urol, 248

Uropherin, 29

Urosin, 248

Urotropin

in lithiasis phosphaturica, 139

in pyelitis and pyelonephritis, 142

in scarlet fever, 581

\section{Valerian}

in broken compensation, 26

in diabetes insipidus, 233

Validol, 430

Vallet's mass, 159

Valvular diseases of the heart. therapy of compensated lesions, I compensated symptomatic, 68

Vaporin, 602 uncompensated lesions, 11

Varicella (see Chicken-pox), 588

Vasogen, 345

Vegetables

digestibility of, 376

in diabetes, 221

in uric acid diathesis, 245

Venesection, 61

accidents, 64

in chlorosis, 63,168

in pneumonia, 62,315
Venescetion

in poisoning. 63

in pulmonary aderna, 62, 320

in threatesed cerebral betsurrtiage and inno Iation. 62

in transfueion of bleod, 63

in true plethora, 62, 183

in uratriak, 62, 124, 862

in venous congention in broken compensen tion, 61

indieations, 61

technic, 63

Venous puncture, 64

Veratrin, 265

Veronal, 30

Vensels, blood, therepy of the (seo Blood rewels, therapy of the), 72

Vigoral, 372

Vinecear, 186

Volvulus, 483

Vomiting

in cancer of the stomech. 452

in gastritis, scute, 43

in uramin, 124

in whooping-cough, 611

nervous, therspy of, 462

\section{Water}

albumin, 374, 832

by rectum, 396

in a normal diet, 210

Waters, mineral (see Mineral waters)

Weights and heights, normal. tables of

$$
\text { Landois's, } 236 \text { 236, } 238
$$

Quetelet's, 235

Weir Mitchell's rest cure (see Rest cure)

Werlhol's disease (sec Purpurs bemorrhecica), 185

Whey cure

in bronchitis, chronic, 303

in diseares of the stomnch and intertines, 390

Whooping-cough, therspy of, 601

anticatarrhal treatment, 610

antiveptic treatment, 601

inhalations, 601

internal antiseptics, 603

antipyrin. 604

antispasmin, 605

aristoquin, 604

basicin, 604

creosote, 605

euquinine. 604

quinine, 603

tussol, 604

local treatment, 602

insuffiation, 602

painting the throat. 603

antispasmodic treatment, 606

manipulation, 610

medication, 606

belladonna, 606

bromoform, 608

chloral hydrate, 607

codeine, $60 \%$

morphine, 607

opium, 607

pasterin, 600

peronin, 607

paychical treatment, 600

complications 61

gastric and intestinal, 611

vomitink. 6is

mechanical results of serere spasms 611

$$
\begin{aligned}
& \text { hemorrhace. } 612 \\
& \text { hermia, } 612 \text { of rectum. } \\
& \text { prolapse of } \\
& 612
\end{aligned}
$$

nervous, 611

sublingual ulcer, 612 
Whooping-cough, complications, nervous laryngeal spasm, 611 respiratory, 611 emphysema, 611

general treatment, 612

diet, 612

hydrotherapy, 612

hygiene, 612

change of scene, 614

clothing, 614

quarantine, 614

Wine

diuretic, 35

huckleberry, 379,471

in diseases of the stomach and intestines, 379

of iron and quinine, 90

Wintergreen, oil of

in gout, 251

in rheumatism, acute articular, 547

Winternitz's use of Leiter's coils, 405, 434

Witch hazel, 356

Xeroform, 588, 597

Yeast

as an intestinal antiseptic, 427

in diabetes, 227

in scurvy, 187

in typhoid fever, 539

Zander's gymnastics

in compensated valvular lesions, 5 in rheumatism, chronic, 264

Zematone, 308

Zine

cyanide, 96

peroxide, 353

valerianate, 96 




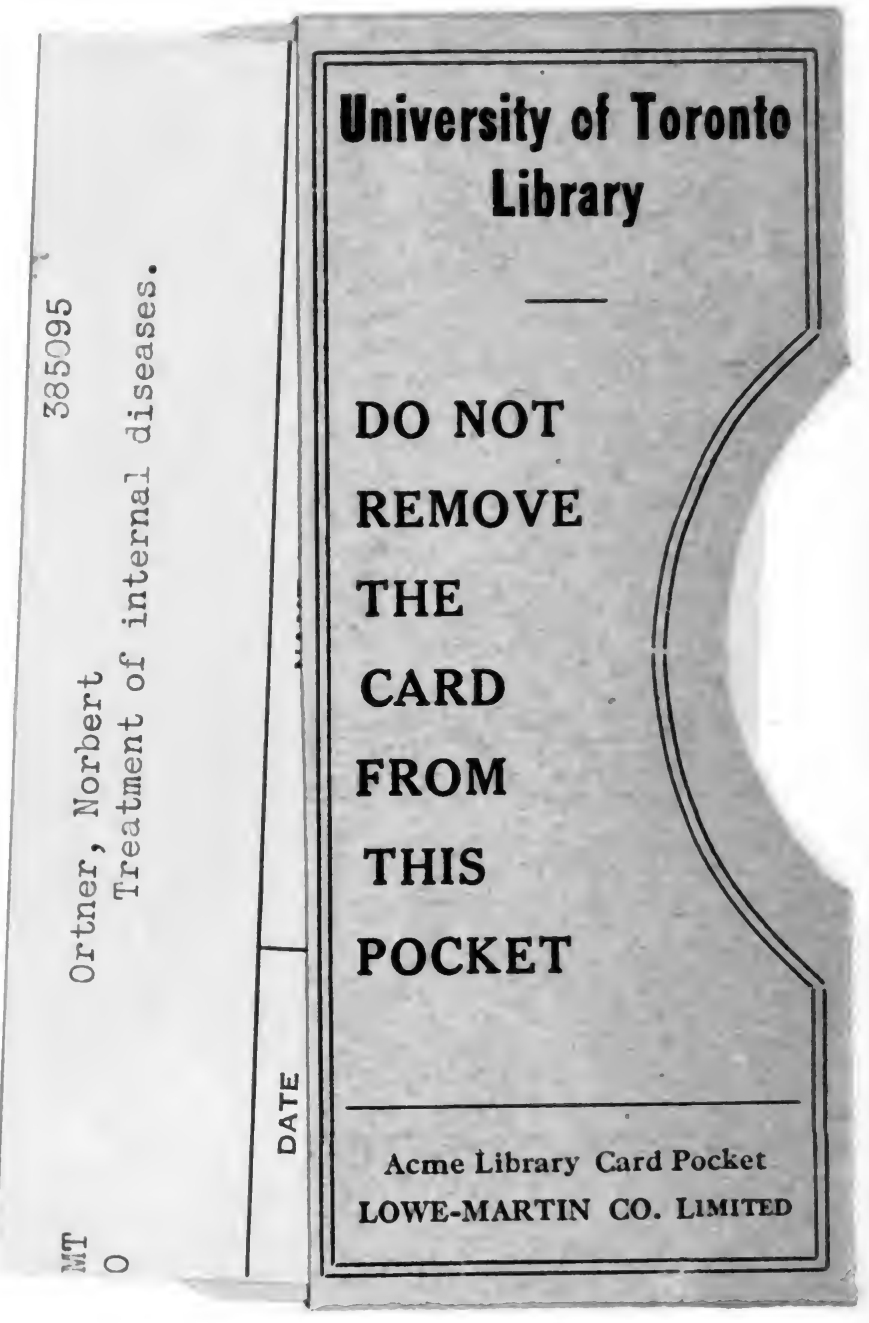



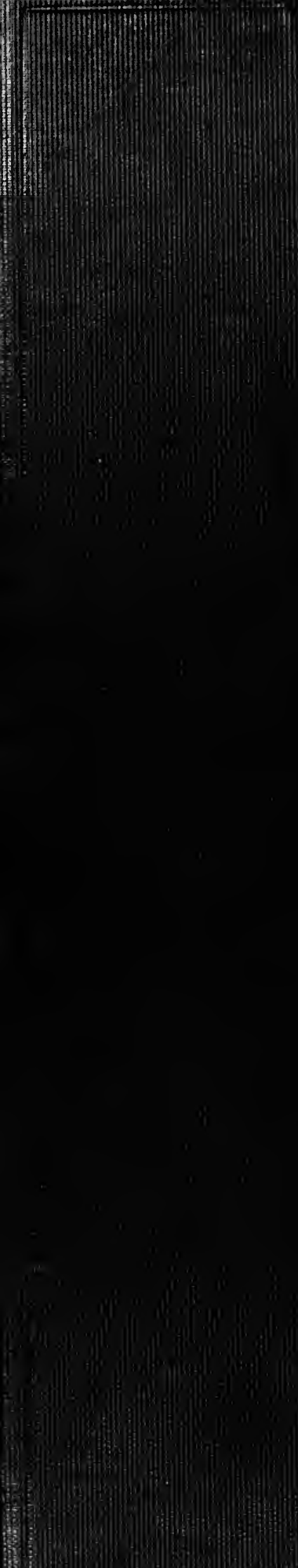

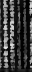

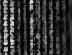

.

(1)
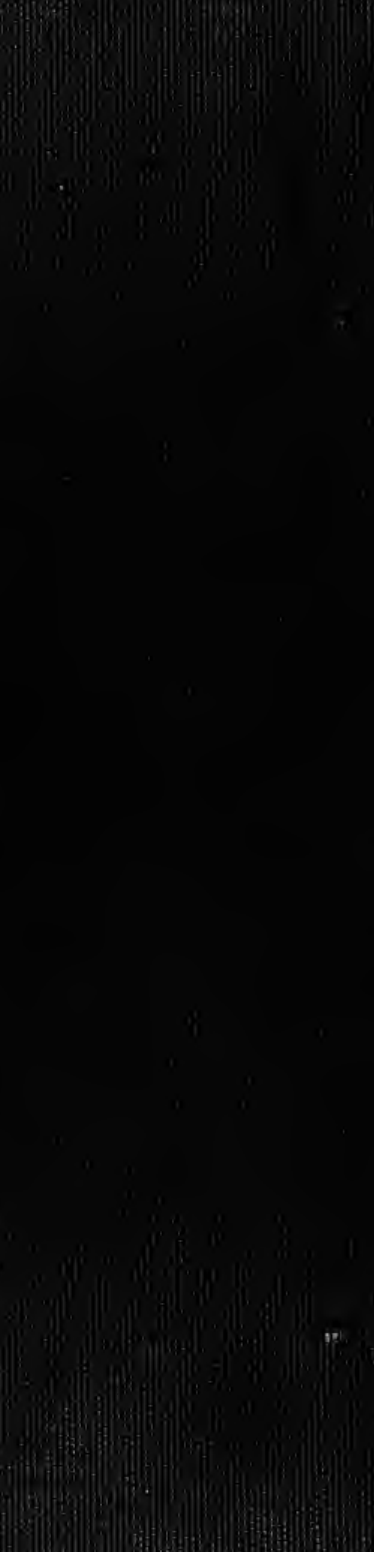